archives-ouvertes

\title{
L'Avenir du contrôle par les pairs dans la publication scientifique face au défis du libre accès
}

\author{
Hachani Samir
}

\section{To cite this version:}

Hachani Samir. L'Avenir du contrôle par les pairs dans la publication scientifique face au défis du libre accès. Sciences de l'information et de la communication. Université d'Alger II, 2013. Français. $<$ tel-00922600>

\section{HAL Id: tel-00922600 \\ https://tel.archives-ouvertes.fr/tel-00922600}

Submitted on 28 Dec 2013

HAL is a multi-disciplinary open access archive for the deposit and dissemination of scientific research documents, whether they are published or not. The documents may come from teaching and research institutions in France or abroad, or from public or private research centers.
L'archive ouverte pluridisciplinaire HAL, est destinée au dépôt et à la diffusion de documents scientifiques de niveau recherche, publiés ou non, émanant des établissements d'enseignement et de recherche français ou étrangers, des laboratoires publics ou privés. 


\begin{abstract}
Université Alger II
Faculté des Sciences Humaines et Sociales Département de Bibliothéconomie et des Sciences Documentaires
\end{abstract}

\title{
L'Avenir du contrôle par les pairs dans la publication scientifique face aux défis du libre accès
}

Thèse présentée pour l'obtention du grade de Docteur en Bibliothéconomie

Par l'étudiant

Samir Hachani

Sous la direction du Professeur

Larbi Icheboudene 


\section{Université Alger II}

Faculté des Sciences Humaines et Sociales Département de Bibliothéconomie et des Sciences Documentaires

\section{L'Avenir du contrôle par les pairs dans la publication scientifique face aux défis du libre accès}

Thèse présentée pour l'obtention du grade de Docteur en Bibliothéconomie

Par l'étudiant

Samir Hachani

Sous la direction du Professeur

Larbi Icheboudene 


\section{Remerciements}

Ce travail n'a pas été, loin s'en faut, dépourvu d'embuches (aussi bien logiques qu'autres ...) mais a été par la même un travail collectif dans le sens où sans l'aide de certaines personnes, il n'aurait jamais vu le jour. Les citer tous et toutes seraient fastidieux et peut être aussi fatigant et chronophage que la thèse en elle-même mais j'essayerai de rendre à tout un chacun (e) son dû

Les quelques années que ce travail a pris de ma vie ont étés parsemés d'événements qui auraient facilement pu me faire abandonner. Ce n'est que grâce à l'inébranlable et indéfectible foi que j'ai en mon Créateur que ce travail a pu être mené à son terme.

De son coté, mon encadreur le Professeur Icheboudene a été tout au long de ce travail un guide et un conseil sans pareil. Il a su me laisser la liberté de développer mes idées et ma manière de travailler tout en m'inculquant et me montrant les rudiments de méthodologie et de sérieux dans le travail. Si ce travail à un quelconque mérite, une grande part lui en revient.

Ce travail investigue, entre autres, la relation d'Internet et son influence sur la Science en devenir et en construction. Il a été pour moi l'occasion de rencontrer un nombre de personnes (la plupart du temps et en premier lieu virtuellement et ensuite et accessoirement en chair et en os). Mr. Hervé Le Crosnier de l'Université de Caen a été, depuis un premier mail que je lui ai adressé le 29 Decembre 2006, un véritable ami et une aide précieuse et désintéressée qui ne s'est jamais départie. Il a toujours répondu à mes questions et surtout a eu le mérite de me guider dans mes premiers pas dans un sujet très nouveau et pas si évident à traiter .Il a su m'orienter et m'aider et ceci n'est pas étonnant d'une personne aux idées progressistes et ouvertes sur le monde, preuve en est ces publications et ses positions sur le sujet. Merci Hervé !!!!

De son coté, Le Professeur Aissa Kadri a été au niveau de l'Université de Paris 8 (Saint Denis) une aide sincère qui ne s'est jamais départie. Combien de fois a t il facilité les démarches administratives dans une administration connue pour être tatillonne et bureaucratique.

Lors de mon passage en France entre 2009 et 2011, j'ai eu l'occasion d'utiliser la BNF Site François Mitterrand. Dans cette imposante bâtisse toute dédiée au savoir, j'ai eu l'immense plaisir de connaitre des professionnels aimant leur métier et aussi le faisant avec abnégation et sérieux. Anne de la salle X, Valérie, Jean Claude et surtout Joachim de la salle T ont étés des compagnons au cours des longues journées passées à la bibliothèque à travailler. Je n'oublierai pas mon ami Slimane Tounsi qui m'a aidé dans la mesure du possible.

Je ne terminerai pas sans citer Nacer Omrane, son épouse Aida dont l'aide m'a été précieuse et incontournable dans la conduite de ce travail. Mon ami et mon frère Rachid Bouarour avec ses connaissances en informatique m'a fait découvrir le sens du partage et la technologie. N'étais ce pour " Teamviewer " et la maitrise de Rachid de cet important outil de partage, nombre de passages de ce travail n'auraient pas pu être rédigés. De son coté, la mise en page, la frappe et surtout l'arrangement physique de ce travail sont l'œuvre incontestable de Mounir Medaci qui m'a fait découvrir les subtilités et les astuces de l'utilisation du traitement de textes. Si ce travail est du point de vue esthétique présentable, l'entier mérite lui en revient. Je n'oublierai pas aussi ses collègues de travail Samir et Hakim qui m'ont aidés au-delà de ce que la relation commerciale impose. 
Enfin last but not least ma petite famille qui a du endurer tout au long de ces années, mon accaparement par le travail et mon manque de disponibilité. Mon épouse Nissa qui a dû supporter mes sautes d'humeur, mon caractère et mes affaires envahissant la maison. Elle a été sans conteste le pilier qui m'a permis de tenir cette dure épreuve qu'elle soit remerciée de tout mon cœur et je ne pourrais jamais lui rendre toute l'aide qu'elle a pu me donner. Comment ne pas aussi remercier mes deux anges Kawthar et Brahim et dont la seule présence était pour moi le plus grand des stimulants et la meilleure des récompenses.

Enfin, je ne cesserais de remercier les membres de ma famille, mes collègues, mes amis et toute personne ayant prononcé ne serait ce qu'un seul mot d'encouragement. Je les remercie du fond de mon cœur et demande pardon à quiconque je n'ai pas cité, ils n'en sont pas moins dans mes pensées et mes remerciements. 
A la mémoire de mes parents, mes frères et sœurs disparus. Et à toute personne œuvrant pour le Savoir avec désintérêt. 


\section{Table des matières}

Liste des tableaux et figures......

Introduction générale.

\section{PREMIERE PARTIE \\ BREVE HISTOIRE DES SUPPORTS DE TRANSMISSION DE LA PENSEE}

Introduction

\section{CHAPITRE I \\ DIFFERENTES ETAPES D'ENREGISTREMENT DE L'INFORMATION}

1-Les différentes écritures

1.1- L'iconographie

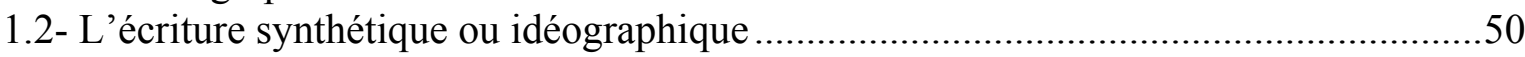

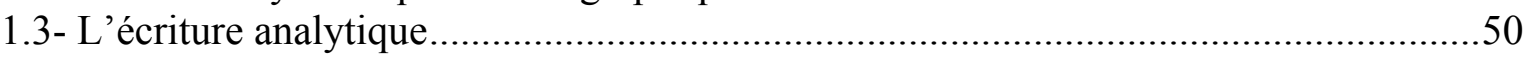

2-L'alphabet

2.1-Définitions

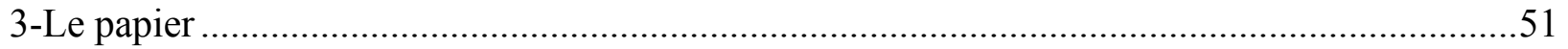

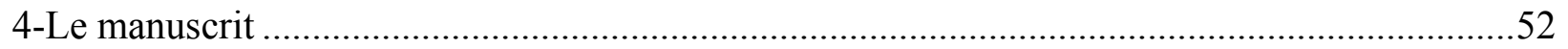

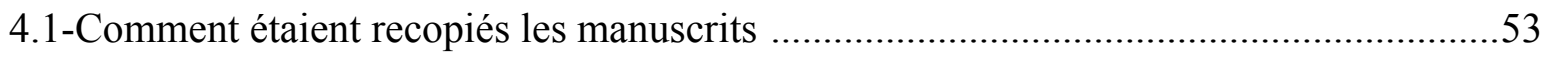

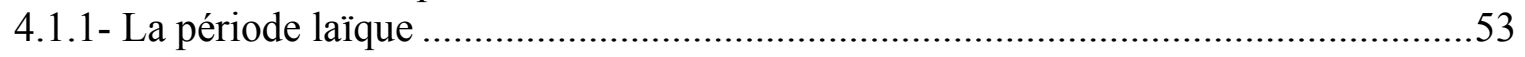

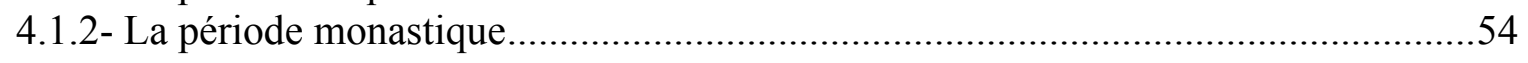

5- L'imprimerie et les éléments constitutifs de son avènement .............................................54

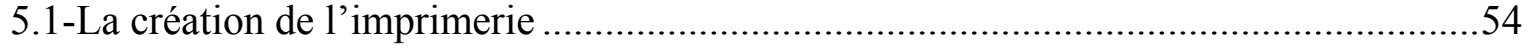

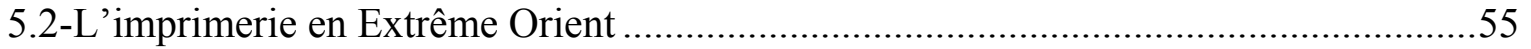

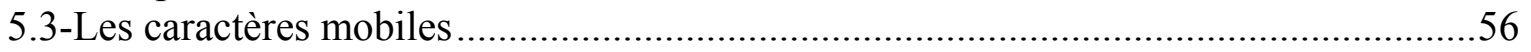

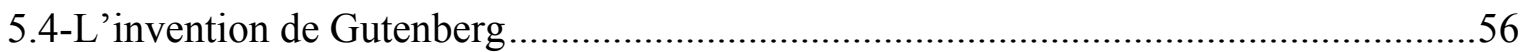

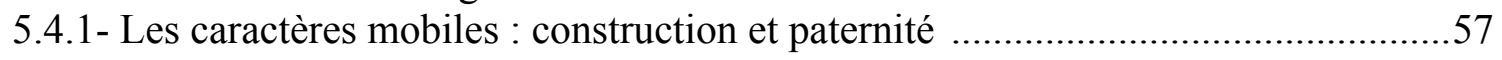

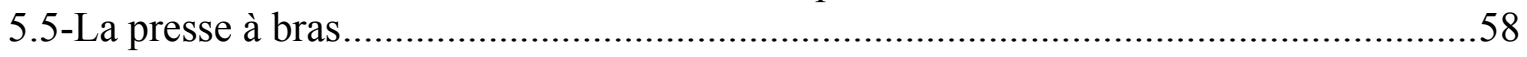




\section{CHAPITRE II \\ COMMUNICATION DE LA SCIENCE PRE AVENEMENT DU PERIODIQUE}

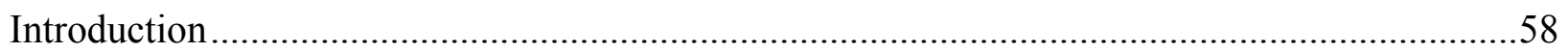

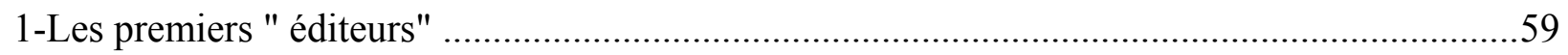

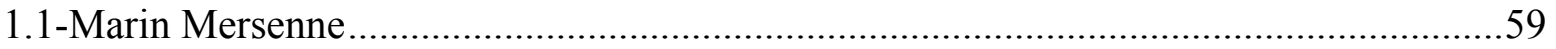

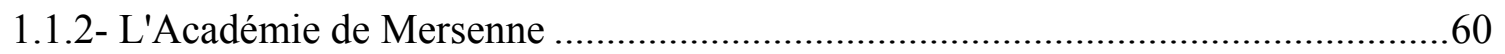

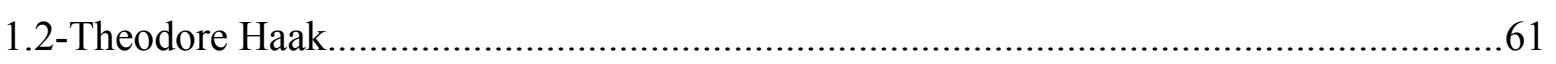

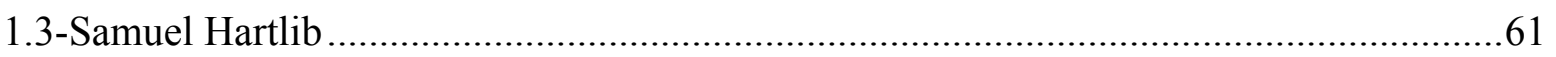

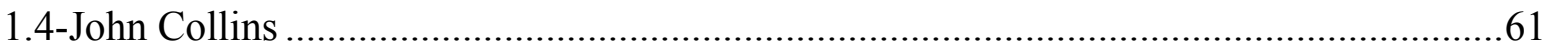

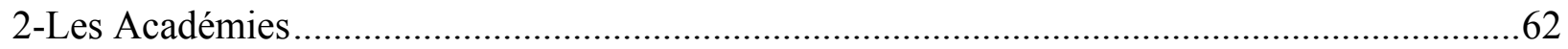

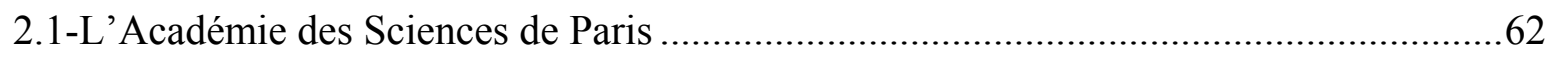

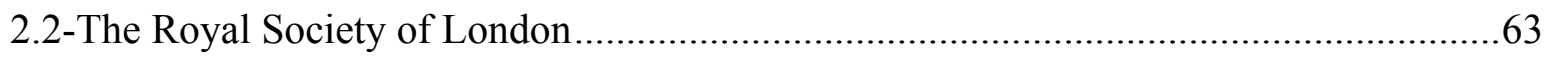

\section{Chapitre III \\ L'AVENEMENT DU PERIODIQUE}

Introduction .66

1-Difficulté de discernement livre - périodique .66

2-Les deux premiers périodiques scientifiques : Le Journal des Sçavans et The Philosophical Transactions

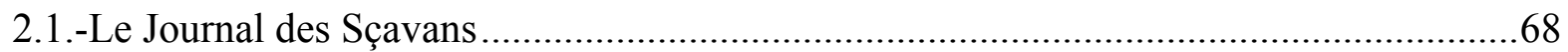

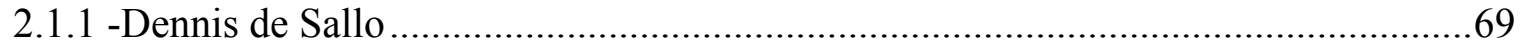

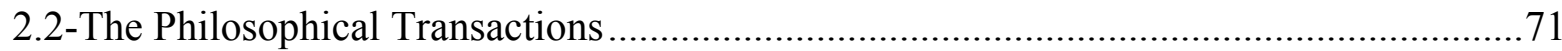

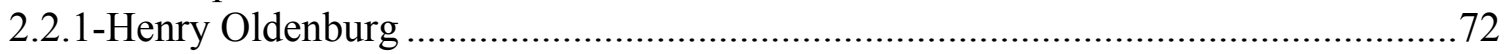

3-Publications périodiques scientifiques précurseurs des journaux scientifiques modernes..................... 74

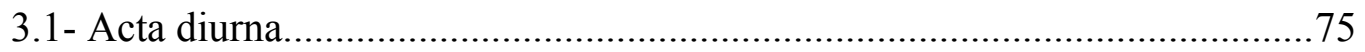

3.2- La Gazette de Pékin.......................................................................... 75

3.3- Relation Aller Fuernemmen und gedenckwuerdigen Historien ....................75

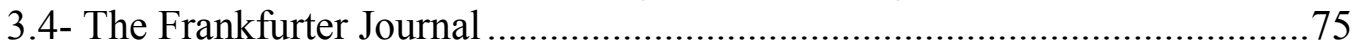

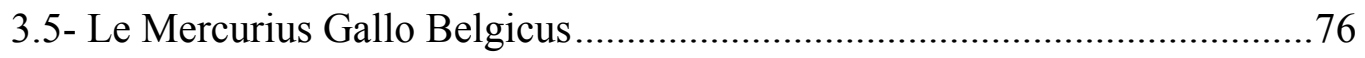

4- Un genre peu connu et peu médiatisé : Le Bureau d'adresse de Théophraste Renaudot...................... 76 
5- Les premiers périodiques scientifiques post Le Journal des Sçavans et The Philosophical Transactions

5.1-Développement des périodiques scientifiques au $17^{\mathrm{e}}, 18^{\mathrm{e}}$ et $19^{\mathrm{e}}$ siècle ..............................79

5.1.1-Deux exemples symptomatiques de l'époque

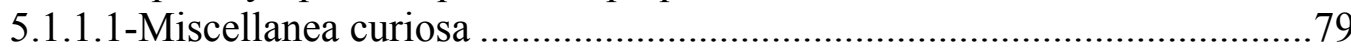

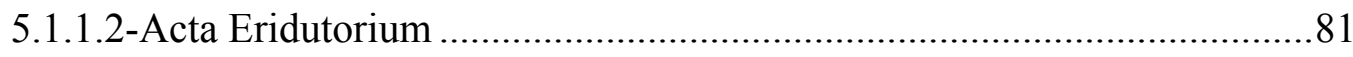

5.1.2- Le Recueil des mémoires et conférences sur les arts et les sciences ......................81

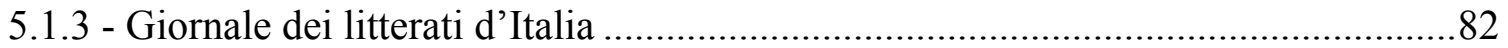

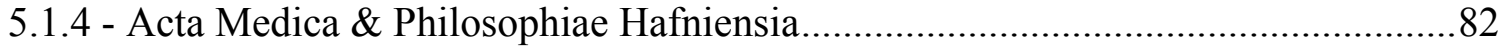

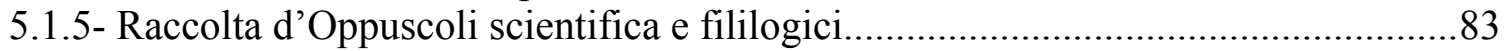

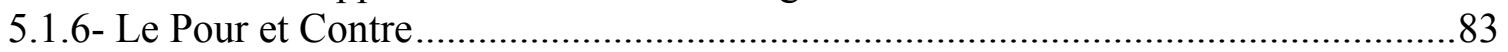

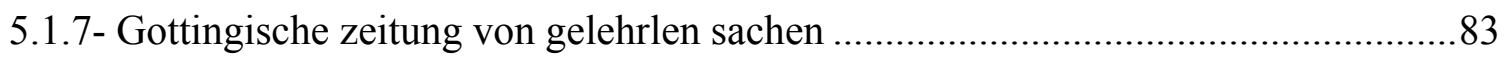

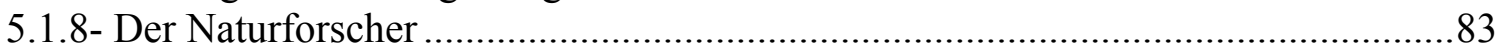

5.1.9- Observations sur la Physique, sur l'Histoire Naturelle et sur les Arts.....................84

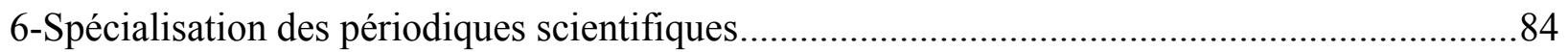

6.1- Les premiers journaux en chimie et en physique.........................................84

6.1.1- Chemische Annalen für die Freunde der Naturlehre, Arzneygelahrtheit,

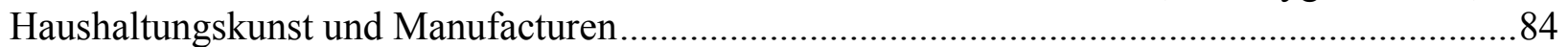

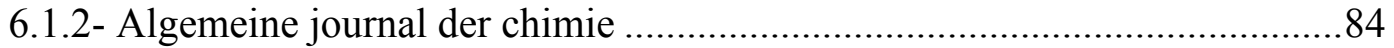

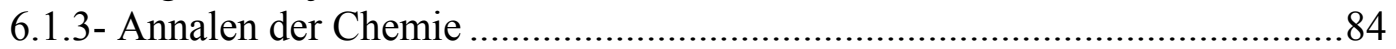

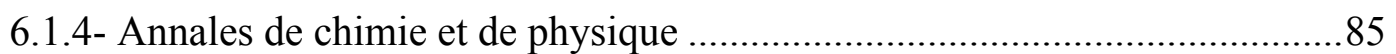

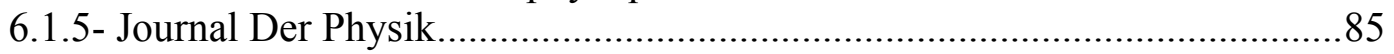

6.2-Philosophical magazine : un magazine philosophique à contenu scientifique 85

6.3-The botanical magazine : une révolution dans les illustrations .......................86

6.4- Les sociétés savantes : sponsors des journaux scientifiques

6.4.1 - The Proceedings of the Royal Physical Society of Edinburg ...................87

6.4.2- The Proceedings of the London Mathematical Society in London ................87

6.4.3- The Proceedings of the Physical Society of London ................................8 88

7-Exemple de prototype de périodique scientifique contrôlé par les pairs moderne :

Nature

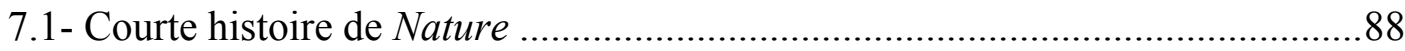

7.1.1-Magazines scientifiques et journaux ayant précédés Nature .................88

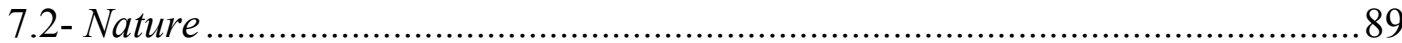

7.3- Comment le magazine Nature fut créé ..........................................................90

7.4- Les éditeurs

7.4.1 - Norman Lockyer..................................................................... 90

7.4.2- Richard Gregory ......................................................................90

7.4.3- A. J. V. Gale et L. J. F. Brimble ......................................................91

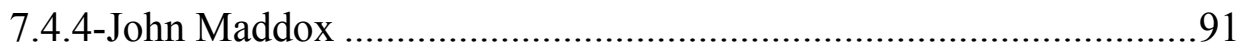

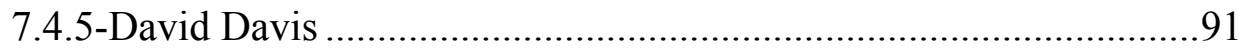

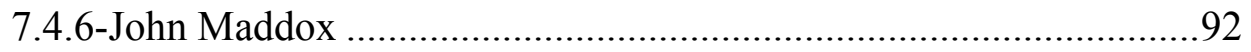

7.4.7-Philip Campbell .........................................................................992

7.5- L'expansion de Nature et son développement ............................................92 


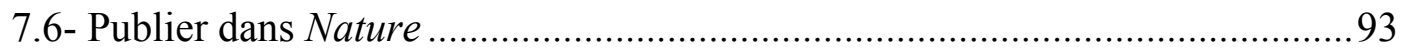

7.6.1- Recherches qui ont fait date.........................................................93

7.6.2- Anomalies du contrôle par les pairs..............................................94

7.7.- La publication dans Nature et ses journaux assimilés .................................95

8-Exemple de prototype de périodique scientifique contrôlé par les pairs moderne :Science .....96

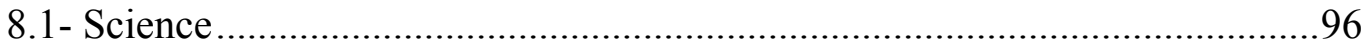

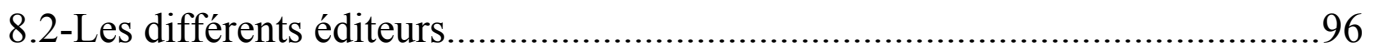

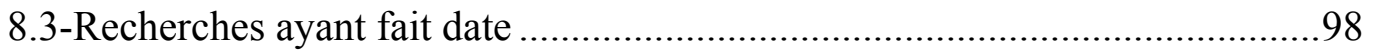

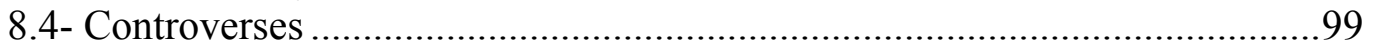

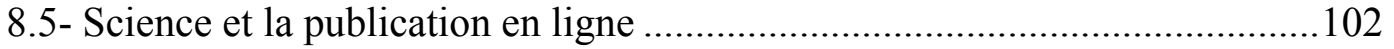

\section{Chapitre V \\ DE QUELQUES EXEMPLES DE DESCRIPTION, TYPOLOGIE \\ ET CARACTERISATION DU PERIODIQUE SCIENTIFIQUE A TRAVERS LES AGES}

Introduction .102

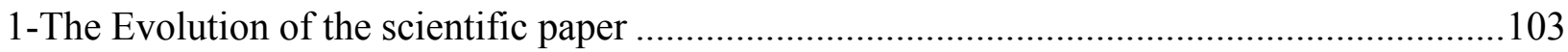

1.1-Les origines et les années de formation (gestation) 1665-1765 …...............103

1.2-Spécialisation et professionnalisation (1765-1865) ..................................105

1.3- Développement de l'article scientifique moderne (1865-1965) ..................107

1.4- Hyperspécialisation et informatisation (1965- ?) .......................................109

2-The Scientific article: from Galileo's new science to the human genome ..............................111

2.1-Précurseurs et rivaux

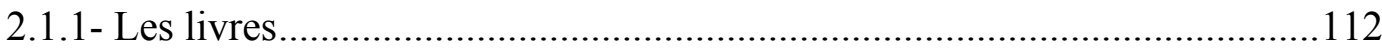

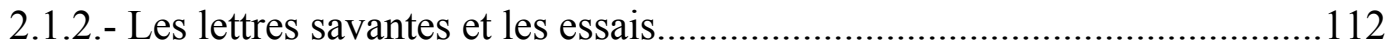

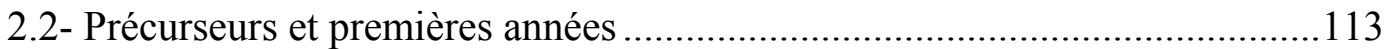

2.2.1- Le premier périodique anglais et la Société Royale de Londres

(Royal Society.

2.2.2- Les premiers périodiques français et l'Académie Royale .........................114

2.2.3- Les premiers journaux d'autres pays ........................................................115

2.2.4- Premier pas de la communication scientifique en Amérique ......................115

2.2.5- La mission de collecte des faits des débuts de la science ............................116

2.3-Spécialisation et maturité accrue

2.3.1- Origine de la littérature spécialisée.......................................................117

2.3.2-Les différents types d'articles scientifiques..........................................117

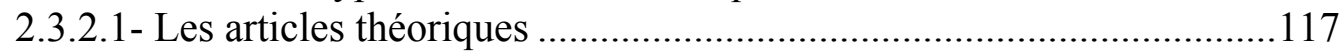

2.3.2.2- Les articles expérimentaux .............................................................117

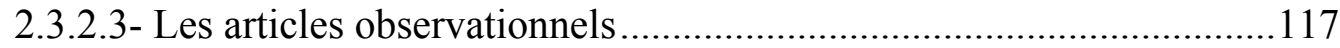

2.3.2.4- Les articles méthodologiques .......................................................... 118

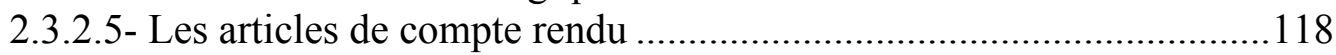

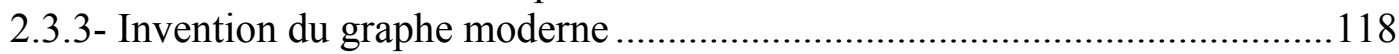

2.3.4- Invention de la table périodique des éléments .........................................118

2.3.5- Les femmes et les minorités en science ...................................................119 
2.4- Communications choisies de quelques ingénieux scientifiques

2.4.1- Newton et l'optique

2.4.2- Darwin et la théorie de l'évolution.....

121

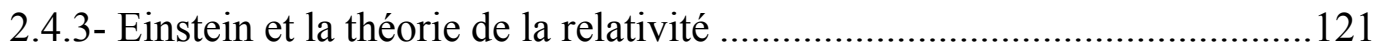

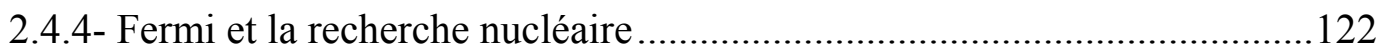

\section{5- Curiosités diverses}

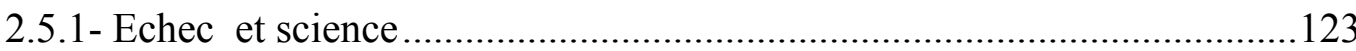

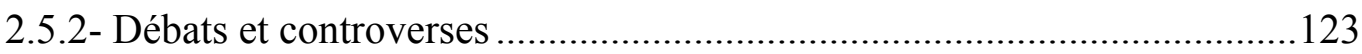

2.5.3- Curiosités littéraires : pour le meilleur et pour le pire ? ..........................124

2.6-Conclusion et que nous réserve le futur

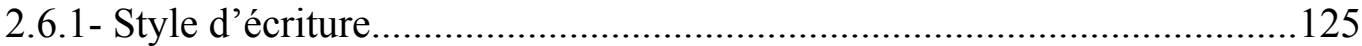

2.6.2- Caractéristiques de présentation ...........................................................126

2.6.3- Présentations visuelles ....................................................................126

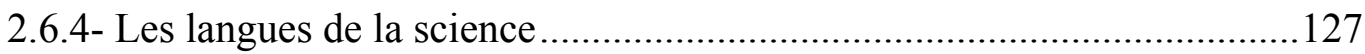

2.6.5- Que nous réserve le futur ? ...............................................................127

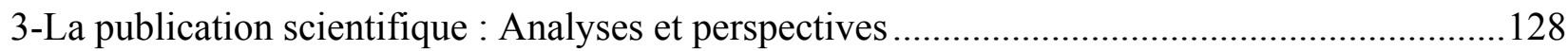

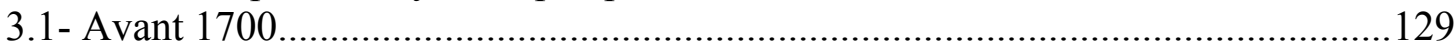

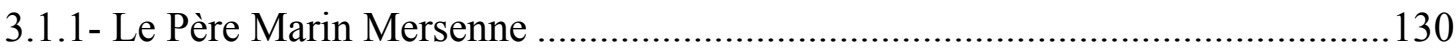

3.1.2- Henry Oldenburg ....................................................................................... 131

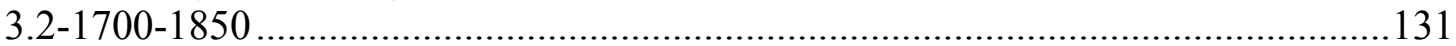

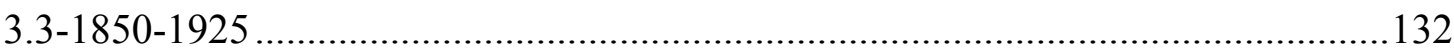

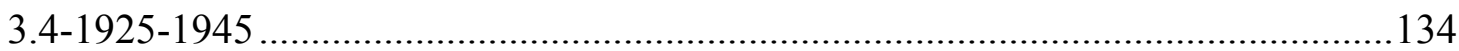

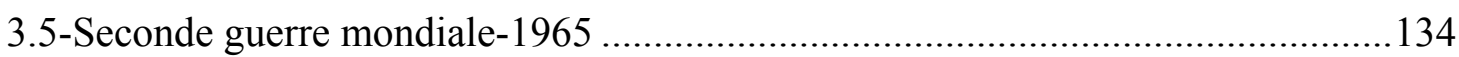

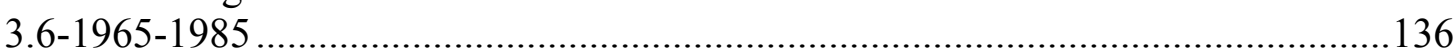

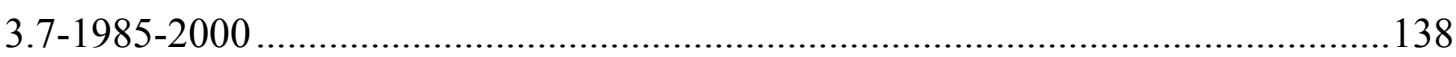

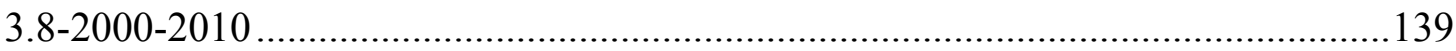

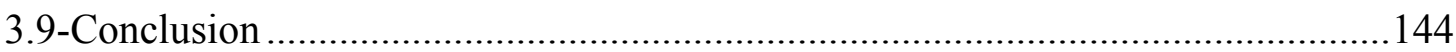

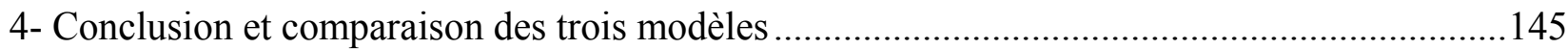

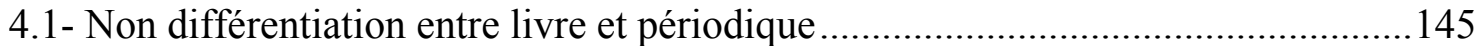

4.2- Développement de la fonction archive du périodique ..........................................145

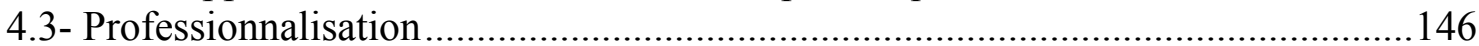

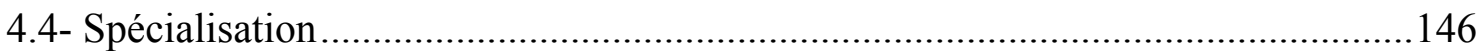

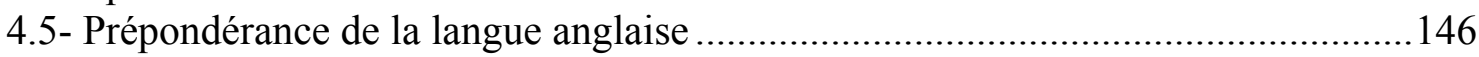

4.6- Augmentation exponentielle de la masse de l'information ....................................147

5- Courte présentation des développements post deuxième guerre mondiale ............................. 147

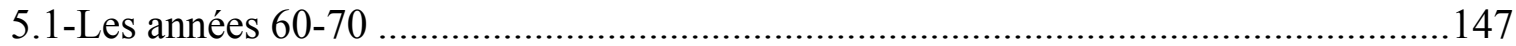

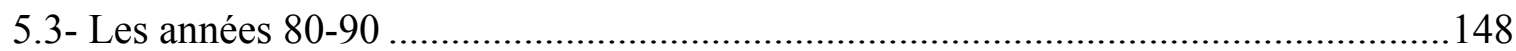

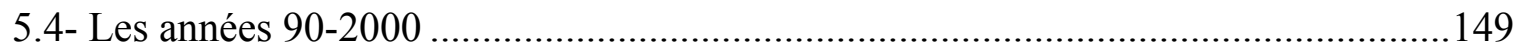

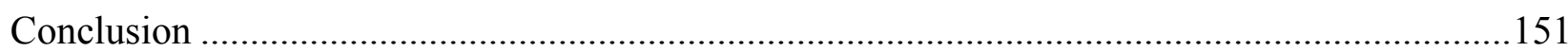

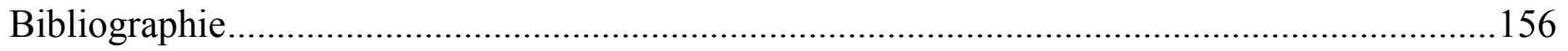




\section{DEUXIEME PARTIE EVOLUTION ET OUTILS DU LIBRE ACCES}

Introduction 160

\section{Chapitre I \\ CONDITIONS CONSTITUTIVES DE L'AVENEMENT DU LIBRE ACCES}

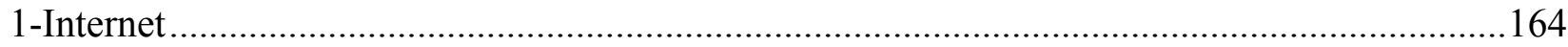

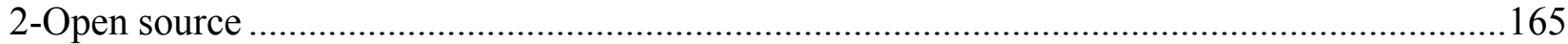

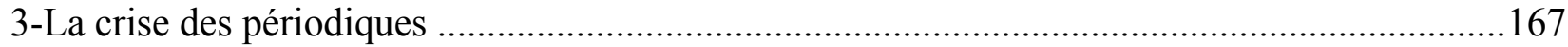

4-Les Appels fondateurs du libre accès :

4.1-L'Appel de Budapest.... 169

4.2-La Déclaration de Béthesda ...................................................................171

4.3-La déclaration de Berlin.............................................................................173

5-Deux pays anglo saxons pionniers du libre accès

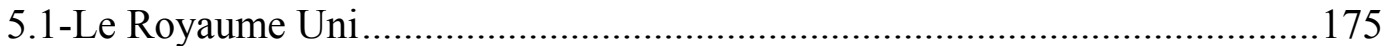

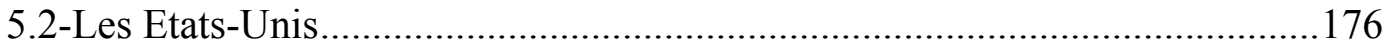

6-Définitions de certains concepts du libre accès

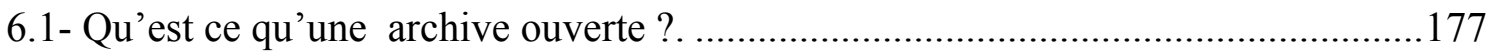

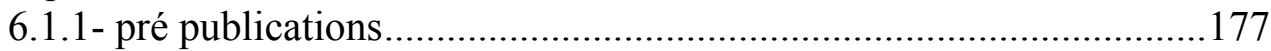

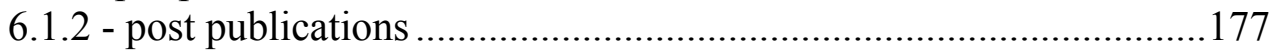

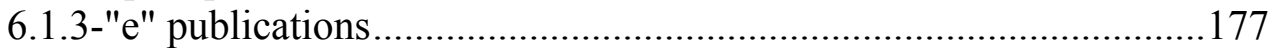

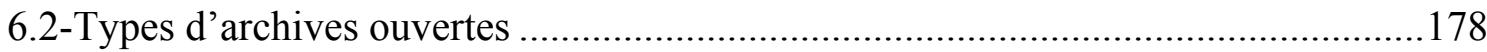

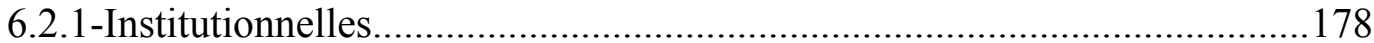

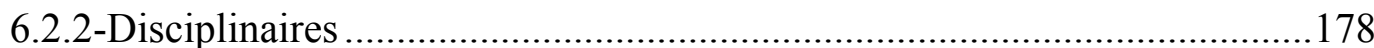

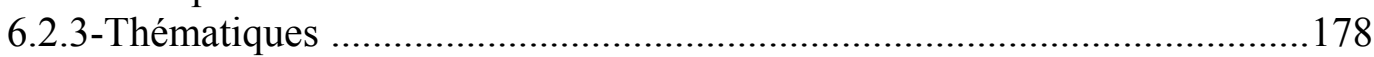

7-Premières et plus importantes archives :

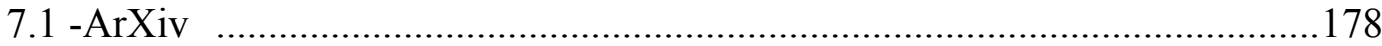

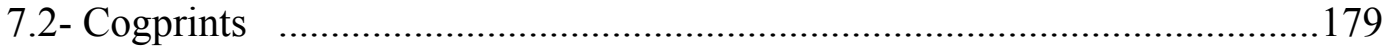

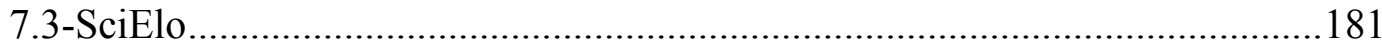

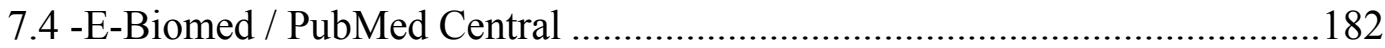

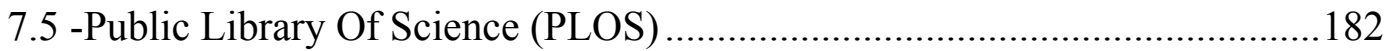

7.6 -Biomed Central........................................................................................... 183

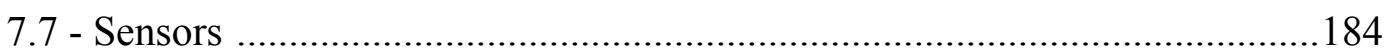




\section{Chapitre II \\ DE QUELQUES EXEMPLES D'ENTREPOTS D'ARCHIVES OUVERTES}

Introduction .185

\section{The Core Metalist of Open Access EPRINTS Archives}

Présentation 185

1.1-Listes générales de publications électroniques en libre accés (texte intégral)

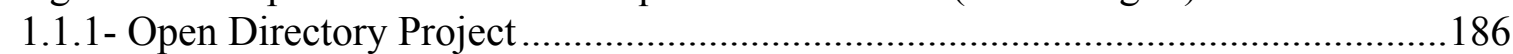

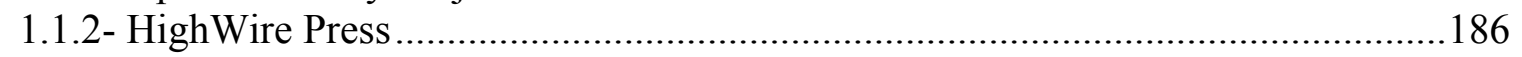

1.1.3-University of Maryland Libraries, Virtual Technical Reports Center:

EPrints, Preprints, \& Technical Reports on the Web. 186

1.1.4- University of Virginia Science and Engineering Libraries, Preprint

Servers and Databases. .186

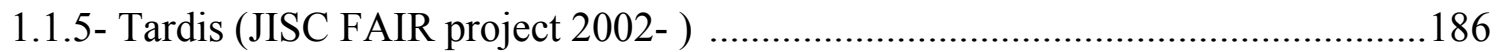

1.1.6- Aardvark, Asian Resources for Libraries ............................................................. 186

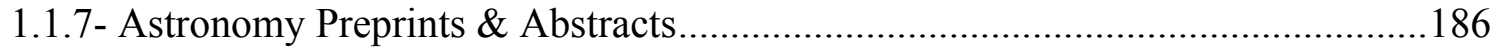

1.2.1-Les archives répondant aux critères OAI

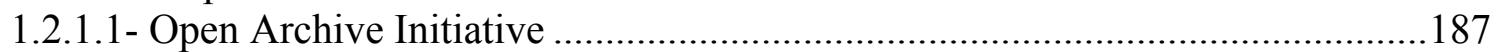

1.2.1.2- Open Archives Forum............................................................................... 187

1.2.2- Liste des archives dont les services sont basés sur l'O.A.I ................................................. 187

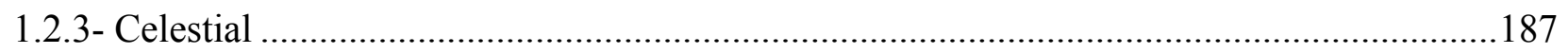

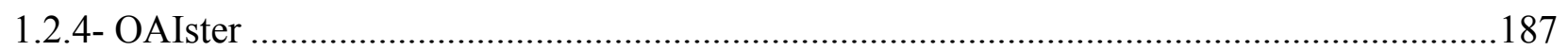

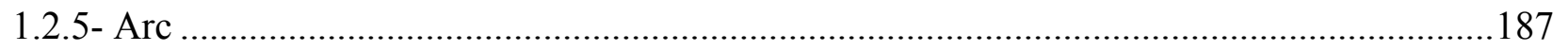

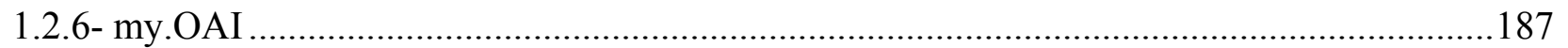

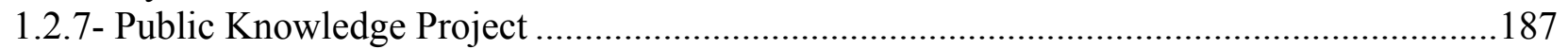

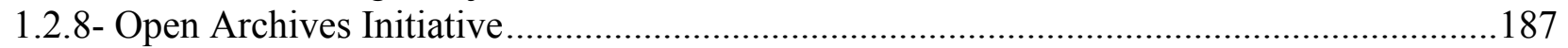

1.3-Liste des archive institutionnelles

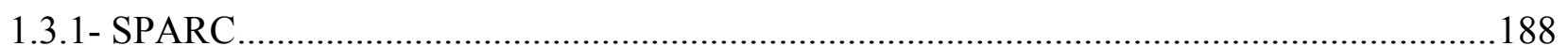

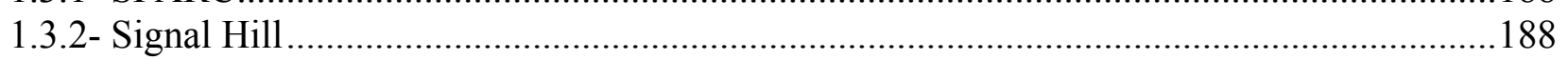

1.3.3.- Les archives institutionnelles

1.3.3.1-University of California, California Digital Library e Scholarship Repository .. 188

1.3.3.2-Caltech, Collection of Open Digital Archives (CODA) ....................................188

1.3.3.3-US Department of Energy (DOE), the Information Bridge................................. 188

1.4-Les archives "Eprints.org"

1.4.1- GNU Eprints

1.5-Les portails (Indexes, recherche unifiée et feuilletage des sites couverts)

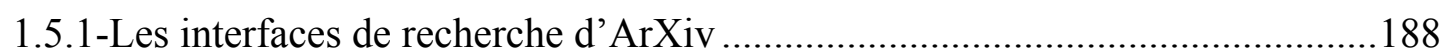

1.5.1.1- Front for the Mathematics ArXiv, interface alternative d'ArXiv ...............188

1.5.1.2- NASA, Astrophysics Data System (ADS ............................................. 188

1.5.1.3- NASA ADS Harvard-Smithsonian Center for Astrophysics

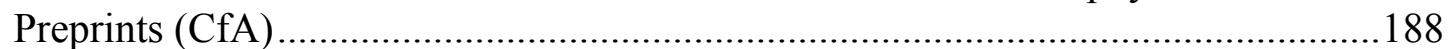

1.5.1.4- The Stanford Linear Accelerator Center (SLAC), SPIRES HEP .................188 


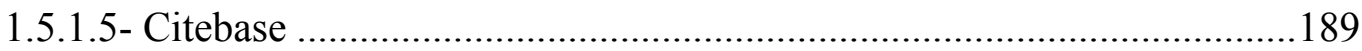

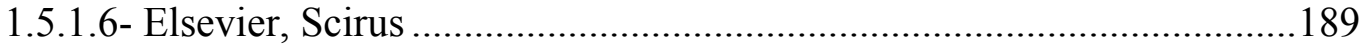

1.5.1.7- CERN Document Server (CDS) …............................................ 189

1.5.1.8- PhysDoc - Physics Documents Worldwide ...........................................189

1.5.1.9- MPRESS, the Mathematics Preprint Search System .............................189

1.5.1.10- US Department of Energy (DOE), PrePRINT Network......................189

1.5.1.11- NTRS, NASA Technical Reports Server ........................................189

\subsection{2- Portails d'archives décentralisées}

1.5.2.1-Networked Computer Science Technical Reference Library (NCSTRL)189

1.5.2.2- Networked Digital Library Of Theses And Dissertations (NDLTD) ......189

1.5.2.3- Open Language Archives Community (OLAC) .....................................190

1.5.3- L'exemple de " The Economics network (RePEc) "

1.5.3.1 - IDEAS (Internet Documents in Economics Access Service) ..................................190

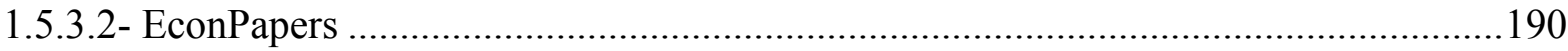

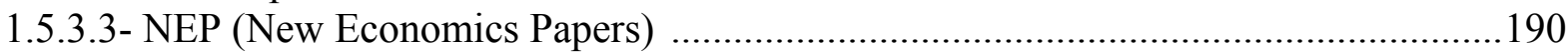

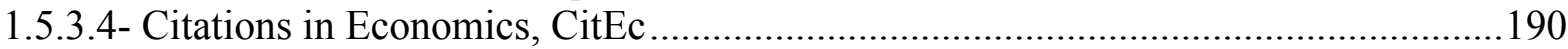

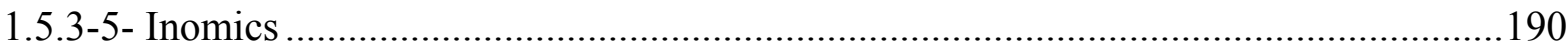

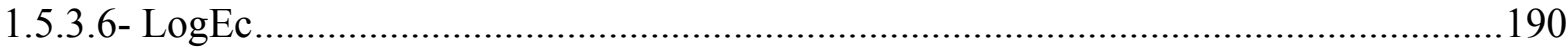

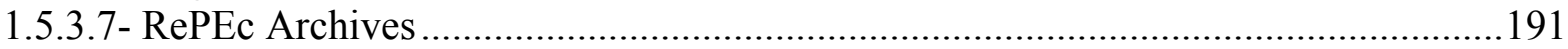

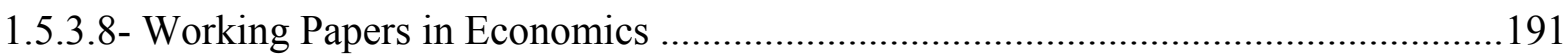

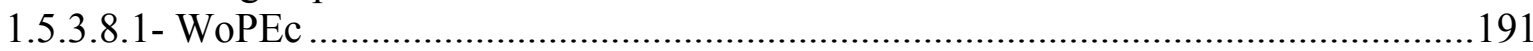

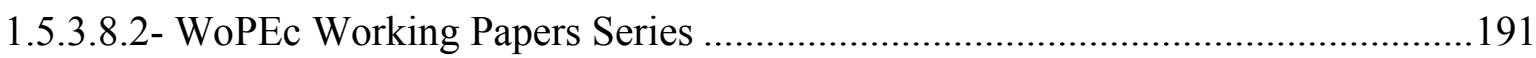

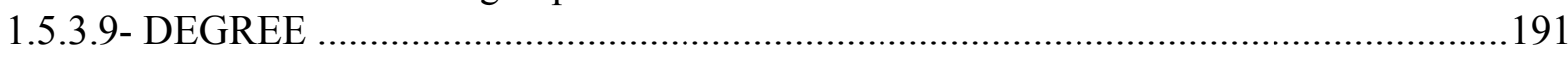

1.5.3.9.1 - International Monetary Fund (IMF) 191

1.5.3.9.2- EconWPA (USA), Economics Working Paper Archive......................................191

1.5.3.9.3-Cowles Foundation, Yale University, Discussion Papers ....................................191

1.5.3.9.4-Center for Mathematical Studies in Economics and Management

Science,Northwestern University (USA), Discussion Papers.....................................................191

1.5.3.9.5- Caltech Division of Social Sciences (USA), Working Papers On-Line ..............191

1.5.3.9.6 - UCLA Department of Economics (USA), Working Papers ................................191

1.5.3.9.7- S-WoPEc, Scandinavian Working Papers in Economics ....................................191

1.5.3.10- Archives sur le modèle RePEc autres qu'économie

1.5.3.10.1 - Documents in Information Science (DoIS) ...................................................191

1.5.3.10.2- R.C.L.I.S. (Research in Computing, Library and Information Science) .........191

1.6-Les archives des journaux en libre accès

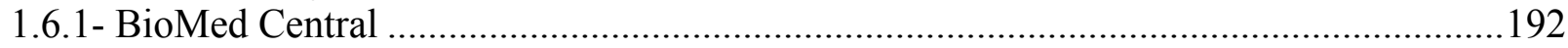

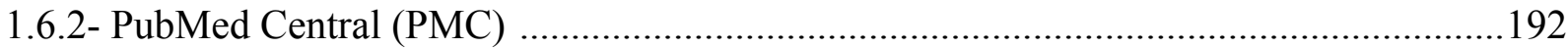

1.6.3- HighWire Press Free Online Full-text Articles .............................................................192

1.6.4- Free Online Full-text Articles (Earth's Largest Free Full-Text Science Archives) ........192

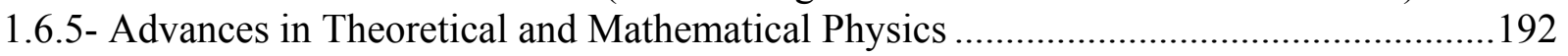

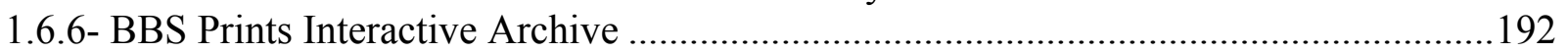

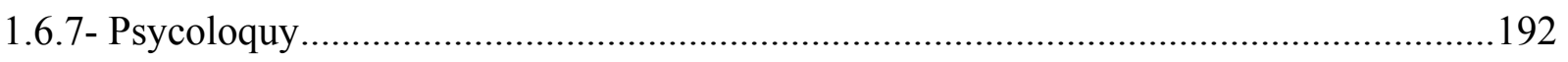

1.7-Les archives disciplinaires

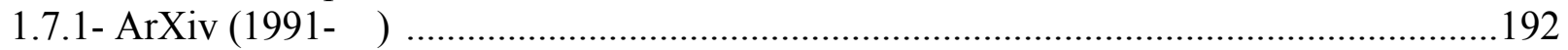




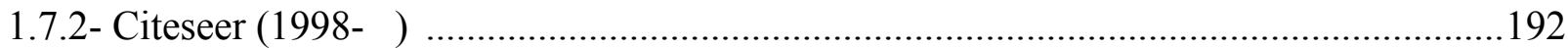

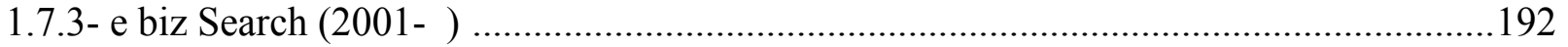

1.7.4- Mathématiques

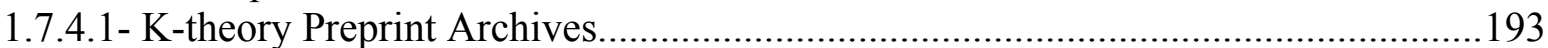

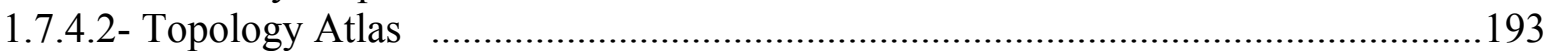

1.7.4.3- Algebraic Number Theory Archives .................................................................. 193

1.7.4.4- Mathematical Physics Preprint Archive, mp_arc ................................................ 193

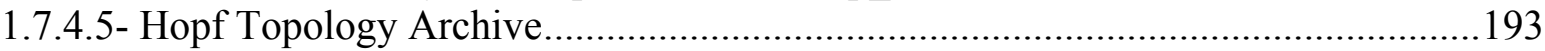

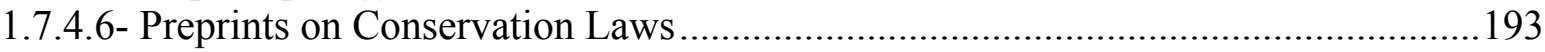

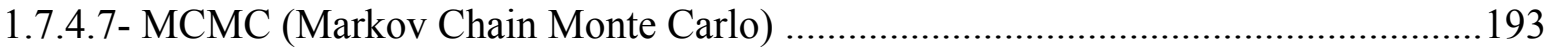

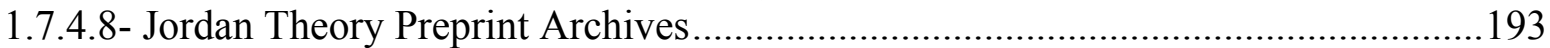

1.7.4.9- Groups, Representations and Cohomology Preprint Archive.................................. 193

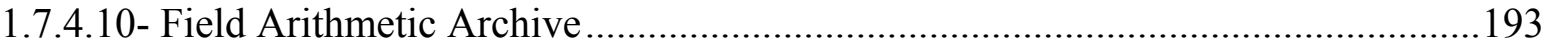

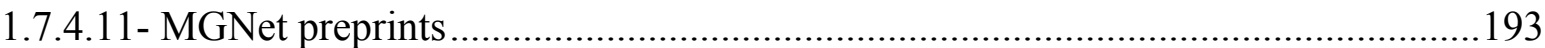

1.7.5- Sciences cognitives

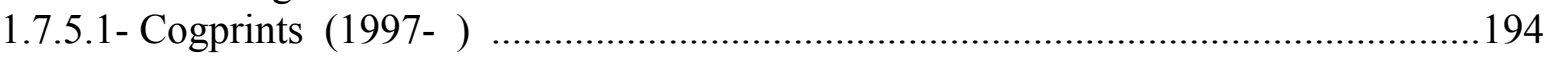

1.7.6- Libray and information sciences

1.7.6.1- E-LIS, E-Prints in Library and Information Science ........................................... 194

1.7.6.2- DList, Digital Library of Information Science and Technology

(October 2002- )

1.7.7- Archives de prépublication encouragées par les éditeurs (auteurs auto archivant)

1.7.7.1- NetPrints Clinical Medicine and Health Research (December 1999- ) ................194

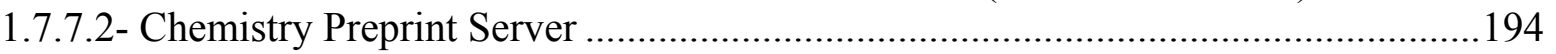

1.7.7.3- Computer Science Preprint Server (November 2001- ) .......................................194

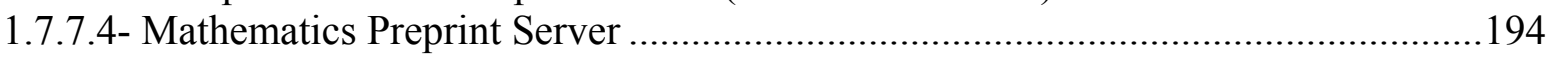

1.7.8- Autres archives disciplinaires

1.7.8.1- HTP Prints, the History \& Theory of Psychology Eprint Archive .............................194

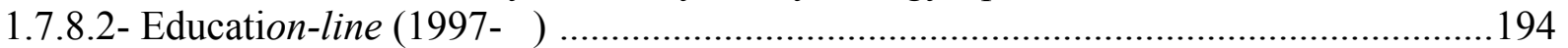

1.7.8.3- Social Science Research Network (SSRN), Social Science Electronic Publishing...195

1.7.8.4-ArchiveSIC (Archive ouverte en Sciences de l'Information et de la Communication)...195

1.7.8.5- Electronic Colloquium on Computational Complexity (1994- ） ...........................195

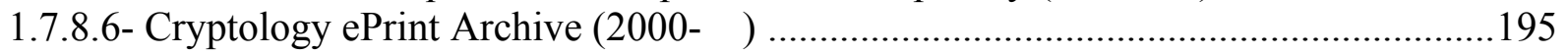

1.7.8.7- The Digital Library of the Commons (DLC), Indiana University ............................. 195

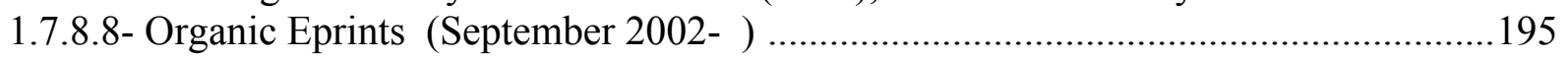

1.7.8.9- University of California International and Area Studies (UCIAS) Digital Collection

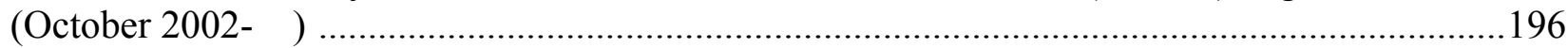

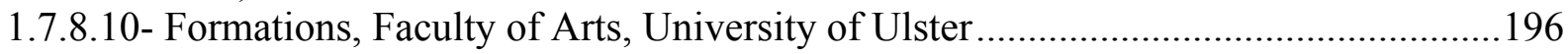

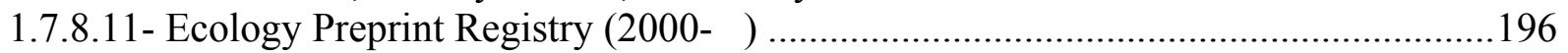

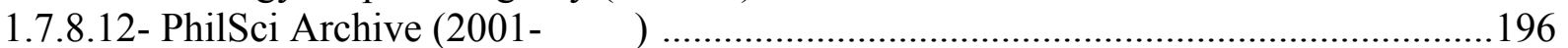

2. Open DOAR et Open ROAR : Deux outils incontournables des entrepôts d'archives

Introduction

\section{1- The Open DOAR (Directory Of Open Access Repositories )}

Introduction 196 


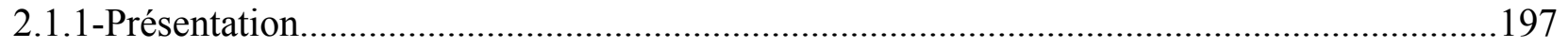

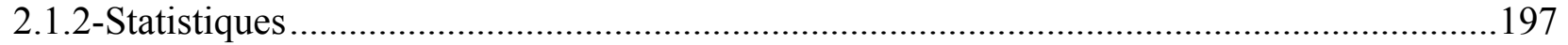

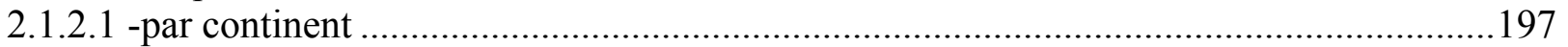

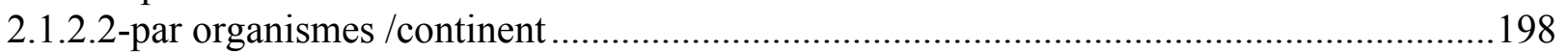

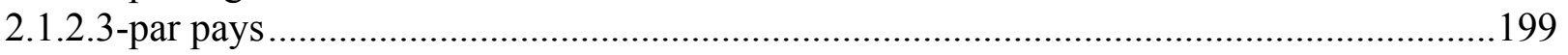

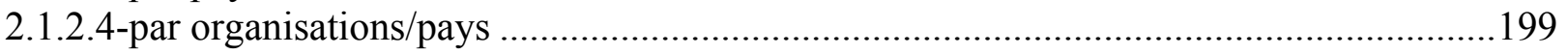

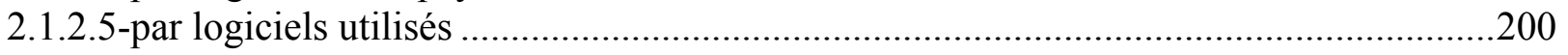

2.1.2.6-par type d'entrepôts en libre accès.........................................................................201

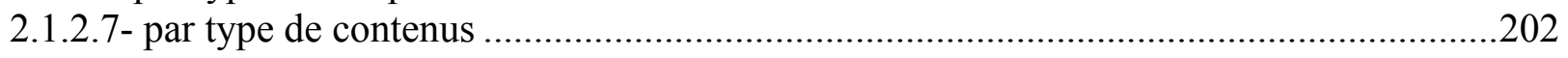

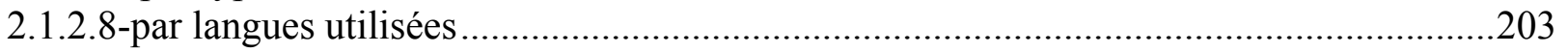

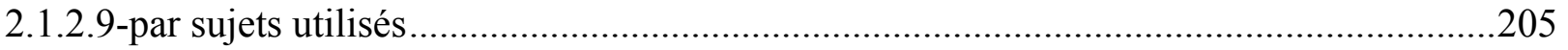

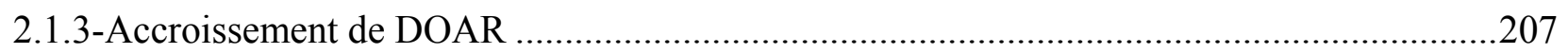

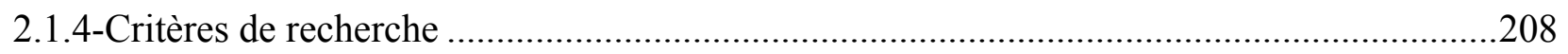

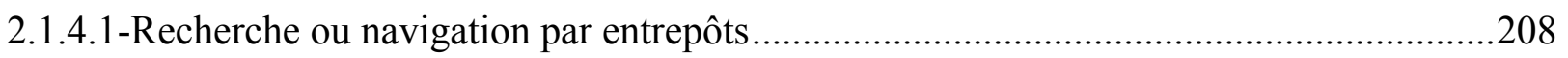

2.1.4.2-Recherche par contenus d'entrepôts ......................................................................208

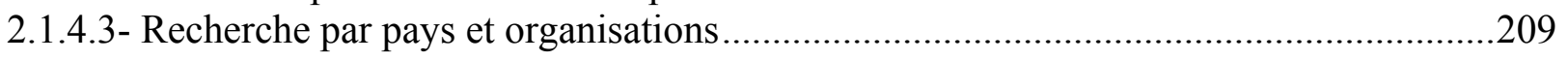

\section{2- Registry Of Open Access Repositories (ROAR)}

2.2.1-Présentation

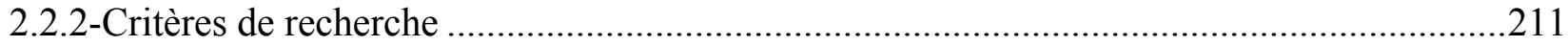

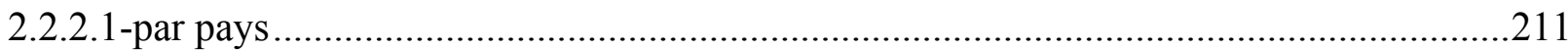

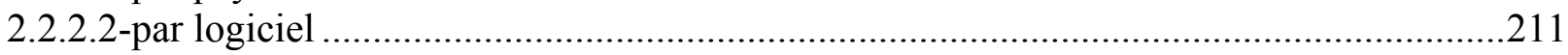

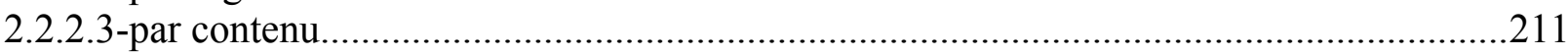

2.2.3-Filtrage :

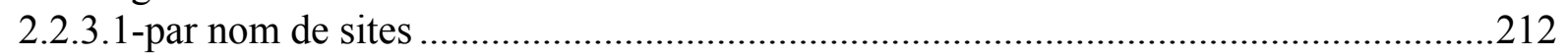

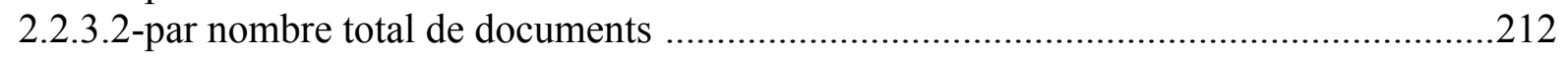

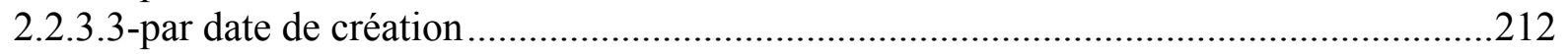

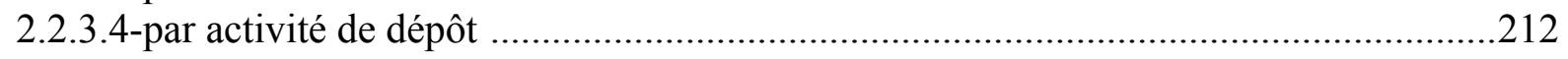

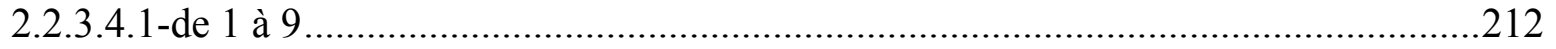

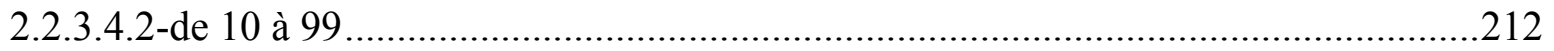

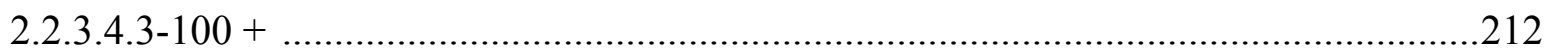

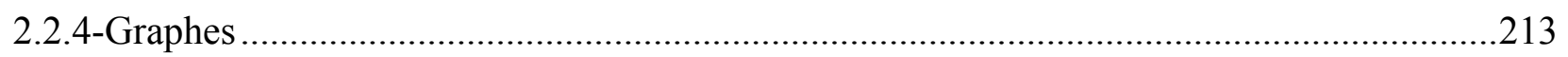

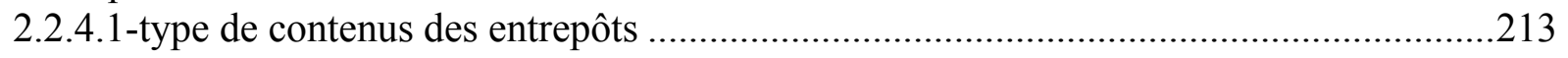

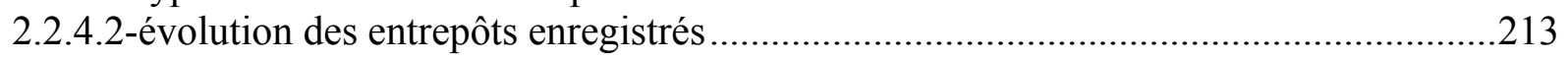

2.2.5-Conclusion commune à l'examen des trois entrepôts....................................................214

\section{Chapitre III \\ LES JOURNAUX ELECTRONIQUES EN LIBRE ACCES SUR INTERNET}

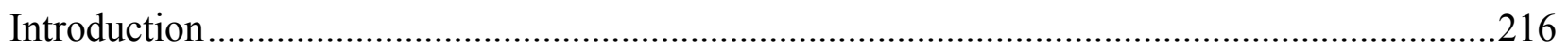

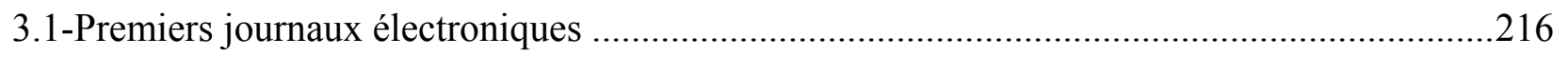

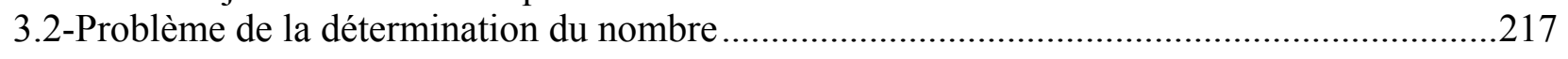




\section{3-The DOAJ (Directory of Open Access Journals)}

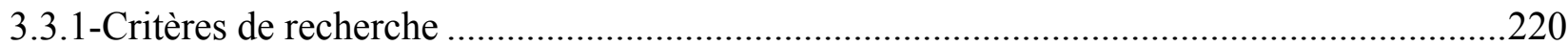

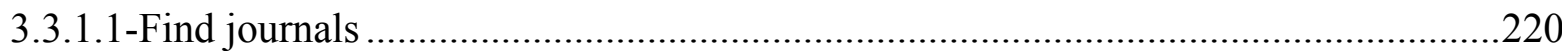

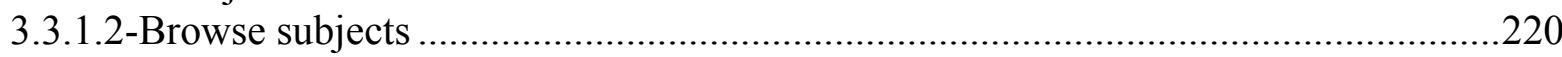

3.3.1.2.1-Agriculture and Food Sciences (Agriculture et sciences nutritives) ...............220

3.3.1.2.2-Arts and Architecture (Arts et Architecture) ...............................................220

3.3.1.2.3-Biology and Life Sciences (Biologie et sciences de la vie) .............................220

3.3.1.2.4-Business and Economics (Commerce et Economie) .....................................220

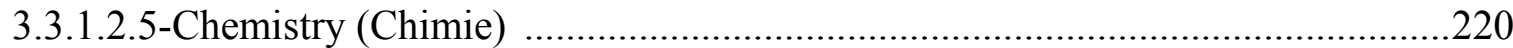

3.3.1.2.6-Earth and Environmental Sciences (Terre et sciences environnementales)......221

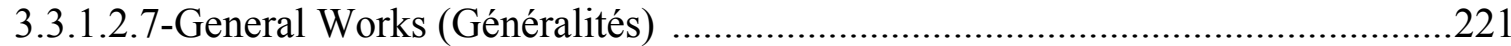

3.3.1.2.8-Health Sciences (Sciences médicales) ..........................................................22

3.3.1.2.9-History and Archaeology (Histoire et Archéologie) ......................................221

3.3.1.2.10-Languages and Literatures (Langages et Littératures) ................................221

3.3.1.2.11-Law and Political Science (Droit et Sciences politiques) ..............................221

3.3.1.2.12-Mathematics and Statistics (Mathématiques et Statistiques) ..........................221

3.3.1.2.13-Philosophy and Religion (Philosophie et Religion) ...................................221

3.3.1.2.14-Physics and Astronomy (Sciences Physiques et Astronomie) .......................221

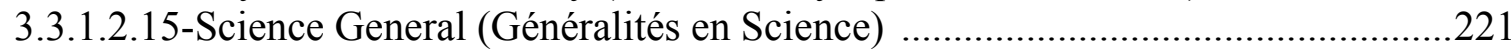

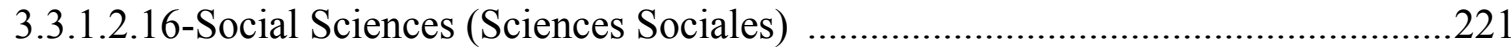

3.3.1.2.17-Technology and Engineering (Technologie et Sciences de l'ingénieur) ........221

3.3.2- Différentes fonctionnalités offertes par le site ..........................................................222

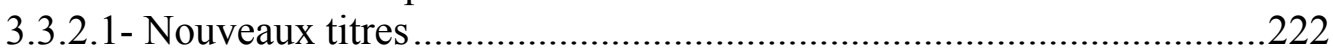

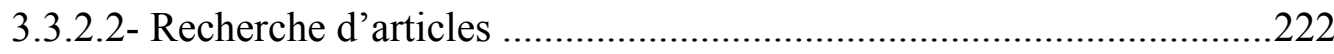

3.3.2.3- Suggérer un journal................................................................222

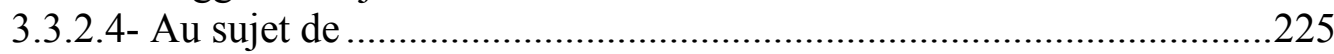

3.3.2.5-Questions fréquemment posées Information ...............................225

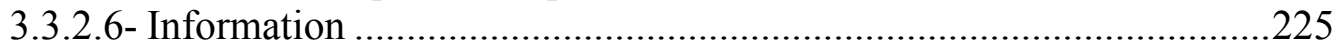

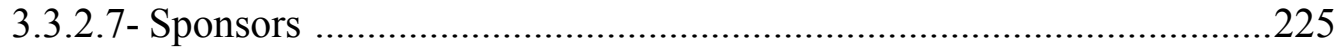

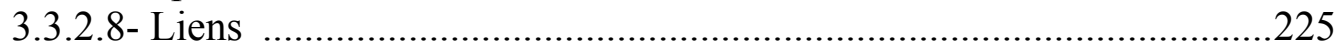

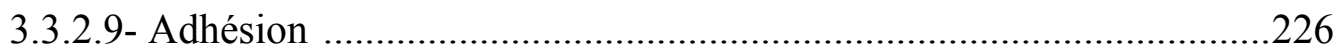

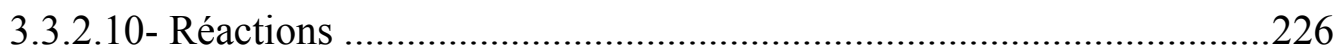

3.3.2.11 - Pour les éditeurs de journaux ..................................................226

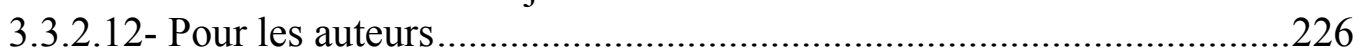

\section{4- African Journal On Line ( AJOL)}

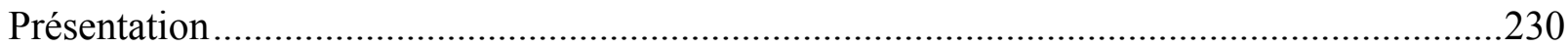

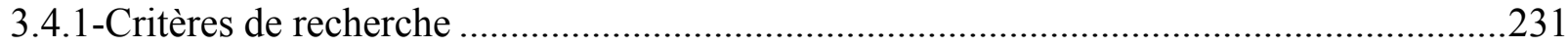

3.4.1.1 -Chercher des journaux

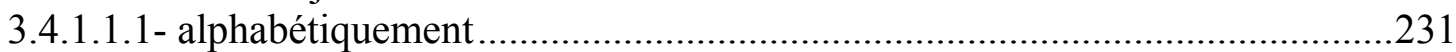

3.4.1.1.2- par sujet........................................................................................231

3.4.1.1.2.1- Sciences de l'agriculture et administration des ressources...............231

3.4.1.1.2.2- Arts, Culture, Langues et littérature ..............................................231

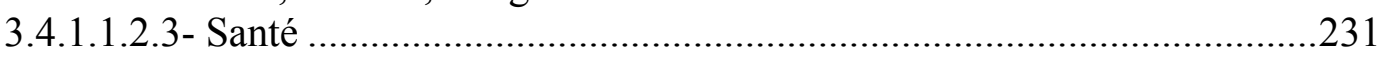

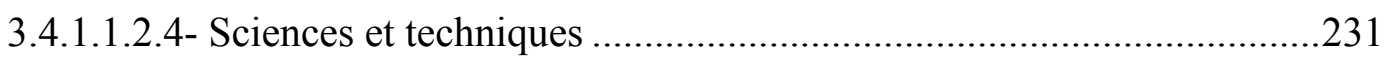

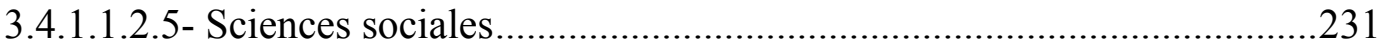




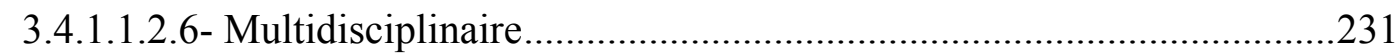

3.4.1.1.3-par pays

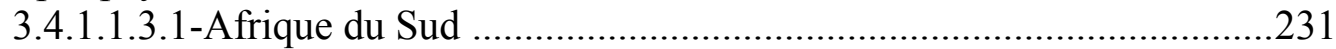

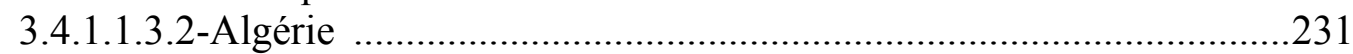

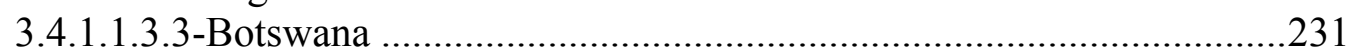

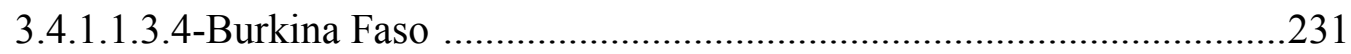

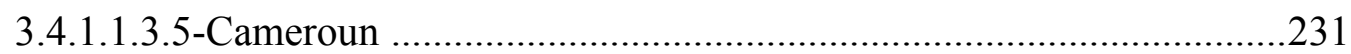

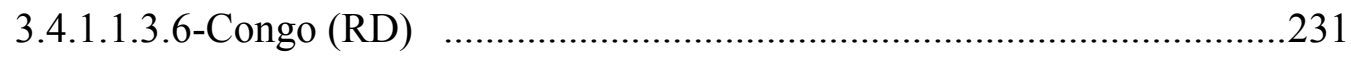

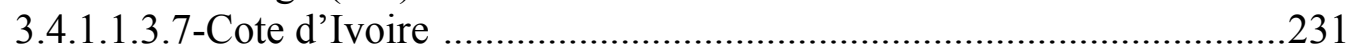

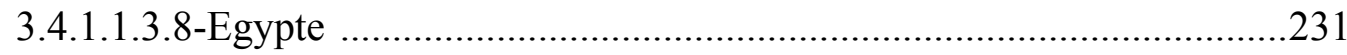

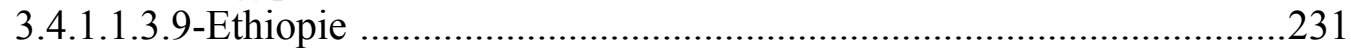

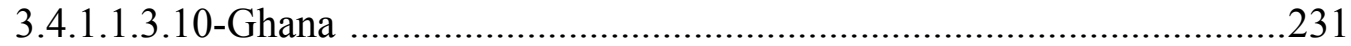

3.4.1.1.3.11-Ile Maurice .............................................................................2231

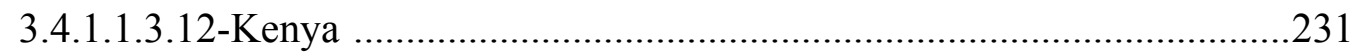

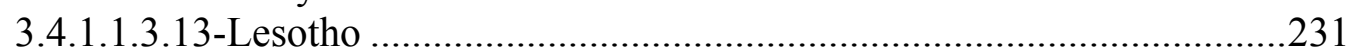

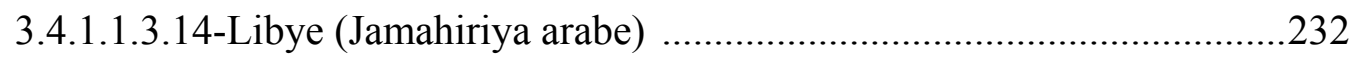

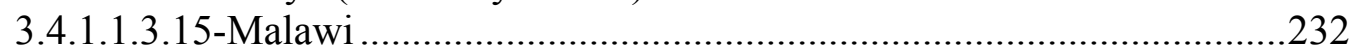

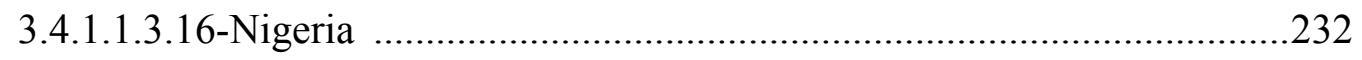

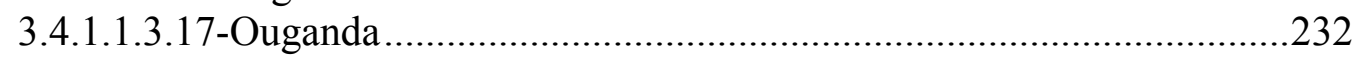

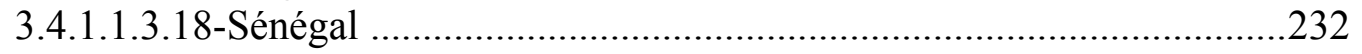

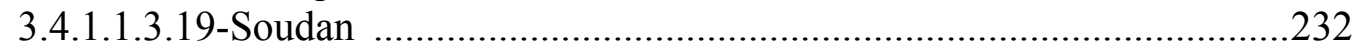

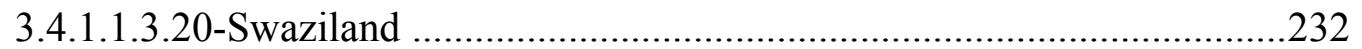

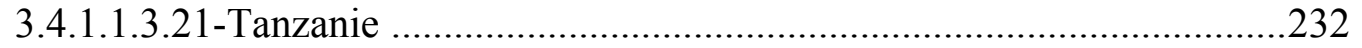

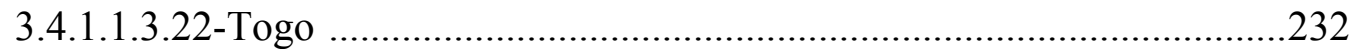

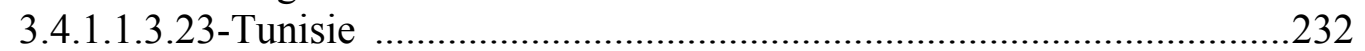

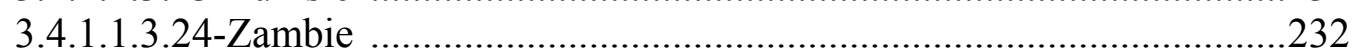

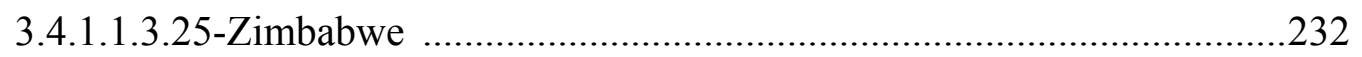

3.4.2-L'accès à l'information

3.4.2.1 - par le moteur de recherche Google ................................................................23

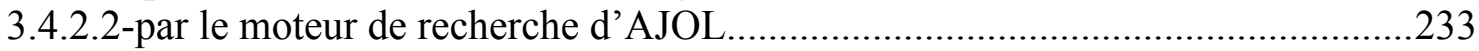

3.4.2.3-Proportion journaux en libre accés /journaux payant ....................................233

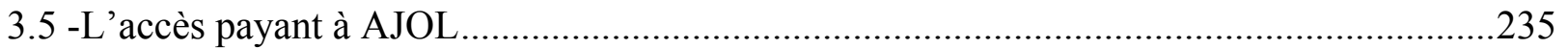

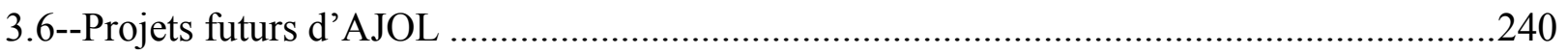

3.7-Projets analogues .....................................................................................................240

3.7.1- INASP (The International Network for the Availability of Scientific Publications) 240

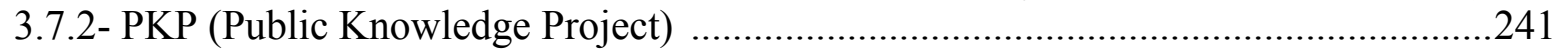

3.7.3 - NISC (National Inquiry Service Center) ..............................................................241

Conclusion

\section{Chapitre IV \\ LIBRE ACCES ET DROITS D'AUTEURS}

Introduction 
4.1-SHERPA ( Securing a Hybrid Environment for Research Preservation and Access ) : Opening access to research

4.1.1- RoMEO: un complément incontournable de SHERPA

4.1.1.2-Recherche par :

4.1.1.2.1-titre de journal .246

4.1.1.2.2-nom d'éditeur.

4..1.1.3-RoMEO News

4.1.1.3.1 -Editeurs permettant l'utilisation de leur PDF

dans les entrepôts institutionnels .249

4.1.1.3.2-Editeurs avec Option de libre accès payant .250

4.2- SHERPA JULIET : organismes financeurs et libre accés .255

\section{Chapitre V \\ DE QUELQUES ASPECTS DU LIBRE ACCES EN ALGERIE}

Introduction. .262

5.1-Le Portail global du libre accés (Global Open Access Portal-GOAP) de l'UNESCO. .262

5.1.1-Global Open Access Portal -Algeria .263

5.1.1.1 -Dépôt numérique en libre accès. .263

5.1.1.1.1 - Dépôt numérique de l'Université d'Alger

5.1.1.1.1.1- Communities \& Collections (communautés et

collections)

5.1.1.1.1.2-Issue date (date de parution) 264

5.1.1.1.1.3-Author (auteur)

5.1.1.1.1.4-Title (titre)

5.1.1.11.5-Subject (sujet)

5.1.1.2-Journaux électroniques en libre accès

5.1.1.2.1-IBScientific magazine. .265

5.1.1.2.2-IBScientific Journal of Science .......................................2.265

5.1.1.2.3-Synergies Algérie .266

5.1.1.2.4- Webreview : un site fédérateur de revues scientifiques

électroniques algériennes

5.1.1.2.5-Les différents titres de Webreview

5.1.1.2.5.1- Archives de l'Institut Pasteur d'Algérie .268

5.1.1.2.5.2- Al-Lugha Wal Adab .268

5.1.1.2.5.3- Annales de l'Institut National Agronomique

El Harrach (Alger) .268 
5.1.1.2.5.4- Bulletin des Sciences Géographiques .......................268

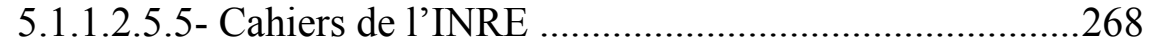

5.1.1.2.5.6- COST (COmmunication Science et Technologie) ...268

5.1.1.2.5.7- Courrier du Savoir ....................................................2. 268

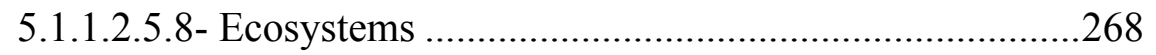

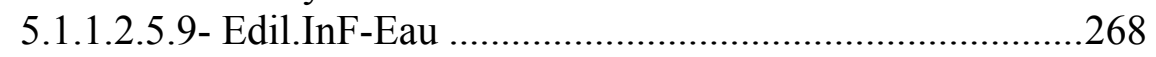

5.1.1.2.5.10- El Bahith Al-Ijtimai .............................................269

5.1.1.2.5.11- Al Maktabat Wa El Maâloumat............................269

5.1.1.2.5.12- El -Tawassol......................................................269

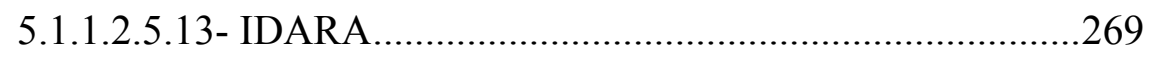

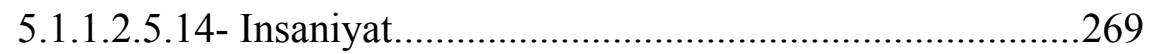

5.1.1.2.5.15- Journal de Neurochirurgie .....................................269

5.1.1.2.5.16- Journal of Electrical Systems..................................269

5.1.1.2.5.17- Journal of the Algerian Chemical Society ..............269

5.1.1.2.5.18- La Revue des Sciences Commerciales ....................269

5.1.1.2.5.19- LARHYSS Journal ................................................269

5.1.1.2.5.20- Le Journal de l'Eau et de l'Environnement .............269

5.1.1.2.5.21- Les Cahiers du CREAD .........................................269

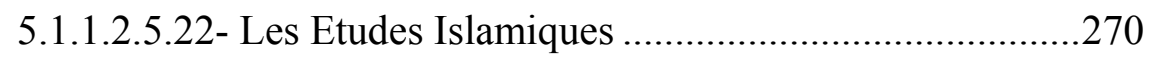

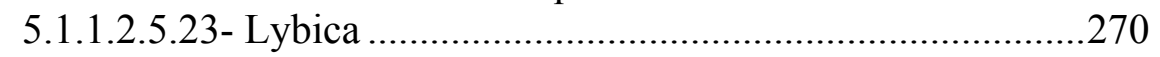

5.1.1.2.5.24- NATURE \& TECHNOLOGIE.............................270

5.1.1.2.5.25- Recherche Agronomique ......................................270

5.1.1.2.5.26- Revue Académique des Etudes Sociales

et Humaines

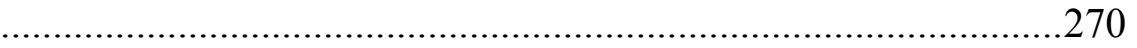

5.1.1.2.5.27- Revue Algérienne des Sciences Juridiques,

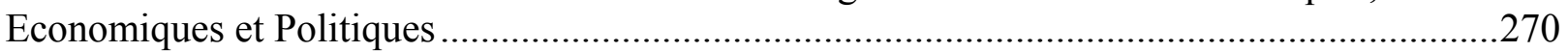

5.1.1.2.5.28- Revue Algérienne du Travail..................................2270

5.1.1.2.5.29- Revue des Energies Renouvelables ........................270

5.1.1.2.5.30- Revue des sciences humaines ...............................2271

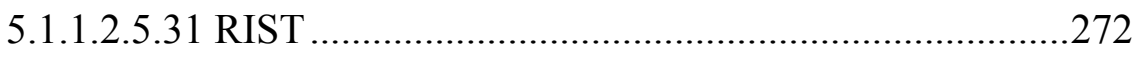

5.1.1.2.5.32- Sciences et Technologie ........................................2 273

5.1.1.2.5.33- Sciences Humaines .................................................2273

5.1.1.2.5.34- Social and Human Sciences Review.......................273

5.1.1.2.5.35- Synthèses ...........................................................273

5.1.1.2.5.36- Technologies Avancées .........................................273

5.2-Internet en Algérie .......................................................................................................2 275

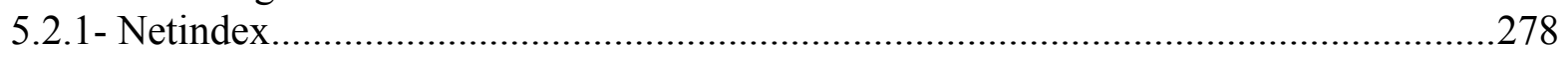

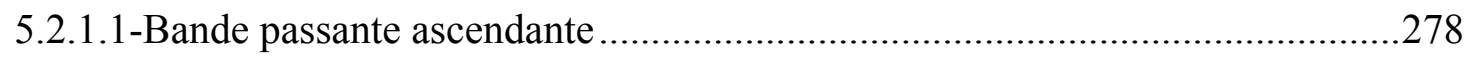

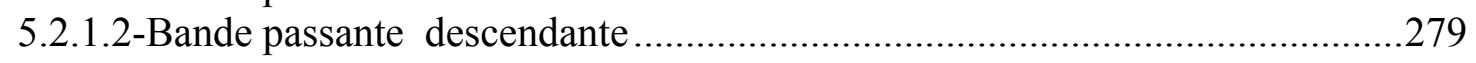

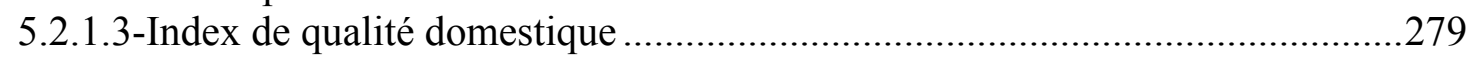

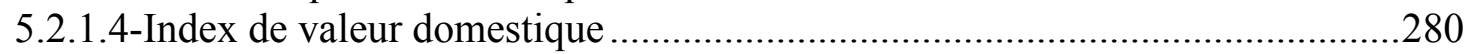

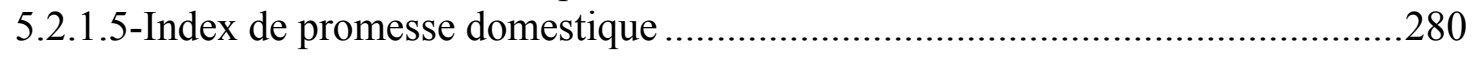

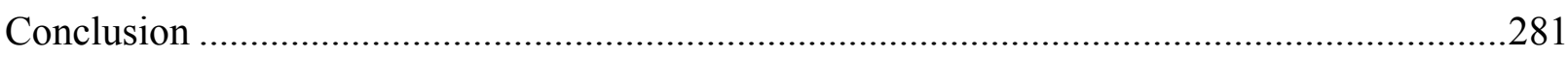

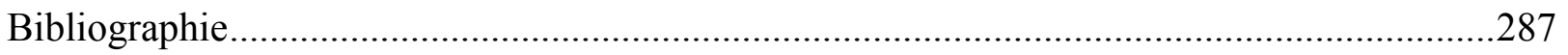




\section{TROISIEME PARTIE}

\section{CONTROLE PAR LES PAIRS ET LIBRE ACCES}

Introduction

\section{Chapitre I \\ PREMIERES MANIFESTATIONS, FORMES ET EVOLUTION DU CONTROLE PAR LES PAIRS}

1.1-Premières formes du contrôle par les pairs

1.2- Premières manifestations du contrôle par les pairs de l'ère moderne .....................................300

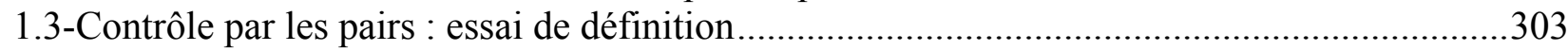

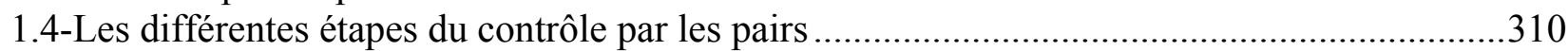

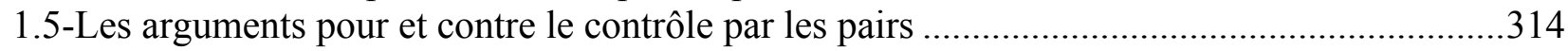

1.5.1-Un exemple de réaction extrême au contrôle par les pairs .............................................315

1.6- Les différents et plus courants préjugés du contrôle par les pairs selon Shatz....................317

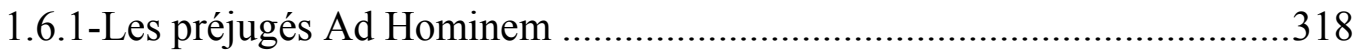

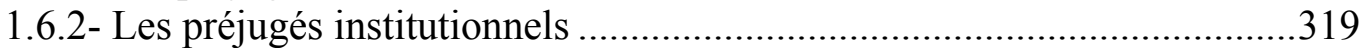

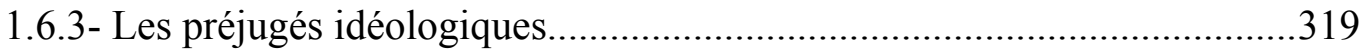

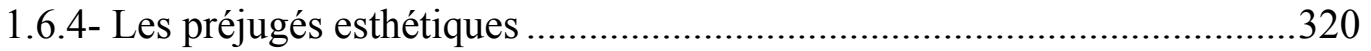

1.7-L'étude de Ceci et Stephen et le préjuge affiliationnel......................................................320

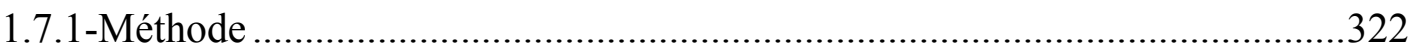

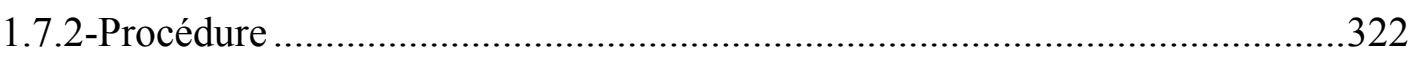

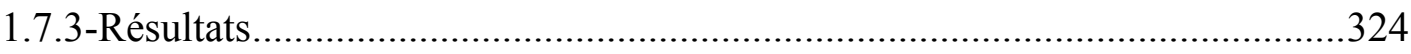

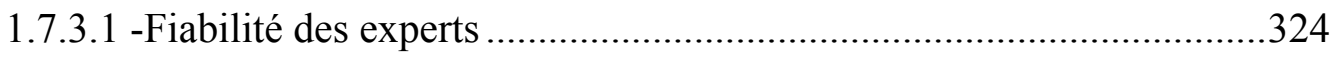

1.7.3.2- Evaluations des resoumissions non détectées.................................324

1.7.3.3- Commentaires critiques émis par les experts .................................325

1.7.3.4- Reconnaître de la recherche déjà publiée .........................................326

1.7..3.5- Changement dans les taux de rejet et les critères de publication..........327

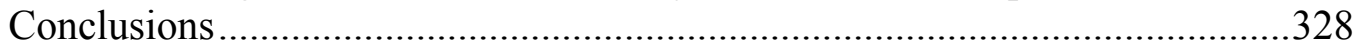

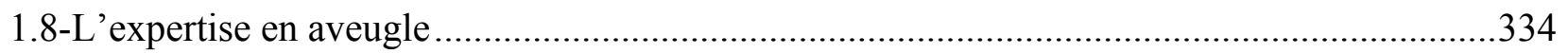

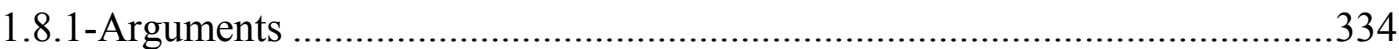

1.8.1.1-Faire partie d'une communauté .......................................................334

1.8.1.2-La thèse de l'omniprésence du préjugé ..............................................335

1.8.1.3-L'argument " pas de mérite intrinsèque " de Fish ..............................335

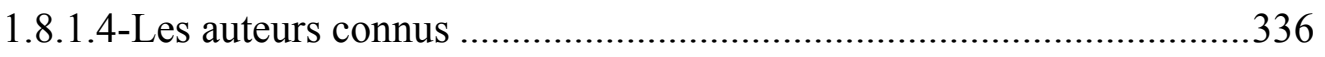

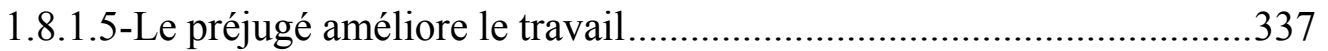

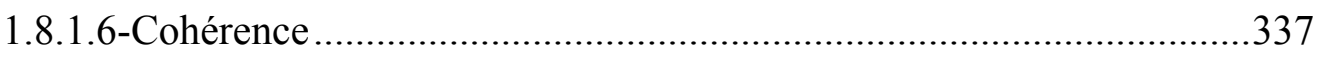

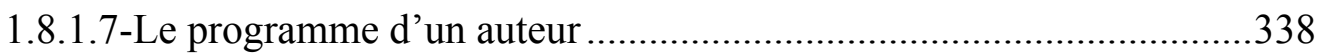

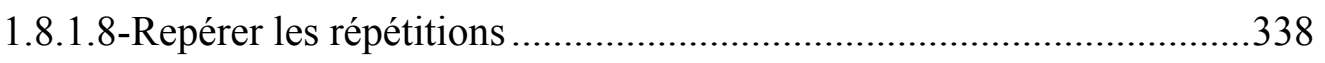

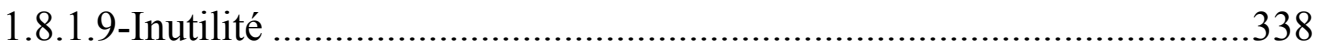




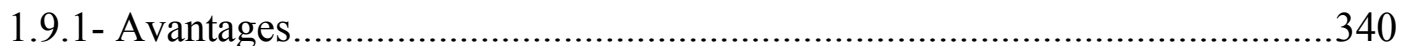

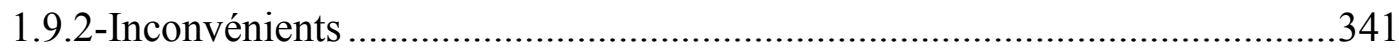

\section{Chapitre II \\ L'EXPERIENCE DU MAGAZINE NATURE EN MATIERE DE CONTROLE PAR LES PAIRS OUVERT}

Introduction .343

2.1-Débat .343

2.2-Vue d'ensemble .343

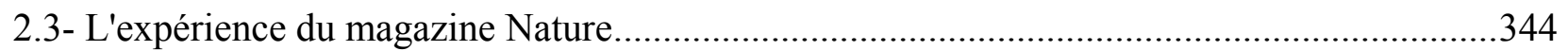

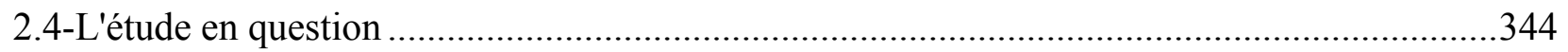

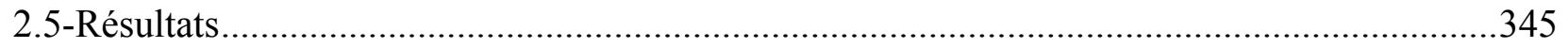

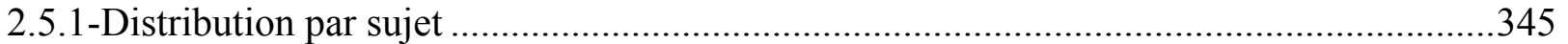

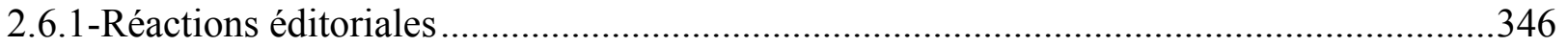

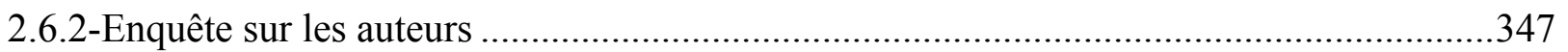

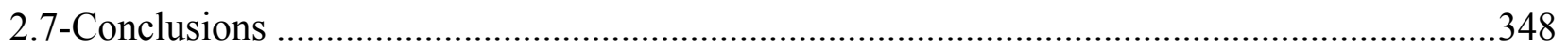

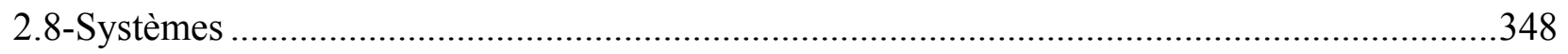

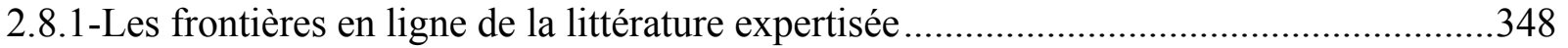

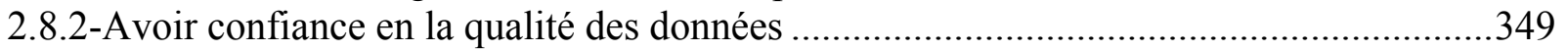

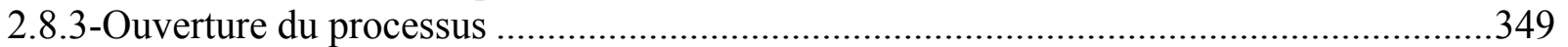

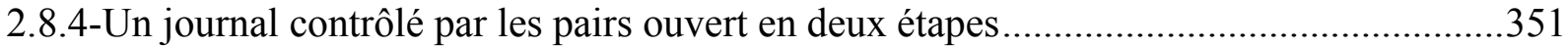

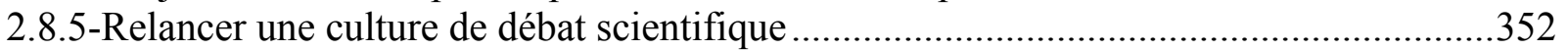

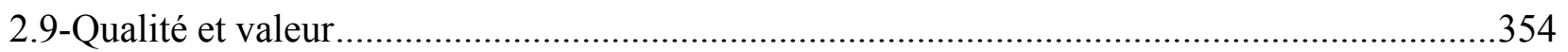

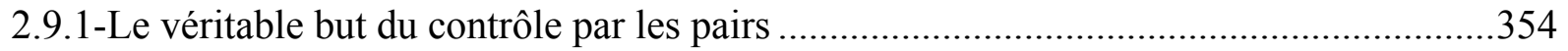

2.9.2-Modèles du contrôle de la qualité pour la recherche scientifique ..................................356

2.9.3-Comment pouvons-nous tirer le maximum du contrôle par les pairs ? .........................357

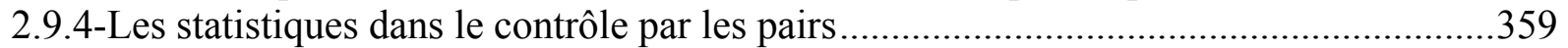

2.9.5-Comment pouvons-nous investiguer le contrôle par les pairs ? ...................................360

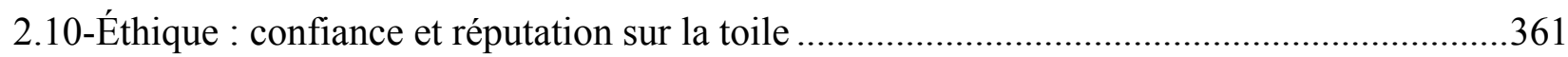

2.10.1-Les publications en ligne ont plusieurs manières pour accéder à la respectabilité....361

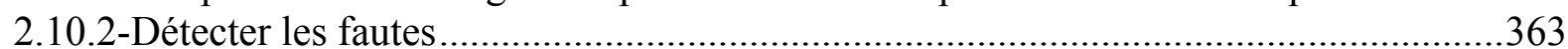

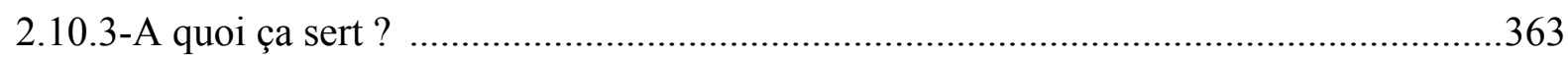

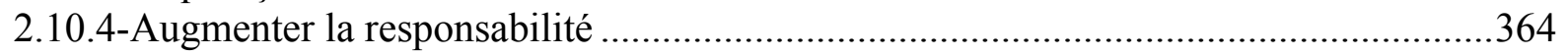




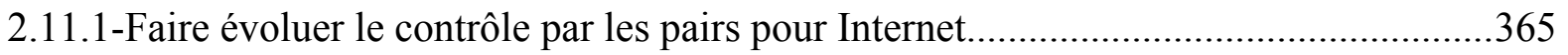

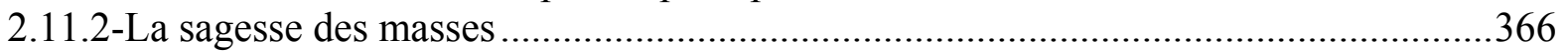

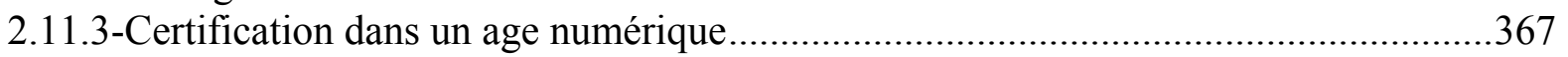

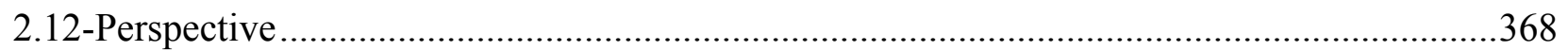

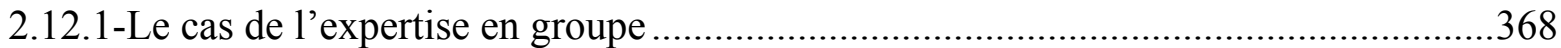

2.12.2-Le contrôle par les pairs des recherches scientifiques interdisciplinaires..................369

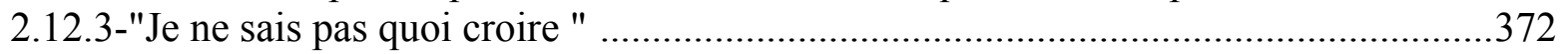

2.12.4-Les avantages et les inconvénients du contrôle par les pairs .......................................373

2.12.5-Est-ce que le contrôle par les pairs a le même sens pour le public

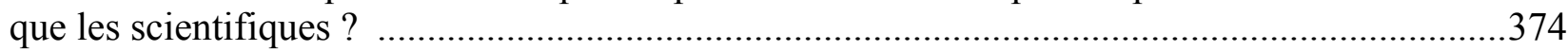

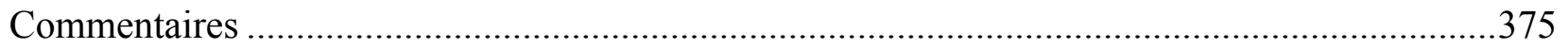

\section{Chapitre III}

CONTROLE PAR LES PAIRS OUVERT

Introduction

3.1-Deux expériences pionnières : BBS et Psycholoquy ...............................................................377

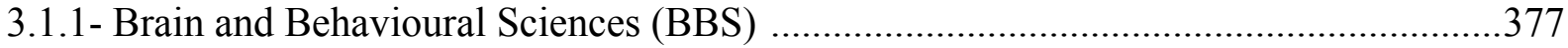

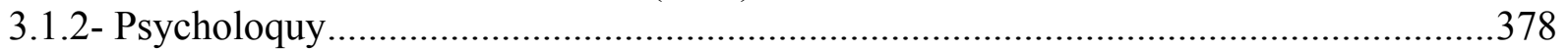

3.2-Quelques exemples d'expériences de contrôle par les pairs ouvert .....................................379

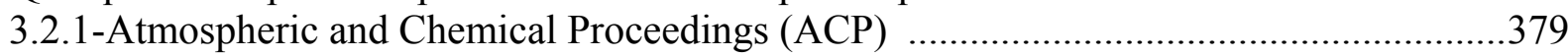

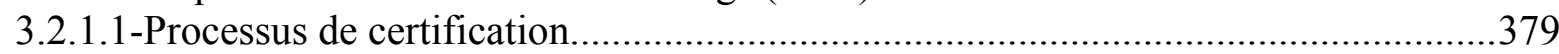

3.2.1.1.1-A.C.P.D. (Atmospheric and Chemistry Physics Discussion) ...........................379

3.2.1.1.1.1-Soumission du manuscrit original et désignation de l'éditeur....................380

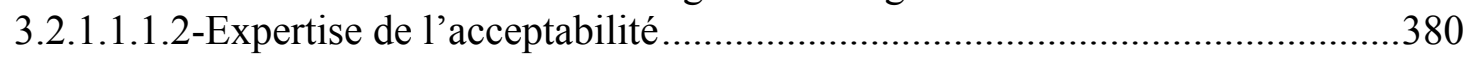

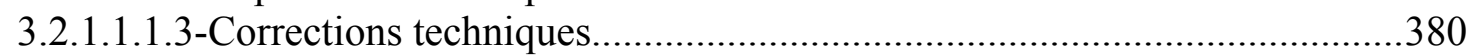

3.2.1.1.1.4-Publication et discussion de la recherche dans ACPD .............................380

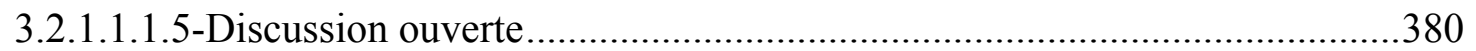

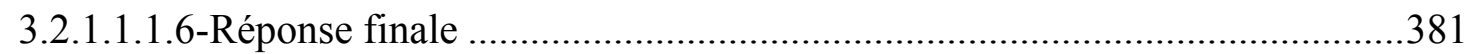

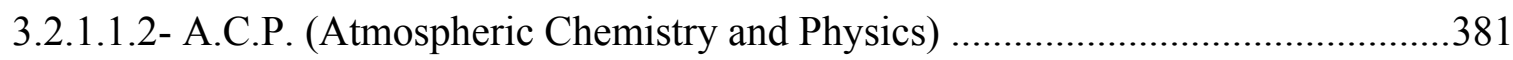

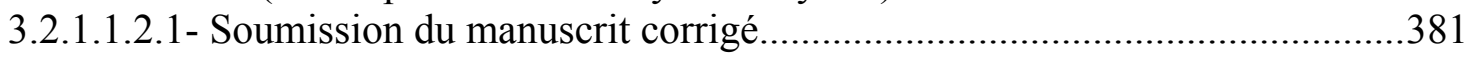

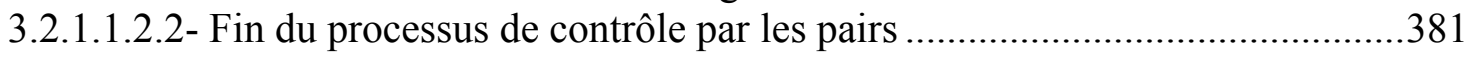

3.2.1.1.2.3- Publication de la recherche finale corrigée dans ACP.............................................381

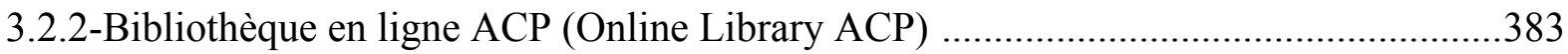

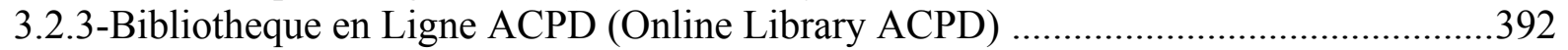

3.3-Faculty of 1000 (F 1000)

Introduction 


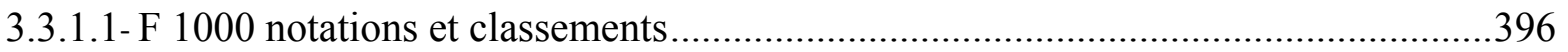

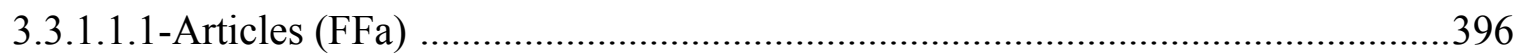

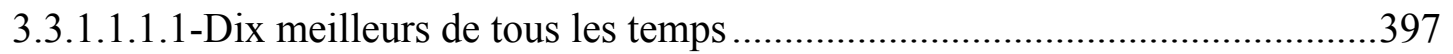

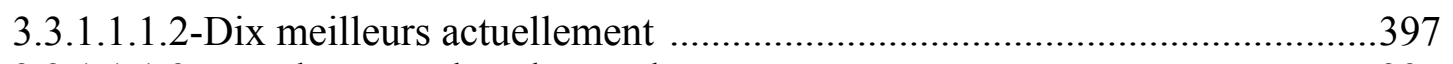

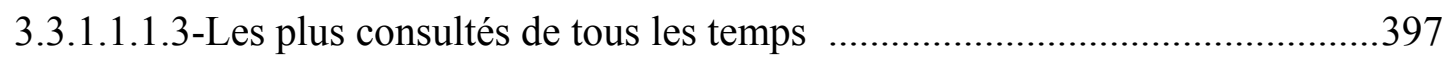

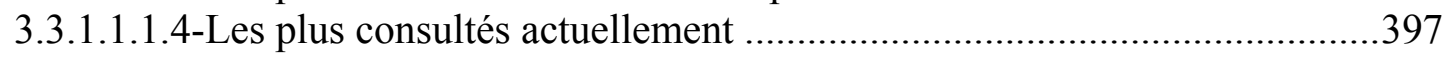

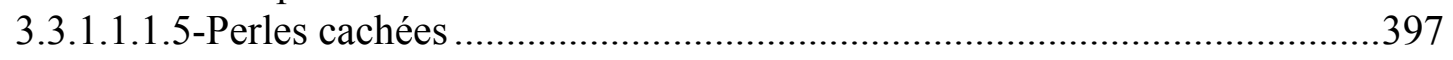

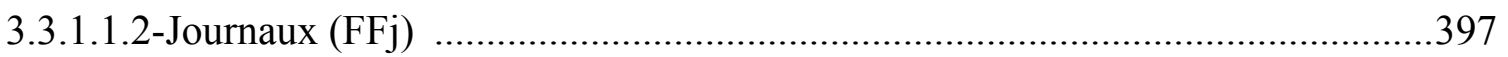

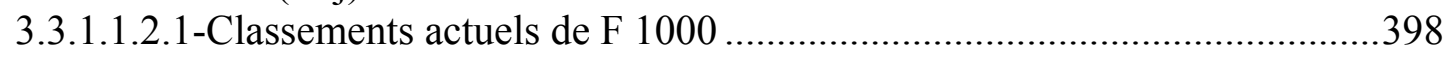

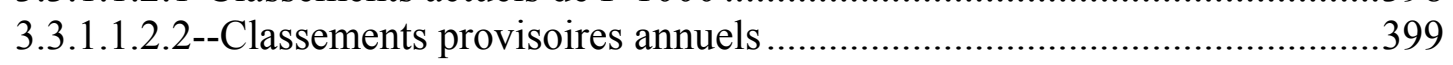

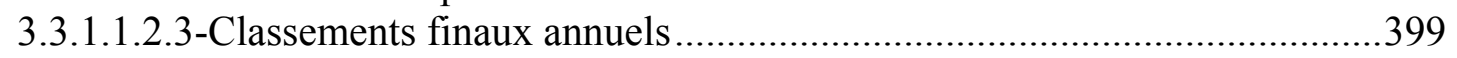

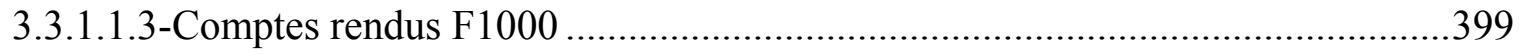

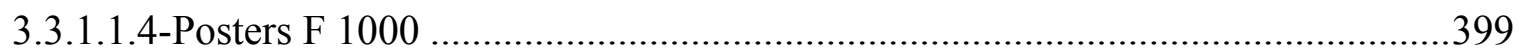

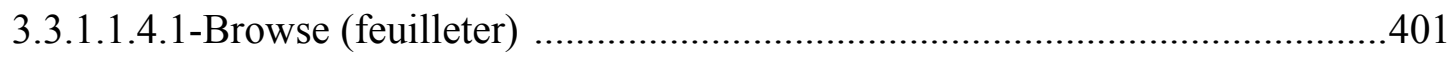

3.3.1.1.4.2-For depositors (pour les déposants) .....................................................401

3.3.1.1.4.3-For Societes/Conferences organizers (pour les associations /organiseurs

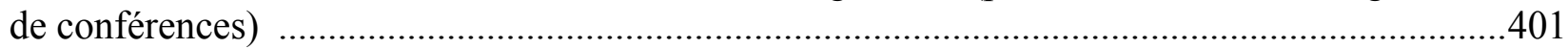

3.3.1.1.4.4-Submission of the week (soumission de la semaine) ............................401

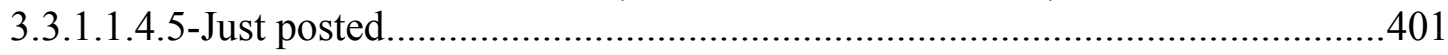

3.3.1.1.4.6-Récent meetings (réunions récentes) ..................................................401

3.3.1.1.4.7-Upcoming meetings (réunions à venir) ................................................401

3.3.1.1.4.8-Naturally selected (naturellement sélectionnées) ..................................402

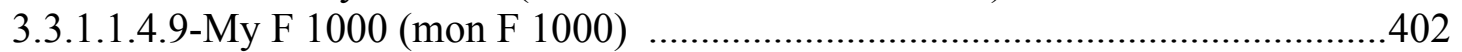

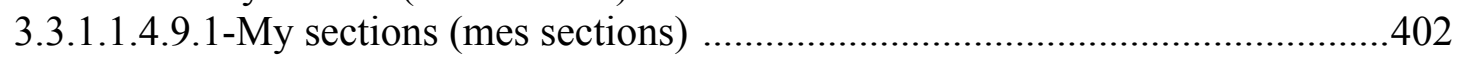

3.3.1.1.4.9.2- My saved articles (mes articles enregistrés) ....................................402

3.3.1.1.4.9.3-My stored searches (mes recherches sauvegardées) ...........................402

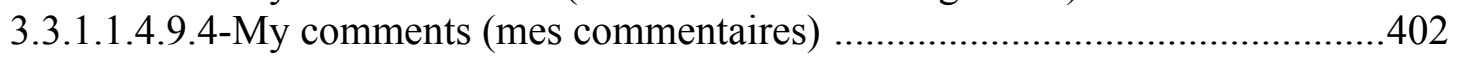

3.3.1.1.4.9.5- My e-mails alerts (mes alertes e-mails) .........................................402

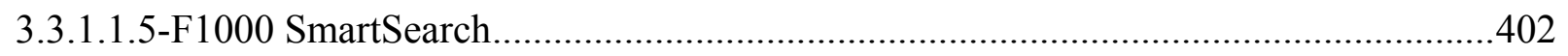

3.4--Electronic Transactions on Artificial Intelligence (ETAI)

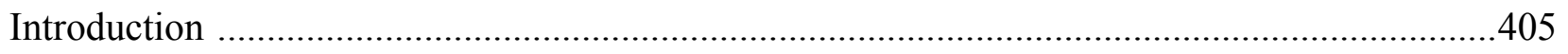

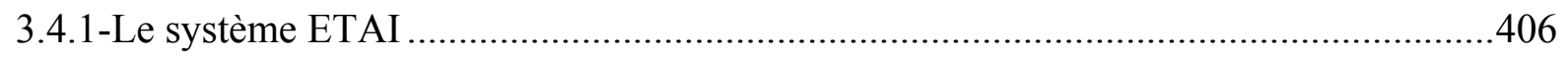

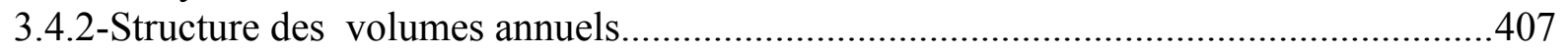

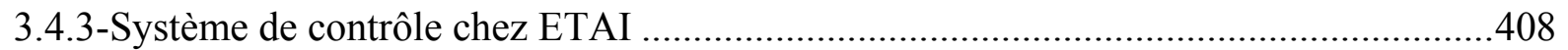

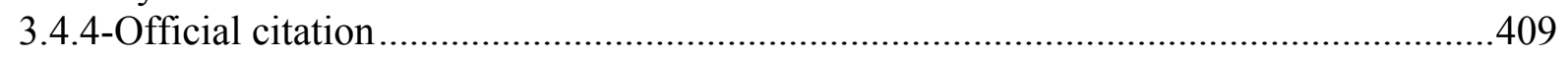

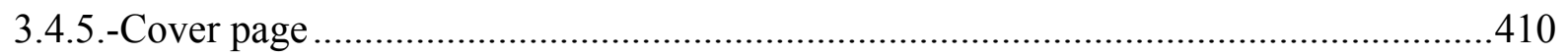

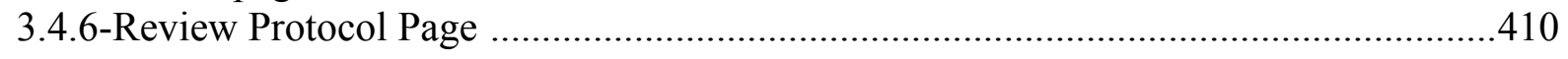




\section{5-Philica}

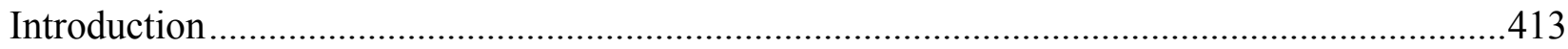

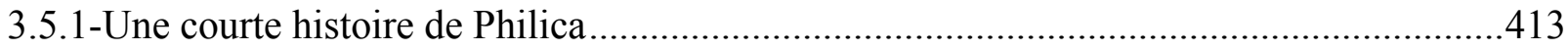

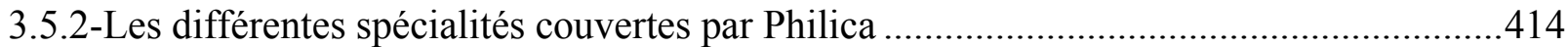

3.5.3-Le problème de la publication scientifique selon Philica ............................................415

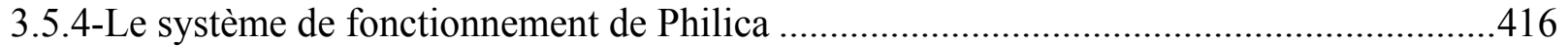

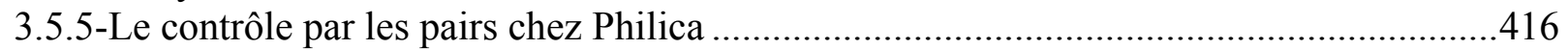

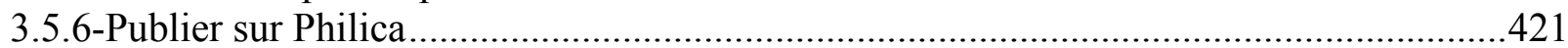

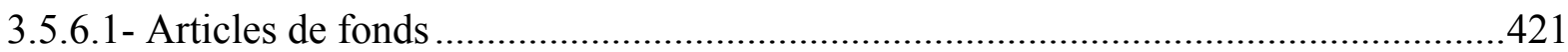

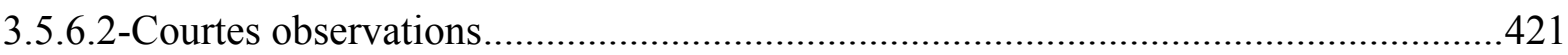

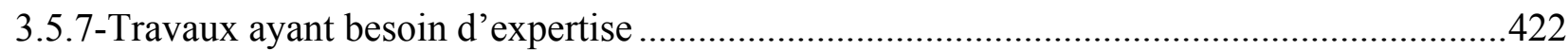

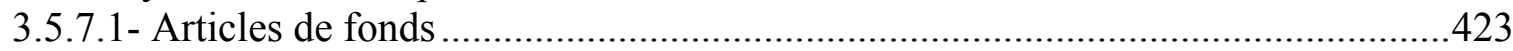

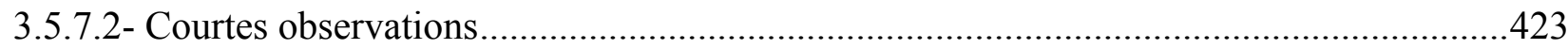

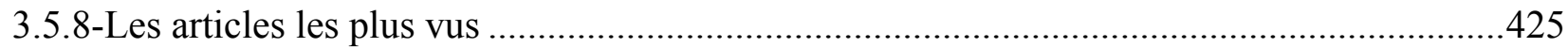

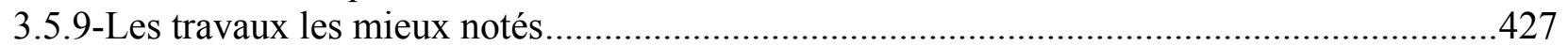

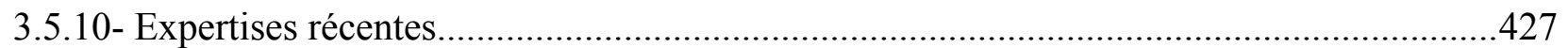

3.5.11- Conclusion et comparaison des quatre modèles examinés ...........................................429

\section{Chapitre IV}

\section{QUESTIONNAIRE CDTA et CERIST}

Introduction

4.1. Présentation des deux centres

4.1.1- Le CERIST

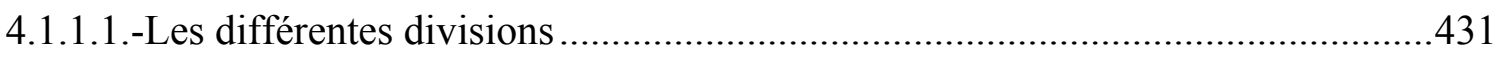

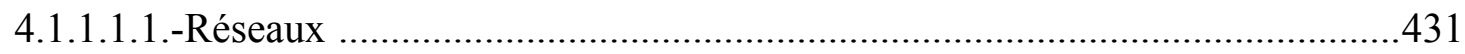

4.1.1.1.2.-Système d'information et système multimédia .......................................431

4.1.1.1.3-Recherche et développement en science de l'information ........................432

4.1.1.1.4-Théorie et ingénierie des systèmes informatiques ....................................432

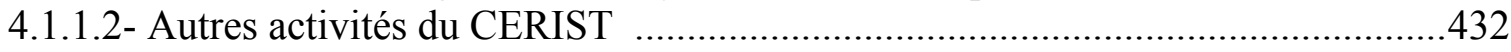

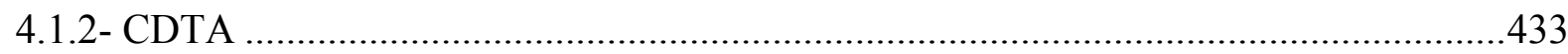

4.2-Présentation des axes, parties et buts du questionnaire ......................................................435

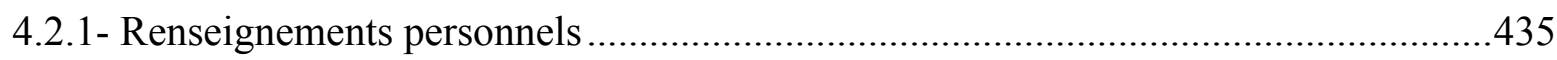

4.2.2- Degré de connaissance du libre accès..................................................................435

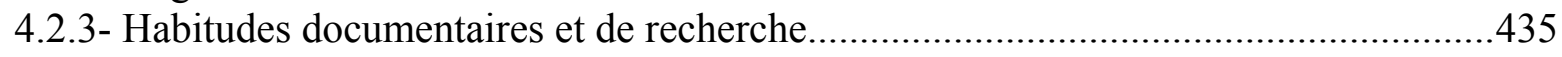

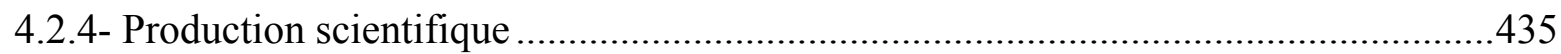

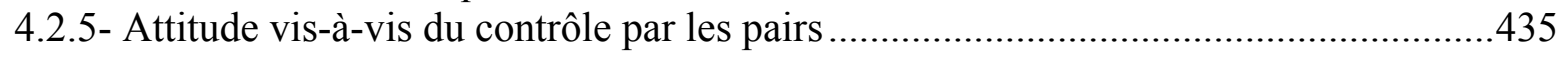

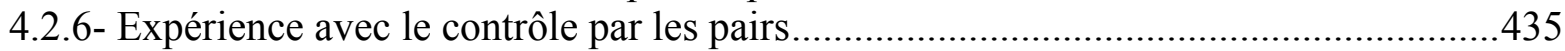

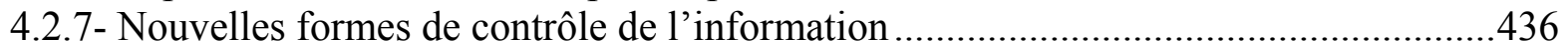

4.2.8- Position des chercheurs vis-à-vis des nouvelles formes de contrôle de l'information. 
4.3-Analyse des réponses du questionnaire adressé aux chercheurs du CERIST ......................436

4.3.1- Renseignements personnels (Q.1, 2, 3,4 et 5) ...................................................437

4.3.2- Degré de connaissance du libre accès (Q.6, 7, 8, 9, 10, 11, 12,13 et 14) ...................437

4.3.3- Habitudes documentaires et de recherche (Q.15, 16,17, et 18) .................................440

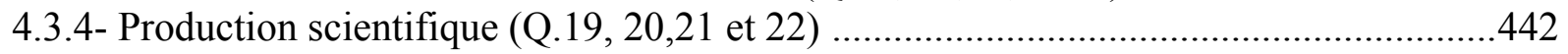

4.3.5- Attitude vis-à-vis du contrôle par les pairs (Q.23 et 24) ...........................................444

4.3.6- Expérience avec le contrôle par les pairs (Q. 25, 26, 27, 28, 29 et 30) .....................445

4.3.7- Nouvelles formes de contrôle de l'information (Q.31, 32,33 et 34) ...........................448

4.3.8- Position des chercheurs vis-à-vis des nouvelles formes de contrôle de l'information

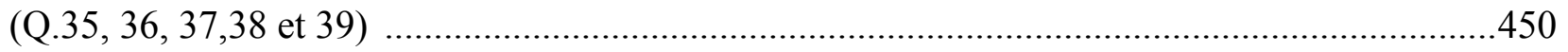

4.4-Analyse des réponses du questionnaire adressé aux chercheurs du CDTA.........................453

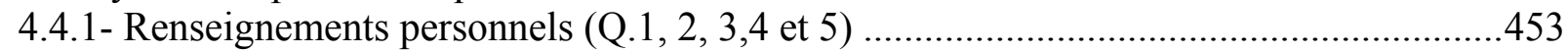

4.4.2- Degré de connaissance du libre accès (Q.6, 7, 8, 9, 10, 11, 12,13 et 14) ....................455

4.4.3- Habitudes documentaires et de recherche (Q.15, 16,17, et 18) ..................................458

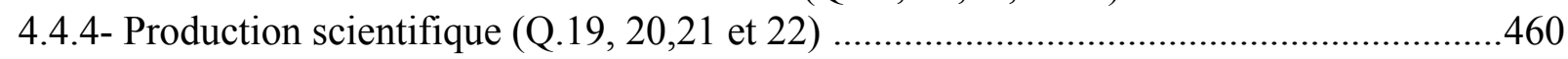

4.4.5- Attitude vis-à-vis du contrôle par les pairs (Q.23 et 24) ............................................461

4.4.6- Expérience avec le contrôle par les pairs (Q. 25, 26, 27, 28, 29 et 30)

4.4.7- Nouvelles formes de contrôle de l'information (Q.31, 32,33 et 34) ............................465

4.4.8- Position des chercheurs vis-à-vis des nouvelles formes de contrôle de l'information

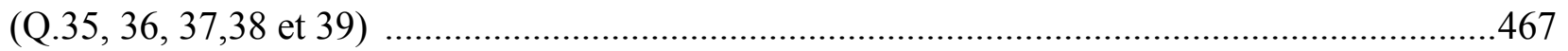

4.5-Tableau comparatif des réponses des chercheurs du CERIST et du CDTA..........................470

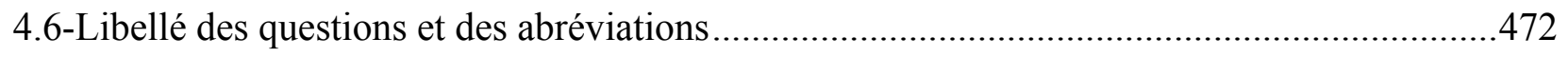

4.7-Conclusion générale sur les réponses du CERIST et du CDTA..........................................488

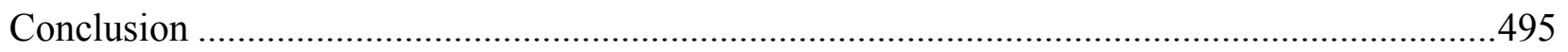

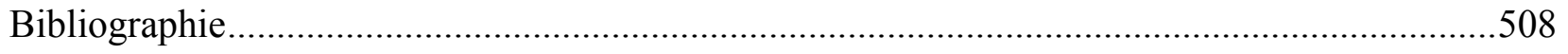

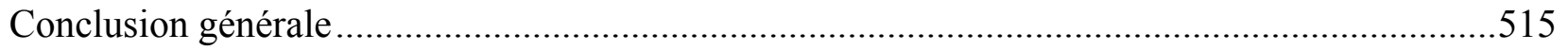

Annexe : Questionnaire 


\section{$\underline{\text { Liste des tableaux et figures }}$}

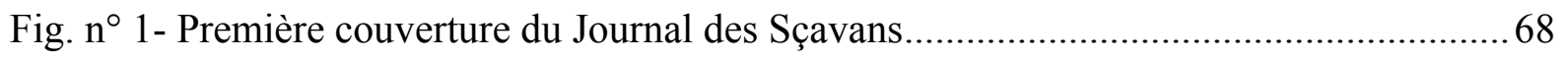

Fig. $\mathrm{n}^{\circ}$ 2-Couvertures du Journal des Savants (1909 et 2009) ............................................ 71

Fig. $n^{\circ} 3$ - Couvertures Philosophical Transactions (1665 et 2011) ...................................... 72

Fig. $\mathrm{n}^{\circ}$ 4-Tableau des augmentations des prix des périodiques (ARL) ............................... 160

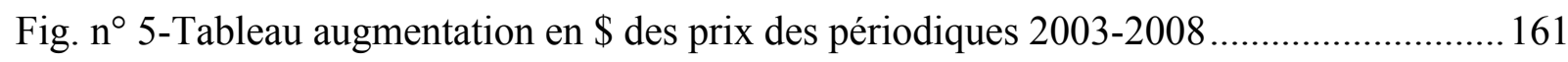

Fig. $\mathrm{n}^{\circ}$ 6-Tableau représentant le pourcentage d'augmentation des prix du tableau $\mathrm{n}^{\circ} 5$........ 162

Fig. $n^{\circ}$ 7-Tableau des prix des périodiques scientifiques (Library Journal 2011) ................... 163

Tableau ${ }^{\circ}{ }^{1}$.-Statistiques population et utilisateurs Internet (31 Decembre 2000) ............... 168

Tableau $^{\circ}{ }^{2}$.-Statistiques population et utilisateurs Internet (30 Juin 2012) ........................ 168

Fig. $n^{\circ} .8$ - Entrepôts par continent- Directory of Open Access Repositories (DOAR) ......... 197

Fig. $\mathrm{n}^{\circ}$ 9- Proportion par continent des organisations ayant un (ou des) entrepôts (DOAR) .. 198

Fig. $\mathrm{n}^{\circ} 10-$ Proportion des entrepôts par pays (DOAR) ......................................................... 199

Fig. $\mathrm{n}^{\circ} 11$ - Proportion des entrepôts d'organisation par pays (DOAR) .................................. 199

Fig. $n^{\circ} 12-$-Utilisation des logiciels d'entrepôts en libre accès(DOAR) ..................................200

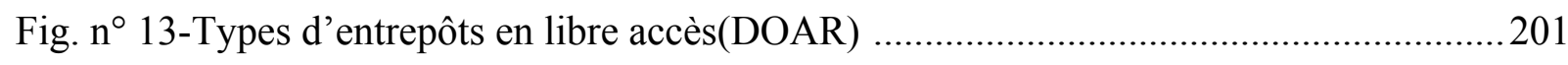

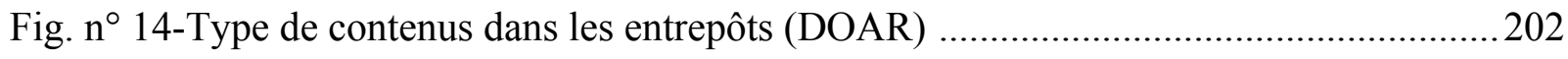

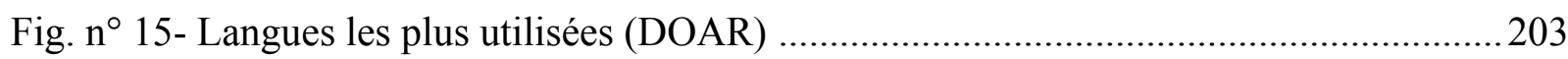

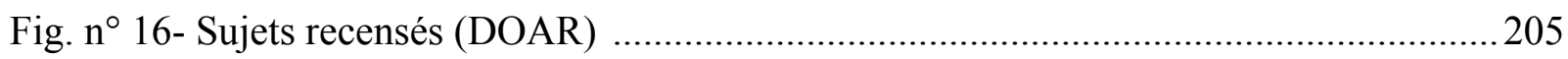

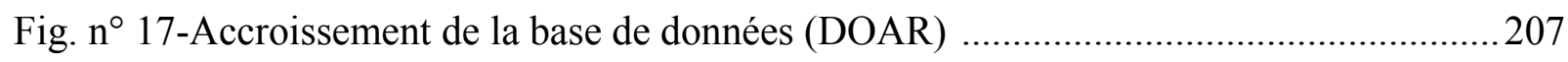

Fig. $\mathrm{n}^{\circ} 18$ - Recherche ou navigation par entrepôts (DOAR) ...............................................2208

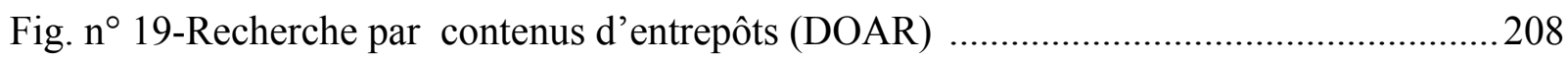

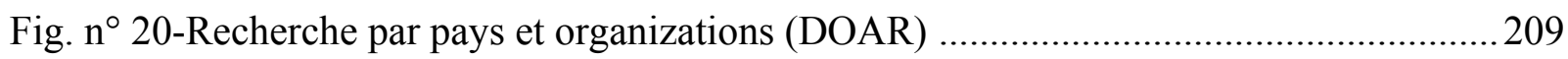

Fig. $n^{\circ}$ 21-Graphe du type de contenus des entrepôts- Registry of Open Access Repositories 
Fig. $\mathrm{n}^{\circ}$ 22- Camembert du type de contenu (ROAR)

Fig. $n^{\circ}$ 23- Graphe gradué de la progression des sites Mai 1989 -Octobre 2007 (ROAR)...... 214

Fig. $\mathrm{n}^{\circ} 24$ - Les deux premiers journaux électroniques (New Horizons in Adult Education et

EJournal ) 216

Fig. $\mathrm{n}^{\circ}$ 25-Différents types de périodiques en ligne dans la base de données

UlrichWeb.com. 219

Tableau $n^{\circ}$ 3-Proportion des titres en libre accès African Journal On Line (AJOL) ..............234

Tableau $\mathrm{n}^{\circ}$ 4- Répartition des pays selon le revenu (AJOL) .239

Tableau $\mathrm{n}^{\circ} 5$ - Forfaits offerts pour l'achat d'articles selon le revenu (AJOL) .240

Fig.n ${ }^{\circ}$ 26- Exemple de transfert des droits d'auteurs (copyright transfer agreement) pour American Library Association (SHERPA RoMEO) 246

Fig.n ${ }^{\circ} 27$ - Exemple de transfert des droits d'auteurs ( copyright transfer agreement ) pour Elsevier ( SHERPA RoMEO) 247

Fig.n ${ }^{\circ}$ 28-Statistiques des autorisations des éditeurs selon les couleurs RoMEO 249

Fig.n ${ }^{\circ} 29-$ Editeurs avec Option de libre accès payant (SHERPA RoMEO) 251

Fig.n ${ }^{\circ}$ 30-Autorisations données par les organisations finançant la recherche pour la publication en libre accès (SHERPA JULIET) 256

Tableau $n^{\circ} 6$ - proportion des articles en libre accès sur Webreview .274

Tableau ${ }^{\circ}$ 7- Statistiques d'utilisation Internet en Afrique (31 Decembre 2011) .277

Tableau ${ }^{\circ}{ }^{8}$ - Les dix premiers utilisateurs Internet en Afrique ( 31 Decembre 2011) .........278

Fig.n ${ }^{\circ}$ 31- Classement de l'Algerie selon la bande passante ascendante (Netindex) ...........279

Fig.n ${ }^{\circ}$ 32- Classement de l'Algerie selon la bande passante descendante (Netindex) .........279

Fig.n ${ }^{\circ}$ 33- Classement de l'Algérie selon l'index de qualité domestique (Netindex) ..........280

Tableau $n^{\circ} 9$ - Paramètres de publication des journaux scientifiques (1975-1995) .294 
Tableau $\mathrm{n}^{\circ} 10$-Taux de rejet des manuscrits soumis à la publication pour les journaux scientifiques et en sciences humaines (1967) 308

Fig.n³4-Cheminement d'un manuscrit à travers le processus de contrôle par les pairs éditorial (Weller) 312

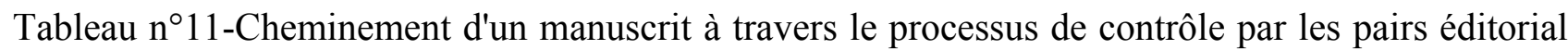
(British Academy) 313

Tableau ${ }^{\circ} 12$ - Fiabilité des experts (selon l'étude de Stephen et Ceci) 324

Tableau $\mathrm{n}^{\circ} 13$-Détection des resoumissions par les experts (selon l'étude

de Stephen et Ceci) 327

Tableau $n^{\circ}$ 14-Stabilité des taux de rejet entre la publication originale et la resoumission (selon l'étude de Stephen et Ceci) 328

Fig.n ${ }^{\circ}$ 35- Recherches par spécialité (Enquête Nature sur le contrôle par les pairs

ouvert 2006) 345

Fig.n ${ }^{\circ}$ 36- Commentaires par recherche et par discipline (Enquête Nature sur le contrôle

par les pairs ouvert 2006) 346

Fig. $n^{\circ}$ 37-Processus de contrôle par les pairs public et discussion publique interactive

(ACPD) 382

Fig.n³8-Processus de contrôle par les pairs, publication et discussion interactive (ACP) .... 382

Tableau $\mathrm{n}^{\circ}$ 15-Classement actuel des journaux expertisés par F 1000 .398

Tableau ${ }^{\circ} 16-$ Tableau comparatif des réponses des chercheurs du CERIST et du CDTA...470 


\section{Introduction Générale}


Le sujet du contrôle par les pairs, étape indispensable et incontournable de la publication scientifique et technique, occupe ces dernières années le devant de la scène dans le monde académique et universitaire. En effet, d'un processus très inégalement utilisé avant la deuxième guerre mondiale, il est devenu un point focal des chercheurs pour qui il représente l'examen suprême de leurs recherches, examen à l'issue duquel ils peuvent publier, obtenir des financements pour leurs travaux, des postes ou des promotions . Cette soudaine et quasi unanime application du processus a résulté d'une donne que la fin de la deuxième guerre mondiale a imposé. Cette période (post deuxième guerre mondiale) s'est caractérisée par une augmentation et une injection massives d'investissements alloués aux fonds de recherche. L'Europe et surtout les Etats Unis au sortir d'une guerre destructrice se devaient de relancer l'appareil de production et cela passait par des programmes de recherche tout azimut. Une exponentielle et constante augmentation des publications scientifiques sur papier rendant compte de ce processus s'ensuit. Aussi l'inflation de publications devait être canalisée, diffusée afin de profiter à ceux qui en avaient besoin à travers le medium le plus approprié : le périodique scientifique et technique .En effet, la monographie ne répondait pas aux critères de rapidité de publication et dissémination de l'information (surtout dans les sciences exactes et médicales) comme le périodique, créé spécialement dans cette optique un peu moins de trois siècles auparavant.

C'est alors qu'un nombre de titres en augmentation constante et de plus en plus spécialisés vit le jour répondant à des besoins aussi variés que les nouvelles spécialités qui en découlent. Avec la spécialisation ${ }^{*}$ de plus en plus accrue de la science, le nombre des titres est allé crescendo pour répondre à de nouvelles spécialités de plus en plus pointues et de plus en plus hyperspécialisées. Cette augmentation a atteint un seuil tel que le traitement manuel de l'information s'est avéré inopérant et dépassé. Alors intervient le contrôle par les pairs avec deux fonctions principales imposées : une fonction qualitative et une fonction quantitative. Face à cette opération déluge tel que l'avait décrit le bibliothécaire de la Marine américaine à l'époque, une sorte de filtre devait être mis en place afin que la masse d'information ne devienne pas incontrôlable du point de vue numérique. Il était devenu, déjà à l'époque, clair que les systèmes de contrôle de l'information arriveraient à saturation et auraient besoin d'une limitation quantitative. L'autre fonction, beaucoup plus au cœur de la problématique du contrôle par les pairs, et en fait est sa quintessence, se résume à un contrôle qualitatif de ce qui est publié et ce qui est rejeté. En effet, si le coté quantitatif du contrôle par les pairs est juste une opération purement arithmétique de ce qui est possible de publier, le coté qualitatif touche quant à lui au devenir de la science, à sa construction et son futur. Autrement dit, la science étant par définition méritocratique et compétitive et non pas ploutocratique ${ }^{*}$, il est normal et même indispensable qu'une sélection se fasse sur la base de critères universels et

\footnotetext{
* La spécialisation est devenue une caractéristique de plus en plus affirmée de la science depuis les premiers périodiques. Cette situation a induit une situation acceptée et de fait de nos jours : la quasi-totale disparition des périodiques scientifiques généralistes à part pour des exceptions très rares et très spéciales tels que Nature, Science, etc....

*La ploutocratie (qui vient du grec "ploutos, richesse et kratos, pouvoir , autorité" ) est un système politique dans lequel le pouvoir politique est dévolu aux détenteurs de la richesse On peut voir à travers cette définition à quel point la science telle qu'elle devrait être est méritocratique , quoique sur le terrain , ceci n'est pas si clairement défini qu'il n'y parait. (http://www.larousse.fr/dictionnaires/francais/ploutocratie/61759 ). Cette idée est développée par Jean Claude Guédon et dans laquelle il remet en cause les nouvelles orientations que prend la publication scientifique au regard des nouvelles restrictions imposées par les éditeurs soucieux de préserver leur acquis face aux avancées sans cesse constantes du libre accès qui, de plus en plus, rognent sur le quasi monopole qu'ils avaient à l'ère papier
} 
admis par tous. De plus, ce deuxième filtre a pour but de préserver la science de la pseudo science par des mécanismes de filtrage et de choix basés sur la scientificité, la rigueur méthodologique et un ensemble de critères sur lesquels la science s'appui. Il serait pour le moins hasardeux de ne pas faire passer une information dont dépendrait la santé publique par un filtre qui la validerait et la rendrait non nocive. Nombre de cas ont eut lieu dans lesquels le contrôle par les pairs a permis la publication d'articles controversés (nonobstant leur valeur scientifique) réfutés plus tard à cause de leur impact potentiel sur la santé publique ${ }^{*}$. C'est dire l'importance du contrôle par les pairs qui diffère dans son application, ses résultats, son influence selon les domaines. Ce qui d'ailleurs est reflété dans les taux d'acceptation qui varient entre les sciences dures et exactes et les sciences humaines et sociales. Les premières (les sciences dures et exactes) ont des taux d'acceptation plus élevés que celles des sciences humaines et sociales. A cet effet, Zuckerman et Merton ont, dans une recherche séminale publiée en $1971^{(1)}$ mais qui reste d'actualité, analysé les taux de rejection dans un échantillon de 83 journaux dans 15 spécialités. Le résultat fut résumé dans la règle de base suivante : plus le journal est orienté vers les sciences humaines, plus le taux de rejection est haut et plus le journal est orienté vers l'observation et l'expérimentation mettant l'accent sur la rigueur de l'observation et l'analyse, plus le taux de rejection est bas. En d'autres termes, les sciences sociales et humaines ont des taux de rejection plus haut que ceux des sciences exactes et techniques (on oppose et utilise généralement les sigles de STM - Science, Technique, Médecine - à celui de SHS - Sciences Humaines et Sociales).Bormann et Daniel ${ }^{(2)}$ expriment une idée quasi analogue et qui est le concept d'erreurs de type I et de type II ${ }^{*}$. Toujours dans le même registre, Cole abonde dans le même sens en ces termes sans équivoque: " les journaux en physique préfèrent faire des erreurs de type I d'acceptation de travaux non significatifs et banals plutôt que des erreurs de type II et rejeter des travaux potentiellement importants. Cette politique conduit souvent à la publication d'articles triviaux avec une signification théorique moindre ou nulle, défauts ayant été fréquemment cités par les experts dans les domaines des sciences sociales pour justifier le rejet d'articles .D'autres spécialités, tels que la sociologie aux Etats-Unis, ont pour critère de rejeter un article à part s'il représente une contribution significative au Savoir. Les sociologues préfèrent faire des erreurs de type II " (3).Enfin une autre contribution au titre des plus explicite se pose la question de la raison de l'acceptation des travaux médiocres qui arrivent à se faire publier. L'auteur exprime sa rancœur dans les termes suivants : " les journaux en physique préfèrent faire des erreurs de type I d'acceptation de travaux non significatifs et banals plutôt que des erreurs de type II et rejeter des travaux potentiellement importants. Cette politique conduit souvent à la publication d'articles triviaux avec une signification théorique moindre ou nulle, défauts ayant

\footnotetext{
* A titre d'exemple, un article qui en 2009 avait argumenté du fait qu'il n'y avait pas encore de preuve que le VIH causait le SIDA avait été critiqué et soulevé un tollé général qui avait conduit à sa rétractation. Le même article fut ressoumis à un autre périodique moins connu et accepté. Ceci fut aussi l'occasion de dénoncer cette acceptation car pour reprendre l'éditorial ..." Il contenait [l'article] des opinions au sujet du SIDA qui pouvaient être potentiellement nuisible à la santé publique en général "(http://www.nature.com/news/paperdenying-hiv-aids-link-secures-publication-1.9737 ).

(1) Zuckerman H.,Merton R.K., Patterns of evaluation in science: institutionalization, structure and functions of the referee system, Minerva, Vol. 9, n 1, p.66-100, 1971

(2) Bornmann, Lutz, Daniel ,Hans-Dieter, Extent of type I and type II errors in editorial decisions: a case study on Angewandte Chemie International Edition, Journal of Informetrics, $n^{\circ} 3$, p . 348-352, 2009

${ }^{*}$ Les erreurs de type I sont des erreurs où l'expert accepte une soumission de qualité douteuse alors que l'erreur de type II est quand une soumission de bonne qualité est refusée. Bien que cette anomalie ne soit pas très documentée, il semblerait qu'elle ait principalement une raison : les experts échaudés par de nombreux cas de type II préfèrent commettre une erreur de type I qui ne les exposerait pas à la vindicte de la communauté (ou alors moins qu'une erreur de type II)

(3) Cole, S., Making science: between nature and society, Cambridge, [Ma., USA]: Harvard University Press, 1992, p. 114
} 
été fréquemment cités par les experts dans les domaines des sciences sociales pour justifier le rejet d'articles .D'autres spécialités, tels que la sociologie aux Etats-Unis, ont pour critère de rejeter un article à part s'il représente une contribution significative au Savoir. Les sociologues préfèrent faire des erreurs de type II " ${ }^{(4)}$.

Cette situation nous conduit à poser une question importante qui découle de quelques citations des auteurs principaux ayant abordé la problématique.Si l'idée de la prépondérance et l'inéluctabilité du contrôle par les pairs dans le monde de la publication scientifique au sujet duquel Ziman a dit " l'expert est le pivot autour duquel tout le processus [d'exercice] de la Science est bati " (5), que Campanario a comparé à " une Olympiade mentale pour le prestige et la reconnaissance " (6) alors que Crane les a appelé [les experts et les membres du comité éditorial] " les gardiens du temple de la Science " (7) et qu'enfin Lederberg a comparé à " la colle qui maintenait l'institution scientifique unie " (8) n'a pas été sujet à discussion ni à des critiques jusqu'à un passé très récent, quelle alors est la raison de ce soudain intérêt pour le sujet? Bien que certains auteurs aussi au fait du sujet que Weller et Shatz pensent qu'il n'a pas été assez abordé, les dernières années ont vus une profusion d'écrits qui n'a fait que répondre au malaise que traverse l'institution de la publication scientifique et technique. Cette soudaine accélération a répondu à des questionnements d'ordre épistémologique des chercheurs. Le contrôle par les pairs fonctionne t-il ? Est-ce le meilleur moyen de filtrer les résultats de la recherche scientifique? N'est il pas en fait un outil aux mains de certains pour perpétuer le status quo et être une manière de bloquer les résultats qui sortiraient des sentiers battus ? En d'autres mots est-il intrinsèquement conservateur? L'ouverture des réseaux et à leur tête Internet représente t-elle la panacée telle que semble le présenter certains inconditionnels du Web ? Sa permissivité (supposée ou réelle), son ouverture et sa propension à mettre l'information au bout d'un clic de souris ne sont ils pas propices à des dépassements et des dérives que certaines affaires largement relayées par les medias ont dévoilés ? Ou au contraire cette même ouverture n'est elle pas l'antidote, l'arme " fatale " contre ces affaires à répétition qui ont entachés l'image noble du chercheur en dévoilant (universellement) toute tentative de fraude, plagiat, manipulation etc....? Comme dans toute entreprise nouvelle, il est encore trop tôt pour porter un jugement définitif vu la rareté (relative) des recherches comparativement à d'autres domaines. Certains chercheurs comme Weller et Shatz se sont en fait étonnés de cet état de fait sachant surtout que le dit sujet ne concerne pas un domaine mais est appliqué à tout le Spectrum de la science. Malgré cela, une littérature importante et fournie a essayé de mettre le doigt sur les dysfonctionnements du contrôle par les pairs. Ainsi,

\footnotetext{
${ }^{(4)}$ Rousseeuw, P. J., Why the wrong papers get published, Chance: new directions for statics and computing, Vol.4, n²1, p. 41-43, 1991.

* L'article cité commence par cette quasi complainte située en haut du titre (déjà évocateur) de l'article. Il y est dit: " Ne vous êtes vous jamais posé la question de savoir pourquoi est ce que les meilleurs journaux scientifiques contiennent tant de recherches horribles alors qu'ils rejettent bêtement des articles vraiment excellents, vous forçant de les resoumettre à de très obscurs endroits [périodiques ] " ?

${ }^{(5)}$ Ziman, John, Public knowledge: an essay concerning the social dimension of science, Cambridge, Cambridge University Press, 1968, p.111

(6) Campanario, Juan Miguel, Peer review for journals as it stands today-Part 1, Science Communication, Vol. 19, $\mathrm{n}^{\circ} .3$, March 1998, p. 181

${ }^{(7)}$ Crane, Diana. The Gatekeepers of science: some factors affecting the selection of articles for scientific journals, The American Sociologist, Vol. 2, n¹ November, 1967, p. 195-201.

${ }^{(8)}$ Lederberg J., Digital communication and the conduct of science : the new literacy, Proceedings of the IEEE, Vol. 66 , p. 1315
} 
Campanario dans deux contributions importantes ${ }^{(9)}$ a analysé le contrôle par les pairs et sa situation actuelle au sujet duquel il a conclut ...." qu'il était un mécanisme de contrôle douteux " (10). Enfin, il termine par citer certains titres (tels que Speculations in Science and Technology. International Journal of Forecasting et The Journal of Management and Iconoclastic Papers) qu'il voit adéquats afin de combattre le coté conservateur de la science qui n'accepte peu (ou prou) les idées révolutionnaires et sortant des sentiers battus. On remarquera que Companario n'a pas (ou peu) abordé le coté électronique du contrôle par les pairs, orientation dont les premiers prémices ne s'étaient pas encore manifestés complètement à l'époque.

\section{Contrôle par les pairs à l'ère d'Internet}

Si à l'ère papier, les différents griefs et autres critiques du contrôle par les pairs étaient définis, des solutions proposées et expérimentées, l'avènement d'Internet dans les années $90 \mathrm{a}$ rendu ce changement révolutionnaire considérée comme un pas en avant vers plus de responsabilisation des experts, l'augmentation du nombre des experts, le choix des experts par les soumissionnaires dans certaines spécialités, etc........avaient essayé de rendre le processus plus démocratique, moins inique et ésotérique, Internet va lui donner une autre dimension plus que révolutionnaire. En effet, d'un processus où le secret était de mise, le contrôle par les pairs avec Internet devient complètement ouvert et accessible à toute personne dotée d'une connexion Internet. A cet effet, Stevan Harnad a contribué à expliquer et à rendre ce concept nouveau et révolutionnaire accessible et compris par la communauté des chercheurs. Une de ces premières et plus importantes contributions au sujet est "Implementing peer review on the net: scientific quality control in scholarly electronic journals " ${ }^{(11)}$ dans laquelle il présente la situation d'Internet et aborde la place du contrôle par les pairs en ces termes : " le filtrage du travail académique et scientifique par une forme de contrôle de qualité a toujours été implicite dés le début dans la publication papier mais il n'est pas, et n'a jamais été, en aucune façon propre au papier ". Il poursuit dans la même optique en ces termes :" le potentiel communicatif savant des réseaux électroniques est révolutionnaire. Il existe un seul secteur dans lequel le Net devra être traditionnel (c'est nous qui soulignons) et qui est la validation des idées savantes et les résultats par le contrôle par les pairs. L'expertise peut être mise en œuvre plus rapidement, plus équitablement et d'une manière efficace sur le Net, mais elle ne peut pas être supprimée comme un nombre de naïfs (qui l'assimilent à la censure ) semble le penser ". Harnad, pourtant un ardent défenseur de l'outil électronique et de ces applications à la communication scientifique, affirme que le contrôle par les pairs est la seule étape qui, dans

\footnotetext{
(9) Campanario, Juan Miguel, Peer review as it stands .Part I, Science Communication, Vol. 19, n .3 . March 1998, p. 181-211

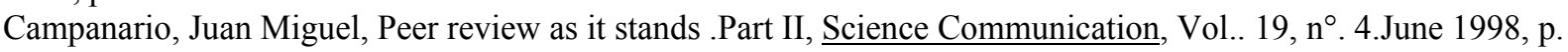
277-306

(10) Campanario, Juan Miguel, op cit Part II , p.299 .

Companario a particulièrement investigué cette problématique (le rejet d'articles s'avérant par la suite des articles très importants et même révolutionnaires) et a conclut que le contrôle par les pairs peut des fois rejeter ce genre d'articles. L'article le plus acerbe est: Have referees rejected some of the most-cited articles of all times ?, Journal of the American Society for Information Science, Vol.47, n4,p 302-310. 1996. La problématique est abordée plus en détails sur le site de Campanario http://www2.uah.es/jmc/papers2.html (Accédé le 12/06/2011)

(11) Harnad, Stevan, Implementing peer review on the net: scientific quality control in scholarly electronic journals, In: Peek, R. \& Newby, G. Eds. Scholarly publication: the electronic frontier, Cambridge [Ma, USA]: MIT Press, 1996, p. 103-108.

(Disponible à http://eprints.soton.ac.uk/252900/1/harnad96.peer.review.html )
} 
un monde pourtant post gutenberguien, ne doit pas changer. Dans une autre contribution ${ }^{(12)}$, tout aussi importante et fondatrice du contrôle par les pairs électronique, Harnad parle de la main invisible du contrôle par les pairs. Abondant dans le même sens que la précédente contribution, il fait ressortir la place qu'occupe le contrôle par les pairs dans le monde actuel de la publication scientifique. Il est intéressant de remarquer le distinguo entre " le contrôle par les pairs " et " le commentaire par les pairs ".Si le premier est, comme si abondement relaté, le filtre aussi bien qualitatif que quantitatif par lequel la Science progresse, le deuxième est le résultat de ce qu'on appellerait une publication scientifique en constante évolution. En effet, Harnad donne l'exemple de Behavioral and Brain Sciences (BBS) et Psycholoquy dont il a été l'éditeur et qui pratiquent ce qui a été aussi appelé contrôle par les pairs post publication. .Enfin, il cite l'exemple, maintenant classique, d'ArXiv qui a connu et connaît toujours un succès remarquable et dont les critères de contrôle ne sont pas très astreignants mais dont les membres font preuve d'auto contrôle et de retenue. D'ailleurs, Harnad indique que nonobstant un contrôle plus ou moins lâche, les physiciens soumettent ces mêmes pré publications à des journaux (ils deviennent alors des publications) où le processus se passe de manière traditionnelle. Nous pensons en fait qu'en plus des spécificités de la recherche en sciences physiques qui demandent des connaissances très pointues, la recherche est déjà " contrôlée " en amont par l'octroi d'un équipement onéreux qui n'est attribué qu'après examen des besoins nécessaires à la recherche.. On peut dire que les physiciens s'autocontrôlent et sont contrôlés quant aux moyens dont ils ont besoin pour travailler.

\section{Importance du sujet}

A l'orée de ce $21^{\mathrm{e}}$ siècle, un fait s'impose quant à l'importance, la prépondérance et l'inéluctabilité même des réseaux dans la vie de tous les jours mais aussi et surtout dans la vie des chercheurs et auteurs de recherches scientifiques. Il est devenu normal pour le chercheur de concevoir, appliquer et publier sa recherche et la disséminer de manière électronique. Ce qui nous parait de nos jours comme routinier, ne date que de deux décennies durant lesquelles le processus de communication scientifique a radicalement changé. Déjà en 1994, Gary Stix écrivait : " les scientifiques transmettent des rapports de recherche - de la première inspiration aux résultats finaux - par l'intermédiaire des réseaux électroniques. Même des expérimentations faites en direct peuvent être vues en ligne. Les éditeurs et les bibliothèques pourraient ne jamais être les mêmes " ${ }^{(13)}$. Cette citation des années 90 tout au début de ce qu'on pourrait appeler la révolution électronique est, deux décennies plus tard, on ne peut plus vraie. Nous réalisons nos expérimentations, communiquons nos résultats, présentons nos travaux, envoyons nos fichiers, répondons à nos " courriers ", etc.... électroniquement. Il serait pour le moins bizarre pour un chercheur de ne pas avoir une boite électronique, de ne pas posséder un (ou plusieurs) ordinateur, un scanneur, une imprimante, un disque dur externe, etc.... Cette panoplie devenue normale a été suivie par la manière dont cette masse d'information est régulée, filtrée et contrôlée. Le contrôle par les pairs est aussi devenu électronique. Il est devenu électronique, à notre avis, à deux niveaux. Il est électronique dans sa conduite journalière dans le sens où l'auteur soumet son travail, l'éditeur le reçoit, lui fait passer un premier filtre, l'envoi aux experts, communique avec eux et enfin rend son verdict (nous avons sciemment schématisé le circuit de la soumission, qui sera abordé plus en détails en infra) électroniquement sans qu'il y ait pratiquement manipulation physique du contenu. Plus important encore, il est aussi électronique dans son déroulement et processus

\footnotetext{
${ }^{(12)}$ Harnad, Stevan, The Invisible hand of peer review, Exploit Interactive, $n^{\circ}$ 5, avril 2000

(Disponible à : http://www.exploit-lib.org/issue5/peer-review )

${ }^{(13)}$ Stix, Gary, The Speed of write Scientific American, Vol. 271, n 6, p. 72-77, Decembre 1994
} 
scientifique. Cette phase du contrôle touche en fait à la quintessence non seulement de la science entrain de se faire mais aussi, on peut l'affirmer (quoique les expériences soient très récentes) à l'ethos de la science. La science n'est plus ce monde ésotérique auquel, seuls quelques initiés, ont accès elle est devenue plus ouverte, plus démocratique, plus citoyenne* Alors que l'univers papier était (et est toujours jusqu'à un certain point) prisonnier de son medium, Internet a libéré le contrôle par les pairs et l'a rendu plus juste, moins sujet aux dérives et moins inique. Cette nouvelle manière de procéder, non seulement change le processus, tel qu'il a été connu et appliqué les 50 à 60 dernières années mais change la science et son déroulement. En d'autres termes, si Internet ne touche pas au but primordial du contrôle par les pairs et qui est de filtrer, sélectionner, choisir et récompenser les meilleurs, il change par contre son processus et ses résultats. D'une étape, dont la transparente n'a jamais été la vertu cardinale, il devient complètement ouvert et change le visage de la science. Elle le change en ce sens qu'elle devient plus citoyenne, plus démocratique et même plus " wiki " . Ce changement en plus de l'ouverture dont il se prévaut et dont nous avons eu cesse de répéter les avantages, comporterait en fait des désavantages par son coté rapidité. En effet, le medium papier était connu, entre autres maux, pour sa lenteur (une des raisons qui a précipité l'avènement des réseaux) mais cette lenteur avait un coté positif en ce sens que cette lenteur permettait de vérifier la véracité, l'applicabilité et aussi la non dangerosité (dans le cas des publications médicales) de l'information avant qu'elle soit acceptée par la communauté et mise à la disposition du public en général. Ceci n'est plus possible avec les réseaux où une information peut être relayée universellement en temps réel (et même en direct) et, peut être, causer des dégâts. Cette dernière donne apporterait de l'eau au moulin de ceux qui voient en l'ouverture du processus un danger à la science. D'un autre coté, ces nouvelles fonctionnalités (ouverture, rapidité, responsabilisation etc...) ont induit un autre aspect que le medium papier n'a jamais connu : le statut de la publication. Dans ce medium, une publication après avoir passé le filtre aussi bien qualitatif que quantitatif est admise dans ce qui est la science publiée $^{* * *}$. Il en est autrement dans l'univers numérique où l'article est " vivant " et non statique. C'est le cas des remarques, expertises, ajouts post publication. Cet aspect nouveau pose le problème de la version finale du texte. L'article n'est plus figé tel qu'il a paru la première fois mais est constamment changé par les remarques, suggestions émises sur Internet. C'est le cas, par exemple, du site A.C.P. ( Atmospheric Chemistry and Physics ) ou F1000 (qui seront examinés plus en détails en infra dans la troisième partie ) et qui pratiquent pour le premier un contrôle par les pairs continu et pour le deuxième une seconde expertise d'un article déjà publié et expertisé. Nous avons cité ces deux cas car se pose la question importante suivante : quelle version de l'article est validée et fait figure d'étalon ? Certains chercheurs ont parlé pour décrire cette nouvelle donne d'article 2.0, en claire analogie aux

\footnotetext{
* Ce concept de citoyenneté, de biens communs, de patrimoine de l'humanité et d'ouverture dépasse, et de loin, la science. Elle est le résultat direct d'un monde de plus en plus ouvert, connecté et surtout responsabilisé. Les différentes affaires ayant entaché le monde des affaires, de la politique et aussi de la science, où la moindre incartade est magnifiée et portée à la connaissance de tout un chacun, en sont les meilleures preuves

** Le mot wiki est d'origine hawaïenne et veut dire " rapide ". Pour certains, wiki serait l'acronyme de l'expression anglaise " What I Know Is " ("Voici ce que je sais").Il est raconté que c'est l'informaticien nord américain Ward Cunnigham qui a le premier utilisé ce mot lorsqu'il fut invité à l'aéroport de Honolulu de prendre le wikiwiki bus T52 (http://gsintranet22.blogs.letelegramme.com/archive/2006/12/13/etymologie-dumot-wiki.html). Depuis le mot a été utilisé dans différentes spécialités comme wikimedia, wikistrike, wikimapia, wikileaks etc.... et bien sur Wikipedia qui est l'encyclopédie à laquelle toute personne peut participer. Nous utilisons ce mot typiquement Internet à escient car la science de nos jours est devenue de par son essence, son déroulement, sa certification très (peut être trop...) rapide

${ }^{* * *}$ La seule instance où l'article, la recherche sur papier est " changée " est le cas des rétractations à cause généralement de problèmes éthiques (la plupart du temps du plagiat, de falsification, etc....)
} 
logiciels (ou même du Web) qui passent de la version 1.0 à 2.0 à 3.0 etc .... en se posant la question suivante :" la science peut-elle adopter sans risque l'esprit bêta du web 2.0 et l'idée d'une remise en cause perpétuelle dans une volonté d'amélioration " ${ }^{14)}$ ? Cette " perpétuelle " remise en cause serait elle préjudiciable à la science ? Peut on comparer l'article (ou même le chercheur tel que l'ont osé Gallezot et Le Deuff) à un logiciel dont la dernière version plus performante annule la précédente? Rien n'est moins sûr car l'article est une unité qui véhicule une idée, une théorie, une affirmation scientifique qui ne saurait s'accommoder de " remise en cause perpétuelle". Là est tout le défi posé par ces nouvelles technologies qui bousculent nos habitus de recherche. Et c'est surtout dans cette partie primordiale, nodale de la construction de la science qu'est le contrôle par les pairs que s'exerce cette révolution et qui sera le centre de notre étude.

\section{État de l'art de la question}

Bien que récent dans son application, le contrôle par les pairs a donné une littérature assez fournie. les références sont souvent des articles alors que les monographies sont moins nombreuses et surtout plus récentes. Stephen Lock, ${ }^{(15)}$ fut le premier en 1985 à étudier le processus. Lock en tant qu'éditeur du British Medical Journal examine le contrôle par les pairs et présente une analyse complète du processus s'adressant aux éditeurs, auteurs et experts du domaine biomédical. Il fait ressortir les défauts et les mérites du contrôle par les pairs. Son étude est provocatrice et remet en question les idées préconçues que les différents acteurs ont au sujet du processus. L'analyse de Lock soulève un nombre de questions dont " quel est le problème avec le contrôle par les pairs ? " à laquelle il répond en analysant les différents composants spécifiques du processus et les résultats en étudiant les réponses des experts ainsi que les réponses des auteurs. Dans "Editors as gatekeepers " (16), les auteurs analysent une autre facette du travail éditorial et qui est celle du " Gardien du temple ". Certaines questions posées sont : pourquoi certains manuscrits sont publiés et d'autres pas ? Qui prend les décisions dans les journaux scientifiques et comment ces décisions sont elles faites? D'un autre coté, cette monographie réunit des éditeurs de journaux en sciences sociales (sociologie, anthropologie, science politique, psychologie, etc....) et donne une vue des rôles qu'ils assimilent ainsi qu'aux relations entre éditeurs, auteurs, experts et lecteurs. Enfin, cette monographie donne des conseils quant aux journaux auxquels il faut soumettre, comment lire les lettres des éditeurs concernant les révisions et aussi comment resoumettre. Les auteurs donnent une vue d'ensemble des expériences qu'ils ont vécu en tant que " Gardien du temple ". Cette fonction (et appellation) est très importante car les éditeurs se considèrent comme les dépositaires des critères les plus sérieux de la science. Ils se considèrent, pratiquement, comme investis d'une mission d'où l'appellation un peu mystique. A la même période, H.D. Daniel publie un livre dans la même optique intitulé " Guardians of science : fairness and reliability of peer review " ${ }^{(17)}$. On remarquera que l'idée développée par Daniel est la même que la référence précédente et qui a trait à l'idée de gardien de la science (le mot science ayant remplacé le mot temple). Dans cette monographie, Daniel présente les résultats d'une enquête détaillée du système de contrôle de Angewandte Chemie, l'un des périodiques en chimie les plus prestigieux au monde pour l'année 1984. En particulier son

\footnotetext{
${ }^{(14)}$ Gallezot, Gabriel, Le Deuff, Olivier, Chercheurs 2.0 ? Les Cahiers du Numérique , Vol 5 , n², p.29., 2009 Disponible à : http://hal.archives-ouvertes.fr/docs/00/39/62/78/PDF/Chercheur2.0-Gallezot LeDeuff-2009.pdf

${ }^{(15)}$ Lock, Stephen, A Difficult balance : editorial peer review in medicine, London,Nuffield Provincial Hospitals Trust, 1985, $172 \mathrm{p}$

${ }^{(16)}$ Simon, Rita J., Fyfe, James J., Editors as gatekeepers, Lanham [Md]. Rowman and Littlefield, 1994, 280 p

(17) Daniel, H.D., Guardians of science: fairness and reliability of peer review, Mannheim [Allemagne], VCH Verlagsgesellschaft, 1994, 118p.
} 
analyse se concentre sur le contenu et le niveau d'accord des rapports des experts, le devenir et l'impact des soumissions rejetés par Angewandte Chemie et publié ailleurs ainsi que niveau de partialité dans les décisions éditorial et des experts et basé sur des aspects d'importance secondaire, tels que la nationalité, titre universitaire et la spécialité de la soumission de l'auteur. Ceci est du vraisemblablement à la nouveauté du processus qui s'impose lentement et progressivement. " Peer review in health sciences " (18) en 1999 se veut une sorte de guide du contrôle par les pairs mais se cantonne au domaine médical. En 2003, une deuxième édition parait : elle inclut un chapitre sur le contrôle par les pairs électronique. C'est dire l'importance de plus en plus grandissante de la problématique en question. En 2001, parait " Editorial peer review : its strengths and weaknesses " ${ }^{(19)}$ un ouvrage qu'on pourrait considérer de référence par Ann C Weller. Dans cette étude, Weller fournit une revue systématique des études empiriques du processus du contrôle par les pairs entre 1945 et 1997.Malgré cela un chapitre intitulé "Peer Review in an Electronic Environment " est inclut à la fin démontrant l'importance du sujet pour le contrôle par les pairs. Enfin, l'auteur remarque que le nombre d'études présentées dans son travail est du à une simple raison : le contrôle par les pairs bien que dans certains cas est présent plus dans certaines spécialités que d'autres (la médecine par exemple), est une discipline qui touche tout le Spectrum de la science. Shatz dans un livre tout aussi séminal ${ }^{(20)}$ aborde quant à lui du point de vue de la philosophie contemporaine. Il aborde dans ce sens des angles de recherche aussi variés que les arguments pour et contre l'expertise à l'aveugle, le prétendu conservatisme du contrôle par les pairs, le statut anormal des publications non expertisées telles que les articles invités ou les publications sur Internet pour les cas de promotion et de titularisation et enfin le futur du contrôle par les pairs à l'âge d'Internet. On remarquera seulement que cette étude malgré son orientation philosophique n'en est pas moins une œuvre de référence dans le sujet. La dernière contribution au sujet est datée de $2007^{(21)}$. C'est une monographie complète qui peut être lu comme un guide pratique du processus d'expertise. Le livre offre des instructions essentielles pour les éditeurs à tous les niveaux, alors qu'il peut être aussi bénéfique de par sa lecture à tout auteur potentiel. Ce livre réussit à fournir des conseils aux experts ainsi que les rédacteurs chargés de la gestion d'un manuscrit dans une revue scientifique donné.

Si les monographies sur le sujet sont rares, les articles de périodiques par contre font légion et font la littérature du sujet. La raison en serait, en plus de la nouveauté de l'étude du phénomène, le manque de travaux précédents. En effet, comme il a été déjà dit, le sujet est relativement nouveau à cause du secret qui l'a toujours entouré qui a influé sur la production de recherches. Zuckerman et Merton ${ }^{(22)}$ ont sans doute en 1971, écrit la première et très importante ${ }^{*}$ contribution au sujet Merton y décrit trois axes principaux de recherches ainsi que leurs résultats. Premièrement, l'émergence et l'institutionnalisation du système de contrôle par les pairs au $17^{\mathrm{e}}$ siècle, deuxiememnt une comparaison du taux d'acceptation de 83 journaux contemporains dans 15 domaines de la science et le savoir et troisièmement enfin analyse les pratiques des auteurs, experts et éditeurs. Cette étude bien que vieille quatre décennies est toujours d'actualité et a été citée abondamment dans la littérature du sujet, preuve en est les chiffres du Social Sciences Citation Index (SSCI) et le Science Citation

\footnotetext{
${ }^{(18)}$ Goodlee, Fiona, Jefferson, Tom, Ed. Peer review in health sciences, London, BMJ Books, 1999, $271 \mathrm{p}$.

(19) Weller, Ann C., Editorial peer review: its strengths and weaknesses, Medford [N.J.], Information Today, 2001, 344p.

${ }^{(20)}$ Shatz, David, Peer review: a critical inquiry, Lanham [Md]. Rowman and Littlefield, 2004,249p.

${ }^{(21)}$ Hames , Irene, Peer review and manuscript management in scientific journals: guidelines for good practice, Hoboken [N.J.],Wiley-Blackwell,2007,306p.

${ }^{(22)}$ Zuckerman, Harriet, Merton Robert K., op.cit.

${ }^{*}$ Le Social Sciences Citation Index(SSCI) et le Science Citation Index (SCI) indiquent que cette recherche a été citée dans plus de 165 publications.
} 
Index qui la classent parmi les études les plus citées. Avant Merton, Crane avait étudié plus spécifiquement le rôle des gardiens du temple ${ }^{(23)}$ en posant, entre autres, la question suivante : le diplôme, l'origine et l'institution influent ils sur la probabilité d'avoir un article accepté par l'une des revues importantes de la sociologie?

Nous remarquerons que les contributions de Merton et Crane émanent de deux sociologues pour qui l'aspect méthodologique est primordial. En 1982, l'étude de Stephen et Ceci ${ }^{(24)}$ a causé un mini séisme dans le landernau de la publication scientifique par les conclusions aux quelles elle est parvenue. En effet, cette étude avait resoumis aux mêmes journaux des articles déjà acceptés. Ces derniers furent refusés en deuxième instance, ce qui poussa les auteurs à conclure à un préjugé institutionnel et personnel. L'affaire Sokal ${ }^{(25)}$ avait aussi abondé dans la même direction et avait prouvé que le contrôle par les pairs pouvait être manipulé et même ridiculisé $^{*}$. Si le sujet du contrôle par les pairs n'a pas été abondement abordé à cause de raisons multiples, l'avènement d'Internet et ses fonctionnalités l'a révolutionné et a donné lieu à de multiples écrits étudiant les avantages et les défauts de cette ouverture. C'est sans conteste Stevan Harnad, figure connue du libre accès qui a le plus écrit sur le sujet. Si parmi ces innombrables publications, les deux publications précédemment citées ( Implementing peer review on the net: scientific quality control in scholarly electronic journals et The invisible hand of peer review) font référence en abordant spécifiquement le sujet du contrôle par les pairs sur Internet, une autre publication non moins importante ${ }^{(27)}$, Harnad utilise le concept révolutionnaire de " scholarly skywriting " ** dans lequel il démontre les fonctionnalités révolutionnaires du Net dans le continuum de la science qui associe, toujours selon Harnad, la rapidité de la pensée à celle d'Internet. Harnad prétend que le " scholarly skywriting " est important car il combine les avantages de la communication orale et écrite, la restauration de la nature synchrone, bilatérale et interactive de la tradition orale à la permanence de la tradition écrite.

\footnotetext{
${ }^{(23)}$ Crane, Diana, The Gatekeepers of science: some factors affecting the selection of articles for scientific journals, The American Sociologist, Vol. 2, p. 195-201, Novembre 1967.

${ }^{24)}$ Peters, Douglas P., Ceci,Stephen J., Peer-review practices of psychological journals: The fate of published articles, submitted again, Behavioral and Brain Sciences, Vol. 5, nº 02, p 187-195, June 1982

${ }^{(25)}$ Sokal, Alan D.,Transgrerssing the boundaries : toward a transformative hermeneutics of quantum gravity, Social Text,n ${ }^{\circ} 46-47$, p.217-252, Spring -Summer 1996

"Sokal pour prouver l'inanité du contrôle par les pairs avait soumis une recherche assaisonnée d'inepties. Après qu'elle eut été acceptée, il envoya une lettre à un autre journal pour dénoncer le manque de contrôle et surtout dénoncer des prises de positions d'une certaine gauche américaine. Il conclut en ces termes : "....estimant que l'absurdité de son article était évidente, Sokal en conclut que la revue méconnaît les règles de la rigueur intellectuelle puisqu'elle s'est permis de publier un article sur la physique quantique sans prendre la précaution de consulter un spécialiste du domaine ". Voir à cet effet, Sokal, Alan D., A Physicist experiments with social studies (http://www.physics.nyu.edu/faculty/sokal/lingua franca v4.pdf ). Il est à signaler que la revue en question ne pratiquait pas de contrôle parcequ ..." elle visait à publier des idées novatrices, et estimait que l'absence de comité encourage plus de recherches originales et moins de recherches conventionnelles ". Ce dernier point est très important car le contrôle par les pairs a été accusé, entre autres, de ne pas accepter d'idées novatrices et d'être intrinsèquement conservateur.

(27) Harnad, Stevan, Scholarly skywriting and the prepublication continuum of scientific inquiry, Psychological Science, Vol. 1: 342 - 343 ,November 1991.

"Expression traduite tantôt en " écriture scientifique dans le ciel " ou " scielographie savante " mais qui ne nous semblent pas adéquates car le concept est nouveau. L'idée est que le Net permet à tout un chacun de voir et de commenter comme si le message était écrit dans le ciel à la manière des avions qui affichent des bannières dans le ciel.
} 
Il faut savoir qu'Harnad en plus de l'idée révolutionnaire de " l'écriture scientifique dans le ciel " avait déjà contribué au sujet de la société " post gutenberguienne " qui serait celle que nous vivons. Harnad se propose d'aborder l'application du contrôle par les pairs sur Internet. Il explique que les réseaux électroniques ont permis à l'édition scientifique de passer d'un modèle commercial dans lequel l'auteur vend son travail sur support papier à un modèle collaboratif. Pour cela, le contrôle par les pairs devra être mis en œuvre sur le Net, recréant ainsi les hiérarchies de revues papier qui permettent à la communauté de juger. Le Net offre également la possibilité de mettre en œuvre plus efficacement et équitablement le contrôle par les pairs, et de le compléter avec ce qui est réellement la dimension révolutionnaire du Net : la publication interactive sous forme de commentaires des pairs ouvert sur les travaux publiés et en cours. Cette contribution ${ }^{(28)}$ est fondatrice des idées de Harnad dans laquelle il avance que la société post gutenberguienne est la société actuelle débarrassée des liens papier. Cette quatrième révolution vient après celle de la parole, l'écriture et l'impression. Elle libérerait l'homme des contraintes dues à l'univers papier et le propulserait dans un monde numérique dominé par" l'écriture scientifique dans le ciel ".

Si Harnad a sans conteste contribué à la recherche de solutions face aux défis que pose le libre accès à la publication scientifique au point où il a hérité du surnom de "archivangeliste" (29), d'autres contributions non moins importantes ont essayé d'aborder le sujet. Arms ${ }^{(30)}$ aborde la problématique des méthodes par lesquelles le contrôle de la qualité est établi et quels sont les indicateurs qui permettent à un utilisateur de reconnaître un bon travail sur Internet ? Pöschl ${ }^{(31)}$ quant à lui aborde la problématique de l'assurance de la qualité scientifique par l'intermédiaire du contrôle par les pairs interactif et la discussion publique. Pöschl est l'éditeur d'A.C.P. que nous verrons plus en détails en infra. Piolat et Vauclair ${ }^{(32)}$ abordent la façon dont l'expertise est transformée par Internet en analysant la manière de favoriser une bonne gestion du huis clos ou ouvrir l'expertise et rendre possible les échanges entre les différents protagonistes de l'expertise. Dominy, Bradley et Bhatt ${ }^{(33)}$ explorent la problématique de l'irruption de Google et comment cela a eu un impact sur la manière dont la science est faite, expertisée et publiée. Suls et Martin ${ }^{(34)}$ examinent les critiques du système traditionnel et décrivent plusieurs alternatives afin de faciliter la discussion et le débat ainsi que la question du nombre d'experts et la différence des taux de rejet entre sciences naturelles et sociales surtout en psychologie. Enfin Sandewall ${ }^{(35)}$ relate l'expérience de son journal ETAI (qui sera aussi examiné plus en détails en infra) et dans laquelle il identifie certaines des difficultés que le contrôle ouvert rencontre. Il est à signaler que sur le site de

\footnotetext{
(28) Harnad, Stevan Post-Gutenberg galaxy: the fourth revolution in the means of production of knowledge, Public-Access Computer Systems Review, Vol. 2, $n^{\circ} 1$, p. 39-53.1991

${ }^{(29)}$ Richard Poynder, The OA Interviews: Stevan Harnad, Open and shut , Sunday, July 2007

(Disponible à : http://poynder.blogspot.com/2007/07/oa-interviews-stevan-harnad.html

(30) Arms, William Y., What are the alternatives to peer review? Quality control in scholarly publishing on the Web, Journal of electronic publishing, Vol.8, $\mathrm{n}^{\circ} 1$ August 2002

(Disponible à : http://quod.lib.umich.edu/cgi/t/text/textidx?c=jep; view=text;rgn=main;idno=3336451.0008.103)

(31) Pöschl, Ulrich, Open access : scientific quality assurance by interactive peer review \& public discussion http://www.atmospheric-chemistry-and-physics.net/pr_acp_open_access_scientific_quality_assurance.pdf

(32) Piolat A., Vauclair J.,Le processus d'expertise éditoriale avant et avec Internet, Pratiques psychologiques, Vol. 10, n 3 , p.255-272, Septembre 2004

${ }^{(33)}$ Dominy,Margaret, Bradley, Jean-Claude, Bhatt, Jay, Peer review in the google age: is technology changing the way science is done and evaluated ?, 2006 [Presentation Power Point]

(Disponible à http://eprints.rclis.org/7411/)

(34) Suls, Jerry, Martin, René, The Air we breathe: a critical look at practices and alternatives in the peerreview process, Perspectives on psychological science, Vol. 4, ${ }^{\circ}$. 1, p.40-50, January 2009

${ }^{(35)}$ Sandewall,Eric, Maintaining live discussion in two-stage open peer review, Frontier in computational neuroscience, 21 Februray 2012
} 
Frontier in computational neuroscience qui publie l'article, les noms des trois experts et leur affiliation sont cités avec l'article*. Ceci est une des conséquences de l'ouverture qu'Internet a permise et qui aurait été tout simplement impensable dans les schémas traditionnels où le secret le plus complet était observé.

\section{Problématique}

Les raisons ayant guidé notre choix du sujet sont multiples et variées. Elles peuvent être résumées dans l'importance sans cesse grandissante du libre accès qui en quelques années est devenu incontournable dans le domaine de la publication scientifique et de l'importance accrue qu'occupe le contrôle par les pairs dans un monde où l'abondance de l'information (la fameuse explosion documentaire) impose un filtre aussi bien qualitatif que quantitatif. La combinaison de ce deux questions et leur actualité sous tend notre travail de recherche.

Le libre accès étant une réalité nouvelle (il n'est vieux que de deux décennies et a admirablement épousé les contours d'Internet. Nous pouvons même affirmer qu'il est - le libre accès - Internet), il est un sujet abondamment abordé et étudié. Sa nouveauté et les promesses qu'il recèle en ont fait un sujet à la mode induisant par delà une abondance de littérature. De son coté, le contrôle par les pairs, bien que présent sous une forme ou une autre dans la publication scientifique n'a connu son apogée qu'avec le fin de la deuxième guerre mondiale et le formidable effort de recherche qui en a résulté. Si la littérature sur le sujet est clairement abondante (beaucoup plus sous forme d'articles et peu de monographies), elle est pratiquement anglo-saxonne et peu d'écrits le sont en d'autres langues (surtout en français). Nombre d'explications ont étés donnés mais il semblerait que la domination du monde de l'édition scientifique par les anglo saxon en soit la raison principale. D'un autre coté, il est de notoriété que le monde de la publication scientifique en raison surtout des politiques de dérèglement des années 80 est devenu fortement perturbé et l'arrivée massive d'éditeurs commerciaux (ayant quelque peu remplacé ou du moins concurrencé les éditeurs des sociétés savantes et universitaires) n'a fait exacerber une crise latente. La fameuse crise des prix des périodiques a été le catalyseur qui a poussé les chercheurs, les bibliothécaires et même les éditeurs à chercher une solution à ce qui s'apparentait à une sorte de cartellisation du savoir. En effet, il est bon de savoir que cette fameuse crise avait conduit l'auteur à la situation incongrue suivante : ayant vendu les droits de sa recherche au journal contre sa publication, il ne peut plus y accéder par l'intermédiaire de sa bibliothèque et doit des fois payer pour voir son propre travail. Face à cette situation pour le moins bizarre, la réaction des bibliothèques afin de juguler les prix de maisons d'éditions aux visées oligopole ${ }^{*}$ a été de former des consortia afin de juguler la hausse des prix. Les éditeurs, forts de leur expérience, répondirent par ce qui est connu sous l'appellation de " Big Deal" (bouquets) et qui est un ensemble de titres électroniques d'un éditeur donné et dont le prix global est inferieur au prix individuel des abonnements aux titres achetés séparément. Si cette option parait de prime abord avantageuse, elle comporte nombre d'inconvénients dont le plus évident est le fait que la bibliothèque se trouve pratiquement condamnée à recevoir des titres dont elle n'a pas besoin mais surtout cette option comporte une clause qui lie la bibliothèque à l'éditeur pour des années et surtout avec une augmentation annuelle fixe qui à la fin grève les budgets et élimine tout avantage ${ }^{(30)}$.C'est à partir de ces données en termes de l'apparition du libre accès

\footnotetext{
* (http://www.frontiersin.org/Computational_Neuroscience/10.3389/fncom.2012.00009/abstract).

*Forme de marché dans lequel un très petit nombre d'entreprises ont le monopole de l'offre d'une marchandise ou d'un service et sont ainsi soustraites au régime de libre concurrence (http://www.cnrtl.fr/definition/oligopole )

${ }^{(30)}$ Bivens-Tatum, Wayne, The Big Deal's damage, Library Journal, 9 Mai 2013, Disponible à : http://lj.libraryjournal.com/2013/05/opinion/peer-to-peer-review/the-big-deals-damage-peer-to-peer-review/\#
} 
comme medium incontournable de la recherche scientifique, de l'irruption d'Internet concurremment comme adjuvant mais aussi moteur du libre accès et enfin de l'influence de ces mutations profondes sur le contrôle par les pairs que se formule notre problématique.

Construction de l'objet

Le contrôle par les pairs, étape incontournable et nécessaire de la science publiée et de ce fait labélisée, est au centre de débats et de critiques ayant touché à sa viabilité et existence même. Si d'un point de vue scientifique, méthodologique et même éthique, il serait pour le moins hasardeux d'en nier l'importance et la prépondérance, il s'est élevé des voix pour en critiquer le déroulement. Il a été, entre autres, décrit à la fois de ",....sujet à des abus,...ayant des experts qui sont incompétents, .....lent et couteux ...." (36) dénotant un profond malaise qui touche aux fondements de la science. Nous disons de la science car en plus d'être méritocratique et même élitiste dans son approche, la science se construit par incrémentations qui s'appuient les unes sur les autres à l'image de l'imbrication des parties d'une maison. Nous utilisons cette analogie car la science est une et indivisible et ne peut se faire par une seule personne, groupe ou entité. Le ciment - pour rester dans l'analogie de la maison - qui lie ces différentes parties est le contrôle par les pairs qui agit en tant contrôleur de la qualité du travail. Ce ciment était, à l'époque papier, omnipotent et surtout secret et l'avènement du libre accès (et d'Internet) en a complètement changé les effets. Nous ne nous posons plus la question de l'amélioration du contrôle par les pairs (qui reste d'actualité) mais plutôt de son adaptation, de son déroulement et l'influence que le libre accès aura sur lui. La question est la suivante : le contrôle par les pairs peut il survivre, sous sa forme actuelle, aux profondes mutations que connait la publication scientifique ? Est-il condamné à disparaitre comme certains l'appellent de tous leurs vœux, arguant du fait que l'une de ces deux fonctions (filtre quantitatif) n'a plus sa raison d'être dans un monde numérique pratiquement infini ? Devrions-nous accepter de " laisser des millions de fleurs éclore ?" (37) comme l'ont déclarés certains en une claire allusion à une sorte égalité absolue pour tout un chacun de publier à charge pour " la sagesse des masses " ( wisdom of the crowd) de repérer la (ou les fautes) ? Ou alors devrions-nous accepter la loi de Linus Torwald qui dit " avec suffisamment d'yeux, les bugs sont minimisés " ( with enough eyeballs, all bugs are shallow) arguant du fait que l'exposition des idées au plus grand nombre ne peut qu'aider à repérer et éventuellement corriger fautes et autres défauts.

Il est clair que l'avènement d'Internet a clairement changé les règles du jeu de la compétition scientifique. Il l'a changé aussi bien dans son déroulement, sa philosophie, son but et ses règles. Au risque de paraitre élitiste, le contrôle par les pairs s'est " peopolisé " et n'est plus l'apanage d'une caste, ni d'un groupe sélectionné. La question qui se pose : cette abaissement des critères est elle préjudiciable à la science, plus ne menace t elle pas le contrôle par les pairs en tant qu'institution " gardienne du temple " [du savoir] ? C'est autour de ce cadre, cette question que s'articule notre problématique du devenir du contrôle par les pairs face aux défis que lui pose le libre accès et Internet.

On peut sans risque de se tromper arguer du fait que cette soudaine (ou du moins rapide) irruption du modèle électronique a eu pour effet de radicaliser le débat (déjà tendu) sur le contrôle par les pairs. Il est devenu dichotomique entre ceux qui, d'un coté, croient que ce changement de paradigme, de Weltanschauung moderne est à même de solutionner tous les

\footnotetext{
(36) Schmelkin , Laura, Perr review : standard or delusion, Division 5, Presidential address to the American Psychological Association, 3 Aout 2003

(37) Perloff, Richard M. and Perloff, Robert, Improving research on and policies for peer review practices $\underline{\text { In }}$ : Harnad, Stevan Ed. Peer commentary on peer review, Cambridge , Cambridge University Press, p.48-49
} 
maux dont est accablé le contrôle par les pairs. Une sorte de vision techniciste qui ferait la part belle à l'expression maintenant culte de Charles Clark " La réponse à la machine est dans la machine" (The answer to the machine is in the machine). De l'autre coté, se trouve ce qui s'apparenterait à des traditionnalistes qui verraient dans l'immixtion du tout technique, une sorte de déviance du coté humain du travail de l'expert. Ces derniers seraient considérés comme des mandarins soucieux des acquis, avantages qu'ils ont acquis dans le système traditionnel. C'est autour de ces deux groupes, orientations, point de vue, générations, approches que ce cristallise le débat autour du contrôle par les pairs et son devenir. Pour notre part, en appréhender les tenants et aboutissants c'est faire la synthèse de deux volets d'études complémentaires et qui sont :

\section{Internet :}

Il est pratiquement devenu impossible de ne pas citer Internet dans notre vie .Il est devenu en un court laps de temps un medium qui a envahi notre vie de tous les jours. Etant relativement nouveau, son influence, ses résultats et ses retombées ne sont pas, et de loin, encore appréhendés. Ce qui est sur, c'est que la communauté scientifique se l'est approprié et en a fait un usage qui, globalement, peut être décrit de positif quant à la conduite de la recherche scientifique. Nous nous en apercevons peut être pas (à cause d'un manque de recul du à la vitesse à laquelle ces changements se produisent) mais nos habitus de recherche ont globalement et irrémédiablement changés. Qui d'entre nous utilise encore, à titre d'exemple, un dictionnaire papier avec la multitude de dictionnaires sur la Toile ? Qui n'a pas recours à Google (malgré toutes les critiques que ce géant essuie) pour trouver une information? Enfin, qui d'entre nous n'a pas recours à Wikipedia, ne serait que pour trouver - rapidement - une information sur un sujet donné ? Internet a ouvert de nouveaux horizons mais par la même a produit des effets sur notre manière de faire la recherche dont le présent travail se propose d'en voir les prémices

\section{$\underline{\text { Le libre accès : }}$}

Il est difficile parfois de distinguer libre accès et Internet .En effet, les deux sont tellement imbriqués et dépendant l'un de l'autre qu'il est difficile de savoir qui a le plus promu l'autre. Est-ce le libre accès qui en mettant en première ligne la crise des prix des périodiques a accéléré l'avènement d'Internet ou est ce au contraire Internet qui en se développant a permis l'éclosion du libre accès ? Il est bizarre de constater que la même question s'est posé entre le papier et l'imprimerie pour savoir qui a le plus contribué à l'avènement de l'autre. Nous pencherons plutôt dans notre cas à l'hypothèse d'Internet ayant ante daté le libre accès et ayant aussi permis les avancées qui de nos jours nous paraissent évidents mais qui avec le recul ne le sont pas. Qui d'entre nous aurait pensé qu'il était possible d'envoyer un "courrier" à l'autre bout du monde en quelques secondes ou recevoir un article sous forme électronique en cliquant simplement sur un lien ou, encore plus, présenter une communication à une distante conférence tout en étant chez soi ? Cette assertion est tellement présente que certains chercheurs se sont posé la question :" Est-ce qu'Internet tue la distance ? " en allusion au rétrécissement des distances dans un monde hyper connecté.

A la lumière de toutes ces données, ces nouveautés et autres innovations, que s'articule la problématique que nous investiguons et qui pourrait être schématiquement formulée en une question nodale qui est : le contrôle par les pairs qui dans sa configuration actuelle est sujet à des questionnements quant à son déroulement, ses pratiques, ses résultats, sa viabilité même peut il être amélioré par l'ouverture d'Internet et de son corollaire immédiat dans le recherche 
scientifique, le libre accès ? A partir des constats des données du monde académique et de son segment publication contrôlée par les pairs et de ces incohérences nous formulons nos hypothèses de travail .Nous en avons retenu trois :

-le contrôle par les pairs tel qu'il a été pratiqué restera, globalement, le même dans son déroulement étant donné qu'il a donné satisfaction malgré les déficiences dont il est accusé. Cette situation résulte de son importance et surtout qu'aucune autre alternative viable n'a été trouvée, il reste la seule solution acceptable.

-le contrôle par les pairs est une partie indissociable de la conduite de la science. Il ne peut de ce fait en être en être dissocié ni dans sa conception ou sa conduite. La science évoluant dans ses pratiques, le contrôle par les pairs se doit d'évoluer de la même manière. Cette évolution ne se fera pas d'une manière radicale ni chronologiquement (elle sera progressive) ni dans la manière (certains taches resterons humaines, manuelles et ne pourrons pas être automatisées)

-le contrôle par les pairs devra évoluer radicalement à l'instar de la pratique de la science devenue très technique. Les différentes affaires et scandales, les tendances de favoritisme, les préjugés imposent cette révolution. Le contrôle par les pairs deviendra entièrement ouvert, dynamique et surtout démocratique et moins inique.

Ces hypothèses s'articulent autour de la question contrôle par les pairs et conduite moderne de la science (Internet et libre accès), question qui étudiera la faisabilité et la viabilité d'un contrôle par les pairs électronique. D'autre part, il est important de se poser la question quelle sera le nouveau visage de la science à l'aune de ces changements qui sont, comme nous avons eu l'occasion de le signaler, d'ordre épistémologique et paradigmatique et touchent à la quintessence de la conduite de la science.

\section{Présentation des trois parties du travail}

Nous avons partagé, pour des raisons méthodologiques et pratiques, notre travail en trois parties distinctes .Ceci a été motivé par un besoin d'en faire un travail qui s'inscrit dans un contexte historique et surtout de continuité. En effet, si l'objet de notre travail est de savoir la place du contrôle part les pairs dans un monde tendant à être de plus en plus électronique et de moins en moins papier (ou pour être plus en accord avec les nouvelles appellations, orientations et notions : de plus en plus numérique et de moins en moins analogique), il aurait été pour le moins présomptueux de ne pas parler de l'origine de ces formidables mutations qui se passent en direct sous nos yeux. Le périodique électronique moderne disponible 24 heures sur 24, partout, au bout du clic d'une souris et avec des fonctionnalités très avancées est la résultante des mutations qu'a vu depuis 1665 la fondation pratiquement simultanée du Journal des Sçavans et des Philosophical Transactions. L'avènement du périodique au $17^{\mathrm{e}}$ siècle répondait à un besoin pressant de rapidité de communication que le livre n'arrivait pas à satisfaire. Cette première partie sera de ce fait, une sorte de récapitulatif des développements que la communication a connus. Des premiers embryons primitifs tels que les pierres, les murs de cavernes, des bouts de bois, l'argile et certains matériaux des plus hétéroclites, l'avènement ou du moins l'introduction du papier a constitué un révolution des plus formidables dans l'histoire de l'humanité. En effet, alors que le support était des plus varié et ne permettait pas une normalisation (aussi primaire soit elle), le papier permit une première unification du support. L'imprimerie de son coté, représente, peut être plus que le papier, une révolution et a fait passer l'Homme, la civilisation, le savoir à un autre tempo qui, de nos jours et avec les moyens sophistiqués dont nous disposons, ne peut être compris. 
L'association des deux occurrences (papier et imprimerie) ont permis à l'Homme de faire un grand bon en avant et dont les effets se font sentir jusqu'à nos jours. C'est ces développements que nous aborderons dans le premier chapitre de la première partie.

Après l'avènement du papier et de l'imprimerie, la production des livres atteint un niveau très appréciable pour l'époque. Les chiffres de la production livresque associés au progrès de l'Europe post Moyen Age font faire un bond aux innovations techniques et à la science en général. C'est dans ce deuxième chapitre que nous essayerons de voir comment la transition du livre au périodique s'est opérée. C'est une période assez mal documentée vu la quasi cohabitation du livre et du nouveau medium qui n'arrivait pas à s'imposer et qui des fois étaient confondus. Le lancement des deux premiers périodiques scientifiques (Le Journal des Sçavans et The Philosophical Transactions) et les autres titres les ayant suivis au cours du $18^{\mathrm{e}}$ et surtout $19^{\mathrm{e}}$ va consacrer le périodique comme le vecteur le premier et le medium de prédilection de transmission du savoir. Cette partie sera abordée dans une énumération des différents périodiques, leur spécialisation et enfin la présentation de deux prototypes représentatifs de périodiques scientifiques modernes : Science et Nature. Ces deux titres sont deux périodiques qui en plus de leur importance et leur prestige reconnu universellement, sont parmi les seuls périodiques généralistes encore existants. En effet, l'une des caractéristiques prépondérante du périodique scientifique moderne contrôlé par les pairs est son extrême spécialisation. En même temps cause et effet, la spécialisation est devenue la norme dans la science publiée. Enfin cette première partie sera aussi l'occasion de comparer les différentes typologies faites du périodique scientifique .Cette comparaison de trois typologies choisies car représentant des points de vue différents fera ressortir les analogies et les différences entre différents chercheurs.

La deuxième partie de cette étude quant à elle se propose de présenter le libre accès depuis son inception jusqu'à ses derniers développements sans cesse croissants. La trame de notre travail s'appuie très fortement sur cette partie car le libre accès y est prépondérant. Non seulement, il l'est car notre étude s'appuie dessus mais aussi et surtout pour une autre raison plus prosaïque : le grand déficit quant à la connaissance de cet outil de recherche surtout au niveau national. En effet, malgré ses avantages et ses avancées très conséquentes de par le monde, notre pays se trouve à la traine même comparé aux pays dont le niveau économique, scientifique et de développement est loin d'égaler le notre. Notre étude et présentation de ces majeurs accomplissements serviront à, nous l'espérons, faire connaitre ce medium dont aucune nation ne peut se passer dans son développement scientifique et technique. Après un bref mais nécessaire chapitre destiné à faire connaitre ce mouvement avec les raisons ayant conduit à son avènement, ses différentes étapes, ses appels, ses définitions ${ }^{*}$ et ses résultats, on abordera un chapitre dans lequel on présentera les entrepôts d'archives ouvertes. On ne parle pas de libre accès sans parler des entrepôts d'archives (appelés aussi voie verte) qui sont des sites dans lesquels un nombre de sites d'archives ouvertes est entreposé. Ces entrepôts sont au cœur du pourquoi du libre accès et aussi une claire preuve que les seules les capacités de plus en plus grandes des entrepôts d'archives ouvertes peuvent faire face à cette explosion documentaire car l'explosion documentaire est au centre des préoccupations des chercheurs

\footnotetext{
* Surtout dans sa partie archive ouverte dont le concept est encore mal compris, le mot - archive - étant pris dans son sens premier et originel et de ce fait très sujet à débat de la part surtout des archivistes qui y voient une sorte de déviation du sens archive. Le problème se situe au niveau du concept archive qui dans le monde papier renvoi à la fonction conservation et qui dans le monde numérique renvoi à la fonction dépôt électronique.
} 
qui ne peuvent plus gérer la masse extraordinaire d'information qui les assaille ${ }^{*}$. Ces outils sont diverses et variés mais ROAR et DOAR sont parmi les plus connus et les plus inclusifs et donnent une idée des progrès que ces outils font réaliser au libre accès. Nous aborderons aussi dans cette partie, une autre manière de faire la science, de publier : les journaux électroniques en libre accès. Ce segment de la publication scientifique en libre accès est plus connu, prisé et accepté que les archives ouvertes. Cette " voie dorée " bénéficierait des faveurs des scientifiques pour de multiples raisons dont les plus importantes sont: une meilleure visibilité, le contrôle de l'information (les entrepôts d'archives étant connus - des fois à tort comme manquant de contrôle et de ce fait moins " sérieux "), la motivation et l'incitation à être visible, etc..... A cet effet, deux sites DOAJ et AJOL, représentent deux approches différentes quant à leur couverture. Le DOAJ (Directory of Open Access Journals ) est résolument international et recense les journaux électroniques de tous pays selon certains critères. L'AJOL ( African Journal On Line ) quant à lui est régional et ambitionne de recenser et faire sortir la recherche africaine de son isolement et en des termes africains . Ce genre d'initiative est, à ne pas en douter, bénéfique pour la recherche dans les pays en voie de développement qui se trouvent, d'une certaine manière ostracisés et marginalisés.

L'un caractéristiques des nouvelles technologies et le libre accès est le problème des droits d'auteurs. Si ce problème n'est nullement spécifique à Internet et l'univers numérique, il n'en pose pas moins la question sous une autre dimension. En effet, l'approche du document papier diffère complètement de celle du document numérique. Dans l'univers papier, le droit d'auteur vieux de plus de quatre siècles (The Statute of Anne, considéré comme la première forme de copyright fut promulguée en 1710 en Grande Bretagne) est, malgré certaines dispositions, bien en place. L'irruption du numérique, avec ses spécificités a imposé de nouvelles lois. La publication sur un support numérique ne peut être appréhendée, traitée ni être la propriété d'une personne, institution ou autre entité de la même manière qu'un support papier tangible. C'est à cet effet, que les sites SHERPA, RoMEO et JULIET se proposent de combler ce vide. Ces sites indispensables recensent et énumèrent les accords et les permissions qu'octroient les éditeurs aux auteurs en ce qui concerne leurs publications. En effet, ces derniers soucieux de ne pas trop antagoniser les chercheurs, et en même temps lâcher du lest face aux avancées du libre accès, ont établis des règles permettant aux chercheurs d'avoir un minimum de contrôle sur leur production scientifique. Malgré, ceci la question des droits d'auteur dans l'univers numérique est loin d'être circonscris vu la difficulté de la tache de définir l'objet numérique comparativement à l'analogique.

Il est de notoriété contrairement au monde papier, que le numérique est omniprésent et sans frontières. Tous les pays, même les plus répressifs et appliquant la censure la plus complète, ne peuvent échapper au réseau des réseaux. De ce fait, Internet impose sa présence dans tous les pays et c'est à l'aune de se présence sur la Toile, qu'un pays est jugé quant à place dans le concert des nations. Ce travail comportera donc un chapitre débâtant et présentant les différents programmes en libre accès en Algerie. Devant l'indigence des écrits locaux sur le sujet, la source la première est le GOAP (Global Open Access Portal) de l'UNESCO qui recense au niveau mondial les ressources en libre accès. Certaines expériences algériennes en libre accès (nommément Webreview du CERIST et le Dépôt Numérique de l'Université d'Alger I) seront aussi présentées De son coté, le site internetworldstats recense les différents

\footnotetext{
* Pour donner une idée de grandeur de la taille du web, Netcraft.com parle de 227225642 sites recensés en septembre 2010 (http://news.netcraft.com/archives/category/web-server-survey/). Il faut garder à l'esprit que ces chiffres sont tout ce qu'il ya d'approximatif et il est pratiquement impossible de savoir le nombre exact. Pour corroborer cette idée, le web invisible (partie du web qui n'est pas indexée par les moteurs de recherche classiques) est estimé, selon certaines sources, à au moins 500 fois la taille du Web visible !!!!
} 
taux et indicateurs Internet ainsi que netindex qui donne des indicateurs quant à la bande passante (et différents autre données sur Internet) des différents pays.

La troisième partie de ce travail quant à elle se concentre sur la contrôle par les pairs et de son avenir face aux nouvelles technologies et Internet. Notre problématique s'articule autour de cette question primordiale : le contrôle par les pairs et à la lumière de tous les changements introduits dans le monde de la publication scientifique par Internet est- il entrain de changer radicalement ? Quelles sont les segments de la publication scientifique qui sont le plus touchés ? Plus important, peut être, que la question des segments que l'introduction d'Internet a changé, la question de quelles manières ces segments de la publication scientifique sont changés ? Notre question s'articule et se dirige plus vers une étude des nouveaux paradigmes induits par les nouvelles technologies. La science telle qu'elle a été toujours connue ne sera jamais, pour paraphraser Stix, peut être la même. Un de ces segments les plus importants, le plus secret, le plus conservateur et moins sujet à changements du processus scientifique, le contrôle par les pairs, est de ce fait irrémédiablement changé. Ceci résulte d'une longue période où le processus était enveloppé d'un voile de mystère, d'ésotérisme encouragé par le secret qui l'a toujours entouré. Nous avons réservé un chapitre dans cette partie à une introduction et définition de concepts généraux concernant le contrôle par les pairs. Ce chapitre nous a paru indispensable face à l'ignorance du sujet, de la mauvaise compréhension et aussi l'ignorance du sujet par ceux là même qui en sont les premiers concernés: les chercheurs. En effet, par ignorance ou simplement désintérêt, les chercheurs ne semblent pas être au courant des tiraillements qui agitent la publication scientifique. Les différents et principaux griefs faits au contrôle par les pairs seront examinés afin d'en tirer les conclusions ainsi que les solutions proposées. Parmi celles-ci, le contrôle en double aveugle qui, de prime abord, devrait être accepté par la communauté parce que plus juste et moins inique et qui rencontre lui aussi des réticences de la part de certains chercheurs confirmés dont l'anonymat ne leur sied pas.

La solution idoine est représentée par une ouverture complète du processus, ouverture dont le magazine Nature a lancé une expérience en 2006 sondant les chercheurs, les experts et les éditeurs sur la question. Le résultat fut, nous pensons, étonnant car une majorité des personnes ayant répondu n'était pas très ni au courant ni d'accord pour cette ouverture. Nature a conclu à la non adéquation de l'expérience vu sa nouveauté quoique la porte n'est pas fermé aux expérimentations futures selon les éditeurs de Nature. Si Nature a conclu à la non adéquation de cette ouverture, d'autres expériences ont eu lieu et ont, par contre, enregistrés des résultats plus que probants. A.C.P., E.T.A.I. et F 1000 sont parmi ces sites ayant promu une politique d'ouverture du processus avec des degrés de réussite assez probants. Si ETAI et ACP pratiquent un contrôle par les pairs pré publication ouvert sur Internet, F 1000 pratique quant à lui un nouveau contrôle que seul Internet permet : un contrôle post publication. Ce contrôle pose la question (surtout que F 1000 concerne la médecine et la biologie) du statut de la publication qui devient une publication vivante, évolutive, ouverte à enrichissement mais qui pose la question nodale suivante : quelle est la version finale acceptée de l'article ? Quels sont les résultats finaux acceptés et entrés comme science certifiée ? Il semblerait que l'ouverture du processus si ardemment voulue à ouvert une boite à Pandore dont on ne voit pas les futures développements. Preuve en est le site Philica qui applique un contrôle, le moins que l'on puisse dire, bizarre. Dans ce site, toute personne qui le désire devient expert, toute personne soumettant à sa soumission acceptée, etc.... Nous avons parlé de Philica car contrairement à A.C.P., E.T.A.I. et F 1000, son processus de certification est tout ce qui a de plus lâche et ne sert en rien la science. S'il est vrai que le contrôle par les pairs a été critiqué pour son extrême fermeture (entre autres), l'autre extrême (une ouverture débridée) est tout aussi préjudiciable. 
La question primordiale qui se pose est : entre l'ouverture tout azimut et les griefs portés à l'encontre du processus, existe t- il un point de balance ? Là est la question qui sous tend l'étude que nous menons. Il est malheureusement impossible et trop tôt pour répondre à cette question à cause du manque de recul d'un processus qui n'a été ouvert que depuis moins d'une décennie.

Enfin, et afin de donner un ancrage local à ce travail, nous avons distribué un questionnaire à deux institutions algériennes de recherche et qui sont le CERIST (Centre de Recherche sur l'Information Scientifique et Technique) et le CDTA (Centre de Développement des Technologies Avancées) censées représenter deux pôles de la recherche scientifique nationale. Le questionnaire distribué a pour but d'investiguer les habitudes et fréquence de soumission des chercheurs, de leur connaissance des concepts du libre accès et surtout leur expérience avec le contrôle par les pairs aussi bien traditionnel qu'électronique. Ce questionnaire viendra à ne pas en douter combler un vide quant à ces problématiques qui sont d'un intérêt certain pour la recherche dans notre pays qui doit s'insérer dans une dynamique certes prometteuse mais dont les retards accumulés seront très difficiles à rattraper.

Ce sont ces trois axes (contrôle par les pairs traditionnel, ouverture et modernisation du processus grâce à Internet et enfin le questionnaire au niveau local) que nous examinerons dans notre quête à répondre à cette question qui sous tend tout notre travail : le contrôle par les pairs, et face aux changements introduits par le libre accès et Internet, changera t- il (sera t’il obligé ?) afin de répondre aux différents griefs qui lui sont imputés ? 


\section{PREMIERE PARTIE}

\section{BREVE HISTOIRE DES SUPPORTS DE LA TRANSMISSION DE LA PENSEE}




\title{
BREVE HISTOIRE DES SUPPORTS DE LA TRANSMISSION DE LA PENSEE
}

\author{
Introduction
}

Depuis que l'Homme existe, il a toujours essayé de communiquer. Si de nos jours, il est arrivé à des moyens de communication dont la sophistication, la diversité et l'étendue n'étaient pas imaginables il y a de cela seulement 20-25 ans, il n'en demeure pas moins qu'un long chemin a été parcouru depuis les premiers embryons de communication de l'homme préhistorique. Ce dernier incapable d'écrire, utilisait la parole aidée en cela par les gestes. Ceci était l'apanage des guérisseurs et conteurs qui étaient sélectionnés surtout pour leur capacité de mémorisation et leur personnalité. De toute évidence, cette manière rudimentaire de communiquer répondait aux besoins de communautés restreintes mais avait le désavantage de ne pas transmettre une information précise et fiable car la mémoire humaine était loin d'être infaillible en plus du fait que le mythe y occupait une place prépondérante. Par exemple, des événements étaient omis, changés et même enjolivés selon l'humeur du conteur ou même pour plaire au Seigneur du moment. De là, s'est imposé le besoin d'enregistrer et de préserver les événements et l'histoire de la tribu, d'où l'avènement d'un semblant d'écriture (enregistrement serait un mot plus adéquat) permettant une transmission plus rigoureuse et plus exacte des événements .Il est probable que les premiers écrits étaient des traces faites à la main sur le sol ainsi que des éraflures faites sur les écorces d'arbres ou la pierre. Malheureusement, la nature même de ces traces (surtout celles faite sur le sol) en a rendu la transmission jusqu'à nos jours impossible. A partir de là, sont nés les premiers embryons ou stages de l'écriture et dont les premiers spécimens sont probablement les peintures recouvrant les murs des caves que l'on a trouvées au Sud de l'Europe (principalement en Espagne et en France) datés entre 20000 et 5000 av. J.C. Ces peintures, représentant des animaux chassés, sont admirablement conservées car elles sont situées dans des endroits où la lumière ne pénètre pratiquement pas.

\section{Chapitre I \\ DIFFERENTES ETAPES D'ENREGISTREMENT DE L'INFORMATION A TRAVERS L'HISTOIRE}

\section{Les différentes écritures}

Avec la sophistication sans cesse accrue de la société primitive, s'est imposée une sorte d'écriture et de consignation de l'information. Si les signes et traces, précédemment décrits, étaient suffisants pour la communication, l'écriture (ou plutôt les différentes écritures) introduisirent un bond qualitatif dans un monde qu'on pourrait décrire de primitif. La pictographie, ou écriture par les images, quant à elle peut être schématiquement classifiée en : l'iconographie, l'écriture synthétique ou idéographique et l'écriture analytique ${ }^{(1)}$

(1) Diringer David, The Book before printing : ancient, medieval and oriental, New York, Dover Publishing, 1982, p. $19-20$ 


\section{1-L'iconographie :}

Sa représentation, connue sous le nom de pictogramme, montre les objets dans une situation d'absence de mouvement. Ainsi, un rond peut représenter un soleil, un animal l'animal en question, etc.... Mais cette représentation manque d'animation et de suite car chaque pictogramme représente une image sans relation avec celle qui la précède ou celle qui la suit.

\section{2-L'écriture synthétique ou idéographique :}

Dans l'écriture synthétique, on passe à un stage supérieur car chaque image est reliée à une autre et forme une idée synthétique. Par exemple, un soleil sombre avec une multitude d'étoiles tout autour représente une éclipse solaire ${ }^{(2)}$. Il y a donc deux images (soleil et étoiles pour notre exemple) pour avoir une seule idée synthétisant les deux images : éclipse solaire.

\section{3-L'écriture analytique :}

Ni l'écriture iconographique ni l'écriture synthétique ne constituent un système complet comme c'est le cas dans l'écriture analytique. Dans cette écriture, des représentations conventionnelles définies à partir d'un nombre de dessins deviennent des symboles qui représentent des objets ou des mots. On peut citer parmi ces écritures analytiques, les écritures sumériennes (qui ont précédé l'écriture cunéiforme), les écritures hiéroglyphiques égyptiennes, etc.... ${ }^{(3)}$.

Découlant de ce bref aperçu sur l'histoire de la transmission orale et de l'écriture qui a conditionné l'apparition du livre", on peut conclure que le faible volume d'information à transmettre, la rudimentarité de la vie n'imposaient pas une consignation des événements par écrit. Ce n'est qu'avec un semblant de sophistication et l'augmentation du volume de savoir à transmettre, couplées à l'inéluctabilité d'avoir à l'enregistrer sous une forme sûre et fiable, que s'impose la création d'un embryon d'écriture qui de l'iconographique à l'idéographique et enfin l'analytique mène l'homme vers de nouvelles formes de communication. Le prochain stade de l'homme dans sa quête à mieux communiquer et surtout laisser une trace sera l'alphabet.

\section{2-L'alphabet :}

Contrairement aux différentes écritures où le signe (ou signes) représente une idée, l'alphabet introduit une sophistication dans le sens où à chaque son correspond un signe. Ceci implique qu'avec un nombre limité de " signes " (ou lettres), on peut écrire un nombre illimité de mots et de concepts. Les systèmes précédant l'invention de l'alphabet étaient, schématiquement, incomplets et, à la limite, pouvaient induire le destinataire en erreur. Ainsi l'histoire que relate Hérodote au sujet du Roi Darius et les Scythes est tout ce qui de plus explicite ${ }^{*}{ }^{(4)}$. Cet

\footnotetext{
(2) Diringer, op. cit. p.21

(3) Diringer, op. cit. p.23

* Par livre nous entendons tous les supports qu'ils soient d'argile, de papyrus, de parchemin, de cuir ou d'autres supports beaucoup plus rares tels que les os, les écorces d'arbres, les branches de palmiers ou même, tel que rapporté par l'antiquaire français Bernard de Mantfauçon qui trouva à Rome à la fin du $17^{\mathrm{e}}$ siècle, un livre fait entièrement en plomb.

(4) Henri Jean Martin, Histoire et pouvoirs de l'écrit, Paris, Albin Michel, 1996 p.23

* Darius reçut des Scythes une grenouille, un rat, un oiseau et des flèches. Il crut que ceci était un hommage mais son beau père, plus sage et plus perspicace, lui en donna la signification. Le message voulait tout simplement dire : "Si tu ne te caches pas dans les marais comme la grenouille, sous terre comme le rat, ou si tu ne t'enfuis pas dans les airs comme l'oiseau, nous te transpercerons de nos flèches".
} 
exemple montre à quel point le système iconographique avait atteint (et montré) ses limites. Cette "écriture" pêchait par un manque flagrant de précision et était sujet à l'interprétation personnelle du destinataire tel que le démontre l'exemple du Roi Darius. Il serait présomptueux (et en dehors du sujet de ce travail) de vouloir présenter l'histoire de l'alphabet mais un bref survol s'impose afin de mesurer l'impact de l'évolution que cela a représenté dans l'histoire de l'Humanité.

\section{1-Définitions :}

L'alphabet est défini comme ..." série de caractères qui, dans l'écriture des différents peuples, peignent les sons et les articulations de leur langues " (5) ou " une collection de signes graphiques qui correspondent à autant de sons vocaux dans une langue ou un groupe de langues données, la lettre ou caractère représente l'unité ultime dont se composent les phonèmes " ${ }^{(6)}$.Malgré la multitude des alphabets existants et surtout les différentes ramifications que chaque alphabet a pu produire, il est communément accepté que le premier connu est l'alphabet phénicien *.

Voila donc l'Humanité dotée d'un outil qui nous parait au $21^{\mathrm{e}}$ siècle tout ce qu'il y a de plus banal mais qui avait représenté pour l'époque un bond qualitatif et quantitatif extraordinaire. Pour schématiser à l'extrême, on peut dire qu'il fallait un nombre illimité de signes pour arriver à un résultat très aléatoire (l'exemple du Roi Darius cité précédemment en est la preuve), on n'a plus besoin, avec l'alphabet, que d'un nombre limité de signes qui correspondent à autant de sons ( quoique l'association de deux signes peut donner un son différent du son de chacune des deux signes - ph par exemple ) et dont la combinaison donne un mot qui désigne un objet et plus important un concept ce qui était quasiment impossible avec les autres écritures

Ce bond qualitatif, nonobstant son importance dans l'amélioration de la communication humaine, a induit de nouveaux besoins et de nouvelles donnes. En effet, cette nouvelle sophistication a élargi le public potentiel et a fait sortir le savoir du domaine de l'ésotérique et l'a ouvert au grand public. De ceci a résulté un autre bond, quantitatif cette fois, qui s'apparenterait à ce que nous appellerions de nos jours " une explosion documentaire " (toute proportion gardée). Cet accroissement exponentiel de la masse documentaire a fait ressortir de son coté, le coté anachronique et inadapté des différents supports de transmission de la pensée. Il est de toute évidence pour le moins difficile de transmettre le savoir, communiquer ou tout simplement envoyer un message avec des matériaux aussi encombrants que la pierre, les os, les écorces d'arbres et d'autres matériaux qui ne pouvaient pas assurer une transmission ni rapide ni pratique.

De là s'est imposé l'utilisation d'un matériau plus pratique, plus léger et surtout plus disponible. Le papier répondait à ces critères surtout que le parchemin et le vélin, jusque là utilisés, étaient chers. D'un autre coté, cette invention préfigurait l'apparition d'une autre technologie qui allait bouleverser et révolutionner le monde et qui était l'imprimerie.

\footnotetext{
(5) La Grande encyclopédie : inventaire raisonné des sciences des lettres et des arts Paris, H .Lamirault, t.2,[s.d.] p.489

(6) Encyclopedia Universalis, Paris, Encyclopaedia Universalis, 2012, t.1, p.1009

* L'alphabet phénicien est considéré comme ayant donné quatre familles principales d'alphabet et qui sont : Les premiers alphabets linéaires d'Arabie et de Syrie, l'alphabet araméen et sa descendance, l'Alphabet grec et l'alphabet latin ( Encyclopedia Universalis, op cit .)
} 


\section{3-Le papier}

L'apparition du papier remonte bien au-delà de son utilisation en Europe en 1150 à Jativa exactement .Sa surface plane et moins rugueuse que le velin et surtout le parchemin en faisait le parfait complément de cette nouvelle technologie (pour l'époque) et y était en fait si lié que se pose la question nodale suivante: l'utilisation du papier a $t$ elle favorisé l'essor de l'imprimerie ou est ce que l'essor de l'imprimerie a favorisé la production du papier ? Mais en fait ce qui est d'intérêt pour nous maintenant est de savoir par qui et quand a été crée le papier?

Contrairement à l'imprimerie où le nom de Gutenberg est fortement concurrencé par les Coster, Waldfogel, etc...., il est établi que le papier a été utilisé la première fois en Chine par Tsa'i Lun. Avant cela, le bambou et la soie étaient utilisés mais ils furent abandonnés pour respectivement leur lourdeur et leur cherté. Tsa'i Lun utilise alors différents matériaux tels que les écorces d'arbre, le chanvre, les chiffons et les fils de pêche. Mais le papier finit par les supplanter et a été depuis utilisé en Chine.

Depuis cette période, on peut suivre les pérégrinations du papier vers l'Ouest de la manière suivante. On retrouve sa trace à Tun-Huang au Turkestan chinois en l'an 150 et en l'an 200 à Lou-Han toujours au Turkestan chinois mais plus à l'ouest. En 399, il atteignit Tourfan au Xinjiang et Gilgit au Pakistan actuel au $6^{\mathrm{e}}$ siècle. En 751, on trouve sa trace à Samarcande et à Baghdâd en 793. On le signale en Egypte en 900 mais pas avec certitude. En 1100, il est signalé à Fès avant de faire sa première apparition en Europe à Jativa en l'an 1150. Fabriano, grand centre de production de papier, fut atteint en l'an 1270 et de là il atteignit Nuremberg en 1390. Ce n'est que vers la fin du $15^{\mathrm{e}}$ siècle que le papier traversa la Manche .Quant à l'Amérique, c'est l'an 1690 qui le vit arriver à Philadelphie ${ }^{(7)}$

Il a fallut donc plus de quinze siècles pour que le papier atteigne ou plutôt recouvre le monde connu d'alors. Mais si le papier dans son long voyage de Chine vers l'Ouest n'atteignit l'Europe que vers le $12^{\mathrm{e}}$ siècle, c'est à Samarcande au $8^{\mathrm{e}}$ siècle que se situe la cause de son passage vers 1'Ouest. En effet, les Arabes lors d'une bataille en 751 firent prisonniers des fabricants de papier chinois qui leur apprirent leur art. En 793, Haroun Rachid employa des fabricants de papier chinois à Baghdâd. Par la suite Damas devint, des siècles durant, un centre d'approvisionnement central du papier pour le monde entier. Et c'est sous les auspices du Royaume arabe de Valence que furent construit des moulins à papier à Jativa en 1150 .

On remarquera que la progression de la production du papier n'a pas connu pas l'envolée que l'on aurait attendue d'un medium aussi pratique et révolutionnaire pour l'époque. Bon nombre de théories ont étés émises mais la plus plausible semble être en relation avec le niveau d'instruction de l'époque. En effet, le niveau d'instruction durant le Moyen Age européen n'est pas très élevé ce qui a induit une absence de demande pressante de livres.

\section{4-Le manuscrit :}

Avant le livre dans sa forme actuelle (quoique le livre de nos jours diffère dans sa texture, sa forme et son contenu des premiers livres) et qui était appelé le codex, existait le volumen. Contrairement au codex où les feuilles étaient coupées et collées, le volumen se présentait

\footnotetext{
(7) Mac Murtrie, Douglas C., The Book : the story of printing [and] bookmaking, New York, Oxford University Press, 1967, p.63
} 
sous la forme d'un rouleau de papyrus ou de parchemin qu'il fallait dérouler pour lire. On imagine les difficultés inhérentes à ce genre de medium ( difficulté de lecture dans le cas d'un long texte, l'extrême difficulté de rangement dans les rayonnages-dans le cas d'une bibliothèque-, l'impossibilité d'écrire sur les deux cotés du matériau et détérioration du matériau à force d'être enroulé et déroulé).

Donc, la forme du codex en elle-même représentait un saut aussi bien qualitatif que quantitatif dans l'histoire des supports de la pensée. Lire un message (le mot message s'entend ici dans le sens le plus large du terme) en déroulant un long rouleau de parchemin ou de papyrus est de toute évidence moins pratique que de " feuilleter " un livre dans la forme classique qu'on lui connaît de nos jours. Nonobstant le fait qu'ils soient enroulés (volumen) ou collés (codex), les feuilles étaient écrites, recopiés à la main. C'est ce qu'on appelle les livres manuscrits (du latin libri manu scripti ou livre écrits à la main).

\section{1-Comment étaient recopiés les manuscrits?}

Au début de la période monastique, le monastère avait le quasi monopole de la production du livre. A partir de la fin du $12^{\mathrm{e}}$ siècle, la période laïque voit de profondes mutations sociales et intellectuelles dont la fondation des universités et le développement de l'instruction chez les laïques. A cela, s'ajoute l'apparition d'une nouvelle classe bourgeoise avide de savoir qui a influé sur les conditions de production du livre en général. Comme on peut le voir, les deux périodes précédemment citées (monastique et laique) sont le reflet du passage d'" une société d'élite [qui] va de plus en plus s'effacer devant une société de masse" ${ }^{(8)}$, préfigurant la démocratisation du savoir qui, exponentiellement, est allée crescendo .

Si comme nous l'avons vu, la fin de la période monastique au $12^{\mathrm{e}}$ siècle voit la diminution de l'importance des centres religieux au profit des centres scientifiques, il n'en demeure pas moins qu'elle n'implique nullement leur disparition complète. En effet, les règles des ordres monastiques prévoient - entre autres charges - la copie des manuscrits, produits par les scriptoria * et qui concernent des ouvrages d'études et des manuscrits liturgiques. A partir du $13^{\mathrm{e}}$ siècle, le centre de la vie intellectuelle se déplace vers les universités et avec lui naîtra un commerce des livres destiné à répondre aux besoins des savants, des professeurs et des étudiants.

\subsection{1-La période laïque}

Cette période voit le développement d'un commerce de livres dans les universités fraichement établies.Nous avons d'un côté, les professeurs qui ont besoin de textes, d'ouvrages de référence et de commentaires. De l'autre coté, les étudiants qui, malgré une faculté de mémorisation développée, avaient eux aussi besoin de supports de cours. Ils pouvaient soit les copier eux même, ce qui impliquait une perte de temps, ou se les faire recopier, s'ils en avaient les moyens, par des copistes professionnels.

Peu a peu se développe autour des universités, une corporation de professionnels du livre qui furent considérés comme faisant partie de l'université. Ils jouissaient de ce fait de certains privilèges et relevaient directement des autorités universitaires. Ces privilèges, par contre, leur

\footnotetext{
${ }^{(8)}$ Febvre Lucien [et] Martin Henri-Martin, L'Apparition du livre, Paris, Albin Michel, 1971, p.14

${ }^{*}$ Pluriel de scriptorium et qui vient du verbe latin scribere et signifiait écrire ou celui qui écrit http://fr.wikipedia.org/wiki/Scriptorium (consulté le 06 Novembre 2008)
} 
imposaient des contraintes et un certain contrôle de la part des autorités universitaires. A cet effet, ils ne pouvaient pas travailler à leur propre compte .Ils exerçaient ce qu'on peut appeler un " service publique " ${ }^{(9)}$.Ils étaient nommés après qu'une enquête eut attesté de leur bonne moralité et de leurs capacités professionnelles et ils devaient fournir une caution et prêter serment à l'université. Une fois établies, leurs activités étaient contrôlées et délimitées par leur tutelle. Ainsi, le libraire était beaucoup plus un dépositaire qu'un marchand de livres : il ne pouvait agir à sa guise pour la vente des manuscrits qui avaient transités pendant des générations entre professeurs et étudiants. Il devait annoncer les ouvrages en sa possession (afin d'éviter une augmentation inconsidérée des prix) et sa tarification était fixée par l'université et elle était différente selon que l'acheteur était un maître ou un étudiant ou était étranger à l'université.

A coté de ce contrôle axé sur l'exercice de la profession, l'Université a aussi imposé une sorte de contrôle beaucoup plus intellectuel par l'intermédiaire des stationnaires ( ou libraires), l'institution de la " pecia " * et enfin et surtout la circulation des " exemplaria".Ce contrôle donc du contenu et de sa concordance avec l'original certifié par les autorités de l'Université se faisait de la manière suivante : à partir de l'exemplar que les pecarrii ( ou autorités de l'Université) avaient soigneusement revu et corrigé était fait des copies à des prix fixes ( ce qui était connu sous le nom de taxation).L'exemplar, modèle unique, revenait au stationnaire après la copie et était de nouveau loué. Cette manière de faire, ingénieuse pour l'époque, l'avait fait comparer par H.V. Shooner à " un premier essai de production industrielle du livre avant l'invention de l'imprimerie " ${ }^{(10)}$.Elle avait surtout l'avantage de produire des copies à partir d'un modèle unique et aussi à ce que des fautes et des altérations ne se propagent pas de copies à copies grâce au travail de vérification des pecarii .D'ailleurs si un exemplar était reconnu défectueux, il était retiré de la circulation. Enfin, le prix de location de ces " peciae " (ou cahiers) était fixés par l'Université et devaient être prêtés à quiconque en faisait la demande.

\subsection{2-La période monastique}

Quant aux monastères, leur organisation se faisait au sein du scriptorium du monastère. Le scriptorium était une grande pièce et chaque scribe avait un bureau. Il était strictement interdit d'utiliser la lumière artificielle, ce qui réduisait les heures de travail aux heures diurnes. Pour permettre aux scribes de travailler tranquillement, il était strictement interdit à quiconque de pénétrer au scriptorium. La personne en charge du scriptorium (l'armarius) devait veiller à fournir aux scribes tout ce dont ils avaient besoin pour mener leur travail dans les meilleures conditions possibles (parchemin, encre, règles, etc.....).Le scribe monastique travaillait généralement six heures par jour et était assisté par un rubricateur, un illuminateur et un miniaturiste. A l'instar de la période laïque où " l'exemplar " était la référence, le monastère faisait relire les manuscrits par une deuxième personne pour éviter que des fautes ne s'y glissent surtout pour des textes classiques. Il était d'ailleurs interdit au scribe de corriger des fautes et ceci afin d'assurer une totale concordance entre l'original et la copie.

\footnotetext{
${ }^{(9)}$ Febvre, Lucien [et] Martin, Henri-Martin, op.cit.p.24

" Qui consistait à ne pas louer le manuscrit en entier mais une partie appelée cahier à celui qui voulait le copier afin de ne pas l'immobiliser trop longtemps par une seule personne.

(10) Bataillon, Louis J., Guyot, Bertrand G., House, Richard H. Ed., La Production du livre universitaire au Moyen Age : exemplar et pecia

(Disponible à

http://bbf.enssib.fr/sdx/BBF/frontoffice/1989/02-03/document.xsp?id=bbf-1989-02-0221-005/1989/02-03/famcritique/critique \&nDoc $=69 \&$ statutMaitre $=$ non\&statutFils $=$ non\&tri $=($ consulté le 06 Novembre 2008$)$
} 


\section{5-L’imprimerie et les éléments constitutifs de son avènement :}

De toute évidence, la période manuscrite, (qu'elle soit laïque ou monastique) répondait à une période où le savoir n'était pas très répandu. La fin du Moyen Age a vu une démocratisation du savoir qui a résulté du passage d'une société élitiste (1'Eglise) à une société beaucoup plus ouverte. L'accès de nombres de plus en plus croissants de personnes au savoir a crée un déséquilibre entre l'offre (le manuscrit) et la demande (le nombre croissant de lecteurs). Face à l'impossibilité qu'avaient les livres manuscrits à répondre aux besoins, s'est imposée l'inéluctabilité de la création d'un outil à même d'accélérer la cadence de production des manuscrits. Cette " mécanisation" de l'outil de production eut, schématiquement, deux évolutions ayant conduits à la création de ce qui a été considéré parmi les plus importantes créations de l'histoire de l'Humanité : l'imprimerie. Les deux créations furent premièrement la gravure sur bois et ensuite les caractères mobiles. La première technique (connue aussi sous l'appellation de xylographie) fut considérée pour l'époque une technique révolutionnaire car elle permettait d'écrire sans le recours au scribe quant à la deuxième technique, plus révolutionnaire encore, elle permettait de multiplier le nombre de pages à imprimer, car contrairement à la gravure sur bois où chaque planche de bois équivalait à une page, l'avènement des caractères mobiles introduisait un autre saut qualitatif cette fois. En effet, cette technique permettait par l'intermédiaire de la combinaison de différentes lettres d'imprimer des textes et surtout de réutiliser indéfiniment les mêmes caractères* .

\section{1-La création de l'imprimerie :}

Bien que le nom de Gutenberg soit intimement lié à l'invention de l'imprimerie (quoique contesté par des chercheurs qui évoquent les noms de Laurens Janszoon Coster, Pamfilo Castaldi, Procopius Waldfoghel, etc...), la seule information sûre quant à la création de l'imprimerie est sa date encore qu'elle n'est pas précise. Elle est située au milieu de $15^{\mathrm{e}}$ siècle en Europe, entre 1436 et 1450 . S'il est fastidieux d'évoquer les différentes péripéties ayant conduit aux premiers livres imprimés, il nous parait adéquat d'en relater les principes les plus élémentaires afin de voir qu'elle a été la portée que cette invention qui nous parait de nos jours tout ce qu'il y a de plus banale.

A l'instar du papier découvert en Chine au $2^{\mathrm{e}}$ siècle, on imprimait déjà en Chine aux environs des $5-6^{\mathrm{e}}$ siècles. L'avènement des caractères mobiles au $15^{\mathrm{e}}$ siècle est considéré par beaucoup comme ayant révolutionné le monde. En effet, si le cheminement de la transmission de la pensée depuis les premiers balbutiements oraux des hommes préhistoriques en passant par les différents messages griffonnés sur les différents supports et l'enregistrement sur papier est linéaire, les caractères mobiles en sont le couronnement. Nous utilisons le mot couronnement car bien avant sa création effective, l'imprimerie existait déjà en Chine avant que la paternité n'en soit réclamée par l'Europe. Nous voulons faire ressortir par cela que si le papier qui a été découvert en Chine au $2^{\mathrm{e}}$ siècle et est parvenu en Europe beaucoup plus tard, ceci est dû à des contraintes d'ordre matériel alors que le fait que l'imprimerie ait vu le jour en premier toujours en Chine mais que ceci n'ait pas eu le même retentissement est du à d'autres raisons que nous allons essayer de développer brievement dans ce qui suit.

\footnotetext{
* Il est intéressant de remarquer que la xylographie qui a été découverte beaucoup plus tôt en Chine au $8^{\mathrm{e}}$ ou $9^{\mathrm{e}}$ siècle, répond beaucoup plus à l'écriture idéographique qu'à l'écriture alphabétique. Même si plus tard les caractères mobiles furent introduits, ils n'eurent pas l'effet escompté comparativement à la xylographie.
} 


\section{2-L’imprimerie en Extrême Orient}

Les premiers livres imprimés en Chine étaient des livres gravés sur bois aux environs des $5-6^{\mathrm{e}}$ siècles. Mais c'est sous le règne de l'Impératrice Shotuku du Japan (748 - 769) que furent imprimés un million d'amulettes bouddhistes achevées en 770.Ces spécimens sont considérés comme le plus ancien spécimen d'impression connu et aussi le plus ancien spécimen de papier existant au Japon. La gravure sur bois était de son coté aussi connue en Chine. C'est sous la dynastie T'ang que la Chine connut une des ses plus fastes périodes. Bien que les exemples abondent indiquant que l'impression sur bois était pratiquée notamment entre 835 et 845, la date de 868 est considérée comme la date la plus exacte de la trace d'un livre imprimé complet avec frontispice. Il fut découvert en 1900 par un prêtre taoïste dans la province du Turkestan, près de la ville de Tun-Huang. Près de cette ville se trouve la " Cave des Mille Bouddhas " où furent trouvés des documents datant d'entre 406 et 997. Parmi les nombreuses liasses découvertes se trouve ce qui est considéré comme la plus ancienne copie existante d'un livre imprimé .C'est une version chinoise des "Diamond Soutra", une des plus respectées des scriptures bouddhistes. A la fin du document se trouve une information tout ce qu'il y a de plus étonnant de précision et surtout de normalisation pour l'époque. Elle est formulée de la manière suivante :" Imprimé en date du 11 Mai 868 par Wang Chich pour distribution générale gratuite en profonde vénération pour perpétuer la mémoire de ses parents " ${ }^{(11)}$.

\section{3-Les caractères mobiles :}

Il parait intéressant de noter que le papier et surtout l'imprimerie (sous ses deux variantes : xylographie et caractères mobiles) bien qu'ayant été découverts en Chine, n'ont reçu leur " lettre de noblesse " qu'en Europe. Le nom de Gutenberg est unanimement lié à l'invention de l'imprimerie et des caractères mobiles qui en constituent le couronnement, alors que les noms de Tsa'i Lun ,Wang Chich ou l'Impératrice Shotuku sont d'illustres inconnus pour le commun des mortels. Si des raisons d'ordre technique déjà citées peuvent être évoquées pour cela ( dont la plus importante est sans conteste le fait que la xylographie était, et de loin, beaucoup plus adaptée à l'écriture idéographique ), il n'en demeure pas moins que l'Europe était à l'époque de la création des caractères mobiles, et par delà l'imprimerie, dans un état propice à l'acceptation de ces innovations. On pourra citer l'introduction du papier, son abondance, la fin du Moyen Age, l'essor de l'éducation, etc...Tous ces progrès et innovations d'une Europe en pleine renaissance ont fait que le nom de Gutenberg restera à jamais attaché à l'invention de ce qui avait été appelé à l'époque " l'art divin de l'imprimerie " par Berthold de Henneberg alors archevêque de Mayence, ville qui vit naître le même Gutenberg ou encore " l'art admirable."

\section{4-L'invention de Gutenberg}

Né entre 1394 et 1400 à Mayence, Johann Gensfleish dit Gutenberg (et dont le vrai nom est Johan Gensfleish zur Laden zum Gutenberg), est le fils d'un négociant en draps qui semble jouir d'une relative aisance. Il est partie en 1439 dans un procès où il est question d'une demande de remboursement émise par le frère de l'un des deux associés avec lesquels il s'était engagé. Cette association stipulait que Gutenberg devait enseigner à ses deux associés des métiers différents. Bien que les documents qui nous soit parvenus soient incomplets, il en ressort que " Gutenberg était engagé dans un processus ressemblant l'imprimerie ${ }^{(12)}$ ". En

\footnotetext{
(11) Mc Murthrie, p.88

${ }^{(12)}$ Op. cit., p.139
} 
1455 , il est encore défendant dans un procès duquel il ressort qu'en 1450 , il prêta la somme (considérable pour l'époque) de 800 guldens d'un orfèvre connu de Mayence du nom de Johann Fust et dont le but était de " terminer le travail " .Vers la fin de 1452, Fust prêta une autre somme de 800 gulden avec l'accord qu'il devait devenir partenaire dans le travail de Gutenberg. Dans le procès de 1455, Fust demanda le remboursement d'un peu plus de 2000 guldens, représentant la somme des 1600 guldens prêtés plus les intérêts dûs. Il semblerait que c'est durant cette période qu'il imprima la fameuse Bible à quarante deux lignes avec l'aide technique de Peter Schöffer, gendre de Fust. Ce dernier demanda aussi qu'on lui remette l'équipement qui fut acheté avec les premiers 800 guldens du prêt. Contrairement au cas précédant, ce procès indique clairement qu'on est en présence d'une entreprise d'impression. Dans la réponse de Gutenberg à Fust, il est clairement fait mention des salaires des travailleurs, de loyer de maison, de parchemin, de papier et d'encre. Plus important encore, on parle de l'argent réservé au travail des livres. Toujours est il que l'affaire donne l'impression qu'il s'agit d'un prêt d'une large somme avancée pour financer un projet d'impression important. Bien qu'encore une fois, il n'y ait pas de traces des suites de ce procès, il parait très probable que Fust devint propriétaire d'au moins l'équivalent en équipement des 800 premiers guldens qu'il prêtât .Après cela, il semble que Gutenberg fit banqueroute et, s'il opéra un petit atelier d'impression, il n'en demeure pas moins qu'il fut loin d'être prospère. En 1465, l'Archevêque de Mayence, l'anoblit et s'engagea à lui fournir un certain nombre de privilèges. Enfin en date du 26 Février 1468, un certain Conrad Humery de Mayence donna une décharge à l'Archbishop pour " certaines formes, lettres, instruments, outils et autres choses appartenant au travail d'impression que Johann Gutenberg laissa après sa mort et qui était et sont encore à moi " (13).

Cette information est doublement importante car d'un coté, elle nous indique une date approximative du décès de Gutenberg et qui serait quelque temps avant 1468 et de toute évidence après 1465 ( date de son anoblissement par l'Archbishop de Mayence ).Plus important encore, elle prouve que malgré les déboires qu'il a enduré ( preuve en est les différents procès dans lesquels il était partie), il n'a eu cesse de pratiquer ce qui avait été appelée l'écriture artificielle ou l'art d'écrire artificiellement (ars artificialite scribendi).

\subsection{1-Les caractères mobiles : construction et paternité}

Nous avons vu que la paternité de l'invention de l'imprimerie est attribuée à Gutenberg non pas parce qu'il a inventé l'imprimerie mais parce que sa mise au point des caractères mobiles répondait à un besoin pressant qu'avait la société européenne d'une quantité de plus en plus grande d'ouvrages face à la démocratisation du savoir au sortir du Moyen Age et permettait avec un nombre limité de caractères de composer une infinité de mots et de concepts mais aussi et surtout de composer la page indéfiniment autant de fois que le demandait le travail d'impression et ceci à l'instar de l'alphabet qui avait permis de transcrire une infinité de concepts avec un nombre limité de signes (lettres).

Mais en fait comment étaient confectionnés les caractères mobiles et comment étaient ils assemblés pour imprimer? Contrairement à la xylographie où chaque planche ne servait qu'à une seule utilisation et devait être chaque fois taillé de nouveau, on s'essaya à graver des caractères mobiles en bois. Cette solution, si elle se rapprochait du principe des caractères mobiles tel que conçu par Gutenberg plus tard, avait cependant des défauts multiples et s'est imposée l'obligation de trouver un autre matériau qui n'avait pas les défauts du bois. Ouvrons

${ }^{(13)}$ Op. cit, p.141 
une parenthèse pour dire que si Gutenberg est concurrencé par de nombreux inventeurs dont Laurens Janszoon dit Coster de Haarlem à qui on a attribué l'invention des caractères mobiles ou encore Procope Waldfogel dont on dit qu'il inventa une machine à écrire rudimentaire, il n'en demeure pas moins qu'il fut celui dont " la solution répondait à toutes les données du problème, rigoureusement, logiquement et ingénieusement " ${ }^{(14)}$.Donc à Gutenberg ne peut être enlevé le mérite d'avoir introduit les caractères mobiles métalliques, contrairement à Coster qui aurait utilisé des caractères mobiles en bois. Il faut dire, qu'étant orfèvre et issu d'une famille de monnayeurs, il était à même de travailler les métaux, de les fondre et d'en faire des alliages.

On peut, d'un autre coté, résumer les différentes étapes de la confection des caractères mobiles métalliques tels que l'a ébauché Gutenberg en trois opérations principales .On taille la lettre à l'envers dans un matériau assez dur. On inscrit la lettre à l'endroit dans un matériau moins dur résistant à la chaleur. Cette gravure est placée au fond d'un moule et un métal. Quant à l'alliage utilisé, il était sans doute composé de plomb, d'étain et d'antimoine et ces trois métaux donnaient le matériau voulu.

\section{5-La presse à bras :}

Cette invention révolutionnaire n'aurait pas eu le retentissement qui fut (et est toujours quoique sous d'autres formes) le sien, s'il ne lui fut pas adjoint une autre invention par le même Gutenberg : la presse. En effet, contrairement à la xylographie où on utilisait la brosse et le frotton pour transférer le texte ou l'image de la planche de bois sur le papier, l'utilisation des caractères mobiles était tributaire d'une sorte de mécanisation que représentait la presse *

Cette technique, aussi rudimentaire qu'elle nous parait de nos jours, n'en constituait pas moins, et pour l'époque, une révolution. Entre autres améliorations par rapport à la xylographie, on peut citer la rapidité, la netteté de l'impression, la possibilité d'imprimer recto verso.L'imprimerie subit après cela de profondes mutations et développements aussi bien quantitatifs que quantitatifs qui l'ont mené à ce qu'elle est de nos jours.

\section{Chapitre II \\ LA COMMUNICATION DE LA SCIENCE AVANT L'AVENEMENT DU PERIODIQUE SCIENTIFIQUE}

Introduction

Avant que le périodique et ses premières manifestations n'apparaissent et qu'il ne devienne le medium de communication de l'information scientifique et technique par excellence que l'on connaît, les savants devaient communiquer entre eux et se faire passer les informations et les découvertes qu'ils faisaient. A part les livres, qui après l'avènement de l'imprimerie, étaient le

\footnotetext{
(14) Idem

* Son principe, simple, peut être résumé en les étapes suivantes :

On place la forme, qui est l'ensemble des caractères constituant le texte, sur le marbre, face plate et lisse. La forme est ensuite encrée. On pose le papier sur la forme. Il est alors fortement pressé sous une platine. Enfin le texte est imprimé à l'endroit.
} 
seul medium qui, à la faveur de la démocratisation du savoir, permettaient la circulation de l'information, il n'existait pas une manière par laquelle le savant communiquait, recevait, enregistrait l'information dont il avait besoin pour avancer dans sa recherche ou alors revendiquer la paternité d'une découverte ( fonction très importante que les périodiques scientifiques actuels assurent ).La période à laquelle se situe cette importante période de l'histoire de l'Humanité est le début du $16^{\mathrm{e}}$ siècle et où l'Europe se réveillait d'un long sommeil ankylosant qu'avait été le Moyen Age .La période connue sous le nom de Renaissance et que l'on décrit par une multitude d'événements dont le premier (et à notre avis parmi les plus important ainsi que de grande importance pour notre travail) est l'invention de l'imprimerie en Europe $(\sim 1450)$ et la réalisation du premier livre (la Bible de Gutenberg, 1454/1455). Cette période voit donc un changement radical dans la structure de la société qui, pour reprendre une expression moderne, " se démocratise ".Ceci a induit de nouveaux besoins et de nouvelles manières de procéder pour gérer cette nouvelle masse documentaire. Nous allons voir quelles étaient les conditions de cette période pré-périodique.

Avant les premiers périodiques de De Sallo et Oldenburg, il existait d'autres formes ou antécédents et qui étaient les correspondances, les catalogues de foire et les calendriers. Mais pour les savants, et avant l'apparition des périodiques scientifiques, la correspondance personnelle était la seule manière de faire circuler l'information. La lettre érudite était utilisée pour échanger l'information et les idées ainsi qu'une sorte de " publication primaire ".Cette forme de correspondance des savants servait à développer de nouvelles idées qui étaient examinées d'une manière critique avant d'être enregistrées, ce qui se faisait dans soit un livre ou une brochure. Ensuite ces lettres étaient envoyées à d'autres savants, qui en retour, envoyaient d'autres lettres qui contenaient des découvertes, des informations de leur partie du monde. Elles étaient alors lues et circulaient entre savants. Quand elles sont envoyées à un membre d'une société savante ou au secrétaire d'une Société, elles étaient lues devant l'assemblée et enregistrées.

Apparaissent alors des personnes qu'on pourrait appeler les ancêtres des éditeurs qui allaient apparaître avec les premiers périodiques scientifiques de la première moitié du $17^{\mathrm{e}}$ siecle.Bien que la période ne soit pas très bien documentée (à l'instar de la période qui allait suivre et les périodiques qui allaient apparaître à partir de 1665), il n'en demeure pas moins que certains noms apparaissent et se détachent. On citera, entre autres, Nicolas-Claude Fabri de Peiresc, Athanasius Kircher, Théophraste Renaudot, mais une personnalité, un nom se détache et c'est celui du Père Marin Mersenne qui allait devenir, selon l'expression de l'un des spécialistes des sciences du $17^{\mathrm{e}}$ siècle Cornelis De Waard, " le secrétaire de l'Europe savante de son temps ".

\section{Les premiers éditeurs}

\section{1-Père Marin Mersenne}

"Jamais mortel ne fut plus curieux que le P. Marin Mersenne pour pénétrer tous les secrets de la nature et pour porter toutes les sciences et tous les arts à leur perfection. Peu de gens furent plus industrieux à satisfaire ceste insatiable curiosité par des expériences de toutes manières, par ses propres méditations et par les relations continuelles qu'il avoit avec tous les scavants et curieux de l'Europe... " ${ }^{(15)}$.C'est ainsi que l'historien de Descartes, André

\footnotetext{
(15) Baillet, André, Vie de Monsieur Des Cartes, T. II, Paris, 1691 p. 352 Cité par A.Beaulieu, Mersenne et Peirsec : une amitié constructive.Disponible à : http://www.peiresc.org/Amitie.html ( Consulté le 14/03/2011)
} 
Baillet s'exprimait concernant le Père Pierre Mersenne qui, a côté de sa fonction religieuse, avait pu réaliser la gageure d'être une sorte de courroie de transmission et d'entrepôt, en quelque sorte, des nouvelles scientifiques de l'Europe de l'époque. Avant 1665, il n'y avait pas d'autre moyen de communiquer que par l'entremise de ces personnes qui se chargeaient (entre autres fonctions) de distribuer les nouvelles découvertes, intervenir dans la résolution des grandes controverses, etc....Cette fonction n'était pas de tout repos sachant les problèmes logistiques de l'époque ainsi que l'éclectisme dont devait faire preuve la personne en question vu , qu'à l'époque, la specialisation n'était pas encore connue. Les philosophes (noms donnés aux savants à l'époque) avaient une érudition et un savoir général qu'il serait impossible d'imaginer de nos jours.

Marin Mersenne, né le 8 septembre 1588, mort le $1^{\text {er }}$ septembre 1648, est un religieux français appartenant à l'ordre des Minimes, érudit, mathématicien et philosophe. On lui doit les premières lois de l'acoustique, qui portèrent longtemps son nom, il établit concomitamment avec Galilée la loi de la chute des corps dans le vide.

Toute sa vie, Mersenne a entretenu une très riche correspondance avec les plus grands savants de son temps : Fermat, Pascal, Gassendi, Descartes, Torricelli, Desargues,etc... A une époque où les journaux scientifiques n'éxistaient pas, il est une sorte de messager qui recueille et fait connaitre les découvertes nouvelles. Il crée en 1635 une Académie, ancêtre de l'Académie des Sciences, où les érudits discuteront librement et passionnément.

C'est cette volonté qui va présider l'année suivante à la création de l'Academie Parisiensis, qui préfigure celle créée par Colbert en 1666. Mersenne est resté célèbre car, à une époque où la presse scientifique n'existait pas encore, il fut, le centre d'un réseau d'échange d'informations, prémisse de la future Académie des Sciences. Sa très volumineuse correspondance (en latin comme en français) avec d'autres mathématiciens et scientifiques de nombreux pays, comme Descartes, en témoigne.

\subsection{2-L'Académie de Mersenne}

Il ne s'agit pas à proprement parler d'une institution. Elle se tient dans les maisons de chacun de ses membres, puis, ne pouvant se déplacer qu'avec difficulté, le père minime reçoit ses amis dans sa cellule, à des jours fixés à l'avance. Par ce biais, il fait connaître les uns aux autres. Il provoque les disputes entre les points de vue de ses amis savants. On compte dans cette académie nombre de mathématiciens, mais aussi quelques astronomes, des philosophes, des imprimeurs, des jésuites, des conseillers d'État, des médecins, des ingénieurs, des artisans, etc.... Ces personnes entrent en correspondance entre eux par l'entremise du père minime. Une liste en sera dressée qui comportera jusqu'à 180 noms, classées en cinq catégories selon un ordre parfois surprenant.

Enfin, Mersenne n'était pas seulement le premier éditeur ou responsable de société savante mais il avait aussi une riche liste de livres qu'ils avaient rédigés et qui dépassaient et de loin son rôle ecclésiastique.

A côté de Mersenne, qui de sa cellule faisait office de centre de tri et de distribution des informations scientifiques ainsi que de leur évaluation, l'Angleterre vivait elle aussi, à l'orée de ce siècle, une révolution intellectuelle. Avant qu'Oldenburg ne lança Philosophical Transactions en 1665, il y avait une forte activité intellectuelle. A l'instar de la France où le 
nom de Mersenne apparait comme le catalyseur, un nombre de savants activaient en Angleterre à l'époque et faisaient office de clearinghouse ${ }^{*}$ de l'information. Il serait fastidieux de citer les différents noms qui ont marqué cette période mais les différentes sources consultées citent trois personnes comme ayant marqué cette période charnière dans l'Europe du début du $17^{\mathrm{e}}$ siècle. Ce sont : Theodore Haak, Samuel Hartlib et John Collins.

\section{2-Theodore Haak}

Il est beaucoup plus connu pour la première traduction de Paradise lost de Milton. Son engagement actif avec d'autres savants au $17^{\mathrm{e}}$ siècle a grandement facilité l'expansion et la diffusion de la " Nouvelle science" à travers toute 1'Europe. Ses dons linguistiques furent utilisés dans la traduction et fournirent les bases de la fondation du "Groupe 1645" considéré comme le précurseur de la Royal Society.

\section{3-Samuel Hartlib}

C'était une personne aux intérêts éclectiques, contemporain de Robert Boyle. Il est souvent décrit comme étant au centre d'un vaste réseau de contacts et de discussions dont le but était de promouvoir la connaissance et l'échange d'information à travers l'Europe. "Il était un secrétaire correspondant du groupe [de Mersenne].Ces lettres étaient des bulletins de secrétaires non officiels de sociétés qui avaient le même but .Il y a ici une claire évidence d'un échange systématique d'information scientifique entre Paris et Londres vingt ans avant que The Royal Society ne mette en place son comité de correspondance étranger " (16) . L'un de ses objectifs était " d'enregistrer tout le savoir humain et le mettre à la disposition de tous pour l'éducation de l'humanité " (17).

\section{4-John Collins}

John Collins était un mathématicien anglais dont la correspondance montre son activité en tant que " intelligencer " ${ }^{* *}$.Il avait l'ambition de fonder une papeterie et imprimer des livres. Parmi ces livres, certains qu'il avait l'intention de rédiger et spécialement un qui concernait les avancées modernes des sciences mathématiques et aussi une liste des meilleurs auteurs de cette spécialité. Beaucoup de ces écrits témoignent de son savoir concernant le commerce ainsi que son intérêt dans les affaires de tous les jours.

\footnotetext{
* Nous utilisons cette expression anglo-saxonne qui veut dire en terme bancaire "Comptoir general de virement " (In. : Harrap's shorter french and english dictionnary, Ed.1991, p.150) car elle fait ressortir toute la fonction exercée.En effet, ces premiers savants, la plupart des illustres amateurs (qui seront plus tard exclus de la recherche du fait de son hyperspecialisation) etaient des sortes des agents qui faisaient dispatcher l'information et la rendaient disponible à tous ceux qui en avaient besoin et meme à ceux qui etaient juste curieux et voulaient savoir.Ils etaient, en quelque sorte et pour rester dans le meme jargon, des gestionnaires du stock d'information qu'ils avaient ammassé.

(16) Harcourt, Brown, Scientific organizations in seventeenth century France, Baltimore, The Willams \& Wilkins Co., 1934, p.44

(17) http://www.hartlibian.org/Hartlibian-research/Samuel-hartlib (Consulté le 19/03/2011)

** Mot anglais venant d'intelligence et dont l'equivalent en français est pratiquement inexistant.Il pourrait etre traduit par" personne traffiquant (dans le sens noble du terme) en choses intelligentes " autrement dit personne qui travaillait sur la science en la faisant circuler, echanger entre personnes savantes.Cette definition est strictement personnelle et nous avons vu qu'il etait opportun de la donner et que nombre de concepts sont difficilement ( pour ne pas dire impossible) à traduire et encore plus à apprehender en dehors de la langue originelle.
} 
Si ces personnes ont, avant l'avènement du périodique, joué un rôle très important en canalisant, recevant, dispatchant l'information scientifique et technique à qui de droit, un autre point mérite d'être relevé en ce qui concerne cette période cruciale. C'est l'avènement des sociétés savantes et leurs appendices (du moins à l'époque) les périodiques. En effet, l'action des scientifiques, telle qu'elle a été brièvement décrite, manquait d'un cadre formel où elle pouvait s'exprimer. C'est alors que se constituèrent un nombre d'Académies ou Sociétés savantes dans lesquelles les savants (ou philosophes) avaient un cadre formel dans lequel il pouvait s'exprimer et publier étant donné que ces académies avaient pour " porte parole " leurs périodiques. Les deux des academies qui avaient, en terme de fondation, épousé la date de lancement du Journal des Sçavans et des Philosophical Transactions sont l'Académie des Sciences de Paris et la Royal Society of London respectivement. Le début du $17^{\mathrm{e}}$ siècle avait quant à lui vu la fondation de deux autres académies qui avaient antidaté The Royal Society of London et l'Academie des Sciences de Paris. Ce sont respectivement :

\section{-Accademia dei Lincei fondé in 1603 par Federico Cesi}

- Academia Naturae Curiosorum fondée en 1652 par trois savants dont le plus proéminent est Johann Laurentius Bausch. Elle est la plus ancienne académie des trois académies encore existantes. The Royal Society of London et l'Académie des Sciences de Paris sont les deux autres.

Avant cela, L'Académie de Platon fut fondée en 387 Av JC et d'ailleurs c'est de là que vient le mot Academie*.

\section{2-Les Académies}

\section{1-L'Académie des sciences de Paris}

L'idée de la fondation de l'Académie des Sciences de Paris eut un nombre de sources mais une de ces sources fut sans doute l'Académie de Mersenne précédemment citée. Le Père Mersenne rencontrait fréquemment et entretenait une riche correspondance avec d'éminentes figures telles que Descartes, Pascal et Galillé. Ce qui a été connu aussi sous l'Académie de Montmor (par rapport à Henri Louis Habert de Montmor) est aussi créditée comme une des sources de la création de l'Académie des Sciences de Paris.

C'est en 1666 que Colbert, crée une Académie qui se consacre au développement des sciences et conseille le pouvoir en ce domaine. Il choisit des savants, mathématiciens (astronomes, mathématiciens et physiciens) et des physiciens (anatomistes, botanistes, zoologistes et chimistes) qui tiennent leur première séance le 22 décembre 1666 dans la Bibliothèque du Roi à Paris. Pendant ses trente premières années, l'Académie fonctionne sans statuts. Dés le début, l'Académie avait en son sein des savants étrangers. Ainsi, Huygens, le célèbre mathématicien hollandais, était un des membres fondateurs.

Le 20 janvier 1699, Louis XIV donne à l'Académie son premier règlement et la place sous sa protection. L'Académie Royale des Sciences siège au Louvre. Ses membres sont nommés par

\footnotetext{
* Le mot académie ou académique vient du nom du lieu où Platon enseignait. Academus ou Hecademus, un héro mythique laissa un jardin et une oliveraie aux citoyens d'Athènes pour l'exercice de la gymnastique. Des festivals s'y tenaient ainsi que des événements athlétiques dans lesquels les participants courraient entre les autels. Des jeux funéraires y étaient aussi programmés. C'est là que Platon commença à enseigner en en $387 \mathrm{Av}$ $\mathrm{JC}$ et de ce fait le mot Académie vient du lieu où Platon enseignait et non pas de l'institution de savoir que ce mot a de nos jours. (http://www-history.mcs.st-and.ac.uk/Societies/Plato.html)
} 
le Roi, après présentation par l'Académie. Ils sont au nombre de 70 auxquels s'ajoutent 85 correspondants. L'Académie était considérée comme un forum pour le développement de la science mais aussi comme une institution étatique qui avait la main haute sur les brevets et la technologie. Le nombre de ses adhérents actifs n'était pas très élevé et se composait de 56 adhérents qui étaient divisés en deux grands catégories: Sciences mathématiques qui se subdivisaient en géométrie, mécanique et astronomie et Sciences physiques qui se subdivisaient en chimie, botanique et anatomie.

D'un point de vue administratif, l'Académie avait un Secrétaire Perpétuel à sa tête qui s'occupait de la distribution des tâches, des rapports annuels sur les progrès de l'Institution, les nécrologies, etc....

Une réforme majeure de la structure de l'Académie fut entreprise par Lavoisier en 1785.La structure générale des sciences mathématiques et des sciences physiques fut retenue mais elles furent (les sciences) divisée en quatre. A la géométrie, la mécanique et l'astronomie dans les sciences mathématiques fut ajoutée physique générale. Ceci devait inclure des sujets tels que l'optique, l'électricité, le magnétisme, l'acoustique et la chaleur qui étaient encore largement des sujets expérimentaux à cette époque mais commençaient à devenir de nature plus mathématique. Cette classification était bonne étant donné qu'elle anticipait ces sujets qui devenaient des mathématiques appliquées et en fait contribuèrent à cette tendance .Les trois Sciences physiques de chimie, botanique et anatomie devinrent sujets : chimie et métallurgie; minéralogie et histoire naturelle; botanique et agriculture et anatomie.

L'avènement de la Révolution Française en 1789 fut un période difficile pour l'Académie qui essaya de se soustraire aux remous qu'avait connus le pays en ces temps. La période vit aussi la création de l'Institut de France en 1795 qui engloba (en plus de l'Académie des Sciences) d'autres académies sous son égide (L'Académie Française, L'Académie des Inscriptions et des Belles Lettres, L'Académie des Beaux Arts et L'Académie des Sciences Morales et Politiques). En 1835, paraissent les Comptes Rendus des séances de l'Académie des Sciences qui deviennent rapidement un instrument de première importance pour diffuser les travaux scientifiques français et étrangers. Contrairement à d'autres publications d'autres académies, les CRAS n'ont qu'une très faible reconnaissance scientifique internationale et visibilité en raison en partie de leur publication en français. Enfin, l'Académie octroie chaque année un Grand Prix très prisé par la communauté scientifique.

\section{2-The Royal Society of London}

Plus que l'Académie des Sciences de Paris, The Royal Society of London est intimement lié à ce qui etait connu comme The Invisible College *.En effet, aux environs des années 1645, un

\footnotetext{
Le terme de " Collège Invisible " représentera à partir des années 1960 l'idée d'un réseau informel de scientifiques correspondant entre eux pour discuter sérieusement du phénomène OVNI. J. Allen Hynek commence à en parler dans un chapitre de son livre en 1972 :" S'il ressort, de mes conversations et de mes échanges de lettres avec nombre de confrères, que l'intérêt des scientifiques ne cesse de croître, celui-ci n'en demeure pas moins, dans la plupart des cas, bien caché. On assiste véritablement à la formation d'un "Collège Invisible" dont les membres, savants ou techniciens, accepteraient volontiers d'entreprendre l'étude du phénomène ovni s'ils en avaient le temps et les possibilités matérielles. Ils constituent un groupe international, qui serait prêt à relever le défi. Il semble qu'a partir de 1973 une version plus formelle de ce collègue ait été fondée par Jacques Vallée et Hynek, où des experts et scientifiques du monde entier correspondent se rencontrent à l'occasion de leurs voyages pour discuter du sujet, sans pour autant croire forcément aux soucoupes volantes. Disponible à http://rr0.org/org/int/association/CollegeInvisible.html
} 
nombre de savants commencèrent à avoir des réunions informelles régulières.Ils avaient pour interêt commun l'acquisition du Savoir par des investigations expérimentales. Leur Collège Invisible (ou leur Collège Philosophique tel qu'il l'avait aussi appelé) discutait la philosophie naturelle, les sciences mécaniques et l'agronomie. Ces rencontres durèrent jusqu'aux environs des années 1658 où la situation politique s'étant détérioré, les savants durent les interrompre. A partir de 1660, la situation se stabilisa et les savants purent reprendre leurs rencontres à Gresham Collège. Ils décidèrent alors de donner à leur institution un caractère officiel. Le Roi fut approché et il donna son accord. L'Académie porta alors le nom de " The Royal Society of London for Promoting Natural Knowledge ".La Société décida de ce fait qu'elle avait besoin d'une publication pour consigner ses travaux. Cette idée bien qu'elle ait été déjà discuté en 1661 n'en a pas moins été mise en application qu'en 1665.C'est la naissance des Philosophical Transactions qui devint - officieusement au début - l'organe de The Royal Society. Officieusement car c'est Henry Oldenburg qui en assura la viabilité financière et ceci jusqu'à son décès. Ce fut le cas pour tous les numéros de Philosophical Transactions qui furent tous financés par les successifs secrétaires de la Royal Society. Ce n'est qu'en 1750 que The Philosophical Transactions fut supporté financièrement, et d'une manière officielle, par la The Royal Society. A travers ses trois siècles et demi d'existence, La Royal Society vit un nombre non négligeable de découvertes trés importantes faites sous ses auspices. Les citer tous serait fastidieux .Nous en citerons quelques uns comme témoin de son importance ${ }^{(18)}$ :

1665 : Micrographia de Robert Hooke est publié contenant des dessins importants utilisant un microscope et contenant la fameuse puce. Le livre utilise pour la première fois le mot " cellule" comme terme biologique.

1677 : Antoni van Leeuwenhoek observe pour la première fois de petits animaux sous le microscope, c'est la première observation de micro-organismes.

1687 :Le livre de Isaac Newton " Principia Mathematica" décrivant l'action de la gravité est publié par The Royal Society. Il est considéré comme l'un des livres les plus importants de tous les temps.

1714 : La première narration anglaise de l'inoculation contre la maladie apparait dans The Philosophical Transactions.

1752 : Benjamin Franklin démontre la nature électrique de l'éclair utilisant un cerf volant et une clé dans une recherche adressée à la Royal Society.

1781 : William Herschel découvre un nouveau corps dans le système solaire qui fut plus tard renommé Uranus.

1823 : The Royal Society approuve la machine à différence de Charles Babbage, qui calcule des tables mathématiques.Babbage est considéré comme l'un des précurseurs de l'informatique.

1864 : Charles Darwin reçoit the Copley Medal pour son livre L'Origine des espèces par le moyen de la sélection naturelle, ou la préservation des races favorisées dans la lutte pour la vie (1859)

${ }^{(18)}$ http://trailblazing.royalsociety.org/?from=footer 
1904 : Le Comité des maladies tropicales de la Royal Society commence à investiguer le malaria et d'autres maladies tropicales, surtout en Afrique.

1919 : Des astronomes confirment la théorie de la relativité générale à la Royal Society à partir des observations faites durant l'éclipse totale de cette année. Albert Einstein est élu en tant que membre en 1921.

1932 : James Chadwick découvre le neutron et publie sa recherche avec The Royal Society.

1936 : Sigmund Freud est élu en tant que membre.

1953 : Francis Crick and James Watson déterminent la structure de l'ADN détaillant leur découverte dans une recherche publiée par The Royal Society. C'est le secret de la vie qui est découvert, radicalement changeant la science pour les décennies à venir.

1956 : La Royal Society établit une base de recherche à Halley Bay, en Antarctique. C'est ici en 1985, que des pertes dramatiques dans la couche d'ozone sont observées. La base est toujours un important point dans la recherche climatique.

2001 : Sir Tim Berners-Lee est élu en tant que membre. Sa proposition d'un projet hypertexte global en 1989 a conduit à la création de la Toile Mondiale (WorldWide Web).

2004 :L'étude conjointe de la Royal Society's sur les nanosciences et les nanotechnologies est publiée.Elle examine les applications de la nouvelle technologie, des impacts environnementaux possibles et aussi son potentiel futur de grande importance

2010 : La Royal Society célèbre son $350^{\mathrm{e}}$ anniversaire.

Il est clair que les trois cent cinquante années depuis la fondation de la Royal Society ont vu des avancées scientifiques notables (pour ne pas dire révolutionnaires) qui ont marqués la Science. Il semblerait que The Philosophical Transactions qui est intimement lié à The Royal Society (bien que ce n'est qu'en 1750 qu'elle en prit la responsabilité financière) ait contribué dans de larges proportions à en faire une des plus prestigieuses académies (en plus d'être parmi les plus anciennes toujours existantes).De son coté, l'Académie des Sciences de Paris bien qu'ayant été fondé à la même période n'a pas le même prestige .Cela serait du peut être dû au fait qu'elle a été incluse dans l'Institut de France ce qui en fait une Academie à l'intérieur d'un Institut et aussi, et surtout, le fait qu'elle n'ait pas un périodique la représentant qui soit d'une stature internationale comme The Philosophical Transactions. En effet, le CRAS (Compte Rendus des séances de l'Académie des Sciences) n'a pas la même stature ni la réputation des Philosophical Transactions. D'un autre coté, le titre lui-même des CRAS a maintes fois changé et a connu maintes scissions alors que la couverture elle-même des différentes sciences a changé à plusieurs reprises.Ceci a engendré que le titre CRAS n'a pu avoir le prestige qu'ont eu les Philosophical Transactions qui lui n'a changé qu'à une seule reprise (pour se scinder en deux spécialisations tout en gardant le même titre). Enfin, le fait que la langue de publication des CRAS soit le français a aussi influé sur sa notoriété étant donné le clair avantage qu'a la langue anglaise dans la publication scientifique. 


\section{Chapitre III \\ L'AVENEMENT DU PERIODIQUE}

Introduction

Après l'avènement de l'imprimerie et de son corollaire direct l'accroissement du nombre de livres disponibles, une spectaculaire augmentation du nombre de livres fut enregistrée .A cet effet, il a été calculé que pour la seule Europe, et entre 1450 et 1500, il a été produit quelques vingt millions d'exemplaires se décomposant en 30 à 35000 impressions différentes représentant 10 à 15000 textes différents ${ }^{(19)}$. De son coté, Kronick, estime que la moyenne mondiale des livres produits annuellement a augmenté de 420 titres entre 1436 et 1536 à 5750 au cours du siècle suivant ${ }^{(20)}$.Même par rapport à nos standards actuels, ces chiffres sont impressionnants surtout si on y ajoute que la population européenne était, à l'époque , loin d'être aussi nombreuse et que surtout le nombre et le niveau des gens instruits ne peuvent être comparés à la population actuelle.

\section{1-Difficulté de discernement livre-périodique}

Mais progressivement, le périodique s'impose face aux besoins d'information de plus en plus mis à jour quoique cette transition ne fût pas aussi abrupte et nette qu'on l'aurait imaginé. Charles Bazerman l'a décrit de la manière suivante : " l'apparition du journal scientifique en 1645 [sic] n'a pas immédiatement remplacé les livres en tant que source première de communication des découvertes scientifiques .Les livres restèrent les sources les plus importantes de l'information scientifique pour de nombreuses années " (21) .De son coté, A.J. Meadows voit que " les recherches les plus importantes continuèrent a être écrites sous forme de monographies pendant le $18^{\mathrm{e}}$ siècle, mais l'habitude commença à s'estomper au $19^{\mathrm{e}}$ siècle du moins dans les sciences physiques " ${ }^{(22)}$. Il faut aussi remarquer que si le passage du medium livre au medium périodique ne peut être clairement établi dans le temps car les deux media ont pratiquement cohabités et coexistés en tant véhicule de transmission des découvertes scientifiques, la forme elle-même du périodique scientifique telle qu'on la connaît de nos jours était pratiquement inconnue. Ainsi, on n'arrivait pas à différencier entre un livre (monographie) et un périodique. D'ailleurs, Bazerman remarque que " plus on examine de prés le passage du livre à l'article scientifique, plus on s'aperçoit que la situation est complexe, avec des découvertes différentes et des travaux différents chacune allant vers des directions différentes. " ${ }^{(23)}$. Il ajoute concernant cette non différentiation tôt dans la vie du périodique scientifique : ".....d'ailleurs, les formes des livres et des articles ne sont pas toujours distinctes et isolées les unes des autres .Quoique les articles de périodiques débutèrent généralement assez courts, certains devinrent plutôt longs, tel que par exemple New pneumatical experiments about respiration de Robert Boyle qui, en 1470, remplit la plupart des numéros 62 et 63 du cinquième volume des [Philosophical] Transactions. De tels longs articles ressemblaient dans la forme à des brochures de la période. A l'orée du $18^{\mathrm{e}}$ siècle, le long article devint commun avec, par exemple, le volume 90 [des Philosophical

\footnotetext{
${ }^{(19)}$ Febvre Lucien [et] Martin Henri-Martin, op. cit, p.350

${ }^{(20)}$ Kronick, D.A., A History of scientific and technical periodicals, New York, Scarecrow, 1962, p.60

(21) Bazerman, Charles, Shaping written knowledge: the genre and activity of the experimental article in science, Madison, [Wisconsin, U.S.A.], University of Wisconsin Press, 1988, p. 80. Disponible sous forme électronique téléchargeable à : http://wac.colostate.edu/books/bazerman shaping/

${ }^{(22)}$ Meadows, A. J, Communication in Science, London, Butterworths, 1974, p.67

${ }^{(23)}$ Bazerman, Charles, op.cit., p.81
} 
Transactions] qui comprenait seulement 18 articles, avec une moyenne, des articles qui dépassaient les 25 pages. D'ailleurs, Kronick signale des journaux du $18^{\mathrm{e}}$ siècle qui avaient une forte ressemblance avec les livres avec chaque numéro consacré à un seul sujet et peut être écrits par un seul auteur" (24). Il conclut en disant : "Ainsi, il semble qu'il y ait un nombre de type de livres et un nombre de types d'articles avec des relations complexes les uns avec les autres. La plupart du travail textuel et historique reste à faire avant qu'une image claire [de la différentiation entre livre et périodique] n'émerge ". ${ }^{(25)} \mathrm{Il}$ abonde dans le même sens en écrivant ....." Durant le $17^{\mathrm{e}}$ et $18^{\mathrm{e}}$ siècle, il est difficile de différencier les titres qui sont périodiques et ceux qui ne le sont pas. La distinction entre livres et périodiques n'était pas aussi ferme et rigide au $17^{\mathrm{e}}$ et $18^{\mathrm{e}}$ siècle qu'elle l'est de nos jours " ${ }^{(26)}$. A ce mélange dans les genres, somme toute normal pour un medium nouveau, s'ajoute une quasi absence d'écrits sur le raisons, les étapes ainsi que les circonstances du passage (ou du moins l'introduction) du périodique. A cet effet, Kronick, un chercheur dont les recherches sur la période font référence reconnaît cette cohabitation et va même plus loin pour faire ressortir que peu est connu sur la période :" ....mais parce que peu a été écrit sur cet important et intéressant sujet " (27)

\section{2-Les deux premiers périodiques scientifiques :Le Journal des Sçavans et The Philosophical Transactions}

Si la période du passage du livre au périodique n'a pas eu lieu d'une manière abrupte et soudaine mais a vu pendant une certaine période une cohabitation et un chevauchement des deux genres, il n'en demeure pas moins que les spécialistes du domaine s'accordent à dire que Le Journal des Sçavans et The Philosophical Transactions sont les deux premiers spécimens qui ont fondés le périodique scientifique et technique des temps modernes. On aura remarqué que deux périodiques sont cités simultanément alors que l'un (Le Journal des Sçavans) parut le 5 Janvier 1665 et a donc précédé le deuxième (The Philosophical Transactions) qui parut le 6 Mars 1665 de deux mois. Cette controverse est en fait dûe à une sorte de classification du degré de "scientificité " de chacun des deux périodiques. Le Journal des Sçavans était plus un périodique qui relatait les activités mondaines et comptes rendus de livres alors que The Philosophical Transactions était (officieusement au début) l'organe de The Royal Academy of London et présentaient des communications scientifiques originales présentées lors des séances de cette Académie. La quasi simultanéité de date de fondation des deux périodiques a induit cet amalgame exacerbé par une sorte de rivalité franco-britannique qui ne dit pas son nom. Nonobstant ces querelles de clocher, ces deux périodiques peuvent se targuer d'être les deux premiers à avoir fondé une nouvelle forme de transmission du savoir qui allait révolutionner le monde de la communication scientifique. Nous allons donc examiner comment et dans quelles conditions ils ont étés fondés ainsi que la personnalité de leurs fondateurs.

\footnotetext{
(24) Idem

(25) Ibidem

${ }^{(26)}$ Kronick, D.A., Ed., Scientific and technicals periodicals of the seventeenth and eighteenth centuries : a guide, Metuchen [New Jersey, USA] ; London, The Scarecrow Press, 1991, p.V

(27) Kronick, D.A., " Devant le déluge " and others essays on early modern scientific communication, Lanham [Maryland, USA] ; Oxford, The Scarecrow press, 2004, p.V
} 


\section{1-Le Journal des Sçavans}

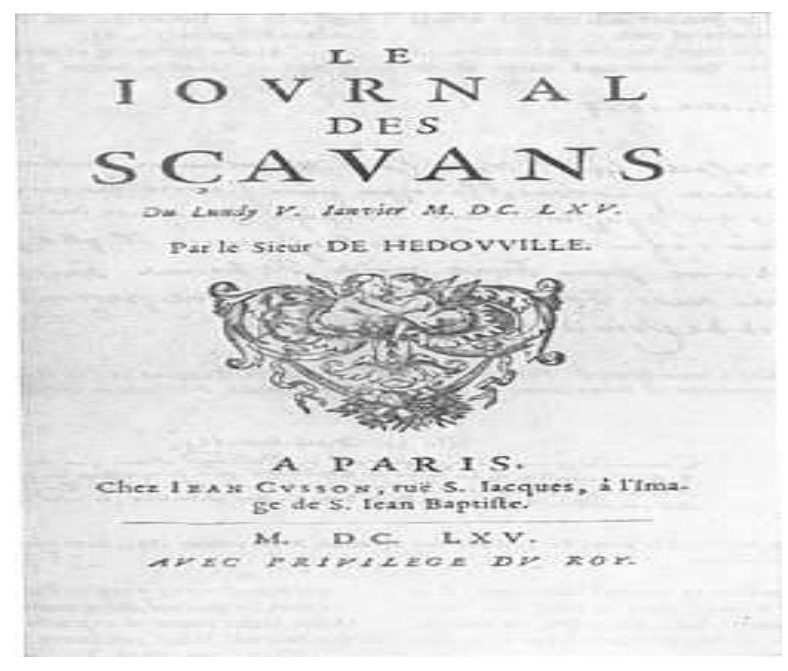

Fig.1-Couverture du premier numéro du Journal des Sçavans

Le Journal des Sçavans fut fondé en 1665 par Denis de Sallo, conseiller au Parlement de Paris, il bénéficia du patronage royal en 1701. Supprimé en 1792, il fut rétabli et réorganisé en 1816 .Il fut édité jusqu'en 1900 aux frais de l'État par un bureau présidé par le Garde des Sceaux, puis le ministre de l'Instruction publique et réserva ses colonnes aux membres de l'Institut. Voué de nouveau à disparaître pour des raisons de restrictions budgétaires, c'est tout naturellement que l'Institut de France, qui avait pris à sa charge les frais d'impression pour les années 1901 et 1902, se substitua à l'État. Néanmoins, ne pouvant consacrer de manière continue des fonds nécessaires à la publication du Journal des Savants, 1'Institut de France proposa à l'Académie des Inscriptions et Belles-lettres d'en accepter la charge, ce qu'elle assura à partir de 1909.

A la charge exclusive de l'Académie depuis cette période, la Journal des Savants accueille des articles originaux marquant des avancées significatives dans les disciplines relevant de sa compétence, tant en raison de leurs résultats que pour l'aspect nouveau de leur méthode. ${ }^{(28)}$

Dans sa préface du premier numéro, Dennis De Sallo, le fondateur du Journal des Sçavans présente de cette manière les " desseins " de son journal :

"Le dessein de ce journal estant de faire sçavoir ce qui se passe de nouveau dans la Republique des Lettres, il sera composé de :

Premierement d'un catalogue exacte des principaux livres qui s'imprimeront dans l'Europe.Et on se contentera pas de citer les simples titres comme jusques à à present la pluspart des Bibliographies : mais de plus on dira de quoy ils traitent, et à quoy ils peuvent estre utiles.

${ }^{(28)}$ http://www.aibl.fr/fr/public/catalogue/journal.html 
Secondement, quand il viendra a mourir quelque personne celebre par sa doctrine et par ses ouvrages, on en fera l'Eloge, et on donnera un Catalogue de ce qu'il aura mis a jour, avec les principales circonstances de sa vie.

En troisieme lieu on fera sçavoir les experiences de Physique et de Chymie, qui peuvent servir a expliquer les effets de la Nature: les nouvelles decouvertes qui se font dans les Arts et les Sciences, comme les machines et les inventions utiles ou curieuses qui peuvent fournir les Mathematiques: les observations du Ciel, celles des Meteores, et ce que l'Anatomie pourra trouver de nouveau dans les animaux.

En quatrieme lieu, les principales decisions des Tribunaux Seculiers et Ecclesisastiques, les censures de Sorbonne et Universitez, tant de ce Royaume que des Pays étrangers

Enfin, on taschera de faire en sorte qu'il ne se passe rien dans l'Europe digne de la curiosité des Gens de Lettres, qu'on ne puisse apprendre par ce Journal "(29).

Comme on peut le voir, les buts du Journal des Sçavans étaient, le moins que l'on puisse dire, ambitieux. Manten continue :" De ce fait, le but du Journal des Sçavans était clairement de servir les intérêts en même temps des communautés scientifiques et scholastiques en Europe. C'était une entreprise qui allait vite s'avérer impossible à exécuter, malgré la grande énergie dépensée par De Sallo et l'aide de scribes qui préparaient des résumés et des notes à partir d'information qui leur parvenait "(30).D'ailleurs ce premier numéro comportait , entre autres, un rapport sur la naissance d'un monstre à Oxford, une note sur les nouveaux télescopes de Giuseppe Campani, un commentaire sur la nouvelle édition du traité de Descartes, De l'homme, et un compte rendu des dernières parutions sur l'histoire de l'Église d'Afrique ${ }^{(31)}$. Bien que le contenu était très éclectique et des fois même trivial (la naissance d'un monstre à Oxford), ce qui en faisait pour une large part une gazette littéraire plutôt qu'un journal scientifique, le Journal des Sçavans n'en joua pas moins un rôle considérable dans la diffusion des connaissances scientifiques en permettant la communication entre savants. Le nombre d'articles consacrés aux sciences, souvent illustrés de gravures sur bois, s'accrut au fil des années. Aussi les lecteurs du Journal furent-ils les premiers à être informés de la parution du Micrographie de Robert Hooke, de la mise au point du premier navire à double coque par William Petty, de l'invention de la balance arithmétique par Cassini, de l'utilisation que fit Robert Holmes des horloges de Huygens à bord de la Réserve, ou encore des expériences menées par Ole Roemer pour déterminer la vitesse de la lumière.

\section{$\underline{\text { 2.1.1- Dennis de Sallo }}$}

Dennis de Sallo fut le fondateur et le premier éditeur du Journal des Sçavans. Il naquit Denis de Sallo, Seigneur de la Coudray, d'une veille famille noble du Poitou en 1626 à Paris. Il ne fut pas un brillant élève mais quand il prit des cours de rhétorique au Collège des Grassins, il obtint tous les prix de sa classe. L'année d'après, il devint un pupille distingué en philosophie et après avoir remarquablement soutenu en publique des thèses en Grecque et en Latin, il se consacra avec ardeur à des études en droit. Il fut si brillant qu'il réussit à succéder à son père,

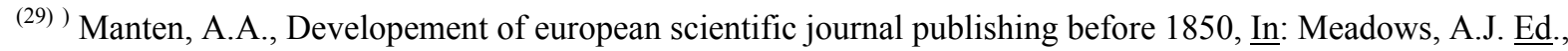
Development of science publishing in Europe, Amsterdam, Elsevier, 1980, p.5

${ }^{(30)}$ Idem

${ }^{(31)}$ http://fr.wikipedia.org/wiki/Journal_des_savants
} 
Jacques de Sallo, en tant que conseiller au Parlement de Paris. Il mourut le 14 Mai 1669 d'apoplexie

Quant à son parcours et son travail, nous nous baserons sur Le Grand Dictionnaire historique ou mélange curieux de l'histoire sacrée et profane... de Louis Moreri ${ }^{(32)}$. On apprend que De Sallo lisait toutes sortes de livres avec une incroyable attention et qu'il avait un nombre de secrétaires qu'il employait afin de transcrire ses réflexions et les passages qu'il avait soulignés. C'est de cette manière qu'il parvint à écrire toutes sortes de traités dans différentes spécialités. Il est très probable que c'est cette considérable quantité de matériaux qu'il amassait de cette manière qui lui a donné l'idée de faire profiter le public de ces extraits et dont il avait reconnu l'utilité à travers ses expériences. Il associa à ses travaux, colossaux pour l'époque, un nombre de gens de lettres et de sciences afin de l'aider. Mais (et ceci mérite d'être souligné pour l'époque et certaines assertions quant aux premières manifestations du contrôle par les pairs) il révisait tous les - peu nombreux- articles que ses collaborateurs lui fournissaient et en écrivait la plupart.

Les autorisations ayant étés obtenues avec l'aide de Jean-Baptiste Colbert (Contrôleur général des finances à l'époque), le plan et les périodes de publication fixées, le Journal des Sçavans put paraître le Lundi 5 Janvier 1665 en une feuille et demi in quarto sous le pseudonyme de Sieur de Houdeville (le nom de l'un des ses servants).La publication continua à paraître chaque lundi jusqu'au 30 Mars de la même année quand l'autorisation (de publier) fut retirée. La raison était, et ceci bien que ces critiques étaient toujours modérées et justes, que sa publication eut beaucoup d'ennemis parmi les gens de lettres et parmi eux les Jésuites, au summum de leur puissance, qui ne voyaient d'un bon œil un tribunal littéraire et philosophique qu'ils n'avaient pas mis en place. Ils demandèrent l'aide des autorités religieuses et le Journal fut suspendu. Le prétexte cité était un passage dans lequel De Sallo critiquait un décret des Inquisiteurs dont " les délicates oreilles demandaient une si grande circonspection".

Colbert, qui était derrière l'autorisation de publier le Journal, compensa cette perte à De Sallo en lui offrant un poste à la Trésorerie et réalisant la pleine mesure de son travail, commissionna l'Abbé Gallois de continuer le travail. Le Journal réapparut le 4 janvier 1666 et devint illustré. L'Abbé Gallois qui fut responsable pendant neuf ans ne fut pas un exemple de régularité : il n'y eut qu'un seul numéro en 1670 et pas un seul en 1673.Après avoir été successivement sous la responsabilité de différents éditeurs sans aucun succès, le Journal fut acquis par l'Etat en 1701 et sa gestion fut confiée à un groupe de personnes au lieu d'une seule. Il réapparut en date du 2 Janvier 1702 et dura jusqu'à 1792 quand les événements politiques le firent encore une fois suspendre. Durant cette période, le seul notable changement fut la périodicité qui d'hebdomadaire changea en mensuel avec des suppléments chaque six mois et ce en 1764.Après maintes tentatives de ressusciter le Journal qui furent toutes d'une manière ou d'une autre vouées à l'échec, ce n'est qu'en date du $1^{\text {er }}$ Septembre 1816 qu'il fut rétabli sur proposition du Garde des Sceaux et du Chancelier sur la base d'un rapport du Secrétaire General du Ministère de la Justice. La présidence du comité éditorial fut de ce fait attribuée aux Gardes des Sceaux à partir de cette date jusqu'au décret impérial du 4 Mai 1857 qui la transféra au Ministère de l'Instruction Publique. Voué de nouveau à disparaître pour des raisons de restrictions budgétaires, c'est tout naturellement que l'Institut de France, qui avait pris à sa charge les frais d'impression pour les années 1901 et 1902, se

${ }^{(32)}$ Moreri, Louis, Grand dictionnaire historique..., Drouet, Paris, 1759, t.IX, p.96, in- folio 
substitua à l'État. Néanmoins, ne pouvant consacrer de manière continue des fonds nécessaires à la publication du Journal des Savants, l'Institut de France proposa à l'Académie des Inscriptions et Belles-Lettres d'en accepter la charge, qu'elle assura à partir de 1909 grâce à des crédits prélevés sur la Fondation Dourlans.
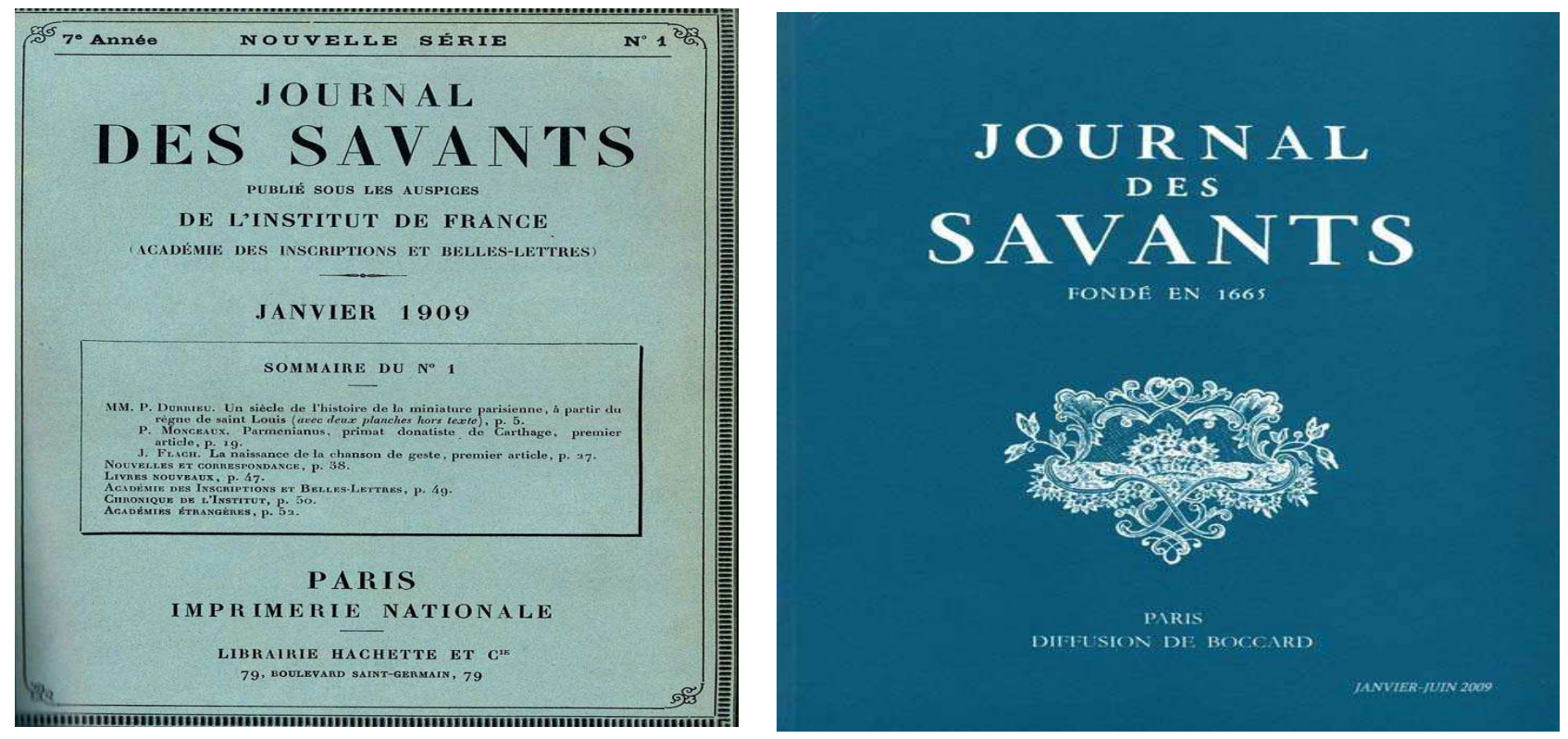

Fig.2-Couvertures du Journal des Savants (1909 et 2009)

\section{2-The Philosophical Transactions .}

Quelque deux mois seulement après la création du Journal des Sçavans à Paris, Henry Oldenburg, alors secrétaire de la Royal Society of London for Improving Natural Knowledge lança The Philosophical Transactions (dont le titre complet est : Philosophical Transactions giving some accompts of the present undertakings, studies, and labours of the Ingenious in many considerable parts of the world). Les premiers volumes de ce qui a été considéré comme le premier périodique scientifique étaient très différentes des numéros que l'on connait de nos jours mais servaient en quintessence la même fonction: informer les membres de la Société et d'autres lecteurs intéressés des dernières découvertes scientifiques. De cette manière, The Philosophical Transactions a jeté les fondements des plus importants principes du périodique scientifique moderne : la priorité scientifique et le contrôle par les pairs. En 1886, l'ampleur et l'importance de la découverte scientifique a augmenté au point qu'il devint nécessaire de diviser le journal en deux parties: The Philosophical Transactions of the Royal Society A: Physical, Mathematical and Engineering Sciences et The Philosophical Transactions of the Royal Society B: Biological Sciences.

Contrairement à De Sallo qui, bien qu'ayant lancé le premier périodique scientifique au monde, ne bénéficie pas de la notoriété qui sied à cette découverte, la personnalité du premier éditeur (on utilise cette fonction qui n'existait pas à l'époque car Henry Oldenburg en était le précurseur) des The Philosophical Transactions, Henry Oldenburg est à plus d'un titre 
intéressante à explorer. En effet, les écrits sur Oldenburg sont nombreux et en font l'archétype de ce qui allait devenir plus tard l'éditeur scientifique moderne tel qu'il est connu de nos jours. Qui était donc Henry Oldenburg et quelles ont étés les conditions qui ont conduit à sa fondation des The Philosophical Transactions et la notoriété qui en a découlé ?.

1

$\therefore$ PHILOSOPHICAL

T R A N S A C l'IONS:

G I V I N G S O M B

A C C O MP T

O P T II P P E S I N T

Undertakings, Studies, and Labours

o 1: T II I:

I NG E N I O U S

I $\mathrm{N}$ M A N Y

CONSIJ E R A BLE PARTS

O F T H E

W O R L D.

Vol $I$.

For Anno 1665 , and 1666 .

Fig 3-

Couverture du premier numéro

Philosophical Transactions

$(1665)$

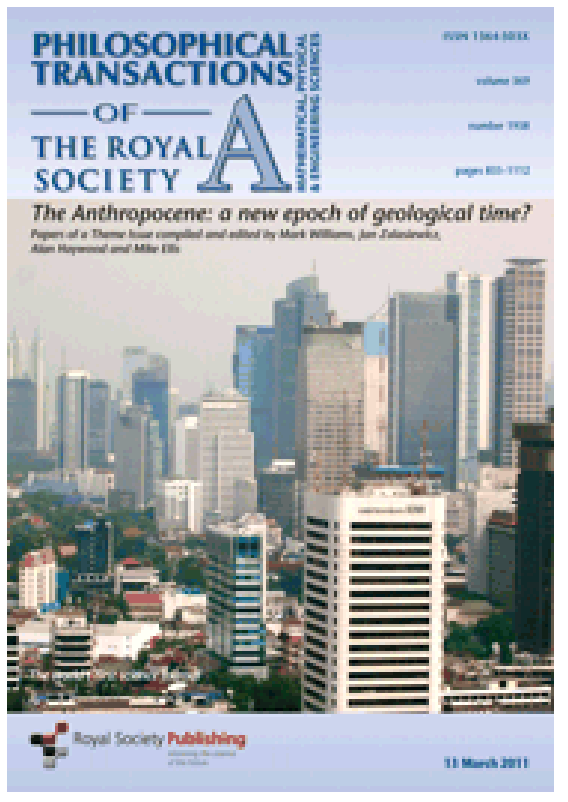

Couverture Philosophical Transactions

(2011)

\subsection{1-Henry Oldenburg}

Né Heinrich Oldenburg aux environs de 1618-19 à Brême, son père était professeur d'Université. Il entreprend des études de théologie à l'Université d'Utrecht et ensuite il devint tuteur dans différentes villes d'Europe. Au cours de ces pérégrinations, il apprit l'Anglais, l'Italien et le Français. Après une mission diplomatique à Londres qui le vit négocier la sécurité des navires de sa ville natale, il s'y établit. Il devint alors l'un des premiers membres de la Royal Society dont il devint en 1662 le premier secrétaire. Il fonda à son compte et sur ses propres deniers The Philosophical Transactions deux mois seulement après le Journal des Sçavans. S'il n'en tira aucun avantage pécuniaire, il n'en demeure pas moins qu'il établit une longue liste de savants avec lesquels il entretenait une correspondance .Parmi les plus connus, on peut citer, entre autres, Rasmus Bartholin, Robert Boyle, Comenius, Samuel de Fermat, Thomas Hobbes, Robert Hooke, Huygens, Leibniz, Spinoza, Melchisédech Thévenot, Isaac Vossius. C'est en leur nom qu'il formule de cette manière l'ambition de la science : " Pousser jusqu'à l'extrême limite des choses et s'efforcer de pénétrer l'antichambre de la Nature pour atteindre son cabinet secret " ${ }^{(33)}$.En 1667, il ne se découragea pas lorsqu'il fut

${ }^{(33)}$ Hall, A.R., and Hall, M.B., Ed, The correspondance of Henry Oldenburg, Madison, University of Wisconsin Press, 1965-73, p.114, Cité par Avramov, Iordan, An apprenticeship in scientific communication: the early correspondence of Henry Oldenburg (1656-63), Notes and the Records of the Royal Society, Vol.53, n², p.187201,22 Mai 1999 
brièvement incarcéré à la Tour de Londres à cause de son abondante correspondance avec l'étranger qui avait éveillé les soupçons. Au contraire, il continuera par tous les moyens à déjouer la censure pour faciliter les communications entre chercheurs et à user de ses talents diplomatiques pour apaiser les tensions et désamorcer les querelles entre savants. Hall résume assez bien la place d'Oldenburg en ces termes :" Pendant quinze ans, de 1662 jusqu'à sa mort en 1677, Henry Oldenburg servit la Royal Society comme second secrétaire et avait la charge de presque toute sa correspondance, interne et externe. Il fut durant cette période, un centre de communication des informations scientifiques, cherchant de nouvelles sources d'information, encourageant les hommes, où qu'ils étaient, à rendre leur travaux publique, agissant comme un intermédiaire entre scientifiques et fournissant, à travers The Philosophical Transactions, un medium pour la publication de courtes recherches scientifiques. La contribution d'Oldenburg à la communication scientifique fut unique durant le $17^{\mathrm{e}}$ siècle, non seulement parce qu'il représentait The Royal Society (dont il était un membre fondateur) et aussi servit tous ses membres avec impartialité. Il ne serait pas exagéré de dire qu'il a inventé les professions d'administrateur scientifique et de journaliste scientifique " ${ }^{(34)}$.Le même auteur, spécialiste par ailleurs d'Oldenburg, écrit dans une autre contribution : " [mais].......parce qu'il vécut jusqu'à la fin de l'année 1667, décédant approximativement à l'age de 58 ans, il a atteint une réputation à travers de l'Europe. Durant les quinze dernières années de sa vie, il était connu dans le monde scientifique et comme un des secrétaires de la Royal Society et le fondateur de Philosophical Transactions, le premier et le véritable plus ancien (c'est nous qui soulignons ) journal scientifique devenant de ce fait le premier administrateur scientifique professionnel.....Pendant quinze ans, son énorme correspondance permit au monde du savoir d'être au courant de ce qui se passait dans la science anglaise et permit aux philosophes naturels [nom donné aux savants en ces temps] anglais d'être au courant de ce qui se passait dans le monde du savoir et de la science en Europe. En établissant un étonnant réseau de communication scientifique à travers aussi bien son journal que sa correspondance ( plus de trois mille lettres qu'il avait écrit ou qu'on lui avait adressé et qui ont survécut en partie ou en entier édité et publié en treize volumes sous le titre de " La correspondance d'Henry Oldenburg"), il a non seulement aidé la Royal Society à fonctionner sans problèmes et efficacement mais a aussi laisser un monument durable de ses aptitudes et applications vu qu'en tant que Secrétaire, il a préservé les recherches et les lettres de la Royal Society et de ce fait a mis les fondations de son actuelle superbe archive, source unique pour l'histoire de la Science depuis $1661 "{ }^{(35) *}$.

${ }^{(34)}$ Hall, Marie Boas, Oldenburg and the art of scientific communication, The British Journal for the History of Science, Vol.2, p 277-290,1965

${ }^{(35)}$ Hall, Marie Boas, Henry Oldenburg : shaping the Royal Society, Oxford University Press, New York, 2002, p.XI

"Comme on peut le voir, l'œuvre et l'héritage d'Oldenburg fut à la fin d'un $17^{\mathrm{e}}$ siècle où l'Europe se réveillait d'un long et ankylosant Moyen Age, une œuvre qui a, et de loin, dépassé celle de De Sallo et sert de modèle à ceux qui étudient l'évolution non seulement des périodiques scientifiques mais de l'étude même de l'évolution de la transmission du Savoir. De même que Philosophical Transactions, bien qu'anté datant le Journal des Sçavans, reste le prototype et l'exemple type du périodique scientifique. Bien que la personnalité d'Oldenburg et son parcours (le fait qu'il était, par exemple, polyglotte) soient important dans la proéminence acquise par les Philosophical Transactions, il semblerait que ce soit la quintessence même des Philosophical Transactions qui en font, le premier et le véritable plus ancien périodique scientifique (pour citer Marie Boas Hall).C'est le contenu véritablement et foncièrement scientifique et dénué de toutes orientations idéologiques ajouté au fait que l'un (Journal des Sçavans) faisait dans la littérature secondaire en publiant des revues et des compilations de recherches déjà publiées et que le second (Philosophical Transactions) reportait sur des recherches originales faites devant les membres de la Royal Society. Cette aristocratisation de la recherche (entre information primaire et information secondaire) s'est perpétué jusqu'à nos jours où des périodiques scientifiques publiant des recherches fondamentales sont plus "cotés" que ceux qui ne publient que l'information secondaire. D'ailleurs, nombre de périodiques dans les sciences pures affichent leur facteur d'impact comme preuve de l'importance de leurs revues par rapport à celles qui font dans l'information secondaire. 
Nous terminerons cette querelle sur le plus important et premier périodique scientifique en reprenant Kronick et dont l'expertise sur le sujet ne peut être mise en cause .Dans une de ces nombreuses contributions, il dit au sujet des Philosophical Transactions et le Journal des Sçavans: " Le Journal des Sçavans tel qu'on l'appelait à l'époque apparut à Paris. L'Académie des Sciences [de Paris] fut fondée quelque temps après dans la même ville et, quoiqu'elle n'avait pas d'association officielle avec le Journal [des Sçavans], les informations au sujet de ces activités apparaissaient sur ces pages. Bien qu'il signalait des découvertes scientifiques telle que les curieuses expérimentations de Jean Denis concernant la transfusion sanguine et d'autres nouvelles scientifiques glanées de leurs correspondants c'était plus un journal littéraire généraliste qu'un journal scientifique (c'est nous qui soulignons).Il était beaucoup plus concerné par la revue de livres dans toutes les spécialités exception faite pour les livres en religion et en politique que les éditeurs traitaient avec beaucoup de précaution. Le Journal fut très vite réimprimé à Amsterdam et d'autres lieux et devint un modèle pour les journaux littéraires en Hollande, Italie, Allemagne et à travers toute l'Europe. The Philosophical Transactions et Le Journal des Sçavans ont tous les deux célébrés en 1965 leur $300^{\mathrm{e}}$ anniversaire quoique dans une forme assez altérée. Le Journal des Savants est aujourd'hui un journal littéraire généraliste et la Royal Society utilise maintenant The Philosophical Transactions pour publier deux séries de monographies hautement spécialisées, l'une en mathématiques et physique et l'autres dans les sciences biologiques" ( ${ }^{(36)}$.Il est clair que le Journal des Sçavans bien qu'ayant précèdé les Philosophical Transactions dans le temps, il n'a pas le titre de premier périodique scientifique qui échoit aux Philosophical Transactions de par sa composante.

Après avoir présenté les deux premiers périodiques scientifiques de l'histoire, et ayant essayé, ne serait ce que d'une manière superficielle, de voir leur influence, les personnages clés qui ont étés prépondérant dans leur fondation, les conditions de leur lancement, la prochaine étape de ce travail va essayer de voir quelles ont étés les conséquences de cette innovation qui à la fin du $17^{\mathrm{e}}$ siècle avait bouleversé les fondements de la transmission du Savoir.

Après que Le Journal des Sçavans et The Philosophical Transactions (chacun à sa manière et dans des registres différents) aient tracé la voie en 1665 pour une nouvelle manière de communiquer la Science et les résultats de la recherche, nombre de périodiques ont commencés à voir le jour à travers toute l'Europe. Les différentes sources s'accordent à faire de Giornali dei litterati en Italie et de Miscellanéa Curiosa en Allemagne les deux premiers périodiques après Le Journal des Sçavans et les Philosophical Transactions et ce respectivement en 1668 et 1670 (quoique pour Miscellanéa Curiosa certaines sources la situe en 1668 aussi).Vu la proximité de la date de fondation de ces deux périodiques, ils ont étés décrits comme des imitations du Journal des Sçavans pour Giornali dei litterati et de Philosophical Transactions pour Miscellanea Curiosa. Ce dernier périodique est aussi considéré, par certains, comme le plus ancien périodique spécialisé.

\section{3-Publications périodiques scientifiques précurseurs des journaux scientifiques modernes}

Il faut signaler que les écrits sur le sujet des périodiques sont tous d'accord sur les premiers périodiques universellement reconnus comme tels, mais qu'il a existé des antécédents qui

\footnotetext{
(36) Kronick, David A., Litterature of the life sciences : the historical background, Bulletin of the New York Academy of Medecine, Vol 60, n9, p.866-867, Novembre 1984
} 
n'ont pas étés comptabilisés car ils ne répondaient pas aux canons de ce qui est considéré comme un périodique. Les plus anciens de ces spécimens étaient :

3.1-Acta diurna qu'on peut traduire par faits du jour, désignait à Rome un recueil officiel qui contenait le récapitulatif des évènements quotidiens : décisions et délibérations du Sénat et du peuple, exécutions capitales, naissances, mariages, divorces, funérailles des personnes illustres, annonce des jeux publics. Il fut rendu public vers l'an 131 av. J.-C. Par leur contenu, les acta diurna préfigurent les premiers journaux; en revanche, leur support de diffusion s'apparente plus à l'affichage public ${ }^{(37)}$.

3.2- La Gazette de Pékin (Jingpao ou Jingbao littéralement Le Témoignage de la Capitale) qui était une publication de la cour impériale chinoise et dont la fondation remonte au $8^{\mathrm{e}}$ siècle à la dynastie Tang. Elle est souvent présentée comme le plus ancien journal au monde. Valravens y voit une certain forme de périodique mais il poursuit " ils [les écrits occidentaux] ne prennent pas en ligne de compte que cette gazette avait une circulation limitée et qu'elle ne contenait que des édits et des décrets - de ce fait elle ne répond pas à la définition moderne d'un journal. Mais c'était sans aucun doute un précurseur du journal ${ }^{(38)}$ ".

3.3- Relation Aller Fuernemmen und gedenckwuerdigen Historien (Communication de toutes les histoires importantes et mémorables) daté de 1605 et publié en 1609 par Johan Carolus. " Le recueil promet des nouvelles de l'ensemble de l'Europe centrale et occidentale..... Le genre suscite contrefaçons et imitations dans d'autres villes allemandes comme l'Avisa Relation oder Zeitung publiée à Augsbourg en 1609 par Lucas Schulte " ${ }^{(39)}$.Le Relation Aller Fuernemmen und gedenckwuerdigen Historien a été reconnu en 2005 par The World Association of Newspapers (Association Mondiale des Journaux) comme étant le plus ancien journal existant qui avait célébré son $400^{\mathrm{e}}$ anniversaire à cette date, alors que les spécialistes fixaient généralement 1609 comme étant l'année où les premières traces de " Relation Aller Fuernemmen und gedenckwuerdigen Historien " étaient disponibles ${ }^{(40)}$.

3.4- The Frankfurter Journal qui fut fondé en 1615 à Francfort sur le Main par Egenolph Emmel, un libraire de la même ville. Cette publication fut imitée, avec quelques améliorations, par le Frankfurter Oberpostamtszeitung qui continua à paraître en tant que Frankfurter Postzeitung et ce jusqu'à $1866^{(41)}$.Il faut signaler que le Frankfurter Journal est considéré le premier journal en Allemagne alors que ..." les premières collections distinctement périodiques des " informations du jour " qu'on distingue des " brochures d'information" isolées et dont il existe au moins un exemple à une date aussi ancienne que $1498 \ldots . . . .$. et qu'on peut trouver dans des bibliothèques toujours existantes" ${ }^{(42)}$

\footnotetext{
${ }^{(37)}$ http://fr.wikipedia.org/wiki/Acta Diurna

(38) Valravens, Hartmut Ed., Newspapers of the world online: U.S. and international perspectives ,Munchen, K.G. Saur, 2006, p.159

(39) Haffemayer, Stephane, Transferts culturels dans la presse européenne du XVIIeme siècle, Le Temps des medias, Vol. 2, n¹1, p.27-28

${ }^{(40)}$ La Presse : quatre siècles de jeunesse ! Disponible à : http://www.wan-press.org/article6468.html (Consulté le $12 / 02 / 2011)$

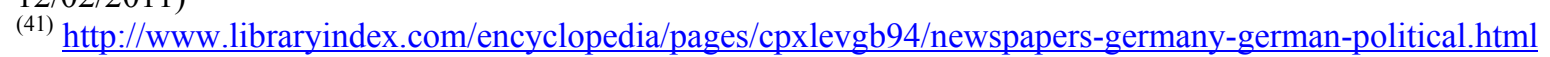

(Consulté le 18/02/2011)

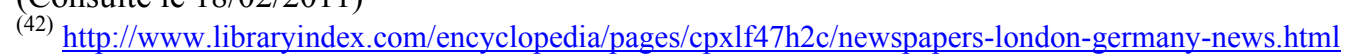

(Consulté le 18/02/2011)
} 
3.5-Le Mercurius Gallo Belgicus, [qui] " a été longtemps considéré comme le plus ancien journal fut fondé en 1598 par Mr. Jansen et le premier volume qui parut in $8^{\circ}$ fut imprimé à Cologne et parut cette même année en 650 pages .C'était plutôt un registre annuel qu'un journal. Il était orné avec la gravure sur bois représentant Mercure sur un globe avec ses attributs habituels .Il fut publié jusqu'au $17^{\mathrm{e}}$ siècle..... "(43).$I 1$ est considéré comme " le plus ancien journal périodique connu de continuité définie que l'on distingue des furtives et éphémères feuilles de bulletins..... et qui couvrait les informations sur l'Europe de 1588 à 1594 mais fut transféré à Francfort sur le Main où il fut publié jusqu'à 1630 "(44).

Nous voudrions aussi citer un cas qui nous a paru très intéressant et en même temps symptomatique des actions de l'époque et qu'on pourrait classer comme précurseur et aussi représentatif des efforts des hommes lettrés de l'époque. Il s'agit du Bureau d'adresse de Théophraste Renaudot.

\section{4- Un genre peu connu et peu médiatisé : Le Bureau d'adresse de Théophraste Renaudot.}

Renaudot était un docteur, un journaliste et un philanthrope qui, entre 1630 et 1641, organisait une série de conférences hebdomadaires publiques sur divers sujets, y compris la science, appelé Conférences du Bureau d'Adresse. Ils furent publiés sous le titre de "Questions traitées ès Conférences du Bureau d'Adresse " en 5 volumes entre 1633 et 1641.Renaudot fonda le Bureau d'Adresse à Paris en 1630.

" Le Bureau était ce qui pourrait être considéré comme une agence d'emploi combiné avec une clinique de consultations externes. Quiconque s'y inscrivait (de 0 à 3 sous chacun selon ses moyens) recevait un traitement médical gratuit, et de l'aide pour la recherche de travail, d'habits pas chers, le gite et l'ameublement. Le Bureau accordait aussi à ses clients des petits crédits et les aidait dans leur relation avec le gouvernement et la loi. Il gardait une carte index de personnes qui cherchaient des services ou offraient de l'aide. De même qu'il avait un index des prix courants. Graduellement, il se diversifia en une agence de publicité, une agence de voyage, un service de messagerie, une agence de louage de chevaux et un magasin où presque tout pouvait être acheté ou loué : bibelot, objets antiques, animaux domestiques maisons, domaines, généalogies, services de tuteurs privés, funérailles........Le Bureau arrangeait les mariages, recrutait des soldats, trouvait des moines pour les monastères et avait même l'intention de s'intéresser aux diplômes académiques .

Ce trafic en biens et services impliquait naturellement aussi un trafic en information. Avec des clients de toutes sortes de milieux et à travers un système de correspondants, le Bureau collectait systématiquement des informations locales et de l'étranger qui s'avérèrent très précieuse pour le gouvernement.

En effet, ceci était la raison principale de la protection continue qu'il recevait de la part du Père Joseph et du Cardinal de Richelieu. Non seulement, ils récupérèrent la meilleure partie de l'information mais ils l'utilisèrent pour influencer l'opinion publique.....

\footnotetext{
(43) Timperley, C.H., A Dictionnary of printers and printing, with the progress of literature ancient and modern, biblographical illustrations, etc. etc., H.Johnson, London, 1849, p.428

${ }^{(44)}$ Ebert, Myrl, The Rise and Development of the American Medical Periodical 1797-1850, Bulletin of the Medical Library Association, Vol. 40, n³, p.245, Juillet 1952
} 
Renaudot transforma aussi le Bureau en un centre d'activité intellectuelle. A partir de 1633, il organisa des conférences hebdomadaires dans ses appartements sur l'Ile de Saint Louis. Comme c'était le cas dans les académies de la Renaissance précédente, quaestiones étaient proposées à ces rencontres qui provoquèrent l'échange d'opinions mais n'étaient pas décidé par de la recherche empirique.....A certains égards, ces " conférences " lorgnaient vers les sociétés scientifiques de la seconde moitié du $17^{\mathrm{e}}$ siècle, les discussions étaient en langage vernaculaire (en Français et non pas en Latin), il était interdit de citer les " autorités ", les sujets politiques et religieux devaient être évités .De temps à autre, des expérimentations même étaient faites afin de démontrer certains point de discussion. En 1640, Renaudot créa un laboratoire chimique. Malgré tout, son intérêt principal n'était pas la science pure, mais son application pédagogique et humanitaire. Selon les principes philanthropiques de Renaudot, les "conférences" étaient ouvertes à toute personne qui était intéressée et, de ce fait, n'étaient pas considérées très prestigieuses parmi l'élite intellectuelle ".

Les conférences hebdomadaires de Renaudot ont une certaine ressemblance avec celles du College Invisible qui précéda la Royal Society. Cependant, elles étaient fréquentées par une audience considérablement plus grande et elles étaient plus assimilables à la science populaire dans leur orientation et leurs intervenants demeuraient anonymes dans les rapports publiés.

Les Conférences précédèrent Le Journal des Sçavans et The Philosophical Transactions de 30 ans. Elles furent collectées sous forme de livre plutôt que publiées en tant que périodique et furent publiées en une traduction anglaise en 1664-1665 alors que la Royal Society était entrain d'être formé ${ }^{(45)}$.

Nous avons cité ces quelques exemples de périodiques (et de publications qui avaient l'aspect périodique comme pour les Conférences de Renaudot) ayant précédé la date de 1665 et qui est la date acceptée de la fondation du périodique scientifique, c'est pour faire ressortir que le $15^{\mathrm{e}}$ et $16^{\mathrm{e}}$ siècle (et même avant avec les Acta Diurna et la Gazette de Pékin) avaient vu des embryons de publications périodiques qui n'avaient rien de scientifique mais étaient beaucoup plus des sortes de brochures, des pamphlets, d'affiches et des feuilles volantes dont le but était de faire passer l'information, ces périodiques n'avaient aucunement la prétention de présenter des découvertes scientifiques mais étaient plutôt des journaux dans le sens du quotidien ou hebdomadaire de nos jours, quoique la périodicité n'y était respectée comme elle l'est de nos jours .On remarquera d'un autre coté, les titres même de ces périodiques étaient différents de ce qui allait être les périodiques scientifiques et en fait ces mêmes titres étaient une indication de la population ciblée. Ainsi ......" les titres adoptés différenciaient ces journaux des publications politiques, mondaines ou de vulgarisation, puisque Journal, Transactions, Acta, Giornale, Bibliothek (Biblioteca, Bibliotheque, Boekzaal), Abhandlungen, Miscellanea, suggèrent l'enregistrement ou la conservation, alors que les feuilles d'informations politiques jouaient plutôt sur la rapidité de communication (par exemple les divers " courriers ", " correo ", ou les divers " Merkur ", " mercurio " etc.). De plus, l'introduction de qualificatifs permettait de préciser la cible, que ce soit les déclinaisons de savant - Journal des savants, Giornale de 'letterati, Actae ruditorum, ou encore Boekzaal der geleerde Wereld -, ou plus avant dans le siecle des variations sur l'amour des sciences et des arts, ainsi des Wöchentliche Anzeigen zum Vortheil der Liebhaber der Wissenschaften und Kunste. Les titres qu'affichent les périodiques savants constituaient donc des signaux d'orientation pour les clients des

${ }^{(45)} \mathrm{http}: / /$ www.historyofinformation.com/index.php?era=1600 (consulté le 28/02/2011) 
librairies qui pouvaient ensuite trouver dans les préfaces et avis des indications sur les visées de leurs auteurs. ${ }^{(46) " .}$

On peut considérer la période qui a suivi l'apparition des deux premiers journaux scientifiques comme une période très riche ${ }^{*}$ car elle a permis l'apparition d'une multitude de titres de périodiques scientifiques dans toutes les spécialités et dont l'énumération, la classification et la consistance sont, à plus d'un titre, importantes pour voir quelle a été leur influence dans les nouveaux schémas de transmission du Savoir. Nous essayerons de faire une sorte d'énumération de ces périodiques afin de voir :

\section{1- Leur progression numérique}

2- Leur évolution quant au contenu (qualitative)

3- Leur évolution quant au nombre (quantitative)

4- Leur évolution quant à l'influence sur la transmission et la conservation du savoir.

\section{5-Les premiers périodiques scientifiques post Journal des Sçavans et Philosophical Transactions}

L'avènement du Journal des Sçavans et The Philosophical Transactions presque simultanément n'a pas tardé à faire des émules et l'Europe s'est trouvée submergé par un nombre de plus en plus croissant de périodiques. Il est peut être exagéré de dire que l'Europe a été submergée mais il est indéniable que nombre d'entreprises furent lancées. Comme déjà signalé , la période est peu et même mal documentée ${ }^{* *}$ et peu de chercheurs se sont aventurés à essayer de faire une cartographie de cette période si importante mais si mal documentée. Au cours de nos recherches bibliographiques, nous avons pu vérifier de visu cette lacune et avons dû adapter notre travail à cet état de fait. D'un autre coté, la plupart des travaux englobant cette période sont le fait d'auteurs anglo-saxons bien que les auteurs européens (non anglo saxons) aient aussi publiés mais pas au rythme des Anglo- Saxons .

(46) Pfeiffer, Jeanne, Vittu, Jean-Pierre, Les Journaux savants, formes de la communication et agents de la construction des savoirs $\left(17^{\mathrm{e}}-18^{\mathrm{e}}\right.$ siècles $)$, Dix-huitième siècle, Vol.1, n ${ }^{\circ} 40$, p.283.2008.

*En même temps très confuse quant au cheminement des différents périodiques qui comme nous l'avons vu auparavant n'avaient pas encore complètement ni assis leur prépondérance dont ils se prévalent de nos jours ni même acquis une forme définitive car, ils étaient en concurrence avec le medium qui était de mise en ces temps le livre, et de ce fait leur ressemblaient énormément.

** A titre d'exemple le premier numero de "Relation Aller Fuernemmen und gedenckwuerdigen Historien" n'a été officiellement daté de 1605 que 400 ans plus tyarden 2005 alors que la dte généralement acceptée etait celle de 1609.C'est le Dr.Martin Welke, fondateur du Musée allemend de la presse et le Pr. Jean Pieerre Kintz à qui la paternité de cette decouverte est attribuée.Ce "certificat de naissance ", pour citer Timothy Balding le Directeur Gaénéral de l'Association Mondiale des Journaux, a été decxouvert dans les archives municipales de la ville de Strasbourg qui faisait partie, à l'époque du " Reichland".Le Directeur Générald el'AMJ a exprimé decouverte de la maniere suivante: "Cette preuve est incontestable et je pense que nous pouvons tous souhaiter un Bon et Heureux $400^{\mathrm{e}}$ anniversaire cette année au journal imprimé !Notre Bureau Executif a éxaminé toius ces faits et est persuadé que cette histoire tient debout ".Disponible à : http://www.wan-press.org/articles64668.html. ( consulté le 21/02/2012) 
De ce fait, la plupart des références sont en langue anglaise. Nous avons basé notre liste de cette période sur les écrits de David Kronick dont trois importantes et incontournables références pour la période et qui sont :

-Scientific and technicals periodicals of the seventeenth and eighteenth centuries: a guide, publié en 1991

-History of scientific and technical periodicals: the origins and development of the scientific and technical press, 1665-1790, publié en 1976.

"Devant le deluge" and others essays on early modern scientific communication, publie en 2004.

5.1-Développement des périodiques scientifiques au $17^{\mathrm{e}}, 18^{\mathrm{e}}$ et $19^{\mathrm{e}}$ siècle.

L'établissement et le développement des sociétés savantes furent un facteur instrumental dans l'émergence du journal comme le medium principal de la communication scientifique. Durant les premières cinquante années du $16^{\mathrm{e}}$ siècle, les collèges invisibles (qui étaient un ensemble de philosophes en contact mais d'une manière informelle) se développèrent via le contact personnel et les communications écrites privées. Ces collèges devinrent, plus tard, les académies et sociétés savantes officielles qui commencèrent à enregistrer et disséminer le résultat des expérimentations de leurs membres dans des livres de compte rendus et aussi via les communications écrites entre leurs membres. Mais la pratique de communication sous la forme de lettre était très restrictive en ce sens que l'information ne pouvait être divulguée, révélée qu'à une seule personne à la fois ou un nombre limité si les copistes étaient utilisés. A cet effet, Nancy Fjällbrant nous cite le cas des anagrammes qu'utilisaient les savants afin de sauvegarder la paternité d'une découverte et éviter qu'elle leur soit subtilisée par un concurrent. Elle dit que : " Au début du $17^{\mathrm{e}}$ siècle, les savants établissaient la priorité de la découverte par le biais d'un anagramme. Une phrase annonçant une découverte était encryptée en un anagramme et déposée chez un témoin officiel. Le savant pouvait alors travailler en toute tranquillité. Si un quelconque compétiteur revendiquait publiquement la même découverte, le savant pouvait se référer à son témoin pour déchiffrer l'anagramme et de cette façon établir sa priorité ".Elle poursuit :.... " "Galilée ecrivit en 1610 l'anagramme suivant à Kepler : smaismrmilmepoetalevmibunenugttaviras. Kepler fut incapable de le déchiffrer et Galilée lui dit plus tard que cet anagramme voulait dire : " altissimum planetam tergeminum observari" (j'ai observé les triples les plus haut de la planète). Ceci avait trait aux anneaux de Saturne que Galilée avait vu pour la première fois. Par ces moyens, il fut capable de gagner du temps pour vérifier ses observations avant de faire une annonce publique officielle ". ${ }^{(47)}$

\subsection{1- Deux exemples symptomatiques de l'époque : Miscellanea curiosa et Acta Eruditorum}

\subsubsection{1-Miscellanea curiosa (1670-1705)}

Le nom complet est " Miscellanea curiosa sive ephemeridum medico-physicarum Germanicarum Academiae Caesareo-Leopoldinae Naturae Curiosorum". En 1652, quatre médecins fondèrent l'Academia Naturae Curiososum à Schweinfurt, la plus ancienne des

\footnotetext{
${ }^{(47)}$ Fjällbrant, Nancy, Scholarly communication: historical developements and new possibilities, [Proceedings], Scholarly Communication in Focus, June-July 1997, IATUL conference, Trondheim. (Disponible à : http://educate.lib.chalmers.se/IATUL/proceedcontents/fullpaper/nfpaper.html )
} 
universités pour la recherche scientifique en Allemagne et près d'un demi-siècle plus ancienne que l'Académie de Berlin. L'Académie, aujourd'hui connue sous la Leopoldina, avait à l'origine pour but principal d'améliorer à la fois la pratique médicale et les soins médicaux. Aujourd'hui, après 350 années, l'Académie se compose de 27 sections avec plus de 1.000 scientifiques, qui représentent presque tous les domaines de l'activité scientifique.

L'Académie publie son propre journal, le premier au monde pour la médecine scientifique, depuis 1670: Miscellanea curiosa medico-physica Academiae Naturae Curiosorum sive Ephemeridum medico-physicarum Germanicarum curiosarum. Le long titre baroque a vu beaucoup de changements au fil des siècles, mais l'accent sur la médecine scientifique y est resté jusqu'en 1928. Jusque-là, environ 150 volumes avaient paru plus ou moins régulièrement.

Le Président de l'Académie a édité les premiers volumes, mais à partir de 1683 un Directeur Ephemeridum fut nommé hors du cercle des membres du comité. La première personne nommée a été Johann Georg von Volckamer, élu président en 1685. Son successeur en tant que Directeur Ephemeridum a été Lucas Schroeck qui lui a également succédé comme Président en 1693. Andreas Elias Büchner et Christoph Jacob Trew furent deux autres éditeurs importants de l'Ephemeriden au $18^{\mathrm{e}}$ siècle.

L'emplacement de l'Académie et, plus tard, sa bibliothèque ainsi que la collection a changé avec la ville natale du président, en commençant par Schweinfurt puis à Nuremberg, Augsbourg, Altdorf, Erlangen et d'autres villes jusqu'à ce que finalement Halle soit devenue le siège permanent de l'association en 1878 .

Christian Gottfried von Nees Esenbeck servi le plus long mandat à la présidence (1818-1858) et sous sa direction 47 volumes de Nova Acta furent publiés. En raison des intérêts de Mr.Nees, l'accent fut mis sur une évolution très nette vers la botanique dans cette période. La concurrence des autres académies et les journaux, la perte de membres, la réforme des structures internes et de la spécialisation et de professionnalisation des différents champs ont été toutes étés des questions débattues au sein de la Leopoldina.

Sous les présidences de Wilhelm Behn (1870-1878) et Carl Hermann Knobloch (1878-1895) l'accent a a été mis sur une représentation égale de toutes les disciplines dans la revue. Au moins un article de chaque section devait être publié chaque année. Au tournant du siècle, le tirage de l'excellent produit du Nova-acta était de 500 dont, contrairement aux précédentes, la majorité n'avait pas besoin d'être vendus, mais était utilisé pour le troc ce dont profitait la Bibliothèque de l'Académie.

La Leopoldina se considéra dès sa fondation au même niveau que The Royal Society et l'Académie des Sciences et se tint en contact avec les deux institutions. Son Ephémérides survécu aux épreuves et les tribulations de plus de 300 ans.Sa fonction principale résidait dans la répartition des connaissances, mais d'importantes découvertes et des nouvelles publications ont d'abord trouvé leur place dans ses pages. Le fait que la moitié de 157 des lauréats du prix Nobel aient été élus à la Leopoldina avant d'avoir reçu cette haute distinction indique la sélection intelligente et de haute qualité à la fois de cette institution et son journal. 


\subsubsection{2- Acta Eruditorum, (1682 à 1782)}

C'est une revue scientifique mensuelle allemande publiée de 1682 à 1782 à Leipzig par le savant Otto Mencke à l'initiative de Leibniz.

Créés à l'imitation du Journal des savants, les Acta Eruditorum sont la première revue scientifique dans l'histoire savante de l'Allemagne.

Rédigés en latin, les Acta Eruditorum comprenaient des résumés de nouveaux écrits, des critiques, des sommaires, de courts essais et des notes, essentiellement dans le domaine des sciences naturelles et des mathématiques, mais également dans le domaine de la théologie et de la philosophie. La collaboration de savants connus de l'époque a assuré la qualité de la nouvelle revue et, de ce fait, encouragé le développement de l'esprit critique en Allemagne.

À la mort d'Otto Mencke, les Acta furent repris par son fils, Johann Burckhardt Mencke jusqu'à son décès en 1732, lui-même remplacé par son propre fils Friedrich Otto Mencke, date à laquelle la revue changea de nom pour devenir les Nova Acta Eruditorum. Les troubles de la Guerre de Sept Ans et la négligence du rédacteur en chef de l'époque, Karol Andrej Bel, qui avait repris la rédaction en 1754, causèrent de sérieux retards dans la parution mensuelle des tomes, à tel point que l'édition de la dernière partie de l'an 1776 n'est parue qu'en 1782.

En 1712, Friedrich Otto fonda le pendant germanophone des Acta Eruditorum, les Deutsche Acta Eruditorum, qui se consacraient plus particulièrement aux écrits historiques et aux polémiques du moment.

Dès ses débuts, nombre d'éminents scientifiques, tels que Isaac Newton, Gottfried Leibniz, Jacob Bernoulli, Humphry Ditton, Ehrenfried Walther von Tschirnhaus, Denis Papin, PierreSimon Laplace, Jérôme Lalande, Giovanni Domenico Cassini, John Flamsteed, Jacob Hermann, Christiaan Huygens ou mais également des philosophes et des humanistes comme Christian Thomasius, Christian Wolff, Veit Ludwig von Seckendorff, Christian Wagner ou Stephan Bergler ont publié dans les Acta.

C'est dans le numéro d'octobre 1684 des Acta que Leibniz publia sa Nova Methodus pro maximis et minimis, itemque tangentibus, et singulare pro illis calculi genus ; outre la solution définitive du problème des tangentes, Leibniz y inaugurait le calcul intégral par la solution du problème de Beaune : Trouver une courbe dont la sous-tangente soit constante.

Il faut signaler que ces deux publications allemandes étaient, de part la date de leur fondation, très proche de 1665, des imitations, des copies de respectivement The Philosophical Transactions (pour Acta Eruditorium) et Journal des Sçavans (pour Miscellanea Curiosa).

Après ces deux périodiques, le medium connut un développement qui ne s'est nullement departit depuis et a conduit à en faire le canal préféré des scientifiques. Il serait fastidieux (et quasi impossible) d'essayer de les citer mais certains sortent du lot et méritent d'être cités. Ce sont :

5.2-Le Recueil des mémoires et conférences sur les arts et les sciences et qui était publié sur le modèle et comme supplément du Journal des Sçavans. Il consistait généralement de revues 
de livres, quelques contributions originales, des lettres et extraits d'autres journaux notablement des The Philosophical Transactions de la Royal Society.

5.3-Giornale dei litterati d'Italia (1668 -1682) c'est le premier périodique italien de l'époque. Il fut fondé en date du 28 Janvier 1668 à Rome, il eut pour responsable Francesco Nazzari et Sarvatore Serra qui, respectivement, s'occupèrent de la revue des livres français et italiens.A l'instar des periodiques de la periode, il prend comme exemple The Philosophical Transactions et le Journal des Sçavans. Y contribuèrent les hommes de culture de l'époque tels que : Michelangelo Ricci, Giovanni Giustino Ciampini, Francesco Serra, Giovanni Luci, Tommaso de Giuli, Giovanni Patrizi.En mars 1675,Nazzari changea d'imprimeur et commença à faire publier le Giornale, ce qui n'empêcha pas le précédant imprimeur à continuer de publier lui aussi le Giornale, on eut de ce fait deux journaux portant le même titre paraissant concurremment.Il était de periodicité trimestrielle et pendant une trentaine d'année , il exerça une forte influence sur toute la péninsule, jouant un role décisif dans la fondation de l'identité nationale et la définition de la littérature italienne.Son contenu érudit embrassait l'histoire, la théologie, la science et le droit. Il fut imité en Italie par un titre homonyme à Parme en 1686 et à Venise en 1710.

5.4-Acta Medica \& Philosophiae Hafniensia (1673-1680) et qui se spécialisait dans la recherche médicale et dont l'éditeur, Thomas Bartholin, professeur d'anatomie, était ce qui est considéré comme l'archétype de l'éditeur critique du $17^{\mathrm{e}}$ siècle. En effet, Bartholin ne se contentait pas de publier les communications en sciences médicales qu'il recevait, il y adjoignait souvent des commentaires et des notes.

Il est à noter que la plupart des premiers périodiques du milieu du $17^{\mathrm{e}}$ et début du $18^{\mathrm{e}}$ siècle disparurent au bout d'une année ou deux ou même, dans certains cas, au bout d'un ou deux numéros. En plus du fait que le medium était neuf, il avait besoin d'adaptation, les journaux n'étaient pas sous la coupe de sociétés savantes dont l'apparition et l'influence ne furent prépondérantes qu'à partir de la première moitie du $18^{\mathrm{e}}$ siècle. Les rares sociétés (ou ce qui y ressemblaient) qui existaient se contentaient de publier leur travaux dans des journaux qui étaient généralement financés par des individus ou alors publiaient leurs travaux sous forme de collection rétrospective de recherches à des intervalles longs et surtout irréguliers. Ceci est dû, il ne faut pas l'oublier, aux conditions sociales de l'époque qui ne se prêtaient nullement à la production scientifique. Il n'y avait pas matière à publier à cause du problème de communication et aussi à une nouvelle tradition qui n'avait pas encore pris racine et qui allait voir au $18^{\mathrm{e}}$ siècle un envol qui ne s'est plus arrêté depuis ce qui a induit des problèmes de gestion de cette extraordinaire masse d'information.

Au début du $18^{\mathrm{e}}$ siècle, il est estimé que 801 titres allaient voir le jour alors qu'entre la relativement courte période entre 1665 et la fin du $17^{\mathrm{e}}$ siècle n'a vu la création que de quelque 30 titres scientifiques et médicaux environ ${ }^{(47)}$.A la première moitié du $18^{\mathrm{e}}$ siècle, le journal scientifique était devenu le medium accepté de la communication scientifique et technique et ses fonctions clairement définis. Ce sont :

1-Pourvoir à la communauté scientifique et les amateurs intéressés avec des informations dans la langue vernaculaire et qui étaient auparavant en langues étrangères.

\footnotetext{
${ }^{(47)}$ Fielding, Garrison, H., The medical and scientific periodicals of the $17^{\text {th }}$ and $18^{\text {th }}$ centuries, Bulletin of the History of medecine, Vol.2, n5, p.296, Juillet 1934
} 
2-Fournir les moyens aux chercheurs scientifiques et en sciences sociales afin de discuter sur un travail scientifique sans l'avoir lu en entier

3-Conserver le matériel scientifique qui aurait autrement été dispersés en tracts individuels et en pamphlets.

4-Aider la recherche en fournissant des canaux de communication à la portée de tout le monde.

5-Encourager les scientifiques à publier leurs travaux.

6-Offrir un forum pour l'examen critique continue des hypothèses et théories scientifiques. ${ }^{(48)}$

Parmi les quelques 800 titres que Fielding a recensé au $18^{\mathrm{e}}$ siècle, on peut citer parmi ceux qui avaient marqué leur époque :

5.5-Raccolta d'Oppuscoli scientifica e fililogici qui parut entre 1728 et 1757 et qui fut continué sous le titre de Nuova Raccolta d'Oppuscoli scientifici entre 1855 et 1887.

5.6-Pour et Contre qui parut entre 1733 et 1740 et eut comme titre alternatif " Le Pour et Contre, ouvrage périodique d'un goût nouveau ".Il fut l'œuvre d'un certain François Prévost (Antoine François Prévost d'Exiles, dit l'abbé) qui n'en publia qu'une vingtaine de volumes. Il est décrit comme " [C'est] une feuille périodique dont la manière n'était point calquée sur les ouvrages du même genre. C'est un assemblage d'anecdotes, de récits, de traductions, de jugement sur les productions de la littérature anglaise : tous ces morceaux sont disposés avec un désordre qui en rendait la lecture encore plus piquante ; et l'impartialité du critique ne s'y dément jamais. Les vingt volumes se composent de 296 numéros, à la fin du tome X est une table des dix premiers volumes; à la fin du tome XX, une table des dix derniers volumes "(49).

5.7-Gottingische zeitung von gelehrlen sachen qui commença à paraître à partir de 1739.C'est une revue littéraire et de recension allemande. Elle est considérée comme la plus vieille revue scientifique apparaissant encore dans l'aire linguistique allemande. Dans cette revue spécialisée, des scientifiques s'efforcent d'examiner d'un œil critique des travaux scientifiques contemporains. Les premiers volumes de Göttingische sont apparus en 1739 et furent à partir de 1747 sous la direction du médecin Albrecht von Haller. Depuis 1753, la revue a été publiée par l'académie des sciences fondée en 1751 à Goettingen et en portait jusqu'en 1801 le titre. En 1802, le titre actuel a été introduit, la revue est publiée par la maison d'édition Vandenhoeck \& Ruprecht, jusqu'aux années de 1896.En 1935, elle a été reprise par la maison d'édition Verlag Weidmann de Berlin. Les Gottingische eurent en outre une interruption entre les années $1960-1961$ et $1944-1953$.

5.8-Der Naturforscher qui parut entre 1774-1804. Il se distingua d'entre les premiers périodiques scientifiques par le fait qu'il publiait un nombre signifiant de contributions originales, contrairement à dupliquer ou réimprimer à partir d'autres sources. Il était le premier à se limiter aux sciences naturelles. L'esquisse de la page de titre montre un

\footnotetext{
${ }^{(48)}$ Houghton Bernard, Scientific periodicals : their historical development, characteristics and control, London, Clive Bingley, p.19

${ }^{(49)}$ Querard, Joseph Marie, La France littéraire : ou dictionnaire bibliographique des savants...... Paris, Firmin Didot, 1836, p.343
} 
gentleman recevant des papiers de la part d'angelots dépeints entrain d'observer et de collecter des spécimens botaniques et zoologiques. L'estampe pliable, une gravure colorée à la main représentant des minéraux et des pierres précieuses, est une illustration typique des périodiques de la fin du $18^{\mathrm{e}}$ siècle.

5.9-Observations sur la Physique, sur l'Histoire Naturelle et sur les Arts qui parut de 1773 à 1794 est une autre publication qui, à l'orée de cette période clé de la communication, avait marqué de son empreinte le monde scientifique. L'Abbé Rozier, scientifique et agronome, édite le journal de physique qu'il achète et renomme. Il dirigea la revue pendant 10 ans et fit paraître des articles de Lavoisier, de Berthollet ou de Parmentier et s'emploie à y réunir des scientifiques de tous les pays. La revue devient de ce fait l'un des rendez-vous de l'Europe savante. Elle a été décrite comme : "..... illustre revue, prédécesseur de " SCIENCE ET VIE ".L'influence de ce journal fut grande à l'époque en ce sens qu'elle ne publiait que des recherches originales. Elle était le réceptacle de la science plutôt qu'un organe d'éducation, ce qui était le cas pour la plupart des journaux existants à l'époque.

\section{6-Spécialisation des journaux scientifiques}

La prochaine étape verra une spécialisation accrue de la science et l'élimination du caractère " amateur " des chercheurs curieux de la nature qu'avait connu le monde scientifique jusque là. Au lieu du caractère généraliste qui avait caractérisé la science (et les scientifiques), une progressive, constante et inexorable spécialisation s'installa. Elle allait devenir d'ailleurs le caractère prédominant du journal scientifique moderne.

\section{1- Les premiers journaux en chimie et en physique}

Les premiers journaux de chimie furent publiés par des individus plutôt que par des sociétés savantes. Ainsi Lorenz Von Crell (1744-1816), professeur de médecine théorique à l'Université de Helmstedt en Allemagne édita le premier périodique exclusivement réservé à la Chimie:

6.1.1-Chemische Annalen für die Freunde der Naturlehre, Arzneygelahrtheit, Haushaltungskunst und Manufacturen et ce à partir de 1778.Le journal continua publication sous ce nom jusqu'à 1781.11 reprit la publication en 1781 sous le titre de Chemische Annalen et il cessa de paraître une deuxième fois en 1803. Le journal est souvent connu sous le titre de Crell's Annalen. Il publia aussi Der Neuesten endteckugenin der chimie et ce entre 1781 et 1786.

6.1.2-Algemeine journal der chimie (1798-1803) et qui se spécialisait en chimie pure et appliquée toujours en Allemagne, publié par Alexander Niklaus Scherer entre autres nombreux titres, et qui deviendra Journal fur praktische chimie en 1834 et qui est toujours en activité sous le titre de Advanced Synthesis \& Catalysis.

On peut aussi citer The Chemist et qui ne dura pas longtemps et parut en 58 numéros entre 1824 et 1825 .

6.1.3-Annalen der Chemie qui furent publiées par Justus Liebig à partir de 1832 et qui paraissent toujours. Ils changèrent successivement de titres de la manière suivante : Annalen der pharmacie (vols. 1-32. 1832-1839), Annalen der chemie und pharmacie (1840-1873), Justus Liebig annalen der chemie und pharmazie (1873-1874), Justus Liebig annalen der 
chemie (vol.173/1874-1978), Liebig annalen (1995-1996), Liebig annalen- Recueil (1997) et enfin European journal of organic chemistry (1998 - ).

6.1.4- Annales de chimie et de physique. La plus ancienne revue de chimie encore en existence est Annales de chimie et de physique qui est une revue scientifique fondée en 1789 à Paris sous le titre Annales de chimie. L'un des premiers éditeurs du périodique fut le chimiste Français Antoine Lavoisier. En 1816, la revue changea son nom pour Annales de chimie et de physique, un nom qu'elle gardera jusqu'en 1913 avant de se voir divisée en deux périodiques distincts nommés Annales de chimie et Annales de physique. La revue Annales de physique est toujours publiée sous ce nom, tandis que Annales de chimie est devenue Annales de chimie - Science des matériaux en 1978. Depuis 2004, c'est Lavoisier qui continue la publication. Bien que les noms aient évolués et que la revue fût séparée en deux périodiques distincts, la numérotation des volumes maintient toujours une continuité entre les différents titres, et ceci, aussi bien pour Annales de chimie que pour Annales de physique. Cette importante revue est connue pour avoir publié nombre de recherches très importantes et surtout pour avoir défendu la nouvelle chimie de Lavoisier qui avait douté de la théorie du Phlogistique*.

6.1.5- Journal Der Physik est le premier périodique spécialisé en physique à être publié et qui commença à paraître à Halle et Leipzig à partir de 1790.C'est la plus ancienne publication de physique, publiée depuis. C'est une revue qui publie des articles dans tous les domaines reliés à la physique. Elle a été tout d'abord publiée de 1790 à 1794 sous le nom de Journal der Physik, puis de 1795 à 1797 sous celui de Neue Journal der Physik. Au début du XX $\mathrm{X}^{\mathrm{e}}$ siècle, l'allemand est la langue de référence dans le domaine de la physique, et la revue, alors publiée dans cette langue est la revue de référence. L'anglais a depuis pris le dessus, et Annalen der Physik est publiée de nos jours en langue anglaise. De nombreux travaux majeurs y ont initialement été publiés. Parmi les articles célèbres, figurent notamment les quatre recherches d'Albert Einstein publiés en 1905, ainsi que des travaux de Heinrich Rudolf Hertz sur l'effet photoélectrique et Max Planck sur le corps noir. Le journal a plusieurs fois changé de titre au cours de son histoire: Journal der Physik, (1790-1794 ) ,Neues Journal der Physik, (17951797), Annalen der Physik, (1799-1808), Annalen der Physik und der physikalischen Chemie, (1819-1824), Annalen der Physik und Chemie, (1824-1899), Annalen der Physik, (1900- ).

6.2-Philosophical Magazine est l'une des plus anciennes publications scientifiques commerciales du monde. Créé en 1798 par Richard Taylor, et depuis publié de façon continue par Taylor and Francis, il fut le journal de prédilection de scientifiques tels Faraday, Joule, Maxwell, J.J. Thomson, Rayleigh et Rutherford. La majeure partie du développement scientifique de ces deux cents dernières années se retrouve dans les pages de cette publication. Le nom du journal date de l'époque où le terme natural philosophy se rapportait à l'ensemble des sciences exactes: physique, chimie, médecine, astronomie, botanique, biologie et géologie. En 1814, le Philosophical Magazine fusionna avec le Journal of Natural Philosophy, Chemistry and the Arts, connu sous le nom de Nicholson's Journal, pour former

\footnotetext{
* La théorie du phlogistique est une théorie scientifique obsolète concernant la combustion. Elle a été développée par J.J. Becher à la fin du XVII siècle et fut prolongée et développée par Georg Ernst Stahl.La théorie phlogistique est devenue caduque après la découverte de l'implication de l'oxygène de l'air dans le processus de combustion par Antoine Laurent de Lavoisier au XVIII ${ }^{\mathrm{e}}$ siècle. La théorie affirme que tous les matériaux inflammables contiennent du phlogiston, une substance incolore, inodore, impondérable qui serait dégagée en brûlant. Une fois brûlée, la substance "déphlogistifiée" apparaîtrait sous sa vraie forme. http://fr.wikipedia.org/wiki/Phlogistique (consulté le 27/02/2011)
} 
le Philosophical Magazine and Journal. D'autres fusions avec les Annals of Philosophy et le Edinburgh Journal of Science conduisirent à un nouveau changement de titre en 1840 pour donner le London, Edinburgh and Dublin Philosophical Magazine and Journal of Science En 1949 le journal redevint le Philosophical Magazine pour une raison de commodité de référencement. En 1987 le petit frère du journal, Philosophical Magazine Letters fut lancé dans le but de publier rapidement de courts articles dans le domaine de la physique de la matière condensée. Phil. Mag. Letters est édité par E.A. Davis, qui coordonne les deux parties du journal.

Le $18^{\mathrm{e}}$ et $19^{\mathrm{e}}$ siècle ont vu une profusion de journaux qui ne faisaient que répondre à de nouvelles branches de savoir qui se créaient. On peut situer à cette période le début de la grande époque des journaux spécialisés et qui allait être une des constantes de la publication et de la communication scientifique et technique. Cette donnée allait est allé crescendo au point où il est pratiquement impossible de trouver de nos jours une publication scientifique qui soit de type généraliste. On citera parmi les plus connus :

6.3-The Botanical Magazine (1745- ) Aussi connu sous l'appellation de Flower-Garden Displayed. C'est une publication illustrée qui commença en $1787^{*}$.Il est considéré comme le plus ancien périodique en botanique et est plus connu sous l'appellation de Curtis's Botanical Magazine.

Chaque numéro contient une description en un langage formel mais accessible et est renommé comme ayant présenté le travail de deux siècles d'illustrateurs botaniques. Nombre de plantes furent connues la première fois dans la publication et la description donnée était agrémentée par les illustrations vivement colorées.

Le premier numéro fut lancé par William Curtis, comme en même temps un journal illustré en jardinage et botanique .Curtis était un apothicaire et un botaniste qui travaillait chez Kew Gardens qui avait publié quelques années auparavant le très acclamé (mais au succès commercial mitigé ) Flora Londinensis.

La publication familiarisait ses lecteurs aux plantes ornementales et exotiques qu'elle présentait in $8^{\circ}$. Les artistes qui auparavant donnaient leur peinture de florale à une audience aisée, voyaient maintenant leur travail publié en un format accessible à une plus grande audience. Les illustrations étaient initialement des impressions colorées à la main, à partir de gravure sur cuivre et dont le but était de compléter le texte. C'était ensuite accompagné par une page ou deux de texte décrivant les propriétés de la plante, son histoire, caractéristiques de croissance et quelques noms communs de la plante.

Les premiers trente volumes utilisèrent de la gravure sur cuivre pour fournir le cliché et dont la coloration à la main nécessitait jusqu'à trente personnes Un numéro pouvait avoir une circulation de 3000 copies avec trois clichés dans chaque numéro . Devant l'augmentation du cout de production ainsi que de la demande, les résultats deviennent variables pour chaque

\footnotetext{
* Il est à signaler que la différence entre les deux dates signalées est symptomatique de cette période où nous avons remarqué que pour une publication donnée deux (et des fois plus) dates sont données. Il est loisible de penser que l'époque n'était pas très documentée et aussi et surtout que l'état des collections qui nous sont arrivées ne permettent pas de situer avec précision les dates .L'exemple du "Relation Aller Fuernemmen und gedenckwuerdigen Historien " que nous avons cité en infra est à ce sens des plus symptomatique de la difficulté à préciser une date de ces premiers périodiques.
} 
numéro. L'utilisation après cela de machines à colorier donnera une uniformité au travail de l'artiste, quoique le processus ne donne pas les mêmes détail des années durant. The magazine a été considéré comme le modèle des premiers journaux en termes d'illustration botanique

La valeur scientifique des figures et des illustration, qui étaient une source de fierté et de prééminence pour le magazine exigeait une formation attentive des illustrateurs .L'artiste travaillait de concert avec le botaniste pour décrire le spécimen, l'utilisation de détails explosés entourant la description donnait au numéro un appel pratique aux botanistes , horticulteurs et jardiniers.

Le magazine est considéré comme la plus grande collection d'illustrations botaniques produites à ce jour et la qualité consistante de ses clichés et son autorité en font le journal le plus cité de sa catégorie. Les clichés colorés manuellement était un processus de travail intensif qui fut remplacé en 1948 par un processus photomécanique.

Il a été publié sans interruption depuis avec un changement dans l'appellation (il devint The Kew Magazine de 1984 à 1994) et en 1995 il reprit le nom communément utilisé de Curtis's Botanical Magazine. Il continue à être publié par Royal Botanic Gardens, Kew comme une publication pour ceux qui sont intéressé en horticulture, écologie ou l'illustration botanique. La forme d'abréviation standard utilisée est Curtis's Bot. Mag. or "Botanical Magazine " dans les citations de la littérature botanique.

\section{4-Les sociétés savantes : sponsors des journaux scientifiques}

Tous ces journaux qui ont jalonnés surtout le $18^{\mathrm{e}}$ siècle et ont jetés les fondations des journaux scientifiques et techniques (nombre d'entre eux sont d'ailleurs sont toujours en existence et représentant parmi les meilleurs périodiques de leur spécialités) ont étés lancés et édités par des personnes qui étaient des scientifiques qui avaient traduits leur amour pour la science en lançant des périodiques avec des fortunes diverses. Il faut dire que si nombre de ces périodiques ont réussis et perdurés des fois jusqu'à nos jours, le nombre de ceux qui n'ont pas duré plus qu'un ou deux numéros (ou même furent morts - nés) est assez important. C'est alors que s'imposa l'idée de sponsoriser les journaux par des sociétés savantes et ce à partir de la seconde moitie du $19^{\mathrm{e}}$ siècle. Parmi les plus importantes de ces sociétés savantes on peut citer :

6.4.1-The Proceedings of the Royal Physical Society of Edinburg en 1783 (qui fit suite à The Philosophical Society)

6.4.2- The Proceedings of the London Mathematical Society in London en 1865. Les activités initiales de la Society comprenaient des conférences et la publication d'un journal. La Society publies des livres et des périodiques, organise des conférences mathématiques; fournit des financements pour promouvoir la recherche et la formation en mathématiques et décerne un nombre de prix et de bourses pour l'excellence en recherche en mathématiques. Les publications périodiques de la Society's comprennent trois journaux imprimés qui sont: the Proceedings, Journal, and Bulletin.

6.4.3- The Proceedings of the Physical Society of London en 1874.C'etait un journal qui traitait de la physique et qui était au debut associé avec the Physical Society of London, England. 
Aux environs du milieu du $19^{\mathrm{e}}$ siècle, un peu plus de 1000 titres de journaux scientifiques et techniques étaient publiés dans le monde ${ }^{(50)}{ }^{*}$. Chiffre qu'il faut prendre avec beaucoup de circonspection ne concorde pas avec d'autres sources qui en donnent des figures complètement différentes. Ce problème (le nombre exacte des périodiques) ne semble pas être prêt d'être résolu (ni même est ce faisable ?) et ceci est du à un nombre données dont les plus importantes sont :

-La définition d'un périodique.

-La différentiation entre périodique et livre surtout au début de la période du passage entre les deux media.

-Les critères de sélection

-Le problème des titres mort-nés

\section{7-Exemple de prototype de périodique scientifique contrôlé par les pairs moderne : Nature}

Cette période avait vu une profusion de titres qui avaient essayés de captiver l'attention de lecteurs d'une communauté scientifique en pleine croissance et avide aussi de connaissance. Parmi ces périodiques, un journal fondé à cette période est considéré comme l'archétype du périodique scientifique moderne qui perdure encore de nos jours .C'est le magazine Nature.

\section{1- Courte histoire de Nature}

\subsection{1-Magazines scientifiques et journaux ayant precedés Nature}

Le $19^{\mathrm{e}}$ siècle avait vu en Grande Bretagne une grande activité scientifique spécialement sa deuxième partie où de grands changements technologiques et industriels avaient eu lieu. $\mathrm{A}$ cette époque, les journaux les plus respectés étaient ceux publiés par la Royal Society qui avait publié les travaux de sommités telles que Newton, Faraday et Darwin. Cette période avait aussi vu le nombre de périodiques de science populaire doubler entre 1850 et 1860. Selon les éditeurs de ces magazines, ces publications étaient destinées à servir comme organe de la science, en quintessence un moyen pour connecter le public au monde scientifique.

\footnotetext{
${ }^{(50)}$ Houghton, op cit. p.26

"Kronick dans " Litterature of the life sciences: the historical background, Bulletin of the New York Academyof Medecine, Vol 60, n 9, p.873, Novembre 1984 " citant " Brodman, E., The Development of Medical Bibliography, Medical Library Association, Baltimore, 1954 " donne le chiffre de 900 périodiques en 1800 et qui aurait atteint presque 60000 en 1901. Comme on peut le voir ces chiffres ne font que confirmer la difficulté (pour ne pas dire la quasi impossibilité) d'avoir un chiffre exact. Pour corroborer cet état de fait, la seconde édition du World List of Scientific Periodicals de la fin de l'année 1933, donne le chiffre exact de 36, 380 titres et ce à la fin du mois de juin de la même année (Nature, n¹33, 26 mai 1934, p.792). Enfin, une autre source cite "approximativement 100 titres en journaux scientifique avant 1800, ce chiffre a augmenté à 1000 titres en l'an 1850 et à 10000 en 1900. Disponible à : (http://www.lib.ncsu.edu/exhibits/tippmann/scijo.html ) Consulté le $02 / 03 / 2011$
} 
Un journal qui fut fondé en 1859 précéda Nature dans le genre .C'etait Recreative Science: $A$ Record and Remembrance of Intellectual Observation, qui commença en tant que magazine d'histoire naturelle et progressa pour englober plus de sujets techniques et d'observation physique et moins d'histoire naturelle. Le nom du journal fut changé du titre original vers Intellectual Observer: A Review of Natural History, Microscopic Research, and Recreative Science et plus tard à The Student and Intellectual Observer of Science, Literature, and Art. Alors que Recreative Science avait essayé plus de sciences physiques telles que l'astronomie et l'archéologie, The Intellectual Observer élargit plus les sujets abordés pour inclure aussi la littérature et l'art.

Similaire à Recreative Science, Popular Science Review journal scientifique créé en 1862, et qui abordait différentes spécialisations scientifiques en créant des sous sections titrées " Résumés scientifiques " ou " Rétrospective trimestrielle " avec des revues de livres et commentaires sur les dernieres publications et les derniers travaux scientifiques.

Deux autres journaux virent le jour en Angleterre, Quarterly Journal of Science et Scientific Opinion, respectivement en 1864 et 1868 et antidataient Nature. Le journal qui avait le plus de ressemblance avec Nature du point de vue de la ligne éditoriale et du format était The Reader, crée en 1864. C'était un amalgame de science, de littérature et d'art qui essayait d'atteindre une audience en dehors de la communauté scientifique et qui était similaire à Popular Science Review. Tous ces journaux qui précédèrent Nature ne purent durer et cessèrent de paraitre chacun avec des fortunes différentes quant à la durée de leur vies et qui oscilla entre deux (Scientific Opinion) et vingt ans (The Popular Science Review).

\section{2-Nature}

Fondé en date du 4 novembre 1869, Nature est parmi les périodiques les plus cités et le journal interdisciplinaire le plus prestigieux au monde .Il est l'un des derniers périodiques scientifiques publiant des recherches originales dans un large spectre des spécialisations scientifiques. Nombre de découvertes et de recherches originales en différentes spécialisations en science ont étés faites et publiées dans des articles et des lettres dans Nature. Les autres exemples connus sont respectivement Science et Proceedings of the National Academy of Sciences.

Les scientifiques faisant de la recherche scientifique sont les premiers lecteurs et contributeurs du magazine mais des résumés et des articles les accompagnant sont publiés et dont le but est de les mettre à la portée des scientifiques des autres spécialités et aussi du public en général. Au début de chaque numéro, un éditorial est rédigé, ainsi que des informations et des articles d'intérêt général pour les scientifiques, y compris les affaires courantes, le financement de la recherche, les affaires en général, l'éthique scientifique ainsi que les percées révolutionnaires. Il existe aussi des sections sur les livres et les arts. Le contenu du reste du journal consiste en des articles en recherche scientifique généralement dense et très technique.A cause de la stricte limite concernant la longueur des articles, très souvent le texte imprimé est en fait un résumé du travail en question avec la plupart des détails relégués au matériel supplémentaire qui l'accompagne sur le site web du journal. 


\section{3-Comment le magazine Nature fut créé}

Juste après la cessation de The Reader, un de ces éditeurs, Norman Lockyer, décida de lancer un nouveau magazine scientifique qu'il appellera Nature. Ce magazine était similaire à ces prédécesseurs dans son but et qui était de fournir aux lecteurs cultivés un forum accessible afin être au courant des avancées du savoir scientifique. Il a été dit que Nature a été crée pour servir des buts de polémiques scientifiques. En effet, dés le début le magazine opposait des groupes dont les orientations et les avis étaient diamétralement opposés et dont le débat conduisait à de riches discussions qui faisaient avancer la science. A cet effet, nombre des premiers numéros de Nature consistaient d'articles écrits par des chercheurs aux idées considérées libérales pour le moment comme par exemple un groupe qui défendait la théorie controversée de Darwin et qui était à cette époque de la deuxième moitié du $19^{\mathrm{e}}$ siècle , attaquée et réfutée par des groupes aux idées plus conservatrices .C'est ce libéralisme dans les idées qui a permis à Nature de perdurer et, contrairement à ces concurrents, devenir de nos jours la référence parmi les magazines scientifiques.

\section{4- Les Editeurs}

La personnalité de l'éditeur a toujours eu une grande importance dans l'orientation et le devenir d'un périodique scientifique. On a vu comment la personnalité d'Henry Oldenburg, considéré l'archétype et le premier éditeur scientifique selon nos critères au $20^{\mathrm{e}}$ siècle, a été très importante dans la perduration et le prestige qu'a The Philosophical Transactions jusqu'à nos jours. De même Nature, a eu dans sa longue histoire des éditeurs qui ont imprégné le magazine de leurs personnalités. Ce sont respectivement

\subsection{1-Norman Lockyer}

Sir Joseph Norman Lockyer ou Norman Lockyer (17 mai 1836-16 août 1920) est un scientifique, vulgarisateur, et astronome britannique. Il est crédité avec Jules Janssen de la découverte de l'hélium. Il a comme autre particularité d'être un scientifique " amateur", son métier originel étant d'être fonctionnaire au ministère de la défense britannique. C'était un professeur à The Imperial Collège. Après sa découverte, il donna de nombreuses conférences et écrit de nombreux articles. Il édita Nature pendant cinquante ans de 1869 à 1909 quand il prit sa retraite.

\subsection{2-Richard Gregory}

Bien qu'il faisait partie du staff éditorial, ce n'est que la fin de la première guerre mondiale et le décès de Norman Lockyer qui permit à Richard Gregory, devenu entretemps Sir Richard Gregory, de vraiment imprégner Science de son empreinte et de le faire rentrer dans la période post deuxième guerre mondiale. Il y eut des changements significatifs dans le magazine et c'est à cette époque que Nature commença à évoluer vers la forme qu'il a a prise actuellement. A titre d'exemple, les éditoriaux qui étaient souvent sous Norman Lockyer des revues de livres devinrent à partir de 1919 beaucoup plus axés sur la discussion des problèmes d'ordre politique et sociaux. Gregory continua à imprégner le journal avec la passion qu'avait Lockyer pour l'éducation mais les problèmes politiques et sociaux de la science étaient les sujets à travers lesquels le zèle et la passion de Gregory pour la science comme agent curatif des maux de la société étaient exprimés. Pour parvenir à cette fin, il diminua le nombre de personnes qui rédigeaient des éditoriaux à quelques personnes bien connues. 


\subsection{3-A. J. V. Gale et L. J. F. Brimble}

C'est la seule fois que Nature eut deux coéditeurs et ceci arriva en 1920 quand Richard Gregory se retira à l'age de soixante quinze ans et qu'A.J.V. Gale rejoint Nature en tant que collaborateur. Il fut rejoint par L.J.F. Brimble en 1931 toujours en tant que collaborateur au même titre qu'A.J.V. Gale. C'est en 1938 et la retraite de Richard Gregory qu'ils devinrent officiellement coéditeurs. Ils cumulaient entre eux plus de vingt cinq ans de service à Nature. Ils formèrent un tandem assez homogène avec Gale s'occupant des sciences physiques et Brimble les sciences biologiques. A.J.V. Gale se retira à la fin de l'année 1961 après avoir travaillé pour Nature plus de quarante ans. Son coéditeur resta alors le seul maitre à bord. L'histoire retiendra que ce tandem pu tenir toute cette période car si l'un (Gale) était travailleur et discret, l'autre (Brimble) était beaucoup plus extraverti et les deux communiquaient par écrit car ils avaient peu de temps pour se voir. En 1965, Brimble décéda.

\subsection{4-John Maddox}

Après le décès de Brimble, il fallut un peu plus de six mois avant qu'un éditeur ne fut désigné. Mc Millan News annonça au mois de juin 1966 que John Maddox avait été désigné comme le nouvel éditeur de Nature. Maddox était un scientifique, chercheur en physique théorique à l'Université de Manchester et journaliste au Manchester Guardian (qui devint plus tard the Guardian).Maddox utilisera beaucoup son expérience en tant que journaliste et scientifique pendant son mandat. Il introduisit nombre de changements dont un staff plus étoffé pour s'occuper des manuscrits qui étaient en retard pour la publication. Il est admis qu'il a, avec son successeur Davis, modernisé Nature qui avait été pendant presque 40 ans dirigé par Brimble et Gale d'une manière traditionnelle. Parmi les plus importants problèmes était le contrôle par les pairs qui, sous Gale et surtout Brimble, fait de manière très bizarre ${ }^{*}$ et avait occasionné à cet effet des retards considérables dans les délais de publication, ce qui est admis très préjudiciable surtout pour les techniques de pointe. Maddox entreprit alors une véritable révision de ces rouages (entre autre un contrôle par les pairs plus officiel) ainsi qu'une modernisation de ses innombrables départements.

\subsection{5-David Davies}

Au mois d'août 1973, un nouvel éditeur prit la place de Maddox. David Davies était seulement le cinquième à occuper le poste en un peu plus d'un siècle. Il était déjà connu au sein du magazine, étant son correspondant des affaires géophysiques des années durant. Il avait passé les dernières années, avant son intronisation, aux Etats Unis en charge d'un organisme appelé " Seismic Discrimination Group " où il travaillait sur la détection des tests d'armes nucléaires souterrains. D'ailleurs, durant la période où il était éditeur, nombre de ces écrits eurent une relation avec le sujet, d'autant plus qu'elle coïncidait avec le plus haut niveau de la guerre froide. Il est connu pour avoir définit une recherche publiée par Nature comme:..." Quelque chose qui la mettrait [ la recherche] à part, aidant à voir le monde à travers une nouvelle et meilleure fenêtre ".

\footnotetext{
* Il est raconté que Brimble, en allant déjeuner dans un club très select à Londres, emmenait avec lui dans ses poches les manuscrits soumis qu'il montrait à certaines personnes pour savoir s'il était digne d'être publiés, ce qui a occasionné quelques fautes mémorables face à cette manière peu orthodoxe d'expertiser les recherches surtout comparé à ce qui se fait de nos jours à travers Internet
} 


\subsection{6-John Maddox}

Au mois de mai 1980, Maddox retourna en tant qu'éditeur de Nature une seconde fois. Son retour ne fut pas accueilli d'une manière enthousiaste mais le style trop académique de Davies avait favorisé son retour car son amour du style journalistique était populaire. Il est dit que l'une des raisons de son départ une premiere fois était dû au fait que Nature en 1971, sous la responsabilité de Maddox, s'etait scindé en trois publication séparées: Nature Physical Sciences, Nature New Biology et Nature, publiés respectivement les lundi, mercredi et vendredi. Il voyait Nature comme un quotidien dont chaque parution se spécialiserait dans un champ scientifique donné. Cette idée n'eut pas l'effet escompté et c'est ce qui explique que son premier départ a coïncidé avec cette " réunification ".

\subsection{7-Philip Campbel}

Une année après avoir célébré son $125^{\mathrm{e}}$ anniversaire avec faste, Nature intronisa Phillip Campbell en tant que éditeur en chef terminant de ce fait la deuxième et dernière fonction de Maddox. Campbell était seulement le $7^{\mathrm{e}}$ éditeur (incluant coéditeur Gale) à avoir assumé la position en exactement 152 ans. Il était l'éditeur de la section des sciences physiques et est toujours en poste. La période à laquelle il a pris ses fonctions coïncide avec la généralisation des technologies de publication en ligne que Nature a parfaitement englobé en lançant nombre de périodique en ligne, mais aussi par le scandale du généticien coréen Woo Suk Hwang qui, dans un article publié dans Nature, prétendait avoir réussi à cloner un embryon humain pour la recherche scientifique (production de cellules-souches).Il fut prouvé plus tard que ces expérimentations n'avaient pas respectés certaines règles éthiques. Les articles incriminés furent rétractés et retirés mais cette polémique eut des effets sur la manière dont sont publiés et certifiés les résultats scientifiques et a ravivé la polémique, qui ne s'est nullement calmé, de la justesse, la faisabilité et surtout l'objectivité du contrôle par les pairs.

\section{5-L'expansion de Nature et son développement}

A partir de 1970, Nature ouvrit son premier bureau à Washington; d'autres branches ouvrirent à New York en 1985, Tokyo et Munich en 1987, Paris en 1989, San Francisco en 2001, Boston en 2004, et Hong Kong enn 2005. A partir des années 80, le journal connu une grande expansion, lançant plus de 10 nouveaux journaux. Ces nouveaux journaux comprennent the Nature Publishing Group, qui fut crée en 1999 et comprend Nature, Nature Research Journals, Stockton Press Specialist Journals et Macmillan Reference (renommé NPG Reference).

En 1997, Nature crea son propre site web (www.nature.com), et en 1999, Nature Publishing Group commença sa série de Reviews. Certains articles et recherches sont disponibles gratuitement sur le site web de Nature. D'autres requièrent l'achat d'un accès premium au site.

Nature revendique un lectorat de quelques 424,000 lecteurs. The journal a une circulation d'environ 53,000 copies et des études ont conclus qu'en moyenne une seule copie est lue par un nombre avoisinant les 8 lecteurs. 


\section{6-Publier dans Nature}

Avoir un article publié dans Nature est très prestigieux parce que les articles sont fréquemment cités et ceci peut amener des promotions, des financements de recherche et l'attention des media. A cause de ces effets positifs, la compétition entre les scientifiques pour publier dans des journaux de haut niveau tel que Nature et d'autres à l'instar de Science, peut être très féroce. Le facteur d'impact de Nature, qui est la mesure de combien de citations un journal génère dans d'autres recherches était de 34.480 en $2009^{*}$ (tel que mesuré par Thomson ISI), parmi les plus hauts de tous les journaux scientifiques.

Comme c'est le cas pour la plupart des autres journaux scientifiques, les articles sont soumis à une sélection initiale par l'éditeur suivi par un contrôle par les pairs (dans lequel d'autres scientifiques, choisis par l'éditeur pour leur expertise dans le sujet mais qui n'ont aucune connexion avec la recherche contrôlée, liront et critiquerons l'article en question), avant publication. Dans le cas de Nature, ils sont envoyés pour expertise à condition d'aborder un sujet de spécialisation et être à la pointe du sujet en particulier. En conséquence, la majorité des articles soumis à Nature sont rejetés sans expertise.

\subsection{1-Recherches qui ont fait date}

Nombre des plus importantes découvertes et percées scientifiques ayant fait date ont étés publiées pour la première fois dans Nature. Ce qui suit est une liste de ces découvertes révolutionnaires qui ont toutes eut des répercussions et des conséquences bien au delà de l'aspect purement scientifique. Ces découvertes sont présentées brièvement suivi des références (date, numéro, pagination, etc....) de l'article publié.

Wave nature of particles - C. Davisson and L. H. Germer (1927). "The scattering of electrons by a single crystal of nickel". Nature 119: 558-560. doi:10.1038/119558a0.

The neutron - J. Chadwick (1932). "Possible existence of a neutron". Nature 129: 312. doi:10.1038/129312a0.

Nuclear fission - L. Meitner and O. R. Frisch (1939). "Disintegration of uranium by neutrons: a new type of nuclear reaction". Nature 143: 239-240. doi:10.1038/143239a0.

The structure of DNA - J. D. Watson and F. H. C. Crick (1953). "Molecular structure of Nucleic Acids: A structure for deoxyribose nucleic acid". Nature 171 (4356): 737-738. doi:10.1038/171737a0. PMID 13054692.

First molecular protein structure (myoglobin) -J. C. Kendrew, G. Bodo, H. M. Dintzis, R. G. Parrish, H. Wyckoff and D. C. Phillips (1958). "A three-dimensional model of the myoglobin

\footnotetext{
* Le facteur d'impact, est une mesure de l'importance d'une revue scientifique. Il est calculé chaque année par l'Institute for Scientific Information (ISI) pour chaque revue suivie. Il est publié dans le Journal Citation Reports. Les facteurs d'impact ont une grande influence dans le domaine de la publication scientifique car ils servent souvent de critères d'évaluation quantitatifs. Le facteur d'impact est calculé sur une période de trois ans. Par exemple, le facteur d'impact de 2003 pour une revue $\mathrm{X}$ est calculé ainsi : $A=$ le nombre de fois que des articles publiés dans X durant la période 2001-2002 sont cités dans l'ensemble des revues indexées durant l'année 2003. $B=$ le nombre d'articles, résumés étendus ou notes publiés dans X dans la période 2001-2002.Le facteur d'impact de la revue X pour 2003 sera $A / B$
} 
molecule obtained by X-ray analysis". Nature 181 (4610): 662-666. doi:10.1038/181662a0. PMID 13517261.

Plate tectonics - J. Tuzo Wilson (1966). "Did the Atlantic close and then re-open?". Nature 211: 676-681. doi:10.1038/211676a0.

Pulsars - A. Hewish, S. J. Bell, J. D. H. Pilkington, P. F. Scott \& R. A. Collins (1968). "Observation of a Rapidly Pulsating Radio Source". Nature 217: 709-713. doi:10.1038/217709a0.

The ozone hole -J. C. Farman, B. G. Gardiner and J. D. Shanklin (1985). "Large losses of total ozone in Antarctica reveal seasonal ClOx/NOx interaction". Nature 315 (6016): 207210. doi:10.1038/315207a0.

First cloning of a mammal (Dolly the sheep) - I. Wilmut, A. E. Schnieke, J. McWhir, A. J. Kind and K. H. S. Campbell (1997). "Viable offspring derived from fetal and adult mammalian cells". Nature 385 (6619): 810-813. doi:10.1038/385810a0. PMID 9039911.

The human genome - International Human Genome Sequencing Consortium (2001). " Initial sequencing and analysis of the human genome" Nature 409 (6822): 860-921. doi:10.1038/35057062. PMID 11237011.

\subsection{2-Anomalies du contrôle par les pairs}

Malgré le haut degré de vérification et la sélectivité que connaissent les recherches soumises à Nature et aussi, et en conséquence de cela, le haut degré de rejet ${ }^{*}$ qui caractérise Nature, un certain nombre de recherches s'avérèrent frauduleuses et relancèrent le débat au sujet de la certification de l'information par les pairs publiés par les revues scientifiques.

Une série de cinq recherches frauduleuses par Jan Hendrik Schön furent publiés par Nature durant la période 2000-2001. Les recherches dont le sujet était la superconductivité s'avérèrent contenir des données falsifiées et d'autres fraudes scientifiques. En 2003, les recherches furent rétractées par Nature. Ce qui a été connu sous le scandale Schön n'était pas limité à Nature. D'autres journaux proéminents tels que Science et Physical Review furent aussi victimes et rétractèrent les recherches de Schön.

Avant de publier une de ses plus importantes découvertes, qui etait la structure du DNA par Watson et Crick en 1953, Nature n'envoya pas la recherche pour expertise extérieure. John Maddox, l'éditeur de Nature déclara que la recherche de Watson and Crick ne fut pas expertisé par Nature...[parce que],il ne pouvait pas être expertisé[étant donné que] l'importance de ces résultats ne pouvait être mise en cause et pas un seul expert aurait pu se taire une fois qu'il avait vu les résultats ".

\footnotetext{
* Il est une règle quelque peu acceptée mais non écrite que les journaux qui ont le plus haut degré de rejection sont de ce fait les plus prisés par les scientifiques pour publier leurs recherches. La publication dans ces journaux est un garant de la qualité de la recherche et assure aux chercheurs célébrité et respect parmi leur pairs. Il a été cependant prouvé que cette règle n'était pas toujours respectée après les différents cas célèbres de fraude et de comportement inappropriés signalés ( dans Nature entre autres ) et dont nous aborderons certains aspects dans la troisième partie de ce travail et qui traitera du contrôle par les pairs.
} 
Plus tôt en 1934, une autre erreur fit la une des journaux scientifiques en terme de rejet et non publication de résultats de recherche. Ceci arriva quand Enrico Fermi soumis sa recherche révolutionnaire dont le sujet était la théorie de la désintégration $\beta$ du noyau. Nature rejeta la découverte parce quelle la considérait trop éloignée de la réalité. En conséquence, la recherche de Fermi fut publié par Zeitschrift für Physik en 1934, et finalement publiée par Nature cinq ans plus tard après que le travail de Fermi devint universellement accepté.

Quand Paul Lauterbur et Peter Mansfield reçurent le Prix Nobel en Physiologie et en Medicine pour une recherche initialement rejetée par Nature et publiée seulement après que Lauterbur contesta la rejection,Nature reconnut plus tard ses errements dans le rejet de certaines recherches importantes dans un éditorial intitulé " Coping with Peer Rejection " (51) (Faire face au rejet des pairs):

" Il y a eut, sans aucun doute, des faux pas dans notre histoire. Elles comprennent le rejet des radiations de Crenkov, le travail sur la photosynthèse de Johann Deisenhofer, Robert Huber et Hartmut Michel et le rejet initial (mais avec l'éventuelle acceptation) de la recherche au sujet des radiations des trous noir de Stephen Hawking ".

\section{7-La publication de Nature et de journaux assimilés}

Nature est édité et publié dans le Royaume Uni par Nature Publishing Group, un groupe subsidiaire de Macmillan Publishers lui même propriété de Georg von Holtzbrinck Publishing Group. Nature a des bureaux à Londres , New York, San Francisco, Washington, D.C., Boston, Tokyo, Hong Kong, Paris, Munich, et Basingstoke. Nature Publishing Group publie aussi des journaux spécialisés comme Nature Neuroscience, Nature Biotechnology, Nature Methods, et la serie de journaux de The Nature Clinical Practice, Nature Structural \& Molecular Biology, Nature Chemistry, et la serie de journaux de The Nature Reviews .

Depuis 2005, chaque numéro de Nature est accompagné par un Nature Podcast ${ }^{*}$ représentant les lignes générales du numéro et des interviews avec les auteurs des articles et les journalistes couvrant la recherche. Présenté par Adam Rutherford et Kerri Smith, et présentant des interviews avec des scientifiques au sujet des dernieres recherches, ainsi que des reportages des éditeurs et journalistes de Nature, elle comprend aussi des créneaux réguliers appelés the " PODium ", qui est une tranche hebdomadaire d'opinion de 60 secondes ,ainsi que "Sound of Science ",un créneau régulier qui passe de la musique en relation avec la science et d'autres enregistrements audio à caractère scientifique. En 2007 ,Nature Publishing Group commença à publier Clinical Pharmacology \& Therapeutics " le journal official de The American Society of Clinical Pharmacology \& Therapeutics " et Molecular Therapy,le journal officiel de The American Society of Gene Therapy ainsi que The International Society for Microbial Ecology (ISME) Journal. Nature Publishing Group lança Nature Photonics, Nature Geoscience et Nature Chemistry respectivement en 2007, 2008 et 2009.Enfin au mois de septembre 2010, Nature New biology et Nature Physical Science qui étaient publiés en complement au journal principal, et ce de 1971 à 1973, furent disponibles en ligne.

\footnotetext{
${ }^{(51)}$ Coping with peer rejection, Nature, Vol. 425, $\mathrm{n}^{\circ}$ 6959, p.645, 16 Octobre 2003

* Le podcasting est un moyen de diffusion de fichiers (audio, vidéo ou autres) sur Internet appelés " podcasts ". Par l'entremise d'un abonnement aux flux RSS ou Atom, le podcasting permet aux utilisateurs l'écoute ou le téléchargement automatique d'émissions audio ou vidéo pour les baladeurs numériques en vue d'une écoute immédiate ou ultérieure.
} 


\section{8-Exemples de prototypes du périodique scientifique contrôlé par les pairs moderne : Science}

\section{1- Science}

Le magazine Science est un autre exemple de périodique scientifique dont le rayonnement, le prestige et la scientificité en font un journal très prisé pour la publication par les scientifiques de tous bords. Il est le journal de " The American Association for the Advancement of Science" et est considéré, au même titre que Nature, comme l'un des journaux scientifiques les plus prestigieux au monde. Ce journal scientifique hebdomadaire, contrôlé par les pairs, a été publié la première fois en 1880, et a une base d'abonnés en version papier de 130000 . Etant donné que les abonnements institutionnels et l'accès en ligne servent une plus grande population, il est estimé que son lectorat est plus proche du million de lecteurs .

Le centre d'intérêt et le but principal de Science est de publier des recherches scientifiques originales importantes et des revues de ces mêmes recherches. Science publie aussi des informations relatives à la science, des opinions sur la politique scientifique et d'autres affaires concernant les scientifiques ainsi que les personnes ayant une implication avec la science et la technologie. Quoique de nos jours la plupart des revues scientifiques se focalisent sur une spécialité donnée, Science et son rival Nature couvrent l'ensemble des disciplines scientifiques. A cet effet, le facteur d'impact de Science était de 29.747 en 2009 (tel que mesuré par The Institute for Scientific Information).

Bien que c'est le journal du The American Association for the Advancement of Science, l'adhésion à l'AAAS n'est pas requise pour publier dans Science .Les recherches sont acceptées d'auteurs provenant de tous les pays .De ce fait, la compétition pour publier dans Science est très intense, et un article publié dans un tel journal très cité conduit à une attention et un avancement de la carrière des auteurs. Moins de 10\% des articles soumis aux éditeurs sont acceptés pour la publication et tous les articles de recherche sont soumis au contrôle par les pairs avant d'apparaitre dans le journal.

\section{2-Les différents éditeurs}

Science fut fondé par le journaliste New Yorkais John Michaels en 1880 avec le soutien financier de Thomas Edison et plus tard Alexander Graham Bell. Cependant, le magazine n'a jamais eu assez d'abonnés pour réussir et cessa de paraitre en mars 1882. L'entomologiste Samuel H. Scudder ressuscita le journal une année plus tard et eut quelques succès en couvrant les réunions de sociétés savantes américaines proéminentes dont l'AAAS .Malgré cela, Science était de nouveau en difficulté financière en 1894 et fut vendu au psychologue James McKeen Cattell pour \$500.Dans un accord signé entre Cattell et le secrétaire d' AAAS Leland O. Howard, Science devint le journal de The American Association for the Advancement of Science (AAAS) en 1900.

Après le décès de Cattell en 1944, la propriété du journal fut transférée à l'AAAS. Malgré cela, le magazine n'avait toujours pas une présence éditoriale continue et constante jusqu'en 1956 quand Graham DuShane devint éditeur.Il fut remplacé en 1962 par le physicien Philip Abelson, codécouvreur du neptunium, et dont les 22 ans de fonction éditoriale furent marqués surtout par le changement dans les habitudes de contrôle par les pairs. En effet, entre autres, il innova pour la distribution des recherches à expertiser en imposant que les experts soient 
appelés au téléphone plutot que joints par lettre*. A la même époque et durant l'exercice de sa fonction, Abelson publia (ou fit publier en tant qu'éditeur) des recherches sur le programme des missions Apollo et fut surtout parmi les premiers à faire publier des écrits sur le SIDA et ce dés 1982 alors que la maladie ( ou plutôt la pandémie ) n'était pas encore connue, encore moins documentée ${ }^{(52)}$. Cette recherche était l'une des premières sur le sujet dans un journal scientifique contrôlé par les pairs. Science publia aussi des résultats des recherches des Professeurs Robert Gallo et Luc Montagnier (avec en toile de fond la fameuse controverse concernant la paternité de la découverte du HIV entre l'équipe française de Luc Montagnier et celle américaine de Robert Gallo) ainsi que de Max Essex. Il faut noter que pour le cas de la paternité de la découverte du virus du SIDA, Science joua un role assez important car il publia les deux recherches qui chacune faisait part de ses découvertes ${ }^{(53)}{ }^{(54)}$.Les années 80 virent une intense controverse qui aboutit, d'une manière indirecte, à donner la paternité de la découverte du SIDA au Professeur Luc Montagnier en lui attribuant en 2008, avec sa collègue Françoise Barre-Sinoussi,le Prix Nobel en Physiologie ou Médecine. En 2002, les deux protagonistes publièrent chacun de son coté une rétrospective des années où ils étaient au cœur de la recherche en ce qui concerne le SIDA ${ }^{(55)(56)}$.Le même numéro de Science permis enfin aux deux chercheurs de co-publier un article dans lequel ils parlent des perspectives pour l'avenir concernant le virus du SIDA ${ }^{(57)}$.Comme on peut le voir, Science a joué dans cette bataille scientifique un rôle de premier plan en donnant la parole et en publiant des résultats de la plus grande importance et surtout, nonobstant la controverse, qui allaient s'avérer fondateur de la recherche dans le domaine. A l'instar de Nature précédemment présenté, Science se place toujours aux avant postes de la recherche en publiant les résultats qui font avancer la science.

Un autre éditeur qui a marqué le magazine Science fut le biochimiste Daniel E. Koshland, qui fut éditeur de 1985 à 1995.Il améliora le contrôle par les pairs, les contenus éditoriaux et aussi la production des articles en ligne. Il avait vu l'importance des changements qui prenaient place dans le domaine de la biologie et la biologie moléculaire, dont il était d'ailleurs un spécialiste occupant une position importante à l'UC Berkeley, et avait tout fait pour que le magazine en soit un acteur majeur en publiant des recherches. D'un autre coté, il insista sur les sciences physiques dont il fit Science un acteur majeur, inaugura la section Perspectives

\footnotetext{
* Ouvrons juste une parenthèse pour dire que si cette " révolution " nous parait en ce début de $21^{\mathrm{e}}$ siècle dérisoire, il n'en demeure pas moins qu'il faut signaler l'extrême rapide changement dans les us et coutumes du contrôle par les pairs et voir que de nos jours un recherche parvient à l'expert à l'autre bout du monde en pratiquement temps réel par Internet. Même chose pour la réponse qui peut être postée et renvoyée électroniquement - en temps réel. Si nous relevons cette extrême rapidité dans la communication scientifique en général et, en ce qui concerne le sujet de notre travail, le contrôle par les pairs il n'en demeure pas moins que pour reprendre l'expression de Hillary Clinton le monde est devenu un petit village. Internet a vraiment rétrécit le monde et accéléré notre rythme de vie et nous n'en citerons que l'exemple malheureux des guerres que nous vivons pratiquement en direct sur nos écrans de télévision ou d'ordinateur.

${ }^{(52)}$ Marx, Jean L., New disease baffles medical community, Science, Vol.217, ${ }^{\circ} 4560$, p.618-621,13 Aout 1982

(53) Barré-Sinoussi, F. ... [et al], Isolation of a T-lymphotropic retrovirus from a patient at risk for acquired immune deficiency syndrome (AIDS), Science, Vol 220, n4599, p.868-871, 20 Mai 1983

(54) Popovic, M. ... [et al], Detection, isolation, and continuous production of cytopathic retroviruses (HTLVIII) from patients with AIDS and pre-AID, Science, Vol.224, n4648, p.497-500, 4 Mai 1984

(55) Montagnier, Luc, A History of HIV Discovery, Science, Vol. 298, nº 5599, p. 1727-1728, 29 Novembre 2002

(56) Gallo, Robert C., The Early Years of HIV/AIDS, Science, Vol. 298, nº 5599, p. 1728-1730, 29 Novembre 2002

${ }^{(57)}$ Gallo, Robert C. and Montagnier, Luc, Prospects for the Future, Science, Vol. 298, n ${ }^{\circ}$ 5599, p. 1730-173, 29 Novembre 2002
} 
du journal, lança Science International à Cambridge ainsi que " Molécule de l'année " connu maintenant sous l'appellation de " Percée de l'année ".

Il introduisit de profonds changements dans le processus du contrôle par les pairs. A son arrivée, les soumissions étaient postées sur un tableau avec différents couleurs pour indiquer leur progression dans le processus. Il remplaça ce système ,un tant soit peu archaïque, par une base donnée sophistiquée de repérage du cheminement de la recherche. Pour ce faire, il ajouta aussi un niveau de triage des soumissions, technique qui a été adoptée par les autres dans le domaine de la publication scientifique dans nombre de spécialités. Sa théorie de l'ajustement induit avait à l'origine reçu des réactions mitigées et, en tant qu'éditeur à Science, il a toujours maintenu que les recherches les plus importantes et les plus innovatrices sont souvent sujettes à controverse mais méritaient l'attention de l'éditeur au point qu'il encourageait les auteurs qui hésitaient à publier dans Science ( de peur d'être rejeté) à le faire. La plupart des actions de modernisation dont s'enorgueillit Science prirent place pendant les fonctions de Koshland. Enfin dans sa section éditoriale connue sous l'appellation de " Dr. No it all " (contraction de " Dr. Know it all"- " Dr. sait tout " où d'une manière sarcastique et amusante , il aborde les problèmes d'ordre différents qui des fois n'ont pas de relations directes avec la science et la publication scientifique mais abordent des problèmes d'ordre général), il eut le mérite de provoquer les réactions des concernés comme lorsqu'il critiqua les services postaux pour leur lenteur dans la livraison aux abonnés et qui reçut une réponse et une amélioration de ces mêmes services.

En 1995, Koshland fut remplacé par le neuroscientifique Floyd E. Bloom. En 2000, Floyd E. Bloom fut remplacé par le biologiste Donald Kennedy Enfin en 2008, le biochimiste Bruce Alberts devint le dernier éditeur en date de Science.

\section{3-Recherches ayant fait date}

$\mathrm{Au}$ cours des premières années du $20^{\mathrm{e}}$ siècle, Science publia nombre de résultats de recherches importantes et de pointe .On citera :

-Le role du chromosome dans l'hérédité chez la mouche du vinaigre par Thomas Hunt Morgan, Lauréat du Prix Nobel de physiologie en 1933

- L'existence du mirage gravitationnel par Albert Einstein dans un article publié en 1936.

- L'existence de galaxies spirales par Edwin Hubble en 1936

En February 2001, les résultats préliminaires du génome humain ont été simultanément publiés par Nature et Science. Science publiant la recherche des Celera Génomics et Nature publiant le projet du génome humain financé publiquement

Malgré cette excellence et sérieux dans le travail éditorial, il n'en demeure pas moins que certaines controverses ont vus le jour et qui ont quelque peu terni la réputation des périodiques concernés (surtout quand il s'agit de journaux scientifiques prestigieux comme Nature, Science, Lancet, New England Journal of Medecine ou Journal of the American Medical Association qui se targuent de publier les recherches les plus sérieuses et les plus sélectives du point de vue qualité scientifique).De même que ces cas ( qu'ils soient des cas de plagiat, fraude ou même de faute et lacunes méthodologiques dans la conception de la 
recherche) ont apporté de l'eau au moulin de ceux qui voient le contrôle par les pairs sous un angle de suppression des avis dissidents et qui, à l'ère de l'Internet et de sa capacité incommensurable de stockage, n'a plus sa raison d'être. La troisième partie de ce travail examinera plus en détails les tenants et aboutissants de la place du contrôle par les pairs, de ses avantages, défauts et autres problèmes qu'il pose. Nous nous contenterons pour le moment d'examiner quelques cas qui ont étés relevé dans Science et qui eurent un retentissement vu leur gravité.

\section{4-Controverses}

En 2002, le journal Science retira huit recherches ${ }^{(58)}$ publiées entre 2000 et 2001 et dont l'auteur était Jan Hendrik Schön après qu'il eut été prouvé qu'il avait fabriqué une grande partie des données. Jan Hendrik Schön est un physicien allemand, qui connut une brève célébrité après une série d'apparentes avancées scientifiques qui finirent par s'avérer être des fraudes.

Les travaux de Schön tournaient principalement autour de la physique de la matière condensée et les nanotechnologies. Bell Labs lui offrit un poste en 1997 à la fin de l'année de l'obtention de son doctorat à l'Université de Constance.

Répertorié, en 2001, comme auteur d'un article de recherche tous les huit jours en moyenne, Schön annonce, la même année, qu'il a réussi à produire un transistor au niveau moléculaire, en déclarant avoir utilisé une fine couche de molécules organiques pour assembler un circuit électrique qui, activé par un courant électrique, fait fonction de transistor. Les retombées potentielles de cette découverte étaient très importantes dans la mesure où elles auraient permis de dépasser l'électronique basée sur le silicium vers l'électronique organique. Ceci aurait permis aux puces de continuer la miniaturisation après le niveau où le silicium ne résiste pas, et ainsi de valider la loi de Moore plus longtemps que ce qui était prédit. Cela aurait aussi eu comme implication une réduction drastique du coût des appareils électroniques.

Mais face au scepticisme exprimé par des collègues au sujet de certaines données (dont certaines étaient dupliquées dans deux expérimentations différentes), son employeur Bell Labs, commandita une enquête pour déterminer les éventuelles malversations commises par Schön. Devant les preuves accablantes, il fut confondu et ne put fournir les données brutes de

${ }^{(58)}$ J. H. Schön, S. Berg, Ch. Kloc, B. Batlogg, Ambipolar pentacene field-effect transistors and inverters, Science 287, 1022, (2000)

J. H. Schön, Ch. Kloc, R. C. Haddon, B. Batlogg, A superconducting field-effect switch, $\underline{\text { Science } 288,656}$ (2000)

J. H. Schön, Ch. Kloc, B. Batlogg, Fractional quantum Hall effect in organic molecular semiconductors, $\underline{\text { Science }}$ 288,2338 (2000)

J. H. Schön, Ch. Kloc, A. Dodabala-pur, B. Batlogg, An organic solid state injection laser, Science 289, 599 (2000)

J. H. Schön, A. Dodabalapur, Ch. Kloc, B. Batlogg, A light-emitting field-effect transistor, Science 290, 963 (2000)

J. H. Schön, Ch. Kloc, H. Y. Hwang, B. Batlogg, Josephson junctions with tunable weak links, Science 292, 252 (2001)

J. H. Schön, Ch. Kloc, B. Batlogg, High-temperature superconductivity in lattice-expanded C60, Science 293, 2432, (2001)

J. H. Schön, H. Meng, Z. Bao, Field-effect modulation of the conductance of single molecules, Science 294, 2138, (2001) 
ses expérimentations ni ceux qu'il aurait dû avoir sur son ordinateur (il évoqua pour se défendre que ses fichiers de données avaient été effacés de son ordinateur parce que, selon lui, les capacités de stockage du disque dur de son ordinateur étaient insuffisantes à conserver ces fichiers et que tous ses échantillons expérimentaux avaient été soit jetés soit irrémédiablement endommagés). Schön perdit son travail et retourna en Allemagne et obtint un poste dans une entreprise d'ingénierie. En juin 2004, l'université de Constance publia une déclaration de presse selon laquelle le doctorat de Schön était révoqué pour " conduite déshonorante ". Le porte-parole du département de physique, Wolfgang Dieterich, appela cette affaire " la plus grande fraude en physique des 50 dernières années " et déclara que le " discrédit a été jeté sur la crédibilité de la science ".

Un autre cas dans lequel Science était acteur est un article paru en $2002^{(59)}$ et dont le sujet était la neurotoxicose de la drogue MDMA (Methylenedioxymethamphetamine nom chimique de la drogue psychotrope connue sous l'appellation d'écstasy). La controverse se situait dans le fait que l'auteur principal de l'article, George A Ricaurte, après avoir déclaré que ladite drogue pouvait causer des dommages irréversibles même dans le cas d'une utilisation unique, a dû se rétracter face au scepticisme des spécialistes. Il publia une rétractation dans laquelle il expliquait que les résultats étaient dû, non pas à l'administration de Methylenedioxymethamphetamine mais de methamphetamine et que ceci résultait d'une confusion dans les deux fioles envoyées par le laboratoire chargé d'envoyer les deux produits ${ }^{(60)}$.Toujours est il que cette confusion avait coïncidé avec la discussion au niveau du Congres américain d'un projet de loi connu sous l'acronyme de RAVE (Reducing American's Vulnerability to Extasy) et certains chercheurs avaient accusé les auteurs de la recherche d'avoir, d'une certaine manière, abondé dans le sens de ceux qui finançaient la recherche et le combat contre les drogues ${ }^{(61)}$ Dans une interview au magazine britannique The Scientist ,les chercheurs Colin Blakemore and Leslie Iversen décrivirent comment ils exprimèrent leur inquiétude au sujet de l'article incriminé avec les éditeurs de Science. "C'est un scandale sans précédent " déclara Iversen au The Scientist." C'est un autre exemple d'un certain genre de chercheur qui de toute évidence font de la recherche sur les drogues illégales dans le but principal de montrer ce que le gouvernement veut qu'ils montrent. Ils prennent de grandes sommes des allocations de recherche gouvernementales pour faire ce genre de travail teint de parti pris ". Cette affaire mis aussi la question du contrôle par les pairs au cœur de la discussion comme nous l'avons déjà présenté. D'un autre coté, ceux qui voient le contrôle par les pairs comme un mécanisme adéquat, argumentent que la faute qui a conduit à la rétractation (l'utilisation d'une fiole autre que celle programmée) n'aurait pas pu être, de toute façon, détectée par le contrôle par les pairs et que le processus scientifique fonctionna parfaitement étant donné que l'article fut rétracté.

Une autre controverse, qui eut un plus grand retentissement et une plus grande couverture médiatique, est celle du chercheur sud coréen Hwang Woo-Suk qui en 2004 et 2005 publia en deux recherches apparemment révolutionnaires. En 2004, Hwang et son équipe publient un article dans le magazine Science ${ }^{(62)}$ annonçant qu'ils sont les premiers au monde à réussir à cloner un embryon humain pour la recherche scientifique (production de cellules-souches).

\footnotetext{
${ }^{(59)}$ Ricaurte, George A.[et al], Severe dopaminergic neurotoxicity in primates after a common recreational dose regimen of MDMA ("Ecstasy"), Science,Vol.297,n5590, p. 2260-2263, 27 septembre 2002

(60) Ricaurte, George A.[et al],Retractation ,Science, Vol. 301, no. 5639 ,p. 1479,12 septembre 2003

(61) Blakemore, Colin, Iversen, Leslie, Retracted ecstasy paper "an outrageous scandal", The Scientist, 16 septembre 2003

${ }^{(62)}$ Hwang ,Woo Suk, .[et al], Evidence of a pluripotent human embryonic stem cell line derived from a cloned blastocyst, ,Science, Vol. 303, n. 5664, p. 1669-1674, 12 mars 2004
} 
En mai 2005, Hwang publie un second article toujours dans Science ${ }^{(63)}$ affirmant qu'il a réussi à produire 11 lignées de cellules souches, chacune à partir d'une personne différente. Ce travail laisse alors entrevoir l'avènement d'une nouvelle ère thérapeutique, au plus près du malade et de la maladie, caractérisée par la production de cellules souches issues de et destinées à des personnes malades, dans le but de guérir leurs organes dysfonctionnels sans risques de rejets par exemple. Peu après ces publications qui ouvraient de nouvelles perspectives dans la recherche médicale, des voix commencèrent à s'élever pour dénoncer des manquements éthiques à la recherche scientifique.

En novembre 2005, le Pr. Gerald Schatten de Pittsburgh, avec qui Hwang Woo-Suk travaillait depuis deux ans, l'accuse de ne pas respecter certaines règles éthiques : il aurait utilisé des ovocytes de jeunes femmes rémunérées, sans expliquer l'emploi qui devait en être fait. Cette prise de position marque le début d'une polémique qui va s'amplifier rapidement jusqu'à invalider les résultats les plus spectaculaires obtenus par Hwang sur le clonage de cellules humaines.

Après avoir brièvement nié les faits qui lui sont reprochés, devant l'accumulation de témoignages accablants, et après que le rapport du comité d'enquête de l'Université nationale de Seoul (Investigation Committee Report, Seoul National University) ${ }^{(64)}$ eut conclu que ses travaux étaient entachés d'irrégularités, Hwang les reconnaît en décembre 2005 et démissionne dans les semaines qui suivent de toutes ses fonctions officielles

Entre autres choses, l'un de ses collaborateurs avait reconnu que plusieurs photos de cellules clonées étaient fausses. En conséquence, Hwang et les autres signataires ont demandé au magazine Science de retirer l'un de ses articles ${ }^{(65)}$.A la suite de ce scandale, Science mis au point un comité pour étudier les suites de cette affaire. Le comité conclut que le journal avait suivi les procédures usuelles et qu'il n'y avait rien qu'il eut pu faire pour éviter la fraude délibérée. Cependant, le comité recommanda que les recherches reçues devaient dorénavant être classées comme non controversée ou controversée, ces derniers devraient être examinées plus attentivement. Par la même, Science suggéra que cette procédure devait être aussi adoptée par Nature (peut être parce que les deux prestigieuses publications ayant étés victimes de ce genre d'agissement ou peut être aussi comme une sorte de défi vis-à-vis d'un rival).

Enfin, dans un éditorial au sujet de cette affaire, Donald Kennedy, éditeur en chef de Science à l'époque, défendit le contrôle par les pairs, en remarquant que démasquer la fraude demanderait une "surveillance couteuse et offensante de la grande majorité des chercheurs afin d'attraper le tricheur occasionnel" (66).

\footnotetext{
${ }^{(63)}$ Hwang, Woo Suk, .[et al], Patient-specific embryonic stem cells derived from human SCNT blastocysts, Science, Vol. $308, n^{\circ} .5729$, p. 1777-1783, 17 Juin 2005

${ }^{(64)}$ Summary of the Final Report on Professor Woo Suk Hwang's Research Allegations by Seoul National University Investigation Committee, New York Times, 10 Juillet 2010.Disponible à : http://www.nytimes.com/2006/01/09/science/text-clonereport.html?pagewanted=1\&_r=1

${ }^{(65)}$ Kennedy, Donald, Retraction of Hwang, [et al], Science 308 (5729) 1777-1783 [and] Retraction of Hwang, [et al], Science, 303 (5664) 1669-1674, Science, Vol.311, n 5759, p.335, 20 janvier 2006.

On remarquera à toutes fins utiles que sept des quinze auteurs de la première recherche (2004) rétractèrent leur travaux alors que pour la deuxième (2005), tous les auteurs rétractèrent les résultats de leur recherche.

${ }^{(66)}$ Kennedy, Donald, Good news-and bad, Science, Vol. 311, n ${ }^{\circ} .5758$, p. 145, 13 Janvier 2006
} 


\section{5-Science et la publication en ligne}

Généralement, les versions en texte intégrale des archives en ligne ne sont pas accessibles au public gratuitement. Elles sont disponibles en ligne aux membres de A.A.A.S. à partir du site web principal du journal. Les abonnements individuels et institutionnels sont disponibles contre payement (mais il est beaucoup plus avantageux de joindre AAAS et recevoir le magazine gratuitement).Le site web de Science donne aussi un accès gratuit à certains articles (principalement des articles de recherche originaux et des éditoriaux) ainsi que les tables des matières complètes des numéros actuels et passés, une année après leur publication. L'accès à tous les articles sur le site web de Science est gratuit si la demande vient d'une adresse IP d'une institution abonnée. Les articles ayant parus entre cinq et six ans et les articles récents de plus d'une année sont disponible via ProQuest. En plus, l'adhésion à AAAS inclut un accès illimité aux archives du site web de Science où il est appelé " Science Classic ".Les institutions peuvent décider d'ajouter "Science Classic" à leur abonnement pour une somme additionnelle.

\section{Chapitre V \\ DE QUELQUES EXEMPLES DE DESCRIPTION, TYPOLOGIE ET CARACTERISATION DU PERIODIQUE SCIENTIFIQUE A TRAVERS LES AGES}

Intoduction

D'après tout ce qui a précèdé, il est loisible de dire que le périodique scientifique contrôlé par les pairs, et malgré presque 350 ans de présence, n'est pas clairement défini et encore moins cerné. Il est vrai qu'il a traversé ces trois siècles et demi et qu'il est devenu une condition sine qua none dans le monde de la recherche scientifique au point qu'il est l'aune à laquelle un chercheur est jugé. Plus le CV d'un chercheur est rempli d'articles publiés, plus sa cote, son aura dans sa communauté est rehaussée. Cet état de fait est encore plus rehaussé si la publication est dans l'une des revues ou publication de rang A ( core periodicals ).Malgré toute cette normalisation et critères obéissant aux canons de la publication scientifique, l'histoire du périodique scientifique et technique contrôlé par les pairs est encore mal connue et mal cernée. Ceci s'est matérialisé dans notre difficulté à trouver une classification normalisée, unifiée et acceptée de tous. Nous avons déjà vu que ce qui est considéré périodique est lui même sujet à controverses quant au nombre, l'inclusion dans la catégorie, la classification, etc..., sans parler du début du périodique où le medium avait du mal à se singulariser et à se détacher du livre. Malgré ces carences, il existe quelques classifications du périodique scientifique qui ont essayé de présenter et d'expliquer son cheminement depuis les premiers pionniers jusqu'au périodique actuel dans sa forme électronique dématérialisée. Nous en avons choisi trois qui nous ont paru les plus complètes et les plus inclusives. Ce sont : 


\section{Evolution of the scientific paper}

(67)

La première est celle de Joseph E. Harmon intitulée " Evolution of the scientific paper " et dans laquelle (pour des raisons de maniabilité et de facilité à notre avis), il départage la période de 1665 à nos jours (en fait à 1965 car la quatrième période commence à cette date) en des périodes de 100 ans .Il nous parait, personnellement, que cette classification prêche par une sorte de schématisation car on ne peut départager l'histoire des périodiques en des périodes aussi égales. De toute évidence, cette classification ne peut être rejetée mais le périodique dans sa brève histoire de 350 ans, n'est pas passé d'une étape à une autre en des bonds aussi égaux dans la durée .D'ailleurs, Kronick dans son livre référence " A History of scientific and technical periodicals : the origin and développement of the scientific and technical press : 1665-1790" retrace le cheminement du périodique et s'arrête à la fin du $18^{\mathrm{e}}$ siècle car bien qu'il reconnaît que cette période ne peut nullement prétendre à une unicité dans le contenu, il reconnaît que ceci est fait dans un but de maîtrise de l'information qui , comme il est communément connu, a connu une explosion exponentielle que seule l'informatisation et l'automatisation peuvent gérer.

Harmon départage donc l'histoire et l'évolution de l'article scientifique en :

\section{1-Les origines et les années de formation (gestation) 1665-1765}

Cette période débute avec la fondation des Philosophical Transactions et dont le titre complet (très représentatif de la manière dont étaient écrits les titres de l'époque et qui est en même temps une présentation des buts de la publication) est "Philosophical Transactions : giving some accompt of the present undertakings, studies and labours of the ingenious in many considerable parts of the world ( Philosophical Transactions: relater certaines des entreprises, études et travaux actuels des ingénieux dans un nombre considérable des contrées du monde). The Philosophical Transactions, qui est toujours en activité, a été en fait fondé quelques mois après une publication française Le Journal des Sçavans à qui la paternité de la première publication scientifique est attribuée. Bien que le laps de temps de la parution entre les deux publications soit minime (le premier fut en janvier 1665 et le deuxième en mars 1665), The Philosophical Transactions est considéré comme étant le véritable prototype de la publication scientifique alors que le Journal des Sçavans est généralement considéré comme étant plus ce qui s'apparenterait de nos jours à un magazine. Ces deux périodiques ayant étés les pionniers, s'en est suivi une polémique au sujet de la primauté de celui qui fut le premier. Il nous suffira de citer Francis André ${ }^{(68)}$ pour voir desquelles des deux est le premier .Il dit " Le premier [le Journal des Sçavans] se voulait le reflet de l'activité des sciences et des arts, en proposant des résumés analytiques des ouvrages récents ainsi que des éléments d'actualité scientifique. Le second [Philosophical Transactions], véritable organe de communication de la Société royale de Londres, publiait les communications originales présentées lors des séances publiques ".Dans la même veine et pour faire taire toute polémique, il poursuit: "Nous ne rentrerons pas dans la querelle stérile de savoir laquelle des deux revues fut la première revue savante. Il nous suffit de constater que le Royaume Uni est aujourd'hui le premier pays

\footnotetext{
${ }^{(67)}$ Harmon, J.E. , Evolution of the scientific paper, IPCC 92 Santa Fe, Crossings Borders, Conference Record, 29 sept.-03 oct.1992, p.468-475.Disponible à :

http://ieeexplore.ieee.org/Xplore/login.jsp?url=http\%3A\%2F\%2Fieeexplore.ieee.org\%2Fiel4\%2F5507\%2F1479 4\%2F00673079.pdf\%3Farnumber\%3D673079\&authDecision=-203

(68) Francis André, Libre accès aux savoirs =open access to knowledge, Paris, Futuribles, 2005, p.9
} 
d'édition scientifique au monde pour déduire lequel des deux a su faire fructifier le mieux ces idées de départ." De son coté, Jean Claude Guédon dans une contribution qui est devenue un classique de la littérature du libre accès et la communication scientifique au XXe siècle, déclare au sujet de la rivalité entre le Journal des Sçavants et Philosophical Transactions que : ...." régulièrement, la revue est comparée au 'Journal des Sçavants', comme si les deux publications étaient des sœurs jumelles - j'ai moi-même commis cette erreur il y a quelques années. Alors qu'il est évident que toutes deux sont des périodiques, traitent de la philosophie de la nature et furent lancées à quelques mois d'intervalle seulement, il n'est pas si évident qu'elles poursuivaient exactement les mêmes buts. La publication française reproduisait un modèle d'échanges épistolaires manuscrits, typique de la République des Lettres, quelque peu cancanier et porté sur l'actualité ; de ce fait, elle se rapproche plus de 'Scientific American' que d'une revue scientifique moderne et apparaît ainsi fermement ancrée dans le domaine du journalisme scientifique, alors émergeant. Bien que le Journal publiât de temps à autre quelques papiers originaux, ceux-ci apparaissaient comme une expression particulière de l'actualité parmi d'autres types de nouvelles ; par contraste, 'Phil Trans', bien que diffusant aussi des informations récentes, avait véritablement pour but de créer un registre public des contributions originales à la connaissance. En d'autres termes, la publication parisienne suivait la mode tandis que la revue londonienne servait à valider l'originalité. Là réside la différence significative (et profonde) entre les deux périodiques "(69).Philosophical Transactions a ainsi publié les travaux de sommités du $16^{\mathrm{e}}$ et $17^{\mathrm{e}}$ siècle tels que Newton, Bacon, Halley pour ne citer que les plus connus.

Il faut dire que l'époque avait vu l'émergence de ce qui est communément connu sous " la méthode scientifique " et qui se basait sur l'expérimentation ou l'observation systématique pour faire avancer le savoir des mécanismes de la nature. Les résultats de cette méthode sont présentés de la meilleure manière sous la forme de courts articles et leur dissémination se fait par la publication d'un ensemble de ces articles dans un seul volume en des intervalles réguliers ou même irréguliers.

Nombre des périodiques importants du $17^{\mathrm{e}}$ et $18^{\mathrm{e}}$ siècle étaient associés à des sociétés savantes, la plus connues étant " The Royal Society of London " alors que des organisations similaires existaient à Paris, Berlin, Stockholm, et Saint Petersburg. Les sociétés savantes sponsorisèrent aussi la publication d'actes qui étaient l'ensemble des recherches présentées aux rencontres de la société savante ou qui résultaient de ces activités expérimentales. Avant cela, les sociétés savantes désignaient un comité ou un secrétaire qui se chargeait de :

- recevoir les lettres des correspondants détaillant les derniers résultats des recherches

- répondre à leurs lettres et les informer des recherches qui pouvaient les intéresser

- lire à haute voix les correspondances d'importance aux réunions de la société savante

Les recherches qui se publiaient dans les premiers périodiques ou les actes de conférences des sociétés savantes couvraient une variété de sujets et visaient une large audience et pas seulement des chercheurs engagés dans une recherche similaire. C'était le Siècle des

\footnotetext{
(69) Guédon, Jean Claude, In Oldenburg's Long Shadow: Librarians, Research Scientists, Publishers, and the Control of Scientific Publishing, In: Creating the Digital Future, Proceedings of 138th ARL Membership Meeting,Toronto [Ontario], May 23-25 2001, Baker, Shirley K. Ed.

Disponible à : http://www.arl.org/resources/pubs/mmproceedings/138guedon.shtml
} 
Lumières et chacun - des roturiers jusqu'aux rois - était intéressé d'apprendre et contribuer aux dernières avancées en science et technologie .Par exemple, le premier numéro de Philosophical Transactions qui se composait de 16 pages avait des contributions de - entre autres - " l'éminent savant Robert Hooke, un astronome, un docteur de nature curieuse et un marin audacieux et connaisseur ". Comme on peut le voir, les premiers périodiques et à leur tête le prototype du périodique scientifique - Philosophical Transactions - ne se distinguait pas par une spécialisation outrancière mais alliait ce qui pourrait être conçu de nos jours comme une forme d'éclectisme.

Le style de langage utilisé était, comparativement à ce qui est de mise de nos jours, celui familier d'une lettre. Ces lettres étaient envoyées aux secrétaires des sociétés savantes et aux éditeurs des périodiques avec l'assomption qu'elles seraient publiées telles que rédigées par l'auteur ou réécrites par le secrétaire ou l'éditeur. Généralement, les auteurs décrivaient les résultats de leur recherche (ou ceux d'une autre personne) dans un langage simple d'un reportage technique d'information, émettant des fois des observations personnelles qui n'étaient pas, le moins que l'on puisse dire, en relation avec le sujet discuté .La plupart de ces articles étaient courts comparativement aux standards modernes et ne dépassaient pas des fois un paragraphe ou deux et signalaient des observations directes telles que les observations de comètes, descriptions de faunes et flores exotiques, histoires de curiosités médicales et les narrations de voyages à des lointaines terres D'un autre coté, une minorité distincte , à l'image de Newton en 1672 avec sa fameuse recherche au sujet de la lumière et la couleur et qui parut dans le numéro 80 de Philosophical Transactions, présente une description assez minutieuse des détails expérimentaux ou des interprétations théoriques des résultats pour attirer une audience aussi large que possible .La plupart des premiers périodiques scientifiques et comptes rendus de conférences utilisaient la langue vernaculaire au lieu de la langue internationale de l'époque et qui était le Latin.

Quelques périodiques et comptes rendus de conférences avaient un système de certification et de contrôle afin de se prémunir de recherches qui n'avaient pas le niveau requis alors que la majorité des périodiques et comptes rendus de conférences ne l'avaient pas. A cet effet, John Hill ${ }^{(70)}$ critiqua " The Royal Society " en 1751 pour publier " des articles carrément insensés et frivoles " dans son Philosophical Transactions. Il cita en tant qu'évidence, des recherches sur un triton* découvert dans la jungle de Virginie, une plante miraculeuse qui guérit les blessures récentes (" mais la toucher, c'est être guéri "), la corne de l'unicorne et la transformation de l'eau en vers. Peu de temps après ces critiques, The Royal society mis en place un comité de cinq membres pour lire et sélectionner les recherches à publier. Cependant, le contrôle externe par des experts ne fut établi et devint une pratique standard pour les périodiques et les comptes rendus de conférences que bien plus tard dans la troisième étape à partir de 1865 .

\section{2-Spécialisation et professionnalisation (1765-1865)}

Cette étape démarra au milieu du $18^{\mathrm{e}}$ siècle quand les scientifiques et leurs sociétés savantes durent devenir plus spécialisés afin de faire face aux rapides avancées en science, spécialement en physiques et chimie. Parmi les périodiques qui parurent au début de cette

\footnotetext{
${ }^{(70)}$ Hill, John, A Review of the Works of the Royal Society of London; containing Animadversions on such of the Papers as deserve particular Observation....... London : R. Griffith., 1751, 286p.

* Animal de la mythologie grecque et qui est une divinité descendant du Dieu Triton, représentée avec un corps d'homme barbu, une queue de poisson, tirant le char des dieux de la mer.
} 
époque, on peut citer Der Naturforscher, Annalen der Physik, Annales de Chimie et de Physique, Botanical Magazine, The Chemist, Journal of the Chemical Society et Observations sur la Physique. Avant cette étape, les seuls périodiques reconnus apparurent dans les spécialités de médecine et d'agriculture où une audience assez importante existait déjà.

$\mathrm{Au}$ cours de cette seconde étape, l'époque du généraliste éclairé fut remplacée par celle du spécialiste industrieux. Une augmentation régulière des scientifiques professionnels et les institutions qui les supportaient (telles que les sociétés savantes spécialisées, les périodiques, les équipements de recherche et les programmes d'enseignement) accompagnèrent cette spécialisation. En plus, les universités et les laboratoires de recherche récemment fondés tels que l'Ecole Polytechnique à Paris, le laboratoire de chimie Justus Von Liebig à Giessen, The Royal College of Chemistry, The Cavendish Laboratory et les instituts du Kaiser Wilhelm GesselSchaft tous devinrent des centres de recherche et de formation des scientifiques sur une grande échelle.

Une des conséquences de cette spécialisation et professionnalisation fut que le profane curieux commença à être écarté en tant que lecteur potentiel et de la littérature technique et même en tant que participant dans les sociétés savantes. Certains chercheurs proposèrent que ne soit acceptés dans les nouvelles sociétés savantes que ceux qui auraient publiés des recherches écrites dans les annales d'une quelconque société savante.

La population des scientifiques professionnels augmentant durant la deuxième étape, les chercheurs pouvaient rédiger leurs recherches en tenant compte du fait qu'ils avaient une population spécialisée en face, spécialement dans les spécialités qui progressaient rapidement telles que les sciences physiques et chimiques. Ce qui veut dire qu'ils étaient sûrs que leurs lecteurs auraient les compétences requises pour comprendre des concepts mystérieux ainsi qu'une connaissance du sujet discuté. D'un autre coté, étant donné que l'expérimentation étant devenue une opération qui se passait dans le laboratoire de recherche particulier contrairement à une rencontre publique en face d'un groupe de scientifique de la société - la recherche expérimentale dans les publications les meilleures devint plus longue et plus techniquement détaillée de telle sorte que le lecteur peut indirectement voir l'expérimentation à travers l'exposé .Pour plus d'autorité, les auteurs très souvent reliaient les expérimentations et les observations à des places, des circonstances et époques spécifiques .

Quoique ces sujets spécialisés répondent à une audience de plus en plus spécialisée, les auteurs de ces recherches retinrent le style d'écriture personnel en y incorporant anecdotes, humour et un langage décoré dans leur prose technique caractéristiques des lettres savantes du premier stage cité. Leurs recherches n'étaient pas normalement, en ce temps, écrites par un groupe mais par un ou deux auteurs étant donné que la pratique de faire des recherches en groupe n'a débuté sérieusement qu'au $20^{\mathrm{e}}$ siècle. Le pronom personnel " Je ", pratiquement disparu de la littérature technique moderne, est commun et la littérature abonde de passages dans lesquels la personnalité de l'auteur transparait.

D'un autre côté, il est important de mentionner que le rôle du scientifique - éditeur était d'une certaine manière différent dans cette seconde étape. Non seulement il pouvait donner la primauté à ces propres recherches et publier des réfutations de la contribution d'autres chercheurs avec lesquels il ne serait pas d'accord, mais il pouvait aussi émettre un commentaire éditorial directement dans une recherche qu'il jugeait offensante, commissionner ou composer des satires de style et de contenu sarcastiques et réimprimer des 
articles pour le seul but de commenter leurs défauts. A cet effet, en 1839 apparut dans un périodique scientifique prestigieux une des recherches les plus étranges et qui satirisait des expérimentations de toute évidence fausses sur " le secret de la fermentation alcoolique ".Cet article humoristique mais néanmoins nullement à propos ridiculisait l'observation faite par Theodore Schwann et Charles Cagniard-Latour que la levure est un organisme vivant capable de convertir le sucre en alcool. Cette parodie quoique non signée est, à ce qui était dit, l'œuvre de deux géants de la chimie au $19^{\mathrm{e}}$ siècle, Friederich Wöhler et Justus Von Liebig (qui croyaient que la levure est une catalyse inanimée). Ce dernier se trouve être aussi l'éditeur du périodique dans lequel cet article est apparu, Annalen der Chemie.

La fin de la deuxième étape a introduit l'émergence des périodiques consacrés aux résumés des recherches publiés et les rétrospectives de la littérature. C'était des publications dont le but le premier était d'aider les scientifiques à faire face à l'accroissement rapide de la littérature.

\section{3-Développement de l'article scientifique moderne (1865-1965)}

$\mathrm{Au}$ début de cette troisième étape, il y avait plus de 1500 périodiques scientifiques et un nombre d'actes de congrès de sociétés savantes alors qu'un siècle auparavant il n'y en avait qu'une centaine. A cette date, ce qui était une courbe de progression linéaire des périodiques scientifiques vit une augmentation spectaculaire et le phénomène de ce qui est communément connu sous le vocable de l'explosion documentaire fit son apparition.

De la constante professionnalisation et spécialisation de la science à la fin du $19^{\mathrm{e}}$ siècle, a résulté l'apparition des recherches qui ressemblent les recherches modernes en style et contenu, du moins dans les journaux scientifiques les plus réputés .Une des raisons ayant contribué à ce changement est la sophistication accrue dans les méthodes statistiques (i.e. analyse régressive, méthode du moindre carré, et le test t-étudiant) et le design expérimental. Un des premiers livres les plus complets traitant du design, l'exécution des expérimentations et l'analyse statistiques de leur résultats à paraître fut The principles of science : a treatise on logic and scientific method par un certain Jevons, un savant en logique et économie connu de l'époque et ce en 1874. Les meilleures recherches de la fin du $19^{\mathrm{e}}$ siècle reflètent la sophistication accrue dans le design expérimental et l'interprétation des résultats qu'a abordés le livre de Jevons.

La rigueur intellectuelle accrue dont devait faire preuve la recherche scientifique donna naissance un style d'écriture plus formel dans lequel les auteurs évitèrent le style humoristique, le langage ornemental, ou les observations personnelles pour ne s'en tenir qu'aux faits essentiels. Sans nul doute, les recherches à plusieurs multiples contribuèrent de plus en plus à l'impersonnalité de la recherche moderne.

Avec ce style de rédaction plus formel vint aussi une structure normalisée pour présenter les résultats expérimentaux. Cette structure (connue des fois sous l'appellation de " structure de sujet ") suit les étapes suivantes :

- une introduction qui présente le problème de recherche ou un besoin

- une méthode expérimentale et les matériaux utilisés pour résoudre ce problème ou répondre à ce besoin 
- des résultats découlant de l'application de la méthode expérimentale.

- une argumentation des résultats avec les conclusions éventuelles.

C'est une manière logique et efficace d'organiser l'information technique détaillée attendue dans une recherche scientifique : elle impose à chaque chercheur de faire face aux mêmes questions de base et de d'essayer généralement les mêmes analyses et ceci même pour les analyses les plus différentes possibles. Un des premiers guides de style décrivant cette structure est Préparation of scientifical and technical papers par Trelease et Yule en 1927. Depuis la publication de ce manuel, nombre de livres et guides de styles ont recommandés la structure de sujet pour démontrer les expériences expérimentales. Les recherches qui démontrent le développement de nouveaux matériaux ou de nouvelles méthodes suivent aussi cette même structure de base.

La mathématisation de la science débute durant la dernière partie de la seconde étape .Ceci résulta en des théories mathématiques englobant un nombre de phénomènes découverts ou expérimentalement recherchés durant le $18^{\mathrm{e}}$ siècle ou plus tôt y compris les champs magnétiques, les vagues électromagnétiques, les forces attractives et répulsives, chaleur, et énergie. Ceci signala l'apparition régulière de recherches strictement théoriques en dehors des mathématiques durant la troisième étape. Avant, les savants avec une inclinaison théorique, comme Newton et Huygens, faisaient aussi presque toujours des travaux expérimentaux.

Une structure normalisée pour la recherche théorique n'est pas aussi facilement identifiable comme c'est le cas pour les recherches expérimentales. Pour cette raison, les guides de styles et livres concernant la manière de rédiger les recherches scientifiques sur le sujet sont rares et ne disent pas grand chose sur le sujet. Harmon dans une analyse de 40 recherches théoriques révèle qu'une recherche typique de ce genre débute par une introduction qui présente un problème à solutionner ou un besoin à identifier et les hypothèses principales que l'auteur émet pour solutionner le problème ou le besoin à identifier. Ceci est suivi par le théorème ou le cadre conceptuel qui découle de ces hypothèses et des facteurs additionnels tels que les hypothèses secondaires, les définitions et les conditions. Vient ensuite la preuve du théorème par raisonnement logique ou la validation par comparaison par ce qui déjà établi ou accepte d'être établi. La recherche se termine par des conclusions y compris des recommandations sur un futur travail qui développerait ou vérifierait le théorème.Cette troisième étape a aussi vu une importance accrue des parties d'une recherche qu'on peut considérer comme annexe au texte principal telles que les résumés d'en-tête, les figures et tables, les remerciements et les références.

Un des premiers périodiques à fournir des instructions aux auteurs au sujet de la préparation des résumés d'en tète fut Astrophysical journal et ce en 1920.Mais ces résumés ne devinrent une partie universellement appliquée des recherches que vers les années 60.

Dans la première et la plus grande partie de la seconde étape, les périodiques et les actes de congrès, avaient relativement peu de représentations, des fois pas plus qu'une ou deux douzaines dans toutes les recherches apparaissant dans toute l'année. Les illustrations qui apparaissaient étaient typiquement des dessins d'appareillage expérimental ou d'autres objets (flore ou faune, corps astraux, anatomie humaine). Assez curieusement, étant donné leur évidente utilité, les graphes de données sont pratiquement absents jusqu'au début du $19^{\mathrm{e}}$ siècle. Mais ce n'est que vers la fin du $18^{\mathrm{e}}$ siècle que Lambert et Playfair inventèrent des 
graphes statistiques pour la représentation visuelle des données. Au début du $20^{\mathrm{e}}$ siècle, le flux de l'argument dans la section résultats et discussion commença à se focaliser autour des représentations et des tables de données.

Les remerciements et les références peuvent être trouvés dans les premières recherches et actes de congrès. Cependant, les remerciements d'aide et de conseils de la part des autres ne commencèrent à apparaître régulièrement qu'au milieu du $19^{\mathrm{e}}$ siècle quand les chercheurs quittèrent leurs greniers et cuisines pour les laboratoires spécialisés. Quant aux références, les auteurs du $17^{\mathrm{e}}-18^{\mathrm{e}}$ siècle ne citaient pas régulièrement les travaux pertinents qui les avaient précédés ou même les idées présentées avant eux par des autres. Les références devinrent plus utilisées durant le milieu du $19^{\mathrm{e}}$ siècle quand une plus grande érudition s'imposa dans les recherches.

\section{4-Hyperspécialisation et informatisation (1965- ?)}

La quatrième étape nous amène au présent - l'âge de la Grande Science. Cette étape peut être mieux décrite par les performances des équipes de recherche qui sont habituellement financées par les gouvernements ou l'industrie. Le véritable moment critique à cet égard fut pendant la deuxième guerre mondiale, quand les gouvernements supportèrent financièrement des efforts de recherche à grande échelle pour développer la bombe atomique, le radar, les roquettes à propulsion liquide et la pénicilline. Le financement pour ces entreprises hasardeuses était bien au delà que ce que pouvait offrir les sources privées qui avaient étés jusque là les principales sources de financement. Le nombre de périodiques scientifiques augmenta pour atteindre 60000 et est encore en augmentation constante et ceci, indirectement, grâce aux énormes dépenses dans la recherche et le développement des gouvernements à travers le monde.

La spécialisation qui a débuté dans le $19^{\mathrm{e}}$ siècle a conduit à l' " hyperspécialisation " dans le $20^{\mathrm{e}}$ siècle. Nous avons de nos jours une multitude de périodiques très spécialisés tels que Macromolecules et Journal of alloys and compound ainsi que les actes de la conférence annuelle de la spectroscopie de Raman. Pour une compréhension complète d'un nombre de ces recherches dans ces publications, le lecteur doit avoir assimilé un corpus ou plus de savoir technique et le vocabulaire spécialisé qui lui est associé. Ce genre de recherche est généralement rédigé pour des spécialistes dans la même spécialisation et non pas le public en général ou même les scientifiques travaillant dans d'autres spécialités.

Il est malheureux de constater que même les spécialistes ont des difficultés à comprendre la littérature de leur propre spécialité. P.J.Abelson qui a occupé le poste d'éditeur du magazine Science a remarqué que les scientifiques de nos jours " ne sont pas de bons communicateurs". Les erreurs les plus communément faites incluent enterrer les faits les plus importants dans les méandres du texte, mal juger le degré de compréhension des lecteurs du sujet, ( soit en sous estimant ou sur estimant ), rabâcher des faits bien connus comme s'ils étaient nouveaux, et ne pas parvenir à relier chaque idée avec la prochaine en un flux d'argument facilement expliqué .D'un autre coté , Abelson note que, du moins dans sa propre expérience en tant qu'éditeur du magazine Science, la plupart des manuscrits soumis sont " intrinsèquement bons et publiables ". On peut conclure à partir des arguments d'Abelson que la plupart des recherches actuelles sont en général, d'assez bonne facture - de toute évidence meilleure que celles de la période des potions magiques et des tritons - alors que la qualité de présenter les faits laisse, des fois, à désirer. 
Une autre critique adressée à la recherche de nos jours est le fait que son style est aussi ennuyant que celui d'un rapport de police .En effet, les tendances dans la rédaction des recherches scientifiques qui ont parus durant la troisième étape sont devenues des normes établies. La bienséance dicte de nos jours que la recherche scientifique doit fondamentalement suivre la structure de sujet et doit être rédigée en utilisant un langage technique aussi plat que possible et un ton de voix discret.

Malgré cela, il y a des exceptions .En réaction à l'homogénéité de la recherche scientifique moderne, nombre de chercheurs ont composé leur recherches sous la forme de vers .Un chercheur présenta sa recherche sous la forme d'une chanson à un meeting scientifique à Chicago y ajoutant même une partition musicale pour la publication des actes .De toute évidence, ces tactiques sont d'une certaine manière faites pour attirer l'attention et les auteurs courent le risque de voir leur travail ne pas être considéré sérieux à cause de leur approche novatrice. Un exemple moins choquant mais qu'on peut considérer une déviation de la norme est le premier chapitre attirant l'attention du livre de Peebles et Silk (Cosmic books of odds) et dont le but déclaré est d' "enrichir, éclairer et amuser " ses lecteurs au sujet des théories en compétition sur la formation des galaxies.

Une autre caractéristique de la recherche moderne est qu'elle est normalement écrite en langue anglaise, même quand elle n'est pas la langue maternelle de l'auteur. Selon Watson ${ }^{(71)}, 88.5 \%$ des articles indexés par le Science Citation Index en 1978 étaient rédigés en anglais et les auteurs dont la langue maternelle n'était pas l'anglais en représentaient $30 \%$. De toute évidence, l'anglais est de nos jours la langue internationale pour la science et la technologie. Dans les années 80 , les premiers périodiques scientifiques électroniques furent fondés pour profiter des remarquables avances en informatique. Ils distribuent, pour la plupart, les recherches écrites conventionnellement en un format électronique. Cependant, les périodiques électroniques sont actuellement envisagés pour remédier aux restrictions actuelles de style et de contenu dans les publications scientifiques sur papier et qui peuvent considérablement être changés. Par exemple, les articles dans ces périodiques peuvent être des rapports techniques complets qui présenteraient tous les détails théoriques et les résultats y compris les longues tables de données qui sont généralement non incluses dans les recherches actuelles sur papier. Les auteurs peuvent même être permis d'inclure des informations au sujet d'expérimentations ratées et les impasses conceptuelles, les définitions de termes techniques donnés, des narrations décrivant qu'est ce qui a orienté les auteurs vers cette manière de procéder, des spéculations intuitives sur les implications des résultats, etc..., si les auteurs (ainsi que les éditeurs et les pairs qui contrôlent l'information ) jugent ce genre d'information appropriée .Les auteurs ne seraient pas ici sous la contrainte de rationaliser et d'expurger qu'impose la recherche scientifique actuelle pour garder la longueur des recherches à un niveau acceptable .Idéalement, ces rapports complets satisferaient le désir du spécialiste de savoir ce qui a permis d'arriver à toute la série d'expérimentations, de développements ou une nouvelle théorie .Ce genre d'information peut aussi être inclu dans une version hypertexte de la version papier de la recherche.

Ce nouveau genre de recherche scientifique ne supplantera probablement pas la recherche traditionnelle. Il servira seulement en tant qu'un véhicule adéquat pour publier périodiquement des rapports scientifiques qui méritent d'être présentés d'une manière plus en

\footnotetext{
${ }^{(71)}$ Watson, J.J., English, the international language of science, Journal belge de radiologie, Vol 69, n³, p 195200,1986
} 
détails. Que ces périodiques portent fruit ou non, il y a peu de doute que la technologie informatique est entrain de changer la manière par laquelle la recherche scientifique est écrite, reproduite et distribuée.

\section{2-The Scientific article: from Gallileo's new science to the human genome ${ }^{(72)}$}

Cette analyse est en fait un séminaire dont l'origine est une exposition du " Special Collections Research Center of the University of Chicago's Joseph Regenstein Library " et qui s'est tenu entre le 5 mai et le 21 août 2000. Les deux auteurs, Joseph E. Harmon et Alan E. Gross à travers une présentation Internet, partagent l'évolution de l'article scientifique en six parties et qui sont :

1-Précurseurs et rivaux.

2- Origines et premières années

3-Spécialisation et maturité accrue

4-Communications sélectionnées de quelques ingénieux scientifiques.

5-Curiosités diverses

6-Conclusion et que nous réserve le futur.

Dans l'introduction, les deux auteurs présentent l'évolution de l'article scientifique de ses modestes débuts au $17^{\mathrm{e}}$ siècle à Paris et Londres (Journal des Sçavans et Philosophical Transactions) jusqu'à son rôle central dans la communication de la science dans le monde en ligne de nos jours. Cette introduction relatera l'importance accrue des journaux scientifiques, le développement de l'article et les différentes manières par lesquelles les scientifiques utilisèrent le texte, les images et les graphes pour communiquer leurs dernières découvertes. Seront présentés des sommités scientifiques telles que Newton, Darwin et Einstein ainsi que d'autres importants mais néanmoins moins connus chercheurs, éditeurs et observateurs amateurs. D'un autre coté, cette introduction examinera quelques unes des avancées scientifiques les plus mémorables ainsi que quelques unes des plus grandes fautes scientifiques. Enfin, en regardant les images de ces mêmes articles annonçant ces découvertes, on peut apprécier la nature du changement dans la communication scientifique ainsi que la pratique journalière de la science et ce depuis plus de 300 ans.

Cette présentation a pour objectifs de :

- Décrire le rôle crucial que jouent les journaux scientifiques dans la dissémination des découvertes et l'émergence d'une communauté scientifique internationale.

- Comparer comment des sommités scientifiques - telles que Newton, Darwin et Einstein - utilisèrent les articles scientifiques publiés pour présenter des découvertes importantes à leurs pairs.

${ }^{(72)}$ www.fathom.com/course/21701730/index.html 
- Evaluer l'impact des tableaux, graphes et images et autres illustrations dans les journaux scientifiques.

- Comprendre comment la structure de l'article a évolué et analyser quelles nouvelles fonctionnalités le World Wide Web permet.

\section{1-Précurseurs et rivaux :}

\subsection{1-Les livres}

Bien avant que l'article scientifique ait fait son apparition vers la fin du $17^{\mathrm{e}}$ siècle, les livres existaient. Il ne fait aucun doute que les livres et non pas les articles communiquèrent les nouvelles révélations au sujet du monde naturel et qui donnèrent naissance à la révolution scientifique. Parmi ceux qui furent les précurseurs on peut citer, De revolutionibus orbiumde coelestium de Copernic, Novum organum de Bacon, Dioptice de Kepler, Sidereus nuncius de Galilée, Discours de la méthode de Descartes, et Principia de Newton.

Quoiqu'avec le $18^{\mathrm{e}}$ siècle, l'article se fût transformé d'un compétiteur insignifiant vers rival sérieux dans la communication de la nouvelle science, les livres restèrent un moyen important de la communication scientifique jusqu'à tard dans le $19^{\mathrm{e}}$ siècle. Bien que des fois fortement concurrencé, et ce même au $20^{\mathrm{e}}$ siècle, le livre n'a pas été supplanté comme moyen de communiquer les découvertes de recherches originales ou même faire une contribution à une théorie. A cet effet, des livres comme Adaptation and natural selection de George William ou Darwin finches de David Lack sont des livres du $20^{\mathrm{e}}$ siècle qui ont rapportés des découvertes révolutionnaires de la biologie évolutionniste.

\subsection{2-Les lettres savantes et les essais}

En plus de livres, l'autre prédécesseur majeur et rival de la communication de la science au $17^{\mathrm{e}}$ siècle est " la lettre savante " et dont l'exemple le plus fameux sont les lettres de Galilée sur les taches de soleil et les orbites des planètes. Alors que les idées de la révolution scientifique se propageaient en Angleterre et dans le Continent, le rythme accéléré de l'activité scientifique obligea les philosophes à communiquer leurs récentes découvertes à travers des correspondances à l'intérieur et entre les pays. Ces lettres n'étaient pas des messages personnels à des amis ou des collègues, mais ressemblaient des essais où étaient exposés les expérimentations et observations récentes au sujet la nature des choses à d'autres personnes intéressés dans ce sujet. Pour disséminer l'information de ces lettres savantes d'une manière plus efficiente, des savants industrieux devinrent des centres de propagation des dernières informations techniques localement et à l'étranger. Leur travail consistait à recevoir les lettres, en faire des copies et ensuite les passer à d'autres scientifiques intéressés. Après l'émergence des sociétés savantes, le travail de " trafficker in intelligence " devint plus formalisé du fait que les sociétés savantes elles même désignèrent un secrétaire pour s'occuper de la correspondance et transmettre le lettres savantes d'importance aux membres de la société et aux amis.

Un exemple de ce genre de lettre est celle que rédigea Evangelista Torricelli, assistant de Galilée et père de l'hydrodynamique en 1664 et qu'il adressa à Michelangelo Ricci un cardinal romain et mécène de la science. Après une salutation typique de l'époque, Torricelli commence brièvement par citer le sort d'une autre lettre savante qu'il avait écrite sur un sujet 
complètement différent, et qui était une preuve mathématique, qu'il avait envoyé à une autre personne .A partir de ce potin personnel d'information, il passe à une discussion du type introductif au sujet d' expérimentations et de conjonctures passées au sujet du vide qui comprenait la brillante métaphore " on vit immergé au fond d'une mer d'élément air ....." Torricelli écrivit sa lettre savante à un moment où la théorie d'Aristote qu'un vide ne pouvait exister était encore acceptable, spécialement au sein de certains influents jésuites. De manière intelligente, il contourne ce problème controversé en argumentant au début que certains réfutent l'existence d'un vide et d'autres non mais parmi ces derniers, personne n'a nié qu'il existe sans " difficulté " dans la nature: donc il a conduit certaines expérimentations pour déterminer est ce que le poids de l'air causait la résistance de la nature au vide, dans le cas où vraiment il en existait un.

La lettre savante n'a jamais vraiment disparu en tant que medium de la communication scientifique : les lettres personnelles entre les scientifiques demeurent un élément vital dans toute reconstruction historique de leur science. Et tel qu'indiqué par les lettres dans Nature, Science, Physical review letters et d'autres périodiques contemporains, la lettre publiée est toujours un medium significatif de la communication scientifique. Bien plus, la relation entre la lettre savante et l'article est directe : nombre d'articles dans les premiers périodiques sont des lettres savantes légèrement révisées pour publication par l'éditeur. C'est par exemple le cas pour le fameux premier article de Newton sur l'optique publié en 1672 dans Philosophical Transactions. Etaient aussi importants durant le $17^{\mathrm{e}}$ et $18^{\mathrm{e}}$ siècle, les livres qui contenaient un nombre de lettres et des articles courts sur des sujets techniques par un seul auteur tel que les lettres publiées de Leeuwenhoek à la Société Royale de Londres et le splendidement illustré livre de Hooke intitulé Micrographia.

\section{2-Précurseurs et premières années}

\subsection{1-Le premier périodique anglais et la Société Royale de Londres (Royal Society)}

C'est en date du mois de mars 1665 qu'Henry Oldenburg, le secrétaire de la Royal Society of London lança le premier périodique scientifique anglais, Philosophical Transactions. Ces comptes rendus de séances relataient les informations de l'étranger ainsi que des articles présentés à la Royal Society et des expérimentations qui y étaient faites - ou selon les mots utilisés sur la page de titre, "donner certains acomptes (....) des entreprises, études et travaux actuels des ingénieux dans nombre de considérables parties du monde ". Les comptes rendus de séance initiaux, paraissant mensuellement, étaient à peu prés de 20 pages et tirés à 1200 copies. Le premier numéro contenait des contributions de, entres autres, l'éminent savant et membre de la Royal Society Robert Hooke, un astronome, " un physicien curieux " et un " marin connaisseur et courageux ". Ce premier modeste mais historique numéro, se termina par une notice nécrologique du mathématicien français Pierre de Fermat. L'index des auteurs sous la direction d'Oldenburg entre 1665 et 1677 est un véritable who's who des savants du $17^{\mathrm{e}}$ siècle en Angleterre et en Europe: Newton, Boyle, Hooke, Wren, Leeuwenhoek, Huygens, Hevelius, Leibniz, Cassini et Halley.

Le journal d'Oldenburg était associé d'une manière officieuse avec la Société Royale et ses membres et leurs amis à travers l'Europe étaient les principaux contributeurs. Selon le livre de Bishop Sprat intitulé History of the Royal Society, les membres de la société essayèrent de rejeter les " amplifications, digressions et les styles non appropriés....pour ramener toute chose aussi prés que possible à la simplicité mathématique et préférer le langage de 
l'intelligence et la science à celui des artisans, des campagnards et des marchands ".Malgré cela, le style d'écriture ne fut pas toujours aussi d'une " simplicité mathématique " et certaines transgressions furent observées à l'image de la métaphorique description de Martin Lister, un physicien intéressé en entomologie et en botanique et ce dans un des premiers numéros de Philosophical Transactions. Mais si Philosophical Transactions a vu différents styles d'écriture durant sa longue histoire, il n'en demeure pas moins qu'il a toujours mis l'accent sur la " simplicité mathématique " et ce jusqu'à nos jours.

\subsection{2-Les premiers périodiques français et l'Académie Royale}

L'autre périodique savant important du $17^{\mathrm{e}}$ siècle, Le Journal des Sçavans, fut lancé au mois de janvier 1665 par Dennis de Sallo. Avant de lancer cet hebdomadaire, Sallo employait des scribes pour transcrire des passages particulièrement intéressants de livres et de lettres savantes afin qu'il puisse rapidement accéder à l'information sur un large canevas de sujets. Reconnaissant que d'autres savants pourraient aussi profiter de cette accumulation diverse d'information, il décida de publier des revues de livres hebdomadaires et des informations sur la science ainsi que sur le droit et la théologie. Parce que Le Journal des Sçavans débuta généralement comme une revue des travaux techniques et autres, certains chercheurs attribuent à Philosophical Transactions le titre de " premier " journal scientifique bien que Sallo lança son journal deux mois avant Philosophical Transactions. Selon les propres mots de Sallo, son journal fut institué " pour soulager ceux qui sont trop paresseux ou trop occupé pour lire un livre en entier. C'est un moyen de satisfaire la curiosité et devenir savant à moindre effort ". Sous sa direction et celle de ces successeurs, le journal signala quelques unes des découvertes les plus importantes au $17^{\mathrm{e}}$ siècle et parmi celles-ci la théorie ondulé de la lumière de Huygens et la mesure de sa vélocité par Roemer.

Avant ses compagnons membres de l'Académie Royale Française et ce en date du mois de septembre 1676, le Danois d'origine Ole Roemer prédit que l'éclipse d'une des lunes de Jupiter serait en date du 6 Novembre 1676 à cinq heures trente cinq minutes et quarante cinq seconde, ce qui veut dire exactement dix minutes après le temps calculé sur la base d'une vitesse instantanée de la lumière. Roemer utilisa le modèle régulier dans ses écarts observés au moment des éclipses d'une lune de Jupiter (Io) sur une certaine période pour argumenter que ceci résultait du fait que la lumière avait une vitesse définie. Cette lune et la terre s'éloignant l'une de l'autre ensuite se rapprochant, le temps que la lumière prit pour atteindre la terre changea en conséquence. Sa prédiction fut confirmée par l'Observatoire de l'Académie à Paris. Selon les calculs de Roemer, publiées dans le numéro de 1776 du Journal des Sçavans (et traduit l'année d'après dans Philosophical Transactions), la vitesse de la lumière était telle qu'il fallait à peu près vingt deux minutes pour voyager le diamètre de l'orbite annuelle de la terre, ce qui équivaut à approximativement 215000 kilomètres /seconde ce qui est raisonnablement prés de l'actuelle valeur de 299792 kilomètres /seconde.

Jusqu'au tout début du $18^{\mathrm{e}}$ siècle, Le Journal des Sçavans était le périodique qui publiait les activités de recherche de l'Académie des Sciences Royale de Paris. Ceci changea en 1702 quand l'Académie Royale commença la publication régulière de ces recherches dans son Histoire et Mémoires. Histoire contenait des résumés de Mémoires et qui étaient des articles complets sélectionnés et, grosso modo, arrangés par ordre chronologique et écrits par les membres de l'Académie. On considère généralement Mémoires comme le journal scientifique le plus important au $18^{\mathrm{e}}$ siècle. 


\subsection{3-Les premiers journaux d'autres pays}

Les premières sociétés savantes - et qui étaient tout à la fois des clubs sociaux, des instituts de recherche et des maisons d'édition - constituaient le facteur institutionnel majeur dans la naissance de l'article scientifique et ce à, et juste après, ses débuts. Ces organisations néophytes avaient en leur sein la plupart des auteurs, lecteurs et éditeurs de la science des $17^{\mathrm{e}}$ $18^{\mathrm{e}}$ siècle en Angleterre et sur le Continent .Elles créèrent aussi les réseaux sociaux voulus pour établir ce qui constituait la science certifiée et la prose scientifique acceptable.

L'élégant Saggi di naturali esperienzi (Essais d'expérimentations naturelles) écrit en Italien et publié en 1667 par l'éphémère Academia del Cimento, est le premier enregistrement important dévolu exclusivement aux activités d'une société savante. Ce chef d'œuvre des premiers spécimens de confection de livres décrit les instruments et les procédures et donne les résultats d'une collection éclectique d'expérimentations faites par les membres de la société entre 1657 et 1662.Le Saggi était la seule publication de cette société savante pionnière et devint le laboratoire manuel pour les expérimentations en physique durant les $17^{\mathrm{e}}$ et $18^{\mathrm{e}}$ siècles.

Les deux premiers périodiques des terres germaniques- Acta eruditorium en 1682 et Miscellanea curiosa medico-physica en 1670 - adoptèrent le latin pour atteindre l'audience internationale la plus large possible .Parmi d'autres inventions importantes, Acta eruditorium publia la plupart des articles dans lesquels Leibniz annonça et articula son invention du calcul. Le Miscellanea curiosa medico-physica, produit du Collegium Naturae Curiosorum, était vraiment un pot-pourri de curiosités .Les 160 observations du volume de la première année comprenait un remède pour une atrophie de l'œil, la narration d'un serpent pétrifié dans l'estomac d'un cerf, la relation d'une substance cornée qui poussait de la cage thoracique d'un homme, des remèdes pour la surdité et les maux de tête, une manière de " rendre les hommes nains " et des histoires de femmes et d'hommes prêts à manger d'étranges choses. Aux environs du milieu du $18^{\mathrm{e}}$ siècle, la plupart des villes importantes savaient leurs propres actes, commentaires, journal, comptes rendus, transactions, mélanges ou mémoires.

\subsection{4-Premier pas de la communication scientifique en Amérique}

Les sociétés savantes et leurs publications apparurent en Amérique avant même la Déclaration de l'Indépendance .La première société savante américaine fut The American Philosophical Society et qui fut lancée à Philadelphie par Benjamin Franklin en 1743 pour poursuivre " toutes les expérimentations philosophiques qui laissent la lumière dans la nature des choses ".Après avoir disparu au bout de moins de quatre ans à cause d'un manque d'intérêt, elle fut relancée d'une manière permanente en 1768. Le premier volume de ces Transactions en 1771 contenait un nombre d'articles qui relataient des observations et des mesures du transit de Venus à travers le Soleil en 1769 et a permis d'établir une réputation internationale non seulement pour The American Philosophical Society mais la science en Amérique en général. Thomas Jefferson servit en tant que troisième président de la société (1797-1815) et publia un article paléontologique sur un fossile paresseux dans le troisième volume de ses Transactions.

Les premiers périodiques et livres scientifiques américains sont remplis avec les découvertes de la nouvelle flore et faune trouvées à travers l'Amérique du Nord et ramenées à la vie dans des descriptions verbales et des gravures artistiques. L'œuvre de John James Audubon sur les oiseaux est l'exemple le plus connu .D'autres, autochtones et étrangers, contribueront aussi à 
l'énorme entreprise de documentation systématique des splendeurs des espèces variées habitant le Nouveau Monde.

Comme dans nombre des premiers journaux, les publications américaines combinèrent des observations scientifiques avec des fabuleux morceaux d'oui dire.Par exemple, dans le même volume où apparut l'article de Thomas Jefferson " Un mémoire sur la découverte de certains os d'un quadrupède du genre griffé dans les contrées ouest de la Virginie " apparut aussi " Une lettre de Mr. John Heckewelder à Benjamin Smith Baron, Docteur, contenant la relation d'un animal appelé le Grand Ours $\mathrm{Nu}$ ".

D'autres journaux qui parurent à l'époque incluent Memoirs of the American Academy of arts and sciences en 1785, Journal of the Academy of natural Sciences en 1817 et American Journal of Sciences and Arts en 1818.

\subsection{5-La mission de collecte des faits des débuts de la science}

La communauté scientifique du $17^{\mathrm{e}}-18^{\mathrm{e}}$ siècles avait une large base amateur, ces scientifiques enthousiastes n'avaient pas spécialement pour but de concocter des explications mathématiques ou mécaniques à leurs observations et résultats expérimentaux. Bon nombre d'articles étaient simplement des observations mal assimilées telles que l'observation de comètes ou d'éclipses, des descriptions d'organismes microscopiques et de faune et flore exotiques ainsi que la narration de curiosités médicales et d'autres " faits étranges ".Ne voulant pas théoriser ni spéculer au delà des faits observables, nombre des philosophes de cet âge se virent comme participant dans le grand rêve non réalisé de Francis Bacon d'un musée contenant un spécimen de chaque fait scientifique.

Une des manifestations concrètes du rêve de Bacon fut un journal rempli de faits intitulé Curtis' Botanical Magazine et qui parut en 1787. Chaque numéro de ce magazine contenait des séries de descriptions d'aquarelles de couleurs vives décrivant des plantes dont les détails sont reproduits d'une manière extraordinairement exacte. Jusqu'à 1948, le magazine produisait ces perles artistiques en produisant en masse une ligne de dessin par un artiste professionnel ou un auteur ensuite des groupes de femmes et d'enfants coloriaient à la main chaque illustration. Chaque illustration est accompagnée par une page ou deux de description verbale comprenant des détails techniques tels que l'habitat de la plante, la couleur, le parfum, la taille, l'arrangement de la fleur et la méthode de pollinisation.

Une autre manifestation du rêve de Bacon fut la monumentale Encyclopédie française du $18^{\mathrm{e}}$ siècle intitulée Encyclopédie : ou Dictionnaire raisonné des sciences, des arts et des métiers. La ressemblance du titre en entier aux noms de journaux de l'époque - tels que Observations et mémoires sur la physique, sur l'histoire naturelle et sur les arts - atteste de leur parenté. Comme dans Curtis' Botanical Magazine, les entrées dans l'encyclopédie appréhendent le sujet en une approche systématique en ce qui concerne le style, le format et le contenu ainsi que la représentation visuelle sur laquelle elle s'est beaucoup basé et qui, dans ce cas, est représentée par presque 3000 gravures à l'eau forte en taille douce. 


\section{3-Spécialisation et maturité accrue}

\subsection{1-Origine de la littérature spécialisée}

Au début était le journal scientifique général. Et le journal scientifique général engendra le journal spécialisé et le journal spécialisé engendra le journal à sujet unique soit selon la classe des composants, maladie spécifique ou méthodologie. Et le journal à sujet unique engendra le journal interdisciplinaire pour relier les spécialités à une date évolutionnaire plus récente. Et la communauté scientifique vit que les journaux étaient importants et ils furent productifs et multiplièrent

Durant les dernières décennies du $18^{\mathrm{e}}$ siècle, les scientifiques avec leurs sociétés et leurs publications devinrent plus spécialisés afin de répondre au flux du savoir technique particulièrement dans les domaines de la physique et de la chimie.

Un des premiers journaux scientifiques généraux destiné aux chercheurs sérieux fut " Observations et mémoires sur la physique, sur l'histoire naturelle et les arts " fondé en 1773 par François Rozier .Tel que l'a présenté Rozier d'une manière éloquente, pour ne pas dire brusque, dans la préface du premier volume : " Nous n'offrirons pas à des amateurs oisifs des travaux simplement agréables ou la fausse illusion de se croire initiés dans la science de laquelle ils ne savent rien..... Nous offrons cette collection à ceux qui vraiment savent ". Il affirma plus loin que le journal lui même " rejetterait tout ce qui n'est qu'une compilation non assimilée et qu'il ne voudrait accepter que ce qui est nouveau et avis pratiques ".A la fondation de la British Association for the Advancement of Science en 1831, William Whewell suggéra que l'adhésion soit restreinte à ceux " qui ont publiés des recherches écrites dans les mémoires de n'importe quelle société savante ". Il voulut exclure de l'adhésion tous ceux qui n'étaient pas, tel que l'a exprimé un critique de la Royal Society, " des laboureurs du vignoble " de la science. Ce désir général pour des critères professionnels élevés en science a mené à un influx d'articles individuels globalement destinés aux experts dans la spécialité. Ceci donna naissance aussi aux premiers grands journaux spécialisés dans les sciences naturelles et physiques en Allemagne, en France et en Angleterre.

\subsection{2-Les différents types d'articles scientifiques}

A travers les siècles, cinq types d'articles scientifiques ont émergés : théorique, expérimental, d'observation, méthodologique et compte rendu. Ces types d'articles sont distincts, chacun ayant un but différent, quoiqu'ils soient très proches les uns des autres.

2.3.2.1-Les articles théoriques se concentrent sur l'explication des événements naturels, en suggérant souvent des expérimentations ou des observations qui pourraient confirmer les explications. Ils offrent les nouvelles variations conceptuelles qui guident l'évolution continue de la science.

2.3.2.2-Les articles expérimentaux relatent les manipulations des objets naturels généralement dans des environnements artificiels tels que les laboratoires de recherche. Ils fournissent l'information empirique essentielle pour l'évolution conceptuelle continue de la science.

2.3.2.3-Les articles observationnels décrivent des objets naturels, généralement à l'extérieur du laboratoire. Ils incluent des activités telles que décrire un nouveau oiseau-mouche ou un 
fossile ou mesurer le spectre d'une nouvelle étoile .Ils ne comprennent pas, de prime abord, la manipulation d'objets naturels sous des conditions contrôlées. Les articles observationnels complètent l'expérimental; ils existent parce qu'une partie de la mission de la science comprendra toujours la description du monde naturel en dehors du laboratoire .En plus, tel que la cosmologie et la paléontologie le démontrent, certains des phénomènes qui intéressent la science seront toujours fermées à l'expérimentation directe.

2.3.2.4-Les articles méthodologiques ne font habituellement pas de nouvelles revendications au sujet du monde naturel mais présentent de nouvelles manières pour faciliter et créer des expérimentations et améliorer les observations. Ils concernent les outils utilisés pour créer la nouvelle science.

2.3.2.5-Les articles de compte rendu décrivent et évaluent la littérature récente dans une spécialité. Ils ne contiennent généralement pas de revendications majeures ou présentées dans des articles précédents. Alors que leurs buts est d'interpréter la science passée, non pas inventer la nouvelle science, ils servent une fonction indispensable - séparer la bonne graine de l'ivraie- comparativement aux quatre autres types .Ils constituent une seconde étape du contrôle par les pairs, une étape beaucoup plus sélective que la première.

\subsection{3-Invention du graphe moderne}

Il est difficile pour le lecteur de nos jours d'imaginer des articles scientifiques sans graphes. En fait le graphe moderne, n'a été inventé que vers la fin du $18^{\mathrm{e}}$ siècle, une centaine d'années après que le journal scientifique ait été inventé .Il était beaucoup utilisé en ce temps par le physicien allemand Johann Heinrich Lambert et l'économiste anglais William Playfair.

Dans cette forme visuelle, l'auteur trace des variables indépendantes telles que le temps sur l'abscisse (axe horizontal) et des variables dépendantes telles que la propriété physique ou chimique qui change avec le temps sur l'ordonnée (axe vertical). Cet arrangement convient idéalement pour communiquer d'un coup d'œil des points de données multiples, représentant visuellement cause et effet, découvrant des tendances dans une large masse de données et faisant des comparaisons entre des groupes de données différents. L'astronome J.F.W. l'a noté dans un article en 1833, " ce genre de graphe [vides avec des axes pré dessinés] sont tellement pratique dans une grande variété de desseins que chaque personne engagée dans ...des recherches physico-mathématique de n'importe quelle description trouvera son compte en ayant un stock de ces graphes toujours à portée de main." L'utilisation continuellement accrue des graphes durant le $19^{\mathrm{e}}$ et $20^{\mathrm{e}}$ siècles a grandement contribué à faire changer l'article scientifique du descriptif et qualitatif vers le mathématique et quantitatif. Le graphe doit être considéré maintenant comme la forme la plus importante pour la représentation visuelle scientifique, supplantant les diagrammes géométriques et les dessins réalistes des objets naturels et des équipements de recherche.

\subsection{4-Invention de la table périodique des éléments}

Similaires aux graphes durant le $19^{\mathrm{e}}$ et $20^{\mathrm{e}}$ siècles, les tables devinrent aussi un ingrédient de plus en plus utilisé de l'article scientifique comme moyen de faire face à la dépendance de plus en plus grande sur le quantitatif .Une des tables les plus remarquables et magnifiques de toutes les tables en science doit être la table périodique de Dimitri Mendeleïev et qui est considérée comme le colonne vertébrale de la chimie moderne. En trois articles publiés en 
1869 et le livre Les principes de chimie, Mendeleïev publia les premières versions de sa table qui organisèrent les 65 éléments de chimie connus en rangées et colonnes guidé par l'ordre croissant de leur poids atomique et propriétés chimiques .Selon Mendeleïev, " la définition de la masse atomique donna un moyen d'analyser et comprendre les transformations chimiques des substances et pour arriver à l'atome alors que la masse de l'atome était montré par la loi périodique influant toutes ses propriétés chimiques principales ". Plus important, dans un article en 1871, il prédit que les vides dans la table seraient remplis par la découverte de nouveaux éléments avec des propriétés prévisibles. La prédiction de Mendeleïev fut confirmée par la découverte du gallium en 1875, du scandium en 1879 et du germanium en 1885.En 1913, Henry Mosley déclara que le nombre de protons dans un élément donné (nombre atomique) était meilleur qu'une masse atomique comme base pour arranger la table périodique. Durant le $20^{\mathrm{e}}$ siècle, les scientifiques découvrirent un nombre de nouveaux éléments prédits par la loi périodique et inventèrent nombre de nouveaux arrangements de la table périodique, y compris des versions spirales et triangulaires ainsi que d'autres faites spécialement pour le World Wide Web .Depuis la moitié des années 90, les scientifiques ont accueilli dans le facteur périodique six autres éléments, les plus lourds étant les éléments 116 et 118 (nouveau en date du mois de Juin 1999).

\subsection{5-Les femmes et les minorités en science}

$\mathrm{Au} 17^{\mathrm{e}}$ siècle, The Royal Society Of London était ouverte à pratiquement tout gentille homme qui pouvait payer sa cotisation : non seulement les chercheurs à plein temps mais aussi tous ceux qui avait une curiosité moyenne au sujet des choses scientifiques et mathématiques, à l'image de John Dryden, un poète lauréat d'Angleterre. Une femme savante, Margaret Cavendish, Duchesse de Newcastle, a même assisté à un meeting en 1667. Malheureusement, la première femme " membre " ne fût élue qu'en 1945.

Alors que les femmes sont notoirement absentes des sociétés savantes et des pages des journaux scientifiques jusqu'au début du $20^{\mathrm{e}}$ siècle, quelques unes travaillèrent en tant qu'assistantes techniques (la plupart du temps sans être payées ni même reconnues), alors que d'autres jouèrent un rôle important dans la communication du savoir scientifique à travers des livres publiés à partir du $17^{\mathrm{e}}$ jusqu'au $19^{\mathrm{e}}$ siècle. A la fin du $18^{\mathrm{e}}$ siècle, Marie-Anne Lavoisier prépara les illustrations techniques pour les publications chimiques de son époux, l'aida dans son laboratoire, et traduisit un livre scientifique sur le phlogistique. A peu prés la même période, Caroline Herschel publia un Catalogue of Stars sous les auspices de The Royal Society Of London. En fait, Herschel observa huit nouvelles comètes dans sa longue vie, parmi lesquelles trois qu'elle consigna dans trois très brefs articles dans Philosophical Transactions.

On peut affirmer que Marie Curie est la première femme à publier des articles scientifiques d'importance majeur, et de ce fait, se frayant une place parmi les scientifiques les plus importants mondialement. En 1898, à l'âge de 30 ans, elle publia avec son époux deux articles importants, chacun annonçant la découverte d'un élément radioactif différent détecté dans le minerai de l'uranium appelé pitch blende. Le premier article fut au sujet du polonium, nommé après le pays d'origine de Curie (la Pologne) et le second, le radium, nommé d'après le nom latin du mot" rayon ".

Une autre femme physicienne d'importance est Lise Meitner .En 1938, Otto Hahn et Fritz Strassmann, travaillant au département de physique du Kaiser Wilhelm Institute fissionnerent 
l'atome de l'uranium. Peu de temps par la suite, Meitner et son neveu Otto Frisch fournirent une explication pourquoi lorsque Hahn et Strassmann bombardèrent l'uranium (nombre atomique de 92) avec des neutrons ils formèrent des isotopes de baryum (nombre atomique de 56) alors qu'un élément avec un nombre atomique plus prés de l'uranium était escompté.

Pour solutionner ce problème, Meitner et Frisch appliquèrent le modèle de la chute liquide du noyau proposé la première fois par Niels Bohr. Dans ce modèle, ils représentèrent l'uranium sous la forme d'une chute liquide devenant instable quand elle est touchée par un neutron, s'allonge ensuite en une forme d'un haltère et enfin se divise en deux petites gouttes. Ce processus conduit alors à la formation de deux noyaux plus petits (baryum et krypton), dégageant une formidable énergie (200 millions d'électrons volts) conforme avec la fameuse équation d'Einstein $\mathrm{E}=\mathrm{MC}^{2}$.Ils appelèrent ce processus " fission", empruntant un terme de biologie .

A la fin du $19^{\mathrm{e}}$ siècle, les portes des universités, des sociétés savantes et des laboratoires de recherche s'ouvrirent graduellement aux femmes. En conséquence, au $20^{\mathrm{e}}$ siècle, on trouve nombre de femmes auteurs faisant des contributions majeures à la littérature scientifique.

D'autres groupes furent aussi écartés de la pratique de la science, comme d'autres professions. Ernest Everett Juste, un des premiers proéminents biologistes africains - américains, quitta les Etats-Unis au début des années 30 pour travailler en Europe parce qu'il sentit qu'il n'était pas estimé à sa juste valeur et victime de discrimination en sa terre natale .Durant sa longue mais tumultueuse carrière, il publia aux environs de 70 articles et deux livres techniques.

\section{4-Communications choisies de quelques ingénieux scientifiques}

\subsection{1-Newton et l'optique}

Le $18^{\mathrm{e}}$ numéro de Philosophical Transactions comporte un article par un jeune et prometteur scientifique du nom d'Isaac Newton dont c'était la première publication scientifique et le premier important article scientifique de tous les temps. Henry Oldenburg, l'éditeur de Philiosophical Transactions, reconnut de toute évidence l'importance de cet article de 12 pages par le relativement obscur professeur de Cambridge University parce que c'est le seul article dans le $18^{\mathrm{e}}$ numéro et il fut imprimé seulement quelque deux semaines après sa réception et ce en date du 2 février 1672 sous sa forme manuscrite .Nous présentons ici l'introduction approfondie précédant l'article en question :

Une lettre de Mr. Isaac Newton, professeur de mathématiques à l'Université de Cambridge comprenant sa nouvelle théorie au sujet de la lumière et les couleurs : où la lumière est déclarée comme n'étant pas similaire ou homogène, mais consistant de rayons différents dont certains sont plus réfrangibles que d'autres et les couleurs sont déclarées comme n'étant pas des qualifications de la lumière dérivées de réfraction de corps naturels (comme il est généralement accepté )mais des propriétés générales et connotatives qui en divers rayons changent où des observantins et des expérimentations diverses sont supposées prouver la dite théorie.

L'affirmation principale de Newton était que la lumière blanche, loin d'être simple, comme on le croyait avant, était un composite de toutes les couleurs du spectre, un composite qui pouvait être décomposé en passant la lumière blanche à travers un prisme et recomposé par 
passage dans l'autre sens dans un second prisme. Cette affirmation avait deux composants de base : premièrement,les résultats expérimentaux de Newton avec les prismes et deuxièmement son éxplication mécanique de ces mêmes résultats - à savoir que la lumière semble être un corps matériel composé de particules et de rayons de lumière colorés différemment qui se réfractent selon les différents angles.

La réaction initiale à l'article de Newton par les membres de la Royal Society fut largement positive. Son article eut un impact sur la manière de penser au sujet de la lumière et de la couleur en Angleterre et sur le Continent et fut sérieusement considéré par deux des plus proéminentes autorités de la physique de la lumière, Robert Hooke et Christiaan Huygens. Cependant, tout ceci ne fut sans quelques anicroches .Un certain nombre de virtuoses remirent en question ses résultats expérimentaux et Hooke et Huygens offrirent des explications alternatives plausibles à l'affirmation de Newton que les rayons de lumière agissent comme des vibrations ou des vagues. Cette controverse marque la première différence d'opinion publique importante à l'intérieur de la communauté émergente de la science .La réponse finale de Newton à ses critiques vint sous la forme d'un livre, Opticks, publié la première fois en 1704.

\subsection{2-Darwin et la théorie de l'évolution}

La révolution darwinienne commence par l'histoire d'un jeune homme dont le père est à juste titre anxieux au sujet de l'avenir de son fils et qui cependant accepte avec une grande inquiétude, de lui permettre de prendre la mer avec le Beagle, un navire dont la mission en faisant le tour du globe était de dresser une carte des côtes de l'Amérique du Sud et de recueillir des spécimens de flore et de faunes. Pour Darwin, le voyage qui dura cinq ans (1831-1836) fut un voyage de découverte intellectuelle. Mais c'était un voyage seulement débuté sur le Beagle, et ce n'est qu'un quart de siècle plus tard que Darwin publia sur l'évolution et même alors ses motivations pour publier n'était pas son sens accru de la certitude mais l'envoi d'un article par Alfred Russell Wallace qui avait discerné dans un éclair de perspicacité le dogme central de l'évolution - que sa force motrice était la sélection naturelle opérant sur une variété d'espèces dans un environnement aux ressources limitées.

La révolution darwinienne ne fut couronnée de succès que rétrospectivement seulement. Même avec l'aide d'alliés, à l'instar du plus fameux, Thomas Henry Huxley, la théorie de Darwin rencontra une opposition considérable, non seulement sur des bases religieuses mais aussi scientifiques. Par exemple, la théorie de Darwin présupposa l'héritabilité de meilleurs traits adaptés mais sa théorie originale n'a pas adressé d'une manière satisfaisante son problème crucial. Ironiquement, ce n'est que six ans après que les articles de Darwin et Wallace et le livre De l'Origine des espèces par la sélection naturelle ou la préservation des races favorisées dans la lutte pour la vie parurent qu'une solution aux problèmes de Darwin vit le jour .Le premier article de Gregor Mendel sur l'héritabilité dans le pois commun fut publié en 1866. Mais il apparut dans un obscur périodique et fut écrit par un scientifique également obscur .Il n'eut pas d'influence immédiate et dut être " redécouvert " au début du siècle suivant.

\subsection{3-Einstein et la théorie de la relativité}

L'année 1905 doit être considérée comme annus mirabilis (année mémorable) dans la longue histoire de l'article scientifique .Parce que c'est l'année où Albert Einstein - un expert 
technicien au bureau des brevets helvétique travaillant de manière indépendante sur les problèmes des physiques théoriques - publia quatre articles révolutionnaires dans Annalen Der Physik. Ils comprenaient non seulement les deux premiers articles sur la théorie de la relativité mais aussi un article présentant une explication mathématique du mouvement Brownien et un autre proposant une explication mécanique de l'effet photoélectrique.

$\mathrm{Au}$ cours de la décennie suivante, Einstein continua à développer son " principe de la relativité " dans une série remarquable d'articles changeant pour toujours nos notions au sujet du temps, de l'espace, du mouvement, de la gravité, la lumière, la masse et l'énergie. L'article de 1905 intitulé " Est-ce que l'inertie d'un corps dépend de son contenu d'énergie ?" est typique des articles d'Einstein au sujet de la relativité : une déduction euclidienne à partir de certains postulats populaires mène à une prédiction renversante - l'équivalence de la masse et de l'énergie, $\mathrm{E}=\mathrm{MC} 2$. Cette révélation est une expression mathématique en direct accord avec la philosophie de la science d'Einstein. Selon cette philosophie, la physique est la recherche des lois qui décrivent la relation parmi des entités fondamentales qui déterminent complètement les lois physiques. " Je crois toujours dans la possibilité d'un modèle de la réalité ", écrit-il en 1954, " ce qui veut dire une théorie qui représente les choses ellesmêmes".

\subsection{4-Fermi et la recherche nucléaire}

C'est le matin du 2 Décembre 1942 et la première pile atomique est sur le point d'entrer dans sa phase critique. C'est en ces termes que Fermi décrivit les événements de cette journée :... " les indications étaient que les dimensions critiques avaient été légèrement dépassées et que le système n'a pas réagit en chaîne seulement à cause de l'absorption [neutronique] des bandes de cadmium .Au cours de la matinée, toutes les bandes de cadmium, à l'exception d'une, furent prudemment retirées, ensuite cette dernière bande fut graduellement extraite, tout en gardant un œil sur l'intensité .A partir des mesures, il est attendu à ce que le système devienne critique en enlevant une longueur d'à peu prés huit pieds de la dernière bande.En réalité, quand quelque sept pieds furent retirés, l'intensité augmenta à un très haut niveau mais se stabilisa après quelques minutes à un niveau limité .C'est avec une certaine trépidation que l'ordre fut donné de retirer un pied et demi additionnel de la bande. Cette opération nous ramènera au sommet .Quand le pied et demi fut retiré, l'intensité commença à monter doucement, mais à un rythme croissant et continua à augmenter jusqu'à ce qu'il devienne évident qu'elle allait actuellement diverger .C'est alors que les bandes de cadmium furent de nouveau insérées dans la structure et l'intensité baissa à un niveau insignifiant".

Juste après, Arthur Holly Compton, le chef du projet à Chicago, téléphona à James Bryant Conant à Washington. Etant donné que l'appel n'était pas sécurisé la conversation fut codée. " Jim, dit Compton, tu seras intéressé de savoir que le navigateur italien a juste atterri dans le nouveau monde ". Conant posa la question : "est ce que les autochtones étaient amicaux ?" La réponse de Compton fut " tout le monde a atterri en bonne santé et est content ".A travers son illustre carrière, Fermi publia beaucoup sur ses recherches nucléaires, gagnant le Prix Nobel en 1938 pour sa découverte de " nouveaux éléments radioactifs produits par radiation neutronique, et....les réactions nucléaires causées par les neutrons lents ". De toute évidence, ces publications dans des journaux s'arrêta abruptement en 1941 et résuma après la guerre. 


\section{5-Curiosités diverses}

\subsection{1-Echec et science}

L'histoire des sciences est autant une histoire de faux tournants, de lignes de questionnements avortées, d'échec d'épanouissement et tout simplement plus d'échec que de succès. Même les grands scientifiques se trompent de temps en temps comme le commun des mortels .En fait, à moins d'être sélectif au point de non représentativité , on doit admettre qu'une grande partie de la science passée est une suite d'impasses et des fautes bien intentionnées éventuellement abandonnées .Ces échecs peuvent prendre différentes formes. Cela peut être des découvertes qui s'avèrent après un court laps de temps erronées comme c'est le cas les rayons $\mathrm{N}$, la fusion à froid et l'eau polymerisée.Ils peuvent être aussi des concepts avec une histoire longue et réussie qui finalement s'avèrent invalide ou d'une certaine manière théoriquement inadéquats tels que le phlogistique, les particules légers et l'éther universel. Ils peuvent aussi être des projets de recherche ambitieux qui apparaissent avec le recul du temps futiles parce que les connaissances courantes et les techniques de recherche d'alors étaient trop primitives pour permettre une percée majeure tels que dans les tentatives avortées d'Einstein à construire une théorie de champs unifiée. Certains projets de recherche n'arrivent pas survivre à cause de la perte du financement comme c'était le cas pour la construction d'un énorme collisionneur à haute conductivité au Texas. D'un autre coté, les expérimentations, les observations ou les théories peuvent être défectueuses à une grande ou petite échelle : se tromper est humain et fait partie du processus de découverte.

Les erreurs mineures, une fois détectées, peuvent être annoncées dans la section " Erratum " d'un journal scientifique .Ces entrées sont généralement courtes et directes. Un exemple montre un auteur dénigrer impitoyablement son propre travail publié dans un entrefilet dans le Journal of Chemical Physics (vol 47, 1967) intitulé " Erratum : échange de charge entre les molécules diatomiques mononucléaires ". R.G. Beene, Jr. résume son avis sur un article qu'il avait publié dans le Journal l'année précédente : " Le matériel que contenait cet article est dénué des sens et devrait être rejeté in toto (en entier). Comme il sera montré ailleurs, un des défauts les plus sérieux est la transformation inadéquate qu'a donnée Eq (9)".On doit insister sur le fait que l'imprimeur produisit le manuscrit exactement comme l'auteur l'a concocté.

\subsection{2-Débat et controverse}

Pratiquer la science implique soutenir que quelque chose est vrai dans le monde naturel et d'être prêt à défendre cette affirmation devant une communauté de pairs. Plus simplement, pratiquer la science veut dire débattre et discuter. Et nulle part n'est la nature argumentative de la science plus apparente que dans les échanges d'une controverse. C'est durant ce genre d'épisode que les débats pour ou contre une nouvelle revendication du savoir sont le plus sévèrement testés. On peut aussi trouver que les élans émotifs ne cadrent pas avec l'image du scientifique comme le chercheur dépassionné de la vérité.

N'importe quelle courte liste des controverses majeures du $20^{\mathrm{e}}$ siècle devrait inclure la théorie des dérives des continents - qu'à un certain moment la surface de la terre était un supercontinent et qu'elle se désintégra il y a de cela quelque 225 millions d'années. Cette courageuse nouvelle théorie fit sa réelle apparition dans deux articles du météorologiste allemand Alfred Wegener. Pendant des décennies, la théorie de Wegener demeura " un beau rêve " parce qu'elle manquait d'un quelconque mécanisme plausible capable de propulser de 
telles large masses de terre sur des milliers de miles. Elle fut rejetée à l'intérieur de la communauté scientifique comme " théorie allemande ", " hérétique " et " bizarre ". Tout ceci changea dans les années 60 avec la théorie de l'écartement de la surface de la mer et des plaques tectoniques qui offrirent un mécanisme plausible pour la dérive des continents .Au même moment, un petit groupe d'opposants à la théorie emmené par un géophysicien soviétique renommé , V. V Beloussov, et une duo père-fils d'Oklahoma, les Meyerhoffs, combattirent avec ténacité cette vague montante de la théorie de la dérive et proposèrent leur propre variante mais sans résultats à la fin.

\subsection{3-Curiosités littéraires : pour le meilleur et pour le pire ?}

Le poète et le romancier divertissent leurs audiences en utilisant une grande variété de stratagèmes littéraires telles que la métaphore et la comparaison, l'onomatopée, l'ironie et la satire et les jeux de mots. De son coté, le scientifique est supposé, autant que peut se faire, transmettre l'information avec précision et sans ambiguïté à ses lecteurs et non pas les éblouir avec la virtuosité littéraire. Tout un chacun lisant la littérature scientifique n'est que trop conscient que le style tend à être très terne et sans surprise. Malgré ces contraintes sur la créativité individuelle, des scientifiques se sont arrangés pour glisser dans leur prose des occasionnelles prestidigitations littéraires. L'instance la plus fameuse est le fait de deux des plus grands chimistes allemands du $18^{\mathrm{e}}$ siècle, Justus Liebig et Friedrich Wöhler qui publièrent en 1839 un article anonyme dans Annalen der pharmacie et qui relatait des expérimentations fictives dont le but était de ridiculiser des observations microscopiques récentes qui indiquaient que la levure est un organisme vivant capable de convertir le sucre en alcool. Une phrase de cet article donne une idée de cette étrange parodie : " pour résumer, le sucre est aspiré dans l'estomac de ce petit animal, un courant de fluides alcooliques est coulé de manière continue de l'anus et de courts jaillissements de carbone dioxyde giclent en un immense organe génital ". D'autres scientifiques ont construit des jeux de mots et des métaphores élaborés, importé des citations littéraires et des peintures célèbres dans leurs textes, caché des images étranges dans des illustrations techniques, écrit des articles en vers, et même fourni des notes musicales pour chanter avec. Ce dont il s'agit dans ce genre d'articles n'est en aucun cas la science de tous les jours mais la science en vacances.

Dans le cas de Lord Kelvin, c'est l'expérience d'un scientifique en vacances. Un des sujets qui intéressait Lord Kelvin était les propriétés physiques de la lumière et sa perception par les humains .Un jour au mois d'août 1899, sûrement en vacances dans les Alpes, il se réveilla avant l'aube pour faire certaines observations sur la première lueur de l'aube. Heureusement pour nous, il consigna ces observations dans un article du magazine Nature qui est une combinaison de prose de poème et de rapport scientifique :

\section{Hôtel du Mont-Revard, 27 Aout 1899}

" En regardant à 4 heures ce matin à partir d'un balcon de cet hôtel, 1545 mètres au dessus du niveau de la mer et aux environs de 68 kilomètres ouest, $18^{\circ}$ sud à partir du Mont Blanc, j'avais cette vue magnifique des chaines alpines de Suisse, Savoie et Dauphiné, parfaitement claire et nette dans le ciel matinal en demi jour. Ceci me donna une opportunité que j'attendais depuis cinq - six ans : voir les toutes premières lueurs instantanées du lever de soleil à travers un air très clair et découvrir est ce qu'elles étaient perceptiblement bleues .J'ai décidé de ce fait de regarder une heure jusqu'au lever du soleil et je fus amplement récompensé par toutes les splendeurs que 
je vis. N'ayant qu'une vague idée de l'orientation de l'hôtel, je ne pouvais pas au début juger d'où le soleil allait se lever, mais au bout d'une demi heure des teintes roses de chaque coté de la place où le demi jour était le plus visible, m'indiqua qu'il serait visible du balcon. Je fus amené à faire cette conclusion par les brosses de Haidinger quand l'illumination de l'air à des hautes altitudes par une brillante demilune presque au dessus des têtes était écrasée par la lumière du soleil ascendante au delà des montagnes. Un peu plus tard, des rayons de la lumière du soleil et les ombres des montagnes distantes convergèrent clairement à un point profond sous le sommet du Mont Blanc lui-même. Au cours des cinq à dix minutes suivantes, je pus voir le point de convergence voyager obliquement d'une manière ascendante jusqu'à ce qu'en un instant, je vis une lumière bleue contre le ciel sur le profil sud du Mont Blanc qui, en moins d'un vingtième de seconde, devint d'un blanc éblouissant comme un brillant arc de lumière électrique .Je n'avais pas de lunette de soleil sous la main et de ce fait je ne pus plus regarder le soleil levant".

On peut se demander que veut dire le terme " les brosses de Haidinger ". Selon une conférence sur la théorie des optiques que Kelvin donna à l'Académie de Musique de Philadelphie en 1884: " l'auteur de la découverte est bien connu à Philadelphie en tant minéralogiste et le phénomène dont je parle porte son nom. Regardez le ciel dans une direction de quatre vingt dix degrés à partir du soleil, et vous verrez une croix bleu et jaune avec le jaune en direction du soleil et à partir du soleil, étendu comme deux queues de renards avec le bleu au milieu et ensuite deux brosses rouges dans l'espace à des angles droit par rapport au bleu .Si vous ne le voyez pas, c'est parce que vos yeux ne sont pas assez sensibles mais un léger entraînement leur donnera la sensitivité voulue. Ces dessins ressemblant à des brosses apparaissent aussi quand on regarde une surface brillante à travers un polariseur ".

\section{6-Conclusion et que nous réserve le futur}

\subsection{1-Style d'écriture}

Les premiers articles scientifiques sont typiquement des courts rapports (rarement plus de dix pages, des fois un seul paragraphe) décrivant la rencontre d'un individu avec la nature à travers l'observation et l'expérimentation .En Angleterre en particulier, ils ont tendance à être une suite de faits baconien intégrés en une narration connectée d'une manière ou une autre, et pas tout le temps, à une théorie explicative. En général, ils essayent d'établir la crédibilité par l'intermédiaire de témoignages fiables plutôt que des détails techniques soigneux et par expérience qualitative plutôt que des expérimentations quantitatives et l'observation pour supporter la théorie. Les premiers articles s'appuyaient aussi largement sur le langage de tous les jours et sont écrits généralement en un style conforme aux normes compositionnelles de la prose expositoire et non pas des normes spécifiques à la science .Pour la plupart, l'audience ciblée est une communauté disparate d'amateurs et de professionnels unie par la curiosité de savoir comment la nature fonctionne. En contraste, l'article scientifique moderne a évolué vers un langage et un style de prose spécialisés adaptés à une communication efficace à d'autres professionnels engagés dans une recherche similaire. Le style impersonnel de ces articles est conçu pour concentrer l'esprit du lecteur sur les faits du laboratoire et le monde naturel, plutôt que d'attirer son attention au texte lui-même ou l'auteur. 


\subsection{2-Caractéristiques de présentation}

Les caractéristiques de présentation de l'article scientifique ont évolué de deux manières primaires. En premier lieu, le développement d'un arrangement et une organisation plus uniforme du contenu de l'article en général. Cet arrangement représente un tribut à l'efficacité de la méthode scientifique comme moyen d'explorer la nature .Pour ce faire, l'article scientifique moderne est typiquement divisé en :

- une introduction qui place les lecteurs dans le contexte scientifique dans lequel ses auteurs travaillent

- une section méthode et matériaux qui esquisse les procédures utilisées.

- une section sur les résultats qui présente les données générées et le contexte intellectuel de leurs acquisitions.

- une section discussion qui interprète les données et adresse les recherches futures qui prolongeraient les résultats présents.

- Une conclusion qui réitère l'argument central dans un ou deux paragraphes et termine l'argumentation centrale.

L'article scientifique a aussi développé un système d'organisation des résultats et d'organisation .Ce système compartimente les traits essentiels dans les articles par le biais de l'utilisation des sommaires et des résumés, des rubriques et des sous rubriques ,des tables et des figures intégrés dans le texte et des citations qui fournissent le contexte pour des affirmations à n'importe quel point du texte et ainsi de suite. Ce système permet aux scientifiques de lire les articles d'une manière opportuniste plutôt que séquentielle, scannant les différentes sections à la recherche de parties utiles de méthode, théorie et fait.

La dernière moitié du $20^{\mathrm{e}}$ siècle a vu une grande floraison de manuels et de guides de styles scientifiques qui ont codifiés aussi bien les caractéristiques de présentation que de style dans l'article scientifique et ont contribué à sa conformité grandissante au-delà des frontières nationales et de disciplines.

\subsection{3-Présentations visuelles}

A l'origine de l'article scientifique en 1665, différents types de représentations visuelles avaient déjà atteint le stade de la maturité : les tables de données étaient depuis un certain moment devenues des produits de première nécessité de la littérature astronomique, les dessins tridimensionnels des traits anatomiques avaient atteints un haut degré de détails techniques et artistiques tels que les graphiques des travaux de Vesalius et Leonardo Da Vinci le montrent, l'établissement des cartes de la terre et du ciel était une entreprise de longue haleine et les diagrammes géométriques ont étés utilisés depuis Euclide. Bien plus, les illustrations de la flore et de la faune rivalisaient avec n'importe quelle production des artistes graphiques actuels. 
Cependant, les illustrations et les tables sont relativement rares au $19^{\mathrm{e}}$ siècle, en partie à cause des dépenses inhérentes à la reproduction. Il n'était pas rare de trouver, par exemple, toutes les illustrations annuelles d'un journal, isolées dans une petite section à la fin du volume. Le graphe, inventé à la fin du $19^{\mathrm{e}}$ siècle, montra le pouvoir des visualisations pour transmettre des masses de données d'un coup d'œil et en découvrir les tendances. Aux $19^{\mathrm{e}}$ et $20^{\mathrm{e}}$ siècles, les scientifiques ont inventé nombre de nouvelles formes de visualisations scientifiques, dont certaines demandent des capacités de décodage hautement spécialisées de la part de l'audience ciblée. A cause de leur utilité dans la création et la communication de la science, et combinées avec le progrès dans la reproduction photographique et la technologie informatique, les représentations visuelles jouent maintenant un rôle central dans l'article scientifique.

\subsection{4-Les langues de la science}

L'Anglais, le Français et le Latin étaient les langages dominants de l'article scientifique aux $17^{\mathrm{e}}$ et $18^{\mathrm{e}}$ siècles. Malgré les importantes contributions par les scientifiques à travers l'Europe, il est établi que les travaux par des chercheurs de pays tels que l'Italie, l'Espagne, le Danemark, la Russie et la Suède, quoique souvent publiés dans leurs langues indigènes respectives, n'en étaient pas moins communiqués à l'Europe et au monde, dans l'une des trois langues majeures de la science. De plus, les deux centres de l'activité scientifique durant le début de cette période, préférèrent clairement la publication dans la langue indigène au lieu du Latin reflétant un tournant décisif d'une érudition scholastique insensible et froide vers une littérature fait d'un mélange scientifique / technologique basée sur l'expérience de l'artisan, le pratiquant, le voyageur. Avec l'émergence des états allemands comme centre au 18 siècle, l'Allemand devint une langue majeure de la science à côté de l'Anglais et le Français.

$\mathrm{Au} 20^{\mathrm{e}}$ siècle, la science étant devenue un phénomène global, les articles scientifiques en Chinois, Japonais et Russe sont aussi devenus proéminents .Cependant, il n'y a pas eu un seul moment dans la longue histoire de l'article scientifique où une langue a dominé à ce point ce genre de publication. Les périodiques les plus importants de la science, nonobstant leurs nationalités, publient maintenant en Anglais. Même l'Académie française des Sciences dans ses comptes rendus a commencé à publier des résumés et des articles complets en anglais .Ce n'est pas par hasard que lorsque The National Research Council of Japan lança un nombre de nouveaux périodiques spécialisés dans les années 20, il choisit l'Anglais pour atteindre l'audience internationale la plus large possible.

\subsection{5-Que nous réserve le futur?}

" De nos jours, au $20^{\mathrm{e}}$ siècle, nous sommes impatient pour appliquer la technologie électronique à la communication scientifique .Il est certain que les éditeurs et les scientifiques qui ont débuté tout ce processus il y a de cela un peu plus de 300 ans, seraient stupéfaits de l'étendue de l'aventure scientifique mondiale, mais je doute qu'ils aient une quelconque difficulté à reconnaître les caractéristiques de base similaires du système ils lancèrent " (73)

L'article scientifique est au milieu d'une transformation radicale stimulée par des avancées dans la technologie informatique et en particulier le traitement de texte et logiciels graphiques, le courrier électronique et le World Wide Web. Cette technologie est entrain de

\footnotetext{
${ }^{(73)}$ Garfield Eugene, Has scientific communication changed in 300 years ? , Current contents, $n^{\circ} 8$, p. 11, 25 Février 1980
} 
changer la manière par laquelle le manuscrit scientifique est préparé par l'auteur, passe à travers le contrôle par les pairs, produit en forme finale, distribué aux lecteurs intéressés et lu attentivement par ces mêmes lecteurs .Aux fronts de la recherche de domaines à l'évolution rapide comme la physique des particules, ce changement est déjà arrivé : les articles publiés dans les périodiques en papier sont déjà dépassés. Au siècle prochain, ce que Stevan Harnad a appelé " The Post Gutenberg era " peut très bien voir l'extinction de la recherche originale apparaissant sous forme papier. Les effets à long terme de la préparation et de la publication électronique des manuscrits pourraient être aussi profonds que lorsque l'article scientifique a évolué de l'écriture de la lettre savante et des livres au $17^{\mathrm{e}}$ siècle.

Prenons par exemple, les résultats initiaux de L'International Human Genome Sequencing Consortium, mis en ligne au mois de Février 2001. Pour la publication de ce classique du moment, le magazine Nature suspendit sa manière classique de ne permettre qu'aux abonnés l'accès à son contenu électronique et le rendit accessible au public. Il est très probable que cet article soit le plus long qu'ait jamais publié Nature (62 pages) où les articles ne dépassent que rarement 4 à 5 pages. Son décodage des trois milliards deux cent millions (3 200000000 ) lettres de l'ADN du gène humain est le travail de vingt laboratoires et plus de deux cent cinquante scientifiques et experts informatiques mondiaux .La découverte la plus surprenante du Consortium (quoique très approximative) fut que le gène humain a 30000 - 40000 gènes encodés - protéine - bien loin des 100000 estimés avant En fait, ce nombre n'est pas du tout aussi élevé que celui de formes de vie moins complexes telles que les mouches des fruits (13000), les vers microscopiques ronds (19000) et les graines de moutardes (26000).

Ce long article se termine par une répétition de la première phrase de l'avant dernier paragraphe du fameux article au sujet de l'ADN de Watson et Crick, paru dans le numéro 1953 de Nature : " Finalement, il n'a pas échappé à notre attention que plus on apprend au sujet du gène humain, plus il y a à explorer ".Cette phrase est suivie, bien à propos, par une citation du poème de T.S. Eliot " Les Quatre quatuors " :

Nous ne devons pas nous arrêter d'explorer

Et la fin de toutes nos explorations

Sera d'arriver là où on a débuté

Et connaître la place pour la première fois

\section{La publication scientifique - Analyses et perspectives ${ }^{(74)}$}

La troisième classification que nous aborderons est beaucoup plus récente et plus complète. Elle date d'Octobre 2008 et est un travail collectif ayant abordé la publication scientifique sous différents angles. Composé de 16 chapitres, elle aborde l'histoire de l'édition scientifique au début de ce travail collectif et lui réserve un chapitre de 26 pages où sous le titre de " Une brève histoire de l'édition scientifique " (75) (et non pas publication scientifique et ce glissement sémantique aura, comme on le verra, une influence sur le contenu et l'orientation des informations contenues dans cette partie surtout comparé aux deux précédentes classifications ) est départagée en huit périodes de durée inégales et qui sont :

\footnotetext{
(74) Schöpfel Joachim, Ed., La Publication scientifique : analyses et perspectives, Paris : Lavoisier, 2008, 368 p.

${ }^{(75)}$ Fredriksen, Einar H., Une brève histoire de l'édition scientifique, In Op.cit., p.23-49
} 
1- Avant 1700

2- $1700-1850$

3- $1850-1925$

4- 1925-1945

5- Seconde guerre mondiale-1965

6- 1965-1985

7- $1985-2000$

8- 2000-2010

Du propre aveu de l'auteur lui-même, cette classification [division] est " ......subjective et reflète des innovations technologiques dans l'industrie graphique ou dans les TIC, une guerre importante ou une politique publique - ou souvent lié - au développement de la recherche scientifique ${ }^{(76)}$. On remarquera que les périodes tendent à se rétrécir plus on avance dans le temps et ceci est du ......" à l'augmentation du nombre de chercheurs, de leurs publications et de l'investissement global - public et privé - dans la recherche ${ }^{(77)}$ ". De notre côté, nous pensons que ces périodes de temps de plus en plus courtes sont dues entre autres, à une accélération des bouleversements technologiques qui tendent à arriver de plus en plus rapidement. En effet, pour ne prendre que l'exemple par excellence et qui est l'ordinateur, qui aurait cru que cet outil si banal et surtout si abordable de nos jours aurait les succès qui est le sien aujourd'hui ? Il ne faut pas oublier que les premiers mainframes des années 60 étaient d'immenses mastodontes qui utilisaient quantité d'énergie et d'espace pour des résultats qui nous paraîtraient ridicules de nos jours. De même qu'Internet, qu'on ne pourrait dissocier de l'ordinateur, n'était connue au début des années 90 que dans une élite universitaire américaine. Quelque vingt ans plus tard, il est devenu un phénomène de société dont personne ne peut prévoir les conséquences et surtout les fonctionnalités qu'il recèlera. Alvin Toffler dans son fameux " The Third Wave " ${ }^{(78)}$ (la troisième vague) * avait prédit cette accélération du changement en disant que ....." le changement est non linéaire, il peut être vers l'arrière, vers l'avant et sur les cotés " ${ }^{(79)}$. Nous allons examiner cette division et voir quelles ont été les périodes, les faits et les personnages importants l'ayant jalonné.

\section{1-Avant 1700}

Le choix de cette date est sûrement dû au fait qu'en 1700, et bien que le périodique scientifique avait déjà trente cinq ans d'âge, il n'en était pas moins qu'au de but de sa vie et

(76) Op.cit., p.24

(77) Ibid

(78) Toffler Alvin, The Third wave, Londres, Morrow, 1980, 544p.

* La théorie des trois vagues d'Alvin Toffler divise l'évolution de la civilisation en trois vagues et qui sont :

- la première vague est la société post agraire et remplace celle des chasseurs qui se réunissaient pour affronter les dangers de la nature.

- la deuxième vague est la vague de la société durant la Révolution industrielle (fin du $17^{\mathrm{e}}$ au milieu du $20^{\mathrm{e}}$ siècles) et qui se distingue par la massification de la société (production de masse, consommation de masse, distribution de masse, etc....). La combinaison de ces massifications combinées à la normalisation, la centralisation, la concentration et la synchronization conduit à un style d'organisation appelé "bureaucracie."

- la troisieme vague est la société post industrielle. Selon Toffler depuis la fin des années 50, la plupart des pays ont amorcés le passage de la seconde vague vers la troisieme vague.Il inventa nombre de mots pour la decrire (tels que société super industrielle). Il a aussi emprunté à d'autres personnes des expressions (tels que le village global, revolution scientifico - technologique, etc...) qui ont conduit, à des degrés differents, à la demassification, la diversité,la production basé sur le savoir et l'accélération du changement. http://en.wikipedia.org/wiki/Alvin_Toffler (consultée le 14/06/2009) .

${ }^{(79)}$ http://en.wikipedia.org/wiki/Alvin_Toffler (consultée le 14/06/2009) 
que son influence ne s'était pas encore fait ressentir à l'image de ce que les siècles suivants allaient observer. L'invention de l'imprimerie vers 1440 et l'existence du papier bien avant cette date (on situe le premier moulin à papier à Jativa en Espagne en 1056 et qui fut introduit par l'intermédiaire des Arabes) peuvent être considérés comme la base de la production de masse des livres et de l'édition. Il a été estimé que quelques vingt millions d'incunables ont étés produits en Europe ce qui est un chiffre étonnant pour une population estimée à cent millions d'habitants.

Il ne semble pas qu'il y ait eu d'éditeur dans le sens moderne du terme avant le $16^{\mathrm{e}}$ siècle. On observe plutôt, avec l'avènement de l'imprimerie, à un transfert des fonctions de scribes vers la reliure et l'impression, ce qui peut être considéré comme une première automatisation et surtout une accélération des moyens de production. Les libraires scientifiques qui travaillaient de concert avec les universités peuvent être considérés comme les premiers éditeurs car ils vendaient livres anciens et nouveaux.

Nombre d'éditeurs et d'imprimeurs donnèrent à la ville d'Anvers au milieu du $16^{\mathrm{e}}$ siècle le titre de ville commerciale la plus importante des Pays Bas et aussi de capitale du monde de la finance. A cet effet, plus de la moitié des livres produits aux Pays Bas entre 1500 et 1540 l'étaient dans cette ville. Si les universités, les instituts avaient crées des maisons d'édition, il n'en demeure pas moins qu'à cette époque c'était les imprimeurs et libraires (ayant hérité des manuscrits que les scribes avaient abandonnés après l'avènement de l'imprimerie) qui étaient ce qui pourrait être considérés les premiers éditeurs .Pour ce faire, ils parcouraient l'Europe et acquéraient les nouvelles publications qu'ils redistribuaient dans de nouveaux territoires. C'est à cette époque que les foires du livre (dont la plus importante, celle de Francfort, existe toujours et est considérée comme la plus importante et la plus grande au monde) ont vu le jour et elles représentent de nos jours un jalon important dans la communication et distribution du savoir.

C'est à cette époque en 1665 que naquirent, presque simultanément, à quelque mois de distance, les deux premiers périodiques scientifiques .Ces deux périodiques dont la simultanéité de parution est étonnante surtout dans une Europe où la vitesse de communication ne pouvait pas être grande, n'en représentent pas moins le prototype et l'ancêtre de notre périodique scientifique moderne. Deux personnalités ont brillé à l'époque et ont posé les jalons de la communication scientifique telle qu'on la connaît de nos jours $\mathrm{Ce}$ sont :

\subsection{1- Le Père Marin Mersenne}

Il transforma sa cellule de monastère en un véritable centre de savoir européen. Il collecta et commenta les idées reçues d'endroits divers et variés sous forme de correspondance ou de conversations. Au $16^{\mathrm{e}}$ siècle, la poste étant devenu plus fiable, les correspondances devinrent une partie importante de la propagation des idées et complémentèrent les voyages et réunions entre chercheurs et dont la faisabilité étaient, le moins que l'on puisse dire, difficile. Le Père Mersenne voyagea beaucoup en Europe et agit comme registre des découvertes de l'époque. Il agit aussi en se faisant l'intermédiaire dans des disputes sur la primauté de certaines découvertes que certains savants revendiquèrent à l'instar de Descartes et Isaac Beeckman en se rendant aux Pays Bas pour rencontrer les deux protagonistes. Il a été comparé par Fredriksen à un blog master de l'enseignement scientifique européen qui assurait l'évaluation (ancêtre du contrôle par les pairs) et de la traduction. 


\subsection{2- Henry Oldenburg}

Il fut nommé premier secrétaire de la Royal Society quand elle fut fondée en 1662, et ceci trois ans seulement avant le lancement de Philosophical Transactions qui fut une revue primaire contrairement au travail de Mersenne qu'on peut qualifier de travail de synthèse car il récoltait les informations et les analysait. La fin du $17^{\mathrm{e}}$ siècle vit l'émergence de quelques journaux en Italie, Allemagne et Danemark. Mais force est de reconnaître que la durée de vie de ces périodiques du début de l'ère du périodique scientifique fut la plupart du temps éphémère et certains ne dépassèrent pas la première année après un ou deux numéros.

C'est à cette époque que l'émergence de bibliothèques collectionnant les revues eut lieu. Elle était liée à la création d'académies et de sociétés savantes. Les bibliothèques universitaires traditionnellement collectaient les références en relation avec les cours dispensés alors que les sociétés savantes attiraient des chercheurs non universitaires et étaient souvent liées à des applications commerciales. Vu les nouvelles donnes concernant l'accroissement de l'intérêt porté envers la science et la technologie, les académies reçurent de nouveaux investissements autrefois réservés aux universités. Cette nouvelle situation est la résultante de la localisation des académies et sociétés savantes dans les grandes villes et de la facilité de communication routière précédemment signalée.

\section{$3.2-1700-1850$}

Cette période verra, avec le lancement des premières revues au milieu de la deuxième moitié du $17^{\mathrm{e}}$ siècle, la croissance continue du commerce des livres mais aussi la montée en puissance et l'émergence des revues. Au lieu de les concurrencer, ces dernières en sont le complément et cette partie va analyser cette cohabitation.

C'est au $18^{\mathrm{e}}$ siècle que la croissance des revues se manifesta. Si au début, les revues scientifiques se caractérisaient par un nombre pratiquement insignifiant et un contenu qu'on pourrait décrire de généraliste, le $18^{\mathrm{e}}$ siècle vit au contraire un accroissement en nombre et aussi une sorte de spécialisation dans les articles primaires dans des domaines bien définis et aussi de synthèses et des résumés. Vers la fin du $18^{\mathrm{e}}$ siècle, le nombre de revues publiés avoisinait les 755 dont 401 en Allemagne, 96 en France , 50 en Grande Bretagne, 43 aux Pays Bas et 37 en Suisse ${ }^{(80)}$.Ces revues étaient publiées dans les principales langues ( le Français , l'Allemand, l'Anglais , etc....) alors que le Latin dont l'utilisation avait commencé à décliner au $14^{\mathrm{e}}$ siècle n'était utilisé que par des auteurs de petits pays.

C'est à cette époque aussi que la course vers la primauté des résultats s'accentua entre les chercheurs et que cette manière de faire fut universellement acceptée. C'est les revues qui assuraient ce rôle pour donner la primauté ; elles agirent en tant qu'archive faisant office de dépôt de validation et ce rôle a été depuis le leur. Concurremment, le nombre de revues et de leur numéros, et par de là le nombre d'articles, ont augmentés. Il a été estimé que le nombre d'articles publiés avant 1850 était d'environ 200000.

Mais si cette période voit une indéniable envolée du périodique scientifique, le livre ne fut pas supplanté complètement (surtout qu'au début, et tel qu'on l'a vu au début de cette partie, il était difficile de différencier l'article du livre à cause de la nouveauté du medium) et c'est à cette période que les plus grandes maisons d'éditions (dont certaines ont encore pignon sur

\footnotetext{
${ }^{(80)}$ Manten, A.A.,op.cit.
} 
rue de nos jours) telles que Taylor \& Francis (Londres 1796), Wiley \& Sons ( New York 1807 ) et Springer-Verlag ( Berlin, 1842) virent le jour .

A la même période, les bibliothèques durent s'adapter à cette exponentielle augmentation de la masse de la littérature scientifique et la rendre accessible aux utilisateurs .De cette période date la spécialisation des bibliothèques où les bibliothèques universitaires servant des populations en grande partie constituée d'étudiants et de chercheurs tendent à acquérir des livres alors que les bibliothèques spécialisées davantage de périodiques.

Enfin et à partir du $18^{\mathrm{e}}$ siècle, la professionnalisation de la recherche scientifique impose de nouvelles donnes surtout pour ce qui est du financement. Les grandes nations industrialisées créent des laboratoires spécialisés et ceci afin de donner à tous leurs chercheurs les moyens de faire de la recherche et aussi d'en faire profiter la communauté .C'est l'époque où le chercheur acquière un statut social important.

\section{$3.3-1850-1925$}

Cette période voit le développement du rôle sans cesse grandissant de l'éditeur qui s'est imposé comme un maillon central inévitable entre le lecteur et le chercheur et ce surtout depuis le $18^{\mathrm{e}}$ siècle. On peut dire que nombre d'œuvres importantes n'auraient vu le jour sans l'apport de ces éditeurs pionniers modernes.Le début du $20^{\mathrm{e}}$ siècle voit le secteur de l'édition scientifique arriver à un degré de maturité jamais atteint auparavant. En Angleterre et aussi et surtout en Allemagne, le rôle des sociétés savantes ne cesse de croître alors que le secteur de l'édition scientifique privé commence à se développer et à éditer des revues de renom.

Springer est l'une de ces maisons les plus importantes du secteur de l'édition scientifique privée. Leurs stratégies et plans qui résumaient en fait le début de l'édition scientifique à grande échelle, étaient exprimés en ces termes par leur directeur Ferdinand Springer :

" On pouvait constater dés 1907 les débuts d'une grande spécialisation et différentiation de la science. Il était donc nécessaire d'établir un plan pour l'organisation de la littérature scientifique dans mes domaines d'activités. Voici ce plan :

\section{1-Publication et dissémination des résultats de recherche .Se fait dans les revues et archives}

2-Communication et rapports sur les résultats de recherche de notre pays vers l'étranger et ceux de l'étranger vers notre pays. Se fait par les Zenralblätter (bulletins bibliographiques signalant les articles parus) qui couvrent de manière objective l'ensemble de la littérature mondiale .Le chercheur décidera si ces rapports lui suffisent ou s'il a besoin de la publication originale (voir point 1).

3-Tandis que les Zenralblätter rapportent objectivement le contenu de la littérature mondiale, les Ergebnisse (résultats) contiennent des synthèses critiques d'une question d'actualité rédigée par des chercheurs.

4- A une époque de spécialisation et émiettement de la science, il me paraissait judicieux de publier des synthèses exhaustives de la science sous forme de manuels qui couvrent l'ensemble de la littérature scientifique. 
5-Il semblait également nécessaire d'éditer des monographies où l'auteur - chercheur présente de nouveaux résultats scientifiques qui ont trouvés un certain aboutissement.

6- Un certain nombre de magazines hebdomadaires et mensuels ont étés crées pour l'ensemble de la médecine et certaines spécialités médicales. Ces publications ont surtout un but pratique mais elles maintiennent aussi le lien avec la science pour ceux qui ont fait des études scientifiques mais n'exercent plus d'activité de recherche ....." ${ }^{(81)}$

Comme on peut le voir cette vision commerciale relatée par le Directeur de la maison d'édition Springer pour la période du début du siècle n'est en fait qu'une préfiguration de ce que ce secteur allait voir comme bouleversements drastiques l'ayant conduit à son état actuel. On remarque que l'auteur parle " de spécialisation et d'émiettement de la science ", phénomène qui va caractériser la recherche scientifique et s'accentuer de plus en plus jusqu'à être une des caractéristiques influençant l'orientation même des nouveaux périodiques .Nous réitérons l'idée que nous avons exprimé avant selon laquelle bon nombre de changements observés dans l'histoire de la transmission de la pensée ne sont en fait que des " répétitions " de successifs bonds en avant réalisés mais à un niveau plus élevé. Par exemple, si l'imprimerie a bouleversé (et le mot n'est pas assez fort) le monde à la fin du $15^{\mathrm{e}}$ siècle, des révolutions successives ont, depuis, eut les mêmes incidences à des degrés plus accentués ce qui confirme une des idées de Toffler selon laquelle le rythme de changement s'accélère et ce qui prenait, par exemple, 10 ans pour changer au $18^{\mathrm{e}}$ siècle ne prendrait à notre époque qu'une unité de temps infiniment plus courte. On citera par exemple, l'ordinateur et son excroissance naturelle Internet. Ou encore le téléphone cellulaire qui de nos jours est présent même dans les contrées les plus reculées et les plus défavorisées économiquement.

La même période voit aussi des innovations dans l'industrie de l'imprimerie prendre place. Nous en citerons les machines Linotype en 1886 et les Monotype en 1897 qui permettaient, respectivement, la saisie par lignes blocs (ou matrices) et de produire des lignes justifiées en caractères mobiles à l'aide d'une fondeuse. Ces machines, les Monotypes, ont étés utilisés jusqu'à la fin des années 60 pour la publication des revues scientifiques.

Dans la même optique, face à l'accroissement du volume des publications scientifiques, les éditeurs qui n'avaient pas de lien directs avec les grands imprimeurs ressentirent ce manque et ils entreprirent de s'y investir. Ainsi Springer s'investit à partir de 1909 avec un imprimeur qui travaillait sur la dernière machine Monotype. Ainsi cette société passa de 250 employés à 660 entre 1908 et $1914^{(82)}$, ce qui indique la fulgurante augmentation de son volume de travail en ce début de siècle.

En médecine, des projets d'indexation et de signalement de la littérature scientifique eurent lieu. A coté des Zenralblätter déjà évoqués en Allemagne, les Etats Unis furent précurseurs en la matière avec le Surgeon General's Office / Army Medical Library / National Library of Medecine en partenariat avec l'American Medical Association. En 1855, le catalogue américain contenait 168537 livres et 511112 articles ${ }^{(83)}$

\footnotetext{
${ }^{(81)}$ Sarkowski, Heinz, Der Springer Verlag, Teil I, 1842-1945, Berlin, Springer-Verlag, 1992, XI, 445 p., Cité par Fredriksen, Einar H., p.30

${ }^{(82)}$ Idem

${ }^{(83)}$ Ibidem
} 
A partir de 1879, Index Medicus, publication qui signale mensuellement les publications internationales en médecine d'une manière structurée, est créée. L'American Medical Association lança aussi un index médical concurrentiel, le Quarterly Cumulative Index to Current Medical literature ainsi que les Chemical Abstracts qui recensent les publications en chimie .D'autres projets similaires en d'autres domaines scientifiques et d'ingénierie sont lancés aux Etats Unis. Cette période a consolidé les acquis de la recherche et de l'édition scientifique qui avaient émergés au $19^{\mathrm{e}}$ siècle et avaient créés les conditions idoines pour les transformations qu'allait connaitre le $20^{\mathrm{e}}$ siècle.

\section{$3.4-1925-1945$}

Cette période de l'entre deux guerres est très importante pour la période d'après guerre et la suite des changements qu'ont connus les dernières décennies. Les revues allemandes, malgré la situation pré guerre en Allemagne, étaient toujours prévalentes mais cela n'allait pas durer longtemps.L'arrivée au pouvoir des Nazis allait changer la donne et la plupart des publications se faisaient à l'étranger (aux Pays Bas et aux Etats-Unis principalement).

A la même période, le Quarterly Cumulative Index to Current Medical Lliterature et Index Medicus fusionnèrent pour devenir The Quarterly Cumulative Index Medicus et ce en 1927. Cette période connut aussi certaines innovations techniques majeures telles que la télécopie, le photocopieur et la télévision. Ces inventions ne changèrent pas, à quelques exceptions prés (la rapidité et la fiabilité des transmissions) la manière dont la communication scientifique se faisait. En fait, on remarque un ralentissement de la vitesse des découvertes scientifiques ce qui était normal vu la situation qui prévalait pour l'époque .En fait, les chercheurs étaient beaucoup plus investis dans la préparation militaire et ceci laissait peu de temps à des recherches extra militaires.

\section{5-Seconde guerre mondiale-1965}

Après la deuxième guerre mondiale, et en conséquence des changements géopolitiques, l'Allemagne perdit de sa proéminence dans l'édition scientifique, principalement au profit des Etats-Unis qui sortirent vainqueurs de la guerre militairement mais aussi et surtout scientifiquement. Il ne faut pas oublier que le père de la bombe atomique américaine était d'origine allemande et que nombre de scientifiques allemands, nonobstant leur passé durant la deuxième guerre mondiale, furent accueillis par les Etats-Unis et offerts tous les moyens pour impulser la recherche dans le cadre de la guerre froide. Donc, dans un monde en pleine guerre froide, le centre de la science et de la recherche avait changé d'une Europe ayant subit les affres de la guerre vers une Amérique triomphante.

C'est dans ce cadre que le plus grand programme scientifique public fut lancé sous l'égide de l'US Office of Scientific Research and Development - OSRD - (Bureau Américain de la Recherche Scientifique et du Développement) et ce au début de la guerre. L'OSRD géra plusieurs projets (dont le fameux projet top secret " Manhattan " qui abouti à la première bombe atomique et ce après qu'Einstein eut averti Roosevelt des tentatives nazies de construire une bombe atomique et employa plus de 10000 chercheurs. Dans un article célèbre ${ }^{(84)}$, Vannevar Bush, qui avait été le premier Directeur de l'O.S.R.D, détailla les avantages que pouvait avoir la recherche militaire sur les différents aspects de la vie de tous les jours.

\footnotetext{
${ }^{(84)}$ Bush, Vannevar, As we may think, The Atlantic, July 1945.

Disponible à : http://www.theatlantic.com/doc/194507/bush
} 
Dans cette contribution qui était, le moins que l'on puisse dire, prémonitoire il imagina une manière de gérer la quantité sans cesse grandissante des publications.De nouvelles technologies pouvaient y remédier et en tête le microfilm qu'il imaginait comme la solution aux problèmes de stockage qui déjà se posaient avec acuité. Le précurseur qu'il était imagina une sorte de lien entre les différents éléments d'une bibliothèque mécanique qu'il appela Memex (Memory extension) et qui permettait de naviguer entre les différents documents. Ce qu'il avait imaginé à l'époque est considéré comme l'ancêtre de l'actuel l'hypertexte (terme utilisé par Ted Nelson quelque vingt ans plus tard). En 1950, il devint le premier directeur de la National Science Foundation, fondation phare de la recherche scientifique aux Etats-Unis. Cette période vit aussi une prolifération de projets surtout en médecine où des index exhaustifs et des listes de la littérature médicale récente furent lancés ainsi que les premières tentatives d'automatisation.

Quant à l'Europe, se relevant difficilement de la guerre, il lui était difficile d'avoir une politique de recherche commune. En fait, les deux pays qui concentraient les efforts de recherche étaient l'Union Soviétique et le Royaume Uni. Mais vite, les Européens comprirent qu'il était primordial d'avoir une politique de recherche commune afin de pouvoir contrecarrer l'hégémonie des Etats-Unis qui se profilait déjà au sortir de la deuxième guerre mondiale. C'est en 1954 que fut créé, à Genève, le CERN (Centre d'Etude et de Recherche Nucléaire) et qui fut le précurseur d'un nombre de centres de recherche fondamentale (tels que l'ESO (European Southern Observatory - European Organisation for Astronomical Research in the Southern Hemisphere ), l'ESA (European Space Agency-Agence Spatiale Européenne, l'EMBL ( European Molecular Biology Laboratory-Laboratoire Européen de la Biologie Moléculaire ) et l'ESRF (European Synchrotron Radiation Facility- Installation Européenne de Rayonnement Synchrotron).

A la même époque, et concurremment avec la montée en puissance des Etats Unis en tant que première puissance mondiale, il est enregistré un déclin de l'Allemand et du Français, comme langues scientifiques internationales. Ceci fut accentué par les restrictions imposées à 1'Allemagne et plus précisément aux éditeurs qui n'avaient pas le droit d'avoir des contacts directs avec les clients étrangers après la deuxième guerre mondiale. Toutes ces raisons ont impulsé les maisons d'édition anglo-saxonnes (surtout britanniques) devenues des leaders dans leurs spécialités. Francis André considère d'ailleurs que "...le Royaume Uni est aujourd'hui le premier pays d'édition scientifique au monde .....", ${ }^{85)}$ ce qui nous donne une idée sur la prépondérance de la langue dans la domination du marché de l'édition.

En conséquence de cet ostracisme de fait, les publications les plus importantes (quantitativement et qualitativement) furent celles des presses universitaires et surtout des sociétés savantes. Ceci est dû au fait que cette période voit l'émergence et la prépondérance des universités et des centres de recherche d'où émanent ces recherches. Il est à signaler que le développement de la recherche post deuxième guerre mondiale a imposé cette (première) explosion qui ne pouvait être contenue que par la création de revues de plus en plus nombreuses et spécialisées. C'est à cette époque que les grandes maisons d'éditions (Pergamon Press, Blackwell, Taylor \& Francis, Wiley et autres) qui dominent le marché actuellement virent le jour. Cette combinaison d'entreprises privées et publiques eut un effet très positif sur la production scientifique qui trouva en ce foisonnement un moyen de voir ses recherches publiées dans les meilleures conditions. De même que ceci entraîna l'internationalisation de la recherche qui, comme nous le verrons plus tard, tombera

\footnotetext{
${ }^{(85)}$ Francis, André, op.cit.p. 6
} 
progressivement dans l'escarcelle de véritables multinationales de la publication scientifique et qui amènera l'avènement du libre accès et des archives ouvertes dont est le sujet de ce travail et qu'on traitera plus loin dans le présent travail.

Enfin cette période verra aussi l'avènement des services d'indexation surtout médicaux dont l'archétype est l'Exerpta Medica .On peut aussi citer la création en 1960 de l'I.S.I. (Institute for Scientific Information) par Eugène Garfield et qui a vraiment donné une impulsion à la recherche bibliographique en général et a été le précurseur des grandes bases de données qu'ont permis la puissance de l'ordinateur d'abord et de l'Internet ensuite.

\section{6-1965-1985}

Si après la deuxième guerre mondiale, il y eut une certaine période de battement dûe aux conséquences désastreuses de la guerre et à la restructuration des sociétés durement éprouvées par presque six ans de guerre, les années 60 sont considérées comme les années de l'avènement des états - providences qui, pour répondre à l'activité sans cesse croissante de la recherche scientifique, y injectent des fonds considérables. Cette nouvelle donne a pour effet non seulement de donner à la recherche scientifique des fonds mais a aussi profondément modifié le monde des bibliothèques et de l'édition et dont les résultats et les conséquences sont visibles, palpables et en constant développement de nos jours. Il est indéniable que cette période a vu une profonde et radicale orientation de l'industrie de l'édition scientifique qui passa de la maison d'édition traditionnelle (des fois et très souvent familiale) à des entreprises d'édition internationales de taille moyenne préfigurant les grands conglomérats de la fin du $20^{\mathrm{e}}$ siècle. Ceci est en partie le résultat de l'investissement des pays industrialisés dans la recherche et dont l'un des résultats est la place prépondérante occupée par la revue qui est devenue le vecteur incontournable de la publication de la recherche. Cette période vit aussi, et face à l'accroissement exponentiel et des revues et de pages les composant, l'introduction des volumes annuels qui se proposait de relier en un seul document les différents numéros parus l'année précédente. Cette technique avait l'avantage (pour l'éditeur) de multiplier le nombre de volumes et de fixer un prix pour chaque volume. Il en résulta un développement économique sans précédant du secteur de l'édition scientifique qui en combinant réductions des coûts, délocalisation, et utilisation des nouvelles technologies a pu parvenir à maintenir des marges bénéficiaires très importantes.

De son côté, le monde des bibliothèques a subit de plein fouet cette évolution et les prémices de ce qui est communément appelé de nos jours " la crise des prix des périodiques " étaient latents et allaient se manifester d'une manière tout ce qu'il y a de plus dramatiques avec la période des dérégularisations qu'ont connus les années 80.Les restrictions budgétaires qui ont découlés de cette dérégularisation ont obligés certaines institutions à l'image de la British Library et son Lending Division ( Service de prêt) à investir le terrain du PEB (Prêt Entre Bibliothèques ). Cette forme précoce de libre accès ${ }^{*}$ s'est faite par la vente (à bas prix) de photocopies d'articles aux scientifiques britanniques et ensuite à d'autres scientifiques au-delà de la Grande Bretagne tels que les scientifiques des pays scandinaves. A la même époque, et face à ses entreprises, il y eut des réunions entre la British Library et les éditeurs britanniques pour trouver une solution compensatoire à cette forme de prêt et qui ne donnèrent aucun

\footnotetext{
* Il est de mon devoir de dire que cette expression m'a été dite au cours d'une discussion que j'ai eu avec Mr. Hervé Le Crosnier au mois de mars 2008 au cours des différentes fois où il a eu l'immense gentillesse de m'orienter dans ce travail (son terme exact est le prêt entre bibliothèques est le premier libre accès).
} 
résultat.Comme nous pouvons le voir, l'idée, la quintessence, les prémices du libre accès étaient présentes bien avant son avènement mais sous des formes différentes et n'avaient pas aboutis seulement parce que ni le timing ni le medium qui pouvaient les impulser n'existaient.

Le début des années 60 peut être aussi considéré comme le début de l'automatisation des services d'indexation et de l'introduction de cette même automatisation dans les bibliothèques dont l'index KWIC (Key Word In Context - mot clé dans le contexte) est l'exemple le plus connu. Cet index (appelé aussi index permuté) est formé par le triage et l'alignement des mots à l'intérieur d'un titre d'article pour permettre à chaque mot dans les titres (à part les mots vides) d'être recherché alphabétiquement dans l'index. Cette méthode d'indexation était très utile avant que l'avènement de la recherche automatisée en texte intégral ne devienne la norme. Les systèmes automatisés les plus connus de cette époque sont :

- MARC :(MAchine Readable Cataloging) qui est un format d'échanges de données bibliographiques permettant d'informatiser les catalogues de bibliothèques .Il a été créé en 1965 par la Bibliothèque du Congrés et a engendré un nombre de format MARC (USMARC, CAN/MARC, MARC 21 qui est une fusion d'US MARC, d' AUS MARC, d' UK MARC et de CAN MARC, et reconnu par l'IFLA comme format d'échange et dont la maintenance est effectuée par la Bibliothèque du Congrès, INTERMARC utilisé par la Bibliothèque Nationale de France et enfin UNIMARC créé par l'IFLA en 1977).

- CDS/ISIS :(Computerised Documentation Service / Integrated Set of Information Systems).C'est un système développé par l'UNESCO en 1965 et était utilisé sur un mainframe IBM. En 1985, une version programmée pour les mini et micro ordinateurs en langage Pascal fut développée utilisant un PC IBM sous MS DOS. En 1995, WinIsis, la version Windows peut fonctionner sur un seul ordinateur ou un système local. Les composants du Java/ISIS client/serveur permettent la manipulation à distance des bases de données sur Internet et est disponible pour Windows, Linux et Mc Intosh. GenISIS de son coté, permet à l'utilisateur de produire des formes HTML pour la recherche des bases de données CDS/ISIS. L'ISIS_DLL fournit une interface de programmation pour développer des applications CDS/ISIS. Enfin, la bibliothèque OpenIsis fournit une autre interface de programmation pour développer les applications CDS/ISIS avec des améliorations.

-MEDLARS (MEDical Literature Analysis and Retrieval System) est un systeme de recherche bibliographique biomédical informatisé .Il fut lancé par The National Library of Medecine en 1964 .En 1971, une version en ligne appelée MEDLINE (" MEDLARS Online " ) devint disponible. Il fait suite à Index Medicus qui depuis 1879 publiait un guide mensuel de la littérature médicale dans des milliers de journaux compilés manuellement. C'est en 1957 que les employés de la NLM commencèrent à planifier l'automatisation d'Index Medicus. En 1960, des spécifications détaillées furent préparées et en 1963, General Electric délivra l'ordinateur qui devait faire fonctionner MEDLARS. La configuration originale fonctionna de la date de réception jusqu'à son remplacement par MEDLARS II en 1975.A partir de 1972, plus de cinquante villes américaines avaient un accès à distance à la base à partir de terminaux et ce via le réseau téléphonique. En Europe, Excerpta Medica connut un développement similaire et devint accessible en ligne à partir des années 70 .

Malgré tous ces efforts, ce n'est que vers le milieu des années 90 que la recherche en ligne est lancée à grande échelle. Avant les années 80, les bases de données en texte intégral, la messagerie électronique et même Internet étaient techniquement faisable mais se heurtaient 
soit au scepticisme ou au manque de moyens (les ordinateurs n'étaient pas aussi abordables ni aussi performants qu'ils le sont de nos jours ).

\section{$3.7-1985-2000$}

Cette période est, sans contexte, celle de l'invention du World Wide Web (www) plus communément connu sous l'appellation de Web (ou toile en langue française).Cette invention (ou plutôt cette consécration car le web existait mais n'était réservé qu'à une élite pour des raisons déjà évoquées) eu un impact très palpable sur les services des bibliothèques, la communication scientifique et surtout les éditeurs commerciaux de cette même littérature scientifique. C'est l'époque de l'agrégation des articles de revues en une seule plateforme à l'image de IDEAL d'Academic Press en 1995, de SpringerLink en 1996, de ScienceDirect d'Elsevier en 1998 et d'autres qui n'ont en fait percé qu'au début du troisième millénaire.

C'est à cette époque que l'idée du libre accès voit le jour car il était indéniable que l'utilisation de nouvelles technologies change complètement la donne et ouvrait de nouveaux horizons devant les chercheurs et aussi les éditeurs commerciaux scientifiques qui au lieu d'adopter une position de refus (ce qu'ils ont fait au début afin de garder leurs avantages) ont plutôt essayé de prendre des décisions tendant à accompagner le mouvement tel que les exemples précédents le démontrent. C'est à cette époque aussi que l'utilisation de la messagerie électronique et des moteurs de recherche oriente la recherche beaucoup plus vers une philosophie de réseau, idée qui sera la base de toute recherche à l'avenir. L'année 1991 voit l'apparition d'ArXiv, première expérience du genre et qui verra par la suite un nombre sans cesse croissant de projets similaires dans les différentes spécialités avec une prépondérance pour les sciences exactes au détriment des sciences sociales. Les fonctions d'ubiquité et de décentralisation des plateformes donnent lieu à des projets qui s'orientaient vers une internationalisation des entreprises. De cette donne a découlé, le phénomène des concentrations et de la cartellisation dont sont accusées les grandes sociétés d'édition mondiales qui, à coup de rachats, de fusions et autres montages financiers, bâtissent de véritables multinationales dont les ramifications ne sont pas clairement définies et dont les conséquences (à l'image de la bibliothèque numérique mondiale de Google et qui de par ses velléités de mainmise sur le Savoir et l'orientation anglophone qu'elle a voulu lui donner a suscité les réactions les plus négatives sans compter le problème du droit d'auteurs qui a fait de ce projet un projet pratiquement mort-né suite aux plaintes des auteurs et éditeurs ayant eu leurs documents numérisés ) ne sont pas toujours ni appréhendées ni même projetées.

Les bibliothèques de leur coté et face à ces changements radicaux dans le monde de l'édition entreprirent des actions à même de sauvegarder leurs intérêts et ceux de leurs lecteurs. En plus des restrictions budgétaires auxquelles elles faisaient face, la transition du papier vers le numérique introduisait une nouvelle donne commerciale car les éditeurs ne proposent plus de titres individuels mais des packages ou ensemble de titres et dont le prix global est (apparemment) avantageux par rapport à la somme de chaque titre individuellement pris. Cette nouvelle politique des éditeurs a fait réagir les bibliothèques qui, afin de constituer une force, ont fondé des consortia pour être en position de force lors des négociations. De son coté, Jean Claude Guédon dans une de ces recherches ${ }^{(86)}$ sur le sujet, a fait ressortir que loin de représenter une aubaine, ces bundlings ou ensemble de titres tendaient à imposer des titres (dont la bibliothèque n'a peut être pas besoin) et à orienter la tendance des recherches effectuées par les lecteurs (et même le facteur d'impact, les chercheurs utilisant ces

${ }^{(86)}$ Guédon, Jean Claude,op.cit. 
ressources auront tendance à citer les mêmes auteurs qui publient dans ces revues ) étant donné que si une bibliothèque acquiert un ensemble de titres du même éditeur ou ensemble d'éditeurs, il y a de fortes chances que ces titres soient de la même école ou orientation qu'elle soit linguistique, idéologique ou autre.

C'est à la suite de ces développements et surtout l'avènement de l'Internet grand public (qu'on peut schématiquement situer au milieu des années 90 surtout dans les pays développés) que les conditions du lancement du mouvement du libre accès sont réunies. L'un de ces porte parole le plus actif, The Scholarly Publishing and Academic Ressource Coalition (SPARC) naquit en 1998 et est considéré comme l'une des plus importantes organisations demandant l'affranchissement de la littérature scientifique et technique des pesanteurs commerciales. Si le mouvement débuta timidement, il n'en demeure pas moins qu'il a accompli des avancées que d'aucuns n'aurait cru possibles surtout en un si court laps de temps.Son vecteur essentiel, Internet, étant toujours en constante évolution, il est clair que le mouvement épousera les contours des avancées que ce dernier enregistrera.

\section{$3.8-2000-2010$}

Il est clair que la décennie que nous vivons se caractérise par une évolution rapide des techniques de diffusion de l'information. C'est surtout à partir de 1995 que la commutation de paquets (technique créée dans les années 60 et qui consiste à " diviser " l'information et à l'envoyer par différentes routes afin qu'elle voyage vite et ensuite l'assembler à l'arrivée) a commencé à avoir une influence sur l'édition et ce avec Internet qui a permis un accès bien plus grand à l'information. De plus de nouveaux outils de recherche qui n'existaient pas à la fin du dernier siècle ont pratiquement envahi le monde la recherche. Ainsi les bases de données accessibles en ligne, les logiciels d'édition, les moteurs de recherche (certains spécialisés à l'image de Google) sont devenus des outils indispensables aux chercheurs.

De leur côté, les maisons d'édition surtout les grandes ont su s'adapter aux nouvelles donnes et de nouvelles presses universitaires voient le jour. D'un autre côté, le secteur public s'intéresse à l'édition scientifique ce qui ouvre de nouvelles opportunités grâce aux financements et l'ouverture de nouvelles niches .Contrairement aux idées reçues, il semblerait qu'on s'oriente plus vers un encouragement de l'établissement d'archives institutionnelles dépendant d'universités, d'institutions ou de sociétés savantes que l'établissement de licences d'accès aux articles de périodiques vu la difficulté à arriver à un consensus entre éditeurs et auteurs.

L'imprimerie, quant à elle, traverse elle aussi une période de profondes mutations. Le papier n'est plus, et de loin, le support le premier de l'information. Les nouvelles techniques d'impression et leur qualité de plus en plus sophistiquée (par exemple la technologie de l'impression à la demande) ont permis d'éliminer les contraintes des stocks non écoulés, de minimiser les investissements non rentables ainsi que la création de librairies sur la Toile. Le rôle de l'éditeur dans la mise en forme est devenu congru grâce aux nouveaux logiciels très performants et très techniques utilisés par les chercheurs pour préparer leur travail.

Enfin, l'industrie de l'édition scientifique a très rapidement répondu à l'évolution des TIC, acquis des compétences numériques et s'est investie dans la qualité de l'information publiée. Cette recherche de l'excellence est au cœur du travail éditorial ainsi que l'une des plus importantes fonctions de la bibliothèque. De cette collaboration peut découler une relation 
fructueuse aussi bien pour l'industrie de l'édition scientifique que pour les bibliothèques de part, d'un coté pour le tri et la sélection de la grande masse d'information et aussi pour la normalisation des formats, balises et le volume de stockage. On voit ici l'opportunité qu'offrent les TIC en matière de complémentarité des rôles :

- les bibliothèques qui se doivent d'offrir gratuitement l'information.

-les éditeurs de promouvoir et vendre des produits de qualité.

Mais cette avancée de l'économie numérique n'est pas sans conséquences sur les autres industries connexes telles que les télécommunications, la musique et l'édition tout en ouvrant de nouveaux horizons. Par exemple, les agrégateurs proposent des plates-formes de revues et de livres. De leur côtés, les grands groupes éditoriaux ainsi que les grandes institutions ont mis en place des archives institutionnelles. Des outils tels qu'EndNote ${ }^{(87)}$ pour l'importation des références et aussi ScholarOne ${ }^{(88)}$ pour la gestion du contrôle des soumissions sont venus s'ajouter et sont utilisés aussi bien par des administrations universitaires que des bibliothèques ou même des éditeurs. Des moteurs de recherche spécialisés tels que Google Scholar ou Scopus ${ }^{(89)}$ offrent de nouvelles alternatives et concurrencent les bases de citations classiques qui avaient jusqu'alors un quasi monopole (ISI par exemple). Dans le secteur de l'agrégation des revues scientifiques, les deux leaders Swets ${ }^{(90)}$ et surtout EBSCO ${ }^{(91)}$ ont changé leur positionnement afin de s'adapter aux nouvelles donnes du marché. Du côté des livres, les éditeurs ont profités de la disponibilité et des différentes fonctionnalités des catalogues électroniques telles que la recherche et la prise de commande pour pénétrer un marché jusque là difficile et le cibler du point de vue régional et des groupes.

Face à la cherté de l'information surtout pour les pays en voie de développement, un nombre de programmes internationaux ont étés initiés afin de permettre l'accès selon les pays et leur Revenu National Brut par habitant. On citera, HINARI ${ }^{92)}$ (Health InterNetwork Access Initiative), AGORA ${ }^{(93)}$ (Access to Global On Line Research in Agriculture) et OARE ${ }^{(94)}$ (Online Access to Research in the Environment) regroupés sous le nom commun de Research4Life ${ }^{(95)}$ et subventionnés par l'O.M.S. Ces programmes prennent en ligne de compte pour l'accès et l'éligibilité les chiffres de la Banque Mondiale ${ }^{(96)}$ en ce qui concerne le PNB.

Le programme HINARI a été mis en place par l'OMS en collaboration avec les six plus grands éditeurs du monde. Il vise à fournir un accès gratuit ou à très bas prix, aux grands périodiques couvrant les domaines de la médecine biologique et des sciences sociales aux établissements publics et à but non lucratif dans les pays en voie de développement. Près de 7,500 sources d'information sont accessibles aux établissements de santé dans 105 pays et fournissent au personnel de santé publique, aux chercheurs et aux législateurs un accès à

\footnotetext{
${ }^{(87)}$ www.endnote.com/

(88) WwW.scholarone.com/

(89) Www.scopus.com/scopus/home.url

(90) WwW.swets.com/

(91) Www.ebscohost.com/

(92) Www.who.int/hinari/fr/index.html

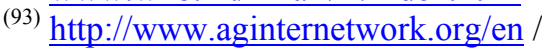

(94) http://www.unep.org/oare/en/

(95) www.research4life.org/

(96) $\underline{\mathrm{http}: / / \mathrm{www} . \text { worldbank.org/worldbank.org }}$
} 
l'information dans le domaine de la santé, contribuant aussi à une meilleure situation de la santé dans le monde.

Le programme AGORA, mis en place par l'Organisation des Nations Unies pour l'Alimentation et l'Agriculture (FAO) et par de grands éditeurs, permet aux pays en développement d'accéder en ligne à une collection bibliographique exceptionnelle dans les domaines tels que l'alimentation, l'agriculture, la science environnementale et les sciences sociales apparentées. AGORA fournit aux institutions de 107 pays une collection de 1900 revues. AGORA est conçue afin d'améliorer dans le monde en développement les études de milliers d'étudiants, d'enseignants et de chercheurs dans les domaines de l'agriculture et des sciences de la vie.

Le service d'accès en ligne pour la recherche sur l'environnement (OARE) est un partenariat international public privé lancé par le Programme des Nations Unies pour l'Environnement (PNUE), l'Université de Yale, et d'éminents éditeurs. L'objectif est de permette aux pays en voie de développement d'accéder gratuitement à l'une des plus importantes collections de littérature scientifique sur l'environnement. Plus de 2,990 titres publiés par plus de 340 maisons d'édition prestigieuses et des sociétés de recherche scientifique sont maintenant disponibles dans 107 pays en voie de développement. OARE permet d'accéder à la littérature scientifique internationale, dans les disciplines aussi diverses que la biologie, la biochimie, la microbiologie, la biotechnologie, la génétique et les espèces génétiquement modifiées, la botanique et la biodiversité, la climatologie, la météorologie, le changement climatique, l'écologie et la conservation de la nature, la conservation de l'énergie et les sources d'énergie renouvelables, la chimie, les ressources économiques naturelles, l'ingénierie de l'environnement, le droit de l'environnement, la politique et la planification, la foresterie, la géographie, l'étude de la population et la migration, la géologie et les sciences de la terre, les désastres naturelles, l'océanographie et la biologie marine, la pollution et la toxicologie environnementale, les sciences du sol et de la désertification, la gestion des déchets, l'eau, l'hydrologie et les zones humides, la zoologie et la biodiversité animale environnementale, les sciences du sol et de la désertification, la gestion des déchets, l'eau, l'hydrologie et les zones humides, la zoologie et la biodiversité animale.

Ainsi, les pays sont divisés en termes d'accès en deux groupes selon le RNB :

-Le premier groupe a un accès gratuit a tous les périodiques et c'est celui dont les pays ont un RNB de - de 1600 \$ pour HINARI et AGORA et de - 1250 \$ pour OARE

-Le deuxième groupe bénéficie d'un accès gratuit mais en payant une contribution de $1000 \$$.Ce sont les pays dont le RNB est compris entre 1601 et $4700 \$$ pour AGORA et HINARI et de 1250 et $3500 \$$ pour OARE.

Ces programmes (et d'autres qu'il serait pratiquement impossible de citer tant ils apparaissent à un rythme qui ne fait que suivre le rapide et exponentiel accroissement des programmes d'accès libre) sont un des plus importants aspects des nouvelles politiques éditoriales du libre accès. Ils tendent à mettre à la disposition des pays en voie de développement un accès qui résorberait l'immense fracture numérique qui existe entre les pays de l'Hémisphère Nord et ceux de l'Hémisphère Sud. Nous pensons personnellement que l'idée et la philosophie du libre accès est, d'une certaine manière, dévoyé de son sens originel car le libre accès devrait permettre, comme son nom l'indique, un accès, si ce n'est complètement libre (nombre 
d'auteurs ont souligné que le libre dans libre accès ne signifiait pas gratuit) mais du moins un plus grand accès. Malheureusement, cette fracture numérique tant documentée ne permet pas à cette noble idée d'être utilisée, exploitée de la manière la plus efficiente possible.

Cette cherté de l'information a conduit les bibliothèques à négocier des licences avec les grands groupes éditoriaux (Elsevier, Wiley \& Sons, etc.....).Devant la multiplicité des contrats et des différences dans l'offre, les contrats ne sont pas uniformes mais répondent aux spécificités de chaque institution. Nombre d'institutions se sont regroupés en consortia (pluriel de consortium défini comme "...un groupement d'entreprises juridiquement indépendantes, réunissant sous une direction unique des moyens financiers ou matériels, en vue d'exécuter en commun des opérations déterminées. En l'occurrence, ... c'est un ensemble de bibliothèques ou d'institutions qui se regroupent pour acheter mais aussi pour utiliser de la documentation électronique en commun " ${ }^{(97)}$ ) pour obtenir les prix les meilleurs et profiter des avantages de ce genre de contrat. A titre d'exemple, une bibliothèque peut proposer de nos jours un accès à quelques 20000 titres au même prix que ce qu'elle offrait il y a de cela $10-15$ ans.

On citera à titre d'exemple, L'OhioLINK ${ }^{(98)}$ (Ohio Libraries and Information NetworK) aux Etats-Unis qui regroupe 88 bibliothèques de collège et d'universités en plus de la bibliothèque de l'Etat de l'Ohio. Il dessert plus de 600000 étudiants, enseignants et chercheurs de l'Etat. Les 88 institutions qui y sont membres se départagent de la manière suivante : 16 Universités publiques, 23 Collèges techniques, 49 Collèges privés en plus de la bibliothèque de l'Etat de 1'Ohio. Ce consortium donne accès à :

1-48 millions de livres,

2-Des millions d'articles électroniques,

3-14,000 journaux électroniques,

3-150 bases de données de recherches électroniques,

4-62,000 livres électroniques,

5-Des milliers d'images, vidéo et de sons,

\section{6-26000 thèses et dissertations d'étudiants de l'Etat d'Ohio}

$\mathrm{Au}$ Royaume-Uni, le Joint Information Systems Committee (JISC) du Higher Education Funding a confié à un consortium réunissant Swets \& Zeitlinger et le département informatique de l'Université de Manchester la responsabilité d'un programme expérimental de fourniture de revues électroniques dénommé NESLI (National Electronic Site Licence Initiative) ${ }^{(99)}$. Le programme comprend la négociation avec les éditeurs et les calculs d'équilibre économique. L'accès à ce service est effectif depuis janvier 1999 pour les bibliothèques participantes. En 2004, NESLI 2 fut lancé et il faisait suite à NESLI qui fut lancé au milieu des années 90 pour répondre à l'offre numérique qui venait d'arriver sur le

\footnotetext{
${ }^{(97)} \mathrm{http} / / /$ eprints.rclis.org/bitstream/10760/4370/1/meurs12000.html

(98) $\mathrm{http://www.ohiolink.edu/}$

${ }^{(99)} \mathrm{http://www.jisc-collections.ac.uk/nesli2/}$
} 
marché. Il couvre 17 éditeurs scientifiques parmi les plus prestigieux qui donnent accès à quelques 7000 journaux en ligne disponibles aux membres du consortium. Selon le site de NESLI 2, ce dernier a pu économiser pour la seule année 2010 13,5 millions $£$ et depuis sa fondation en 2004 plus de 40 millions $£^{(100)}$.Nous voyons ici la force du consortium surtout dans un pays considéré comme le premier dans l'édition scientifique technique à l'échelle mondiale. Les économies réalisées sont très importantes et surtout permettent à un nombre accru d'institutions d'avoir un accès qu'ils n'auraient pas eu si elles avaient négociés toutes seules.

$\mathrm{Au}$ niveau international, les différents consortia se regroupent en une " Coalition Internationale de Consortiums de Bibliothèques" (International Coalition of Library Consortia).Sa première réunion eut lieu en 1997 et s'appelait alors Consortium Of Consortia (COC). L'ICOLC rassemble de manière informelle plus de 200 consortia de bibliothèques nord-américaines, mais aussi anglaises, hollandaises, allemandes et australiennes. Cette coalition s'est formée pour informer les autres futurs consortia et discuter sur les contrats, les négociations, les produits. Elle se définit comme "...servant les organisations participantes en facilitant la discussion entres les membres des consortia au sujet des problèmes d'intérêt communs. Durant l'année, l'ICOLC peut organiser des réunions dont le but est de permettre aux membres des consortia participantes d'être à jour en ce qui concerne les nouvelles ressources d'information électroniques, les pratiques de facturation des vendeurs et fournisseurs d'information électroniques et autres problèmes concernant les directeurs, les conseils d'administration et bibliothèques de consortia. Au cours de ces meetings, l'ICOLC peut se réunir avec des fournisseurs d'information donnés, leur fournissant un forum afin de présenter leurs offres et engager un dialogue avec les leaders des consortia autour des problèmes d'intérêt communs. " D'autres sites du même genre voient le jour et sont là pour aider les bibliothèques qui voudraient passer le même genre de contrat. Ainsi Liblicense , sous la direction de Ann Okerson de l'Université de Yale, présente de manière détaillée et commentée les contrats de licences américains passés avec les éditeurs de documents électroniques. Un logiciel d'aide à la négociation et à la rédaction de contrat est téléchargeable. Nous voudrions remarquer une anomalie dans cette liste et elle concerne les pays qui y participent : une infime partie provient des pays en voie de développement (en fait seuls quatre pays possèdent leur propres consortium affilié à l'ICOLC et ce sont: le Botswana, l'Egypte, le Liban et le Lesotho) Nous nous permettons de poser la question suivante : l'idée de consortium a germé pour aider les bibliothèques à faire face à ce qui communément connu sous le vocable anglo saxon du " serial price crisis " ( traduit adéquatement par Jean Claude Guédon en " la crise des prix des périodiques").N'aurait il pas été plus logique, plus adéquat que les pays dont les ressources sont les plus faibles qui en profitent? Une bibliothèque nord américaine par exemple est moins sujette, de toute évidence, à des contraintes budgétaires qu'une bibliothèque malienne, ougandaise ou rwandaise. Ceci n'est pas dit pour rabaisser ces pays mais pour faire ressortir l'incongruité d'une situation où les riches font des économies et les pauvres ne profitent pas des avantages qu'offre ces nouveaux medias. D'après ce qui nous a été permis de remarquer, c'est un manque chronique d'information concernant ce sujet qui se pose. En effet, paradoxalement alors que toutes les nouvelles approches dont est sujet ce travail (archives ouvertes, journaux et portails en libre accès, consortia, etc.....) tendent à faciliter l'accès et l'utilisation de l'information grâce à Internet, et que nombre de programmes sont spécifiquement faits pour répondre à ces besoins dans les pays en voie de développement (en plus des exemples récemment cités, des programmes tels qu'INASP, PERII, SciDev.Net, etc.....), c'est ces

${ }^{(100)}$ http://www.jisc-collections.ac.uk/nesli2/ (Rubrique About us) 
derniers qui en profitent le moins . Un travail de sensibilisation doit être entrepris afin que la quintessence de cette philosophie (le libre accès) ne soit pas perdue.

\section{Conclusion}

L'édition scientifique et technique, contrairement à l'édition en général, a toujours été axée sur la recherche et se caractérise par une forte demande et un public très ciblé. Si l'invention du papier et de l'imprimerie avait créé une industrie basée sur le papier, l'avènement de la Toile mondiale a imposé de nouveaux éléments qui demandent une adaptation quasi-totale à de nouvelles règles. Les deux protagonistes (éditeurs et bibliothèques) doivent revoir leur place dans ce schéma mais c'est surtout les éditeurs qui font face à la lancinante question de leur devenir. Tant qu'ils peuvent faire un profit, ils investiront dans de nouveaux créneaux et les bibliothèques bénéficieront de ces investissements.

Après la deuxième guerre mondiale, il y a eu un intérêt grandissant pour la recherche qui a amené de grands investissements du point de vue financier dans le monde occidental en général mais surtout aux Etats-Unis. Deux secteurs agissant en vase communiquant ont en bénéficié : le monde de l'édition et celui des bibliothèques. Ils ont toujours travaillé de concert mais les nouvelles donnes numériques font qu'ils sont beaucoup plus complémentaires, contrairement à l'idée généralement acceptée qu'ils tendent à agir de manière adverse ou du moins que la politique de l'un (l'édition) allait à l'encontre de l'autre (bibliothèque).En effet, le nombre croissant des articles scientifiques produits (on estime qu'entre 1995 et 2010, le nombre d'articles produits a pratiquement doublé) entre autres en conséquence d'articles payés par les auteurs, leurs institutions ou générés par des nouvelles archives institutionnelles et cette masse doit être gérée par une institution (la bibliothèque) qui voit son rôle non seulement changé mais aussi et surtout qui devient plus primordiale afin d'absorber ce flux qui , comme répété à maintes fois, progresse exponentiellement.

L'avènement des réseaux (à leur tête Internet) a permis une plus grande disponibilité et un plus grand pouvoir d'achat des bibliothèques qui doivent maîtriser de nouveaux mécanismes d'acquisition de l'information. Il faut s'attendre à d'autres offres tarifaires de la part des éditeurs qui ont dû s'adapter en présentant de nouvelles propositions technologiques et tarifaires .Encore une fois, on voit que la complémentarité plutôt que l'adversité est le nouveau mode de fonctionnement des deux pôles.

Le modèle de l'édition scientifique a besoin pour pouvoir présenter les résultats de la recherche d'un modèle stable et pérenne. Les éditeurs, les bibliothèques, le secteur de la recherche développement ainsi que les lecteurs ont tous besoin d'une industrie éditoriale qui puisse assurer un accès durable et un stockage à long terme à l'information. Les données n'étant pas les mêmes que dans le domaine du papier, de nouvelles règles d'archivage de l'information sont demandées. En effet, le papier pouvait se détériorer mais était " lisible " alors que de nos jours nombre de fichiers ne sont pas lisibles car sont dépassés, de formats obsolètes ou carrément abîmés. On touche du doigt, peut être, le vrai challenge que la sphère des éditeurs devrait adresser : la pérennisation et l'archivage à long terme de l'information sans oublier le tant redouté 404 not found que rencontre tout chercheur d'information sur Internet.

Publier n'a jamais sans induire des dépenses et il est difficile de comprendre cela pour la publication scientifique et technique. L'objectif de la recherche étant de rendre publique des 
découvertes bénéficiant ( $\mathrm{du}$ moins en principe) la société, il apparaît pour le moins inacceptable de la faire payer. Si dans l'univers papier, ces dépenses étaient inclues dans la publication en chargeant tant par page, dans l'univers numérique, c'est le chercheur qui paye pour sa publication (option auteur payeur).Enfin, cette augmentation révèle le travail, incontournable (même dans l'univers numérique), des éditeurs dans la mise en page et surtout du contrôle de la qualité des publications.

Enfin, les développements dans les universités et centres de recherche continueront à avoir un impact sur la création de bibliothèques numériques, les nouveaux éditeurs scientifiques et les conditions de travail des chercheurs et étudiants.De plus, les chercheurs, les réseaux scientifiques et les sociétés savantes seront toujours des acteurs indispensables des bibliothèques, maisons d'éditions et intermédiaires alors que les lois du marché ( sous d'autres formes et données ) resteront globalement les mêmes que dans l'univers papier.

\section{4-Conclusion et comparaison des trois modèles}

L'examen ou la présentation de ces trois modèles de l'évolution de l'article scientifique et technique a été faite afin de voir de quelle manière les chercheurs voient cette évolution ( ou révolution ). Bien que chaque chercheur voit cette évolution selon son propre prisme et grille de lecture, nous pouvons, de prime abord, voir que leur description a des similitudes que l'on peut résumer dans les points suivants :

\section{1-Non différentiation entre livre et périodique}

Au moment de la création des premiers périodiques scientifiques, la différentiation entre le livre et le périodique n'était pas si clairement établi que vers le milieu du $18^{\mathrm{e}}$ siècle. Ce n'est qu'au bout d'un certain temps que ce nouveau paradigme s'impose mais le périodique n'a nullement remplacé le livre d'une manière définitive il a tout simplement pris de l'importance alors que le livre est toujours présent. Ceci était du au fait qu'étant un nouveau medium, il " cohabitait " avec le livre et nous avons le cas d'articles qui étaient aussi longs que des livres et aussi de périodiques qui n'avait qu'un seul sujet ou article d'un même auteur. Thomas Kuhn ${ }^{(101)}$ a évoque cette manière qu'ont les nouvelles inventions, innovations (il a utilisé le mot paradigme) à venir se greffer progressivement et de remplacer le paradigme précédant. Il dit que le "...le progrès scientifique n'est pas évolutionnaire mais c'est plutôt une série d'interludes pacifiques ponctués par des révolutions intellectuellement violentes [et dans ces révolutions] une vue conceptuelle du monde est remplacée par une autre" ${ }^{(102)}$.

\section{2-Développement de la fonction archive du périodique}

Les périodiques n'ont en fait que fait qu'archiver et officialiser la lettre savante, qui était la seule manière de faire circuler l'information. En effet, en ces temps où les moyens de communication et de déplacement n'étaient pas très développés, la seule manière de faire parvenir et diffuser les découvertes était d'écrire à un collègue et la lui faire lire. De toute évidence, cette manière de faire avait de nombreux inconvénients comme la lenteur, l'incertitude quant à l'arrivée de l'information, les problèmes de paternité d'éventuelles découvertes, etc.... D'ailleurs en ce qui concerne ce dernier point, en vue d'éviter d'éventuels

\footnotetext{
${ }^{(101)}$ Kuhn, Thomas, The Structure of scientific révolution, Chicago, University Of Chicago Press, 1962, 226p. ${ }^{(102)}$ Op.cit.
} 
plagiats, nombres de savants utilisaient des sortes de codes ( anagrammes) quand ils communiquaient avec leurs collègues afin de rendre le texte intelligible aux autres.

\section{3-Professionnalisation}

Les périodiques (faisant suite à la lettre savante) qui se distinguaient par un langage familier de tous les jours - des fois même, et à la limite, poétique - pleins de formules de politesse, ont du adopter un langage normalisé, technique, impersonnel et moins dithyrambique. Ceci avait pour but de normaliser les résultats de la recherche et de la mettre à un niveau où l'amateur ne pouvait se permettre de participer aux débats de la Science. La science s'étant professionnalisé (hyper professionnalisé diront certains), il fallait adopter un langage qui réponde à cette situation. C'est dans cette optique que, par exemple, les instructions aux auteurs sont présentes dans tous les périodiques sollicitant une contribution. Cela procède de cette normalisation, et par delà l'internationalisation, du Savoir.

\section{4-Spécialisation}

Les premiers périodiques étaient ce qui serait considéré de nos jours comme généralistes et s'adressaient de ce fait à tout un chacun. Le monde découvrait ce nouveau medium et chacun y allait de sa contribution. C'est avec leur augmentation que s'est imposée une spécialisation et une sélection du contenu, des contributeurs, des moyens de contrôle et de certification de l'information, etc......Les deux premiers périodiques spécialisés le furent en médecine et c'était: Miscellanea Curiosa (1670-1705) et Acta Medica et Philosophia Hafniensia (1673$1680){ }^{(103)}$.Ils furent suivis en cela par d'autres spécialités plus tard et les périodiques ont eut depuis tendance (suivant en cela la Science) à se spécialiser. Il existe de nos jours très peu (à part des exemples assez particuliers que nous avons présentés) de périodiques scientifiques généralistes. De nos jours, l'hyperspécialisation a complètement fait disparaître les périodiques généralistes.

\section{5-Prépondérance de la langue anglaise}

Bien qu'au début le Latin était utilisé comme Lingua Franca (langues véhiculaire ) à la place des langages vernaculaires et que plus tard le Français et l'Allemand occupèrent une place importante dans les langues de publication, l'Anglais, depuis le début du $20^{\mathrm{e}}$ siècle surtout, domine la publication scientifique et technique. Ceci est vrai au point où même les auteurs dont la langue maternelle ou de publication n'est pas l'anglais sont obligés de rédiger en cette langue afin d'avoir une plus grande exposition et d'être lus par le plus grand nombre. Selon Watson $^{(104)}, 88.5 \%$ des articles indexés par le Science Citation Index en 1978 étaient rédigés en anglais et les auteurs dont la langue maternelle n'était pas l'anglais en représentaient 30 $\%$., ce qui nous donne plus du quart de ceux qui publient le font en anglais alors que ce n'est pas leur langue maternelle. Ces chiffres datant de plus de 30 ans, il est sur que cette tendance, à la lumière de la mondialisation, s'est accentuée. En effet, en 2000 cette proportion avait atteint $95.9 \%$ après être passé de $84.5 \%$ en 1980 et à $90.5 \%$ en $1990^{(105)}$. On remarquera que la portion, déjà congrue, des autres langues a tendance, contrairement à l'anglais, à se rétrécir. A titre d'exemple, et toujours pour la même période (entre 1990 et 2000) le Français est passé

\footnotetext{
${ }^{(103)}$ Manten, A.A.,op.cit. p.18.

(104) ) Watson, J.J., op cit.

${ }^{(105)}$ Bordons, Maria, Gomes, Isabel, Towards a single language in science? A Spanish view, Serials, Vol.17, $n^{\circ} 2$, p.189, Juillet 2004
} 
de $3.8 \%$ à $1.9 \%$ et enfin à $1.0 \%$, L'Allemand de $5.1 \%$ à $2.5 \%$ et enfin $1.1 \%$, l'Espagnol de $0.7 \%$ à $0.4 \%$ et enfin $0.3 \%$, , le Japonais de $0.7 \%$ à $0.5 \%$ et enfin $0.3 \%{ }^{(106)}$

\section{6-Augmentation exponentielle de la masse de l'information}

L'extraordinaire accroissement de la masse documentaire en général et des périodiques en particulier pose de toute évidence d'immenses problèmes de gestion de cette masse. Cet accroissement qui est allé crescendo et a existé dès le début du $17^{\mathrm{e}}$ siècle avant même l'avènement des deux premiers périodiques précités. Ainsi dès 1613, un certain Barnaby Rich se plaignait de cette " maladie " en ces termes peu équivoques : "Une des maladies de cet âge est la multiplicité des livres, ils sont tellement nombreux qu'on n'est pas capable de digérer l'abondance de matière inanimée qui est déversée et présentée chaque jour au monde " ${ }^{(107)}$.Cette " explosion", " pollution ", " inondation ", " crise " ${ }^{108)}$, etc.....s'est accéléré au $20^{\mathrm{e}}$ siècle et surtout après la deuxième guerre mondiale sous l'impulsion des programmes de recherche et a conduit aux systèmes d'automatisation de la recherche documentaire.

\section{5- Courte présentation des développements post deuxième guerre mondiale}

\section{1-Les années 60-70}

Les années 60-70 verront avec les programmes de recherche spatiaux américains " la naissance d'une informatique centralisée [qui] est associée aux premiers services bibliographiques en ligne. L'essor d'une informatique lourde et centralisée (1960) puis l'essor des systèmes d'ordinateurs à temps partagé (1963) ont permis le développement des premiers services de recherche documentaire automatisés dans le domaine scientifique " (109). A cet effet, on peut citer Dialog ${ }^{(110)}$ qui fut fondé en 1967 par Roger Summit et qui devint en 1972 le premier service commercial en ligne. Suivront d'autres banques en ligne qui ne sont en fait que le résultat de l'informatisation de produits papier: Science Citation Index en 1965, Scientific and Technical Aerospace Reports en 1966-1968 et Nuclear Science Abstracts et US Government Reports Abstracts en 1969-1970 ${ }^{(111)}$.

Les années 70 verront quant à elles les premiers balbutiements d'Internet en 1974 avec le definition du protocole TCP-IP (Transmission Control Protocol/Internet Protocol) qui avait été précédé par le systeme ARPANET (Advanced Research Projects Agency Network) en 1969.Le développement de TCP-IP par Vincent E. Cerf et Robert E. Kahn avait signifié pratiquement le debut de l'Internet civil.En effet, en 1980, Arpanet se divise en deux réseaux distincts, l'un militaire (MILNET, de l'anglais Military Network, qui deviendra le DDN Defense Data Network) et l'autre, universitaire, The National Science Foundation Network (NSFnet), que les militaires abandonnèrent au monde civil. Débarrassé de la tutelle militaire, Internet prend son envol qui associé à la puissance des Etats-Unis où il a été créé, deviendra

\footnotetext{
(106) Idem

(107) Braun T., Zsindely, S.,Growth of scientific litterature and the Barnaby Rich effect, Scientometrics, Vol. 7, $\mathrm{n}^{\circ} 3-6$, p.529,1985

(108) Idem.

(109) Chartron, Ghislaine, Produits et services de la documentation scientifique numérique. Stratégies des acteurs de la chaîne éditoriale, p. $21 \underline{\mathrm{In}}$ : Les Chercheurs et la documentation numérique : nouveaux services et usages, Chartron, Ghislaine, Ed., Edition du cercle de la librairie, Paris, 2002, 271p.

(110) www.dialog.com

(111) Vickery, Brian, A Century of scientific and technical information, Journal of documentation, Vol 55, $\mathrm{n}^{\circ} 5 \mathrm{p}$. 503 Cité par Chartron, Ghislaine, Op Cit.p.21
} 
un phénomène qui n'a pas fini d'être étudié et qui, plus important pour notre recherche, va révolutionner le monde de la publication scientifique et technique .Ceci se matérialisera grosso modo par les développements suivants :

-L'accès distant conversationnel par le biais de terminaux et ensuite par le biais de microordinateurs

-Les réseaux de commutation par paquets. En plus de TCP-IP , X 25 est aussi lancé .C'est un protocole de communication normalisé par commutation de paquets en mode point à point.

-Les premiers logiciels documentaires sont commercialisés pour les gros systèmes. Ils ont pour nom : BASIS, STAIRS, MISTRAL, STATUS.

-L'année 1973 voit l'arrivée des premiers micro-ordinateurs. Ce développement qui sera primordial pour la suite, verra apparaître le Micral de Bull en 1973, le PC de IBM en 1975 et l'Apple II en 1977.Bien que d'une capacité qui, de nos jours, nous paraitrait ridicule, l'Apple II avait un MOS Technology 65028 bit à $1 \mathrm{MHz}$ et un Bus système de $1 \mathrm{MHz}$ et une RAM de $4 \mathrm{Ko}$, extensible à 64 Ko. Mais un problème de prix empêche leur grande utilisation ce qui fait qu'ils sont beaucoup plus présents dans les institutions documentaires surtout qu'une industrie logicielle dédiée se développe. Cette soudaine accélération des offres technologiques donne lieu à la création de nouveaux produits et services pour l'information électronique qui déjà commençait à poindre et s'imposer. Les bibliographies les plus importantes et majeures sous forme papier sont mises en lignes sur des terminaux dédiés. A cet effet, c'est aux EtatsUnis que démarre cette opération avec Medline, Cain (Bibliography of the Northern Ireland Conflict),Chemical Abstracts, Compendex (Computer Engineering Index), American Petroleum Institute Abstracts, Georef (de l'American Geological Institute),Inspec (Information Service for Physics, Electronics, and Computing), etc...En 1972, les banques de données Pascal et Francis sont créées en médecine et en sciences sociales.

La mise en ligne de ces produits entraine la création de serveurs dont la fonction est d'assurer un bon hébergement et une bonne gestion de toute cette masse documentaire. La National Library of Médecine aux Etats-Unis et 1'European Space Agency en Europe font figure d'institutions pionnières dans ces dispositifs.

\section{2-Les années 80-90}

Les années 1980-90 ont vus de leur coté le perfectionnement et la diversification des technologies qui conduisent à multiplier les modes de diffusion et les interfaces d'interrogations des produits documentaires. On notera parmi les plus importants développements :

-Les réseaux se développent dans une optique économique et on note aussi la multiplication des serveurs de banques de données .Parmi ceux là , les plus importants sont : Blaise (British Library), DIMDI (Deutsches Institut für Medizinische Dokumentation und Information), Infoline (Pergamon), STN (Scientific and Technical Networks) et Datastar (Radio suisse).

-La mise en place et la démocratisation des micro-ordinateurs ainsi que la mise en place d'une industrie logicielle y afférente. Ainsi les SIGB (Systèmes Intégrés de Gestion de Bibliothèques), les logiciels documentaires, le développement d'interfaces conviviales 
simplifiant l'interrogation des banques de données, les logiciels hypertexte sont tous développés.

-L'apparition de nouveaux supports de stockage dont le cédérom et qui vont induire des changements radicaux dans la manière dont est perçue la disponibilité des produits documentaires. Les centres de documentation et bibliothèques ont intégrés ces données en créant des réseaux et des postes de consultation cédérom. Ceci aura pour résultat de mettre à portée d'un plus grand nombre l'accès aux banques de données et aussi une plus grande autonomie dans la recherche documentaire.

-La décennie voit aussi et surtout l'importance de plus en plus accrue des réseaux de la recherche scientifique au niveau international. Parmi ces premiers réseaux, on peut citer :

*des réseaux disciplinaires : SPAN (The Space Physics Analysis Network) en Astronomie, HEPNET (High-Energy Physics NETwork) en physique des hautes énergies qui ont étés toujours à la pointe de l'utilisation des informations à travers les réseaux.ArXIv qui de nos jours fait référence en la matière est l'héritier de ces réseaux pionniers.

*des réseaux nationaux : NSFNET (National Science Fondation NETwork) aux Etats-Unis, JANET (Joint Academic NETwork) au Royaume Uni, DFN (Deutsches ForschungsNetz) en Allemagne, NORDUNET (Nordic research and education network) dans les pays scandinaves, SURFNET aux Pays Bas

\section{*des réseaux internationaux: EARN-BITNET (Because It's Time NETwork)}

-Les premiers balbutiements de l'édition électronique avec la norme SGML (Standard Generalized Markup Language)- langage normalisé de balisage généralisé. C'est un langage de codage de données dont l'objectif est de permettre, dans un échange entre systèmes informatiques, de transférer, en même temps, des données textuelles et leurs structures. Il est essentiellement dirigé vers la lecture des documents techniques.

-Enfin en 1989, Tim Berners -Lee lance le World Wide Web au CERN (Organisation Européenne pour la Recherche Nucléaire) à Genève et ce en 1989.Cette invention, ou du moins l'application des hypertextes, va définitivement lancer l'édition électronique .

\section{3-Les années 90-2000}

Les années 1990-2000 voient pratiquement l'envol du Web avec les améliorations successives qui en découlent. La recherche est devenue facilement diffusable et éditable à travers le Web.Cette étape (les années 90 jusqu'au début du $21^{\mathrm{e}}$ siècle) peut être considérée comme cruciale car l'accélération et la disponibilité des moyens vont rendre l'accès et la gestion éditoriale de plus en plus sophistiqués. En Europe, les réseaux de la recherche se renforcent à l'image de RENATER (Le REseau NAtional de télécommunications pour la Technologie l'Enseignement et la Recherche).Le Web après sa création va s'imposer comme protocole de communication sur Internet. Mosaic ensuite Netscape et enfin Internet Explorer apparaissent successivement en tant que client Web. Ils vont permettre une plus grande facilité d'utilisation et une plus grande interactivité. La création par Tim Berners - Lee au CERN en 1991 du langage HTML (Hypertext Markup Language) - langage de balisage hypertexte - qui est un format de données conçu pour représenter les pages web. Les différentes versions de ce 
standard donnent en 1996 un langage plus structuré et qui est le XML (Extensible Markup Language) - langage extensible de balisage - qui est devenu le langage par excellence. A partir de 1995, les moteurs moteur de recherche tels Alta Vista suivi peu après par Google en 1998 prennent leur envol et deviennent des outils indispensables (quoiqu'après la mainmise de Goggle - et son gigantisme - sur l'information ait soulevé des craintes concernant l'orientation linguistique et culturelle qu'il voulait donner à l'information et ses répercussions sur la liberté de tout un chacun de choisir ses références). D'un autre coté, et à l'instar de Facebook, il semblerait que Google utilise les historiques de recherche des lecteurs pour bâtir une base de données des habitudes de ces mêmes utilisateurs, mine d'information qui peut - si ce n'est déjà fait- être vendue aux publicitaires pour cibler leur clientèle. Toute cette masse d'information ne pouvait être exploité sans une uniformisation des normes d'interopérabilité de formats différents. La norme Z39.50 qui est un protocole de communication informatique client-serveur pour rechercher à travers un réseau informatique des informations dans des bases de données a donc été produit afin de permettre cette interopérabilité. En 1995, le Dublin Core qui est un schéma de métadonnées génériques qui permet de décrire des ressources numériques ou physiques et d'établir des relations avec d'autres ressources est lancé par 1'OCLC (Online Computer Library Center) et la NCSA (National Center for Supercomputing Applications) en vue de normaliser la description des ressources sur Internet. Cette même période voit les premiers périodiques électroniques en ligne ainsi que les premières tentatives des versions électroniques des périodiques papier. Les différentes fonctionnalités du Web permettent de nouveaux modèles alternatifs dans la communication scientifique .A cet effet, ArXiv, le serveur de preprints de Los Alamos National Laboratory est lancé en 1991 par Paul Ginsparg. Il est considéré comme l'un des premiers ( si ce n'est le premier) serveur de prépublication et qui a été possible grâce aux fonctionnalités d'Internet. Les physiciens ont toujours été une population utilisatrice de nouvelles technologies ainsi que sa propension à échanger les prépublications librement avant publication. L'avènement d'Internet n'a fait qu'accélérer une habitude qui existait avant sous forme papier (ou par téléphone) et l'a transposé sur le Web. Les Etats-Unis, qui avec le Royaume Uni sont considérés les deux pays ayant le plus fait pour le libre accès bien qu'il soit de création récente, lancent par l'intermédiaire des NIH (National Institutes of Health) PubMed et PubMed Central respectivement en 1998 et 1999.Ils visent à archiver l'ensemble de la production de la littérature médicale. L'Europe de son coté, lance E-Biosci (Portail européen d'accès à l'information factuelle et en texte intégral en sciences du vivant) de l'EMBO (European Molecular Biology Organization). Ces trois exemples parmi les premiers ne sont en fait que le prélude à une croissance exponentielle des différents modèles d'accès libre et dont l'Internet et ses différentes fonctionnalités sont les vecteurs principaux. Ces développements que nous allons essayer de voir dans la prochaine partie de ce travail et qui concerne la naissance du libre accès sous toutes ses formes. Il faut dire que les différents développements que nous avons évoqués ont eu pour culmination l'avènement du libre accès et qui de part ses fonctionnalités diverses est supposé solutionner les problèmes documentaires des chercheurs. 


\section{Conclusion}

Le besoin vital pour la survie et la perpétuation de la race humaine a suivi de toute évidence la progression (nous serons tenté de dire la sophistication) des moyens que l'homme a lui-même crée afin d'arriver à ses fins. L'Homme primitif n'avait pas une langue avec laquelle il communiquait. Il se contentait de gestes pour faire parvenir son message, gestes qui étaient de toute évidence loin d'être sophistiqués et limitait le pouvoir de faire parvenir un message clair et encore moins de le distribuer à une grande communauté. D'ailleurs, partager un message, une connaissance, un Savoir n'étaient pas des premières priorités des hommes à cette époque étant donné l'extrême limitation des contacts et déplacements d'un monde aux besoins et moyens rudimentaires. Après cette période où l'Homme menait une vie très primaire et dont les besoins étaient en conséquence simples, vint une étape où il apprit à parler et s'exprimer avec des sons et des mots ( ou ce que nous appellerions mots dans notre langage actuel).Cette sophistication par rapport à l'étape précédente où le geste pouvait être interprété différemment, était un jalon supplémentaire dans sa quête vers une sophistication et une abstraction de plus en plus grande. Malgré toute cette progressive avancée, l'Homme était toujours sous la menace de ne pas pouvoir perpétuer cette information étant donné que la mémoire humaine était faillible. C'est alors que vint l'étape des premiers enregistrements de cette information sur différents supports et qui n'étaient pas des supports dont la maniabilité était le point fort. Ces messages étaient à ce stade représentés non pas par des mots (ce qui était encore loin de la sophistication en cours) mais des signes faits à la main au sol ou sur des écorces d'arbres. Ces signes fugaces n'ont pas, de toute évidence survécus, et la seule trace est représentée par des peintures trouvées dans des grottes. Il nous reste de ces dessins de très beaux spécimens dont les peintures rupestres du Hoggar par exemple sont un bel exemple. Apres cette étape, la pictographie en passant de l'iconographie à l'écriture synthétique et enfin l'écriture analytique représenta une autre avancée qui elle-même était graduelle. Tous ces développements successifs qui ont conduit l'Homme de l'état primaire vers une sorte de synthétisation de sa pensée ont culminé avec l'avènement de l'alphabet sous ses différentes formes et présentation. En effet, on peut utiliser le mot culmination car le bond représenté par l'avènement de l'alphabet par rapport aux systèmes précédents est un bond qualitatif. Il est qualitatif dans le sens où, nonobstant les avantages inhérents à ce genre de système, il représente un grand pas vers l'abstraction de la pensée. En effet, l'alphabet a permis à l'Homme d'améliorer sa communication en passant d'un système d'approximation extrême où un signe, un dessin ou une association de plusieurs de ces mêmes représentations pouvait être interprété de différentes manières à un système où chaque signe représente un son. Cette amélioration aussi bien quantitative et aussi qualitative n'a pas été, loin s'en faut, uniforme, les différents alphabets ayant vu des améliorations et des changements successifs et récurrents.

Ces premiers jalons que l'Homme a utilisé pour communiquer et dont l'alphabet est la culmination indurent de ce fait d'autres besoins auxquels il fallait répondre. Parmi ces nouveaux besoins, une plus grande masse d'information à la portée d'un plus grand nombre et avec une (relative) plus grande disponibilité et auxquels les supports utilisés jusque là ne pouvait répondre ni quantitativement ni qualitativement. Quantitativement car les différents matériaux tendaient à se raréfier et à être cher (comme la soie) et surtout qualitativement car ils ne répondaient pas à la maniabilité dont on se serait attendu. La prochaine étape dans cette continuelle amélioration des moyens de communication concernera la recherche de matériaux adéquats à utiliser pour stocker cette nouvelle masse d'information. Le parchemin et surtout le vélin étant extrêmement cher et demandant de grandes quantités pour des résultats pas très effectifs, la recherche d'un autre matériau s'imposa. Le papier, bien qu'inventé en Chine au $2^{\mathrm{e}}$ siècle, n'atteignit l'Europe et les Amériques qu'en 1150 et 1690 respectivement. Son 
introduction constitua sans nul doute une grande avancée tant dans une sorte de démocratisation du savoir que du point de vue de sa maniabilité qui dépassait de loin les autres supports que nous avons cité. Sa surface plane et lisse permettait d'y " écrire " d'une manière bien plus confortable et sur de plus grandes surfaces. Cette invention qui avait mis tant de temps avant d'atteindre le monde occidental allait s'avérer un support dont la longévité, le coté pratique et économique sont toujours d'actualité et ce n'est pas les différentes prophéties ayant pronostiqué sa disparition et qui reviennent cycliquement qui nous contredirons.

Si l'utilisation du papier a constitué une grande avancée en ce qui concerne le support sur lequel était recopié l'information, il n'en demeure pas moins que la manière dont ceci était fait ne se distinguait pas par une grande rapidité, ni conformité. Les manuscrits étaient recopiés à la main .On devine sans peine que cette manière de faire comportait des lacunes dont la plus importante était la cadence avec laquelle ces manuscrits étaient recopiés sans parler de l'exactitude des textes recopiés et qui étaient sujets à des omissions et même des changements (des fois intentionnels) dont les raisons étaient diverses. Cette période transitoire avait connu une sorte de flottement dû à l'apparition d'un nouveau support qui permettait une meilleure transmission de l'information mais qui était freiné par une technique très lente qui ne profitait pas de cette avancée. Les scribes de l'époque n'arrivaient pas, de par leur technique à imprimer une cadence plus rapide face aux besoins de plus en plus grand d'une société qui sortait d'une période de léthargie représentée par le Moyen Age. C'est alors que pour répondre à cette demande de plus en plus pressante, des tentatives de mécanisation et automatisation du processus d'écriture manuelle furent entreprises. Ces tentatives peuvent être schématisées en deux techniques qui eurent le mérite de préparer le terrain à une automatisation complète du processus d'écriture .Ce sont la xylographie et ensuite les caractères mobiles dans cet ordre car ils représentaient deux facettes d'une même technique. Pour être plus clair, la xylographie ( ou gravure sur bois ) était une technique de reproduction qui utilisait le bois. Les caractères mobiles quant à eux représentaient un bond aussi bien qualitatif que quantitatif. En effet, ils pouvaient être réutilisés indéfiniment, contrairement à la xylographie. Cet acquis technique peut être comparé, toutes proportions gardées, au passage de l'écriture idéographique à l'alphabet où un signe équivalait à un concept à un système alphabétique où chaque lettre équivalait à un son et l'ensemble de lettres donnaient un mot. Ces deux techniques qui ont suivis l'une l'autre ont abouti à ce qui est considéré, à juste titre d'ailleurs et une unanimité quasi consensuelle, comme l'une des plus importantes innovations de l'histoire de l'Humanité : l'imprimerie. Cette invention a, au-delà des considérations techniques, permis de rendre le savoir plus accessible à un plus grand nombre après qu'il eut été réservé à une caste de privilégiés. Si cette idée nous parait en ce $21^{\mathrm{e}}$ siècle une idée pratiquement suranné et qui va de soit, il n'en demeure pas moins qu'elle constituait à l'époque un concept révolutionnaire. Elle a définitivement sorti le savoir de la mainmise d'une minorité (qui l'utilisait pour dominer) et aussi d'une caste (l'Eglise).Depuis et jusqu'en 1665 , l'imprimerie a servi à transcrire sous différentes formes des livres ou ce qui est plus professionnellement appelé des monographies. C'était la seule manière pour un chercheur de communiquer le résultat de ces recherches et de les faire connaître. Mais ceci avait le défaut de ne pas faire parvenir rapidement ni d'assurer la paternité d'une découverte à celui qui le meritait. Dès le début, s'est posé cette problématique de la vitesse de la communication surtout dans des spécialités comme la physique, l'astronomie ou la médecine où une découverte appartient à celui qui l'a annoncé à ses collègues le premier. On a vu que les savants devaient se résoudre à des expédients pour s'assurer de la primauté des découvertes scientifiques. Parallèlement, à cette période et à ces premiers balbutiements de communications organisés, s'organisait au sein de ces groupes de savants une sorte de groupe 
de contact dont le but était justement de diffuser et disséminer ces découvertes, ces experimentations. Si au début ,les philosophes, tels qu'ils étaient appelés ,s'organisaient d'une manière informelle *, la moitié du $17^{\mathrm{e}}$ siècle a vu la fondation des plus importantes sociétés savantes qui allaient canaliser, en quelque sorte, ces efforts et leur donner un cachet plus officiel que celui des collèges invisibles qui n'étaient que des réunions de savants discutant et expérimentant leurs découvertes. Deux de ces académies fondées au milieu du $17^{\mathrm{e}}$ siècle (The Royal Society of London et l'Académie des Sciences de Paris) ont joué un rôle important et le jouent encore de nos jours quoique chacune dans un registre différent. $\mathrm{Au}$ début ces académies se contentaient de faire lire durant leurs séances les différentes recherches qui leur avait été envoyées. Les représentants de ces mêmes académies dans les différentes villes où elles avaient une sorte d'antenne en faisait de même. On voit d'ici les problèmes qui se posent dans un tel schéma et dont le plus évident est le manque d'exhaustivité de la transmission des informations étant donné que les problèmes de transports, de disponibilité, etc...faisaient de cette opération une opération très aléatoire quant aux résultats et en fait avait l'effet contraire que celui de diffuser et disséminer l'information et surtout assurer le rôle d'archivage et pérennité de l'information sans compter le rôle primordial de la primauté de la découverte.

Devant ces difficultés de coordination, de disponibilité et de scientificité même, les scientifiques se devaient de trouver un moyen plus rapide, plus sûr et plus à même de répondre à leur attente. Il existait alors des personnes qui de par leur position pouvaient faire ce travail d'éditeurs (quoique ni la nomination ni la fonction n'existaient officiellement à l'époque).C'est alors que des érudits comme Renaudot, Mersenne, De Sallo, Haak, et surtout Henry Oldenburg entreprirent de fonder des publications périodiques paraissant à des intervalles réguliers. La date de 1665 est unanimement acceptée comme étant la date de fondation des premiers périodiques scientifiques contrôlés par les pairs ${ }^{* *}$ Bien que deux périodiques aient parus pratiquement à la même période, c'est The Philosophical Transactions qui est considéré comme le premier périodique scientifique bien qu'il ait

\footnotetext{
* C'est à partir de ces réunions informelles qu'est née l'expression "College invisible".Cet appelation a été utilisé la première fois en 1646 par Robert Boyle (le célèbre physicien et chimiste, considéré comme le père de la philosophie naturelle) dans une de ces correspondances ayant précédé la creation de la Royal Society de London. ( Stimson, Dorothy, Dr. Wilkins and the Royal Society, Journal of. Modern History, ${ }^{\circ}{ }^{\circ} 111, p .539-63,1931$ ) Plus recemment, Derek De Solla-Price en 1963 (Little science, big science, Columbia University Press, New York, XVI-119 p.) et Diana Crane en 1972 (Invisible college :diffusion of knowledge in scientific communities,Chicago University Press, X-213 p. ) ont abordé le sujet. Crane montre l'existence de réseaux de communication informels entre des groupes chercheurs restreints seniors ayant accès à l'information privilégiée. Cette information était diffusée rapidement grâce à des réseaux de contact et à un échange de littérature grise. Nous verrons que les nouveaux réseaux numériques permettent de rendre plus visibles, plus rapides et " plus démocratiques " les collèges invisibles. (Teasdale, Guy, Vers l'édition e-savante ? Etude des defis du passage à l'Internet, p.168-182 In :La Publication en ligne, Charlotte Nikitenko [et]Peter Stockinger Ed., Les Cahiers du numérique, $\operatorname{Vol} 1, \mathrm{n}^{\circ} 5,223 \mathrm{p}, 2000$

Nous ouvrons une parenthèse pour clarifier un point. Nous parlons dans notre recherche des journaux scientifiques contrôlés par les pairs car contrairement aux journaux périodiques que l'on appelle la presse (quotidienne et autres), les périodiques scientifiques incluent dans leur processus de publication un point important et qui est la certification de l'information. Par certification, nous entendons le fait que l'information publiée est contrôlée par des scientifiques spécialistes dans le domaine et qui jugent est ce que la recherche en question ajoute au savoir humain .Les experts délivrent donc un quitus de conformité aux critères acceptées de la Science. De part cette définition, on peut voir les points de divergence que ceci peut engendrer étant donné que la Science est supposée être neutre, logique, objective et reposant sur des faits scientifiques avérés mais nous verrons dans la troisième partie de ce travail que ce n'est pas toujours le cas et que la nature humaine étant ce qu'elle est , nombres de fautes ( intentionnelles et non intentionnelles ) peuvent être commises.
} 
antidaté Le Journal des Sçavans de deux mois. Après cette innovation, le nombre de périodiques s'accrut en nombre et surtout en genre. Pour être plus clair, si le nouveau medium de part sa facilité et surtout sa rapidité de publication séduit et les éditeurs et les auteurs et de ce fait induit une augmentation en nombre, l'autre caractéristique qui lui est inhérente est sans conteste la spécialisation. Il faut signaler que les " philosophes " de l'époque avait un éclectisme qui paraitrait incongru de nos jours. Il n'était pas bizarre de trouver un philosophe et qui était mathématicien, philosophe et en même temps médecin !!! Cet état de fait n'était pas sans déplaire aux savants qui voyaient en cet amateurisme une dégradation du niveau et firent tout pour en exclure les chercheurs occasionnels .A partir de ce moment (aux environs du $18-19^{\mathrm{e}}$ siècle) la science a connu une tendance à la spécialisation qui ne s'est pas démentie et qui en fait ne fait qu'augmenter de plus en plus de nos jours .D'ailleurs, le nombre de plus en plus grand de périodiques ne serait, selon certaines hypothèses, que le résultat de cette hyperspécialisation, selon une expression utilisée par des chercheur. Cela se passerait schématiquement de la manière suivante: dans chaque spécialité se créent des sous spécialités, résultant de la recherche. Ces sous spécialités entreraient en conflit (scientifique) avec la spécialité à laquelle elles appartiennent et fondent une nouvelle spécialité qui lancerait un nouveau périodique. Cette profusion et accroissement est allé crescendo jusqu'à atteindre un niveau qui ne pouvait plus être géré par les moyens classiques. Cet accroissement a vraiment décollé avec la fin de la deuxième guerre mondiale et l'essor de la recherche scientifique entrepris par les pays occidentaux au sortir de la guerre. C'est alors l'essor des différents efforts d'automatisation et d'informatisation qui ont vus le jour surtout dans les années 60 et qui ont culminé avec les bases de données bibliographiques censées gérer le flot presqu'incontrôlable de la masse documentaire. Tous ces développements ont eu de multiples répercussions sur la manière dont est appréhendé l'accès à l'information qui tout en devenant plus facile, plus accessible n'en a pas moins induit d'autres problématiques inhérentes à cette même facilité dont entre autres les nouvelles restrictions de l'accès à l'information qui n'existaient pas dans l'univers papier (voir les différentes Directives Européennes d'accès à l'information, le Digital Millenium Copyright Act aux Etats-Unis,le DOI,etc....).L'avènement d'Internet grand public aux environs du milieu des années 90 a permis une ouverture et une plus grande démocratisation de l'accès à l'information car elle a mis l'information à portée d'un plus grand nombre ( les dernières statistiques donnent $28.7 \%$ de la population mondiale qui a accès à Internet. Ces chiffres doivent être pondérés, il est vrai, avec des taux de pénétration qui varient grandement de région à région : ils sont respectivement de $77.4 \%$ en Amérique du Nord à $10.9 \%$ en Afrique ${ }^{(112)}$. Ce dernier développement que nous vivons de

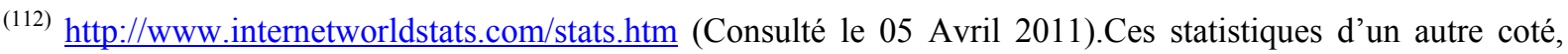
peuvent etre sous un autre angle.En effet, si un grand ecart dans le taux de penetration entre le Nord et le Sud existe de meme que dans le pourcentage de ceux qui utilisent Internet mondialement - les $2 / 3$ sont localisés en Europe et en Asie-, il est un chiffre qui reconforterait l'idée qu'Internet tendrait à se democratiser.En effet, on remarque que la progression entre 2000 et 2010 est beaucoup plus importante dans ce qui pourrait etre appelé le monde en voie de developement ( Afrique, Moyen Orient et Amerique latine /Caraibes ) que ce qu'il est dans le monde developé.Ainsi l'Afrique a enregistré une progression de 2,357.3\%, le Moyen Orient $1,825.3 \%$ et l'Amerique latine /Caraibes $1,032.8 \%$.Ces chiffres encourageants sont à comparer aux statistiques du monde developé à l'instar de l'Amerique du Nord qui n'enregistre qu'une progression de 146.3\%.Deux lectures peuvent etre faites de ces chiffres diametralement opposés : soit le monde en voie de developement fait des progrés dans son rattrapage du retard accumulé ou le monde developé a atteint une sorte de saturation du taux de penetration. A titre d'exemple les pays scandinaves ont pratiquement atteint ce taux de saturation et de quasi couverture de toute le population, ils affichent $92.5 \%, 94.8 \%, 97.6 \%$ pour respectivement la Suéde, la Norvege et l'Islande.D'ailleurs les autres pays scandinaves affichent eux aussi des chiffres tout aussi impressionnants (Danemark $86.1 \%$, Finland 85.3\%).De toute evidence, ces chiffres de quelque angle examiné montrent une preponderance d'Internet dans la vie des nations et son importance quant à son developement et sa politique de recherche.
} 
nos jours a conduit à une complète et profonde refonte de la manière dont est conduit la recherche .Le chercheur de nos jours a beaucoup plus tendance à consulter Internet ne serait ce que pour avoir une idée du sujet qu'il investigue. Si au début, se référencer à Internet était considéré comme une solution de facilité, de nos jours le chercheur est obligé, ne serait que par le degré de facilité que cela permet, d'utiliser Internet. Nombre de bases de données et de périodique ne sont disponibles que sur Internet et certains sont nés électroniques et n'ont pas de contrepartie papier (comme Psycoloquy).Le résultat de cet état de fait est que le périodique électronique ( et la recherche sur Internet en général) est devenu en peu de temps une nécessité du chercheur moderne car ils permettent un gain de temps, de déplacement, de communicabilité entre chercheurs dont n'auraient surement jamais rêvé les premiers érudits et scientifiques qui avaient fondé les premiers périodiques au $17^{\mathrm{e}}$ siècle. 


\section{Bibliographie}

Bazerman, Charles, Shaping written knowledge: the genre and activity of the experimental article in science, Madison, [Wisconsin, U.S.A.], University of Wisconsin Press, 1988, 400p.

Bordons, Maria, Gomes, Isabel, Towards a single language in science ? A Spanish view, Serials, Vol.17, n², p.189, Juillet 2004

Boyer, Jacques, The Founder of the first scientific journal,The Popular science monthly, p.690,Mars 1894

Braun T., Zsindely, S.,Growth of scientific litterature and the Barnaby Rich effect, Scientometrics, Vol. 7, n³-6, p.529I

Bush, Vannevar, As we may think, The Atlantic, Juillet 1945. Disponible à : http://www.theatlantic.com/doc/194507/bush

Chartron, Ghislaine, Produits et services de la documentation scientifique numérique. Stratégies des acteurs de la chaîne éditoriale, $\underline{I n}$ : Les Chercheurs et la documentation numérique : nouveaux services et usages, Chartron, Ghislaine, Ed., Edition du cercle de la librairie, Paris, 2002, 271p.

Diringer David, The Book before printing: ancient, medieval and oriental, New York, Dover Publishing, 1982, p.624p.

Ebert, Myrl, The Rise and Development of the American Medical Periodical 1797-1850, Bulletin of the Medical Library Association, Vol. 40, n³, p.245, Juillet 1952

Febvre Lucien [et] Martin Henri-Martin, L'Apparition du livre, Paris, Albin Michel, 1971, $600 \mathrm{p}$.

Fielding, Garrison, H., The medical and scientific periodicals of the $17^{\text {th }}$ and $18^{\text {th }}$ centuries, Bulletin of the History of medecine, Vol.2, n5, p.296, Juillet 1934

Fjällbrant, Nancy, Scholarly communication : historical developements and new possibilities, [Proceedings], Scholarly Communication in Focus, June-July 1997, IATUL conference, Trondheim.

Disponible à : http://educate.lib.chalmers.se/IATUL/proceedcontents/fullpaper/nfpaper.html

Francis André, Libre accès aux savoirs =open access to knowledge, Paris, Futuribles, 2005, $74 \mathrm{p}$.

Garfield Eugene, Has scientific communication changed in 300 years ? , Current contents, nº, p. 11, 25 Février 1980

Guédon, Jean Claude, In Oldenburg's Long Shadow: Librarians, Research Scientists,

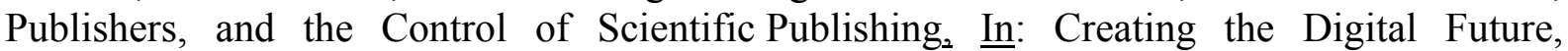
Proceedings of 138th ARL Membership Meeting,Toronto [Ontario], May 23-25 2001, Baker, Shirley K. Ed. 


\section{Disponible à : $\underline{\text { http://www.arl.org/resources/pubs/mmproceedings/138guedon.shtml }}$}

Haffemayer, Stephane, Transferts culturels dans la presse européenne du XVIIeme siècle, Le Temps des medias, Vol. 2, n¹1, p.27-28

Hall, Marie Boas, Henry Oldenburg : shaping the Royal Society, Oxford University Press, New York, 2002, 386p.

Hall, Marie Boas, Oldenburg and the art of scientific communication, The British Journal for the History of Science, Vol.2, p 277-290,1965

Harcourt, Brown, Scientific organizations in seventeenth century France, Baltimore, The Willams \& Wilkins Co., 1934, 306 p.

Harmon, J.E., Evolution of the scientific paper, IPCC 92 Santa Fe, Crossings Borders, Conference Record, 29 sept.-03 oct.1992, p.468-475.

Disponible à l'adresse suivante http://ieeexplore.ieee.org/Xplore/login.jsp?url=http\%3A\%2F\%2Fieeexplore.ieee.org\%2Fiel4 \%2F5507\%2F14794\%2F00673079.pdf\%3Farnumber\%3D673079\&authDecision=-203

Hill, John, A Review of the Works of the Royal Society of London; containing Animadversions on such of the Papers as deserve particular Observation. London: R. Griffith., 1751,292 p.

Houghton Bernard, Scientific periodicals : their historical development, characteristics and control, London, Clive Bingley, 135 p.

Jean Martin ,Henri, Histoire et pouvoirs de l'écrit, Paris, Albin Michel, 1996, 546 p.

Kennedy, Donald, Good news-and bad, Science, Vol. 311, n . 5758, p. 145, 13 Janvier 2006

Kronick, D.A., A History of scientific and technical periodicals, New York, Scarecrow, 1962, $274 \mathrm{p}$.

Kronick, David A., Litterature of the life sciences : the historical background, Bulletin of the New York Academy of Medecine, Vol 60, nº 9, p.866-867, Novembre 1984

Kronick, D.A., Ed., Scientific and technicals periodicals of the seventeenth and eighteenth centuries : a guide, Metuchen [New Jersey, USA] ; London, The Scarecrow Press, 1991, 332 p.

Kronick, D.A., " Devant le déluge " and others essays on early modern scientific communication, Lanham [Maryland, USA] ; Oxford, The Scarecrow press, 2004, 335 p.

Kuhn, Thomas, The Structure of scientific révolution, Chicago, University Of Chicago Press, 1962, 226p.

Lechene, Robert, L'Imprimerie: de Gutenberg à l'électron, [Paris], La Farandole, 1972, 205 p. 
Mac Murtrie, Douglas C., The Book: the story of printing [and] bookmaking, New York, Oxford University Press, 1967, 710 p.

Manten, A.A., Developement of european scientific journal publishing before 1850, In: Meadows, A.J. Ed., Development of science publishing in Europe, Amsterdam, Elsevier, $1980,269 \mathrm{p}$

Meadows, A. J, Communication in Science, London, Butterworths, 1974, 248 p.

Moreri, Louis, Grand dictionnaire historique..., Drouet, Paris, 1759, t.IX, p.96, in- folio

Newspapers of the world online : U.S. and international perspectives, Valravens, Hartmut Ed. ,Munchen, K.G. Saur, 2006, p.159

Pfeiffer, Jeanne, Vittu, Jean - Pierre, Les Journaux savants, formes de la communication et agents de la construction des savoirs ( $17 \mathrm{e}-18^{\mathrm{e}}$ siècles), Dix-huitième siècle, Vol.1, $\mathrm{n}^{\circ} 40$, p.283.,2008

Presse (La) : quatre siècles de jeunesse !

Disponible à : http://www.wan-press.org/article6468.html (Consulté le 12/02/2011)

Querard, Joseph Marie, La France littéraire : ou dictionnaire bibliographique des savants......, Paris ,Firmin Didot, 1836, 568 p.

Schöpfel Joachim, Ed., La Publication scientifique: analyses et perspectives, Paris : Lavoisier, 2008, 368 p

Timperley, C.H., A Dictionnary of printers and printing, with the progress of literature ancient and modern, biblographical illustrations, etc. etc.,H.Johnson, London, 1849, 996 p.

Toffler Alvin, The Third wave, Londres, Morrow, 1980, 544p.

Vickery, Brian, A Century of scientific and technical information, Journal of documentation, Vol 55, n ${ }^{\circ}$ p. 503

Watson, J.J., English, the international language of science, Journal belge de radiologie, Vol 69, $\mathrm{n}^{\circ} 3, \mathrm{p} 195-200,1986$ 


\section{DEUXIEME PARTIE}

EVOLUTION ET OUTILS DU LIBRE ACCES 


\section{EVOLUTION ET OUTILS DU LIBRE ACCES}

Introduction

Bien que de création récente (la première archive ouverte lancée par Paul Ginsparg, ArXiv, date de 1991), le mouvement pour le libre accès à l'information scientifique et technique a remarquablement épousé, chronologiquement parlant, l'avènement d'Internet. Les prémices de la création de ce dernier remontant aux années 60 sous une forme militaire, Internet n'est, cependant, devenu le phénomène incontournable que l'on connaît de nos jours ( et dont l'influence va crescendo et l'omniprésence dans notre vie de tous les jours est sans cesse plus grande et plus présente ), qu'au début des années 90.Cette quasi simultanéité de l'occurrence a, d'un autre coté, coïncidé avec un accroissement vertigineux des prix des périodiques scientifiques surtout dans la filière S.T.M. (Science, Technique, Médecine).Cet accroissement aussi vertigineux qu'handicapant a entrainé des mouvements de désabonnement tels que les bibliothèques se sont retrouvées prisonnières d'une spirale incapacitante de politiques de désacquisition.Ces deux événements (augmentation incontrôlée des prix des périodiques et même des fois des monographies et avènement d'Internet) ont précipité le dit mouvement. Les bibliothèques, partie prenante du libre accès de part leur position de réceptacle de la production scientifique des chercheurs, se sont retrouvées aux avant postes de cette révolution dans la publication scientifique. Elles ont étés les premières à souffrir, et en même temps supporter, cet état de fait. Elles se trouvaient entre le marteau (de l'ire des lecteurs) et l'enclume (des prix exorbitants imposés par les grands groupes éditoriaux oligopoles).Nombre de recherches ont documenté cet aspect et essayé de l'expliquer avec un seul résultat: il devenait insupportable et la communauté se devait de réagir. L'A.R.L. (Association of Research Libraries), association américaine très active dans la défense des bibliothèques académiques a calculé les augmentations sur une période cruciale de cinq ans (1995-2000). Le tableau suivant représente cette situation de la manière la plus claire ${ }^{(1)}$ :

\begin{tabular}{|l|r|r|c|}
\hline & \multicolumn{1}{|c|}{$\mathbf{1 9 9 5}$} & \multicolumn{1}{|c|}{$\mathbf{2 0 0 0}$} & \multicolumn{1}{c|}{$\begin{array}{c}\text { Change } \\
\mathbf{1 9 9 5 - 2 0 0 0}\end{array}$} \\
\hline Brain Research & $\$ 10,181$ & $\$ 16,344$ & $60.5 \%$ \\
\hline Biochim. Biophys. Acta & $\$ 7,555$ & $\$ 11,362$ & $50.4 \%$ \\
\hline Chem. Phys. Letters & $\$ 5,279$ & $\$ 9,029$ & $71.0 \%$ \\
\hline Tetrahedron Letters & $\$ 5,119$ & $\$ 8,859$ & $73.1 \%$ \\
\hline Eur. Jrnl. of Pharmacology & $\$ 4,576$ & $\$ 7,329$ & $60.2 \%$ \\
\hline Gene & $\$ 3,924$ & $\$ 6,974$ & $77.7 \%$ \\
\hline Inorganica Chim. Acta & $\$ 3,611$ & $\$ 6,302$ & $74.5 \%$ \\
\hline Intl. Jrnl. of Pharmaceutics & $\$ 3,006$ & $\$ 5,589$ & $85.9 \%$ \\
\hline Neuroscience & $\$ 3,487$ & $\$ 5,875$ & $68.5 \%$ \\
\hline Theoretical Computer Science & $\$ 2,774$ & $\$ 4,317$ & $55.6 \%$ \\
\hline Jrnl. of Exp. Marine Bio. \& Eco. & $\$ 1,947$ & $\$ 3,280$ & $68.5 \%$ \\
\hline
\end{tabular}

Fig 4-Tableau des augmentations des prix des periodiques (ARL)

Comme on peut le voir, les augmentations sont très sensibles et dépassent, et de loin, les niveaux d'inflation de la période 1995-2000.On peut pousser plus loin l'analyse et voir que la moyenne de ces augmentations a été de $67,80 \%$ soit une augmentation de $2 / 3$ des prix. C'est la filière de sciences pharmaceutiques qui a le plus souffert avec un taux de $85.9 \%$ pour International Journal of Pharmaceutics, alors que d'autres filières comme la génétique (avec Gene ) avec un taux de $77.70 \%$ n'ont pas été épargnées. Il est intéressant de remarquer que

(1) www.arl.org/create/archive/ressources/Tables_Pages/JusteTables/TABLE_STM.html 
Brain Research, le périodique scientifique le plus cher au monde, n'a augmenté que de 60.50 $\%$, passant de $10181 \$$ à $16344 \${ }^{*}$ ce qui est déjà un prix et une augmentation mirobolante mais n'a pas atteint les sommets d'augmentation de International Journal of Pharmaceutics qui a pratiquement doublé (augmentation de 85.9\%).

D'un autre coté, un travail plus récent et plus exhaustif portant sur la même problématique montre en fait que la situation est loin de se résoudre mais en fait tend à s'aggraver tel que le montre le tableau suivant :

\begin{tabular}{|l|c|c|c|c|c|c|c|}
\hline \multicolumn{1}{|c|}{ Spécialité } & 2002 & 2003 & 2004 & 2005 & 2006 & 2007 & 2008 \\
\hline Agriculture & 631 & 647 & 773 & 833 & 889 & 964 & 1034 \\
\hline Anthropologie & 300 & 415 & 385 & 415 & 432 & 474 & 530 \\
\hline Arts \& Architecture & 154 & 144 & 176 & 188 & 210 & 222 & 243 \\
\hline Astronomie & 1256 & 1123 & 1289 & 1340 & 1488 & 1551 & 1671 \\
\hline Biologie & 1089 & 1207 & 1292 & 1406 & 1536 & 1674 & 1810 \\
\hline Botanique & 880 & 830 & 1059 & 1165 & 1256 & 1364 & 1491 \\
\hline Business \& Economie & 527 & 618 & 677 & 733 & 781 & 830 & 897 \\
\hline Chimie & 2432 & 2635 & 2582 & 2748 & 2965 & 3187 & 3490 \\
\hline Education & 300 & 309 & 379 & 419 & 460 & 509 & 545 \\
\hline Ingénierie & 1305 & 1561 & 1452 & 1561 & 1652 & 1767 & 1919 \\
\hline Sciences de l'Alimentation & 897 & 978 & 1133 & 1239 & 1337 & 1409 & 1554 \\
\hline Généralités sciences & 810 & 865 & 910 & 975 & 1040 & 1142 & 1213 \\
\hline Travaux généraux & 181 & 186 & 121 & 132 & 137 & 147 & 158 \\
\hline Géographie & 746 & 808 & 812 & 855 & 909 & 989 & 1089 \\
\hline Géologie & 1012 & 1079 & 1147 & 1245 & 1312 & 1413 & 1521 \\
\hline Santé & 808 & 846 & 932 & 1010 & 1105 & 1207 & 1330 \\
\hline Histoire & 132 & 143 & 171 & 184 & 197 & 215 & 238 \\
\hline Langage et littérature & 120 & 129 & 159 & 173 & 185 & 200 & 221 \\
\hline Droit & 159 & 176 & 198 & 206 & 231 & 257 & 275 \\
\hline Bibliothéconomie et sciences de & 286 & 319 & 360 & 403 & 424 & 453 & 487 \\
l'information & & & & & & & \\
\hline Mathématiques et Informatique & 981 & 1039 & 1109 & 1181 & 1235 & 1323 & 1411 \\
\hline Sciences militaires et navales & 346 & 472 & 454 & 512 & 598 & 623 & 634 \\
\hline Musique & 96 & 98 & 101 & 125 & 129 & 141 & 161 \\
\hline Philosophie et religion & 156 & 146 & 210 & 452 & 483 & 529 & 584 \\
\hline Recréation & 358 & 389 & 437 & 467 & 509 & 551 & 598 \\
\hline Psychologie & 146 & 116 & 200 & 227 & 247 & 277 & 322 \\
\hline Sociologie & 332 & 361 & 411 & 450 & 487 & 533 & 586 \\
\hline Technologie & 1151 & 1232 & 1330 & 1432 & 1535 & 1640 & 1776 \\
\hline Zoologie & 973 & 977 & 958 & 1032 & 1115 & 1206 & 1311 \\
\hline & & & & & & & \\
\hline
\end{tabular}

Fig.5-Tableau augmentation en $\$$ des prix des périodiques 2003-2008 ${ }^{(2)}$

\footnotetext{
* Le prix de l'abonnement annuel de Brain Research avait atteint en date du 02/02/2012, 24047 \$ pour tous les pays à part pour le Japon, les pays européen et l'Iran. Les prix en Europe étaient, à la même date, de $21440 €$ ou 28433.79 \$.Enfin, son prix au Japon était de 2853700 Yen ou 37109.01 \$. Informations obtenues du site d'Elsevier qui gère ce périodique.

http://www.elsevier.com/wps/find/journaldescription.cws home/622287/bibliographic

A titre d'exemple, cette somme en Yen représente en monnaie nationale la somme de 2804 961,85 DA !!!! On imagine mal l'acquisition en Algerie d'un periodique dont le prix avoisinerait la somme de 300000000 de nos centimes. (http://www.xe.com/ucc/convert/?Amount=2853700\&From=JPY\&To=DZD)

(2) Greco, Albert, Ed., The State of scholarly publishing: challenges and opportunities, New Brunswick [N.J., U.S.A] Transactions publisher, 2009, p.XI
} 
Ce qui nous donne encore pour ce deuxième tableau une augmentation constante des prix qui d'année en année, enregistrent des courbes ascendantes qui ne se démentent pas, à part pour certaines spécialités comme Anthropologie, Astronomie, Botanique, Chimie, etc.. qui enregistrent de faibles baisses sur une année et aussitôt reprises l'année d'après. La seule exception est " Travaux généraux " qui enregistre non pas une baisse mais plutôt des prix en dents de scie tantôt augmentant et tantôt baissant. D'ailleurs, c'est la seule spécialité dont les prix ont baissé entre 2002 et 2008.Ces prix sont représentés dans le tableau suivant avec le pourcentage d'augmentation (ou baisse pour " Travaux généraux ") entre les années 2002 et 2008.

\begin{tabular}{|c|c|c|c|}
\hline Spécialité & 2002 & 2008 & $\%$ augmentation \\
\hline Agriculture & 631 & 1034 & $+64 \%$ \\
\hline Anthropologie & 300 & 530 & $+77 \%$ \\
\hline Arts \& Architecture & 154 & 243 & $+58 \%$ \\
\hline Astronomie & 1256 & 1671 & $+33 \%$ \\
\hline Biologie & 1089 & 1810 & $+66 \%$ \\
\hline Botanique & 880 & 1491 & $+70 \%$ \\
\hline Business \& Economie & 527 & 897 & $+58 \%$ \\
\hline Chimie & 2432 & 3490 & $+43 \%$ \\
\hline Education & 300 & 545 & $+82 \%$ \\
\hline Ingénierie & 1305 & 1919 & $+47 \%$ \\
\hline Sciences de l'Alimentation & 897 & 1554 & $+73 \%$ \\
\hline Généralités sciences & 810 & 1213 & $+50 \%$ \\
\hline Travaux généraux & 181 & 158 & $-12 \%$ \\
\hline Géographie & 746 & 1089 & $+46 \%$ \\
\hline Géologie & 1012 & 1521 & $+50 \%$ \\
\hline Santé & 808 & 1330 & $+65 \%$ \\
\hline Histoire & 132 & 238 & $+80 \%$ \\
\hline Langage et littérature & 120 & 221 & $+84 \%$ \\
\hline Droit & 159 & 275 & $+73 \%$ \\
\hline Bibliothéconomie et sciences de l'information & 286 & 487 & $+70 \%$ \\
\hline Mathématiques et Informatique & 981 & 1411 & $+44 \%$ \\
\hline Sciences militaires et navales & 346 & 634 & $+83 \%$ \\
\hline Musique & 96 & 161 & $+68 \%$ \\
\hline Philosophie et religion & 156 & 584 & $+274 \%$ \\
\hline Recréation & 358 & 598 & $+67 \%$ \\
\hline Psychologie & 146 & 322 & $+120 \%$ \\
\hline Sociologie & 332 & 586 & $+77 \%$ \\
\hline Technologie & 1151 & 1776 & $+54 \%$ \\
\hline Zoologie & 973 & 1311 & $+35 \%$ \\
\hline
\end{tabular}

Fig.6-Tableau représentant le pourcentage d'augmentation des prix du tableau $n^{\circ} 5$

Ce qui nous donne une moyenne d'augmentation sur la période de $68.93 \%$ (qui avait atteint pour certaines spécialités des sommets d'augmentation de $274 \%$ et $120 \%$ pour Philosophie et religion et Psychologie et une seule légère baisse de $-12 \%$ pour les travaux généraux), qui est approximativement la même que celle du tableau précédent qui était de $67.80 \%$. Cet accroissement, que ces deux tableaux représentent d'une manière tout ce qu'il y a de plus claire, a drastiquement réduit les titres que pouvait acquérir les bibliothèques. Enfin en 2011, Library Journal a calculé les prix moyens des abonnements aux périodiques dans 15 spécialités différentes. Ces prix sont representés dans le tableau suivant : 


\section{TABLE 1 AVERAGE 2011 PRICE FOR SCIENTIFIC DISCIPLINES}

\begin{tabular}{|c|c|c|c|}
\hline DISCIPLINE & $\begin{array}{l}\text { AVERAGE PRICE } \\
\text { PER TIME }\end{array}$ & DISCIPLINE & $\begin{array}{l}\text { AVERAGE PRICE } \\
\text { PER IIILE }\end{array}$ \\
\hline Chemistry & $\$ 4,044$ & Math \& Computer Science & $\$ 1,593$ \\
\hline Physics & 3,499 & Food Science & 1,564 \\
\hline Biology & 2,167 & Health Sciences & 1.470 \\
\hline Engineering & 2,035 & Technology & 1,374 \\
\hline Astronomy & 2,008 & General Science & 1,333 \\
\hline Geology & 1,791 & Geography & 1.155 \\
\hline Botany & 1,731 & Agriculture & 1,103 \\
\hline Zoology & 1,647 & SOURCE: LUPERIODICALS PF & VEY 2011 \\
\hline
\end{tabular}

Fig.7-Tableau des prix des periodiques scientifiques ( Library Journal 2011)

Cet amalgame de raisons (avènement d'Internet, montée vertigineuse des prix des périodiques, besoin de se réapproprier les résultats de la recherche par ceux la même qui la produisent) a constitué le ferment ayant aidé au lancement et à l'acceptation d'une idée , somme toute révolutionnaire, et qui consiste pratiquement court-circuiter le maillon central d'une relation tripartite ( auteur-éditeur-lecteur) faisant de l'éditeur la condition sine qua none de la publication scientifique contrôlée par les pairs. Il faut dire aussi que les éditeurs commerciaux (à l'image d'Elsevier) affichent toujours des bénéfices mirobolants et une excellente santé financière face à un marché inélastique (un marché inélastique est défini par Jean-Claude Guédon comme un " marché où l'augmentation du prix influe peu sur la demande, parce que la marchandise demandée est indispensable. En l'absence de contrôles étatiques, cette situation conduit généralement à des surprofits...... " ${ }^{(3)}$ ) qui se double d'une dérive oligopole qui fait qu'un nombre réduit de maisons d'éditions contrôle la majorité de ce qui est publié ( à titre d'exemple, il y a deux millions d'articles publiés par quelque deux mille éditeurs et la moitié de ces articles est publiée par seulement six éditeurs ultra puissants (4) $)$.Cette non adéquation des règles économiques classiques de l'offre et de la demande a été favorisée et encouragée par la politique des fusions (mergers) qu'ont connu les années 80 et a débouché sur une quasi position de cartel. Tant que le savoir faire des éditeurs commerciaux était indispensable, l'envolée des prix était, si on peut dire, justifiée mais les fonctionnalités, l'ubiquité, la rapidité et la facilité dont se prévaut Internet fait de chaque auteur un éditeur en puissance sous réserve d'un équipement adéquat et d'un minimum de savoir informatique.

Nous allons étudier dans les lignes qui suivent, avant de voir le développement du mouvement du libre accès, quelles ont été les différentes étapes qui ont conduit à faire d'Internet l'outil omnipotent qu'il est devenu de nos jours, et qui représente à notre avis une révolution comparable ( si ce n'est plus importante ) à l'avènement de l'imprimerie et de son association au papier au $15^{\mathrm{e}}$ siècle ainsi qu'une condition indispensable de son évolution et développement.

\footnotetext{
(3) Jean-Claude Guédon, Repenser le sens de la communication scientifique : l'accès libre, Médecine / Sciences, vol. 24, ${ }^{\circ}$ 6-7, Juin Juillet 2008, p.642

${ }^{(4)}$ Francis André, Libre accès aux savoirs = Open access to knowledge, Paris, Futuribles perspectives, 2005 , p.19
} 


\section{Chapitre I \\ Les conditions constitutives de l'avenement du libre accès}

Il est indéniable que le libre accès n'aurait vu le jour sans d'Internet. Cet outil qui a envahi la vie de tous les jours et dont les ramifications et les influences ne sont pas encore pleinement étudiées, est une condition sine qua none du libre accès .Si avant, l'automatisation des différents étapes, outils et fonctions de recherche dans les bibliothèques se faisait off line ( elles ne se faisaient pas de concert avec d'autres institutions, personnes en dehors de l'institution qui l'utilisait ), elles sont devenues avec Internet universelles et ne se cantonnent plus à un seul poste mais à l'ensemble des différents ordinateurs reliés les uns aux autres. D'ailleurs le mot Internet est la contraction de deux mots" international" et "network " ou réseau international. Il est donc primordial, pour ne pas dire incontournable, de prendre le coté universel et surtout ouvert d'Internet pour en comprendre le fonctionnement et les résultats. De là, l'obligation de présenter ne serait que les contours de ce nouveau phénomène afin d'en appréhender les influences sur le libre accès.

\section{1-Internet}

L'idée de créer un réseau des réseaux (tel qu'est aussi connu Internet) ne date pas des années 80 ou 90 mais remonte aux années de guerre froide. En effet, le Department of Defence des Etats-Unis voulait créer "...un système de communication flexible et décentralisé capable de résister à une attaque nucléaire " ${ }^{(5)}$ et "....dont la mission était de mobiliser les ressources de la recherche, en particulier universitaire pour permettre aux Etats-Unis d'avoir une supériorité militaire sur l'URSS et ceci au lendemain du lancement de Spoutnik en 1957" (6) .Pour ce faire, le Department of Defence avait crée en 1958 l'Advanced Research Project Agency (ARPA) qui dépendait de l'Information Processing Technique Office (IPTO).Tel que définit par Joseph Licklider, l'objectif de l'IPTO était de simuler la recherche sur l'informatique interactive et eu recours à la transmission des communications appelée " commutation par paquets " où le message est décomposé en paquets voyageant indépendamment les uns des autres en empruntant les voies disponibles pour être reconstitués à l'arrivée. Cette technique a été mise au point par Donald Davis du National Physical Laboratory en Grande Bretagne et Paul Baran de la Rand Corporation, think thank connue pour ses étroites relations avec le Department of Defence des Etats Unis.

Les premiers ordinateurs connectés furent ceux de quatre centres universitaires tous situés au Sud Ouest des Etats-Unis et qui sont: University of California at Los Angeles (UCLA), Stanford Research Institute, University of California at Santa Barbara et Utah University. A ces quatre pionniers et entre Décembre 1969 et Janvier 1971 s'ajoutèrent une quinzaine d'organismes dont la plupart sont universitaires et nous citerons entre autres et parmi les plus connus: Stanford University, Massachusetts Institute of Technology (M.I.T), Mitre et Burroughs ${ }^{(7)}$. Après cela, un nombre croissant d'institutions est ajouté aux premiers établissements et ce chiffre n'a cessé de croître d'une façon exponentielle depuis ce jour.

L'un des obstacles les plus récurrents (pour ne pas dire l'obstacle) qui se dressait devant la propagation d'Internet est le langage par lequel communiquent les différents ordinateurs du réseau. A cet effet, deux chercheurs en théories informatiques, Robert Kahn de l'ARPA et Vint Cerf de Stanford University publient en 1974 un article posant l'architecture fondamentale d'Internet ${ }^{(8)}$. La même année, Vint Cerf, Gerard Lelann (du groupe français

\footnotetext{
${ }^{(5)}$ Castells Manuels, La Galaxie Internet, Paris, Fayard, 2000, p.19

(6) Idem

(7) http://www.walthowe.com (consulté le 5 Juin 2008)

(8) Cerf V., Kahn R., A Protocol for packets network interconnections. IEEE Transactions on communications, Vol. COM-22, p.637-648, May 1974
} 
Cyclades) et Robert Metcalf (alors étudiant à Harvard) élaborent le TCP (Transmission Control Protocol) qui est scindé en 1978 en deux pour mettre au point un protocole interréseau (Inter-network Protocol) et on obtient ainsi le protocole TCP/ IP sur lequel repose la structure d'Internet à ce jour. Le protocole est adopté en 1980 par le Defence Department, remplaçant le Network Control Protocol (N.C.P.) et aux environ de 1983, il est universellement accepté.

En 1983, le Department of Defence, inquiet que des fuites puissent avoir lieu dans son système, scinde le réseau en deux : MILNET, qui se spécialise dans l'utilisation militaire et ARPA-INTERNET consacré à la recherche. En 1984, la National Science Foundation (NSF) crée la NSFNET, et quatre ans plus tard, elle commence à utiliser ARPA-INTERNET comme épine dorsale Enfin, en 1990 ARPANET est démilitarisé .

Il est à noter que les années 80 voient, surtout aux Etats-Unis, une large politique de privatisation des télécommunications précédée par une politique de subventions de la part du Department of Defence visant aider les fabricants d'ordinateurs à intégrer TCP/IP dans leur protocole. De ce fait, la base était jetée pour la gestion privée d'Internet. En 1995, NSFNET disparaît, ouvrant ainsi la porte grande ouverte à la privatisation complète d'Internet.

Mais c'est Tim Berners - Lee qui en 1990 met au point le World Wide Web ou la Toile mondiale (www) au Laboratoire européen des physiques de particules (CERN) à Genève et à qui est attribuée la paternité de la création d'Internet. Sa particularité était l'hypertexte qui permet de lier des textes à d'autres textes à travers le réseau. Si le nom de Tim Berners - Lee est à jamais lié au nom d'Internet en créant le www, il faut dire qu'il avait l'avantage de l'existence effective d'Internet et qu'il a profité pour mettre au point le logiciel permettant de récupérer et d'envoyer l'information de et vers tout ordinateur connecté à la Toile, d'autres pionniers ont rêvé et contribué à la construction d'un accès mondiale à l'information. Il serait long et fastidieux d'essayer de les citer tous ici, car chacun a à sa manière contribué d'une manière ou une autre à faire Internet. Parmi ces précurseurs, nous citerons entre autres :

Vannevar Bush: Ce chercheur qui travaillait à " The Office of Scientific Research and Development ", proposa en 1945 (déjà) Memex (contraction de memory extender) qui est un système se proposant de récupérer et d'annoter les données Il est considéré, à juste titre d'ailleurs, comme le prototype théorique de l'hypertexte actuel.

Ted Nelson : Il proposa en 1965 un système utopique appelé Xanadu qui se proposait de réunir toutes les informations passées et futures et de la planète.

Douglas Engelbert : Il conçut On-line en 1968 et qui comprenait une interface graphique et ceci à partir du Augmentation Research Center.

Bill Atkinson: Il élabora en 1980, un système d'informations interconnectées appelé Hypercard au sein de d'Apple Computer.

\section{2-Open source :}

L'histoire d'Internet et son exponentiel développement, sa facilité d'utilisation et ses innombrables et révolutionnaires fonctions ne seraient pas possibles sans le mouvement de l'Open Source qui est venu en réaction aux velléités des logiciels propriétaires et à leur tête Microsoft. Son principe est simple : toute personne utilisant un logiciel en accès libre est tenu en retour d'en diffuser le code source sur Internet et ceci afin qu'il soit continuellement amélioré. Nous verrons plus tard que l'idée d'Open Source (Logiciel Libre) et Open Access 
(Libre Accès) " convergent " vers un même but ${ }^{(9)}$ et procèdent de la même philosophie tendant à mettre à la disposition de tout un chacun l'information scientifique et technique moyennant une connexion Internet, quoique cette vision idyllique (utopique même) n'est pas sans soulever certaines réserves, d'ordre juridique surtout, que nous aurons l'occasion d'aborder dans ce travail. Aussi anecdotique qu'elle puisse paraître *, la démission de Stallman exprimait le fait qu'il ne voulait pas que le travail qu'il faisait " devienne inutilisable dans le cadre dans lequel il était fait : créer une communauté nouvelle de partage de logiciels (10)".D'autre part, elle exprime une sorte de ras le bol qu'éprouvait les informaticiens lorsqu'ils voulaient améliorer un logiciel dont le code source était verrouillé. Stallman fonda alors la Free Software Foundation (FSF) ${ }^{(11)}$ pour substituer le copyleft ${ }^{* *}$ au copyright (qui est par la même un double jeu de mots faisant référence d'une part au copyright traditionnel opposition right (droit,) dans le sens légal du terme et left (gauche) et d'autre part à l'expression copy left (copie laissée ou copie autorisée). Il est parfois traduit de la même façon en français par gauche d'auteur (par le projet GNU ou la FSF) en opposition à droit d'auteur, mais en perdant la visibilité de la notion de copie autorisée. Il est aussi parfois traduit copie laissée dans le sens droits de reproduction abandonnés ).D'ailleurs, Stallman créa GNU ${ }^{(12)}$ (Gnu Not Unix), logiciel Open Source qui remplacera UNIX ( d'où le nom " GNU et non pas UNIX ").Fidèle à sa philosophie, il le publie sur Internet et permet son libre changement tant que le copyleft est respecté .L'autre figure de proue de l'Open Source est le Finlandais Linus Torvald, qui étudiant à l’Université de Helsinki, créa Linux ${ }^{(13)}$., basé sur UNIX et le met sur Internet en libre d'accès. Ceci eu pour effet que les innombrables ajouts des hackers et utilisateurs en ont fait un des systèmes d'exploitation les plus avancés spécialement tout ce qui a trait à l'informatique liée à Internet. L'Open Source, d'un autre coté, a suscité de nombreux autres programmes dont le plus connu est Apache ${ }^{(14)}$ et dont sont équipés plus de $60 \%$ des serveurs du Web et ceci en $2001^{(15)}$.De l'autre coté de la barrière, se trouvent les logiciels propriétaires dont la figure de proue est Microsoft et son fondateur Bill Gates. Son système d'exploitation Windows et son logiciel de navigation Internet Explorer (à commencer par Windows 98 lancé en Juin 1998 et qui intègre Internet Explorer dans le système d'exploitation) ont eu un énorme succès commercial aux antipodes des buts des protagonistes du logiciel libre. La domination outrancière du système Windows associé à certaines velléités de cartellisation ont eu pour résultat un nombre de procès ayant la plupart aboutit à la condamnation de Microsoft sans pour autant diminuer de sa suprématie

\footnotetext{
(9) Willinsky John. The Unacknowledged convergence of open source, open access and open science, First Monday, Vol. 8, n 8, Aout 2005. (Accessible à : http://first Monday.org/ issues 10-8/Willinsky/index.html . *Richard Stallman, alors programmeur à l'Artificial Intelligence Agency du fameux M.I.T., n'ayant pu accéder au code source du logiciel de son imprimante qui lui posait problème, décida de démissionner de son poste en signe de protestation contre le fait que le code source ne soit pas livré avec l'imprimante

${ }^{(10)}$ Stallman Richard. Copyright and globalisation in the age of computer network In_Rishab Aiyer Ghosh (Ed.), CODE: Collaborative Ownership and Digital Economy. Cambridge [Mass.]: MIT Press, 2005, p.317-335, cité par Willinsky, op.cit.

(11) www.fsf.org

** Le copyleft est la possibilité donnée par l'auteur d'un travail soumis au droit d'auteur (œuvre d'art, texte, programme informatique, etc.) de copier, d'utiliser, d'étudier, de modifier et de distribuer son œuvre dans la mesure où ces possibilités restent préservées. L'auteur n'autorise donc pas que son travail puisse évoluer avec une restriction de ce droit à la copie, ce qui fait que le contributeur apportant une modification (une correction, l'ajout d'une fonctionnalité, une réutilisation d'une œuvre d'art, etc.) est contraint de ne redistribuer ses propres contributions qu'avec les mêmes conditions d'utilisation. Autrement dit, les créations réalisées à partir d'éléments sous copyleft héritent de cette caractéristique (http://fr.wikipedia.org/wiki/Copyleft)

(12) www.gnu.org

(13) Www.linux.org

(14) www.apache.org

(15) Castells Manuel, op. cit, p.24
} 


\section{3-La crise des périodiques}

Si les deux conditions précédentes (avènement d'Internet, communauté open source très active) furent le ferment ayant préparé le terrain au libre accès, une autre occurrence non moins importante précipita son avènement. Cette situation est ce qui est communément connu sous la crise des prix des périodiques .Entre 1975 et 1995, les prix des périodiques connurent une augmentation spectaculaire (englobant même les monographies) ayant conduit à un mouvement de désabonnement et ce même dans les bibliothèques des pays développés tels que les Etats-Unis , la Grande Bretagne alors que le budget de leur universités équivaut, dans certains cas, au budget de l'enseignement supérieur de pays développés comme la France ( à cet effet un dossier de l'hebdomadaire Le Point fait ressortir que " le capital de Harvard est de 29 milliards de dollars, soit autant que le budget global de l'enseignement supérieur français " (16)). La question ayant été largement abordée et surtout documentée, le foisonnement de statistiques fait qu'il est difficile de retrouver dans les chiffres. Nous en citerons deux qui, à notre avis, résument toute l'étendue du problème :

- l'abonnement au périodique scientifique le plus cher au monde " Brain research" édité par Elsevier est passé de 10181 à 16344 \$ entre 1995 et 2000 (voir tableau ci-dessus) et est proposé en date du 19 Novembre 2008 à $23361 \$^{(17)}$, ce qui fait que son prix a plus que doublé en treize ans enregistrant une augmentation de $129.45 \%$.

- le taux d'augmentation des prix des périodiques entre 1975 et 1995 (cette dernière date étant considéré comme la date approximative du début de la " révolution scientifique " associée à Internet) est de l'ordre de $300 \%$ au-delà de l'inflation pour les éditeurs commerciaux et les sociétés savantes, et d'un peu plus de $200 \%$ pour les presses universitaires ${ }^{(18)}$.

Ces trois conditions ou occurrences, combinées et réunies quasi simultanément ont accéléré l'avènement du libre accès .Mais, c'est l'insupportable et incapacitante augmentation des prix des périodiques qui l'a mis au monde .En effet, si on peut considérer Internet et l'open source comme des occurrences reliées (il n'y aurait pas eu d'Internet - ou du moins Internet ne serait pas devenu ce qu'elle est - sans la culture ouverte et de partage des promoteurs des logiciels libres), la crise des prix des periodiques a agit comme un révélateur et aussi comme un accelerateur. En de termes plus clairs, la crise des periodiques dont les effets se faisaient (et se font toujours) sentir de maniere de plus en plus aigue, a en même temps profité de l'avènement et developpement d'Internet mais a aussi rendu ces fonctionnalités (celles Internet) utilisables dans le cadre de ce mouvement (combattre les augmentations immodérées des prix des periodiques) .

Mais à mesure qu'Internet se démocratise - des chercheurs tels que Manuel Castells ont estimé qu'en l'an 2000, il y aurait 2 milliards d'utilisateurs ${ }^{(19)}$ alors qu'à la fin Juin 2008, le site Internetworldstats en recensait $1463632361^{(20)}$ tel que le montre le tableau suivant :

\footnotetext{
${ }^{(16)}$ Universités : autopsie d'un gâchis, Le Point, 5 Juillet 2007, p.54, n 1816

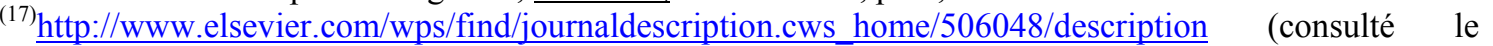
le 19 Novembre 2008)

(18) European Commission, Study on the economic and technical revolution of the scientific publication markets in Europe, Brussels, European Union, 2006, p.7

${ }^{(19)}$ Castells ,Op. cit., p. 11

(20) $\mathrm{http}: / /$ www.internetworldstats.com/stats.htm
} 


\begin{tabular}{|c|c|c|c|c|c|c|}
\hline \multicolumn{7}{|c|}{ WORLD INTERNET USAGE AND POPULATION STATISTICS } \\
\hline World Regions & $\begin{array}{l}\text { Population } \\
\text { ( } 2008 \text { Est.) }\end{array}$ & $\begin{array}{l}\text { Internet Users } \\
\text { Dec/31, } 2000\end{array}$ & $\begin{array}{l}\text { Internet Usage, } \\
\text { Latest Data }\end{array}$ & $\begin{array}{l}\text { \% Population } \\
\text { ( Penetration ) }\end{array}$ & $\begin{array}{l}\text { Usage } \\
\% \text { of } \\
\text { World }\end{array}$ & $\begin{array}{c}\text { Usage } \\
\text { Growth } \\
2000- \\
2008\end{array}$ \\
\hline Africa & $955,206,348$ & $4,514,400$ & $51,065,630$ & $5.3 \%$ & $3.5 \%$ & $1,031.2 \%$ \\
\hline Asia & $3,776,181,949$ & $114,304,000$ & $578,538,257$ & $15.3 \%$ & $39.5 \%$ & $406.1 \%$ \\
\hline Europe & $800,401,065$ & $105,096,093$ & $384,633,765$ & $48.1 \%$ & $26.3 \%$ & $266.0 \%$ \\
\hline Middle East & $197,090,443$ & $3,284,800$ & $41,939,200$ & $21.3 \%$ & $2.9 \%$ & $1,176.8 \%$ \\
\hline North America & $337,167,248$ & $108,096,800$ & $248,241,969$ & $73.6 \%$ & $17.0 \%$ & $129.6 \%$ \\
\hline $\begin{array}{l}\text { Latin } \\
\text { America/Caribbean }\end{array}$ & $576,091,673$ & $18,068,919$ & $139,009,209$ & $24.1 \%$ & $9.5 \%$ & $669.3 \%$ \\
\hline Oceania / Australia & $33,981,562$ & $7,620,480$ & $20,204,331$ & $59.5 \%$ & $1.4 \%$ & $165.1 \%$ \\
\hline WORLD TOTAL & $6,676,120,288$ & $360,985,492$ & $1,463,632,361$ & $21.9 \%$ & $100.0 \%$ & $305.5 \%$ \\
\hline
\end{tabular}

Tableau $n^{\circ} 1 .-$ Statistiques population et utilisateurs Internet (31 Decembre 2000)

Les dernières statistiques (30 Juin 2012) du même site donnent les chiffres suivants :

\begin{tabular}{|c|c|c|c|c|c|c|}
\hline \multicolumn{7}{|c|}{$\begin{array}{l}\text { WORLD INTERNET USAGE AND POPULATION STATISTICS } \\
\text { June } 30,2012\end{array}$} \\
\hline World Regions & $\begin{array}{l}\text { Population } \\
\text { ( } 2012 \text { Est.) }\end{array}$ & $\begin{array}{l}\text { Internet Users } \\
\text { Dec. 31, } 2000\end{array}$ & $\begin{array}{l}\text { Internet Users } \\
\text { Latest Data }\end{array}$ & $\begin{array}{l}\text { Penetration } \\
\text { (\% Population) }\end{array}$ & $\begin{array}{c}\text { Growth } \\
2000-2012\end{array}$ & $\begin{array}{l}\text { Users \% } \\
\text { of Table }\end{array}$ \\
\hline Africa & $1,073,380,925$ & $4,514,400$ & $167,335,676$ & $15.6 \%$ & $3,606.7 \%$ & $7.0 \%$ \\
\hline Asia & $3,922,066,987$ & $114,304,000$ & $1,076,681,059$ & $27.5 \%$ & $841.9 \%$ & $44.8 \%$ \\
\hline Europe & $820,918,446$ & $105,096,093$ & $518,512,109$ & $63.2 \%$ & $393.4 \%$ & $21.5 \%$ \\
\hline Middle East & $223,608,203$ & $3,284,800$ & $90,000,455$ & $40.2 \%$ & $2,639.9 \%$ & $3.7 \%$ \\
\hline North America & $348,280,154$ & $108,096,800$ & $273,785,413$ & $78.6 \%$ & $153.3 \%$ & $11.4 \%$ \\
\hline $\begin{array}{l}\text { Latin America I } \\
\text { Caribbean }\end{array}$ & $593,688,638$ & $18,068,919$ & $254,915,745$ & $42.9 \%$ & $1,310.8 \%$ & $10.6 \%$ \\
\hline Oceania / Australia & $35,903,569$ & $7,620,480$ & $24,287,919$ & $67.6 \%$ & $218.7 \%$ & $1.0 \%$ \\
\hline WORLD TOTAL & $7,017,846,922$ & $360,985,492$ & $2,405,518,376$ & $34.3 \%$ & $566.4 \%$ & $100.0 \%$ \\
\hline \multicolumn{7}{|c|}{$\begin{array}{l}\text { NOTES: (1) Internet Usage and World Population Statistics are for June } 30,2012 \text {. (2) CLICK on each world region name for } \\
\text { detailed regional usage information. (3) Demographic (Population) numbers are based on data from the US Census Bureau } \\
\text { and local census agencies. (4) Internet usage information comes from data published by Nielsen Online, by the International } \\
\text { Telecommunications Union, by GfK, local ICT Regulators and other reliable sources. (5) For definitions, disclaimers, navigation } \\
\text { help and methodology, please refer to the Site Surfing Guide. (6) Information in this site may be cited, giving the due credit to } \\
\text { www. internetworldstats.com. Copyright ( } 92001-2013, \text { Miniwatts Marketing Group. All rights reserved worldwide. }\end{array}$} \\
\hline
\end{tabular}

Tableau $n^{\circ}$ 2.-Statistiques population et utilisateurs Internet (30 Juin 2012)

la recherche s'oriente elle vers de nouveaux services tels que les connexions sans fil, où chacun peut se connecter sur son ordinateur portable où la connexion est disponible à l'image des aéroports, les hôtels dont certains offrent même une connexion gratuite. On peut aussi citer les différents gadgets qui abondent sur le marché et qui sont commercialisés de telle sorte à répondre aux nouveaux besoins tels les petites tablettes, les ordinateurs de poche (plus connu sous l'acronyme de PDA ou Personnal Digital Assistant), les smart phones, les consoles de jeux, etc..., qui peuvent tous se connecter à Internet sans fil.

Quant à l'avenir d'Internet, il est très difficile de prédire quoi que ce soit tant la vitesse à laquelle il évolue, les fonctionnalités nouvelles qu'il recèle (certains réfrigérateurs en sont équipés), les nouvelles capacités de la bande passante (un chercheur chez Sisco a pu lancer une connexion à 40 GB qui permet de regarder 1500 téléviseurs Haute Définition en 
simultané ou télécharger un DVD Haute Définition en 2 secondes) ${ }^{(21)}$ en font un medium d'avenir mais dont les influences et effets sont loin toutes étudies ni même appréhendés tellement ce qui était du domaine de la science fiction est devenu réalité en un très court laps de temps .

Après ce bref et obligatoirement superficiel survol des conditions constitutives de l'avènement du libre accès et de son importance non seulement dans le monde académique mais aussi globalement ${ }^{*}$ et où la place d'Internet en tant que vecteur essentiel a été démontré et ce malgré sa nouveauté, nous allons présenter les différentes étapes, expériences actions et acteurs ayant conduit au développement (on ne peut parler de succès pour le moment car un véritable affrontement - guerre pour reprendre un chercheur ${ }^{* *}$ - oppose les protagonistes de chaque camps) du mouvement du libre accès à l'information scientifique technique et de ses deux corollaires et versions immédiats : les archives ouvertes et les journaux électroniques en libre accès sur Internet.

\section{4-Les appels fondateurs du libre accès}

Si Internet, open source et crise des périodiques furent les ferments ayant accéléré l'avènement du libre accès, il fallait un cadre l'officialisant. Bien qu'un grand nombre d'appels, chartes, déclarations et autres conférences aient été lancés depuis le début, il n'en demeure pas moins que trois événements majeurs et fondateurs eurent lieu au début des années 90 qui lancèrent le libre accès sous ses différentes formes. Il est à signaler que ces actions furent au début le fait de personnes militantes du libre accès. Ces actions furent plus tard rejointes par des institutions et enfin ces actions furent adoptées et sponsorisées par des gouvernements ce qui leur donna plus de poids. Ces trois evénements sont :

\section{1-L'Appel de Budapest ${ }^{(22)}$}

A l'instigation de George Soros, financier et philanthrope, se tient à Budapest au cours du mois de Décembre 2001, une réunion des acteurs les plus actifs et influents du mouvement (tels que Jean-Claude Guédon, Peter Suber, Michael Eisen et Stevan Harnad).Y sont aussi représentées les bibliothèques entre autres la S.P.A.R.C. ( Scholarly Publishing and Academic Ressource Coalition) qui se distingue par des positions revendicatives dans le domaine du libre accès .En date du 14 Février 2002, la " Budapest Open Access Initiative" est publiée sur Internet et dans les grands quotidiens . Le texte tel que présenté sur le site Internet comporte différentes rubriques dont nous citerons :

- Lire l'Initiative

-Signer l'initiative

-Liste des signataires

-Aide aux revues en accès libre

-L'OSI encourage la participation des éditeurs

\footnotetext{
(21) www.washingtonpost.com du 18 Juillet 2007

* A titre d'exemple, le magazine américain Nature, dans " 2003 in context " le classa parmi les sujets les plus importants avec le clonage humain, les organismes génétiquement modifiés (OGM) et l'accès global à l'eau potable et ce en posant la question cruciale suivante: " Est-ce que la littérature scientifique du futur sera dominée par les journaux qui ne font pas payer leur lecteurs?" (Voir Nature du 18 Décembre 2003) alors que le magazine Science dans Breakthrough of the year - Percée de l'année - y voit une des spécialités à surveiller. (Voir Science du 19 Décembre 2003)

** Le terme n'est pas exagéré entre les différents protagonistes et ceci est pris d'un article d'un des plus ardents défenseurs du libre accès Richard Poynder qui dans " War in Europe " présente la position des différents parties en commençant par " alors que la bataille pour l'accès libre à la littérature scientifique s'intensifie, différents fronts de conflit s'ouvrent " Disponible à (http://richardpoynder.co.uk/Essays\%20on\%200pen\%20Access.htm ) ${ }^{(22)}$ http://www.soros.org/openaccess/fr/read.shtml
} 
Etant la première des réunions du libre accès, l'Initiative de Budapest définit dans l'introduction les buts et la convergence de deux événements l'un ancien " ...la volonté des scientifiques et universitaires de publier sans rétribution les fruits de leur recherche dans des revues savantes, pour l'amour de la recherche et de la connaissance " et l'autre beaucoup plus récent : l'Internet. La rencontre de ces deux événements a donné lieu à la naissance de l'accès libre qui y est définit comme suit: " Par libre accès à cette littérature, nous entendons sa mise à disposition gratuite sur l'Internet public, permettant à tout un chacun de lire, télécharger, copier, transmettre, imprimer, chercher ou faire un lien vers le texte intégral de ces articles, les disséquer pour les indexer, s'en servir de données pour un logiciel, ou s'en servir à toute autre fin légale, sans barrière financière, légale ou technique autres que celles indissociables de l'accès et l'utilisation d'Internet. La seule contrainte sur la reproduction et la distribution, et le seul rôle du copyright dans ce domaine devrait être de garantir aux auteurs un contrôle sur l'intégrité de leurs travaux et le droit à être correctement reconnus et cités ".

D’un autre coté, l'Initiative définit les deux stratégies complémentaires à adopter :

1- Auto-archivage : en premier lieu, les savants ont besoin d'outils et d'assistance pour déposer leurs articles de revues à comité de lecture dans des archives électroniques ouvertes, une pratique communément appelée auto-archivage. Lorsque ces archives sont conformes aux standards définis par l'Open Archives Initiative, des moteurs de recherche et autres outils peuvent traiter des archives distinctes comme un seul et unique fonds d'archive. L'utilisateur n'a alors plus besoin de savoir quelle archive existe, ni où elle est localisée, pour accéder à son contenu et l'utiliser ". Cette stratégie est aussi connue sous l'abréviation de BOAI 1 (Budapest Open Access Initiative 1)

2- Revues Alternatives : en second lieu, les savants ont besoin des moyens pour lancer une nouvelle génération de revues alternatives engagées dans le libre accès et pour aider les revues existantes qui choisissent d'opérer la transition vers le libre accès. Puisque les articles de revues devraient être diffusés aussi largement que possible, ces nouveaux périodiques n'invoqueront plus le droit d'auteur pour restreindre l'accès et l'utilisation du matériel qu'ils publient. Puisque le prix constitue un obstacle à l'accès, ces nouvelles revues ne factureront pas l'abonnement ou l'accès, et se tourneront vers d'autres méthodes pour couvrir leurs frais. Il existe, pour cette fin, de nombreuses sources de financement alternatives, parmi lesquelles les institutions et les gouvernements qui financent la recherche, les universités et laboratoires qui emploient les chercheurs, les dotations allouées par discipline ou par institution, les amis de la cause du libre accès, les profits générés par la vente d'enrichissements apportés aux textes de base, les fonds libérés par la transformation ou la disparition des périodiques facturant un abonnement traditionnel ou un prix d'accès, voire les contributions des chercheurs eux-mêmes. Nul besoin de favoriser une solution plutôt qu'une autre pour toutes les disciplines et toutes les nations, ni d'arrêter de chercher de nouvelles alternatives originales. Cette stratégie est aussi connue sous l'abréviation de BOAI 2 (Budapest Open Access Initiative 2)

Il semblerait que la deuxième stratégie (la publication dans des revues en libre accès ) ait les faveurs des chercheurs pour les avantages qu'elle présente ( " visibilité, un nouvel impact et un nouveau public élargis et quantifiables " tel qu'il est stipulé dans l'Initiative), alors que l'auto archivage, peu connu et surtout peu compris, est d'une certaine manière délaissé et n'a pas les faveurs des chercheurs. Il semblerait en fait, que la publication dans des " revues alternatives " procure des avantages immédiats alors que l'auto archivage est beaucoup plus une action à long terme. 


\section{2-La Déclaration de Bethesda ${ }^{(23)}$}

En date du 11 Avril 2003 se sont réunis à Bethesda dans l'Etat du Maryland aux Etats-Unis des chercheurs de la communauté biomédicale pour discuter des problèmes inhérents à leur spécialité. Le préambule présente l'objectif de ce document et qui est "d'encourager les débats au sein de la communauté de la recherche biomédicale sur les moyens à employer afin de concrétiser le plus rapidement possible ce souhait largement partagé qu'est le libre accès à la littérature scientifique primaire. Notre objectif était de nous mettre d'accord sur les mesures concrètes nécessaires à la promotion d'un passage rapide et efficace à la publication en libre accès ; ces mesures pourraient être appliquées par toutes les parties concernées, à savoir, les organismes de soutien et de financement de la recherche scientifique, les chercheurs qui génèrent les résultats, les éditeurs par l'intermédiaire desquels les résultats de la recherche sont évalués par les pairs et distribués, et enfin les chercheurs, bibliothécaires et toute autre personne ayant besoin d'accéder à ce savoir ". Le document comporte quatre grandes parties et qui sont :

1-Définition de la publication en libre accès et qui est comme suit : " Une publication en libre accès est une publication qui remplit les deux conditions suivantes :

1. Le/les auteur(s) ainsi que les titulaires du droit d'auteur accordent à tous les utilisateurs un droit d'accès gratuit, irrévocable, mondial et perpétuel et leur concèdent une licence leur permettant de copier, utiliser, distribuer, transmettre et visualiser publiquement l'œuvre et d'utiliser cette œuvre pour la réalisation et la distribution d'œuvres dérivées, sous quelque format électronique que ce soit et dans un but raisonnable, et ce à condition d'en indiquer correctement l'auteur; ils accordent également aux utilisateurs le droit de faire un petit nombre de copies papier pour leur usage personnel.

2. La version complète de l'œuvre, ainsi que tout document connexe, dont une copie de l'autorisation ci-dessus, réalisée dans un format électronique standard approprié, est déposée dès sa publication initiale dans au moins un réservoir en ligne subventionné par un établissement d'enseignement supérieur, une société savante, une agence gouvernementale ou tout autre organisme reconnu œuvrant pour le libre accès, la diffusion sans restriction, l'interopérabilité, et l'archivage à long terme (PubMed Central est un exemple de ce type de réservoir en sciences biomédicales) .

\section{Notes :}

1. Le libre accès est l'attribut de travaux individuels et pas nécessairement celui des revues ou des éditeurs.

2. Les règles de la communauté, plutôt que les lois sur le droit d'auteur, continueront à fournir les mécanismes garantissant une bonne attribution de la paternité de l'œuvre et une utilisation responsable de l'œuvre publiée, comme elles le font déjà aujourd'hui."

Nous remarquons qu'à coté des critères classiques de la publication en libre accès, le document évoque la place du postprint, l'interopérabilité et l'archivage à long terme

2-Déclaration du groupe de travail Institutions et Agences de financement: Dans cette partie sont énoncées quelques unes des recommandations que les organismes et institutions de financement font pour que le libre accès soit effectif. Tout en reconnaissant le changement du paradigme de recherche, l'appel énonce quatre recommandations nécessaires au succès du libre accès. Ce sont :

(22) www.earlham.edu/ peters/fos/bethesda.htm (version originale)

La version en langue française est disponible à : http://openaccess.inist.fr/spip.php?article20 
-encouragement des chercheurs bénéficiaires de subventions à publier selon le principe du libre accès et à le promouvoir.

-engagement à aider financièrement (sous réserve de rester "dans les limites raisonnables déterminées par les conditions du marché et les services fournis " ) les chercheurs à supporter les frais de publication inhérents aux nouvelles formes de publication. Il est à signaler que cette aide est conditionnée par la publication des revues à comité de lecture.

- Affirmation de la primauté de l'article pris en tant que tel et non pas de la revue lors des nominations, les promotions, les primes et l'attribution et les demandes de subventions.

-La présence d'une publication en libre accès comme une preuve de service rendu à la communauté des chercheurs lors des nominations, les promotions, les primes et l'attribution et les demandes de subventions.

3- Déclaration du groupe de travail Bibliothèques et Editeurs : Les deux pôles (bibliothèques et éditeurs) étant primordial dans la réussite du libre accès, ils s'engagent à :

-Pour les bibliothèques :

* Travailler à promouvoir le passage au libre accès et le présenter à la communauté des utilisateurs.

* Prioriser la sensibilisation des utilisateurs quant au libre accès.

* Mettre en exergue les revues en libre accès dans les catalogues.

-Pour les éditeurs :

* Fournir une option de libre accès à tout article publié sous forme papier.

* Fixer un calendrier précis pour le passage au modèle en libre accès

* Collaborer à uniformiser les moyens d'interrogation et d'archivage des modèles en libre accès

* Assurer aux chercheurs, notamment à ceux originaires de pays en voie de développement, qui n'ont pas les moyens de payer dans la modèle auteur payeur, des tarifs préférentiels.

4-Déclaration du groupe de travail Chercheurs et Sociétés Savantes : Les chercheurs et les associations professionnelles les représentant reconnaissent et encouragent la dissémination des résultats de la recherche découlant de la publication électronique. De ce fait, ils :

* Cautionnent les principes du libre accès

* Reconnaissent la place primordiale de la publication scientifique dans le processus de recherche et l'obligation d'inclure les coûts de la recherche dans la recherche elle-même.

* Apportent leur soutien au libre accès et s'engagent à l'appliquer à l'ensemble des travaux qu'ils publient.

* S'engagent à publier, contrôler, relire dans les revues en libre accès ou celles qui s'engagent dans cette voie. 
* S'engagent (l'expression " se faire l'avocat " est utilisée) à défendre l'idée que les méthodes d'évaluation prennent en ligne de compte les articles publiés dans les revues en libre accès et non pas seulement la revue en elle-même.

* S'engagent à informer collègues, membres et le public en général sur le libre accès et les informer des raisons de leur soutien et de leur engagement.

Comme on peut le voir, l'Initiative de Bethesda, qui a été lancée par la communauté biomédicale, beaucoup plus touchée par la crise des périodiques car elle enregistre l'un des des taux d'augmentation les plus grands des prix des périodiques, est plus complète et plus précise que l'Initiative de Budapest. En effet, si l'Initiative de Budapest a mis les bases du libre accès et a permis de lancer ses préceptes, l'Initiative de Bethesda profite des expériences passées et détaille un peu plus les différents buts que le libre accès poursuit en y incluant différents groupes que l'Initiative de Budapest n'avait pas inclut. La prochaine étape verra la déclaration de Berlin parachever cette étape dans la libération de l'information scientifique et technique de la puissante emprise des éditeurs commerciaux.

\section{3-La Déclaration de Berlin ${ }^{(24)}$}

Entre le 20 et le 22 Octobre 2003 , se tient à Berlin une conférence connue sous l'appellation de " la Déclaration de Berlin sur le libre accès à la connaissance en sciences exactes, sciences de la vie, sciences humaines et sociales ".Elle fait suite aux Déclaration de Budapest et Bethesda ainsi que la Charte ECHO (European Cultural Heritage Online) qui en était une conférence satellite qui s'est tenu le 18 et 19 Octobre 2003 à Berlin ${ }^{(25)}$.Etant une suite logique des deux Déclarations précités, il n'en demeure pas moins que cette Déclaration se distingue par le fait qu'elle est signée par les institutions ( au nombre de 255 dont la totalité est du monde développé à part une seule organisation signataire du monde en développement et qui est Bibliotheca Alexandrina) et non plus des individus. Cette différence est importante car les institutions de toute évidence ont plus de poids que les individus. D'un autre coté, la Déclaration étend le modèle du libre accès à l'ensemble des résultats de la recherche (les donnés brutes, les documents graphiques et multimédia et à l'héritage culturel). La Déclaration reprend grosso modo les mêmes idées que les deux précédentes Déclarations et comporte :

- Une préface

- Des objectifs

- Définition d'une contribution en libre accès que nous reproduisons ci-dessus car nous estimons qu'elle est d'une importance primordiale et qu'elle est plus ou moins différente des autres définitions non pas dans la quintessence mais dans l'évolution et aussi le contexte.

" Les contributions au libre accès doivent satisfaire deux conditions :

1. Leurs auteurs et les propriétaires des droits afférents concèdent à tous les utilisateurs un droit gratuit, irrévocable et mondial d'accéder à l'œuvre en question, ainsi qu'une licence les autorisant à la copier, l'utiliser, la distribuer, la transmettre et la montrer en public, et de réaliser et de diffuser des œuvres dérivées, sur quelque support numérique que ce soit et dans quelque but responsable que ce soit, sous réserve de mentionner comme il se doit son auteur (les règles usuelles de la collectivité continueront à disposer des modalités d'attribution

\footnotetext{
(24) http://oa.mpg.de/openaccess-berlin/berlindeclaration.html (Version originale). La version française est disponible à :http://oa.mpg.de/openaccess-berlin/BerlinDeclaration wsis fr.pdf

${ }^{(25)}$ http://oa.mpg.de/openaccess-berlin/program echo.html
} 
légitime à l'auteur et d'utilisation responsable de l'œuvre publiée, comme à présent), tout comme le droit d'en faire des copies imprimées en petit nombre pour un usage personnel.

2.Une version complète de cette œuvre, ainsi que de tous ses documents annexes, y compris une copie de la permission définie dans ce qui précède, est déposée (et, de fait, publiée) sous un format électronique approprié auprès d'au moins une archive en ligne, utilisant les normes techniques appropriées (comme les définitions des Archives Ouvertes [Open Archives]), archive gérée et entretenue par une institution académique, une société savante, une administration publique, ou un organisme établi ayant pour but d'assurer le libre accès, la distribution non restrictive, l'interopérabilité et l'archivage à long terme "

Cette réunion initiale a été suivie par un nombre de conférences de suivi dont le but est la mise en œuvre des recommandations de la Déclaration de Berlin .Ce sont respectivement :

-La Conférence qui s'est tenu à Genève au CERN le 12 et 13 Mai 2004 et dont l'intitulé était

" Etapes vers la mis en œuvre de la Déclaration de Berlin sur le libre accès à la connaissance en sciences exactes, sciences de la vie, sciences humaines et sociales ${ }^{(26)}$ " (connue aussi sous l'appellation de Berlin 2).

-La Conférence qui s'est tenu à Southampton le 28 Février et 1er Mars 2005 et dont l'intitulé était " Progrès dans la mise en œuvre de la Déclaration de Berlin sur le libre accès à la connaissance en sciences exactes, sciences de la vie, sciences humaines et sociales (27)" (connue aussi sous l'appellation de Berlin 3).

-La Conférence qui s'est tenu à Golm (près de Potsdam) en Allemagne entre le 29 et 31 Mars 2006 et dont l'intitulé était " De la promesse vers la pratique (28)" (connue aussi sous l'appellation de Berlin 4).

-La Conférence qui s'est tenu à Padoue entre le 19 et 21 Septembre 2007 et dont l'intitulé était "Berlin 5 Libre accès : De la pratique à l'impact : Conséquences de la dissémination du Savoir ${ }^{(29) " ~(c o n n u e ~ a u s s i ~ s o u s ~ l ' a p p e l l a t i o n ~ d e ~ B e r l i n ~ 5) . ~}$

-La Conférence qui s'est tenu à Düsseldorf entre le 11 et le 13 Septembre 2008 et dont l'intitulé était " Changer la communication savante dans la Société du Savoir ${ }^{(30)}$ "(connue aussi sous l'appellation de Berlin 6).

Depuis les conférences se sont tenues annuellement dans différentes villes dans le monde ( malgré que le nom de la conference conserve toujours "Berlin " suivi d'un nombre et dont la dernière est Berlin 10 s'est tenue à Stellenbosch en Afrique du Sud entre le 6 et 8 Novembre 2012.La prochaine conférence Berlin 11 aura lieu le 19 et 20 Novembre 2013 au " Max Planck Society" à Berlin et sera une opportunité de faire le bilan à l'occasion du dixième anniversaire de la déclaration ) et sont devenues des manifestations incontournables non seulement des protagonistes et des militants du libre accès mais aussi des éditeurs, des bibliothécaires ( partie prenante et primordiale du libre accès ) et même des hommes

\footnotetext{
(26) http://oa.mpg.de/openaccess-cern/index.html

(27) $\mathrm{http://www.eprints.org/events/berlin3/index.html}$

(28) $\mathrm{http}: / /$ oa.mpg.de/openaccess-golm/index.html

(29) $\mathrm{http}: / /$ oa.mpg.de/openaccess-padua/index.html

(30) http://oa.mpg.de/openaccess-dus/index.html

* Surtout celle de Southampton où sous la rubrique " recommandation acceptée ", il est clairement stipulé :

"Afin de mettre en œuvre [les recommandations de] la Déclaration de Berlin, les institutions doivent mettre en œuvre une politique qui :

-requiert tous leur chercheurs de déposer une copie de tous leurs articles publiés dans un dépôt en libre accès et -encourage leurs chercheurs à publier leurs articles de recherche dans journal en libre accès quand un journal approprié existe (et faire de telle sorte que cela soit possible) Texte disponible en anglais à :

http://www.eprints.org/events/berlin3/outcomes.html
} 
politiques conscients de l'importance de ce mouvement .Toutes ces conférences ont étés autant de jalons dans la progression du libre accès qui de nos jours jouit d'une acceptation, d'un poids que même les plus acharnés défenseurs des schémas traditionnels de l'édition commerciale ne sauraient ni contester ni denier.

Ces trois Déclarations font désormais partie des textes fondateurs du libre accès et ont fait avancer sa cause avec le poids des institutions après que des individus dédiés (à l'image de Stevan Harnad dont la hargne ,constance et les écrits en faveur du libre accès et surtout l'auto archivage n'a pas d'égale et que Richard Poynder a appelé " un prophète dont le temps est venu " (31) ) aient portés le mouvement par la seule force de leur dévouement et abnégation et ceci au niveau international. Au niveau national, deux pays ont enregistrés des progrès notables et initié des projets de loi qui en font les pays leaders dans le monde du libre accès. Ce sont la Grande Bretagne et les Etats-Unis.

\section{5-Deux pays pionniers du libre accès : Le Royaume Uni et Les Etats-Unis}

\section{1- La Grande Bretagne}

Grâce à l'action de nombreux scientifiques et à sa présence dans les universités britanniques, le libre accès a considérablement progressé en Grande Bretagne. Ainsi en Décembre 2003, the " House of Common Science and Technology Commitee " ${ }^{(32)}$ a lancé une enquête concernant les publications scientifiques afin de cerner les problèmes de prix, d'accès et de disponibilité. Des témoignages écrits ${ }^{(33)}$ (au nombre de 143 émanant de chercheurs, bibliothécaires et d'éditeurs) furent soumis au Comite durant le mois de Février 2004, suivis de témoignages oraux à partir du mois de Mars 2004.En date du 7 Juillet de la même année, le rapport du Comité - sous le titre évocateur de " Scientific publications : Free for all ?" (34) (Publications scientifiques: gratuites pour tout le monde ?) - fut publié. Il était très favorable aux propositions, il était question d'encourager l'auto archivage (qui était et est toujours considéré le parent pauvre du libre accès) en obligeant les chercheurs britanniques à déposer leurs articles dans des archives institutionnelles et préconiser la publication facultative dans des revues en libre accès.

Malheureusement, cette position courageuse et novatrice du House of Common Science and Technology Commitee fut rejetée par le Gouvernement ${ }^{(35)}$ qui fit un amalgame malheureux entre auto archivage et libre accès. Pour ne pas être en reste, le House of Common Science and Technology Commitee répliqua et accusa ouvertement le gouvernement ${ }^{(36)}$ (ou plus exactement The Department of Trade and Industry ou Département du Commerce et de l'Industrie) d'avoir favorisé le secteur (en fait ils utilisèrent des mots assez durs tel que " se prosterner ") de l'industrie de l'édition scientifique. En date du 9 Novembre 2004, le très respecté et écouté " The Financial Times " dans un éditorial dénonça la position ambiguë et non conforme aux principes du libre accès en prenant faits et cause avec la position du " House of Common Science and Technology Commitee" ${ }^{(37)}$ ceci à coté de réactions dans des périodiques scientifiques de référence aussi respectés que Science, The Daily Telegraph, The

\footnotetext{
${ }^{(31)}$ Richard Poynder, Ten Years after, Information Today, Vol. 21 No. 9 - October 2004

Disponible à : http://www.infotoday.com/it/oct04/poynder.shtml

${ }^{(32)}$ www.parliament.uk/parliamentary committees/science and technology committee.cfm

(33) $\mathrm{http}: / /$ www.publications.parliament.uk/pa/cm200304/cmselect/cmsctech/399/399we01.htm

(34) $\mathrm{http://www.publications.parliament.uk/pa/cm200304/cmselect/cmsctech/399/39902.htm}$

(35) $\mathrm{http}: / /$ www.publications.parliament.uk/pa/cm200304/cmselect/cmsctech/1200/120006.htm\#a1

(36) $\mathrm{http://www.publications.parliament.uk/pa/cm200304/cmselect/cmsctech/1200/120003.htm}$

(37) $\mathrm{http}: / /$ www.ft.com/cms/s/98f16604-31f4-11d9-97c0-

00000e 2511c8,Authorised=false.html?_i_location=http $\% 3 \mathrm{~A} \% 2 \mathrm{~F} \% 2 \mathrm{Fwww} . \mathrm{ft} . \mathrm{com} \% 2 \mathrm{Fcms} \% 2 \mathrm{Fs} \% 2 \mathrm{~F} 0 \% 2 \mathrm{~F} 98 \mathrm{fl}$ 6604-31f4-11d9-97c0-

$00000 \mathrm{e} 2511 \mathrm{c} 8 . \mathrm{html} \&$ ___referer=http $\% 3 \mathrm{~A} \% 2 \mathrm{~F} \% 2 \mathrm{Fwww}$. biomedcentral.com $\% 2 \mathrm{Fopenaccess} \% 2 \mathrm{Finquiry} \% 2 \mathrm{~F}$
} 
Guardian, Guardian Education, The Scientist, Managing Information. Enfin, Biomed Central $^{(38)}$ publia à l'occasion de ce débat un très important dossier ${ }^{(39)}$ où à coté de sa très pertinente analyse des débats s'étant déroulé au cours de la l'enquête de la " House of Common Science and Technology Commitee", il y inclu une autre analyse où il battit en brèche les allégations que firent les éditeurs commerciaux quant à la faisabilité du libre accès. Dans cette analyse ${ }^{(40)}$, il est question de démystifier les déclarations des éditeurs commerciaux pour ne pas avoir à appliquer le libre accès. De son coté, le non moins respecté magazine Nature publia un dossier " Access to literature : the debate continues" (Accès à la littérature : le débat continue) en date du 13 Septembre $2004^{(41)}$ où il examina les différentes options surtout celle de l'auteur payeur où les frais de publications seraient assumés par l'auteur et son unité de recherche. Comme on peut le voir, les débats ont fait rage dans un pays considérée comme le premier pays d'édition scientifique au monde et où " The Wellcome Trust" ${ }^{(42)}$, (considérée comme la deuxième plus riche fondation mondiale de charité après la Fondation Bill et Melinda-Gates) dépense plus de 600 Millions de $£$ par an sur la recherche biomédicale.

\section{2-Les Etats-Unis :}

A l'instar de la Grande Bretagne, les Etats-Unis sont un pays où la cause du libre accès a le plus été défendue et débattue grâce surtout au travail d'organisations telles que l'A.R.L. (Association of Research Libraries) ${ }^{(43)}$.Ainsi en Juillet 2004 est né un projet d'auto archivage des publications des " National Institute of Health" ${ }^{(44)}$. Ces publications étant financées par l'argent du contribuable, des associations de malades, des chercheurs (dont des Prix Nobel en médecine et en biologie) virent qu'il était logique qu'elles soient disponibles publiquement sans aucun délai. Ils avaient annoncé, au début, que toutes ces publications devaient être déposées dans PubMed Central ${ }^{(45)}$ qui est l'archive digitale gratuite des NIH en sciences biomédicales et de la vie et ceci dans un délai ne dépassant pas les six mois. Mais là aussi, les dernières dispositions indiquent un retour en arrière, à l'image des décisions prises en Grande Bretagne. Non seulement l'auto archivage devient facultatif mais surtout il ne se ferait que douze mois après la date de publication au lieu des six mois initiaux. Cette dernière disposition quasi annihile les bienfaits de l'auto archivage .En effet, dans une science aussi sensible au facteur temps que la biologie, des résultats disponibles un an après leur parution sont démunis de tout bienfaits.

L'analyse de ces deux exemples (pris car représentant les deux pays où le libre accès a le plus progressé) montre à quel point la " guerre " - pour reprendre Richard Poynder - entre chercheurs et éditeurs commerciaux fait rage .Chaque protagoniste essaye des présenter les avantages de son point de vue tout en mettant en exergue les failles dans le système préconisé par son opposant. Il semblerait, à première vue, que le camp des éditeurs commerciaux a pu manœuvrer de telle sorte que les lois, même si elles passent, sont amputés de leurs substances (à l'image du degré de latence comme dans le cas des NIH qui des six mois initiaux est passé à douze mois rendant de ce fait la mesure pratiquement caduc et sans effet).Il est à signaler que les grands groupes éditoriaux ( à l'image de Elsevier ou Springer ) sont des véritables multinationales dont les chiffres d'affaires sont de l'ordre de milliards de dollars et peuvent de ce fait se permettre un intense lobbying qui très souvent fait pencher la balance de leur coté.

\footnotetext{
${ }^{(38)} \mathrm{http}: / / \mathrm{www} \cdot$ biomedcentral.com/

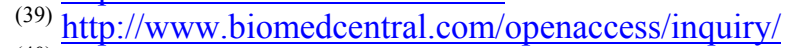

(40) $\mathrm{http}: / /$ www.biomedcentral.com/openaccess/inquiry/myths/

(41) $\mathrm{http} / / / \mathrm{www} \cdot$ nature.com/nature/focus/accessdebate/

(42) WwW.wellcome.ac.uk

(43) http://www.arl.org

(44) http://www.nih.gov

(45) $\underline{\text { http://www.pubmedcentral.nih.gov }}$
} 


\section{6-Définitions de certains concepts du libre accès}

\section{1-Qu'est ce qu'une archive ouverte?}

Le mot " archive " dans " archive ouverte " a un sens différent de celui traditionnellement utilisé. S'il est vrai que l'aspect " conservation " utilisé dans le sens du mot archive pris traditionnellement est présent, il n'en demeure pas moins que c'est le sens de " dépôt " qui y est plus prévalent. Donc on peut simplifier à l'extrême en disant que l'archive ouverte est un serveur Internet dans lequel sont déposés des documents. La première définition en a été donné par la de Déclaration de Budapest ${ }^{(46)}$. Nous la reproduisons intégralement car elle est très importante et permet de lever certaines équivoques liées principalement à la nouveauté du medium. Elle dit: "Par accès libre, nous entendons sa mise à disposition gratuite sur l'Internet public, permettant à tout un chacun de lire, télécharger, copier, transmettre, imprimer, chercher ou faire un lien vers le texte intégral de ces articles, les disséquer pour les indexer, s'en servir de données pour un logiciel, ou s'en servir à toute autre fin légale, sans barrière financière, légale ou technique que celles indissociables de l'accès et l'utilisation d'Internet. La seule contrainte sur la reproduction et la distribution, et le seul rôle du copyright dans ce domaine devrait être de garantir aux auteurs un contrôle sur l'intégrité de leurs travaux et le droit à être correctement reconnus et cités ".Comme on peut le voir que le libre accès est total (lecture, téléchargement, transmission, impression, etc...) à condition de respecter le droit d'auteurs qui, nous le verrons, est au cœur de la bataille que se livrent les éditeurs et les chercheurs pour voir qui est a droit à l'usufruit de la recherche.

D’un autre coté, les archives contiennent deux types de publications et qui sont :

6.1.1- les pré publications et qui sont des documents non encore soumis au contrôle par les pairs. C'est aussi ..."un texte finalisé par son (ou ses) auteur(s) mais non encore publié (évalué, corrigé, etc.) par un éditeur .Sous forme papier, la prépublication circulait généralement entre pairs et, depuis que les serveurs d'archives ouvertes existent, elle peut y être déposée " (47)

6.1.2- les post publications et qui sont des documents ayant été certifiés et contrôlés par les pairs. C'est aussi..." [Une publication] qui résulte du processus de publication géré par les éditeurs: le chercheur envoie sa prépublication, celle-ci est évaluée par un comité scientifique, des corrections sont souvent demandées à l'auteur qui renvoie un texte définitif (quelques aller/retour de ce genre sont possibles), une mise en page est réalisée par l'éditeur (avec généralement l'aide de l'auteur qui respecte une feuille de style), la diffusion est à la charge de l'éditeur" ${ }^{(48)}$.

6.1.3- les " e - publications ", les deux genres de publication précédentes sont connues sous l'appellation publications électroniques et étaient au début déposées simultanément dans 1'archive. Cette simultanéité de présence pose problème car le chercheur ne sait pas quelle est la version certifiée et finale de l'article. Ce qui peut vraiment poser problème dans des spécialités aussi sensibles que la médecine par exemple où une information non contrôlée peut conduire à des résultats graves. D'ailleurs, dans leur argumentaire contre la faisabilité du système, les éditeurs commerciaux évoquent cette faiblesse (l'absence ou du moins la non solidité du contrôle) comme une raison de leur inamovibilité dans le schéma classique de transmission du savoir (Chercheu-lecteur- $\rightarrow$ Editeur - certification $-\rightarrow$ Lecteur -

\footnotetext{
${ }^{(46)}$ http://www.soros.org/openaccess/fr/read.shtml

(47) Aubry Christine, Janik Joanna (Ed.), Les Archives ouvertes : enjeux et pratiques : guide à l'intention des professionnels de l'information et de la documentation, Paris, Association des professionnels de l'information et de la documentation (ADBS), p.99

${ }^{(48)}$ Idem
} 
chercheur).Nous verrons plus tard, que si le contrôle par les pairs est différent dans les archives ouvertes en particulier et dans l'environnement numérique en général, il n'en demeure pas moins qu'il est la condition de l'avancement de la science ( " Il n'est de science que publiée " ) ${ }^{(49)}$

\section{2-Types d'archives ouvertes}

D'un autre coté, les archives ouvertes se subdivisent en :

6.2.1- Archives institutionnelles : ce sont de archives qui dépendent d'institutions telles que les universités, les grandes écoles, les organismes de recherche, etc...

6.2.2- Archives disciplinaires: ce sont des archives qui regroupent des communautés scientifiques de domaine recherche assez large.

6.2.3- Archives thématiques : ce sont des archives focalisant sur un sujet ou un type de document particulier.

Après ce bref rappel de définitions relatives à des concepts soit nouveaux, soit dont le sens prend dans le nouveau medium des connotations différentes (nous pensons ici au mot archive qui pour les archivistes est la préservation sur le long terme associée à une politique institutionnelle alors que dans le contexte de la communication scientifique électronique, c'est un réservoir d'information de documents), nous allons présenter les plus importantes archives, les appels ( ou initiatives), les institutions et les actions ayant jalonnés la courte histoire des archives ouvertes .

\section{7-Premières et plus importantes archives :}

Il serait présomptueux de vouloir présenter la totalité (ni même la majorité) des archives ouvertes ayant foisonné ces dernières années depuis ArXiv en 1991.La plupart des pays et en particulier les pays développés ont mis en place des entrepôts d'archives qu'ils soient institutionnelles, disciplinaires ou thématiques L'impossibilité de recenser ces projets tient à la nature du medium qui sous tend le mouvement du libre accès : Internet. Sa facilité d'utilisation, ses fonctionnalités sans cesse développées et surtout sa grande démocratisation ont littéralement fait de la création et de la gestion d'une archive une opération de routine. Devant ce foisonnement et perpétuel accroissement, il existe quelques références exhaustives ayant essayé de recenser ces entrepôts. Nous allons essayer de les présenter par ordre chronologique et essayer de voir quelles sont leurs fonctionnalités et leurs contenus.

7.1-ArXiv ${ }^{(50)}$ :

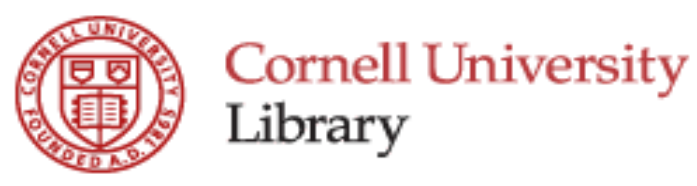

arXiv.org

Dés les années 80, des chercheurs ont commencés à déposer leurs articles sur Internet (premièrement sur les sites FTP ensuite sur le Web). Mais ce n'est qu'en 1991 que Paul Ginsparg, physicien du Laboratoire National de Los Alamos, créa ArXiv, base de données dans la théorie des cordes et la gravité bidimensionnelle. Elle a évolué et compte aujourd'hui

\footnotetext{
${ }^{(49)}$ Francis André, op.cit., p.7

${ }^{(50)} \mathrm{http}: / /$ arxiv.org
} 
des articles en physique, mathématiques, sciences non linéaires, informatique, la biologie quantitative et les statistiques. Elle comptait en date du 23 novembre 2008 508,978 e-prints, alors qu'elle avait dépassé le cap des 500000 e- prints en date du 3 Octobre 2008.Par la même, l'archive est maintenant hébergée par l'Université de Cornell et elle est partiellement subventionnée par la National Science Foundation (NSF). On peut rechercher dans le site de différentes manières dont les plus importantes sont:

-Nouvelles soumissions

-Soumissions les plus récentes

-Trouver (find) qui est une sorte de recherche libre où il est possible de rechercher par les critères classiques de recherche (sujet, années, auteur, titre, résumé).Il est aussi donné des conseils comment chercher et optimiser les résultats (opérateurs booléens, méta caractères, exactes phrases, etc...).Il est a remarquer que le succès d'ArXiv (en plus d'être la plus ancienne, elle est la plus mise à jour car quand on la consulte les dépots sont du même jour ou, au plus, du jour précédent ), n'est en fait que la transposition d'une ancienne habitude qu'avaient les physiciens, les informaticiens et, à un degré moindre, les économistes, à échanger leurs prépublications. L'avènement des réseaux n'a fait qu'accélérer considérablement la vitesse d'échange.

\section{2-Cogprints ${ }^{(51)}$}

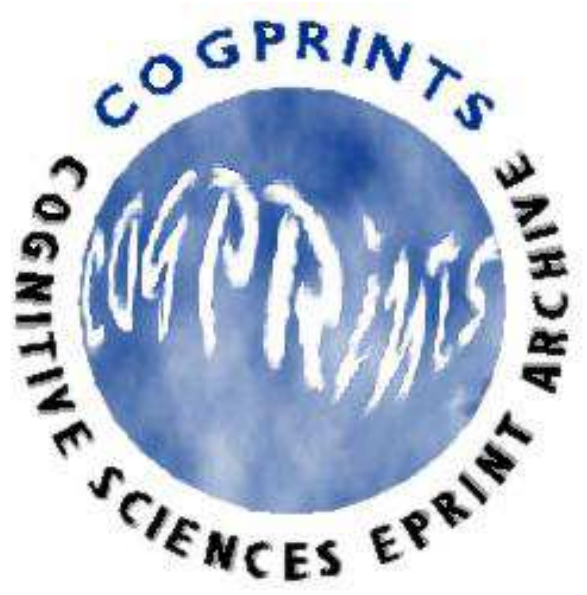

Créée par Stevan Harnad, sans aucun doute le plus critique des manœuvres des éditeurs commerciaux et le plus incisif défenseur des principes du libre accès et des archives ouvertes, Cogprints se définit comme " une archive électronique pour les recherches auto - archivées dans n'importe quelle spécialité de la psychologie, la neuroscience, et la linguistique et nombre de spécialités en informatique, la philosophie, la biologie, la médecine, l'anthropologie ainsi que toutes les autres spécialités des sciences mathématiques, sociales et physiques qui sont pertinentes à l'étude de la cognition ".L'archive est sous le logiciel Eprints 3 développé par School of Electronics and Computer Science de Université de Southampton. Elle comptait en date du 23 Novembre 20083236 documents dont 2468 ont été déposés entre 1997 et 2008 et 768 entre 1950 et 1997 (ces derniers dépôts étant sûrement des recherches effectuées et non pas déposés entre ces deux dates car l'archive n'existait pas et doivent aussi être des recherches effectués par des chercheurs de Université de Southampton dont dépend l'archive).Il est possible de rechercher l'archive de plusieurs manières dont les plus proéminentes sont :

${ }^{(51)}$ http://cogprints.org/ 
-par sujet: où on remarque qu'en plus des sujets qui traitent des sciences cognitives, il y est ajouté deux rubriques : " electronic publishing " et " journals "

-par année : où on remarque que l'archive remonte à l'année 1950 et aussi que la majorité des dépôts ont eu lieu entre 1991 et 2008 (2739 dépôts).Avant cette date, les dépôts n'étaient pas très nombreux.

Le site offre d'autres fonctionnalités et qui sont :

-Derniers ajouts : qui recense les derniers dépôts fait au niveau de l'archive

-Chercher le dépôt : qui offre différentes fonctionnalités de recherche telles que sujet, mots clés, types de supports, le statut de la publication (non publié, publié, sous presse), format (HTML, PDF), etc...

-Feuilleter (browse) le dépôt : qui fait en fait double emploi avec recherche par sujet car les mêmes informations sont obtenus lorsqu'on clique sur recherche par sujet.

-Au sujet de ce dépôt : qui donne des informations sur le site telles que, entre autres, l'adresse de contact.

Contrairement à ArXiv dont le succès est pris comme exemple par tous les concepteurs d'archives, il semblerait que Cogprints n'ait pas eu le succès escompté (ne serait ce du point de vue nombre de dépôts) et ceci est dû (succès des archives en sciences exactes versus quasi échec des archives en sciences sociales et humaines) à :

- manque de culture dans le partage des prépublications en sciences cognitives.

- absence de raison d'être d'une archive centrale et disciplinaire.

-absence d'une politique institutionnelle d'auto archivage ${ }^{(52)}$.

Personnellement, nous pensons que cette différence dans l'approche est la résultante, en plus des raisons précédemment citées, à deux raisons et qui sont :

1) la langue : l'utilisation de la langue dans les sciences humaines et sociales est d'une importance primordiale. Il en a même résulté des " bantoustans" linguistiques entre les principales langues utilisées (avec en toile de fond une rivalité franco-américaine que Jean- Noel Jeanneney, Président de la Bibliothèque Nationale de France à l'époque, a typifié dans un pamphlet retentissant ${ }^{(53)}$ ) s'auto excluant et pour reprendre l'expression de Jean Michel Salaün " chacun cultive son jardin " ${ }^{(54)}$.Contrairement, les sciences exactes n'utilisent pas la rhétorique autant et ont tendance à utiliser non pas des phrases mais beaucoup plus des formules scientifiques universelles. Cette manière de procéder facilite les échanges et le partage car la " langue " est une et n'a pas besoin d'interprétation.

2) La formation : les chercheurs en sciences exactes utilisent au cours de leurs recherches l'outil informatique et en fait il leur est indispensable. Il est pratiquement impossible pour un mathématicien, un physicien ou un statisticien de se passer de la puissance et la facilité d'utilisation de l'ordinateur. D'autre part, les scientifiques ont, de par leur formation, manipulé l'outil informatique plus tôt que les littéraires qui n'y ont recours qu'en des rares occasions.

${ }^{(52)}$ Aubry Christine, Janik Joanna (Ed.), op. cit. p.36

(53) Jeanneney Jean - Noël, Quand Google défie l'Europe, Paris, Mille et une nuits, 2006, 347 p.

${ }^{(54)}$ Aubry Christine, Janik Joanna (Ed.), op. cit. p. 284 


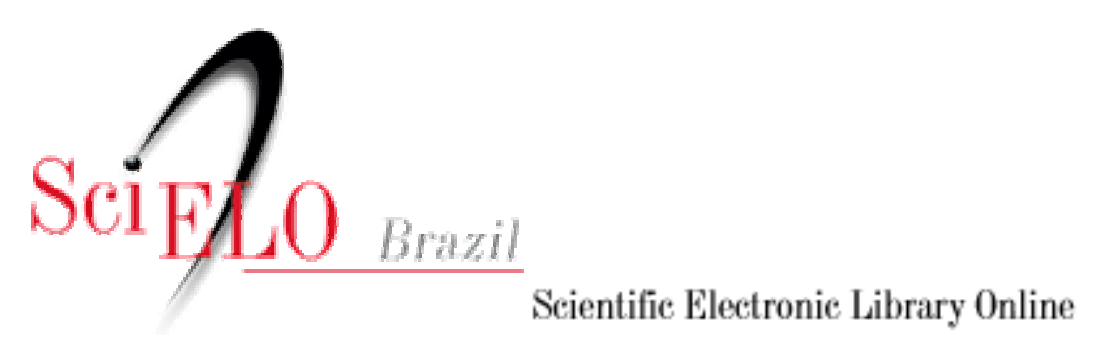

Cette archive (Scientific Electronic Library On Line) est une bibliothèque électronique d'un nombre selectif de périodiques scientifiques brésiliens. Elle a été fondée en 1998 par le Chili et le Brésil, et est une réponse à l'exclusion des recherches non anglophones (principalement hispanophones et lusophones) de l'exposition donnée aux recherches faites aux recherches en langue anglaise. Certaines études, en effet, placent le pourcentage des sites Internet en langue anglaise sur la Toile à $82 \%{ }^{(56)}$, ce qui met les autres langues en position de faiblesse et surtout accentue la fracture numérique .D'un autre coté, le fait de ne pas avoir une exposition suffisante induit un manque dans les citations ce qui implique que les auteurs n'écrivant pas en langue anglaise seront moins cités donc globalement moins reconnus. Les initiateurs du projet ont considéré ceci de " la science perdue ". Cette archive permit à des chercheurs peu exposés d'être plus visible et se sachant lus et (re)connus, ils commencèrent à écrire en langue anglaise et s'ouvrirent de nouveaux horizons. Depuis l'Espagne, Cuba, la Colombie, le Costa Rica, le Mexique, le Pérou et le Venezuela s'y joignirent. L'archive comptait en 2005, 25000 documents librement consultables. Elle compte en date du 23 Novembre 2008, 594 titres de périodiques, 12809 numéros de périodiques, 191722 articles de périodiques ${ }^{(57)}$.

On peut rechercher le site par les fonctionnalités suivantes :

1) Recherche par périodiques :

- liste alphabétique de périodiques

-liste alphabétique des sujets

-formulaire de recherche

2) Recherche par sujets

-Index auteurs

-Index sujets

-Formulaire de recherche

Le site comporte aussi une autre section qui concerne :

-l'utilisation du site (demande de périodiques, demandes de numéro de périodiques, les dix titres les plus demandés et le nombre d'articles demandés par mois)

-citation des périodiques

-co autorage (où il est possible de chercher les auteurs ayant publiés ensemble par pays, date et titre de périodique).

Contrairement aux deux autres archives dont l'origine (Etats-Unis et Grande Bretagne) implique des moyens matériels (surtout) assez conséquents, SciELO est le prototype de l'archive des pays en voie de développement (ou peut être émergents) et qui à coté de son application du principe du libre accès, combat aussi l'isolement (linguistique, technologique, économique et même politique) dont sont victimes les pays émergents. Nous pensons personnellement que l'idée, la philosophie même du libre accès a été dévoyée de son but

\footnotetext{
${ }^{(55)} \mathrm{http}: / /$ www.scielo.br

${ }^{(56)}$ Auh Taik-Sup, Promoting multilinguism on the Internet : the Korean experience http://www.unesco.org/webworld/infoethics_2/fr/summaries.htm

${ }^{(57)} \mathrm{http}: / / \mathrm{www}$. scielo.org/php/index.php?lang=en
} 
initial (l'accès sans restriction à l'information sans autre condition qu'une connexion Internet) par la persistance d'une fracture numérique au point où ses éventuels bénéfices sont pratiquement annihilés. Ce genre d'initiatives peut contribuer à l'amenuiser car le fait de pouvoir publier et être lu atténue l'inégalité d'accès.

\section{4-E-Biomed/PubMed Central ${ }^{(58)}$}

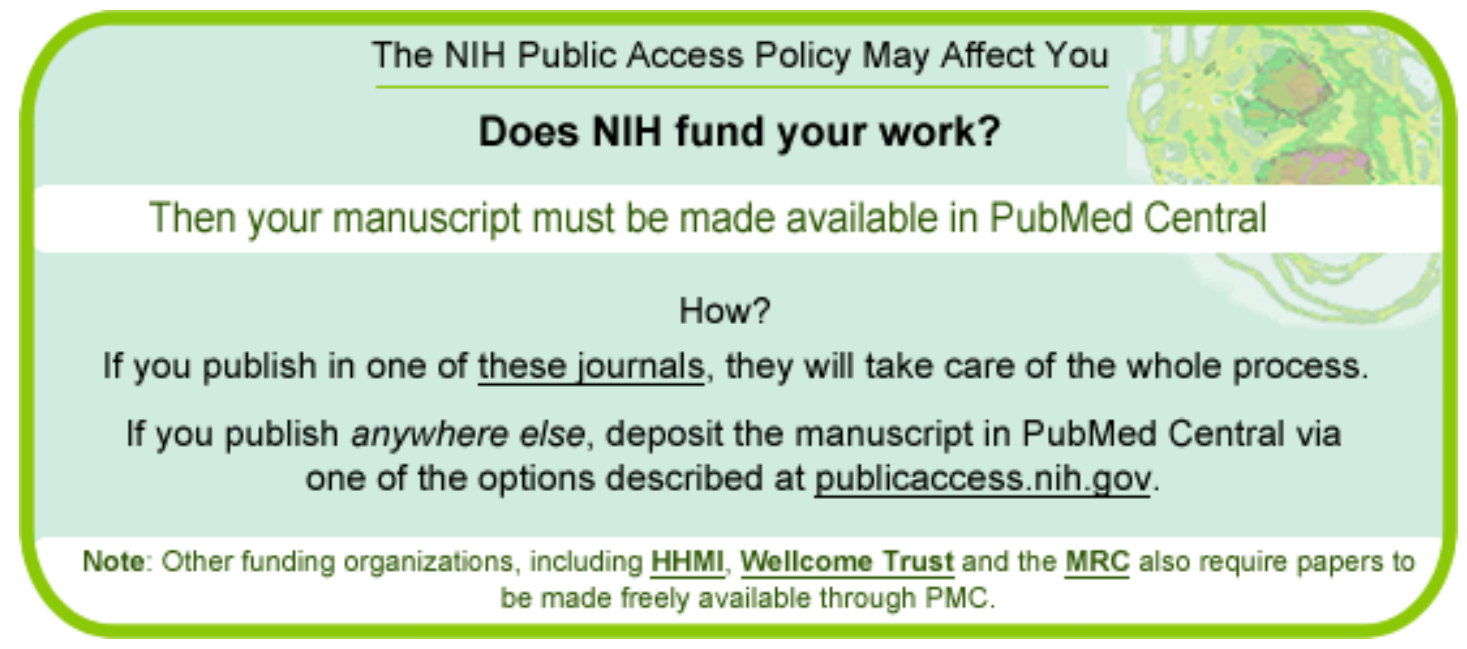

En 1997, la base de données de la National Library of Medecine ${ }^{(59)}$, a été mise en ligne accessible à tous sur Internet. Elle donne en plus de la référence, le résumé de l'article. Néanmoins, Harold Varmus, Prix Nobel et à l'époque Directeur des puissantes National Institutes of Health ${ }^{(60)}(\mathrm{NIH})$ lance l'idée qu'il faudrait étendre l'information au-delà du résumé. Ainsi est lancée l'idée d'une archive biomédicale où les pré publications ainsi que les post publications seront déposées librement et en texte intégral sur l'archive. Des débats houleux où le problème du contrôle par les pairs fut âprement discuté s'ensuivirent, car une information non certifiée, non contrôlée surtout dans des spécialités aussi sensible que le domaine biomédical, peut s'avérer dangereuse. Les critiques se concentrèrent sur les pré publications oubliant que l'essentiel est dans la post publication. A la fin, il fut décidé que les pré publications devront être soumises au contrôle avant publication alors que les post publications des périodiques participant à l'archive seront fixées à six mois. Le projet sera lancé en Février 2000 sous l'appellation de PubMed Central.

\section{5-Public Library of Science (PLoS) ${ }^{(61)}$}

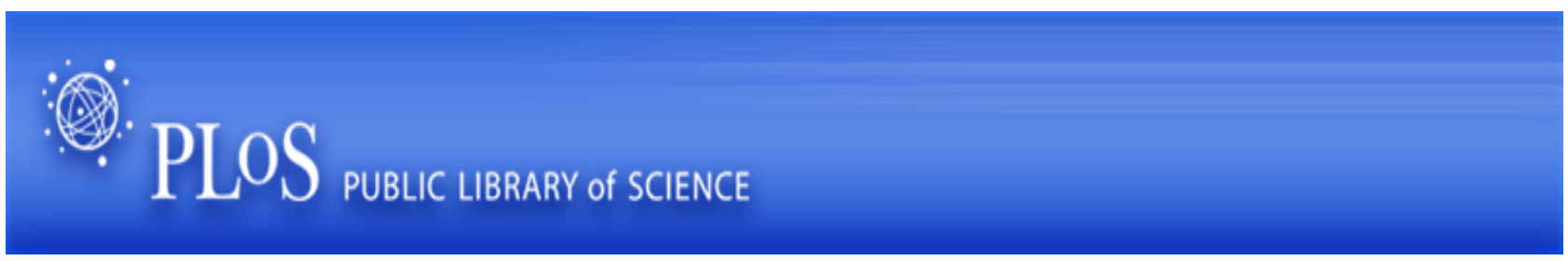

Face au tollé que cette proposition souleva, et sans surprise, dans le monde des éditeurs (au point où l'un d'entre eux utilisa la réflexion suivante : " c'est comme si on invitait les dindes à venir se faire rôtir à Noël " et qui en dit long sur l'animosité que cette idée provoque), qui n'adhérèrent pas au projet (seules 161 revues y adhérèrent et ce après quatre ans de son

(58) www.nih.gov/about/director/pubmedcentral/ebiomedarch.htm

(59) http://www.nlm.nih.gov/

(60) www.nih.gov/

(61) $\underline{\mathrm{http}: / / \mathrm{www} \cdot \mathrm{plos} . o r g /}$ 
lancement) car il mettait en danger leur juteux business, Harnold Varmus lança PLoS début 2001 une pétition sur Internet qui demandait aux éditeurs de mettre leur texte sur l'archive au bout de six mois. Les signataires menacent, dans le cas où ceci n'est pas appliqué, de ne pas acheter, publier ni expertiser dans ces revues. La pétition récolta au bout d'un an 34000 signatures mais ceci n'eut pas l'effet escompté. En fait ceci est du à une sorte de " frilosité " de la part des chercheurs qui face à l'inconnu que représente le nouveau medium, préférèrent plutôt publier dans des revues à haut facteur d'impact, facteur important, s'il en est, dans l'avancement de la carrière d'un chercheur. Les éditeurs adoptèrent un profil bas et laissèrent passer l'orage.
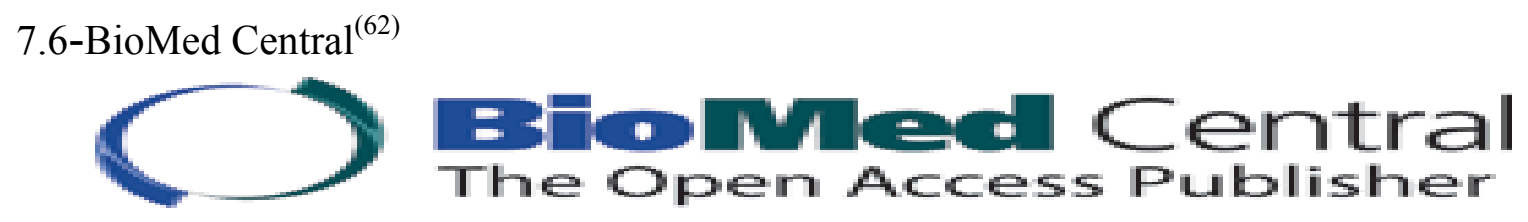

\author{
Welcome to BioMed Central \\ Publisher of 196 peer-reviewed open access journals
}

A la même période, le Current Science Group, sentant un intérêt dans ce genre de publication et d'archives en libre accès créa une nouvelle maison d'édition, BioMed Central. Elle a proposé au début d'assurer le " peer reviewing " (contrôle par les pairs) ainsi que la traduction des prépublications déposées dans PubMed Central. Les articles seront mis dans l'archive une fois qu'il aient passé le contrôle par les pairs. La nouveauté de BioMed Central réside dans un nouveau mode de publication qui reporte sur l'auteur (ou l'institution qui le finance) le payement des coûts associés à la publication de sa recherche. Ce modèle novateur (auteur payeur vs lecteur - payeur) s'il est acceptable du point de vue financier (il est de notoriété que l'un des problèmes posé par l'accès sans restrictions aux résultats de la recherche doit répondre à la question de " qui va payer les frais de publication "), n'en pose pas moins un nombre de problèmes d'ordre moral et d'équité. Les sommes demandées * sont loin d'être à la portée même d'un chercheur ou d'une institution du monde développé, encore moins d'un chercheur d'un pays en voie de développement. Cette disparité tendra, à notre avis, à accentuer (tel que nous l'avons déjà fait remarquer) cette fameuse fracture numérique qui met pratiquement les chercheurs des pays développés et ceux des pays en voie de développement dans deux mondes différents.D'un autre coté, cette manière de procéder peut conduire, si on n'y prend garde, à " acheter " sa communication. En effet, rien n'empêcherait un chercheur, pour les besoins d'une promotion ou un financement, de soumettre son article à un périodique pas trop regardant sur la qualité tant que le chercheur paye pour permettre sa communication d'être en libre accès.

Pour illustrer notre point, le cas du périodique électronique " Sensors " ci-dessous.

\footnotetext{
${ }^{(62)}$ http://www.biomedcentral.com/

* C'est le cas, entre autres, pour Springer avec Open Choice qui demande 3000 \$ et Online Open de Blackwell qui demande 2500 \$.D'un autre coté, certaines sociétés savantes proposent un modèle hybride où le chercheur qui paye voit sa publication disponible gratuitement alors que le non payement implique l'application du régime classique des publications à abonnement payant. (C'est le cas de The American physiological Society, Oxford University Press et The Company of Biologists)
} 


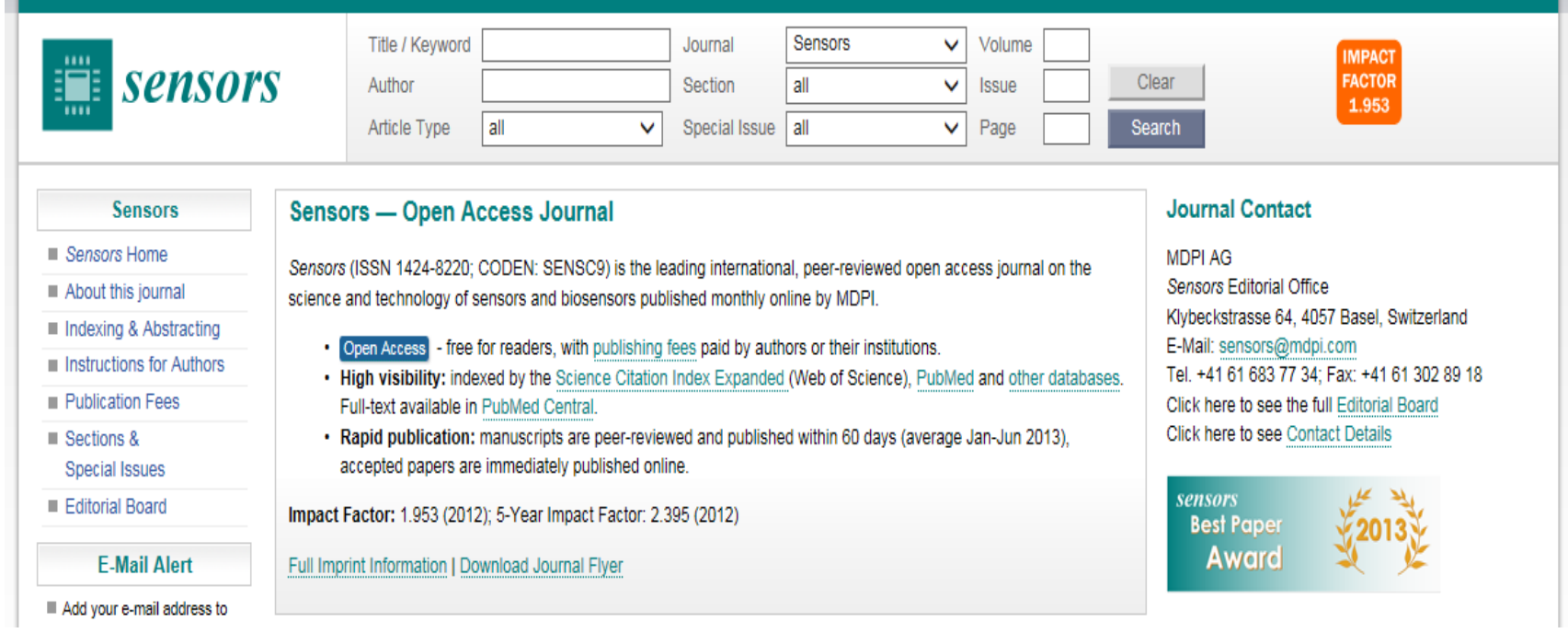

\section{7- " Sensors " (63)}

" Sensors " se définit comme " un périodique scientifique contrôlé par les pairs (l'expression " peer-refereed " est utilisée alors que c'est " peer reviewed " qui est d'habitude utilisée) publié en ligne mensuellement depuis 2001 par Molecular Diversity Preservation International (MDPI) ${ }^{(64)}$ et dont le siège est à Bale en Suisse". A la consultation du site, il est clairement stipulé à la section Open access " qu'il [le libre accès] est gratuit pour les lecteurs avec des coûts de publication bas payés par l'auteur ou son institution. Les coûts de publication en libre accès sont de l'ordre de 1050 Francs Suisses par recherche pour les recherches bien écrites (c'est nous qui soulignons) et de 1300 Francs Suisses pour les recherches qui nécessitent un formatage additionnel approfondi et/ ou des corrections en Anglais". Il est ajouté un peu plus loin à la section Rapid publication que les recherches acceptées sont immédiatement publiées. Loin de nous de critiquer cette approche mais il nous semblerait que cette manière de présenter le processus prêche par une sorte de commercialisation qui ne dit pas son nom. Est ce que la correction dans la langue n'est elle pas un des critères de publication et dans le cas où cela n'est pas présent est ce que cela nécessite 150 Francs Suisses additionnels ? Si le libre accès est en soi un une occasion de pouvoir accéder à l'information, ceci ne devrait pas être au détriment de la qualité et du sérieux du savoir publié.

Toutes ces initiatives qu'on peut considérer comme fondatrice du mouvement du libre accès furent au début portées par des individus travaillant dans le giron universitaire ou de la recherche .Ces personnes étaient (et sont toujours) les premiers que la rareté et le non accès à l'information pénalisent. A partir de la une autre étape, plus importante, verra l'implication d'institutions internationales de chercheurs qui à travers des initiatives (et à leur tête l'Appel de Budapest suivi de celui de Bethesda et enfin celui de Berlin) imposera un certain nombre de concessions de la part des éditeurs et adoptera des positions qui vont dans le sens de la libération de l'information scientifique et technique.

\footnotetext{
(63) http://www.mdpi.org/sensors/

(64) $\underline{\text { http://www.mdpi.org }}$
} 


\section{Chapitre II \\ De quelques exemples d'entrepots d'archives ouvertes}

Introduction

" L'entrepôt d'archives (des fois appelé reservoir) a dans le contexte de la communication électronique scientifique, ses origines dans la communauté " E-prints" et de ses pionniers, il est ici synonyme de réservoir d'information (appelé aussi parfois " entrepôt "). Le terme est très lié au mouvement "Open Archive Initiative", qui a pour objectif des finalités d'interopérabilité technique entre ces réservoirs (interrogation transversale par des moteurs de recherche) ${ }^{(65)}$.En de termes plus clairs, l'entrepot d'archives ouvertes est un ensemble d'archives ouvertes regroupées dans un seul reservoir ou entrepôt. Ils sont le resultat des avancées et augmentations numeriques des archives et se proposent de les regrouper.Parmi les plus importantes, nous citerons :

1-The Core Metalist of open access Eprints archives ${ }^{(66)}$

OpCit

project

The Open Citation Project - Reference Linking and Citation Analysis for Open Archives

Home | About | News | Partners and people | Papers and presentations | Research

This page last updated Monday, 30-Jun-2003 21:28:22 BST

\section{Explore Open Archives}

\section{Core Metalist of Open Access Eprint Archives}

Présentation

La dernière mise à jour de cette méta liste date du 30 Juin 2003. Elle est tenue par Steve Hitchcock, chercheur anglais connu pour ces nombreuses et pertinentes publications et recherches sur le sujet des archives ouvertes. Elle se départage en sept grandes subdivisions et qui sont :

-Listes générales des publications électroniques en libre accès (texte intégral)

-Les Archives répondant aux critères de 1'O.A.I. (Open Archives Initiatives- Initiative des archives ouvertes).

-Listes des archives institutionnelles.

- Les Archives de " Eprints.org ".

-Les Portails.

-Les archives des journaux en libre accès.

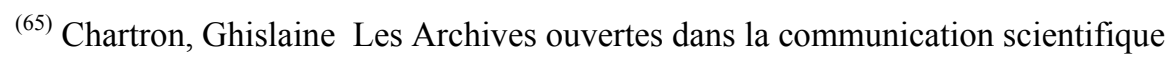

Disponible à http://urfist.enc.sorbonne.fr/anciensite/archives-ouvertes.htm

${ }^{(66)} \mathrm{http}: / /$ opcit.eprints.org/explorearchives.shtml 


\section{-Les archives disciplinaires}

Etant donné que cette méta liste fut la première à essayer de recenser la totalité des archives, il nous a paru important de présenter ses différents subdivisions car elles représentent la première tentative de recensement des différentes archives et de ce fait elle peut être prise en ligne de compte pour de futures recherches.

\section{1-Listes générales des publications électroniques en libre accès (texte intégral)}

1.1.1-Open Directory Project ${ }^{(67)}$ et qui sont des archives en accès libre en ligne (60 archives recensées en date du 16 mars 2003).C'est des archives électroniques donnant un accès libre et inconditionnel aux recherches scientifiques revues par les pairs ainsi qu'à des publications académiques.

1.1.2-HighWire Press ${ }^{(68)}$ et qui se présente comme " les archives en sciences en texte intégral les plus complets sur Terre " (20 archives) et qui est une " liste produite pour mettre en relief " HighWire's Free Online Full-text Articles" et qui est la plus complète de ce genre d'archive.

1.1.3-University of Maryland Libraries, Virtual Technical Reports Center: EPrints, Preprints, $\&$ Technical Reports on the Web ${ }^{(69)}$.Les institutions qui sont dans cette archive fournissent soit des rapports en texte intégral ou des larges extraits de leurs rapports techniques.Classée alphabétiquement par nom d'institutions (dernière mise à jour le 05 Mars 2003).

1.1.4-University of Virginia Science and Engineering Libraries, Preprint Servers and Databases ${ }^{(70)}$ (33 archives, dernière modification le 13 Janvier 2003).C'est un site qui indique un nombre de ressources électroniques de prépublications dans tous les domaines de la science et de l'ingénierie.

1.1.5-Tardis (JISC FAIR project 2002- _ $\quad{ }^{(71)}$ qui recèle des publications électroniques et des archives apparentées avec les catégories institutionnelles et les sujets identifiés (113 archives, premiers affichage Janvier 2003).

1.1.6-Aardvark, Asian Resources for Libraries ${ }^{(72)}$ qui recèle des prépublications libres de droits et des archives en sciences en texte intégral (115 archives en date du 15 Mars 2003).

-American Mathematical Society (AMS) ${ }^{(73)}$, qui est une liste des prépublications et des serveurs de publications électroniques en mathématiques.

1.1.7-Astronomy Preprints \& Abstracts ${ }^{(74)}$, hébergée par " The National Radio Astronomy Observatory " qui est relié à une liste de sites .Il comprend des serveurs de prépublications institutionnels.

1.2.1-Les Archives répondant aux critères de l'O.A.I.

\footnotetext{
${ }^{(67)} \mathrm{http}$ ///www.dmoz.org/Science/Publications/Archives/Free_Access_Online_Archives/

(68) $\mathrm{http} / / /$ highwire.stanford.edu/lists/largest.dtl

${ }^{(69)} \mathrm{http}: / /$ www.lib.umd.edu/ENGIN/TechReports/Virtual-TechReports.html

${ }^{(70)} \mathrm{http}: / /$ viva.lib.virginia.edu/science/guides/s-preprn.htm

(71) $\mathrm{http} / / /$ tardis.eprints.org/discussion/eprintarchivessubjecttable9103.htm

${ }^{(72)} \mathrm{http}: / / \mathrm{www}$.aardvarknet.info/user/subject19/index.cfm?all=All

${ }^{(73)} \mathrm{http}: / /$ www.ams.org/global-preprints

(74) $\mathrm{http} / /$ www.cv.nrao.edu/fits/www/yp_preprint.html
} 
1.2.1.1-Open Archive Initiative ${ }^{(75)}$, fournisseurs de données enregistrées et qui sont des "dépôts [d'archives] conformes"(77 archives en date du 27 mars 2003).

1.2.1.2-Open Archives Forum ${ }^{(76)}$, liste d'entrepôts [d'archives] (20 archives en date du 20 mars 2003).

\subsection{2-Liste des archives dont les services sont basés sur l'O.A.I.}

1.2.3-Celestial ${ }^{(77)}$, portail d'archives ouvertes qui moissonne et place les métadonnées en mémoire cache des dépôts se conformant au protocole OAI- PMH et rend ces données accessibles à d'autres services pour les moissonner. Il comprend un nombre d'enregistrements dans les dépôts d'archives et les métadonnées des noms de domaine.

1.2.4-OAIster ${ }^{(78)}$ qui renferme 1093,169 enregistrements provenant de 144 institutions (mis à jour le 21 Février 2003).

1.2.5-Arc ${ }^{(79)}$ qui est un service expérimental de recherche d'archives croisées. Il est utile pour enquêter sur les problèmes de moissonnage des dépôts conformes à 1'O.A.I. et les rend disponibles par l'intermédiaire d'une interface de recherche unifiée.

1.2.6-my.OAI ${ }^{(80)}$ qui est un engin de recherche personnalisable selon l'utilisateur et qui englobe des métadonnées de bases de données sélectionnées qui proviennent de l'O.A.I., voir la liste de bases de données sur l'interface de recherche invitée.

1.2.7-Public Knowledge Project ${ }^{(81)}$ qui est un moissonneur d'archives ouvertes où les archives doivent requérir le moissonnage (12 archives en date du 20 March 2003).

1.2.8-Open Archives Initiative ${ }^{(82)}$ qui est un explorateur de dépôts [d'archives], l'université de Virginia Tech.y joue le rôle d'interface pour tester les archives d'une manière interactive à leur conformité à l'O.A.I.-P.M.H. (60 archives, en date du 4 Avril 2003)

\section{3-Listes des archives institutionnelles}

1.3.1-SPARC ${ }^{(83)}$ qui sélectionne une liste des dépôts institutionnels et liste le genre de contenus (généralement des prépublications et des recherches publiées).Il recense les logiciels utilisés et les URL des dépôts (la plupart des dépôts utilisent EPrints.org, dernière mise à jour le13 Février, 2002)

1.3.2-Signal Hill ${ }^{(84)}$ qui est un partenariat européen pour la publication établi par les bibliothèques universitaires d'Utrecht, Delft et les presses universitaires de Florence et dont les archives institutionnelles sont classées par pays.

\footnotetext{
${ }^{(75)} \mathrm{http}: / / \mathrm{www}$.openarchives.org/Register/BrowseSites.pl

${ }^{(76)} \mathrm{http}: / /$ www.oaforum.org/oaf_db/list_db/list_repositories.php

${ }^{(77)} \mathrm{http}: / /$ celestial.eprints.org/cgi-bin/status

${ }^{(78)} \mathrm{http} / / /$ oaister.umdl.umich.edu/o/oaister/viewcolls.html

${ }^{(79)} \mathrm{http}: / /$ oaister.umdl.umich.edu/o/oaister/viewcolls.html

${ }^{(80)} \mathrm{http} / /$ www.myoai.com/search/Search.cgi/LoginForm?Login=guest\&Password=guest

${ }^{(81)} \mathrm{http}: / /$ www.pkp.ubc.ca/harvester/archives.php

${ }^{(82)} \mathrm{http} / / /$ oai.dlib.vt.edu/cgi-bin/Explorer/oai2.0/testoai

${ }^{(83)} \mathrm{http}: / / \mathrm{www}$.arl.org/sparc/core/index.asp?page $=\mathrm{m} 1$

${ }^{(84)} \mathrm{http}: / /$ www.signal-hill.org/archives/institutions.html
} 


\subsection{3-Les archives institutionnelles}

1.3.3.1-University of California, California Digital Library eScholarship Repository ${ }^{(85)}$ offre aux enseignants un point central pour déposer toute sorte de recherche ou production savante jugée digne d'intérêt par leurs unités de recherche, centres ou départements participant y compris des travaux de recherche et des prépublications.

1.3.3.2-Caltech ${ }^{(86)}$, Collection of Open Digital Archives (CODA), comprend plus de 10 dépôts en cours de réalisation ou actifs.

1.3.3.3-US Department Of Energy ${ }^{(87)}$ (DOE), the Information Bridge. Ce site fournit l'Open Source aux enregistrements bibliographiques et aux textes intégraux des rapports de développement et des recherches du DOE en physique, matériaux, biologie, sciences environnementales, technologie des énergies, l'ingénierie, informatique et science de l'information, énergies renouvelables, et autres sujets. Comprend des textes intégraux produits et fournis par les Laboratoires Nationaux du DOE et les bénéficiaires de bourses et ceci à partir de 1995. Les documents de succession sont fournis à mesure qu'ils deviennent disponibles.

\section{4-Eprints.org archives}

1.4.1-GNU Eprints ${ }^{(88)}$, logiciel pour le développement d'archives de publication électroniques institutionnelles mais peut être aussi utilisé pour bâtir d'autres types d'archives avec d'autres types de contenus. Tous les entrepôts qui ont étés bâtis en utilisant les deux premières versions de ce logiciel sont dans ces deux listes.

1.5-Portails (Indexes, recherche unifiée et feuilletage des sites couverts)

1.5.1-Les interfaces de recherche d'ArXiv

1.5.1.1 - Front for the Mathematics ArXiv ${ }^{(89)}$, interface alternative d'ArXiv

1.5.1.2- NASA, Astrophysics Data System (ADS) ${ }^{(90)}$, formulaire de question au sujet des prépublications ArXiv.Die Pro-Physik Findemaschine ${ }^{(91)}$, moteur de recherche allemand spécialisé, comprend ArXiv parmi ses ressources interrogeables. Il utilise des taxinomies flexibles pour permettre des recherches thématiques cross disciplinaires.

1.5.1.3-NASA ADS Harvard-Smithsonian Center for Astrophysics Preprints (CfA) formulaire de questions au sujet des prépublications.

1.5.1.4-The Stanford Linear Accelerator Center (SLAC), SPIRES HEP ${ }^{(93)}$, base de données qui contient plus de 500,000 articles relevant de la physique des hautes énergies y compris des recherches publiées dans les journaux, des prépublications, des publications électroniques, des

\footnotetext{
${ }^{(85)} \mathrm{http}: / /$ repositories.cdlib.org/escholarship/

${ }^{(86)} \mathrm{http}: / /$ library.caltech.edu/digital/

${ }^{(87)} \mathrm{http}: / /$ www.osti.gov/bridge/

${ }^{(88)} \mathrm{http}: / /$ software.eprints.org/\#ep1 (29 archives) http://software.eprints.org/\#ep 2 (37 archives)

${ }^{(89)} \mathrm{http}: / /$ front.math.ucdavis.edu/

${ }^{(90)} \mathrm{http} / / /$ adsabs.harvard.edu/preprint_service.html

(91) $\mathrm{http}: / /$ findemaschine.pro-physik. $\overline{\mathrm{de}} /$ ?language $=\mathrm{e}$

${ }^{(92)} \mathrm{http} / / /$ adsabs.harvard.edu/cfa/preprints.htm

${ }^{(93)} \mathrm{http}: / /$ www.slac.stanford.edu/spires/hep/

(94) $\mathrm{http}: / /$ citebase.eprints.org/help/coverage.php
} 
rapports techniques, des recherches présentées à des conférences et des thèses qui sont indexées par le bibliothèques de SLAC et de Deutsches Elektronen Synchotron (DESY) et ce depuis 1974.

1.5.1.5-Citebase ${ }^{(94)}$, site de recherche classé par citation et par impact de découverte pour ArXiv ( comprend aussi CogPrints et BioMed Central).

1.5.1.6-Elsevier, Scirus ${ }^{(95)}$, qui est décrit comme " le moteur de recherche spécialisé en science le plus complet sur Internet ".Il couvre plus de 135000,000 pages en relation avec les sciences et qui représentent 120000,000 de pages Web de sources payantes ainsi que des archives de publications électroniques importantes .

1.5.1.7-CERN Document Server (CDS) ${ }^{(96)}$ interface Web interrogeable de plus de 550,000 enregistrements bibliographiques, et qui comprend 220,000 documents en texte intégral en physique des particules et les spécialités connexes. Il comprend aussi des prépublications, des articles, des livres, des journaux et des photographies.

1.5.1.8-PhysDoc - Physics Documents Worldwide ${ }^{(97)}$, présente une liste de liens aux sources des documents, tels que les prépublications, les rapports de recherche, les rapports annuels, la liste internationale distribuée des publications des institutions de physique ainsi que celle des physiciens .Cette liste est classée par continent, pays et ville.

1.5.1.9-MPRESS, the Mathematics Preprint Search System ${ }^{(98)}$, index interrogeable de prépublication de 10 serveurs qui couvrent généralement des serveurs géographiques mais aussi des serveurs disciplinaires de mathématiques dont " Topology Atlas, Algebraic Number Theory Archives and K-theory Preprint Archives " ainsi que la partie mathématiques du site miroir d'ArXiv à Augsburg.

1.5.1.10-US Department Of Energy (DOE), PrePRINT Network ${ }^{(99)}$ portail interrogeable vers les serveurs de prépublications en relation avec les disciplines techniques et scientifiques qui intéressent le DOE telles que la physique, les matériaux, la chimie ainsi que certaines spécialités en biologie, sciences environnementales et médecine nucléaire.

1.5.1.11-NTRS, NASA Technical Reports Server ${ }^{(100)}$, interface de recherche pour 18 bases de données.

\subsection{2-Portails d'archives décentralisées}

1.5.2.1-Networked Computer Science Technical Reference Library (NCSTRL) ${ }^{(101)}$ qui est dans le processus d'être développé en une architecture qui supporte la conformité à l'OAI dans un projet collaboratif qui comprend " NASA Langley, Old Dominion University, University of Virginia and Virginia Tech ".La liste des archives participantes peut être consultée à : http://www.ncstrl.org:8900/ncstrl/body.html

1.5.2.2-Networked Digital Library Of Theses And Dissertations (NDLTD) ${ }^{(102)}$.Elle comprend beaucoup plus des thèses que des publications électroniques. Elle est présentée comme

\footnotetext{
${ }^{(95)} \mathrm{http}: / /$ www.scirus.com/about/\#content

${ }^{(96)} \mathrm{http}: / /$ weblib.cern.ch

${ }^{(97)} \mathrm{http}: / /$ de.physnet.net/PhysNet/physdoc.html

(98) http://mathnet.preprints.org/

(99) http://www.osti.gov/preprints/ppnbrowse.html

${ }^{(100)} \mathrm{http}: / /$ ntrs.nasa.gov/

(101) http://www.ncstrl.org/

(102) http://www.ndltd.org/
} 
l'exemple d'une archive dont le but est de rendre disponible le libre accès aux résultats des recherches en texte intégral.

1.5.2.3-Open Language Archives Community (OLAC) ${ }^{(103)}$ qui est une bibliothèque virtuelle internationale des ressources en langage. Vingt et une archives y participent ainsi que trois fournisseurs de services dont " OLAC Aggregator, Swahili Language Resources" et un fournisseur de services virtuel. Open Language Archives sont des entrepôts de données sur les langues, la documentation et la description .Ils comprennent des textes, des enregistrements, des dictionnaires, des notes de grammaire et des notes de champs qui tendent à mettre les informations disponible ouvertement y compris des dépôts qui ont un composant numérique accessible, même si c'est juste des catalogues en ligne ou quelque composants numériques. C'est beaucoup plus un service de préservation et de secours pour les ressources de langages qu'une archive de publications électroniques.

\subsection{3-L'exemple de " The Economics network (RePEc) "}

RePEc ${ }^{(104)}$ est une importante base de données de recherches, d'articles de journaux et de composants logiciels avec des enregistrements sur plus de 177,000 articles dont plus de 86,000 sont disponibles en ligne.

Les services suivants fournissent accès à la totalité ou à une partie de la base de données RePEc pour la recherche ou le feuilletage :

1.5.3.1-IDEAS (Internet Documents in Economics Access Service) ${ }^{(105)}$, recherche la base de données RePEc en entier.

\subsubsection{2-EconPapers ${ }^{(106)}$, fournit l'accès à RePEc}

1.5.3.3-NEP (New Economics Papers) ${ }^{(107)}$, service d'annonces e-mail pour des nouvelles recherches téléchargeables concernant plus de 40 champs spécifiques.

1.5.3.4-Citations in Economics, CitEc ${ }^{(108)}$ : citations d'articles dans la base de données RePEc.

1.5.3.5-Inomics ${ }^{(109)}$, recherches téléchargeables intégrées à l'information sur des conférences économiques, des informations sur l'emploi ainsi qu'une recherche Internet pour les économistes.

1.5.3.6-LogEc ${ }^{(110)}$, statistiques d'accès détaillés pour les services participants à RePEc.

\footnotetext{
${ }^{(103)} \mathrm{http}: / / \mathrm{www}$. language-archives.org/index.html/

${ }^{(104)} \mathrm{http}: / /$ repec.org/

${ }^{(105)} \mathrm{http}: / /$ ideas.repec.org/

${ }^{(106)} \mathrm{http}: / /$ econpapers.hhs.se/

${ }^{(107)} \mathrm{http}: / /$ netec.wustl.edu/NEP/

(108) http://netec.ier.hit-u.ac.jp/CitEc/

${ }^{(109)} \mathrm{http}: / /$ www.inomics.com/cgi/show

(110) http://logec.hhs.se/
} 
1.5.3.7-RePEc ArchivesFournisseurs actuels de l'archive à RePEc (111) .Les archives participantes fournissent plus de 1000 collections RePEc (nombre des collections les plus importantes sont des collections de périodiques ou de base de données de moindre importance). $\operatorname{LogEc}$ liste les 25 meilleurs collections du dernier mois.

\subsubsection{8-Working Papers in Economics}

1.5.3.8.1-WoPEc ${ }^{(112)}$, toutes les recherches dans WoPEc sont téléchargeables mais pas nécessairement gratuites ( contient plus de 80, 000 documents sous format électronique: 53035 Working Papers, 41895 articles de journal).

\subsubsection{2-WoPEc Working Papers Series ${ }^{(113)}$ (liste des archives indexées)}

1.5.3.9-DEGREE ${ }^{(114)}$ : recherche sous forme électronique en texte intégral sur l'économie et l'économie des affaires de neuf universités en Hollande.

1.5.3.9.1 - International Monetary Fund (IMF): recherches classées par date.

1.5.3.9.2-EconWPA (USA), Economics Working Paper Archive ${ }^{(115)}$

1.5.3.9.3-Cowles Foundation, Yale University, Discussion Papers ${ }^{(116)}$

1.5.3.9.4-Center for Mathematical Studies in Economics and Management Science, Northwestern University (USA) ${ }^{(117)}$, Discussion Papers

\subsubsection{5-Caltech Division of Social Sciences (USA) ${ }^{(118)}$, Working Papers On-Line}

\subsubsection{6-UCLA Department of Economics (USA) ${ }^{(119)}$, Working Papers}

1.5.3.9.7-S-WoPEc, Scandinavian Working Papers in Economics ${ }^{(120)}$.

\subsubsection{0- Archives sur le modèle RePEc autres qu'économie}

1.5.3.10.1-Documents in Information Science (DoIS) ${ }^{(121)}$, base de données d'articles et d'actes de conférence publiés sous format électronique dans la spécialité bibliothéconomie, contient près de 10042 articles et 3045 actes de conférence dont 6928 sont téléchargeables (en date du 28 Février 2003).

1.5.3.10.2-Une base de données plus étendue, R.C.L.I.S. ${ }^{(122)}$ (Research in Computing, Library and Information Science) est en cours de réalisation.

1.6-Archives de journaux en libre accès

\footnotetext{
${ }^{(111)} \mathrm{http}: / /$ ideas.repec.org/archives.html

${ }^{(112)} \mathrm{http}: / /$ netec.mcc.ac.uk/WoPEc.html

${ }^{(113)} \mathrm{http}: / /$ netec.mcc.ac.uk/WoPEc/data/PaperSeries.html

${ }^{(114)} \mathrm{http}: / /$ drcwww.uvt.nl/dbi/instructie/degree/

${ }^{(115)} \mathrm{http}: / /$ econwpa.wustl.edu/

${ }^{(116)} \mathrm{http}: / /$ cowles.econ.yale.edu/P/cd/cfdpmain.htm

${ }^{(117)} \mathrm{http}: / / w w w . k e l l o g g . n w u . e d u /$ research/math/discussion_papers.htm

${ }^{(118)} \mathrm{http}: / / \mathrm{www} . h s s . c a l t e c h . e d u / \mathrm{ss} /$ working-papers/recent

${ }^{(119)} \mathrm{http}: / /$ econweb.sscnet.ucla.edu/research/workingp.htm

(120) $\mathrm{http}: / /$ swopec.hhs.se/

${ }^{(121)} \mathrm{http}: / /$ dois.mimas.ac.uk/

${ }^{(122)} \mathrm{http}: / /$ rclis.org/about.html
} 


\subsection{1- BioMed Central ${ }^{(123)}$ (120 journaux en date du 20 Février 2003)}

1.6.2 - PubMed Central (PMC) ${ }^{(124)}$, c'est l'archive numérique du Journal des Sciences de la Vie de la bibliothèque nationale de Médecine des Etats-Unis ( 52 journaux participants en date du 20 Février 2003).

1.6.3- HighWire Press Free Online Full-text Articles ${ }^{(125)}$ (liste limitée aux journaux publiés en ligne avec l'assistance de HighWire Press).En date du 28 Février 2003, des 1, 358,713 articles que recelait l'archive 472,871 étaient disponible gratuitement et en texte intégral.

\subsection{4-Free Online Full-text Articles est l'entrée principale à Earth's Largest Free Full-Text} Science Archives (une liste produite par HighWire Press).

1.6.5-Advances in Theoretical and Mathematical Physics ${ }^{(126)}$, recouvre les archives d'ArXiv. Toutes les archives sont archivées à L.A.N.L. (Los Alamos National Laboratory) et ses sites miroirs. ATMP a seulement des liens avec l'archive ci-dessus et de ce fait réalisant un des premiers journaux électronique qui recouvre l'archive de publication électronique globale.

1.6.6-BBS Prints Interactive Archive ${ }^{(127)}$ du journal Behavioral and Brain Sciences qui contient des recherches originales " cibles " certifiées par les pairs, des commentaires ouverts et des réponses par les pairs

1.6.7-Psycoloquy ${ }^{(128)}$, articles et commentaires par les pairs dans toutes les spécialités de la psychologie ainsi que les sciences cognitives, la neuroscience, la biologie comportementale, l'intelligence artificielle, la robotique/vision, la linguistique et la philosophie (Eprints.org archive).

\section{7-Archives disciplinaires :}

1.7.1 -ArXiv (1991- $)^{(129)}$, . le site de l'administration centrale est situé à Cornell University, des sites miroirs partout dans le monde, administre l'accès à plus de 230,000 recherches, résumés. Comprend des liens aux analyses de citation pour les recherches par SLAC

1.7.2-Citeseer ${ }^{(130)}$ (1998- , aussi connu sous l'appellation de ResearchIndex), archive développé au NEC Research Institute, NJ, USA, cache des textes intégraux de recherche accessible gratuitement sur la Toile en Informatique au format Postscript et PDF par une indexation de citation autonome. Prétend indexer plus de 500,000 recherches.

1.7.3-ebizSearch (2001- $)^{(131)}$, administré par the eBusiness Research Center à Pennsylvania State University, basé sur le logiciel Citeseer .Crée d'une manière autonome des citation d'index de la littérature e-commerce. Le moteur de recherche cherche des sites Web d'universités, d'organisations commerciales, d'instituts de recherche et de départements gouvernementaux pour extraire des articles émanant d'universités, des recherches, des livres blancs, des rapports de consultance, des articles de magazine et des statistiques et des faits publiés. Ce n'est pas la totalité des articles qui son hébergés par ebizSearch, qui fait une analyse de citation de tous les articles accessibles.

\footnotetext{
${ }^{(123)} \mathrm{http}: / / \mathrm{www} \cdot$ biomedcentral.com/start.asp

${ }^{(124)} \mathrm{http}: / /$ pubmedcentral.nih.gov/

${ }^{(125)} \mathrm{http}: / /$ highwire.stanford.edu/lists/freeart.dtl

${ }^{(126)} \mathrm{http}: / / \mathrm{www}$. intlpress.com/journals/ATMP/

(127) http://www.bbsonline.org/

${ }^{(128)} \mathrm{http}: / /$ psycprints.ecs.soton.ac.uk/

${ }^{(129)} \mathrm{http} / / /$ www.arXiv.org/

${ }^{(130)} \mathrm{http}: / /$ citeseer.nj.nec.com/cs

(131) http:/gunther.smeal.psu.edu/
} 


\subsection{4-Mathématiques}

1.7.4.1-K-theory Preprint Archives ${ }^{(132)}$ (à partir de 1995), administré par " Mathematics Department, University of Illinois at Urbana-Champaign".*

1.7.4.2-Topology Atlas ${ }^{(133)}$ serveur de prépublications (recherches à partir de 1995) était le plus actif en 1996 et 1997, accepte toujours des soumissions mais suggère l'utilisation de " the Mathematics Archive" (arXiv.org ou son interface) pour distribuer et trouver des prépublications, hébergé à " York University, North York, Ontario "*

1.7.4.3-Algebraic Number Theory Archives ${ }^{(134)}$ (à partir de 1996, gelée à partir de Janvier 2003), hébergé par " The Mathematics Department, University of Illinois at UrbanaChampaign ".

1.7.4.4-Mathematical Physics Preprint Archive, mp_arc ${ }^{(135)}$ (recherches à partir de 1991), hébergé par " Mathematics Department, University of Texas at Austin ".

1.7.4.5-Hopf Topology Archive ${ }^{(136)}$ (recherches à partir de 1997), hebergée par The Department of Mathematics, Purdue University.

1.7.4.6-Preprints on Conservation Laws ${ }^{(137)}$ (recherches à partir de 1996), administrée au " Department of Mathematical Sciences, Norwegian University of Science and Technology (NTNU), Trondheim ".*

1.7.4.7-MCMC (Markov Chain Monte Carlo) ${ }^{(138)}$ methodology Preprint Service (recherches à partir de 1993), administrée par " The Statistical Laboratory, University of Cambridge".

1.7.4.8-Jordan Theory Preprint Archives ${ }^{(139)}$ (recherches à partir de 1996), hébergée au " Institut fur Mathematik, Universitat Innsbruck ".

1.7.4.9-Groups, Representations and Cohomology Preprint Archive ${ }^{(140)}$ (recherches à partir de 1995), administré par " Department of Mathematics, University of Georgia, USA ".

1.7.4.10-Field Arithmetic Archive ${ }^{(141)}$, située à Ben Gurion University in Be'er-Sheva, Israël, héberge des prépublications électroniques en arithmétique des champs,la Théorie de Galois ,la théorie modèle des champs et des disciplines connexes .

1.7.4.11-MGNet preprints ${ }^{(142)}$ (recherches à partir de 1991, dernière recherche déposée en 2001), Department of Computer Science, Yale University, entrepôt pour l'information concernant les méthodes de décomposition des tubes multi grille, à plusieurs niveaux, à plusieurs échelles, d'agrégation, correction de défauts et de domaine.

\footnotetext{
(132) http://www.math.uiuc.edu/K-theory/

${ }^{(133)} \mathrm{http}: / /$ at.yorku.ca/topology/preprint.htm

${ }^{(134)} \mathrm{http}: / / w w w . m a t h . u i u c . e d u / A l g e b r a i c-N u m b e r-T h e o r y /$

${ }^{(135)} \mathrm{http}: / /$ rene.ma.utexas.edu/mp_arc/index.html

(136) $\mathrm{http}: / /$ hopf.math.purdue.edu/

${ }^{(137)} \mathrm{http}$ ///www.math.ntnu.no/conservation/

* - Ces trois sites sont interrogeables via MPRESS

${ }^{(138)} \mathrm{http}: / /$ www.statslab.cam.ac.uk/ mcmc/index.html

${ }^{(139)} \mathrm{http}: / /$ mathematik.uibk.ac.at/mathematik/jordan/index.html

${ }^{(140)} \mathrm{http}: / / w w w . m a t h . u g a . e d u / a r c h i v e . h t m l$

${ }^{(141)} \mathrm{http}: / / w w w . c s . b g u . a c .11 /$ research/Fields/

${ }^{(142)} \mathrm{http}: / /$ casper.cs.yale.edu/mgnet/www/mgnet-papers.htm
} 
En date du mois de de Mai 2001, l'Union Mathématique Internationale adopta une résolution encourageant les mathématiciens à rendre leur travail disponible en ligne :" Le libre accès à la documentation mathématique est un but important .... Notre action va élargir, d'une manière substantielle, le réservoir du matériel primaire librement disponible aidant les scientifiques qui travaillent sans accès adéquat aux bibliothèques ".

\subsection{5-Sciences cognitives}

1.7.5.1-Cogprints ${ }^{(143)}$ (1997- ), archive électronique pour l'auto archivage de recherches dans toutes les spécialités de psychologie, neuroscience, linguistique et un certain nombre de spécialités relatives à l'informatique, la philosophie, la biologie, la médecine, l'anthropologie ainsi qu'un nombre d'autres spécialités pertinentes à l'étude de la cognition.C'était initialement un projet dans le programme du JISC Electronic Libraries (eLib) Programme, administré par " The IAM Group, University of Southampton ".

\subsection{6- Libray and information sciences}

\subsubsection{1 -E-LIS, E-Prints in Library and Information Science ${ }^{(144)}$}

1.7.6.2-DList, Digital Library of Information Science and Technology ${ }^{(145)}$ (October 2002), administrée par " School of Information Resources and Library Science and Arizona Health Sciences Library, University of Arizona ".

\subsection{7-Archives de prépublications encouragées par les éditeurs (auteurs auto archivant)}

1.7.7.1-NetPrints Clinical Medicine and Health Research ${ }^{(146)}$ (December 1999- ), BMJ Publishing Group et HighWire Press, un entrepôt de recherches originales non contrôlées par les pairs.

1.7.7.2-Chemistry Preprint Server (August 2000- ), ChemWeb.com ${ }^{(147)}$, Elsevier (640 recherches en date du 3 Mars 2003).

1.7.7.3-Computer Science Preprint Server (November 2001- ), Elsevier ${ }^{(148)}$ (409 recherches en date du 24 Mars 2003).

1.7.7.4-Mathematics Preprint Server ${ }^{(149)}$ (May 2001- ), Elsevier, un fournisseur de données enregistré, répond aux normes OAI v 2.0 (842 recherches en date du 25 Mars 2003.

\subsection{8-Autres archives disciplinaires}

1.7.8.1- -HTP Prints, the History \& Theory of Psychology Eprint Archive (September 2001), administrée par York University, Toronto ${ }^{(150)}$

1.7.8.2-Education-line ${ }^{(151)}$ (1997- ), base de données libre d'accès de textes intégraux de recherches présentées à des conférences, des travaux de recherche et littérature sous forme

\footnotetext{
${ }^{(143)} \mathrm{http}: / / \operatorname{cog}$ prints.ecs.soton.ac.uk/

${ }^{(144)} \mathrm{http}: / /$ eprints.rclis.org/

${ }^{(145)} \mathrm{http}: / / \mathrm{dlist} . \operatorname{sir}$. arizona.edu/

${ }^{(146)} \mathrm{http} / / / \mathrm{clinmed} . n e t p r i n t s . o r g / h o m e . d t l$

${ }^{(147)} \mathrm{http}: / / \mathrm{www} . c h e m w e b . c o m /$ preprint

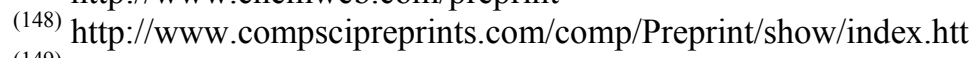

${ }^{(149)} \mathrm{http}: / /$ www.mathpreprints.com/math/Preprint/show/

${ }^{(150)} \mathrm{http}: / /$ htpprints.yorku.ca/

${ }^{(151)} \mathrm{http}: / / w w w . l e e d s . a c . u k / e d u c o l /$
} 
électronique qui encourage la recherche, les politiques et les pratiques en science de l'éducation. C'était initialement un projet dans le cadre du " JISC Electronic Libraries (eLib) Programme, administrée par" The Brotherton Library, University of Leeds ".

1.7.8.3-Social Science Research Network (SSRN), Social Science Electronic Publishing, Inc. ${ }^{(152)}$. Les travaux de recherche et les résumés sont fournis par journal, éditeur et institutions pour distribution à travers eLibrary du SSRN et qui consiste en deux parties :

-Une base de données qui contient des résumés sur plus de 49200 travaux de recherche savants et des recherches en préparation.

-Une " Electronic Paper Collection " contenant plus de 308000 documents en texte intégral téléchargeables.

Social Science Research Network se compose de journaux / réseaux de recherche spécialisés dans les sciences sociales: Comptabilité, Economie, Economie financière, Sciences juridiques, Administration et Négociations. C'est un curieux amalgame d'un point de vue de publication électronique. Ce n'est pas une archive de publication électronique à $100 \%$, c'est plus un service basé sur l'abonnement .Le modèle économique et le but ne sont pas clair. Est ce que les recherches téléchargeables le sont librement? De toute évidence, certaines le sont, mais quelle proportion l'est, si proportion il y a, ceci n'est pas clair .Il semble que les bases peuvent être feuilletées séparément mais pas recherchées séparément alors qu'il n'est pas possible de rechercher seulement les travaux librement accessible.

1.7.8.4-ArchiveSIC (153) (Archive ouverte en Sciences de l'Information et de la Communication), recherche en texte intégral sur les sciences de l'information et de la communication (site bilingue en français et en anglais).

1.7.8.5-Electronic Colloquium on Computational Complexity ${ }^{(154)}$ (recherches à partir de 1994) sous la responsabilité de la chaire de l'informatique théorique et les nouvelles applications à l'Université de Trier .Rapports de recherche, enquêtes et livres en complexité informatique.

1.7.8.6-Cryptology ePrint Archive (155) (2000- ), administrée par " The International Association for Cryptologic Research " (IACR), incorpore les contenus de " The Theory of Cryptology Library 1996-1999 ".

1.7.8.7-The Digital Library of the Commons (DLC), Indiana University ${ }^{(156)}$, contient une archive de recherches soumises par les auteurs, ainsi que des recherches publiées dans des conférences en texte intégral, des dissertations, des travaux de recherche et des prépublications (le terme "The Commons " est un terme générique pour les ressources partagées dans lequel chaque dépositaire à un intérêt égal. Les études sur les " Commons " comprennent les informations sur les "Commons" avec des questions sur le savoir publique, le domaine public, la science ouverte et le libre échange des idées)

1.7.8.8-Organic Eprints ${ }^{(157)}$ (September 2002- ), fondée par " The Danish Research Centre for Organic Farming (DARCOF), archive en accès libre pour des recherches en agriculture organique.

\footnotetext{
${ }^{(152)} \mathrm{http}: / /$ www.ssrn.com /

${ }^{(153)} \mathrm{http}: / /$ archivesic.ccsd.cnrs.fr/

(154) http://www.eccc.uni-trier.de/eccc /

(155) http://eprint.iacr.org /

(156) $\mathrm{http}: / /$ dlc.dlib.indiana.edu /

${ }^{(157)} \mathrm{http}$ ://orgprints.org/
} 
1.7.8.9-University of California International and Area Studies ${ }^{(158)}$ (UCIAS) Digital Collection (October 2002- ), partenariat de l'University of California Press, the California Digital Library (CDL) et des unités de recherche à orientation internationale sur huit campus de l'Université de Californie (UC). Publie des articles, des monographies et des volumes édités contrôlés par les pairs selon des critères mis au point par le comité éditorial interdisciplinaire d'UCIAS Editorial Board et approuvé par the University of California Press

1.7.8.10-Formations, Faculty of Arts, University of Ulster ${ }^{(159)}$, accueille des prépublications en études sur les media et des forums d'apprentissage électroniques sur la base de courtes recherches. Initialement un projet du programme " The JISC Electronic Libraries (eLib)".

1.7.8.11-Ecology Preprint Registry ${ }^{(160)}$ (recherches à partir de Juillet 2001), hébergée au " The National Center for Ecological Analysis and Synthesis ", dissémination des résultats de nouvelles recherches destinées à la publication (i.e. pas de recherches ou de la littérature grise), seulement des prépublications avec une base théorique peuvent être soumises, la couverture peut être étendue pour inclure des soumissions de toute la discipline de l'écologie.

1.7.8.12-PhilSci Archive (161) (Janvier 2001- ), hébergée au " The Departments of Philosophy and of History and Philosophy of Science ", University of Pittsburgh, prépublications en philosophie de la science.

\section{2-Open DOAR et Open ROAR : Deux outils incontournables des entrepots d'archives}

Introduction

Face à l'accroissement exponentiel et à l'explosion du nombre de sites d'archives ouvertes et après l'arrêt de The Core Metalist of Open Access EPRINTS Archives en 2003 pour des raisons inconnues (mais dues, à notre avis, à une raison principale et qui serait l'accroissement incontrôlé du nombre de sites), une nouvelle génération d'entrepôt vit le jour et qui répondait plus aux nouvelles formes d'entreposage des archives en libre accès .Ces deux sites, Open ROAR et Open DOAR, développés à partir de 2004 et 2005 sont quasi identiques à la différence que ROAR fournit des informations sur les données et les informations contenues dans le site. Nous allons les présenter en détails afin de voir quelles en sont les fonctionnalités et les informations qu'ils présentent

\footnotetext{
${ }^{(158)} \mathrm{http}: / /$ repositories.cdlib.org/uciaspubs/

${ }^{(159)} \mathrm{http}: / /$ formations2.ulst.ac.uk/

${ }^{(160)} \mathrm{http}: / /$ www.nceas.ucsb.edu:8504/esa/ppr/ppr.Query

(161) http://philsci-archive.pitt.edu/
} 


\title{
OpenDOAR
}

\author{
Directory of Open Access Repositories \\ Home | Find | Suggest | Tools | FAQ | About | \\ Contact Us
}

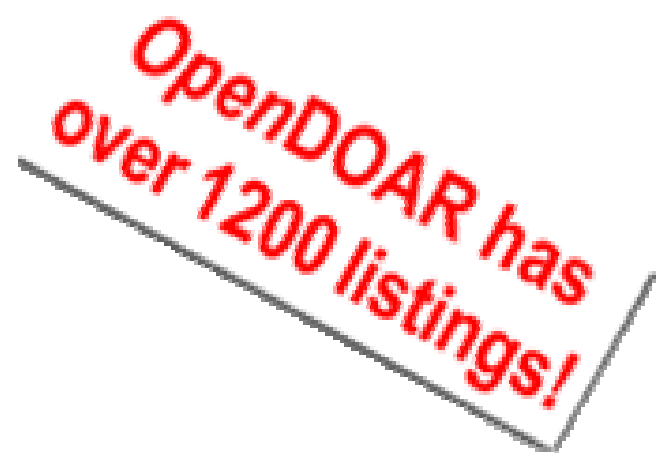

\subsection{1-Présentation}

Open DOAR, est une liste de référence des entrepôts académiques en libre accès .En plus, elle permet aussi de faire des recherches par entrepôt ainsi que par contenu d'entrepôt. La liste actuelle énumère les entrepôts et permet une subdivision et une sélection par une variété de critères dont les plus importants sont :

- Spécialisation.

- Pays.

- Langue.

- Type de contenu.

- Type de logiciel.

- Type d'entrepôt.

\subsection{2-Statistiques}

Le site offre aussi une suite de tableaux statistiques et dont les plus importants sont :

2.1.2.1-Entrepôts par continent :

\section{Proportion of Repositories by Continent - Worldwide}

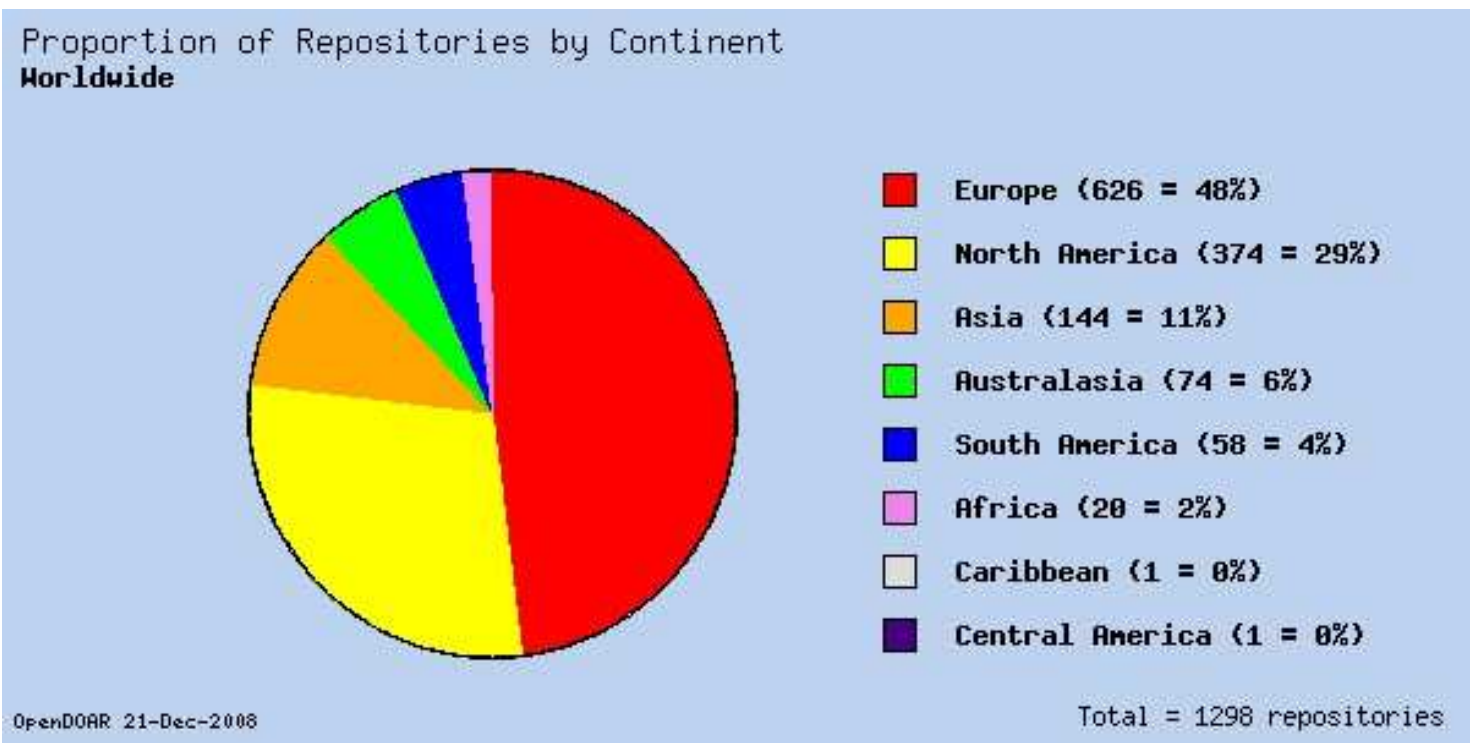

${ }^{(162)}$ http://www.opendoar.org/ 
Ce tableau montre à quel point le déséquilibre est patent entre les pays développés et ceux en voie de développement. En effet, sur les 1298 entrepôts recensés en date du 21/12/2008, les continents nord américains et européens recèlent plus que les trois quart des entrepôts (77\%). Seul l'Asie avec $11 \%$ des entrepôts semble relever le défi. Le reste des continents recensés (l'Asie australe, l'Amérique du Sud, les Caraïbes et l'Afrique) quant à eux ne représentent que $12 \%$ de l'ensemble alors qu'ils représentent l'immense majorité de la population mondiale. S'il est vrai que les moyens dont dispose le monde développé et le monde en voie de développement ne peuvent être comparés, il n'en demeure pas moins que c'est le chercheur du Sud qui profiterait de cette manne plus que le chercheur d'un pays du Nord. A coté du manque de moyens, il semblerait aussi que les responsables (et même des fois les concernés eux même) ignorent (ou feignent d'ignorer) la formidable opportunité qu'offre ce medium.

\subsubsection{2-Proportion par continent des organisations ayant un (ou des) entrepôts:}

\section{Proportion of Repository Organisations by Continent - Worldwide}

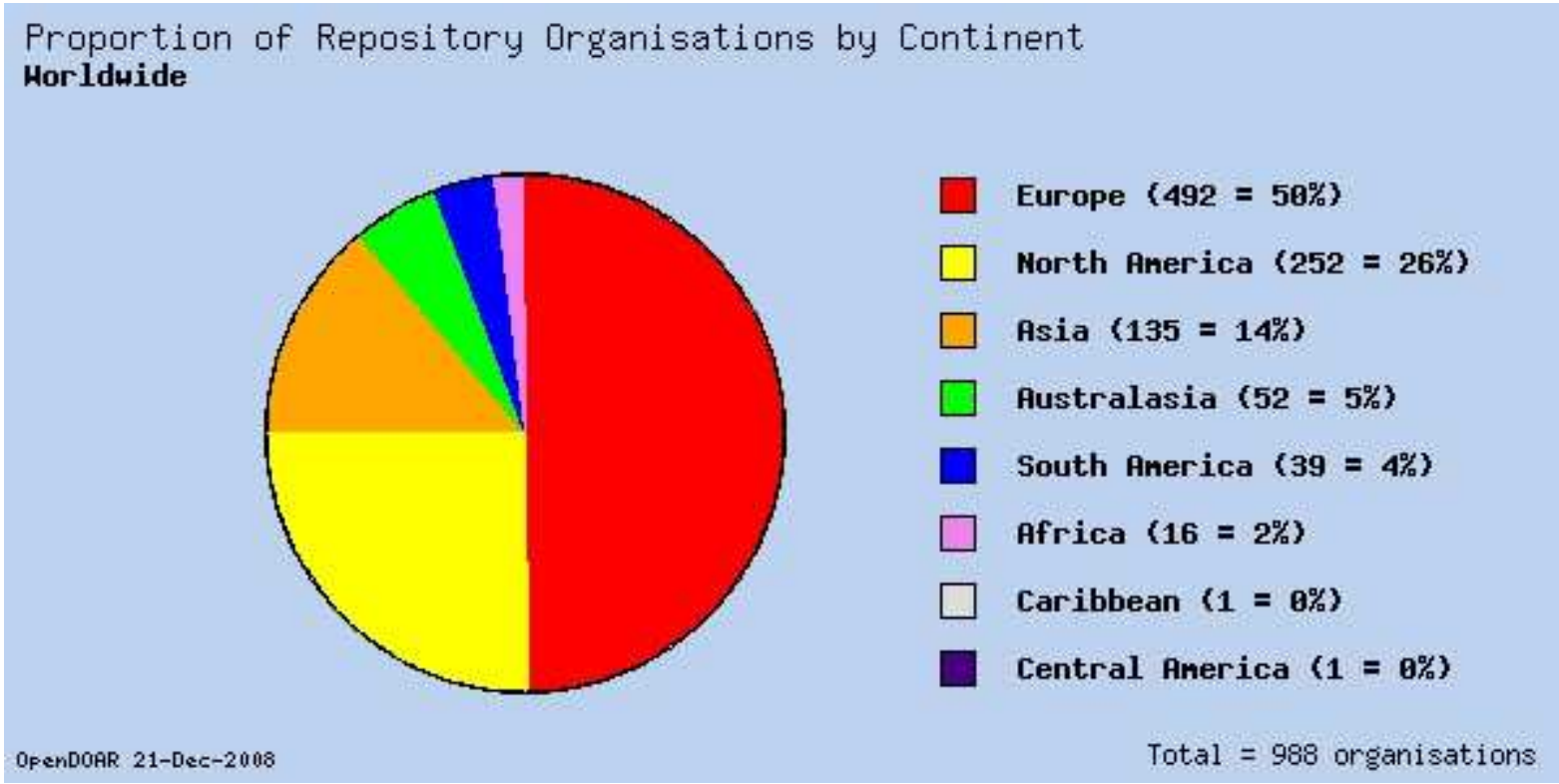

Ce tableau représente le nombre d'organisations par continent ayant un (ou plus) d'entrepôt. A peu près le même déséquilibre est constaté avec des petites variations dûes au fait que ce tableau recense le nombre d'organisation sans tenir compte du nombre d'entrepôts. Certaines de ces organisations ayant plus d'un entrepôt dans certain cas (on en dénombre certains qui en possède jusqu'à 20).Ainsi sur les 988 organisations recensées en date du 21 Décembre 2008, l'Europe recèle la moitié des organisations et l'Amérique du Nord, un peu plus du quart $(26 \%)$, l'Asie en recèle quant à elle presque le $1 / 7^{\mathrm{e}}(13 \%)$. Le reste est presque équitablement reparti (si on peut dire) entre l'Asie australe, l'Amérique du Sud et l'Afrique qui en fait totalisent moins que le pourcentage de l'Asie. Les Caraïbes et l'Amérique centrale quant à elles font figure de parents pauvres avec seulement 2 entrepôts $(0.20 \%)$ sur les 988 recensés à la date du recensement. 


\section{Proportion of Repositories by Country - Worldwide}

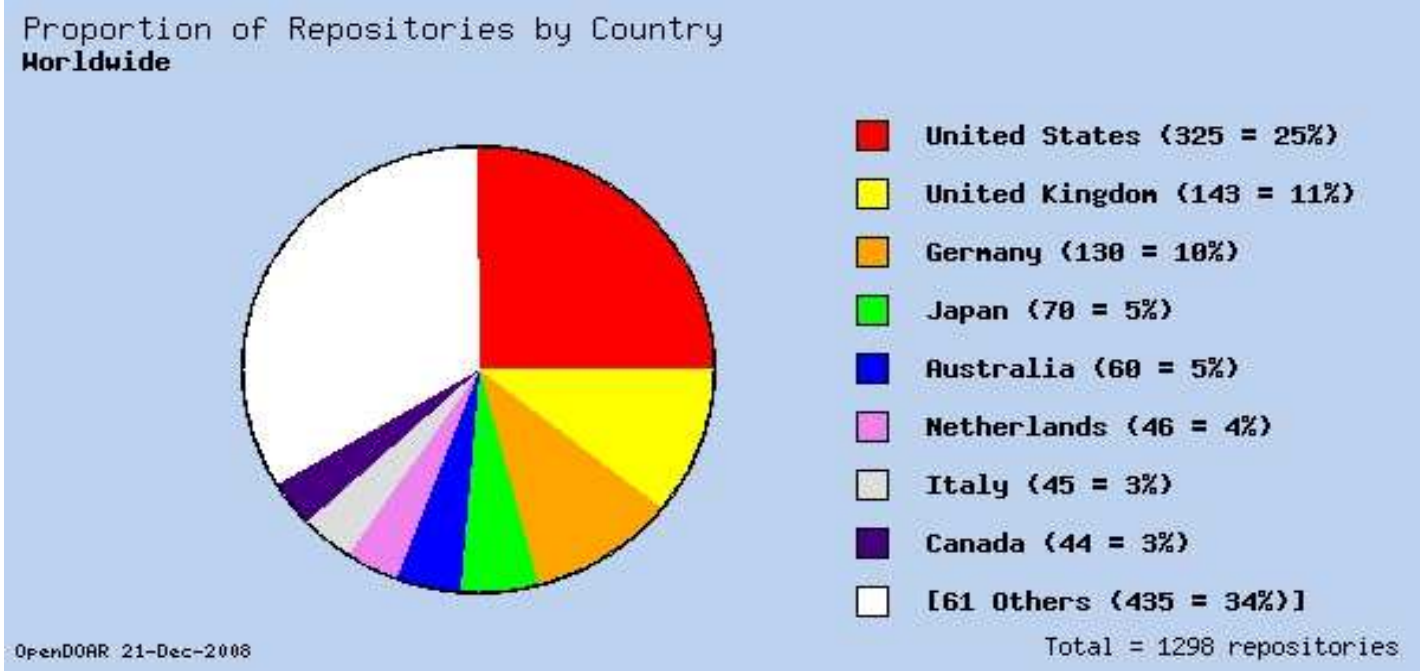

A l'image des statistiques de deux tableaux précédents, ce tableau ne fait que confirmer la domination des Anglo-Saxons dans le domaine des archives ouvertes.A partir d'ArXiv en 1991 et les travaux de Stevan Harnad à l'Université de Southampton en 1993 ( notamment Cogprints ), les Anglo-Saxons ont toujours été à la pointe de la défense du libre accès et de l'établissement d'entrepôts d'archives. Ceci se vérifie dans le tableau par pays où la Grande Bretagne et surtout les Etats Unis représentent un peu plus du $1 / 3 \mathrm{e}$ du total des entrepôts recensés ce qui dépasse même le total des 61 autres pays réunis $(34 \%)$.On remarque que l'Allemagne elle aussi rivalise avec la Grande Bretagne et totalise $10 \%$.Quant au reste des entrepôts, ils sont presque équitablement distribués entre le Japon, l'Australie, la Hollande, l'Italie et le Canada qui totalisent $20 \%$ du total des entrepôts recensés .Quant aux autres pays , tel que cela a été maintes fois signalé, les pays qui pourraient le plus en profiter ne semblent pas accorder une importance quelle qu'elle soit à ce medium facilitant la communication et rendant l'information disponible à moindre frais à l'image de l'Algérie qui ne possède aucune archive ouverte ni un entrepôt d'archives réunissant les différentes archives du pays, d'une institution donnée ou d'une discipline donnée tel que c'est le cas pour nombre de pays.

\subsubsection{4-Proportion des entrepôts d'organisation par pays:}

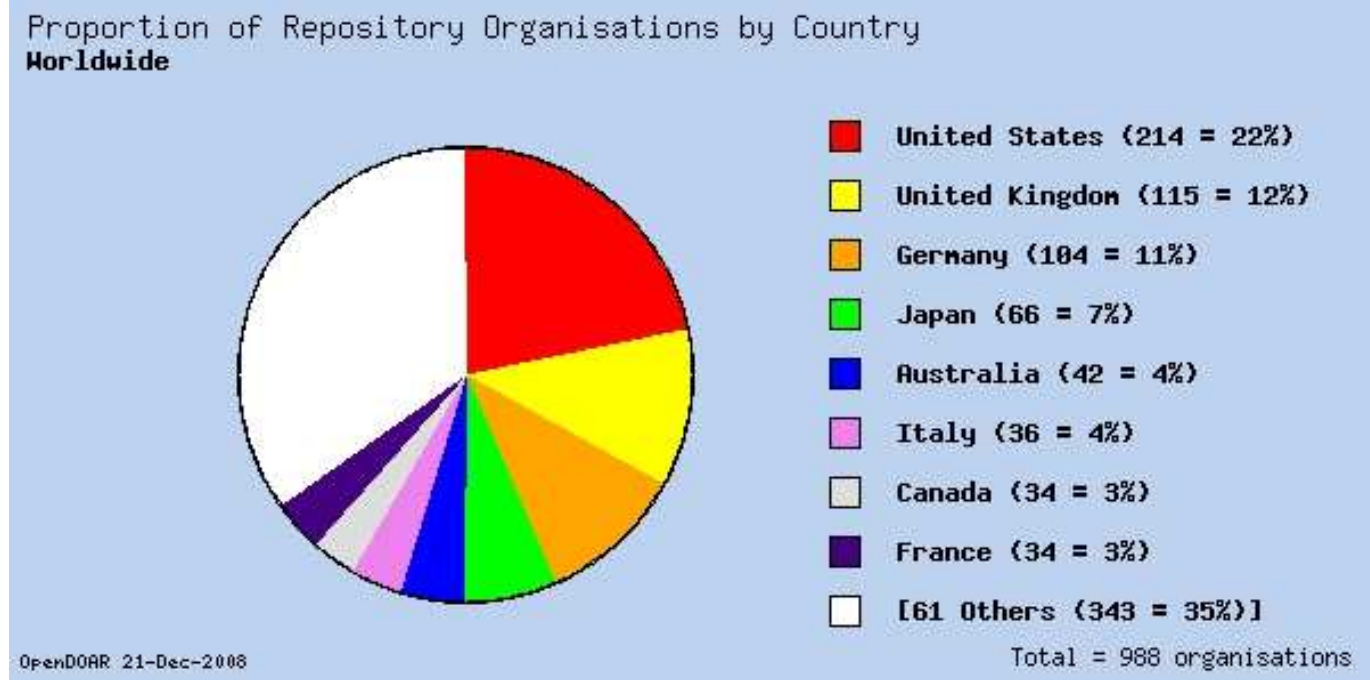


Ce tableau représente le nombre des entrepôts d'organisations dans chaque pays .Vu que plusieurs pays ont plus d'un entrepôt d'organisation, le nombre total d'organisations recensées était de 988 en date du 21 Décembre 2008. Le même " classement " des pays fournisseurs d'entrepôts se confirme avec les Etats Unis et la Grande Bretagne toujours en pole position. L'Allemagne se maintient avec $11 \%$ des entrepôts d'organisation .Le reste des entrepôts d'organisations sont repartis entre le Japon (7\%), l'Australie et l'Italie (4\%), le Canada et la France (3\%).Quant aux autres pays, au nombre de 61 , ils ne représentent que $35 \%$ soit à peu près le même pourcentage que les des deux pays qui dominent la scène: Les Etats Unis et le Grande Bretagne .Ceci illustre de manière tout ce qu'il y a de plus clair l'énorme fossé qui, malheureusement, ne fait que se creuser de plus en plus chaque jour qui passe.

\subsubsection{5-Utilisation des logiciels d'entrepots en libre accès:}

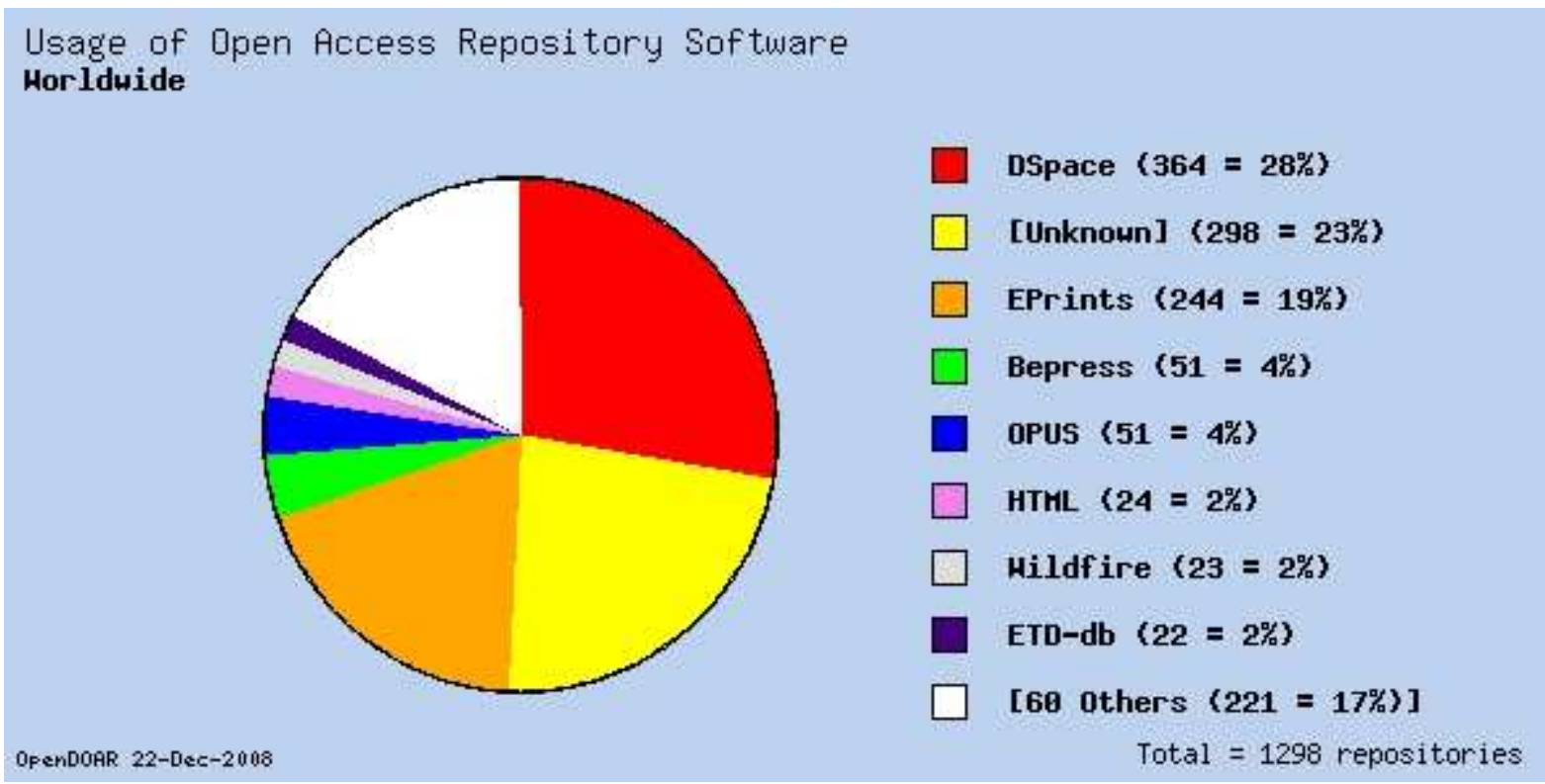

Pratiquement, la moitié des logiciels utilisés pour le fonctionnement de ces entrepôts sont d'origine anglo-saxonne. En effet, Dspace (163), développé par les bibliothèques du Massachusetts Institute of Technology (MIT) et les laboratoires de Hewlett Packard (HP) et Eprints ${ }^{(164)}$, développé par " The School of Electronic and Computer Science " de 1'Université de Southampton représentent $47 \%$ des logiciels utilisés. Presque le quart $(23 \%)$ est d'origine inconnue et sont sûrement des logiciels maisons que les organisations ont elles même developpé. Le reste des logiciels utilisés comme Bepress ${ }^{(165)}$, OPUS ${ }^{(166)}$, Wildfire ${ }^{(167)}$, ETD-db ${ }^{(168)}$ etc...et qui représentent $14 \%$ des logiciels utilisés sont aussi d'origine anglo-saxonne. Une proportion presque analogue (17\%) représente les autres logiciels et qui sont d'origine diverses. Ce qui nous donne que presque les $2 / 3$ des logiciels utilisés (61\%) sont d'origine anglo saxonne .Plus que tous les autres tableaux qui décrivent les différents statistiques au sujet d'Open DOAR, ce tableau démontre la domination des anglo-saxons dans la création et l'application des logiciels de gestion des entrepôts d'archives ouvertes. Ceci peut être considéré comme logique car le monde anglophone et les Etats Unis en particulier sont le berceau de la révolution informatique (surtout pour ce qui est des logiciels libres et qui

\footnotetext{
(163) Www.dspace.org

(164) $\mathrm{http}: / /$ www.eprints.org

(165) $\mathrm{http://bepress.com}$

(166) $\mathrm{http://www.bath.ac.uk/library/eprints/index.html}$

(167) http:// www.wildfirehq.org

${ }^{(168)} \mathrm{http}: / /$ scholar.lib.vt.edu/ETD-db/index.shtml
} 
sont une des composantes les plus importantes sur laquelle s'appuie la philosophie du mouvement du libre accès) et il est normal que les outils de gestion y soit développés.

\subsubsection{6-Types d'entrepôts en libre accès:}

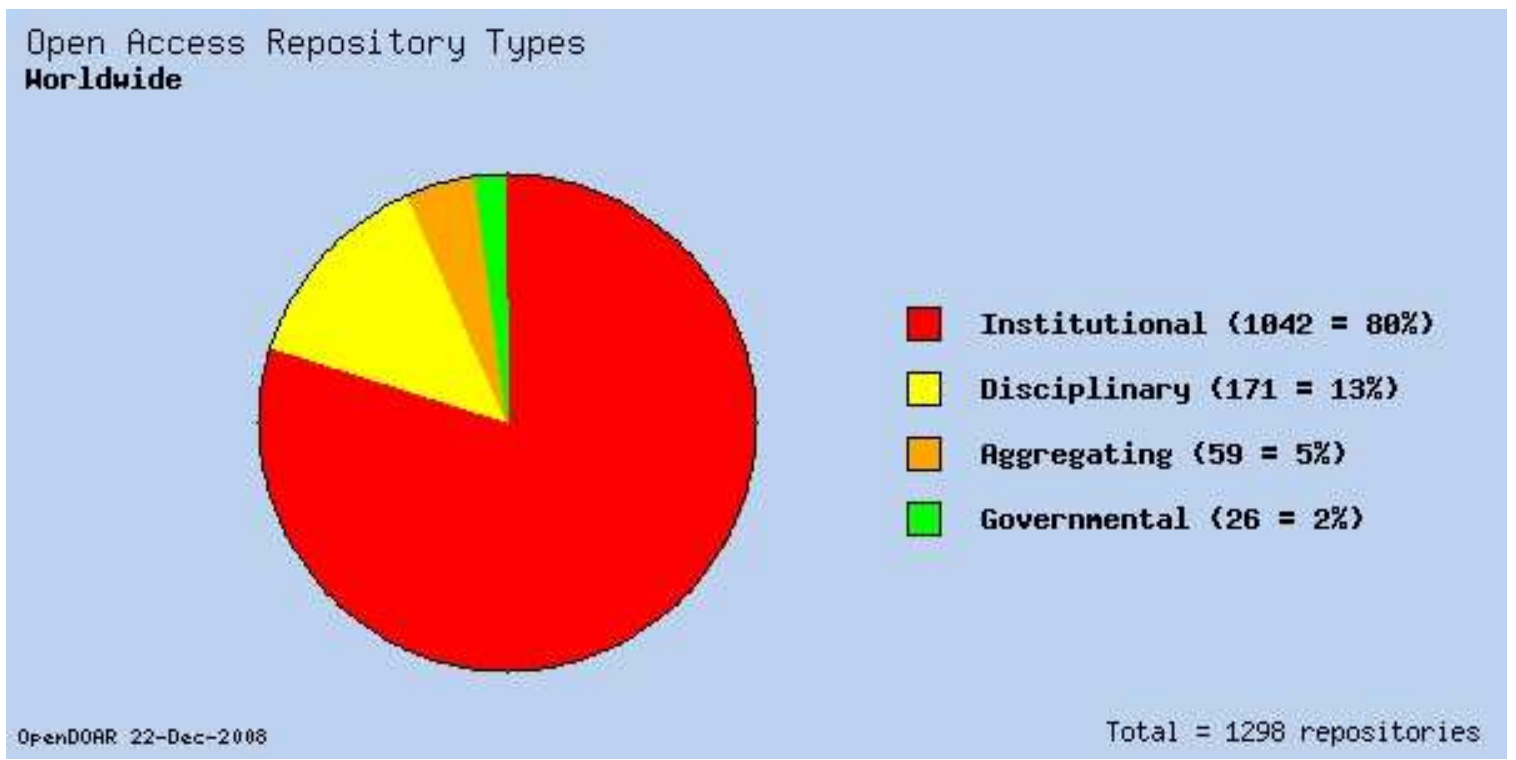

Plusieurs recherches ont fait ressortir la relative appréhension de l'utilisation des archives ouvertes par les chercheurs et ceci est dû à plusieurs raisons et dont les plus évidentes sont : la nouveauté du medium, l'ignorance des bienfaits de déposer dans une archive, la méconnaissance même du medium, la peur de se voir déposséder du fruit de son travail,l'absence de contrôle par les pairs etc....Une autre raison est la non obligation faite au chercheur de déposer son travail dans une archive ouverte. Cette hypothèse est corroborée par les résultats du tableau précédant où sur les 1298 entrepôts recensés en date du 22 Décembre $2008,80 \%$ des entrepôts sont des entrepôts institutionnels qui sont des entrepôts où seul le chercheur affilié à l'institution (université, unité de recherches, laboratoire, etc...) peut ( et dans certains cas doit) déposer les résultats de sa recherche .Cette obligation tient à une raison simple : l'institution finançant la recherche, il est normal que le chercheur y dépose son travail et lui donne la primeur de sa découverte ( surtout pour les sciences exactes où le facteur temps est autrement plus sensible que le sciences humaines et sociales ). Cette manière de voir a des conséquences positives pour le chercheur, son institution et aussi la recherche en général. Le deuxième type d'entrepôt est l'entrepôt disciplinaire qui regroupe les recherches d'une seule discipline mais réunit plusieurs institutions à l'image d'ArXiv (physique, informatique, statistiques, etc...) et ArchiveSic (sciences de l'information et de la communication en général).Ce genre d'entrepôt a l'avantage d'attirer des chercheurs d'horizons différents mais dont la spécialité est une. Il représente $13 \%$ de l'ensemble des entrepôts recensés. Le troisième type d'entrepôt est l'entrepôt agrégateur et qui est un entrepôt qui rassemble les données qui se trouvent dans des entrepôts subsidiaires. Il représente $5 \%$ des entrepôts recensés. Enfin le dernier type d'entrepôt est l'entrepôt gouvernemental et qui représente $2 \%$ des entrepôts recensés .Il s'agit d'entrepôt où sont déposées les recherches émanant d'un gouvernement donnée à l'image des NIH aux Etats Unis. Il nous parait tout de même étonnant que ce genre d'entrepôts ne représente pas une plus grande proportion car les gouvernements même les plus libéraux, financent les recherches surtout celles qui ont trait à la santé et on se serait attendu à ce qu'ils représentent un plus grand pourcentage.

Il est loisible de dire que les types d'entrepôts qui existent au niveau d'OpenDOAR reflètent la prévalence de chaque type d'entrepôts dans la réalité. L'immense majorité (les $\left.4 / 5^{\mathrm{e}}\right)$ des entrepôts sont institutionnels et ceci est du au fait à l'obligation qu'impose l'institution à ses chercheurs de déposer leur travaux dans une archive qu'elle gère. Les entrepôts d'archives 
disciplinaires représentent $13 \%$ du total, c'est des entrepôts qui se singularisent par le fait que bien qu'ils ne soient pas reliés organiquement à une institution, ils regroupent les recherches qui ont trait à une même discipline et de ce fait sont très utiles au chercheur dont les centres d'intérêt sont définis. Le reste des entrepôts (agrégateurs et gouvernementaux) ne représentent pas une grande proportion (seulement $8 \%$ ) et ceci est dû à leur spécificités qui ne semblent pas très propice à leur succès comparativement aux deux autres types d'entrepôts cités.

\subsubsection{7-Type de contenus dans les entrepôts DOAR}

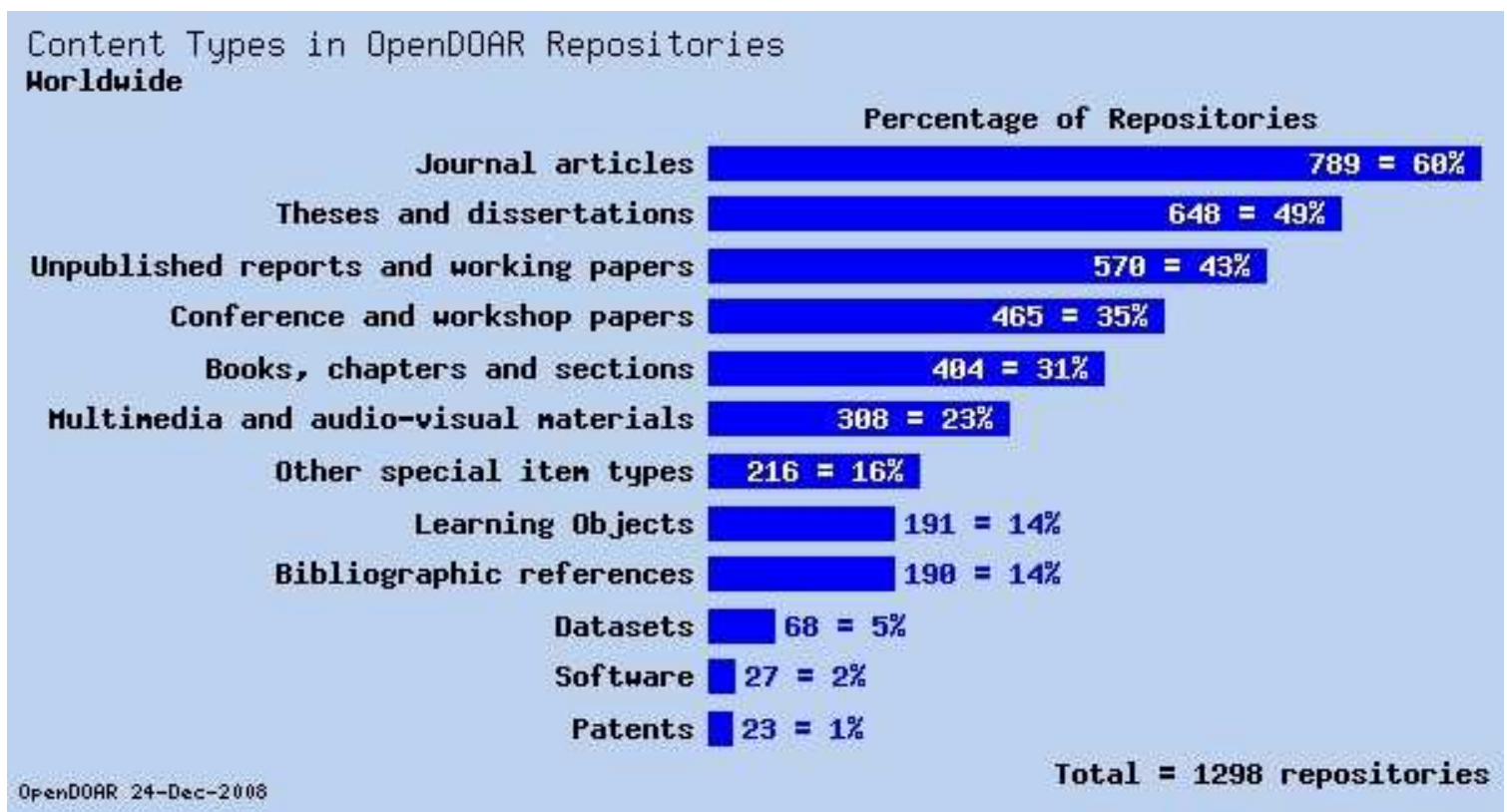

Ce tableau nous indique quel type de documents les entrepôts DOAR recèlent. La première remarque qui s'impose est que sur les douze types de documents que contient DOAR (articles de journaux, thèses et dissertations, rapports non publiés et travaux de recherche, recherches présentées à des conférences et ateliers, chapitres et sections de livres, matériel multimédia et audiovisuel, autres types d'objets spéciaux, objets d'apprentissage, références bibliographiques, ensemble de données, logiciels et brevets), neuf types de documents (articles de journaux, thèses et dissertations, rapports non publiés et travaux de recherche, recherches présentées à des conférences et ateliers, chapitres et sections de livres, matériel multimédia et audiovisuel, autres types d'objets spéciaux, objets d'apprentissage, références bibliographiques) y sont prévalent et représentent des pourcentages assez élevés surtout pour le quatre premiers (articles de journaux, thèses et dissertations, rapports non publiés et travaux de recherche, recherches présentées à des conférences et ateliers). On peut expliquer cette prévalence par le fait que ces types de documents (surtout les articles de périodiques) se prêtent à ce type de medium du fait de sa rapidité et de son influence par le facteur temps. En effet, les recherches apparaissant dans les articles de périodiques (surtout en science médicales et exactes) ont besoin d'être connues le plus vite possible pour un nombre de raisons dont la plus importante est l'utilisation de la découverte et son application dans les délais les plus bref et qui peut (dans le cas de la médecine) sauver des vies. On remarque aussi que sur le quatre premiers types de documents, les thèses et dissertations, les rapports non publiés et travaux de recherche, recherches présentées à des conférences et ateliers représentent un segment du savoir qui a longtemps souffert (et souffre toujours quoiqu'à un degré moindre) d'une sorte de non exposition .La littérature grise a toujours été confinée du fait de sa spécialisation à un public restreint et n'être que peu connue et que par ceux qui on en besoin : les chercheurs. Les archives ouvertes se développant de plus en plus, il semblerait qu'elles soient appelées à servir de nouveau medium par lequel cette masse non négligeable et de haute facture scientifique de données se popularise et soit plus visible.Enfin, on 
remarquera dans ce tableau que les pourcentages ne représentent pas le pourcentage par rapport au tout (l'entrepôt) mais est le pourcentage du type de document dans l'entrepôt en général. Ceci est dû au fait que plusieurs types documents se retrouvent dans différentes archives.

\subsubsection{8-Les langues les plus utilisées dans Open DOAR}

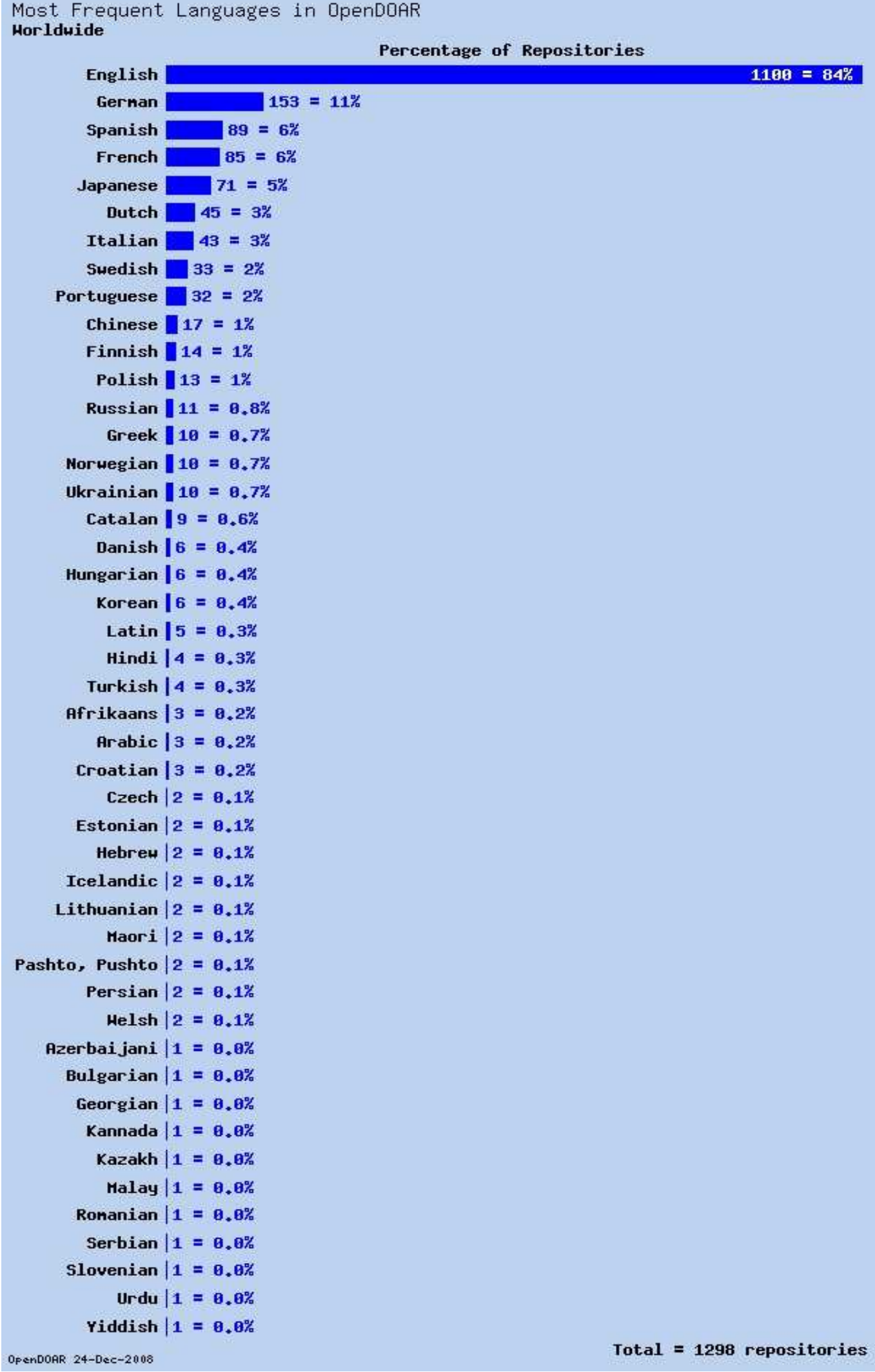


Etant donné l'avance prise par les pays anglo-saxons dans l'établissement des entrepôts d'archives ouvertes, il est logique que cette avance se traduise au niveau de l'utilisation de la langue. D'un autre coté, il est de notoriété que la langue anglaise est la langue de la science par excellence en plus du fait qu'elle est utilisée dans toutes les organisations internationales en tant que langue première. De ce fait, certains pays même si leur langue officielle n'est pas l'anglais l'utilisent toujours et ceci pour avoir un plus de visibilité. C'est le cas de SCIELO, l'archive ouverte lancée par les Sud Américains et qui après son lancement a poussé les chercheurs sud américains à faire des recherches en langue anglaise. D'ailleurs, une analyse plus détaillée des sites recensés par DOAR, montre que la grande majorité des sites ont une fonctionnalité permettant de passer généralement à la langue anglaise (et des fois aux autres langues).Ainsi le site de l'Université de Saint Gallen ${ }^{(169)}$ en Suisse est en langue allemande et a une version en langue anglaise alors que les langues les plus utilisées en Suisse sont le Français et l'Italien à coté de l'Allemand. Même les sites français (à l'image de " Mémoires en Sciences de l'Information et de la Communication (mémSIC)" ${ }^{(170)}$ ) pourtant chatouilleux sur le plan de la langue, ont généralement des sites où la langue anglaise est représentée . D'autres sites cependant ne sont présentés qu'en langue autochtone à l'image de ArchitekturInformatik ${ }^{(170)}$ et qui est en Allemand. De toute évidence, la langue anglaise occupe dans le domaine de la recherche une place prépondérante qui fait que les langues minoritaires ont peu de se voir représenter d'une manière équitable. D'ailleurs, nous avons cité une précédemment une étude de l'Unesco et qui situait le pourcentage des sites en langue anglaise sur la Toile à $82 \%$.

Quant aux autres langues, on les citera par ordre décroissant de nombre de sites (nous éviterons de citer des pourcentages car à l'image du tableau précédant représentant les types de contenus, les pourcentages dépassent - et de loin - les 100\% car nombre d'entrepôts contiennent plus d'une langue et ceci a été comptabilisé) :

- Allemand : 153 entrepôts.

- Espagnol : 89 entrepôts.

- Français : 85 entrepôts.

- Japonais : 71 entrepôts.

- Hollandais : 45 entrepôts.

- Italien : 43 entrepôts.

Il serait intéressant de savoir est ce que la prépondérance et la domination accentuée de la langue anglaise sont dûes au fait que le mouvement du libre accès est né et s'est développé dans les pays anglo- saxons ou est ce que d'autres raisons et paramètres entrent en ligne de compte. De même, qu'il serait instructif de voir d'ici à quelques années est ce qu'une démocratisation des entrepôts (linguistiquement parlant) se produira et est ce que les langues dites minoritaires referont leur retard surtout qu'une langue comme le Chinois, parlé par plus d'un milliard d'êtres humains (et aussi par un pays dont la croissance est à l'orée de ce $21^{\mathrm{e}}$ siècle la plus rapide du point de vue économique) ne représente que le $1 \%$ du total des entrepôts recensés.

\footnotetext{
${ }^{(169)} \mathrm{http}: / /$ www.unisg.ch/hsgweb.nsf/wwwPubhomepage/webhomepageger?opendocument

(170) $\mathrm{http}: / /$ memsic.ccsd.cnrs.fr /

${ }^{(171)} \mathrm{http}: / /$ architektur-informatik.scix.net/cgi-bin/works/OAI
} 


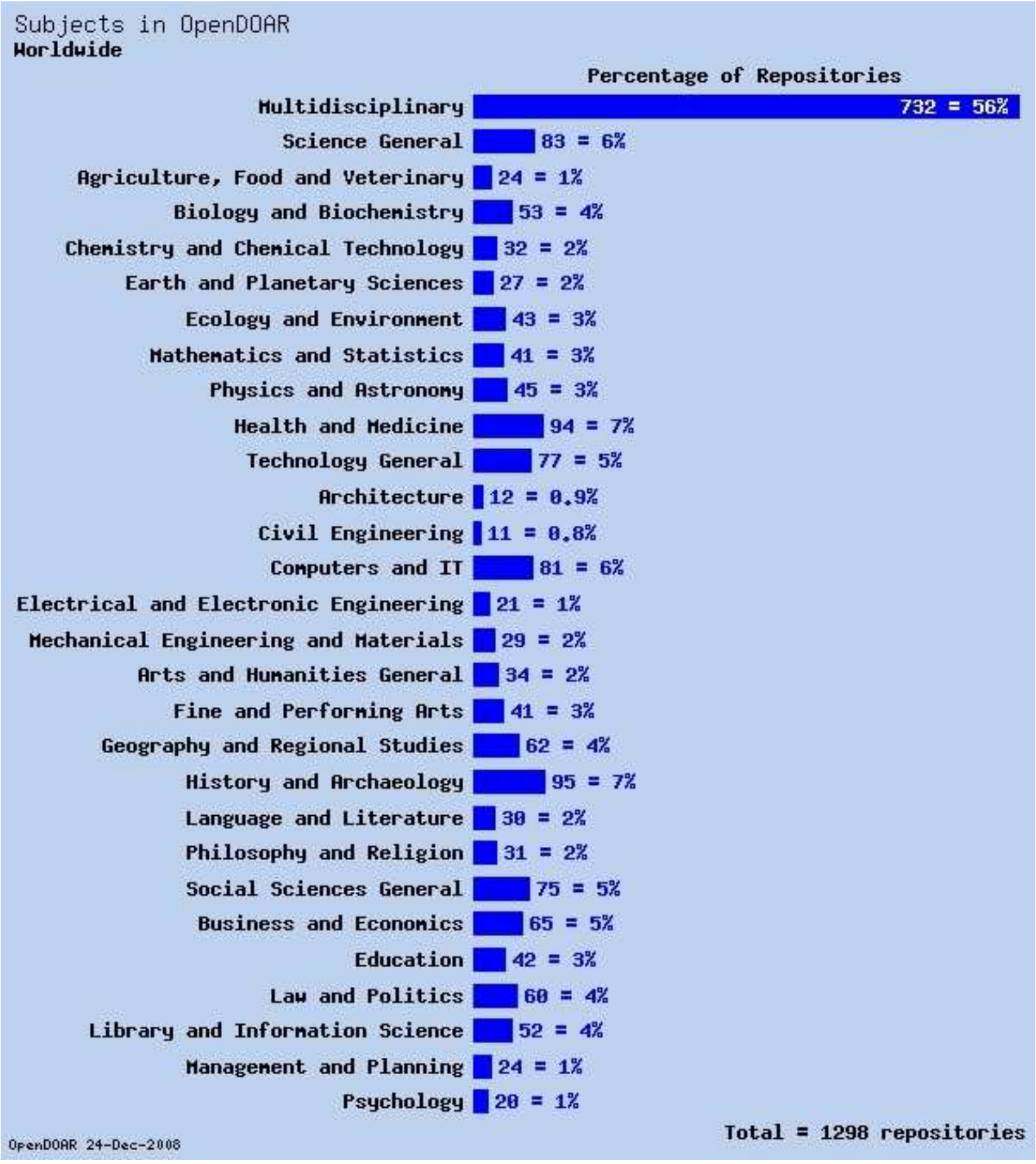

Nous remarquons de prime abord dans ce tableau que les entrepôts multidisciplinaires forment la majorité des entrepôts soit $56 \%$.On peut y voir une certaine logique si nous comparons ce pourcentage avec le pourcentage des types d'entrepôts qui sont majoritairement institutionnels et qui représentaient dans le tableau un pourcentage de $80 \%$. En effet, les entrepôts institutionnels (surtout ceux d'une université à titre d'exemple) ont tendance à recenser les différents travaux que les différents départements, instituts, centres de recherche ou laboratoires de la dite institution et ceci a induit une sorte de catégorie où la difficulté de déterminer la spécialité la fait rentrer dans la catégorie de multidisciplinaire. D'un autre coté, face la difficulté de déterminer les spécialités dans chaque entrepôt, les entrepôts disciplinaires ne représentent que $13 \%$ et ceci toujours dans le tableau qui traite des types d'entrepôts. On peut toujours y voir la même logique étant le fait que les entrepôts institutionnels (non spécialisés) sont la majorité, il en a résulté que les entrepôts disciplinaires (spécialisés) sont une minorité. A titre d'exemple, la spécialité Bibliothéconomie (dont la dénomination a d'ailleurs connu sous l'influence des nouvelles technologies qui en sont 
devenues une partie intégrale de la spécialité une pléthore de dénominations comme sciences de l'information, technologies de l'information, technologies de la communication, sciences documentaires etc...), peut être difficilement classée dans une spécialité bien déterminée. Elle peut être dans les sciences sociales, humaines, histoire, manuscrits, archéologie et surtout informatique avec l'avènement de l'ordinateur comme outil indispensable au travail du bibliothécaire (appellation réductrice face aux nouvelles taches que ce dernier est appelé à exécuter dans le cadre de son travail).Les concepteurs de cet entrepôt reconnaissent, d'ailleurs la faiblesse de leur classification et recommandent de consulter les entrepôts multidisciplinaires en premier lieu vu que les grandes institutions tendent à y inclure la totalité de leur production. Nous remarquons de notre coté, que certaines dénominations des sujets traités dans Open DOAR, prêchent par un excès de généralités. En effet, des appellations telles que Généralités en Science, Généralités en Technologie, Généralités en Arts et Humanités ou Généralités en Sciences Sociales ne donnent pas la pleine mesure et ne décrivent pas le contenu car, comme son nom l'indique, le contenu est général. Il est très difficile de voir dans une rubrique telle que Généralités en Science une rubrique significative étant donné que le mot Science est très vague. D'ailleurs, nous avons cru déceler une anomalie qui résulte de cette approximation. Il est pour le moins curieux (et sûrement contraire aux orientations et contenus des archives ouvertes) qu'Open DOAR recense 95 entrepôts en Histoire et Archéologie, 62 en Géographie et Etudes Régionales, 41 en Beaux Arts et Arts du Spectacle, 30 en Langage et Littérature et seulement 45 en Physique et astronomie , 21 en Ingénierie Electrique et Electronique, 12 en Architecture, 11 en Ingénierie Civile .Beaucoup plus, la spécialité par laquelle ont démarré les archives ouvertes étant les sciences physiques, il est pratiquement inconcevable qu'une spécialité aussi peu demandeuse de vitesse de transmission des données qu'est l'Archéologie et l'Histoire puisse avoir plus du double des entrepôts d'archives que la Physique et l'Astronomie. Notre hypothèse est que les sciences exactes en général sont très compartimentées et très spécialisées ce qui a conduit à ce que les différents entrepôts n'aient pas des chiffres d'archives élevés et en fait, il existe une multitude d'entrepôts chacun spécialisé dans une branche très spécialisée. A titre d'exemple, arXiv qui se spécialise en Physiques, Mathématiques, Informatique, Biologie quantitative et Statistiques a en tant que spécialisation en sciences physiques par exemple douze sous groupes et qui sont :

1-Astrophysique

2-Matière condensée

3-Relativité générale et cosmologie quantique

4-Physiques des hautes énergies (expérimentation)

5-Physiques des hautes énergies (treillage)

6-Physiques des hautes énergies ( phénoménologie)

7-Physiques des hautes énergies (théorie)

8-Physique mathématique

9- Expérimentation nucléaire

10-Théorie nucléaire

11-Physiques (qui se subdivisent en 22 sous groupes très spécialisés)

12-Physiques quantiques.

La spécialisation Mathématiques a de son coté 22 subdivisions et Informatique 38 subdivisions .Si nous examinons maintenant l'entrepôt d'archives en Bibliothéconomie 
" The Public Knowledge Project " (172) fondé par John Willinsky, et qui dépend de la Faculté des sciences de l'éducation de l'Université de la Colombie britannique, on s'aperçoit que les sujets qu'il aborde ne sont pas de la même précision que celle qu'a ArXiv. En effet, les sujets abordés sont ceux qui ont trait au libre accès sous ses différentes formes, étant donné que PKP est un programme tendant à vulgariser et à présenter les différents produits que ses chercheurs ont mis au point (dont " Open Journal System " logiciel libre conçu pour la gestion des journaux électroniques et " Open Conference Systems " qui est un logiciel libre permettant la mise en place de site Web pour les conférences).Donc, cet entrepôt a tendance a être plus étoffé qu'ArXiv parce qu'il contient des spécialités qui dans ArXiv seraient distribués en de multiple sous groupes .

\subsection{3-Accroissement de la base de données OpenDOAR}

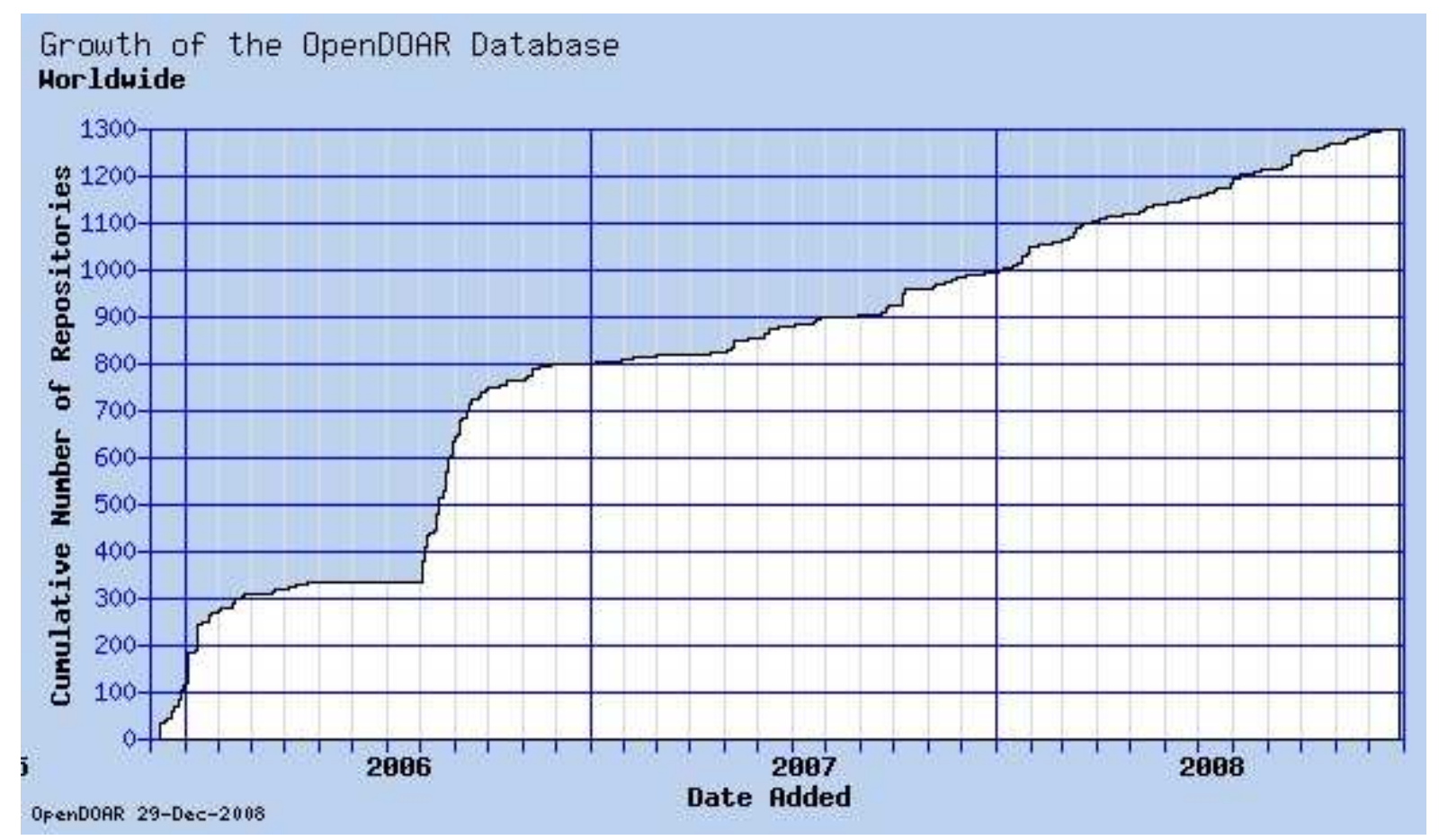

Depuis son lancement officiel en date du 15 Février 2005 par l'Université de Nottingham en Grande Bretagne et l'Université de Lund en Suède et fort du sponsoring d'un nombre d'institutions connues pour leur défense du libre accès telles que l'Open Society Institute ${ }^{(173)}$, The Joint Information Systems Committe ${ }^{(174)}$ ainsi que SPARC Europe ${ }^{(175)}$, le projet OpenDoar avait atteint en date du 29 Décembre 20081298 entrepôts ( le nombre d'entrepôts était en date du 28 Août 20081218 entrepôts).On remarque que le site a modestement débuté en Décembre 2005 et a régulièrement progressé depuis pour atteindre le chiffre actuel. Si au mois de Décembre 2005, le site ne recensait qu'une centaine d'entrepôts, il a enregistré à la mi Janvier 2006 un accroissement assez important qui l'a mené vers les 200 entrepôts .Ce n'est qu'a partir de Juillet 2006 et jusqu'au début Décembre 2006 qu'il connaît une spectaculaire augmentation de quelque $425 \%$ en passant de 320-325 à 800 entrepôts. Ceci n'est pas dû à un soudain accroissement des enregistrements des entrepôts mais, étant donné qu'un arriéré des enregistrements s'était constitué aux premières phases du lancement du projet, cette augmentation ne représente que le véritable et effectif nombre des entrepôts enregistrés. Ce n'est qu'à partir de Janvier 2007, qu'on peut voir la véritable progression des entrepôts. D'ailleurs, on remarquera que la progression à partir de cette date suit une courbe

\footnotetext{
${ }^{(172)}$ http://pkp.sfu.ca/biblio

(173) WwW.soros.org/

(174) Www.jisc.ac.uk/

(175) Www.sparceurope.org/
} 
beaucoup plus logique et moins erratique en passant de 800 à 1000 en 2007 et ensuite à 1298 à la fin 2008.

A coté de ces tableaux qui représentent de la meilleure manière les différentes fonctionnalités et statistiques d'Open DOAR, on peut rechercher le site par plusieurs paramètres et représentés dans les tableaux suivants :

2.1.4- Criteres de recherche

2.1.4.1-Recherche ou navigation par entrepôts

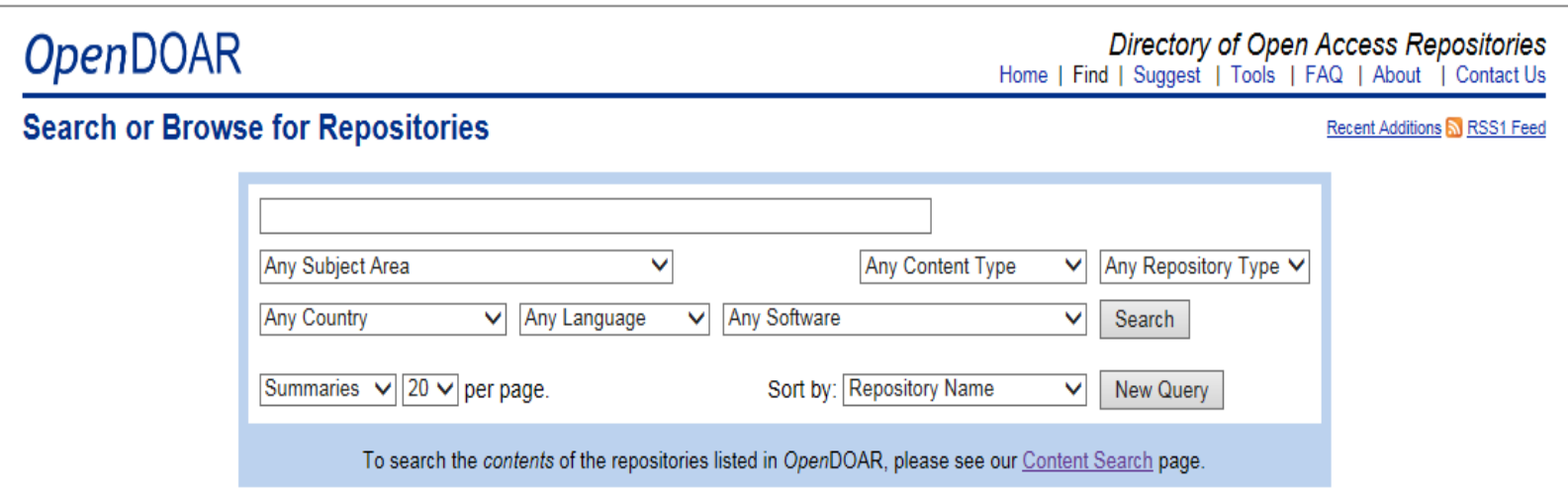

Cette fonctionnalité permet de rechercher

-par entrepôts (le site affiche tous les entrepôts que recèle le site)

-par paramètres dans les entrepôts (par sujets, par type de contenu, par type d'entrepôt, par pays, par langue, par logiciel utilisé)

\subsubsection{2-Recherche par contenus d'entrepôts}

\section{Search Repository Contents}

This service, based on the Google Custom Search engine, lets you search the contents of the repositories listed in OpenDOAR for freely available academic research information. This quality assured approach minimises (but does not eliminate!) spurious or junk results, and leads more directly to useful and relevant information. Full texts are available for most results.

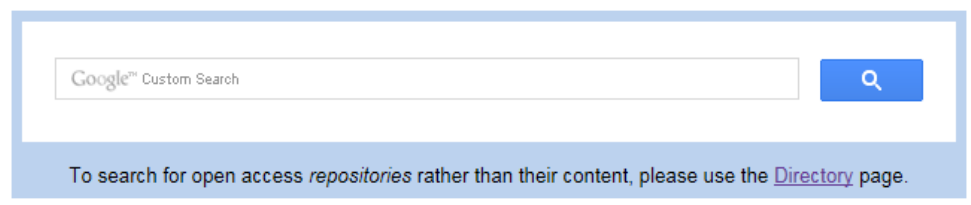

This service relies on Google's indexes, which in turn rely on repositories being suitably structured and configured for the Googlebot web crawler. If you are an administrator and your material is not being retrieved, first check that your repository is listed in OpenDOAR. If it is listed, you may need to review your set-up against Google's Guidelines for Webmasters and see the related pages in the Webmaster Help Center, especially the FAQ on how Google crawls sites. There is also excellent advice on $\underline{\text { How to Facilitate Google Crawling prepared by Peter Suber. }}$

Cette fonctionnalité permet de rechercher les contenus en texte intégral des entrepôts recensés par Open DOAR. Ceci est possible grâce à un service de recherche expérimental avec le moteur de recherche personnalisé de Google ${ }^{(176)}$ et dont la particularité est d'utiliser la puissance et l'expérience de Google et de l'allier à la base de données d'OpenDOAR. Ce qui, d'après le site, minimisera les résultats superflus, et donnera des informations plus utiles et plus pertinentes.

${ }^{(176)}$ http://www.google.com/coop/cse/ 


\section{OpenDOAR - Countries and Organisations}

\section{Africa | Asia | Australasia | Caribbean | Central America | Europe | North America | Oceania | South America | [Unspecified]}

\section{Click on a name to see the corresponding OpenDOAR summaries, or on a URL to visit the relevant website.}

C'est une liste d'entrepôts que recèle Open DOAR classée par continent (Afrique, Asie, Asie australe les Caraïbes, Amérique centrale, Europe, Amérique du Nord, Amérique du Sud).La recherche se fait soit par continent (après avoir cliqué sur le continent, on obtient une liste des pays qui ont lancé des entrepôts d'archives ouvertes) ou par nom d'organisation (et ceci en cliquant directement sur l'URL de l'institution).

\section{2-Registry of Open Access Repositories (ROAR) ${ }^{(177)}$}

${ }^{(177)}$ http://roar.eprints.org 


\section{Registry of Open Access Repositories (ROAR) $\square$ seach}

- Home Browse Search Content Search Register a Repository Help Login Register in ROARMAP

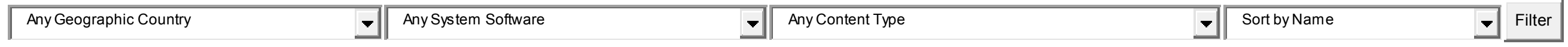

Registry of Open Access Repositories (ROAR) resulted in 1239 repositories

View this result set as a chart:

-
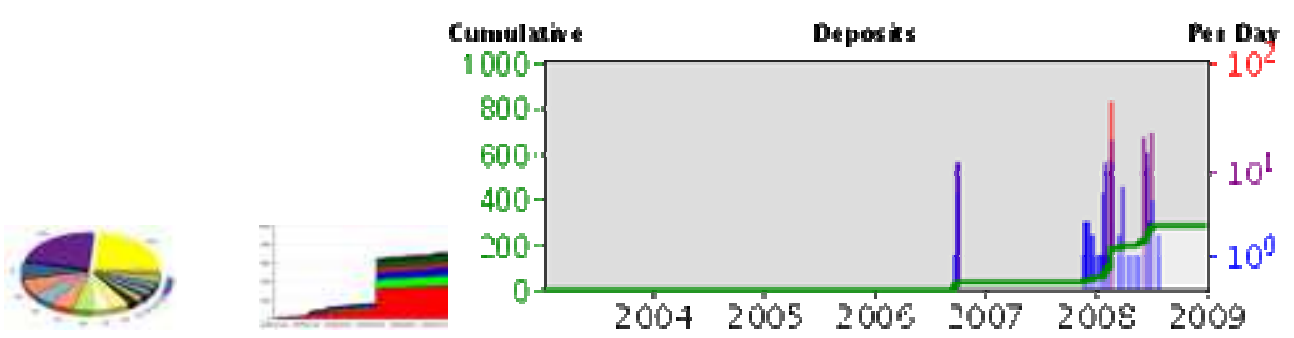

Export this result set as:

- Comma-Separated Format

Analyse the content of these repositories (ㅁveats

- Google Earth Overlay (Thumbnails / Bars)

- $\quad$... other formats )

Export repository records summary as: Cumulative Graph $\underline{\text { Cumulative Table File Format Graph }}$

View repository contents as:

Copyright 2005-2007 Tim Brody <tdb01r@ecs.soton.ac.uk $>$, University of Southampton, UK. (c)

This work is licenced under a Creative Commons Licence. 


\subsection{1-Présentation}

ROAR est une autre liste d'entrepôts en libre accès qui a commencé à fonctionner en 2004 et était appelée originellement " Institutional Archives Registry ".Elle fait suite à " GNU Eprints listings " qui était en place avant. ROAR a été créé pour avoir une meilleure maîtrise du listing de " GNU Eprints repositories " mais a évolué en un entrepôt de libre accès général. D'ailleurs, le site présente ROAR de la manière suivante : " Nous promouvons le libre accès à la littérature de recherche pré et post contrôlée par les pairs à travers l'auto archivage de l'auteur dans des archives institutionnelles de publications électroniques. Le libre accès à la recherche maximise l'accès à la recherche et, par delà, aussi l'impact de la recherche ce qui la rend plus productive et effective ". Ce registre à deux fonctions :

- Surveiller la croissance générale du nombre des archives de publications électroniques.

- Maintenir une liste des sites de " GNU Eprints " (logiciel créé par l'Université de Southampton pour faciliter l'auto archivage)

Donc, ROAR est devenu, à l'instar de UIUC (University of Illinois at Urbana-Champaign) registry of OAI-PMH ${ }^{(178)}$ ou de The Openarchives.eu ${ }^{(179)}$, un des innombrables entrepôts que la Toile a permit de développer.

\subsection{2-Critères de recherche:}

Il est possible de rechercher le site utilisant plusieurs paramètres dont les plus importantes sont :

2.2.2.1-Pays : classé alphabétiquement par nom de pays, il comporte aussi une rubrique " autres " qui recense quatre sites (arXiv.org e-Print archive, Elpub : Digital Library, RePEc: Research Papers in Economics et University Papers) qui ne sont attribués à aucun pays car ce sont des sites décentralisés ( à titre d'exemple RePEc: Research Papers in Economics est un site qui héberge un effort collaboratif de volontaires de 64 pays ).

Quant aux autres pays qui composent la liste, nous retrouvons les Etats-Unis et le Royaume Uni avec respectivement 260 et 130 entrepôts, alors que l'Allemagne (97 entrepôts), le Japon (63 entrepots), le Brésil (62 entrepôts) forment les autres pays où les entrepôts sont présents avec force.

2.2.2.2-Logiciel : cette partie du site recense les logiciels utilisés par les entrepôts pour faire fonctionner leurs sites. A coté de différents logiciels au nombre de 348 et qui sont des logiciels développés par les institutions, il existe encore 154 différents logiciels (Fedora, HAL, Greenstone, etc...) qui administrent différentes institutions .Enfin, la plupart des entrepôts sont administrés par des logiciels ayant fait leur preuve comme Dspace (373 entrepôts - 30,10 \%), Eprints (287 entrepôts -23,16\%) ou Bepress (77 entrepôts -06,21\%). On remarquera le fait qu'Eprints soit présent dans pratiquement le quart des entrepôts est normal car ROAR a débuté comme une liste des sites d'Eprint

2.2.2.3-Contenu : cette partie comporte les différents types de contenus que l'entrepôt contient. Il est composé dans son immense majorité de sites de recherche institutionnels ou départementaux au nombre de 671 (54.15\%), de sites de recherche interinstitutionnels au nombre de 113 (09.12\%), de sites de thèses électroniques au nombre de $110(08.87 \%)$ et de 107 sites contenant des journaux et des publications électroniques $(08.63 \%)$. A ces sites qui représentent une proportion dépassant les

\footnotetext{
${ }^{(178)}$ www.uiuc.edu/

(179) $\mathrm{http://www.openarchives.eu/}$

* En plus de Home : qui est l'interface du site. Elle présente le nombre d'entrepôts que recèle le site qui était de 1239 en date du 31 Décembre 2008) :
} 
$80 \%$, s'ajoutent 185 sites (soit 14,93\%) classés sous la rubrique " autres " et qui sont des sites qui ne peuvent être classés avec les autres sites car ils cumulent les paramètres de tous les autres sites.

\subsection{3-Filtrage :}

Cette rubrique comprend quatre sous rubriques et qui trient les différents sites par :

\subsubsection{1 -Noms de sites : et qui présente les différents sites classés alphabétiquement.}

2.2.3.2 -Nombre total de documents : cette sous rubrique présente les différents sites et les classe du plus fourni (en date du 31 Décembre 2008, PubMed Central avait 1508613 documents) jusqu'à certains nouveaux sites n'ayant pas encore enregistré de dépôt de documents

2.2.3.3-Date de création : cette sous rubrique présente les 1239 sites du plus recent au plus ancien. Comme on peut le voir, les sites de dépôt dans des archives ouvertes, quoique de manière peut être rudimentaire au début, avaient commencé bien avant qu'Internet ne devienne l'outil indispensable qu'il est devenu de nos jours. D'un autre coté, bien que les pays anglo-saxons aient été (et sont toujours) les premiers à s'investir dans le mouvement du libre accès et des archives ouvertes, c'est un site italien (" Archeologia e Calcolatori " et dont la création remonte au $1^{\text {er }}$ Janvier 1990) qui a le premier commencé à archiver des articles et surtout dans une spécialité qui n'est pas connue pour sa propension à l'utilisation des archives ouvertes. D'un autre coté, le deuxième plus ancien site est brésilien "Informaçao e Sociedade : Estudios et il date du $1^{\mathrm{er}}$ Janvier 1991. Encore une fois, c'est un site de sciences humaines (le titre se traduirait par " Information et Société " ) et une publication en série qui est parmi les premiers sites en archives ouvertes .Ceci est contraire aux idées générales reçues au sujet des archives ouvertes (prépondérance des pays anglo-saxons et aussi des sites en sciences exactes et médicales plutôt que les sites en sciences sociales et humaines) et mériterait, à ne pas en douter, un approfondissement pour savoir le pourquoi et les raisons de cet état de fait .

2.2.3.4 -Activité de dépôt : cette sous rubrique analyse les statistiques de dépôt par nombre et par jour chaque fois qu'un dépôt a eu lieu. La sous rubrique trie les dépôts selon trois paramètres et qui sont :

2.2.3.4.1-Dépôts de 1 à 9 : recense par ordre décroissant le nombre précité ( 1 à 9 dépôts / jour) et combien de fois ceci est arrivé .A titre d'exemple, en date du 31 Décembre 2008, le site de " E-Lis: Research in Computing and Library and Information Science" et " University of Southampton: Department of Electronics and Computer Science" avaient tous les deux respectivement 238 et 200 jours où la cadence de dépôt était entre 1 et 9 dépôts.

2.2.3.4.2-Dépôts de 10 à 99 : recense par ordre décroissant le nombre précité (de 10 à 99 dépôts/ jour) et combien de fois ceci est arrivé. A la même date, "HAL: Hyper Article en Ligne" et "Archive Ouverte INRIA" avaient respectivement 254 et 242 jours où la cadence de dépôt avait oscillé entre 10 et 99 dépôts.

2.2.3.4.3-Dépôt $100+:$ recense par ordre décroissant le nombre précité (plus de 100 dépôts/ jour) et combien de fois ceci est arrivé .A titre d'exemple, " arXiv.org e-Print archive (physics, mathematics, related fields) " et " PubMed Central " avaient respectivement 255 et 219 jours où la cadence de dépôt avait dépassé les 100 dépôts. Il est à remarquer que cette barre des plus de 100 dépôts n'est pas très commune. D'ailleurs, si on examine les sites qui l'ont atteint, très peu peuvent se prévaloir d'avoir des statistiques aussi élevées. Une analyse plus attentive, nous révèle 224 sites l'ont atteint mais l'immense majorité ne l'ont atteint que peu de fois .A la même date, 67 sites ne l'avaient atteint qu'une seule fois, et l'immense majorité ne l'avaient atteint que peu de fois. Si nous insistons sur ces chiffres, ceci est dû au fait que seul les sites connus et consommateurs de prépublications surtout peuvent se prévaloir de cette formidable quantité de documents déposés et on peut dire que " arXiv.org e-Print archive" (physics, mathematics, related fields) a eu pendant 
toute l'année des dépôts dépassant les 100 documents ce qui est tout simplement ahurissant car si on enlève les week-ends et les jours fériés, on s'aperçoit que les physiciens et les mathématiciens qui utilisent arXiv le font tout le temps.Ceci n'est que la preuve que les spécialisations en sciences exactes et médicales (à l'image de " PubMed Central " qui a eu 219 jours où les documents déposés ont dépassé les 100 dépôts) ont besoin de ce medium pour progresser alors que les sciences humaines et sociales elles n'ont pas auatant besoin de la rapidité du medium.

\subsection{4-Graphes}

A côté de ces paramètres de recherche, on peut aussi visualiser les résultats et les statistiques par l'intermédiaire de graphes et qui se présentent de trois manières suivantes :

\subsubsection{1- Graphe qui représente le type de contenus des entrepôts}

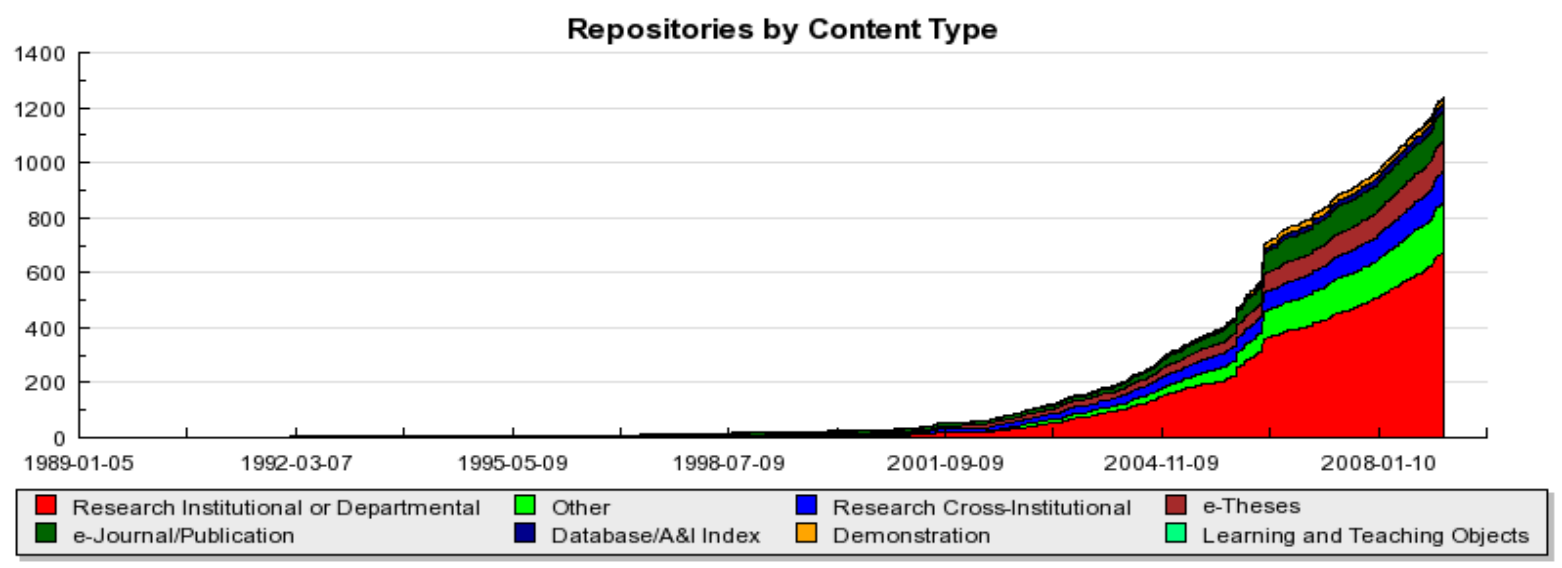

2.2.4.2-Un " camembert " représentant toujours le type de contenu

Repositories by Content Type
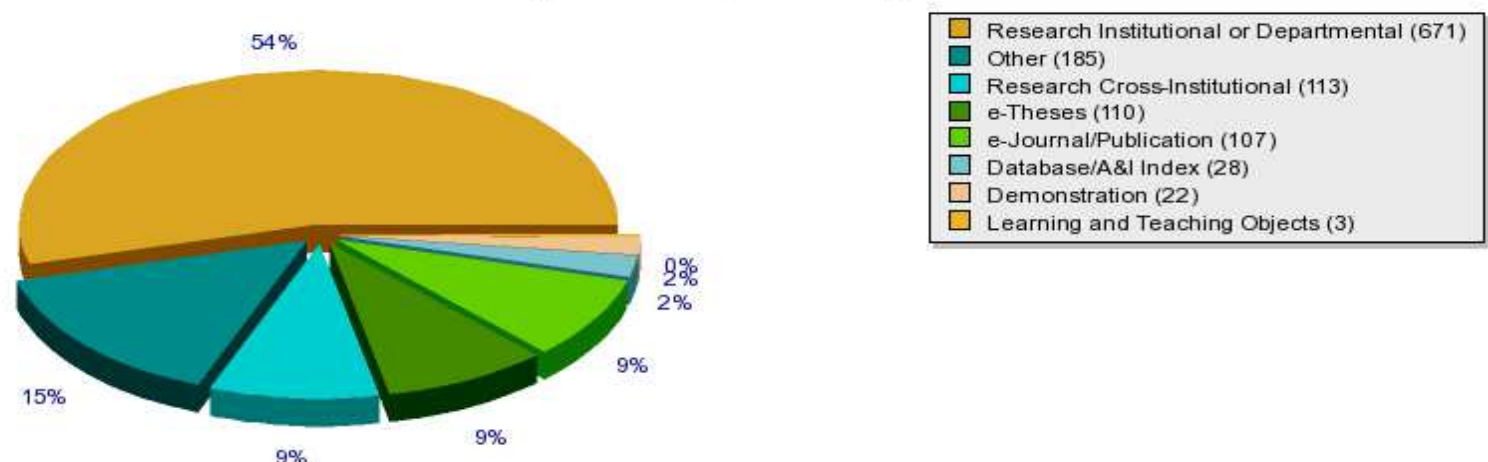

$9 \%$

2.2.4.3-Un graphe gradué représentant la progression des sites qu'héberge ROAR depuis Mai 1989 jusqu'à Octobre 2007. On remarque que le décollage effectif de l'entrepôt se situe aux environs de Septembre 2001 et que depuis cette date sa progression est constante. 


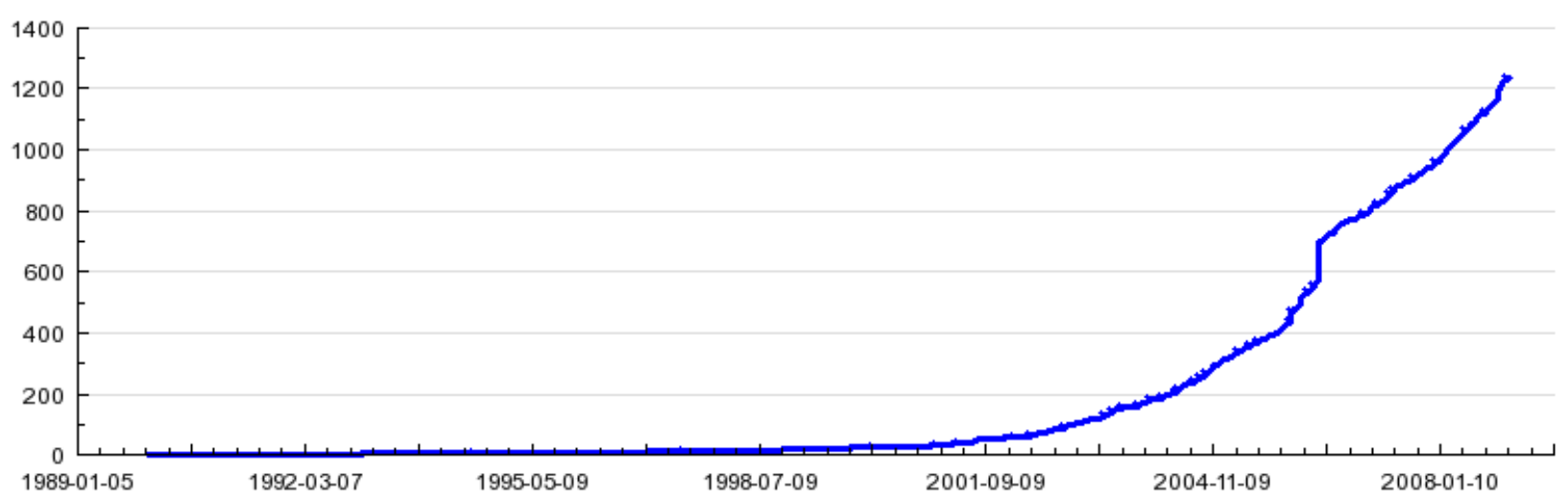

\subsection{5-Conclusion commune aux trois entrepots}

Nous avons choisi et présenté ces trois entrepôts entre tous les entrepôts qui se créent à travers le monde pour des raisons multiples dont nous citerons :

1-Les trois entrepôts représentent pour l'un (The Core Metalist of open access Eprints archives) la première tentative de mettre au point une liste exhaustive des différentes archives qui ont poussé après l'avènement d'Internet et pour le deuxième et troisième (" Open DOAR: The Directory of open access repositories " et " Registry of Open Access Repositories (ROAR)) l'exemple de ce que le mouvement a accompli au cours des dernières quinze années en plus du fait que ces deux derniers sites représentent une mise à jour constante des différents entrepôts ( contrairement à " The Core Metalist of open access Eprints archives " dont la dernière mis à jour date du 30 Juin 2003)

2-Ils représentent, d'après nous, trois exemples symptomatiques de la situation des archives ouvertes.

3-Ils se situent l'un au début et les autres sont plus récents (en fait sont constamment mise à jour car les additions et corrections sont presque journalières) ce qui permet de faire une comparaison quantitative mais beaucoup plus qualitative.

4-Ils permettent d'un autre coté de voir quels sont les progrès accomplis par les archives ouvertes et les nouvelles fonctionnalités qu'Internet a introduites dans le traitement des archives ouvertes.

En conclusion, on peut dire que les archives de publications en libre accès et leur conséquences directes, les entrepôts d'archives en libre accès, sont des entrepôts où les auteurs, ayant publiés ou en phase de publication, peuvent déposer leurs travaux .Si le chercheur qui auto archive veut la reconnaissance de ses pairs par la publication en libre accès, il n'en demeure pas moins que cette reconnaissance doit passer par une rigoureuse certification par ses pairs. L'amélioration de l'impact qui résulte du libre accès n'est pas antinomique avec le contrôle par les pairs mais en est, en fait, une condition sine qua none. On peut voir dans les listes précédentes que la progression est constante et que les moyens qu'a permit Internet font qu'il est de plus en plus possible de regrouper un nombre de plus en plus grand de données. Si le premier entrepôt que nous avons examiné prêchait par une sorte de rusticité (si on le compare avec les fonctionnalités Internet de nos jours) et aussi par le fait que les chiffres qu'il a présenté se sont arrêtés en 2003, les deux derniers représentent les derniers développements qu'ont atteints les archives ouvertes. L'énormité de la tâche que s'assigne le mouvement à rendre disponible l'information scientifique et technique (malgré la puissance de plus en plus accrue et de plus en plus financièrement abordable de l'outil informatique) est quasi impossible. En effet, l'augmentation exponentielle des dépôts d'archives et 
leur corollaire immédiat la création des entrepôts d'archives, l'acceptation par un nombre de plus en plus grands de chercheurs, d'organismes de recherche, d'institutions gouvernementales, d'organisations intergouvernementales et aussi (quoique sous la pression) d'éditeurs a littéralement fait explosé le nombre de dépôt d'archives qui ne peuvent plus être recensés exhaustivement. D'ailleurs, cette situation ressemble à s'y méprendre aux tentatives faites à la fin du $19^{\mathrm{e}}$ siècle par Paul Otlet et Henri Lafontaine qui créèrent " le Répertoire Bibliographique Universel " et qui atteignit jusqu'à 15000,000 notices. Cette expérience qui se proposait de réunir toutes les informations de tous les pays et de tous les temps ne fut pas menée à terme à cause de l'impossibilité de recenser tout ce qui avait été écrit. De nos jours, la formidable puissance de 1'outil informatique a facilité le traitement de l'information mais a aussi induit une augmentation extraordinaire des sites à traiter. On peut dire que la progression qu'a permis Internet a été effacée par l'accroissement des sites qu'a permis ce même Internet.

L'avenir des archives ouvertes, un des pans du libre accès, est à ce jour sujet à de multiples débats quant à sa faisabilité, ses conséquences et ses résultats. La question n'est pas de savoir est ce que la communication scientifique se fera avec (dans ?) les archives ouvertes mais plutôt quelle en sera le nouveau visage, à quel point l'ubiquité, la vitesse, les fonctionnalités, les nouveautés d'Internet, vecteur incontournable du libre accès en général et des archives ouvertes en particulier, vont-elles influencer le continuum et la pérennité du Savoir? Bien que ce mouvement n'en soit qu'à ses débuts, tout observateur attentif au sujet voit bien que la communication scientifique contrôlée par les pairs, est irrémédiablement changé par le libre accès .Si les archives ouvertes avec leur foisonnement, leur différentes étapes (prépublications, contrôle ou certification, post publications) ont toujours été considérées comme le parent pauvre du libre accès en raison de multiples causes dont la plus importante l'ignorance même de l'existence du medium, ainsi que l'ignorance des bénéfices que peut en tirer le chercheur, l'appréhension face à un medium somme toute nouveau ainsi que le forcing des éditeurs afin de faire le minimum de concessions légales permettant au chercheur de garder le contrôle de sa propre production, il est un autre pan du libre accès qui semble jouir d'une plus grande popularité chez les chercheurs et qui est la publication dans les périodiques électroniques librement accessible sur la Toile. 


\section{Chapitre III}

\section{LES JOURNAUX ELECTRONIQUES SUR INTERNET :}

\section{Introduction}

A coté des archives ouvertes (connues aussi dans le jargon sous l'appellation de route verte ou green road) et dont nous en avons vu quelques exemples et aussi les entrepôts qui en représentent une autre étape car ils ne sont qu'une des conséquences du développement exponentiel des archives ouvertes, nous allons examiner une autre facette du libre accès : les journaux électroniques librement accessibles sur la Toile (connus aussi sous l'appellation de route dorée ou golden road).

\section{1- Premiers journaux électroniques}

Il est fort probable que " New horizons in adult education," (figure 1.1) fondé à l'automne 1987 par Michael Ehringhaus and Bird Stasz, tous deux de l'Unversité de Syracuse soit le premier journal scientifique contrôlé par les pairs à etre publié sous forme électronique. Le premier numéro fut envoyé sur The Adult Education Network (AEDNET). Ce titre est toujours en parution. Au mois de Mars 1991, Ted Jennings de " The University at Albany " (State University of New York) lança "EJournal" (figure 1.2) qui avait été décrit comme étant un journal qui s'intéressait aux implications des réseaux électroniques et les textes. Enfin au mois de septembre 1991," The Online journal of current clinical trials ", publié à partir de septembre 1991 fut décrit comme " le premier journal électronique contrôlé par les pairs en médecine " (180)

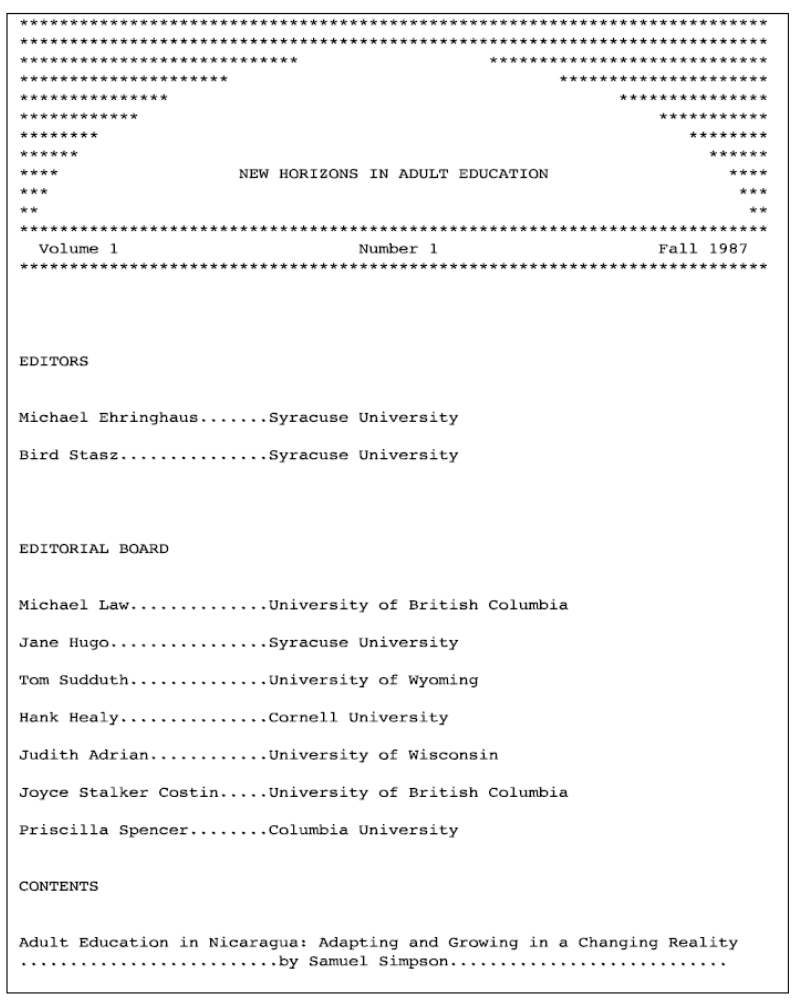

Figure 1.1: The first electronic journal: New horizons in adult education

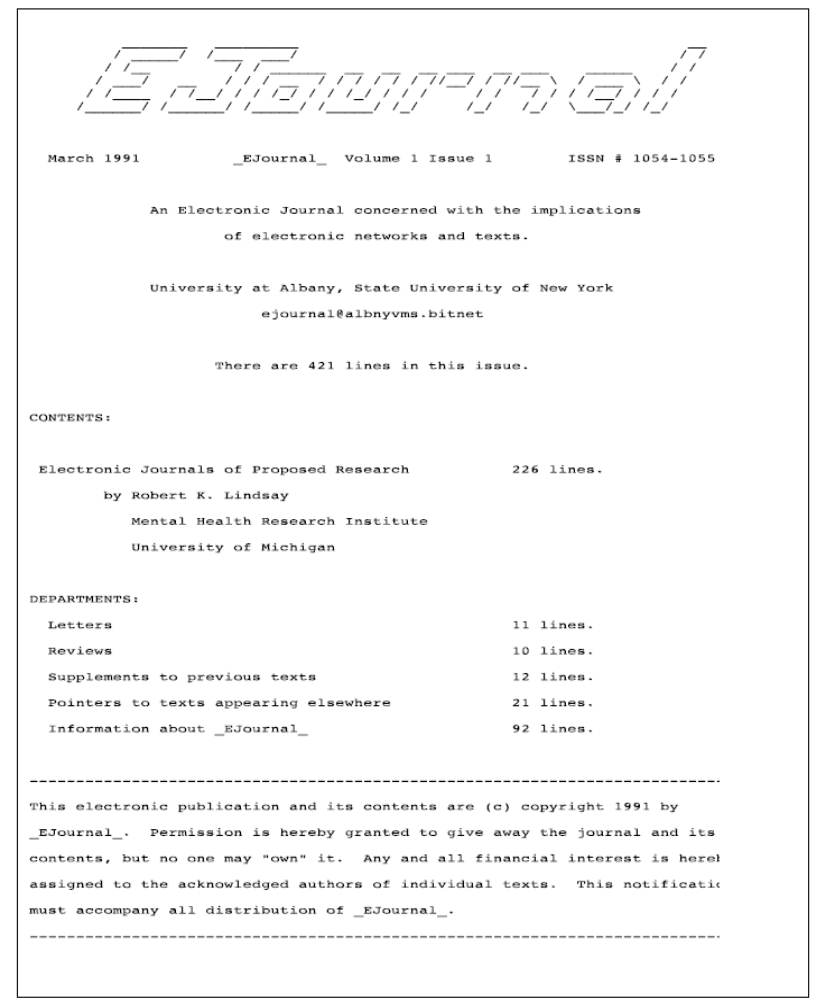

Figure 1.2: The first issue of Ejournal

Si ces exemples qu'on peut désigner comme les ancêtres de nos journaux électroniques actuels et qui à l'époque n'était connus et n'intéressaient qu'une poignée de pionniers, de nos jours le chercheur peut difficilement se passer des journaux électroniques. A cet effet, Gary Stix écrivait en 1994 : " Les scientifiques rapportent maintenant leur recherche - depuis la première inspiration

(180) ) Mackenzi Owen, John Stewart, The Scientific article in the age of digitization, [s.l.:s.n.], 2005, p.1

Academic Proefschrift : Science de l'information : Université d'Amsterdam : 2005

Disponible à : http://dare.uva.nl/document/17843 
jusqu'au résultat final - par les réseaux électroniques .Même les expériences peuvent suivies en direct, en ligne. Les éditeurs et les bibliothèques pourraient bien ne jamais être les mêmes "(181) ${ }^{\text {Si }}$ cette affirmation pouvait paraître un tant soit peu révolutionnaire et futuriste pour l'époque, il est clair que cette projection sur le futur s'est avérée tellement vraie et s'est concrétisée qu'en fait de nos jours on voit que les nouvelles fonctionnalités des réseaux ne sont pas complètement appréhendées ni peuvent être pronostiquées tellement ce qui paraissait comme de la science fiction il y a de cela seulement quelques décennies s'est concrétisé en entier

\section{2-Problème de la détermination du nombre}

Au-delà de ces projections, il est un point que nombre de chercheurs ont abordé mais qui reste sujet à controverse : le nombre exacte des journaux électroniques se trouvant sur la Toile. Une abondante littérature y a été consacrée mais vu la nouveauté du medium, il est pratiquement impossible de savoir le nombre exacte mais juste des chiffres qui ne sont pas, le moins que le puisse dire, très précis. Carol Tenopir, chercheur américain qui se spécialise dans la communication scientifique présente sa thèse de la manière suivante : " Je peux dire avec certitude qu'à la fin 2003, il y avait juste un peu moins de 50000 périodiques savants et entre un tiers et un peu plus de la moitié sous forme électronique .J'ai appris une chose : c'est que ces chiffres sont une cible mouvante et parfois même suspecte. Il faut continuellement vérifier et garder vague les affirmations définitives " ${ }^{(182)}$. Un simple calcul place donc le nombre de journaux électroniques entre 17000 et 25000 titres ce qui est, vu la nouveauté du medium, peut être considéré comme une percée formidable. Ce chiffre est corroboré par une autre étude, académique celle là, qui situait le nombre à " au moins 17000 journaux " (183) .Dans la même source, Tenopir donne des chiffres qui varient entre 11000 et 14600 titres ainsi qu'une autre évaluation qui situait les chiffres à " un peu plus de 43500 titres ". La question qui se pose est: comment le même auteur dans la même référence peut il donner des chiffres aussi dissonants? A notre avis, ceci tient à deux raisons inextricablement liées :

1-Le medium étant nouveau, il est pratiquement impossible de savoir quelles sont les nouveaux titres qui sont lancés surtout que le mouvement prend de l'ampleur.

2-Malgré les différentes recherches et la littérature abondante produite (surtout anglo saxonne), il semblerait que la définition du périodique académique (scientifique, savant sont d'autres appellations utilisées) ne soit pas normalisée ou du moins ne jouit pas d'un accord entre les chercheurs et même les éditeurs. Interrogée à ce sujet, Yvette Diven, director, product management, serials, R.R. Bowker LLC et qui produit Ulrich's la célèbre base données qui recense toutes les publications en série au monde affirma qu'Ulrich's a deux appellations qui désignent les publications scientifiques : "savant / académique" " et " revue par les pairs". Si certains éditeurs académiques savent l'exacte signification de ces concepts, d'autres malheureusement entendent par "revue par les pairs" le fait que "la revue est fréquemment consultée par des lecteurs faisant partie des pairs dans le marché des publications ..." D'un autre coté, certains de ces éditeurs pensent qu'un revue est automatiquement revue par les pairs si elle a un comité éditorial. Comme on peut le voir, cette ignorance, ou du moins cette confusion sur les définitions, a déteint sur les résultats des chiffres auxquels le chercheur s'attendrait.

Ceci en plus du fait que Ulrich's recense selon Ulrichsweb.com ${ }^{(184)}$ sa base de données en ligne et qui est la plus mise à jour, " un quart de million de titres bibliographiques" .Tenopir en recense 180,200 qui sont toujours actifs et ce toujours dans la même référence. Il est évident que cette myriade de chiffres a été présentée pour prouver une seule chose: la quasi impossibilité d'avoir un

\footnotetext{
${ }^{(181)}$ G.Stix," The Speed of write", Scientific American, Vol.271, n6,1994, p.72

(182) Tenopir, Carol, Online: scholarly journals: how many? Library Journal, 129 (2) :2, 2004 Disponible à : http://www.libraryjournal.com/article/CA374956.html

${ }^{(183)}$ Mackenzi Owen, John Stewart, op.cit., p.5

${ }^{(184)} \mathrm{http}: / /$ ulrichsweb.com/ ulrichsweb
} 
chiffre précis et exacte et pour paraphraser Tenopir, [il faut] "......garder vague les affirmations définitives ".

Quant au nombre ou du moins le pourcentage de ces périodiques qui sont en libre accès, on peut affirmer que la confusion est encore plus grande car en plus de la nouveauté déjà évoqué,il est difficile de définir un périodique en accès libre face aux différentes formes ( " saveurs " pour reprendre l'expression utilisée par John Willinsky ${ }^{(185)}$ ) du libre accès qui ne sont pas complètement établies et qui font l'objet de divers expérimentations dont seul l'avenir peut en pronostiquer le succès ou l'échec. Une étude récente ${ }^{(186)}$ utilisant des critères différents a conclu que $19.4 \%$ des articles de l'année 2006 était accessible gratuitement. On remarquera que l'étude parle de pourcentage d'articles et non pas de pourcentage de titres de périodiques. Il est évident que cette étude est intéressante de par les paramètres qu'elle prend en compte (s'appuyant sur les bases de données de l'Institute of Scientific Information (ISI) ${ }^{(187)}$ et de l'Ulrich's International Periodicals Directory, elle a estimé que le nombre d'articles publiés en 2006 par 23750 journaux était approximativement de 1350 000, dont $4.6 \%$ étaient dans des revues électroniques disponibles gratuitement et $3.5 \%$ étaient disponibles après un embargo d'une année. A ces $8.1 \%$ constitués d'articles disponibles à différents niveaux, s'ajoute $11.3 \%$ d'articles déposés par les auteurs dans les entrepôts de publication électronique ou les pages personnelles ${ }^{* *}$ car connaître le pourcentage d'articles en libre accès est plus quantifiable et précis que le pourcentage des titres de périodiques qui est moins précis étant donné qu'un titre ou un numéro de périodique peut contenir un nombre variable de recherches, il n'en demeure pas moins qu'il nous parait plus méthodologiquement important, scientifique et dans l'optique de notre travail d'étudier le nombre et le pourcentage des

\footnotetext{
** Dans cette analyse des différentes formes du libre accès, John Willinsky distingue neuf formes et qui sont :

- E Prints archives (Archive de publication électronique) le chercheur archive sa prépublication et / ou sa post publication dans une archive en libre accès (exemple ArXiv.org Eprint Service)

- Unqualified (Indéterminé) publication immédiate et en totalité de journaux en libre accès (First Monday)

- Dual mode (Mode Duel) mode où est offert en même temps un abonnement papier et un libre accès des éditions du journal (Journal of Postgraduate Medicine )

- Delayed OA (Libre accès avec délai) édition en libre disponible quelques mois après la publication initiale

- Author Fee (Payement par l'auteur) l'auteur paye pour participer à la publication en libre accès Central)

- Partial OA (Libre accès partiel) certains articles dans le numéro sont en libre accès (New York Review of Books )

- Per Capita( Par produit individuel brut) libre accès disponible pour certains pays sur la base du revenu par personne (HINARI- Organisation Mondiale de la santé )

- Abstract ( Resumé ) libre accès à la table des matières et le résumé (Science Direct)

- Co Op (Cooptation) les membres de l'institution promeuvent les journaux en libre accès (German Academic Publishers )

On peut voir d'après l'analyse de John Willinsky, figure importante dans le mouvement du libre accès et dont le livre " The Access principle : the case for open access to research and scholarship " (MIT Press , 2005) fait autorité dans le mouvement que les différentes formes du libre accès ne sont pas complètement ni définitivement établis et que le forcing des éditeurs a un tant soit peu retardé le mouvement malgré quelques concessions dictées beaucoup plus par les événements qu'une soudaine philanthropie de ces mêmes éditeurs. Nous signalerons, juste à titre d'exemple, que le livre cité, malgré qu'il vendu par son editeur (MIT Press) est aussi disponible et librement téléchargeable à l'adresse suivante :

http://mitpress.mit.edu/catalog/item/ebook.asp?ttype=2\&tid=10611

${ }^{(185)}$ Willinsky, John, The Nine flavors of open access scholarly publishing, Journal of posgraduate medicine , 2003, 49 ,p.263-267

Disponible à : http://www.jpgmonline.com/temp/jpgm493263-216091_060009.pdf

${ }^{(186)}$ Bjork, Bo-Chrisler; Roosr, Anniki ; Lauri, Mari, Global annual volume of peer reviewed scholarly article and the share available via differents access options, ElPUB 2008.Open scholarship : authority, community and sustainability in the age of web 2.0- Proceedings of the 12th International Conference on Electronic Publishing, held in Toronto, 25-27 June 2008/ Edited by Leslie Chan and Susanna Mornatti, p.178-186

Disponible à : http://elpub.scix.net/cgi-bin/works/Show?178 elpub2008

${ }^{(187)} \mathrm{http}: / /$ scientific.thomson.com/

** On remarquera qu'une certaine cacophonie caractérise parfois le nombre de périodiques en libre accès .La figure dans cette page représente les différents types de périodiques en ligne que la base de données UlrichWeb recense. Sur les 4686 périodiques recensés, 1120 sont en libre accès ce qui nous donne un pourcentage de $23.90 \%$. , loin des $8.1 \%$ des périodiques en libre accès immédiat ou en période de latence. Il est vrai que d'autres paramètres ont du sûrement rentrer en ligne de compte mais, encore une fois et au risque de nous répéter, nous paraphraserons Tenopir pour la justesse de son analyse "....... ces chiffres sont une cible mouvante et parfois même suspecte ".
} 
titres gratuitement disponibles sur Internet. Nous prendrons pour ce faire comme base de travail deux sites de journaux en libre accès sur Internet et qui font reference dans le monde de la publication electroniques des periodiques.Ce sont le D.O.A.J. (Directory of Open Access Journal) et A.J.O.L. (African Journal On Line) car representant l'un une couverture au niveau international et l'autre une couverture au niveau regional. Ce dernier est intéressant à plus d'un titre car comme il est connu les pays en voie de développement souffrent d'une fracture numérique plus que conséquente qui grève sérieusement leur effort développement. Le libre accès est, à notre avis, une opportunité plus qu'importante par lequel les pays en voie de développement peuvent rattraper ou du moins rétrécir le fossé qui ne fait que s'approfondir entre eux et les pays développés.

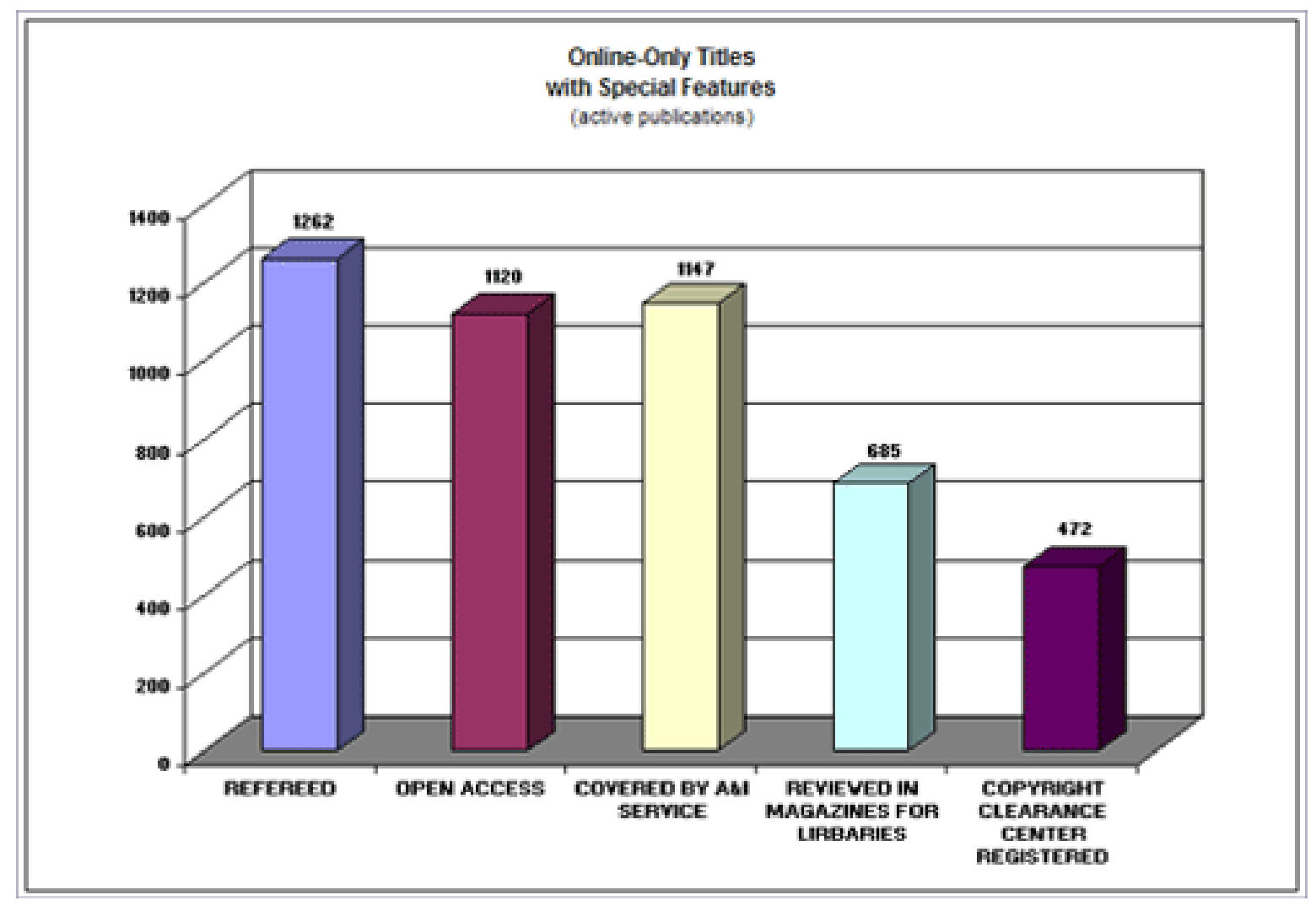

Fig. $n{ }^{\circ}$ 25-Tableau représentant les nombres concernant les différents types de périodiques en ligne dans la base de données UlrichWeb.com

3.3-" The Directory of Open Access Journals " (188)

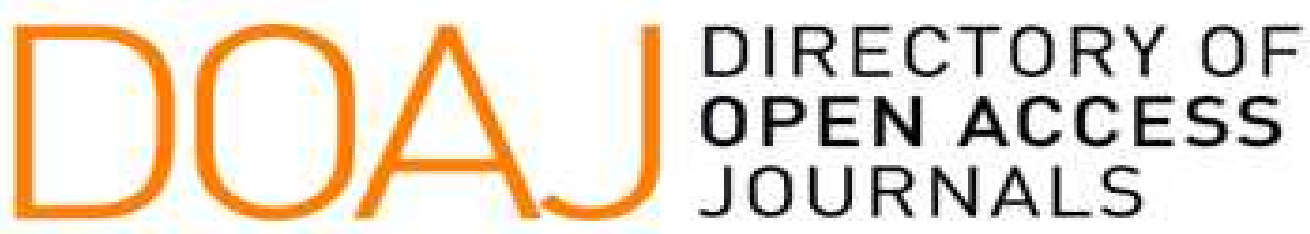

${ }^{(188)}$ http://www.doaj.org 


\subsection{1- Critères de recherche}

Ces informations sont présentées sur l'interface du site. Le site peut être aussi recherché de plusieurs manière dont :

3.3.1.1-Find Journals (trouver des périodiques): on peut rechercher à travers cette fonctionnalités les périodiques que recèle la liste et ceci de deux manières :

- en écrivant le nom du périodique dans la case réservée à cet effet.

- en cherchant les titres alphabétiquement par la première lettre du titre.

3.3.1.2-Browse subjects (chercher par sujets) : tel que le stipule le site, les sujets qu'il recense sont au nombre de 17 sujets. Ce sont :

3.3.1.2.1-Agriculture and Food Sciences (Agriculture et sciences nutritives)

3.3.1.2.2-Arts and Architecture (Arts et Architecture)

3.3.1.2.3-Biology and Life Sciences (Biologie et sciences de la vie)

3.3.1.2.4-Business and Economics (Commerce et Economie)

3.3.1.2.5-Chemistry (Chimie)

3.3.1.2.6-Earth and Environmental Sciences (Terre et sciences environnementales)

3.3.1.2.7-General Works (Généralités)

3.3.1.2.8-Health Sciences (Sciences médicales)

3.3.1.2.9-History and Archaeology (Histoire et Archéologie)

3.3.1.2.10-Languages and Literatures (Langages et Littératures)

3.3.1.2.11-Law and Political Science (Droit et Sciences politiques)

3.3.1.2.12-Mathematics and Statistics (Mathématiques et Statistiques)

3.3.1.2.13-Philosophy and Religion (Philosophie et Religion)

3.3.1.2.14-Physics and Astronomy (Sciences Physiques et Astronomie)

3.3.1.2.15-Science General (Généralités en Science)

3.3.1.2.16-Social Sciences (Sciences Sociales)

3.3.1.2.17-Technology and Engineering (Technologie et Sciences de l'ingénieur)

Ces 17 sujets sont représentés dans la liste suivante selon chaque spécialité et donnent aussi le nombre de journaux dans chaque spécialité

$\underline{\text { Agriculture and Food Sciences }}$

Agriculture (General) (75 journals), Animal Sciences (57 journals) Aquaculture and Fisheries (11 journals) Forestry (20 journals) Nutrition and Food Sciences (19 journals) Plant Sciences (31 journals)

Arts and Architecture

Architecture (13 journals) Arts in general (26 journals) History of arts (5 journals) Music (30 journals) Performing Arts (16 journals) Visual Arts (7 journals)

\section{Biology and Life Sciences}

Biology (147 journals) Anatomy (5 journals) Botany (50 journals) Cytology (6 journals) Genetics (30 journals) Microbiology (34 journals) Physiology (25 journals) Zoology (61 journals) Biochemistry (30 journals) Biotechnology (25 journals)

$\underline{\text { Business and Economics }}$

Business and Management (75 journals) Economics (78 journals)

Chemistry

Analytical Chemistry (8 journals) Chemical Engineering (10 journals)Chemistry(General) (62 journals) Inorganic Chemistry (2 journals) Organic Chemistry (7 journals) 
Earth and Environmental Sciences

Earth Sciences (58 journals) Ecology (37 journals) Environmental Sciences (68 journals) Geography (42 journals) Geology (59 journals) Geophysics and Geomagnetism (6 journals) Meteorology and Climatology (15 journals) Oceanography (19 journals)

General Works

Multidisciplinary (53 journals)

$\underline{\text { Health Sciences }}$

Dentistry (38 journals) Medicine (General) (285 journals) Allergy and Immunology (17 journals) Anesthesiology (6 journals) Cardiovascular (41 journals) Dermatology (12 journals) Gastroenterology (20 journals) Gynecology and Obstetrics (22 journals) Internal medicine (166 journals) Neurology (63 journals) Oncology (41 journals) Ophthalmology (13 journals) Otorhinolaryngology (12 journals) Pathology (20 journals) Pediatrics (39 journals) Pharmacy and materia medica (21 journals) Psychiatry (33 journals) Sports Medicine (12 journals) Surgery (58 journals) Therapeutics (44 journals) Urology (15 journals) Nursing (25 journals) Public Health (119 journals)

History and Archaeology

Archaeology (21 journals) Diplomatics. Archives. Seals (2 journals) History (117 journals)

Languages and Literatures

Languages and Literatures (144 journals) Linguistics (102 journals)

Law and Political Science

Law (66 journals) Political Science (103 journals)

Mathematics and Statistics

Mathematics (132 journals) Statistics (27 journals)

Philosophy and Religion

Philosophy (93 journals) Religion (56 journals) The Bible (5 journals)

Physics and Astronomy

Astronomy (General) (15 journals) Physics (General) (52 journals) Acoustics (6 journals)

Electricity (5 journals) Heat (1 journals) Mechanics Nuclear Physics (5 journals) Optics and Lights (11 journals)

$\underline{\text { Science General }}$

Information theory (3 journals) Science (General) (44 journals)

$\underline{\text { Social Sciences }}$

Anthropology (53 journals) Education (260 journals) Ethnology (12 journals) Gender Studies (24 journals) Library and Information Science ( 90 journals) Bibliography (1 journals) Media and communication (58 journals) Psychology (99 journals) Social Sciences (169 journals) Migration (8 journals) Sociology (72 journals) Social and Public Welfare (25 journals) Sports Science (13 journals)

Technology and Engineering

Chemical Technology (9 journals) Computer Science (135 journals) Construction (6 journals) Electrical and Nuclear Engineering (27 journals) Environmental Engineering (3 journals) Environmental Technology (4 journals) General and Civil Engineering (68 journals) Hydraulic engineering ( 1 journals) Industrial Engineering (9 journals) Manufactures (6 journals) Materials (14 journals) Mechanical Engineering (16 journals) Military Science (1 journals) Mining and Metallurgy (6 journals) Technology (General) (50 journals) Transportation (11 journals) 
A la lecture de ces chiffres quelques remarques s'imposent. Nous les résumerons en:

1- Sur les 126 spécialisations et qui constituent les 3812 titres recensés, 12 spécialisations (Biology -147 journaux - , Médicine générale - 285 journaux- Médicine Interne -166 journaux- Santé Publique - 119 journaux- Histoire -117 journaux- Langues et Littératures -144 journauxLinguistique -102 journaux- Sciences Politiques -103 journaux- Mathématiques -132 journauxEducation -260 journaux- Sciences Sociales -169 journaux et Informatique 135 journaux) à elles seules englobent 1879 titres presque la moitie (49.29\%) des titres recensés .

2- De ces douze spécialisations, la spécialisation Sciences médicales recèle 570 titres soit $14.95 \%$ de l'ensemble des titres ou $30.35 \%$ des 12 spécialisations précitées.

3-Malgré la prépondérance des sciences exactes dans le domaine des publications électroniques, les sciences sociales et humaines sont présentes. Ainsi, Education, Sciences sociales, Sciences politiques, Littérature et Langues, Linguistique et Histoire totalisent 895 titres soit $23.47 \%$ du total des titres de la liste ou $47.63 \%$ des douze spécialités déja citées.

4- La spécialité Physique et Astronomie de son côté malgré que les premières tentatives des publications électroniques se soient déroulés en son sein, ne semble pas (du moins dans DOAJ) jouir d'une grande appropriation. Ainsi, les titres qui y sont inclus au nombre de 95 ne représentent que $2.49 \%$ du total des titres. Notre hypothèse est que cette spécialité a besoin d'une rapidité de transmission telle que le journal électronique malgré sa rapidité comparativement au journal papier ne peut être utilise par les physiciens qui lui préfèrent les archives ouvertes .En effet, les archives ouvertes permettent (grâce à Internet) une quasi instantanéité de la transmission et cette manière de procéder répond parfaitement aux besoins de chercheurs pour qui la notion temps est primordiale.

\subsection{2-Différentes fonctionnalités}

3.3.2.1-New titles (Nouveaux titres): Cette rubrique se propose de présenter les derniers titres qui ont intégrés le site. En cliquant sur le lien, on obtient ce qui suit :

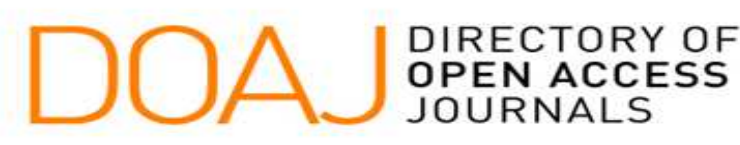

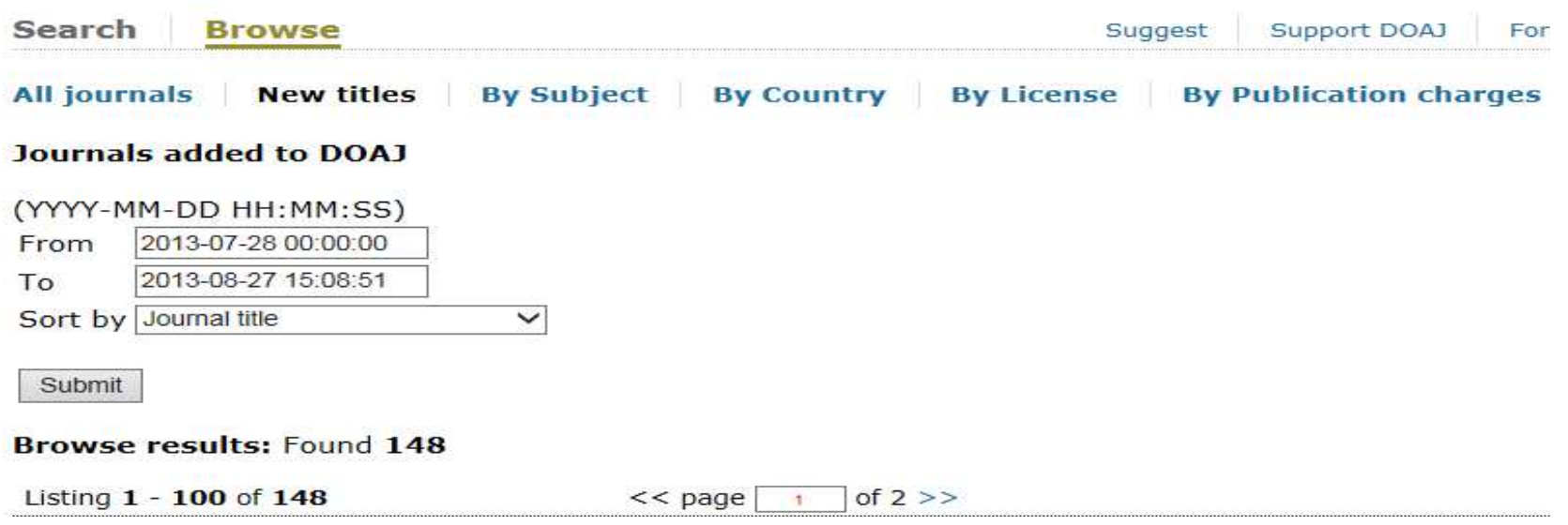

On peut voir qu'en date du 12 Janvier 2009, 27 Journaux furent ajoutés entre le $1^{\text {er }}$ Décembre 2008 à minuit et le 12 Janvier 2009 à $14: 25: 44$. Il existe trois fonctions pour trier et voir les mises à jour des nouveaux titres : quotidienne, hebdomadaire et mensuelle .Enfin, on peut trier les nouveaux titres de deux manières : soit selon le titre du journal ou selon la date et l'heure de l'ajout à DOAJ

3.3.2.2-Find articles (Recherche d'articles): Cette fonction permet de rechercher des articles utilisant des mots clés .Cette recherche s'appuie sur les opérateurs booléens ( " et ", " ou " et "pas" ) et associe en même temps une recherche par champs ( titre, titre du périodique, auteur, mots clés et résumé ou tous ces champs combinés ) 
(more than one word in a field will be treated as phrase)

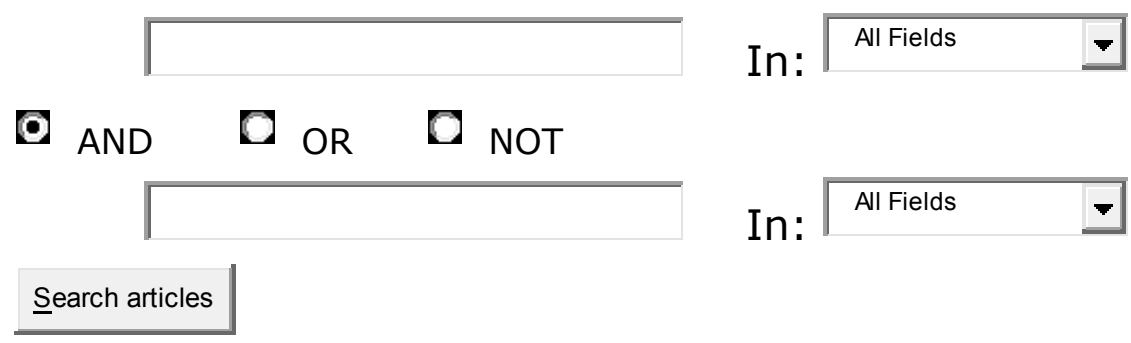

Nous avons fait une recherche en utilisant et en combinant les mots clés suivants : " electronic journals " et " open access " et en combinant avec tous les champs ${ }^{*}$. Le résultat fut :

Find articles
Found 5 documents matching your query :
All Fields=electronic journals AND All Fields=open access
Presenting page 1/1
Local Purchasing of Journals is Required in Addition to a Nationally Purchased Collection
to Meet the Information Needs of NHS Staff. A review of: Glover, Steven William, John
Addison, Colette Gleghorn, and John Bramwell. "Journal Usage in NHS Hospitals: A
Comparison Report of Total Usage at an Acute NHS Trust and a Specialist NHS Trust in
the North West of England." Health Information and Libraries Journal 24.3 (2007):
193 $\square$.
Author: Jennie Kelson Journal: Evidence Based Library and Information Practice Year:
2008 Vol: 3 Issue: 1 Pages/record No.: $68-71$
View record Fulltext

Arab Researchers Promotions of Open Archives and Free Electronic Journal : Sultan

Qabus University Faculty as a Model

Author: Abd Al-Majid Salih Bu Azza Journal: cybrarians journal Year: 2006 Vol: Issue:

10 Pages/record No.:

Vieu record Fulltext

Portal elektroničkih časopisa Open J-Gate

Author: Mayer, M. Journal: Kemija u Industriji Year: 2006 Vol: 55 Issue: 09 Pages/record

No.: $382-383$

View record Fulltext

Arab Researchers Promotions of Open Archives and Free

Author: Abd Al-Majid Salih Bu Azza Journal: cybrarians journal Year: 2006 Vol: Issue:

10 Pages/record No.:

View record Fulltext

Economics and quality estimation of open access journals

Author: Francke, Helena Journal: Svensk Biblioteksforskning Year: 2005 Vol: 15 Issue: 1

Pages/record No.:

View record Fulltext

\begin{abstract}
* A titre indicatif, lors de l'utilisation des mêmes mots en langue française, le résultat fut nul. De même, lors de l'utilisation des mots clés suivants : "contrôle par les pairs" et "communication scientifique", le résultat fut encore une fois nul alors qu'en langue anglaise, " peer review " et " scientific communication " donna trois références. Ceci indique clairement l'hégémonie maintes fois signalée de la langue anglaise dans la recherché scientifique en général et dans le libre accès en particulier. Il ne faut pas oublier que l'immense majorité des logiciels de gestion sont en langue anglaise et ceci explique le fait que les mots en anglais soient plus reconnus .D'ailleurs, nous avons tenté une recherche en recherchant les mêmes termes (journaux électroniques) dans Bulletin des Bibliothèques de France qui est inclut dans DOAJ. Cette recherché a donné 106 références ce qui nous a oblige à sélectionner la période entre 2000 et 2008 (tel que demandé par le moteur de recherché) et ceci a donné 43 références. Donc, comme on peut le voir, les sites de libre accès semblent privilégier les références en langue anglaise et ne pas moissonner les références en langue française tel que l'exemple cité le prouve. La question que nous nous posons est: si des langues dites " majeures " comme le français sont ignorées, que dire des langues dites " mineures " telles que l'Arabe ou autre. En fait, l'hégémonie de certaines langues (l'Anglais surtout) risque en fait de conduire à une plus grande fracture entre ceux qui ont accès à l'information et ceux qui peinent à y arriver. Ceci est antinomique avec la mission et le but du libre accès dont le but le premier est de permettre à tous d'accéder à l'Information scientifique et technique sans aucune discrimination de quelque sorte que ce soit à part la disponibilité d'une connexion Internet .
\end{abstract}


3.3.2.3- Suggest a journal (suggérer un journal): Cette rubrique permet d'inclure et d'ajouter un nouveau titre à la liste de DOAJ. En cliquant sur le lien, on obtient l'interface suivante qui en plus des informations demandées sur le périodique, informe sur les conditions d'inclusion et les critères d'inclusion.

\section{Suggest a new title}

Please use this form to suggest a journal to be included in DOAJ. The directory contains fulltext, open access scientific and scholarly journals that use an appropriate quality control system to guarantee the content.

What we don't include in DOAJ?

- Newsletters that mainly inform about an institution.

- Journals that charge any access or subscription fees.

- Journals that have an embargo period.

For more information See our selection criteria.

Note: Please make sure that the journal you are suggesting is not already in DOAJ.

Note: Fields with the ${ }^{*}$ sign are mandatory.

Haut du formulaire
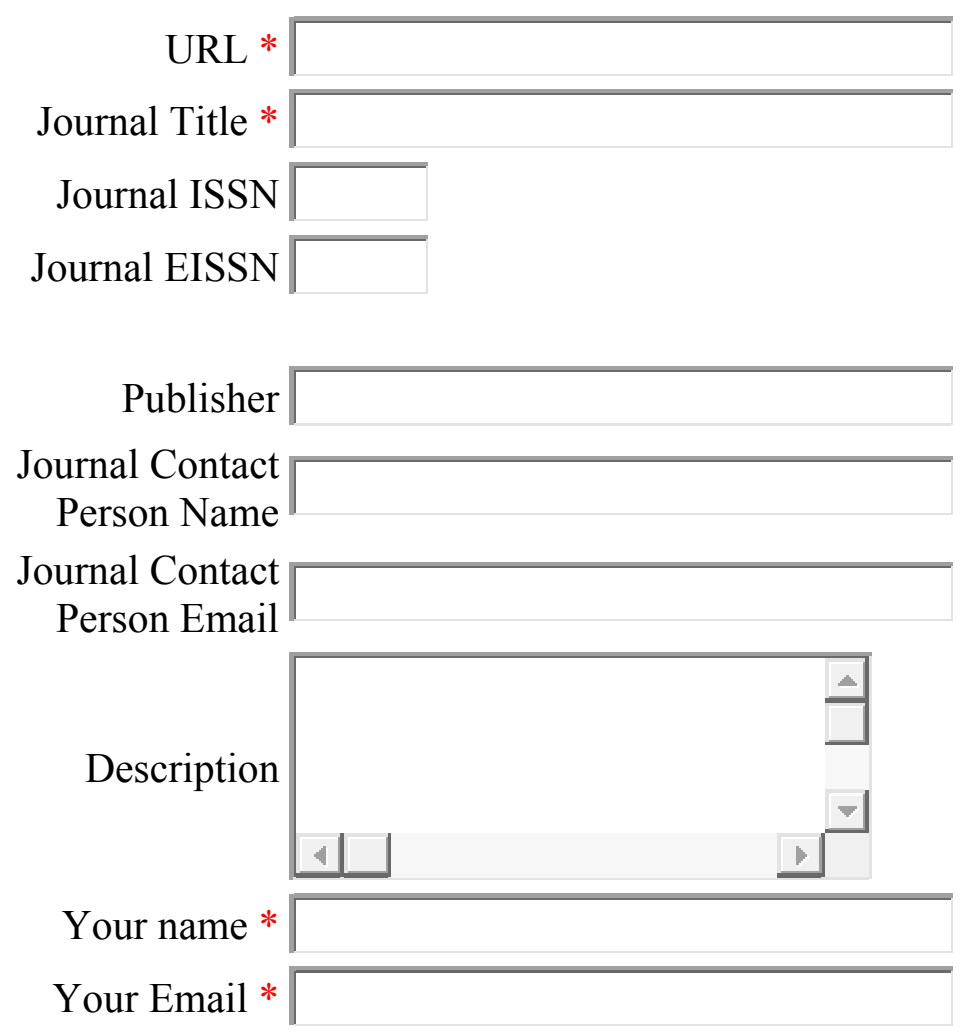

To avoid automated posting software, please type in the word

Security ${ }^{*}$ displayed in the image 
3.3.2.4-About (au sujet de): Cette rubrique se propose de présenter le site et ses différentes fonctionnalités. Elle comprend :

1- But et couverture

2- Définitions

3- Critères de sélection

4- Informations sur les métadonnées

5- Historique du projet

3.3.2.5-FAQ (Questions fréquemment posées) : A l'instar d'autres sites, cette rubrique se propose d'éclairer l'utilisateur sur les questions les plus à même d'être posées. A cet effet, dix sept questions allant de " Qu'est ce que le DOAJ ? " à " Comment puis je supporter ou être un sponsor de DOAJ ?" sont posées et des réponses succinctes et claires (avec lien hypertexte pour certains points tels que des définitions ou par exemple les parties ayant subventionnées le projet) sont donnée afin de guider l'utilisateur .

3.3.2.6-Information (Information) : Cette rubrique relate les différentes informations inhérentes au DOAJ telles que les nouvelles fonctions ajoutées (la fonction " Journaux par pays " et dont nous reproduisons ci-dessus les 10 premiers pays avec le nombre par année et ce depuis 2002, date de la fondation de DOAJ)

\begin{tabular}{|c|c|c|c|c|c|c|c|c|c|c|c|c|c|c|c|c|c|}
\hline \multirow{2}{*}{$\#$} & \multirow{2}{*}{ Country } & \multicolumn{8}{|c|}{ Number of journals added into DOAJ } & \multicolumn{8}{|c|}{ Total number of journals in DOAJ } \\
\hline & & 2002 & 2003 & 2004 & 2005 & 2006 & 2007 & 2008 & 2009 & 2002 & 2003 & 2004 & 2005 & 2006 & 2007 & 2008 & 2009 \\
\hline 1 & $\begin{array}{l}\text { United } \\
\text { States }\end{array}$ & $\underline{20}$ & $\underline{217}$ & $\underline{97}$ & $\underline{97}$ & $\underline{73}$ & $\underline{113}$ & $\underline{211}$ & 0 & $\underline{20}$ & $\underline{237}$ & $\underline{334}$ & $\underline{431}$ & $\underline{504}$ & $\underline{617}$ & $\underline{828}$ & $\underline{828}$ \\
\hline 2 & Brazil & 0 & $\underline{8}$ & $\underline{118}$ & $\underline{48}$ & $\underline{53}$ & $\underline{61}$ & $\underline{74}$ & 0 & 0 & $\underline{8}$ & $\underline{126}$ & $\underline{174}$ & $\underline{227}$ & $\underline{288}$ & $\underline{362}$ & $\underline{362}$ \\
\hline 3 & $\begin{array}{l}\text { United } \\
\text { Kingdom }\end{array}$ & $\underline{3}$ & $\underline{117}$ & $\underline{47}$ & $\underline{43}$ & $\underline{43}$ & $\underline{33}$ & $\underline{35}$ & 0 & $\underline{3}$ & $\underline{120}$ & $\underline{167}$ & $\underline{210}$ & $\underline{253}$ & $\underline{286}$ & $\underline{321}$ & $\underline{321}$ \\
\hline 4 & Spain & 0 & $\underline{5}$ & $\underline{21}$ & $\underline{63}$ & $\underline{53}$ & $\underline{27}$ & $\underline{58}$ & 0 & 0 & $\underline{5}$ & $\underline{26}$ & $\underline{89}$ & $\underline{142}$ & $\underline{169}$ & $\underline{227}$ & $\underline{227}$ \\
\hline 5 & Germany & 0 & 12 & 19 & $\underline{38}$ & $\underline{23}$ & $\underline{30}$ & $\underline{25}$ & 0 & 0 & 12 & 31 & $\underline{69}$ & $\underline{92}$ & 122 & 147 & 147 \\
\hline 6 & Canada & 0 & $\underline{29}$ & $\underline{13}$ & $\underline{11}$ & $\underline{17}$ & 16 & $\underline{25}$ & 0 & 0 & $\underline{29}$ & 42 & $\underline{53}$ & $\underline{70}$ & 86 & $\underline{111}$ & $\underline{111}$ \\
\hline 7 & India & 0 & $\underline{\underline{16}}$ & $\underline{\underline{20}}$ & $\underline{15}$ & $\underline{18}$ & $\underline{21}$ & $\underline{17}$ & 0 & 0 & $\underline{\underline{16}}$ & $\underline{\underline{36}}$ & $\underline{51}$ & $\underline{69}$ & $\underline{\underline{90}}$ & $\underline{107}$ & $\underline{107}$ \\
\hline 8 & Turkey & 0 & $\underline{4}$ & $\underline{9}$ & $\underline{\underline{25}}$ & $\underline{13}$ & $\underline{27}$ & $\underline{25}$ & 0 & 0 & $\underline{4}$ & $\underline{13}$ & $\underline{38}$ & $\underline{51}$ & $\underline{78}$ & $\underline{103}$ & 103 \\
\hline 9 & Japan & $\underline{2}$ & $\underline{21}$ & 45 & $\underline{23}$ & $\underline{3}$ & 0 & $\underline{3}$ & 0 & $\underline{2}$ & $\underline{23}$ & $\underline{68}$ & $\underline{91}$ & $\underline{94}$ & $\underline{94}$ & $\underline{97}$ & 97 \\
\hline 10 & Chile & 0 & $\underline{3}$ & $\underline{41}$ & $\underline{19}$ & $\underline{13}$ & $\underline{7}$ & $\underline{13}$ & 0 & 0 & $\underline{3}$ & 44 & $\underline{63}$ & $\underline{76}$ & $\underline{83}$ & $\underline{96}$ & $\underline{96}$ \\
\hline
\end{tabular}

Les différentes importantes étapes que le site à accompli (tel que les nombres de journaux qui ont étés intégrés à la liste et qui sont passés de 2000 journaux en date du 13 Janvier 2006 à 2500 le 15 Décembre de la même année et enfin à 3000 journaux en date du 12 Décembre 2007) ainsi que d'autres informations pertinentes sur la vie du site. Cette rubrique est importante car elle permet de suivre les différentes phases que le site a traversé depuis sa fondation en date du 14 Février 2004 jusqu'à ce jour.

3.3.2.7-Sponsors (Sponsors) : Les quatre sponsors de DOAJ sont

1- Lund University en Suède

2- EBSCO Information Services

3- La Bibliothèque Nationale de Suède

4- INASP (International Network for the Availability of Scientific Publications).

3.3.2.8-Links (Liens): Cette rubrique propose des liens vers cinq questions très importantes concernant le libre accès. A ces questions des réponses sont données avec des liens hypertexte. Ces questions sont :

1-Qu'est ce que le libre accès ?

2- Publication en accès libre pour les propriétaires de journaux

3- Systèmes de publication 
4- Publication en accès libre pour les auteurs

5-Informations et débats sur le libre accès

3.3.2.9-Membership (Adhésion): Cette rubrique présente les parties qui sponsorisent financièrement le DOAJ (Open Society Institute, The National Library of Sweden, SPARC, SPARC Europe et Axiell) ainsi que les bénéfices de l'adhésion. Deux importantes recommandations en faveur de cette adhésion sont rapportées. L'une émane de Peter Suber, une des figures les plus connues du libre accès et une autre émanant du Président du LIBER (LIgue des Bibliothèques Européennes de Recherche) Hans Geleijnse. Les frais d'adhésion vont de 100 Euros pour une adhésion individuelle, à 400 Euros pour les bibliothèques, les universités et les centres de recherche, 4000 Euros pour les consortia de bibliothèques et les associations de bibliothèques, et enfin à 5000 Euros pour les agrégateurs et autres fournisseurs de service.

3.3.2.10-Feedback (réaction): Cette rubrique permet de contacter la partie responsable pour d'éventuelles questions. Ayant contacté la responsable, nous reçûmes une réponse le jour d'après que nous reproduisons à titre indicatif

\section{SV: Feedback for doaj: OA AND HYBRID JOURNALS}

Lundi 18 Août 2008 11h11mn 02s

De:

"Sonja Brage" <Sonja.Brage@lub.lu.se>

Ajouter l'expéditeur dans les contacts

À:

sam_hac1@yahoo.fr

Dear Dr. Hachani,

The journals we include are open access and free of charge for the users. See the BOAI definition of open access: the right of users to "read, download, copy, distribute, print, search, or link to the full texts of these articles" as mandatory for a journal to be included in the directory. The fee you talk about is for authors to publish their article in a journal. Some open access and some not open access journals charge fees for authors and yes, if you have no department behind you or grant it are sure hard. You have to search for journals at http://www.doaj.org/doaj ?func=forAuthors and fill in "No publication fee", which does not mean that those journals are of a lower quality than those which charge you.

Sincerely, Sonja Brage.

Notre but en reproduisant cette réponse est de faire ressortir la rapidité avec laquelle Internet a révolutionné la communication scientifique dans le monde académique. Cette réponse nous est parvenue un jour après l'envoi de la question et cette vitesse est au cœur du mouvement du libre accès après la liberté et la gratuité de consultation de l'information.

3.3.2.11-For Journal owners (pour les éditeurs de journaux): Cette rubrique est réservée aux éditeurs de journaux. Un nom d'utilisateur et un mot de passé sont requis pour y accéder.

3.3.2.12-For authors (pour les auteurs) : Cette rubrique se propose d'aider la personne qui veut publier sur le DOAJ à suivre les étapes que cela induit .Il est à signaler que l'interface de cette rubrique n'est pas la meme car si dans les autres interfaces, il est écrit " Directory of Open Access Journals ", dans cette interface, il est écrit " Directory of Open Access and Hybrid Journals for Authors ".D'ailleurs c'est ce changement qui nous a poussé à correspondre avec la personne citée 
précédemment .Le mot " Hybrid " indique en fait les publications pour lesquelles l'auteur ( ou son unité de recherche) paye une somme pour que sa publication soit librement accessible (Système auteur- payeur au lieu de lecteur - payeur ).Nous avons déjà évoqué cette nouvelle donne induite par le libre accès et qui nous semble contraire aux préceptes du libre accès qui suppose un accès complet, en plus du fait que les sommes demandées ne sont pas à la portée de tout un chacun. L'interface se présente de la manière suivante :

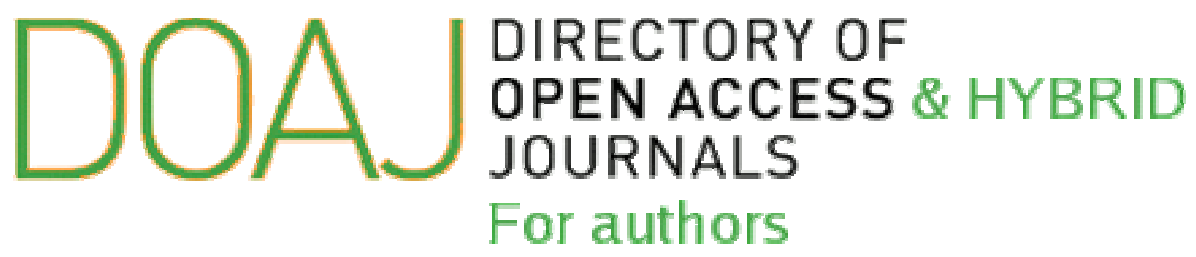

\section{Welcome to the author service of DOAJ - The Directory of Open Access \& Hybrid Journals. Here you can look up where to publish your research as Open Access (OA).}

On remarque que les trois critères de publication sont indiqués. Ce sont :

- $\quad$ "All " et qui indique tous les périodiques que recèlent la liste et les manières d'y publier

- " No publication fee " et qui indique les périodiques qui publient gratuitement

- $\quad$ " Publication fee " et qui indique les périodiques qui publient moyennant un payement

On trouve aussi une rubrique FAQ (Questions fréquemment posées) et qui consiste en une série de 7 questions et réponses qui répondent aux questionnements du chercheur voulant publier dans une publication en libre accès.

Quant à la recherche dans cette rubrique, il n'est pas possible de rechercher séparément les périodiques selon leur accessibilité. Le chercheur doit premièrement choisir une spécialité ensuite, l'interface lui affiche les différents titres avec leur statut. A titre d'exemple, nous avons choisi dans l'arborescence des sujets, la spécialité " Sciences sociales " qui inclut " Bibliothéconomie et Sciences de l'information ". Il existe 93 journaux (14/01/2009) dans la spécialité. ll existe, selon la liste fournie, quatre types de publication :

Libre accès sans payement (78 titres ou $83.87 \%$ )

Libre accès avec payement ( 5 titres ou $5.37 \%$ )

Libre accès sous licence Creative Commons ( 7 titres ou $7.52 \%$ )

Journal Hybride (qui demande un payement) (3 titres ou $3.22 \%$ )

Nous avons reproduit, à titre d'exemple dans la page qui suit, les différents titres et leur statut d'accessibilité ainsi que les informations fournies par le site.

D'un autre coté, nous avons fait une recherche avec la spécialité " Sciences médicales " qui inclut " Neurologie". A la même date, il existait 94 titres et qui se départageaient comme pour la spécialité "Bibliothéconomie et Sciences documentaires" de la manière suivante" :

\footnotetext{
* On remarque que le nombre total et par delà les pourcentages dépassent les 94 titres et les $100 \%$.Ceci est du au fait que certains titres sont en même temps en libre accès et aussi sous licence Créative Commons alors que pour d'autres titres, il est que stipulé que les informations ne sont pas disponibles. Nous les avons inclus avec l'en tête qui les précédait.
} 
Libre accès sans payement (37 titres ou 31.91\%)

Libre accès avec payement (21 titres ou $22.34 \%$ )

Libre accès sous licence Creative Commons (13 titres ou $13.82 \%$ )

Journal Hybride (qui demande un payement) (31 titres ou $32.97 \%$ )

\section{Browse by subject}

\section{Expand Subject Tree}

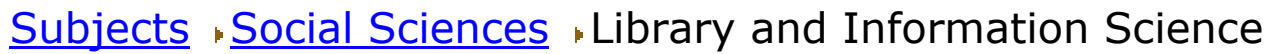

Bibliography (1 journals)

93 journals belonging to subject: Library and Information Science

Open Access: The content is available for free to all

Hybrid journal: If you pay the publisher your individual article will be freely available while other articles in the same issue can require subscription for access

ACIMED Open Access

ISSN: 10249435

EISSN: 15612880

Subject: Library and Information Science --- Medicine (General)

Publisher: Centro Nacional de Información de Ciencias Médicas

Language: Spanish

Keywords: health sciences

Publication fee: No --- Further Information

Anales de Documentación Open Access

ISSN: 15752437

EISSN: 16977904

Subject: Library and Information Science

Publisher: Universidad de Murcia

Language: Spanish, English, French

Keywords: documentation, archival science, librarianship, documental restoration, information literacy

Publication fee: Yes --- Further Information

DIGITHUM Open Access

ISSN: 15752275

Subject: Library and Information Science --- Social Sciences

Publisher: Fundació per la Universitat Oberta de Catalunya (FUOC)

Language: Catalan, Spanish, English

Keywords: humanities, social science, digital culture, information society, ICT

Publication fee: No --- Further Information

License: $(\mathrm{cc}) \mathrm{EY}-\mathrm{NC}-\mathrm{ND}$

Learned publishing Hybrid journal

ISSN: 09531513

Subject: Library and Information Science

Publisher: Association of Learned and Professional Society Publishers

Publication fee: Yes --- Further Information 
Si on compare les chiffres des deux spécialités, il apparaît clair que le système auteur - payeur (qui est décrit ici sous l'appellation hybride) est plus applicable et est plus accepté par les sciences médicales et biologiques que dans les sciences sociales et humaines. De toute évidence, une abondante littérature a été produite pour expliquer ce déséquilibre qui se vérifie presque à chaque occurrence " et qui peut se résumer sur la prépondérance des sciences exactes et médicales dans l'appropriation des nouvelles technologies par rapport aux sciences sociles et humaines. Mais il est une autre raison qui, à notre avis, explique cette prépondérance Les budgets des laboratoires de recherche en biologie et sciences médicales sont autrement plus importants que ceux des unités de recherche en sciences sociales. Il est admis que les Etats investissent beaucoup plus dans des secteurs qui touchent au devenir de leur concitoyen (à l'image de l'épidémie du SIDA qui a mobilisé surtout dans les années 80 d'immenses budgets pour arriver à une solution) qu'à des spécialités dont l'urgence ne peut être comparu avec l'urgence d'une pandémie (on peut aussi penser plus récemment à la menace de la grippe aviaire). Cette urgence dans l'information et aussi les budgets conséquents qui ont en résulté à induit une sorte d'inflation dans la communication scientifique. Les éditeurs, face aux pressions qu'exerce sur leur juteux commerce le libre accès, et afin de ne pas perdre leurs avantages, ont imaginé cet échappatoire qui leur permet d'une pierre deux coups. D'un coté, ils accompagnent un mouvement qu'ils savent inéluctable et d'un autre coté ils chargent un droit d'entrée. D'un autre coté, cette manière de procéder, nonobstant les différents désavantages qu'elle comporte, n'en est pas moins une assurance pour le chercheur de voir sa publication disponible .Il ne faut pas oublier que la publication dans une revue à facteur d'impact élevé est très important pour l'avancement d'un chercheur sans oublier le coté " égo " qui existe en chaque personne. Etre publié dans une revue référence dans la spécialité fait de la personne en question une référence, sans compter les avantages que cela induit en avancement et prestige. Toutes ces raisons sont savamment utilisées pour amener le chercheur à publier (n'oublions pas la fameuse expression " publish or perish ") quitte à le faire à payer.

En conclusion, on peut dire que le DOAJ représente au niveau international une référence dans le monde des journaux électroniques disponibles en ligne. A l'image des entrepôts d'archives qui regroupent les différentes archives ouvertes, les listes de journaux en ligne permettent de recenser les journaux disponibles selon des classifications et des critères que nous avons eu l'occasion de voir en étudiant le DOAJ. Si cette liste est indéniablement un outil de travail de première importance et est un exemple du tout nouveau créneau dans la communication scientifique, car elle permet d'un coté de rassembler un nombre important de périodiques et d'un autre coté, elle permet aussi de les classifier par spécialisation, pays, etc... il n'en demeure pas moins que la sélection des journaux qui demandent des payements pour publier (surtout pour les sciences médicales) ne répond pas aux préceptes de base du libre accès. Il est vrai que l'expression " hybride " est utilisée mais le libre accès devrait être sans restriction surtout si les sommes demandées (que nous aurons l'occasion d'aborder plus loin dans le présent travail) ne sont pas à la portée d'un chercheur (quoique " l'astuce " consiste à faire payer l'entité à laquelle est rattaché le chercheur) même d'un pays développé. Si DOAJ se propose de lister les périodiques sur une base internationale, il existe une autre liste qui elle se propose de sélectionner les périodiques au niveau régional et (comme nous allons le voir) qui est une des manières de faire sortir les chercheurs des pays en voie de développement (ceux de l'Afrique pour le cas qui nous intéresse) d'une sorte d'ostracisme et de marginalisation qui ne dit pas son nom. Cette liste est "African Journal On Line " (AJOL)

\footnotetext{
* Nous avons vérifié dans la spécialité " Physiologie " et qui dépend de " Biologie et Sciences de la vie", les chiffres étaient beaucoup plus accentués car il y avait sur les 91 titres que recelait la spécialité, 66 titres ou $70.96 \%$ qui étaient classés sous la rubrique hybride qui demandait un payement. En poussant plus loin l'étude, il s'est avéré que sur le reste des titres, seuls 9 ou $9.89 \%$ étaient en libre accès intégral .Le reste des titres étaient en libre accès mais demandaient un payement.
} 


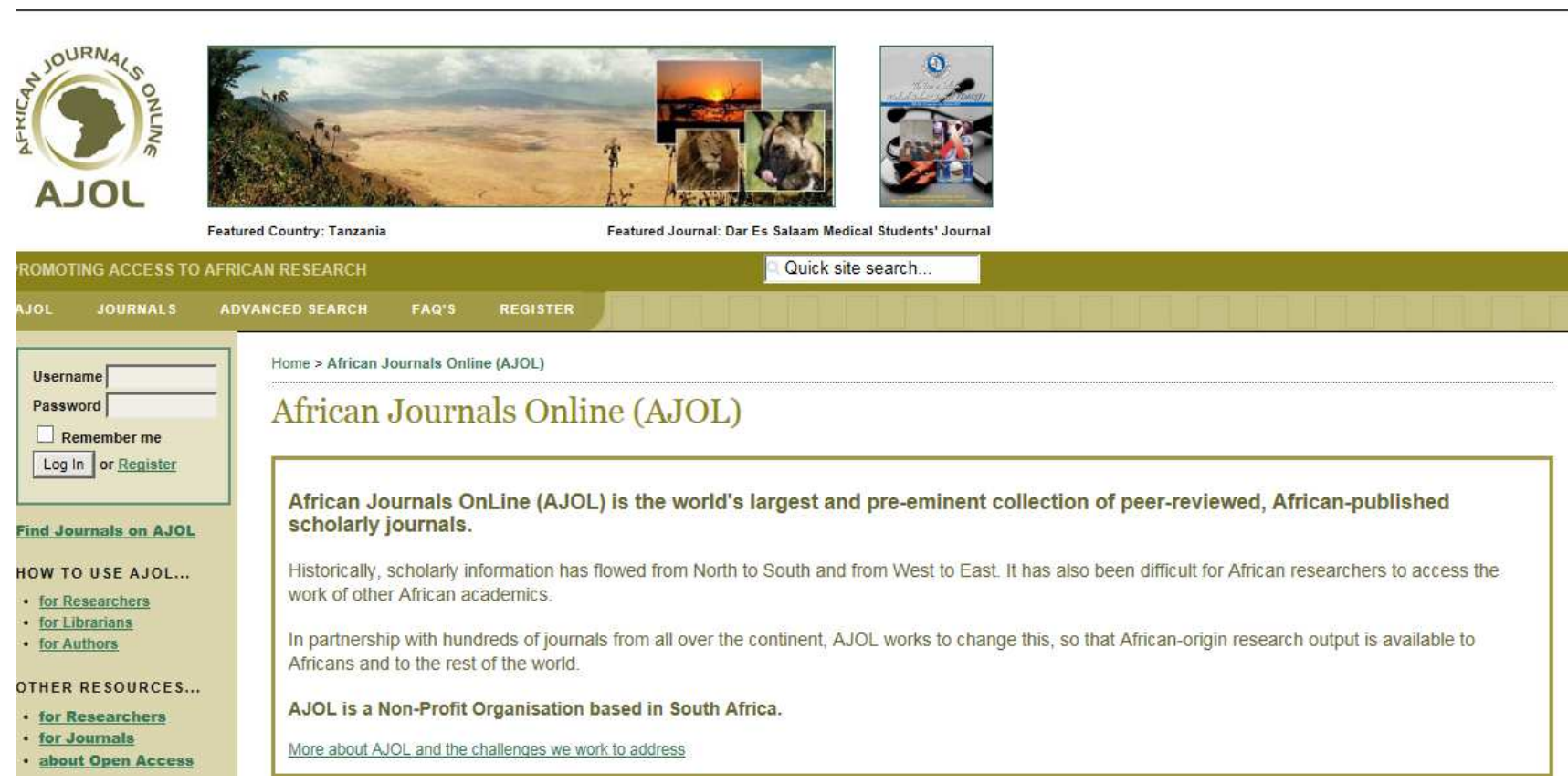

Comme indiqué par son nom, AJOL est une liste des périodiques scientifiques, publiés en Afrique disponibles en ligne. L'interface du site affiche la phrase suivante: " Promouvoir l'accès à la recherche africaine ".Cette phrase décrit de la manière la plus claire possible l'isolement et le manque de moyens dont souffre le chercheur en Afrique. D'ailleurs dans la partie présentant le site, AJOL est présenté comme " un service permettant l'accès à la recherche publiée africaine qui augment la visibilité internationale du savoir indigène" ainsi que " la plus grande collection au monde de périodiques savants en ligne contrôlés par les pairs publiés en Afrique ". Il est explicitement dit qu'à cause de ressources limitées et de la fracture numérique, nombre de périodiques scientifiques contrôlés par les pairs ne sont pas assez cités, utilisés ni évalués et de ce fait ne parviennent pas à ceux qui on en le plus besoin. En plus, si la recherche provenant des pays développés est disponible à travers des programmes tels que HINARI, AGORA et OARE, la réciprocité (recherche en provenance d'Afrique) n'existe pas .Les pays africains ont besoin de jouer un plus grand rôle dans l'environnement scientifique global en ligne.

AJOL fut initié en Mai 1998 (avec au début 8 titres) comme un projet pilote administré par " The International Network for the Availability of Scientific Publication" (INASP) ${ }^{(190)}$ et dont les buts initiaux étaient de promouvoir l'utilisation des périodiques publiés en Afrique dans les sciences et ceci en mettent les tables de matières sur Internet. A la fin de l'année 1999, et après évaluation, il fut recommandé qu'AJOL s'étende à plus de périodiques et à plus de sujets et qu'il inclue aussi les résumés. En Août 2000, il incluait 50 titres anglophones publiés en Afrique et qui concernait les sciences agricoles, sciences et technologie, santé et sciences sociales. En plus, il fournissait des résumés quand ils étaient disponibles et a introduit un service de recherche par mots clés et un service de livraison de photocopies de documents pour tous les titres. Durant la période 2001/2002, des titres additionnels furent ajoutés surtout d'Afrique du Sud et des pays francophones. A la fin 2003, les tables de matières et les résumés de 175 journaux provenant de 21 pays dont certains francophones furent ajoutés. En Mars 2004, à AJOL fut adjoint un système de base de données sophistiqué et ceci afin d'augmenter sa visibilité et faire de telle sorte que la liste soit compatible avec les autres services offerts par le Web entre autres les moteurs de recherche de l'Open Archive Initiative qui moissonnent les métadonnées de chaque article et les mettent à la disposition des

(189) http://www.ajol.info/

(190) $\underline{\text { http://www.inasp.info/ }}$ 
chercheurs au niveau international. Au début de 2009, AJOL héberge plus de 331 journaux revus par les pairs en provenance de 25 pays et le site reçoit plus de 50000 visites de toutes les parties du monde.

3.4.1-Critères de recherche

Quant à la manière de rechercher le site, il se fait de plusieurs manières et qui sont :

3.4.1.1-Browse journals. (Rechercher les journaux) Ceci peut être fait de trois manières :

3.4.1.1.1-Alphabétiquement

3.4.1.1.2-Par Sujets et qui se subdivise en :

3.4.1.1.2.1-Sciences de l'agriculture et administration des ressources (50 titres soit 13.22\%)

3.4.1.1.2.2-Arts, Culture, Langues et littérature (25 titres soit $06.61 \%$ )

3.4.1.1.2.3-Santé (111 titres soit $29.36 \%)$

3.4.1.1.2.4-Sciences et techniques (10 titres soit $02.64 \%$ )

- Généralités (34 titres soit $08.99 \%$ )

- Informatique (0 titre)

- Sciences de la terre (4 titres soit $1.05 \%$ )

- Ingénierie (6 titres soit $1.58 \%$ )

- Science nutritive et technologie (4 titres soit $1.05 \%$ )

- Sciences de la vie (34 titres soit $8.99 \%$ )

- Sciences physiques ( 8 titres soit $2.11 \%$ )

3.4.1.1.2.5-Sciences sociales (3 titres soit $0.79 \%$ )

- Généralités (26 titres soit $6.87 \%$ )

- Anthropologie, sociologie, psychologie ( 11 titres soit $2.91 \%$ )

- Economie, finance, administration (9 titres soit $2.38 \%$ )

- Education (12 titres soit $3.17 \%$ )

- Sciences de l'information et de la communication (13 titres soit $3.43 \%$ )

- Droit (3 titres soit $0.79 \%$ )

-Politique (4 titres soit $1.05 \%$ )

3.4.1.1.2.6-Multidisciplinaire (11 titres soit $2.91 \%$ )

3.4.1.1.3-Pays : les vingt cinq pays recensés et qui sont par ordre alphabétique totalisent chacun :

3.4.1.1.3.1-Afrique du Sud (63 titres soit $18.47 \%$ )

3.4.1.1.3.2-Algérie (2 titres soit $0.58 \%$ )

3.4.1.1.3.3-Botswana (2 titres soit $0.58 \%$ )

3.4.1.1.3.4-Burkina Faso (2 titres soit $0.58 \%$ )

3.4.1.1.3.5-Cameroun (6 titres soit $1.75 \%$ )

3.4.1.1.3.6-Congo (RD) ( 1 titre soit $0.29 \%$ )

3.4.1.1.3.7-Cote d'Ivoire (4 titres soit $1.17 \%$ )

3.4.1.1.3.8-Egypte (9 titres soit $2.63 \%)$

3.4.1.1.3.9-Ethiopie (14 titres soit $04.10 \%$ )

3.4.1.1.3.10-Ghana (16 titres soit $04.69 \%$ )

3.4.1.1.3.11-Ile Maurice (1 titre soit $0.29 \%$ )

3.4.1.1.3.12-Kenya (20 titres soit $05.86 \%$ )

3.4.1.1.3.13-Lesotho (3 titres soit $0.87 \%$ ) 
3.4.1.1.3.14-Libye (Jamahiriya arabe) (1 titre soit $0.29 \%$ )

3.4.1.1.3.15-Malawi (3 titres soit $0.87 \%$ )

3.4.1.1.3.16-Nigeria (149 titres soit $43.69 \%$ )

3.4.1.1.3.17-Ouganda (8 titres soit $2.34 \%$ )

3.4.1.1.3.18-Sénégal ( 6 titres soit $1.75 \%$ )

3.4.1.1.3.19-Soudan (2 titres soit $0.58 \%$ )

3.4.1.1.3.20-Swaziland (4 titres soit $1.17 \%$ )

3.4.1.1.3.21-Tanzanie (12 titres soit $03.51 \%$ )

3.4.1.1.3.22-Togo (1 titre soit $0.29 \%$ )

3.4.1.1.3.23-Tunisie (1 titre soit $0.29 \%$ )

3.4.1.1.3.24-Zambie (1 titre soit $0.29 \%$ )

3.4.1.1.3.25-Zimbabwe (10 titres soit $2.93 \%$ )

Nous remarquons que la liste des pays participants à AJOL n'est pas complète car certains pays n'y figure pas (Maroc, Tchad, Gambie,Niger, etc...). Nous pensons que la raison est le manque de sensibilisation de la part des pays non inclus de l'importance de ce genre d'outil dans le concert mondial des chercheurs (point sur lequel le site insiste lorsqu'il parle de la participation des travaux de recherches en provenance des pays africains ).D'un autre coté, deux pays ( l'Afrique du Sud et le Nigeria) dominent cette liste d'une manière claire. En effet, ces deux pays totalisent $62.16 \%$ de l'ensemble des titres et seuls quelques pays (Kenya, Ghana, Zimbabwe) rivalisent un tant soit peu avec cette dominance de ces deux pays. Ces cinq pays (Nigeria, Afrique du Sud, Kenya, Ghana, Zimbabwe) totalisent les trois quart (75.64 \% pour plus précis) des titres que recèle AJOL. De cette constatation, découle une conclusion : étant donné que ces cinq pays sont anglophones, il est normal que leur titres soit en langue anglaise. Nous avons de ce fait calculé les taux des revues selon les langues et le résultat fut :

Anglais : 347 titres (soit $97.47 \%$ )

Français : 9 titres (soit $2.52 \%$ )

Il est à signaler que plusieurs revues publient en plusieurs langues mais que l'Anglais y est toujours présent. D'un autre coté, même certains pays dont la langue officielle est le Français (comme le Cameroun ) publient des périodiques dont le contenu des fois est plus anglophone que francophone ( par exemple "African Anthropologist " publié au Cameroun et dont la majorité des numéros des recherches publiées le sont en Anglais et dont les deux premiers numéros - Volume $6, \mathrm{n}^{\circ} 1$ et 2 (1999) - sont exclusivement en Anglais ) .Il est a signaler aussi que certains périodiques sud africains comportent des recherches en langue Afrikaner. De ces chiffres découle une conclusion que nous avons déjà évoquée : la prépondérance de la langue anglaise dans la recherche scientifique à un point où même des pays dont la langue officielle est le Français promeuvent des publications en langue anglaise. Nous ne terminerons pas cette courte analyse par pays sans indiquer la peu reluisante place qu'occupe l'Algérie et qui avec 2 titres (Revue d'Information Scientifique et Technique, publiée par le Centre de Recherche sur l'Information Scientifique et Techniques et Technologies Avancées, publiée par le Centre de Développement des Technologies Avancées) se trouve distancée par des pays comme le Swaziland (4 périodiques), Le Lesotho (3 périodiques), et le Malawi (3 périodiques). Loin de nous d'amoindrir le mérite de ces pays, mais ils sont loin d'avoir les moyens matériels et surtout humains que l'Algérie possède. Comme dans toute Révolution, le timing est très important et il nous semble qu'on n'est conscient de l'opportunité qu'offre ce genre de moyen. Pour illustrer ce que nous disons, le dernier numéro disponible de RIST est daté de Janvier 2003 alors que le dernier numéro de Technologies Avancées date de Janvier 2006.Il serait adéquat que ces institutions (et d'autres) s'investissent plus dans le mouvement car tout retard sera très difficile à rattraper.

\footnotetext{
*On aura remarqué que les chiffres que nous avons analysés sont contradictoires .En effet, le site annonce par exemple en date du 17 Janvier 2009, 331 périodiques sur AJOL. Nos calculs personnels sur le site à la même période ont donné respectivement : 341 titres selon les pays et 378 selon les spécialités et 356 selon les langues. Ces écarts seraient du, d'après nous, à des chevauchements (par exemple dans la catégorie " Multidisciplinaire ") et aussi à certains titres qui n'auraient pas étés encore enregistrés. Toujours est il que les chiffres cités sont des chiffres que nous avons nous même calculés directement sur le site.
} 


\subsection{2-L'accès à l'information dans AJOL}

L'accès à l'information dans AJOL se fait globalement de deux manières :

3.4.2.1-Par l'intermédiaire du moteur de recherche Google où l'introduction d'un mot clé donne le résultat de ce mot dans la base de données d'AJOL.

3.4.2.2-Directement sur le site où il est possible de rechercher par sites le contenu. Si le site d'AJOL donne le nombre de sites qu'il recèle, il ne donne pas ceux qui sont en libre accès car, certains titres sont en libre accès alors que d'autres demandent un payement selon des critères que nous allons voir.

\subsubsection{3- Proportion journaux en libre accès /journaux payant :}

Nous avons recherché individuellement chaque site pour voir ceux qui offraient un libre accès intégral et ceux qui demandaient un payement .Les résultats ont étés :

Titres en libre accès : 86 (soit $22.75 \%$ )

Titres demandant un payement : 290 (soit $76.71 \%$ )

Le total est de 376 titres au lieu des 378 titres précédemment calculés et ceci est du au fait que les sites de deux titres et qui sont "African natural history " et "African plant protection " ne sont pas fonctionnels. Ces mêmes chiffres se départagent selon les spécialités selon les chiffres suivants :

1- Sciences agricoles et administration des ressources: Libre accès : 08 Accès contre payement : 42

2- Arts, culture, langue et littérature : Libre accès : 04 Accès contre payement : 21

3- Santé : Libre accès : 40 Accès contre payement : 71

4-Science et technologie : Libre accès : 02 Accès contre payement : 08

-Généralités : Libre accès : 05 Accès contre payement : 29

- Informatique : Libre accès : 0 Accès contre payement : 0

- Sciences de la terre : Libre accès : 01 Accès contre payement : 03

- Ingénierie : Libre accès : 0 Accès contre payement : 06

-Science nutritives et technologie : Libre accès : 02 Accès contre payement : 02

-Sciences de la vie : Libre accès : 11 Accès contre payement : 21 (2 titres ne sont pas fonctionnels dans cette spécialité et nous n'avons pu savoir leur statut).

-Sciences physiques : Libre accès : 01 Accès contre payement : 07

5-Sciences sociales : Libre accès : 01 Accès contre payement : 02

-Généralités : Libre accès : 04 Accès contre payement : 22

-Anthropologie, Psychologie, Sociologie : Libre accès : 0 Accès contre payement : 11

-Economie, Finance, Administration : Libre accès : 02 Accès contre payement : 07

-Education : Libre accès : 02 Accès contre payement : 10

-Sciences de l'information et de la communication : Libre accès : 0 Accès contre payement : 13

-Droit : Libre accès : 02 Accès contre payement : 01

-Politique : Libre accès : 0 Accès contre payement : 04

6- Pluridciplinaire : Libre accès : 01 Accès contre payement : 10 
Ces chiffres sont représentés par le tableau suivant :

\begin{tabular}{|l|c|c|c|}
\hline \multicolumn{1}{|c|}{ Spécialités } & Libre accès & $\begin{array}{c}\text { Accès contre } \\
\text { payement }\end{array}$ & $\begin{array}{c}\text { Pourcentage des } \\
\text { titres en libre accès }\end{array}$ \\
\hline Sciences agricoles et administration des & 08 & 42 & $16 \%$ \\
ressources & 04 & 21 & $16 \%$ \\
\hline Arts, culture, langue et littérature & & & \\
\hline Santé & 40 & 71 & $36.03 \%$ \\
\hline Science et technologie & 02 & 08 & $20 \%$ \\
-Généralités & 05 & 29 & $14.70 \%$ \\
-Informatique & 00 & 00 & --- \\
-Sciences de la terre & 01 & 03 & $25 \%$ \\
-Ingénierie & 00 & 06 & --- \\
-Sciences nutritives et technologie & 02 & 02 & $50 \%$ \\
-Sciences de la vie & 11 & $21 *$ & $34.37 \%$ \\
-Sciences physiques & 01 & 07 & $12.50 \%$ \\
\hline Sciences sociales & 01 & 02 & $33.33 \%$ \\
-Généralités & 04 & 22 & $15.38 \%$ \\
-Anthropologie, Psychologie et Sociologie & 02 & 11 & --- \\
-Economie, Finance et Administration & 02 & 07 & $22.22 \%$ \\
-Education & 00 & 10 & $16.66 \%$ \\
-Sciences de 1'Information et & & 13 & ---- \\
Communication & $1 a$ & 01 & $66.66 \%$ \\
-Droit & 01 & 10 & $09.09 \%$ \\
-Politique & 01 & 10 & $09.09 \%$ \\
\hline Multidisciplinaire & & & \\
\hline
\end{tabular}

Tableau n ${ }^{\circ}$-Proportion des titres en libre accès

Ces chiffres sont importants à plus d'un titre et méritent de notre part des commentaires et des remarques que nous résumerons en :

1- Il semblerait qu'AJOL n'est pas un site qui applique le libre accès tel que défini par les différentes Déclarations. Nonobstant les raisons, le fait que moins du quart des titres soient en libre accès intégral n'aide pas à encourager la recherche surtout qu'AJOL est supposé aider la recherche africaine à s'insérer dans le concert universel de la recherche. S'il est vrai que le site se borne à héberger les titres et que la politique d'accès est laissée à la discrétion du journal, il n'en demeure pas moins que le payement pour l'accès n'est pas pour faciliter à combattre, ni combler la fameuse fracture numérique.

A part les spécialités " Santé" et " Sciences de la vie " qui avec des pourcentages assez respectables de respectivement $36.03 \%$ et $34.37 \%$, les autres spécialités ne semblent pas œuvrer pour le libre accès .Nous remarquons en particulier que la spécialité " Sciences de l'information et de la communication " ne comporte aucun titre en libre accès alors que c'est les bibliothécaires qui sont aux avant postes du libre accès (à l'exemple de "Association of Research Libraries " aux Etats-Unis dont la voix est respectée et écoutée). Cette absence n'est pas sans conséquences car les premiers à souffrir de la crise des périodiques sont bien les bibliothèques dont les budgets n'arrivent pas à répondre à la spirale de l'augmentation et ce dans les pays développés que alors dire des pays en voie de développement en général et de l'Afrique en particulier.

\footnotetext{
* Deux titres sont non comptabilisés car leurs sites étaient non fonctionnels. La direction d'AJOL nous a informé que l'un " East African Natural History" est en libre accès pour la période 1984-2004 alors "African Plant Protection" n'est plus hébergé par AJOL et que son statut leur est inconnu
} 
2- La faiblesse des titres en Sciences exactes (Informatique, Sciences nutritives et technologie et Sciences physiques) en plus de la faiblesse du taux en libre accès .S'il est loin de notre propos d'amoindrir le mérite des sciences sociales et humaines, il n'en demeure pas moins que les sciences exactes sont très importantes dans le décollage économique des pays en voie de développement.

La classification des différentes spécialités ne nous semble pas très au point et prêche par une sorte de confusion. En effet, comment expliquer que des titres tels que " Journal of Science and Technology " soit classé dans la spécialité " Arts, culture, langue et littérature " ou que " Journal of East African Natural History " soit classé dans la spécialité " Sciences et techniques " ou que " Ghana Journal of Forestry " soit classé dans les " Généralités " en " Sciences Sociales" .S'il est vrai que des fois ces titres peuvent comporter des sujets qui touchent à plusieurs spécialités connexes ( et qui pourrait être inclus dans la spécialité Multidisciplinaire ), il n'en demeure pas moins, qu'à notre avis, la dite classification gagnerait à être revue et affinée.

Si ces titres sont en libre accès (sous différentes conditions tel que nous l'avons vu), il est possible pour les autres titres qui n'appliquent pas le principe du libre accès d'y accéder en contrepartie d'un payement. Nous allons voir de quelle manière cette opération est réalisée.

\section{5-L'accès payant à AJOL}

Lorsque le titre n'est pas en libre accès, l'utilisateur est orienté vers les fonctionnalités qui lui permettent d'accéder à l'article voulu en payant .Prenons un exemple pour illustrer cette opération. " Africa Insight " est un titre qui n'est pas en libre accès. Lorsque l'utilisateur, clique sur le site, il obtient la page suivante :

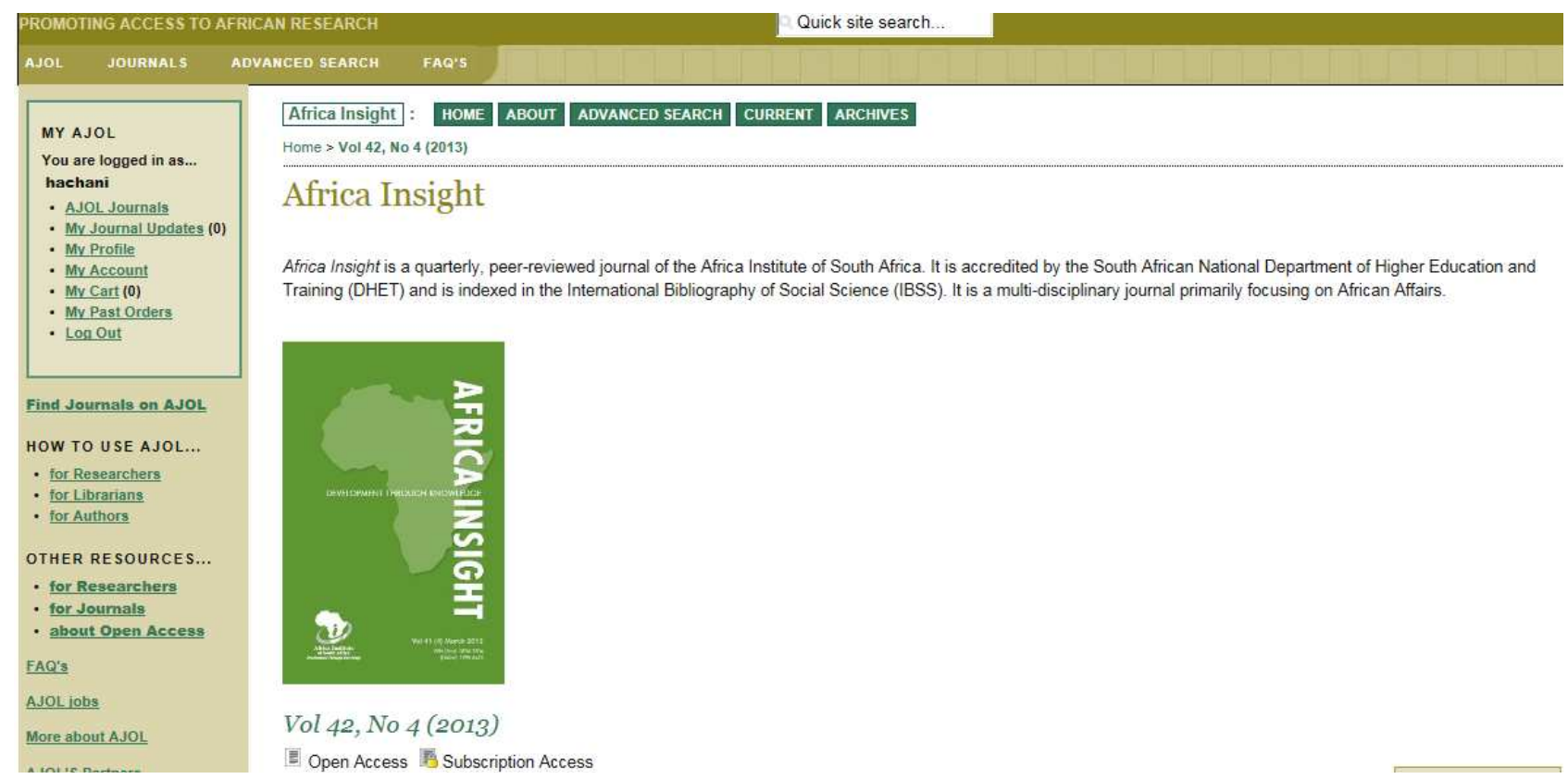

On remarque au bas de la page en dessus de la couverture les mentions " open access " (libre accès) et " subscription access " (accès par abonnement ou payant représenté par B ).Les articles de ce titre étant payant, en cliquant sur le premier titre par exemple du numéro, on obtient ce qui suit : 
를 Open Access

South Africa in the BRICS: Opportunities, Challenges and Prospects1

HBesada, E Tok, KWinters

Abstract

This study investigates the participation of South Africa in the BRICS group from an analytical perspective. It is argued that South Africa's membership to this group entails both opportunities and challenges for South Africa, the continent and the system of global governance. We seek to flesh out the potential mediumand long-term implications as well as the benefits and risks of South Africa's membership in the areas pertaining to trade, foreign policy and development

Full Text:

EMAIL FULL TEXT 屌

DOWNLOAD FULL TEXT F

Africa Insight. ISSN: 02562804

Dans cette page, le résumé est présenté et le texte intégral est soit envoyé sur la boite électronique) ou alors télécharg. En cliquant sur " download full text " (télécharger le texte intégral), on obtient la page suivante :

\section{\begin{tabular}{|l|l|l|l|l|}
\hline Africa Insight : & HOME & ABOUT ADVANCED SEARCH & CURRENT & ARCHIVES \\
\hline
\end{tabular}}

Home > Contents of your cart > Contents of your cart

\section{Contents of your cart}

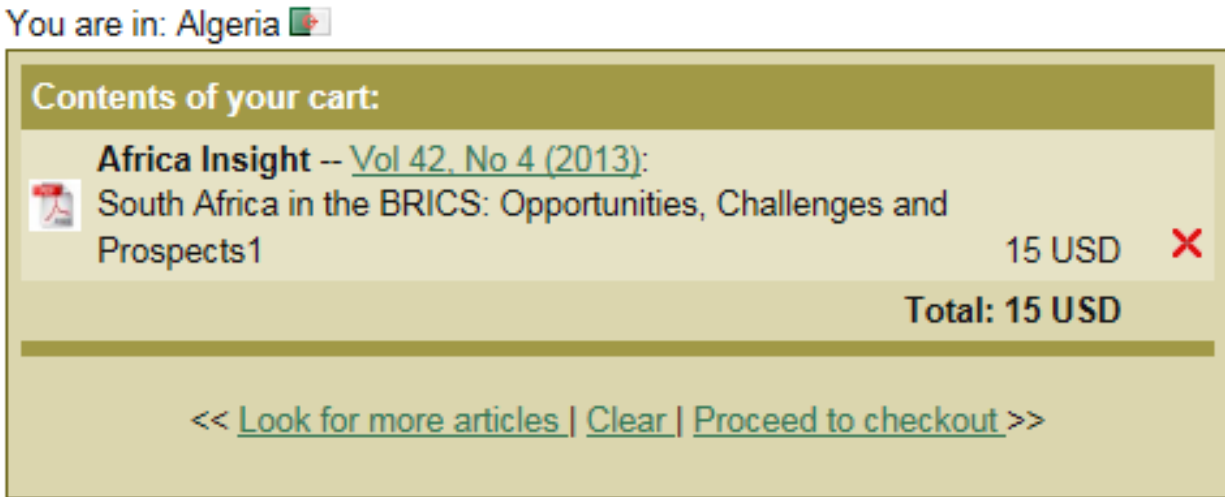

Africa Insight. ISSN: 02562804

On remarquera que, grâce à l'adresse IP, le pays est détecté AJOL pratiquant un accès selon les chiffres de la Banque Mondiale. En cliquant sur " Proceed to check out " (passer la commande), on obtient la page suivante : 


\section{Checkout}

You have several options to proceed with your checkout. Readers from low income countries are entitled to 3 articles per month. You can also set up a pre-paid account with us or have your university library buy a subscription. Pay-per-view is also available though online payment via 2 checkout.

Use an existing account code.

ENTER YOUR ACCOUNT CODE AND CLICK HERE

Pay by Credit Card

» You can use any major credit card to pay for your order. USE YOUR CARD

REVEW YOUR CART'S CONTENT

Africa Insight. ISSN: 02562804

Comme on peut le voir, il est possible de payer en créant un compte ou alors en payant par une carte de crédit. La première option impose de créer un compte (généralement institutionnel) et se présente de la manière suivante :

\section{\begin{tabular}{|l|l|l|l|l|l|}
\hline Africa Insight $:$ & HOME & ABOUT & ADVANCED SEARCH & CURRENT & ARCHIVES \\
\hline
\end{tabular}}

Home $>$ Contents of your cart $>$ My Account

\section{My Account}

\section{Open Access:}

Open Access Articles are free to download.

Low Income Country Credits:

Readers from Low Income countries are entitled to 3 free articles per month.

- You are in Algeria, classified by the World Bank as Upper-Middle Income (UMIC).

Institution-based Prepaid Account:

Institutions can set up a Prepaid Account with us, which all their users can access via an Account Code. Click here for information.

ENTER YOUR ACCOUNT CODE AND CLICK HERE.

Or purchase prepaid Article credits using a credit card (via our payment gateway partner 2checkout.com) by selecting one of the following:

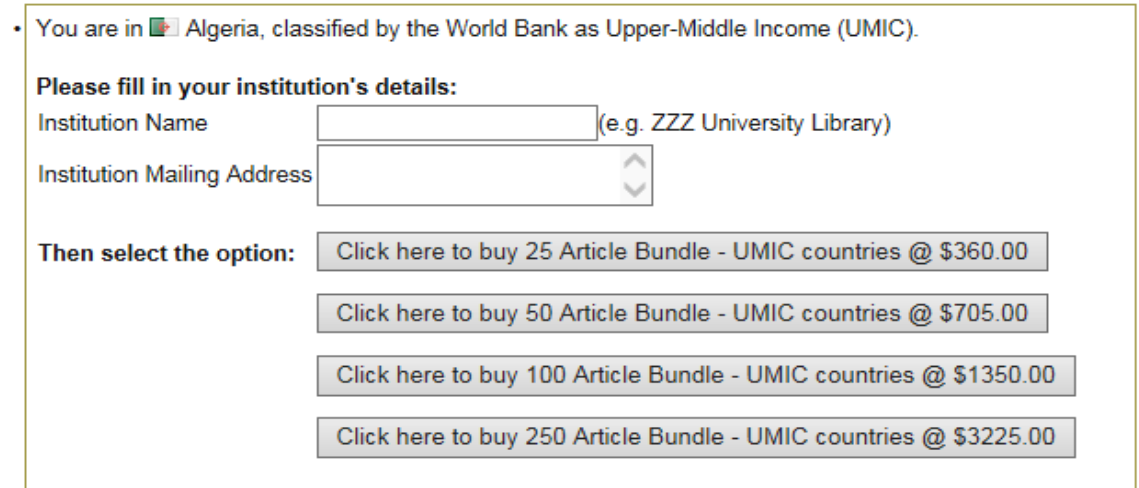

Pay by Credit Card

Articles can be purchased using online credit card payments via 2checkout.

On peut résumer cette opération en la création d'un compte où l'institution peut puiser le quota d'articles qu'elle a acheté * . La deuxième consiste au payement par carte de crédit et elle se présente de la manière suivante :

\footnotetext{
* Nous ne pouvons décrire cette étape étant donne qu'elle est réservée aux institutions et impose la création d'un compte au nom de l'institution, ce qu'il nous est impossible de faire
} 
Home $>$ Contents of your cart > Pay by Credit Card

\section{Pay by Credit Card}

You are about to purchase 1 articles. Each article costs 15 USD. Order Total: 15 USD.

\section{CHECKOUT.COM}

You will be redirected to the following page at our trusted e-commerce partner 2checkout.com, where you can complete your purchase.

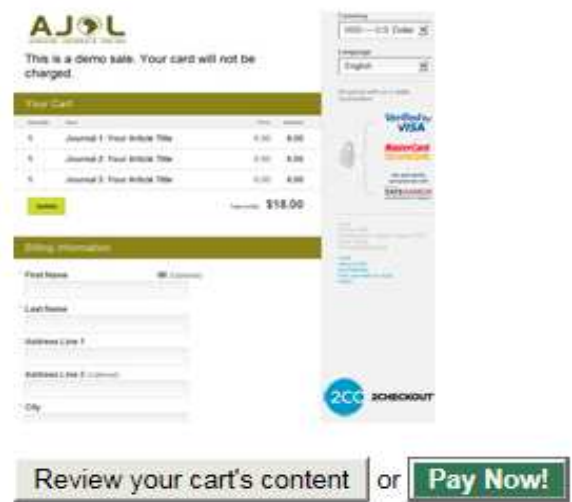

Africa Insight. ISSN: 02562804

En cliquant sur " Pay now ", on obtient ce qui suit :

\section{AJOL}

\section{Secure Checkout}

\section{(1) Review Cart}

\begin{tabular}{|c|c|c|c|}
\hline Item & $\begin{array}{r}\text { Unit } \\
\text { Price }\end{array}$ & Quantity & Subtotal \\
\hline Al: South Africa in the BRICS: Opportunities, & $\$ 15.00$ & 1 & $\$ 15.00$ \\
\hline
\end{tabular}

Current Total (USD): $\$ \mathbf{1 5 . 0 0}$
This website is secure, Your personal details are safe.

Verified by MasterCard. VISA SecureCode.

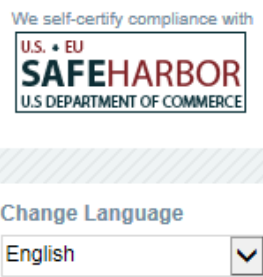

Change Currency USD - U.S. Dollar 


\section{CHECKOUT}

En cliquant sur " billing information" et "payement method", la personne est conduite à travers les étapes connues de payement par carte de crédit

Comme on peut le constater, la procédure de commande d'articles est assez compliquée et se résume en :

La recherche du pays où réside le chercheur pour accéder à l'information. En cliquant sur " voir pays ayant droit de participer ", il obtient une liste de 243 pays dérivée des chiffres de la Banque mondiale et partagée en trois catégories :

Les pays a bas revenus au nombre de 60, les pays à revenus intermédiaire au nombre de 91 et les pays à haut revenus au nombre de 9

Les pays du premier groupe ne sont assujettis a à aucun payement et reçoivent les articles demandés gratuitement .Cette gratuité est toutefois sujette à certaines conditions : il n'est livré que 3 articles gratuits par mois et les demandeurs de ces articles ne doivent pas être citoyen du pays dont est originaire le titre (Un chercheur algérien ne peux pas, à titre d'exemple, demander un article de Technologies avancées du CDTA ou Revue de l'Information Scientifique et Technique du CERIST). D'un autre coté, les étudiants ne peuvent pas demander des articles en leur nom propre mais doivent pour ce faire les demander par l'intermédiaire de leurs bibliothèques. Enfin, le maximum d'articles qu'une bibliothèque peut commander mensuellement ne peut excéder 12 articles gratuits.

Les pays du deuxième groupe peuvent quant à eux peuvent accéder aux articles à des prix subventionnés ( 09 \$ l'article)

Les pays du troisième groupe enfin sont assujettis à un payement intégral ( $15 \$$ l'article). Le tableau suivant illustre cette répartition des pays et des payements

\begin{tabular}{|c|c|c|}
\hline Catégorie de pays & Catégorie de livraison & Payement en US\$ \\
\hline Pays a bas revenus & Financé par AJOL & $\$ 0$ \\
\hline Pays a revenus intermédiaires & Subventionnée par AJOL & $\$ 5$ \\
\hline Pays a hauts revenus & Payement complet & $\$ 15$ \\
\hline
\end{tabular}

Tableau ${ }^{\circ}{ }^{4}$ - Répartition des pays selon le revenu

Il est à noter que généralement, le fichier est envoyé par courrier électronique à l'adresse du chercheur ou de la bibliothèque soit sous forme d'un fichier PDF ou Microsoft Word (à condition qu'il ne dépasse pas un poids de $1 \mathrm{Mb}$ ). Dans le cas où le fichier n'est pas disponible électroniquement, il est envoyé par fax s'il ne dépasse pas 10 pages et par voie postale s'il les dépasse et ce dans un délai ne dépassant pas 2 jours à partir de la date de réception. 
Quant aux méthodes de payement, il existe deux formules :

- Par carte de crédit

- En ouvrant un compte de livraison de documents prépayé dans lequel le chercheur ou l'institution paye une certaine somme pour un nombre donné d'articles par an. Les différents forfaits se présentent de la manière suivante :

\begin{tabular}{|c|c|c|c|c|}
\hline Type de Compte & $\begin{array}{c}\text { Jusqu'à } \\
25 \text { articles }\end{array}$ & $\begin{array}{l}\text { Jusqu'à } \\
50 \text { articles }\end{array}$ & $\begin{array}{c}\text { Jusqu'à } \\
100 \text { articles }\end{array}$ & $\begin{array}{c}\text { Jusqu'à } \\
250 \text { articles }\end{array}$ \\
\hline $\begin{array}{l}\text { Pays à revenus } \\
\text { intermédiaires }\end{array}$ & $\begin{array}{c}\text { US\$220 } \\
\text { (US\$8.80 par } \\
\text { article**) }^{*} \text {. }\end{array}$ & $\begin{array}{c}\text { US\$428 } \\
\text { (US\$8.56 par } \\
\text { article*) }\end{array}$ & $\begin{array}{c}\text { US\$828 } \\
\text { (US\$8.28 par } \\
\text { article*) }\end{array}$ & $\begin{array}{c}\text { US } \$ 1980 \\
\text { (US\$7.92 par } \\
\text { article*) }\end{array}$ \\
\hline $\begin{array}{l}\text { Pays à hauts } \\
\text { revenus }\end{array}$ & $\begin{array}{c}\text { US\$390 } \\
\text { (US\$15.60 par } \\
\text { article*) }\end{array}$ & $\begin{array}{c}\text { US\$752 } \\
\text { (US\$15.04 par } \\
\text { article*) }\end{array}$ & $\begin{array}{c}\text { US\$1440 } \\
\text { (US\$14.40 par } \\
\text { article*) }\end{array}$ & $\begin{array}{c}\text { US\$3440 } \\
\text { (US\$13.76 par } \\
\text { article*) }\end{array}$ \\
\hline
\end{tabular}

Tableau $\mathrm{n}^{\circ} 5$ - Forfaits offerts pour l'achat d'articles selon le revenu

\section{6-Projets futurs d'AJOL}

Les buts les plus importants d'AJOL pour le futur sont de faciliter un accès universel au texte intégral des journaux africains soit en l'hébergeant en ligne ou en orientant l'utilisateur vers le site Web. Le futur d'AJOL est envisagé en tant que fournisseur d'un large éventail d'accès en ligne des textes intégraux des journaux allant d'un accès libre total à un accès par abonnement avec des modèles hybrides d'accès restreint. Le modèle actuel d'AJOL permet déjà un certain accès libre et envisage de porter sa couverture pour permettre aussi des modèles d'accès restreints et d'abonnement aux textes intégraux.

\section{7-Projets analogues}

3.7.1-INASP (The International Network for the Availability of Scientific Publications) ${ }^{(191)}$

Sponsor principal d'AJOL, INASP a établi d'autres projets analogues dans d'autres régions surtout au Sud et au Sud Est asiatique. INASP assiste aussi des journaux africains à publier leur texte intégral en ligne et le " Programme for the Enhancement of Research Information " (PERI) ${ }^{(192)}$ qui est un programme dépendant d'INASP fournit de l'aide aux chercheurs dans le monde entier par le biais de l'accès à l'information, la formation et l'aide à l'accès à l'information.

\subsection{2-PKP (Public Knowledge Project $)^{(193)}$}

La base de données d'AJOL fut développée en utilisant le logiciel libre de management de journaux connu sous l'appellation de " Open Journal System " (194) développé par le PKP à L'Université de la Colombie Britannique au Canada. Le travail collaboratif d'AJOL avec ces organisations a permit de mettre au point un site de qualité supérieure avec des fonctionnalités avancées.

\footnotetext{
* Les taux standard pour la livraison de documents sont US\$9.00 par article et US\$16.00 par article pour les pays à revenus intermédiaires et les pays à haut revenu respectivement. Les taux escomptés par article montrés s'appliquent quand la quantité maximum d'articles pour cette option de compte est demandée

${ }^{(191)} \mathrm{http}$ ://www.inasp.info

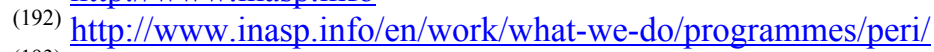

(193) http://pkp.sfu.ca

(194) $\underline{\text { http://pkp.sfu.ca/?q=ojs }}$
} 
NISC est une compagnie spécialisée dans les bases de données bibliographiques et la littérature scientifique africaine. Quoique d'essence commerciale, NISC est fière de sa mission qui consiste à promouvoir la recherche africaine dans le monde et fournit l'Afrique avec des produits d'information internationaux de niveau élevé.

\section{Conclusion}

En conclusion, on peut dire qu'AJOL est un projet répondant aux besoins de visibilité des chercheurs africains qui se trouvent marginalisés dans le concert universel des chercheurs .En effet, les chercheurs africains en particulier et ceux du monde en voie de développement en général se heurtent à des difficultés d'accès à l'information résultant d'une multitude de raisons ( prix des périodiques, cherté de l'accès à Internet, fracture numérique, etc...).Cette situation est aggravée par un manque d'exposition des travaux de ces même chercheurs car les critères de publication, les sujets abordés et même les moyens dévolus ne peuvent être comparés à ceux des pays développés. Plusieurs recherches et études ont fait ressortir que le taux de publication des chercheurs des pays en voie de développement est inférieur à celui des chercheurs des pays en voie de développement et ceci n'a rien a à voir avec la valeur intrinsèque des chercheurs .Plus grave, le co- autorage se fait à sens unique et le chercheur du pays en voie de développement se voit " colonisé de nouveau " par le chercheur du Nord. .Farid Dahdouh -Guebas a utilisé l'expression de " neo colonial science " ${ }^{(196)}$ où le chercheur du pays en voie de développement n'est pas traité d'égal à égal par son confrère du Nord. Pire, si l'expression de " neo colonial science " est en elle-même symptomatique d'un état d'esprit de domination (pour ne pas dire de condescendance), elle est aggravée, selon le même auteur, par une attitude où le chercheur du pays en voie de développement n'est pas pris en ligne de compte dans la publication même lorsque le sujet du travail est le pays en question et que le chercheur du Sud participe. La même idée est développée par Benjamin Acosta Cazeres qui parle en fait de " colonialisme scientifique et safari recherche" ${ }^{(197)}$ et où il prouve que les chercheurs des pays en voie de développement ont moins accès aux journaux médicaux, soumettent moins d'articles, ont un taux de rejet beaucoup plus élevé que celui des chercheurs des pays développés et sont moins représentés dans les comité éditoriaux sans qu'il y ait une claire indication quant à la différence dans le niveau des travaux résultant de leur statut de chercheur d'un pays en voie de développement).Toutes ces raison militent en faveur de l'établissement de sites à l'image d'AJOL * et qui peuvent avoir une quadruple fonction :

\footnotetext{
(195) Www.nisc.co.za/

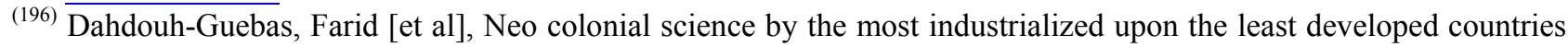
in peer reviewed publishing, Scientometrics, Vol 56, n³2003, p.329-343

${ }^{(197)}$ Acosta-Cazares, Benjamin[...et al ], Scientific colonialism and safari research.

Disponible à : http://clinmed.netprints.org/cgi/content/full/2000010008v1

* INASP sponsorise nombre de Journaux en ligne tels que NepJOL, BanglaJOL, VJOL, PhilJOL et SLJOL qui tous répondent aux besoins de chercheurs de pays ( Népal, Bangladesh,Vietnam, Philippines et Sri Lanka) ) qu'on pourrait même décrire d' " enclavés scientifiquement ".Bien que n'ayant pas atteint les chiffres d'AJOL qui concernent ceux d'un continent, ces différents sites ont tout de même atteint :
}

-Pour NepJol : 31 titres de journaux comprenant 85 tables de matière. Sur les 1341 articles que comprennent ces tables de matière, 899 sont en libre accès (http://www.nepjol.info/)

-Pour BanglaJOL : 25 titres comprenant 109 tables de matière. Sur les 1518 articles que comprennent ces tables de matière, 1009 sont en libre accès (http://www.banglajol.info/)

-Pour VJOL : 23 titres comprenant 142 tables de matière. Sur les 1473 articles que comprennent ces tables de matière, 1010 sont en libre accès (http://www.vjol.info/)

-Pour PhilJOL : 26 titres comprenant 70 tables de matière. Sur les 610 articles que comprennent ces tables de matière, 462 sont en libre accès (http://www.philjol.info/)

-Pour SLJOL : 6 titres comprenant 29 tables de matière. Sur les 272 articles que comprennent ces tables de matière, 271 sont en libre accès (http://www.sljol.info/) 
2- Permettre aux chercheurs africains de publier dans des sujets qui leur sont importants et en leur terme

3-Donner une plus grande visibilité aux chercheurs indigènes et leur permettre d'intégrer le concert universel des chercheurs

4-Avoir une plus grande représentation dans les comités de lecture par l'intermédiaire de cette visibilité accrue (c'est l'une des raisons évoquée par Benjamin Acosta Cazeres concernant cette faiblesse d'acceptation et qui résulterait, entre autres, d'une faible représentativité au niveau des comités éditoriaux des grand titres )

D'un autre côté, bien qu'AJOL réponde à un besoin tout ce qu'il y a de plus légitime des chercheurs africains, il n'en demeure pas moins que sa philosophie dérive un tant soit peu de la philosophie du libre accès .Le fait de payer (entre $\$ 9$ et $\$ 15$ l'article) en plus du payement par carte de crédit ne fait qu'isoler et rend l'accès à l'information aléatoire pour le chercheur africain. Si cette somme peut paraître insignifiante pour un chercheur du Nord, elle peut représenter un pourcentage assez important du revenu de la majorité des chercheurs africains. Le fait qu'une compagnie privée (NISC) ait pris la direction et le management du site explique peut être cet anachronisme .Il ne faut pas oublier que INASP et PKP sont les deux sponsors les plus importants d'AJOL (l'un financier et l'autre logiciel) et qu'ils oeuvrent pour le libre accès surtout PKP qui en mettant sur le marché un logiciel libre aide à la vulgarisation des journaux électroniques, vecteur du libre accès. De même que 1'UNESCO, le Comité pour la Dissémination de l'Information Scientifique, The National Academy of Sciences, NORAD, Danida et Sida: DFID et qui sponsorisent aussi AJOL sont des organisations dont les buts est d'aider les chercheurs du Sud à s'affranchir des contingences financières qui entravent, et même stoppent, ses efforts. Malgré cela, l'expérience d'AJOL (et celles de NepJOL, BanglaJOL, VJOL, PhilJOL et SLJOL) est en elle même encourageante et prouve que les pays du Sud peuvent briser la fatalité d'un sous développement endémique pour peu qu'un minimum leur est fournit.

\section{Chapitre IV LIBRE ACCES ET DROITS D'AUTEURS}

\section{Introduction}

Toutes les tentatives que nous avons vues, et qui ne représentent en fait qu'un nombre infime de sites parmi la myriade des sites que l'extraordinaire vitesse et fonctions d'Internet ont permis, se heurtent en fait à une question ou plutôt à un problème auquel l'auteur doit faire face en déposant sa recherche soit dans une archive ouverte ou un journal électronique : les droits d'auteur. En effet si dans l'univers papier, les règles étaient (et sont toujours) bien définies, l'explosion des archives ouvertes en particulier et du libre accès en général ont fondamentalement changé les règles. Nous pesons bien nos mots quand nous disons fondamentalement, car pour schématiser à l'extrême nous pourrions dire que la recherche publiée dans le périodique traditionnel (papier) appartient à l'éditeur qui en contrepartie de la publication de la recherche pouvait la revendre à des prix prohibitifs étant donné que le chercheur était satisfait de l'exposition (surtout dans le cas des revues importantes et prestigieuses appelées " revues noyaux " traduction littérale de "core titles " ou de rang A et dont l'acceptation d'une recherche était un précieux sésame vers la célébrité scientifique et autres avantages liés à la fonction de chercheur ) et de la reconnaissance des pairs car toute recherche passait par le tamis du contrôle par les pairs qui lui donnait toute sa validité et lui permettait d'être acceptée comme élément constitutif de la Science et du Savoir. Quant à la recherche qui est 
présente sur Internet (sous forme de publications électroniques dans une archive ouverte ou sous forme d'article dans une revue électronique en ligne) à qui appartienne t-elle ? Est-ce que le chercheur qui postule à une publication est propriétaire de sa recherche étant donné qu'il lui est loisible de la mettre sur son site personnel, celui de son université (son laboratoire ou équipe de recherche) ou est ce que l'éditeur en est propriétaire ? Comme on peut le voir, les règles même de publication sont changées et cette situation a poussé les éditeurs à lâcher du lest afin de ne pas perdre l'emprise qu'ils ont toujours (mais pour combien de temps ?) sur la publication scientifique contrôlée par les pairs. De leur coté, les chercheurs sont toujours ignorants et réticents vis-à-vis d'un medium somme toute nouveau et dont ils appréhendent les conséquences. On peut résumer ces réticences en :

1-La nouveauté du medium

2- La peur de se voir déposséder de son travail (plagiat) dans un medium qui a été décrit comme un Far West et une Terra Nova ( ou Terra incognita comme l'on décrit d'autres chercheurs) de l'information.

3- La peur de voir ses travaux " noyés" dans la multitude des sites de la Toile.

4-L'ignorance des règles régissant la publication sur Internet et quels sont les droits que garde l'auteur déposant sa recherche dans une archive ouverte.

A cet effet, le site SHERPA (Securing a Hybrid Environment for Research Preservation and Access) est considéré comme le site que l'auteur voulant publier doit impérativement consulter pour connaître ses droits, les éditeurs qui permettent le dépôt (pré et post publications) etc.....Le site presente aussi nombre d'autres droits, avantages et obligations que l'auteur se doit de savoir dans un nouveau medium et par lesquels il peut donner à sa publication toute l'exposition dont elle a besoin.

4.1- SHERPA (Securing a Hybrid Environment for Research Preservation and Access) ${ }^{\text {(198) }}$

${ }^{(198)}$ http://www.sherpa.ac.uk/ 
Award winning SHERPA is investigating issues in the future of scholarly communication. It is developing open-access institutional repositories in universities to facilitate the rapid and efficient worldwide dissemination of research.

\section{SHERPA Services}

- RoMEO - Publisher's copyright \& archiving policies

- JULIET - Research funders archiving mandates and guidelines

- OpenDOAR worldwide Directory of Open Access Repositories

- SHERPA Search - simple full-text search of UK repositories

\section{SHERPA Resources}

- Forthcoming Events - including speaking engagements

- Guidance on depositing material; copyright; and open access for authors

- Advocacy Materials for administrators, including past SHERPA presentations

- Links to other initiatives and related background information

\section{Current SHERPA \& Allied Projects}

- RSP (Repositories Support Project) - support service for UK repository growth

- DRIVER - developing a cross-European repository network
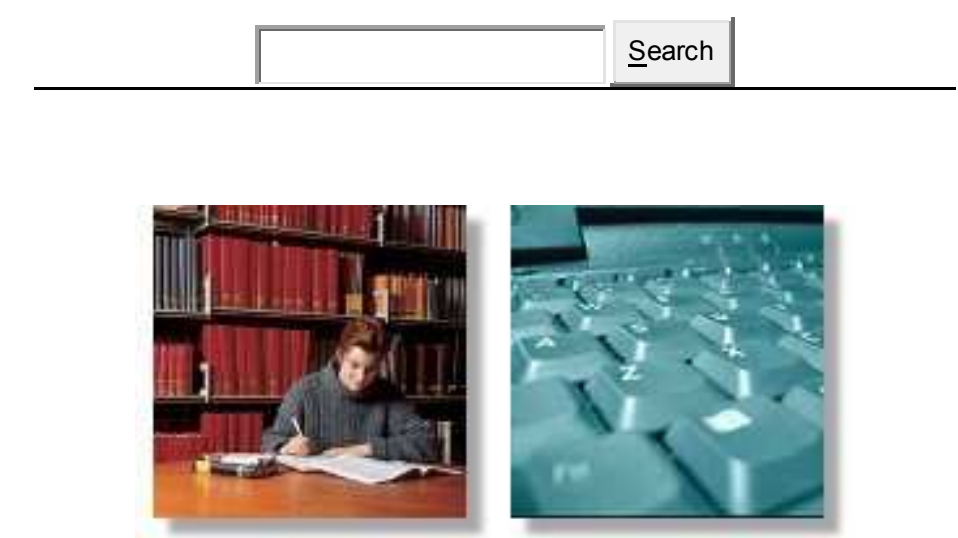

News

$\underline{\text { Selected research funders' grant expenditure }}$

$\underline{\text { JORUM event in December }}$

RSP Events Programme 2008/09

Publishers with Paid Options for Open Access

more news...

$\underline{\text { Jobs }}$

Programmer/Repository Web Developer, The 
infrastructure

- UKCoRR - United Kingdom Council of Research Repositories

- Intute: Repository Search - searches across 90 UK academic repositories

- The Depot a keep-safe repository for UK academics

- SHERPA DP 2 - continuing to look at preservation models

- SHERPA/RoMEO - listing publishers' copyright agreements

\section{Completed SHERPA \& Allied Projects}

- SHERPA Project - which helped develop archives in research-led institutions

- SHERPA Plus - expanding the repository network in the UK

- SHERPA DP - looking at preservation models

- PROSPERO - scoping The Depot keep-safe service

- EThOS - developing a national eTheses service

- Copyright Knowledge Bank - copyright investigation

\section{SHERPA: Securing a Hybrid Environment for Research} Preservation and Access
Library and Learning Resource Centre, The Open University

Digital Preservation Specialist: Parliamentary Archives, House of Lords

Research Officer (Text Digitisation), UKOLN, University of Bath

\section{עISC OPEN SOCIETY INSTITUTE RLUK Research Libraries UK

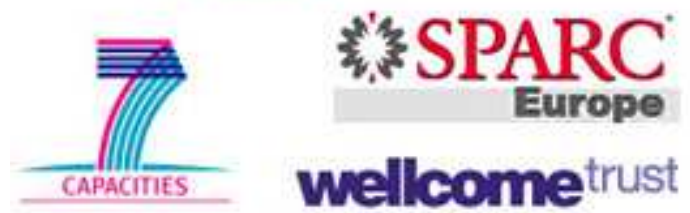

We gratefully acknowledge the generous support given to SHERPA projects by our funders

SHERPA is hosted by the University of Nottingham

(c) 2006, University of Nottingham Contact us 
Le site de SHERPA (qu'on peut traduire par " Assurer un Environnement Hybride pour la Préservation de la Recherche et l'Accès ") se définit comme ..." étudiant les problèmes du futur de la communication scientifique. Il développe les dépôts institutionnels en libre accès dans les universités pour faciliter la dissémination, globale, rapide et efficace de la recherche". Parmi les différents services offerts par le SHERPA, on peu citer :

\subsection{1-ROMEO ${ }^{(199)}$}

Ce service résume les permissions que donne chaque éditeur lors de ce qui est appelé le " copyright transfer agreement " ou le transfert des droits d'auteurs. A cet effet, et face à la pression des protagonistes du libre accès, nombre de maisons d'éditions ont du lâcher du lest et permettre, à des degrés variés, le dépôt dans des archives ouvertes. La recherche se fait de deux manières :

\subsubsection{1-Par titre de journal :}

Lors de la recherche, nous avons cherché " American libraries " (le journal de l'American Library Association-A.LA.) et l'affichage du résultat fut de la manière suivante :

\section{SHERPA ROMEO \\ ...opening access to research

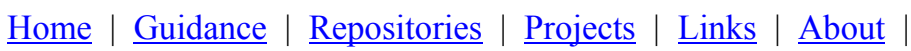 Contacts \\ Publisher copyright policies \& self-archiving}

One journal found when searched for American libraries:

Journal: American Libraries (ISSN: 0002-9769)

Publisher: American Library Association

These summaries are for the publishers default policies and changes or exceptions can often be negotiated by authors.

All information is correct to the best of our knowledge but should not be relied upon for legal advice.

Publisher: American Library Association

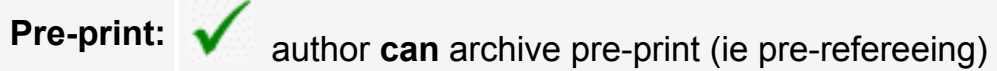

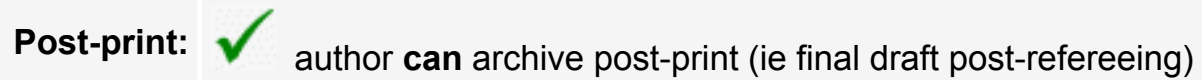

Conditions: - On author or institutional web pages and institutional repositories

- Published source must be acknowledged

Mandated OA: $\underline{\mathrm{AHRC}}$ (Compliant);

$\sqrt{\text { JISC }}($ Compliant)

Copyright: view guidance and licence form and ACRL FAQs

RoMEO: This is a RoMEO green publisher

Updated: $01-O c t-2008$. Suggest an update for this record

Fig.n`26- Exemple de transfert des droits d'auteurs ( copyright transfer agreement ) pour American Library Association)

${ }^{(199)}$ http://www.sherpa.ac.uk/romeo 
Cette manière nous donne les informations pertinentes quant à ce que permet l'éditeur quant à l'utilisation des différentes étapes par lesquelles passent la publication .Pour notre exemple, l'auteur qui publie sur "American Librairies" peut :

-archiver la version pré publication (i.e. pré - expertise ou non revue) -archiver la version post publication (i.e. mouture finale post expertisée) -archiver sur la page web personnelle ou institutionnelle ainsi que dans les entrepôts institutionnels -obligation de citer la source

\subsubsection{2-Par nom d'éditeurs :}

Lors de la recherche, nous avons cherché " Elsevier " et le résultat fut :

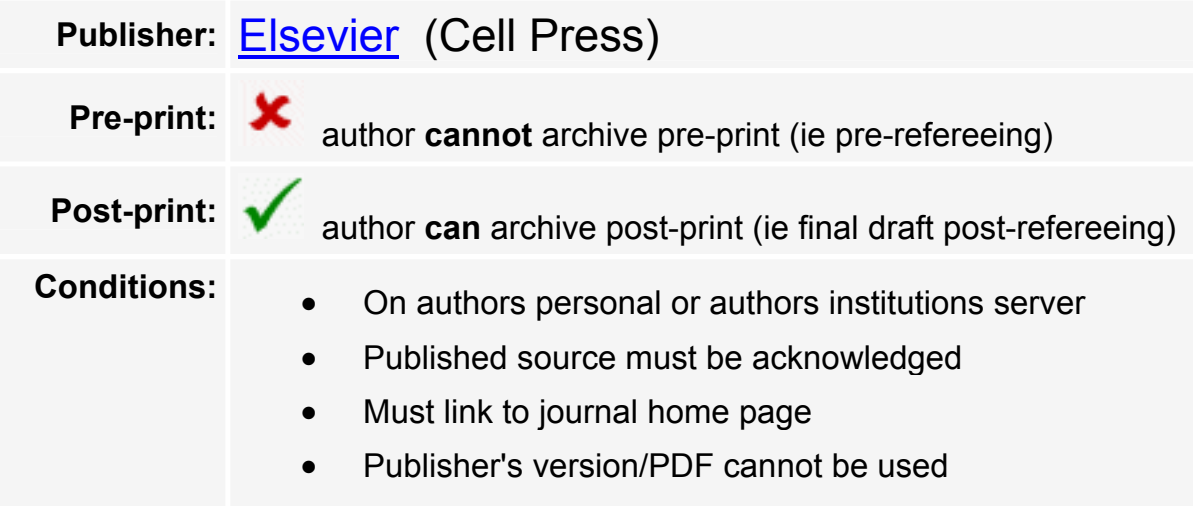

Mandated OA:

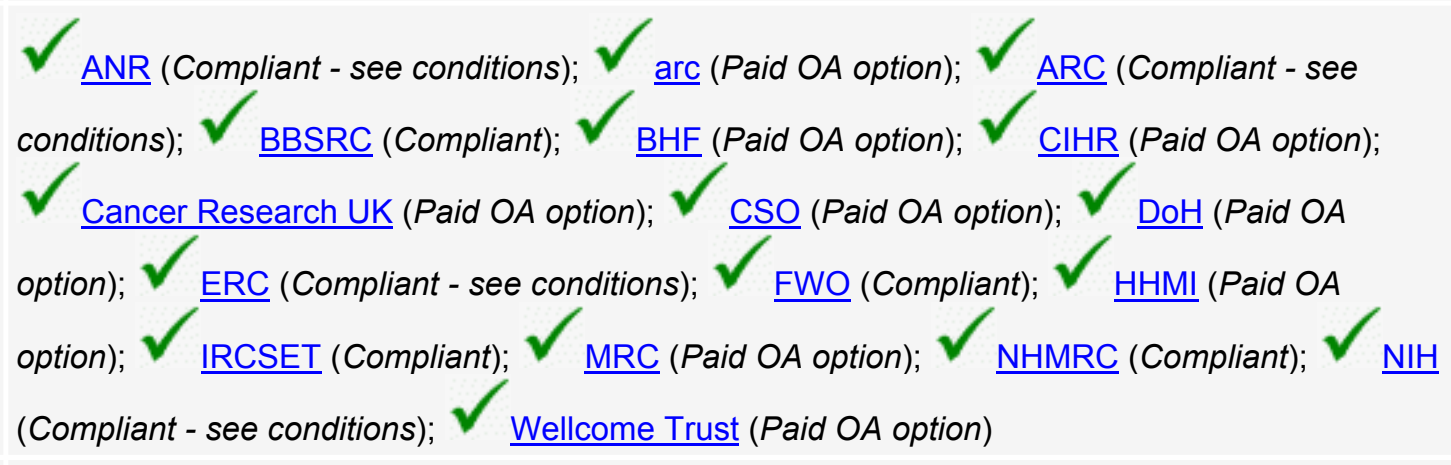

Paid access: Sponsorship Option

Copyright: view policy

RoMEO: This is a ROMEO blue publisher

Updated: $07-J a n-2009$. Suggest an update for this record

Fig.27- Exemple de transfert des droits d'auteurs ( copyright transfer agreement ) pour Elsevier

On remarque qu'avec Elsevier (Cell Press), l'auteur a les droits suivants :

-archiver la version finale

-archiver sur le serveur personnel ou des institutions auxquelles est membre l'auteur

-signaler la source publiée

-faire un lien à la page principale du journal

Mais 
-interdiction d'archiver la version non contrôlée

-interdiction d'utiliser la version PDF de l'éditeur.

On peut aussi chercher les conditions posées par les éditeurs en feuilletant leur liste de cinq manières différentes :

-Voir la liste alphabétique des éditeurs qui font partie de Romeo

-Voir la liste alphabétique des éditeurs verts (qui permettent l'archivage de la pré publication et la post publication)

-Voir la liste alphabétique des éditeurs bleus (qui permettent l'archivage de la post publication seulement)

-Voir la liste alphabétique des éditeurs jaunes (qui permettent l'archivage de la version non contrôlée seulement)

-Voir la liste alphabétique des éditeurs blancs (qui ne permettent aucune forme d'archivage).

Il est intéressant de noter que les 487 éditeurs qui figuraient sur la liste en date du 29 Janvier 2009 se repartissent de la manière suivante :

- $\quad 151$ étaient verts soit un pourcentage de $31 \%$

- 107 étaient bleus soit un pourcentage de $21.97 \%$

- 60 étaient jaunes soit un pourcentage de $12.32 \%$

- 169 étaient blancs soit un pourcentage de $34.70 \%$

Chiffres que la figure suivante représente de la meilleure manière :

\section{Statistics for the 487 publishers on this list}

\begin{tabular}{|c|c|c|}
\hline ROMEO colour & \multicolumn{1}{|c}{ Archiving policy } & Publishers \\
\hline$\underline{\text { green }}$ & can archive pre-print and post-print & 151 \\
\hline$\underline{\text { blue }}$ & can archive post-print (ie final draft post-refereeing) & 107 \\
\hline yellow & can archive pre-print (ie pre-refereeing) & 60 \\
\hline white & archiving not formally supported & 169 \\
\hline
\end{tabular}

Summary: $\mathbf{6 5 \%}$ of publishers on this list formally allow some form of self-archiving. 


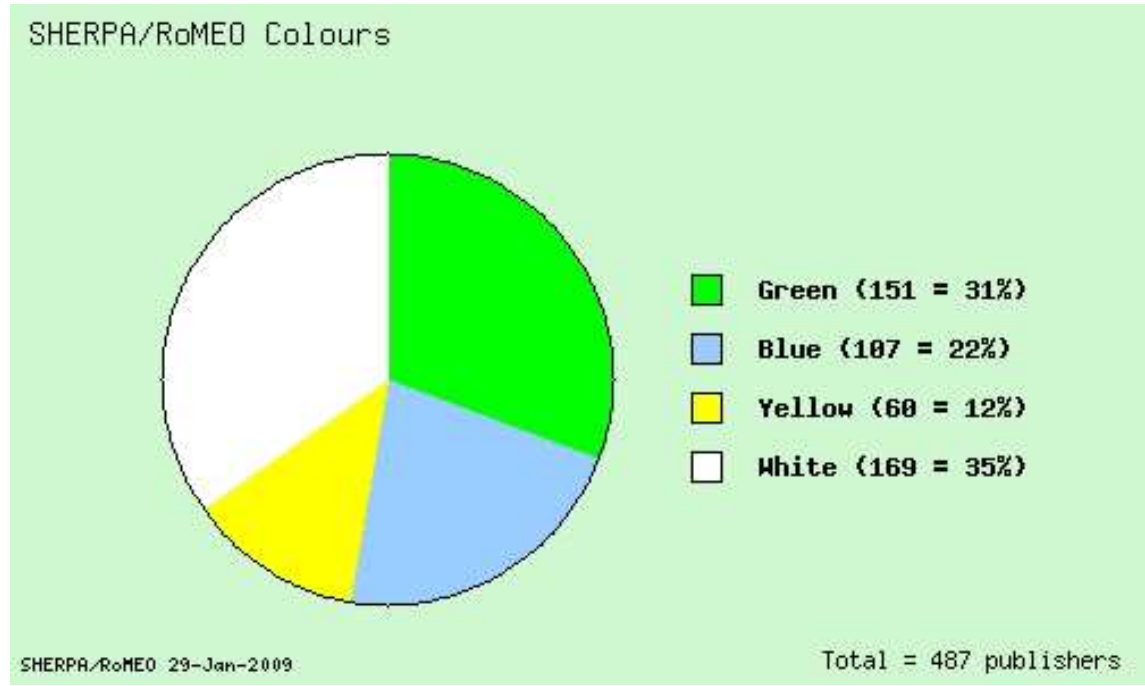

Fig. $n^{\circ} 28$ - Statistiques des autorisations des éditeurs selon les couleurs

Ces chiffres peuvent être interprétés et lus de différentes manières .On peut y voir une avancée inéluctable du mouvement du libre accès à l'Information Scientifique et Technique étant donné que $65.29 \%$ des éditeurs permettent l'auto archivage à différents paliers ou que le mouvement n'a pas encore atteint son rythme de croisière puisque le reste (soit $34.70 \%$ ) ne le permettent pas. Toujours est-il qu'il nous semble que les éditeurs conscients des avancées du libre accès ont dû faire un minimum de concessions afin de ne pas voir leur échapper un commerce très lucratif.

D’un autre coté, le site peut être recherché de différentes manières dont les plus importantes sont :

\subsubsection{3-Ro MEo News et qui liste :}

La dernière mise à jour de RoMEo et qui énumère les nouveaux éditeurs ayant rejoints RoMEo (avec la " couleur " et la date d'inclusion).Les mises à jour et qui concernent principalement le changement de couleur de l'éditeur avec la date ainsi que les changements de nom, d'URL ou des erreurs dans l'écriture ou l'épellation du nom.

\subsubsection{1-Editeurs permettant l'utilisation de leur PDF dans les entrepôts institutionnels :}

Le sort des versions PDF de l'éditeur est très souvent sujet à controverse et leur statut n'est pas clairement indiqué, la plupart des auteurs croyant que ceci est strictement prohibé. En fait, la réalité est différente et a besoin d'éclaircissement. A cet effet et en date du 28 Aout 2008, un nombre d'éditeurs a permis l'utilisation sans restriction de la version PDF de leurs articles dans les entrepôts institutionnels de l'auteur. En plus de ces éditeurs au nombre de 56 (en date du 29 Janvier 2009), un nombre d'autres éditeurs permettaient l'utilisation mais la faisait assortir soit d'un embargo ou un payement ou les deux à la fois. Ils se décomposent de la manière suivante :

- Publication après une période d'embargo :

* 6 mois : 5 titres

* 12 mois : 7 titres

* 18 mois : 1 titre

* 24 mois : 4 titres

* 48 mois : 1 titre 
* 60 mois : 1 titre

* Période d'embargo diverses : 1 titre

Publication avec payement et période d'embargo :

* 12 mois : 1 titre

* 24 mois : 1 titre

Comme on peut le voir, les éditeurs sentant la pression des protagonistes du libre accès font des concessions et celles que nous avons cités n'en sont qu'un exemple Il est vrai qu'une période d'embargo de 5 ans peut paraître excessive mais le sujet n'étant pas très sensible (jardinage pour notre cas), les conséquences de ce délai ne peuvent pas être très graves. On peut dire la même chose de la période d'embargo de 4 ans et qui concerne elle aussi une spécialité (sciences marines et aquatiques) dont le facteur de latence n'est pas très important. Contrairement, on remarque que les titres dont la période de latence est la plus basse (entre 6 et 12 mois) sont généralement des titres qui traitent de sujets où le facteur temps est primordial (American Heart Association, Society of Systematic Biology, Society for Endocrinology, etc...). Les facteurs ayant conduit à cette situation sont facilement explicables : une information en médecine est très sensible au facteur temps car elle peut sauver des vies tandis qu'une information en jardinage est moins (pour ne pas dire pas du tout) sujette à l'urgence (de même que pour les sciences sociales et humaines où une théorie, les résultats d'une enquête peuvent peut prendre des années à être vérifié ou compris).

\subsubsection{2-Editeurs avec Option de libre accès payant}

A coté de ces éditeurs qui permettent sous différentes formes et conditions le dépôt des recherches dans un entrepôt institutionnel, certains éditeurs ont innové en permettant le libre accès sans restrictions en contrepartie du payement d'une somme par l'auteur (ou sa tutelle). Nous avons déjà eu l'occasion de parler de cette option et - à notre avis - des dérives qu'elle peut engendrer ainsi que l'obstacle financier que cela représente en général mais surtout pour le chercheur d'un pays en voie de développement. En plus de cet obstacle financier, cette manière d'agir peut aussi ouvrir la porte à d'autres dérives car la liberté que permet le medium est propice à ce genre de dépassement. En effet, rien n'empêcherait un chercheur de cibler un périodique dont les critères de publication ne sont pas très exigeants acceptant cette manière de publier pour les besoins d'une promotion, d'un projet de recherche ou tout autre raison. D'ailleurs, comme nous allons le voir plus tôt dans ce travail en plus des sommes extrêmement prohibitives de certains périodiques, une sorte de rejet des travaux de recherche des chercheurs des pays en voie de développement est observé (état de fait stigmatisé par Farid Dahdouh -Guebas et Benjamin Acosta Cazeres cités plus haut).

Toujours est-il que les éditeurs recensés étaient en date du 24 Février 2009, 59 éditeurs qui offraient l'option du payement qui permettait la publication immédiate des recherches et qui sont représentés dans le tableau suivant : 


\section{ROMEO}

Publishers' paid open access options, allow authors to deposit their articles immediately in open access repositories upon payment of a fee. The same publishers may also allow authors to deposit after an embargo period without payment of a fee.

Where a publishers' standard policy does not allow an author to comply with their funding agency's mandate (see JULIET), paid open access options may enable an author to comply.

\begin{tabular}{|c|c|c|c|c|c|}
\hline Publisher & Paid Option Name & \multicolumn{3}{|c|}{ Price per Article } & Remarks \\
\hline Akademiai Kiado & $\underline{\text { Optional Open Article }}$ & $\$ 562-\$ 1125$ & $(£ 302-£ 604)$ & $€ 450$ - €900 & Fee depends on Institution \\
\hline AlphaMed Pres & [Unnamed] & $\$ 2000$ & (£1075) & - & - \\
\hline $\begin{array}{l}\text { American Association of Pharmaceutical } \\
\text { Scientists }\end{array}$ & Open Choice & $\$ 3000$ & $(£ 1612)$ & - & Part of Springer Group \\
\hline American Chemical Society & ACS Author Choice & $\$ 3000$ & $(£ 1612)$ & - & $\begin{array}{l}\text { Discounts due to } \\
\text { membership }\end{array}$ \\
\hline American Dairy Science Association & $\begin{array}{l}\text { Paid Open Access } \\
\text { option }\end{array}$ & $\$ 1000$ & $(£ 537)$ & - & - \\
\hline American Geophyscial Union (AGU) & $\begin{array}{l}\text { Author Choice for Open } \\
\underline{\text { Access }}\end{array}$ & \multicolumn{3}{|c|}{ fee based on article length and number of figures } & - \\
\hline American Institute of Physics & Author Select & $\$ 1500-\$ 2500$ & $(£ 806-£ 1343)$ & - & Fee depends on journal \\
\hline American Physical Society & Free to Read & $\$ 975-\$ 1300$ & $(£ 524-£ 698)$ & - & Fee depends on journal \\
\hline American Physiological Society & Author Choice & $\$ 2000-\$ 3000$ & $(£ 1075-£ 1612)$ & - & $\begin{array}{l}\text { Fee varies if review or } \\
\text { research article }\end{array}$ \\
\hline American Psychological Association & [Unnamed] & $\$ 2500-\$ 4000$ & $(£ 1343-£ 2149)$ & - & - \\
\hline $\begin{array}{l}\text { American Society for Biochemistry and } \\
\text { Molecular Biology }\end{array}$ & Author Choice & $\$ 1500-\$ 2000$ & $(£ 806-£ 1075)$ & - & Fee depends on membership \\
\hline American Society for Nutrition & Open Access & $\$ 3000$ & $(£ 1612)$ & - & - \\
\hline
\end{tabular}




\begin{tabular}{|c|c|c|c|c|c|}
\hline & Publication Option & & & & \\
\hline American Society of Hematology & [Unnamed] & $\$ 2500$ & (£1343) & - & - \\
\hline American Society of Neuroradiology & [Unnamed] & $\$ 500$ & $(£ 269)$ & - & - \\
\hline $\begin{array}{l}\text { American Society of Tropical Medicine and } \\
\text { Hygiene }\end{array}$ & [Unnamed] & $\$ 2500$ & $(£ 1343)$ & - & Benefits unclear \\
\hline Arnold Publishers & Sage Open & $\$ 3000$ & (£1612) & - & Part of SAGE \\
\hline $\begin{array}{l}\text { Association of Learned and Professional } \\
\text { Society Publishers (ALPSP) }\end{array}$ & ALPSP Author Choice & $\$ 2500-\$ 3000$ & $£ 1250-£ 1500$ & - & Fee depends on membership \\
\hline Blackwell & Online Open & $\$ 3000$ & $(£ 1612)$ & - & - \\
\hline British Medical Journal Publishing & BMJ Unlocked & $\$ 2220-\$ 3145$ & $£ 1200-£ 1700$ & - & Fee depends on journal \\
\hline Cambridge University Press & $\begin{array}{l}\text { Cambridge Open } \\
\text { Option }\end{array}$ & $\$ 2700$ & $£ 1500$ & - & - \\
\hline Company of Biologists & [Unnamed] & & No info on cost & & - \\
\hline CRC Press & iOpenAccess & $\$ 3250$ & $(£ 1746)$ & - & $\begin{array}{l}\text { Part of Taylor \& Francis } \\
\text { Group }\end{array}$ \\
\hline Elsevier & Sponsorship Option & $\$ 3000$ for Lancet & $\begin{array}{l}£ 400 \text { per page for } \\
\text { Lancet }\end{array}$ & - & - \\
\hline Elsevier (Cell Press) & Sponsorship Option & $\$ 5000$ & $(£ 2686)$ & - & - \\
\hline Elsevier Masson & Sponsorship Option & $\$ 3000$ & (£1612) & - & - \\
\hline FASEB & Open Access Option & $\$ 2500$ & $(£ 1343)$ & - & - \\
\hline HFSP Publishing & [Unnamed] & $\$ 2000$ & $(£ 1075)$ & - & - \\
\hline Inderscience & Author Open Access & $\$ 3000$ & $(£ 1612)$ & $€ 2300$ & - \\
\hline Informa Healthcare & iOpenAccess & $\$ 3250$ & $(£ 1746)$ & - & $\begin{array}{l}\text { Part of Taylor \& Francis } \\
\text { Group }\end{array}$ \\
\hline Inter Research & [Unnamed] & & No info on cost & & - \\
\hline International Union of Crystallography & [Unnamed] & $\$ 150-\$ 1000$ & $(£ 81-£ 537)$ & - & Fee depends on journal \\
\hline John Wiley \& Sons & Open Choice & $\$ 3000$ & $(£ 1612)$ & - & - \\
\hline Journal of Rheumatology & $\begin{array}{l}\text { Full Release Publication } \\
\text { Option }\end{array}$ & $\$ 3500$ & $(£ 1880)$ & - & - \\
\hline
\end{tabular}




\begin{tabular}{|c|c|c|c|c|c|}
\hline Karger & Authors Choice & $(\$ 2509)$ & $(£ 1348)$ & CHF2750 & - \\
\hline Kluwer & Open Choise & $\$ 3000$ & $(£ 1612)$ & - & Part of Springer Group \\
\hline Landes Bioscience & Open Access Policy & $\$ 500-\$ 750$ & $(£ 269-£ 403)$ & - & $\begin{array}{l}\text { Fee depends on Institutional } \\
\text { subscription }\end{array}$ \\
\hline Longwoods Publishing & Longwoods Open & $\$ 2500$ & $(£ 1343)$ & - & - \\
\hline Magnolia Press & [Unnamed] & $\$ 20$ per page & (£11 per page) & - & - \\
\hline Marcel Dekker & $\underline{\text { iOpenAccess }}$ & $\$ 3250$ & $(£ 1746)$ & - & $\begin{array}{l}\text { Part of Taylor \& Francis } \\
\text { Group }\end{array}$ \\
\hline Mary Ann Liebert & Liebert Open Option & $\$ 3000$ & (£1612) & - & $\begin{array}{l}\text { For deposit on PMC. Fee } \\
\text { reduces on subsequent } \\
\text { papers }\end{array}$ \\
\hline Mineralogical Society of America & $\underline{\text { Self-archiving fee }}$ & $\$ 250$ per page & (£134 per page) & - & - \\
\hline National Academy of Sciences & $\begin{array}{l}\text { PNAS Open Access } \\
\text { Option }\end{array}$ & $\$ 850-\$ 1200$ & $(£ 457-£ 645)$ & - & $\begin{array}{l}\text { Fee depends on Institutional } \\
\text { membership }\end{array}$ \\
\hline Nature & $\begin{array}{l}\text { Author Pays Hybrid } \\
\text { Model }\end{array}$ & $\$ 3000$ & $£ 2000$ & $€ 2400$ & - \\
\hline Nature (British Journal of Pharmacology) & BJP Open fee & $\$ 2500$ & $£ 1250$ & $€ 1800$ & - \\
\hline Nature (EMBO) & EMBO Open Charges & $\$ 2540$ & $£ 1350$ & $€ 2000$ & - \\
\hline Oxford University Press & Oxford Open & $\$ 1500-\$ 2800$ & $£ 800-£ 1500$ & - & $\begin{array}{l}\text { Fee depends on whether } \\
\text { institution subscribes (lower } \\
\text { fee if they do) }\end{array}$ \\
\hline Portland Press & Opt2Pay & $\$ 3000-\$ 3500$ & $£ 1500-£ 1750$ & $€ 220-€ 2590$ & - \\
\hline Professional Engineering Publishing (IME) & $\begin{array}{l}\text { Engineering Open } \\
\text { Choice }\end{array}$ & $(\$ 1864)$ & $£ 1700$ & - & - \\
\hline Psychology Press & $\underline{\text { iOpenAccess }}$ & $\$ 3250$ & $(£ 1746)$ & - & $\begin{array}{l}\text { Part of Taylor \& Francis } \\
\text { Group }\end{array}$ \\
\hline Radiation Research Society & [Unnamed] & Page charges & Page charges & - & Must use Publishers' PDF \\
\hline Routledge & $\underline{\text { iOpenAccess }}$ & $\$ 3250$ & $(£ 1746)$ & - & $\begin{array}{l}\text { Part of Taylor \& Francis } \\
\text { Group }\end{array}$ \\
\hline Royal College of Psychiatrists & [Unnamed] & $\$ 4500$ & $£ 2500$ & - & - \\
\hline
\end{tabular}




\begin{tabular}{|c|c|c|c|c|c|}
\hline Royal Society & $\begin{array}{l}\text { EXIS Open } \\
\text { Choice }\end{array}$ & $\begin{array}{l}\text { \$278-416 per page for first 6-10 pages, } \\
\text { \$93 for subsequent pages }\end{array}$ & $\begin{array}{l}£ 150-225 \text { per page for first } 6-10 \text { pages, } \\
£ 50 \text { for subsequent pages }\end{array}$ & - & Fee depends on journal \\
\hline Royal Society of Chemistry & $\begin{array}{l}\text { RSC Open } \\
\underline{\text { Science }}\end{array}$ & $(\$ 1096-\$ 2741)$ & $£ 1000-£ 2500$ & - & $\begin{array}{l}15 \% \text { discount to members; } \\
\text { depends on article type }\end{array}$ \\
\hline Sage & $\underline{\text { Sage Open }}$ & $\$ 3000$ & $£ 1600$ & - & - \\
\hline Society for Endocrinology & $\underline{\text { Free Access Fee }}$ & $(\$ 2192)$ & $£ 2000$ & - & - \\
\hline Society for General Microbiology & Open Option & $(\$ 1644)$ & $£ 1500$ & - & - \\
\hline $\begin{array}{l}\text { Society for Reproduction and } \\
\text { Fertility }\end{array}$ & Free Access Fee & $(\$ 2192)$ & $£ 2000$ & - & - \\
\hline Society of Systematic Biology & $\underline{\text { iOpenAccess }}$ & $\$ 3250$ & $(£ 1746)$ & - & Part of Taylor \& Francis Group \\
\hline $\begin{array}{l}\text { Society of the European Journal } \\
\text { of Endocrinology }\end{array}$ & Free Access Fee & $(\$ 2192)$ & $£ 2000$ & - & - \\
\hline $\begin{array}{l}\text { Society of Vertebrate } \\
\text { Paleontology }\end{array}$ & [Unnamed] & \multicolumn{3}{|c|}{ No info on cost } & - \\
\hline Springer & Open Choice & $\$ 3000$ & $(£ 1612)$ & - & - \\
\hline Taylor and Francis & $\underline{\text { iOpenAccess }}$ & $\$ 3250$ & $(£ 1746)$ & - & - \\
\hline Walter de Gruyter & $\frac{\text { WdG Open }}{\underline{\text { Access }}}$ & $(\$ 1475-\$ 2950)$ & $(£ 792-£ 1585)$ & $\begin{array}{l}€ 1000- \\
€ 2000\end{array}$ & - \\
\hline
\end{tabular}

Last Updated: 20/02/2009. The prices in brackets are estimates that have been converted from the publishers' preferred currencies using rates on: 20/08/2008 .

(c) University of Nottingham, 2008-2009 Contact us

Fig.n²9- Editeurs avec Option de libre accès payant 
La liste comprend les éléments suivants :

- le nom de l'éditeur classé alphabétiquement

- le nom de l'option telle qu'elle est choisie par l'éditeur. Bien qu'un nombre d'editeurs n'attribuent pas de nom spécifique (unnamed) à leur option, ceux qui en attribuent utilisent des noms qui essayent et de les démarquer et d'attirer l'attention. Ainsi, on trouve " Free to read " de l'American Physical Society, " Open Choice " de John Wiley and Sons ou encore " Opt 2 Pay " de Portland Press .

- Les sommes demandées (en Dollars américains ou en Livres sterlings). On remarque :

- l'extrême différence entre des prix qui vont de 150 \$ pour " The International Union of Christallography" à $5000 \$$ pour Elsevier ( Cell Press) ou de $81 £$ pour " The International Union of Christallography " à $2500 £$ " The Royal College of Psychiatrists " et " The Royal Society of Chemistry"

- l'option offerte de payer par page et qui, comme nous allons le voir brièvement, comporte des différences de prix pour le moins aberrantes. A titre d'exemple :

*American Geophysical Union qui précise que le prix est fonction de la longueur de l'article et du nombre de figures.

* Elsevier qui demande soit 3000 \$ pour publier dans le prestigieux Lancet ou encore $400 £$ par page pour publier dans le même périodique médical.

*Radiation Research Society qui stipule que le prix est fonction du nombre de pages.

*Royal Society dont l'offre est entre 278 et $416 \$$ par page (ou 150 à $225 £$ par page) pour les 610 premières pages ensuite un tarif dégressif est appliqué pour les pages subséquentes et qui est de $93 \$$ ou $50 £$

Comme on peut le voir, cette option de payer pour publier est entrée dans les mœurs des chercheurs à l'heure d'Internet. Les éditeurs y voient une manière de répondre aux demandes pressantes des chercheurs et ces mêmes chercheurs y voient une manière rapide de voir le fruit de leur travail publié. Il semblerait que cette option soit - du moins pour le moment - l'option qui réponde le plus aux nouvelles donnes de la publication scientifique. A cet effet, des institutions universitaires ont innové en offrant de payer les sommes demandées à leur chercheurs .Parmi ces exemples, 1'Université de Berkeley à travers son programme " Berkeley Research Impact Initiative "(200) se propose de sponsoriser les chercheurs de l'Université et ceci afin de maximiser le rayonnement de la recherche émanant de l'institution. Cette initiative co - sponsorisée par le Vice Recteur chargé de la recherche et le Bibliothécaire de 1'Université et qui date du 21 Janvier 2008 est appelée à être dupliquée ailleurs car, il nous semble que c'est vers cette direction que s'oriente le futur de la publication scientifique. Ceci est du au fait que l'expérience (du libre accès) étant relativement récente, il est impératif qu'un juste milieu soit trouvé afin que ne soient pas lésés les deux parties en présence, quoiqu'il nous paraîtrait que les éditeurs aient su manœuvrer de telle sorte à ce que les avancées du libre accès soient le plus diluées afin de ne pas toucher à des avantages pécuniaires très importants.

\section{2-SHERPA JULIET}

D'un autre coté, le site de Sherpa offre aussi à coté de RoMEO, une autre rubrique appelée JULIET et qui elle se propose de présenter un résumé des autorisations données par les organisations qui financent la recherche vis-à-vis de la publication en libre accès soit dans des entrepôts d'archives en libre accès ou alors dans les revues électroniques en libre accès. Le tableau se présente de la manière suivante :

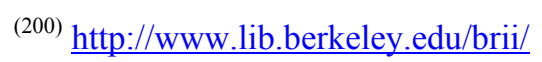




\section{SHERPA JULIET}

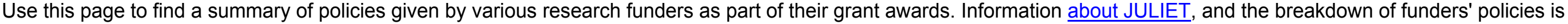
given in the key below the table.

\begin{tabular}{|c|c|c|c|c|}
\hline \multicolumn{5}{|c|}{ Click on the relevant column heading to re-sort by Country } \\
\hline \multirow{2}{*}{ Funding Organisation (linked to their JULIET summary) } & \multicolumn{2}{|c|}{ Publications Policy } & \multirow{2}{*}{$\begin{array}{c}\text { Data Archiving } \\
\text { Policy }\end{array}$} & \multirow{2}{*}{$\underline{\text { Country }}$} \\
\hline & $\begin{array}{c}\text { Open Access } \\
\text { Archiving }\end{array}$ & $\begin{array}{c}\text { Open Access } \\
\text { Publishing }\end{array}$ & & \\
\hline Agence Nationale de la Recherche (ANR) & 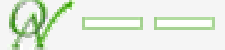 & & & France \\
\hline Arthritis Research Campaign (arc) & $\longrightarrow$ & $\Longrightarrow$ & & United Kingdom \\
\hline Arts and Humanities Research Council (AHRC) & 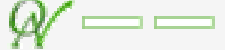 & & & United Kingdom \\
\hline Australian Research Council (ARC) & $\square \square$ & & $\square \square$ & Australia \\
\hline$\underline{\text { Autism Speaks }}$ & $\longrightarrow$ & & & United States \\
\hline Biotechnology and Biological Sciences Research Council (BBSRC) & 尸凹 & & & United Kingdom \\
\hline British Heart Foundation (BHF) & ב & $\Longrightarrow$ & & United Kingdom \\
\hline Canadian Breast Cancer Research Alliance (CBCRA) & $\square \square \square$ & $\longrightarrow$ & & Canada \\
\hline Canadian Institutes of Health Research (CIHR) & & A & $A / \square$ & Canada \\
\hline$\underline{\text { Cancer Research UK }}$ & 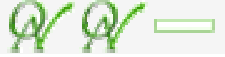 & $\square$ & & United Kingdom \\
\hline 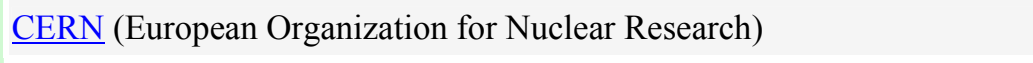 & $\longrightarrow$ & & $\square \square$ & [International] \\
\hline Chief Scientist Office, Scottish Executive (CSO) & $\square$ & & $\square \square$ & United Kingdom \\
\hline Department of Health (DoH) & 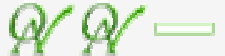 & & & United Kingdom \\
\hline
\end{tabular}


Deutsche Forschungsgemeinschaft (DFG)

Economic and Social Research Council (ESRC)

Engineering and Physical Sciences Research Council (EPSRC)

European Commision

European Research Council (ERC)

Fonds Wetenschappelijk Onderzoek (Vlaanderen) (FWO)

Fonds zur Förderung der wissenschaftlichen Forschung (FWF)

Fraunhofer-Gessellschaft

Genome Canada

Gordon and Betty Moore Foundation (GBMF)

Health Research Board (Ireland) (HRB)

Higher Education Authority (HEA)

Howard Hughes Medical Institute (HHMI)

Inserm (Institut national de la santé et de la recherche médicale)

Instituto Superiore di Sanita (ISS)

IRCSET (Irish Research Council for Science, Engineering and Technology)

JISC (Joint Information Systems Committee)

MacArthur Foundation (John D. and Catherine T. MacArthur Foundation)

Medical Research Council (MRC)

National Cancer Institute of Canada (NCIC)

National Health and Medical Research Council (NHMRC)

National Institutes of Health (NIH)

National Research Council Canada (NRC)

Natural Environment Research Council (NERC)

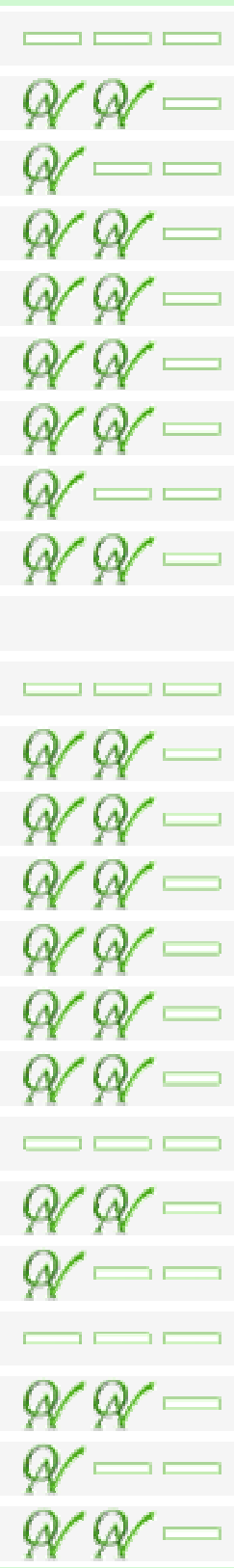

Germany

United Kingdom

United Kingdom

[International]

A $A$

[International]

Belgium

Austria

Germany

Canada

Q

Q

United States

Ireland

Q

Ireland

United States

France

Italy

Ireland

United Kingdom

United States

United Kingdom

Canada

Australia

United States

Canada

United Kingdom 
Ontario Institute for Cancer Research (OICR)

\section{$\underline{\text { Principado de Asturias }}$}

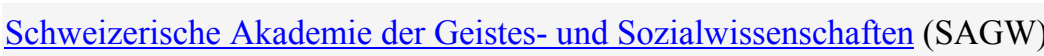

Schweizerischer Nationalfonds zur Förderung der Wissenschaftlichen Forschung (SNF)

Science and Technology Facilities Council (STFC)

Science Foundation Ireland (SFI)

$\underline{\text { Stroke Association }}$

$\underline{\text { Wellcome Trust }}$

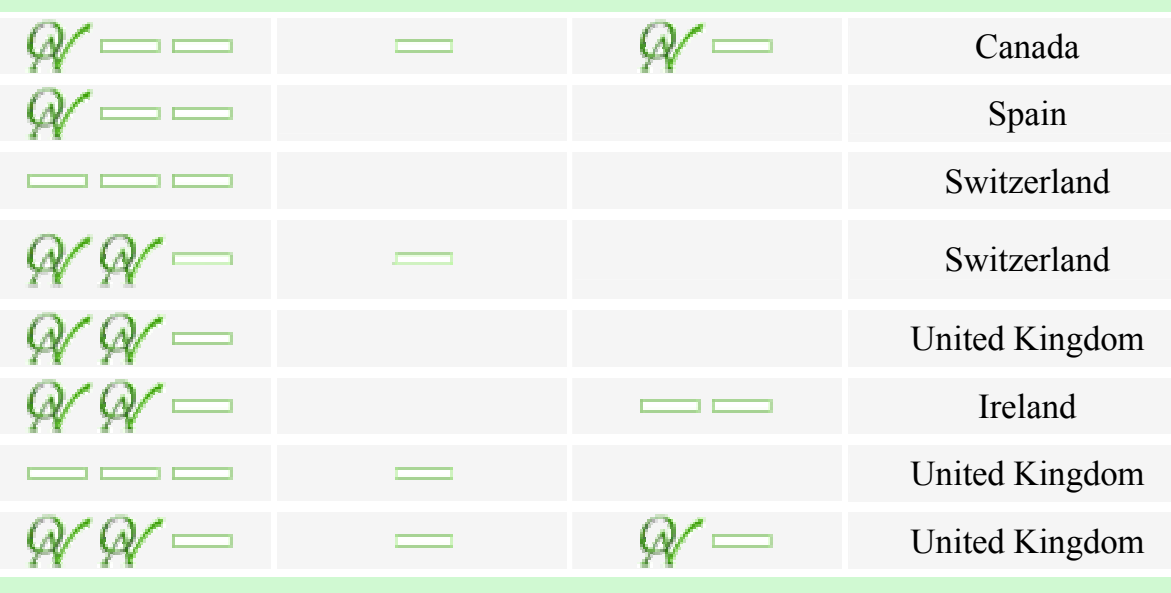

This information is accurate to the best of our knowledge, but should not be relied upon for legal advice.

JULIET and Support for Open Access to Research $A$

\section{Publications Policies}

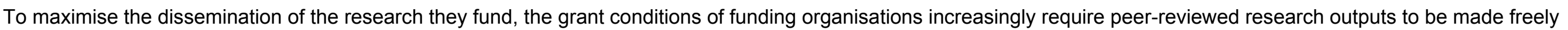

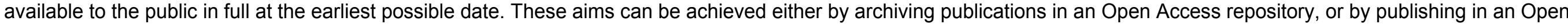

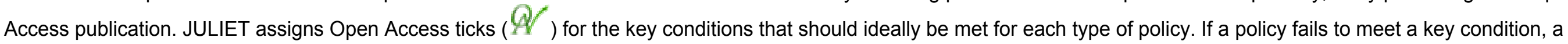
dash $(\square)$ is shown instead

\section{Self-Archiving}

The ideal publications archiving policy has three key parts:

$\begin{array}{lll}\text { Whether to archive: } & \text { Deposit required } \\ \text { What to archive: } & \text { The full final version } \\ \text { When to archive: } & \text { When accepted for publication }\end{array}$

to be made available free of charge without any access restrictions the published version or the author's final peer-reviewed version although toleration of publishers' embargos negates this 


\section{Open Access Publishing}

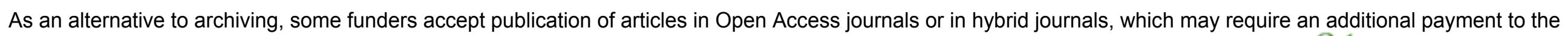

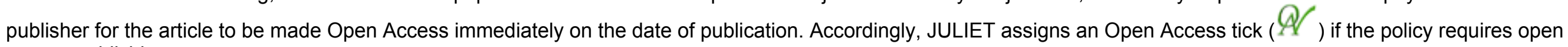
access publishing.

\section{Data Archiving Policies}

Funding organisations are also increasingly requiring grantees to deposit their raw research data in appropriate public archives or stores, in order to facilitate the validation of results and further work by other researchers. The practicalities of providing access to large data-sets can be costly for data archives. Therefore, an Open Access tick may still be given if the archive recovers the costs of access provision on a not-for-profit basis.

Because it can take some time to write up results after a work has been completed, policies may allow a reasonable period of grace during which the original researchers have exclusive access to the data before it has to be deposited in a public archive. The lengths of such periods vary by subject discipline. For instance six months might be suitable for a chemistry project, but an archaeological dig could require several years. However, there clearly needs to be an outer limit.

JULIET assigns up to two Open Access ticks for data archiving policies:

Q Data archiving is required.

Q Data must be deposited within five years.

\section{Statistical Analysis}

For an indication of how UK research funders have implemented Open Access policies and level of funds affected, please see: Selected research funders' grant expenditure.

\section{Updates and Feedback}

The information in JULIET is updated by community contributions. If a funding organisation with an open access or data archiving policy is not listed here, please tell us about it. Please submit any updates or feedback to the JULIET Administrators.

JULIET complements the ROMEO service provided by SHERPA for authors and repository administrators, which lists summaries of publishers' copyright transfer agreements as they relate to archiving. Further information on Open Access is available for authors, including links to contacts and repositories which may be able to take eprints to fulfil funders' requirements and recommendations. Further information on repositories is available from OpenDOAR.

Further information about RoMEO \& JULIET [PDF files] - Leaflet | Large Poster (A1) | Small Poster (A3)

\section{curl}

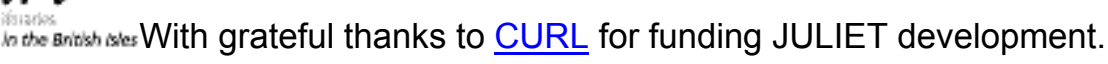

(C) 2006-2009, University of Nottingham Contact u

Fig.n ${ }^{\circ}$ 30- Autorisations données par les organisations finançant la recherche pour la publication en libre accès (SHERPA JULIET) 
Comme on peut le voir le nombre d'agences qui financent la recherche est de 45 agences classées alphabétiquement et ce en date du 24 Février 2009. Elles se subdivisent selon les pays de la manière suivante :

Royaume uni : 15

Etats-Unis : 05

France : 02

Confédération helvétique : 02

Australie : 02

Irlande : 04

Canada : 06

Italie : 01

Autriche : 01

Belgique : 01

Allemagne : 02

Espagne : 01

Enfin 03 sont classées sous " International " et qui sont des organisations continentales telles que le CERN, la Commission européenne et le Conseil de recherche européenne. Cette liste ne fait que confirmer la prépondérance et l'avance prise par les pays anglo-saxons (nommément le Royaume Uni les Etats-Unis qui totalisent à eux seuls $44.44 \%$ des organismes finançant la recherche. On peut aussi ajouter le Canada qui fait aussi d'une certaine manière partie du monde anglo-saxon et qui ajouté aux deux autres pays totalisent à eux trois 57.77\%) dans le mouvement du libre accès. Cette avance qui s'est vérifiée dans les autres composantes (entrepôts d'archives et périodiques électroniques en libre accès, etc...) est corroborée en quelque sorte par cette donne (les organismes de recherches financeurs de la recherche) et on peut dire que ceci est en même cause et effet. Pour être plus claire, on peut dire que le fait que les organismes de financement soient majoritairement anglo - saxons a automatiquement encouragé la création et le lancement d'entrepôts, de périodiques en libre accès dans la sphère anglo-saxonne et ceci a en contrepartie encouragé ces mêmes organismes à financer de plus en plus la recherche et à la sponsoriser.

D’un autre coté, le tableau précité comporte les éléments suivants :

-Le nom de l'organisme financeur

-La politique de publication et qui comprend :

* La politique d'archivage en libre accès

* La politique de publication en libre accès

-La politique d'archivage des données 
Ces trois rubriques résument en fait le but de JULIET. En effet " optimiser la dissémination de la recherche qu'ils financent, les conditions d'attribution de financement des organisations requièrent de plus en plus que les résultats de la recherche contrôlée par les pairs soit accessibles librement au public dans les plus brefs délais .Ces buts peuvent être atteints soit par l'archivage des publications dans des entrepôts en libre accès ou en publiant dans un périodique en libre accès JULIET assigne des coches d'accès libre pour les conditions principales qui devraient être idéalement atteint pour chaque type de publication. Dans le cas une politique ne répond pas à une des conditions importantes, un tiret est mis" ${ }^{(201)}$.De cette manière est définie la politique de publication de JULIET. D'un autre coté, le site donne aussi des explications sur :

\section{L’Auto-archivage :}

Défini comme étant la politique d'archivage idéale des publications et qui se compose de trois importantes parties :

- Archiver ou pas ? - dépôt requis - doit être disponible gratuitement sans aucune restriction d'accès.

- Archiver quoi ? -la version finale complète- la version publiée ou la version finale contrôlée de l'auteur.

- Quand archiver? - à l'acceptation pour publication - quoique l'embargo de l'éditeur ne le permet pas.

\section{$\underline{\text { Publication dans des journaux en libre accès }}$}

Une autre option examinée par JULIET est la publication dans des journaux en libre accès ou des journaux hybrides .Cette dernière option peut être sujette à un payement additionnel pour permettre le libre accès immédiat et sans restrictions .De ce fait, JULIET assigne une coche accès libre si la politique de l'institution qui finance la recherche requiert la publication en libre accès.

$\underline{\text { Politique d'archivage des données. }}$

Les organisations qui financent la recherche requièrent aussi de plus en plus les chercheurs de déposer les données de recherche brutes dans des archives publiques adéquates ou des dépôts. Ceci a pour but, de faciliter la validation des résultats de la recherche et son utilisation par d'autres chercheurs, et étant donné que les résultats de la recherche pour être finalisés et présentables prennent du temps, la politique d'archivage de l'organisation qui finance la recherche peut permettre une période de grâce durant laquelle les chercheurs ont un accès exclusif aux données avant qu'elles ne soient rendues publiques. A cet effet, JULIET donne deux coches libre accès lorsque :

-l'archivage des données est requis

- les données doivent être déposées au bout de cinq ans

On peut dire que les deux rubriques de SHERPA, RoMÉO et JULIET constituent une mine d'informations pour le chercheur et les organismes qui financent la recherche .Si le premier guide le chercheur dans les dédales de la publication électronique tout en lui donnant et présentant ses droits et obligations dans un medium somme toute nouveau et auquel la plupart des chercheurs ne sont pas habitués ( et dont ils sont même ignorants dans certains cas ), le deuxième recense les positions des organismes financeurs de la recherche et leurs positions vis-à-vis de la dissémination des résultats de la recherche qu'ils sponsorisent.Ils peuvent être considérés comme des outils incontournables de

${ }^{(201)}$ http://www.sherpa .ac.uk/juliet/i,dex.php\#key 
la nouvelle maniere de conduire la Science et la publication scientifique .Pour preuve de cette importance sans cesse accrue, les chiffres et statistiques en constant changement temoignant des évolutions de la scène du libre accès.

\section{Chapitre V \\ De quelques aspects du libre accés en Algérie}

\section{Introduction}

Il est vrai que les prémices et premiers frémissements du libre accès ont eus lieu en Occident et plus particulièrement dans les pays anglo saxons (Etats Unis et Royaume Uni).Entre les premières expériences aux Etats Unis et l'état de la recherche scientifique dans le monde existe un grand bond en avant et dont, on ne cessera de le répéter, Internet est le vecteur principal. Il est malheureux de le dire mais les pays en voie de développement, bien que le libre accès leur ouvre la porte de l'accès à l'information, autrefois fermée et payante dans l'univers papier, ne semblent pas tellement avoir conscience des avantages qui en découlent et qui peuvent en être tirés. Ceci s'applique particulièrement à l'Algérie qui, malgré son potentiel humain et matériel, ne semble pas être très impliquée dans le mouvement du libre accès ni par sa participation dans les archives ouvertes ni dans les périodiques électroniques. De plus, nous avons eu, à plusieurs fois, l'occasion de nous apercevoir que nos institutions, au delà des affirmations d'intentions et du discours officiel, ne profitent pas (au sens noble du terme) des avantages offerts par le libre accès .Il existe une myriade de programmes, d'organisations, de formations qui permettent de s'insérer dans le concert de cette nouvelle manière d'accéder à l'information. Pour essayer de voir quelle est la position, la situation de l'Algérie et ses avancées dans ce domaine, nous avons consulté certaines sources (majoritairement électroniques) pour dresser, autant que peut se faire, un tableau exhaustif. Nous allons citer ces ressources et voir est ce que l'Algérie ou du moins la communauté des chercheurs algériens est intégrée dans un mouvement, on n'aura cesse de le dire, très ouvert, collaboratif mais aussi, et surtout, très rapide dans ses évolutions et dont les retards éventuels accumulés sont très difficiles à rattraper.

\section{1-Le Portail global du libre accés (Global Open Access Portal-GOAP) de 1’UNESCO ${ }^{(202)}$}

Ce portail de création récente et lancé le $1^{\mathrm{er}}$ Novembre 2011 " ......présente un état des lieux du libre accès à l'information scientifique à travers le monde et propose les ressources suivantes :

- informations par pays sur le statut du libre accès

- principales organisations engagées dans le mouvement du libre accès dans les Etats membres

- grandes thématiques du secteur

- ouvrages de référence publiés dans les différentes régions du monde

- évaluation critique des principales barrières au libre accès dans chaque pays

- potentiel du libre accès dans les Etats membres de l'Unesco

- financement et dépôts en libre accès

- liens vers les initiatives sur le libre accès à travers le monde " (203).

Le site est financé par les gouvernements de la Colombie, le Danemark et la Norvège et aussi le Département d'Etat des Etats Unis d'Amérique. Les pays sont subdivisés, en deux catégories et qui sont :

- les pays qui ont eu plus de succès à mettre en œuvre le libre accès, le portail met en évidence les facteurs cruciaux de succès et les aspects de l'environnement habilitant.

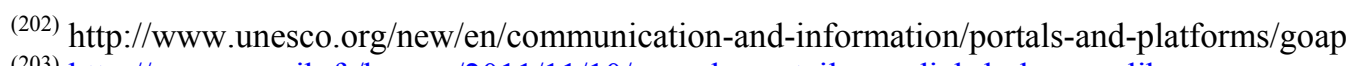

${ }^{(203)} \mathrm{http}$ ://www.enssib.fr/breves/2011/11/10/goap-le-portail-mondial-de-l-acces-libre 
- les pays et les régions qui en sont encore dans les premiers stades du développement du libre accès, le portail identifie les acteurs principaux, les barrières potentielles et les possibilités.

Pour notre cas, l'Algérie est classée dans le deuxième groupe parmi les pays dont le mouvement du libre accès en est encore aux premiers stades. Nous nous proposons voir en détail ce que ce site recense comme réalisations en matière de libre accès.

\subsection{1-Global Open Access Portal -Algeria ${ }^{(204)}$}

" Le mouvement du libre accés est un mouvement récent en Algérie. Cependant, il y a eu un retour d'écoute positif et constant de la part des différentes parties prenantes : bibliothécaires, universitaires et chercheurs. Tous supportent et participent dans le mouvement du libre accés ".C'est en ces termes qu'est présentée la situation du libre accés en Algérie par le site de l'UNESCO. Nous avons essayé de savoir quelle est, effectivement et sur le terrain, la situation du libre accés en Algérie.

\subsubsection{1-Dépôt numérique en libre accès}

\subsection{Dépôt numérique de l’Université d'Alger}

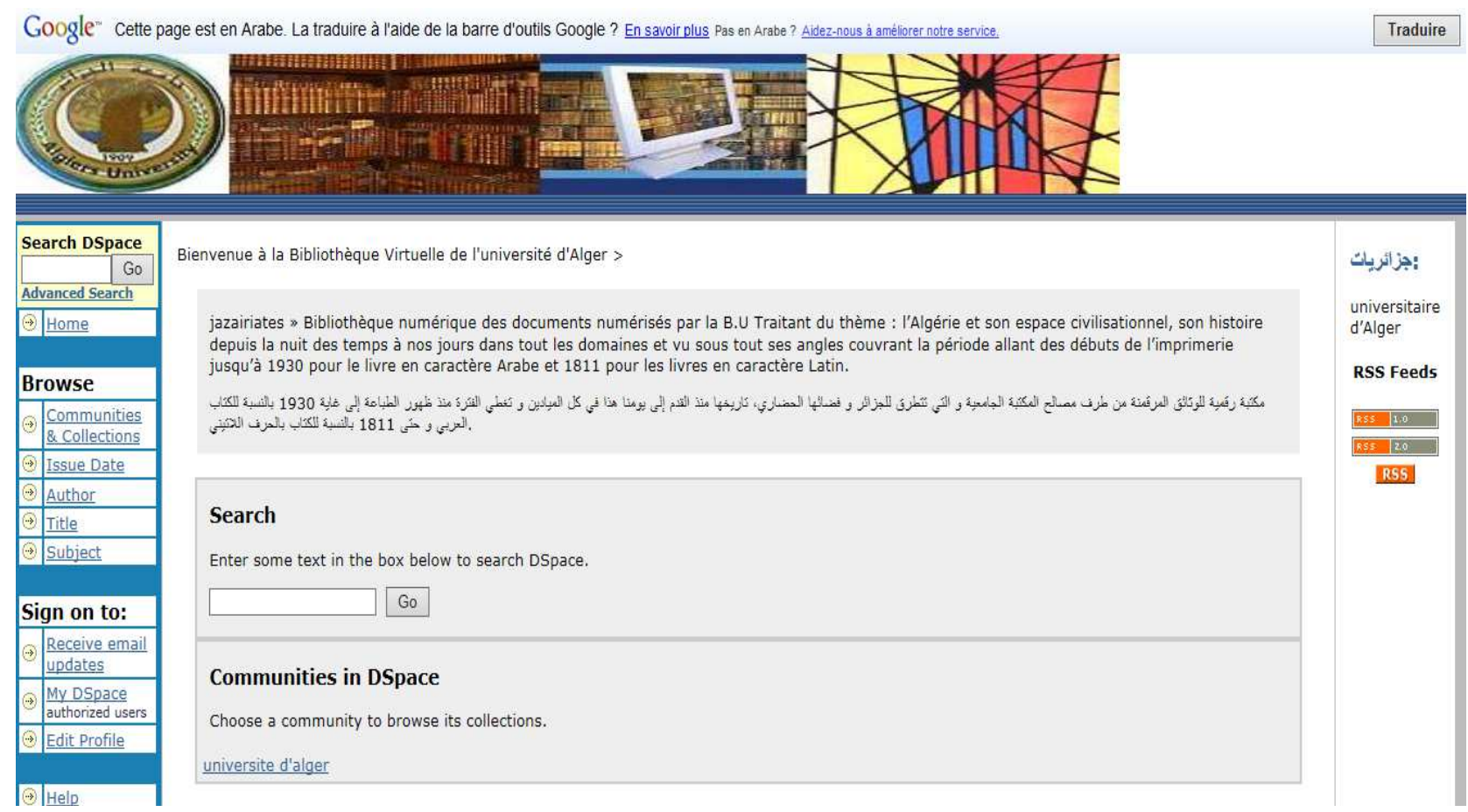

Le seul entrepôt en libre accès est celui de l'Université d'Alger I ${ }^{(205)}$, précédemment Benyoucef Benkhedda. Il semble que le site soit récent et pas assez fourni quant aux dépôts. La page principale comporte deux rubriques: recherche (dans laquelle on peut rechercher par mot clés) et communautés du site (qui comporte les communautés du site).

Il comporte d'autre part plusieurs autres rubriques et qui sont :

-Communities \& Collections (communautés et collections)

-Issue date (date de parution)

${ }^{(204)} \mathrm{http}$ ///www.unesco.org/new/en/communication-and-information/portals-and-platforms/goap/access-by-region/arabstates/algeria /

${ }^{(205)} \mathrm{http://193.194.83.98/jspui} \mathrm{/}$ 
-Author (auteur)

-Title (titre)

-Subject (sujet)

\subsection{1- Communities \& Collections (communautés et collections)}

Quant à la première rubrique " Communities \& Collections", elle comporte une liste de communautés et leur collection. La seule communauté existante est " Université d'Alger " et comme collection une seule intitulée " Livre ". En cliquant sur " Livre ", on obtient un nombre de choix :

-dans (qui affichent " livre", " all of Dspace " et " Université d'Alger ")

-rechercher (avec des mots clés libres)

-feuilleter (avec des mots clés comme sujet, titre, auteur et date de parution)

La même page affiche une colonne " soumissions récentes " dans laquelle un nombre de soumissions sont affichées. Il ne semble pas qu'elles soient récentes vu que nous obtenons " Contribution à l'étude du coma hépatique ", Thèse de doctorat en médecine soutenue en 1954 par Maouche Mohand Amokrane ou encore " Recherches sur plusieurs ouvrages de Léonard de Pise " par M.F. Woepcke et publiées en 1861 ou alors " La Colonisation officielle en Algérie : des essais tentés depuis la conquête et de la situation actuelle " par le Comte d' Haussonville et édité en 1883, ou encore " Contribution à l'étude de la fréquence des formes cliniques et de l'évolution de la tuberculose pulmonaire au cours de la grossesse et du post-partum : Résultats du traitement par les antibiotiques associés ou non à la collapsothérapie " Thèse pour le Doctorat en Médecine présentée et soutenue publiquement le 12 juillet 1955 par Mme Larbaoui nee Belloucif Mimia. D'un autre coté, une des recherches dans le site concerne un travail beaucoup plus récent paru dans Annales de chirurgie plastique esthétique en 2007 sous le titre de " Traitement des séquelles de brûlures du cuir chevelu par expansion cutanée : mise au point $=$ Tissue expansion in surgical treatment of burn scars of the scalp " et dont les auteurs sont tous issus du Service des brûlés et de chirurgie réparatrice, Centre Hospitalier Saint Luc Saint Joseph, Lyon, France. D'autres soumissions récentes, telles qu'elles sont labellisées dans le site, et auxquelles nous n'avons pas pu accéder le site étant très rarement opérationnel et paraissant être en construction et dont certaines numérisations ne semblent pas aussi réussies (fichiers endommagés), se trouvent sur le site et il ne semble pas qu'il y ait une unicité de sujet ni de date dans les soumissions et les recherches mises sur le site. Il semblerait aussi que ce qui est mis en ligne sont surtout des ouvrages numérisés de la bibliothèque plutôt que des recherches émanant des enseignants de l'Université (ce qui en aurait fait un entrepôt institutionnel dans le sens où il est universellement compris).

\subsection{2-Issue date (date de parution)}

La deuxième rubrique " Issue date " (date de parution), quant à elle, recense aussi des documents (au nombre de 73) et dont la date de parution s'échelonne entre le 11 mai 2010 et le 29 novembre 2010.De prime abord nous remarquons que la date de parution telle qu'elle est sur le site ne correspond pas à la date de parution effective du document mais sûrement à celle de mise sur le site car tous les documents sont des documents anciens et même très anciens ( un de ces documents est intitulé "Avantages pour la France de coloniser la Régence d'Alger " par un certain D.J. Montagne et publié en 1830) et ne peuvent avoir paru à cette date.

\subsection{3-Author (auteur)}

La quatrième rubrique est "Author" (auteur), elle comporte 63 entrées et qui sont classées par ordre alphabétique de aa à Zerdani Salah. Les 63 entrées ne sont en fait que les 73 documents signalés plus haut dans les rubriques " Title " et " Issue date". 


\subsection{4-Title (titre)}

La troisième rubrique est " Title " (titre) recense elle aussi des documents (au nombre de 73) et dont la date de parution s'échelonne entre le 11 mai 2010 et le 29 novembre 2010 .Il semble que c'est les mêmes notices mais classées par titre.

\subsection{5-Subject (sujet)}

La cinquième rubrique "Subject" (sujet) quant à elle ne comporte aucune information étant donné qu'elle est toujours en cours de construction et qu'il n'existe pas de vedettes.

On peut conclure que ce site, le premier (à notre connaissance) en Algérie est un premier pas encourageant vers l'intégration de l'Algérie dans le concert du libre accès surtout que ce genre d'entrepôt n'a pas bonne presse et n'est pas très connu. Le fait qu'il soit le seul en Algérie (encore une fois à notre connaissance) alors qu'il en existe 3 à Chypre ,3 au Ghana, 4 au Kenya et 2 au Lesotho ${ }^{(206)}$ est symptomatique du retard que l'Algérie est entrain d'accumuler. Nous avons cité ces pays, majoritairement africains et moins bien nantis financièrement, humainement et techniquement que l'Algérie (ayant omis de citer d'autres pays africains et du Tiers monde comme le Népal, le Soudan ,le Zimbabwe ou encore le Kirghizistan qui eux comptabilisent tous plus de dépôt numérique) pour faire ressortir le fait que notre pays tend à prendre un retard qui , au risque encore de nous répéter, pourrait très vite s'avérer insurmontable ou du moins difficile à combler.

\subsubsection{2-Journaux électroniques en libre accés}

Il existe, d'après le site de l'UNESCO, deux journaux " algériens " en libre accès .Ces deux journaux sont édités par la même maison d'édition, IB Scientific Publishing ${ }^{(207)}$ (IB signifiant Ibn Badis) située dans le Royaume Uni. Ce sont :

\subsubsection{1-IBScientific magazine ${ }^{(208)}$ :}

C'est un magazine multidisciplinaire qui publie des articles, des critiques, des informations et des essais dont le but est de faire comprendre au public la science et de s'engager dans à la defendre. Il semblerait qu'IBScientific magazine ne soit pas à jour car le dernier numéro disponible est le Vol 3, $\mathrm{n}^{\circ} 1$ (2007).Les autres volumes disponibles sont les numéros de l'année 2005 (Vol 1, $\mathrm{n}^{\circ} 1$ et 2) et ceux de l'année 2006 ( Vol 2, n¹, 2,3 et 4).On remarquera que l'équipe éditoriale est composée de chercheurs issus d'institutions britanniques telles que l'Université de Cambridge principalement.

\subsubsection{2-IBScientific Journal of Science ${ }^{(209)}$ :}

C'est un périodique trimestriel multidisciplinaire publiant des recherches originales dans un large spectre de disciplines allant des sciences à l'ingénierie aux sciences humaines.La même remarque s'applique en ce qui concerne la régularité de parution de IBScientific Journal of Science. En effet, les volumes 1 et 2 (2006 et 2007) comportent 3 numéros (1,2 et 3) alors que les volumes subséquents 3 et 4 (2008 et 2009) ne comportent que deux numéros (1 et 2).L'équipe éditoriale est pratiquement la même que celle de IBScientific magazine à un ou deux membres prés.

Ces deux revues qui sont signalées comme algériennes par le site de l'UNESCO, sont signalées comme des revues du Royaume Uni par le site Intute ${ }^{(210)}$ qui se définit comme un site web .... " Aidant à trouver les meilleurs sites pour les études et la recherche ".D'ailleurs le site de DOAJ qui est la référence même en la matière ne cite pas ces deux périodiques comme algériens ${ }^{(211)}$ mais

\footnotetext{
${ }^{(206)}$ http://roar.eprints.org/ (en date du 3 Mars 2012)

(207) $\mathrm{http://ibscientific.net/main/}$

(208) $\mathrm{http}: / /$ publications.ibscientific.net/Magazine

(209) $\mathrm{http} / / /$ publications.ibscientific.net/JournalOfScience

(210) $\mathrm{http}: / / w w w . i n t u t e . a c . u k / c g i-b i n / f u l l r e c o r d . p l$ handle $=20070622-114432$

(211) $\mathrm{http}$ ://www.doaj.org/doaj?func=byCountry\&uiLanguage $=\mathrm{en}$
} 
comme provenant du Royaume Uni pour IB Journal of Science et ce en $2006^{(212)}$ alors qu'IBScientific magazine n'est même pas cité..... Loin de nous de critiquer pour critiquer, mais ces deux périodiques scientifiques n'ont (apparemment) d'Algériens que le staff éditorial composé de chercheurs travaillant au Royaume Uni dans des institutions de recherche. De plus, ils gagneraient à être plus connus et mis à la disposition des chercheurs algériens. Enfin, leur périodicité et fréquence de parution ne semblent pas être très régulières sachant l'extrême importance de ces critères dans la notoriété d'un journal scientifique contrôlé par les pairs.

\subsubsection{3-Synergies Algerie :}

Le site de l'UNESCO cite aussi Synergies Algérie comme un périodique algérien en libre accés. Contrairement aux deux périodiques précédemment cités et dont nous avons fait ressortir l'incongruité de l'appartenance, Synergies Algérie qui se définit comme " une revue francophone de recherche en sciences humaines, particulièrement ouverte aux travaux en langues-cultures, aux approches linguistiques et culturelles de l'espace algérien. Sa vocation est de mettre en œuvre en Algérie le Programme Mondial de Diffusion Scientifique Francophone en Réseau du GERFLINT, Groupe d'Etudes et de Recherches pour le Français Langue Internationale " ${ }^{(213)}$ est présenté sur le site du DOAJ comme un périodique algérien. Il faut dire que le réseau GERFLINT se compose de 22 revues appelées toutes SYNERGIES suivies par le nom du pays (Synergie Corée, Synergies Venezuela, etc....), de la division linguistique (Synergies Pays Germanophone) ou régional (Synergies Monde Arabe ou Synergies Monde Méditerranéen).

La revue se définit comme quadrimestrielle et est indexée dans les répertoires suivants : Index Islamicus , Ulrich's, DOAJ, SHERPA/ROMEO, MIAR, Mir@bel et Ent'revues. Elle se compose d'un comité éditorial dont la moitie (en fait 8 sur 17) est algérienne et ne semble pas être très régulière elle aussi quant à sa parution. En effet, sur les 13 numéros depuis 2007, seule l'année 2010 a trois numéros, les autres années enregistrent des parutions irrégulières .Ainsi l'année 2007 enregistre 1 numéro, l'année 2008 enregistre 2 numéros, l'année 2009 enregistre 5 numéros, l'année 2010 enregistre 3 numéros et enfin l'année 2011 enregistre quant à elle 2 numéros. A delà de ce constat d'irrégularité, les différents numéros de Synergies Algérie sont effectivement en libre accès et on peut y accéder directement en cliquant sur l'année en question ensuite une suite de titres est affichée et à partir de laquelle on peut avoir le texte intégral. On remarquera que les revues Synergies sont éditées sur papier par le GERFLINT et qu'elles ont disponibles électroniquement sur le site de l'Université de Franche Comté au Centre de Linguistique Appliquée de Besançon ${ }^{(214)}$.D'un autre coté, les sponsors des revues Synergies sont: le Ministère Français des Affaires Etrangères , le Ministère Français de l'Enseignement Supérieur et de la Recherche, la Fondation Maison des Sciences de l'Homme, The Directory of Open Access Journal (DOAJ),le projet Mir@bel ${ }^{(215)}$ (Mutualisation d'informations sur les revues et leurs accès dans les bases en ligne ) et enfin " Akademia Pedagogiczna " de Cracovie en Pologne.

On remarquera que pour ces deux revues (ou plutôt trois car la première maison d'édition IB Scientific Publishing édite deux revues) les sujets, les auteurs (des fois) sont Algériens et non pas la revue qui est édité soit à l'étranger par des chercheurs algériens ( et est considéré comme c'est le cas de IB Journal of Science ou est édité par l'entremise d'organismes de recherche étrangers et font partie "... de revues animées par des équipes locales en liaison avec un comité scientifique international " ${ }^{(216)}$.Loin de nous de denier à ces revues leur appartenance au revues algériennes mais force est de reconnaître qu'elles se situent loin du contexte algérien et ne représentent, d'après nous ,que des tentatives, certes louables, mais qui ne sont pas représentatives de la vérité du terrain en Algérie.

\footnotetext{
${ }^{(212)} \mathrm{http}$ ://www.doaj.org/doaj?func=journalsByCountry\&cId=206\&year=2006\&added $=1$ \&uiLanguage $=\mathrm{fr}$

(213) $\mathrm{http}: / /$ gerflint.eu/publications/synergies-des-pays/synergies-algerie.html

(214) http://ressources-cla.univ-fcomte.fr/gerflint/revues.html

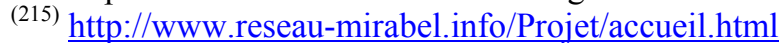

(216) $\underline{\text { http://ressources-cla.univ-fcomte.fr/gerflint/revues.html\#base }}$
} 


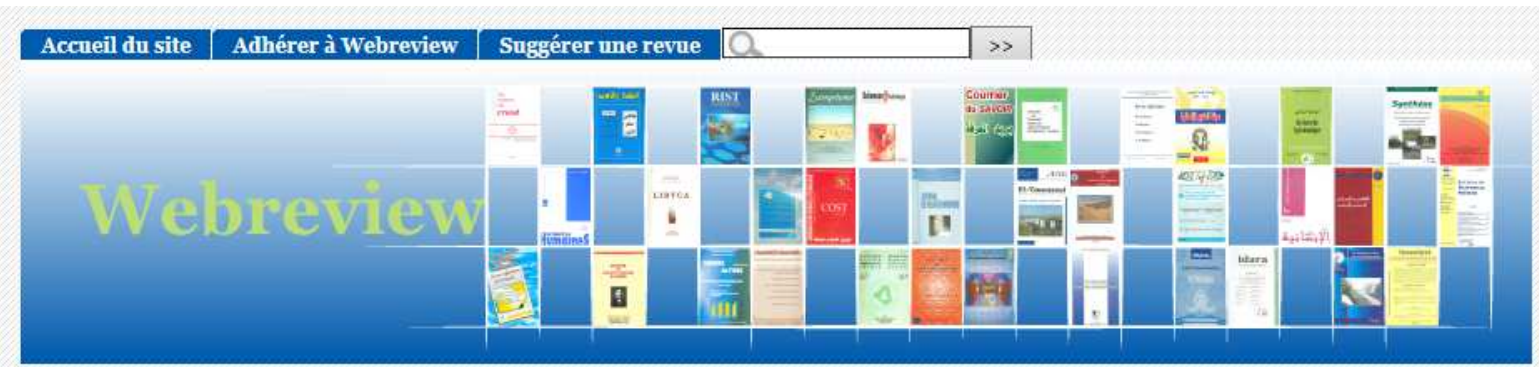

D'un autre coté, le Centre de Recherche sur l'Information scientifique et Technique (CERIST) offre Webreview ${ }^{(217)}$ qui est un panorama des revues algériennes offerte sur la Toile. Il comporte une liste de 36 revues scientifiques accessibles sur le Web. Ces revues sont toutes éditées en Algérie et offrent un large spectre de spécialités. Il offre les types d'accès suivants :

-Accès aux résumés et aux mots clés

-Accès aux articles en textes intégral.

La recherche à travers un moteur de recherche interne qui permet de faire une recherche simple par domaine, par titre, par éditeur,...

Les 36 revues sont présentées de la manière suivante :

-le titre de la revue

-l'ISSN

-l'éditeur

-le site web

-un court résumé sur la revue, sa couverture, ses buts, etc......

-le sommaire

A titre d'exemple, la page web de la première revue indexée "Archives de l'Institut Pasteur d'Algérie " se présente de la manière suivante :

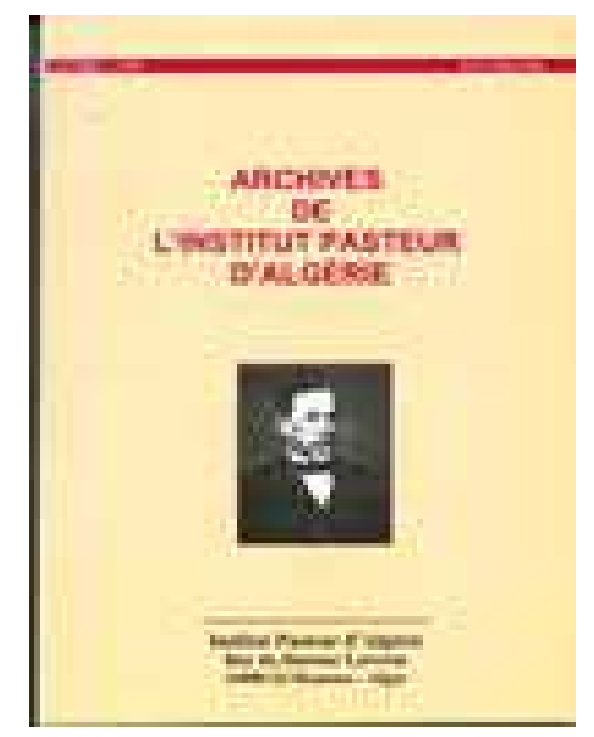

${ }^{(217)}$ http://www.webreview.dz 
5.1.1.2.5.1-Archives de l'Institut Pasteur d'Algérie

- ISSN : 0020-2460

- Editeur : Institut Pasteur d'Algérie

- Site web :http://www.ands.dz/ipa/pageaccueil.htm

C'est une revue éditée par l'Institut Pasteur d'Algérie. Elle publie des comptes rendus de travaux de recherches, articles originaux, revues d'articles faisant le point des connaissances actuelles sur un sujet donné, des mises au point techniques, des notes, des correspondances, lettres à l'éditeur, réflexions faisant état d’opinions sur les aspects de la santé, éditoriaux, etc.

Le sommaire

\section{(1) Tome 63-1999}

Pour cette revue, il n'existe qu'un seul numéro sur le site et qui est le Tome 63 daté de l'année 1999.Sur les 8 articles que ce numéro présente, aucun n'est en libre accés, le site se contentant de mettre un résumé de la recherche.

5.1.1.2.5.2-Al-Lugha Wal Adab et qui est la revue de l'Institut de langues et de littérature arabe de l'Université d'Alger dont aucune des 4 recherches du numéro 15 ne sont en libre accés et qui ne sont signalés que par le résumé.

5.1.1.2.5.3-Annales de l'Institut National Agronomique El Harrach (Alger) et qui est la revue de 1'Institut National Agronomique dont les 9 recherches sont toutes en libre accés.

5.1.1.2.5.4-Bulletin des Sciences Géographiques et qui est la revue de l'Institut national de Cartographie et de Télédétection (INCT) et dont les 7 numéros $(11,12,14,15,16,18$ et 21) ne sont pas tous en libre accés. Ainsi le $\mathrm{n}^{\circ} 11$ contenant 8 recherches est complètement en libre accès, les numéros 12,14, 15, 16 avec respectivement 7, 6, 6 et 10 recherches et dont aucune n'est en libre aces. Le numéro 18 avec 8 recherches est complètement en libre accès alors que le numéro 21 n'a que 2 recherches en libre accès sur les 8 recherches sur le site.

5.1.1.2.5.5-Cahiers de l'INRE et qui est la revue de l'Institut National de Recherche en Education et qui ne comporte qu'un seul numéro $\left(n^{\circ} 1\right)$.Ce numéro ne comporte que 3 recherches toutes en libre accès.

5.1.1.2.5.6-COST (COmmunication Science et Technologie) et qui est la revue de l'Ecole Normale Supérieure d'Enseignement Technique d'Oran, elle ne comporte qu'un seul numéro (14) et ce numéro comporte 14 articles dont 10 sont en texte intégral.

5.1.1.2.5.7-Courrier du Savoir et qui est la revue de l'Université Mohammed Khider à Biskra, elle comporte 8 numéros (de 1 à 8 ) et toutes les recherches sont en libre accés. Ainsi le $n^{\circ} 1$ comporte 12 recherches, le $n^{\circ} 213$ recherches, le $n^{\circ} 313$ recherches, le $n^{\circ} 414$ recherches, le $n^{\circ} 516$ recherches, le $n^{\circ} 623$ recherches, le ${ }^{\circ} 79$ recherches et le $n^{\circ} 817$ recherches.

5.1.1.2.5.8-Ecosystems qui est la revue du Laboratoire d'Eco développement des Espaces/Unité de recherche de l'INRAA de Sidi Bel Abbés, elle comporte 9 recherches dont aucune n'est en libre accès.

5.1.1.2.5.9-Edil.InF-Eau qui est la revue d'Engineering Environment Consult, elle comporte 10 numéros de parution irrégulière et qui se présentent de la manière suivante :

- $\mathrm{n}^{\circ} 6$ - 3 articles dont 2 en libre accès

- $n^{\circ} 12-3$ articles dont 2 en libre accès

- $\mathrm{n}^{\circ} 15-2$ articles en libre accès

- $\mathrm{n}^{\circ} 17-1$ article dont seul le résumé est présenté 
- ${ }^{\circ} 18-1$ article dont seul le résumé est présenté

- n¹9- 1 article dont seul le résumé est présenté

- n²0- 1 article dont seul le résumé est présenté

- $\mathrm{n}^{\circ} 21$ - 1 article dont seul le résumé est présenté

- n²6- 1 article dont seul le résumé est présenté

- n²7- 3 articles dont seul le résumé est présenté

5.1.1.2.5.10-El Bahith Al-Ijtimai qui est la revue du Département de sociologie de l'Université Mentouri de Constantine, et dont seul le ${ }^{\circ} 4$ est en ligne. Ce numéro recèle 7 articles dont 5 en texte intégral.

5.1.1.2.5.11-Al Maktabat Wa El Maâloumat qui est la revue du Département de bibliothéconomie de l'Université Mentouri- Constantine et dont les deux numéros en ligne sont le 1 et le 2.Le premier recèle 9 articles tous en libre accès et le deuxième 10 articles dont 9 sont en ligne.

5.1.1.2.5.12-El -Tawassol qui est une revue éditée par l'université Badji Mokhtar d'Annaba. Elle ne comporte qu'un seul numero qui est le 17 .Ce numéro comporte 16 recherches et dont 15 sont en libre accés.

5.1.1.2.5.13-IDARA qui est la revue du Centre de documentation et de recherches administratives de l'École Nationale d'Administration. Le seul numéro disponible en ligne est le 25 qui comporte 14 recherches et dont 12 sont en libre accés.

5.1.1.2.5.14-Insaniyat qui est la revue de Centre de Recherche en Anthropologie Sociale et Culturelle à Oran. Le seul volume disponible est le volume 6 qui comporte deux numéros (17 et 18).Ces deux numéros recèlent 17 recherches et dont 16 sont en libre accès.

5.1.1.2.5.15-Journal de Neurochirurgie qui est la revue de la Société Algérienne de Neurochirurgie. Elle comporte 5 numéros (de 00 à 04 ) et qui comportent respectivement 10, 10, 10,7 et 9 recherches et dont aucune n'est en libre accès.

5.1.1.2.5.16-Journal of Electrical Systems qui est la revue de Département d'Électronique de l'Université d'Oum El-Bouaghi. Il ne comporte qu'un seul volume qui recèle 2 numéros (1 et 2) avec respectivement 5 et 3 recherches toutes en libre accès.

5.1.1.2.5.17-Journal of the Algerian Chemical Society qui est éditée par l'Université d'Alger. Seuls 2 volumes sont en ligne (13 qui comporte le $\mathrm{n}^{\circ} 2$ et 14 qui comporte le $\left.\mathrm{n}^{\circ} 1\right)$.Ces deux volumes totalisent respectivement 9 et 12 recherches dont aucune n'est en libre accès.

5.1.1.2.5.18-La Revue des Sciences Commerciales qui est la revue de l'Institut National du Commerce. Elle ne comporte que le numéro 4 et dont seules 4 des 7 recherches sur le site sont en libre accès.

5.1.1.2.5.19-LARHYSS Journal qui est la revue du LAboratoire de Recherche en Hydraulique Souterraine et de Surface de l'Université Mohammed Khider à Biskra. Le site recèle 6 numéros (de 1 à 6) comprenant respectivement $9,10,15,13,19$ et 10 recherches. Tous les numéros offrent les recherches en libre accés à part le numéro 6 dont 2 recherches ne sont pas accessibles.

5.1.1.2.5.20-Le Journal de l'Eau et de l'Environnement qui est la revue de l'Ecole Nationale Supérieure de l'Hydraulique de Blida. Elle ne comporte que le numéro 6 et dont toutes les 6 recherches sont en libre accès.

5.1.1.2.5.21-Les Cahiers du CREAD qui est la revue du Centre de Recherche en Economie Appliquée au Développement. Seuls 2 numéros (45 et 48) sont en ligne, ils comportent respectivement 7 et 4 recherches dont aucune n'est en libre accès. 
5.1.1.2.5.22-Les Etudes Islamiques qui est la revue du Haut Conseil Islamique. Elle ne comporte que le numéro 2 et dont seule la moitié des 8 recherches peut être accédée en ligne.

5.1.1.2.5.23-Lybica qui est la revue du Centre National d'Études Historiques. Seuls les tomes 30 et 31 sont en ligne et dont les 5 recherches sur le site ne sont pas en libre accès.

5.1.1.2.5.24-NATURE \& TECHNOLOGIE qui est une revue éditée par l'Université Hassiba Benbouali de Chlef. Elle comporte 3 numéros (de 1 à 3 ) qui recèlent respectivement 9,7 et 7 recherches. A part une recherche dans le numéro 1, toutes les autres recherches sont en libre accès.

5.1.1.2.5.25-Recherche Agronomique qui est la revue de l'Institut National de la Recherche Agronomique (INRA).Le site comporte 13 numéros (de 00 à 11 et ensuite le numéro 14) et dont le degré en terme d'accessibilité au texte intégral est très irrégulier. Les différents numéros se présentent de la manière suivante :

$\mathrm{N}^{\circ} 00-11$ recherches dont aucune n'est en libre accès $\mathrm{N}^{\circ} 01-06$ recherches dont aucune n'est en libre accès $\mathrm{N}^{\circ}$ 02-05 recherches dont aucune n'est en libre accès $\mathrm{N}^{\circ}$ 03-04 recherches dont aucune n'est en libre accès

$\mathrm{N}^{\circ}$ 04-06 recherches dont aucune n'est en libre accès

$\mathrm{N}^{\circ}$ 05-05 recherches dont aucune n'est en libre accès

$\mathrm{N}^{\circ} 06-07$ recherches toutes en libre accès

$\mathrm{N}^{\circ} 07-08$ recherches toutes en libre accès

$\mathrm{N}^{\circ} 08-08$ recherches dont aucune n'est en libre accès

$\mathrm{N}^{\circ} 09-05$ recherches toutes en libre accès

$\mathrm{N}^{\circ} 10-05$ recherches toutes en libre accès

$\mathrm{N}^{\circ} 11-06$ recherches dont 5 en libre accès

$\mathrm{N}^{\circ}$ 14-07 recherches dont aucune n'est en libre accès

5.1.1.2.5.26-Revue Académique des Etudes Sociales et Humaines qui est une revue éditée par l'Université Hassiba Benbouali de Chlef. Elle ne comporte sur le site que 4 numéros (00 à 03) et dont le nombre recherches est respectivement de 7, 10, 09 et 11.Toutes les recherches sont en libre accès.

5.1.1.2.5.27-Revue Algérienne des Sciences Juridiques, Economiques et Politiques qui est une revue éditée par la Faculté de Droit de l'Université d'Alger. Seul le volume 36 est sur le site, il recèle 4 numéros $(1,2,3$ et 4$)$ avec respectivement $5,4,8$ et 9 recherches. Seule une recherche dans le numéro 3 est en libre accès.

5.1.1.2.5.28-Revue Algérienne du Travail qui est la revue de 1'Institut National du Travail.Les seuls numéros sur le site sont le 23, 28 et 29 avec respectivement 4,5 et 5 recherches. Les numéros 23 et 29 n'ont aucune recherche en libre accès alors que le numéro 28 avec 5 recherches est entièrement en libre accès.

5.1.1.2.5.29-Revue des Energies Renouvelables qui est la revue du Centre de Développement des Energies Renouvelables.Elle comporte sur le site 12 volumes numérotés de 01 à 12.Chacun de ces volumes comporte 2 numéros ( 01 et 02 ) à part le volume 9 qui comporte 4 numéros et le volume 11 qui ne comporte qu'un seul numéro. L'état de la collection en termes d'accessibilité peut être décrit de la manière suivante :

$\underline{\text { Vol.1 }}$

$\mathrm{N}^{\circ} 1-7$ recherches dont 4 en libre accès

$\mathrm{N}^{\circ} 2-4$ recherches toutes en libre accès 
$\underline{\text { Vol.2 }}$

$\mathrm{N}^{\circ} 1-6$ recherches toutes en libre accès $N^{\circ} 2-6$ recherches dont 5 en libre accès

$\underline{\text { Vol.3 }}$

$\mathrm{N}^{\circ} 1-6$ recherches toutes en libre accès

$\mathrm{N}^{\circ} 2-6$ recherches toutes en libre accès

$\underline{\text { Vol.4 }}$

$\mathrm{N}^{\circ} 1-13$ recherches dont 1 en libre accès*

$\mathrm{N}^{\circ} 2-6$ recherches dont 5 en libre accès ${ }^{*}$

$\underline{\text { Vol.5 }}$

$\mathrm{N}^{\circ} 1-5$ recherches toutes en libre accès

$\mathrm{N}^{\circ} 2-6$ recherches toutes en libre accès

$\underline{\text { Vol.6 }}$

$\mathrm{N}^{\circ} 1-6$ recherches dont aucune n'est en libre accès

$\mathrm{N}^{\circ} 2-6$ recherches dont aucune n'est en libre accès

$\underline{\text { Vol.7 }}$

$\overline{\mathrm{N}^{\circ} 1-6}$ recherches dont 5 en libre accès

$\mathrm{N}^{\circ} 2-6$ recherches toutes en libre accès

$\underline{\text { Vol.8 }}$

$\mathrm{N}^{\circ} 1-7$ recherches toutes en libre accès

$\mathrm{N}^{\circ} 2-7$ recherches toutes en libre accès

$\underline{\text { Vol.9 }}$

$\mathrm{N}^{\circ} 1-6$ recherches toutes en libre accès $\mathrm{N}^{\circ} 2-7$ recherches toutes en libre accès $\mathrm{N}^{\circ} 3-11$ recherches toutes en libre accès $\mathrm{N}^{\circ} 4-12$ recherches toutes en libre accès

\section{$\underline{\text { Vol.10 }}$}

$\mathrm{N}^{\circ} 1-14$ recherches toutes en libre accès

$\mathrm{N}^{\circ} 2-15$ recherches toutes en libre accès

$\underline{\text { Vol.11 }}$

$\mathrm{N}^{\circ} 1-15$ recherches toutes en libre accès

$\underline{\text { Vol.12 }}$

$\mathrm{N}^{\circ} 1-14$ recherches toutes en libre accès $\mathrm{N}^{\circ} 2$ - 4 recherches toutes en libre accès

5.1.1.2.5.30-Revue des Sciences Humaines qui est la revue de l'Université Mohammed Khider à Biskra. Elle comporte sur le site 6 numéros (de 1 à 6) qui respectivement comportent 16, 21, 16, 17, 23 et 9 recherches. Toutes les recherches sont en libre accès sauf une recherche dans le numéro 06.

\footnotetext{
* En fait, il n'y a que 12 recherches étant donné qu'une recherche est répétée et comptabilisée comme une autre recherche

${ }^{*}$ Ce numéro est en fait la répétition du numéro qui le précède avec exactement les mêmes recherches comptabilisées une deuxième fois
} 
5.1.1.2.5.31-RIST qui est la revue du CEntre de Recherche en Information Scientifique et Technique (CERIST).Le site recèle 16 volumes de 1 à 17 (le volume 6 n'étant pas offert) et chaque volume recèle, généralement, 2 numéros. L'état de la collection en termes d'accessibilité peut être décrit de la manière suivante :

\section{$\underline{\text { Vol.1 }}$}

$\mathrm{N}^{\circ} 1-9$ recherches toutes en libre accès

$\mathrm{N}^{\circ} 2-6$ recherches toutes en libre accès

$\underline{\text { Vol.2 }}$

$\mathrm{N}^{\circ} 1-5$ recherches toutes en libre accès $\mathrm{N}^{\circ} 2-5$ recherches dont 4 en libre accès

\section{$\underline{\text { Vol.3 }}$}

$\mathrm{N}^{\circ} 1-8$ recherches toutes en libre accès

\section{$\underline{\text { Vol.4 }}$}

$\mathrm{N}^{\circ} 1-6$ recherches toutes en libre accès

$\underline{\text { Vol.5 }}$

$\mathrm{N}^{\circ} 1-5$ recherches toutes en libre accès $\mathrm{N}^{\circ} 2-6$ recherches toutes en libre accès

\section{$\underline{\text { Vol.7 }}$}

$\mathrm{N}^{\circ} 1-7$ recherches dont 4 en libre accès $\mathrm{N}^{\circ} 2$ - 13 recherches dont 10 en libre accès

\section{$\underline{\text { Vol.8 }}$}

$\mathrm{N}^{\circ} 1-7$ recherches dont 5 en libre accès $\mathrm{N}^{\circ} 2-7$ recherches toutes en libre accès

\section{$\underline{\text { Vol.9 }}$}

$\mathrm{N}^{\circ} 1-9$ recherches toutes en libre accès

$\mathrm{N}^{\circ} 2-11$ recherches dont 10 en libre accès

$\underline{\text { Vol.10 }}$

$\mathrm{N}^{\circ} 1$ et $\mathrm{N}^{\circ} 2-12$ recherches toutes en libre accès

\section{$\underline{\text { Vol.11 }}$}

$\mathrm{N}^{\circ} 1-9$ recherches toutes en libre accès $\mathrm{N}^{\circ} 2-9$ recherches toutes en libre accès

$\underline{\text { Vol.12 }}$

$\mathrm{N}^{\circ} 1-9$ recherches dont 7 en libre accès

\section{$\underline{\text { Vol.13 }}$}

$\mathrm{N}^{\circ} 1-9$ recherches dont 8 en libre accès $\mathrm{N}^{\circ} 2-9$ recherches toutes en libre accès

\section{$\underline{\text { Vol.14 }}$}

$\mathrm{N}^{\circ} 1-9$ recherches dont 8 en libre accès $N^{\circ} 2-9$ recherches dont 5 en libre accès

\section{$\underline{\text { Vol.15 }}$}

$\mathrm{N}^{\circ} 1$ et $\mathrm{N}^{\circ} 2-9$ recherches toutes en libre accès 
$\underline{\text { Vol.16 }}$

$\mathrm{N}^{\circ} 1-8$ recherches dont 7 en libre accès

$\mathrm{N}^{\circ} 2-9$ recherches toutes en libre accès

Vol. 17

$\mathrm{N}^{\circ} 1$ et $\mathrm{N}^{\circ} 2-10$ recherches toutes en libre accés

5.1.1.2.5.32-Sciences et Technologie est une revue éditée par l'Université Mentouri à Constantine.Elle comporte sur le site 3 numéros $(12,13$ et 14) et qui comportent respectivement 14 , 15 et 9 recherches. Toutes ces recherches sont en libre accès à part le numéro 12 dont 1 recherche n'offre que le résumé.

5.1.1.2.5.33-Sciences Humaines qui est une revue éditée par l'Université Mentouri à Constantine. Elle comporte sur les site 11 numéros ( 1,2,5,7,8,9,11,12,13,14 et 17). L'état de la collection en termes d'accessibilité peut être décrit de la manière suivante :

$\mathrm{N}^{\circ} 1-2$ recherches dont aucune en libre accès

$\mathrm{N}^{\circ} 2-1$ recherches dont seul le résumé est offert.

$\mathrm{N}^{\circ}$ 5- 3 recherche dont aucune en libre accès

$N^{\circ} 7-6$ recherches dont aucune en libre accès

N 8-9 recherches dont aucune en libre accès

$\mathrm{N}^{\circ}$ 9-11 recherches dont aucune en libre accès

$\mathrm{N}^{\circ} 11-20$ recherches dont 6 en libre accès

$\mathrm{N}^{\circ} 12-24$ recherches dont 17 en libre accès

$\mathrm{N}^{\circ} 13-20$ recherches dont 10 en libre accés

$\mathrm{N}^{\circ} 14-23$ recherches dont 6 en libre accés

$\mathrm{N}^{\circ} 17-24$ recherches dont aucune en libre accés

5.1.1.2.5.34-Social and Human Sciences Review est une revue éditée par l'Université de Batna. Le seul numéro sur le site est le numéro 10 dont seul 1 recherche sur les 9 est en libre accés.

5.1.1.2.5.35-Synthèse est une revue éditée par l'Université Badji Mokhtar à Annaba. Seul le $\mathrm{n}^{\circ} 5$ est sur le site. Les 13 recherches sont toutes en libre accès.

5.1.1.2.5.36-Technologies Avancées qui est la revue du Centre de Développement des Technologies Avancées (CDTA).Seuls eux numéros (14 et 17) sont sur le site avec respectivement 6 et 4 recherches. Elles sont toutes en libre accès.

Ces chiffres peuvent être résumés dans le tableau suivant: 


\begin{tabular}{|c|c|c|c|c|}
\hline Titre & $\begin{array}{c}\text { Nombre } \\
\text { d'articles }\end{array}$ & $\begin{array}{c}\text { Texte } \\
\text { integral }\end{array}$ & Résumé & $\begin{array}{l}\text { Pourcentage } \\
\text { en libre accès }\end{array}$ \\
\hline Annales de l'Institut National Agronomique El-Harrach (...) & 09 & 09 & 00 & $100 \%$ \\
\hline Recherche Agronomique & 84 & 31 & 53 & $36 \%$ \\
\hline Lybica & 10 & 00 & 10 & $00 \%$ \\
\hline Journal of the Algerian Chemical Society & 17 & 00 & 17 & $00 \%$ \\
\hline La Revue des Sciences Commerciales & 07 & 06 & 01 & $86 \%$ \\
\hline Les Cahiers du CREAD & 11 & 00 & 11 & $00 \%$ \\
\hline Cahiers de l'INRE & 03 & 03 & 00 & $100 \%$ \\
\hline Bulletin des Sciences Géographiques & 78 & 63 & 15 & $81 \%$ \\
\hline RIST & 248 & 228 & 20 & $92 \%$ \\
\hline $\begin{array}{l}\text { Majallat Al Maktabat Wa El Maâloumat } \\
\text { (مجلة المكتبات و المعلومات }\end{array}$ & 19 & 19 & 00 & $100 \%$ \\
\hline $\begin{array}{l}\text { Al-Lugha Wal Adab } \\
\text { (اللغة والأدب (الغة }\end{array}$ & 04 & 00 & 04 & $00 \%$ \\
\hline $\begin{array}{l}\text { Les Etudes Islamiques } \\
\text { (الدر اسات الإسلامية) }\end{array}$ & 08 & 04 & 04 & $50 \%$ \\
\hline Ecosystem & 09 & 00 & 09 & $00 \%$ \\
\hline Edil.InF-Eau & 15 & 06 & 09 & $40 \%$ \\
\hline LARHYSS Journal & 76 & 74 & 02 & $97 \%$ \\
\hline Le Journal de l'Eau et de l'Environnement & 06 & 06 & 00 & $100 \%$ \\
\hline COST & 14 & 10 & 04 & $71 \%$ \\
\hline Courrier du Savoir & 117 & 117 & 00 & $100 \%$ \\
\hline Journal of Electrical Systems & 14 & 14 & 00 & $100 \%$ \\
\hline NATURE \&TECHNOLOGIE & 54 & 53 & 01 & $98 \%$ \\
\hline Revue des Energies Renouvelables & 402 & 389 & 13 & $97 \%$ \\
\hline Sciences et Technologie & 38 & 37 & 01 & $97 \%$ \\
\hline Synthèse & 13 & 13 & 00 & $10 \%$ \\
\hline Technologies Avancées & 10 & 10 & 00 & $100 \%$ \\
\hline IDARA & 25 & 23 & 02 & $92 \%$ \\
\hline $\begin{array}{l}\text { Revue Algérienne des Sciences Juridiques, Economiques et } \\
\text { Politiques }\end{array}$ & 26 & 01 & 25 & $04 \%$ \\
\hline Archives de l'Institut Pasteur d'Algérie & 08 & 00 & 08 & $00 \%$ \\
\hline Journal de Neurochirurgie & 43 & 00 & 43 & $00 \%$ \\
\hline El -Tawassol & 35 & 33 & 02 & $94 \%$ \\
\hline Insaniyat & 17 & 06 & 11 & $35 \%$ \\
\hline Revue Algérienne du Travail & 14 & 00 & 14 & $00 \%$ \\
\hline Sciences Humaines & 143 & 40 & 103 & $28 \%$ \\
\hline $\begin{array}{l}\text { Revue Académique des Etudes Sociales et Humaines } \\
\text { (الأكاديمبة للار اسات الإجماعية و الإنسانية) }\end{array}$ & 37 & 37 & 00 & $100 \%$ \\
\hline $\begin{array}{l}\text { El Bahith Al-Ijtimai } \\
\text { (الباحث الإجتماعي) }\end{array}$ & 07 & 07 & 00 & $100 \%$ \\
\hline $\begin{array}{l}\text { Revue des Sciences Humaines et Sociales } \\
\text { (الإجتماعية و الإنسانية العلوم مجلة) }\end{array}$ & 09 & 01 & 08 & $11 \%$ \\
\hline $\begin{array}{l}\text { Revue des Sciences Humaines } \\
\text { (مجلة العلوم الإنسانبة) }\end{array}$ & 232 & 228 & 04 & $98 \%$ \\
\hline Total & 1862 & 1468 & 394 & $79 \%$ \\
\hline
\end{tabular}

Tableau $\mathrm{n}^{\circ} 6$-proportion des articles en libre accès sur Webreview

Aprés cet examen en détails, autant que peut se faire, de Webreview on peut faire les remarques suivantes concernant les différentes revues offertes sur le site. Ce sont :

- la faiblesse quantitative de certaines revues en ce qui concerne le nombre et aussi, dans certains cas le nombre de recherches dans chaque numéro. 
- L'extrême irrégularité des parutions (du moins celles offertes sur le site)

- Le nombre de recherches dont l'accès est libre qui dans certains cas est très faible et même inexistant

- L'irrégularité de l'application du libre accès à l'intérieur même d'une même revue qui des fois offre dans le même numéro ou volume des recherches en libre accès et d'autres sont seul le résumé est disponible. Ceci est fait sans, qu'apparemment, une claire politique d'accès que définirait le (ou les) responsable en amont de la parution de la publication. Les parutions ne paraissent obéir à aucun critère de continuité et de pérennité dans la politique de libre accès.

Malgré ces lacunes, somme toute normales pour une première expérience, on peut qualifier Webreview de tentative novatrice et encourageante au niveau national en matière de publication de revues scientifiques sur la Toile. Cette expérience, néanmoins, gagnerait à être plus exhaustive englobant plus de titres dont la communauté nationale des chercheurs est productrice ou participantes et aussi il gagnerait à être plus connu. A titre d'exemple, bien que nous intéressant à ces problématiques, nous n'avons eu connaissance de cet important outil que par l'intermédiaire de sites (celui de l'UNESCO en l'occurrence) qui se spécialisent dans le libre accès. Il serait plus rentable, judicieux et adéquat qu'une campagne de sensibilisation et de marketing soit mise en place afin de faire connaitre ces produits (que ce soit au niveau du CERIST, acteur incontournable dans ce domaine par la richesse de sa composante humaine et technique ou encore les universités à l'instar du dépôt numérique de l'Université d'Alger I ) dans un monde de plus en plus ouvert et dont la vitesse de changement ne laisse aucune chance à ceux qui accumulerait du retard et ne s'inséreraient pas dans un monde en constant et rapide développement.

Nous nous sommes contentés de ces quelques expériences nationales en nous basant sur Internet et sur notre connaissance de l'état des lieux et du vécu du libre accès en Algérie. D'autres expériences et vécus ont étés sciemment non abordés car le libre accès est en même temps très récent et surtout méconnu par ceux là même qui devraient le promouvoir. Il semblerait, qu'au niveau national, toute expérience est faite, non pas d'une manière ouverte, mais en catimini ce qui est en même temps saugrenu et surtout impossible pour le libre accès vu sa propension à être à la portée de chaque personne qui peut accéder à Internet. Outil indispensable s'il en est et dont toute tentative dans ce cadre auquel il n'est pas associé est irrémédiablement voué à l'échec.

\section{2-Internet en Algérie}

Il n'a échappée à personne à travers ces lignes et aussi de par la configuration du libre accès que ce soit dans ce qui est connu sous l'appellation de la route dorée (les journaux électroniques en libre accés) ou encore la route verte (les archives ouvertes) l'importance extrême et primordiale d'Internet et de sa disponibilité. Il est de notoriété aussi que la disponibilité, la fiabilité et surtout performances d'Internet n'est pas la même partout dans le monde et ceci influe sur les performances et les résultats que peut avoir le libre accès sur les projets de mise à la disposition des ressources documentaires. On peut affirmer sans risque de se tromper donc que de la disponibilité effective et performante d'Internet dépend la réussite d'une politique de libre accès. Qu'en est-il de l'Algérie en ce début de $21^{\mathrm{e}}$ siècle ? Nous allons essayer de répondre à cette question importante, s'il en est, en nous référant à un site qui fait office de référence en la matière et que nous avons déjà cité qui est le site de : www.internetworldstats.com .L'Algérie est classée avec les pays africains * et se classe respectivement aux positions suivantes :

$-13^{\mathrm{e}}$ en terme de taux de pénétration (nombre de connectés par habitants) avec un taux de $13.4 \%$.

\footnotetext{
* Contrairement à internetworldstats, le portail global du libre accés de l'UNESCO (Global Open Access Portal) classe l'Algérie, en plus d'être un pays africain, aussi avec les pays arabes.
} 
$-7^{\mathrm{e}}$ position en terme du taux d'utilisateurs en Afrique (nombre de connectés par le nombre de connectés du continent) avec un taux de $3.4 \%$.

$-7^{\mathrm{e}}$ position en nombre de connectés avec 4700000 connectés.

Ces chiffres bruts pris en tant que tel ne reflètent pas les potentialités humaines, infrastructurelles et financières de l'Algérie. En effet, il est tout de même étonnant que des petits pays (dans le sens potentialités et moyens s'entend) tels que le Cap Vert, le Kenya, l'Ile Maurice ou Sao Tomé et Principe aient un taux de pénétration plus haut que l'Algérie ou alors que la Tanzanie ait plus de connectés que l'Algerie. Nous voulons dire que la situation d'Internet en Algérie pourrait être, et de loin, meilleur si une claire politique de vulgarisation de l'outil Internet était mise en place par les pouvoirs publics. En plus de ces chiffres, l'état et la bande passante Internet y est encore très sujette aux multiples pannes et coupures. De plus, malgré les propos lénifiants des pouvoirs publics (surtout la tutelle), Internet n'est toujours pas répandu à grande échelle dans les universités où les étudiants ont en le plus besoin. D'un autre coté, il est de notoriété que l'état des lignes téléphoniques et du réseau téléphonique en Algérie n'est pas de tout premier ordre et que cet état de fait ne peut aider à la propagation de l'outil Internet. Il nous semble que face à l'étendue géographique du pays qui dépasse les $2000000 \mathrm{~km}^{2}$, une solution d'Internet sans fil (Wi Fi) s'impose. En plus de limiter les sempiternelles coupures et de désenclaver les régions lointaines et aussi montagneuses, cette option a l'avantage d'être moins chère et plus fiable. Des pays aussi développés que le Canada et dont la superficie est plus vaste ont opté pour ce genre de solution à même de garantir un accès aussi grand que possible au plus grand nombre même les contrées les plus reculées et les plus isolées. 


\begin{tabular}{|c|c|c|c|c|c|c|}
\hline \multicolumn{7}{|c|}{ INTERNET USAGE STATISTICS FOR AFRICA } \\
\hline \multirow{2}{*}{ AFRICA } & Population & $\begin{array}{c}\text { Internet } \\
\text { Users }\end{array}$ & $\begin{array}{c}\text { Internet } \\
\text { Users }\end{array}$ & Penetration & Users & Facebook \\
\hline & (2011 Est.) & Dec/2000 & 31-Dec-11 & $\begin{array}{c}(\% \\
\text { Population) }\end{array}$ & $\%$ Africa & 31-Dec-11 \\
\hline Algeria & $34,994,937$ & 50 & $4,700,000$ & $13.4 \%$ & $3.4 \%$ & $2,835,740$ \\
\hline Angola & $13,338,541$ & 30 & 744,195 & $5.6 \%$ & $0.5 \%$ & 322,3 \\
\hline Benin & $9,325,032$ & 15 & 744,195 & $3.0 \%$ & $0.2 \%$ & 130,24 \\
\hline Botswana & $2,065,398$ & 15 & 167,18 & $8.1 \%$ & $0.1 \%$ & 167,18 \\
\hline Burkina Faso & $16,751,455$ & 10 & 230,562 & $1.4 \%$ & $0.2 \%$ & 95,38 \\
\hline Burundi & $10,216,190$ & 3 & 176,04 & $1.7 \%$ & $0.1 \%$ & 30,78 \\
\hline Cameroon & $19,711,291$ & 20 & 783,956 & $4.0 \%$ & $0.6 \%$ & 467,34 \\
\hline Cape Verde & 516,1 & 8 & 148,8 & $28.8 \%$ & $0.1 \%$ & 76,98 \\
\hline Central African & $4,950,027$ & 1,5 & 123,8 & $2.5 \%$ & $0.1 \%$ & 123,8 \\
\hline Chad & $10,758,945$ & 1 & 190,863 & $1.8 \%$ & $0.1 \%$ & 38 \\
\hline$\underline{\text { Comoros }}$ & 794,683 & 1,5 & 37,472 & $4.7 \%$ & $0.0 \%$ & 13,84 \\
\hline$\underline{\text { Congo }}$ & $4,243,929$ & 500 & 295,132 & $7.0 \%$ & $0.2 \%$ & 77,1 \\
\hline Congo, Dem. Rep. & $71,712,867$ & 500 & 915,4 & $1.3 \%$ & $0.7 \%$ & 915,4 \\
\hline Cote d'Ivoire & $21,504,162$ & 40 & 968 & $4.5 \%$ & $0.7 \%$ & $\mathrm{n} / \mathrm{a}$ \\
\hline Djibouti & 757,074 & 1,4 & 61,32 & $8.1 \%$ & $0.0 \%$ & 51,24 \\
\hline Egypt & $82,079,636$ & 450 & $21,691,776$ & $26.4 \%$ & $15.5 \%$ & $9,391,580$ \\
\hline Equatorial Guinea & 668,225 & 500 & 42,024 & $6.3 \%$ & $0.0 \%$ & 18,18 \\
\hline Eritrea & $5,939,484$ & 5 & 283,699 & $4.8 \%$ & $0.2 \%$ & 19,18 \\
\hline Ethiopia & $90,873,739$ & 10 & 622,122 & $0.7 \%$ & $0.4 \%$ & 472,46 \\
\hline Gabon & $1,576,665$ & 15 & 108,845 & $6.9 \%$ & $0.1 \%$ & 91,2 \\
\hline Gambia & $1,797,860$ & 4 & 159,012 & $8.8 \%$ & $0.1 \%$ & 74,84 \\
\hline Ghana & $24,791,073$ & 30 & $2,085,501$ & $8.4 \%$ & $1.5 \%$ & $1,146,560$ \\
\hline Guinea & $10,601,009$ & 8 & 95,823 & $0.9 \%$ & $0.1 \%$ & 42,28 \\
\hline Guinea-Bissau & $1,596,677$ & 1,5 & 37,123 & $2.3 \%$ & $0.0 \%$ & $\mathrm{n} / \mathrm{a}$ \\
\hline Kenya & $41,070,934$ & 200 & $10,492,785$ & $25.5 \%$ & $7.5 \%$ & $1,298,560$ \\
\hline Lesotho & $1,924,886$ & 4 & 83,813 & $4.4 \%$ & $0.1 \%$ & 27,7 \\
\hline Liberia & $3,786,764$ & 500 & 20 & $0.5 \%$ & $0.0 \%$ & $\mathrm{n} / \mathrm{a}$ \\
\hline Libya & $6,597,960$ & 10 & 391,88 & $5.9 \%$ & $0.3 \%$ & 391,88 \\
\hline Madagascar & $21,926,221$ & 30 & 352,135 & $1.6 \%$ & $0.3 \%$ & 216,84 \\
\hline
\end{tabular}

\begin{tabular}{|c|c|c|c|c|c|c|}
\hline \multicolumn{7}{|c|}{ INTERNET USAGE STATISTICS FOR AFRICA } \\
\hline \multirow{2}{*}{$\underline{A F R I C A}$} & Population & $\begin{array}{c}\text { Internet } \\
\text { Users }\end{array}$ & $\begin{array}{c}\text { Internet } \\
\text { Users }\end{array}$ & Penetration & Users & Facebook \\
\hline & (2011 Est.) & Dec/2000 & 31-Dec-11 & $\begin{array}{c}(\% \\
\text { Population) }\end{array}$ & $\%$ Africa & 31-Dec-11 \\
\hline Malawi & $15,879,252$ & 15 & 716,4 & $4.5 \%$ & $0.5 \%$ & 112,1 \\
\hline Mali & $14,159,904$ & 18,8 & 414,985 & $2.9 \%$ & $0.3 \%$ & 132,72 \\
\hline Mauritania & $3,281,634$ & 5 & 100,333 & $3.1 \%$ & $0.1 \%$ & 83,26 \\
\hline Mauritius & $1,303,717$ & 87 & 323,494 & $24.8 \%$ & $0.2 \%$ & 298,84 \\
\hline Mayotte (FR) & 209,53 & $\mathrm{n} / \mathrm{a}$ & 10,62 & $5.1 \%$ & $0.0 \%$ & 10,34 \\
\hline Morocco & $31,968,361$ & 100 & $15,656,192$ & $49.0 \%$ & $11.2 \%$ & $4,075,500$ \\
\hline Mozambique & $22,948,858$ & 30 & 975,395 & $4.3 \%$ & $0.7 \%$ & 186,46 \\
\hline Namibia & $2,147,585$ & 30 & 148,414 & $6.9 \%$ & $0.1 \%$ & 134,14 \\
\hline Niger & $16,468,886$ & 5 & 128,749 & $0.8 \%$ & $0.1 \%$ & 44,58 \\
\hline Nigeria & $155,215,573$ & 200 & $45,039,711$ & $29.0 \%$ & $32.2 \%$ & $4,369,740$ \\
\hline Reunion (FR) & 834,261 & 130 & 300 & $36.0 \%$ & $0.2 \%$ & 206,8 \\
\hline Rwanda & $11,370,425$ & 5 & 818,048 & $7.2 \%$ & $0.6 \%$ & 114,74 \\
\hline Saint Helena (UK) & 7,7 & $\mathrm{n} / \mathrm{a}$ & 900 & $11.7 \%$ & $0.0 \%$ & $\mathrm{n} / \mathrm{a}$ \\
\hline Sao Tome \& Principe & 179,506 & 6,5 & 31,012 & $17.3 \%$ & $0.0 \%$ & 3,32 \\
\hline Senegal & $12,643,799$ & 40 & $1,989,396$ & $15.7 \%$ & $1.4 \%$ & 620,26 \\
\hline Seychelles & 89,188 & 6 & 33,9 & $38.0 \%$ & $0.0 \%$ & 19,5 \\
\hline Sierra Leone & $5,363,669$ & 5 & 48,52 & $0.9 \%$ & $0.0 \%$ & 48,52 \\
\hline Somalia & $9,925,640$ & 200 & 106 & $1.1 \%$ & $0.1 \%$ & 55,14 \\
\hline South Africa & $49,004,031$ & $2,400,000$ & $6,800,000$ & $13.9 \%$ & $4.9 \%$ & $4,822,820$ \\
\hline South Sudan & $8,260,490$ & - & $\mathrm{n} / \mathrm{a}$ & $\mathrm{n} / \mathrm{a}$ & $0.0 \%$ & $\mathrm{n} / \mathrm{a}$ \\
\hline Sudan & $45,047,502$ & 30 & $4,200,000$ & $9.3 \%$ & $3.0 \%$ & $\mathrm{n} / \mathrm{a}$ \\
\hline Swaziland & $1,370,424$ & 10 & 95,122 & $6.9 \%$ & $0.1 \%$ & 54,22 \\
\hline Tanzania & $42,746,620$ & 115 & $4,932,535$ & $11.5 \%$ & $3.5 \%$ & 414,54 \\
\hline Togo & $6,771,993$ & 100 & 356,3 & $5.3 \%$ & $0.3 \%$ & 71,2 \\
\hline Tunisia & $10,629,186$ & 100 & $3,856,984$ & $36.3 \%$ & $2.8 \%$ & $2,799,260$ \\
\hline Uganda & $34,612,250$ & 40 & $4,178,085$ & $12.1 \%$ & $3.0 \%$ & 346,98 \\
\hline Western Sahara & 507,16 & $\mathrm{n} / \mathrm{a}$ & $\mathrm{n} / \mathrm{a}$ & $\mathrm{n} / \mathrm{a}$ & $0.0 \%$ & $\mathrm{n} / \mathrm{a}$ \\
\hline Zambia & $13,881,336$ & 20 & 882,17 & $6.4 \%$ & $0.6 \%$ & 177,82 \\
\hline Zimbabwe & $12,084,304$ & 50 & $1,445,717$ & $12.0 \%$ & $1.0 \%$ & $\mathrm{n} / \mathrm{a}$ \\
\hline
\end{tabular}

\begin{tabular}{|l|c|c|c|c|c|c|}
\hline TOTAL AFRICA & $1,037,524,058$ & $4,514,400$ & $139,875,242$ & $13.5 \%$ & $100.0 \%$ & $37,739,380$ \\
\hline
\end{tabular}

Tableau n ${ }^{\circ}$ 7- Statistiques d'utilisation Internet en Afrique ( 31 Decembre 2011) 


\section{Africa Top Internet Countries \\ December 31, 2011}

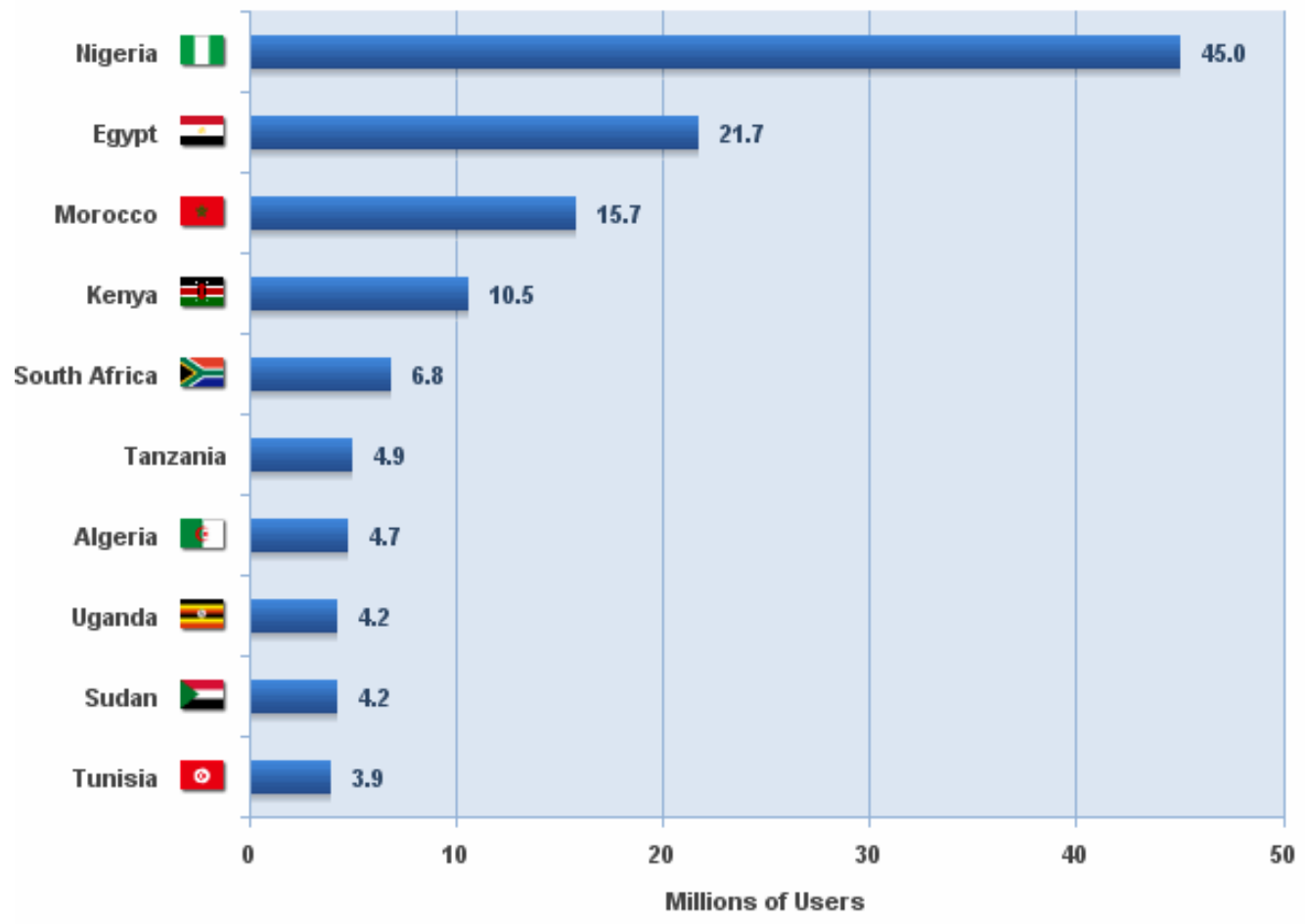

Source: www.internetworldstats.com/stats1.htm

Copyright @ 2012, Miniwatts Marketing Group

Tableau $n{ }^{\circ}$ 8- Les dix premiers utilisateurs Intenet en Afrique

\subsection{1.-Netindex}

D'un autre côté, si les chiffres bruts d'Internet (taux de pénétration, taux d'utilisateurs en Afrique et nombre de connectés) ne sont pas, loin s'en faut, encourageants, les chiffres concernant la bande passante et les statistiques y afférant le sont plus. Quand on parle de la bande passante, nous parlons non pas de l'existence ou non d'Internet mais de sa qualité et de son débit. A cet effet, le site www.netindex.com ${ }^{(218)}$ calcule, sur la bases de données scientifiques, les valeurs de la bande passante ascendante, descendante ainsi que d'autres données spécifiques au site et qui sont l'index de qualité domestique, l'index de valeur domestique et l'index de promesse domestique que nous allons brièvement présenter. Les chiffres concernant l'Algérie sont les suivants en date du $1^{\mathrm{er}}$ septembre 2013 :

5.2.1.1-Bande passante ascendante: avec un débit ascendant (download) de 1.21 Mbps , l'Algérie se classe en $180^{\mathrm{e}}$ position sur 184 pays devançant seulement le Malawi, la RD du Congo,le Bénin et le Burkina Faso ${ }^{(219)}$ tel que le démontre la figure suivante :

(218) Www.netindex.com

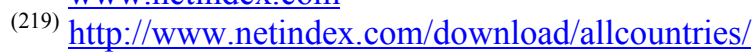




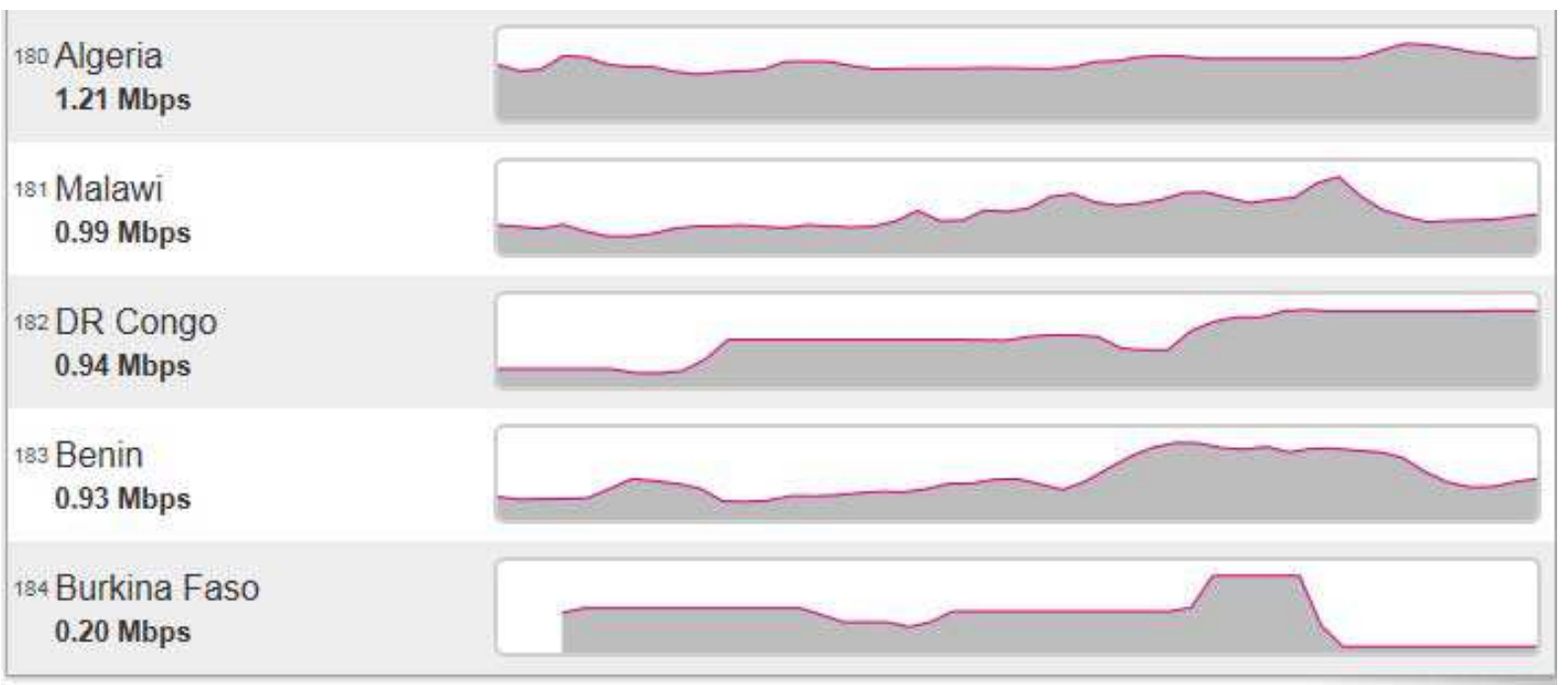

Fig.n ${ }^{\circ}$ 31- Classement de l'Algerie selon la bande bande passante ascendante

5.2.1.2-Bande passante descendante : avec un débit descendant (upload) de $0.52 \mathrm{Mbps}$, l'Algérie se classe en $180^{\mathrm{e}}$ position sur 184 pays devançant seulement le Benin, le Venezuela , la RD du Congo et le Burkina Faso ${ }^{(220)}$ tel que le démontre la figure suivante :
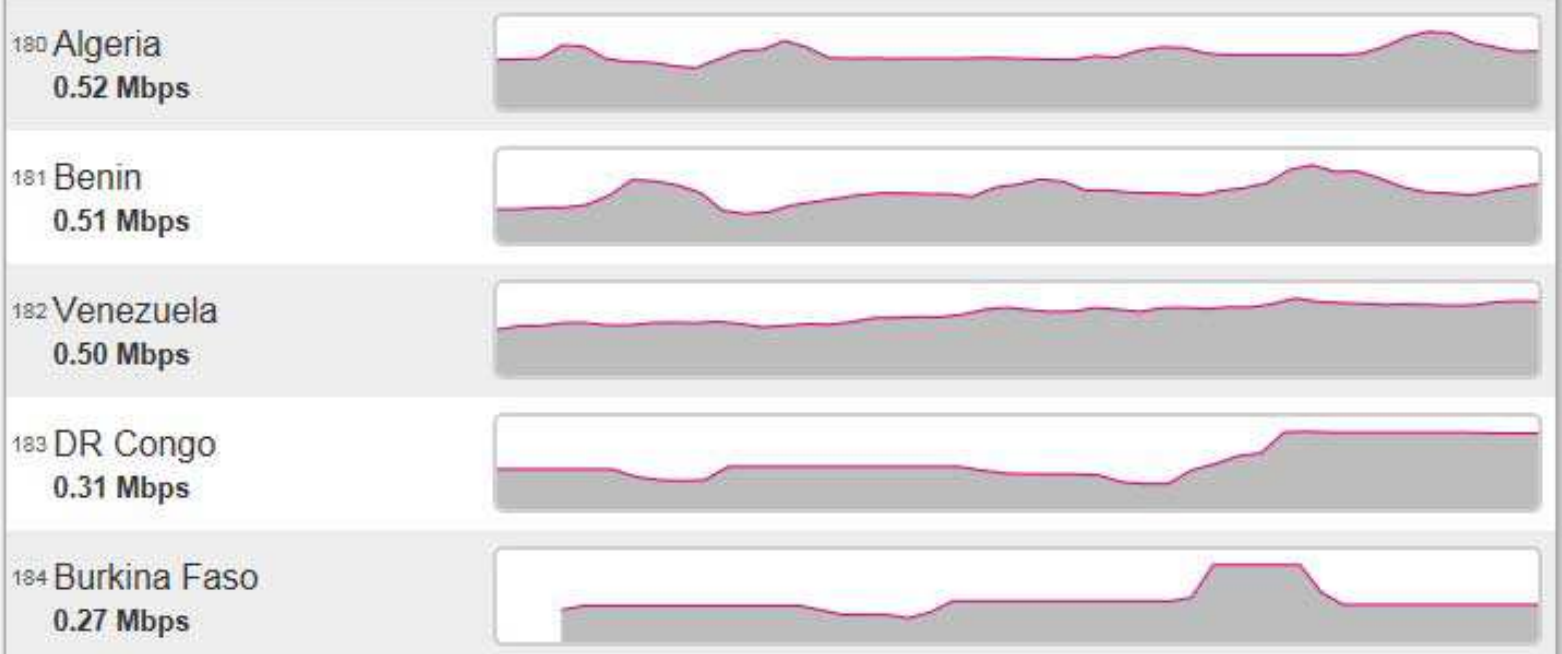

Fig.n ${ }^{\circ}$ 32- Classement de l'Algerie selon la bande bande passante descendante

5.2.1.3-Index de qualité domestique : cet index compare et classe la qualité du haut débit mondialement. L'Algérie se classe en $46^{\mathrm{e}}$ position sur 52 pays avec un index de $75.18^{(221)}$ tel que le démontre la figure suivante : 


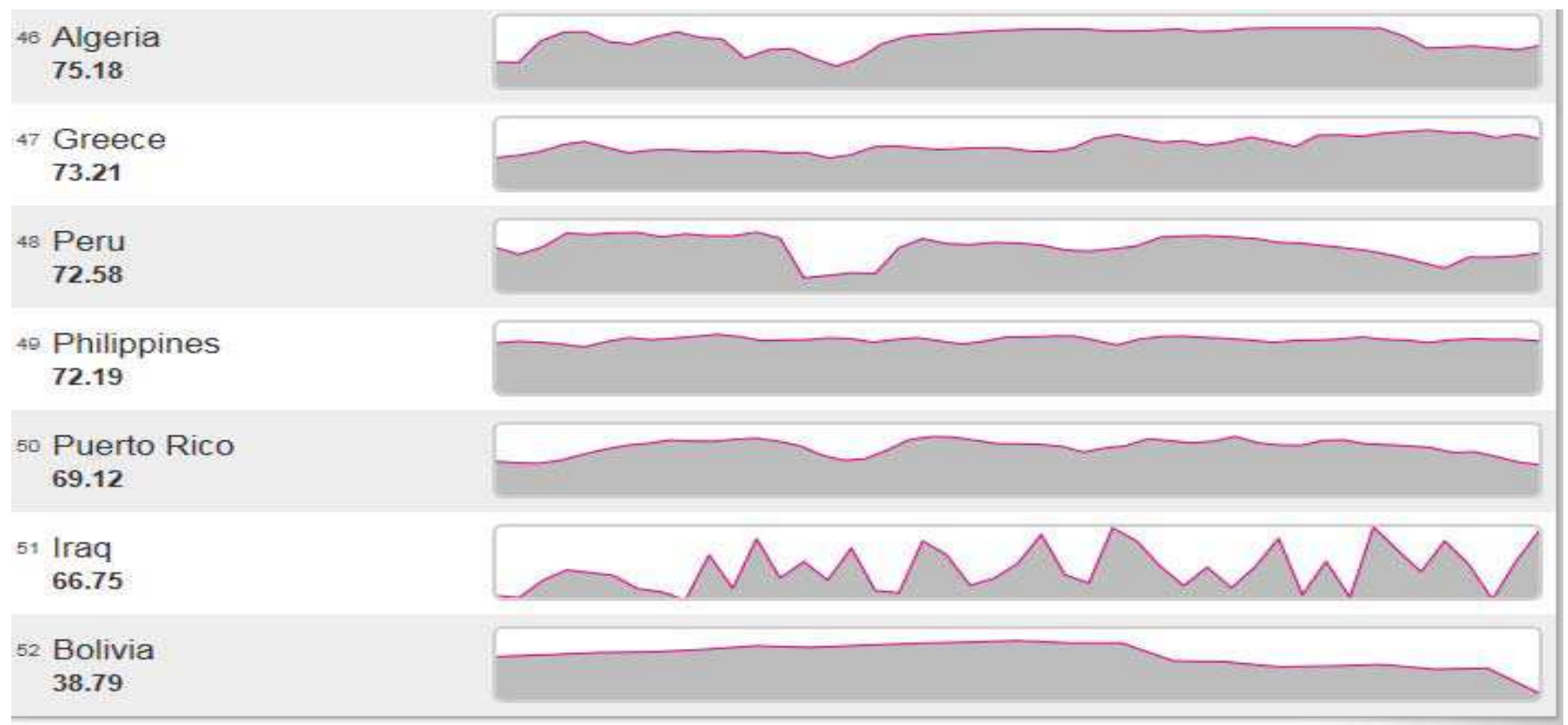

Fig.n ${ }^{\circ} 33-$ Classement de l'Algérie selon l'index de qualité domestique

5.2.1.4-Index de valeur domestique : cet index compare et classe la valeur du haut débit mondialement. La valeur est le coût moyen en \$ par Megabit par seconde. Sur les 62 pays répertoriés et classés, l'Algérie n'est pas citée ${ }^{(222)}$.

5.2.1.5-Index de promesse domestique : cet index compare et classe un aspect important des performances des fournisseurs d'accès à Internet (FAI).La valeur obtenue est la proportion médiane du taux de débit descendant (download) à la vitesse du débit descendant auquel on a souscrit (connu aussi sous l'appellation de " vitesse promise "). En d'autres termes, cet index calcule le débit souscrit au débit effectif. A l'instar de l'index de valeur domestique, sur les 62 pays répertoriés et classés, l'Algérie n'est pas citée ${ }^{(223)}$.

Ces chiffres d'un site référencé ajoutés aux chiffres bruts d'Internet se passent de tout commentaire et devraient interpeller les responsables quant à la situation d'Internet dans notre pays. Nous nous sommes contentés de citer les chiffres les plus importants car d'autres chiffres et données sont encore plus inquiétants ${ }^{*}$. Ces chiffres sont donnés ici à titre indicatif mais devrait être analysés, pour notre travail du moins, à l'aune d'une question quintessentielle : quel libre accès avec des chiffres pareils? Il n'est pas dans les prérogatives ni les limites de ce travail d'y répondre mais il serait temps qu'une politique claire et courageuse tout azimut de promotion d'Internet soit entreprise en Algérie afin de permettre l'accès à la société de l'information si galvaudée mais qui tarde à voir le jour à cause de raisons obscurs et néfastes non seulement à la recherche mais à la société en général.

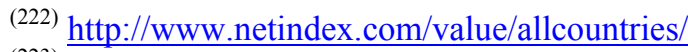

(223) http://www.netindex.com/promise/allcountries/

* Le site en question est très riche en données. Par exemple, il existe une fonctionnalité "Go to your location" qui permet grâce à l'adresse IP de calculer le débit aussi bien ascendant que descendant de la ville. On apprend que le débit ascendant d'Alger est de $1.28 \mathrm{Mbps}$ alors que le débit descendant est de $0.54 \mathrm{Mbps}$ alors que la ville dont le test donne les valeurs les plus hautes est Timisoara en Roumanie avec un débit ascendant de $80.05 \mathrm{Mbps}$ et un débit descendant de 21.28 Mbps (http://www.netindex.com/download/4,2316/Timisoara,-RO/ ) 
Bien que d'histoire récente, le libre accès a accompli en ces vingt dernières années qu'a duré à peu prés son existence, des progrès et des développements extraordinaires que seules les fonctionnalités de l'outil informatique et surtout d'Internet ont rendu possible. On ne peut parler de libre accés (dans ses deux formes archives ouvertes et journaux électroniques) que si on évoque Internet. En effet, si le réseaux des réseaux tel qu'il est aussi connu n'est pas de création récente et n'a pas été créé pour les raisons qui sont les siens de nos jours, il n'en demeure pas moins que son véritable décollage peut être situé à la fin du siècle dernier et plus précisément à la dernière décennie du siècle en question. Cet envolée a coïncidé avec une crise des prix des périodiques ayant atteint des sommets et des proportions tels que les bibliothèques et autres centres de documentation ont dû faire des coupes claires dans leur budget et se désabonner des revues les plus importantes.Cette situation a été encouragé par les différentes politiques de désengagement et de libéralisation outrancière des années 80 surtout aux Etats Unis et en Grande Bretagne. C'est alors que des chercheurs pionniers (tel que Harnad, Guédon, Suber, Odlyzko, Ginsparg, etc..) entreprirent de se réapproprier leur travail, jusque là prisonnier des droits d'auteurs que les éditeurs commerciaux avaient acquis en contrepartie de leur savoir faire et réputation. Il faut dire que ces mêmes chercheurs ne pouvaient accéder à des recherches dont ils avaient besoin parce que, comme on l'a dit, elles étaient protégées par les droits que l'éditeur avait acquis et ne pouvaient donner accés à leurs propres recherches car elles étaient sous le même régime. En plus, ces recherches étaient (et sont toujours) financées par les organismes de recherche nationaux ou internationaux ce qui revient à dire par les chercheurs eux-mêmes, ce qui donnait une situation surréaliste où le chercheur ne pouvait accéder, ni faire accéder sa propre recherche qu'il avait financé lui-même par l'intermédiaire de sa participation à l'effort de recherche. Fort de leur maitrise de l'outil informatique (la plupart sont de formation scientifique et surtout informatique), ces chercheurs entreprirent de ce créer des outils à même de permettre de se diminuer la mainmise des éditeurs et de rendre leur liberté à leur publication. Il n'a jamais été, par ailleurs, question de substituer le rôle de l'éditeur mais de l'adapter aux nouvelles donnes que ce mouvement à engendré. De leur coté, les éditeurs conscients du danger que ce nouvel ordre pourrait engendrer pour leur juteuses affaires et surtout de la liberté qu'Internet donnerait aux chercheurs, ont adaptés leur offres et lâché du lest afin que la confrontation n'ait pas lieu.

Il est un fait admis dans la courte l'histoire du libre accès que la première archive ouverte qui a lancé le mouvement est ArXiv et ce en 1991.Cette archive ouverte qui recensait par exemple en date du 25 Janvier 2012730,347 publications électroniques en Physiques, Mathématiques, Informatique, Biologie quantitative, Finance quantitative et Statistiques est citée par les différents chercheurs comme l'archétype de l'archive ouverte. Les physiciens ont de tout temps eut cette culture du partage de l'information surtout pré publication. Si cette manière d'agir était, avant l'avènement d'Internet, soit cantonnée aux communications personnelles (écrites ou téléphoniques) ou alors lors des contacts informels entre chercheurs lors des conférences et autres réunions scientifiques (ce qui a donné naissance au concept de collège invisible), avec Internet cette pratique est devenue plus rapide, instantanée et surtout permettait de partager des données très volumineuses entre plusieurs chercheurs et ce pratiquement en temps réel. Cette expérience réussie ${ }^{*}$ a été suivie par d'autres exemples dans d'autres spécialités à l'instar de Cogprints en sciences cognitives, SciELO qui elle a essayé de " déghettoïser " la recherche sud américaine surtout du point de vue linguistique où un chercheur hispanophone ou lusophone n'avait pas la même exposition que le chercheur anglophone.A la même période, des tentatives surtout nord américaines d'archives principalement en sciences médicales et biomédicales comme E Biomed/PubMed Central, Public

\footnotetext{
* Preuve en est ces soumissions en constante augmentation de jour en jour - à titre d'exemple entre la date citée plus haut -24 Janvier 2012- et le jour d'après il y a eut une différence de 472 dépôts vu qu'il y avait 730819 publications électroniques déposées. Comme nous l'avons vu plus haut en date du 23 novembre 2008, il y avait 508,978 e-prints, alors que l'archive avait dépassé le cap des 500000 e- prints en date du 3 Octobre 2008.D'un autre coté et afin d'exemplifier la rapidité avec laquelle une soumission est mise sur le site, Astrophysical Models of r-Process Nucleosynthesis: An Update par Yong-Zhong Qian qui avait été envoyée en date du 24 Janvier 2012 à 20:54:08 GMT était sur le site le 25 Janvier 2012 tôt le matin.C'est dire que l'information est relayée en temps réel.
} 
Library Of Science (PLOS) et BioMed Central furent lancées. Bien que soutenues par de puissants lobbys, ces tentatives n'eurent pas le succès escompté et ce pour de multiple raisons dont entre autres le lobbying des puissants éditeurs ( qui, pour arriver à leur fin, engagèrent un certain Eric Dezenhall, connu sous le surnom du " Pit Bull des relations publiques " et dont la mission était d'attaquer et de garder sur la défensive les propositions des militants du libre accès ${ }^{(224)}$.Il utilisa de formules choc telles que " l'accès publique équivaut à la censure gouvernementale ". Selon Peter Suber, Dezenhall demanda entre 500000 et $600000 \$$ pour un période de six mois de travail ${ }^{(25)}$. C'est dire l'importance de la mission qui lui a été assignée) mais aussi - et peut être plus - à cause de la nature des informations non contrôlées que contiendraient ces archives .En effet, contrairement aux sciences physiques, mathématiques, informatique où l'information ne peut conduire à des dégâts sur la santé publique, une information non contrôlée en médecine peut elle conduire à de dangers sachant l'extrême facilité et rapidité avec laquelle les informations circulent sur Internet. De cette vérité, découle le pourquoi du succès et la perduration de certaines archives dans certaines spécialités (notablement ArXiv) et le relatif échec dans d'autres spécialités (notablement en médecine).

Toujours est-il que ces actions pionnières étaient le fait de chercheurs et de militants (on dira même de chercheurs militants) qui avaient vu en l'avènement d'Internet une solution à un problème lancinant qui ne faisait que s'aggraver. Les Harnad, Ginsparg, Guedon, Eisen, Varmus, Van de Sompel, etc... ne pouvaient, malgré toute la hargne et les efforts qu'ils mettaient dans leurs actions, faire changer les choses n'était ce la couverture, l'aide et le support d'organisations. La première de ces actions fut le fameux Appel de Budapest en 2003. Financée par le richissime financier américain d'origine hongroise Richard Soros, elle se tient dans la capitale hongroise et est considérée comme le texte fondateur du libre accès car non seulement elle a bénéficié du support financier de Georges Soros et sa fondation " Open Society Foundation ", mais aussi de la présence des bibliothèques dont le rôle dans le libre accès est très prépondérant. Etant la première de ces réunions, elle définit le libre accès et surtout ces deux " stratégies complémentaires " qui sont l'auto archivage et la publication en libre accès dans les "revues alternatives ".Si l'Appel de Budapest a lancé le libre accès à la fin de l'année 2001, l'Appel de Bethesda au début de 2003 en a été la continuation et aussi le complément. S'étant tenu dans la ville médicale de Bethesda où les spécialités en sciences biomédicales se sont réunis pour discuter des problèmes spécifiques à leur profession, elle a un peu plus décrit en détails les objectifs, les moyens et les différents groupes composant le monde du libre accés ( à l'instar des différents groupes de travail à qui a été assignée des tâches à accomplir chacun dans son domaine ).Le dernier des trois appels (connus aussi sous l'appellation des " 3 B ") est l'Appel de Berlin qui s'est tenu à Berlin ( suivi en cela par des conférences annuelles sur le libre accès portant chacune le nom de Berlin suivi par un chiffre. La dernière en date est Berlin 12 qui s'est tenu à Stellenbosch en Afrique du Sud) Cette réunion se distingue par rapport aux autres Appels ,en plus du fait qu'elle est devenue une réunion annuelle, par le fait qu'elle étend le libre accès à tous les domaines du savoir étant donné qu'elle englobe dans son intitulé "...[ les ] sciences exactes, sciences de la vie, sciences humaines et sociales ".Cette précision est importante en ce sens qu'elle couvre toutes les spécialités et surtout qu'elle étend le libre accès aux donnés brutes, les documents graphiques et multimédia et à l'héritage culturel. De plus elle est signée par des institutions (plus précisément des bibliothèques) ce qui lui donne plus de poids par rapport aux signatures individuelles qui elles n'engagent que les personnes. Ces trois appels devenus les textes fondateurs du libre accès (suivi en cela par d'innombrables autre appels tels que The Declaration of Havana, The Bonn statement, The Valparaiso Declaration for Improved Scientific Communication in the Electronic Medium, The Göttingen Declaration on Copyright for Education and Research et l'Appel de Riadh entre autres ) se sont, en fait, transformé d'Appels en des forums où l'avenir de la publication scientifique numérique avec toutes ses facettes est discutée. D'ailleurs, l'Appel de Berlin s'est transformé depuis en une conférence annuelle (avec chacune son thème) où les plus grands chercheurs du libre accès se retrouvent pour discuter des derniers développements.

${ }^{(224)}$ Giles , Jim, PR’s Pit Bull takes on open access, Nature, n445, p.347, 25 Janvier 2007

${ }^{(225)} \mathrm{http}: / /$ www.earlham.edu/ peters/fos/timeline.htm (accédé en date du 25 Janvier 2012) 
Au niveau national ou gouvernemental, deux pays dominant le monde de l'édition et de la publication scientifique ont été parmi les premiers à mettre au point des législations allant dans le sens des nouvelles donnes que le libre accès a induit. Les Etats Unis et la Grande Bretagne, forts d'une industrie de l'édition florissante ont voulu passer des législations encadrant, encourageant et accompagnant le libre accès. Malheureusement, la puissance des lobbys de l'industrie de l'édition a fait diluer la portée des propositions surtout pour les Etats Unis où le projet des NIH a été oblitéré de sa portée au sujet du degré de latence (appelé aussi barrière mobile), porté de six à douze mois résultant en la quasi nullité du libre accés. En effet, il est de notoriété que l'information médicale et biomédicale (surtout quand il s'agit de certaines maladies pandémiques telles que le SIDA) devient vite obsolète et est plutôt souvent source de survie pour certains malades et maladies. Les grandes maisons d'éditions anglo saxonnes ont su manœuvrer pour faire de ces décisions, pourtant d'utilité publique, des décisions vides de portée et sens en repoussant les délais de publication de recherches pourtant financées publiquement .Il faut dire que dans ces pays, et surtout aux Etats Unis, la puissance financière des multinationales de l'information est tellement grande qu'elle peut même faire passer l'appât du gain avant la santé des citoyens.

Malgré toutes ces péripéties, qu'on pourrait considérer de normales dans un medium qui n'est entré que dans sa deuxième décennie dans sa forme actuelle, le libre accés a accompli des progrès tels et est devenu un sujet qu'il est pratiquement impossible de ne pas l'aborder dans le monde des bibliothèques et de la documentation. Cette augmentation exponentielle de site d'archives ouvertes est telle qu'il n'est plus possible d'avoir un chiffre non pas exact mais approximatif de ces archives et, même plus, de pouvoir les gérer. A partir de là, s'est imposée l'inéluctabilité de créer des outils à même de rassembler et de mettre à la disposition des chercheurs un ensemble des sites (généralement apparentés par le sujet ou tout autre critère) dans une même adresse fin de faciliter la recherche. Les entrepôts d'archives ouvertes sont une conséquence directe de cette énième explosion documentaire induite par les réseaux. Si " The Core Metalist of open access Eprints archives " est le premier de ces entrepôts d'archives regroupant un nombre d'archives ouvertes en libre accés dans différentes spécialités, on peut considérer ERIC (Education Resources Information Center) ${ }^{(26)}$ comme étant la première forme d'entrepôt en libre accés de matériels éducatifs. De toute évidence, cette base de données datant des années 60 (plus exactement 1966), elle ne peut être classée avec une archive ouverte dans le sens que prend ce terme de nos jours. Néanmoins, force est de reconnaître que de par sa structure, sa philosophie, son contenu et sa couverture, elle s'y apparente. Quant à " The Core Metalist of open access Eprints archives ", on peut le considérer comme le premier exemple ou génération de ces entrepôts d'archives dont le but est d'essayer de rassembler différentes archives que la Toile a produit dans un seul site au lieu d'être dispersées dans différentes archives. Cet entrepôt multidisciplinaire a cessé d'être mis à jour en date du 30 Juin 2003 sûrement à cause de l'impossibilité de continuer à recenser des références en constante augmentation et qui deviennent au bout d'un certain moment tout simplement ingérable. L'exemple de " Timeline of the Open Access Movement " (qui était connu aussi sous l'appellation de " Timeline of the Free Online Scholarship Movement ") est à ce sens très symptomatique des difficultés que traverse le mouvement du libre accès à recenser d'une manière exhaustive ses ressources. En effet, son concepteur, Peter Suber, a décidé de transférer, et ce en date du 9 Février 2009,cette liste pourtant très complète et très consultée, car elle recensait tous les événements qui avaient trait au libre accès, à un " wiki " appelé Open Access Directory ${ }^{(227)}$ " où il est ouvert à toute la communauté [des chercheurs] pour l'éditer ".Cette manière de procéder est une sorte d'aveu d'impuissance à gérer des ressources dont la croissance est plus que décuplée par les fonctionnalités de plus en plus puissante et innovante de l'outil informatique. On assiste en fait à une sorte d'explosion documentaire à l'envers dans le sens où l'outil informatique supposé, de par ses fonctionnalités de rapidité, d'ubiquité, de capacité de stockage et facilité d'utilisation, faciliter la recherche a créé tellement de ressources qu'il est devenu impossible de les gérer. Cet aspect mérite d'être étudié étant une des nouvelles (et inattendues) conséquences de la prolifération et de la puissance de l'outil informatique et surtout Internet.

(226) $\mathrm{http}: / / \mathrm{www}$.eric.ed.gov/

(227) $\mathrm{http}: / /$ oad.simmons.edu/oadwiki/Timeline 
Quant aux autres entrepôts tels que DOAR et ROAR on peut sans risques de se tromper, dire qu'ils rendent à la communauté des chercheurs d'immenses services de par leur richesse et leur facilité d'utilisation. Ils permettent, en effet, de regrouper en un seul site un nombre d'archives qui auraient été autrement repartis sur des centaines si ce n'est des milliers de sites. Leurs différentes fonctionnalités que l'on a eu l'occasion de voir sont une mine d'information pour les chercheurs, les organismes de financements, les institutions d'enseignement.

Le site SHERPA et ses deux rubriques RoMEO et JULIET sont de ce fait deux indispensables outils dont le but est d'aider et orienter et le chercheur et les organismes de financements dans leurs rapports quotidiens et continus avec un medium qui est, comme on l'a vu, nouveau et induisant de ce fait des nouvelles approches. Si SHERPA RoMEO sert beaucoup plus le chercheur à se retrouver et à se positionner dans les dédales du libre accès par rapport aux droits qu'il a sur sa publication et ceux l'éditeur, droits qui ne sont plus aussi clairement définis et clarifiés que dans l'univers papier, la deuxième rubrique elle concerne beaucoup plus un aspect financier et de principes étant donné qu'elle clarifie la position des différents organismes financeurs quant à leur position par rapport aux financements qui permettent à la recherche de voir le jour. RoMÉO et JULIET sont donc deux compléments dont le chercheur et les organismes investis dans la recherche ne peuvent s'en passer sans risque d'être dépassés. Malgré cela, nous devons avouer une sorte de confusion face aux différentes informations et positions des différents protagonistes, confusion que nous jugeons personnellement, préjudiciable à la bonne circulation de l'information mais aussi (peut être sciemment) avantageuse aux éditeurs. Ceci est plausible étant donné que le statu quo les arrangeraient et que ces nouvelles fonctionnalités dont le but le premier est d'informer, d'expliciter ne sont pas à leur avantage. Il existe un foisonnement extraordinaire de sites, de législations, de positions, d'approches et de propositions tels qu'il devient quasiment impossible de se retrouver dans les différentes situations que les nouveaux schémas ont induit.

Quant au deuxième volet du libre accès celui concernant les périodiques électroniques et appelé route dorée ( golden road ), il est beaucoup plus prisé, utilisé et connu que les archives ouvertes appelées aussi route verte ( green road ).En effet, il est beaucoup plus gratifiant et rapide (personnellement et surtout professionnellement) de publier dans une revue que de voir son travail pratiquement " balancé " et comme nous l'avons vu ,noyé dans un medium non seulement méconnu mais aussi dont la fiabilité quant au référencement bibliographique est sujet - à tort ou à raison - à caution. D'un autre coté, il semblerait que les archives ouvertes souffriraient d'un manque de confiance de la part des chercheurs étant donné le manque - encore une fois à tort ou à travers - de certification de l'information contrairement aux périodiques où un comité éditorial juge de la pertinence, la scientificité, l'originalité, l'apport ou ajout au corpus de la spécialité et la méthodologie de la soumission avant de la publier. Il faut dire que ce modèle (archives ouvertes) est beaucoup plus usité dans la communauté des chercheurs en physiques (dont est issu ArXiv, la première archive ouverte toujours en activité) qui elle n'a pas besoin d'un contrôle aussi stricte car ce contrôle est opéré en amont et non pas en aval.Tous ces données ont fait des journaux électroniques des outils plus acceptés et surtout connus dans le monde de la recherche scientifique que les archives ouvertes. Si contrairement aux archives ouvertes, les périodiques électroniques sont plus acceptés, connus et surtout intériorisés, il n'en demeure pas moins que le chercheur se trouve confronté au problème de leur nombre. En effet, il est quasiment impossible (pour ne pas dire tout simplement impossible) de connaitre leur nombre. Des chercheurs aussi chevronnés que Carol Tenopir ou Donald King ont reconnu cette vérité face à ce que Tenopir a appelé " une cible mouvante et des fois suspecte ". Cet état de fait est dû à plusieurs raisons dont les plus importantes et les plus plausibles pourraient se résumer à :

-la relative nouveauté du medium (surtout par rapport au livre)

-la définition d'un périodique

-l'extrême foisonnement du support 
-les problèmes de changement de titres, de scission, d'absorption, de fusion, de cessation de parution, de reparution sous un autre titre ou forme, de changement de périodicité, etc....

Malgré tous ces problèmes, le périodique électronique est entré dans les mœurs et les habitus des chercheurs. Il est devenu inconcevable pour le chercheur de nos jours de ne pas accéder depuis son ordinateur à ses revues scientifiques favorites, de ne pas lire ses articles sur ordinateur ou de ne pas soumettre son article par le biais de la messagerie électronique. Ce changement radical, et sans doute irréversible, a même induit de nouvelles manières de citer les références et d'accéder à l'information. De ce fait, alors qu'il était plus que suspect de citer une référence ou un lien électronique ceci et devenu très usité et même dans certain cas la norme (quoique les liens inopérants et si souvent rencontrés et par le fameux 404 pose le problème de la pérennité et l'archivage de l'information).Malgré ces impondérables, le périodique électronique a accompli ce vingt dernières années des progrès et des avancées qui auraient été décrit de futuriste tellement ils auraient été impossible à réaliser ( ni même imaginer ) il y a de cela quelques années .Qui aurait , par exemple, imaginé qu'un article puisse contenir une vidéo de l'expérience ou de l'opération qui serait disponible avec l'article ou alors les sons d'une quelconque espèce d'oiseaux et qui serait intégrés au texte. Toutes ces fonctionnalités et innovations sont maintenant possibles avec les technologies qu'offre Internet.

Si dans DOAJ et AJOL, les deux exemples que nos avons cité ces technologies ne sont pas offertes, il n'en demeure pas moins qu'ils représentent les deux meilleurs exemples de site recensant les périodiques scientifiques contrôlés par les pairs que recèle la Toile. Nous insistons sur le vocable et l'appellation périodiques scientifiques car il existe d'autres sites (dont Ullrich's, NewsJour) qui eux recensent toutes publications à caractère périodique. Ces deux sites bien que de couverture différentes (l'un est international et l'autre régional ou continental) n'en demeure pas moins des références étant donné que le premier recense 7454 titres dont 3586 en texte intégral et un total de 746610 articles et que le deuxième recense 417 titres dont 59566 articles en texte intégral et ce en date du 02 Février 2012.Comme on peut le voir, ces deux sites ( bien que dans un registre et à un niveau différents) regroupent dans un seul site ( comme dans le cas de DOAR et ROAR ) un nombre assez important de périodiques scientifiques et contrôlés par les pairs. Les deux sites se distinguent aussi par une constante et continue augmentation du nombre des périodiques recensés ainsi que l'addition de nombreuses fonctionnalités facilitant la recherche (langues, spécialités, localisation des visiteurs, etc....) et dont l'équivalent dans l'univers papier est tout simplement impossible ( par exemple la localisation des visiteurs et qui se fait par adresse IP n'est pas, de toute évidence, possible sur le support papier ).Toutes ces fonctionnalités, nonobstant certaines qui paraissent à la limite du gadget et triviales, ne sont en fait que le résultat de ce qui est devenu le nouvel article scientifique et technique contrôlés par les pairs, un nouvel article que ne reconnaîtraient pas les premiers philosophes et les premiers " éditeurs " du $17^{\mathrm{e}}$ siècle dont le plus important et plus pressant des besoins était de trouver où publier, faire partager sa découverte à un plus grand nombre, faire parvenir l'information le plus vite possible, notion de rapidité qui de nos jours est non seulement accélérée mais aussi serait complètement inimaginable quelques décennies avant.

Enfin, bien que le libre accès ait été lancé en Occident, il est d'essence universelle de par sa configuration (Internet) et ses ramifications (ouverture universelle du savoir). Malgré cela, nous avons toujours défendu l'idée qu'il (le libre accès) est une aubaine et une occasion pour les pays en développement de sortir de leur déficit informationnel et d'accéder à l'information dont ils ont besoin. Malheureusement, cette opportunité est entravée par un problème technique dont souffrent les dits pays. La fracture numérique en effet, annihile ces avantages car si l'information est présente et surtout orientée et axée vers les pays en voie de développement de par les différents programmes aussi bien nationaux qu'internationaux et qui ambitionnent d'aider les dits pays, elle n'arrive pas à cause d'une bande passante très faible et surtout aléatoire. L'information en libre accès est globalement présente et disponible mais le medium qui la transporte la transmet est inopérant ou en mauvais état. Cet état de fait a pour résultat inattendu d'amoindrir les bienfaits de ces programmes 
et ces efforts. Si ces entraves sont, un tant soit peu, normales dans le contexte des pays en voie de développement qui peinent à assurer le minimum vital pour des raisons connues, le cas de l'Algérie est quant à lui étonnant pour ne pas dire plus. Notre pays se targue d'une santé financière très enviable au point que les dettes des pays les plus pauvres d'Afrique ${ }^{(228)}$ et même arabes ${ }^{(229)}$ ont étés annulées alors que le tout puissant FMI a récemment emprunté à l'Algérie ${ }^{(230)}$. Malgré cette embellie conséquences d'une augmentation du prix du pétrole, la situation d'Internet, devenue une partie et un levier indispensable du développement de toute nation en ce $21^{\mathrm{e}}$ siècle, souffre de dysfonctionnements graves et incompréhensibles. Les chiffres que nous avons présentés se passent de tous commentaires et font partie d'une situation globale * de désintérêt de la part des pouvoirs publiques et politiques qui ne semblent appréhender (ou feignent de le faire) les défis de ce $21^{\mathrm{e}}$ siècle dont les changements sont très rapides et difficiles à rattraper. Ceux en charge de secteurs aussi stratégiques que le Ministère de la Poste et des Technologies de l'Information et de la Communication doivent savoir que l'ère de l'information que nous vivons se caractérise par des changements et des mutations extrêmement rapides sur lesquels il est ( pour utiliser un mot adéquat) impératif de bien surfer afin que la vague porte le secteur vers l'avant plutôt que ne l'engloutisse. Pour cela, il est aussi primordial de faire passer l'intérêt général avant l'intérêt personnel et matériel afin de permettre au pays de profiter des conditions actuelles qui permettent de se développer. Ceci est peut être un vœu pieux mais les conditions requises existent, reste la volonté de le faire.

\footnotetext{
${ }^{(228)} \mathrm{http} / / \mathrm{www}$. afrik.com/l-algerie-annule-la-dette-de-14-pays-africains

${ }^{(229)} \mathrm{http} / /$ www.alterinfo.net/notes/L-Algerie-annule-les-dettes-detenues-sur-1-Irak-et-le-Yemen b5568234.html

(230) $\mathrm{http}: / /$ www.elmouwatin.dz/IMG/article PDF/article a5478.pdf

* Nous pensons ici à la $3 \mathrm{G}$ qui aux dernières nouvelles et tel le personnage de l'Arlésienne, est programmée en Algérie au premier trimestre 2013. Ceci ne prêterait à rire si cette norme (déjà dépassée car le $4 \mathrm{G}$ est déjà opérationnelle dans certains pays) n'était largement disponible dans des pays comme l'Angola, le Bangladesh, le Burundi, le Congo, la Gambie, Haïti, le Kenya, la Corée du Nord, le Malawi, etc.... De plus, certains pays sont directement passés à la 4 G comme le Cameroun, la Jamaïque, le Liberia, le Panama, le Puerto Rico, etc...

Disponible à http://www.worldtimezone.com/gsm.html
} 
Aubry Christine, Janik Joanna (Ed.), Les Archives ouvertes : enjeux et pratiques : guide à l'intention des professionnels de l'information et de la documentation, Paris, Association des professionnels de l'information et de la documentation (ADBS), 333p.

Auh Taik-Sup, Promoting multilinguism on the Internet: the Korean experience http://www.unesco.org/webworld/infoethics_2/fr/summaries.htm

Bjork, Bo-Chrisler ; Roosr, Anniki ; Lauri, Mari, Global annual volume of peer reviewed scholarly article and the share available via differents access options, ElPUB 2008. Open scholarship: authority, community and sustainability in the age of web 2.0- Proceedings of the 12th International Conference on Electronic Publishing, held in Toronto, 25-27 June 2008/ Edited by Leslie Chan and Susanna Mornatti, p.178-186

Disponible à : http://elpub.scix.net/cgi-bin/works/Show?178_elpub2008

Canessa, Enrique, Zennaro, Marco,Ed. Science disemination using open access : a compendium of selected literature on open access, Rome, The Abdus Salam International Centre for Theoretical Physics,2008,207 p.

Disponible à : $\underline{\text { http://sdu.ictp.it/openaccess/SciDissOpenAccess.pdf }}$

Castells Manuels, La Galaxie Internet, Paris, Fayard, 2000, 368 p.

Cerf V., Kahn R., A Protocol for packets network interconnections. IEEE Transactions on communications, Vol. COM-22, p.637-648, May 1974

Chanier, Thierry, Archives ouvertes et publication scientifique: comment metre en place l'accès libre aux résulatas de la recherche ?, Paris, L ' Harmattan,2004, 187 p.

Crawford, Susan Y.,Hurd, Julie M.and Weller, Ann C., From print to electronic: the transformation of scientific communication, Medford [New Jersey], Information Today, 1996, 117p.

Eisenhart, Douglas M., Publishing in the information age: a new management framework for the digital era ,Westport [Connecticut ],Praeger, 1996,306p.

European Commission, Study on the economic and technical revolution of the scientific publication markets in Europe, Brussels, European Union, 2006, 112 p.

Disponible à : http://ec.europa.eu/research/science-society/pdf/scientific-publication-study_en.pdf

Francis André, Libre accès aux savoirs = Open access to knowledge, Paris, Futuribles perspectives, 2005, $74 \mathrm{p}$.

Disponible à : $\underline{\text { www.futuribles.com/fr/viewer/pdf/1772/ }}$

Guédon, Jean-Claude, Repenser le sens de la communication scientifique : l'accès libre, Médecine / Sciences, vol. 24, n 6-7, Juin Juillet 2008, p.642

Greco, Albert, Ed., The State of scholarly publishing : challenges and opportunities, Transactions publishers, New Brunswick [N.J., USA] ; London, 2009, 267p.

Jeanneney Jean - Noël, Quand Google défie l'Europe, Paris, Mille et une nuits, 2006, 347 p

Mackenzi Owen, John Stewart, The Scientific article in the age of digitization, [s.1.:s.n],2005,308 p.

Academic Proefschrift : Science de l'information : Université d'Amsterdam : 2005

Disponible à : http://dare.uva.nl/document/17843 
Negroponté, Nicolas, Being digital,New York,Alfred A. Knopf, 1995,245p.

Peek, Robin P., Newby, Gregory B, Ed., Scholarly publishing : the electronic frontier, Cambridge [Massasuchetts ], MIT Press, 1996, 365 p.

Stallman Richard. Copyright and globalisation in the age of computer network In_Rishab Aiyer Ghosh (Ed.), CODE: Collaborative Ownership and Digital Economy. Cambridge [Mass.]: MIT Press, 2005 , p.317-335

Stix, Gary, The Speed of write, Scientific American, Vol.271, nº6, 1994, p.72

Tenopir, Carol, Online : scholarly journals : how many? Library Journal, 129(2) :2, 2004

Disponible à : http://www.libraryjournal.com/article/CA374956.html

Tenopir, Carol, King, Donald W.,Towards electronic journals : realities for scientists , librarians and publishers, Washington DC ,Special Libraries Association, 2000,489 p.

Willinsky, John, The Access principle: the case for open access to research and scholarship, Cambridge [Massasuchetts ], MIT Press, 2006, 287p.

Willinsky, John, The Nine flavors of open access scholarly publishing, Journal of posgraduate

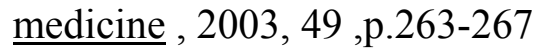

Willinsky John. The Unacknowledged convergence of open source, open access and open science, First Monday, Vol. 8, $\mathrm{n}^{\circ}$ 8, Aout 2005. (Accessible à :

http://first Monday.org/ issues 10-8/Willinsky/index.html

\section{Webographie}

http://www.walthowe.com (consulté le 5 Juin 2008)

www.fsf.org

www.gnu.org

www.linux.org

www.apache.org

http://www.internetworldstats.com/stats.htm

http://arxiv.org

http://cogprints.org/

http://www.scielo.br

http://www.scielo.org/php/index.php?lang=en

www.nih.gov/about/director/pubmedcentral/ebiomedarch.htm

http://www.ncbi.nlm.nih.gov/PubMed/

http://www.nlm.nih.gov/

www.nih.gov/

http://www.plos.org/

http://www.biomedcentral.com/

http://www.mdpi.org/sensors/

http://www.mdpi.org

http://www.soros.org/openaccess/fr/read.shtml

www.earlham.edu/ peters/fos/bethesda.htm

http://openaccess.inist.fr/spip.php?article20

http://oa.mpg.de/openaccess-berlin/berlindeclaration.html

http://oa.mpg.de/openaccess-berlin/BerlinDeclaration_wsis_fr.pdf

http://oa.mpg.de/openaccess-berlin/program echo.html

http://oa.mpg.de/openaccess-cern/index.html 
http://www.eprints.org/events/berlin3/index.html

http://oa.mpg.de/openaccess-golm/index.html

http://oa.mpg.de/openaccess-padua/index.html

http://oa.mpg.de/openaccess-dus/index.html

www.parliament.uk/parliamentary committees/science and technology committee.cfm

http://www.publications.parliament.uk/pa/cm200304/cmselect/cmsctech/399/399we01.htm

http://www.publications.parliament.uk/pa/cm200304/cmselect/cmsctech/399/39902.htm

http://www.publications.parliament.uk/pa/cm200304/cmselect/cmsctech/1200/120006.htm\#a1

http://www.publications.parliament.uk/pa/cm200304/cmselect/cmsctech/1200/120003.htm

http://www.ft.com/cms/s/98f16604-31f4-11d9-97c0-

http://www.biomedcentral.com/

http://www.biomedcentral.com/openaccess/inquiry/

http://www.biomedcentral.com/openaccess/inquiry/myths/

http://www.nature.com/nature/focus/accessdebate/

www.wellcome.ac.uk

http://www.arl.org

http://www.nih.gov

http://www.pubmedcentral.nih.gov

http://opcit.eprints.org/explorearchives.shtml

http://www.dmoz.org/Science/Publications/Archives/Free_Access_Online_Archives/

http://highwire.stanford.edu/lists/largest.dtl

http://www.lib.umd.edu/ENGIN/TechReports/Virtual-TechReports.html

http://viva.lib.virginia.edu/science/guides/s-preprn.htm

$\mathrm{http}$ ://tardis.eprints.org/discussion/eprintarchivessubjecttable9103.htm

http://www.aardvarknet.info/user/subject19/index.cfm?all=All

http://www.ams.org/global-preprints

http://www.cv.nrao.edu/fits/www/yp_preprint.html

http://www.openarchives.org/Register/BrowseSites.pl

http://www.oaforum.org/oaf_db/list_db/list_repositories.php

http://celestial.eprints.org/cgi-bin/status

http://oaister.umdl.umich.edu/o/oaister/viewcolls.html

http://oaister.umdl.umich.edu/o/oaister/viewcolls.html

http://www.myoai.com/search/Search.cgi/LoginForm?Login=guest\&Password=guest

http://www.pkp.ubc.ca/harvester/archives.php

http://oai.dlib.vt.edu/cgi-bin/Explorer/oai2.0/testoai

http://www.arl.org/sparc/core/index.asp?page $=\mathrm{m} 1$

http://www.signal-hill.org/archives/institutions.html

http://repositories.cdlib.org/escholarship/

http://library.caltech.edu/digital/

http://www.osti.gov/bridge/

http://software.eprints.org/\#ep1 (29 archives)

http://software.eprints.org/\#ep 2 (37 archives)

http://front.math.ucdavis.edu/

http://adsabs.harvard.edu/preprint_service.html

http://findemaschine.pro-physik.de/?language=e

http://adsabs.harvard.edu/cfa/preprints.htm

http://www.slac.stanford.edu/spires/hep/

http://citebase.eprints.org/help/coverage.php

http://weblib.cern.ch

http://de.physnet.net/PhysNet/physdoc.html

http://mathnet.preprints.org/

http://www.osti.gov/preprints/ppnbrowse.html

http://ntrs.nasa.gov/

http://www.ncstrl.org/

http://www.ndltd.org/ 
http://www.language-archives.org/index.html/

http://repec.org/

http://ideas.repec.org/

http://econpapers.hhs.se/

http://netec.wustl.edu/NEP/

http://netec.ier.hit-u.ac.jp/CitEc/

http://www.inomics.com/cgi/show

http://ideas.repec.org/archives.html

http://netec.mcc.ac.uk/WoPEc.html

http://netec.mcc.ac.uk/WoPEc/data/PaperSeries.html

http://drcwww.uvt.nl/dbi/instructie/degree/

http://econwpa.wustl.edu/

http://cowles.econ.yale.edu/P/cd/cfdpmain.htm

http://www.kellogg.nwu.edu/research/math/discussion_papers.htm

http://www.hss.caltech.edu/ss/working-papers/recent

http://econweb.sscnet.ucla.edu/research/workingp.htm

http://swopec.hhs.se/

http://dois.mimas.ac.uk/

http://rclis.org/about.html

http://www.biomedcentral.com/start.asp

http://pubmedcentral.nih.gov/

http://highwire.stanford.edu/lists/freeart.dtl

http://www.intlpress.com/journals/ATMP/

http://www.bbsonline.org/

http://psycprints.ecs.soton.ac.uk/

http://www.arXiv.org/

http://citeseer.nj.nec.com/cs

http://gunther.smeal.psu.edu/

http://www.math.uiuc.edu/K-theory/

http://at.yorku.ca/topology/preprint.htm

http://www.math.uiuc.edu/Algebraic-Number-Theory/

http://rene.ma.utexas.edu/mp_arc/index.html

http://hopf.math.purdue.edu/

http://www.math.ntnu.no/conservation/

http://www.statslab.cam.ac.uk/ mcmc/index.html

http://mathematik.uibk.ac.at/mathematik/jordan/index.html

http://www.math.uga.edu/archive.html

http://www.cs.bgu.ac.il/research/Fields/

http://casper.cs.yale.edu/mgnet/www/mgnet-papers.htm

http://cogprints.ecs.soton.ac.uk/

http://eprints.rclis.org/

http://dlist.sir.arizona.edu/

http://clinmed.netprints.org/home.dtl

http://www.chemweb.com/preprint

http://www.compscipreprints.com/comp/Preprint/show/index.htt

http://www.mathpreprints.com/math/Preprint/show/

http://htpprints.yorku.ca/

http://www.leeds.ac.uk/educol/

http://www.ssrn.com/

http://archivesic.ccsd.cnrs.fr/

http://www.eccc.uni-trier.de/eccc/

http://eprint.iacr.org/

http://dlc.dlib.indiana.edu/

http://orgprints.org/

http://repositories.cdlib.org/uciaspubs/ 
http://formations2.ulst.ac.uk/

http://www.nceas.ucsb.edu:8504/esa/ppr/ppr.Query

http://philsci-archive.pitt.edu/

http://www.opendoar.org/

www.dspace.org

http://www.eprints.org

http://bepress.com

http://www.bath.ac.uk/library/eprints/index.html

http:// www.wildfirehq.org

http://scholar.lib.vt.edu/ETD-db/index.shtml

http://www.unisg.ch/hsgweb.nsf/wwwPubhomepage/webhomepageger?opendocument

http://memsic.ccsd.cnrs.fr/

http://architektur-informatik.scix.net/cgi-bin/works/OAI

http://pkp.sfu.ca/biblio

www.soros.org/

www.jisc.ac.uk/

www.sparceurope.org/

http://www.google.com/coop/cse/

http://roar.eprints.org

www.uiuc.edu/

http://www.openarchives.eu/

http://ulrichsweb.com/ ulrichsweb

http://scientific.thomson.com/

http://www.doaj.org

http://www.ajol.info/

http://www.inasp.info/

http://clinmed.netprints.org/cgi/content/full/2000010008v1

http://www.nepjol.info

http://www.banglajol.info/

http://www.vjol.info/

http://www.philjol.info/

http://www.sljol.info/

http://www.sherpa.ac.uk/

http://www.sherpa.ac.uk/romeo 


\section{TROISIEME PARTIE}

\section{LIBRE ACCES ET CONTROLE PAR LES PAIRS}




\section{CONTROLE PAR LES PAIRS ET LIBRE ACCES}

\section{Introduction}

La question (problème selon certains) du contrôle par les pairs des publications scientifiques et techniques est assez récente ( quoique "The Philosophical Transactions " fondé en 1665 comportait une clause imposant un contrôle par les pairs ${ }^{*}$.En effet, au début, les périodiques qui avaient remplacé le livre n'étaient pas nombreux et les savants qui pouvaient y contribuer aussi. De ce fait, la fonction de tamis que joue le contrôle par les pairs de nos jours (de par la qualité et le nombre) n'était pas présente En fait, c'était plutôt le contraire qui se passait et qui faisait que les éditeurs (ou ce qui étaient considérés comme les éditeurs tels qu'on les connait de nos jours) publiaient pratiquement tout qui était soumis sans contrôle aucun et, dans certains cas, sollicitaient les auteurs pour remplir leurs journaux. A cet effet, Ray Spier dit : "A partir du milieu des années 1800, il y avait plus d'espace de journal que d'articles à imprimer. Quand les journaux mettaient au point un comité d'éditeurs assistants, leur première responsabilité était d'attirer des articles et des expertises afin de remplir les pages de la publication. Le contrôle par les pairs, consista alors pour les cent années à venir à publier les opinions des éditeurs, renforcés quand nécessaire par des comités spéciaux, mis en place par les sociétés [savantes] afin d'évaluer les manuscrits envoyés ${ }^{(1)}$ ". C'est le long, continuel et exponentiel accroissement du nombre des chercheurs et des publications, et que Derek De Solla Price à très bien décrit ${ }^{(2)}$ qui a imposé un ralentissement du nombre de publications et une sélection de la qualité de qui était publié. A titre d'exemple, et pour situer ce passage de la science vers la supra science (le tire exact de la traduction de cet ouvrage de référence est " science et supra science " ) et surtout son extrême concentration au $20^{\mathrm{e}}$ siècle, De Solla Price a calculé qu'entre 80 et $90 \%$ des savants ayant vécu étaient vivants en $1963^{(3)}$, que la taille brute de la science en effectifs ou en publication doublait chaque $10 / 15$ ans ${ }^{(4)}$, que le nombre de périodiques scientifiques se situait aux environs de 50000 dont 30000 encore publiés qui publiaient quelques 6000000 d'articles avec une augmentation d'au moins 500 000 articles par an ${ }^{(5)}$.Enfin, il existait à l'époque aux Etats Unis aux environs d'un million de scientifiques. Ce chiffre a été atteint de la manière suivante : il était de 1000 savants en 1800 , 10000 en 1850, 100000 en 1900 pour atteindre enfin 1000000 dans les années 60(date de l'étude) ${ }^{(6)}$. Comme on peut le voir, l'étude de De Solla Price fait ressortir cette accélération extraordinaire qui a eu lieu au milieu du siècle précédant et qui a imposé une limitation quantitative (et par delà qualitative) des publications que les moyens de traitement de l'information n'arrivaient pas (et n'arrivent toujours pas) à gérer et juguler. Plus récemment,

\footnotetext{
* Le premier numéro de Philosophical Transactions qui parut le 15 Mars 1665 stipulait par l'intermédiaire du Conseil des Philosophical Transactions ce qui suit :...." Que les Philosophical Transactions soient composées par Mr. Oldenburg, imprimées le premier lundi de chaque mois, si nous avons assez de matériel pour le faire et que la brochure soit autorisée par le même Conseil [des Philosophical Transactions] " étant premièrement revue par quelques membres de la même institution

${ }^{(1)}$ Spier, Ray, The History of the peer review process in science, Trends in biotechniology, Vol.20, no $8 \mathrm{p} .357-$ 358-8 August 2002

(2) DeSolla-Price, Derek, Little science, big science, Columbia University Press, New York, 1963, XVI-119p.

${ }^{(3)}$ DeSolla-Price, Derek, op.cit. p.1

(4) DeSolla-Price, Derek, op.cit. p.6

${ }^{(5)}$ DeSolla-Price, Derek, op.cit. p. 8

${ }^{(6)}$ Idem
} 
une autre étude ${ }^{(7)}$ a pointé du doigt les mêmes dysfonctionnements de la même fonction et l'inflation qui caractérise le monde de la publication scientifique .Cette étude a conclu aux chiffres suivants :

\begin{tabular}{|l|c|c|}
\hline Paramètre de modèle de coût & 1975 & 1995 \\
\hline Nombre de numéros & 6.5 & 8.3 \\
\hline Nombre d'articles par titre & 85 & 123 \\
\hline Nombre de manuscrits soumis & 90 & 205 \\
\hline Nombre de page articles & 630 & 1439 \\
\hline Nombre de pages de graphiques spéciaux & 114 & 260 \\
\hline Nombre de pages total & 820 & 1728 \\
\hline Nombre (moyen) d'abonnements & 2900 & 1900 \\
\hline
\end{tabular}

Tableau n9-Paramètres de publication des journaux scientifiques ( 1975-1995)

On remarque que ce tableau sur une période de vingt ans démontre et abonde dans le même sens que l'étude de De Solla Price. En effet, des sept paramètres examinés, six voient une augmentation assez importante et c'est:

- Le nombre de numéros publiés qui augmente de $78.31 \%$

- $\quad$ Le nombre d'articles par titre qui augmente de $69.10 \%$

- $\quad$ Le nombre de manuscrits soumis qui augmente de $227.77 \%$

- $\quad$ Le nombre de page articles qui augmente de $228.41 \%$

- Le nombre de pages de graphiques spéciaux qui augmente de $228.07 \%$

- $\quad$ Le nombre de pages total qui augmente de $210.73 \%$

Seul le paramètre des abonnements est en baisse de $65.51 \%$. Cette " anomalie " dans un tableau où tous les indicateurs vont dans le même sens est à notre avis très compréhensible et même logique. En effet, on pourrait expliquer ceci par un paramètre que cette recherche essaye d'examiner et il concerne la crise des prix des périodiques qui a sérieusement grevé le budget des bibliothèques (et des chercheurs) et a induit cette baisse du nombre d'abonnements. D'un autre coté, on pourrait supposer aussi que face aux nouveaux schémas de distribution de l'information ( nommément le libre accès sous ses différentes formes et

\footnotetext{
(7) Tenopir, Carol and King Donald W.,Towards electronic journals : realities for scientist, librarians and publishers, Special Libraries Association, Washington, DC, , 2000, Cité par Miller Cass, T.and Harris, Julianna C.,Conflicting agenda for scholars, scholars, publishers and institutions, p.19 In The State of scholarly publishing : challenges and opportunities, Greco, Albert N. Ed. Transactions Publishers, New Brunswick [ N.J., USA] ,2009, 254p.
} 
l'omniprésence d'Internet) ont aussi influé sur cette baisse des abonnements, l'information étant (en principe) plus disponible sur les réseaux.

La science étant par définition élitiste ou du moins méritocratique, la sélection s'est faite sur la base de la qualité des publications soumises qui a donné naissance à ce qui est connu sous l'appellation générique du contrôle par les pairs. Nous tenons à signaler que si l'expression " contrôle par les pairs" est la plus usitée pour décrire le processus, nombre d'autres expressions, appellations sont utilisées telles que certification, évaluation, révision par les pairs et aussi évaluation ou validation scientifique et enfin validation du contenu par un comité d'experts ${ }^{(8)}$. Par contre en langue anglaise, l'expression" peer review " est de loin l'expression consacrée et la plus utilisée et ceci est dû, à notre avis, au fait que le sujet est beaucoup plus abordé et documenté en langue anglaise qu'en langue française. D'ailleurs, au cours de notre recherche nous n'avons pas trouvé un seul ouvrage spécifiquement dévolu au sujet alors que les ouvrages en langue anglaise, bien que peu nombreux, étaient présents. Quant aux articles de périodiques, là aussi la prépondérance de la langue anglaise est très visible. La seule explication que nous pouvons avancer, étayée par des chiffres, est que le monde anglo-saxon a dominé (et domine toujours) le monde et de la publication et de l'édition scientifique et technique et ce au sortir de la deuxième guerre mondiale et l'émergence des Etats Unis d'Amérique comme première puissance mondiale. D'un autre coté, (mis à part Elsevier qui constitue un cas dans le monde la publication scientifique et technique de par ses moyens et sa tentaculaire domination) les plus grandes maisons d'éditions sont anglaises ou nord américaines ce qui expliquerait qu'elles dominent le marché de la publication scientifique et technique et par delà la documentation sur le sujet. Nous aborderons donc dans cette partie du travail l'histoire du contrôle par les pairs, ses premières formes, comment il s'est imposé, ses tenants et aboutissants, ses critiques et ses supporteurs, ses points faibles et ses points forts ainsi que les différentes enquêtes, études et autres recherches ayant essayé de voir quelle est la place du contrôle par les pairs dans la diffusion de la Science. Nous essayerons à la lumière de ce tour d'horizon de répondre à la question centrale qui sous tend notre travail et qui est: ce que le contrôle par les pairs est changé à l'ère d'Internet et du libre accès sous toutes ses formes ?

\footnotetext{
${ }^{(8)}$ http://openaccess.inist.fr/spip.php?mot28
} 


\section{Chapitre I}

\section{PREMIERES MANIFESTATIONS, FORMES ET EVOLUTION DU CONTROLE PAR LES PAIRS}

\section{1-Premières formes du contrôle par les pairs}

" Dans le sens le plus large du terme, on peut dire que le contrôle par les pairs a existé depuis que l'Homme a commencé à identifier et communiquer ce qu'il pensait être du savoir nouveau. Ceci parce que le contrôle par les pairs (qu'il soit post ou pré publication) est partie intégrale et essentielle $\mathrm{du}$ processus de construction du consensus et est inhérent et nécessaire au développement du savoir scientifique ${ }^{(9)}$. Le contrôle par les pairs a donc anti daté les périodiques et autres manifestations scientifiques et a en fait débuté avec le Savoir partagé de l'Humanité et le besoin de le valider. Comme il a été déjà dit, la trace la plus ancienne et documentée d'un quelconque contrôle de l'information scientifique par les pairs tel qu'il est connu de nos jours est dans le premier numéro de The Philosophical Transactions of the Royal Social of London en 1665 qui dans son premier numéro énonçait que :" ...that the Philosophical Transactions to be composed by Mr.Oldenburg, be printed the first Monday of every month, if we have sufficient matter for it and that the tract be licensed by the Council , being first reviewed by some members of the same " (c'est nous qui soulignons ).Par contre, Dennis De Sallo, le premier éditeur du Journal des Sçavans, lancé deux mois avant The Philosophical Transactions n'avait pas lui cette prétention de garantir ni de contrôler l'information mais tout simplement la signaler. Il avait déclaré :" Nous aspirons à rendre comptes les idées des autres sans les garantir ${ }^{(10)}$.En fait, il est plus juste de dire que la pratique telle qu'on l'a connaît de nos jours date de plus tard, exactement en 1731, et "Medical Essays and Observations" une publication de "The Royal Society of Edinburgh"(11).

De son coté, Ray Spier situe la première description documentée d'une forme de contrôle par les pairs au livre de Ishak Ibn Ali Arrahwi (854-931) de la ville Erraha en Syrie intitulé " Ethique d'un médecin ".Il y décrit le premier processus de contrôle par les pairs médical. Le médecin consultant doit toujours faire des doubles des notes de la condition du patient après chaque visite. Après la guérison ou le décès du patient, les notes du médecin étaient examinées par un conseil médical local composé d'autres médecins qui les contrôlaient pour décider est ce que son exercice répond aux critères requis pour la pratique médicale. Dans le cas leur expertise était négative, le médecin pouvait être poursuivi par un patient mal traité ${ }^{(12)}$. Kronick, dans un des articles ayant abordé ces premières manifestations du contrôle par

\footnotetext{
(9) Kronick D.A., Peer review in 18th-century scientific journalism, Journal of the American Medical Association, Vol. 263, $\mathrm{n}^{\circ}$,p. 1321-1322, 1990

${ }^{(10)}$ Drummond, Rennie, Editorial peer review: its development and rationale,p. 2 In :Peer review in health sciences, Goodlee, Fiona and Tom Jefferson Ed., BMJ Books,London, 2003, VIII-392p.

(11) Katzen, M.F., The Changing appearance of research journal in science and technology: an analysis and case study, p.184 In: Meadows, A.J. Ed., Development of science publishing in Europe, Amsterdam, Elsevier, IX 269 p.

(12) Spier, Ray, op.cit.
} 
les pairs affirme que ...." quoique les débuts du " contrôle par les pairs " sont très souvent associés avec la Royal Society of London lorsqu'elle assuma officiellement la responsabilité des Philosophical Transactions en 1752, des antécédents des pratiques du contrôle par les pairs remontent au $17^{\mathrm{e}}$ siècle. Malgré les différences dans les environnements académiques, sociaux, intellectuels et économiques, entre le $18^{\mathrm{e}}$ siècle et le présent, il existe des similarités instructives et intéressantes dans la manière par laquelle les premiers éditeurs exerçaient et définissaient leurs prérogatives et leurs responsabilités. Ces préoccupations s'étendent aux idées et pratiques qui ont une relation très étroite avec ce qui est connu de nos jours sous l'appellation du contrôle par les pairs ou arbitrage" ${ }^{(13)}$.Kronick, dont l'expertise et l'étendue des publications sur le sujet (les premiers périodiques scientifiques du $17^{\mathrm{e}}$ et $18^{\mathrm{e}}$ siècle) fait référence, affirme dans une autre contribution ce qui suit: "Certaines des pratiques éditoriales, telles que le contrôle par les pairs, commencèrent dans les méthodes que ces premières sociétés savantes inventèrent pour accepter les communications à publier. Booth argumente que la Royal Society of London introduisit la première le concept d'arbitrage en mettant en place un comité pour examiner toutes les recherches avant qu'elles ne soient publiées dans Philosophical Transactions. Il y avait cependant plusieurs antécédents à cette pratique. Oldenburg filtrait les communications qui devaient être présentées à la Royal Society, mais après que les recherches aient étés lues, elles étaient encore une fois réexaminées par des Membres. Au début de son histoire, l'Académie des Sciences de Paris mis en place des comités de sélection afin de déterminer si un membre pouvait ou pas publier sous ses auspices. Le contrôle par les pairs tel qu'on le connait généralement de nos jours est décrit dans la préface de l'édition française de " Medical essays and observations " publiée par une certaine " Society in Edinburgh" en 1731.Les recherches soumises, il y est dit, sont distribuées selon le contenu de leurs sujets aux membres les plus versés dans le sujet afin qu'ils les expertisent. Elle spécifie aussi que l'identité de celui qui expertise la recherche ne doit pas être connue de l'auteur, un des premiers exemples du principe controversé de l'anonymat de l'expert * .La Société Royale de Médecine, juste après sa fondation en 1776 inaugura un système par lequel deux membres examinaient chaque recherche soumise à la Société et qui fournissaient un résumé et une critique aux autres membres. La validation du

\footnotetext{
${ }^{(13)}$ Kronick, David A., Litterature of the life sciences : historical background, Bulletin of the New York Academy of Medicine, $\mathrm{Vol} 60, \mathrm{n}^{\circ}$, p.869-870,9 Novembre 1988

*Nous verrons un plus tard dans ce travail que le problème de l'anonymat de l'expert a engendré un nombre de recherches et dont les résultats sont, le moins que l'on puisse dire, contradictoires. En effet, il ne semble pas qu'il y ait une position claire quant aux conséquences de l'anonymat ou pas (ce qui appelé open peer review vs blind peer review- contrôle par les pairs ouvert ou contrôle par les pairs à l'aveugle) de l'expert. La même chose peut être dite en ce qui concerne l'anonymat de l'auteur. Là aussi les différentes recherches quant à garder le nom de l'auteur secret ou pas n'ont pas donné des résultats penchant vers une position ou une autre. Enfin, une dernière variante consiste à garder le nom de l'auteur et de l'expert secret (double blind peer review ou contrôle par les pairs double aveugle) Ces différentes variantes sont encore plus aggravées par le fait que le contrôle par les pairs se fait dans certaines spécialités (telle que la physique) sur Internet, est ouvert à toute la communauté et, dans certaines cas, en temps réel. Ces fonctionnalités et leurs conséquences sur la qualité du contrôle par les pairs ne sont pas encore connues étant donné qu'Internet, vecteur essentiel de ces nouvelles formes de certification et malgré son exponentiel et extraordinaire développement en peu de temps, n'a pas atteint, bien loin s'en faut, son rendement maximal.
} 
travail scientifique à travers l'expertise et la discussion était en fait une fonction importante des premières sociétés savantes ${ }^{(14)}$.

Comme on peut le constater, le contrôle par les pairs n'était peut être pas celui que l'on connaît de nos jours et qui, généralement, obéit à des règles et a des traditions bien établies, mais existait à l'époque des premières sociétés savantes qui avaient, en quelque sorte, lancés les premiers périodiques au milieu de la deuxième moitié du $17^{\mathrm{e}}$ siècle. Cette imprécision dans la date effective du début du contrôle par les pairs est on ne peut plus clairement expliquée que dans ce qui suit par un spécialiste du sujet: " Il n'existe pratiquement pas de narration historique du contrôle par les pairs. Les journaux biomédicaux apparurent au $19^{\mathrm{e}}$ siècle comme des organes personnels, suivant en cela le modèle du journalisme en général. Les éditeurs de journaux se considéraient avant tout comme des éducateurs. La pratique du contrôle par les pairs éditoriale ne devint monnaie courante que quelque temps après la deuxième guerre mondiale. Contrairement à l'hypothèse communément acceptée, le contrôle par les pairs éditorial n'est pas venu à la suite ou été une interaction du contrôle par les pairs pour l'attribution de subventions pour le financement des projets. Les procédures du contrôle par les pairs éditorial ne se sont pas propagés d'une manière ordonnée, ils ne furent pas développés par les comités éditoriaux et appliqués de journal en journal. Au lieu de cela, des soumissions non contrôlées sur des bases individuelles ont pu être faites à plusieurs reprises tout au long de la période, commençant à partir du début jusqu'au milieu au $19^{\mathrm{e}}$ siècle. Cependant, l'institutionnalisation du processus ne prit place qu'au $20^{\mathrm{e}}$ siècle, soit pour gérer de nouveaux problèmes dans le nombre d'articles soumis ou pour répondre aux demandes pour l'autorité experte et l'objectivité dans un monde de plus en plus spécialisé " (15).

Il a, donc, existé des embryons de contrôle par les pairs depuis 9-10 ${ }^{\mathrm{e}}$ siècle (tel que décrit par Ray Spier pour le contrôle de la pratique de la médecine dans la ville de Erraha en Syrie) et aussi au $17^{\mathrm{e}}$ siècle pour les premiers périodiques (et surtout les Philosophical Transactions qui explicitement conditionnent la publication par l'expertise préalable), mais ce processus ne peut être vraiment décrit comme contrôle par les pairs selon les critères appliqués de nos jours. L'éditeur (ou celui qui assumait la fonction telle qu'on la connaît de nos jours) faisait office de premier tamis (fonction toujours assuré de nos jours) et surtout, point très important expliquant l'imprécision de la date $\mathrm{du}$ ou des premières manifestations du processus, n'envoyait pas la recherche pour expertise à l'extérieur du journal mais se contentait de l'expertiser lui-même et, accessoirement, ensuite demander l'avis de scientifiques qui faisaient partie du comité du journal. De ce fait, le contrôle par les pairs n'a vraiment pris la forme normalisée qui est la sienne que lorsque les manuscrits étaient envoyés pour expertise extérieure. En effet, ce contrôle n'était pas normalisé ni appliqué unilatéralement dans tous les journaux et ne fut adopté comme sceau de qualité et de certification indispensables à la Science (qui ne l'oublions pas progresse par incrémentations et chaque nouvelle incrémentation doit s'appuyer sur des bases justes et solides) que lorsque le volume des publications (et de la masse documentaire en général) atteignirent un niveau ingérable.

\footnotetext{
${ }^{(14)}$ Kronick, David A., op.cit.

${ }^{(15)}$ Burnham, J.C., The Evolution of editorial peer review, Journal of the Amaerican Medical Association, Vol. 263, n¹0, p. 1323-1329,9 Mars 1990
} 
On peut conclure donc que le contrôle par les pairs a eu au début deux fonctions primordiales et qui sont :

-Le filtrage des informations et des recherches sur lesquelles s'appuie la progression de la Science du point de vue l'exactitude des données et de la méthodologie demandée pour la publication scientifique (filtrage qualitatif)

-Le filtrage du nombre de soumissions qui, avec le développement de la science et des budgets recherche et développement, est devenu impossible à contrôler (filtrage quantitatif).

Pour donner une idée de cette explosion documentaire, certains chiffres des années 60 donnent l'amplitude de cette Opération Déluge*. Par exemple, le fameux Weinberg Report ${ }^{\text {(16) }}$ en 1963, nommé d'après Alvin Weinberg, Directeur du Oak Ridge National Laboratory signala que Chemical Abstracts contenait en 193054000 résumés, chiffre qui avait atteint 165000 résumés en 1962. Ce chiffre avait bondit à 200000 résumés en 1970.Les quatre plus grandes bases de données bibliographiques - Chemical Abstracts,Biological Abstracts, Excerpta Medica et MEDLARS - dépassent quant à elles la barre des 200000 résumés ${ }^{(17)}$. Dans la même veine, en 1963 le nombre de résumés et de périodiques avait été estimé à respectivement 1000000 et $10000^{(18)}$. Enfin, une autre estimation place le nombre de périodiques à 41000 et à 1000000 le nombre de d'articles en sciences et en technologie Toutes les autres disciplines totaliseraient quant à elles $1000000 \operatorname{articles}^{(19)}$.

Ces deux raisons (l'une scientifique, méthodologique et l'autre purement statistique) ont donnés forme au contrôle par les pairs qui est devenu de nos jours une condition sine qua none pour qu'une publication puisse être prise au sérieux et, plus important, faire partie du corpus de la Science. A cet effet, le contrôle par les pairs a été appelé " le procès verbal de la science " ${ }^{(20)}$.Comme devant un tribunal qui doit statuer de la véracité des faits qui lui sont exposés, le contrôle par les pairs fait office de tribunal de la Science accordant le quitus de ce qui mérite de figurer dans la Science et ce qui est acceptable selon certains critères, supposés, universels *

\footnotetext{
*Expression utilisée par le bibliothécaire du Département de la Marine Américaine dans les années 50 et qui décrivait une tactique pour contrecarrer le flot pratiquement ingérable de l'information. Il avait calculé par exemple, que les cinq cent dernières années entre 1450 et 1950 (cette dernière date étant la date butoir de cette statistique), il avait été publié plus de douze millions (12 000000$)$ de titres dont dix millions (10 000 000) de ces titres entre 1850 et 1950 (Schultze, R.S.,Literaturflut und mechanisierte Auskunftserteilung unter Berücksichtigung der modernen Einrichlungendes Auslands, Nachr. Dokum, Vol.1, n¹0, Cité par Loos, Th.P., On Documentation of scientific litterature, Butterworth,London, 1973, p.21.)

${ }^{(16)}$ Weinberg, Alvin M., Science, Government and information : the responsabilities of the technical community and the government in the transfer of information, President's Science Advisory Committee, Washington , D.C., 55p., 1963 (Accessible à : http://eric.ed.gov/PDFS/ED048894.pdf )

${ }^{(17)}$ Loos, Th.P., On Documentation of scientific litterature , Butterworth,London, 1973, p.21

(18) Shilling, C.W., Requirement for a scientific mission - oriented information center, American Documentation, Vol.14, n¹, p.49-53, Janvier 1963

${ }^{(19)}$ Bourne , Charles P., The World 's technical journal literature : an estimate of volume , origin , language, field, indexing and abstracting, American Documentation, Vol.13,n2, 159-168, Avril 1962

${ }^{(20)}$ Schaffner, A.C., The Future of scientific journals : lessons from the past, Information Technology Libraries, Vol.13, n4, p.239-248, 4 Dec.1994 Cité par Teasdale, Guy, Vers l'édition e-savante ? Etude des défis du passage à l'Internet, p.170 In : La Publication en ligne, Charlotte Nikitenko [et] Peter Stockinger Ed., Les Cahiers du numérique, Vol. 1, n5, 223 p, 2000

*Ouvrons juste une parenthèse pour dire que nous utilisons supposées parce que si la Science, surtout les sciences exactes, est supposée être neutre, la réalité est toute autre vu que la Science ne se propage pas et n'est pas aussi neutre qu'elle devrait l'être. Ce point sera abordé quand nous parlerons des différents cas de blocages délibérés, de rejets de recherches qui ne vont pas avec les dogmes acceptées ainsi que le rejet de recherches innovatrices qui se sont finalement avérées des recherches ayant des répercussions certaines sur l'avancement de la Science et qui avaient de facto révolutionné le monde en général et le monde de la recherche en particulier.
} 
D'un autre coté, nous tenons à préciser que notre sujet de recherche s'applique au contrôle par les pairs des publications scientifiques et techniques (scientifique veut dire ici Savoir et non scientifique en opposition à sciences sociales) et non pas le contrôle du financement des différents projets de recherche qui lui s'applique à voir si un projet de recherche mérite qu'on lui accorde un financement (publique ou privé) pour qu'il puisse être mené à terme, ni au contrôle par les pairs en ce qui concerne les monographies qui lui est complètement différent et obéit à d'autres critères. On peut dire qu'on a affaire à deux genres de contrôle :

1- l'un concerne la viabilité d'accorder une source de financement

2- l'autre à juger si un travail est digne de faire partie du corpus de la Science.

\section{2-Première manifestations du contrôle par les pairs de l'ère moderne}

Si le contrôle par les pairs n'a pas été appliqué ni de manière uniforme, ni de manière continue ni même appliqué du tout (même de nos jours en des cas rares) avant la moitié du $19^{\mathrm{e}}$ et le début du $20^{\mathrm{e}}$ siècles, il est indéniable qu'il a été formalisé et accepté universellement au $20^{\mathrm{e}}$ siècle tel que le montre cette chronologie ${ }^{(21)}$ des différents journaux et personnes ayant joués un rôle dans l'institution du contrôle par les pairs

-Au $19^{\mathrm{e}}$ siècle, Nature et The British Médical journal adoptèrent tous les deux des formes de contrôle par les pairs similaires à ceux qu'avaient adoptées The Philosophical Transactions.

-En 1905, les éditeurs de Surgery, Gynecology and Obstetrics annoncèrent que les " chirurgiens, les gynécologues et les obstétriciens dirigerons la tendance de la politique [du journal] editorialement ".Comme cela a été expliqué par Burnham " diriger editorialement " veut dire que tout contrôle sera fait par le staff du journal.

-En 1910, The American Journal of Diseases of Children avait un comité éditorial de " quatre à six pédiatres qui auront un contrôle éditorial du journal d'une manière similaire à celle pratiquée par le comité éditorial de The Archives of Internal Medecine

-En 1915, The New England Journal of Medecine (appelé alors The Boston Medical and Surgical Journal) avait deux groupes membres du comité [éditorial]: les éditeurs consultants et le comité consultatif.

-Au cours des années 30 et 40, The Journal of American Medical Association (JAMA) s'appuya sur un petit staff interne pour les décisions éditoriales.

-En 1942, l'expertise éditoriale des manuscrits pour Anesthesiology prenait place dans le bureau éditorial entre l'éditeur et deux éditeurs associés.

-En 1942 aussi, The Journal of Clinical Investigation commença à utiliser le contrôle par les pairs éditorial quand l'éditeur, Gamble " institua la politique qui consistait à envoyer les recherches à des experts externes au comité éditorial pour évaluation ".

(21) Weller, Ann C., Editorial peer review: its strengths and weaknesses, Information Today, Medford [N.J., USA], 2002, p.3-6 
-En 1944, le premier volume en entier d'un nouveau journal The Journal of Neurosurgery fut rédigé par un groupe " d'auteurs des plus selects ".En 1964, " tout manuscrit soumis à la publication dans le Journal est revu par chaque membre du comité éditorial ".

-Ingelfinger ${ }^{*}$ soutient que le premier éditeur de The American Journal of Medecine, qui commença à publier en 1946, utilisa le contrôle par les pairs d'une manière limité et décida de 95\% de toutes les soumissions au bout d'une semaine ou deux de la date de réception du manuscrit.

-Pour la première fois en 1948, The Journal of Pediatrics publia une liste de membres du comité éditorial qui devait " maintenir des critères d'acceptation élevés des articles à publier".

-Dans les années 50, à cause de la vitesse à laquelle étaient découvertes les " particules V" dans la physique des hautes énergies, en plus du besoin pressant de rendre cette information disponible rapidement, les physiciens sentirent que la publication ne pouvait attendre le délai habituel associé avec le processus de publication. Ils commencèrent à envoyer des pré publications à leurs collègues. Lorsque ces nouvelles découvertes furent publiées dans Physical Review, presque toute la communauté était au courant de chaque nouvelle particule.

La solution pour les physiciens, à l'époque était l'addition d'un nouveau journal -Physical Review Letters - qui avait une politique de publication rapide. L'éditeur, Pastenack, admit qu'il était " difficile (mais essentiel) de déterminer si le travail qui nous était soumis avait de l'importance et de l'urgence comme physique de base..... Nous nous attendions à ce que certaines des Lettres que nous publiâmes s'avéreraient contenir des défauts et des fois des erreurs majeures parce qu'elles étaient préparées d'une manière précipitée ".En 1961, Physical Review Letters recevait trop de soumissions au gout de l'éditeur qui avertissait les auteurs que les éditeurs serait " plus stricte et de ce fait plus arbitraire dans notre politique de rejet ".Des années plus tard, l'éditeur de Physical Review Letters concéda que " durant la frénétique période des années 60 nous ne publiâmes jamais sans au moins appliquer les formes les plus élémentaires du contrôle par le pairs ".

-Entre 1954 et 1972, l'éditeur de Southern Medical Journal utilisa un comité éditorial étendu pour un contrôle par les pairs moderne utilisant des experts provenant de The Southern Medical Association.

-En 1958, avec l'annonce d'un nouveau journal, The American Journal of Cardiology, l'éditeur déclara qu'il demanderait au comité éditorial de " guider les éditeurs dans leur sélection du matériel [scientifique] et aider à garder les normes scientifiques du journal à un niveau élevé. Nombre de présentations seront sous la forme de symposia et séminaires qui seront arrangés et édités par les membres du comité éditorial et d'autres autorités dans leurs spécialités respectives ".Il assura aussi les lecteurs que les éditeurs n'auront pas d'à priori quand ils accepteront ou rejetteront un manuscrit.

\footnotetext{
*La règle d' Ingelfinger ( Ingelfinger rule ) instituée en 1969 par Franz Ingelfinger quand il était Editeur en chef de New England Journal of Médecine prohibe la divulgation des résultats des recherches dans les mass media avant qu'ils aient été expertisés et publiés. Cette règle prohibe aussi la soumission simultanée à plusieurs journaux ce qui était acceptable, et même souhaitable, quand le volume des soumissions n'avait pas atteint le niveau actuel.
} 
-En 1958, le nouvel éditeur de Circulation Research informa le lectorat que les manuscrits seraient " revus par un comité d'éditeurs distingués ".

-En 1962, les éditeurs de The Archives of Dermatology s'aperçurent qu'ils avaient besoin de " chercher attentivement pour des sommités avec un savoir détaillé suffisant pour servir comme des experts éditoriaux critiques et constructifs. Durant l'année dernière, le comité éditorial de The Archives of Dermatology a lancé un programme pour la recherche de ce genre d'expertise pour chaque manuscrit soumis.

-En 1962, The International Council of Scientific Union Abstracting Boards conduisit une étude de journaux primaires connus de dix pays. Seize pour cent (16\%) des éditeurs affirmèrent que leurs journaux n'étaient pas contrôlés par les pairs et un autre huit pour cent (8\%) donnèrent des réponses équivoques. Les autres détails de l'étude (critère d'inclusion, titres de journaux, taux de réponses, sujet des journaux, etc...) ne furent pas fournis par Porter.

-De 1965 à 1972, Braceland était éditeur de The American Journal of Psychiatry. Dans une discussion de ces treize années [durant lesquelles il assura le poste d'éditeur en chef], il a sous entendut que le contrôle par les pairs éditorial n'avait pas été utilisé avant qu'il ne prit ses fonctions: " dés le début, nous optâmes pour le système des arbitrages, i.e. envoyer nos manuscrits pour une expertise extérieure ".

-En 1966, une étude portant sur 166 journaux en sciences humaines révéla que $9.6 \%$ des éditeurs prirent la décision d'acceptation ou de rejet eux-mêmes. Le reste des éditeurs ont reconnu qu'en règle générale chaque manuscrit est expertisé par deux ou trois lecteurs [expert].

- En 1974, The Lancet utilisait son staff éditorial pour décider de quatre vingt dix pour cent (90\%). Douglas - Wilson, qui fut longtemps éditeur de The Lancet, questionna ouvertement le postulat que le contrôle par les pairs était essentiel: " Je suis un opposant convaincu du contrôle par les pairs routinier ". Il tempérera sa remarque en indiquant les catégories de manuscrits qui devraient être expertisées comme les manuscrits qui prétendent des vertus thérapeutiques de produits pharmaceutiques avec des effets adverses et de ceux qui signalent de nouvelles drogues.

-En 1977, le nouvel éditeur de Parapsychology" changea progressivement The Journal vers une utilisation plus formelle du contrôle par les pairs".L'éditeur précédant était opposé au contrôle par les pairs externe.

-Aux environs de l'année 1985, quelques 30\% des manuscrits soumis au Lancet étaient envoyés pour une expertise externe .En 1989, un éditorial dans Lancet déclara " aux EtatsUnis, il est trop demandé du contrôle par les pairs. Les carrières et la viabilité de départements en entier dépendent maintenant de la publication dans des journaux contrôlés par les pairs ". L'éditorial conclut : " le contrôle par les pairs est à son meilleur quand on ne lui demande pas trop ". 
Nous avons reproduit en intégralité cette très complète chronique du contrôle par les pairs (qui a pratiquement relaté le processus seulement au $20^{\mathrm{e}}$ siècle, date de sa forme moderne) car nous pensons qu'il est primordial de comprendre quelles ont étés les prémices de cet ancien procédé qui n'a gagné ses lettres de noblesse que lorsqu'il a fallut restreindre le flot ininterrompu et ingérable de la masse documentaire. Nous remarquerons dans cette chronique la montée en puissance de l'inéluctabilité du contrôle par les pairs et surtout sa progressive acceptation par l'ensemble de la communauté scientifique. Les différents protagonistes cités, malgré leurs divergences, à l'image de l'éditorial de Lancet qui questionnait l'importance et le rôle démesuré du contrôle par les pairs dans la vie des chercheurs et de leurs institutions, sont d'accord pour dire que c'est un processus indispensable à la bonne marche de la science.

\section{3-Contrôle par les pairs : essai de définition}

Le contrôle par les pairs a été longuement décrit et étudié dans les références en langue anglaise comme il nous a été donné l'occasion de le dire. Nous nous contenterons de citer quelques définitions de monographies ayant exclusivement abordé le sujet. Comme nous l'avons dit précédemment, ces monographies exclusivement dévolues au contrôle par les pairs ne sont pas très nombreuses mais ont le mérite d'avoir abordé et analysé le sujet sous tous ces différents angles, dans les différentes spécialités et ont abordés les différentes écoles de pensée qui l'ont étudiés. Malheureusement, de toutes ces peu nombreuses monographies, nous n'avons pu consulter que deux malgré nos différentes recherches.

La première de ces définitions dit: "....En ce qui concerne les contextes en dehors du domaine académique, le contrôle par les pairs est simplement l'évaluation d'une personne par un pair, en d'autres termes, un autre professionnel. Dans le contexte académique, cette utilisation de " contrôle par les pairs " désigne une évaluation de tous les aspects du rendement d'un professeur y compris non seulement sa production scientifique mais aussi l'enseignement et ses états de service au sein de comités. Dans le monde académique, le mot conjure généralement quelque chose de plus étroit, à savoir, l'expertise d'articles, de livres soumis pour publication, d'articles, de livres déjà publiés et des projets de recherche proposés...... [Y seront inclus] aussi bien le contrôle par les pairs post publication de manuscrits par des experts et des éditeurs et le contrôle post publication par des évaluateurs qui décident de l'embauche, la titularisation, la promotion et les salaires.......En ce qui concerne l'expertise pré publication, " le contrôle par les pairs a un sens restreint et un sens large. Dans le sens restreint, le contrôle par les pairs désigne le processus dans lequel l'éditeur d'un journal envoi le matériel soumis à des spécialistes, des experts dans le sujet de l'article ou le livre de l'auteur. Dans son usage restreint, un article n'est pas expertisé quand l'éditeur prend tout seul la décision pour l'acceptation ou le rejet sans consultation externe. Cependant, dans son usage plus large, l'expertise par tout autre professionnel, y compris l'éditeur du journal, est appelé contrôle par les pairs, même si le " pair " n'est pas vraiment un " pair " parce qu'il ou elle n'est pas aussi expert (e) dans la spécialité que l'auteur. Quand les personnes parlent de l'avènement historique du contrôle par les pairs, ils le datent des fois par le changement de l'expertise par un éditeur vers une expertise par des experts externes. Au moins deux des journaux philosophiques les plus importants, The Journal of Philosophy et 
Philosophical Review sont encore généralement expertisés par les éditeurs (desquels il y en a plusieurs)....." (22).

Nous avons cité d'une manière extensive cette définition (bien qu'on ait supprimé certaines phrases et gardé que ce qui intéressait notre travail) car elle donne une idée de ce qu'est le contrôle part les pairs, ses différentes composantes et variantes. On remarquera aussi que le contrôle par les pairs n'est devenu contrôle par les pairs dans le sens moderne du terme que lorsque l'expertise a commencé à se faire en dehors des murs de la maison d'édition et par d'autres personnes que l'éditeur. Ce point (l'expertise se faisant par des experts externes à la maison d'édition) explique la difficulté à mettre une date précise du démarrage effectif de cette opération. Il peut être situé au $10^{\mathrm{e}}$ siècle comme on l'a vu dans le livre d'Ishak Ibn Ali Errahwi et le contrôle des performances d'un docteur et les résultats de son traitement, ou beaucoup plus tard avec les premiers périodiques et plus précisément The Philosophical Transactions et son premier numéro qui cite nommément le contrôle avant la publication. Ce changement fondamental et important a eu lieu quand la quantité de soumissions est devenue trop importante à gérer par l'éditeur tout seul, que la spécialisation sans cesse croissante a obligé le recours à des experts de plus en plus spécialisés en plus du fait que l'accroissement de la masse documentaire a induit d'autres pratiques dans le jugement du corpus du Savoir. Certains de ces points seront abordés plus tard dans le cadre de ce travail.

La deuxième définition ou plutôt groupe de définition est celle d'une monographie entièrement dévolue au contrôle par les pairs et est citée en tant que référence, l'auteur étant par ailleurs une personne connue dans le domaine. Notons seulement que l'auteur utilise " Définition d'un journal contrôlé par les pairs " et non pas " contrôle par les pairs " dans sa monographie. Cette différence n'est pas, à notre avis, importante car définir un journal contrôlé par les pairs implique automatiquement définir qu'est ce le contrôle par les pairs. La première et " très générale définition " pour reprendre l'auteur est ...." une expertise d'un manuscrit par une personne autre que l'éditeur" ${ }^{(23)}$.Cette définition concise a le mérite d'être clair bien qu'elle prêche par une sorte de simplification. En effet, le contrôle par les pairs a des versions, des applications, des variations différentes qui ne peuvent être englobée par cette définition. Weller cite une autre définition qui fait suite à une étude de journaux dans le domaine infirmier. Cette définition dit : ".... [Un journal contrôlé par les pairs] est un journal qui utilise des individus avec l'expertise professionnelle pour évaluer un manuscrit. Ces experts sont sélectionnés par le staff du journal en accordance avec l'expertise voulue ou sont composés d'un groupe préétabli d'experts. La décision sur le manuscrit est faite sur un minimum de trois expertises et la responsabilité ultime pour la décision concernant [l'acceptation ou le rejet] le manuscrit est celle de l'éditeur .Il partage aussi systématiquement avec l'auteur les raisons du rejet et les commentaires des experts " ${ }^{(24)}$. Comme on peut le constater cette définition (bien qu'elle ait été faite dans le cadre d'une étude dans le domaine infirmier) est plus complète et donne plus de détails quant au processus (les experts, leur choix, leur nombre, la décision finale et aussi les relations entre l'éditeur et l'auteur).Dans la même veine, l'éditeur de Nursing Outlook utilise aussi le concept des experts en dehors du bureau éditorial comme " pierre angulaire " (c'est nous qui soulignons) du journal contrôlé

\footnotetext{
(22) Shatz, David, Peer review : a critical inquiry, Rowman \&Littlefield, Lanham[Md., USA], 2004 ,p.7-8

${ }^{(23)}$ Weller, Ann C., op.cit, p.15

(24) Swanson, E.A., McCloskey, J., The Manuscript review process of nursing journals, Image ,Vol.14, n³, p.72-

76, Octobre 1982 Cité par Weller, Ann C, Op cit p. 15
} 
par les pairs ${ }^{(25)}$.D'un autre côté , le contrôle par les pairs des journaux est défini comme " l'évaluation par des experts (des pairs) de matériel soumis à la publication dans des périodiques scientifiques et techniques " ${ }^{(26)}$.Enfin, Lock considère que " l'évaluation experte d'articles pour la publication était une bonne définition de travail pour le contrôle par les pairs " ${ }^{(27)}$.Le Comité International des Editeurs de Journaux Médicaux (International Committee of Medical Editors) définit un journal contrôlé par les pairs comme : " [un journal] qui a soumis une grande partie de ses articles publiés à la révision par des experts qui ne font pas partie du staff éditorial. Les nombres et les types de manuscrits envoyés à l'expertise, le nombre d'experts, les procédures d'expertise et l'utilisation faite des avis exprimés par les experts peuvent varier. De ce fait, chaque journal devrait divulguer sa politique dans sa section Instructions aux auteurs pour le bénéfice des lecteurs et des auteurs potentiels " (28). Cette définition est importante et instructive à plus d'un titre car, en plus du fait qu'elle émane d'un Comité international d'éditeurs en médecine où le contrôle par les pairs est d'une importance primordiale (pour ne pas dire vitale), elle fixe les différentes procédures quant à l'exercice de cette étape importante de la Science publiée. Weller définit enfin le journal contrôlé par les pairs comme : " Un journal qui a une partie des manuscrits soumis évalués par une personne autre que l'éditeur du journal " (29).

Dans un autre chapitre du contrôle par les pairs, celui des sources des financements des projets de recherche, The British Academy publie un rapport dans lequel elle définit et aborde les différents aspects et raisons du contrôle par les pairs. Nous en retiendrons deux qui nous semble répondre aux axes de notre travail. La première de ces définitions est la suivante : " Le contrôle par les pairs par des évaluateurs experts est utilisé pour juger la qualité des recherches et publications dans nombre de disciplines et est d'une grande importance pour les sciences sociales et humaines. Il fournit des jugements experts qui peuvent former une base pour les décisions soit pour publier un travail scientifique ou financer une proposition de recherche. Ce genre de jugement fournit aux chercheurs et auteurs aussi un retour d'écoute (feedback) pendant qu'ils progressent dans leurs travaux " (30). On remarque que cette définition inclut le financement aussi des projets de recherche qui doivent passer eux aussi par un filtre afin de pouvoir bénéficier du financement indispensables à leur bonne tenue ( ceci s'applique beaucoup plus pour les sciences exactes et dures où la recherche ne peut se faire sans des équipements et des installations que ne peut avoir le chercheur. Généralement ce genre de financement est surtout le résultat d'efforts assuré par les Etats ou sont, des fois, transnationaux comme dans le cas du Large Hadron Collider qui est un effort du " European Organization for Nuclear Research " -CERN).La deuxième définition, plus concise et plus précise est plus en adéquation avec l'une des deux formes du contrôle par les pairs: celui qui concerne le jugement de la possibilité de publier un travail donné." Le contrôle par les pairs est la pratique par laquelle la valeur d'une recherche est évaluée par ceux qui ont des compétences démontrées pour juger. C'est le moyen traditionnel par lequel la qualité de la

\footnotetext{
${ }^{(25)}$ Lewis, E.P. ,A Peerless publication, Nursing outlook,Vol.28, nº 4,p.225-226, Avril 1980 Cité par Weller, Ann C, Op cit p. 15

(26) Bailar, J.C. \& Patterson, K., Journal peer review : the need for a research agenda, New England Journal of Medecine, Vol.312, n 10 , p.654-67,7 Mars 1985 Cité par Weller, Ann C, Op cit p. 15

${ }^{(27)}$ Lock, S., Quality assurance in medical publication, Proceedings of The Royal Society of Edinburgh, Section b-biological sciences, 101, p.359-375, 1993.

(28) International Committee of Medical Journal Editors, Statements on Electronic Publication and on PeerReviewed Journals, Annals of internal medecine, Vol. 116, no. 12, p.1030

${ }^{(29)}$ Weller, Ann C. Op cit. , p.16

${ }^{(30)}$ The British Academy, Peer review : the challenges for the humanities and social science : a British Academy report, The British Academy, London, 2007,p.iii
} 
recherche est garantie dans les études académiques " (31). A la question " Qu'est ce que le contrôle par les pairs scientifique " ? Sense about Science, une organisation de recherche britannique, répond ce qui suit: " Le contrôle par les pairs scientifique est l'évaluation des résultats de la recherche scientifique ou des propositions pour la compétence, la signification et l'originalité, par des experts qualifiés qui font de la recherche et soumettent du travail [de recherche] pour publication dans le même domaine (des pairs) " (32).

Nous nous risquerons, quant à nous, à donner une définition qui exprimera notre point de vue, notre manière de voir mais aussi et surtout synthétisera les différentes lectures et définitions que nous avons pu rencontrer au cours de cette recherche. Le contrôle par les pairs est un filtre quantitatif et qualitatif utilisé par les chercheurs comme outil afin de certifier que l'information, la recherche soumise dans le but d'être publiée répond aux canons scientifiques et méthodologiques pour faire partie du corpus de la Science.

Nous avons présenté plusieurs définitions du contrôle par les pairs et essayé de l'expliquer pour un nombre de raisons dont nous citerons celles qui nous ont paru importantes et qui sont :

Premièrement, l'occasion nous a été donnée durant notre recherche de découvrir le peu d'intérêt accordé à cette importante et indispensable étape de la recherche et ce même par des chercheurs supposés être concernés .En effet, le chercheur, l'enseignant, le cadre même est supposé publier afin soit communiquer ses résultats et obtenir des financements, mettre à jour ses connaissances ou alors aider à faire connaître l'institution qui l'emploie. De là, la célèbre expression de " publish or perish " (publier ou périr), qui aurait été utilisée la première fois par un certain Kimball C.Atwood aux environs de l'année $1950^{(33)}{ }^{*}$ et qui gouverne pratiquement le monde académique surtout en terme de " volume" de publication. Nous avons donc senti une sorte non seulement de désintérêt mais aussi d'ignorance de cette pression au niveau national où la pression de publier n'existe et on pourrait même dire que la culture de

${ }^{(31)}$ The British Academy, Op. Cit., p.ix
${ }^{(32)}$ Brown, Tracey, Ed.,Peer review and the acceptance of new scientific ideas:Discussion paper from a Working
party on equipping the public with an understanding of peer review., November 2002-May 2004, Sense about
Science, London, 2004, p.7.(Disponible sous forme électronique à : http://www.senseaboutscience.org)
(33) Sojka, R.E., Mayland, H.F., Driving science with one eye on the peer review mirror, Forum proceedings,
"Ethics, Values, and the Promise of Science", San Francisco, 25-26-02-1993, p.202 (Disponible à
http://eprints.nwisrl.ars.usda.gov/1062/1/816.pdf)

${ }^{*}$ De son coté, Eugène Garfield donne une autre version de cette expression si célèbre et si citée. Après maintes recherches, il a trouvé deux citations qui sont, l'une contemporaine à celle d'Atwood et l'autre plus ancienne. La première nous ramène au célèbre chercheur en media et société Marshall Mc Luhan qui en date du 22 Juin 1951 en écrivant au poète Ezra Pound cite l'expression en parlant des universités qui feraient n'importe quoi afin de satisfaire les administrateurs de ces institutions .La deuxième occurrence où cette célèbre citation a été utilisé est par un sociologue, un certain Logan Wilson qui en 1942 l'utilisa dans un de ces livres intitulé "The Academic man: a study in the sociology of a profession".Dans les deux cas, Garfield relève le fait que les deux chercheurs ont utilisé les guillemets ce qui supposerait qu'il n'ont pas inventé mais utilisé l'expression. Il cite enfin le célèbre sociologue des sciences Robert K. Merton qui affirme que l'expression était utilisée dans le monde académique pré deuxième guerre mondiale (Eugène Garfield, What is the primordial reference for the phrase "Publish or Perish"? The Scientist, Vol. 10, $\mathrm{n}^{\circ}$ 12, p. 11 (Disponible à: http://www.thescientist.com/article/display/17052/\#ixzz1JnZFmh2X.

On notera que cette phrase célèbre a engendré des écrits qui parodient cette expression .Un livre est même paru et dont le titre est "Publish AND perish" (Hynes, James, Publish and perish: three tales of tenure and terror, Picador, New York, 1998, 338p.).Ce livre satirique, présente le dilemme que vit le chercheur dans sa quête de titularisation et avancement. Le titre est en lui même symptomatique car si publish or perish veut dire que le chercheur doit publier afin de ne pas être exclu, publish and perish veut dire que la course à la publication a des conséquences qui dépassent le cadre scientifique. En fait cette course a conduit à une inflation de publications où le nombre et non pas la qualité prime et où le chercheur publie pour publier et non pas pour participer au développement de sa spécialité. 
publier n'existe pas. L'enseignant, le chercheur une fois en poste, une fois titularisé n'écrit plus, ne cherche plus et n'a pas d'incitation à le faire. A cet effet, Dahdouh - Guebbas dans une contribution qui aborde la problématique de la publication dans les pays en voie développement et sa relation avec les pays développés affirme ce qui suit :" La question qui se pose est : est ce que les scientifiques des instituts de recherche locaux dans les pays visés[pour être bref la recherche en question examine la problématique de la publication des recherches dont le sujet est un pays en voie de développement et qui à la fin ne donne aucun crédit aux auteurs de ces mêmes pays ] croient eux-mêmes que la publication scientifique est importante. D'un coté, les positions fixes que ce genre de chercheurs obtient peuvent être responsables dans le manque de motivation dans la publication scientifique. De l'autre coté, les recherches publiées dans les journaux ou media locaux ( littérature grise) peuvent avoir un résultat plus bénéfique pour eux " ${ }^{(34)}$.Alors qu'ailleurs la longueur (nonobstant la qualité) du CV d'un enseignant est sa carte de visite, il est des enseignants dont la liste des publications équivaut à un ou deux articles publiés dans des revues généralement locales avec une forte dose de favoritisme et au prestige réduit et dont les mécanismes de certification ne sont pas au diapason des grandes revues au fort facteur d'impact.

Deuxièmement, la littérature sur le sujet en plus d'être très restreinte, pratiquement monolingue (anglais), se distingue par l'absence d'un corpus de définitions bien établi et normalisé. Ceci peut être expliqué par différentes raisons dont nous citerons deux et qui sont:

-la nouveauté du processus qui ne date, dans sa forme actuelle acceptée, que de la période post deuxième guerre mondiale.

-les différences dans le processus selon les différentes spécialités et champs de recherche. Il est clair que certifier un travail de recherche en sciences exactes est plus " facile " que certifier un travail en sciences humaines et sociales .Le premier s'appuie sur des règles pratiquement mathématiques et des procédures de recherche universelles et normalisées, alors que dans le deuxième, il est plus difficile de juger, le corpus étant divisé en champs linguistiques, régionaux, philosophiques et autres. Pour schématiser à l'extrême, il est plus facile de dire si une opération mathématique est juste alors que juger une théorie, une analyse ou un point de vue où le côté personnel (affectif même) entre en ligne de compte, relève tout simplement de la gageure. D'ailleurs, les différentes études concernant les taux d'acceptation des soumissions d'articles pointent toutes vers la même tendance que le tableau suivant ${ }^{(35)}$ démontre de la manière la plus claire (l'étude quoiqu'ancienne est toujours d'actualité l'article et ses auteurs sont devenus des références dans le domaine du contrôle par les pairs) :

\footnotetext{
(34) Dahdouh-Guebas, Farid [et al], Neo-colonial science by the most industrialized upon the least developed countries in peer-reviewed publishing, Scientometrics, Vol.56, n³, p.340-341, 2003

${ }^{(35)}$ Zuckerman, Harriet, Merton, Robert K., Patterns of evaluation in science : institutionalisation, structure and functions of the referee system, Minerva, Vol.9, ${ }^{\circ} 1$, p.76, Janvier 1971
} 


\begin{tabular}{|l|c|c|}
\hline & $\begin{array}{c}\text { Taux de rejet moyen } \\
\text { en \% }\end{array}$ & \begin{tabular}{c} 
Nombre de journaux \\
\hline Histoire
\end{tabular} \\
\hline Langage et littérature & 80 & 5 \\
\hline Philosophie & 85 & 5 \\
\hline Sciences politiques & 84 & 2 \\
\hline Sociologie & 78 & 14 \\
\hline Psychologie (à part expérimentale et physiologique) & 70 & 7 \\
\hline Economie & 69 & 2 \\
\hline Psychologie expérimentale et physiologique & 51 & 5 \\
\hline Mathématiques et statistiques & 50 & 2 \\
\hline Anthropologie & 48 & 2 \\
\hline Chimie & 31 & 12 \\
\hline Géographie & 30 & 12 \\
\hline Sciences biologiques & 29 & 2 \\
\hline Physique & 24 & 1 \\
\hline Géologie & 22 & 83 \\
\hline Linguistique & 20 & 5 \\
\hline Total & & 2 \\
\hline
\end{tabular}

Tableau $\mathrm{n}^{\circ}$ 10-Taux de rejet des manuscrits soumis à la publication pour les journaux scientifiques et en sciences humaines (1967)

Comme on peut le voir, les écarts dans les taux de rejet et d'acceptation sont très grands et confirment par l'hypothèse qui dit que les soumissions dans les sciences sociales et humaines ont moins de chance d'être acceptées que les soumissions dans les sciences exactes et dures. Cette stratification, cependant a besoin d'être clarifiée car la lecture du tableau nous fait ressortir qu'en fait les auteurs ont départagé les spécialités en trois domaines (alors que généralement on parle de sciences exactes et sciences sociales comme étant les deux grandes branches du Savoir) et qui sont par ordre décroissant de taux de rejet :

\section{1-Les sciences humaines}

\section{2-Les sciences sociales et comportementales}

3-Les sciences physiques, chimiques et biologiques.

On remarquera que les mathématiques et les statistiques occupent une position intermédiaire et affichent des taux de rejet pratiquement équivalant à ceux de l'acceptation. Les auteurs remarquent que les journaux en sciences physiques et qui affichent parmi les taux les plus bas de rejet (24\% avec une moyenne qui oscillait pour 11 des 12 journaux considérés entre 11 et $25 \%$ ) présentent une anomalie. En effet, le $12^{\mathrm{e}}$ journal American Journal of Physics a un taux 
de rejet de $40 \%$ ce qui est plus près des taux des sciences sociales et comportementales que celui des physiques, chimiques et biologiques selon la classification établie par les auteurs. En fait, American Journal of Physics est un journal non pas en sciences physiques mais sur les sciences physiques. Il ne présente pas de recherches nouvelles en physiques mais des recherches sur les aspects sociaux, historiques, pédagogiques et humains de la physique, ce qui explique ses taux de rejet assez haut. De même, pour ce qui est de l'anthropologie, un cas similaire est présent au sujet des deux journaux sujet de la recherche, American Anthropologist et American Journal of Physical Anthropology qui affichent des taux éloignés (respectivement $65 \%$ et $30 \%$ ) les uns des autres et ceci pour les mêmes raisons que l'exemple précédant. En psychologie la même tendance est relevée entre la psychologie sociale, anormale, clinique et éducative et la psychologie expérimentale, physiologique et comparative. La première affiche des taux de $70 \%$ et la deuxième des taux plus proches de ceux des sciences physiques avec de 51\%.Enfin un dernier exemple concernant ces différences à l'intérieur de tendances bien établies et qui concerne les journaux en langage et littérature et qui ont ( dans la tradition des sciences humaines ) un des taux de rejet les plus haut avec $86 \%$, dépassés seulement dans cette étude par l'histoire avec un taux de $90 \%$.Le journal Linguistics, qui adopte des orientations mathématiques et logiques dans l'étude du langage , a un taux de rejet de $20 \%$ identique aux taux des sciences physiques.

Quelles conclusions peut-on tirer de cette étude ? Il est de prime abord facile de schématiser et de dire que le contrôle par les pairs est plus facile, plus " scientifique " et plus logique dans les sciences exactes et dures que dans les sciences sociales et humaines .Ceci ne prendrait pas en ligne de compte les différences à l'intérieur d'une même spécialité et qui peuvent varier de $20 \%$ à $86 \%$ ou un taux de rejet dépassent quatre fois plus important dans une même discipline. Il serait plus adéquat de dire qu'en fait les sciences exactes avec un coté social, éducatif tendent à avoir des taux de rejet plus haut de même que des spécialités réputées faisant partie des sciences sociales et humaines avec des tendances plus " scientifiques " ont des taux plus proches de ceux des sciences exactes. Cet état de fait a été parfaitement résumé par les auteurs :" La tendance des différences entre champs et à l'intérieur des champs peut être décrite dans le même procédé empirique : plus le journal est d'orientation sciences humaines, plus le taux de rejet de publication des manuscrits est élevé, [et] plus expérimentale et observationnelle est l'orientation [de la publication] avec un accent sur la rigueur de l'observation et l'analyse, [plus] le taux de rejet est bas " ${ }^{(36)}$..Cette même idée est exprimée en d'autres termes par un autre chercheur de la manière suivante: " Dans les sciences, parce que les critères d'évaluation sont très clairs, les articles sont acceptés quand il n'y a pas de raisons à invoquer pour le rejet. Dans les sciences humaines, l'argument à tendance à être le contraire: un manuscrit est rejeté à part s'il y a de fortes raisons pour le publier " (37). Nous avons voulu insister sur ce point en relation avec les différentes définitions qu'a le contrôle par les pairs et les différents taux de rejet selon les disciplines ( point nodal et primordial dans le processus de soumission et d'acceptation des manuscrits soumis au filtre du contrôle par les pairs) pour arriver à un point très important de notre recherche et qui concerne les différentes étapes du contrôle par les pairs en plus clair comment se déroule le processus du contrôle par les pairs.

\footnotetext{
(36) Zuckerman, Harriet, Merton, Robert K, op. cit. p.77

${ }^{(37)}$ Meadows, A.J., Communicating research, Academic Press, London, 1998, p.184
} 


\section{4-Les différentes étapes du contrôle par les pairs:}

Comme nous l'avons vu de par les définitions, le contrôle par les pairs est l'opération par laquelle une publication est contrôlée par les scientifiques spécialisés dans le domaine. Les différentes étapes par laquelle cette opération passe sont multiples et variées selon les spécialités, les éditeurs et les us et coutumes des différents titres mais généralement cette opération se subdivise en trois étapes et qui sont :

-L' (es) auteur (s) soumet (ent) un manuscrit à un journal dans sa (leur) spécialité. Cette soumission peut être non demandée ou alors dans le cadre d'un thème que l'éditeur $(s)$ choisit et propose aux chercheurs potentiels (cas des numéros thématiques ou spéciaux).

-L'éditeur, à la réception du manuscrit, en prend connaissance .Il peut alors prendre trois décisions

* décider à la lecture du résumé (et éventuellement le texte en entier) soit de continuer le processus de certification du manuscrit ou non. Généralement, cette décision est prise (dans un sens ou un autre) pour faire concorder le sujet soumis aux thèmes de la revue. Cette décision est, dans tous les cas, du ressort de l'éditeur.

* expertiser alors le manuscrit lui-même ou avec l'aide du staff du journal. Cette manière de procéder n'est plus très utilisée de nos jours à part pour certains cas assez rares. D'ailleurs, nous avons vu que le contrôle par les pairs est devenu le processus que l'on connait de nos jours après qu'il eut été fait appel à des experts externes au journal en question.

* le faire expertiser par des experts extérieurs au journal.

Cette première phase à la réception du manuscrit est une phase interne où le manuscrit ne quitte pas les bureaux de la revue.

Plus plausiblement, l'éditeur sollicite généralement deux experts à trois externes pour certifier la recherche. Ces experts sont eux même des chercheurs ayant un degré d'expertise dans le sujet de la soumission en question.

Les experts à qui sont adressées ces soumissions doivent juger des mérites de la publication et recommander de sa publication ou de son rejet. Les cas qui, généralement, peuvent se présenter comme résultat de ces expertises sont les suivantes :

1-Acceptation sans corrections (cas très rares).

2-Acceptation avec des corrections légères.

3-Acceptation sous réserves de corrections assez conséquentes.

4-Rejet avec recommandation de faire publier ailleurs.

5-Rejet de la soumission sans aucune recommandation. ${ }^{*}$

Si la description précédente concerne les étapes générales, standards décrits d'une manière assez superficielle, le processus est quant à lui un peu plus varié et comporte en fait d'autres étapes que nous allons décrire dans ce qui suit.

\footnotetext{
*Il existe aussi d'autres cas assez rares (dont nous pouvons témoigner personnellement car cela nous a été demandé une fois pour une soumission) et dans lequel il est demandé au chercheur de présenter un poster au lieu d'un article. Il est vrai que ceci a eu lieu pour une conférence mais le cas est pratiquement le même étant donné que les communications soumis à une conférence sont aussi sujet à un travail de certification. Pour des raisons qui nous échappent, les recherches publiées dans le cadre de conférences ne semblent pas bénéficier de la même " aura " qu'un article publié dans une revue à comité de lecture .Ce point mériterait une étude pour voir les différences dans les critères de sélection et de certification dans une conférence et dans une revue à comité de lecture.
} 
-les éditeurs acceptent ou rejettent, des fois, le manuscrit sans l'aide des experts

-les experts peuvent (rarement) être membre du comité éditorial du journal mais sont plus souvent issus d'une liste d'experts potentiels que l'éditeur garde dans les dossiers du bureau éditorial.

-plus souvent que non, durant le processus d'expertise, les éditeurs utilisent le processus de l'expertise anonyme (les noms des experts ne sont pas connus de l'auteur).

-moins souvent, ils utilisent l'expertise aveugle (les noms des auteurs sont enlevés des manuscrits avant d'être envoyés aux experts).

-les éditeurs peuvent fournir aux experts un formulaire à remplir, des pages de directives ou tout simplement demander des commentaires.

- les rapports des experts peuvent être traités de différentes manières:

* les commentaires peuvent être retournés aux auteurs exactement tels qu'ils ont étés rédigés

* les commentaires peuvent être résumés par l'éditeur

* l'éditeur peut simplement donner à l'auteur les recommandations finales sans les commentaires des experts.

- les experts sont des conseillers seulement; les éditeurs peuvent et, en fait, arrivent souvent à des décisions contraires à toutes les recommandations des experts. Ils peuvent décider de publier un manuscrit parce que le sujet est d'actualité, intéressant, provoquant ou controversé.

-souvent les éditoriaux, actes de conférences, symposia, manuscrits invités, les numéros spéciaux avec un éditeur invité ne sont pas expertisés.

- une fois accepté, le manuscrit est placé en attente de publication.

- si le manuscrit est rejeté, différentes options existent pour l'auteur:

* faire un appel à l'éditeur

* soumettre à un autre journal exactement tel que le manuscrit a été originalement écrit

* réviser le manuscrit et le soumettre de nouveau ou le soumettre un autre journal

* ranger définitivement le manuscrit

Enfin, les éditeurs peuvent changer leur avis et accepter un manuscrit rejeté si l'auteur présente de solides arguments ou s'il y a fait de substantielles altérations

Cette procédure est résumée par le schéma suivant ${ }^{(38)}$ décrivant le processus qui, à quelques nuances prés, est le même pour toutes les soumissions.

${ }^{(38)}$ Weller, Ann C., op.cit. p.2 


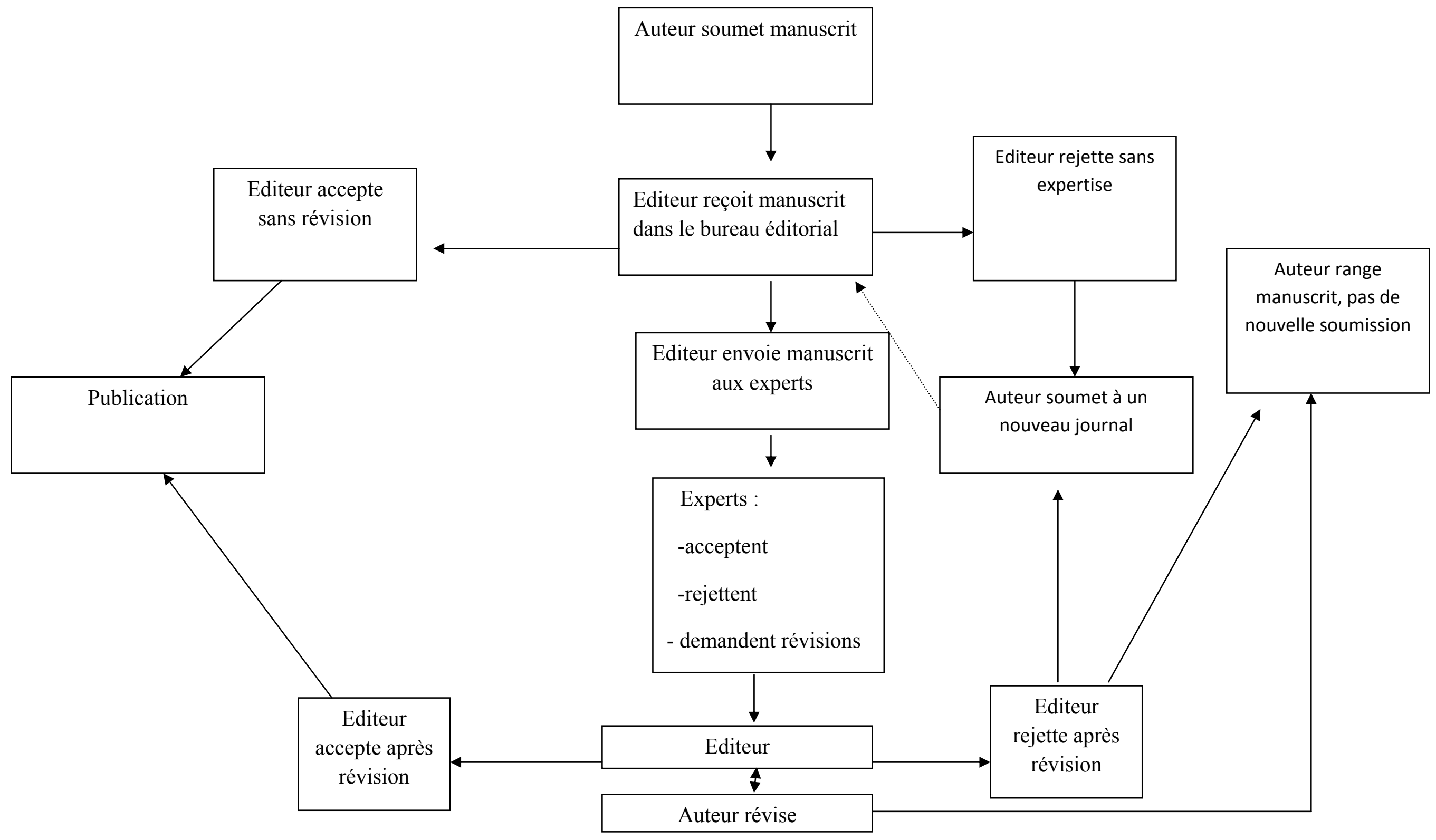

Fig.34-Cheminement d'un manuscrit à travers le processus de contrôle par les pairs éditorial 
Un autre schéma plus simple, plus direct présente ce processus en sept étapes et qui sont détaillées dans le tableau suivant ${ }^{(39)}$ :

Etape 1 Réception du manuscrit par l'éditeur du journal

Etape 2 Le manuscrit est examiné pour voir s'il répond aux critères de la nature/sujet du journal. S'il est jugé inapproprié, il sera rejeté à ce stage

Etape 3 S'il est jugé approprié, le manuscrit sera envoyé aux experts (normalement au moins deux sont désignés) auxquels il sera demandé d'évaluer ses mérites (importance/originalité/validité des résultats de la recherche)

Etape 4 Les experts retournent leurs évaluations quant à la pertinence de la publication pour l'éditeur du journal.

Etape 5 Les éditeurs décident sur la base des expertises soit de publier, rejeter ou demander des révisions et une nouvelle soumission. Si cela leur est demandé (réviser et soumettre de nouveau le manuscrit), les auteurs le font en tenant en ligne de compte les suggestions des experts. Ce processus peut prendre plusieurs mois

Etape 6 Les recherches révisées sont examinées par l'éditeur du journal qui prend une décision finale.

Etape $7 \quad$ Le manuscrit est accepté ou refusé

Tableau $\mathrm{n}^{\circ}$ 11- Cheminement d'un manuscrit à travers le processus de contrôle par les pairs éditorial

Si l'avis des experts est important et de premier ordre même, la décision finale revient toujours à l'éditeur qui doit, dans le cas où les rapports qu'il reçoit ne sont pas concluants pour une soumission, trancher et décider du sort de la publication.

Telles sont les différentes étapes du processus dans son expression la plus simple. Nous avons sciemment schématiquement donné les différentes étapes. Nous disons schématiquement car ces étapes sont entrecoupées d'échanges entre les trois protagonistes (auteur (s), éditeur (s), expert (s)) concernant les très probables changements à apporter avant que le manuscrit ne devienne une publication.

Il serait présomptueux et fastidieux de vouloir présenter ce processus dans les détails étant donné qu'il est loin d'être uniforme et que les écrits sur le sujet ont abordés les différentes phases. Nous préférons plutôt nous intéresser aux différents écrits que ce processus a engendré surtout en ce qui concerne la neutralité , la viabilité , la justesse de ce processus .Si

\footnotetext{
(39) The British Academy, op.cit., p.3
} 
les chercheurs admettent tous qu'il est indispensable à la bonne marche de la Science, il n'en demeure pas moins que durant les cinquante à soixante derniers années où il a été institutionnalisé , le contrôle par les pairs a connu des couacs qui mettent en doute sa validité et, dans certains cas extrêmes, son existence même. En effet, face à la pression de publier afin de progresser, d'obtenir des financements et de se voir promouvoir à des postes importants, les chercheurs doivent passer par ce processus qui authentifie, sélectionne et " sépare la graine de l'ivraie" selon l'expression utilisée par certains chercheurs pour décrire le processus du contrôle par les pairs.

\section{$\underline{1.5-L e s ~ a r g u m e n t s ~ p o u r ~ e t ~ c o n t r e ~ l e ~ c o n t r o ̂ l e ~ p a r ~ l e s ~ p a i r s ~}$}

Le sujet du contrôle par les pairs est devenu à travers les années, et surtout dans les pays occidentaux, par la force des choses un sujet de polémiques et de contentions ayant atteint dans certains cas des formes n'honorant nullement le monde académique. Cette situation est le résultat d'une sorte de compétition entre chercheurs qui se disputent positions, financements, postes de responsabilité, promotions et titularisation à coup de publications et de l'autre coté les journaux et leurs éditeurs qui eux délivrent l'imprimatur de ces mêmes sésames vers la gloire. Si certains cas manifestes de blocages, de fraudes, de jugements erronés ont étés enregistrés (dont nous avons parlé en bref dans la deuxième partie de ce travail et que nous aborderons sous certains angles dans cette partie), il n'en demeure pas moins que le contrôle par les pairs est unanimement accepté par les différentes composantes de la communauté académique et scientifique comme indispensable à la bonne marche de la science et qui sans sa présence mettrait les journaux scientifiques au même niveau que les journaux hebdomadaires qui publient ce qui vend et non pas ce qui est vrai et reconnu. Là se situe la différence entre les journaux que nous lisons chaque jour (de plus en plus sur Internet d'ailleurs et ce point est important à plus d'un titre pour notre présent travail et surtout sa partie expertise sur Internet) et le périodique scientifique qui lui délivre un travail de recherche, donnant des résultats ayant passé le tamis de l'expertise de personnes supposées être des experts dans la spécialité. Selon Bailar," le contrôle par les pairs des journaux est un lointain et mystérieux business pour nombre de chercheurs. Quatre paradigmes semblent capturer la plupart des opinions actuelles au sujet du contrôle par les pairs des travaux scientifiques soumis à la publication. [Ce sont]:

1- Le tamis (le contrôle par les pairs filtre les soumissions qui méritent d'être publiées et celles qui ne le sont pas)

2- La réorientation (un auteur persistant peut éventuellement publier n'importe quoi, mais le contrôle par les pairs décide où il le publie)

3- La mise en forme (les articles seront changés en des formes meilleures et nouvelles entre le marteau du contrôle par les pairs et l'enclume des critères éditoriaux)

4- La prise de risque (le contrôle par les pairs est essentiellement imprévisible et non reproductible et, de ce fait, aléatoire) " ${ }^{(40)}$.

\footnotetext{
(40) Bailar, John C. III, and Patterson, Kay, Journal peer review: the need for a research agenda, New England Journal of Medecine, Vol. 312, p.654-657, 7 Mars 1985
} 
Ces quatre paradigmes, pour reprendre Bailar, constituent en fait les quatre fonctions que joue le contrôle par les pairs.

Le premier (la fonction de tamis ou de filtre) est, selon nous, la plus importante et la moins décriée par la communauté scientifique, étant donné qu'un scientifique sérieux veut que le travail qu'il fait soit bâti sur des bases solides. Comme nous l'avons dit, le travail scientifique (la Science en général) avance par incrémentations et ce qui est découvert n'est que le résultat de ce qui a été découvert avant et ceci construit la Science. Le contrôle par les pairs assure cette fonction de tamis et permet de bâtir sur ce qui est acceptée par la communauté scientifique comme savoir expertisé et certifié.

Le deuxième (la réorientation) permet à chaque article de trouver sa niche. Il est de notoriété que les critères de rejet et d'acceptation des articles soumis différent de journal en journal. Une sorte de classification qui va du plus journal le sélectif au plus permissif s'est établi entre les différents titres. Le facteur d'impact (qui schématiquement donne le nombre de fois l'article a été cité) permet cette classification. De ce fait, un auteur dont la recherche a été rejetée par un journal la soumettra à un autre dont le facteur d'impact, les critères de sélection l'aura, la réputation sont moins astringents.

Le troisième (mise en forme) permet à l'article soumis de s'améliorer, de corriger ses erreurs et par delà de faire progresser l'auteur. Bailar utilise la métaphore du marteau et de l'enclume pour faire ressortir que la soumission est tiraillée entre deux pôles divergents qui lui imposent des critères qui ne peuvent que l'améliorer. D'ailleurs, nombre de recherches ont prouvés le bien fondé de l'idée que le processus éditorial améliore le manuscrit. Les experts, étant au courant des dernières recherches qui passent par les comités ont tendance, à apporter un plus que le chercheur, sans recul par rapport à sa propre recherche, ne voit pas.

Le quatrième (la prise de risque) est quant lui un paradigme qui est au centre de toutes les controverses qui agitent le monde la publication scientifique et qui a engendré moult recherches et contributions quant à la viabilité et même l'utilité du contrôle par les pairs .Ce problème qui a fait surface après des décisions contestables de la part de certains experts et éditeurs a introduit une sorte de suspicion quant aux décisions prises et les raisons de certaines positions de la part des éditeurs. Nous aborderons certaines de ces positions lorsqu'on abordera les différents griefs portés à l'encontre du contrôle par les pairs.

\subsection{1-Un exemple de réaction extrême au contrôle par les pairs}

"a) sujet aux préjugés, y compris les préjugés des experts, les préjugés de l'éditeur, des différentes formes de préjugés

b) non scientifique et manquant de preuves quant à son importance

c) n'ayant pas de résultat mesurable, et quand la recherche est conduite, c'est généralement sur la qualité de l'expertise plutôt que la qualité du manuscrit

d) conservateur, tendant à accepter pour la publication des articles qui sont moins controversés et moins innovateurs

e) lent et onéreux

f) produisant des recherches qui sont souvent scandaleusement imparfaites 
g) incapable détecter les fraudes

h) imprécis

i) subjectif

j) sujet au secret

k) ayant un nombre d'experts incompétents

1) produisant un accord relativement bas parmi les experts d'un même manuscrit

m) ayant des difficultés à affronter la dissidence [scientifique]

n) inutile

o) conduisant à des cas de malhonnêteté potentielle entre les experts qui, des fois, volent des idées des manuscrits ils expertisent

p) étouffant la communication scientifique et de ce fait ralentissant la progression du savoir

q) sujet à des pressions politiques diverses

r) incestueux [sic] avec un petit groupe de d'experts jugeant les travaux les uns des autres, surtout dans des petites spécialités étroitement définies.

s) ayant des experts qui sont acerbes, méchants, trop critiques, arbitraires, égoïstes, sauvages [sic], impolis, irresponsables, arrogants, inappropriés " et il existe probablement quelques autres adjectifs choisis dans la littérature " [du sujet]."(41)

C'est en ces termes peu amènes et à la limite de la caricature et de l'invective que ce chercheur a décrit le contrôle par les pairs. Si le coté théâtral de cette énumération, dont le but est sans doute de frapper l'imagination, est évident, il n'en demeure pas moins que les chercheurs, surtout Outre Atlantique, dans leur écrit expriment une sorte de ras le bol et désillusion quant au contrôle par les pairs qui est devenu la cible de toutes les critiques et même, selon ces mêmes chercheurs, la raison de leurs échecs (supposés).

Selon Weller ${ }^{(42)}$, idéalement un expert devrait aborder la recherche avec l'intention de la juger impartialement et d'évaluer le manuscrit seulement sur la base de ses mérites scientifiques. Les seuls facteurs pertinents que l'expert doit juger sont la qualité et l'importance du manuscrit, sa conception, sa méthodologie, son analyse, la discussion des idées, les conclusions et la logique du processus de pensée. D'autres critères incluent la rigueur logique, la contribution au savoir, la conception de la recherche, la méthodologie, l'objectivité, le choix du sujet, le style d'écriture et l'organisation. Elle insiste sur le fait que le prestige de l'auteur, l'affiliation institutionnelle, le pays, le sexe, l'ethnicité, les sources de financement ou toute autre information sans rapport avec le projet de recherche ne devraient pas influer sur les commentaires ou recommandations de l'expert, de même pour les intérêts et les points vue personnel de l'auteur.

Il ne faut pas oublier que la fonction de gardiens du temple (Gatekeeping pour reprendre le terme exact utilisé en langue anglaise) a donné aux gatekeepers des pouvoirs obnubilant avec

\footnotetext{
${ }^{(41)}$ Schmelkin, L. P., Peer review: Standard or delusion? Presidential Address delivered at The annual meeting of the American Psychological Association, Division 5, Toronto, Canada (August 2003).

${ }^{(42)}$ Weller, Ann, C. , op.cit., p.207
} 
lesquels ils peuvent soit impulser ou au contraire briser la carrière d'un chercheur. Parmi ces innombrables (et parfois injustifiées) raisons, une semble presque faire l'unanimité au sein des chercheurs et elle concerne les préjugés".Quand on parle de préjugé, on parle de l'action qui fait du gardien du temple une personne dont le travail de sélection, de choix, d'évaluation, de correction ne se fait pas d'une manière objective, ni impartiale et obéit à des raisons que l'objectivité et le bon sens répriment. Cet aspect a été largement abordé par de nombreux chercheurs qui ont voulu prouver que le contrôle par les pairs au lieu d'agir comme un tamis sélectionnant le bon et éliminant le mauvais, est devenu en fait une arme politique permettant d'arriver à de substantiels avantages découlant des différents financements que la publication (ou non publication, c'est selon le prisme duquel on parle) permet d'atteindre. Bien que les griefs à l'encontre du contrôle par les pairs soient nombreux et variés, on peut affirmer sans risque de se tromper dire que le problème du préjugé et de l'à priori est le problème qui cristallise la plupart des critiques. Nous essayerons de l'examiner en premier lieu vu sa prépondérance et son importance et aussi l'abondante documentation qu'il a engendré.

\section{6-Les différents et plus courant préjugés du contrôle par les pairs selon Shatz}

Il est de notoriété que le contrôle par les pairs a engendré une foultitude de critiques quant à son déroulement mais la littérature du sujet a particulièrement fait ressortir le problème des préjugés à l'encontre d'une soumission donnée. Ces préjugés sont très nombreux et différents mais les recherches en la matière ont fait ressortir quelques uns dont 1'occurrence est plus régulière et insistante. A cet effet, Shatz dans son séminal travail sur le contrôle par les pairs en distingue quatre types: ${ }^{(43)}$

-Préjugé Ad hominem (préjugé à l'encontre d'une personne donnée)

-Préjugé institutionnel (préjugé à l'encontre d'une affiliation donnée)

-Préjugé idéologique (préjugé à l'encontre d'une idéologie donnée)

-Préjugé esthétique (préjugé à l'encontre d'une esthétique donnée)

Etant donné l'extrême sensibilité de ce problème et de ces conséquences (effectives ou supposées) sur l'avenir et le devenir des chercheurs, il est d'une importance primordiale d'examiner en plus en détails ces quatre préjugés qu'auraient les experts quand ils jugent les soumissions de leurs collègues (et parfois rivaux).

\footnotetext{
* Ouvrons ici une parenthèse pour signaler que nous avons choisi d'utiliser le mot " préjugé " (au lieu d'à priori, penchant ou favoritisme) car il nous a semblé le plus adéquat pour décrire cette état de fait .De l'autre coté, le terme " bias " en anglais semble faire le consensus et est utilisé pratiquement à l'exclusion de tout autre terme. Encore une fois, il semblerait que le fait que le sujet est beaucoup plus abordé dans la littérature anglo-saxonne a induit une sorte de consolidation, standardisation et unification des concepts. D'ailleurs, tout au long de cette recherche, l'un des problèmes les plus épineux que nous avons rencontré a eu trait au vocabulaire pratiquement en anglais exclusivement et qu'il a fallut traduire. La même chose s'applique au mot "gatekeeper" que Jean Claude Guédon a utilisé la première fois (dans le sens qui nous intéresse) dans " Open Access Archives: from Scientific Plutocracy to the Republic of Science" (http://archive.ifla.org/IV/ifla68/papers/guedon.pdf) et qui a été adéquatement traduit en gardien du temple donnant par delà l'idée de la personne (ou personnes) ayant la main haute sur l'accès à quelque chose d'important. D'autres traductions que nous avons pu rencontrer ont donné : gardien, portier et même éclusier !!!

(43) Shatz, David, op.cit., p.37-45
} 


\subsection{1-les préjugés Ad Hominem}

Le préjugé Ad Hominem est le préjugé qu'aurait un expert à l'encontre d'une personne donnée. Ce préjugé peut être positif ou négatif. Dans le premier cas, il peut s'agir d'une amitié ou alors une sympathie pour la situation personnelle de l'auteur (comme par exemple la personne doit être confirmée dans son poste et la décision peut soit briser ou relancer sa carrière ).Le préjugé peut être négatif quand par exemple, il peut s'agir de jalousie ou , plus plausiblement, quand ce même auteur est en compétition avec celui qu'il expertise pour un poste important ou une récompense ou un honneur ( un prix, une distinction, etc....).Dans ce cas en particulier, l'expert et l'auteur sont généralement dans la même spécialité et il est dans l'intérêt du premier à ce que la soumission du deuxième soit rejetée (un cas flagrant de conflit d'intérêt qui arrive très souvent dans le domaine biomédical où un brevet vaut des millions de dollars et où la primauté dans la découverte est primordiale, cas qui ne se présente pas dans les sciences sociales et humaines à part, peut être, dans des cas extrêmement rares).De même, l'expert peut faire preuve de favoritisme vis-à-vis de ces amis ( old boy's network) et promouvoir leurs étudiants et vice versa ne pas examiner et expertiser les soumissions des autres ne faisant pas partie de cette fratrie d'une manière objective. L'expert peut, dans d'autre cas, avoir des attitudes diamétralement opposées quant au même travail selon qu'il cite ou non leur propre travail. Dans ces cas, le préjugé Ad Hominem peut prendre des formes particulièrement crues car l'expert ne fait pas son travail scientifique mais est entrain d'exprimer ses sentiments personnels à l'encontre du soumissionnaire. Quand l'expert ressent soit de la jalousie, de la sympathie ou alors de l'amitié, ce n'est pas le travail qu'il expertise qu'il examine mais sa propre approche et point de vue à partir de prismes définitivement non scientifiques, quoiqu' il tend à ne pas reconnaître cet état de fait. Une autre forme de préjugé Ad Hominem, plus pernicieuse à notre avis, concerne l'impression (favorable ou défavorable) que l'expert a du travail qu'il a sous les yeux et ceci à partir, non pas de sa valeur intrinsèque actuelle, mais à partir de l'opinion qu'il en a eu au cours d'une soumission précédente. Pour être plus clair, le préjugé (encore une fois favorable ou défavorable) de l'expert s'exprime dans son jugement non pas sur la base du travail qu'il est entrain d'expertiser mais sur l'idée qu'il a de l'auteur et de sa compétence basé sur une évaluation dans le passé. Ce préjugé est beaucoup plus prépondérant dans le cas défavorable (où l'auteur qui aurait, dans le passé, fait une soumission estimée médiocre serait " condamné " à ne pas progresser) que favorable (où l'auteur qui aurait été bien jugé dans le passé - ou est devenu une référence dans sa spécialitéserait automatiquement attribué un avis favorable, sans prendre en ligne de compte que ce même auteur peut avoir régressé ou s'est endormi sur ses lauriers). Nombre de faits et d'expérimentations ont prouvés que des travaux de tous bords, lorsqu'ils sont soumis par d'illustres inconnus sont automatiquement rejetés alors que ces mêmes travaux soumis par des personnes connues, sont presque automatiquement acceptés. Le célèbre sociologue américain, Robert K. Merton, a décrit cette situation dans un article (44) désormais célèbre sous l'appellation désormais célèbre aussi de " l'effet Matthieu" tiré de l'évangile selon Mathieu et qui dit :" Car on donnera à celui qui a, et il sera dans l'abondance, mais à celui qui n'a pas on ôtera même ce qu'il a ".Pour paraphraser Shatz, l'effet Matthieu donne plus de pouvoir aux puissants et affaiblit ceux qui n'ont n'en pas. 


\subsection{2-Les préjugés institutionnels}

Les préjugés institutionnels sont des préjugés que l'on aurait au sujet d'une institution (affiliation) donnée. Dans ce cas, l'expert assumerait que le travail d'un auteur issu d'une institution donnée serait de qualité supérieure (ou inférieure) par rapport aux autres institutions de part son appartenance à cette institution. Dans la même veine, on peut évoquer les préjuges d'ordre sexiste ou racial dans lesquels l'expert jugerait la soumission non pas sur sa valeur intrinsèque mais jugerait qui soumet (femme - homme, blanc -noir, etc....).

Les chercheurs d'institutions prestigieuses comme par exemple Harvard ou MIT aux Etats Unis, Princeton en Grande Bretagne, La Sorbonne en France auraient donc un préjugé favorable en tant que membre de cette institution par rapport à un chercheur dont l'institution n'aurait la même aura, nonobstant la qualité du travail. On remarquera que ce préjugé s'apparente, d'une certaine manière, au préjugé Ad Hominem et à l'effet Matthieu, dans le sens où les chercheurs des institutions " huppées " seraient encore plus avantagés de par leur appartenance et récolteraient de ce fait plus de dividendes (publications $\rightarrow$ avancements).En fait, ce préjugé, en plus de l'effet Matthieu dont il est le produit, est dû à l'approche que l'expert a vis à vis la soumission qui lui est proposée. En effet, l'expert a tendance à avoir eu la même formation et à utiliser les mêmes méthodes que le soumissionnaire en plus du fait qu'il a de fortes chances que son appréhension de ce qui est important ou pas soit la même. Cette manière de voir s'apparente au préjugé idéologique (en fait c'est ce préjugé idéologique - qui influe sur le processus plutôt que le contraire). L'éditeur de son côté peut (et est souvent sujet) à ce genre de préjugé quand il a tendance à privilégier la publication de recherches portant " l'en tête " d'une prestigieuse institution ajoutant de ce fait à l'aura de son journal.

\subsection{3- Les préjugés idéologiques}

Les préjugés idéologiques sont des préjugés qui tendent à influer positivement ou négativement la position d'un expert. Dans les deux cas, l'expert peut soit accepter et être attentif aux arguments qui confirment les siens ou contrairement être contre les arguments qui vont à l'encontre de ses propres points de vue. De plus, le préjugé idéologique a été décrit comme un préjugé " à la mode " en ce sens que les recherches qui vont à l'encontre des théories acceptées et ayant " pignon sur rue " ont de fortes chances de se voir rejeter alors que les recherches qui abondent dans le sens de ces théories ont de fortes chances de se voir accepter . Ceci a engendré un total déséquilibre dans les taux des acceptations en conséquence d'une autre donnée : les experts étant choisis parmi les plus reconnus des scientifiques, il y a de fortes chances à ce que ces derniers abondent dans le sens du statu quo et acceptent les soumissions ne sortant pas des sentiers battus .Enfin, il est ( presque ) normal pour un scientifique de ne pas prendre de risques et d'adouber une idée qui n'a pas eu le consensus de la communauté. Shatz parle de "conservatisme épistémique" et de " conservatisme méthodologique " et qui consisterait et mandaterait une personne à défendre ses points de vue ultérieurs même si l'évidence (ou les évidences) ne semble pas supporter de manière adéquate l'idée qu'il défend. Ce genre de préjugé ressemble en bien des points au préjugé ad hominem précédemment exposé. 


\subsection{4-les préjugés esthétiques}

Est ce qu'un article imprimé impressionne plus le lecteur qu'un simple manuscrit? Est-ce que la substance d'un article imprimé impressionne plus quand c'est réimprimé dans une police agréable à voir ? Si la réponse est positive, alors Shatz affirme que les préjugés esthétiques existent. Il poursuit que l'apparence affecte le jugement du contenu " les gens jugent les livres par leur couverture ".Si ces critères sont importants dans la recherche d'un emploi (les candidats à un poste de travail sont souvent conseillés de faire une présentation - aussi bien vestimentaire que de leur curriculum vitae - aussi propre et ordonnée que possible car ceci impressionnerait positivement leur éventuels employeurs), il n' y a pas d'indication que cela soit important pour la rédaction d'un article. L'expert n'accorde pas une importance démesurée au fait que le chercheur soit une personne négligée dans sa tenue ou même dans sa vie de tous les jours. Bon nombre de scientifiques distingués sont connus pour être très désordonné "...leurs bureaux donnent l'impression qu'ils viennent juste d'être frappés par un ouragan ".Cette manière de voir les choses résume à elle seule l'image désormais célèbre mais caricaturale du scientifique génie mais aussi personne un peu désordonnée et tête en l'air.

\section{7-L'étude de Stephen et Ceci et le préjugé institutionnel}

Ce préjugé (institutionnel) a cristallisé l'attention des chercheurs sur le sujet car en plus des retombées académiques, il est passé à un niveau politique dans le sens où publication équivaut à augmentation de salaires, postes de responsabilité, promotions etc....(et ce surtout dans les pays développés) et nul doute que ces retombées positives ne laisseraient pas les acteurs insensibles. A cet effet, une recherche menée en 1982 par deux psychologues américains ${ }^{(45)}$ et qui fait référence dans le domaine a démontré la justesse de cette thèse (quoique la conception de l'expérimentation, sa méthodologie et même les conclusions ont suscité chez les chercheurs du domaine des réserves qu'il serait hors de sujet d'aborder en détails ici).De quoi s'agit il exactement? Tel que l'indique le titre de la recherche, il s'agit du sort d'articles en psychologie acceptés pour la publication et qui furent resoumis pour voir quelle sera la réaction des mêmes journaux les ayants publié en premier lieu. Bien que l'étude ait suscité des remarques de par ses faiblesses méthodologiques et autres, il semblerait qu'elle confirme la thèse du préjugé institutionnel. En effet, les auteurs ont ressoumis 12 articles déjà publiés par des chercheurs prestigieux et très productif appartenant aux 12 plus prestigieuses universités américaines dans le domaine. Ces articles publiés le furent dans 12 des plus prestigieux journaux américains en psychologie dont le taux de rejet avoisinait les $80 \%$.Il est à signaler que ces journaux pratiquaient le processus d'expertise non aveugle. Les chercheurs qui soumirent une deuxième fois les manuscrits altérèrent les noms et les institutions originels ainsi que le contenu (titre, mots clés, méthodologie, etc.....) mais pas d'une manière radicale. La dernière étape de la première partie de cette étude fut l'envoi aux mêmes périodiques qui les avaient acceptés dix huit à trente deux mois auparavant. Les résultats obtenus furent, le moins que l'on puisse dire, étonnant. Qu'on en juge :

\footnotetext{
(45) Peters, Douglas P. and Ceci , Stephen C., Peer-review practices of psychological journals: The fate of published articles, submitted again, Behavioral and Brain Sciences, Vol. 5, n² , June 1982, pp 187-195
} 
-de l'échantillon des trente huit éditeurs et experts, seul trois détectèrent la resoumission.

-ce résultat permit à neuf des douze resoumissions de continuer le processus de contrôle par les pairs.

-de ces neufs resoumissions, une seule fut acceptée et huit furent rejetées.

- seize des dix huit experts recommandèrent le rejet et les éditeurs les suivirent dans leur décision.

-dans la plupart des cas, les raisons ayant entraîné le rejet étaient décrits comme " défauts méthodologiques sérieux ".

Cette étude a depuis été analysée, critiquée car elle était, d'après ses détracteurs, méthodologiquement non fiable pour nombre de raisons. Face au tollé que cette étude provoqua dans le monde académique et ses répercussions sur le processus, Behavioural and Brain Science (le périodique qui accepta la recherche) publia une série de réponses (cinquante six exactement) où les chercheurs en général et les chercheurs dans le domaine de la publication scientifique et sa certification en particulier exprimèrent leurs griefs et critiques. Ils peuvent être résumés en :

-En plus de la manière trompeuse avec laquelle l'étude a été menée, la plupart des critiques avaient trait la taille de l'échantillon qui était trop petite. De ce fait, les différences peuvent être aléatoires et dues à la chance.

-D'autre part les experts avaient de fortes chances d'être critiques étant donné que ces journaux avaient de toute façon un haut taux de rejet.

-De plus ces experts étaient différents de ceux qui avaient expertisé la première fois et de ce fait il y avait une différence d'âge, de personnalité, niveau d'expérience et de compétence.

-D'autres chercheurs de leur coté soulignèrent le fait que deux années s'étaient écoulées depuis la première publication et qu'en conséquence, il était presque normal qu'il y ait rejet vu que les experts étaient moins impressionnés par une recherche qui pourrait paraitre dépassée, périmée et désuète.

-De plus l'utilisation d'appellation vagues, triviales et à la limite du condescendant telles que " Tri-Valley Center for Human Potential " a pu, peut être, influé sur la décision. La mention " Université " même accolée à un nom obscur aurait eu un résultat différent.

-Enfin, la remarque la plus intéressante - et qui a trait au statut du soumissionnaire - a été de dire qu'il était presque normal que le prestige de l'institution d'un chercheur soit un garant de la qualité du travail.

Nous remarquons, personnellement que ce dernier point est un cas avéré de préjugé institutionnel que nous avons eu l'occasion d'aborder.

Nous nous proposons d'examiner plus en détails cette étude afin de voir quelles ont en étés la méthode, la procédure et enfin les résultats. 


\subsection{1-Méthode}

Au début, cette étude devait inclure treize articles publiés dans des périodiques scientifiques contrôlés par les pairs. Cependant, les auteurs apprirent durant les premières phases de l'étude, qu'un journal avait changé ses critères de publication en conséquence de l'arrivée d'un nouvel éditeur. Ce journal ne fut pas inclut dans les journaux de l'étude .Ceci a été fait pour éviter de fausser les résultats, l'arrivée d'un nouveau éditeur étant, généralement, synonyme de changement de la politique générale du journal.

Les douze journaux représentent un large spectre des spécialités de la psychologie avec chacune ayant une représentation dans l'American Psychological Association. Le taux global de rejet de ces journaux avoisinait les $80 \%$ au moment de l'acceptation des articles en première instance. Ces journaux étaient aussi considérés comme des journaux sérieux, faisant partie du courant dominant et hautement considérés par les chercheurs en terme où publier et où ils s'attendent à ce que les résultats les plus importants dans la spécialité soient publiés. De plus une analyse de l'impact de ces journaux, révèle que des douze journaux, dix étaient classés dans les vingt premiers à partir d'une liste de soixante dix sept journaux en psychologie. En outre, la moyenne annuelle de fréquence de citation par journal et article dans le Journal Citation Report pour les douze journaux était de 1.15 ce qui place ces journaux dans le top 75\% des journaux recensés par le Journal Citation Report en plus du fait qu'ils représentent quelque 70\% des journaux " prestigieux" en psychologie. Enfin, point important, tous les journaux utilisent le principe de contrôle par les pairs non aveugle.

\subsection{2-Procédure}

Un article de chacun des douze journaux fut choisi au hasard. La seule contrainte quand à la sélection était que l'article devait se conformer au critère " prestige" et qui était dans cette étude qu'au moins un des auteurs soit affilié à une institution dont le département de psychologie était parmi les meilleurs en terme de classement, productivité et les citations des membres du département. Les articles choisis avaient les caractéristiques suivantes :

a) à part un cas, tous les auteurs originaux étaient parmi les dix plus prestigieux départements de psychologie aux Etats Unis.

b) les auteurs furent sélectionnés à partir des départements de psychologie qui avaient les trente plus hauts classements de production et $75 \%$ des auteurs faisaient partie des dix meilleurs départements de psychologie dans leurs spécialités respectives.

c) Chaque article avait au moins un auteur qui faisait partie des meilleurs vingt cinq institutions en terme de citation de production académique dont 50\% dans les six meilleurs.

d) Les articles furent choisis à partir de recherches publiées entre dix huit et trente deux mois avant la resoumission pour l'étude. 
e) Le nombre annuel moyen de citations dans le Social Science Index* pour les douze articles était de 1.5 aussi bien une année que deux années après publication.

f) Etant donné, tel que précisé auparavant, que le nombre moyen des citations par an des articles dans ces journaux durant cette période était de 1.15, il semble que les articles sélectionnés étaient au dessus de la moyenne en terme de qualité, si on utilise une mesure de décompte des citations de leur journaux respectifs. Il est à signaler que le nombre moyen de citations pour tous les articles en sciences sociales dix ans après leur parution est de 1.4 .

Avant la resoumission de l'article comme si il l'était pour la première fois, différentes modifications furent introduites. Premièrement les noms (mais pas le sexe) et l'affiliation institutionnelle des auteurs originaux furent changés en de fictifs noms sans sens ou statut en psychologie. Par exemple, Dr.Wade M.Johnson (un nom fictif) au Tri-Valley Center for Human Potential (une institution fictive).Les titres des articles originaux, les résumés, et le début des paragraphes de l'introduction furent légèrement modifiés. Les auteurs de l'étude espérait que ces quelques changements superficiels masquerait suffisamment les resoumissions dans le cas l'éditeur ou un expert utilisait un système mécanique de classement qui résulterait dans une détection automatique (titre, mots clés ou résumés, etc....).Les modifications apportées aux articles originaux étaient toujours minimales et purement cosmétiques ( changement de l'ordre des mots ou des phrases, substituer les synonymes pour des mots non techniques et ainsi de suite ).Le sens des titres des articles originaux, les résumes, et les premiers paragraphes ne furent jamais modifiés .Le reste des sections de l'introduction et toute la méthode, les résultats et la discussion furent imprimés exactement de la même manière qu'ils apparurent sur papier. Pour se prémunir un peu plus contre la détection superficielle, le format de présentation des données fut aussi occasionnellement modifié en changeant les graphes en tables et vice versa.

Chaque manuscrit fut préparé conformément avec les instructions aux auteurs de chaque journal respectif et ensuite envoyé au journal qui l'avait publié originellement avec une lettre de motivation demandant qu'il soit considéré pour publication. Les éditeurs et les experts n'étaient pas au courant des efforts .Ils furent informés au sujet de la nature du projet soit quand ils détectèrent que le manuscrit était une resoumission ou quand l'étude fut terminée. Etant donné que ces événements se passèrent à différentes périodes pour différents journaux, il fut demandé à tous les participants de garder l'existence de l'étude confidentielle jusqu'à ce qu'elle soit terminée.

\footnotetext{
* Le Social Science Citation Index est un index de citation interdisciplinaire. Cette base de donnée de citation couvre quelque 2474 parmi les journaux en sciences sociales les plus important dans le monde et ce dans plus de cinquante disciplines. Cette base de données fournit l'information pour identifier les articles les plus fréquemment cités et par quel éditeur et quel auteur.
} 


\subsection{3-Résultats}

\subsubsection{1-La fiabilité des experts}

Neuf des douze manuscrits soumis ne furent pas détectés (ni par les éditeurs ni par les experts) comme ayant été publiés auparavant. Ces articles ayant reçu récemment (entre deux et trois ans) de positives évaluations qui a résulté en leur publication, une seconde expertise par le même journal aurait dû donner les mêmes résultats i.e. reconnaissance du caractère scientifique et recommandation de publication. Cependant comme on peut le voir dans le tableau suivant ${ }^{(46)}$ ceci était l'exception plutôt que la règle :

\begin{tabular}{|c|c|c|c|c|}
\cline { 2 - 5 } \multicolumn{1}{c|}{} & \multicolumn{2}{c|}{ Experts seuls } & Editeurs et Experts \\
\hline Journal & Rejet & Acceptation & Rejet & Acceptation \\
\hline A & 2 & 0 & 3 & 0 \\
\hline B & 3 & 0 & 5 & 0 \\
\hline C & 1 & 0 & 1 & 0 \\
\hline D & 2 & 0 & 2 & 0 \\
\hline E & 2 & 0 & 4 & 0 \\
\hline F & 2 & 0 & 3 & 0 \\
\hline G & 2 & 0 & 4 & 0 \\
\hline H & 2 & 0 & 4 & 4 \\
\hline I & 0 & 2 & 0 & $4(13 \%)$ \\
\hline
\end{tabular}

Tableau n ${ }^{\circ}$ 12- Fiabilité des experts ( Stephen et Ceci)

\subsubsection{2-Evaluations des resoumissions non détectées}

Huit des neuf articles [non détectés] furent rejetés. Seuls $13 \%$ des éditeurs et des experts combinés recommandèrent publication dans leurs journaux. Il faut ajouter que chaque éditeur ou éditeur associé inclus dans l'échantillon indiqua qu'il avait examiné le manuscrit et qu'il avait avalisé les recommandations des experts. Quand seules les évaluations des experts furent examinées - ce qui pourrait être plus significatif, les décisions des éditeurs n'étant pas indépendantes des évaluations des experts -, il fut découvert qu'une plus petite proportion (11\%) jugèrent les recherches acceptable pour la publication. Les experts pour sept des journaux (A-G) émirent des recommandations claires et sans équivoque concernant la publication (par exemple, journal A : "Il y a trop de problèmes associés à ce manuscrit pour recommander son acceptation pour ....").

${ }^{(46)}$ Peters, Douglas P. and Ceci, Stephen C,op.cit. p.197 
Deux experts pour le huitième journal dans la liste $(\mathrm{H})$ n'émirent aucune recommandation explicite quant l'acceptabilité de la publication mais leurs expertises étaient exclusivement négatives qu'on pourrait peut être mieux caractériser par une liste d'erreurs, de faiblesses [méthodologiques] et de lacunes. Des copies ces deux expertises indécises sans l'identification des journaux furent envoyées à six psychologues (qui tous exerçaient en tant que consultants éditoriaux ou éditeurs) à qui on demanda d'évaluer le manuscrit en question sur la base de ces commentaires seulement. On utilisa une échelle de cinq points et qui consistait en :

a) Contribution majeure : profonde, théoriquement importante, très bien conçue et exécutée, acceptée sans question

b) Publication justifiée : travail sérieux, contribution solide, à accepter avec des révisions mineures

c) Assez sérieux et important: publication justifiée si l'espace le permet.

d) Mal rédigé : utilité limitée, pourrait être publiable si certaines aspects sont améliorés ou développés, nécessite des révisions importantes.

e) Pas assez sérieux ou important pour justifier la publication

Les notes moyennes pour les deux expertises furent 4.8 et 5.0.Vu que ces chiffres supportent interprétation propre (ainsi que celle de l'éditeur) qu'un message clair de rejet était implicite, il était justifié de classer ces expertises comme " rejet ".

\subsubsection{3-Commentaires critiques émis par les experts}

Les objections les plus fréquentes que les experts avaient à l'encontre des manuscrits avaient trait aux conceptions des études et recherches et les analyses statistiques. Plusieurs experts détectèrent des faiblesses méthodologiques dans les recherches resoumises. Par exemple, Journal B : " un sérieux problème se situe au niveau de la portée de la difficulté du matériau de ......... à l'intérieur des groupes. Il n'est pas fourni de compte rendu de cette portée et pas de contrôle de ses effets possibles n'est fourni. De même, la compatibilité des matériaux à travers les groupes est inconnue ". Journal D : " les analyses séparées de chaque test basées sur l'hypothèse que les groupes sont équivalents et déduire des gains peut être trompeur ...spécialement quand les analyses peuvent être faites directement " ; Journal E : " Je ne pense pas que cette recherche soit publiable, elle a besoin d'un travail expérimental plus poussé " ; Journal H : "Il n'est pas clair qu'est ce que les résultats de cette recherche démontrent, en partie parce que la méthode et les procédures ne sont pas décrits avec suffisamment de détails mais surtout à cause de plusieurs défauts méthodologiques dans la conception de l'étude "; Journal G : " Quelques autres problèmes étaient ......l'utilisation d'A.N.O.V.A. (analyse de la variance) .....perte de 3 enfants d'expérimentation 2 à expérimentation 3".

A part quelques changements cosmétiques mentionnés plus tôt, les manuscrits resoumis étaient identiques aux versions originales qui avaient apparus sur papier. Nombre d'experts des recherches resoumises critiquèrent les capacités de rédaction et de communication des 
auteurs. Par exemple, Journal G : " Resserrez l'orientation théorique dans l'introduction. Elle parait imprécise et remplie de généralisation excessive (ou du moins de conclusions non documentées) à l'heure actuelle "; Journal E: "De même, je ne sais pas ce que veut dire les joueurs avaient la capacité d'avoir différents numéros de marqueurs au but, étant donné que le jeu tel que décrit en page cinq était tel que chaque joueur avait un seulement un marqueur. Tout est très confus......Je pense que toute la présentation des résultats a besoin d'être planifiée plus soigneusement et réorganisée" ; Journal F : " Apparemment, ceci est supposé être un résumé. Cependant, le style de rédaction laisse beaucoup à désirer en termes de communiquer au lecteur. Elle exige de l'auteur d'aller d'un résultat positif à une double négation, à une qualification, à une négation des résultats et enfin à deux phrases (avant dernier dans ce paragraphe) dont l'interprétation n'est juste pas claire ".

\subsubsection{4-Reconnaître de la recherche déjà publiée}

Quand les articles furent ressoumis aux journaux qui les avaient originalement publiés, combien furent détectés ou reconnus? La réponse peut être trouvé dans le tableau suivant. Les résultats sont examinés par:

a) L'inclusion de toutes les personnes représentant chaque journal (éditeur, assistant éditeurs et experts) qui avaient un quelconque contact avec le manuscrit et de ce fait auraient pu détecter la supercherie et

b) L'inclusion des experts seulement étant donné qu'on peut raisonnablement soutenir qu'il serait injuste de s'attendre à ce que les éditeurs soient au courant de toute la littérature de chaque spécialité couverte par leur journal.

Les résultats montrent qu'il y a peu de différence quel groupe est étudié car les résultats sont essentiellement identiques. Aussi surprenant que cela puis paraître, la majorité écrasante des éditeurs et des experts ( $92 \%$ ou $35 / 38$ des éditeurs et des experts ensemble et $87 \%$ ou 20/23 des experts tout seuls) ne réussirent pas reconnaître les manuscrits comme des articles ayant apparu dans la littérature du courant dominant du domaine de recherche approprié à cette recherche durant les derniers dix huit à trente deux mois. Il est à souligner que seulement deux journaux ( $\mathrm{C}$ et $\mathrm{I})$ avaient changé les éditeurs durant cette période 


\begin{tabular}{|c|c|c|c|c|}
\cline { 2 - 5 } \multicolumn{1}{c|}{} & \multicolumn{2}{c|}{ Editeurs et experts } & \multicolumn{2}{c|}{ Experts seuls } \\
\hline Journal & Détectée & Non détectée & Détectée & Non détectée \\
\hline A & 0 & 3 & 0 & 2 \\
\hline B & 0 & 5 & 0 & 3 \\
\hline C & 0 & 1 & 0 & 2 \\
\hline D & 0 & 2 & 0 & 2 \\
\hline E & 0 & 4 & 0 & 2 \\
\hline F & 00 & 3 & 0 & 2 \\
\hline G & 0 & 4 & 0 & 2 \\
\hline H & 0 & 4 & 0 & 1 \\
\hline I & 0 & 2 & 1 & 1 \\
\hline J & 1 & 2 & 1 & 0 \\
\hline K & 1 & 1 & 1 & $20(87 \%)$ \\
\hline L & 1 & $35(92 \%)$ & $3(13 \%)$ & 2 \\
\hline
\end{tabular}

Tableau ${ }^{\circ} 13$-Détection des resoumissions par les experts (Stephen et Ceci)

\subsubsection{5-Changement dans les taux de rejet et les critères de publication}

Etant donné que les changements dans les critères de publication d'un journal pourraient expliquer pourquoi les articles ressoumis ne furent pas acceptés une deuxième fois, les journaux et les autres sources d'information furent examinés pour voir quels changements, le cas échéant, furent introduits. La vérification des déclarations de rédaction et les sujets abordés dans chaque journal ne révéla aucun changement dans les axes de recherche (avec l'exception explicite de Journal M qui fut supprimé de toutes les analyses, tel qu'indiqué précédemment).Le tableau suivant montre la variation nette dans les taux de rejet pour chaque journal à partir du moment l'article original apparut jusqu'à la date de resoumissions pour cette étude .Il est clair que les chiffres de rejet globaux pour les neuf journaux qui expertisèrent les articles ressoumis étaient restés assez constant ( et haut, en moyenne autour des $80 \%$ ) à travers les deux périodes d'expertise. 


\begin{tabular}{|c|c|}
\hline Journal & $\begin{array}{c}\text { Taux de rejet original dont on a soustrait le taux de rejet au moment de la } \\
\text { resoumission du manuscrit }\end{array}$ \\
\hline A & $16 \%$ \\
\hline B & $7 \%$ \\
\hline C & $-2 \%$ \\
\hline D & $-15 \%$ \\
\hline E & $-6 \%$ \\
\hline F & $0 \%$ \\
\hline G & $15 \%$ \\
\hline H & $3 \%$ \\
\hline I & $5 \%$ \\
\hline J & $5 \%$ \\
\hline K & $0 \%$ \\
\hline L & $7 \%$ \\
\hline M & $-2 \%$ \\
\hline
\end{tabular}

Tableau $n^{\circ}$ 14-Stabilité des taux de rejet entre la publication originale et la resoumission (Stephen et Ceci)

Ce tableau des écarts des taux de soumission entre les deux périodes nous montre qu'à part deux ou trois journaux ( plus précisément trois journaux et qui sont A ,D, G ), l'écart dans ces taux est globalement stable et est même stable à 100\% dans deux journaux (F et L).D Quant aux autres journaux, le taux de variation est inférieur à $10 \%$ ce qui serait une preuve de la constance des critères de publication chez ces journaux et, par delà, éliminerait un quelconque changement à cause de critères soit plus de sévères ou plus lâches. Ce tableau démontre donc, à l'instar des tableaux précédents, que les journaux qui ont étés choisis pour cette étude n'ont pas changé leur critères d'acceptation d'une manière dramatique ce qui aurait expliqué un quelconque changement radical dans leur politiques.

\section{Conclusions :}

Il paraîtrait de prime abord que cette étude a prouvé le bien fondé du caractère partial du contrôle par les pairs. En plus, elle avaliserait l'idée que le contrôle par les pairs n'est pas capable de détecter les écrits de la littérature d'un domaine donné publiés dans des journaux à haut facteur d'impact (ce qui en dit long sur la capacité des journaux et leur éditeurs et experts - les fameux gardiens du temple - quant à la détection des fraudes et autres actes délictueux tels que le plagiat qui défraient de temps à autre le monde scientifique).

Mais tel que cela a été relevé par les chercheurs, cette étude prêche par un certain nombre de faiblesses méthodologiques qui ont en, peut être, amoindri la portée. Parmi ces critiques 
portées à l'encontre de cette étude, nous citerons quelques unes qui nous ont parues les plus importantes .Nous insistons sur cet aspect car depuis sa publication, cette étude n'a cessé d'être citée que ce soit pour être présentée comme la preuve de l'inutilité (ou du moins la non fiabilité) du contrôle par les pairs ou alors vilipendée comme ayant été bâtie sur des bases de recherche non scientifiques. Nous citerons quelques unes de ces critiques dans ce qui suit :

1) Des neufs journaux en question (de $\mathrm{A}$ à $\mathrm{H}$ ) examinés, un seul a répété son évaluation positive faite en premier lieu et a accepté la publication. Les articles sélectionnés avaient étés publiés par des journaux scientifiques prestigieux et reconnus qui avaient en premier lieu passé un rigoureux système de certification qui en moyenne rejetait $80 \%$ des soumissions. Comment peut ton expliquer qu'en deuxième expertise par le même journal, ils ne soient pas acceptés? Il existe un nombre d'hypothèses pour expliquer cette question.

2) Un changement dans la politique concernant le type de soumission considérée acceptable ou une augmentation substantielle (aux environs de 25 à $30 \%$ ) du taux de rejet impliquant une plus grande difficulté à faire accepter une recherche pourraient expliquer pourquoi les articles ressoumis aient étés rejetés. Cependant tel que mentionné plus tôt, aucun de deux facteurs cités (changement dans la politique d'acceptation ou changement substantiel dans les taux de rejet) ne fut observé ce qui élimine cette hypothèse.

3) On peut aussi argumenter que les experts pour les resoumissions n'ont pas reconnus la conception spécifique ou les détails de la recherche originelle, mais peuvent s'être rappelé les points soulevés expérimentalement. Ces articles ayant été publiés quelque temps après la complexion du travail, il est possible que leurs résultats aient été déjà incorporés dans ce qui est considéré, pour l'expert, comme connu dans le domaine. Ils auraient pu donc rejeter le travail sur la base de la redondance de ce qu'ils auraient pu se rappeler de la littérature. Cette éventualité aurait été accepté si les experts avaient indiqués dans leur rapports des objections et des griefs du genre " Ce point est dépassé et sans intérêt " ou " Ce travail n'ajoute rien de nouveau au domaine " ou alors " Des découvertes similaires ont étés signalées par des chercheurs préalablement ".Aucune des expertises n'offrit ce genre de raisons pour le rejet, ou même quelque chose de vaguement analogue. Les manuscrits furent rejetés essentiellement, pour des raisons de méthodologie et de traitement statistique et non parce que les experts jugèrent le travail non original ou nouveau.

4) Une autre hypothèse que les auteurs de la recherche ont évoqué serait ce qui est connu sous l'appellation d' " effets de régression ".Les articles choisis pour l'étude ayant été tous publié, la seule direction possible dans le cas d'une resoumission serait un taux d'acceptation moindre (moins de 100\%) et jamais supérieur, certains articles pouvant être rejetés. Quoique la régression vers la moyenne (Phénomène statistique signifiant que des mesures répétées dans une même population tendront davantage vers la moyenne ${ }^{(47)}$ ) n'était pas contrôlée (les manuscrits rejetés n'étant pas resoumis une

\footnotetext{
${ }^{(47)} \mathrm{http}: / / \mathrm{www}$. minerva-ebm.be/articles/FR/woordenlijst fr/regression vers moyenne.htm
} 
deuxième fois), il est toujours possible de se demander combien de régression serait probable. Même si les chiffres du taux d'acceptation originaux de l'article sont pris au minimum de $67 \%$ (i.e. avec l'éditeur et au moins un des deux experts recommandant publication), et même si la corrélation entre les pairs d'experts était de zéro (la possibilité la plus extrême), la régression attendue serait seulement vers le taux de base, à savoir le pourcentage d'experts recommandant la publication initialement ceci de toute évidence est loin des 11\%, tel que montré dans fig. 10. En d'autres termes, on devra émettre l'hypothèse d'une grande négative fiabilité de la régression pour tenir compte du considérable changement dans le jugement des experts. En fait, il a y aurait des raisons plus plausibles pour expliquer ces résultats.

Entre autres, les auteurs de la recherche évoquent un à priori systématique vis à vis des auteurs et des institutions. (à priori institutionnel et à priori du statut) qui peut être une source d'influence dans le cas de l'expertise des journaux scientifiques. Les auteurs des articles de l'étude faisaient tous partie d'institutions prestigieuses, reconnues et productives en psychologie. Les noms des auteurs et de leurs institutions étaient dans les deux cas (première et deuxième soumission) identifiés, la seule différence étant que les noms des auteurs et des institutions dans la resoumission avaient été changés. Les évaluations négatives prédominantes des resoumissions pourraient refléter une sorte d'à priori positif vis-à-vis des auteurs originaux par rapport à leurs appartenance à une institution prestigieuse. Ces auteurs pourraient avoir reçu une évaluation plus complaisante et moins critique que l'évaluation dont furent sujet les auteurs inconnus d'institutions aux noms peu connus (en fait de noms fictifs). Les experts s'attendent à ce que les manuscrits (et les recherches) émanant d'auteurs de prestigieuses universités soient supérieur en qualité en général que les manuscrits d'auteurs travaillant dans des environnements moins prestigieux. En conséquence donner plus d'évaluations variables aux auteurs d'institutions prestigieuses peut servir en tant que prophétie auto réalisatrice (prophétie qui modifie des comportements de telle sorte qu'ils font advenir ce que la prophétie annonce).D'un autre côté, les experts dans leur travail de certification font face à deux cas de figure diamétralement opposés et qui sont :

1) faire une évaluation " négative fausse " quand ils examinent des recherches soumises par des auteurs d'institutions prestigieuses autrement dit rejeter des soumissions qui sont de qualité supérieure.

2) faire une évaluation " positive fausse " quand ils examinent des recherches soumises par des auteurs d'institutions moins prestigieuses autrement dit accepter des soumissions qui sont de qualité médiocre.

Si les experts basent leurs critères d'évaluation sur l'expérience ou la conviction que les recherches de qualité proviennent en grande partie d'institutions et d'auteurs prestigieux, alors on a affaire à un cas clair d'à priori personnel et institutionnel.

En plus de ces griefs somme toute normaux car il ne faut pas oublier que ce travail est fait par des humains, un autre problème à commencé à être discuté dans le système d'expertise des journaux .Il s'agit de la responsabilité des experts vis-à-vis des auteurs. Les chercheurs de sciences sociales et comportementales étant sujet à des taux de rejection des plus prestigieux 
journaux de leur domaine avoisinant ou dépassant les $70 \%$, et considérant le nombre considérable de manuscrits soumis que les éditeurs et experts doivent certifier chaque année, il est normal que beaucoup ait été dit de la part des défenseurs et détracteurs du système de contrôle par les pairs concernant le besoin de responsabilisation.

Les éditeurs et les expert, se plaignent de leur coté de ce qui a été connu sous le vocable du " fléau d'expertiser l'auteur à répétition " ou " le récidiviste ".Résultant du désespoir, de l'insensibilité ou de l'entêtement, une portion significative du temps de l'expert est une suite de répétition - une succession de mauvaises recherches du même individu. Il a été suggéré qu'une banque centrale automatisée, comportant les titres et les auteurs rejetés, soit mise en place. Ces deux données (titres et noms des auteurs) seraient divisée en deux groupes :a) rejetées b) acceptable mais pas suffisant ou approprié pour le journal. Ces informations seraient alors distribuées aux éditeurs ou mieux seraient accessible sur des cassettes mises à la disposition de la communauté des chercheurs. L'hypothèse sous jacente à ce point est que " l'auteur à répétition " est une personne qui, au fond, sait que son manuscrit est de qualité douteuse mais espère qu'avec un coup de chance, tel que des experts indulgents, le manuscrit est accepté. Par conséquent, les resoumissions sont envoyées jusqu'à ce que soit " l'auteur à répétition " trouve une niche (comme c'est probablement le cas étant donné le grand nombre de publications existantes) ou que la liste des périodiques visés soit épuisée. L'existence d'une telle base de données fournissant le nom des auteurs rejetés aux éditeurs potentiels est destiné à dissuader " la soumission à répétition ". Il est clair que peu d'auteurs voudraient la potentielle et répandue notoriété d'être identifiés comme un " un raté de la publication".

De l'autre coté de ce problème de responsabilisation, on trouve les auteurs qui pensent que les éditeurs et les experts doivent être tenus plus responsable de la qualité des expertises ils performent dans le cours de leur décision quant à la publicabilité d'une recherche. Très souvent, les auteurs de manuscrits rejetés soutiennent que leurs recherches ont étés rejetées non parce qu'elles n'avaient pas assez de mérite mais parce qu'elles sont victimes de la faible fiabilité du jugement des experts (de toute évidence plus probable dans le cas de décisions partagées), leur incompétence, ou alors leur à priori ou préjugé.. Une récente proposition concernant la responsabilisation des experts a suggéré la création d'une " expertise des auteurs " des experts de journaux ${ }^{(48)}$.L'éditeur du journal enverrait à l'auteur avec la lettre de décision et les expertises un questionnaire qui solliciterait une évaluation de chaque expertise par l'auteur. Cette évaluation porterait sur l'attention qui lui est portée, l'équité dans le jugement et le coté constructif de l'expertise ainsi qu'une partie comportant des commentaires. L'éditeur classerait les questionnaires sous le nom de l'expert, notant la décision éditoriale et la disposition finale du manuscrit. A intervalles réguliers, peut être une fois par an, l'éditeur pourrait examiner ces questionnaires. Dans le cas, un expert est sujet à des réclamations répétées, il pourrait être mis fin à ses fonctions ou être réprimandé par l'éditeur. On peut penser qu'avec une telle procédure d'évaluation des experts, les éditeurs auraient une base à partir de laquelle ils peuvent éliminer les experts qui ne sont pas à jour par rapport à la littérature, rejettent constamment des articles sur la base de critères particuliers ou alors qui généralement favoriseraient les auteurs qui appartiennent à la même institution ou partagent des traditions de recherche similaires aux leurs. Les experts face à un registre de

\footnotetext{
${ }^{(48)}$ Hall, J., Author review of reviewers, American Psychologist, Vol .34, 1979, p.798
} 
plaintes (comparable à un registre des doléances mis à la disposition des usagers dans une administration) des auteurs en constante augmentation deviendraient, on l'espère, plus consciencieux dans leurs évaluations des manuscrits.

Il semblerait, d'après nombre d'études sur le sujet de l'anonymat de l'expertise que le contrôle par les pairs anonyme pose plus de problèmes qu'il n'en résout. Un système qui permettrait à l'expert de proférer des remarques excessives, insultantes, hors sujet et mal informées sur le travail et la personne du chercheur sans être tenu responsable ne peut être décemment décrit comme équitable. Dans une certaine mesure, l'expert est effectivement responsable par rapport à l'éditeur mais il existe de fortes chances pour qu'il y ait abus et ceci ne devrait pas être ignoré.

L'étude se termine par un nombre de suggestions quant à l'amélioration du contrôle par les pairs. Elles peuvent être résumées, mais non circonscrites, en :

-Adopter une norme de notation standardisée et unifiée dans laquelle un ensemble explicite de critères d'évaluation est mis en place.

- Former des experts de telle sorte que leurs expertises soient de meilleure qualité et plus sûres.

- Donner aux experts potentiels des échantillons d'évaluations éditoriales actuelles et leurs décisions de publications subséquentes. Les éditeurs auraient en charge la responsabilité d'expliquer les attributs spécifiques des manuscrits soumis et leur publicabilité. Une collection d'expertises et d'articles sur lesquelles les experts peuvent être d'accord (i.e. grande qualité, très publiable, qualité douteuse, rejeté) pourrait être très utile comme dispositif de formation ou de sélection pour les experts.

- Instaurer un système de contrôle plus responsabilisant pour les experts, l'idée de base étant une forme d'expertise des experts par des juges, des auteurs et des éditeurs. Surveiller systématiquement la qualité des rapports des experts devrait avoir un effet correcteur sur les pratiques d'expertise des journaux.

- Encourager les " commentaires ouverts des pairs ".L'idée de base de cette nouvelle forme de certification ouverte est de compléter le système traditionnel fermé de contrôle en donnant aux auteurs de manuscrits acceptés (expertisés) une chance de répondre ouvertement aux critiques. Les lecteurs du journal peuvent voir, avec l'article, les commentaires (des premiers experts et autres) et la réponse formelle de l'auteur publiée dans son entier avoir une chance d'examiner et évaluer ce processus de " désaccord créatif " et former leur propre opinion quant au mérite du travail d'un chercheur.

- Etablir le contrôle par les pairs en aveugle comme une politique d'expertise standardisée et normalisée. Nous avons déjà vu que l'à priori personnel et institutionnel posait problème dans certains cas et influençait (positivement ou négativement) le résultat de l'expertise. Il est clair que l'expertise à l'aveugle peut aussi engendrer des problèmes additionnels et même être contournée (l'identité de l'auteur pouvant être devinée facilement par un expert chevronné selon ses citations ou références) mais elle semble, en l'état actuel des choses, assurer un minimum d'équité. Il semble aussi que face aux différents scandales et critiques qu'elle soit devenue la manière la plus équitable et de ce fait, dans un monde aspirant à l'égalité et la responsabilisation, la seule voie à suivre. 
On peut conclure en disant que cette étude a touché du doigt et démontré que le contrôle par les pairs est traversé et est sujet à des pratiques qu'il est supposé combattre ou du moins éviter. En effet, tel que nous avons essayé de le faire ressortir, l'une des fonctions les plus importantes du contrôle par les pairs réside en sa qualité de sélection du meilleur de la Science pour qu'il soit publié. Cette fonction de tamisage ne peut être adéquate que si cette étape primordiale est appliquée et mise en vigueur de manière aussi neutre, impartiale que possible. Il semblerait que cette étude prouve le contraire, la resoumission des mêmes manuscrits aux mêmes journaux mais sous des noms et affiliations assumés a donné des résultats étonnants tendant à accréditer la thèse du préjugé personnel et institutionnel. Il est vrai que cette étude a été critiquée pour de nombreuses failles méthodologiques relevées par les chercheurs mais il n'en demeure pas moins que ses résultats sont de nature à jeter un voile de suspicion sur la neutralité des décisions rendues par les experts. Nous pensons personnellement que cette " anomalie " est somme toute normale. Nous disons normale car le travail de certification fait appel à des humains et ne pourra jamais être (malgré toutes les projections futuristes) assuré par une machine dans un monde dominé par l'automatisation. Ceci implique, malheureusement, des positions qui se démarquent de la neutralité, les personnes pouvant être sujet à des penchants et des inclinaisons dont les raisons sont d'ordre humain et affectif. D'ailleurs dans un célèbre échange entre deux chercheurs, Stanley Fish et Jeffrey Skoblow chacun présenta un avis divergeant quant à la justesse de la soumission en aveugle. Le premier plus âgé et plus connu que le premier argua du fait que le jugement et la certification de son travail devait prendre en ligne de compte son nom et sa stature car il avait acquis et construit (lui et ses semblables dans le même niveau scientifique) au fil des années une expérience, un savoir faire qui le distinguait des autres et ceci devait être pris en ligne de compte ${ }^{(49)}$. Il évoque même l'idée qu' " il n'y a quelque chose de telle appelée mérite intrinsèque " et que sa contribution devait être expertisée en connaissance de son identité. De son coté, Jeffrey Skoblow dans une réponse à cet article ${ }^{(50)}$ argue du contraire et défend le contrôle par les pairs en aveugle comme protégeant les " pauvres ouvriers " (i.e. les chercheurs débutants) contre la prépondérance et le traitement de déférence qu'auraient les soumissions des " lumières scientifiques " (i.e. les chercheurs chevronnés).Il trouve injuste l'accentuation du pouvoir de ceux qui, déjà, sont très puissants au détriment de ceux qui aspirent à les rejoindre. Il va plus loin en la comparant aux fameuses et infamantes "Reaganomics " *. Il termine sa réplique en affirmant que démystifier un mythe est une chose mais affaiblir les bas échelons de l'échelle académique est une autre chose.

Cet échange entre deux chercheurs du même domaine montre à quel point la publication scientifique est devenue au fil des années un véritable business dans lequel chaque aspirant

\footnotetext{
${ }^{(49)}$ Fish, Stanley, No bias, no merit : the case against blind submission, Publications of the Modern Language Association, Vol.103, n5, p. 739-748, October 1988

${ }^{(50)}$ Skoblow, Jeffey Fish on blind submission, Publications of the Modern Language Association,Vol.104, n`2 p.215-216, 1989

*Terme populaire qui fait référence à la politique économique adoptée par Ronald Reagan.Cette politique se fondait sur une réduction générale des impôts, une réduction des dépenses sociales, une augmentation du budget de la défense et la déréglementation des marchés intérieurs. La reaganomie s'appuyait en partie sur l'économie de l'offre et sur la théorie de la percolation. Ces théories avançaient que la baisse des impôts, en particulier pour les entreprises, est le meilleur moyen de stimuler la croissance économique. L'idée est que les économies d'impôt réalisées par les entreprises seront réinjectées dans l'économie via l'investissement et ainsi encourageront la croissance.
} 
chercheur cherche à obtenir sa part du gâteau. Ce qui a fait que les débats ont parfois débordé pour prendre des accents de règlements de compte et dépasser tous les règles de bienséance et virer à l'invective, l'insulte, l'accusation et l'anathème.

\section{8-L'expertise en aveugle}

Appelée aussi " expertise anonyme " ${ }^{(51)}$, cette procédure est supposée alléger et surtout diminuer les instances de pré jugement que l'expertise traditionnelle (où l'identité et l'affiliation de l'auteur sont connues) a toujours engendré .En effet, l'expertise à l'aveugle est supposée certifier le travail en tant que tel et non pas la personne qui en est l'auteur ou l'institution auquel il appartient. De prime abord, cette manière d'agir ne devrait rencontrer que des échos favorables vu qu'elle introduit une dose d'impartialité et de " démocratie" dans un processus dont, le moins que l'on puisse dire, les rouages n'en sont pas une bonne exemplification. Aussi paradoxal que cela puisse paraître le processus (de l'expertise à l'aveugle) a suscité nombre de critiques et de réactions (dont la fameuse controverse et échange entre Fish et Skoblow - voir infra) qui selon Shatz ..." peuvent nous rendre sceptiques au sujet de la nécessité de l'expertise à l'aveugle dans tous les cas " (52) (c'est nous qui soulignons).Il est intéressant de remarquer dans ce contexte, encore une fois, que le contrôle par les pairs est fondamentalement différent et s'applique aussi de manière différente selon les spécialités.

\subsection{1-Arguments}

\subsubsection{1-Faire partie d'une communauté}

Rendre le contrôle par les pairs aveugle (impersonnel nous dirions) revient à enlever le sens d'appartenance à une communauté qui est au cœur de la vie académique. Quand les chercheurs dans une spécialité donnée sont au courant qui sont les collègues travaillant sur un sujet et quelles sont la substance et les avancées de ce travail, ils ressentent un sens d'appartenance et de communauté. Sens qui ne serait pas avec l'expertise à l'aveugle.

D'un autre coté, si l'expertise à l'aveugle est, dans certains cas, largement utilisée, certains journaux utilisent aussi l'expertise double aveugle et dans laquelle en plus de garder le nom de l'auteur secret, le nom de l'expert l'est aussi. De nouveau cette manière de procéder a soulevé des critiques multiples (dont la plus prépondérante est celle largement relayée et très souvent citée de l'impunité de l'expert qui se trouverait de ce fait protégé et pourrait agir sans avoir à rendre compte à quiconque à part l'éditeur peut être ...) et a accentué en fait l'accusation précédente de la non appartenance à une communauté étant donné qu'un anonymat complet règne durant l'expertise. Shatz parle dans cette optique de différence entre l'équité (pour celui qui défend l'expertise à l'aveugle) et l'utilité (pour celui qui serait contre l'expertise à l'aveugle). La question qu'il pose est de peser le pour et le contre entre l'équité et le sens de l'utilité pour la communauté scientifique .Difficile de répondre quand on sait les profondes divergences entre chercheurs.

\footnotetext{
(51) Shatz, op.cit., p.48

${ }^{(52)}$ Shatz, op.cit., p.49
} 
On peut aussi avancer que l'argument de la communauté versus l'argument l'expertise à l'aveugle est battu en brèche quand un expert ayant des préjugés exclu de facto les auteurs des institutions non cotées. En effet, cette (très plausible) éventualité aurait l'effet contraire de l'appartenance à une communauté car l'expert serait sous le coup d'un préjugé institutionnel. Dans ce cas, l'expertise à l'aveugle promeut ceux qui n'auraient pas l'occasion d'accéder au cercle fermé de ceux qui publient.

\subsubsection{2-La thèse de l'omniprésence du préjugé}

Cette thèse soutient que le préjugé est omniprésent dans chaque expertise et de ce fait il ne sert à rien d'essayer de le combattre (entre autre par l'expertise à l'aveugle) .Ceci serait dû selon les protagonistes de cette thèse - au fait que l'expertise étant humaine, il est impossible qu'elle soit dénuée de préjugés. On argumentera que cette position impliquerait que les problèmes du contrôle par les pairs étant insoluble, il n'y a pas lieu de faire des efforts.

Tel qu'elle est présentée cette argumentation n'est ni méthodologiquement ni moralement défendable. L'expertise à l'aveugle n'a pas pour mission d'éliminer tous les préjugés mais certains comme le préjugé personnel, institutionnel, racial et sexuel alors que le préjugé idéologique ne peut être, de toute évidence, combattu par l'expertise à l'aveugle étant donné que c'est le coté idéologique qui est différent selon les chercheurs. L'expertise à l'aveugle ne vise pas la perfection mais vise à instaurer un système juste où les préjugés qui peuvent être combattu le sont, ce qui est meilleur qu'un système où aucun effort n'est fait. On peut affirmer qu'un expert fait un travail plus juste non pas s'il suit la directive disant " éliminer tous les préjugés éliminables " mais plutôt " éliminer les préjugés selon un modèle qui conduira à un résultat qui sera globalement plus juste " ${ }^{(53)}$.On peut résumer cette thèse de l'omniprésence du préjugé en disant qu'elle (la thèse) ne rend pas l'expertise à l'aveugle inutile. Elle (l'expertise à l'aveugle) élimine certains préjugés surtout personnels et institutionnel ainsi que les préjugés sexuels et raciaux. De plus, même en tenant compte de la thèse, les experts peuvent (et doivent) faire tout leur possible afin d'éliminer les autres préjugés qu'ils ont ce qui conduirait les autres préjugés à avoir moins d'effet sur l'expertise vu qu'ils (les préjugés) auraient tendance à s'auto éliminer. Pour synthétiser : on doit faire de son mieux et l'élimination de certains préjugés est un pas positif même si d'autres préjugés demeurent.

\subsubsection{3-L'argument " pas de mérite intrinsèque " de Fish}

Stanley Fish est un universitaire et théoricien de la littérature américain, souvent perçu ou qualifié comme l'un des représentants du postmodernisme. Dans l'une de ces célèbres contributions ${ }^{(54)}$, il affirme que le principe dans une expertise à l'aveugle est que la recherche est jugée sur ses mérites intrinsèques, loin de considérations étrangères au travail. Ces considérations étrangères incluent " le rang, l'affiliation, le statut professionnel, les réalisations passées, l'identification idéologique, le sexe ou toute chose qui peut être connue

\footnotetext{
${ }^{(53)}$ Shatz, op.cit., p.52

${ }^{(54)}$ Fish, Stanley, op. cit.
} 
sur l'auteur " ${ }^{(55)}$.Fish voit en ces " considérations étrangères " non pas des données qui devraient être enlevées ou du moins séparées de par l'expertise à l'aveugle mais il affirme en fait qu'elles font partie (et selon lui sont même essentielles) du processus de contrôle. L'identité d'un auteur et les différentes informations le concernant font partie de l'évaluation du mérite .Il termine en disant " Je voudrais insister, en conclusion, qu'il n'existe pas ce qui serait appelé mérite intrinsèque et en fait, si je peux paraphraser James I, pas de préjugé, pas de mérite " ${ }^{(56)}$.La position controversée de Fish, allant à l'encontre de la plupart des positions généralement acceptées ,a donné lieu à de multiples réactions ( dont une de Jerry Skoblow qui défendait lui la position des chercheurs novices et qui étaient pour l'expertise à l'aveugle afin d'avoir un traitement sans préjugé) la plupart négatives. Nous avons ici un cas non pas de conflit de génération mais de statut politique où les " nouveaux " essayent de se frayer un chemin et où les " anciens" essayent garder intact leur position. La logique de Fish est que son travail doit être jugé en tant que travail émanant de Fish avec ce que cela implique comme respect et traitement spécial alors que la position exprimée par Skoblow est que tout travail doit entre jugé intrinsèquement sur ses mérites propres et non en tant que émanant d'un chercheur connu et de ce fait traité différemment. Fish pour répondre à ces différents détracteurs que cette position a suscité a répondu que lui a gravi les échelons et a obtenu cette stature, cette réputation et ces avantages après avoir " peiner dans le vignoble " selon son expression. Il demande aux novices de faire leurs preuves avant de pouvoir bénéficier du traitement de faveur dont lui et ses semblables (en stature) jouissent. Cette position représente pour nous un cas très clair d'abus de position et en même temps elle est contradictoire. Abus de position de demander un traitement de faveur et surtout contradictoire car si Fish et ses pairs sont si sûrs de leur niveau, une expertise à l'aveugle ne devrait pas leur faire peur et en fait il devrait l'encourager car quoi que l'on pense l'expertise à l'aveugle est la solution la plus juste et la plus démocratique dans un monde de plus en plus ouvert et scrutateur.

\section{$\underline{1.8 .1 .4-L e s ~ a u t e u r s ~ c o n n u s ~}$}

Les éditeurs ont affaire à un sérieux challenge quand un auteur connu et fameux soumet un travail à leur journal. Dans ce cas, les lecteurs ne seraient pas intéressés par l'article en luimême mais par l'auteur qui le soumet. Est ce que dans ce cas, on applique l'expertise à l'aveugle? Il ne faut pas oublier que publier le travail d'une personne qui a fait ses preuves dans une spécialité quelconque a des retombées positives sur la réputation du journal. Dans ce cas, une sorte de préjugé personnel ( ad hominem ) est appliqué car on penserait qu'une soumission par un auteur connu est automatiquement bonne et celle d'un auteur moins connu moins bonne de ce fait augmentant encore plus les possibilités de publication pour ceux qui sont connus et diminuant les chances des chercheurs novices n'ayant pas encore atteint le stade de célébrités et de ce fait ne pouvant s'imposer juste sur leur réputation ( une sorte d'effet Mathieu que nous avons l'occasion de présenter).Malgré cela, il semblerait que l'application de l'expertise à l'aveugle ne peut être appliquée unilatéralement car pour reprendre Richard T.De George et Fred Woodward :....." les éditeurs doivent prendre en

\footnotetext{
${ }^{(55)}$ Fish, Stanley, Doing what comes naturally : change, rhetoric and the practice of theory in literature and legal studies,[Durham, N.C.]: Duke University Press, 1989, p.164

${ }^{(56)}$ Idem
} 
ligne de compte plusieurs considérations quand ils décident de publier un manuscrit - la qualité étant seulement l'une d'elles " ${ }^{(57)}$.Cette affirmation va à notre avis à l'encontre de que le contrôle par les pairs est supposé promouvoir et faire : sélectionner et évaluer le meilleur manuscrit du point de vue scientifique et méthodologique et rejeter ce (et ceux ) qui ne l'est ( le sont ) pas. Le contrôle par les pairs étant traversé par d'innombrables controverses et courants, cette approche ne représente qu'une vue que d'autres ne partageraient pas (voir le débat Fish-Skoblow en supra).

\subsubsection{5-Le préjugé améliore le travail}

Aussi paradoxal que cette affirmation puisse paraître, elle se vérifie dans les faits .En effet, un expert avec des préjugés (généralement personnels ou institutionnels) aurait tendance à trouver d'éventuelles faiblesses (qu'un expert sans préjugés ne remarquerait peut être pas) et ceci conduirait l'auteur à améliorer son travail à travers les corrections qui lui auraient été demandé. C'est ce qui a été appelé " un préjugé négatif ", qu'en est-il lorsque le préjugé est positif? Un préjugé positif est dans ce cas un préjugé où l'expert pour différentes raisons (telles qu'une amitié avec l'auteur ou alors le besoin d'être en bon terme avec l'auteur qui est connu et enseigne dans une institution prestigieuse - cas avéré de préjugé institutionnel positif différent du préjugé institutionnel négatif -), l'expert accorde à la soumission une plus grand attention et peut même aider à l'améliorer en construisant des arguments et même remarquer des évidences (qu'il n'aurait pas fait normalement ) pour apporter de l'eau au moulin des arguments de l'auteur ayant soumis. Certains chercheurs ont comparé cette manière de procéder à faire de l'expert un coauteur. Est ce que ce préjugé à l'envers vaut la peine d'être maintenu contre l'expertise à l'aveugle ? .Il semblerait que oui étant donné que contrairement au préjugé ad hominem qui rejetterait un travail sur une base personnelle, cet aspect tendrait à améliorer une soumission à cause d'une expertise sans concession.

\subsubsection{6-Cohérence}

Cet argument part de l'idée qu'appliquer l'expertise à l'aveugle crée une incohérence dans les pratiques académiques. Ainsi, les embauches et les promotions sont faites tout en sachant où le postulant a enseigné auparavant. De même, l'achat des livres est fait en connaissance de cause des travaux précédents du postulant et, dans le cas d'une anthologie, de l'identité des auteurs ayant participé. L'évaluation académique se fait avec la possibilité qu'ont les experts d'être au courant des post publications d'un auteur donné ainsi que de son identité, son université et dans quel journal son travail a été publié et aussi de juger les travaux publiés dans des journaux en particulier. Tous ces aspects militent en faveur de la non application de l'expertise à l'aveugle pour les prépublications académiques sous le chapitre de la cohérence des pratiques de contrôle de l'information scientifique et technique. Ces deux poids deux mesures font de l'expertise à l'aveugle une pratique difficilement acceptable seulement pour les soumissions aux journaux scientifiques.

(57) De George, Richard T. and Woodward, Fred, Ethics and manuscript reviewing, Journal of scholarly publishing, Vol..25, n³, 1994, p.134-142 


\subsubsection{7-Le programme d'un auteur}

Connaître les publications d'un auteur peut légitimement aider un expert. La soumission qui est sous expertise peut (et est très souvent) reliée à d'autres publications. Dans ce cas, la qualité de la recherche ne peut être évaluée que dans le contexte général du programme du postulant. Plus, elle ne peut être jugée que si les autres composantes du programme sont connues. Ces composantes font partie d'une suite de travaux dont la connaissance aide à mieux juger. Eliminer le contrôle à l'aveugle dans ce cas et faire ressortir la place de la publication objet du contrôle conduit à une expertise plus juste quoique les travaux d'un chercheur puissent être de qualités différentes. Il est vrai que le contrôle à l'aveugle peut paraître désuet car généralement l'auteur a tendance à s'auto citer dans nombre de cas ce qui le rend pratiquement inopérant. Une des solutions proposées consiste pour l'auteur à signaler à l'éditeur (et non pas l'expert) que le travail fait partie d'un programme plus large. Il peut alors prendre deux décisions : soit lever le voile de l'anonymat et faire expertiser le travail en tant partie d'un plus grand programme ou alors il peut garder l'anonymat mais prendre la décision finale (qui, il ne faut pas l'oublier, lui revient) en tenant compte de ce critère. Quelque soit la décision, la prépondérance et la place de la soumission dans un programme plus large militent contre le contrôle à l'aveugle.

\subsubsection{8-Repérer les répétitions}

Eliminer le contrôle à l'aveugle peut aider à repérer et démasquer les duplications que certains auteurs font de leur propre travail. Ceci est fait dans le but évident de gonfler et faire paraitre leur CV plus étoffé que ce qu'il est vraiment. En plus d'être moralement répréhensible, elle a le défaut de prendre la place d'autres publications plus méritantes surtout dans l'univers papier où l'espace est très souvent sujet à compétition (ce qui n'est pas le cas pour l'univers numérique où cette contrainte est quasi inexistante).C'est pour cela qu'il est plus facile pour un expert (à condition qu'il soit bien au courant de ce qui se publie dans son domaine) de démasquer une duplication s'il connaît le nom de l'auteur. Théoriquement, l'éditeur peut faire ce travail mais comme il est de toute façon moins " expert " que l'expert auquel il assigne le travail, ceci est difficile. Il est clair que lever le voile sur l'identité de l'auteur donne à l'expert un meilleur contexte d'évaluation que si l'expertise était faite à l'aveugle. Une des solutions proposées est de garder l'identité de l'auteur secrète mais de demander à l'auteur de déclarer est ce que l'une de ses publications ressemble celle qui est sujette à expertise ou même de joindre une copie des publications en question. Une partie indépendante pourrait alors s'occuper de cette opération avant même que l'expert ne juge le travail sur ces mérites propres. Il est clair que le délai causé par cette opération de vérification ne devrait pas dépasser quelques jours afin d'éviter (et ne pas exacerber) les retards généralement associés au contrôle par les pairs.

\subsubsection{9-Inutilité}

Expertiser à l'aveugle n'arrive toujours pas à accomplir le but qui lui est assigné .Ceci n'empêcherait pas un expert de deviner l'auteur de la soumission surtout si ce dernier a déjà abondamment publié et qu'il travaille (comme c'est très souvent le cas) dans la même spécialité. Le succès de l'expertise à l'aveugle est très mitigé car il est estimé entre 50 et $76 \%$ 
en science selon une étude ${ }^{(58)}$ qui y avait cependant inclut aussi les autos citations ( qui permettent à un chercheur de citer ses propres travaux mais par la même lèvent le voile sur son identité ).Mais selon Shatz, " confronté à un choix entre tout expertiser à l'aveugle ou son contraire, il est préférable de tout expertiser à l'aveugle ${ }^{(59)}$ ".Position qu'il faudrait peut être , à notre sens, mitiger étant donné que les différences d'approche des spécialités, des situations et aussi des personnes en charge de ce processus ( éditeurs et experts) ne peuvent conduire à une catégorisation stricte de l'application ou non du principe de l'expertise à l'aveugle. Un autre problème se pose et c'est celui non pas de savoir qui est l'auteur mais a trait au fait de penser savoir qui est l'auteur. Pour être plus, clair l'expertise à l'aveugle étant basé sur la non divulgation du nom de celui qui a écrit et soumis l'article, elle crée un autre préjugé car il est (très) possible que supposer qu'un tel a écrit peut s'avérer faux et créer un autre préjugé ( qui ne serait pas de mise dans le cas des soumissions où le nom de l'auteur ne serait pas masqué).C'est un problème que les chercheurs ayant conduit des essais cliniques aléatoires connaissent bien. Dans ce genre d'expériences, les patients ne savent pas s'il leur est donné vraiment une drogue expérimentale ou alors un placebo ${ }^{(60)}$.Les patients essayent de deviner des fois quel genre de drogues ils sont administrés (drogue expérimentale ou placebo) et cet essai de deviner influe sur leur sensation d'une manière ou une autre. Dans les essais cliniques aléatoires à grande échelle, la validité de l'expérience est préservée en choisissant et cet essai de deviner influe sur leur sensation d'une manière ou une autre. Dans les essais cliniques aléatoires à grande échelle, la validité de l'expérience est préservée en choisissant d'une manière aléatoire les patients des deux groupes (ceux recevant la drogue expérimentale et ceux recevant le placebo) et vu que les patients des deux groupes essayerons de deviner, les différences entre les deux groupes de patients reste toujours informative. Malgré ces défauts et ces exceptions, l'expertise à l'aveugle est toujours meilleure que l'expertise où le nom de l'auteur est connu.

\section{9-L'expertise double aveugle : une solution controversée et critiquée}

Si une des manières de combattre ce qui est considéré comme un des plus importants problèmes que rencontre tout chercheur dans sa quête de publier est de masquer son identité afin que le travail soit jugé de la manière la plus objective possible, d'autres propositions ont aussi suggéré aussi de masquer l'identité des experts. Ce qui est connu sous l'appellation

\footnotetext{
${ }^{(58)}$ Godlee F, The Ethics of peer review In Hudson Jones A., McLellan F. Ed., Ethical Issues in Biomedical Publication, Johns Hopkins University Press, Baltimore, 2000

(59) Shatz, p.51

${ }^{(60)}$ Un placebo est une mesure thérapeutique d'efficacité intrinsèque nulle ou faible, sans rapport logique avec la maladie, mais agissant, si le sujet pense recevoir un traitement actif, par un mécanisme psychologique ou psychophysiologique. Dit autrement : «ça marche juste parce que j'y crois », ou l'autosuggestion appliquée à la médecine. Le médicament placebo ne contient a priori aucun composé chimique ayant une activité démontrée. Notons par ailleurs que le placebo ne se présente pas uniquement sous la forme d'un médicament : il peut s'agir d'une opération chirurgicale inadéquate, d'un traitement physio thérapeutique mal conduit ou inutile, et de toute autre intervention thérapeutique dont l'indication est mal posée, ou la réalisation incorrecte. L'effet placebo (du latin: " je plairai ") est défini comme l'écart positif constaté entre le résultat thérapeutique observé lors de l'administration d'un médicament et l'effet thérapeutique prévisible en fonction des données strictes de la pharmacologie. Tout geste thérapeutique, valide ou non, comporte d'ailleurs une part plus ou moins grande d'effet placebo. Cet écart est de l'ordre de $30 \%$ habituellement et peut atteindre 60-70\% dans les migraines ou les dépressions. L'état de certains patients souffrant d'affections réputées " incurables " s'en trouve parfois objectivement amélioré.
} 
d'expertise en double aveugle. Cette pratique n'est pas unanimement pratiquée pour des raisons multiples qui sont en même temps cause et effet de toute la problématique des préjugés dans l'expertise par les pairs. Il faut dire que confrontée à de multiples cas de dépassements, de favoritisme et de scandales même, la communauté scientifique a dû trouver des palliatifs pour y remédier. A coté de l'expertise à l'aveugle dont nous avons vus certaines des avantages et défauts, l'expertise double aveugle a introduit un peu plus de " démocratie " dans le processus vu qu'il est devenu plus responsabilisé et juste.

Il est de toute évidence devenu de plus en plus difficile dans toutes les instances de la vie de tous les jours de se comporter d'une manière où la responsabilisation est absente. Toute personne à quelque niveau qu'elle soit est tenue de rendre des comptes .Dans notre cas (l'expertise des soumissions en vue de leur publications dans des journaux scientifiques) rendre des comptes peut avoir plusieurs sens. Nous en citerons :

1- L'expert peut ne plus être appelé à expertiser si ces expertises passées ont étés de mauvaise qualité et en décalage avec ses collègues experts.

2- L'auteur devrait voir (peut être même expertiser) le rapport de l'expert devenant par la même les évaluateurs de leurs propres experts.

3- L'expert pourrait être obligé de dévoiler son identité et signer ses expertises devenant de ce fait responsable vis-à-vis de l'auteur. En signant ses expertises, il deviendrait plus responsable vis à vis de l'auteur que s'il restait anonyme. L'auteur saurait alors qui tenir responsable des remarques qui ont étés émises.

Cependant, cette responsabilisation publique a un prix. Par exemple, un chercheur novice fraîchement intronisé comme expert aura toutes les peines à signer un rapport négatif surtout si cela concerne un ténor de la spécialité de peur de représailles. La même chose peut être dite pour les chercheurs confirmés qui auraient des difficultés à s'exprimer franchement comme c'est le cas lorsqu'ils doivent rédiger des lettres de recommandations pour d'autres chercheurs. De plus, étant donné que cette procédure est nouvelle, recruter de nouveaux experts pourrait s'avérer difficile et surtout les taux d'acceptation exploseraient, les experts n'étant pas prêt à s'antagoniser des collègues par une expertise négative. Une étude conduite par Cardiovascular Research ${ }^{(61)}$ conclut à la faisabilité de cette mesure et énumère ses avantages et inconvénients. Ce sont :

\subsection{1-Avantages}

1-L'expertise ouverte signée aide les experts à maintenir une balance appropriée entre leur rôle de juge et leur rôle consistant à aider les auteurs. Les expertises signées aident l'auteur et il est beaucoup plus naturel d'atteindre ce rôle constructif de l'expert en signant qu'en restant anonyme .Signer aide aussi l'expert à se rappeler son engagement d'être des auxiliaires et non pas des adversaires des auteurs.

${ }^{\left({ }^{61)}\right.}$ Fabiato, Alexandre, Anonymity of reviewers, Cardiovascular Research, Vol.28, nº 8 , 1994, p.1134-1139 
2-Les compétences des experts ajouteront de la crédibilité à leur commentaire. La recherche citée en exemple montre que les auteurs ne prennent pas en ligne de compte les remarques de leurs recherches rejetées avant de resoumettre à un autre journal. Curieusement, suggérer que connaitre les compétences des experts ajoute de la crédibilité sonne comme un endossement d'un préjugé ad hominem envers certains experts. C'est pourquoi les expertises anonymes ont l'avantage d'être jugées sur leur mérite.

3-Le contrôle ouvert rend les experts plus responsables. Leur réputation est en jeu.

4-Le contrôle ouvert aide à réduire les abus intolérables du système tels que les délais et les comportements abusifs.

5-Le contrôle ouvert peut aider à résoudre les problèmes qui se poseraient dans des domaines controversés de la recherche.

6-Dans une communauté scientifique respectable, il semble qu'il y ait peu de justification pour le secret.

7-Le contrôle ouvert rendra le processus du contrôle par les pairs moins désagréable et plus poli. Les études ont démontré que les expertises signées sont plus courtoises.

8-Les nouvelles technologies pourraient très bien rendre le contrôle ouvert une nécessité.

9-Une expertise signée permet à un expert d'obtenir de la reconnaissance quand elle est dûe. De même un auteur peut inclure maintenant l'expert dans les remerciements par son nom.

\subsection{2-Inconvénients}

1-Les experts novices auront peur des représailles par les auteurs confirmés.

2-Création d'un réseau de copinage (old boy network) qui favoriserait les scientifiques confirmés. Ils accepteraient plus facilement les recherches des personnes de leurs groupes, s'attendant à une réciprocité. Ils pourraient aussi tirer parti de la relation qu'ils ont avec d'anciens étudiants qui expertiseraient leur recherches (et qui seraient complaisant dans leur travail).

3-Création d'un sentiment de rancune et d'animosité.

4-Le contrôle ouvert conduira à un taux d'acceptation plus élevé, étant donné que pour éviter les représailles, les animosités, etc..., les experts ne voudront pas rédiger des rapports critiques. Des études ont clairement démontré que les expertises à l'aveugle sont plus critiques. Alexandre Fabiato remarque les éditeurs pourraient interpréter les formulations faites avec tact des experts comme étant une critique. De leur coté, les auteurs feront remarquer à l'éditeur la formulation plaisante de l'expertise pour le harceler dans le cas d'un rejet.

5-Le contrôle ouvert entraînera plus de travail et de problèmes pour l'éditeur. Ils auront du mal à trouver des experts pour les recherches de qualité douteuse et aussi ils perdraient les experts potentiels qui voudraient rester anonymes. Enfin, ils auront beaucoup plus de mal à 
expliquer et justifier les rejets aux auteurs étant donné que la formulation des expertises négatives sera moins forte.

6-On ne doit pas changer un système qui, globalement, fonctionne bien.

Les résultats de cette étude dont les répondants avaient exprimé majoritairement et respectivement à raison de $83 \%$ pour les experts et $70 \%$ pour les auteurs ${ }^{(62)}$ leur préférence en faveur du maintien du droit de l'expert à décider d'expertiser anonymement ou non, est corroborée par d'autres études ${ }^{(63)}$ surtout dans le domaine médical où la tendance ne semble pas indiquer une différence notable entre expertiser à l'aveugle ou de la manière traditionnelle. Cependant dans des spécialités telles que la psychologie, les résultats ne sont pas les même étant donné que le jugement a plutôt tendance à être plus subjectif que lorsqu'on examine une soumission en sciences en général.

Toutes ces études et expériences n'ont malheureusement pas fait pencher la balance d'un coté ou un autre .La raison principale, à notre avis, est le fait que le contrôle par les pairs est une activité humaine. Activité répondant à des critères qui peuvent faire appel (et le plus souvent le sont) au sentiment, à la subjectivité, aux penchants (idéologique, linguistique, sexuel, régional, national, etc ....) et surtout les préjugés de toutes sortes. En effet, de par sa nature humaine (tout peut être automatisé dans le processus de l'envoi de la soumission à travers les réseaux à la forme finale sauf la certification, le jugement, le contrôle de la recherche), le contrôle par les pairs est entrepris par des personnes auxquelles il ne peut être demandé de se départir de leur sentiment. Si cet aspect est de toute évidence préjudiciable à la bonne marche de la certification de la Science, il est malheureusement peu des solutions pour le combattre (ce qui n'implique pas qu'il faut se résigner mais les différentes recherches n'ont pu trouver de solutions).La nature humaine étant ce qu'elle est et étant peu sujette à changement, les seules solutions ont été celles promouvant une plus grande ouverture .Plus grande ouverture qui rendrait les experts de plus en plus responsables de leur actions au lieu d'être " protégés " par le secret qui a toujours été un des aspects du contrôle par les pairs classique et surtout sur support papier. C'est à cet effet que l'expérience du magazine Nature est venue afin d'analyser quelles seraient les changements, attitudes et aussi expériences dans le domaine. Nous allons examiner cette étude et en voir quels en sont les conclusions et autres résultats que les chercheurs ont pu obtenir. Il est à signaler que cette étude est importante dans le sens où elle est la seule à ce niveau et à ce degré d'exhaustivité à avoir abordé le sujet d'actualité.

\footnotetext{
${ }^{(62)}$ Fabiato, Alexandre, op.cit., p.1134

${ }^{(63)}$ Dont nous citerons

-Van Rooyen, Susan [et al], Effect of open peer review on quality of reviews and on reviewers' recommendations: a randomised trial, British Médical Journal, Vol. 318, 2 January 1999, p.23-27.

-Morrow Brown, Harry, Peer review should not be anonymous, British Medical Journal, Vol.32, 12 April 2003, p.824.

-Van Rooyen S, [et al], The effect of blinding and masking on the quality of peer review: a randomized trial, Journal of the American Medical Association, 1998, Vol.280, p.234-237.

-McNutt R.A., [et al], The Effects of blinding on the quality of peer review. Journal of the American Medical Association, 1990, Vol..263, p.1371-1376.

-Godlee, F, [et al], Effect on the quality of peer review of blinding reviewers and asking them to sign their reports: a randomized controlled trial, Journal of the American Medical Association, 1998, Vol.280,p.37-40 -http://publicationethics.org/case/anonymous-peer-review- $\%$ E2\%80\%93-author-requesting-manuscript-file (accédée le 24 Avril 2012)
} 


\section{Chapitre II}

\section{L'EXPERIENCE DU MAGAZINE NATURE EN MATIERE DE CONTROLE PAR LES PAIRS OUVERT}

\section{Introduction}

Face à toutes ces controverses, suspicions, méfiance et questionnements que le contrôle par les pairs posa et ne cesse de poser et devant la quasi impossibilité pour les chercheurs d'avoir un consensus quant à sa viabilité, le magazine Nature lança une étude ${ }^{(64)}$ dont le but était de voir est ce que le contrôle par les pairs traditionnel (sur papier) avec tous les problèmes qu'il a engendré pouvait être amélioré (ou du moins aidé ) par un modèle plus ouvert connu sous l'appellation de contrôle par les pairs ouvert .Cette version propose ,schématiquement et tel qu'indiqué par son nom, à ce que le contrôle par les pairs ( traditionnellement fermé en ce sens que l'expertise est quasiment secrète) soit plus ouvert et que ces différentes étapes soient plus " démocratiques " afin d'éviter les dépassements qui ont eu lieu et dont la majorité des chercheurs conviennent qu'ils proviennent de cet aspect secret propice à tous les dépassements. Cette étude consista en une enquête qui dura quatre mois sur les habitudes des chercheurs et des experts quant à leurs soumissions et expertises. Ensuite, elle fut suivie par un débat sous la forme de contributions par d'éminents chercheurs qui investiguèrent les différents aspects (avec cinq rubriques principales et qui sont: Systèmes, Qualité et valeur, Ethique, Solutions techniques et Perspective) du contrôle par les pairs insistant sur cette nouvelle approche qui est une résultante de l'introduction d'Internet. Nous allons premièrement voir quelles ont étés les résultats de l'étude et ensuite les débats qui ont découlé des vingt deux articles rédigés par des chercheurs et des experts en la matière.

\section{1-Débat}

Le contrôle par les pairs est généralement accepté comme une partie essentielle de la publication scientifique. Mais les manières par lesquelles il est mis en pratique varient selon les journaux et les disciplines. Quelle est alors la meilleure méthode pour le mettre en œuvre ? Quelles sont les préoccupations d'ordre éthiques? Et comment est ce que les nouvelles technologies peuvent être utilisées pour améliorer les modèles traditionnels?

Cette étude sur le web du magazine Nature consiste en 22 articles d'analyse et perspectives de chercheurs de premier plan, d'éditeurs et autres parties prenantes pour répondre à ces questions.

\section{2-Vue d'ensemble}

L'étude du contrôle par les pairs ouvert du magazine Nature

Malgré l'enthousiasme pour le concept, le contrôle par les pairs ouvert n'était pas très populaire que ce soit parmi les auteurs ou par les scientifiques qui furent invités à commenter.

\footnotetext{
${ }^{(64)}$ http://www.nature.com/nature/peerreview/debate/
} 


\section{3-L'expérience du magazine Nature}

En date du $1^{\text {er }}$ Juin 2006, le magazine Nature lança une expérience, une étude sur le contrôle par les pairs ouvert. L'intention était d'explorer l'intérêt des chercheurs dans un modèle particulier du contrôle par les pairs ouvert soit en tant que chercheurs ou experts. Cette expérience avait aussi pour but de fournir aux éditeurs de Nature avec un test des avantages d'une extension potentielle du contrôle par les pairs traditionnel.

A plusieurs reprises durant cette enquête, des chercheurs et des journalistes se demandèrent est ce que cette enquête reflétait un sens d'insatisfaction vis-à-vis du processus. Au contraire, ce processus fonctionne aussi bien que les autres systèmes de contrôle par les pairs. La plus récente des enquêtes a abondé en ce sens vu que 3/4 des interrogés (74\%) ont estimés que leur recherche avait été amélioré par le contrôle par les pairs, le 1/5 (20\%) était neutres et seulement $6 \%$ estimait que leur recherche n'avait pas bénéficié, ni été amélioré par le contrôle par les pairs.

Cependant, le contrôle par les pairs n'est pas exempt de critiques et il faut le mettre constamment sous la loupe vu l'importance qui lui est accordée par la communauté des chercheurs et aussi les attentes qu'il suscite.

\section{4-L'étude en question}

Le magazine Nature reçoit aux environs de 10000 recherches chaque année et les éditeurs en rejettent aux environs de $66 \%$ sans expertise. Ces recherches qui passent ce premier filtre sont soumises au processus traditionnel de contrôle par les pairs dans lequel deux ou trois experts choisis par les éditeurs sont désignés pour analyser et commenter d'une manière anonyme et confidentielle. Les éditeurs examinent les commentaires et décident du rejet, révision ou acceptation. Seules $7 \%$ des soumissions initiales sont finalement publiées.

Avant que l'étude ne soit lancée, une enquête quant à sa viabilité fut entreprise et elle indiqua un niveau suffisant d'intérêt qui la justifia. C'est alors qu'entre le $1^{\text {er }}$ Juin et le 30 Septembre 2006, les auteurs dont les nouvelles soumissions avaient passé le filtre initial furent invités à les poster dans un serveur ouvert sur Internet pour des commentaires publics. Pour ceux qui l'avaient accepté, leur recherche fut simultanément soumise au processus de contrôle par les pairs traditionnel. Tous les commentaires reçus en affichage ouvert furent vérifiés pour des problèmes légaux ou encore pour un quelconque langage inapproprié. Les commentaires devaient être signés pour être publiés. Une fois le processus standard complété (c'est-à-dire que les commentaires des experts sollicités aient étés reçus), les commentaires reçus sur le serveur furent aussi rassemblés et la recherche retirée du serveur.

Au début de l'étude, et à plusieurs reprises durant, des alertes e-mails furent envoyées à tous ceux qui étaient enregistrés sur Nature.com ainsi que tout lecteur intéressé, qui pourraient s'inscrire afin de recevoir des mises à jour régulières. A plusieurs reprises, les éditeurs contactèrent des groupes de chercheurs dans une discipline donnée qu'ils pensèrent intéressés dans l'expertise ou le commentaire de recherches spécifiques. L'étude était constamment mise au premier plan durant toute la période sur le site web de Nature.com. De ce fait, des efforts 
substantiels furent entrepris afin d'attirer l'attention de contributeurs potentiels au processus de du contrôle par les pairs ouvert.

Après ces quatre mois durant laquelle l'étude fut entreprise, au mois d'Octobre, plusieurs enquêtes furent lancées pour réunir les réactions pendant que les recherches étaient soumises en même temps au contrôle par les pairs ouvert et celui sollicité.

\section{5.-Résultats}

Un total de 1369 recherches fut envoyé pour expertise durant l'étude. Les auteurs de 71 recherches (ou 5\%) acceptèrent que leurs recherches soient sujettes à un commentaire ouvert. De ces 71 recherches, $33(46 \%)$ ne reçurent pas de commentaires alors que les autres 33 (54\%) reçurent un total de 92 commentaires techniques. De ces 92 commentaires, 49 (53\%) l'étaient pour 8 recherches. Le reste des 30 recherches avaient des commentaires qui étaient répartis d'une manière égale. La recherche la plus commentée reçut 10 commentaires (une recherche dont le sujet portrait sur la sélection sexuelle de la période post accouplement).Il n'y avait pas eu de un problème apparent concernant le temps de soumission : les recherches ayant reçus le plus de commentaires étaient reparties d'une manière égale à travers l'étude et les recherches les plus récentes n'avaient pas enregistrés une quelconque baisse d'intérêt.

L'étude reçut un important et conséquent trafic en ligne: une moyenne de 5600 pages html vues par semaine et à peu près les mêmes chiffres de RSS Feeds. Cependant cet intérêt des lecteurs ne se convertit pas en un accroissement significatif des commentaires.

\section{$\underline{\text { 2.5.1-Distribution par sujet }}$}

Les recherches furent catégorisées en 15 spécialités. Le nombre de recherches sur le serveur ouvert dans chaque spécialité était bas. De ce fait, toute extrapolation résultant de ces chiffres était incertaine. La distribution des recherches postées est représentée dans le graphe suivant :

\section{Papers by Field}

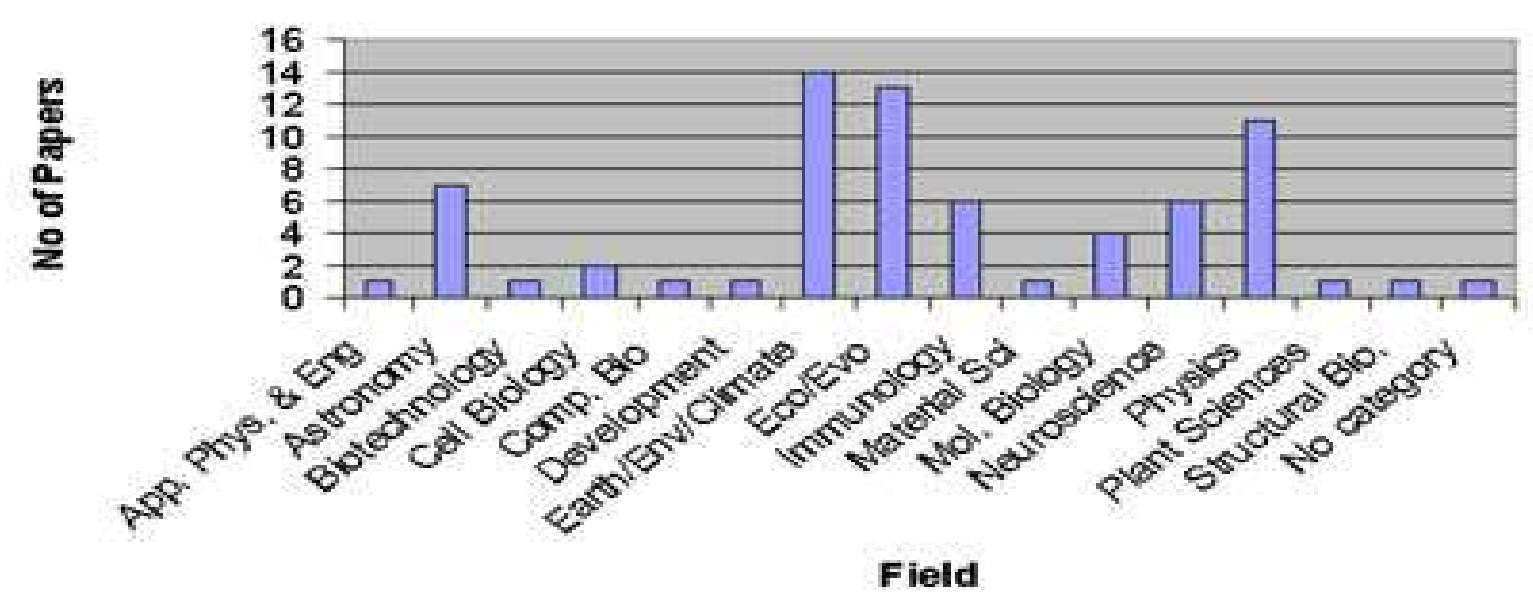

Figure $n^{\circ} 35-$ Recherches par spécialité 
La plupart des recherches étaient dans les spécialités Terre / Environnement / Sciences climatiques et Ecologie / Evolution avec respectivement 13 et 14 recherches, suivies par les Sciences Physiques avec 11 recherches. L'Astronomie, l'Immunologie et les Neuroscience représentaient le reste des spécialités.

Tel que prédit, il y avait moins de recherches dans la plupart des spécialités cellulaires et moléculaires, quoiqu'il faille reconnaître que celles qui étaient soumises au contrôle par les pairs ouvert reçurent autant de commentaires que celles dans les autres disciplines. Il n'y eut pas de recherches postées en biochimie, biologie chimique, génétique / génomique, recherche médicale, microbiologie, paléontologie ou zoologie.

Le tableau suivant montre la distribution du nombre moyen de commentaires reçus par recherche. Dix spécialités reçurent une moyenne de plus d'un commentaire par recherche .Ce sont: Astronomie, Biologie cellulaire, Environnement/Sciences climatiques, Ecologie /Evolution, Immunologie, Biologie moléculaire, Neurosciences, Sciences physiques, Sciences végétales et Biologie structurelle. Toutes ces disciplines comprirent un ou deux recherches qui furent abondamment commentées. On doit cependant garder à l'esprit, que les nombres absolus de recherches sont petits.

\section{Comments per Paper by Field}

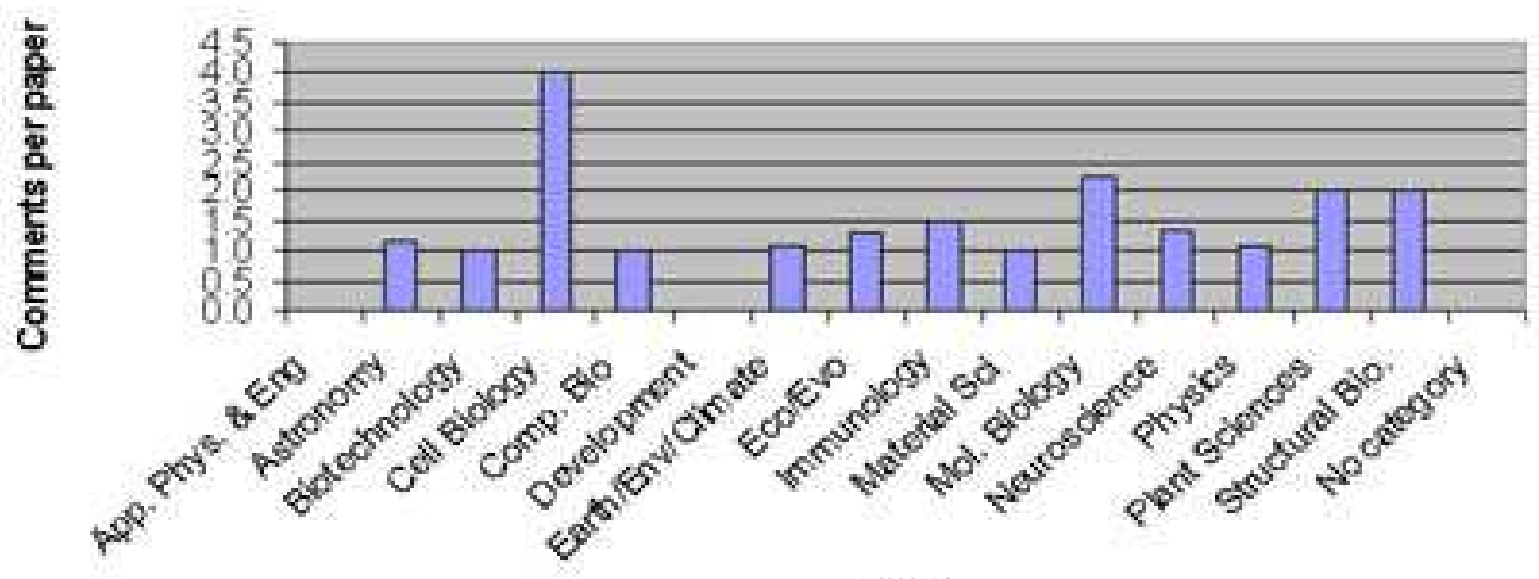

Field

Fig.n ${ }^{\circ}$ 36-Commentaires par recherche et par discipline

\subsection{1-Réactions éditoriales}

Chaque commentaire reçu fut évalué par l'éditeur en charge au moment de l'expertise de la recherche selon l'échelle suivante :

1- Activement inutile

2-Commentaires raisonnables mais pas d'information utile

3-Points valides mineurs et / ou détails. 
4-Points majeurs en ligne avec les commentaires sollicités des experts.

5-A directement influencé la publication au delà et en plus des commentaires des experts.

A chaque commentaire fut donné deux évaluations : une pour la valeur technique et une autre pour la valeur éditoriale (en d'autres termes pour les commentaires en relation avec le contexte et la signification).

Pas un seul éditeur ne jugea que les commentaires sur les recherches qu'il avait en charge méritent plus de 4 de quelque manière que ce soit. Les notes moyennes étaient : technique 2.6 et éditorial 1.8.En d'autres termes, les commentaires furent jugés comme ayant une valeur beaucoup plus éditoriale que technique. Ceci est en partie dû au fait que plusieurs recherches ne reçurent des commentaires que sur des points éditoriaux. Pas un seul éditeur ne déclara que les commentaires influencèrent sa décision en ce qui concerne la publication.

Pour une évaluation qualitative, les éditeurs discutèrent les résultats et reportèrent les avis suivants :

- Une impression générale d'indifférence de la part de leurs contacts dans la spécialité et qu'il était très difficile d'obtenir des commentaires.

- Les tentatives directes de sollicitation des commentaires eurent un succès très limité.

- Les éditeurs en biologie en particulier n'étaient pas surpris que les auteurs dans des spécialités très compétitives ne souhaitaient pas être impliqués.

- D'une manière anecdotique, certains auteurs étaient réticents de participer à cause de la peur du plagiat et des demandes de brevets.

- D’une manière anecdotique, les commentateurs potentiels déclarèrent que le contrôle par les pairs ouvert est " une bonne chose à faire " mais n'étaient pas près à fournir leurs réactions sur les recherches sur le serveur.

- Les éditeurs eurent l'impression que la plupart des commentaires étaient d'une utilisation limitée pour la prise de décision. La plupart étaient des commentaires de caractère général tels que " bon travail " plutôt que d'ajouter au processus de contrôle par les pairs.

\subsection{2-Enquête sur les auteurs}

Tous les auteurs qui participèrent à l'étude reçurent un questionnaire. Soixante quatre personnes furent contactées et vingt sept (42\%) répondirent. Les résultats étaient les suivants :

- Vingt répondirent que l'expérience était intéressante.

- Des quatorze qui reçurent des commentaires ouverts, quatre les décrivent comme " non utiles ", six comme " utiles d'une certaine manière " et quatre " très utiles ". 
- Quoique la plupart des personnes ayant répondu ne reçut pas de commentaires additionnels en prenant part à l'étude (tel qu'e-mail ou appel téléphonique), ceux qui le firent (cinq personnes) les trouvèrent soit " utiles " ou " très utiles " (une personne).

- Certains auteurs exprimèrent leur préoccupation au sujet d'un éventuel plagiat et d'autres furent déçus de ne pas recevoir plus de commentaires.

- Des vingt sept personnes qui répondirent, onze exprimèrent une préférence pour le contrôle par les pairs ouvert.

\section{7-Conclusions}

Malgré l'intérêt palpable dans l'étude, seul un nombre limité d'auteurs opta pour la participation. Il y avait un niveau élevé d'intérêt exprimé dans le contrôle par les pairs ouvert par les auteurs qui optèrent de poster leurs manuscrits d'une manière ouverte et qui répondirent après l'étude, en contraste aux avis des éditeurs. Une petite majorité de ces auteurs qui participèrent reçurent des commentaires, mais typiquement peu malgré un trafic significatif sur le web. La plupart des commentaires n'étaient pas techniquement substantiel. Les réactions suggèrent qu'il y a une réticence marquée au sein des chercheurs à offrir des commentaires ouverts.

Le magazine Nature et ses éditeurs, continueront à explorer des utilisations participatives du web. Mais pour le moment, au moins, ils ne mettront pas en œuvre le contrôle par les pairs ouvert.

Comme nous l'avons déjà signalé, les contributions ont étés départagées en cinq rubriques et qui sont : Systèmes, Qualité et valeur, Ethique, Solutions techniques et Perspective. Chacune de ces rubriques examine un aspect du contrôle par les pairs et présente les plus récentes et plus innovantes expériences en la matière. Etant donné leur importance, elles sont présentées en détails dans ce qui suit.

\section{8-SYSTEMES}

\subsection{1)-Les frontières en ligne de la littérature expertisée}

Cet article présente une vue d'ensemble du contrôle par les pairs. Il s'appuie et présente l'expérience des journaux publiés par BMC (BioMed Central) et la manière dont l'expertise est faite surtout entre les deux branches que couvrent les journaux BMC: médecine et biologie. La première entreprend un contrôle plus ouvert que la deuxième dans le cas de BMC. De plus, l'article aborde un autre aspect typiquement inhérent à la nouvelle nature de l'article électronique: ses différentes versions. Si dans l'univers papier, une fois publié, l'article ne change nullement (à part dans des cas très spécifiques comme par exemple les rétractions), l'univers des réseaux permet à un article d'avoir plusieurs versions et plusieurs " publications ".L'auteur compare, à juste titre, ce nouvel aspect de l'article scientifique aux versions d'un logiciel qui passerait de 1.0 à $1.1,1.2,1.3$ etc..... jusqu'à ce qu'une nouvelle version $(2.0,3.0)$ apparaisse. La comparaison avec le logiciel est très à propos car l'article est 
devenu globalement électronique même si une version papier existe. Cette analogie avec le logiciel interpelle les chercheurs quant au devenir et la forme que prendrait l'article scientifique dans le futur. Il ne sera pas de toute évidence le même et cette nouvelle forme se répercutera sans aucun doute sur le contrôle par les pairs. C'est pour cette raison qu'avec Internet la certification est indubitablement et irrémédiablement changée.

\subsection{2)-Avoir confiance en la qualité des données}

Cet article quant à lui aborde un autre aspect du contrôle par les pairs : les bases de données. Il présente le site Signaling Gateway Molecule Pages, base de données fournissant des informations essentielles sur plus de 4000 protéines de mammifères (souris et humaines) impliquée dans la signalisation cellulaire. La publication de base de données représente un défi unique pour l'expert. Le contrôle par les pairs suit un modèle similaire à celui d'un article d'expertise commissionné. A cause de la nature du matériel, le processus de contrôle par les pairs est conduit en ligne dans son intégralité, avec les experts qui ajoutent leurs commentaires directement sur la page molécule à côté du contenu pertinent. On donne aux experts des comptes d'utilisateurs anonymes et soit ils acceptent ou commentent sur chaque section individuelle qui contient des données que l'auteur a ajouté.

Un aspect fondamental des bases de données scientifiques est que l'information peut être capturée et publiée rapidement, présentant une vue d'ensemble de données en changement constant. Un inconvénient majeur dans l'addition du contrôle par les pairs au processus de corrections des bases de données est la cadence plus lente à laquelle les données peuvent être ajoutées. Cela peut prendre plusieurs mois pour que les articles soient expertisés, révisés et édités. Dans des spécialités qui évoluent très vite, les données peuvent déjà être dépassées au moment de leur publication.

Enfin, les responsables de Signaling Gateway Molecule Pages sont entrain d'introduire des outils wiki de logiciel sociaux aux pages molécules. Les wikis permettent tout utilisateur enregistré de modifier l'information, de telle sorte que l'outil est présent à coté des données expertisées et peut offrir une solution viable au problème de la vitesse de la correction. Il faut que l'utilisateur sache cependant, quelle information expertisée de celle non expertisée. Les wikis dans les pages molécules fourniront une plateforme pour la communauté pour devenir plus impliquée mais leur succès dépendra largement de son utilisation ou non par cette même communauté.

\subsection{3)-Ouverture du processus}

Les auteurs de cet article présentent le système du journal en libre accès " The Electronic Transactions on Artificial Intelligence (ETAI)" comme un système hybride de contrôle par les pairs. Le caractère hybride tient au fait que l'article passe par les deux aspects du contrôle par les pairs : l'un ouvert et l'autre, plus traditionnel, fermé. De plus, une des innovations du système ETAI a trait à la dissociation de deux fonctions précédemment liées : 1 'examen et l'arbitrage. Le but de l'examen est de communiquer avec la communauté des pairs et fournir un retour d'écoute pour les auteurs. Le but de l'arbitrage est de définir un seuil de qualité 
minimale avant l'acceptation au journal. Ceci sépare effectivement les buts traditionnels du contrôle par les pairs en deux fonctions distinctes.

Le processus d'examen commence aussitôt qu'un article est soumis à ETAI et est reçu par un de ces éditeurs, il est alors posté sur le site et annoncé à la communauté des pairs via e-mail. Une période de discussion de trois mois débute où il n'y a pas d'anonymat et tous les commentaires sont faits ouvertement.

Cette discussion peut être prolongé au delà des de trois mois, par exemple pour permettre à une discussion animée de parvenir à une conclusion. Ensuite, aux auteurs est donnée une chance de réviser leur recherche envoyée pour une révision experte dans laquelle l'identité des experts est confidentielle. A ce stade, les experts sont seulement supposés retourner un verdict " accepté " ou " rejeté " étant donné que des commentaires plus détaillés devraient avoir été reçus durant la période de révision. L'étape d'arbitrage est de ce fait rapide comparée aux autres journaux dans la même spécialité. Le temps combiné pour la décision des experts plus la publication sur le site web est souvent moins d'un mois dans la plupart des cas et trois mois dans $70 \%$ des cas. Une fois accepté, l'article est alors inclut dans un numéro du journal.

L'article est en fait publié dès que la discussion débute. La priorité du résultat compte à partir de la date de publication en ce sens. Si notre approche devait être utilisée dans les spécialités au profile haut, les recherches discutées devraient être clairement marqués en tant que telles rendant clair que les résultats ne sont pas encore validés. Le système d'ETAI est plutôt sévère vis-à-vis des auteurs. Ils doivent être prêt à répondre rapidement aux critiques durant la période d'examen et risquent avoir leur article rejeté en public. Cependant, ceux qui ont acceptés d'être soumis à ces épreuves font état d'une expérience favorable. Un commentaire récurant est que le programme fournit une bonne publicité pour leurs travaux. En fait, même les remarques critiques sont les bienvenues. Si l'auteur reçoit une question difficile et peut donner une bonne réponse, il ou elle est alors dans une position avantageuse. Tous les commentaires sont filtrés par un éditeur d'ETAI, qui s'assure aussi bien qu'ils sont pertinents et au dessus d'un certain seuil de qualité de base.

Les auteurs dans les journaux traditionnels sont pressés d'avoir leurs articles publiés aussi vite que possible afin de revendiquer la priorité. Sur ETAI la priorité compte à partir du premier jour de discussion, ce qui rend la date exacte d'acceptation sans importance. Le fait qu'il y ait moins de pression pour faire l'expertise très vite est de toute évidence un avantage du programme d'ETAI : il permet aux expertises d'être faites plus attentivement.

La plupart des journaux traditionnels considèrent aussi que l'anonymat des experts comme essentiel. Est ce que les chercheurs sont prés à rédiger des expertises ouvertes et risquer de se faire des ennemis des auteurs rejetés ? La réponse est qu'un examen critique dans ETAI ne constitue pas une menace, au contraire cela aide à améliorer l'article et à augmenter sa visibilité. Ensemble experts et auteurs ont adopté ceci sans problème.

Dans certains cas, la discussion débute toute seule, mais dans nombre de cas l'éditeur invite spécifiquement quelques pairs à écrire des expertises pour une recherche donnée. Ceci conduit 
souvent à une nouvelle discussion où des contributions d'examen additionnels sont obtenues spontanément. Les discussions sur une recherche donnée impliquent typiquement des commentaires de trois ou quatre personnes avec un maximum de six dans une seule discussion.

De toute évidence, certains articles sont rejetés mais le taux de rejet est relativement bas comparativement aux journaux conventionnels. Ceci ne semble pas représenter une baisse de niveau mais une pratique de soumission restrictive de la part des auteurs. Dans un journal conventionnel, rien n'empêche un auteur de soumettre une recherche prématurément : si elle est rejetée, au moins on obtient des commentaires.

\subsection{4)-Un journal contrôlé par les pairs ouvert en deux étapes}

Dans cette contribution, les éditeurs d'Atmospheric Chemistry and Physics expliquent la manière dont sont expertisées les soumissions.

Des cas assez récents et fréquents de fraudes scientifiques spectaculaires ont alimenté le sujet de contrôle de la qualité scientifique. De plus, il est de notoriété que nombre de recherches sont pour le moins négligemment préparées. Ces deux problèmes sont parmi les plus récurrents du contrôle par les pairs. Un problème similaire, si ce n'est plus sérieux, est la grande proportion de recherches négligemment préparées qui diluent plutôt que ne rehaussent le savoir scientifique.

Les éditeurs du journal pensent qu'une combinaison des avantages du contrôle ouvert et ceux du processus traditionnel peut améliorer le contrôle par les pairs. Ces deux étapes sont schématiquement : une évaluation pré publication (experts) et une évaluation post publication (la communauté des chercheurs)

Atmospheric Chemistry and Physics et un nombre de journaux apparentés publiés par The European Geosciences Union ont un processus de publication à deux étapes avec un contrôle par les pairs publique et une discussion interactive Dans la première étape, les manuscrits qui passent un pré filtrage rapide (examen d'accès) sont immédiatement publiés comme " recherches de discussion " sur le site web du journal. Ils sont alors sujet à une discussion publique interactive durant une période de 8 semaines durant laquelle les commentaires des experts désignés, les commentaires additionnels par d'autres membres de la communauté intéressés et les réponses des auteurs sont publiés à côté de la recherche en discussion. Les experts peuvent choisir de signer leurs commentaires ou de rester anonyme, mais les commentaires par les autres scientifiques doivent être signés.

Dans la seconde étape, la révision du manuscrit et le contrôle par les pairs sont complétés de la même manière que dans les journaux traditionnels (avec d'autres séries de révisions non publiques et expertises quand cela est requis). Si elles sont acceptées, les recherches finales sont publiées dans le journal principal. Pour fournir une trace durable des expertises et assurer la précédence de publication de l'auteur, chaque recherche discutée et les commentaires interactifs restent archivés d'une manière permanente et cités individuellement. 
Etabli en 2001, Atmospheric Chemistry and Physics et en 2005 publia 204 recherches finalisées .En moyenne, une sur quatre recherches reçoit un commentaire de la communauté scientifique en plus des commentaires des experts désignés (dans les journaux traditionnels ceci arrive aux environs de 1 sur 100 recherches).Il y a généralement 0.45 pages de commentaires et réponses par page de discussion de recherche originale.

Les statistiques confirment que le contrôle par les pairs collaboratif facilite et améliore l'assurance qualité. Le journal a un taux de rejet global relativement bas de moins de $20 \%$, mais seulement trois après qu'il eut été lancé, le facteur d'impact ISI du journal place Atmospheric Chemistry and Physics $12^{\mathrm{e}}$ sur un total de 169 en " Sciences atmosphériques et Météorologie " et " Sciences environnementales ".

Ces chiffres soutiennent l'idée que le contrôle par les pairs publique et la discussion interactive découragent les auteurs de soumettre des manuscrits de basse qualité et de ce fait soulage les éditeurs et les experts de la tâche de passer trop de temps sur des soumissions médiocres. Dans une expertise publique, cependant, les arguments des experts sont publiquement entendus et, si les commentaires sont ouvertement signés, les experts peuvent aussi revendiquer la paternité de leur contribution.

Le contrôle par les pairs collaboratif avec un processus de publication en deux étapes et la discussion publique interactive résout le dilemme entre l'échange scientifique rapide et une qualité d'assurance minutieuse. Il favorise la discussion scientifique, dissuade la soumission de manuscrits de basse qualité, conserve les capacités d'expertise et améliore la densité d'information dans les recherches finales.

\subsection{5)-Relancer une culture de débat scientifique}

Le contrôle par les pairs ouvert peut il fonctionner pour les biologistes ? Les éditeurs de Biology Direct sont optimistes. Dans le but d'offrir à la communauté scientifique une alternative au système de contrôle par les pairs actuel, Biology Direct fut lancé récemment.

Dans Biology Direct tout le processus se passe de manière ouverte : les auteurs sélectionnent leurs propres experts à partir du comité éditorial, et les expertises sont non seulement signées mais aussi publiées à côté des réponses des auteurs comme partie intégrale de chaque article. La seule condition est que trois membres du comité éditorial de Biology Direct deviennent assez intéressés dans une soumission pour l'expertiser eux même ou solliciter une expertise extérieure. Inversement, une recherche est rejetée si l'auteur ne peut pas avoir trois expertises.

Les avantages d'un système ouvert

Ce système vient à bout des maux bien connus du contrôle par les pairs anonyme et est juste pour les auteurs et les experts. Les auteurs ont l'occasion de discuter avec les experts ouvertement et de faire face aux suggestions des experts sur les mérites apparents sans avoir peur d'un rejet affectant la réponse. Les experts ont la reconnaissance du public pour leur travail très souvent difficile, avec les résultats rendus disponible aux lecteurs. En outre, le système de Biology Direct est propice à la publication de recherches innovantes, osées qui auraient de la difficulté à être publiées dans le système actuel. Plus important peut être, 
l'approche de Biology Direct par rapport au contrôle par les pairs pourrait aider à revitaliser la culture du débat scientifique qui est entrain de disparaître dans le duel inégal entre des experts omnipotents, anonymes et des auteurs sans défense.

Quelles ont étés les conclusions depuis le lancement de Biology Direct en Janvier 2006 ? Quelques tendances commencent à émerger.Par exemple, un intérêt certain dans l'approche est indiqué par la réponse enthousiaste d'un grand nombre de scientifiques très réputés qui ont accepté de joindre le comité

Biology Direct a déjà publié dix articles (sans compter l'éditorial de lancement) et trois autres ont étés soumis avec des expertises (ce qui signifie acceptation sous le modèle de Biology Direct).Quelque 15 recherches sont actuellement aux différents stades du processus d'expertise.

Il est à noter qu'une recherche a réuni trois expertises qui mettent en doute les conclusions centrales du travail (ce qui aurait certainement été qualifié de négatif par les critères habituels) et deux autres recherches ont eut une ou deux de ce genre d'expertise. De toute évidence, le système de publication dans Biology Direct permet un ou plus de rounds d'expertise durant lesquels non seulement le manuscrit mais aussi les expertises peuvent être modifiés en particulier pour inclure les réactions des experts à la révision du manuscrit original. Toutes les recherches actuellement publiées, sans exception aucune, ont étés révisées et dans certains cas, il y a eut un intense aller-retour.

A partir de ces expériences initiales, on peut dire que le système d'expertise de Biology Direct fonctionne: il offre des discussions intéressantes, constructives et très critiques de chaque recherche publiée, ajoutant une valeur significative à la publication.. Il est concevable que, dans le futur, des amendements au modèle tels que des notations explicites véhiculeront le message des expertises aux lecteurs.

Le mécanisme principal de rejet effectif d'une soumission à Biology Direct (à part auto rejet, ce qui est déjà arrivé une fois) est lorsque l'auteur ne peut trouver trois experts pour un manuscrit et abandonne (dans un cas extrême, ayant sollicité tout le comité éditorial).Dans le scénario du meilleur des cas, ce mécanisme de rejet réduirait le nombre de recherches de routine, même si elles sont acceptables du point de vue technique, qui arriveraient à être acceptées par Biology Direct vu qu'elles pourraient ne pas réussir à provoquer un intérêt suffisant afin de susciter des expertises. D'une manière moins optimiste, ce genre d'échec peut tout simplement refléter un manque d'expertises pertinentes dans le comité éditorial.

Jusqu'à présent, il n'y a eu que de deux tentatives qui ont échoués à avoir leur manuscrit expertisé par Biology Direct. Mais plusieurs auteurs ayant soumis des recherches apparemment dans le cadre de Biology Direct ont eu des difficultés à trouver des experts. Ceci est un rappel que la qualité d'un journal est proportionnelle à la qualité de son comité éditorial.

La plus grande préoccupation avec le modèle libéral d'acceptation de Biology Direct serait qu'il permettrait à plus de pseudoscience d'être publiée que dans les journaux traditionnels. 
Afin d'éviter que ceci arrive, Biology Direct a un système d'alerte qui permet, dans le cas où les éditeurs identifient une soumission qui ne répond pas aux critères scientifiques, d'alerter les experts qui rejettent alors le manuscrit. Un le seul cas a été détecté mais le système a fonctionné sans problème avec trois experts repérant immédiatement des signes de problèmes et notifiant les éditeurs.

\section{Le futur de Biology Direct}

Biology Direct est appelé à devenir un espace pour la publication d'études pionnières, d'hypothèses et de recherches qui pourraient ne pas passer les critères strictes des meilleurs journaux traditionnels mais qui offrent des idées radicales. Mais la plupart des recherches, cependant, sont des recherches tout ce qu'il y a de plus routinier : c'est des travaux sérieux qui auraient facilement pu être publiées dans des journaux plus traditionnels. Peut être que ceci est un signe que Biology Direct attirera un sain mélange de bonne science " normale " et des idées audacieuses.

Il y a un intérêt de la part de la communauté (certains articles ont étés déjà accédés plus de 2000 fois) et ceci est soutenu par un retour de commentaires positif fort de la part de beaucoup d'auteurs. Il est à souhaiter que l'approche de Biology Direct ait un futur comme alternative viable au système actuel du contrôle par les pairs.

\section{9-QUALITE ET VALEUR}

\subsection{1-)-Le véritable but du contrôle par les pairs}

Le contrôle par les pairs a besoin d'indicateurs quantitatifs pour mesurer ses performances. Mais il semble insuffisamment étudié. Une approche plus systématique est de mise si nous voulons comprendre le contrôle par les pairs tel qu'il est actuellement pratiqué ou évaluer les avantages et inconvénients de toute approche alternative.

Etant donné que nombre de recherches ne sont jamais citées (et on se doute rarement lues), il n'est probablement pas tellement important, à part pour l'auteur, que la recherche médiocre soit publiée dans un obscur journal. Beaucoup plus important, est la question du journal où elle est publiée et en fait ceci est la majeure fonction du contrôle par les pairs. Il est généralement compris entre les scientifiques qu'il existe une hiérarchie des journaux. Au sommet de la pyramide se trouve les journaux interdisciplinaires les plus prestigieux; en dessous de ces journaux est le niveau intermédiaire de bons journaux propres à chaque discipline avec des taux variés de sélectivité et spécialisation et à la base se trouve une collection large et hétérogène de journaux dont les compétences sont restreintes, régionales ou tout simplement non sélectives.

Pour réussir en science, on doit gravir cette pyramide : dans le monde académique au moins, la publication dans les journaux les plus prestigieux est la clé à l'avancement professionnel. La compétition est inhérente à la science comme c'est le cas pour toute activité où des individus talentueux visent l'excellence. Il y a aussi une compétition de plus en plus grande pour " l'espace mental " parmi ses collègues scientifiques. Avec plus d'un million de 
recherches par an en constante augmentation, personne n'a le temps de lire chaque recherche à part dans les spécialités les plus restreintes ce qui fait qu'une sélection est indispensable.

Il existe dans cette optique des coûts mesurables du contrôle par les pairs. Ce sont :

-Combien de temps se passe $t$ il entre la soumission et la publication et combien est ceci imputable au contrôle par les pairs? Il serait intéressant d'estimer la valeur monétaire de ce délai : si le savoir nouveau représente le retour sur investissements publiques dans la recherche, quel est la dissémination dilatoire de la réalisation de ce retour?

-Combien de temps est ce que les experts dépensent sur le contrôle par les pairs ? Quoique les experts tirent des avantages en expertisant, cela représente toujours du temps pris aux autres activités (recherche, enseignement) qu'ils auraient autrement priorisés .

-Quel est actuellement le coût actuel d'accès aux recherches expertisées ? De toute évidence, les coûts d'abonnement payent pour d'autres choses à coté du contrôle par les pairs (correction, impression et publication en ligne et ainsi de suite) mais opérer le système d'expertise coûte de l'argent et la plupart des éditeurs de journaux argueront que le contrôle par les pairs représente un composant substantiel de la valeur qu'ils ajoutent. Le coût payé pour un accès potentiel est facilement calculé (les frais de site de licence divisée par le nombre de recherches publiées) mais un nombre plus intéressant serait le coût actuel de l'accès (le coût de la licence divisé par le nombre de téléchargements actuel).

On peut aussi démontrer quelques avantages mesurables du contrôle par les pairs

-Est ce que les recherches publiées différent de la soumission initiale ? En particulier, combien de fois est ce que les auteurs effectuent ils de nouvelles expérimentations ou analyses en réponse aux commentaires des experts?

-Quel est le taux d'acceptation après contrôle par les pairs ? En principe, le contrôle par les pairs agit comme un filtre mais les auteurs expérimentés tendent à cibler leurs soumissions, envoyant seulement leurs recherches les plus importantes aux journaux les plus prestigieux.

-Quelle est la valeur que les lecteurs tirent du système actuel de classement tel que cela est mesuré par leurs habitudes de lecture ? Dans quelle mesure, utilisent-ils l'identité du journal pour décider qu'est ce qu'ils lisent?

-Enfin, la question la plus importante est jusqu'à quel point est ce que le contrôle par les pairs prédit il les jugements à long terme de la communauté scientifique. Une manière d'aborder ceci est à travers les données de citation; les articles qui résistent à l'épreuve du temps devraient être les plus cités comparativement aux autres dans la même spécialité, même plusieurs années après leur publication.

Une tentative de réponse à cette dernière question est suggérée par une étude pilote réalisée par Faculty of 1000, un site web qui cherche à identifier et classer des recherches intéressantes basé sur les votes d'experts triés sur le volet. Pour un échantillon de 2500 recherches en neurosciences énumérées dans Faculty of 1000, il y a avait une forte corrélation entre le facteur F 1000 de la recherche et le facteur d'impact du journal dans laquelle elle est apparue. Cette constatation bien que préliminaire, devrait donner à réfléchir à quiconque croit 
que le système actuel de contrôle par les pairs est fondamentalement imparfait ou qu'une méthode plus distribuée d'évaluation donnerait des résultats différents. .

Le contrôle par les pairs n'est pas la véritable solution de tous les temps, il serait insensé de croire qu'il n'y aura jamais de meilleure solution émergeant au sujet du filtrage de l'information scientifique. Nombre d'alternatives intéressantes ont étés suggérés. Mais le taux impressionnant du progrès scientifique au cours des dernières décennies est en partie un hommage à l'efficacité du système actuel du contrôle par les pairs. Le minimum pour toute nouvelle alternative devrait de ce fait être mis assez haut. N'importe quel nouveau système devrait comprendre les critères suivants :

- être fiable-il doit prédire l'importance d'une recherche avec un niveau de précision comparable à ou meilleur que le système actuel.

-donner une recommandation qui est facilement digestible, permettant aux scientifiques trop occupés de prendre des décisions rapides au sujet de ce qu'ils lisent.

- être économique, non seulement en terme de coûts directs, tels que les opérations web, mais aussi en terme de temps d'expert investi.

- fonctionner rapidement. Le système de contrôle par les pairs produit des décisions claires d'une manière relativement rapide. Peut être qu'un index de popularité (par exemple pour la liste qui reçoit le plus d'emails) fournirait une lecture rapide mais il y danger d'amplification accrue- le soi disant Matthew effect identifié par Robert Merton il y a presque 40 ans et qui risque d'être exacerbé en cette époque de communication digitale.

-être résistant au " jeux " par les auteurs. Bien sûr les auteurs expérimentés savent comment travailler avec le système actuel, mais la séparation des pouvoirs entre les éditeurs et les experts anonymes préserve l'intégrité du système. une chose est sûre : si un système devient influent dans la détermination du succès d'une carrière, les auteurs chercheront des manières de le manipuler à leur avantage.

Il y a de la place pour l'amélioration à l'intérieur du système actuel. Les éditeurs ne naissent pas en tant que tels et les outils électroniques peuvent les aider à faire un meilleur travail. Par exemple, les systèmes de suivi des manuscrits peuvent fournir un retour d'écoute sur les causes de délais et où sont alloués les ressources. Mieux même, les éditeurs bénéficieraient du retour d'écoute sur la qualité de leurs décisions. Il serait intéressant, par exemple, de comparer rétrospectivement les citations des recherches acceptées comparées aux citations des recherches rejetées et voir est ce que les éditeurs varient dans leur capacité à designer le gagnant.

\subsection{2)-Modèles du contrôle de la qualité pour la recherche scientifique}

Le système du contrôle par les pairs suggérerait que ce serait la meilleure solution. Il est utilisé en tant que mécanisme de contrôle de la qualité de la littérature biomédicale. Les variantes du système actuel ou les alternatives complètes ont étés à peine explorées jusqu'à présent. Le système actuel a trois aspects importants et qui sont : l'assurance de qualité par le 
biais des opinions expertes, gérer la compétition pour l'espace de publication et enfin la mission scientifique d'améliorer le savoir scientifique.

Huit disciplines non biomédicales bien définies (agriculture, astronomie, archéologie, sciences de la prise de décision, droit, économie, mathématiques, physiques) et dont dépendait trente et un journaux furent choisis. Leurs éditeurs appliquaient tous un modèle universel dans lequel chaque recherche publiée est expertisée, d'une manière ouverte ou anonymement, par un nombre limité d'experts.

En biomédecine, les modèles de contrôle par les pairs prépublication sur Internet (au Medical Journal of Australia) et le modèle éditorial de Cochrane Collaboration (à la Cochrane Library) représentent des alternatives à examiner

Le contrôle par les pairs pré publication sur Internet

Le contrôle par les pairs ouvert consiste à rendre les commentaires des experts publiques et / ou inviter des commentaires post publication des lecteurs qui sont alors publiés. Ce genre de système fut testé au Medical Journal of Australia, où 60 (81\%) des auteurs et $150(92 \%)$ des experts acceptèrent de prendre part à une étude utilisant un accès site web pour le contrôle par les pairs pré publication. Quoique le retour d'écoute informel de la part des lecteurs fût positif, seul 29\% des commentaires poussèrent les auteurs à changer leur manuscrit.

Le modèle Cochrane Collaboration :

Le modèle Cochrane Collaboration est un effort collaboratif pour évaluer les effets des interventions des soins de santé par la publication et la mise à jour systématique des expertises dans la base de données Cochrane. Le processus éditorial comprend un contrôle par les pairs pré et post publication des protocoles et des expertises complètes. Le processus est complètement ouvert, quiconque qui participe à l'expertise est reconnu et souvent les éditeurs sont aussi auteurs et experts et vice versa.

La caractéristique primordiale de compétition est remplacée par la collaboration et la dépendance sur la publication électronique qui virtuellement enlève les contraintes d'espace.

Il n'existe peut être pas d'alternatives significatives au système actuel, ou est ce que personne n'a pensé à les développer .Quoique, non parfait le système actuel, pourrait être le meilleur possible. Devrions-nous explorer des alternatives partielles? Ceci voudrait dire que la compétition devrait céder la place à la collaboration. Un changement de ce genre aurait des implications majeures pour la science et l'industrie de la publication mais nous pensons que le but (améliorer la science) en vaut la peine.

\subsection{3-)-Comment pouvons nous tirer le maximum du contrôle par les pairs ?}

Améliorer le contrôle par les pairs dépend du fait de rendre ses aspects humains encore plus humains. Les journaux doivent demander à l'expert adéquat d'expertiser l'article adéquat, les aider à le faire vite et soigneusement, faire de telle sorte à ce qu'ils soient content de signer 
leur expertise, les remercier, leur dire comment leur travail a été fait et encourager une reconnaissance large de ce qui est trop souvent une tache ingrate.

Le contrôle par les pairs est le seul contrôle de qualité que nous avons pour approuver et rendre publique les résultats dans les journaux et les conférences. Mais c'est largement un processus amateur : trop souvent incapable de détecter les erreurs, lent, cher et peu fiable. Il n'est pas très bon pour appréhender les problèmes éthiques ou la fraude scientifique. Dans le pire des cas, il bloque l'innovation, est déraisonnablement biaisé et est ouvert à l'abus, tout ceci parce que le contrôle par les pairs est un processus humain, un art plutôt qu'une science.

Comment les journaux peuvent ils améliorer le processus du contrôle par les pairs ? Etre honnête, utiles et raisonnable vis-à-vis des auteurs et experts permet d'en obtenir le maximum. Au British Medical Journal, la totalité du processus de contrôle par les pairs est faite d'une manière aussi transparente que possible en demandant aux auteurs et aux experts de déclarer les conflits d'intérêt, laissant les experts voir les protocoles de recherche aussi bien que les manuscrits complétés, demandant aux auteurs de fournir les documents à l'appui, les liens à leur propre site web et, des fois, leurs données brutes et ceci peut même aller jusqu'à demander aux auteurs comment ils ont répondus aux expertises d'autres journaux qui ont déjà rejeté leur travail. Plus important, un système de contrôle par les pairs complètement ouvert est utilisé dans lequel auteurs et experts connaissent les noms et les adresses les uns des autres, et les experts ne peuvent pas faire des commentaires séparés à l'éditeur ou succomber facilement au favoritisme - par exemple contre des auteurs inconnus d'institutions non prestigieuses et/ou contre les femmes. Les auteurs et les experts aiment ce système, seul un petit nombre des milliers d'experts ont arrêtés d'expertiser depuis son lancement, et il existe de bonnes preuves à partir d'un essai de contrôle aléatoire que cela n'a pas d'effets adverses sur les expertises.

Les éditeurs de journaux ont besoin de choisir les experts adéquats dès la première fois afin de ne pas faire perdre le temps des experts, des éditeurs et des auteurs. Un bon système de suivi des manuscrits en ligne ainsi qu'une large base de données d'experts bien gérée, des invitations envoyées par courrier électronique et des délais stricts aident beaucoup. De même que choisir ses experts pour leur savoir, expertise et crédibilité plutôt que leur distinction aide beaucoup.

Il y a d'autres actions qui amélioreraient le système de contrôle par les pairs de n'importe quel journal. Faire comprendre aux auteurs et aux experts ce que vous attendez d'eux. Publier des instructions complètes et détaillées pour les auteurs pour les aider à fournir tout ce que les experts veulent voir. Donner aux experts des instructions claires, comprenant des orientations (ce qu'ils doivent inclure dans l'expertise, combien d'effort ils doivent mettre dans la recherche de la littérature, expliquer qu'est ce qui est utile du point de vue de la perspective de l'éditeur, etc....).

Encore mieux, demander aux experts qu'est ce qu'ils veulent. Un certain nombre des expert du British Medical Journal ont demandés à être formé dans le contrôle par les pairs, de ce fait nous proposons maintenant un atelier qui couvre l'évidence sur les pièges du contrôle par les pairs et fournit des exercices sur ce que les auteurs et les éditeurs du British Medical Journal 
attendent des experts. Les experts veulent aussi un retour d'écoute sur leurs performances de telle sorte qu'ils apprennent et se perfectionnent.

Qu'en est-il des récompenses ? Pendant de nombreuses années, le British Medical Journal a payé les experts une somme forfaitaire de $50 £$ par expertise. Certains experts disent que ceci est un tracas administratif et qu'ils préféreraient avoir un abonnement gratuit ou la récompense plus significative et durable de la reconnaissance publique et académique pour le travail qu'ils font. les nombreuses heures qu'ils font chaque année ne devraient elles pas être reconnues plus formellement par les panels d'interview et les exercices d'évaluation de la recherche?

\subsection{4-)-Les statistiques dans le contrôle par les pairs}

Les méthodes statistiques sont largement utilisées dans nombre de domaines en sciences naturelles notamment en épidémiologie. Avec la puissance actuelle des outils informatiques et des logiciels, les chercheurs utilisent des méthodes informatiques intensives de grande complexité, menant très souvent à l'utilisation de techniques qui sont de plus en plus sophistiquées et puissantes.

Un autre problème persistant pour les éditeurs de journaux est d'obtenir les services d'experts chevronnés. Il est conventionnel d'avoir au moins deux experts dans le domaine du sujet pour une recherche soumise, et leur expertise tend à ne pas être en statistiques (à part pour des recherches purement méthodologiques).

Obtenir deux experts avec l'expertise appropriée est déjà assez difficile sans demander qu'un autre expert évalue l'utilisation des statistiques dans la recherche. Les statisticiens sont déjà très demandés. Pour ceux qui sont déjà très impliqués dans (et des fois dépassés par) le service et le support des essais cliniques, le travail impayé du contrôle par les pairs est au plus bas de leur liste des priorités.

Il y a aussi des recherches qui présentent de nouvelles méthodes ou des applications inédites de méthodes existantes .L'auteur inclut souvent la ou les personnes qui ont utilisés les premiers la technique en question et qui de ce fait sont très bien placées pour la juger.

Certains journaux à grand tirage, tel que The American Heart Association Circulation,The New England Journal of Medecine et The Journal of the American Medical Association emploient des consultants statistiques ou des éditeurs payés. Mais ceci est au delà des moyens de la plupart des journaux et soulève une autre question : jusqu'à quel point est ce important que les méthodes statistiques soient correctes par la pratique conventionnelle?

Si la réponse est que les erreurs statistiques ne sont pas tant que ça différentes de celles dans d'autres méthodes alors la question devient un problème plus général. Les scientifiques ont besoin de meilleures instructions dans l'interprétation des résultats statistiques.

Peut être qu'à la fin, on devra revenir à l'observation d'un de mes collègues dans laquelle il dit que ...... " le véritable contrôle par les pairs commence après la publication". 


\subsection{5-)-Comment pouvons nous investiguer le contrôle par les pairs ?}

Améliorer le processus du contrôle par les pairs repose sur la compréhension de son contexte et sa culture.

A quoi ressemblerait une investigation empirique de ces problèmes ? Ce genre de question avec des personnes qui pensent faire de la recherche sur la solution des problèmes éthiques basée sur l'évidence est abordé. Parmi les doléances :

-Des expertises décourageantes, y compris des experts insultants, peu serviables ou non qualifiés, des éditeurs qui semblent donner un poids égal aux expertises bonne, mauvaise, ambiguë ou contradictoire, le rejet de recherches qui sont très favorablement expertisées.

-Des expertises malhonnêtes, y compris des expertises qui expriment des préjugés, des conflits d'intérêt ou n'ont pas lus la recherche d'une manier assez minutieuse.

-Des exigences impossibles quand les experts ou les éditeurs sont supposés détecter une quelconque malhonnêteté telle que la contrefaçon, la falsification ou le plagiat.

Est-ce que toutes ces doléances reflètent le même problème ? Est-ce qu'une solution guérirait tous les problèmes ?

-Un débutant soumet une recherche naïve au journal principal du domaine qui est rejetée avec deux expertises qui sont beaucoup plus des coups bas qu'un retour d'information utile. Après les révisions, l'auteur soumet à un journal moins prestigieux et est accepté, à condition de répondre aux suggestions importantes par les experts.

-Un jeune chercheur soumet une recherche complexe et soigneusement élaborée à un journal important. Elle est rejetée avec des suggestions que la recherche a besoin de recherches complémentaires importantes. Une année plus tard, une recherche presque identique est publiée par un autre journal important du domaine par un chercheur bien connu et aux connections bien établies qui était membre du comité éditorial du journal précédant. Afin de limiter les dégâts, le jeune chercheur ne se plaint pas et passe à un autre projet de recherche.

-Un manuscrit est publié avec une méthodologie défaillante et une introduction qui est largement plagiée- problèmes identifiés plus tard par un lecteur.

Imaginons maintenant les perspectives des éditeurs :

-La plupart des expertises peuvent contribuer à améliorer une recherche.

-Trouver des experts compétents, ouverts d'esprit, sans à priori et bien choisis pour chaque recherche est difficile: les meilleurs experts sont des personnes occupées. Contrôler les experts novices se fait par approximations successives. Même les personnes bien connues dans leur domaine peuvent être de piètres experts.

-Les limites en pagination qui imposent un taux de rejet de $90 \%$ peuvent mener à l'élimination de quelques très bonnes recherches. 
-Certains éditeurs manquent les ressources pour donner toute l'attention voulue à chaque recherche.

-Editer un nouveau journal, dans lequel l'éditeur joue un rôle majeur dans le façonnement des soumissions pour un domaine naissant est différent de l'édition d'un journal de courant dominant et bien établi.

-Un journal qui enquêterait sur tous les semblants de mauvaise conduite scientifique augmenterait le temps utilisé pour l'expertise et serait accusé d'outrepasser son rôle.

Et les perspectives des experts :

-Expertiser est une grande quantité de travail pro bono pour un professionnel occupé si c'est fait d'une manière adéquate, mais c'est aussi une responsabilité envers la communauté, une expérience d'apprentissage pour être au diapason du domaine et peut être aussi un symbole de statut.

-Un accord d'expertiser souvent implique un manque de temps, résultant en délais non respectés et des expertises hâtives.

Le plus important à interroger, cependant, sont les éditeurs en chef qui supervisent le processus d'expertise. Notamment, ils devraient être interrogés sur la manière dont ils sélectionnent, utilisent, évaluent et gèrent les experts et comment ils expliquent leurs décisions aux auteurs .D'autres questions pourraient inclure comment les éditeurs différent par la formation de base, les ressources, le taux de rejet, le prestige, l'âge et le domaine de leurs journaux.

On soupçonne le contrôle par les pairs d'être un peu comme la démocratie - un mauvais système mais le meilleur possible. Il parait être celui qui prend différentes formes dans des cultures (scientifiques) différentes et peut être arrangé pour améliorer son opération. Espérons que la recherche dans le futur découvrira et disséminera les meilleurs moyens pour peaufiner le système à l'intérieur des contraintes de chaque type de journal.

\subsection{0-ETHIQUE : Confiance et réputation sur la toile}

\subsection{1)-Les publications en ligne ont plusieurs manières pour accéder à la respectabilité}

Confiance et réputation sont fondamentales pour la publication scientifique. La Toile offre d'alléchantes nouvelles manières de publier, mais celles ci peuvent elles gagner la confiance cruciale à l'acceptation scientifique ? Le contrôle par les pairs est la manière traditionnelle de construire la confiance, mais il est long et cher, certains sujets sont difficiles à expertiser et les experts font des erreurs. Quelles alternatives ont les auteurs?

Une pratique effective surprenante, devenue la norme en informatique, est pour les auteurs de déposer des prépublications de leurs recherches dans leurs propres sites web quand ils les 
soumettent aux journaux pour expertises. A la publication, la version finale remplace la prépublication.

Les entrepôts de prépublications, organisés par discipline ou institution sont une autre manière de distribuer des recherches avant ou sans expertise. Le plus connu est ArXiv, qui domine les sciences physiques et est important en mathématiques et les domaines connexes. Il offre aux chercheurs une distribution immédiate et mondiale de leurs recherches, une occasion pour les autres de les commenter (et pour les auteurs de réviser) une recherche avant qu'elle ne soit soumise à un journal, ainsi qu'une archive complète.

ArXiv fut créée par des physiciens pour des physiciens, mais quoiqu'il ait acquis la confiance de sa communauté, d'autres initiatives similaires n'ont pas eu le même succès. DSpace, par exemple, est une tentative par des bibliothécaires d'universités, d'encourager les membres du corps professoral de placer leur production en ligne mais n'a pas, jusqu'à maintenant, réussi à attirer beaucoup d'auteurs. Une des difficultés est que les universités sont tellement diverses que les entrepôts institutionnels ne peuvent être adaptés à une communauté spécifique de la manière dont l'est ArXiv pour les physiciens.

Une approche plus révolutionnaire est de laisser les lecteurs modifier sans consulter les auteurs originels. Wikipedia utilise cette approche. En respectant de simples directives, n'importe qui peut créer une nouvelle entrée encyclopédie ou d'en modifier une existante. Ce succès ne vient pas facilement et peut créer ces propres problèmes : vandalisme, préjugé et des disputes entre les éditeurs qui inversent les changements des uns les autres. Mais Wikipedia s'efforce de créer un climat de confiance et fait continuellement des petites mais significatives révisions à ces procédures .D'autres tentatives d'écriture collaborative moins bien gérées n'ont pas réussi.

Dans la publication conventionnelle, éditeurs et experts sont choisis pour leurs expertises et la confiance est acquise à travers l'autorité des experts. Sur la Toile, une alternative est de permettre à n'importe qui de soumettre une expertise et construire la confiance sur la base du consensus. Un exemple est le système d'expertise des livres à Amazon. Nombre des expertises d'Amazon sont excellentes, mais des problèmes apparaissent en raison du pouvoir qu'ont les expertises en ligne à influencer les ventes de livres et les réputations des auteurs.

La réputation d'un journal est généralement rattachée à un nom. Dans la publication conventionnelle, cela pourrait être le nom d'une société (The American Physical Society), une université (Oxford University Press) un éditeur (Wiley), ou simplement le nom du journal luimême (Nature).Une université donne son nom à une presse qui attache son nom à une publication qui à son tour fournit des références pour les auteurs et les experts. Sur la Toile, plusieurs nouvelles publications ont réussi à se construire une réputation sans être rattachés à un nom conventionnel. Un exempleest D-Lib Magazine qui est devenue la première publication pour la recherche sur les bibliothèques numériques. Le magazine, qui n'a pas de contrôle par les pairs a construit sa réputation à travers son soutien des auteurs : en fournissant des éditeurs experts, une réponse rapide des manuscrits soumis, ponctualité, libre accès pour les lecteurs et une politique de copyright avantageuse. Nombre d'auteurs connus accordent à ces attributs plus que l'imprimatur du contrôle par les pairs. 
L'avancement professionnel en science est encore basé sur les journaux contrôlés par les pairs, mais dans certaines disciplines les bénéfices de la dissémination rapide et le libre accès, l'abondance d'indices au sujet de la qualité autre que le contrôle par les pairs, et l'excellente qualité de certaines publication non contrôlées ont gagné la confiance du scientifique.

\subsection{2-Détecter les fautes}

Internet a changé la manière dont la science est entreprise, particulièrement en ce qui concerne la publication. Les manuscrits sont soumis, expertisés et les auteurs notifiés électroniquement maintenant. Mais quoique l'efficacité et la vitesse du processus du contrôle par les pairs aient augmenté, un ensemble de problèmes périphériques sont apparus.

Plus précisément, il n'a jamais été plus facile de détecter les manquements au comportement éthique que de nos jours. Comme preuve, le nombre de problèmes éthiques rapportés impliquant The American Physical Society (dans 14 journaux différents) est monté en flèche d'une moyenne de moins d'un par an avant 1999 à plus de 50 en 2004, quand tous les publications de la Society devinrent disponibles en ligne.

Quand il évalue un manuscrit, un expert n'a plus besoin de se déplacer pour fouiller dans les journaux imprimés de la bibliothèque à la recherche d'un paragraphe ou une figure qui semblait familière. Tout ce dont on a besoin de nos jours est de taper quelques mots clés sur un moteur de recherche approprié et de suite, tous les articles pertinents apparaîtront sur votre bureau. Ou alors, pour les plus méticuleux, les logiciels anti plagiat sont disponibles pour le téléchargement gratuitement.

Je ne pense pas que ce soit prudent pour les journaux de devenir des détectives actifs, recherchant les fautes scientifiques potentielles dans chaque manuscrit soumis. En outre, le temps et les ressources considérables dont les journaux auraient besoin pour détecter les fautes potentielles n'en vaudraient pas la peine.

Les journaux sont peut être responsable de veiller à ce que le fait scientifique soit intègre, mais la confiance dans nos collègues scientifiques et nous-mêmes a été et devrait continuer à être les fondements de notre discipline.

\subsection{3)-A quoi ça sert?}

Analyser le but du contrôle par les pairs

La plupart des personnes acceptent le fait que le contrôle par les pairs est très important et devrait être maintenu et protégé, mais peu sont d'accord sur la question : quel but sert il ? La publication de la science, même à son niveau le plus simple, implique des interactions complexes entre les chercheurs (auteurs), les éditeurs de journaux, les experts et les lecteurs.

Pour les auteurs et les organisations finançant, le contrôle par les pairs fournit une importante apparence de respectabilité Les découvertes publiées dans un journal prestigieux prennent l'aura de la réputation du journal. Pourtant même les journaux les plus respectés ont étés pris 
en défaut et malgré un contrôle par les pairs poussé, ils se sont retrouvés à publier du matériel frauduleux ou sérieusement altéré.

Pour les éditeurs, le contrôle par les pairs peut aider à informer le processus de prise de décision. La plupart des éditeurs obtiennent deux ou trois expertises pour chaque article avant de décider. Les journaux prestigieux basent leur réputation sur leur exclusivité et prennent une approche du sommet à la base, écrémant une proportion infime des articles qu'ils reçoivent. Les taux de rejet pour Lancet et The New England Journal of Medecine, par exemple, sont bien au-delà de 90\%. Néanmoins d'autres journaux contrôlés par les pairs adoptent une philosophie différente. Certains journaux électroniques, tels que ceux dans la série de BioMed Central, opèrent " un préjugé envers la publication ", acceptant n'importe quoi qui réponde à un minimum de critères scientifiques et éthiques. Entre ces deux extrêmes, se trouvent les nombreux journaux de spécialité avec des taux de rejet aux environs de $50 \%$. Tous utilisent le contrôle par les pairs, et peuvent même utiliser les mêmes experts alors que les résultats sont clairement différents. On ne peut alors pas regarder le contrôle par les pairs comme un test diagnostique conçu pour produire une réponse correcte, étant donné que la " justesse " des résultats dépend du contexte du journal. Quelques braves journaux ont suggéré que le contrôle par les pairs devrait être vu comme un mécanisme pour décider où une étude se publie plutôt que si elle doit ou non être publiée.

Le contrôle par les pairs adresse t il les besoins de ces intervenants?

Avec tous les différents besoins et utilisations du contrôle par les pairs, pouvons nous dire que c'est un outil de prise de décision d'un objectif incertain et / ou de fiabilité faible ? Il agit comme un filtre conçu pour supprimer la mauvaise science alors qu'il est connu pour être presque inutile, du moins à certaines occasions, pour détecter les fraudes majeures. Les modèles traditionnels, où les experts restent masqués dans l'anonymat, sont aussi ouvert à l'abus, vu que les données ou les idées peuvent être volées d'une recherche en phase d'expertise, ou alors la publication peut être retardée par les compétiteurs.

Malgré la relative faible évidence des études formelles, l'expérience nous dit que la précision, la clarté et justesse des recherches scientifiques tend à s'améliorer avec l'expertise attentive par les collègues ou éditeurs. Si on veut améliorer le processus du contrôle par les pairs, on a besoin de commencer à être d'accord sur son but principal : est ce un filtre, un système de distribution, ou un processus de contrôle de la qualité ?

\subsection{4)-Augmenter la responsabilité}

Le contrôle par les pairs est actuellement conçu pour identifier les imperfections dans la conception, la présentation, l'analyse et l'interprétation de la science et fournir une critique prompte, détaillée et constructive pour améliorer la recherche.

Le système de contrôle par les pairs d'un journal repose sur l'intégrité et la responsabilisation des auteurs, des éditeurs et des experts. Des intérêts concurrents financiers ou personnels peuvent fournir la motivation pour une mauvaise conduite. Un nombre de politiques et de mécanismes que tous les journaux scientifiques pourraient adopter pour améliorer la 
transparence et promouvoir un contrôle par les pairs juste pourraient se résumer dans les points suivants :

1-Améliorer la transparence

2-Promouvoir une expertise juste

3-Faciliter le contrôle par les pairs

Ces recommandations ne sont, en aucun cas, compréhensives mais elles amélioreraient la responsabilisation de ceux qui sont investis dans le processus du contrôle par les pairs: auteurs, éditeurs et experts. De tels changements devraient aider à assurer l'intégrité de la recherche et la dissémination des résultats de la recherche dans la littérature scientifique.

\subsection{1-SOLUTIONS TECHNIQUES}

\subsection{1)-Faire évoluer le contrôle par les pairs pour Internet}

Quoique les formes traditionnelles de la communication scientifique soient toujours utilisées, le volume absolu et le rythme de l'information qu'Internet a permis et les outils de publication imposent des solutions originales.

Un être humain peut filtrer manuellement quelques messages spam par jour mais pas des centaines ou des milliers. Alternativement, plutôt que combattre le système, on peut trouver des manières de le faire travailler pour nous. En d'autre termes, avec une équipe assez large de contributeurs vérifiant un document, toutes les erreurs seront localisées.

On peut penser de Wikipedia comme un système pour générer des articles qui sont résistant aux attaques idéologiques et représenter un instantané du consensus actuel des masses au sujet d'une idée particulière. Mais ce système ne peut être un substitut pour le contrôle par les pairs par les experts.

Alors qu'il ne serait pas utile de séparer le contrôle par les pairs de l'article, une possibilité offerte par Internet est de séparer les articles des journaux qui les publient en particulier. Le rythme de travail scientifique a été décrit comme ayant cinq éléments : enregistrement, certification, sensibilisation, archivage et récompense. Ces services, quoique présentés aux auteurs comme un tout unifié, sont actuellement une combinaison de capacités, fournies par l'éditeur lui-même et des services externes dans laquelle il agit comme un intermédiaire avec des parties tierces.

Pour le rôle de certification, le présent système de contrôle par les pairs a une valeur durable, assurant qu'un article passe certaines normes de qualité et intégrité scientifique. Mais l'article lui-même peut vivre une vie indépendante sur les pages web ou dans les entrepôts institutionnels sans jamais être publié dans un journal.

Mais alors sans le journal remplissant le rôle de sensibilisation, comment est ce que les scientifiques peuvent suivre la trace d'articles d'intérêt pour eux ? Et comment pouvons nous mesurer l'impact d'un article individuel? 
C'est un domaine de recherche stimulant que de comprendre comment la discussion en ligne reflète le discours scientifique. Nous découvrons qu'une discussion beaucoup plus large au sujet d'un article en particulier a lieu sur Internet aussi bien à l'étape de pré impression avant la certification et une fois que l'article a passé le contrôle par les pairs.

Le contrôle par les pairs exclu la désinformation préjudiciable tout en s'adaptant à de nouveaux entrants. Il existe un profond respect sous- jacent pour le contrôle par les pairs qui, en dernier ressort, est un service extraordinaire fourni gratuitement par des scientifiques à la communauté scientifique et la société en général.

\subsection{2)-La sagesse des masses}

Qui sont les pairs dans le contrôle par les pairs ? Ils ont généralement un Ph.D et travaillent dans un domaine pertinent à la recherche examinée. S'ils font partie du monde académique, ils peuvent être des professeurs titulaires. Le processus n'est pas parfait, pour des raisons allant du copinage au caprice alors que la longue expérience a démontré qu'il était meilleur que les alternatives.

Mais maintenant un nouveau genre de contrôle par les pairs est entrain d'émerger en ligne, en dehors de la communauté scientifique.

Wikipedia est peut être l'exemple le plus dramatique, une encyclopédie en ligne écrite et éditée par plus de 1000000 volontaires. Les éditeurs de Wikipedia n'ont pas à avoir des Ph.D ou quelque sorte d'affiliation professionnelle; leurs contributions sont considérées sur leur mérite, indifféremment de qui ils sont ou comment ils sont devenus instruits. Si ce qu'ils écrivent est accepté par l'inspection, il est consigné sinon il est éliminé. Toutes les personneslecteurs et contributeurs ensemble - voient le même " modifiez cette page ", invitant correction et extension, ceci résulte en une amélioration continue de la qualité.

Dominant ce monde publication ouverte est la hiérarchie égalitaire de Google qui établit la pertinence et le mérite sur la base des liens entrants à partir des autres sites. L'autorité d'une citation est déterminée par combien d'autres l'ont cité. Une forme de contrôle par les pairs est utilisée mais pas celle qu'un journal scientifique reconnaîtra.

Sommes-nous en présence de deux différentes utilisations de " pairs " - en science, un professeur ; en ligne, n'importe qui ? La réponse dépend de ce dont on a envie. Tel qu'il est le contrôle par les pairs dans les journaux décide surtout si une recherche est inédite et assez intéressante pour satisfaire les contraintes d'espace d'une publication imprimée et la mission du journal. Son rôle le plus important est de donner à l'éditeur du journal le signal de l'acceptation ou le rejet.

Le contrôle par les pairs scientifique est généralement un processus de " pré filtrage "décidant lesquelles des nombreuses recherches soumises devront être publiée. Par opposition, un site comme Digg par exemple est un " post filtre "décidant lesquelles des nombreuses recherches publiées sont les plus intéressantes pour un groupe de lecteurs. 
Les expériences de publication

Les journaux gratuits en ligne de The Public Library of Science (PLoS) ont l'intention d'étendre ce modèle par l'adoption de conventions à partir de la blogosphere : une zone de commentaires ouverts pour chaque recherche, des " suivis antérieurs " qui montrent quels sites s'y sont liés et peut être un schéma de notation des lecteurs.

Ce genre d'enregistrement aurait pour effet de non seulement de continuer le contrôle par les pairs après la publication, mais aussi de rendre plus facile de trouver un travail important dans une multitude de recherches - celles qui sont sujet à intérêt.

La montée du " pair " en ligne a donc montré qu'il y a une autre manière d'exploiter la sagesse collective. Ceci ne va pas, cependant, éliminer le contrôle par les pairs traditionnel. La raison peut être expliquée dans les termes économiques de la rareté et l'abondance. Le contrôle par les pairs fermé est meilleur dans des environnements rares où beaucoup de recherches se battent pour quelques créneaux convoités dans les journaux. Le contrôle par les pairs ouvert est meilleur dans des environnements des journaux en ligne avec un espace illimité ou un marché de la post publication de l'opinion de l'ensemble des travaux.

Dans le monde rare des pages limitées des plus importants journaux, le prestige est acquis à travers les hauts critères de ces journaux et leur exclusivité. Ceci découle, en partie, du processus, qui implique impressionner la combinaison très discriminante d'un éditeur et quelque chercheurs respectés. Ce n'est toujours pas juste ni efficace, mais dans un monde gouverné par la réputation relever avec succès le défi est une preuve d'au moins quelque genre d'aptitude.

Mais dans le marché de l'abondance des journaux en ligne ou celui du filtrage post publication, où chaque recherche concurrence d'autres recherches dans sa spécialité, le prestige est juste un facteur dans beaucoup de pertinences déterminantes pour un lecteur, et le plus d'aides au filtrage il y a, le mieux c'est.

A partir de cette perspective, nous vivons des moments très passionnants. Les expériences de Nature, les journaux $P L O S$ et autres révèlent où et comment ces techniques fonctionnent le mieux .Mais Wikipedia et Digg ont déjà démontré qu'ils fonctionnent aussi.

\subsection{3)-Certification dans un âge numérique}

The Digital Library Research and Prototyping Team à la bibliothèque de recherche du Los Alamos National Laboratory mène des recherches sur des aspects différents de la communication scientifique à l'ère numérique, y compris le contrôle par les pairs Notre recherche essaye d'analyser simultanément les propriétés du système existant et de formuler des alternatives faisables.

L'environnement numérique permet des changements systémiques dans les procédures de la communication scientifique. Ce potentiel pour un changement fondamental est lié à deux propriétés de l'environnement numérique qui n'étaient pas disponible dans le monde papier. Premièrement, les fonctions fondamentales de notre système de communication scientifique 
peuvent être séparées (du moins théoriquement) dans l'environnement digital. Deuxièmement, nous serons capable $d^{\prime}$ 'enregistrer dans une forme lisible par machine ensuite rassembler et plus tard extraire les données l'ensemble des événements de ce système.

Une des orientations de l'enquête nous a mené à définir un cadre dans lequel le contrôle par les pairs est un service autonome recouvrant les entrepôts scientifiques hébergeant des manuscrits non expertisés avec les entrepôts et les services d'expertises reliés pour une vue intégrée de l'information distribuée. Une autre orientation identifie automatiquement les experts potentiels basés sur une information extractible à partir de l'environnement numérique tels que le domaine de spécialisation du manuscrit et le modèle de citation et l'ensemble de la littérature du sujet en question.

Les limitations d'une communication scientifique basée sur le papier ont conduit à une intégration verticale de toutes les fonctions dans le système traditionnel du journal. Un éditeur enregistre la date d'enregistrement comme la date à laquelle le manuscrit est reçu. Le processus de contrôle par les pairs conduit sous les auspices de l'éditeur du journal, certifie les revendications faites dans le manuscrit. L'article publié remplit la fonction de sensibilisation. Récompense est basé sur le fait de publier dans un journal prestigieux et aussi être cité par d'autres savants .Enfin, dans l'ère papier, les bibliothèques archivent l'article publié, groupé dans un numéro de journal, en le mettant sur les rayons.

L'environnement numérique en réseau permet aux fonctions de la communication scientifique d'être individuellement mis en œuvre par de multiples parties de manières différentes et ensuite combiné comme services d'accompagnement ou alternatives en ce qui peut vraiment être considéré comme une chaîne de valeurs basée sur le réseau.

Ceci peut paraître encore lointain mais des exemples de telles chaînes de valeur déconstruites sont déjà entrain d'émerger. Par exemple, dans la communauté des sciences physiques, ArXiv remplit la fonction registration. Nombre de manuscrits soumis à ArXiv, finissent éventuellement dans des journaux bien établis remplissant la fonction de certification à travers le contrôle par les pairs traditionnel. Ou ils finissent dans ce qui est connu sous l'appellation des journaux de superposition tel qu'Advances in Theoretical and Mathematical Physics qui fournit une certaine forme de certification en listant des manuscrits dans un numéro.

Le contrôle par les pairs tel qu'on le connaît représente seulement une des facettes de la certification. La notre recherche sur la certification a déjà démontré que les différentes propriétés du mécanisme du contrôle par les pairs, telles qu'elles ont émergés dans un environnement de communication basé sur le papier ne devrait pas être nécessairement considérées comme acquise dans l'environnement numérique émergeant.

\subsection{2-PERSPECTIVE}

\subsection{1)-Le cas de l'expertise en groupe}

Le système actuel d'expertise individuel a montré ses limitations. La recherche moderne est en même temps multidisciplinaire et technique et il est souvent difficile de trouver des experts 
qui ont une expertise significative à travers des sous spécialités ensemble avec le savoir faire dans une discipline spécifique. De ce fait, les experts, même ceux dans le même domaine de recherche diffèrent souvent dans leur évaluation d'une recherche. Comme alternative, on peut utiliser un système appelé expertise des pairs en groupe.

Dans l'expertise des pairs en groupe, un éditeur aurait deux options quand il envoie un manuscrit à l'expertise. Un seul expert pourrait évaluer le travail et soumettre une recommandation à l'éditeur sur la base de ces mérites. Ou l'expert pourrait formellement discuter la recherche avec son groupe de recherche, rassemblant des entrants de la part d'étudiants en graduation, les post docs, techniciens et autres chercheurs et alors utiliser cette discussion pour faire une recommandation à l'éditeur. La seconde option est analogue aux clubs de journaux déjà en place dans nombre de laboratoires, quoique d'une manière plus formelle, et est l'idée principale du système d'expertise des pairs en groupe.

Une différence importante entre les clubs de journaux informels et l'expertise des pairs en groupe serait la suppression de l'information non scientifique et non technique du manuscrit pour préserver la confidentialité de la source. Dissimuler le nom des auteurs éviterait le préjugé de publication en faveur des auteurs " stars ".L'expert principal serait responsable d'assurer la confidentialité.

La courte histoire

Il est proposé que les auteurs fournissent deux versions de leur manuscrit à la soumission : une recherche complète et une version plus courte contenant le texte principal et les chiffres importants seulement. La version courte proposée n'aurait pas de références, de financement ou d'autres informations spécifiques à l'enquêteur.

Aux experts seront données les deux versions d'un manuscrit à utiliser soit dans le processus actuel du contrôle par les pairs comme d'habitude ou dans l'expertise des pairs en groupe.

Les journaux pourraient identifier plusieurs centres de contrôle par les pairs en groupe sur la base de leur expertise. De plus, étant donné que les experts ne sont pas actuellement indemnisés et pas toujours appréciés, que ces centres soient annuellement reconnus.

En maintenant l'option d'expertiser la recherche de la manière habituelle, ces experts seraient encore capables de participer au processus du contrôle par les pairs.

Ce système proposé devrait améliorer la consistance et la qualité du processus d'expertise luimême et faire plus pour assurer la publication de recherches de haute qualité .La discussion critique et l'expertise d'un manuscrit avant sa publication plutôt qu'après, aurait évité certains cas de scandale de mauvaise conduite des derniers mois.

\subsection{2)-Le contrôle par les pairs des recherches scientifiques interdisciplinaires}

Aussi bien les universités que les agences de financement ont proclamées le besoin pour la recherche " interdisciplinaire " et plus d'équipes " interdisciplinaires ". Cependant la publication de ce genre de travail soulève un ensemble unique de défis pour le contrôle par les 
pairs auxquels les journaux traditionnels unidisciplinaires ne sont pas complètement préparés. Nombre de domaines de recherches les plus intéressantes sont eux mêmes le résultat hybride de multiples disciplines, alors que le contrôle par les pairs soulève des problèmes dans l'évaluation de la recherche interdisciplinaire.

Dans cet article, " contrôle par les pairs interdisciplinaire «est utilisé pour dénoter le cas spécifique où un expert n'est pas expert dans toutes les domaines des questions de recherche, données et méthodes de la recherche soumise et cela arrive de plus en plus souvent. Ceci peut arriver aussi bien pour une étude hybride qui mélange questions et méthodes de deux domaines auparavant séparés que pour une recherche qui découvre de nouveaux résultats dans un domaine utilisant de nouvelles méthodes développées dans un autre (même si certains chercheurs connaissent des aspects de deux domaines, cette combinaison de découvertes inédites dans un domaine et des méthodes originales dans un autre peut le faire rentrer dans les problèmes déjà définis ).Malheureusement, nombre d'hypothèses du contrôle par les pairs standard ne s'appliquent dans ces cas.

Quelles sont ces hypothèses ? Premièrement, les experts ont besoin d'avoir une autorité globale pour évaluer et l'impact et la validité pour rendre une décision. Deuxièmement, les experts ont besoin d'être choisis à partir du domaine approprié de la recherche, de telle sorte que leur rôle de procureur est naturellement équilibré par un intérêt véritable à accepter les innovations à l'intérieur des domaines de spécialisation.

Dans le contrôle par les pairs interdisciplinaire, l'expert n'est pas au courant de certains domaines pertinents aux questions de la recherche, données ou méthodologie. Ceci peut avoir de nombreux effets. Premièrement, la considération positive de l'impact diminue, tandis que la considération négative de la validité peut augmenter pour devenir dominante. Les experts potentiels qui comprennent les données de la recherche mais pas les détails de sa méthodologie déclineront souvent de l'expertiser. Même s'ils le font, à leur expertise sera donné, souvent, moins de poids par l'éditeur que les évaluations par les experts en méthodologie. Les experts qui travaillent essentiellement sur la méthodologie mais ne sont ne sont pas au courant des données et résultats des domaines de la recherche la critiqueront souvent comme manquant de signification (parce que ces données et résultats ne sont d'aucun intérêt pour eux) ou nouveauté ( si la méthodologie en elle même n'est pas très originale ) ou comme invalide ( si les données actuelles dans ce domaine nécessitent des hypothèses différentes que celles auxquelles ils sont habitués ).Il est à noter que la catégorie la plus importante de recherche interdisciplinaire pour les journaux et les lecteurs (intéressante, résultats originaux rendus possible par une méthodologie nouvelle) serait probablement bloquée par cette situation de perdant-perdant.

Deuxièmement, et plus important, quand les experts n'ont pas une connaissance approfondie de la littérature en détail pertinentes à ces données spécifiques, il ne peuvent plus pratiquer la règle d'or du contrôle par les pairs :" Ce travail représente $t$ il une avancée significative par rapport à la littérature précédente ? " et généralement reviennent à une question plus conservative : " Existe-t-il quelque chose dans la recherche qui me parait incertain ? ".Alors que cette question pourrait être raisonnable si l'arbitre est expert dans tous les aspects de ce travail, elle est souvent fatale pour le contrôle par les pairs interdisciplinaire. Etant donné que les experts sont peu familiers avec certains domaines de la recherche, il est probable qu'ils sentiront de l'incertitude et de la gêne au sujet de certains aspects. Si les experts dans un domaine donné sont plus susceptibles de faire des revendications erronées (déclaration 
contredite par la littérature existante) au sujet de données en dehors de leur domaine d'expertise, qu'au sujet de données à l'intérieur de leur domaine, ceci constitue un préjugé vis-à-vis du domaine. Dans le contrôle par les pairs interdisciplinaire, la bonne politique est de tout simplement dire :" Je ne sais pas dans ce genre de cas " mais sous les hypothèses du contrôle par les pairs traditionnel, ce genre de déclaration pourrait être perçu comme une confession d'incompétence en tant qu' " autorité ".

Quand les spécialistes sont demandés d'expertiser un matériel qui est en dehors de leur domaine d'expertise, le contrôle par les pairs traditionnel change d'une mesure de qualité scientifique à une mesure du confort de l'expert.

Les journaux devraient considérer un nombre de corrections évidentes :

-Premièrement évaluer l'impact et ensuite validité. Ces deux phases ont besoin d'experts différents, de deux domaines différents. La phase d'impact doit être rapide S'il n'y a pas d'audience, il n'y a pas d'impact. Seules les recherches à l'impact élevé continuent vers la validation.

Le contrôle par les pairs interdisciplinaire nécessite discussion avant décision. Le contrôle par les pairs traditionnel nécessite que les experts expriment un jugement comme premier pas. Mais pour le contrôle par les pairs interdisciplinaire, beaucoup de questions ont besoin d'être posées et résolues, intégrant les expertises de tous les experts et les auteurs avant que l'expert puisse juger le manuscrit.

Evaluation soutenue. A cause de la possibilité du préjugé de domaine, le contrôle par les pairs interdisciplinaire ne peut être adjugé par les expressions d'opinions, mais plutôt par des faits démontrés à partir des données ou à partir de la littérature publiée directement pertinentes aux données de la recherche. Parce que la synthèse est requise, les autres experts et auteurs ont besoin de voir des citations spécifiques d'évidence pour une revendication d'expert et répondre de la même manière. Les experts doivent distinguer les critiques mineures des critiques majeures. Les opinions des auteurs et des experts peuvent être changées par cette information. C'est seulement sur cette base que les experts prennent une décision sur la validité finale. Ces critères sont beaucoup plus importants pour le contrôle par les pairs interdisciplinaire que traditionnel

Les journaux devraient déclarer leur politique au sujet du contrôle par les pairs interdisciplinaire. Les journaux qui publient des recherches à l'intérieur de domaines spécifiques doivent clairement déclarer qu'ils n'offrent pas de soutien pour la recherche interdisciplinaire. Les journaux qui publient des recherches qui englobent différentes spécialités devraient publier une politique détaillée décrivant le processus, y compris comment ils gèrent chacune des questions ci-dessus.

Editeurs et auteurs devraient prendre en ligne le préjugé de domaine. "Je ne sais pas " devrait être restauré pleinement à l'honneur comme signe d'ouverture d'esprit et d'objectivité. Les experts devraient être instruits de confiner leurs commentaires à leur domaine d'expertise, de soulever des questions avant de porter un jugement, et devraient être clairement demandés s'ils ont effectués le genre spécifique d'analyse utilisée dans le manuscrit sur le type spécifique de données qu'il présente. Les éditeurs devraient être attentif aux signes classiques de préjugé de domaine, telles que les expertises qui évitent de discuter les données actuelles de la recherche, les critiques en grande partie spéculatives qui ne sont pas étayées par des 
données spécifiques ou la littérature ou le manque d'évidence que les critiques sont actuellement pertinentes aux données spécifiques dans la recherche.

\subsection{3)-"Je ne sais pas quoi croire "}

Comprendre le contrôle par les pairs est primordial pour développer des opinions éclairées au sujet de la recherche scientifique.

Le grand public est présenté avec " des découvertes scientifiques " à partir d'un large éventail de sources, certains plus crédibles que d'autres. Le public a besoin de savoir au sujet du contrôle par les pairs en tant que mécanisme de qualité ce qui veut dire que les scientifiques devraient en parler. Mais quand on leur parle du contrôle par les pairs, la plupart des scientifiques pensent à la recherche qu'ils ont dans le système ou aux recherches entassées dans la boite attendant leur attention ou les deux la fois. Il est rare que les scientifiques prennent du recul par rapport au contrôle par les pairs et y pensent comme un ensemble de principes et d'attentes qui nécessitent une soumission volontaire à l'examen critiques des pairs et aspirent à définir qu'est ce qui est dans l'intérêt de la science comme un tout.

Il y a quelques années, Sense About Science, association caritative d'éducation commença à réfléchir à une manière d'équiper le public avec une compréhension du contrôle par les pairs. Les pratiques de publication scientifique paraissaient étrangement secrètes en comparaison avec ce qui passe à l'examen et la qualité de données dans d'autres parties de la société.

Sense About Science a commencé, à travers des ateliers et des discussions, à réfléchir aux véritables questions que les gens posent au sujet de la science qu'ils rencontrent (" Est-ce que c'est vrai ?" et " Est-ce que cette personne sait de quoi elle parle ? ") .Ceci a conduit à la publication en 2005 d'un guide appelé " Je ne sais pas quoi croire " qui n'émit pas d'hypothèses préalables au sujet du savoir des gens concernant la publication scientifique.

Les journalistes scientifiques dans les media nationaux sont sans doute familiers avec la publication scientifique. Plus généralement, il semble qu'il y ait une reconnaissance croissante du besoin pour plus de commentaires et clarté sur le statut (publié /non publié /répété et ainsi de suite) des découvertes scientifiques. En Grande Bretagne, par exemple, les lignes directrices produites par le conseiller scientifique en chef du gouvernement sont entrain d'être réécrites et l'utilisation par le gouvernement de l'évidence est entrain d'être investiguée.

Parler au public au sujet du contrôle par les pairs représente plus que fournir de l'information au sujet de la manière dont la science fonctionne. Il permet au public de développer une approche plus sceptique et de questionner les histoires par eux même.

La confusion qui souvent entoure les découvertes scientifiques, les risques et les certitudes ne pourraient être une meilleure occasion pour embarquer dans une discussion au sujet de la manière dont la science avance et donner au gens quelque indépendance du flux quotidien de pseudoscience. 


\subsection{4)-Les avantages et les inconvénients du contrôle par les pairs}

Le but de n'importe quel changement dans le système du contrôle par les pairs doit être d'améliorer la qualité de l'expertise et dont la qualité est déterminée par deux fonctions distinctes: filtrer les manuscrits pour publication dans un journal donné, et faire des suggestions constructives sur la manière dont le manuscrit ou étude peut être amélioré. Le contrôle par les pairs ouvert (où les experts signent leurs expertises) accomplirait il ce but ?

L'inconvénient principal du contrôle par les pairs ouvert est l'accumulation probable d'ennemies qui pourraient essayer de se venger plus tard. Que les auteurs soient très souvent mieux informés que les experts sur le sujet qu'ils ont récemment étudié en profondeur peut mener à des situations de conflit.

D'un autre coté, l'expertise anonyme ne stoppe toujours pas les auteurs de garder du ressentiment si leur recherche est rejetée. Dans un domaine restreint, les auteurs sauront probablement qui est l'expert. Même si ce n'est pas évident, la plupart des auteurs essayent de deviner l'identité de leurs experts à partir des commentaires ou des recommandations. Par exemple, un auteur peut accuser un expert d'avoir rejeté son travail parce que les recommandations avaient cité les références de l'auteur injustement accusé.

Mais il y a plusieurs avantages à un système de contrôle par les pairs ouvert. Premièrement, les experts seraient plus subtils et constructifs .L'utilisation du sarcasme dans l'expertise traditionnelle peut indiquer une envie sérieuse d'améliorer le manuscrit

Deuxièmement, les experts avec un intérêt acquis dans la suppression de la publication d'un manuscrit seraient plus facilement démasqués par les auteurs. L'expertise anonyme offre aux experts sans scrupules plus d'occasions de bloquer une publication sans répercussion.

Troisièmement, un système d'expertise complètement ouvert aurait les noms des experts publiés dans une note de bas de page à chaque recherche pour encourager les experts de faire un travail minutieux.

Nonobstant le genre de contrôle par les pairs utilisé, il y a un besoin évident pour un éditeur de servir de médiateur dans l'échange et assumer la responsabilité de la décision finale. Les experts peuvent donner leur opinion experte (honnête, entachée d'émotion, ou représenter une tentative manifeste de supprimer le manuscrit) mais c'est aux éditeurs de déterminer les motifs de l'expert.

Bien que l'idée du contrôle par les pairs ouvert ajoutant de la valeur au processus, il est difficile de l'appliquer dans notre monde compétitif moderne. Une solution de transition, serait que les experts font des suggestions ouvertes constructives pour révision ou travail additionnel sur la recherche et ce seulement après que le manuscrit eut été accepté provisoirement pour publication. Ceci est similaire au système du membre/éditeur utilisé par The Proceedings of the National Science Academy. Le membre expertisant un manuscrit est identifié aux auteurs seulement quand la recherche est acceptée pour publication. Pour les manuscrite rejetés, l'expertise aveugle permet aux experts de rester cachés dans l'anonymat et 
les éditeurs de se cacher derrière le jugement des experts dans leur décision de rejet. Publier les noms des experts à coté des recherches acceptées semble encore valable. La responsabilité de la publication est alors partagée entre les auteurs, le journal et les experts.

\subsection{5)-Est-ce que le contrôle par les pairs a le même sens pour le public que les scientifiques?}

La communauté des chercheurs sait que l'information scientifique qui n'a pas été contrôlée par les pairs ne devrait pas être prise au sérieux. En tant que scientifiques, nous faisons une distinction entre ce qui est dans les communiqués de presse et ce qui est publié dans la littérature scientifique formelle. On connaît aussi la différence entre une recherche primaire contrôle par les pairs et une lettre à l'éditeur non expertisée ou texte d'opinion .En d'autres termes, nous comprenons le système du contrôle par les pairs et nous l'utilisons comme filtre pour séparer la graine de l'ivraie.

Le système du contrôle par les pairs a ses défauts,il est le moins mauvais système qui puisse être imaginé, et malgré qu'il ait besoin d'être arrangé, les notions de base devraient rester intactes. Une manière de considérer son principe de fonctionnement est: " Jugement des scientifiques, pour les scientifiques, pour le peuple ".Mais est ce que le peuple comprend les limitations du processus. Même les journalistes qui devraient agir en tant que liens importants entre les scientifiques et le public semblent des fois ne pas valoriser le rôle du contrôle par les pairs.

Tout ce qui est contrôlé par les pairs n'est pas juste.Les scientifiques professionnel savent généralement comment classer les recherches à l'intérieur de leur propres domaines d'expertise (trop souvent très étroites de nos jours).Nous réalisons que certains journaux sont plus stricts que d'autres et que les normes du contrôle peuvent malheureusement être trop flexible. Une course pour le profit a sans doute conduit à l'apparition de trop de journaux, et il est devenu très facile de trouver où publier un travail de mauvaise qualité.

Mais le terme "contrôle par les pairs " est souvent assimilé à " l'étalon or ".De là, les personnes politiquement motivées, paresseuses et sans scrupules peuvent utiliser la littérature contrôlée par les pairs d'une manière sélective pour présenter des arguments qui sont gravement viciés ou même dommageables pour les politiques publiques. Les scientifiques professionnels peuvent voir à travers cette tactique. Nous savons que la vérité scientifique évolue sur la base d'un consensus incrémental, pas à travers une recherche isolée qui adopte une position non-conformiste même si elle a été " contrôlée par les pairs ".

Dans la recherche sur le SIDA, une petite clique de scientifiques et de profanes scientifiquement ignorants promeuvent la bizarre idée que le HIV ne cause pas le SIDA ou dans une variante particulièrement douteuse du genre, que le HIV n'existe pas actuellement. Ces négationnistes du SIDA sont experts à utiliser d'une manière sélective la littérature contrôlée par les pairs pour renforcer leurs positions. Je pense qu'ils manquent la formation - 
ou l'intégrité - d'apprécier deux choses qui sont compris par des scientifiques professionnels. Premièrement, que la littérature contrôlée par les pairs se développe au fil du temps, de telle sorte que ce qui était légitimement incertain il y a vingt ans est pleinement compris aujourd'hui. Cela signifie que citer des recherches vieilles d'une dizaine d'années et en ignorer de plus récentes est malhonnête. Deuxièmement, qu'ignorer chaque recherche à l'exception de celle qui le plus commodément convient à une position préconçue peut être considéré comme de la malhonnêteté scientifique.

Un problème auquel le public fait face quand il essaye de comprendre la science est que la littérature contrôlée par les pairs n'est toujours pas généralement accessible, malgré les efforts du mouvement de l'accès libre. Les activistes sur le SIDA qui ne peuvent facilement avoir accès aux bases de données de publication ont besoin d'information. Les éditeurs ont besoin de d'être à la page, le public a le droit de voir les recherches que ses impôts payent.

Et ainsi, malgré le fait que les scientifiques professionnels rient à l'idée que le HIV n'est pas la cause du SIDA, certaines personnes vulnérables, récemment infectées qui voudraient croire qu'elles n'ont pas contracté une maladie mortelle, se retrouvent à surfer sur la Toile. Malheureusement, c'est ce que font aussi certains journalistes scientifiques et leurs éditeurs. Ceci pourrait être évité si le public avait un meilleur accès à la littérature contrôlé par les pairs et si les scientifiques de bonne foi étaient prêts à donner au public plus d'assistance à l'interpréter correctement.

\subsection{3-Commentaires :}

Une analyse ne serait ce que superficielle des différentes contributions sur le sujet du contrôle par les pairs ouvert nous montre l'étendue des points de vue, des orientations et des expériences entreprises. Ces expériences sont venues en réponse à un malaise latent concernant le contrôle par les pairs, ce qui a motivé cette expérience unique de par sa couverture et son étendue. Bien qu'elle soit unique et compréhensive, le lecteur a un goût d'inachevé et de déception. Il aurait été attendu que les différents contributeurs proposent des solutions et des remèdes mais, force est de reconnaître, que ce n'est nullement le cas. Ceci est, à notre avis, largement explicable et compréhensible .Le contrôle par les pairs suscite tellement de débats, de controverses et d'affrontements qu'il est difficile de prendre position. Sachant surtout que de ses résultats dépendent des avantages, des promotions et des postes de travail très recherchés. A cela s'ajoute la nouveauté du medium qui en fait une arme à double tranchant: si les fonctionnalités d'Internet permettent une rapidité, une interactivité et une facilité dans la communication, la transmission de l'information qu'il aurait été impossible d'imaginer 10 à 20 ans avant, cette extrême rapidité dans le changement a induit une sorte de peur (peut être assortie de rejet) de cette nouvelle situation. On se retrouve dans la position où ceux qui réclamaient à cors et à cri le changement le refusent car il est trop rapide. D'ailleurs les instigateurs de cette expérience et les contributeurs sont des sommités dans leurs spécialités, ce sont des chercheurs dont le savoir et l'érudition ne peuvent être mis en 
cause. Malgré cela, ils ont déclaré qu'elle ne sera pas reconduite et que Nature se contentera d'appliquer le contrôle par les pairs tel qu'il a été appliqué avant. Loin d'être une reculade, cette décision traduit plutôt une sorte de pause afin de voir quels sont les retours d'écoute (feedback) à cette véritable révolution (et évolution) dans la science publiée.

\section{CHAPITRE III}

\section{LE CONTROLE PAR LES PAIRS OUVERT}

\section{Introduction}

Comme on a pu le voir, ces différentes recherches, études et autres expérimentations n'ont pas pu (et de loin et même dans certains cas elles rendues les choses plus ardues à l'image de l'argumentation de Fish) départager ni même donner une idée claire des remèdes dont le contrôle par les pairs a besoin. Au contraire, alors qu'une analyse découlant de fait (et en même temps superficielle) aurait donné l'équation : ouverture $=$ plus de justice et moins d'injustice, ceci ne s'est pas matérialisé.

Les chercheurs se sont alors orientés vers une forme plus ouverte et en même temps beaucoup plus révolutionnaire de la certification et contrôle de l'information publiée dans des périodiques scientifiques. Il s'agit du contrôle électronique sur Internet où l'information, au lieu d'être confinée entre les canaux créés par le contrôle par les pairs traditionnel et que l'on peut schématiser par le triptyque auteur(s)-éditeur-experts, est ouverte et permet à toute la communauté de participer et de " certifier " l'information. De prime abord, cette manière de procéder (avec des nuances, des approches et des procédures très différentes que nous allons voir en détails dans ce qui suit) devrait gagner la confiance de la communauté des chercheurs qui verraient s'éloigner et s'estomper les accusations de pré jugement, abus et dépassements de toute sortes. Il est sans doute important de dire que ces expériences sont toutes très récentes et ne datent en fait que de quelques deux décennies à peu prés parce qu'elles épousent le medium qui les sous tend : Internet. Bien que de création et de mise en œuvre récentes, cette manière de procéder a donné lieu à une abondante bibliographie et une production des plus soutenues. Le premier qui a abordé le sujet (du moins à notre connaissance) est un des plus grands protagonistes du libre accès : Stevan Harnad qui en $1996^{(65)}$ parlait les premières formes de certification de l'information sur Internet. Cet article peut être considéré comme la première tentative d'étudier le contrôle par les pairs sur Internet étant donné sa date (1996) qui se situe aux premières années du medium concerné. Dans cette contribution, Harnad commence par présenter la manière traditionnelle de publier sur papier et qui, d'après lui, est appelée à disparaître à cause d'un nombre de raisons dont les plus importantes (et évidentes)

\footnotetext{
${ }^{(65)}$ Harnad, Stevan, Implementing Peer Review on the Net: scientific Quality Control in scholarly electronic journals, In: Peek, R., Newby, G, Ed, Scholarly publication: the electronic frontier, Cambridge [Ma, U.S.A.]: MIT Press. p. 103-108.
} 
sont la lenteur du support et le diktat des éditeurs qui, de par la maitrise qu'ils ont acquis dans la publication des recherches, conditionnent cette opération par la cession des droits de publication qu'a l'auteur. L'avènement d'Internet a complètement changé la donne étant donné que chaque auteur (pour schématiser à l'extrême) est un éditeur en puissance et n'a plus besoin que d'une connexion Internet et un savoir minimum en informatique pour " publier ".Ce que Harnad a appelé " les implications de la technologie post Gutenberg de la communication électronique en réseau....." ${ }^{(66)}$ permettent ce passage et cette révolution ${ }^{*}$.

\section{1-Deux expériences pionnières : BBS et Psycholoquy}

Il présente pour ce faire deux expériences de périodiques électroniques qu'il a fondé et dont les formes de certifications sont ouvertes .Ce sont :

3.1.1-Behavioral and Brain Sciences (BBS) ${ }^{(67)}$ et dont les éditeurs le présentent comme " un journal de renommée internationale utilisant le format de certification innovant connu sous l'appellation de " Commentaires ouverts des pairs " (Open Peer Commentary).Les travaux de recherche particulièrement controversés et significatifs dans les domaines de la psychologie, la biologie comportementale ou les sciences cognitives sont publiés avec 10 à 25 commentaires pour chaque article émanant de ces disciplines et des disciplines connexes

${ }^{(66)}$ Harnad, Stevan, op.cit .p.105

* Harnad avait publié auparavant en 1990 et 1991 deux textes qui avaient été considérés comme fondateurs des nouvelles fonctionnalités qu'Internet permet. Ce sont respectivement:

- Post-Gutenberg Galaxy: The Fourth Revolution in the Means of Production of Knowledge, Public-Access Computer Systems Review, Vol.2, n²1: p.39-53, 1991

- Scholarly Skywriting and the Prepublication Continuum of Scientific Inquiry, Psychological Science, Vol. 1, p.342 - 343, 1990

Dans ces deux contributions, Harnad avait fait en quintessence fait ressortir que le monde a connu trois révolutions et qui sont : le langage, l'écriture et enfin l'imprimerie (d'où le titre de " post Gutenberg galaxy ").La quatrième qui à cette date n'avait pas eu lieu (mais qui en rétrospective plus de deux décennies plus tard est une réalité dans le monde occidental du moins) et celle de la révolution de l'information appelé par Harnad " Scholarly Skywriting " (très maladroitement traduit par "écriture scientifique dans le ciel", " écrire dans le ciel " , écriture céleste " ou encore "scielographie". L'expression anglaise fait en réalité référence à l'écriture publicitaire tracée dans le ciel par les avions. L'auteur l'utilise pour indiquer que le Web permet d'écrire à la vue de tout le monde accessible à : http://www.archipel.uqam.ca/83/2/cartierneuf.rtf. ) Dans cette nouvelle forme de communication, débarrassé des contraintes du support papier, le chercheur se verrait " publier " sur la toile et sa publication serait visible dans le ciel et chaque chercheur pourrait y apporter sa contribution grâce à Internet. Harnad pose aussi le problème du contrôle par les pairs et dit que, contrairement au début d'Internet, où le niveau des publications laissait à désirer (en plus d'autres problèmes), la toile est maintenant plus organisé et que cette cacophonie n'est plus de mise .A cet effet il dit:"le potentiel communicatif des réseaux électroniques est révolutionnaire. Il existe seulement un secteur dans lequel la Toile devra être traditionnelle et ceci est dans la validation des idées scientifiques et les résultats par le contrôle par les pairs. Expertiser peut être mis en œuvre beaucoup plus rapidement, équitablement et avec efficacité sur la Toile mais on ne peut s'en passer tel que certains naïfs enthousiastes (qui le mettent sur le même pied d'égalité que la censure) semblent penser " (op.cit p.109).Il faut remarquer que si les exemples souvent présentés comme pionniers (tels que Behavioral and Brain Sciences ou Psycholoquy ) ont l'avantage d'être nés avec Internet, bien avant le lancement de la Toile telle que nous la connaissons de nos jours, existait Current Anthropology fondé en 1959 par Sol Tax . Les manuscrits des anthropologues passaient entre les mains de différents experts, qui ajoutaient leurs commentaires au fur et à mesure. Ces commentaires étaient ensuite communiqués à l'auteur, qui pouvait en tenir compte et ainsi améliorer son article. Le point important ici est que les commentaires opposés étaient publiés avec l'article. Cela permet d'obtenir une autre perspective sur l'article que celle de l'auteur. Cependant, cette procédure s'est ensuite construite en deux étapes. Tout d'abord, la traditionnelle revue par les pairs ; et ensuite seulement, une invitation à des commentaires (et enfin la publication).

http:/homepages.vub.ac.be/ clvidal/writings/Vidal 2005a Le commentaire ouvert.pdf) accédé le 07/05/2012

${ }^{(67)}$ http://journals.cambridge.org/action/displayJournal?jid=BBS 
en plus des réponses de l'auteur à ces commentaires. Le résultat est un forum unique et fascinant pour la communication, la critique et la stimulation et particulièrement l'unification de la recherche dans les sciences comportementales et des neurosciences qui vont de la neurobiologie moléculaire à l'intelligence artificielle et la philosophie de l'esprit ".D'un autre coté, Harnad dans une interview ${ }^{(68)}$ présente BBS de la manière suivante : " un journal traditionnel commence par conduire le processus du contrôle par les pairs et publie alors les articles ayant répondus à ses critères de certification .BBS...... fait circuler premièrement les articles ( acceptés et certifiés ) à environ 100 chercheurs dans toutes les spécialités et dans le monde entier les invitant à soumettre un commentaire de 1000 mots qui critique ou complète l'article cible. L'auteur répond alors aux commentaires (10-20 ou plus) et tout est coédité dans le journal ".Comme on peut le voir, BBS utilise un système de certification ouvert et qui fait participer, en plus des experts " classiques ", d'autres " experts " externes qui contribuent à la certification. De plus, l'aspect plus révolutionnaire (à notre avis) sont les réponses que l'auteur fait et qui sont publiées avec l'article certifié ce qui en fait un article " vivant " étant donné qu'on peut voir son évolution et sa maturation, chose qui n'était pas possible dans les schémas de certification traditionnels et qui étaient (les schémas) fermés et, comme documenté plus tôt, sujet à toutes sortes de manipulations et autres préjugés préjudiciables à la bonne marche de la Science.

3.1.2-Psycholoquy a été fondé en 1989 et a été appelé " l'équivalent en ligne " de BBS ${ }^{(69)}$.Le site du périodique ${ }^{(70)}$ (qui a été suspendu pour des raisons scientifiques et financières *) présente Psycholoquy comme " un journal électronique interdisciplinaire international contrôlé par les pairs parrainé par l'A.P.A. (American Psychological Association).Psycholoquy publie des articles cibles et des commentaires des pairs dans tous les domaines de la psychologie ainsi que les sciences cognitives, les neurosciences, la biologie comportementale, l'intelligence artificielle, robotique/vision, linguistique et philosophie ".

Ces deux périodiques lancés par un pionnier du libre accès et dont un seul (Behavioral and Brain Sciences) est toujours existant et fait figure de référence dans le domaine (à tel point que sur le site les éditeurs affichent - non sans fierté - " le facteur d'impact de BBS a d'avantage augmenté [pour atteindre] 21.952 - le plus haut de tous les journaux de Thomson Reuters JCR ${ }^{\circledR}$ Social Science Edition ${ }^{(71)}$ ).Il faut dire que la personnalité de Harnad y est

\footnotetext{
${ }^{(68)} \mathrm{http}: / /$ richardfmasters.wordpress.com/2011/06/02/the-humble-beginnings-of-peer-review-alternatives-stevanhanard-and-open-peer-commentary/

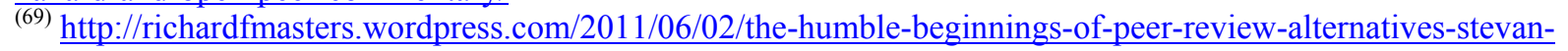
hanard-and-open-peer-commentary/

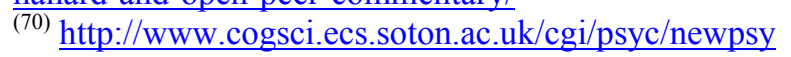

* Raisons qui nous ont été expliquées dans une correspondance personnelle avec Stevan Harnad qui a explicitement dit que " Psycholoquy bien que suspendu, était en voie d'être restauré et que le Docteur Leslie Carr (School of Electronics and Computer Science University of Southampton, chercheur connu dans le domaine de la recherche des problèmes relatifs du libre accès) faisait tout son possible pour qu'elle le soit le plus vite possible ". Dans une deuxième correspondance, Harnad présente Psycholoquy comme un pendant de BBS mais qu'après quelques années il était devenu évident qu'il (Psycholoquy) était prématuré et n'était pas viable financièrement (contrairement à B.B.S. financé par les abonnements). Nous avons voulu parler de cette information dans le cadre de notre travail mais surtout pour faire ressortir l'extrême rapidité par laquelle les réseaux permettent de nos jours de communiquer et de faire passer l'information.

${ }^{(71)}$ http://journals.cambridge.org/action/displayJournal?jid=BBS (accédé le 09/05/2012)
} 
pour beaucoup dans cette notoriété , étant l'un des pionniers et sûrement le plus prolifique des chercheurs du libre accès. Ces deux expériences pionnières du contrôle par les pairs ouvert (avec Current Anthropology en $1959^{*}$ mais qui lui se situait dans l'univers papier et qui de ce fait ne peut prétendre à être classé avec les nouvelles formes de certification qui elles sont basées sur l'utilisation d'Internet en tant vecteur principal) ont été suivies par d'autres expériences les unes plus novatrices (et des fois même farfelues comme nous le verrons pour Philica ) que les autres et dont nous verrons les plus exemples les plus connus et surtout les plus révolutionnaires.

3.2- Quelques exemples d'expériences de contrôle par les pairs ouvert

\subsection{1-ATMOSPHERIC CHEMISTRY AND PHYSICS (ACP) ${ }^{(72)}$}

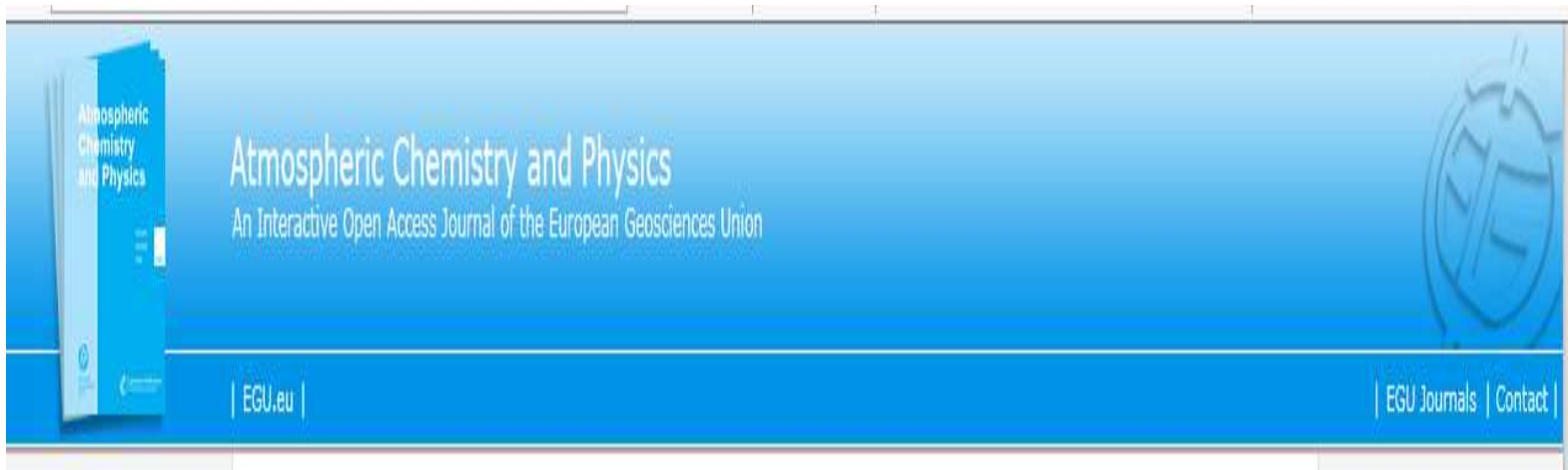

Atmospheric Chemistry and Physics (Journal en libre accès interactif de l'Union Européenne des Géosciences) se définit comme " un périodique scientifique international contrôlé par les pairs consacré à la publication et à la discussion publique de recherche de qualité supérieure concernant l'atmosphère terrestre et les processus chimique et physiques sous jacents ". Atmospheric Chemistry and Physics Discussions (ACPD) et Atmospheric Chemistry and Physics (ACP) sont publiés par The Copernicus GmbH (Copernicus Publications) pour le compte de The European Geosciences Union (EGU).

\subsubsection{1-Processus de certification}

ACP utilise un processus de contrôle assez original très différent du processus traditionnel .Il est subdivisé en deux étapes et qui sont :

\subsubsection{1-ACPD (Atmospheric Chemistry and Physics Discussions)}

Cette étape est subdivisée en :

\footnotetext{
${ }^{*}$ Saul Tax, le fondateur de Current Anthropology était un visionnaire qui avait imaginé un système (sur papier) très ressemblant à ce qui se fait actuellement sur Internet. Dans chaque numéro publié, les éditeurs choisissaient au moins un article et l'envoyait à une liste de lecteurs qui le commentaient, commentaires auxquels l'auteur répondait. Le tout (article, commentaires des lecteurs et réponses de l'auteur) était alors publié. Tax avait décrit cette manière d'agir comme "...une technique combinant les avantages des symposium ( sans avoir à voyager) et les avantages du genre de discussions qu'on trouve dans une lettre aux éditeurs ( sans avoir à attendre ) [ce qui permettait ] de réunir des spécialistes, mettre en commun des moyens dans des domaines de plus en plus difficiles à maitriser par une seule personne et attirer des personnes à la limite de notre spécialité". http://archaeology.about.com/b/2007/01/04/open-peer-review-current-anthropology.htm

${ }^{(72)}$ www.atmospheric-chemistry-and-physics.net/
} 


\subsection{1-Soumission du manuscrit original et désignation de l'éditeur}

La recherche est soumise électroniquement à un contrôle initial par un coéditeur afin de s'assurer de sa qualité scientifique et technique fondamentales et de sa concordance avec les sujets couverts par le journal.

\subsection{2-Expertise de l'acceptabilité}

Le coéditeur doit décider et évaluer est ce que le manuscrit soumis se situe dans les limites des sujets couverts part le journal et est ce qu'il répond à un minimum de qualité scientifique de base. Si nécessaire, il peut demander l'aide d'experts indépendants qu'il choisira. Il peut suggérer des corrections techniques (telles que des fautes typographiques, clarification de tableaux et figures, etc...) avant publication dans ACPD. A ce stade du processus de certification, toute autre demande de révision du contenu scientifique n'est pas permise mais sera permise une fois que la recherche est soumise à la discussion interactive dans ACPD.

\subsection{3-Corrections techniques}

Les auteurs ont l'occasion de faire des corrections techniques qui peuvent être revues par le coéditeur pour vérification et empêcher des corrections plus approfondies qui ne sont pas permises à ce stade.

\subsection{4-Publication et discussion de la recherche dans ACPD}

Après acceptation de la recherche, le manuscrit est typographié par The Copernicus Publications Production Office, corrigé et relu par les auteurs et publié comme recherche en discussion sur le site d'ACPD. L'accessibilité publique, l'archivage et la citabilité sont assurés à partir de ce moment (généralement pour une période de 2 à 8 semaines à partir de la soumission).

\subsection{5-Discussion ouverte (8 semaines)}

Une fois publiée sur Internet, la recherche est ouverte pour l'expertise publique et la discussion, durant lesquels des commentaires interactifs par des experts désignés peuvent être publiés (anonymement ou nommément) et tous les membres de la communauté scientifique intéressés (nommément).Normalement, chaque recherche en discussion devrait recevoir au moins deux commentaires de la part d'experts désignés *

\footnotetext{
* Dans la discussion publique interactive qui suit la publication d'une recherche dans ACPD, les commentaires interactifs suivants peuvent être soumis pour une publication immédiate non contrôlée à coté de la recherche en discussion :

-Courts commentaires : peuvent être postés par toute personne faisant partie de la communauté scientifique enregistré (enregistrement en ligne gratuit).Ce genre de commentaires sont attribués (publié avec le nom du de la personne ayant commenté)

-Commentaires des experts : ne peuvent que postés par les experts impliqués dans l'expertise de la recherche en discussion. Ils peuvent être anonymes ou nommément ( selon les desideratas des experts ).

-Commentaires de l'éditeur : ne peuvent être postés que par l'éditeur de la recherche en discussion.

Commentaires de l'auteur : ne peuvent qu'être postés par l'auteur contact de la recherche en discussion au nom des auteurs.

Tous les commentaires interactifs sont entièrement cités, paginés et archivés comme supplément à ACPD. http://www.atmospheric-chemistry-andphysics.net/review/review process_and interactive_public discussion.html\#interactivepublicdiscussion
} 


\subsection{6-Réponse finale}

Après la discussion ouverte, les auteurs devraient publier une réponse à tous les commentaires (dans un délai de 4 semaines, prolongeable sur demande).L'éditeur peut aussi publier des commentaires ou des recommandations supplémentaires. Normalement, cependant les recommandations formelles et décisions éditoriales ne sont émises qu'après que les auteurs aient eu l'occasion de répondre à tous les commentaires ou qu'ils aient demandés des conseils éditoriaux avant de répondre.

\subsubsection{2--ACP (Atmospheric Chemistry and Physics)}

\subsection{1- Soumission du manuscrit corrigé}

La soumission d'un manuscrit révisé n'est possible qu'après que les auteurs aient répondus d'une manière satisfaisante à tous les commentaires et si le manuscrit révisé répond à tous les critères de qualité élevés d'A.C.P. ( Voir les critères de l'évaluation du manuscrit à http://www.atmospheric-chemistry-and-physics.net/review/ms evaluation_criteria.html ).En cas de doute, les auteurs devraient s'adresser à l'éditeur dans le cas il recommanderait la préparation et la soumission d'un nouveau manuscrit révisé ou non. Normalement le manuscrit révisé devrait être soumis au plus tard 4 à 8 semaines après la fin de la discussion ouverte. Si plus de temps est requis pour la révision du manuscrit, les auteurs peuvent demander une prorogation.

\subsection{2.-Fin du processus de contrôle par les pairs}

Compte tenu de l'accès au contrôle par les pairs et de la discussion publique interactive, le coéditeur soit directement accepte / rejette pour publication dans ACP ou alors consulte des experts de manière traditionnelle. Si nécessaire des révisions additionnelles peuvent être demandées pendant le processus de contrôle par les pairs jusqu'à ce qu'une décision finale au sujet de rejet / acceptation pour ACP soit finalement atteinte.

\subsection{3-Publication de la recherché finale corrigée dans ACP}

Dans le cas où la recherche est acceptée, elle est typographié et corrigée. Elle est alors publiée sur le site web d'A.C.P. avec un lien direct à la recherche originale et à la discussion interactive dans A.C.P.D. Toutes les publications (la recherche originale, les commentaires interactifs et la recherche finale révisée) sont archivées d'une manière permanente et restent accessible au public en général par le biais d'Internet ainsi que la recherche révisée finale accessible en tant qu'exemplaire papier.

On remarquera que ces neuf étapes (subdivisées en deux sous étapes - A.C.P.D. et A.C.P.) comportent une dixième étape qui est en elle-même révolutionnaire par rapport à l'ensemble du processus. En effet, une fois la recherche publiée sur ACP et après avoir passé les différentes étapes, les commentaires post publication de la recherche expertisée sont acceptés et même encouragés. Ces commentaires contrôlés passent par le même processus de certification et publication décrit ci-dessus - discussion et publication dans ACPD -, ils peuvent aussi être publiés dans ACP s'ils sont suffisamment intéressants. 
Ce processus assez compliqué et surtout novateur est résumé par les deux figures suivantes :
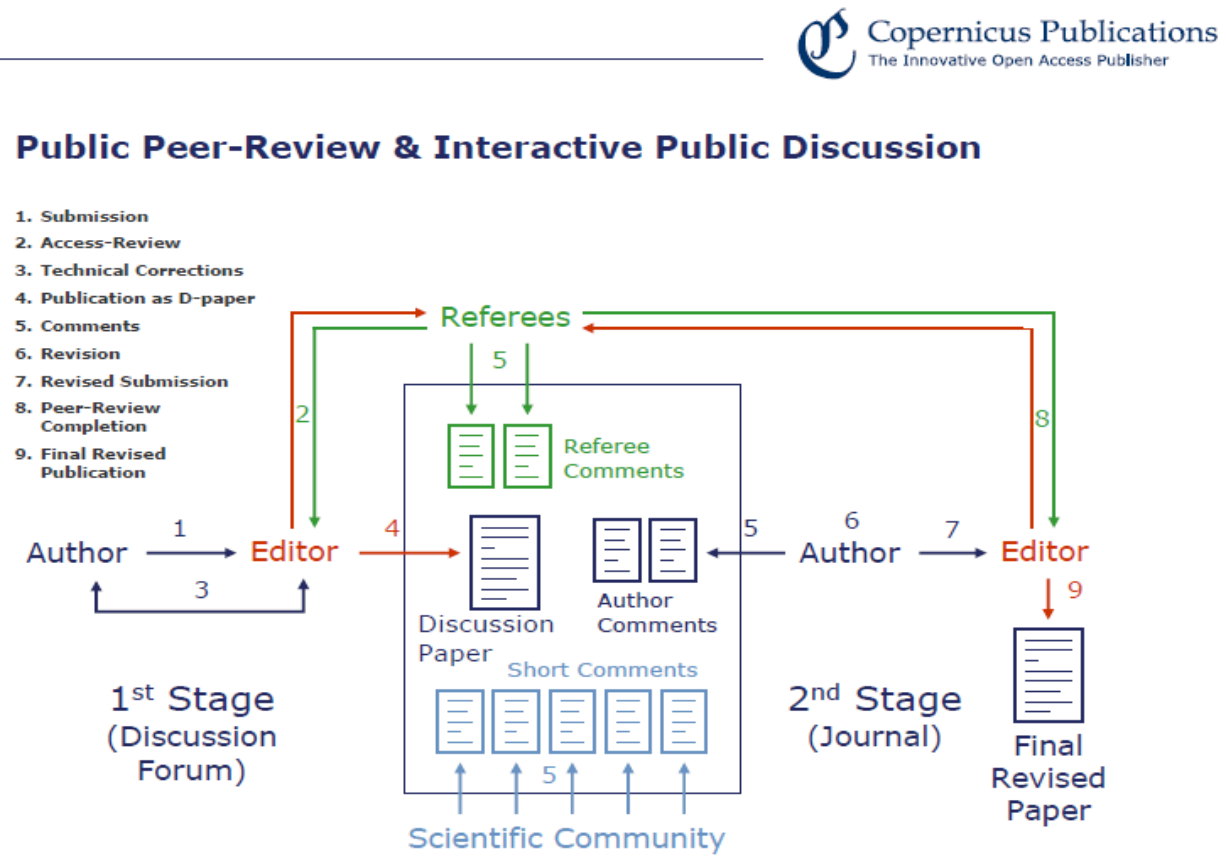

Fig. $n^{\circ}$ 37-Processus de contrôle par les pairs public et discussion publique interactive (ACPD)

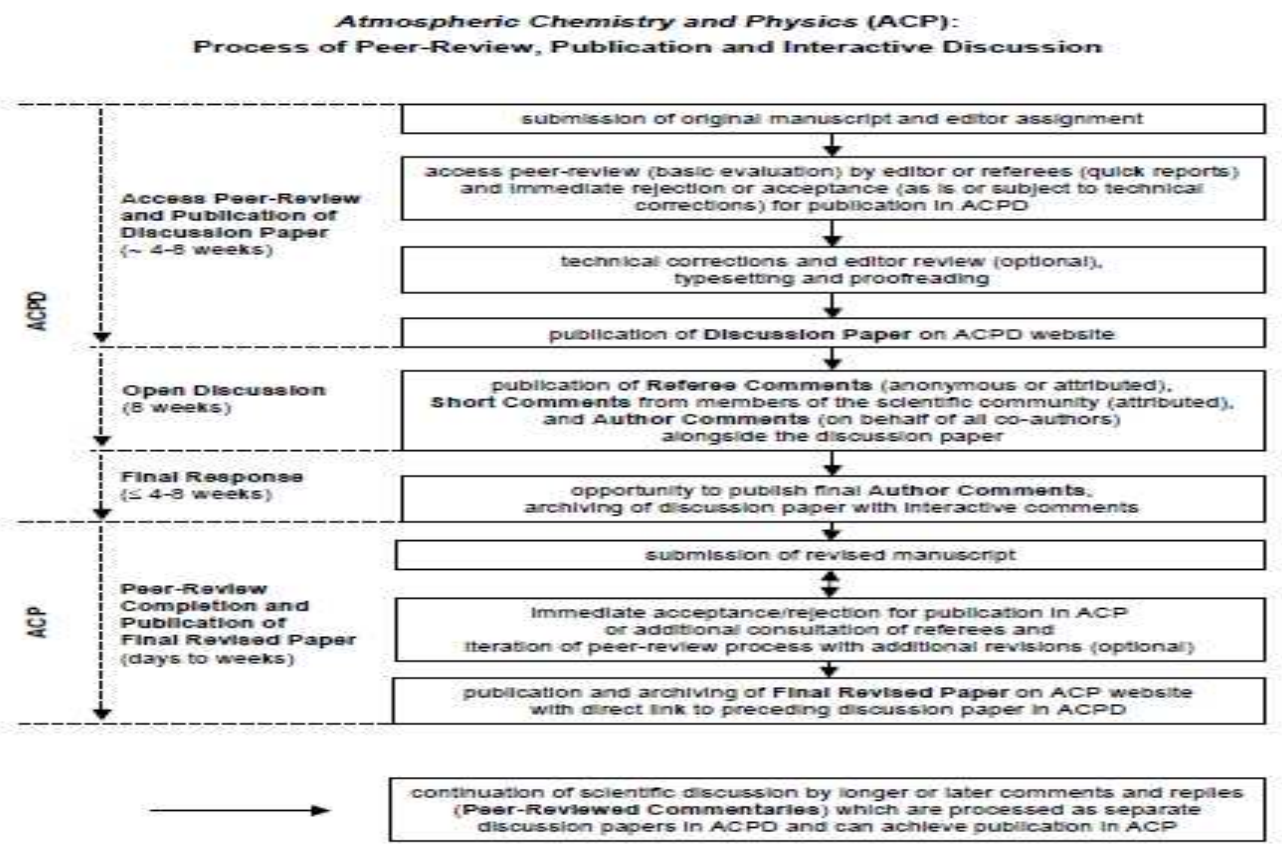

Fig.n³8-Processus de contrôle par les pairs, publication et discussion interactive (ACP)

D'un autre coté, le processus de certification peut être suivi pratiquement en temps réel de par l'interactivité du site.Ainsi, le site comporte une liste des recherches qui se subdivise en : 
3.2.2-Bibliotheque en ligne ACP (Online Library ACP) et qui comporte les recherches récemment expertisées. La recherche expertisée et prête à être publiée dans ACP est présentée de la manière suivante :

\section{ACP - Recent Final Revised Papers}

\section{Megacity ozone ail quality under four a alternative future scenarilos}

T. M, Butler, Z, S, Stock, M, R. Russo, H, A, C, Denier van der Gon, and M, G, Lawerence

Atmos, Chem, Phys, 12, 4413-4428, 2012

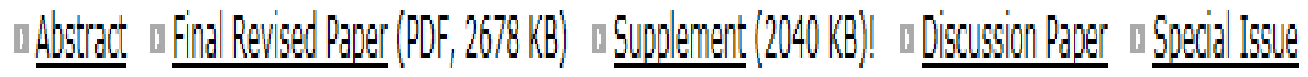

On remarquera que la recherche publiée prise en exemple comporte cinq rubriques :

- résumé (résumé présenté généralement par l' (es) auteur (s))

- recherche expertisée finale (la recherche telle qu'elle sera publiée après avoir passé les différentes étapes de la certification)

- suppléments (informations n'ayant pas été inclues dans la recherche telles que les graphes et autre illustrations- à titre d'exemple les suppléments pour cette recherche s'étalent sur 12 pages et représentent des tableaux, des graphes et des calculs .(Voir http://www.atmos-chemphys.net/12/4399/2012/acp-12-4399-2012-supplement.pdf)

-discussion de la recherche (commentaires que les experts désignés et les chercheurs de la communauté ont émis avec les réponses et qui sont librement accessible par toute personne intéressée)

-numéro spécial (n'apparaît que lorsque la recherche est incluse dans un numéro spécial concernant un sujet donné)

Et dont seules trois (le résumé, la recherche expertisée finale, la discussion de la recherche) sont permanentes, les autres (les suppléments, numéro spécial) n'apparaissant que lorsque le besoin se fait sentir de les inclure.

Le point le plus important dans ce processus a trait à discussion de la recherche et dans lequel l'expertise se fait non seulement par les experts désignés et les membres de la communauté mais surtout où les remarques et les réponses qu'elles ont produits sont publiées. Ainsi pour la recherche citée en exemple, en cliquant sur discussion de la recherche, on obtient la page suivante : 


\section{Megacity ozone air quality under four alternative future scenarios}

T. M. Butler ${ }^{1,2}$, Z. S. Stock ${ }^{3}$, M. R. Russo ${ }^{3,4}$, H. A. C. Denier van der Gon ${ }^{5}$, and

M. G. Lawrence ${ }^{1,2}$

${ }^{1}$ Max Planck Institute for Chemistry, Mainz, Germany

${ }^{2}$ Institute for Advanced Sustainability Studies, Potsdam, Germany

${ }^{3}$ Centre for Atmospheric Science, Department of Chemistry, Cambridge University, UK

${ }^{4}$ NCAS Climate, UK

${ }^{{ }^{T}}$ TNO, The Netherlands
Review Status

This discussion paper has been under review for the journal Atmospheric Chemistry and Physics (ACP). Please refer to the corresponding final paper in ACP.

Abstract. The impact of the megacities of the world on global tropospheric ozone, and conversely, the extent to which megacities are influenced by emissios of ozone precursors from outside of the megacities is examined under the four alternative RCP ("Representative Concentration Pathway") emissions scenarios. Despite accounting for about $6 \%$ of present-day anthropogenic emissions of ozone precursor species, the contribution of emissions from megacities to global tropospheric ozone is calculated to be $0.84 \%$. By 2100 this contribution falls to between 0.18 and $0.62 \%$ depending on the scenario, with the lower value being for the most-polluting of the four future emissions scenarios due to stringent controls on ozone precursor emissions from highly populated areas combined with a stronger tropospheric background ozone field. The higher end of this range is from the least-polluting of the four emissions scenarios, due lower background tropospheric ozone combined with the use of a different downscaling methodology in the construction of the scenario. Although the absolute impact of megacities on global ozone is small, an important result of this study is that under all future scenarios, future air quality in megacities is expected to be less influenced by local emissions within the cities, but instead more influenced by emission sources outside of the cities. Air quality trends in the megacities of the developing world are projected to be similar to observed trends in developed world megacities over the last few decades. Assumptions made when downscaling the emissions scenarios onto the grids used in such modelling studies can have a large influence on these results. Future work should concentrate on the creation of spatially explicit scenarios of urban development for use in global chemical transport models.

Citation: Butler, T. M., Stock, Z. S., Russo, M. R., Denier van der Gon, H. A. C., and Lawrence, M. G.: Megacity ozone air quality under four alternative future scenarios, Atmos. Chem. Phys. Discuss., 12, 129-163, doi:10.5194/acpd-12-129-2012, 2012.

On aura remarqué que cette page est pratiquement une copie de la page précédente avec une addition importante : discussion interactive. Cette étape de la publication et la manière dont elle est entreprise est au cœur du processus d'A.C.P. En effet, en cliquant sur discussion interactive on obtient la page suivante :

Atmos. Chem. Phys. Discuss., 12, 129-163, 2012

www.atmos-chem-phys-discuss,net/12/129/2012/

doi:10.5194/acpd-12-129-2012

(c) Author(s) 2012. This work is distributed

under the Creative Commons Attribution 3.0 License.

\section{Megacity ozone air quality under four alternative future scenarios}

T. M. Butler, Z. S. Stock, M. R. Russo, H. A. C. Denier van der Gon, and M. G. Lawrence

\section{Interactive Discussion}

AC: Author Comment | RC: Referee Comment | SC: Short Comment | EC: Editor Comment

E. Printer-friendly Version $\$$ - Supplement

RC C42: 'Referee Comment', Anonymous Referee \#2, 30 Jan 2012 it

RC C238: 'Referee Comment on "Megacity ozone air quality under four alternative future scenario"', Anonymous Referee \#1, 24 Feb 2012
Review Status

This discussion paper has been under review for the journal Atmospheric Chemistry and Physics (ACP). Please refer to the corresponding final paper in ACP. 
Cette recherche a produit 2 commentaires, tous accessibles en ligne en cliquant sur le lien ainsi que la réponse de l'auteur principal de la recherche. On remarque que les deux commentaires le sont par des experts anonymes (les experts issus de la communauté des chercheurs de la spécialité avaient cette option de faire des commentaires anonymement mais après s'être inscrit sur le site. De leur coté, les experts désignés par le coéditeur ont pour obligation de se faire connaitre par nom, ce qui est, dans le monde fermé du contrôle par les pairs, une avancée très significative ayant pour but de combattre les différents préjugés déjà évoqués). Ces commentaires traduisent l'extrême ouverture du processus et se présentent de la manière suivante :

Atmos. Chem. Phys. Discuss., 12, C42-C45, 2012 www.atmos-chem-phys-discuss.net/12/C42/2012/ (c) Author(s) 2012. This work is distributed under the Creative Commons Attribute 3.0 License.
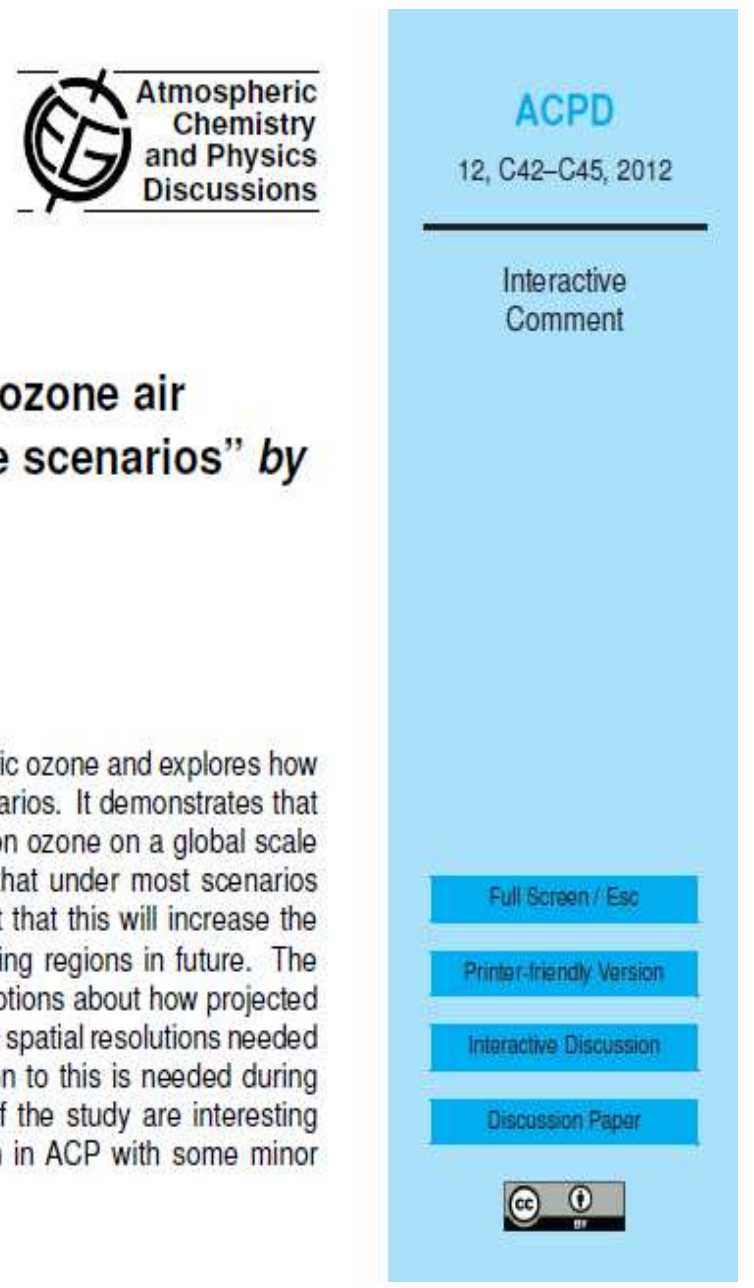

This paper describes the effect of megacities on tropospheric ozone and explores how this is likely to change under realistic future emission scenarios. It demonstrates that emissions in these regions have a relatively small impact on ozone on a global scale in proportion to their share of precursor emissions, and that under most scenarios this is likely to drop further in future. The authors suggest that this will increase the sensitivity of urban air quality to emissions from surrounding regions in future. The paper also examines the sensitivity of the results to assumptions about how projected national-level emission changes are] downscaled to the high spatial resolutions needed for air quality modeling, and highlights that further attention to this is needed during generation of emission scenarios in future. The results of the study are interesting and valuable, and the paper is appropriate for publication in ACP with some minor corrections, detailed below. 


\section{General Comments}

The model studies are performed at relatively coarse resolution that is 3-4 times larger than most megacities at the current time. While this may introduce only a small bias in assessing their global impacts, it is likely to introduce much larger errors in assessment of megacity air quality, a focus of the paper highlighted in the title. The authors need to be clearer in section 3 about the implications of their choice of resolution by noting the potential biases for assessment of air quality in particular.

Many megacities experience substantial titration effects from high NO removing ozone, and these effects are likely to vary by season and location. How much are these effects expected to influence the conclusions drawn about redistribution of emissions? Given that the countries involved vary in size greatly, the influence of redistribution will be dependent on the fractional importance of megacities in different countries as well as on latitude and emission magnitude. It is clear from the lower panel in Fig 5 that the redistribution has different effects for different cities within a single country (e.g., the US) and explanation of this finding is needed in section 4.

\section{Specific Comments}

p.130, I.13: "differing downscaling" - need to be more specific here

p.135, I.23: Were $\mathrm{CH}_{4}$ concentrations assumed fixed, or was some surface boundary condition used? A brief statement of this here would be useful.

p.135, 1.28: "made more objective" is unclear; please be explicit here.

p.136, I.5: Stock et al. and Collins World Atlas are both missing from the reference list.

p.140, 1.21: The section referred to here is unnumbered and appears at the end of the current section (p.141). The section should either be numbered or absorbed into the main body of section 3 here, and the forward reference "in Sect 3 " (p.140,I.21) replaced with "below".

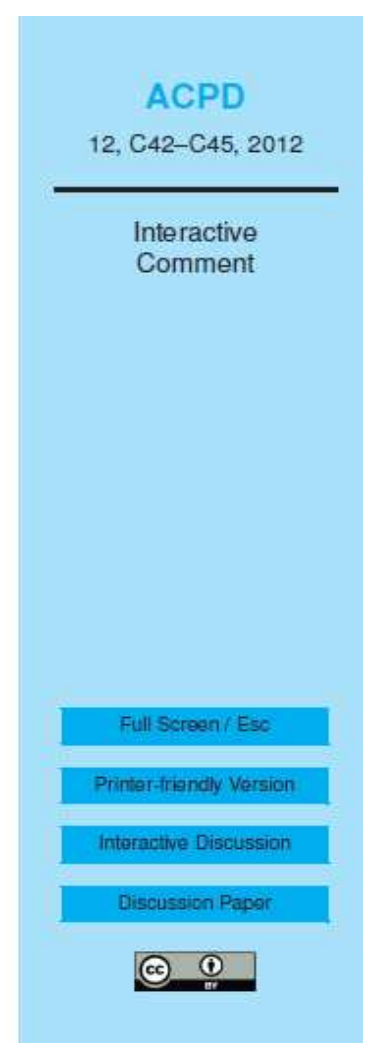

p.141, 1.27: What is the justification for choosing a $25 \%$ emission reduction here? Why not remove the megacity emissions by harmonizing them over the country as a whole? Some additional explanation for these choices is needed here.

p.144, 1.9-10: This finding that the redistribution of emissions has little effect on global ozone could be highlighted in a much more positive way, as it suggests that the exact distribution of emissions isn't critically important for chemistry-climate model studies. The value of this result could also be noted more strongly in the conclusions (p.148, I.16).

p.146, 1.19: The $0.84 \%$ result described here in the conclusions should appear earlier in the text. It would be useful to discuss how this compares with the effect of other surface sources, either from this study (if available) or from previous studies, e.g., under the QUANTIFY project. It would also be interesting to quantify how much smaller the megacity impact would have been if the same emissions were uniformly redistributed.

p.146, 1.21: "Ozone precursor" Should this read "NOx"?

p.146, 1.24: "relatively sophisticated" is unclear, please rephrase this and be more specific.

p.147, I.21: "Similar trends have already been observed..." please supply a reference here.

p.148, 1.11: Emissions are usually generated on a country and sector basis, and the geographical distribution is a separate procedure. The call for finer resolution here is understandable, but given that different users have different needs, approaches and model grids, it would perhaps be more valuable to call for scale-independent emissions (area or vector) which could then be applied or mapped to any geographical model grid.

p.155: Tropospheric ozone column densities are usually presented in Dobson Units, so it would be helpful to note in the caption what the base case $0.75 \times 10^{\prime} 18$ molecules $/ \mathrm{cm} 2$ is equivalent to about $28 \mathrm{DU}$.

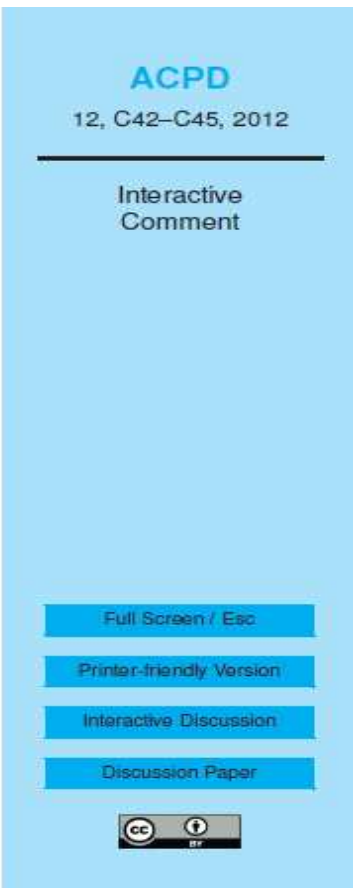


Given the discussion in section 4 it is unnecessary to show data from each city in figure $6-8$. These figures are too crowded. It would be a lot clearer to average the results over each latitude band and show the mean (as a line) and standard deviation (as an error bar), and the resulting five lines would make the seasonality (and spread) much more apparent to the reader.

Typos

p.130,l.12: due $\rightarrow$ due to

p. 131,l.16: increased $\rightarrow$ improved

p.144,l.22: remove "when"

Interactive comment on Atmos. Chem. Phys. Discuss, 12, 129, 2012

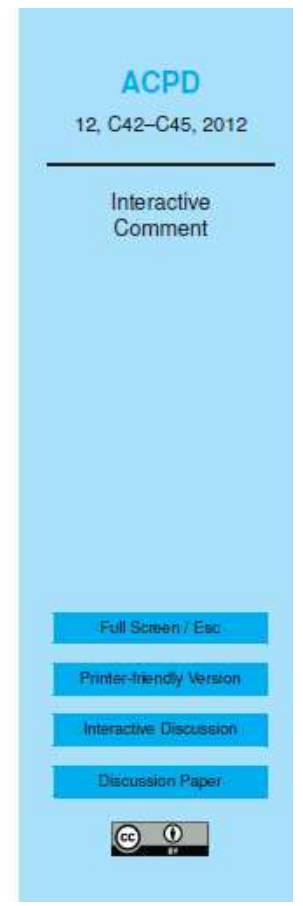

A la suite des commentaires des deux experts anonymes (dont nous avons reproduit une seule à titre d'exemple et dont on peut remarquer la minutie avec laquelle les différents points sont discutés), on peut voir la réponse détaillée et (des fois personnelle) sur 8 pages de l'auteur principal à ces mêmes commentaires de la manière suivante :

Atmos. Chem. Phys. Discuss., 12, C1834-C1841, 2012

www.atmos-chem-phys-discuss.net/12/C1834/2012

(c) Author(s) 2012. This work is distributed under

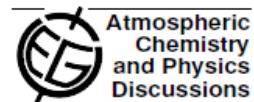

ACPD

the Creative Commons Attribute 3.0 License.

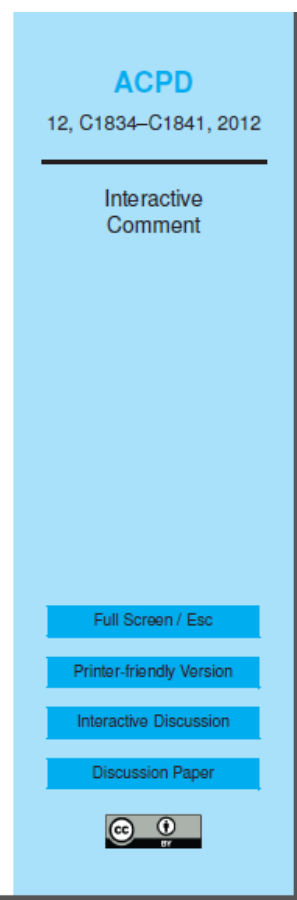

Interactive comment on "Megacity ozone air quality under four alternative future scenarios" by T. M. Butler et al.

\section{T. M. Butler et al.}

tim.butler@iass-potsdam.de

Received and published: 25 April 2012

We thank both anonymous referees for their overall positive assessment of ou manuscript and their helpful comments. Based on these comments we have made changes to our manuscript which we believe have enhanced its quality. Details of the changes and responses to points raised by the reviewers are listed below.

Anonymous referee \#1

General comments

P141L1-17

Referee \#2 has also highlighted similar issues in their general comments. The re- 
sponse to both reviewers is given here. In order to respond to these comments we have altered our discussion in Section 3 to more explicitly describe the bias towards NOx limited conditions expected from using a coarse model resolution, and we have mentioned that these biases are likely to be strongest in our megacity grid cells. We have also clarified the methods by which Wild and Prather (2006) quantified these biases. Referee \#1 also makes the interesting point that it would be good to know specifically how the sensitivity of ozone production regimes to megacity emissions perturbations varies with model resolution. We agree with this, but we would not like to speculate on this without actually having done any simulations investigating this; unfortunately it was not possible to repeat the suite of simulations at higher resolution given the resources available for this study.

P145

We agree that the figures summarising data from many different cities would be better presented as means and standard deviations. Reviewer \#2 also suggested this, and we prefer his suggestion of presenting the standard deviations as error bars on the mean seasonal cycles for each latitude band. The figures have been modified accordingly.

P148L20-25

We already have some discussion about the applicability of the RCP scenarios for air quality studies in Section 2.1, but the reviewer is correct that this should be mentioned again in the conclusions. We added this to the middle of the final paragraph, where we also reiterate other limitations of the emissions we have used.

P131L21-22

The reviewer is correct that none of the referenced papers include a detailed long term trend analysis of the effects of background ozone on urban air quality. They do however identify effects of background ozone, and it is also fair to say that urban emission controls have improved local urban ozone air quality. To respond to this reviewer com-

ACPD

12, C1834-C1841, 2012

Interactive

Comment

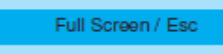

Printer-friendly Version

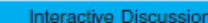

Interactive Discussion

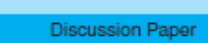

(c) (7)

ment we have toned down the definitiveness of our statement in the introduction, and we have expanded our discussion of this issue in the conclusions. We also reworded a similar sentence in the abstract.

Specific comments

P131L6

We agree that since previous work has minimised the global impact of megacities, that this statement about the potential impact of megacities seems out of place. To convey the point that this is still an area of scientific interest, we have instead mentioned that recently, two major EU research projects have been funded to investigate these issues.

\section{P131L17}

We have added text explaining that toxic pollutants are defined by the US Clean Air Act.

\section{P132}

We do not understand why paragraphs 2 and 3 here are interpreted to be contradictory. P133L15

Yes, the "coupling" in CMIP originally referred to coupled ocean- atmosphere models, and has now grown to include coupled chemistry- climate models. For the CMIP5 project, not all models necessarily include the chemistry component. We have reworded this sentence to better reflect this context.

\section{P133L20-23}

We have not claimed that climate co-benefits contribute to ozone precursor reductions in RCP-8.5. This is clearly attributed to RCP-2.6 in our manuscript. We have slightly reworded our explanation to make this clearer. Additionally the reviewer has requested more information on the technological measures used in the scenarios. We have added

ACPD

12, C1834-C1841, 2012

Interactive

Comment

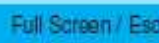

Printer-friandly Version

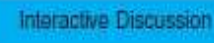

Discussion Paper

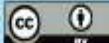


this to the second paragraph of the subsection, where we now mention that RCP- 8.5 includes a continuing reliance on fossil fuels, and the RCP-2.6 includes efficiency improvements, capture and sequestration of carbon dioxide, and increased use of nuclear and renewable energy.

P135L23

Yes, all primary NOx is emitted as NO. We added some text to Section 3 to make this clear.

P136

We already had a sentence early in this paragraph explaining that the megacity grid cells are based only on 2005 data. In order to emphasise this even more, we have added another sentence to the end of the paragraph repeating this point and making it clear that the same mask is used in all cases.

P138L5

We agree that it would be of some interest to plot the megacity proportion of total anthropogenic emissions from RCP-8.5-P in Figure 3, although none of the arguments we make in the paper depend specifically on this. We are reluctant to do this, however, because we do not introduce RCP-8.5-P until after our discussion of Figure 3. Adding this information to the figure would involve either a reorganisation of the sequence of material in the paper, or a forward reference, both of which would detract from the readability.

P144L2

We have added text generalising this point to all scenarios created using proportional downscaling, and we have also added text before this clarifying our additional explanation of why the local megacity impact is especially high in RCP-2.6.

Minor comments

C1837

ACPD

$12, \mathrm{C} 1834-\mathrm{C} 1841,2012$

Interactive

Comment

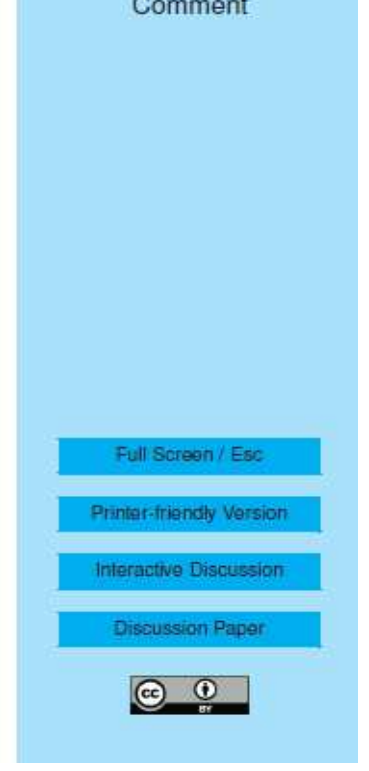

\section{ACPD}

12, C1834-C1841, 2012

We fixed this typo.

P141

We agree with the suggested change in the ordering of the paragraphs, and have made this change.

P142

We have added appropriate subsection headings.

P144

We have removed this stray word, and clarified further that the percentage change is relative to the base run.

P145

We have added an explicit expression for the percentage change.

Figures

We agree that some of the figure titles can seem somewhat cryptic. In order to address this, we have removed the figure titles from Fig. 5 (the panels are already explained well in the caption), and updated the titles of Figs. 6,7 , and 8 to be more human readable. For technical reasons the titles to Fig. 4 were not updated. If desired, we can also update these.

\section{Supplementary Figures}

We have a added a short note to the caption for each of figures S1 to S3 explaining how to read the color scale.

Anonymous referee \#2
Interactive

Comment

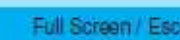

Printer-friendly Version

Discussion Paper

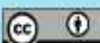

Interactive Discussion 
The first part of the general comments from reviewer \#2 brings up some very similar points to the first general comment of reviewer \#1. Our response to both reviewers regarding this issue can be found in our response to reviewer \#1.

Regarding the rest of this general comment, we disagree with the reviewer that any definitive conclusions about the behaviour of individual cities in our redistribution analysis can be inferred from our Figure 5. Indeed, we caution in Section 4 (P146L13, discussion version) that focusing on any particular individual city in an analysis such as ours is unlikely to be useful.

Specific comments

P130L13

We changed "different" to "simpler", and added text to make it clear that the result of this difference is an increase in the computer emissions associated with the megacities. P135L23

Our treatment of the methane mixing ratios is described in Section 3 . We have added some extra text to this section to make it clearer that methane mixing ratios are fixed throughout the entire model domain.

P135L28

We have changed this around to show that the method is less dependant on the subjective judgement of the person constructing the megacity mask.

P136L5

We understand from ACP style guidelines that manuscripts in preparation are to be referenced only using footnotes, and not included in the main list of references. We added the reference for the Collins World Atlas to the reference list.

\section{C1839}

\section{P140L21}

It is not clear to us why this subsection is unnumbered. It is declared with the same standard latex subsection command as all other subsections in the manuscript. This appears to be a matter for the typesetting staff at the journal to investigate. We thank the referee for pointing this out, and we will make sure that this is fixed during the proofreading stage.

\section{P141L27}

It is not clear to us what the reviewer means by "harmonising (megacity emissions) over the country as a whole", but we suspect that this is likely to be as arbitrary a choice as redistributing the emissions as we have done so in this study. Regarding the choice of the $25 \%$ emissions reduction, we added some text explaining this with reference to the literature at the end of this subsection.

P144L9-10

The redistribution of emissions involved in creating RCP-8.5-P from RCP-8.5 is more extensive than the redistribution of megacity emissions in our redistribution runs. This provides a much better illustration of this effect, which is what we are actually highlighting in our conclusions. In order to make this clearer in the text we have expanded our discussion of Figure S4, and explicitly drawn attention to the similarity between RCP. 8.5 and $\mathrm{RCP}-8.5-\mathrm{P}$ in this figure, with discussion of the relevance to radiative forcing.

\section{P146L19}

The $0.84 \%$ figure is taken from Table 2, and we have added an explicit mention of this number to the text describing this table. It is not immediately clear to us how it would be useful to compare this number, the effect of a wide range of sources which are co-located in small geographical regions, with numbers from other studies such as QUANTIFY which describe the effects of single source categories which are spread over large geographical regions. We do agree with the reviewer that comparison of

\section{C1840}

ACPD

12, C1834-C1841, 2012

Interactive

Comment

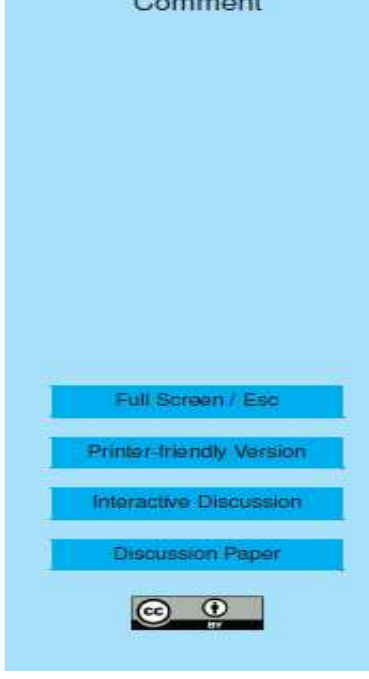

ACPD

12, C1834-C1841, 2012

Interactive

Comment 
different approaches for analysing megacity impacts would be interesting, but we believe that a comprehensive approach to this is beyond the scope of our study; we have explored one possible approach, and future studies may choose differently.

P146L21

The $6 \%$ figure for $\mathrm{NOx}$ applies equally well to present day anthropogenic emissions all ozone precursors, namely CO, NOx, and NMVOC, as seen in our Figure 3.

P146L24

"spatially explicit" describes more specifically the point being made here. We have changed the text appropriately.

P147L21

This is related to the 4 th general comment of referee \#1.

P148L11

We agree completely with this suggestion by the reviewer, but we do not want to dilute our message by calling for a list of technical options for emissions specification. We believe that by calling for "emissions scenarios which are geographically resolved enough to be useful at small scales", that we make our point clearly.

P155

We have added a conversion factor to Dobson Units in the caption.

Figures

See the second general comment from reviewer \#1.

Typos

All fixed. Thanks.

Interactive comment on Atmos. Chem. Phys. Discuss., 12, 129, 2012. C1841

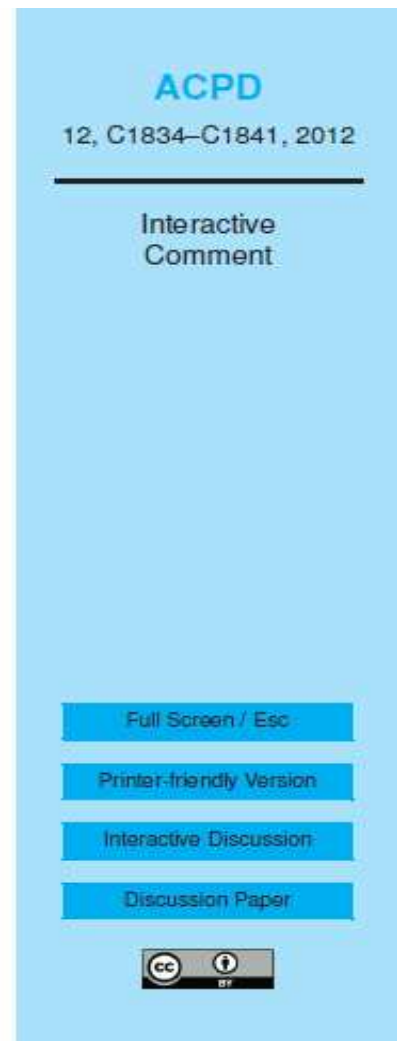

Nous avons reproduit in extenso ces trois parties de l'expertise (de la part de deux experts anonymes et ensuite la réponse - très détaillée - des auteurs) afin de faire ressortit le caractère révolutionnaire du processus de certification d'A.C.P. Révolutionnaire, le processus l'est car il présente un processus connu pour sa propension à être secret et le rend le plus ouvert possible .Il présente les différentes étapes par lesquelles le manuscrit passe et le chercheur ( et même le curieux) peut suivre ( pratiquement ) en temps réel la science en construction. Cette ouverture est, à plus d'un titre, révolutionnaire car en plus de faire faire la science en direct (si l'on peut s'exprimer ainsi), elle peut être un antidote assez déterrant contre les différentes formes de dérives largement documentées par la documentation sur le sujet. Même si l'expérience est assez récente et innovante, et que les retours des opinions quant à sa viabilité ne peuvent être documentés encore, il nous parait que c'est dans cette direction que se dirige l'avenir de la publication scientifique contrôlée par les pairs .Publication qui profitera des différentes fonctionnalités offertes par Internet et qui dans ce cas sont : rapidité et surtout ouverture. On peut voir l'auteur (qui parle au nom des auteurs de la recherche) répondre point par point aux différentes questions et remarques que les experts (anonymes et désignés) ont émis allant jusqu'à corriger même les erreurs de frappes, c'est dire la minutie et la précision des corrections, qui plus est, est faite au vu et au su de la communauté des experts et plus important de la communauté au sens plus large du terme. Cette ouverture qui fait la force (et en même temps la faiblesse car elle - l'ouverture - peut aussi conduire à une sorte d'inflation de l'information déjà existante dans le monde papier et qui se trouve plus qu'exacerbée dans le monde quasiment infini du numérique) d'Internet a prouvé sa propension à mettre fin (ou du moins atténuer) les différents maux dont a été chargé le monde de la certification de la publication scientifique quoique le processus étant essentiellement humain, il ne sera jamais exempt de reproches et de suspicion. 
Si la partie précédente a décrit en détails les différentes étapes que le manuscrit publié traverse ainsi que sa forme finale, cette étape concerne les recherches en cours d'expertise. Ainsi à titre d'exemple, en date du 04 Septembre 2013, il y avait 157 recherches en cours d'expertise s'étalant du 7 Decembre 2012 au 4 septembre 2013.Un grand nombre de ces recherches soumises à expertise ont reçus des commentaires de la part de la communauté et des experts désignés. A titre d'exemple, à la même date une recherche en discussion ouverte se présentait sur le site de la manière suivante :

\section{ACPD - Papers in Open Discussion}

Middle atmospheric changes caused by the January and March 2012 solar proton events

$04 \operatorname{Sep} 2013$

\section{H. Jackman, C. E, Randall, V. L. Harvey, S. Wang, E. L. Fleming, M. López-Puertas, B. Funke, and P. F. Bernath}

Atmos, Chem, Phys, Discuss, 13, 23251-23293, 2013

口Abstract ם Discussion Paper (PDF, 1180 KB) « Interactive Discussion (Open, O Comments)

Manuscript under review for ACP

On remarquera que l'interface est la même que pour " Bibliothèque en ligne ACP " (Online Library ACP) avec la notable différence qu'à la place de " Discussion paper " la mention " Interactive discussion " la remplace avec la mention de " Open " et le nombre de commentaires que la recherche a suscité. Si la recherche que nous avons cité n'avait en date de la rédaction de ces lignes (le même jour) pas reçu de commentaires, une autre soumise en date du 12 Aout 2013 avait reçu un commentaire et qui se présentait de la manière suivante :

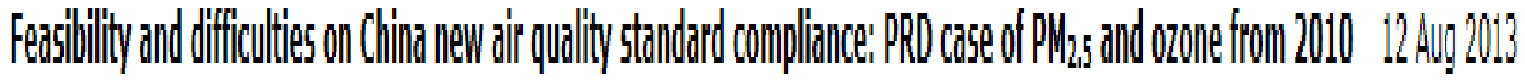

\section{to 2025

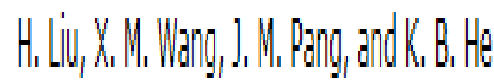

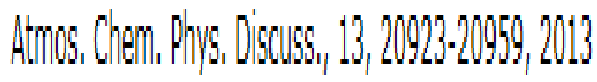

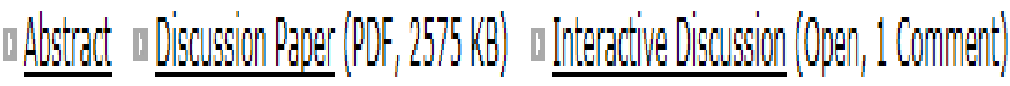

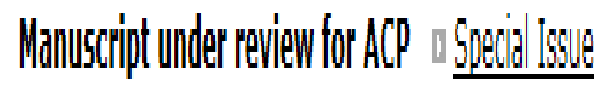

En cliquant sur le lien " Interactive discussion", on obtient la page suivante : 
Atmos. Chem. Phys. Discuss., 13, 20923-20959, 2013 www.atmos-chem-phys-discuss,net/13/20923/2013/ doi:10.5194/acpd-13-20923-2013

(c) Author(s) 2013. This work is distributed

under the Creative Commons Attribution 3.0 License.

Feasibility and difficulties on China new air quality standard compliance: PRD case of PM 2.5 and ozone from 2010 to 2025

H. Liu, X. M. Wang, J. M. Pang, and K. B. He
Review Status

This discussion paper is under review for the joumal Atmospheric Chemistry and Physics (ACP).

Interactive Discussion

Status: Open (until 07 Oct 2013)

AC: Author Comment | RC: Referee Comment | SC: Short Comment | EC: Editor Comment

[Post a Comment] [Subscribe to Comment Alert] - Printer-friendly Version - Supplement

RC C6126: 'First Reviewer Comments', Anonymous Referee \#1, 26 Aug 2013 [reply]

Dans ce " RC " (referee comment), un expert anonyme commente la soumission sur 5 pages le 26 Aout 2013 dont nous reproduirons (à titre d'exemple) la première :

Atmos. Chem. Phys. Discuss., 13, C6126-C6130, 2013 www. atmos-chem-phys-discuss.net/13/C6126/2013/

(1) Author(s) 2013. This work is distributed under

the Creative Commons Attribute 3.0 License.

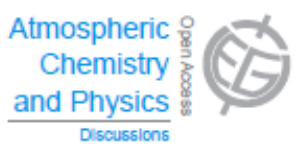

ACPD

13, C6126-C6130, 2013

Interactive

Comment

\section{Interactive comment on "Feasibility and difficulties on China new air quality standard compliance: PRD case of $\mathrm{PM}_{2.5}$ and ozone from 2010 to 2025 " by H. Liu et al.}

\footnotetext{
Anonymous Referee \#1

Received and published: 26 August 2013

First Referee Comment

RE: Feasibility and difficulties on China new air quality standard compliance: PRD case of PM2.5 and ozone from 2010 to 2025

H. Liu, X. M. Wang, J. M. Pang, and K. B. He

Atmos. Chem. Phys. Discuss., 13, 20923-20959, 2013 www.atmos-chem-physdiscuss.net/13/20923/2013/ doi:10.5194/acpd-13-20923-2013

General Comments The present manuscript explores an important issue that would connect science and control policy in China in a timely manner. Naturally, the chal-

\section{C6126}


On remarquera que sous " Review Status " (Statut de l'expertise), il est indiqué que la dite soumission est toujours sous expertise. D'autres fonctionnalités incluent :

-Post a comment (poster un commentaire)

-Reply ( répondre )

En cliquant sur un des deux liens on obtient

\section{Post a Comment to ACPD}

13 Create Account ax Resend Login Data
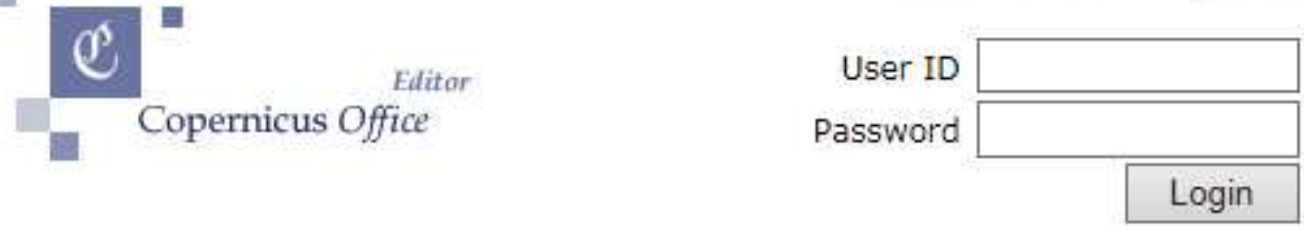

Où on doit être inscrit ou s'inscrire pour poster le commentaire en utilisant la figure précédente

Enfin, on peut dans le lien " suscribe to comment alert " (s'abonner aux alertes de commentaires), on obtient la figure suivante qui obéit aux mêmes schéma :

Copernicus Office Editor

Is Create Account a Resend Login Data
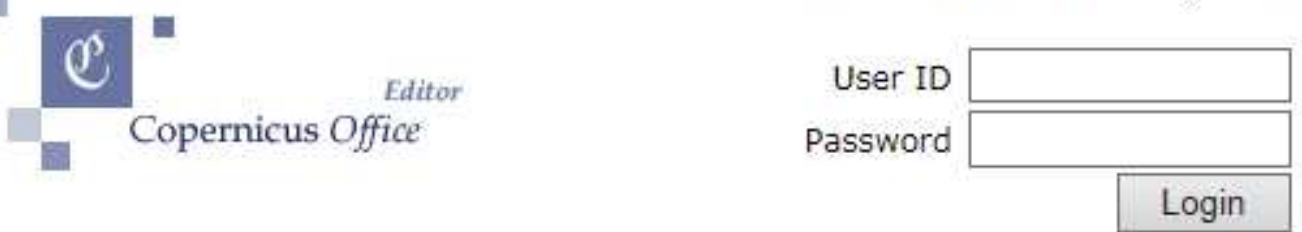

Enfin, le site comporte une rubrique " most commented papers of all times " ainsi que " most commented papers of the last 12 months " (respectivement " les recherches les plus commentées de tous les temps" et " les recherches les plus commentées des 12 derniers mois ").Cette rubrique est intéressante à plus d'un titre car au-delà du coté statistiques ( à titre d'exemple la recherche ayant suscité le plus de commentaires avait reçu 33 commentaires ), elle nous fait découvrir les différentes étapes, commentaires, réponses et échanges et ceci dans une ouverture la plus totale .En plus de les découvrir ,il nous a été permis de découvrir que, par exemple, la recherche en discussion la plus citée de tous les temps ${ }^{(73)}$ ( pour ACP) n'a pas été publiée et plus que ceci , ACP n'a pas l'intention de le faire. Cette soumission ayant suscité ce nombre de commentaires (qui, même en termes d'A.C.P., est inhabituel), elle s'est terminé par un appel des auteurs vers l'éditeur en chef pour revoir sa position .Appel n'ayant pas fait changer d'avis l'éditeur en chef qui a finalement rejeté la recherche et ce après plus d'une année (déposée le 19 Septembre 2009 et rejetée définitivement en date du 14 Octobre 2009) de discussion, ayant été des fois très animées. Si ces étapes ne sont pas à

\footnotetext{
${ }^{(73)}$ A. M. Makarieva, V. G. Gorshkov, and B.-L. Li , On the validity of representing hurricanes as Carnot heat engine

http://www.atmos-chem-phys-discuss.net/8/17423/2008/acpd-8-17423-2008.pdf
} 
proprement parler révolutionnaires en elles même, le caractère révolutionnaire se situe dans l'ouverture du processus où on peut voir en direct l'article et ses différents changements et mutations.

\section{3-FACULTY OF $1000\left(\right.$ F 1000) ${ }^{(74)}$}

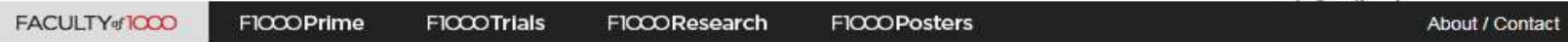

\section{FACULTYof 1000}

\section{Faculty of $\mathbf{1 0 0 0}$ is the publisher of four unique services that support and inform the work of life scientists and clinicians.}

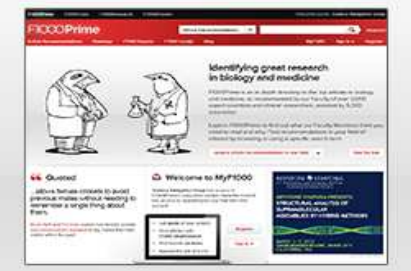

F1000Prime An in-depth directory of top articles in biology and medicine, as recommended by our Faculty of over 5,000 expert scientists and clinical researchers.

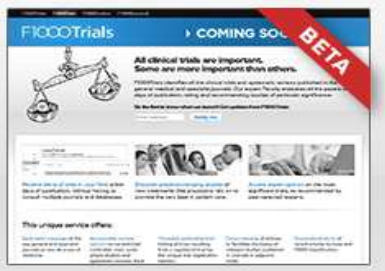

F1000Trials A comprehensive listing of published clinical trials with recommendations and comments on the most important. New from

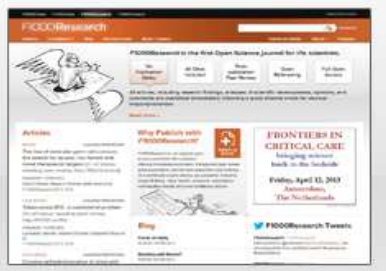

F1000Research An innovative open access journal offering immediate publication and open peer review.

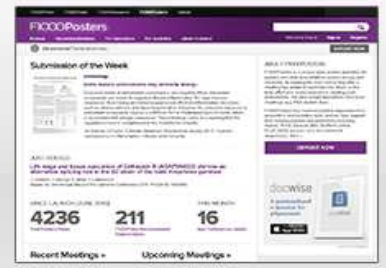

F1000Posters The open access repository for posters and slide presentations in biology and medicine

Introduction

Le site Faculty of 1000 est un site spécialisé en biologie et en médecine et dont la spécificité est de pratiquer un contrôle par les pairs post publication et non pré publication. En effet, le site de F1000 ".....comporte une base de données importante mise à jour quotidiennement et qui se compose de 100000 évaluations des plus importants articles publiés en médecine et biologie. Les articles sont sélectionnés, notés et évalués par notre faculté de plus de 10000 experts scientifiques et chercheurs cliniques et qui couvrent plus de 3000 périodiques contrôlés par les pairs " ${ }^{(75}$. Contrairement à ACP, F 1000 n'expertise pas les articles en médecine et en biologie mais les expertise après leur publication (ou les ré expertisent). Ce qui veut dire que les articles dont il est question, ont déjà passé l'épreuve du contrôle par les pairs, sont publiés et font l'objet maintenant d'un second contrôle (choix serait plus adéquat) pour être " sélectionnés, notés et évalués " de nouveau. Les personnes qui sont responsables de cette deuxième évaluation (ou choix c'est selon), au nombre de 10000 , sont des sommités dans leur spécialités et des personnes reconnues grâce à leur contribution au développement de la science. Ils sont subdivisés en :

-International Advisory Board (Conseil Consultatif International): il aide à orienter F 1000 en ce qui concerne le champ d'application du service et les grandes lignes de la politique de $\mathrm{F}$ 1000. Le Conseil Consultatif International aide aussi dans la sélection des Doyens de Facultés qui composent actuellement le service de F 1000.

\footnotetext{
(74) $\mathrm{http://f1000.com/}$

(75) $\underline{\mathrm{http}: / / \mathrm{f} 1000 . \mathrm{com} / \text { tour }}$
} 
-Heads of faculty (Doyen de faculté): ce sont d'éminents scientifiques et cliniciens nommés par le Conseil Consultatif International qui supervisent les 44 facultés composant F 1000.Ils subdivisent leur sujet de principales sections dans leurs disciplines et participent dans la désignation des chefs de section pour chaque domaine.

-Section Heads (Chef de section) : ce sont des experts reconnus dans leur spécialité et sont responsables de la subdivision de leur section en rubriques et la sélection de membres de Faculté appropriés dont la tache est d'expertiser la littérature dans chaque domaine thématique. Le nombre de membres dans chaque Faculté dépend très largement du nombre d'articles publiés dans chaque domaine thématique.

-Faculty Members and Associate Faculty Members (Membres de faculté et membres de faculté associés) : ils ont pour mission d'évaluer, noter et classer les articles à inclure dans $\mathrm{F}$ 1000.Les membres de faculté associés, désignés par les membres de faculté, aident à scanner les tables de matière de tous les journaux généralistes et spécialisés afin de s'assurer que la littérature dans leur domaine thématique a été adéquatement couverte. La Faculté sélectionne et couvre actuellement plus de 1500 articles chaque mois, ce qui correspond, approximativement, au top $2 \%$ de tous les articles publiés dans les sciences biologiques et médicales.

Donc comme on peut le voir, F 1000 est bâti sur une organisation assez complexe et hiérarchisée, indispensable pour gérer le très important flux d'information qui découle de la production scientifique en biologie et en sciences médicales. .

\subsection{1-Les articles évalués}

Une des particularités de F 1000 est la rubrique appelée " articles évalués " et dans laquelle des articles à évaluer sont rassemblés par les membres de Faculté et de section (pour les sous spécialités).Ils sont ensuite indexés sous différentes vedettes, étiquetés, les rendant de ce fait facile à rechercher et à feuilleter. Les membres de Facultés et leurs membres associés sélectionnent et évaluent ensuite les meilleurs articles qu'ils ont lus dans leurs spécialités. Les évaluations sont sous la forme de commentaires accessibles et brefs, mettant en évidence les découvertes les plus significatives et mettant le travail en contexte. Cette opération est accompagnée par des scores numériques pour permettre le calcul des notations et des classements.

\subsubsection{1-F 1000 notations et classements}

\subsubsection{1-Articles ( FFa)}

Les articles à évaluer rassemblés sont ensuite notés " recommandé " (6), " doit être lu " (8) et enfin " exceptionnel "(10).Ces notes émanant de différents membres sont ensuite additionnées pour calculer et obtenir le " F1000 Article Factors (Ffa) " ou " Facteurs d'articles F 1000 " qui permettent de classer les articles à travers toutes les spécialités " et chacune des spécialités des Facultés. Le plus d'évaluations un article reçoit, le plus haut son score et en conséquence son " Facteurs d'articles F 1000 "augmente et par delà son classement. Le " Facteur d'articles $\mathrm{F} 1000 "$ est calculé à partir du plus haut score donné par un membre de F1000 plus une incrémentation pour chaque score additionnel donné par un membre de F1000. Le " Facteur d'articles F 1000 " est affiché à côté de l'information de l'article et les scores individuels à côté de chaque évaluation individuelle. En utilisant ces valeurs " Facteur d'articles F 1000", on obtient les catégories d'articles suivants:

\footnotetext{
* Il est à signaler que 44 spécialités (allant de l'anesthésiologie et le management de la douleur à l'urologie en passant par les sciences endocrines et métaboliques) sont abordées dans le site.
} 
3.3.1.1.1.1- Dix meilleurs de tous les temps : en date du 23 Mai 2012, l'article intitulé : Genome-wide non-mendelian inheritance of extra-genomic information in Arabidopsis, publié par Nature en 2005 a atteint le " Facteur d'articles F 1000 " le plus haut avec un score de 62. Il avait reçu 20 évaluations.

3.3.1.1.1.2--Dix meilleurs actuellement : en date du 23 Mai 2012, l'article intitulé : Gut flora metabolism of phosphatidylcholine promotes cardiovascular disease, publié par Nature en 2011 a atteint le " Facteur d'articles F 1000 " des 10 meilleurs avec un score de 20. Il avait reçu 5 évaluations.

3.3.1.1.1.3-Les plus consultés de tous les temps : les articles de cette rubrique sont classés quotidiennement et indiquent les articles que les pairs ont estimé être de tout premier intérêt depuis le lancement de F 1000. En date du 23 Mai 2012, l'article intitulé : Genome-wide nonmendelian inheritance of extra-genomic information in Arabidopsis, publié par Nature en 2004 avait atteint le " Facteur d'articles F 1000 " le plus haut avec un score de 62.Il avait reçu 20 évaluations. On remarquera que c'est le même article ayant obtenu le titre de meilleur article de tous les temps (pour F 1000).

3.3.1.1.1.4-Les plus consultés actuellement : les classements des articles de cette rubrique sont générés quotidiennement utilisant seulement les articles évalués dans F 1000 au cours des 14 jours précédents et indiquent les articles que les pairs considèrent actuellement comme les plus intéressants dans F 1000. En date du 23 Mai 2012, l'article intitulé: Gut flora metabolism of phosphatidylcholine promotes cardiovascular disease, publié par Nature en 2011 avait été le plus consulté de cette période et atteint le " Facteur d'articles F 1000 " des plus consultés avec un score de 20 . Il avait reçu 5 évaluations. On remarquera aussi que c'est le même article ayant obtenu le titre des 10 meilleurs articles de l'époque (pour F 1000)

3.3.1.1.1.5-Perles cachées : ce sont des articles qui se sont avérés importants mais qui n'avaient pas au début eu toute l'attention voulue. Ils sont générés quotidiennement et n'incluent que les articles parus dans des journaux spécialisés, évalués dans F 1000 les 14 jours précédents. La liste des journaux est la même pour toutes les facultés composant $\mathrm{F} 1000$ et bien que certains puissent être considérés comme importants dans leur spécialités, ils sont inclus car ils peuvent ne pas être évident aux lecteurs en dehors du domaine de la Faculté. Les articles sont classés selon leur " Facteur d'articles F 1000 ", qui est calculé à partir de tous les classement donnés par la Faculté à cet article .Dans le cas, plusieurs articles ont le même " Facteur d'articles F 1000 ", celui qui est le plus consulté est classé premier. En date du 23 Mai 2012, l'article intitulé :Returning from oblivion : imaging the neural core, publié en 2012 par Journal of Neurosciences avait un " Facteur d'articles F 1000 " de 16. On remarquera que cet article a reçu des trois membres de F1000 l'ayant jugé des notes de 10 ( exceptionnel ) ce qui dénote l'importance de cette rubrique dont le but ( tel qu'indiqué par son appellation ) est de déterrer les articles importants ayant, pour des raisons diverses, échappé le contrôle minutieux de la communauté et son système de repérage des recherches importantes .

\subsubsection{2-Journaux (FFj)}

En plus de classer et noter les articles, F 1000 a aussi innové en classant les périodiques que ses Facultés ont choisis." The F1000 Journal Factor " ou " Facteur de Journal F 1000" est une mesure du degré de réussite d'un journal donné dans $F$ 1000. Elle est calculée à partir des valeurs individuelles du " Facteur d'articles F 1000 "( FFa) et normalisée selon le nombre total d'articles que chaque journal a publié.

Les journaux sont classés du sommet vers la base (de facultés à sections).Ceci permet de voir non seulement où les membres des différentes Facultés pensent que les recherches les plus importantes dans chaque spécialité sont publiées mais aussi où il est le plus adéquat de 
publier. On peut suivre la trace de chaque " Facteur de Journal F 1000 " à travers les évaluations et les notations des membres de F 1000 pour chaque article ce qui permet de voir quels sont les articles qui ont contribué à la place qu'occupe chaque journal dans le classement. A l'instar des rubriques de classement et de notation mises en place pour les articles, les rubriques suivantes sont utilisées pour les journaux :

3.3.1.1.2.1-Classements actuels de $\mathbf{F} 1000$ : ce sont les mesures de performance les plus mises à jour d'un journal. Ils sont calculés mensuellement sur la base des évaluations publiées les 12 derniers mois indépendamment de la date de publication de l'article. A titre d'exemple en date du 24 Mai 2012, le classement des vingt premiers journaux (sur un total de 1120 journaux recensés) était le suivant : ${ }^{(76)}$

\begin{tabular}{|c|c|c|c|c|c|}
\hline Classement & $\begin{array}{l}\text { Facteur de Journal } \\
\text { F } 1000\end{array}$ & Journal & $\begin{array}{c}\text { Articles } \\
\text { sélectionnés }\end{array}$ & Evaluations & $\begin{array}{l}\text { Articles } \\
\text { éligibles }\end{array}$ \\
\hline 1 & 51.39 & Nature & 396 & 729 & 1096 \\
\hline 2 & 49.95 & Cell & 191 & 329 & 337 \\
\hline 3 & 49.17 & Science & 297 & 567 & 1021 \\
\hline 4 & 47.99 & $\begin{array}{c}\text { Proceedings of the } \\
\text { National Academy of } \\
\text { Science. }\end{array}$ & 569 & 674 & 3712 \\
\hline 5 & 45.83 & $\begin{array}{l}\text { New England Journal of } \\
\text { Mdecine. }\end{array}$ & 162 & 297 & 635 \\
\hline 6 & 45.77 & Nature medicine & 94 & 144 & 204 \\
\hline 7 & 45.40 & $\begin{array}{c}\text { Journal.of.Experimental } \\
\text { Medicine }\end{array}$ & 100 & 148 & 217 \\
\hline 8 & 44.35 & Nature Immunology & 63 & 106 & 126 \\
\hline 9 & 44.08 & Nature Cell Biology & 72 & 118 & 170 \\
\hline 10 & 43.83 & Neuron & 92 & 128 & 279 \\
\hline 11 & 43.52 & Nature Neuroscience & 83 & 119 & 251 \\
\hline 12 & 43.21 & Molecular Cell & 89 & 117 & 283 \\
\hline 13 & 43.08 & Immunity & 59 & 93 & 139 \\
\hline 14 & 42.76 & Journal of Cell Biology & 85 & 120 & 288 \\
\hline 15 & 42.28 & $\begin{array}{c}\text { Journal. of Clinical } \\
\text { Investigation }\end{array}$ & 93 & 130 & 405 \\
\hline 16 & 41.10 & Nature Genetics & 62 & 82 & 225 \\
\hline 17 & 40.83 & Anesthesiology & 64 & 94 & 263 \\
\hline 18 & 40.58 & Cancer Cell & 39 & 55 & 112 \\
\hline 19 & 40.22 & $\begin{array}{l}\text { Nature Structural \& } \\
\text { Molecular Biology }\end{array}$ & 55 & 71 & 222 \\
\hline 20 & 40.21 & Developmental Cell & 47 & 66 & 160 \\
\hline
\end{tabular}

Tableau $\mathrm{n}^{\circ}$ 14- Classement actuel des journaux expertisés par F 1000

Cette liste comporte les titres les plus prestigieux de la médicine et la biologie comme Nature, The New England Journal of Medecine, Science, etc..., ce qui semble indiquer le sérieux de sa procédure de sélection qui englobe ces titres.

(76) http://f1000.com/rankings/journals/year/current (Accédé le 24 Mai 2012) 
3.3.1.1.2.2-Classements provisoires annuels : ils sont publiés au mois de Juillet et ils sont calculés à partir des évaluations des articles qui ont étés publiés dans la dernière année civile complète et permettant 6 mois d'évaluations de s'accumuler. Le tableau provisoire décrivant les classements de l'année $2010^{(77)}$ recèle pratiquement les mêmes titres que celui du tableau précèdent ainsi qu'un classement aussi pratiquement analogue (on remarquera aussi la remarquable stabilité des notations attribuées et qui sont très proches de celles du tableau précèdent) ce qui prouverait la justesse des critères appliqués à la sélection des revues incluses dans F 1000.

3.3.1.1.2.3-Classements finaux annuels : ce sont les classements qui permettent au moins à 18 mois d'évaluations de s'accumuler. A titre d'exemple, les classements finaux disponibles sont ceux de $2009^{(78)}$ et ce en date du 24 Mai 2012 ce qui respecte la période minimal de 18 mois énoncée pour la confection des listes finales. Les titres inclus sont aussi pratiquement les mêmes et ainsi que le classement et les notations attribuées.

\subsubsection{3-Comptes rendus F1000}

Les comptes rendus en libre accès F 1000 ajoutent une autre dimension à F1000 en fournissant des comptes rendus uniques et contrôlés par les pairs sur des thèmes émergents en biologie et en médecine. Les chefs de section F 1000 garantissent une couverture des dernières avancées en sélectionnant les sujets et en nommant les experts afin de fournir un contexte à la littérature évaluée.

Ces journaux en libre accès sont disponibles gratuitement en texte intégral sans aucune barrière de quelque genre que ce soit ou d'un abonnement et sont actuellement indexés et/ou résumés par PubMed, PubMed Central, Scopus, Embase, Global Health et CAB Abstracts. Il existe :

-F 1000 Reports Biology et qui date de 2009 avec des parutions mensuelles

- F 1000 Reports Medecine et qui date de 2009 avec des parutions mensuelles

Il semblerait que les Comptes rendus F 1000 différent un tant soit peu de la philosophie de base de F 1000 qui consiste à rassembler, noter et classer des articles (et aussi dernièrement des journaux) et passe au stade de publications contrôlées par un mécanisme interne traditionnel.

\subsubsection{4-Posters F 1000}

Les posters F1000 sont un autre aspect très novateur que F 1000 créé. En effet, le site propose d'héberger dans un entrepôt en libre accès les affiches et les présentations de diapositives ainsi que les présentations orales faites dans des conférences, symposia et autres séminaires. En permettant au travail d'être visible longtemps après sa présentation, il permet de maximiser les bénéfices du point de vue temps, investissement aussi retour d'écoute. Les présentations sont, dans la plupart des cas et une fois la conférence terminée, perdues et ne

(77) $\mathrm{http}: / /$ /f1000.com/rankings/journals/year/2010 
sont vues et utilisées que par un nombre de toute évidence restreint de chercheurs (ceux présents à la conférence). Les réseaux sociaux permettent à la communauté scientifique d'être plus au courant (et en certains cas en temps réel) de ce qui se passe même quand la distance les sépare. Ils permettent de faire parvenir l'information et d'atteindre un plus grand nombre surtout lorsqu'on sait que Twitter et les blogs scientifiques sont très présents dans ce genre de manifestation. F 1000 posters permet d'éviter les longs délais découlant de la non dissémination de ces recherches. Il permet d'éviter aussi la duplication et fait avancer la Science. De même, ils permettent de favoriser un environnement permanent et structuré ainsi qu'un lieu pour des discussions continues et un développement de l'information présentée. D'ailleurs, F 1000 à travers des sondages fait auprès des chercheurs fait ressortir qu'ils étaient à raison de plus de $85 \%$ favorable à l'idée et que $95 \%$ pensent qu'il leur sera utile ${ }^{(79)}$.Quant aux plus grands éditeurs investigués (au nombre de 109), ils ont fait preuve d'une certaine ouverture quant à ce nouveau genre de publication. Ils ont répondu de la manière suivante quant à l'opportunité de publier ou du moins déposer les posters dans F 1000 :

- 66 soit $60 \%$ ne voit aucun problème quant au dépôt dans F 1000

- 4 soit $4 \%$ n'y voit aucun problème tant que le travail soumis contient assez d'informations supplémentaires par rapport à ce qui a été déposé dans F 1000.

- 2 soit $2 \%$ n'y voit aucun problème mais laissent la décision finale à l'éditeur de chaque journal

- 10 soit $9 \%$ recommandent de contacter les éditeurs en premier lieu afin de discuter chaque cas individuellement

- 27 soit $25 \%$ quant à eux verraient le dépôt comme une sorte de prépublication. ${ }^{(80)}$

Il est clair que même les éditeurs, pourtant connus pour être très frileux à l'idée de voir les publications dispersées avant d'être publiés dans leur journaux, ne sont pas contre l'idée novatrice de F 1000 et ce à $75 \%$ qui accepteraient un dépôt dans F 1000 (sous différentes conditions), alors que seul $25 \%$ y sont résolument contre.

On remarquera seulement que ce nouveau service (lancé en 2010) n'assure pas automatiquement le contrôle par les pairs et de ce fait, les recherches ne sont pas certifiées. Cependant, certaines peuvent faire l'objet d'un travail d'expertise et intégrer de cette manière les autres publications du site. A titre d'exemple, en date du 26 Mai 2012, il y avait 3036 affiches et diapositifs déposés dont seulement 160 (soit $5 \%$ ) avaient étés expertisés alors que 43 affiches et diapositives avaient étés postés au mois de Mai ${ }^{(81)}$.

\footnotetext{
${ }^{(79)} \mathrm{http}: / / \mathrm{f} 1000 . \mathrm{com} /$ about/whatis/posters

${ }^{(80)} \mathrm{http://f1000.com/posters/journalresponses}$

${ }^{(81)} \underline{\mathrm{http}: / / \mathrm{f} 1000 . \mathrm{com} / \text { posters }}$
} 
Enfin, la rubrique comporte d'autres renseignements tel que :

3.3.1.1.4.1-Browse (feuilleter) : où on peut voir toutes les affiches et autres diapositives (Power Point) disponibles sur le site .A titre d'exemple, en date du 30 Mai 2012, il y avait 12 présentations de diapositives et 295 affiches et toutes étaient récentes.

3.3.1.1.4.2-For depositors (pour les déposants) : où les différents avantages de déposer les recherches sur F 1000 sont énumérées. Il existe aussi une rubrique " questions les plus fréquemment posées " (FAQs) au sujet des problèmes inhérents au dépôt par rapport à la publication classique. On remarque que sur le coté droit, se trouve une indication " Deposit now " (déposer maintenant).Une fois que l'on clique sur le lien, on obtient une suite d'étapes à suivre pour déposer l'affiche ou les diapositives (Power Point).

3.3.1.1.4.3-For Societes/Conferences organizers (pour les associations /organiseurs de conférences) : où les associations et les organiseurs de conférences sont invités à faire poster le contenu de leur conférences sur le site .De plus, même si la conférence a son propre site pour poster les affiches et diapositifs, F 1000 se propose de réutiliser les présentations et ceci par l'intermédiaire d'une licence de réutilisation. Le site donne une suite d'étapes à suivre afin de guider les associations et les organiseurs de conférence dans ce processus .Enfin, à l'instar des déposants, une suite d'avantages à déposer dans F1000 est énumérée.

3.3.1.1.4.4-Submission of the week (soumission de la semaine): qui est une soumission choisie pour la semaine en cours. Le nom de l'évaluateur (ou évaluateurs) apparaît dans la soumission (ce qui prouverait que cet - ou ces - expert (s) a (ont) choisi la soumission en question et l'a (ont) élu meilleure de la semaine).C'est une autre preuve de l'extrême ouverture et aussi et surtout responsabilisation de ceux qui expertisent, choisissent et élisent les recherches à paraître.

3.3.1.1.4.5- Just posted et qui signale l'affiche la dernière postée sur le site. En date du 30 Mai 2012, cette rubrique contenait " Comparative meta-analysis of proteomic data on extracellular vesicle subsets" et qui était une affiche posté à "1st Annual Meeting of the International Society for Extracellular Vesicles 2012, 18 - 21 Apr. 2012, 1" par Tamas G Szabo [...et al] du Department of Genetics, Cell and Immunobiology, Semmelweis University, Budapest, Hungary. On remarquera que cette affiche avait été présentée en date du 18 Avril 2012 et donc postée sur le site de F 1000 au bout d'un mois. Cette date est extrêmement importante quand on sait que certaines présentations aux conférences, symposia, colloques et autres manifestations scientifiques ne sont jamais publiées et ne sont visibles que dans les actes de ces manifestations. Cette rubrique permet de les faire publier et permettent à la communauté d'en profiter et ce en un temps de latence très court.

3.3.1.1.4.6-Récent meetings (réunions récentes) : qui recensent toutes les réunions que le site a couvertes En date du 30 Mai 2012, il y avait 540 réunions la plupart datant de l'année en cours mais dont la première était datée de l'an 2000.

3.3.1.1.4.7-Upcoming meetings (réunions à venir) : qui recensent les conférences, congrès et autres manifestations scientifiques qui auront lieu en 2012 et 2013 et que site a l'intention de couvrir. 
3.3.1.1.4.8-Naturally selected (naturellement sélectionnées): qui est un blog (il est aussi considéré comme un op ed -contraction de "opposite the editorial page"- et " qui est un article de journal qui exprime les opinions d'une personne qui n'entretient pas habituellement de liens avec le comité de rédaction du journal. Au contraire de l'éditorial, il est signé par l'auteur ${ }^{\left({ }^{(2)}\right)}$ où les scientifiques, les chercheurs et même d'autres personnes intéressées par le sujet peuvent émettre des avis et aussi poster des informations relatives au site. Il met en évidence et signale les recherches de F 1000 les plus intéressantes ainsi que des interviews et des vidéos des personnes qui sont derrière ces recherches. De même qu'il présente des commentaires divertissants et de premier ordre sur les membres des facultés, sur ce qu'ils lisent, leur désaccord et les auteurs qu'ils évaluent, c'est en fait une image de ce qui se discute de plus important dans les articles pertinents dans les sciences biologiques et médicales. Enfin, il présente les dernières nouvelles et les dernières innovations (comme par exemple lorsque le site a atteint 120000 évaluations en date du 13 Avril 2012 et qui fut célébré par les membres des facultés comme un jalon et une étape importants dans la vie du site).

3.3.1.1.4.9- My F 1000 (mon F 1000) : c'est pratiquement un tableau de bord personnel du chercheur où il peut adapter ses recherches et qui comprend les fonctionnalités suivantes :

3.3.1.1.4.9.1-My sections (mes sections) : permet de sélectionner les sections dans F 1000 qui couvrent les domaines d'intérêt / spécialité. Tous les articles évalués dans ces sections seront filtrés dans les sections qui couvrent les domaines d'intérêt / spécialité.

3.3.1.1.4.9.2-My saved articles (mes articles enregistrés): permet après avoir cliquer sur " Add to My F1000 " sur toute page d'article de sauvegarder les détails de l'article en question dans la bibliothèque des articles sauvegardés.

3.3.1.1.4.9.3-My stored searches (mes recherches sauvegardées) : permet de sauvegarder les recherches personnalisées, facilement réutilisées et rediffusées à tout moment.

3.3.1.1.4.9.4-My comments (mes commentaires) : permet de sauvegarder les commentaires faits par le chercheur pour de futures références.

3.3.1.1.4.9.5- My e-mails alerts (mes alertes e-mails) : permet de gérer le flux des alertes de la fonctionnalité My sections (mes sections) ainsi que ceux de My saved articles (mes articles enregistrés).

\subsubsection{5-F 1000 SmartSearch.}

En date du 24 Mai 2012, le site de F 1000 a lancé un nouveau service appelé " F1000 SmartSearch." (Recherche intelligente F 1000) présenté comme une manière révolutionnaire de cibler la recherche d'articles. Selon le site, F1000 SmartSearch se propose d'être ...." un outil de recherche Pub Med intuitif et unique qui " apprend " quels articles sont les plus pertinents pour le chercheur à travers l'entrée des données et l'interaction" ${ }^{83)}$.En de termes plus compréhensibles, ce service se propose de faire la recherche d'articles selon les données que le chercheur a lui même introduit et ensuite, et ceci est le coté révolutionnaire de ce service, il " apprend" selon les intrants que le chercheur lui fournit et de ce fait augmente de plus en plus sa capacité à répondre aux attentes du chercheur plus ce dernier lui donne des

\footnotetext{
${ }^{(82)} \mathrm{http} / / /$ fr.wikipedia.org/wiki/Op-ed (consulté le 04 Juin 2012)

${ }^{(83)} \mathrm{http}: / /$ f1000.com/my/searches/manage
} 
informations. Pour ce faire, le chercheur doit créer un compte qui se présente de la manière suivante :

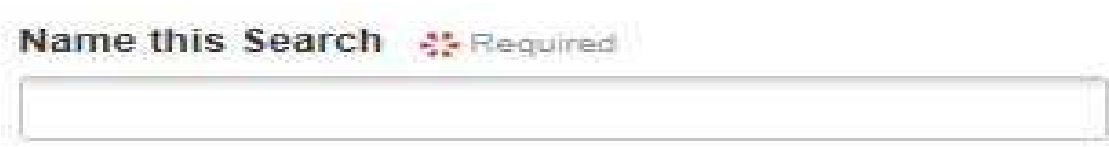

Add Article Seeds $\%$ Required
PMID

\section{PMID}

\section{PMID}

\section{PMID}

\section{PMID}

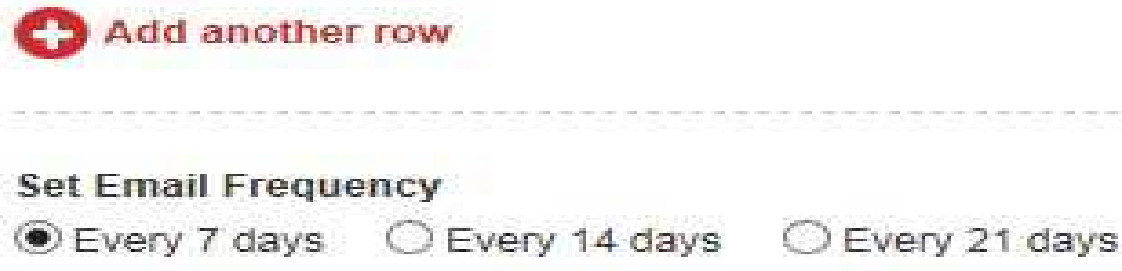

Dans le premier carré (Name this Search), le chercheur doit faire rentrer un mot clé pertinent par rapport à la recherche qu'il a l'intention de faire .Ensuite, il alimente les cinq colonnes (Add article seeds) par un minimum de cinq articles à partir de PubMed (PMIDs) qui correspondent à ces critères. F1000 SmartSearch utilise alors ces groupes d'articles représentatifs comme modèle afin de trouver des articles nouvellement publiés susceptibles d'être pertinents et intéressants pour le chercheur. Les premiers ensembles de résultats apparaîtront immédiatement de la manière suivante : 


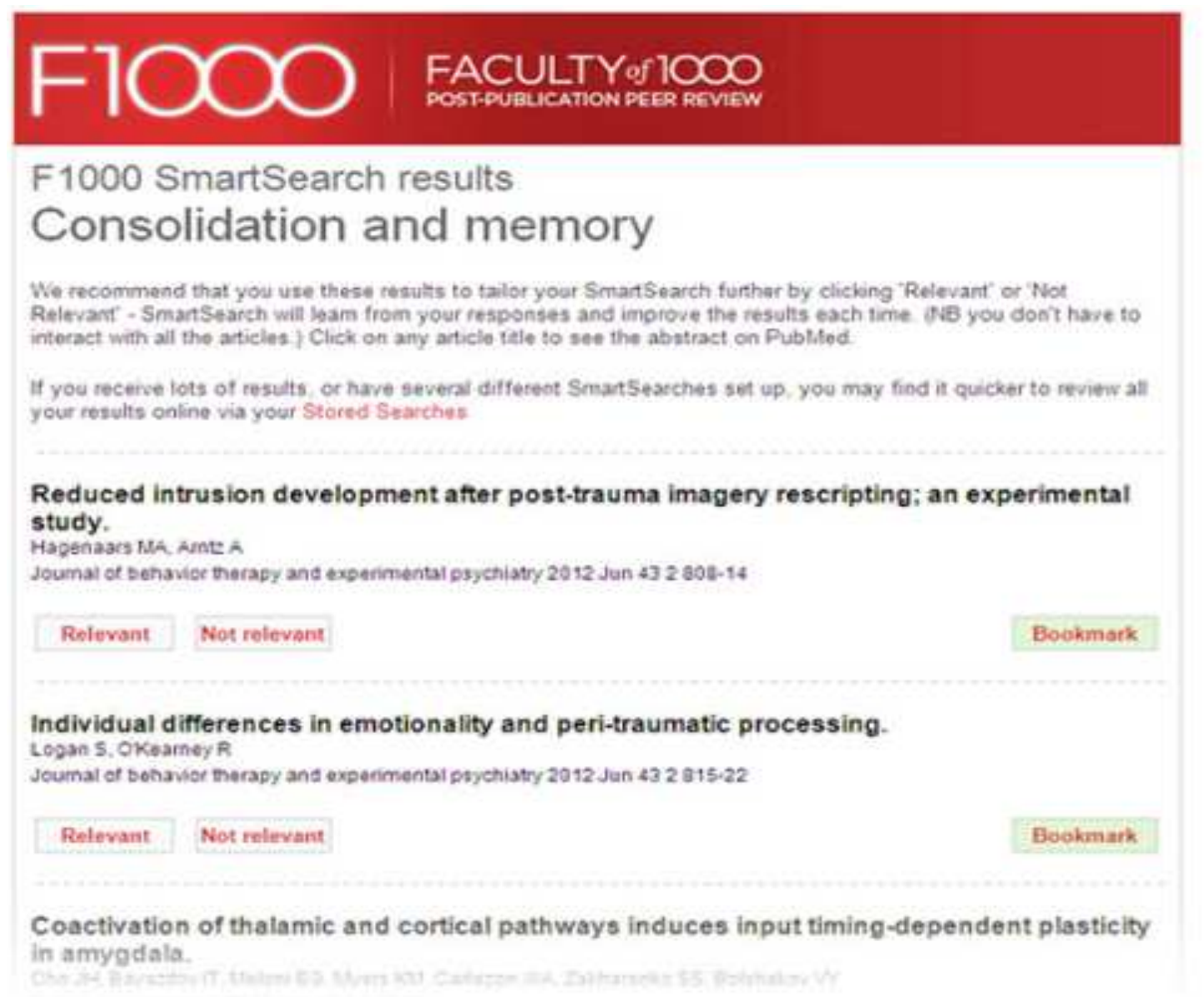

\section{F1000 Smartsearch results}

Le chercheur devra alors (et là est l'aspect révolutionnaire de ce service) devra cliquer sur " pertinent " (relevant) ou alors " non pertinent " (non relevant ). SmartSearch " apprendra " alors à partir de ces réponses et s'améliorera à chaque ensemble de résultats. Le chercheur recevra alors un e- mail d'alerte à la fréquence demandée (une, deux ou trois semaines) mais ne seront envoyées que les résultats considérés pertinents. Etant donné que SmartSearch exclura toutes les requêtes non pertinentes, le chercheur ne recevra qu'un nombre de plus en plus restreint mais plus pertinent d'articles pour chaque alerte. Le chercheur est conseillé de choisir encore soit " pertinent " ou " non pertinent " comme dans les premières requêtes et ceci mènera à des résultats de plus en plus pertinents, raffinés et plus en relation avec le sujet du chercheur*.

Le processus de F 1000 peut être considéré comme révolutionnaire dans le domaine de la publication scientifique et technique et dans son contrôle par les pairs. Non seulement, il applique un contrôle sur Internet ouvert à tous mais (et ceci peut être considéré comme le coté révolutionnaire du processus) il applique un contrôle post publication, faisant des recherches

\footnotetext{
* Nous n'avons pu tenter une recherche et vérifier la véracité des assertions de F 1000 quant à la pertinence des résultats et ceci principalement en conséquence de la difficulté de l'opération et aussi du fait que lors de la rédaction de ces lignes, le service était très récent et il était difficile (pour ne pas dire impossible) d'avoir un feed back plausible, ce genre d'expérience ayant besoin d'un certain temps avant qu'il puisse être jugé d'autant plus que l'on peut dire, sans risque de se tromper, que ces nouveaux schémas sont révolutionnaires.
} 
déjà expertisées des recherches ré-expertisées une deuxième fois et même allant plus loin en essayant de repérer les perles cachées (hidden jewels), recherches qui seraient passées entre les mailles du contrôle par les pairs et qui en seconde ( ou troisième ou même plus ) lecture recevraient le traitement adéquat et reconnu à leur juste valeur. D'un autre coté, F1000 posters peut être considéré comme un service unique en son genre dans le monde de la publication scientifique. En effet, si les recherches publiés étaient (et sont toujours) assez bien indexées et facilement repérables et consultables, les communications et autres affiches présentées aux conférences, congrès, colloques et autres journées d'études ne le sont pas aussi facilement. Ce genre de documents connus sous l'appellation de littérature grise et dont la définition de la $3^{\mathrm{e}}$ Conférence Internationale sur la Littérature Grise en 1997 ( connue sous l'appellation de la définition de Luxembourg ) - a fixé les contours comme : " Document produit à tous les niveaux des institutions gouvernementales, académiques, commerciales et industrielles, sous forme papier ou électronique, et non contrôlé par les éditeurs commerciaux, c'est-à-dire tout document diffusé par des institutions pour lesquelles l'édition n'est pas l'activité première " ${ }^{(84)}$ - échappe aux circuits traditionnels de publications scientifique. F 1000 posters est venu combler un vide et la richesse de ces affiches (posters) et présentations de diapositives (power point) en a fait un outil très intéressant pour les chercheurs qui voient ainsi leur recherches présentées dans des réunions scientifiques, et qui étaient vouées à être peut être pas perdues mais mal indexées, recevoir toute l'attention et la couverture voulues. Enfin, le service F1000 SmartSearch quoique très récent et n'ayant pas encore fait ses preuves (bien que certains chercheurs l'ayant utilisé l'ait décrit en des termes très élogieux ${ }^{(85)}$ ) semble de par son unique interaction et surtout personnalisation un outil de recherche ( comparable à un moteur de recherche interne ) qui rendrait d'énormes services aux chercheurs en sciences médicales et biologiques qui ont besoin de l'information la plus mise à joue et la plus précise ( ce que ce service se propose de faire d'ailleurs ) dans un domaine où la vitesse de publication et de revendication d'éventuels usufruits de brevets et de découvertes n'est plus à prouver.

\section{4- Electronic Transactions on Artificial Intelligence (ETAI) ${ }^{(86)}$}
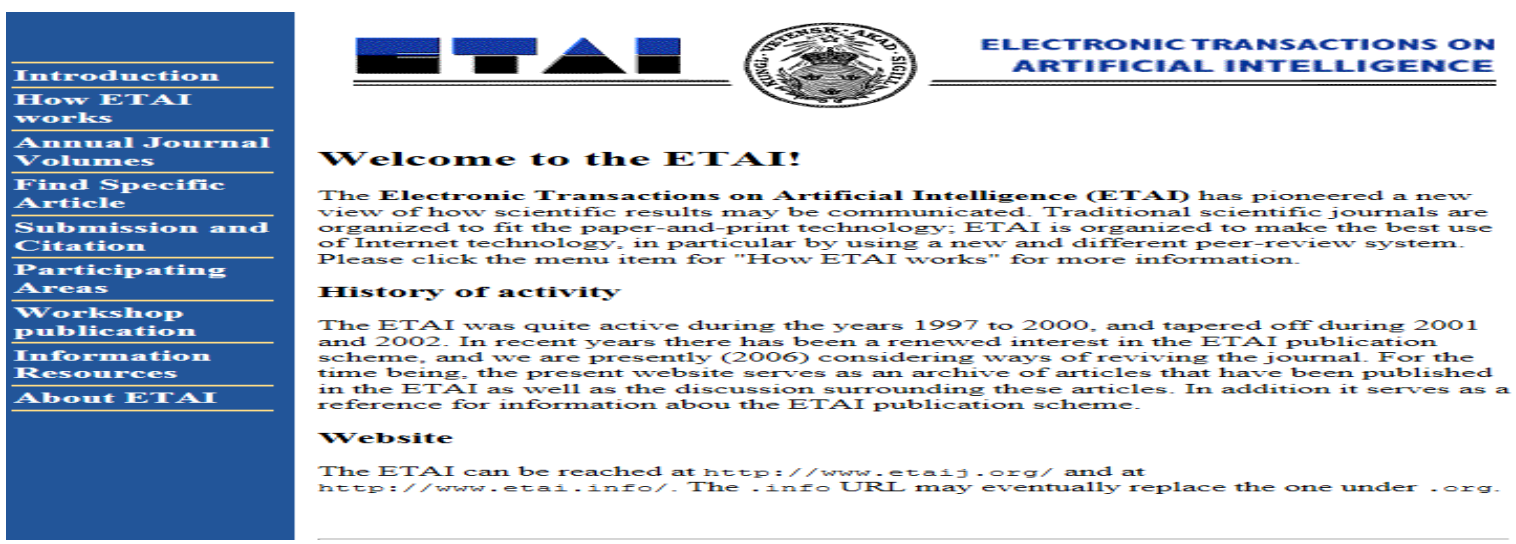

Introduction

Le site " The Electronic Transactions on Artificial Intelligence (ETAI)" est ... " [un journal qui] a innové une nouvelle manière par laquelle les résultats scientifiques sont communiqués. Les journaux scientifiques traditionnels sont organisés de telle sorte à s'adapter à la

${ }^{(84)} \mathrm{http} / / /$ bbf.enssib.fr/consulter/bbf-2010-02-0088-009

${ }^{(85)} \mathrm{http://blog.f1000.com//} \mathrm{(consulté} \mathrm{le} 06$ Juin 2012)

(86) $\underline{\mathrm{http}: / / \mathrm{www} . i d a . \text { liu.se/ext/etai/ }}$ 
technologie du papier et de l'impression ; [alors qu'] ETAI est organisé de telle sorte à faire le meilleur usage de la technologie Internet notamment en utilisant un système de contrôle par les pairs nouveau et différent.......".Ces en ces termes, qui ne laissent aucune place à l'ambiguïté, que se définit ETAI qui se place résolument dans un mouvement d'ouverture de la certification des publications scientifiques. Bien que cela n'apparaît pas clairement, "The Electronic Transactions on Artificial Intelligence“ est édité par The Department of Computer and Information Science - Linköping University en Suéde.

Le site a été assez actif entre 1997 et 2000.A partir de 2001, la parution est devenue de plus en plus erratique et a cessé (du moins d'après les numéros sur les site) ${ }^{\left({ }^{87}\right)}$.Une note (datée du 31 Mai 2006) signale que des efforts sont entrepris pour faire relancer le journal mais il semblerait que ceci n'a pas eu lieu (du moins selon le site) ${ }^{*}$.Malgré l'apparent arrêt de la publication, le contrôle par les pairs appliqué par The Electronic Transactions on Artificial Intelligence mérite que l'on s'y intéresse de par sa manière de procéder et qui s'apparente , plus ou moins, au système de certification d'A.C.P précédemment examiné.

\subsection{1-Le système ETAI}

La manière dont sont expertisées les recherches à ETAI et leurs caractéristiques peuvent être présentées et commentées dans les lignes suivantes :

-Il fournit un processus de discussion ouverte au sujet d'articles et un retour d'écoute pour les auteurs avant même que l'article ne soit publié. Cette discussion est disponible et préservée sur le site web d'E.T.A.I et les participants à la discussion ne sont pas anonymes.

-Contrairement à d'autres journaux qui utilisent le contrôle par les pairs ouvert, ETAI associe une discussion ouverte de l'article soumis avec une expertise confidentielle ultérieure du même article où la décision est prise pour savoir si l'article est accepté ou non.

-En plus de poster les articles acceptés et toutes les discussions à leur sujet (qu'ils aient étés acceptés ou non), le site d'ETAI contient aussi d'autres types d'éléments d'information qui contribuent à ou résultent du processus de recherche.

-Toutes les informations sur le site d'ETAI sont en libre accès : elles peuvent être consultées par toute personne gratuitement et elles le resteront. Le copyright des articles d'ETAI est la propriété de l'auteur et ces derniers s'engagent contractuellement à ce que leur recherche participe dans ce système de libre accès.

-Etant donné qu'il n'existe pas de frais d'abonnement, ETAI n'est sous aucune contrainte de publier un nombre donné de pages par an. Si plus d'articles sont acceptés une année donnée, plus d'articles seront publiés cette année et sans délais de publication supplémentaires, si moins d'articles sont acceptables, moins seront acceptés et de cette manière la qualité des

\footnotetext{
${ }^{(87)} \mathrm{http}: / / \mathrm{www}$. ida.liu.se/ext/etai/

*Dans une correspondance électronique que nous avons eu le $1^{\text {er }}$ Juin 2012 avec Eric Sandewall, l'éditeur en chef d'ETAI, il nous répondu que des efforts étaient entrepris afin de relancer ETAI mais il semblerait que ceci, du moins à notre connaissance, n'ait pas eu lieu.
} 
articles ne sera pas compromise. Les variations de l'importance du volume annuel dans notre système sont naturelles et permises.

\subsection{2-Structure des volumes annuels}

La structure des volumes annuels d'ETAI est expliquée en détails dans la section "Annuals journals volumes ".En effet, le système ETAI adopte une sorte de système hybride entre les caractères électroniques et ceux classiques d'une publication sur papier. Ainsi, il est expliqué qu'ETAI de part sa nature électronique n'est pas automatiquement structurée en volumes se subdivisant en numéros et qu'une numérotation à partir de 1 (un) était possible. Cependant, il a été choisi de conserver la structure usuelle de numérotation par volumes, en partie pour être compatible avec le système de citation et aussi car ETAI parait aussi sous forme papier en livrets reliés.

De ce fait ETAI apparaît en volumes annuels dont le numéro 1 apparut en 1997. Chaque volume est divisé en sections qui vont de $\mathrm{A}, \mathrm{B}, \mathrm{C}$ etc......et chaque section est divisée à son tour en numéros de 1, 2 etc..... La section A est réservée aux articles soumis individuellement et les sections subséquentes pour les numéros spéciaux, la section B étant réservée à " Machine Intelligence Workshops" et ce depuis 1999.

Contrairement aux journaux traditionnels, un numéro d'ETAI d'une année donnée contient les articles soumis l'année en question indépendamment du fait qu'ils aient étés acceptés l'année en question ou plus tard. Ceci est normal pour un système d'expertise ouvert étant donné que l'article est disponible publiquement dés sa soumission. En conséquence, le volume d'une année donnée peut très bien ne pas être complet bien après la fin de l'année.

Chaque numéro est imprimé en un livret séparé. Une section est divisée en plus d'un numéro si le nombre de pages est tellement important qu'il est plus pratique de le scinder en plusieurs livrets tout en maintenant la pagination continue à l'intérieur de chaque section.

Les articles peuvent être ajoutés progressivement à une section dés leur acceptation et le site peut poster des sections incomplètes quand plus de recherches sont attendues dans le futur. La copie imprimée est produite une fois que la section est complète.

En plus d'appliquer un contrôle par les pairs ouvert sur Internet, ETAI pratique aussi une sorte de numérotation déconstruite (c'est nous qui soulignons) qui s'apparenterait à ce que les anglo saxons appellent "overlay journals". Cette appellation (et idée) est pratiquement impossible à traduire mais elle voudrait dire " journaux de recouvrement, de superposition". C'est le cas de journaux qui sont soit :

-" Un journal en libre accès qui prend les soumissions des prépublications déposées dans une archive ....et les fait contrôler par les pairs " (88)

${ }^{(88)}$ Suber, Peter, Guide to the open access movement, 2003 Guide to the Open Access Movement, 2003. Disponible à http://www.earlham.edu/ peters/fos/guide.htm, accédée 12 Juin 2012 
-" Un journal contrôlé par les pairs dont le contenu est déposé dans ou réside dans un ou plusieurs entrepôts en libre accès " ${ }^{(89)}$.

Ces deux définitions font état d'un nouveau genre de périodique scientifique qui ne serait plus un seul support physique continu et compact mais en fait un journal qui se caractériserait par une déconstruction du contenu (regroupant plusieurs articles disparates provenant de différentes sources) mais aussi d'un journal qui physiquement n'existerait pas en tant qu'unité mais serait la somme de plusieurs sous unités ( des journaux différents ). ETAI n'appliquerait pas peut être cette définition d'une manière rigoureuse mais en fait l'appliquerait à l'intérieur de son propre titre (une sorte de journal déconstruit interne).

\subsection{3-Système de contrôle chez ETAI}

A l'instar d'A.C.P que nous avons vu en supra, ETAI utilise un système de contrôle et d'expertise complètement ouvert sur Internet. Les numéros (de 1 à 6 entre 1997 et 2002) et les articles expertisés sont tous présentés de telle sorte à ce qu'un lecteur ou un chercheur puisse suivre la discussion, l'expertise, les réponses, les révisions etc....A titre d'exemple, le volume 1 de l'année 1997 se présente de la manière suivante :

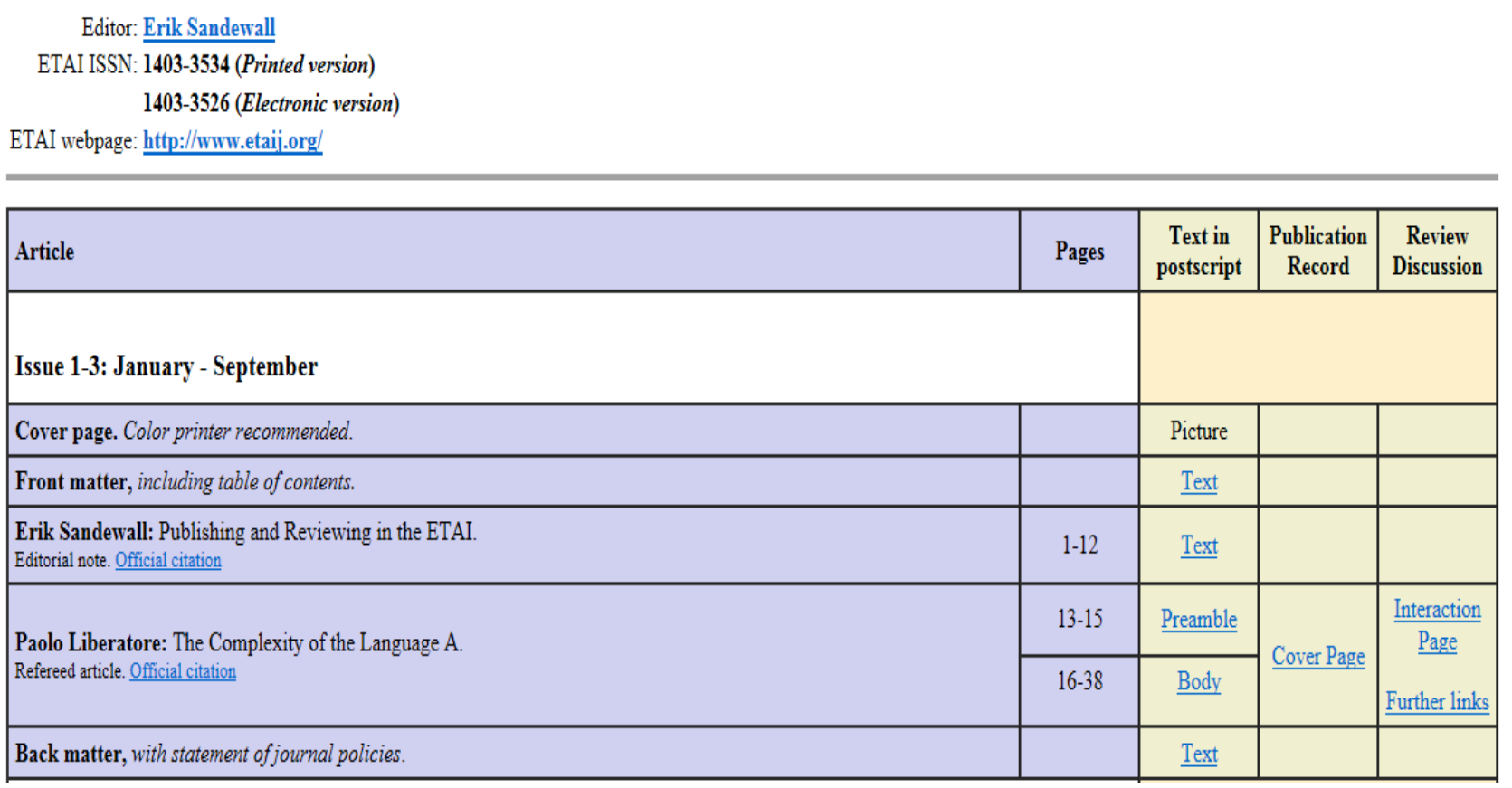

Tel qu'expliqué sur le site, les différentes étapes de la soumission jusqu'à la publication finale sous forme de liens sont clairement indiquées. Ainsi, et selon le site, les différents liens sont :

-Preamble (préface) : contient le résumé, l’historique de publication et le sommaire.

-Body (corps) [du texte]): contient la partie essentielle de l'article. Le contenu combiné de ces deux parties constituent dans la colonne " Text in postscript" exactement le contenu de la présente section du journal.

(89) Repository Interface for Overlaid Journal Archives (RIOJA).Project definition. Disponible à http://www.ucl.ac.uk/ls/rioja/. accédé le 12 Juin 2012 
-Official citation (citation officielle) : conduit vers une page web recommandée quand on cite l'article en question dans la bibliographie d'un autre article par exemple.

-Cover page (page de couverture) : conduit à la (aux) première (s) version (s) de l'article publiée (s) par E-Press pour permettre la discussion du contrôle par les pairs ouverte possible. Elle contient aussi le registre des révisions successives de l'article, s'il y a lieu (dans certains cas elle ne contient que la version finale et non pas la version préliminaire). Le corps de l'article destiné au numéro d'ETAI a été obtenu de la publication E-Press en supprimant ses pages initiales.

-Interaction page (page d'interaction) : conduit à une page contenant l'enregistrement de la discussion d'expertise d'ETAI pour l'article. Les questions additionnelles et supplémentaires au sujet d'un article donné sont toujours bienvenues même après son acceptation par ETAI.

Mais en fait, ces différentes fonctionnalités ne sont pas tous assurées ni opérationnelles et en cliquant sur Preamble (préface), Body (corps [du texte]) ou Text (Texte) par exemple on n'obtient qu'une redirection vers un fichier qui n'est pas reconnu.

\subsection{4-Official citation}

Par contre, quand on clique sur Official citation (citation officielle) pour le premier article dans le numéro, on obtient la page suivante :

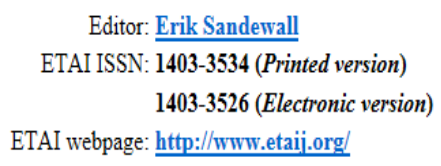

\begin{tabular}{|c|c|c|c|c|}
\hline Article & Pages & $\begin{array}{l}\text { Text in } \\
\text { postscript }\end{array}$ & $\begin{array}{l}\text { Publication } \\
\text { Record }\end{array}$ & $\begin{array}{c}\text { Review } \\
\text { Discussion }\end{array}$ \\
\hline \multicolumn{5}{|l|}{ Issue 1-3: January - September } \\
\hline Cover page. Color printer recommended. & & Picture & & \\
\hline Front matter, including table of contents. & & $\underline{\text { Text }}$ & & \\
\hline $\begin{array}{l}\text { Erik Sandewall: Publishing and Reviewing in the ETAI. } \\
\text { Editorial note. Official citation }\end{array}$ & $1-12$ & $\underline{\text { Text }}$ & & \\
\hline \multirow{2}{*}{$\begin{array}{l}\text { Paolo Liberatore: The Complexity of the Language A. } \\
\text { Refereed article. Official citation }\end{array}$} & $13-15$ & Preamble & \multirow{2}{*}{$\underline{\text { Cover Page }}$} & $\frac{\text { Interaction }}{\text { Page }}$ \\
\hline & $16-38$ & $\underline{\text { Body }}$ & & Further links \\
\hline Back matter, with statement of journal policies. & & $\underline{\text { Text }}$ & & \\
\hline
\end{tabular}

Cette page inclut (comme son nom l'indique) la manière dont l'article doit être cité ainsi que quelques renseignements sur le système ETAI, le texte intégral, l'expertise et la qualité assurance [du système ETAI], la priorité de la recherche ainsi la disponibilité persistante de la citation. Ces deux dernières informations (la priorité de la recherche ainsi la disponibilité persistante de la citation) sont, de nos jours, très importantes car Internet permet de situer très précisément la date de dépôt d'une découverte donnée ainsi que la disponibilité et par delà la pérennité et 1 'archivage de la citation. 


\subsection{5-Cover page}

Par contre, quand on clique sur Cover page (page de couverture) soit à partir de la page où sont présentés les articles du numéro ou alors à partir de " Official citation ", on obtient ce qui suit :

\begin{tabular}{|c|c|c|}
\hline Files: Description & File size & Format \\
\hline Fulltext & & PDF (requires Acrobat Reader) \\
\hline Fulltext, Revised & & PDF (requires Acrobat Reader) \\
\hline Fulltext & & PostScript (requires a PostScript Reader) \\
\hline Fulltext, Revised & & PostScript (requires a PostScript Reader) \\
\hline
\end{tabular}

Author: Paolo Liberatore

Article title: The Complexity of the Language A

Publ. type: Article

Volume: 2

Article No: 6

Language: English

Abstract [en]: In this paper we analyze the complexity of the language A, proposed in [Celfond and Lifschitz, 1993] to formalize properties of actions. We prove that the general language is NP complete, thus intractable, and show some tractable (polynomial) subclasses of it. We also show how states that are unreachable affect the semantics of the language.

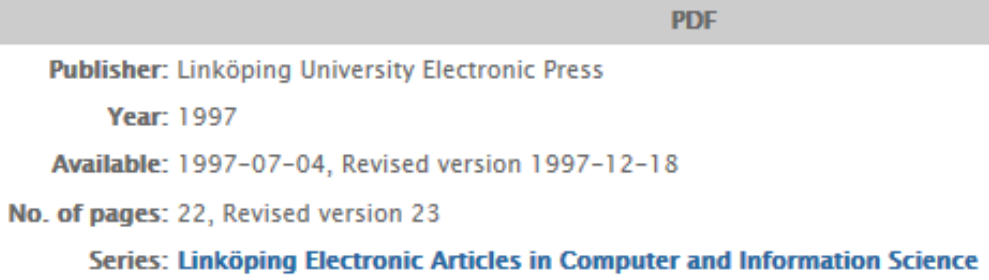

Liberatore, Paolo (1997). The Complexity of the Language A in Linköping Electronic Articles in Computer and Information Science, Vol. 2 http://www.ep.liu.se/ea/cis/1997/006/. ()

Cette page est intéressante à plus d'un titre car elle est une sorte de fiche catalographique de l'article dont elle décrit tous les données telles que :

Les différentes versions du texte, l'auteur, le titre de l'article, le type de publication, le volume, le numéro, la langue, le résumé, l'éditeur, l'année de publication, la disponibilité, le nombre de pages, la collection , l'ISSN et référence de la page en question.

\subsection{6-Review Protocol Page}

La partie la plus importante pour notre travail concerne l'expertise et l'arbitrage, selon les termes utilisés par ETAI, des soumissions directement sur Internet qui est aussi présente sous le nom de Review Protocol Page (Page de protocole d'expertise). Cette page sert non seulement à publier l'article soumis mais aussi et surtout à présenter ce que les responsables d'ETAI ont appelé une " procédure originale d'expertise en deux étapes " et qui sont l'expertise et l'arbitrage. La première ouverte permet à la communauté des pairs de poser des 
questions à l'auteur et de créer une discussion au sujet de la soumission. La deuxième quant à elle, appelée arbitrage, ressemble l'arbitrage conventionnel des journaux à la différence que la majeure partie du retour d'écoute requis est supposée déjà avoir eu lieu dans la première phase d'expertise.

La page du protocole d'expertise est utilisée comme structure de travail pour le processus d'expertise en entier. Durant la première phase d'expertise, elle accumule les contributions successives du débat. Si les experts font des commentaires spécifiques au sujet de l'article durant la phase expertise, ils seront aussi postés dans la page du protocole d'expertise mais sans indiquer l'identité de l'expert. Dans beaucoup de cas, les experts peuvent envoyer une recommandation de " acceptation " ou " rejet " si un retour d'écoute suffisant a déjà été obtenu dans la phase d'expertise. Pour exemple, en cliquant sur Interaction Page (page d'interaction) pour la même soumission prise en exemple, on obtient ce qui suit :

\section{Paolo Liberatore}

\section{The Complexity of the Language $A$}

The article mentioned above has been submitted to the Electronic Transactions on Artificial Intelligence, and the present page contains the review discussion. Click for more explanations and for the webpage of the author, Paolo Liberatore.

Overview of interactions

\begin{tabular}{|r|l|l|l||}
\hline N:o & Question & Answer(s) & $\begin{array}{l}\text { Continued } \\
\text { discussion }\end{array}$ \\
\hline \hline 1 & 11.7 Erik Sandewall & 14.7 Paolo Liberatore & \\
\hline 2 & 22.7 Michael Thielscher & 24.7 Paolo Liberatore & \\
\hline 3 & 22.7 Michael Thielscher & 24.7 Paolo Liberatore & \\
\hline \hline 4 & 28.7 Erik Sandewall & 30.7 Paolo Liberatore & \\
\hline 5 & 21.11 Anonymous reviewer & 24.11 Paolo Liberatore & \\
\hline 6 & 24.11 Thomas Drakengren & 1.12 Paolo Liberatore & \\
\hline
\end{tabular}




\section{Q1. Erik Sandewall (11.7):}

Paolo,

What is the relationship (or is there any) between your results and the results by Nebel and Bäckström [j-aij-66-125] on the complexity of plan validation and temporal projection? Since basic $A$ characterizes updates to the current state, it is at least related to the STRIPS-based framework of classical planning, and deciding the consistency of a set of statements in $A$ would seem to be related to plan validation?

References:

i-aij-66- Bernhard Nebel and Christer Bäckström.

125 On the computational complexity of temporal projection, planning, and plan validation. [abstract] [postscript] [bibtex] Artificial Intelligence, vol. 66 (1994), pp. 125-160.

\section{A1. Paolo Liberatore (14.7):}

This is an interesting question. In general, entailment in reasoning about actions languages such $\boldsymbol{A}$ is strictly related to (deductive) plan validation. Indeed, a plan is a sequence of actions, and it achieves a goal $G$ iff the goal is true after the execution of the sequence (regardless of the initial state).

As for the specific problems of consistency (or entailment) in $A$ and plan validation as defined by Bäckström and Nebel, I think there are substantial differences. Let me explain in short what is the source of intractability of these problems. In A the NP-hardness is due to the incomplete specification of the initial state: one has in general to consider an exponential number of possible initial states. The problems of temporal projection and plan validation analyzed by Bäckström and Nebel are intractable because the possible sequences of actions can be exponentially many. The initial state is fixed (and fully specified),

Comme on peut le voir cette soumission a reçu 6 questions par 4 différents experts (ou du moins des pairs) à la quelles l'auteur a répondu. On remarquera que ces réponses sont pratiquement instantanées (elles ne sont séparés que par deux ou trois jours à part pour une d'entre elle qui a pris une semaine). On peut voir à travers cet échange quasiment en temps réel (dont nous avons reproduit à titre d'exemple le premier entre l'auteur et l'éditeur d'ETAI Erik Sandewall ) les progrès et surtout le changement radical non pas seulement du contrôle par les pairs qui s'affranchit d'un nombre important de défauts tellement décriés ( tels que la lenteur , l'anonymat, le parti pris , etc.....) et dont ces nouveaux schémas sont là pour essayer de les corriger mais aussi changent radicalement la nature de la publication scientifique qui devient une publication dont la progression et l'évolution s'affiche pour tout un chacun à voir et est, en même temps, une publication en constante évolution ce qui peut permettre de corriger certaines fautes ( et même des manipulations de résultats ) qui auraient pu se glisser et échapper à l'examen minutieux du contrôle par les pairs comme cela est arrivé par le passé. 


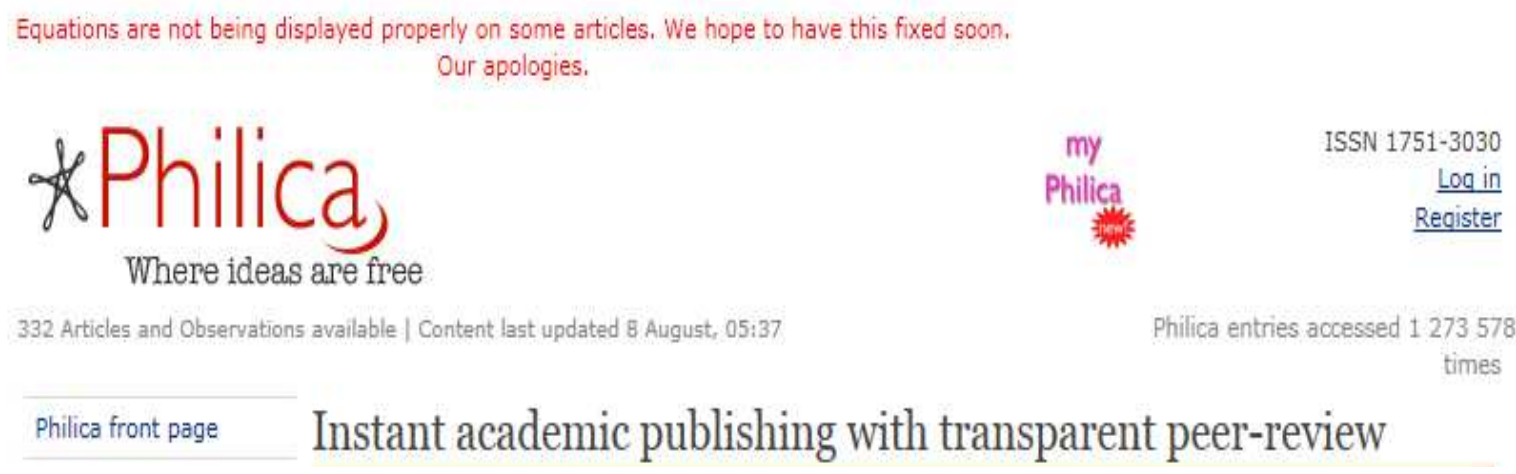

Introduction

Le site de Philica propose une autre approche de la publication scientifique qui diffère complètement des autres sites et journaux électroniques en ligne. En effet, Philica accepte toutes les soumissions et n'impose aucune restriction en amont à tout ce qui lui est soumis. Il est expliqué que le site au lieu de sélectionner lui même par l'intermédiaire d'un système de choix et de tri laisse la communauté des chercheurs soit accepter ou rejeter la soumission. Cette approche est, le moins que l'on puisse dire et nonobstant les résultats, révolutionnaire. En effet, ceci permet à toutes les soumissions de paraître sans aucune barrière que ce soit spatial ou de " qualité ". Nous avons mis " qualité " car le mot clé utilisé par la communauté scientifique dans le contrôle par les pairs est la qualité (on a utilisé l'expression de "séparer le bon grain de l'ivraie" dans ce cas) et les chercheurs (surtout ceux confirmés) tendent à voir dans l'absence - ou du moins l'application lâche - du contrôle par les pairs une sorte de crise de lèse majesté et d'absence de sérieux. Si ceci pouvait être défendu dans l'univers papier (il ne faut pas oublier que le contrôle par les pairs a schématiquement eu deux grands objectifs : limiter la masse de l'information et choisir les meilleures recherches pour la publication) où l'espace était limité, l'univers numérique est quasi infini et permet ce genre d'approche ( certains chercheurs ont même proposé l'abolition pure et simple du contrôle par les pairs et son remplacement par une sorte de certification, de jugement universels et non contrôlés, proposition qui n'a pas reçu l'aval des scientifiques qui, malgré tous leur griefs à l'encontre du contrôle par les pairs, pensent toujours qu'il est indispensable) où tout est publié et où chacun est expert. Ce genre de communisme informationnel est la résultante directe d'Internet et son extrême ouverture et rapidité. Est-il applicable ? Nous allons voir de quelle manière Phillica fonctionne et quelles sont les nouvelles fonctionnalités (s'ils en existent) qu'il introduit dans le monde la publication scientifique contrôlée par les pairs.

\subsection{1-Une courte histoire de Philica}

Philica est un périodique scientifique en ligne acceptant toutes les soumissions. Philica aborde la publication scientifique et le contrôle par les pairs des publications scientifiques d'une

\footnotetext{
${ }^{(90)}$ http://www.philica.com/
} 
manière complètement révolutionnaire. Philica a été créée par deux psychologues britanniques insatisfaits avec la publication scientifique traditionnelle. Cette même industrie de la publication scientifique qui réalise des profits sur les efforts des chercheurs par le biais de restrictions draconiennes du droit d'auteurs limitant considérablement le libre échange d'information aussi bien entre les groupes de chercheurs qu'entre les chercheurs et le public. Philica redonne le contrôle aux chercheurs et leur fournit un forum transparent et ouvert où savoir et réflexion peuvent vivre et croître.

Fondamentalement, Philica fournit toujours un processus de contrôle par les pairs académique permettant un examen critique adéquat des idées et des découvertes scientifiques. Cependant pour la première fois, ce processus est en même temps ouvert et dynamique. Ouvert car les expertises peuvent être vus publiquement, dynamique car les opinions peuvent changer au fil du temps ce qui est reflété dans le processus d'expertise.

Cette courte présentation de Philica ne semble pas différer de prime abord des processus d'expertise précédemment vus car il se fait (le processus) de manière ouverte mais la différence fondamentale se situe au niveau de la soumission qui elle est totalement libre et ne fait pas l'objet d'une pré sélection par une personnalité scientifique (représentée par l'éditeur) mais est totalement libre, la sélection se faisant par toute la communauté qui jugera de la viabilité ou non de la soumission. C'est une approche révolutionnaire mais qui pourrait comporter des écueils et des embûches vu la nouveauté et surtout l'extrême absence de contrôle pré publication qui fait que tout est publié. La science étant méritocratique, cette approche nie cette vérité et n'est pas de toute façon acceptée par tous les chercheurs qui y voient une sorte de clochardisation de la publication scientifique permettant à tout le monde de publier. Cette approche pourrait être acceptée par les chercheurs novices qui y verraient une sorte de démocratisation et de chance à saisir mais pas par les chercheurs chevronnés qui ayant bâtis une réputation ne veulent, peut être, pas être contestés et mis au même niveau que tout le monde.

\subsection{2-Les différentes spécialités couvertes par Philica.}

Le site de Philica englobe 27 spécialités et qui sont : l'anthropologie, l'astronomie et la cosmologie, les sciences biologiques, la chimie et l'ingénierie chimique, l'informatique, l'économie et développement, l'éducation , l'ingénierie ,études environnementales, géographie et géologie, histoire, les sciences humaines, les études internationales, le droit, les langues et la linguistique, les études d'administration et de commerce, les mathématiques, la médecine, la musique, les neurosciences, la philosophie, les sciences physiques, les études politiques, la psychologie, la sociologie et les sciences sociales, les études de transport et enfin la zoologie et les études vétérinaires. Ces spécialités supposées couvrir tout le spectre du savoir ne nous paraissent pas remplir cette mission. En effet, non seulement certaines sciences n'y figurent pas (les sciences nucléaires, l'océanographie par exemple) et d'autres y figurant ne sont soit pas claires (études internationales ou études de transport par exemple) ou alors sont trop imprécises (à l'instar de études d'administration et de commerce). Ces imprécisions et omissions ne sont pas sans effets sur la viabilité et surtout la crédibilité du site. 


\subsection{3-Le problème de la publication scientifique selon Philica}

Comme tout site qui promeut sa manière de procéder, le site de Philica après une brève présentation des avantages et inconvénients (beaucoup plus les inconvénients) du contrôle par les pairs et afin de justifier leur position maximaliste, les concepteurs du site énumèrent les inconvénients du contrôle par les pairs et qu'ils résument en ${ }^{(91)}$ :

-Temps d'expertises inutilement longs

-Recherches rejetées pour des motifs futiles

-Experts ne lisant pas les recherches correctement en raison de contraintes de temps

-Publication bloquée parce qu'un expert travaille sur un sujet semblable

-Experts réagissant d'une manière non professionnelle à la critique de leur travail

-Tendance pour les experts à être des auteurs confirmés et de ce fait d'avoir des préjugés subséquent contre les idées et les méthodologies nouvelles.

-Expertises positives, suivies par " Cependant, je ne suis pas sur que votre soumission soit adéquate pour ce journal - pourquoi ne pas soumettre plutôt à journal X ".

Après avoir cité ces raisons, somme toute classiques, du processus du contrôle par les pairs et des différents problèmes qu'un chercheur rencontre dans sa quête de publier (tels que le temps passé à apporter les correctifs qui résulterait dans l'obtention de la paternité d'une découverte par un autre chercheur, les désaccords avec l'expert qui résulteraient dans le rejet de la soumission, l'envoi de la soumission à un autre journal résultant dans une complète refonte de la soumission et par delà une perte de temps additionnelle, etc.....), Philica affirme :

" Les chercheurs veulent publier "

" Les chercheurs ont besoin de publier " (ont besoin en italique sur le site)

" Philica leur permet de le faire "

Philica se positionne donc, d'après ces déclarations, comme le défenseur des chercheurs injustement traités par les experts et le système de publication scientifique et leur offre un forum où ils peuvent librement publier. Cette position ne prend pas en ligne de compte que la science ne peut être que méritocratique. Que le contrôle par les pairs soit sujet à des dépassements et des comportements le ternissant est un fait avéré que tout chercheur a expérimenté mais de là à simplement l'éliminer et laisser tout se publier avec l'aval de toute personne possédant une connexion Internet nous parait tout simplement dénué de toute légitimité scientifique. Que se passerait-il si un " chercheur " en médecine publiait les résultats de ses recherches sur le SIDA par exemple préconisant la prise de palliatifs médicamenteux non encore testés ? Est-ce que les éventuelles complications que cela entraînerait seraient la responsabilité du site, du chercheur, des " experts " ? Le contrôle par les pairs avec tous ses défauts ne peut être indûment enlevé et remplacé par une sorte sagesse

\footnotetext{
${ }^{(91)}$ http://www.philica.com/tutorial.php
} 
de la foule (wisdom of the crowd ) car selon Ziman, l'expert est la " cheville ouvrière autour de laquelle toute la science est pivotée " ${ }^{(92)}$. C'est dire l'importance du contrôle par les pairs - et de l'expert - pour la bonne marche de la Science qui ne peut se passer de ce qui est un tamis permettant de séparer le bon grain de l'ivraie.

\subsection{4-Le système de fonctionnement de Philica}

La soumission d'articles et d'observations " ${ }^{*}$ se fait part le biais de la page de soumission du site. Le plus intéressant est le fait que le travail est publié instantanément et gratuitement. Il peut être consulté par tout un chacun et peut être expertisé par tous les chercheurs de la profession. Les jours, semaines, mois et même années qui suivent, il sera expertisé par les collègues et a le potentiel d'atteindre toute personne dans le monde connectée à Internet. Philica permet ainsi de mettre les idées dans un espace ouvert comme jamais auparavant. Si le travail soumis est de bonne qualité, il recevra de bonnes expertises par d'autres chercheurs, dans le cas contraire, les avis des chercheurs seront reflétés dans les expertises. Le processus est dynamique et ouvert de telle sorte que les lecteurs peuvent voir non seulement le travail soumis mais aussi l'opinion que les gens ont de ce travail. Jamais les expertises n'ont étés aussi ouvertes que pour cette manière de procéder. Une expertise négative n'empêchera pas de publier mais sera un signe qu'il faudrait peut être penser à reformuler ou alors que les éléments de la soumission devraient être interprétés avec prudence. D'un autre coté, le travail sera expertisé non pas par deux ou trois experts mais par des dizaines et des dizaines de chercheurs.

D'un autre côté, les communications soumises à des conférences ainsi que les travaux en progrès peuvent être soumis à Philica et la communauté peut donner et des retours d'écoute et des conseils. Ces matériaux depuis longtemps relégués à être classés sans suite peuvent revenir à la vie et de fait, à la lumière des nouvelles recherches, d'anciens travaux peuvent prendre une nouvelle signification. Grâce à Philica, le chercheur ne recevra plus cette réponse terrible maintes fois entendue et lue : " Votre recherche est très bonne mais malheureusement ce n'est la place adéquate pour la publier ".

\subsection{5-Le contrôle par les pairs chez Philica}

Le processus de certification de la soumission est comparé à ....eBay ${ }^{*}$ pour les universitaires !!! C'est ainsi que Philica définit son système de contrôle de l'information. Il est expliqué que lorsque quelqu'un expertise votre travail, son impact dépend des certifications que cette même personne a reçu de son propre travail. En effet, le site eBay auquel le système de contrôle de Philica se réfère et se compare a institué un système par lequel les vendeurs qui proposent leur marchandise sur le site sont évalués. Le système est très complexe mais peut être résumé en les avis donnés par les autres e Bayeur et qui comportent :

Est-ce que l'objet proposé est conforme à la description?

\footnotetext{
${ }^{(92)}$ Ziman, John M. , Public knowledge : the social dimension of science, Cambridge [Ma., U.S.A.]: Cambridge University Press, 1968, p.111

* Les observations Philica donnent l'occasion aux chercheurs de décrire des découvertes intéressantes ou curieuses qu'ils ont fait et ceci en pas plus de 2000 mots.

* eBay est une entreprise américaine de courtage en ligne, connue par son site web de ventes aux enchères du même nom. Elle est devenue une référence mondiale dans son secteur et un phénomène de société. En 2009, elle comptait plus de 276 millions de membres inscrits. (http://fr.wikipedia.org/wiki/EBay)
} 
Est ce que la communication avec le vendeur est bonne?

Est ce que les délais de livraison sont respectés?

Est ce que les frais d'expédition et de livraison sont ils acceptables ?

Le vendeur obtient ainsi un taux de satisfaction qui lui est attribué par les différents acheteurs ayant a eu affaire à lui. C'est un système purement économique et commercial d'évaluation auquel Philica se compare et s'identifie. D'ailleurs, le site explique un peu plus loin cette notion en affirmant que ...." Ceci veut dire que l'opinion de quelqu'un dont le travail est tenu en haute estime a plus de poids que l'opinion de celui dont le travail est jugé moins performant. Cette position, ainsi que leur impact sur le jugement qu'ils portent sur le travail des autres change constamment dans le monde dynamique de Philica. Les idées et les opinions changent tout le temps - Philica nous laisse voir cette évolution. Ceci nous montre le monde la publication [scientifique] sous un tout nouveau visage " ${ }^{(93)}$. Le site continue l'explication de la méthode Philica en ces termes: " l'importance d'une expertise donnée dépend de plusieurs paramètres dont les plus importants sont :

-Les évaluations du travail de l'expert sur Philica -les personnes dont le travail est jugé très positivement (et qui de ce fait peuvent être considérés comme de " bons "chercheurs) ont plus de poids.

-Si l'expert a un statut confirmé-les personnes qui nous ont prouvé qu'ils sont de véritables et authentiques chercheurs professionnels sont beaucoup plus influent (plus de détails)

-L'âge de l'expertise -les expertises plus récentes ont un peu plus de poids que les anciennes afin de refléter l'évolution des opinions à l'encontre d'un travail donné. "

Comparativement aux autres systèmes de certification vus en supra, le système de contrôle de l'information soumise souffre, d'après nous, non seulement d'un nombre de défauts mais aussi est basé sur des critères tout ce qu'il y a de plus subjectifs. En effet, si nous analysons la manière dont est faite la certification de la soumission sur Philica, nous nous trouvons en face de schémas qui non seulement sortent des sentiers battus (ce qui est le cas des autres exemples cités en supra) mais en fait dans une sorte de dévalorisation de la fonction la première du contrôle par les pairs sensé sélectionner les meilleures recherches, celles qui méritent d'être publiées. Cette opération - le contrôle par les pairs - avec tous les défauts et la subjectivité qui l'a caractérise et dont l'abondante documentation n'en est que l'expression, a toujours été conduite de la même manière et qu'on peut très schématiquement résumer de la manière suivante : le quitus, l'imprimatur est délivré par ceux qui ont fait leur preuve. Ceux-ci ont dû attendre d'être reconnus pour pouvoir eux même reconnaître. Ces étapes et démarches sont absentes de Philica où chacun expertise, juge, attribue et décide faisant de ce site une sorte de foire aux soumissions où la qualité n'est pas définie par des pairs eux même désignés pour leur expertise. D'ailleurs, nonobstant le caractère un peu trop auto promoteur du site, nous avons remarqué des critères que nous voudrions examiner et les comparer avec les formes traditionnelles et même modernes (telles que vues dans ACP, F1000, etc....) et voir quelles sont les problèmes que ce genre de certification pourrait poser. Examinons les trois critères définissant la publication et la certification sur Philica. Il est dit que " l'importance d'une expertise donnée dépend de plusieurs paramètres dont les plus importants sont :

\footnotetext{
${ }^{(93)}$ http://www.philica.com/tutorial.php
} 
1- Les évaluations du travail de l'expert sur Philica." les personnes dont le travail est jugé très positivement (et qui de ce fait peuvent être considérés comme de " bons " chercheurs) ont plus de poids".

Sur quelles bases peut on considérer une personne comme " un bon chercheur " et qui résulterait (le statut de " bon chercheur ") des évaluations (positives) que ce même " bon chercheur " aurait obtenu en reconnaissance de son bon travail ? Il nous parait que ces définitions souffrent trop d'approximation (définition de " bon chercheur " et " travail jugé positivement ") et sont surtout subjectives. Qui d'ailleurs les délivreraient ? D'un autre coté, cette manière de procéder peut très bien donner lieu à des manipulations pour attribuer le label de " bon chercheur ".On peut très bien imaginer un groupe de chercheurs se liguant afin de s'auto échanger des appréciations de " travail jugé positif " et de label " bon chercheur ".Il est intéressant de voir que ce risque (de voir s'attribuer des bonnes appréciations dans un cadre de "old boy network" - relation de copinage ou d'échange de bon procédé -) a déjà été cité comme l'un des défauts du contrôle par les pairs traditionnel où les chercheurs " se renverraient l'ascenseur " et jugeraient leurs pairs - du moins ceux faisant partie du "old boy network"- d'une manière positive. Comme on peut le voir, le système Philica au lieu de donner la possibilité à chacun de publier en fait augmente cette fracture de publication pour peu que les " bons chercheurs " se liguent et manipulent le système dans le but de faire perdurer le statu quo.

\section{2- Si l'expert a un statut confirmé-les personnes qui nous ont prouvé qu'ils sont de véritables et authentiques chercheurs professionnels sont beaucoup plus influent (plus de détails)}

Ce deuxième critère rejoint l'iniquité du premier critère déjà signalée et en fait l'accentue. En effet, si dans le premier critère les " bons chercheurs " sont désignés selon leur " bon travail " lui même résultat du " jugement positif attribué à ce travail " [par d'autres chercheurs], ce critère est supposé lui donner plus de poids. Mais en fait, la notion de véritables et authentiques chercheurs professionnels (le site utilise le concept de bona fide ${ }^{*}$ ) est une notion créée par Philica appelée "Statut confirmé " attribuée aux chercheurs ayant prouvé leur capacités. Le chercheur qui l'obtiendrait se verrait accordé plus de poids à ces travaux. Il est obtenu en envoyant une lettre signée avec l'en tête de l'institution auquel le chercheur appartient comprenant : nom, adresse, nom d'utilisateur Philica, l'adresse électronique utilisée pour s'inscrire à Philica, le nom et la date de début de travail pour l'institution, poste occupé à l'institution d'appartenance. Aussitôt reçue, le statut de chercheur confirmé sera attribué " si tout est en ordre ". Cette notion de " Statut confirmé " est accompagnée par " une chouette petite icône "[sic] qui accompagnerait toutes les expertises de ceux qui l'obtiendraient. De plus, une fois obtenu, ce statut aura un effet rétroactif, englobant les expertises du chercheur avant l'obtention de ce statut. Cette manière d'agir interpelle quelques remarques. Ce statut tant prisé ne semble pas obéir à des critères scientifiques. Il est obtenu en envoyant des informations les plus élémentaires et accordé " si tout est en ordre " ( si tant est que cette expression veut dire quelque chose dans ce contexte ).Il n'est nullement fait état de publications dans des revues à fort facteur d'impact, de positions académiques, de productions de brevets, d'inventions, etc.... La seule condition est d'envoyer les plus élémentaires des informations et le statut est obtenu avec en prime " une chouette petite icône [sic] ". D'ailleurs, cette rubrique appelée " démontrer son statut " ....." encourage fortement tous les membres de Philica de [nous] prouver qu'ils sont de véritables et authentiques chercheurs

\footnotetext{
* En philosophie, le concept de bonne foi (en latin: la bonne foi, ou de bonne foi pour "bona fide ") désigne une intention sincère, honnête ou une croyance nonobstant l'issue d'une action. Le concept opposé est mala fide qui veut dire mauvaise foi (http://en.wikipedia.org/wiki/Good faith)
} 
professionnels étant donné que ceci aide à instaurer un climat de confiance. Pour les utilisateurs, il existe un nombre d'autres grandes motivations d'obtenir le statut confirmé :

-les expertises des membres confirmés ont considérablement plus de poids que celles des autres membres non confirmés.

-les membres confirmés obtiennent une chouette petite icône $(\checkmark)$ qui apparaît chaque fois que leur nom est cité ainsi que sur leur expertises. Ceci veut dire que les gens ont beaucoup plus de chance de faire confiance en ce qu'ils disent " (94)

Cette manière de distribuer les rôles, les promotions et les lauriers ne semble pas obéir aux plus élémentaires préceptes de la logique. En effet, nous sommes tous, chacun à son niveau, en compétition pour de très prisées - et rares - places dans le Parthénon de la recherche et ceci ne peut être obtenu que par l'intermédiaire du travail et l'effort et non pas l'auto attribution de pseudo titres de chercheurs émérites. Dans une de ces plus importantes contributions ${ }^{(95)}$, Jean Claude Guédon a parlé d'un passage de la " Ploutocratie " (Système politique ou ordre social dans lequel la puissance financière et économique est prépondérante ${ }^{(96)}$ ) vers la République des Sciences dans les archives ouvertes et avait fait ressortir que même dans le contexte de l'Europe du $17^{\mathrm{e}}$ siècle " l'égalitarisme n'avait, bien sur, jamais prévalu du moins pas comme résultat des efforts scientifiques. Au contraire, une très forte atmosphère de compétition - et même de litige et de controverse - a toujours prévalu en science conduisant à un ordre hiérarchique strictement défini de la réputation. Cependant; les règles de jeu étaient aussi égalitaires qu'elles ne l'ont jamais été et on pouvait facilement qualifier la science au $17^{\mathrm{e}}$ siècle comme un système méritocratique d'excellence (c'est nous qui soulignons).Ainsi un ensemble d'éléments homogènes fut obtenu variant de médiocre et insignifiant à excellent et même génial " ${ }^{(97)}$.Cette définition d'un chercheur aussi reconnu que Jean Claude Guédon nous donne une idée sur le fonctionnement et les règles qui régissent la recherche scientifique et qui peut être résumée dans un système méritocratique d'excellence et dont les résultats varient du médiocre au génial. Une sorte d'hiérarchisation s'auto établit sur la base de ces critères qui sont universels et intemporels, hiérarchisation qui n'est nullement établie par le chercheur lui-même mais par ses pairs et son travail. Ces critères ne semblent pas du tout être appliqués chez Philica qui au contraire dans un souci d'extrême démocratisation et dévalorisation de la publication scientifique ne fait pas la différence entre le bon et le mauvais et laisse ce choix au chercheur lui-même, incapable, de toute évidence, de faire objectivement - ce choix.

3-L'âge de l'expertise -les expertises plus récentes ont peu plus de poids que les anciennes afin de refléter l'évolution des opinions à l'encontre d'un travail donné."

Il ne nous semble pas d'après nos lectures sur le sujet que les avis et expertises plus récentes aient plus de poids ou soient plus adéquates dans l'absolu que celles plus anciennes. Bien au contraire, et dans certains cas, les avis et les expertises les plus anciens se sont avérés justes.

\footnotetext{
${ }^{(94)}$ http://www.philica.com/status.php

(95) Guédon, Jean-Claude, Open Access Archives : from scientific ploutocraty to the republic of science, IFLA Journal, Vol 29, n²,p.129-140

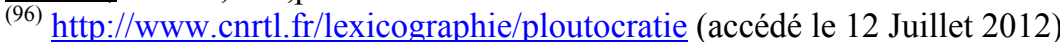

(97) Guédon, Jean-Claude, op.cit., p.129
} 
A titre d'exemple, la célèbre affaire Velikovsky ${ }^{*}$ est tout indiquée pour relativiser et même contredire cette assertion car les différentes étapes par laquelle cette fameuse affaire est passée (ne s'étant éteinte qu'a la mort de Velikovsky en 1979 encore que certains Pro Velikovsky et Anti Velikovsky animent toujours la discussion) ont prouvé qu'en fait les premiers avis émis sont en fait ceux qui ont plus de poids et ont étés acceptés par la communauté scientifique comme ceux qui ont expertisé le travail de Velikovsky de la manière la plus adéquate. Il est vrai d'un autre coté, que les progrès de la science très souvent font faire des découvertes qui contredisent des théories largement acceptées en leur temps à l'image de la théorie du phlogistique émise au $17^{\mathrm{e}}$ siècle. Nous avons cité ces deux exemples diamétralement opposés pour faire ressortir que les tenants et aboutissants concernant le contrôle par les pairs sont loin d'être connus. Comme il a été très largement documenté - dans ce travail et dans la documentation du sujet en général -, cette question est loin d'être solutionné et des recherches indispensables afin de réparer et essayer de rendre plus juste, ouverte cette étape inéluctable de la Science. Ce n'est pas en "démocratisant " à l'extrême (en fait dévalorisant est un mot plus adéquat) et en essayant de mettre des critères iniques que Philica rendra la publication scientifique plus juste et moins sujette à des dépassements. La Science n'accepte ni la médiocrité, ni le laisser aller et encore moins l'auto satisfaction, elle est de quelque angle que l'on se situe et avant tout méritocratique, critère auquel Philica ne semble pas accorder une très grande importance.

\footnotetext{
* L'affaire Velikovsky a son origine dans un livre écrit par Immanuel Velikovsky en 1950 intitulé " Worlds in Collision " (Mondes en collision) et dans lequel il avait, en quintessence, émis des théories sur les catastrophes naturelles ayant jalonné l'histoire de la terre en y incluant des références de différentes sources (psychologie, astronomie, cosmologie, mythologie grecque, hindoue, Ancien Testament, etc....). Ce livre accepté et même applaudit par la critique fut, par contre, rejeté par la communauté scientifique qui y vit une sorte de travail de charlatan dénué de toute approche scientifique. Velikovsky fut traité "d'ignorant et incompétent en science et en scientificité historique : sa méthode était tendancieuse, ses données erronées et ses conclusions impossibles". (Bauer, Henry H., The Velikovsky Affair, La Recherche, p.1448, Décembre 1988).L'affaire ne tarda pas à enflammer la communauté scientifique et de nombreuses réactions qu'elles aient été en faveur ou contre Velikovsky furent enregistrées. Jusqu'à aujourd'hui, ce qui est communément devenue connue comme " L'Affaire Velikovsky " suscite toujours des controverses et des réactions sous formes d'articles, livres et même de journées consacrées à l'étude cette affaire. On en citera quelques uns (parmi les innombrables réflexions) pour ceux qui voudraient s'approfondir et investiguer plus en détails cette affaire qui a enflammé dans les années 50 (et de temps en temps de nos jours) la communauté scientifique. Ce sont :
}

Pearlman, Dale Ann, Ed., Stephen J. Gould and Immanuel Velikovsky: essays in the continuing Velikovsky Affair, Ivy Press, Surrey [England], 1996, 832 p.

De Grazia, Alfred,Ed, The Velikovsky Affair: The warfare of science and scientism, University books, New York ,1966, 260p.

Bauer, Henry H., Beyond Velikovsky: the history of a public controversy: University of Illinois Press, Urbana [Ill., USA] 1999, xiii - 354 p.

Dolby, R. G. A., What Can We Usefully Learn from the Velikovsky Affair ? Social Studies of Science, Vol. 5, No. 2, May 1975, p. 165-175

Ellenberger, Leroy, A lesson from Velikovsky, Skeptical Inquirer, , Vol X, n4, Summer 1986, p. 380-381.

Enfin, en 1974 le thème de la $140^{\mathrm{e}}$ réunion annuelle de la prestigieuse American Association for the Advancement of Science (AAAS) fut " Velikovsky's Challenge to Science ".La parole fut donnée à cette occasion à un nombre de scientifique (dont Velikovsky) pour débattre des théories de ce dernier. Il en résulta un livre dont voici les références :

Goldsmith, Donald W. Ed, Scientists Confront Velikovsky, W. W. Norton, New York, 1977, 188p. 


\subsection{6-Publier sur Philica}

Le site présente de la manière suivante le processus de publication et le genre de publications acceptées par Philica : "Philica offre aux chercheurs un tout nouveau mode de propagation de leurs idées et découvertes au monde. Ici, le travail est publié instantanément et le processus de contrôle par les pairs commence immédiatement après. De cette manière, les expertises aident les lecteurs à interpréter les publications, plutôt que décider qu'elles verront un jour la lumière $\mathrm{du}$ jour ou pas. Publier sur Philica, au même titre que lire les articles, est complètement gratuit " (98).

Philica accepte deux types de soumissions : les articles de fonds et les courtes observations. Ils sont définis de la manière suivante :

\subsubsection{1-Article de fonds :}

Ils correspondent à une recherche soumise à un journal scientifique traditionnel et sont saisis en utilisant un simple traitement de texte en ligne. Ceci permet aux articles d'être importés à partir de traitement de textes autonomes tels que MS Word et peuvent des équations dans le format Tex.

\section{Les articles doivent être complet et non pas des résumés ou des versions abrégées de travaux publiés ailleurs.}

\subsubsection{2- Courtes observations :}

Les courtes observations de Philica permettent en quelques 2000 caractères de décrire des découvertes intéressantes ou curieuses faites par les chercheurs. Elles sont conçues de telle sorte que ces idées puissent être partagées - une autre personne aurait peut être le temps ou l'envie de prendre l'observation et mener un projet de recherche et s'approfondir plus dans le sujet. A ce titre, une courte observation Philica n'est pas faite afin d'être approfondie ou prouvée comme l'est un article. Les équations de format Tex peuvent être utilisées comme c'est le cas pour les articles de fonds.

Les courtes observations Philica sont un débouché idéal pour les études de cas individuels et servent aussi comme un lieu fiable où les idées et les découvertes peuvent être enregistrées de telle sorte que la paternité future ne puisse pas être contestée.

Les observations sont simplement soumises utilisant un formulaire en ligne qui peut prendre des codes standard Tex pour ajouter des formules.

\section{Les observations doivent être autonomes. Ils ne doivent pas être des versions abrégées de travaux publiés ailleurs.}

Cette partie du site est supposée présenter le processus de publication soit disant révolutionnaire de Philica. En fait, il n'est présenté que les types de soumissions (Articles de fonds et courtes observations) et les moyens somme toute banals du côté technique de la soumission. On remarquera cette propension du site à vouloir se présenter comme la solution

${ }^{\left({ }^{98)}\right.}$ http://www.philica.com/submit.php (Accédé le 14 Juillet 2012) 
idoine de tous les chercheurs par son extrême facilité d'utilisation et son extrême ouverture à toutes les idées, ouverture qui en fait, d'après notre étude et surtout comparaison avec les autres sites ayant adopté la même politique, tendrait à le dévaloriser plutôt qu'a le rendre attractif pour les chercheurs. Cette idée d'extrême démocratisation et facilité d'utilisation va être, le moins que l'on puisse dire, infirmé par un lien du site que l'on va examiner dans ce qui suit.

\subsection{7-Travaux ayant besoin d'expertises}

Dans cette partie du site, il est demandé aux experts de bien vouloir expertiser des recherches que leurs auteurs ont mises sur le site. Pour être plus précis, il est dit : " les éléments [articles] suivants ont besoin d'expertises de la part d'experts. Si vous avez des compétences, veuillez évaluer et commenter le travail des [autres] personnes afin de les aider à l'interpréter. Pour expertiser [une soumission], cliquer simplement sur le lien en bas de l'article ou l'observation " ${ }^{(99)}$. Cette page contenait en date du 16 Juillet 2012, exactement 27 articles et 25 courtes observations non encore expertisées. Certaines des soumissions avaient plus de 6 ans depuis qu'elles avaient été déposées mais n'ont pas toujours reçus de réactions. Pourtant, examinant le nombre de fois chacune des soumissions avait été lues (information disponible au bas de la soumission), on s'aperçoit qu'elles l'ont étés d'une manière continue. A titre d'exemple, la soumission la plus ancienne au chapitre des articles date du 26 Septembre 2006 et a été lue 3280 fois ${ }^{(100)}$. Certaines soumissions ont elles été lues entre plus de 6000 et 7000 fois (exactement 7309 fois pour " Exploring the Cross compatibility of the Andreasen (1995) definition of social Marketing and the AMA (2004) definition of Commercial Marketing (101) soumise le 29 Novembre 2006 et 6022 fois pour " The Cosmopolitan Universalism of Aphra Behn's Oroonoko" (102) et soumise le 4 Octobre 2006 ). Quant aux courtes observations, la plus ancienne des soumissions date du 19 Juin 2006 et a été lue 4815 fois. Certaines soumissions au chapitre des courtes observations ont été quant à elles lues entre plus de 6000 et 8000 fois (exactement 6023 pour " Tags Indicate That Open Access Is Flourishing"(103) soumise le 8 Janvier 2007 et 8884 fois pour " What does the term " IT project " actually mean?: a challenge to the IT profession " (104) soumise le 15 Mars 2007).Il est a signaler que le nombre total des articles et courtes observations disponibles était en date du 9 Juillet 2012 de 281 ce qui met le pourcentage de soumissions non expertisées ( malgré le laps de temps qui des fois dépasse 6 ans entre la date de dépôt et la date à laquelle cette observation a été faite) à $18.50 \%$.Philica s'étant [auto] qualifié de " publication académique instantanée avec contrôle par les pairs transparent ", il ne semble pas que ce but soit, le moins que l'on puisse dire , atteint . En effet, le chercheur qui dépose sa soumission dans un tel site s'attend à ce qu'elle soit au moins "expertisée" mais le système mis en place par Philica fait que certaines de ces soumissions ne le sont pas (et ne le seront peut être jamais bien qu'elles soient abondamment

\footnotetext{
${ }^{(99)}$ http://www.philica.com/noreviews.php (Accédé le 16 Juillet 2012)

${ }^{(100)} \mathrm{http} / /$ www.philica.com/display article.php?article id=20 (Accédé le 16 Juillet 2012)

(101) $\mathrm{http}: / /$ www.philica.com/display article.php?article id=62 (Accédé le 16 Juillet 2012)

(102) $\mathrm{http}: / /$ www.philica.com/display article.php?article id=29 (Accédé le 16 Juillet 2012)

(103) $\mathrm{http} / / / \mathrm{www}$.philica.com/display_observation.php?observation id=34 (Accédé le 16 Juillet 2012)

(104) $\mathrm{http}: / /$ www.philica.com/display observation.php?observation_id=36 (Accédé le 16 Juillet 2012)
} 
lues). D'un autre coté, les sujets - et par delà la qualité - de certains sujets sont, pour le moins, douteux. Qu'on en juge :

3.5.7.1- Articles de fonds: nous avons remarqué que sur les 27 articles, 5 l'étaient d'un certain Mitchell M., 4 d'un certain Turtur C. et enfin 3 pour Doglas Y. et 3 pour Nicholson M. De plus, les sujets abordés (tout en respectant les auteurs) ne semblent pas être très " scientifiques " et expliqueraient, peut être, leur non expertise après une si longue période de temps. A titre d'exemple, un certain David Strayhorn a soumis une recherche en Sciences Physiques dont le titre est " Dérivation de la Loi de Born à partir du comptage de résultat et une solution au problème quantitatif de l'interprétation des mondes multiples" (105) alors qu'un autre auteur du nom de Martin Nicholson a soumis une recherche en astronomie et cosmologie dont le titre est " Est-ce que J0233+0105 et J0233+0106 sont des composants d'étoile binaire ?" ${ }^{(106)}$.Au delà de la formulation ( dont nous ne sommes nullement expert pour pouvoir juger de la qualité scientifique ), il ne nous semble pas que ces sujets soient abordés de manière très académique, ni même font partie des sujets intéressant la communauté scientifique ce qui expliquerait ( nous insistons sur le conditionnel) de leur non expertise malgré le nombre élevé de fois qu'elles aient été lues.

3.5.7.2-Courtes observations : de la même manière que pour les articles de fonds, sur les 25 soumissions, 5 l'étaient d'un certain Berman B., 4 du même Turtur C. et enfin 3 pour le même Nicholson M. On retrouve, donc, généralement les mêmes auteurs que dans les articles de fonds et dont le travail n'a suscité aucun commentaire. Les sujets quant à eux ne diffèrent pas quant à la substance et on peut même dire qu'ils " dépassent " les articles de fonds quant à leur étrangeté et bizarrerie. A titre d'exemple, les titres des 5 courtes observations par Berman B., auteur se disant étudier le Temps depuis 40 ans [sic], ne laissent nullement la place au doute. Qu'on en juge :

1-Le Temps et le spectre de l'ordre de grandeur ${ }^{(107)}$, soumise le 29 Avril 2009, consultée 3716 fois et dont l'auteur a mis comme référence : The book "Gravitation", Misner, Thorne, Wheeler.

2-La Loi de l'inverse du carré ${ }^{(108)}$ soumise le 22 Février 2010, consultée 2828 fois et dont l'auteur a ajouté comme " circonstances d'observation " : " Résultat de méditation au dilemme que la Nature abhorre une discontinuité ou une infinité ....."

3- La Physique du temps ${ }^{(109)}$ soumise le 20 Janvier 2011, consultée 1995 fois et dont l'auteur a ajouté comme " circonstances d'observation " : " J'ai étudié le temps plus de 40 ans ".

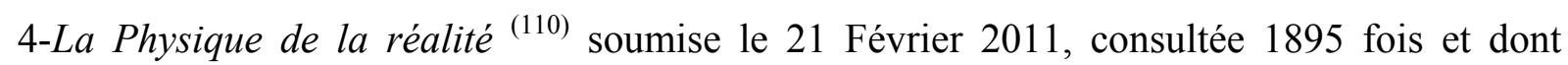
l'auteur a ajouté comme " circonstances d'observation " : " Ceci est une partie d'un article qui sera soumis très prochainement. C'est la culmination de 40 ans d'étude ".

\footnotetext{
${ }^{(105)} \mathrm{http}: / /$ www.philica.com/display article.php?article id=28 (Accédé le 16 Juillet 2012)

(106) $\mathrm{http} / / / \mathrm{www} . p h i l i c a . c o m / d i s p l a y$ article.php?article id=78 (Accédé le 16 Juillet 2012)

(107) $\mathrm{http} / /$ www.philica.com/display observation.php?observation id=53 (Accédé le 17 Juillet 2012)

(108) $\mathrm{http} / /$ www.philica.com/display observation.php?observation id=57 (Accédé le 17 Juillet 2012)

(109) $\mathrm{http} / / /$ www.philica.com/display observation.php?observation id=64 (Accédé le 17 Juillet 2012)

(110) $\mathrm{http}: / /$ www.philica.com/display observation.php?observation id=65 (Accédé le 17 Juillet 2012)
} 
5- Communication de modulation du temps ${ }^{(111)}$ soumise le 16 Mars 2012, consultée 395 fois et dont l'auteur a ajouté comme " circonstances d'observation " : " Etudes en cours du Temps ".

Nous avons cité cet auteur (comme cela aurait pu être le cas pour d'autres) car il a publié le plus d'observations courtes non expertisées (5). En plus du fait que même Philica n'est pas sure de son établissement de rattachement, ni de son statut de chercheur bona fide (le système Philica si friand de " chouette petite icône " accole un " ?" " à coté de son nom ce qui indique qu'il n'est pas un utilisateur confirmé bien que sa soumission la plus ancienne date de plus de 3 ans .....) * , les différentes " circonstances d'observation " (comme celle énonçant " Résultat de méditation au dilemme que la Nature abhorre une discontinuité ou une infinité... ") laissent perplexes et dubitatifs quant à leur utilité. Enfin, le sujet lui-même " l'étude du temps " prêterait à sourire n'était ce le supposé sérieux de l'entreprise. Dans la même veine, dans une autre soumission (non expertisée) intitulée "Induction d'une clairvoyance accrue à travers le jeune" (112) l'auteur examine l'influence qu'à le jeune sur l'acuité visuelle de l'être humain .Il en a conclut qu'à partir du deuxième jour de jeune ( on remarquera que le chercheur dit explicitement en préambule " Au mois de Juillet 2010 , j'ai commencé à essayer de jeûner), il avait expérimenté un accroissement de sa perception des choses : il pouvait voir des choses lointaines qu'il n'aurait pas vues sans jeûne. Sa conclusion est que, l'Homme moderne n'ayant pas à jeûner de longues périodes comme c'était le cas pour l'Homme préhistorique, il acquiert cette faculté en jeûnant de longues périodes. L'auteur avait fait l'expérience plusieurs fois et avait remarqué cette extra sensorialité accrue à partir du deuxième jour. Sa conclusion est que le jeûne augmente la perception et la vue des choses lointaines et que cette fonction était plus présente chez l'Homme préhistorique car il pouvait passer (était obligé même) de longues périodes à jeûner s'il n'arrivait pas à capturer le gibier pour sa subsistance. Nous ne sommes pas spécialiste en psychologie mais cette soumission ne nous parait pas répondre aux critères les plus élémentaires de la méthodologie scientifique et ceci dans les points suivants : -L'auteur a-t-il fait la même expérience avec un groupe de contrôle ?

-L'auteur n'est il pas victime d'un effet placebo ?

-L'auteur a t il jeûné ou " essayer de jeûner " ?

-L'auteur a-t-il répété cette expérience durant les différentes périodes de l'année ?

- L'auteur n'était il pas conditionné à croire ce qu'il voyait? (ou pensait voir)

Autant de questions (et d'autres comme le stimulus par exemple que des chercheurs plus chevronnés en psychologie pourraient détecter) que le chercheur n'adresse pas dans sa soumission et qui font que sa recherche, une recherche méthodologiquement faible.

En conclusion de cette partie concernant les soumissions ayant besoin d'expertises, il nous parait que c'est le système (on dira même la philosophie) de Philica qui est en cause. Cette attitude débridée vis-à-vis du contrôle par les pairs (son absence même comme filtre primaire) a pour conséquence une flopée de soumissions dont la qualité n'est a pas à proprement parler de haute facture.

\footnotetext{
(111) http://www.philica.com/display observation.php?observation id=74 (Accédé le 17 Juillet 2012)

* Toujours dans le même esprit d'accoler de chouettes petites icônes aux noms des chercheurs soumissionnant, Philica a ajouté (en plus des signes $\mathcal{H}$ pour les membres confirmés et ? pour les utilisateurs dont la qualité de bona fide n'est pas confirmée), le signe $\nless$ pour ceux qui ont fait une donation et les appelés "officiellement génial " ( pour être plus précis il est écrit "Cet utilisateur est officiellement génial ".....)

${ }^{(112)} \mathrm{http}: / /$ www.philica.com/display observation.php?observation id=66 (Accédé le 17 Juillet 2012)
} 


\subsection{8-Les articles les plus vus ${ }^{(113)}$}

Cette rubrique se propose d'établir un classement des articles (et courtes observations) les plus vus (non pas les plus lus). C'est une liste des 15 articles et 15 observations les plus lus avec le nombre de fois que cela a été fait .Ainsi, l'article le plus vu est intitulé " La Source d'énergie du soleil n'est pas la fusion nucléaire mais des champs magnétiques du centre de la galaxie. Le soleil convertit l'énergie en masse et non pas la masse en énergie " ${ }^{(114)}$,soumis en date du 11 Décembre 2006 et vu (en date du 18 Juillet 2012) 34782 fois. Malgré ces données statistiques, l'article ne semble pas avoir reçu des expertises très élogieuses car dans une autre rubrique intitulée " Information sur l'article ", la notation à partir de trois expertises n'a donné que les chiffres suivants :

Originalité $=14.74$

Importance $=13.56$

Qualité d'ensemble $=11.50$

Tout en sachant qu'un score de 100 représente un niveau "moyen". D'un autre coté, les expertises sont publiées avec l'article et se présentent de la manière suivante:

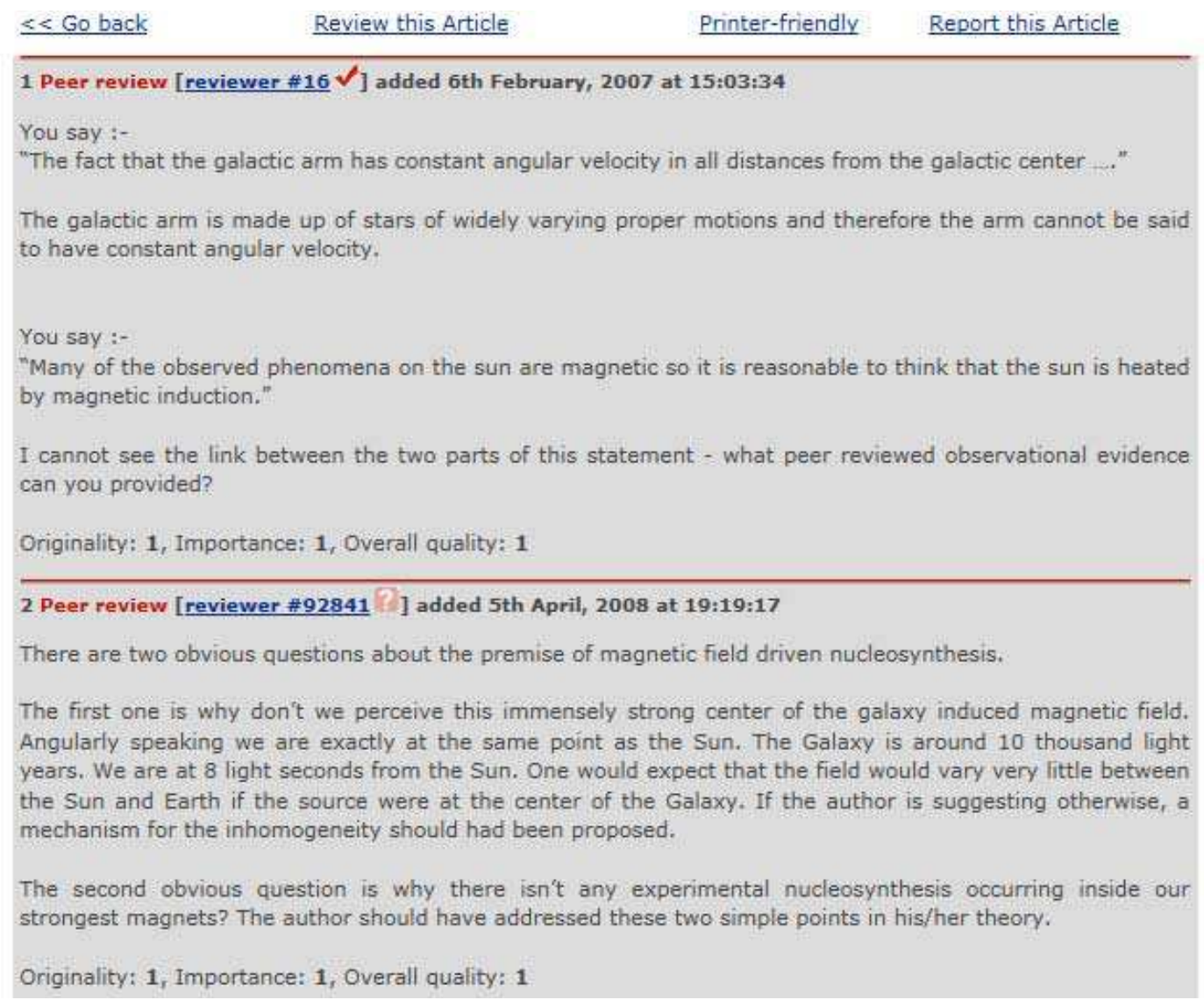

Pour poster une expertise, l'utilisateur doit s'inscrire et obtenir un nom d'utilisateur avec lequel il peut se connecter. Une fois qu'il veut expertiser un article ou une observation, il doit cliquer sur " expertiser cet article " (ou observation) et il obtient le formulaire suivant :

(113) http://www.philica.com/top10pop.php (Accédé le 18 Juillet 2012)

(114) $\mathrm{http}: / /$ www.philica.com/display article.php?article id=65 (Accédé le 18 Juillet 2012) 
Enter your anonymous review of this Article here

Please aim to keep your review objective and informative - good reviews rarely have just one or two lines. Provide constructive comments rather than criticism.

You may use HTML tags if you like.

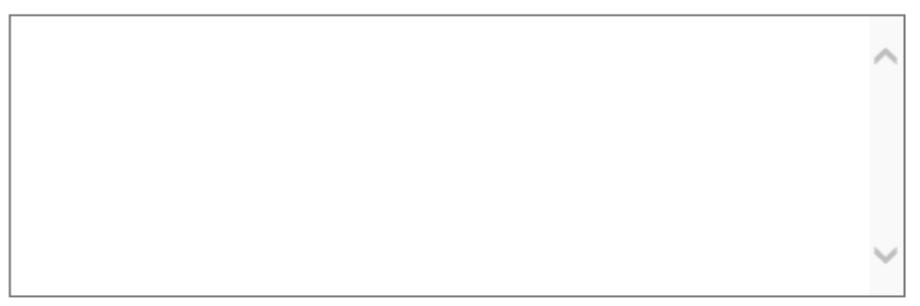

Please rate this Article on the following criteria

where 1 is very poor, 4 is average, and 7 is excellent

Originality

$\mathrm{O}_{1} \mathrm{O}_{2} \mathrm{O}_{3} \mathrm{O}_{4} \mathrm{O}_{5} \mathrm{O}_{6} \mathrm{O}_{7}$

Importance

$\mathrm{O}_{1} \mathrm{O}_{2} \mathrm{O}_{3} \mathrm{O}_{4} \mathrm{O}_{5} \mathrm{O}_{6} \mathrm{O}_{7}$

Overall quality of the Article

$\mathrm{O}_{1} \mathrm{O}_{2} \mathrm{O}_{3} \mathrm{O}_{4} \mathrm{O}_{5} \mathrm{O}_{6} \mathrm{O}_{7}$

Unless you sign your review, which you are welcome to do if you wish, it will be anonymous to the author and to other Philica readers. Nevertheless, the administrators can see who you are if necessary so please be sure your review is not abusive.

Check your comments carefully before submitting - they cannot be edited in the future. Are you completely happy with what you have written? Are you really an expert in this field?

By submitting this review, you agree to license it for general use under the GNU free documentation license.

Submit your opinions

Le formulaire à remplir parait très sommaire quant à la notation (de 1 à 7) et les critères de jugement (Originalité, importance et qualité d'ensemble) le sont aussi". On remarquera seulement cette question surréaliste adressée à l'expert et qui dit:" Etes vous complètement content de ce que vous avez écrit ? Êtes-vous vraiment un expert dans cette spécialité ? ". Est il possible pour un site sérieux de demander à l'expert est ce qu'il est content de ce qu'il a écrit et surtout est ce qu'il vraiment est un expert? Le contrôle par les pairs tel qu'il est connu et pratiqué (que cela soit de la manière traditionnelle ou ouverte tel qu'il se pratique depuis l'avènement d'Internet et dont nous avons présenté quelques exemples dans ce travail) est réservé à ceux qui ont fait leurs preuves et qui n'ont pas besoin (dans la plupart des cas du moins) de se déclarer et justifier de leur qualité d'expert. Tout chercheur, quand il soumet une recherche (que ce soit dans un journal académique ou même pour une conférence) reconnait généralement les noms de ténors de la spécialité qui jugent les travaux des autres parce qu'ils ont publiés, encadrés, enseignés etc.... et non pas à la suite d'une quelconque déclaration ou auto attribution du titre d'expert. Philica, encore une fois, dévalorise la certification de l'information et par delà, ne rend service ni à la Science et encore moins aux chercheurs qu'il est supposé défendre en leur donnant une solution à leur problème de publication. Il leur permet de publier, peut être, mais sans la qualité et ceci ne peut être au service de la Science qui, au risque encore de nous répéter et d'insister, est méritocratique.

\footnotetext{
* On remarquera que les moyens de calcul de la note attribuée pour l'originalité, l'importance et la qualité d'ensemble n'est pas donnée sur le site. Tout ce qu'on sait c'est qu'à l'expert est donné une sorte de barème de 1 à 7 qu'il doit noter. Ceci est d'autant plus intéressant à savoir que les notes attribuées semblent aléatoires et vont des plus basses aux plus hautes
} 


\subsection{9-Les travaux les mieux notés ${ }^{(115)}$}

Cette rubrique recense les travaux ayant obtenu les notes les plus hautes dans les trois critères précédemment signalés. Le site signale que la notation peut changer à tout moment et ceci est affecté par la notation donnée chaque travail de recherche ainsi que l'opinion actuelle à l'encontre du travail de l'expert lui-même. Cette rubrique ressemble à s'y méprendre à la rubrique précédente des travaux les plus vus (ou les plus populaires c'est selon l'appellation du site) et est présentée sous le titre de " le meilleur truc sur Philica en ce moment même ".Les meilleurs recherches (quelles soient articles ou observations) sont présentées, encore une fois, sans qu'il y ait d'indication quant à l'obtention des notes. Notes qui semblent être le résultat d'un calcul assez précis car les notes sont par exemple : $242.37^{(116)}, 193.26$ ${ }^{(117)}$ ou alors $175.43^{(118)}$.Du point de vue pratique, ils (articles et observations ) sont notés selon l'originalité, l'importance et la qualité d'ensemble séparément et il n'existe pas de notation générale des trois critères pour chaque article ou observation. On remarquera enfin, la bizarrerie des sujets abordés comme dans les autres rubriques du site. A titre d'exemple, l'article le mieux noté concerne " La musique durant l'exercice: Est-ce que le tempo influence les réponses psychophysiques " ${ }^{(119)}$ qui a obtenu les meilleurs notes dans les trois critères cités. Quant au deuxième article le mieux noté, il concerne " Les effets du temps de la journée dans le dépassement des bicycles par les conducteurs " ${ }^{(120)}$ qui a été classé deuxième à part dans le critère qualité d'ensemble où il est classé troisième. Quant aux observations, c'est une observation au sujet très similaire et par le même auteur qui obtient le plus haute note .C'est " Dépasser les bicycles au Royaume Uni : voitures par rapport aux véhicules légers [de transport] de marchandises " ${ }^{(121)}$ qui a été classée première en qualité d'ensemble et originalité et seconde en importance. A l'instar des articles, ces observations nous ont parus aussi dénuées de méthodologie scientifique ne serait que par leur énoncés. En effet, quelle est l'importance de la différence entre la manière dont les voitures ou les véhicules légers [de transport] de marchandises dépassent les bicycles ? Encore une fois, cette rubrique donne une impression de répétition et surtout de superficialité des sujets, superficialité qui s'est vérifié de puis le début de notre examen et analyse du site.

\subsubsection{0-Expertises récentes ${ }^{(122)}$}

Cette rubrique énumère les expertises les plus récentes ayant eu lieu sur le site. On remarquera que les dates ne comportent pas d'année dans la date. A l'instar des autres rubriques, un distinguo est fait entre articles et observations qui sont respectivement au nombre de 7 et 6 . On remarquera aussi que certaines observations ou certains articles ont plus d'une expertise et ceci est signalé sur la page.

Le reste des rubriques sont des rubriques d'ordre pratiques et sont :

-Comment citer Philica

-F.A.Q.s (les Questions les plus Fréquemment Posées)

-Supporter Philica [financièrement]

-Nous contacter

${ }^{(115)} \mathrm{http}: / /$ www.philica.com/top10rate.php (Accédé le 21 Juillet 2012)

(116) http://www.philica.com/display article.php?article id=24 (Accédé le 22 Juillet 2012)

(117) $\mathrm{http} / / /$ www.philica.com/display article.php?article id=14 (Accédé le 22 Juillet 2012)

(118) $\mathrm{http://www.philica.com/display} \mathrm{article.php?article} \mathrm{id=161} \mathrm{(Accédé} \mathrm{le} 22$ Juillet 2012)

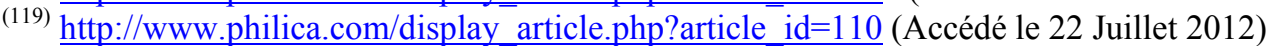

(120) $\mathrm{http://www.philica.com/display} \mathrm{article.php?article} \mathrm{id=24} \mathrm{(Accédé} \mathrm{le} 22$ Juillet 2012)

(121) $\mathrm{http}: / /$ www.philica.com/display observation.php?observation id=10 (Accédé le 22 Juillet 2012)

(122) $\underline{\mathrm{http}: / / w w w . p h i l i c a . c o m / r e c e n t r e v i e w s . p h p}$ (Accédé le 22 Juillet 2012) 
-Obtenir statut confirmé

Conclusion et comparaison des quatre modèles examinés

Les expériences concernant les nouvelles formes de certification de l'information scientifique et technique ont, depuis l'avènement et d'Internet, abondés. Tantôt appelées contrôle par les pairs ouvert (utilisé dans le sens de contrôle par les pairs sur Internet et qui en fait concerne le contrôle par les pairs où le (les) nom (s) de l' (es) auteur (s) et des experts sont connus et qui est un des palliatifs aux nombreuses critiques adressées au contrôle par les pairs traditionnel) ou contrôle par les pairs électronique, ces nouvelles formes ne sont pas encore complètement ni acceptées et encore moins testées de manière conclusive. Ceci est du fait qu'elles n'ont pas eus le temps d'être analysées et étudiées de manière approfondies afin de déterminer leur impact sur la publication scientifique et aussi car elles suscitent de la part des différents acteurs de la publication scientifique (surtout les auteurs soumettant) des réticences et des méfiances somme toute normales. Nous avons dans cette partie de ce travail examiné quelques une des expériences les plus innovantes ${ }^{*}$ au nombre de quatre (ACP, F 1000, ETAI et Philica) et nous pouvons en tirer les conclusions primaires suivantes :

\footnotetext{
*Nous avons choisi ces sites car disponibles et dont le processus est entièrement visible par n'importe quel personne avec une connexion Internet. D'autres expériences qu'on peut citer (liste non exhaustive) sont :

1- Biology Direct (http://www.biology-direct.com/authors/peerreview) et dans lequel l'auteur est responsable du choix des experts potentiellement adéquats pour expertiser son travail et ceci à partir du comité éditorial disponible à http://www.biology-direct.com/about/edboard. Enfin, les commentaires des experts sont publics et publiés avec l'article expertisé. Il est à noter que Biology Direct applique la règle "auteur payeur" qui s'élève à $1250 £(\sim 1960 \$ / 1605 €)$

2- British Medical Journal (BMJ)-Rapid response (http://www.bmj.com/comment/rapid-responses) qui sont " des lettres électroniques à l'éditeur ".Elles permettent aux utilisateurs de débattre des problèmes soulevés dans des articles publiés sur bmj.com. Bien qu'une sélection des réponses rapides sera incluse comme lettres de lecteurs éditées dans le numéro imprimé hebdomadaire de BMJ, leur première apparition en ligne implique que ce sont des articles publiés. Il est à noter que ce service a connu un grand succès passant de 20000 réponses pour la periode 1998-2002 à 50000 en 2005 (http://homepages.vub.ac.be/ clvidal/writings/Vidal_2005a_Le commentaire ouvert.pdf, p.6) En date du 23 Juillet 2012, il y avait 86533 rapides réponses.

3- Journal of Interactive Media in Education (http://www-jime.open.ac.uk/ ).Dans cette variante, le cycle de soumission passé par trois phases fondamentales et qui sont : la revue par les pairs privée (avec trois pairs), la revue par les pairs publique et la publication. Cette forme est représentée par le schéma suivant (http://homepages.vub.ac.be/ clvidal/writings/Vidal_2005a_Le commentaire_ouvert.pdf, p.5)
}

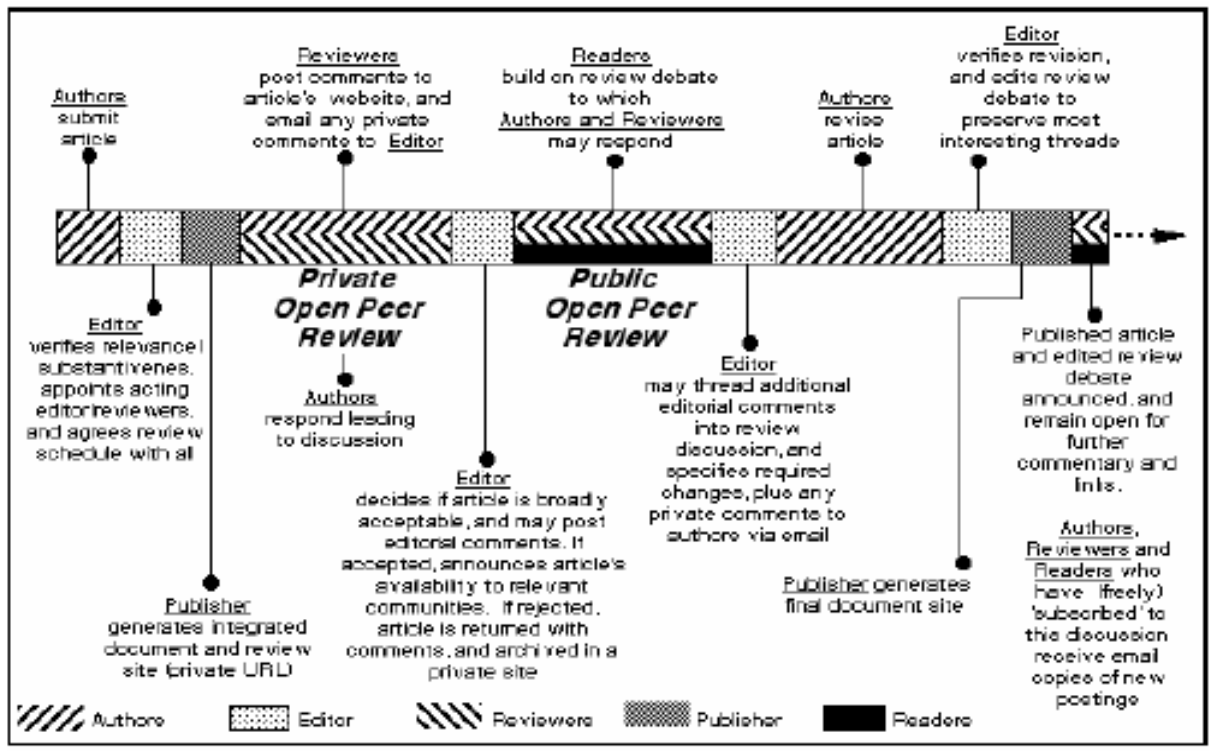

Schéma d'un cycle de soumission d'article au $J M E^{10}$. 


\subsubsection{1-Conclusion et comparaison des quatre modèles examinés}

-Les sites d'A.C.P. et de F1000 semblent être les sites dont le fonctionnement obéit aux règles les plus méthodologiques et les plus professionnelles

-Ces deux sites sont réussis parce qu'ils concernent deux spécialités dans laquelle le contrôle par les pairs est primordial (surtout en ce qui concerne F 1000 qui couvre les sciences biologiques et à un degré moindre $\mathrm{ACP}$ qui concerne les sciences physiques et chimiques. Ce dernier bénéficiant de l'aide et support de l'Union Européenne des Géosciences, ce qui semble lui donner plus de moyens et de certification)

-Le site d 'ETAI quant à lui, bien qu'ayant été suspendu jusqu'à nouvel ordre pour des raisons multiples, ne représente pas moins une expérience originale surtout que le sujet de l'intelligence artificielle est méconnu et peu abordé.

-Le site de Philica quant à lui représente une tentative, que nous jugeons personnellement non fructueuse ni concluante, de libérer totalement la publication scientifique des contraintes du contrôle par les pairs. Si l'idée en elle-même est louable, il n'en demeure pas moins que Philica au lieu de donner une chance à ceux que le contrôle par les pairs traditionnel a lésé et exclu à cause de critères jugés trop draconiens, a, au contraire, dévalorisé la publication scientifique en permettant à toute personne avec une connexion Internet de publier. De plus, la fonction d'expert, nodale dans le système de la Science, a elle aussi été mise à mal et est devenue non pas acquise après l'effort et le travail mais auto attribué par l'expert lui-même.

Malgré toutes ces péripéties, ces différentes tentatives ne répondent qu'à une seule raison : rendre le contrôle par les pairs plus responsable et moins secret en le mettant sur Internet au vu et au su de toute personne avec une connexion. Cette permissivité et surtout ouverture, nouvelle dans le monde de la publication scientifique, ne peut être jugée car trop récente et comme toute expérience sans recul, les résultats ne peuvent être analysés, les chercheurs euxmêmes n'étant pas sur des avantages et inconvénients de cette révolution. Comme il a déjà été souligné, ces expérimentations n'ont étés possible que grâce à Internet et du libre accès qui, malgré leur extraordinaire progrès (surtout Internet), n'ont étés lancés qu'à la fin du siècle dernier et n'ont de ce fait qu'un peu plus de deux décennies d'âge. Période et laps de temps amplement insuffisants pour être jugé sur des bases solides.

\section{Chapitre IV}

\section{QUESTIONNAIRE CDTA ET CERIST}

Introduction

Après avoir examiné tous ces développements, tendances, études et controverses dans le monde occidental, il nous a paru utile et même obligatoire d'essayer de voir quelle est la position des chercheurs algériens face cette nouvelle orientation que prend la recherche scientifique dans le monde. Il n'échappe à personne que la recherche est internationale (ou du moins est supposée l'être) et que les barrières (qu'elles soient linguistiques, religieuses, nationales ou autres) ne devraient pas rentrer en ligne de compte. En fait, ceci n'est pas le cas 
car un certain nombre de critères et de conditions ne sont pas les mêmes pour un chercheur venant du Sud qui doit faire face à des contraintes et obstacles que celui du Nord ne connait pas. De ce fait, alors que la science est supposée être méritocratique et récompenser celui (ou celle) qui est le (ou la) meilleur, il récompense celui (ou celle) qui a le plus de moyens nonobstant son mérite. Nous avons déjà cité Farid Dahdouh-Guebbas et Benjamin Acosta Cazeres qui avaient parlé de respectivement "Neo-colonial science" et "Scientific colonialism and safari research" et dans les quels ils avaient fait ressortir, qu'à armes égales, un chercheur du Sud ne pouvait pas concurrencer son collègue du Nord. Même plus, ces mêmes chercheurs se faisaient d'une certaine manière déposséder du fruit de leur travail et Dahdouh-Guebbas l'a même qualifié " d'exploitation des chercheurs locaux ". Afin de vérifier ces données et ces hypothèses localement, nous avons décidé d'investiguer ces problématiques en soumettant à deux institutions algériennes que nous avons jugé les plus aptes (de par leur orientations et missions ) à répondre à ces questionnements un questionnaire dont le but est d'enquêter sur les habitudes de publication des chercheurs ainsi que leur degré de savoir et d'inclusion dans des schémas de publication très nouveaux et que les chercheurs doivent connaitre afin de s'insérer dans le concert universel de la recherche. Ces deux institutions sont le Centre de Développement des Technologies Avancées (CDTA) et le CEntre de Recherche en Information Scientifique et Technique (CERIST).Ces deux centres ont étés choisis pour leur disponibilité * et le fait qu'ils représentent des domaines opposés de la recherche ceci a été fait afin de voir et de sonder les habitudes de deux communautés de chercheurs différentes et aussi peut être confirmer (ou infirmer ) les différentes hypothèses quant à l'utilisation des nouvelles technologies par ces mêmes communautés dans leur quête de publication. Avant d'analyser et de dépouiller les questionnaires des deux centres précités, nous les présenterons d'une manière brève insistant sur leurs objectifs et missions.

\footnotetext{
*Nous tenons à préciser que cette donnée de la disponibilité a été un des problèmes que ces questionnaires pourraient poser de part et l'échantillonnage et aussi des réponses. En effet, le CDTA et le CERIST ont étés choisis parce qu'ils représentaient deux centres dont les habitudes de publication pouvaient être différentes de part leur domaine de couverture. Malheureusement, après que notre demande de distribution des questionnaires eut été reçue avec une grande joie par les responsables du CDTA (par la responsable de la bibliothèque pour être plus précis), nous fumes traité d'une manière indigne de chercheurs de "Technologies Avancées ", contrairement aux chercheurs du CERIST qui répondirent selon leurs moyens à notre questionnaire. Ce traitement se " matérialisa "- si on s'exprimer ainsi- par une absence de réponses malgré nos relances incessantes et nos innombrables mails. Nous fumes victime de réponses condescendantes de la part des chercheurs qui s'étonnaient que nous nous adressâmes à eux directement alors que nous avions reçu l'aval du Directeur du Centre quant la distribution du questionnaire. Nous eûmes l'occasion de rencontrer la responsable de la bibliothèque qui avait accepté avec tant d'enthousiasme de nous aider et elle nous fit comprendre que ce changement brutal était du à un changement de direction du Centre. Toujours est il que nous manageâmes d'arracher le nombre de 31 questionnaires sur un total de 92 chercheurs toujours au centre (le nombre total à l'époque où nous eûmes à endurer cette épreuve était de 131 inscrits).Nous avons relaté cet épisode malheureux pour expliquer un quelconque défaut méthodologique quant au nombre de répondants mais aussi et surtout pour pointer du doigt une certaine " frilosité " qu'ont certains responsables à communiquer des informations aussi bénignes que les habitudes de publication de chercheurs dont la fonction la première est de .....communiquer. Tous ces péripéties (dont certaines frisant l'insulte) sont disponibles grâce à la fonction " archive " (c'est le cas de le dire pour notre sujet) que peut avoir Internet...... Cette mise au point n'est pas faite dans un but polémique mais pour tout simplement pointer du doigt les dysfonctionnements de la recherche scientifique dans notre pays qui obéit à des critères loin d'être scientifiques.
} 


\subsection{1.-Le CERIST}

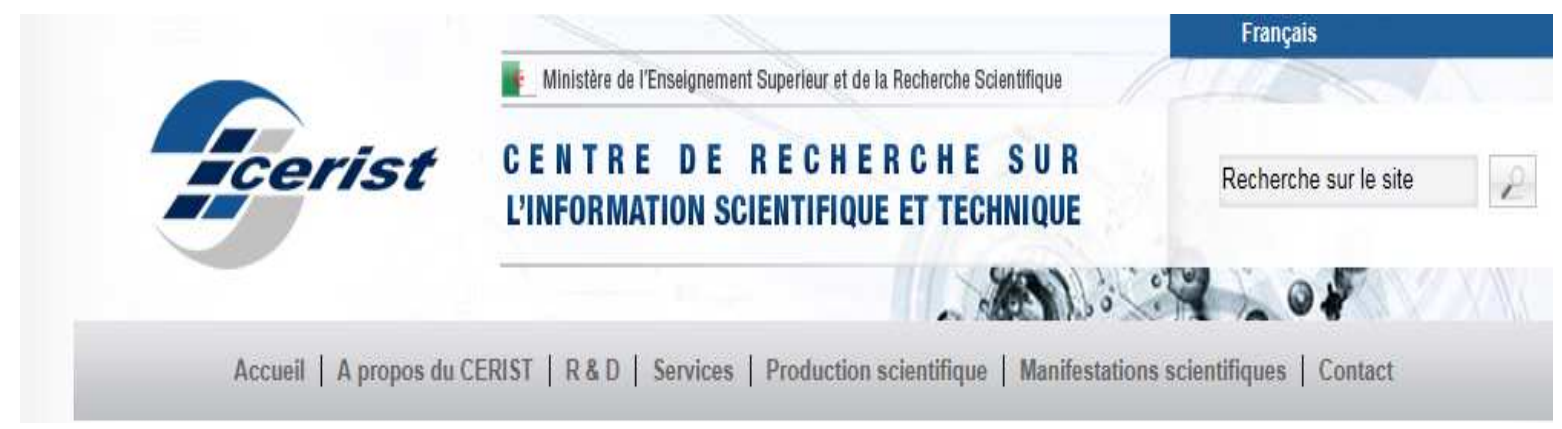

Le CEntre de Recherche sur l'Information Scientifique et Technique, a été créé en date du 16 mars 1985.Il était sous la tutelle du premier Ministère et avait pour mission principale de mener toute recherche relative à la création, à la mise en place et au développement d'un système national d'information scientifique et technique. Il a été, ultérieurement, rattaché au Haut Commissariat à la Recherche. Enfin, il a été déclaré comme étant un Etablissement Public à caractère Scientifique et Technologique à vocation intersectorielle et placé sous la tutelle du Ministre de l'enseignement Superieur et de la Recherche Scientifique par le décret $\mathrm{n}^{\circ}$ 03 - 454 du 1er décembre 2003 Le CERIST est organisé en départements administratifs et techniques et en divisions de recherche. Outre le siège central fixé à Alger, le centre dispose de sites régionaux et de bureaux de liaison répartis géographiquement au niveau de trois pôles principaux du territoire.

\subsubsection{1-Les différentes divisions}

Le centre est divisé en quatre divisions de recherche et qui sont :

\subsubsection{1) RESEAUX}

Les principaux axes de recherche de cette division sont:

Technologie Internet et Réseaux.

Technologie des systèmes collaboratifs et intégrés.

Technologie des systèmes Web et multimédia et de gestion de contenu.

Technologie des systèmes de transfert de connaissance.

\subsubsection{2) SYSTEMES D'INFORMATION ET SYSTEMES MULTIMEDIA}

Cette division est une structure de recherche-développement et dont les axes de recherche peuvent être résumés aux problématiques relatives aux technologies de l'information ( service web , multimédia, gestion des connaissances, ainsi que la qualité et la sécurité des systèmes d'information).Les axes du volet développement de la division quant à eux peuvent être résumés en la conception et développement des systèmes d'information d'aide à la décision 
ainsi que de la réalisation d'études, telle que les schémas directeurs informatique, les cahiers des charges, l'audit informatique, et le développement de logiciels spécifiques.

\subsubsection{3) RECHERCHE ET DEVELOPPEMENT EN SCIENCES DE L'INFORMATION}

Les principaux axes de recherche de la division recherche et développement en sciences de l'information sont :

Ingénierie des systèmes bibliothéconomiques et documentaires.

Gestion et organisation de systèmes bibliothéconomiques et documentaires.

Socio économie, le management et le droit des TIC.

\subsubsection{4) THEORIES ET INGENIERIE DES SYSTEMES INFORMATIQUES}

La division Théorie et Ingénierie des Systèmes Informatiques contribue au développement du réseau national d'information scientifique et technique en menant des recherches fondamentales et appliquées, en contribuant à la formation pour et par la recherche et à la définition de programmes de recherche dans les axes suivants :

Intelligence artificielle et génie logiciel

Recherche d'information

Systèmes distribués et mobiles

Sécurité et qualité de service

\subsubsection{2-Autres activités du CERIST}

A coté de ces quatre divisions de recherche, le CERIST a une production scientifique assez importante se matérialisant en :

-Des articles publiés dans des revues

-Des communications présentées dans des conférences

-Des ouvrages

-Des thèses

-Des rapports internes

Le CERIST est aussi dépositaire de base de données nationales telles que :

-CAT (Catalogue Algérien des Thèses)

-CAP (Catalogue Algérien des Périodiques) 


\section{-ALGERIANA}

- ASA (Algerian Scientific Abstract) : Base de données recensant et signalant les publications scientifiques algériennes.

-ISSN (International Standard Sérial Number)

-PNST (Portail National de Signalement des thèses)

L'une des rubriques que le site du CERIST héberge est " Webreview " et qui est une liste de périodiques scientifiques algériens en ligne. Au nombre de 36, ces périodiques sont pour la plupart en libre accès (certains n'offrent que le résumé) et offrent accès au texte intégral de

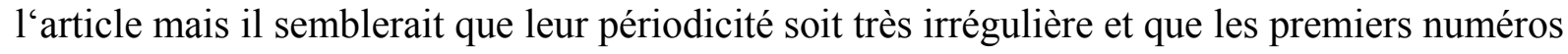
d'un grand nombre soit très récent.

Le CERIST offre aussi un accès local par adresse IP à des bases de données internationales connues telles que JSTOR, ACM Digital Library et les bases de données bibliographiques de l'INIS. De plus, il offre des formations diplômantes en Post-Graduation Spécialisée en Information Scientifique et Technique (en ligne) et en Post-Graduation Spécialisée en Sécurité Informatique.

Enfin, le CERIST édite semestriellement RIST (Revue de l'Information Scientifique et Technique) qui se définit comme " un journal qui s'intéresse à tous les travaux de recherche ayant trait aux sciences de l'information, l'informatique documentaire, l'archivistique et les nouvelles technologies de l'information ".Le site ne présente que les volumes 1 à 17 entre 1991 et 2007.Nous remarquons que l'accès aux texte intégraux est libre alors que cette même publication est payante sur AJOL (African Journal On Line ).D'autre part, il semblerait que sa parution ne soit pas régulière .

Comme on peut le voir, le CERIST de part ses missions, ses infrastructures et sa composante humaine est un acteur important dans la recherche scientifique nationale et son inclusion dans cette recherche répond à ce besoin d'enquêter sur les habitudes de soumission, de publication de ces chercheurs.

\subsection{2-Le CDTA}

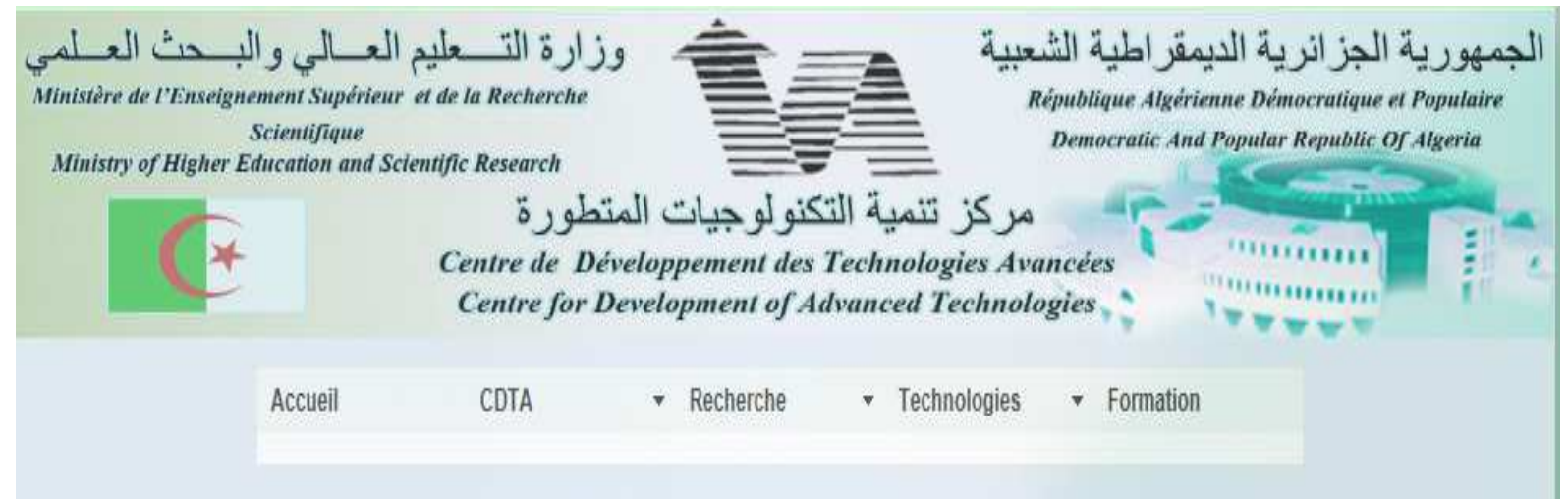


Le Centre de Développement des Technologies Avancées (CDTA) a été crée au sein du Commissariat aux Energies Nouvelles en 1982 .Le Centre était composé des laboratoires suivants :

Architecture des Systèmes

Robotique

Laser

Plasma

Fusion thermonucléaire

Etude spatiale des rayonnements

En date du 22 Mars 1988, le Centre de Développement des Technologies Avancées fut créé en tant qu'entité autonome. Le 29 Novembre de la même année, à la faveur d'une réorganisation interne, les départements de recherche précédemment cités deviennent :

Cybernétique

Milieux Ionisés

Microélectronique

En date du $1^{\text {er }}$ Décembre 2003, le Centre passe au statut d'Etablissement Public à Caractère Scientifique et Technologique. En 2006, le Centre, à la suite d'un arrêté interministériel, voit ses différentes composantes changer et de ce fait il se dote des divisions de recherche suivantes :

Architecture des systèmes et multimédia

Microélectronique et nanotechnologie

Milieux ionisés et laser

Productique et Robotique

Ces mêmes divisions ont une activité de publication assez dense étant donné que dans l'onglet valorisation (articles et communications), une liste assez longue d'articles et de communications présentées dans des conférences aussi bien nationales qu'internationales est répertoriée. Nous remarquons le peu de ces études en libre accès (nous voulons dire que le texte intégral de ces communications et présentations aux conférences n'est pas disponible à part pour un infime pourcentage) sur le site et mises à la disposition des éventuels chercheurs, 
contrairement à la revue " Technologies Avancées " qui elle est en libre accès à partir d'un poste Internet.

Le CDTA édite aussi semestriellement un périodique scientifique international " Technologies Avancées ".Le site du CDTA n'en recense cependant que 6 numéros (14-15-16-17-18-20) couvrant la période Juillet 2002-Decembre 2008.Contrairement à RIST qui offre un accès libre à partir d'Internet mais dont l'accès est payant sur AJOL, " Technologies Avancées " est aussi libre d'accès sur AJOL.

\section{2-Présentation des axes, parties et buts du questionnaire}

Le questionnaire adressé aux deux organismes (voir une copie en annexe) a pour but d'investiguer les habitudes documentaires des chercheurs des deux centres et leur point de vue quant au contrôle par les pairs d'une manière générale mais aussi le contrôle par les pairs à l'ère d'Internet. Pour pouvoir comparer en toute équité et arriver à des résultats qui puissent faire ressortir une éventuelle différence dans les habitudes de publication des deux organismes, le même questionnaire (à part la partie archive ouverte où pour le CDTA nous avons posé la question sur ArXiv et pour le CERIST la question concernait ArchiveSIC) leur a été adressé. Le dit questionnaire a été scindé en cinq parties et qui sont :

4.2.1-Renseignements personnels où nous avons demandé de renseignements concernant l'âge, le sexe, la fonction et le diplôme.

4.2.2-Degré de connaissance du libre accès où les questions posées avait pour but de tester le savoir des chercheurs dans les différentes actions ayant marqué le mouvement du libre accès telles que les appels, les archive ouvertes, les entrepôts d'archives ouvertes et aussi les plus importants et plus connus sites de journaux électroniques en libre accès et leur degré d'utilisation.

4.2.3-Habitudes documentaires et de recherche où nous avons voulu savoir principalement le support utilisé (papier ou électronique) ainsi que la (les) langues utilisée dans les soumissions d'articles

4.2.4-Production scientifique où l'accent a été mis sur le nombre et le genre de soumission (article ou communication à une conférence) ainsi que si ces soumissions étaient nationales ou internationales.

4.2.5-Attitude vis-à-vis du contrôle par les pairs où nous avons mis l'accent sur l'importance qu'a le contrôle pour le chercheur et les raisons ayant poussé le chercheur à (éventuellement) accepter les révisions demandées par les experts.

4.2.6-Expérience avec le contrôle par les pairs où nous avons mis l'accent sur la réaction du chercheur face au contrôle par les pairs et son avis quant à son importance et par delà comment il le voit dans sa configuration actuelle ainsi que le pourcentage d'acceptation de ses soumissions. 
4.2.7-Nouvelles formes de contrôle de l'information où nous avons voulu investiguer et essayer de savoir le degré de connaissance des nouvelles formes de contrôle de l'information ainsi que la position de ces mêmes chercheurs quant à une éventuelle insertion dans ces schémas de part leur éventuelle participation en tant qu'experts.

\subsection{8- Position des chercheurs vis-à-vis des nouvelles formes de contrôle de l'information} où l'accent a été mis sur l'avis des chercheurs face à cette véritable révolution dans le monde académique et celui de la recherche et ceci en insistant sur les cas de plagiat, de subjectivité ayant secoué le monde de la recherche, sans oublier la place de la publication électronique dans l'avancement et sa viabilité quant aux résultats de la recherche

\section{3-ANALYSE DES REPONSES DU QUESTIONNAIRE ADRESSE AUX CHERCHEURS DU CERIST}

\subsection{1- RENSEIGNEMENTS PERSONNELS}

\section{Question n-}

Age :

La tranche d'âge de ceux et celles ayant répondu au questionnaire s'échelonne entre 1955 et 1981 alors que 6 chercheurs n'ont pas jugé opportun de donner d'indication sur leur âge .Ce qui nous donne sur l'ensemble des répondants un taux de 83\%. Dix sept d'entre eux (soit $48 \%$ des chercheurs ou $58 \%$ de ceux et celles qui ont répondu) représente la tranche d'âge des trentenaires et qu'on pourrait considérer comme de potentiels chercheurs ayant des besoins de publication (pour leur avancement entre autres) et surtout ayant atteint un certain degré de maîtrise de leur domaine.

\section{Question n²-}

\section{Sexe :}

Ceux et celles qui ont répondu se repartissent de la manière suivante

-19 chercheurs de sexe masculin soit $54 \%$

-16 chercheurs de sexe féminin soit $46 \%$

Ce qui nous donne un échantillonnage assez équilibré entre chercheurs de sexe masculin et chercheurs de sexe féminin. Le site du CERIST dénombre d'ailleurs 49 chercheurs dont 23 (47\%) sont de sexe féminin et $26(52 \%)$ de sexe masculin. 


\section{Question n³-}

\section{Spécialité :}

Sur les 35 répondants, on dénombre 16 chercheurs de la Division Recherche en Sciences de l'Information soit $46 \%$, 11 chercheurs de la Division Réseaux soit $31 \%$, 6 chercheurs de la Division Systèmes d'Information et Systèmes Multimédia soit $17 \%$ et 2 chercheurs de la Division Théorie et Ingénierie des Systèmes Informatiques soit $6 \%$.

\section{Question n ${ }^{\circ}$ -}

\section{Fonction}

Dans cette question, nous avons voulu savoir quelle était la fonction occupée par les différents chercheurs ayant répondu au questionnaire. Nous avons obtenu 16 attachés de recherche soit $45 \%, 8$ chargés d'études (qui correspond en fait dans l'annuaire des chercheurs du site du CERIST à chargé de recherche) soit $23 \%, 1$ directeur de division soit 3\%, 1 maitre de recherche soit $3 \%, 2$ " chercheurs " soit $6 \%$ et enfin 7 chercheurs n'ont pas répondu à cette question soit $20 \%$.Si nous examinons, l'annuaire des chercheurs précédemment cité, nous avons 2 directeurs de recherche soit 4\%, 7 maîtres de recherche soit $18 \%, 7$ chargés de recherche soit $18 \%$, et enfin 23 attachés de recherche soit $60 \%$, ce qui dans l'ensemble nous donne un échantillon de répondants assez représentatif de la population des chercheurs du CERIST.

\section{Question n5-}

\section{Diplôme}

Le but de cette question est de déterminer le niveau d'instruction des différents chercheurs du CERIST et cela de par les diplômes obtenus. Sur les 35 répondants, 22 chercheurs ont un magister soit $63 \%, 6$ chercheurs sont détenteur d'un ingéniorat soit 17\%, 5 chercheurs ont un doctorat soit $14 \%, 1$ chercheur a une licence soit $3 \%$ et 1 chercheur a le niveau de Master 2 Recherche soit $3 \%$. Ce point est plus ou moins corroboré par la fonction attaché de recherche et qui est détenue par les chercheur ayant le diplôme de magister et qui est la plus représentée dans cette question.

\subsection{2-II-DEGRE DE CONNAISSANCE DU LIBRE ACCES}

\section{Question nº6-}

\section{Êtes-vous au courant des efforts militant pour l'accès libre et gratuit à l'IST ?}

A cette question, les chercheurs ont répondu à $74 \%$ qu'ils étaient au courant des efforts promouvant le libre accès alors que $26 \%$ n'étaient pas au courant. On peut estimer ces chiffres un tant soit peu acceptable car la nouveauté du mouvement peut expliquer que les 
trois quarts des chercheurs ont une connaissance de ce mouvement dont le but est d'accéder gratuitement la documentation dont ils ont besoin. D'un autre coté, on peut aussi voir dans ce taux, peut être, un manque de sensibilisation au niveau national de ce mouvement qui promeut l'accès libre, situation paradoxale s'il en est, dans un pays en développement ayant besoin d'accéder à l'information.

\section{Question n7-}

\section{Connaissez-vous l’O.A.I. ? (Open Access Initiative)}

Cette question a pour but de savoir est ce que les chercheurs qui bénéficient des bienfaits du libre accès en savent les fondements qu'est l'O.A.I. Une quasi parité (49\% contre $51 \%$ ) apparaît entre ceux qui en connaissent l'existence et ceux qui ne la connaissent pas.

\section{Question n⿳8-}

\section{Etes vous au courant de}

-l'Appel de Budapest en 2002

\section{- l'Appel de Berlin en 2003}

\section{- l'Appel de Berlin III en 2005}

Nous avons voulu par cette question savoir le degré de connaissance des textes fondateurs du libre accès par les chercheurs, textes sans lesquels le libre accès n'aurait pas atteint les progrès dont il peut se targuer de nos jours. Nous avons choisi les trois appels car tout chercheur en la matière doit ne serait ce qu'en connaitre l'existence. Les résultats nous donnent un taux de 20 $\%$ au courant de ces appels alors que $80 \%$ ne les connaissent. Ces chiffres dénotent à notre avis une étonnante méconnaissance. De plus si le cinquième seulement connait ces appels, les signataires quant à eux sont pratiquement inexistants, seuls 2 chercheurs ont signé l'Appel de Budapest et un seul a signé les Appels de Budapest et celui de Berlin. Nous pensons, personnellement, que le fait de signer ces Appels permet au chercheur de montrer son engagement dans un mouvement qui somme toute s'appuie sur ce genre de positionnement ce qui ne semble pas être le cas pour les chercheurs investigués.

\section{Question n9-}

\section{Savez vous qu'est ce qu'une archive ouverte?}

Cette question qui paraîtrait anodine, et à la limite inopportune, découle de notre expérience personnelle quant à la réaction que cette appellation provoque chez les chercheurs. En effet, cette appellation pose problème étant donné que le mot " archive " évoque rarement pour le commun des mortels Internet mais plutôt l'univers papier. De ce fait, cette question tend à investiguer le degré de connaissance des chercheurs sur cet aspect. Nous obtenons un taux de 
$29 \%$ de chercheurs qui ne connaissent pas ce qu'est une archive ouverte et un taux de $71 \%$ de chercheurs qui connaissent le sens du terme, taux acceptable vu la nouveauté du medium.

\section{Question n¹0-}

\section{En avez-vous déjà utilisé une en dépôt ou consultation ?}

Nous avons voulu savoir de par cette question est ce que les chercheurs du CERIST utilisent les archives ouvertes juste pour trouver des sources de documentation ou au contraire participer à enrichir les différentes archives ouvertes par leur dépôt. Nous obtenons un taux de $29 \%$ de chercheurs qui utilisent les archives ouvertes principalement pour la consultation dans des sites tels que HAL ( 3 occurrences ), Archive Sic ( 3 occurrences ), CiteSeer, le site de l'ENNSIB alors qu'un chercheur a déposé sa thèse sur le site de Scribd. Le reste des chercheurs qui représentent $71 \%$ n'utilisent les archives ouvertes ni pour le dépôt ni pour la consultation ce qui représente un assez important taux mais qui serait acceptable vu, encore une fois, la nouveauté du medium.

\section{Question n¹1-}

\section{Connaissez-vous Archive SIC?}

Nous avons posé cette question car ArchiveSIC peut être considéré comme le site (francophone du moins) de référence en science de l'information et de la communication. Malgré cela, seuls $34 \%$ des chercheurs en connaissent l'existence alors que 66\% l'ignorent. Une des explications plausibles serait que nombre des chercheurs investigués n'ont pas une formation de base en science de l'information et de la communication mais une formation beaucoup plus sciences exactes, surtout pour les divisions Réseaux et Théories et Ingénierie des Systèmes Informatiques.

\section{Question n²12-}

\section{Connaissez-vous :}

\section{-The Registry of Open Access Repositories (ROAR)}

\section{-The Directory of Open Access Repositories (DOAR)}

Les deux sites précités sont pour les professionnels du libre accès des sites de base recensant et listant d'une manière exhaustive les entrepôts d'archives ouvertes au niveau international. Malgré cette importance, 33 des chercheurs investigués soit $94 \%$ disent ne pas les connaitre et seul $6 \%$ les connaissent ce qui constitue une faille dans les habitudes documentaires de recherche sachant l'extrême richesse de ces sites qui permettent l'accès direct et non restreint à l'information scientifique et technique. 


\section{Question n¹3-}

Avez-vous déjà utilisé des journaux électroniques en libre accès ?

A cette question somme toute normale dans un monde de plus en plus dominé par le medium électronique, une quasi parité ( $49 \%$ contre $51 \%$ ) existe entre ceux qui utilisent les journaux électroniques et ceux qui ne les utilisent pas ce qui est étonnant étant donné la disponibilité d'une connexion Internet au CERIST et donc la possibilité de consulter des journaux directement sur le poste du chercheur.Quant à ceux qui utilisent les journaux électroniques, nous remarquons une grande variété et un grand éclectisme dans les titres. Nous avons par exemple : B.B.F. (Bulletin des bibliothèques de France), Archimag, Documentaliste, RIST, Cybermetrics, Journaux Science Direct, Solaris, MDPI, Cybrarian, D-Lib Magazine, ACM, IEEE Explore, Springer, Lex Electronica (Revue internationale du droit des technologies de l'information), Journal of Classification. On remarquera que B.B.F. (5 fois) et Documentaliste, RIST et Archimag ( 2 fois) sont les plus cités.

\section{Question n¹4-}

\section{Connaissez-vous :}

\section{-African Journal On Line (AJOL)}

- Directory of Open Access Journal (DOAJ)

A cette question, 4 chercheurs soit $11 \%$ ont répondus qu'ils connaissaient ces deux sites de journaux électroniques en libre accès alors que 26 soit $74 \%$ ont répondu qu'ils ne les connaissaient pas. D'un autre coté, 5 chercheurs soit $14 \%$ ont indiqués qu'ils connaissaient AJOL et non pas DOAJ. Ceci peut être interprété de plusieurs manières mais la plus plausible est que RIST, le périodique scientifique du CERIST est indexé par AJOL et non DOAJ. Toujours est il que seul un quart des chercheurs investigués ont indiqués qu'ils connaissaient ces deux sites de références des journaux en libre accès, ce qui nous semble un taux faible surtout que ce sont les deux sites internationaux de référence.

\subsection{3- HABITUDES DOCUMENTAIRES ET DE RECHERCHE}

\section{Question n ${ }^{\circ}$ 15-}

Dans vos activités de recherche et de documentation utilisez-vous plus le support :

-Papier

\section{-Electronique}

\section{-Indifféremment}

Il n'échappe à personne que l'utilisation du support quel le chercheur utilise pendant sa recherche a considérablement changé. Le papier n'est plus le seul et plus utilisé support il est concurrencé (et même des fois supplanté) par le medium électronique. Cet état de fait est amplement vérifié dans notre étude car 24 chercheurs soit $68 \%$ disent préférer le support électronique alors le support papier est préféré par seulement 1 chercheur soit 3\% .D’un autre 
côté, 8 chercheurs soit $23 \%$ disent être indifférent au support et 2 chercheurs soit $6 \%$ utilisent les deux supports en même temps. Ceci nous donne un net avantage au support électronique qui de ce fait est pratiquement plébiscité pour sa facilité et sa maniabilité.

\section{Question $n^{\circ} 16-$}

\section{Quel est le support que vous préférez?}

\section{-Papier}

\section{-Electronique}

\section{-Indifféremment}

Si les chercheurs ont dans leur immense majorité indiqué la primauté de l'utilisation du support électronique, cette tendance n'est pas aussi grande quand les chercheurs sont investigués sur leur préférence. La tendance s'inverse car 15 chercheurs soit $43 \%$ préfèrent le support papier et 14 soit $40 \%$ préfèrent le support électronique, le reste soit 6 chercheurs et qui représentent $17 \%$ indiquent que le support leur est indifférent. Nous touchons ici à une constante chez les chercheurs qui, bien qu'ils apprécient la maniabilité et la rapidité du support électronique, n'en préfèrent pas moins le papier pour des raisons multiples dont l'accessibilité et la facilité d'utilisation (on n'a pas besoin d'une machine pour lire un article papier) ainsi que l'habitude de manier le papier encore présente.

\section{Question nº17-}

\section{Dans quelle proportion estimez-vous votre utilisation des deux supports?}

\section{-Papier}

\section{-Electronique}

Les réponses que nous avons obtenu quant à l'utilisation effective des deux supports sont on ne peu plus explicite. Les chercheurs utilisent le support électronique beaucoup plus que le support papier et ceci à des taux ne souffrant d'aucune contestation. Ainsi 3 chercheurs soit $9 \%$ affirment utiliser le support à raison de $05 \%-95 \%$ en faveur du support électronique, 4 chercheurs soit $11 \%$ l'utilisent à raison de $10 \%-90 \%, 10$ chercheurs soit $29 \%$ l'utilisent quant à eux à raison de $20 \%-80 \%$ et 9 chercheurs soit $26 \%$ ont affirmé que leur utilisation des deux supports était de l'ordre de 30\%-70\%, 2 chercheurs soit $6 \%$ quant à eux ont répondu que ce taux était de 40\%-60\%, 1 chercheur soit 3\% a déclaré que ce taux était de $49 \%-51 \%$ et enfin 1 chercheur utilise le papier et le support électronique à parfaite parité 50\%-50\%. Seuls 3 chercheurs ont déclaré qu'ils utilisaient le papier plus que le support électronique. Ils ont déclaré des taux de $60 \%-40 \%, 70 \%-30 \%$ et $90 \%-10 \%$ en faveur du papier. Comme on peut le voir $86 \%$ des chercheurs affirment utiliser le support électronique plus que le papier. Tendance somme toute normale si on voit les nouvelles orientations de la recherche scientifique qui de nos jours se passe beaucoup plus sur Internet que sur le papier. 


\section{Question n¹8-}

\section{Quelle (s) langue (s) utilisez-vous dans vos soumissions?}

\section{-Français}

\section{- Arabe \\ -Anglais \\ -Autres (précisez)}

Des trois langues proposées dans la question concernant les langues utilisées lors des soumissions (arabe, français et anglais), 16 chercheurs utilisant en même temps l'anglais et le français soit $46 \%$ alors que 10 chercheurs soit $29 \%$ utilisent l'anglais seulement ce qui nous donne un taux de $75 \%$ chercheurs utilisant les deux langues dans leur soumissions. De plus, 3 chercheurs soit $9 \%$ utilisent le français pour leurs soumissions alors que 3 autres chercheurs soit $9 \%$ utilisent quant à eux le français et l'arabe. Enfin, 1 chercheur soit 3\% a affirmé publier en arabe, 1 chercheur soit 3\% a affirmé publier en français - anglais - arabe allemand, enfin 1 chercheur soit 3\% a affirme publier en français - arabe - anglais. Nous pouvons conclure sans risque de nous tromper que la langue anglaise est devenue la langue de communication chez les chercheurs investigués, ce qui est normal sachant l'extrême internationalisation de la recherche qui inclut une exclusion de facto de ceux qui ne maîtriseraient pas la langue de la science par excellence : 1'Anglais. Nous remarquons aussi une assez fréquente utilisation de la langue française et, à un degré moindre, celui de la langue arabe.

\subsection{4-PRODUCTION SCIENTIFIQUE}

\section{Question n¹9-}

\section{Avez-vous déjà publié dans des journaux scientifiques?}

\section{- Oui}

\section{-Non}

Tel que le sujet de cette recherche essaye de prouver, publier est une obligation pour le chercheur dans le cadre de son travail et pour progresser. La publication dans des revues scientifiques (et les promotions inhérentes) permet aussi d'accéder à un nombre d'avantages qui en découlent. Dans ce cadre, 16 chercheurs du CERIST soit $46 \%$ ont indiqué ne pas avoir publié, alors que 19 chercheurs soit $54 \%$ ont positivement répondu à cette question. Cinq de des chercheurs ayant publié soit $14 \%$ ont publié dans des revues internationales et 6 chercheurs soit $17 \%$ dans des revues nationales. Huit autres chercheurs soit $23 \%$ ont publié en même temps dans des revues nationales et internationales. On peut estimer ce taux de publication des chercheurs du CERIST comme étant assez bas comparativement à d'autres organismes (bien qu'il n'existe pas de chiffres précis) mais remettre ce taux dans le contexte algérien est plus adéquat étant donné l'absence (ou du moins la faiblesse) de la culture de publication dans notre environnement. 


\section{Question n²0-}

\section{Quel est le nombre de ces publications ?}

$$
\begin{array}{r}
-1-3 \\
-3-5 \\
-5+
\end{array}
$$

Cette question tend à investiguer le nombre donc la productivité des chercheurs du CERIST. Des 19 chercheurs ayant déclaré qu'ils ont publié dans la question précédente ,11 soit 58\% ont publié entre 1 et 3 articles, 4 chercheurs soit $21 \%$ ont publié entre 3 et 5 articles et enfin 4 chercheurs soit $21 \%$ ont publié plus de 5 articles. Ces chiffres ne peuvent être interprétés dans l'absolu mais on notera le même constat des $46 \%$ des chercheurs n'ayant pas publié qui revient.

\section{Question n²1-}

Avez-vous déjà participé à des conférences dans votre spécialité ?

-Oui

-Non

Les chercheurs du CERIST ont participé à des conférences à raison de 29 sur les 35 répondants soit $83 \%$ alors que 6 chercheurs soit $17 \%$ n'ont pas participé à aucune conférence. Des 29 chercheurs ayant participé à des conférences, 12 soit $35 \%$ ont participé à des conférences nationales et internationales, 11 soit $31 \%$ n'ont participé qu'à des conférences internationales et enfin 6 chercheurs soit $17 \%$ n'ont participé qu'à des conférences au niveau national. Si le taux de publication dans des revues scientifiques, comme nous l'avons vu, est un tant soit peu faible, le nombre des participations des répondants à des conférences, qu'elles soient nationales ou internationales, peut être estimé acceptable et même encourageant.

\section{Question n²2-}

\section{Quel est le nombre de ces publications ?}

$$
\begin{array}{r}
-1-3 \\
-3-5 \\
-5+
\end{array}
$$

Des 29 répondants ayant participé à des conférences, 12 soit $35 \%$ ont participé à des conférences entre 1 et 3 fois , 6 soit $17 \%$ ont participé à concurrence de 3 à 5 fois et enfin 11 chercheurs soit $31 \%$ ont participé à plus de 5 conférences. De même que le nombre des chercheurs ayant participé à des conférences, le nombre de ces participations est aussi encourageant et prouve que les chercheurs du CERIST participent (soit au niveau local ou international) au développement de leur spécialité en soumettant aux conférences. 


\subsection{5-ATTITUDE VIS-A-VIS DU CONTROLE PAR LES PAIRS}

Question n²3-

Au cours de vos soumissions saviez-vous si elles étaient contrôlées par les pairs?

-Oui

-Non

Il est connu que les revues scientifiques et les articles qu'elles publient n'ont pas le même " poids " ni chez les chercheurs ni chez ceux qui doivent les évaluer pour d’éventuels promotions ou financements. De ce fait, une véritable hiérarchie des revues s'est imposée dans laquelle le contrôle par les pairs joue un rôle important pour ne pas dire primordial. Pour être plus clair, une véritable dichotomie s'est auto imposé entre les revues certifiées " sérieuses " et celles qui publient n'importe quelle information ou du moins dont les critères de publication sont plus astreignant. C'est d'ailleurs la raison du tant décrié facteur d'impact et autres outils tels que " The Institut for Scientific Information "(ISI) ou Scopus qui recensent les citations des articles. Quant aux chercheurs du CERIST, on peut considérer qu'ils ont intégré cette donnée dans leur effort de publication puisque 24 chercheurs soit $69 \%$ l'ont pris en ligne de compte alors que seuls 7 soit $20 \%$ n'ont en pas tenu compte, 4 chercheurs soit $11 \%$ n'ayant répondu à cette question.

\section{Question n²4-}

Avez-vous soumis vos recherches en tenant compte de ce paramètre?

-Oui

-Non

Les raisons pour lesquelles les chercheurs publient peuvent être variées et multiples .De ces raisons, l'avancement dans le cadre du travail surtout et le prestige d'être publié entrent en ligne de compte. Vingt six des chercheurs investigués soit $74 \%$ ont répondu qu'ils ont tenu compte de ce paramètre quand ils ont soumis alors que 9 chercheurs soit $26 \%$ n'en ont pas tenu compte. De ces 26 chercheurs ayant soumis en tenant compte de ce paramètre, 14 chercheurs soit $54 \%$ des ces chercheurs l'ont fait pour des raisons d'avancement alors que 2 chercheurs soit $8 \%$ l'ont fait pour le prestige et que 2 autres soit $8 \%$ l'ont fait pour l'avancement et le prestige en même temps. Trois chercheurs soit $11 \%$ n'ont pas donné de raison (bien qu'ils aient répondu oui à la question) et 5 autres soit $19 \%$ n'ont pas répondu à la question. Il apparaît clair que le célèbre " publish or perish " (publier ou périr ) commence à guider les chercheurs algériens (ou du moins du CERIST) dans leur travail de recherche. 


\subsection{6-EXPERIENCE AVEC LE CONTROLE PAR LES PAIRS}

\section{Question n²5-}

Estimez vous que le contrôle par les pairs est indispensable à l'avancement de la Science ?

-Oui
-Non

Bien que le contrôle par les pairs ait atteint un consensus quant à son importance et son inéluctabilité dans l'avancement de la Science, il existe certains courants de pensée qui y voit une sorte de suppression de nouvelles pensées (ce qui lui a été reproché à juste titre comme nos l'avons vu) et y voient une manière d'asseoir plus la mainmise des ténors de la spécialité. Cette question est destinée à investiguer la position des chercheurs quant à l'importance de cette opération. Des 32 chercheurs ayant répondu à la question (3 chercheurs n'ont pas répondu), 31 soit $97 \%$ croient que le contrôle par les pairs est indispensable alors qu'un seul chercheur soit 3\% ne le voit pas indispensable. On peut penser sans risque de nous tromper que les chercheurs du CERIST voient dans le contrôle par les pairs une étape indispensable et importante de la science publiée.

\section{Question n²6-}

Avez-vous eu l'impression au cours de vos soumissions que votre travail a été jugé d'une manière objective ?

$$
\begin{aligned}
& \text {-Oui } \\
& \text {-Non }
\end{aligned}
$$

L'une des reproches fait au contrôle par les pairs est - entre autres - d'être sujet à des expertises subjectives. Cette subjectivité peut prendre plusieurs formes (personnelle, institutionnelle, culturelle, etc.....) et est sujet à de multiples critiques. Dans leur réponses, sur les 28 chercheurs du CERIST ayant répondu à la question (7 chercheurs n'y ont pas répondu), 22 soit $79 \%$ ont affirmé avoir été jugés d'une manière objective alors que 5 chercheurs soit $18 \%$ ont estimé avoir été jugé d'une manière subjective. Notons qu'un chercheur soit 3\% a jugé que son travail a été jugé de manière objective et aussi d'une manière subjective selon les cas. On peut juger que dans l'ensemble les chercheurs du CERIST n'ont pas eu à souffrir des multiples griefs que portent nombre de chercheurs à travers le monde à l'encontre du contrôle par les pairs. 


\section{Question n²7-}

Estimez vous normal que dans les formes traditionnelles de certification, le nom de l'expert soit tenu secret?

-Oui

-Non

Un des aspects les plus décriés dans le contrôle par les pairs est l'anonymat dont jouit l'expert alors que, généralement, le nom du soumissionnaire est connu. Cette approche impartiale a été le sujet de nombreuses recherches ayant fait ressortir que l'anonymat pouvait remédier à cet état fait alors que d'autres avaient en fait ressortir le contraire. Certains auteurs avaient même pris position contre l'anonymat arguant du fait qu'ils voulaient être jugés comme étant les auteurs de la recherche car ceci rentrait dans les critères inhérents au jugement de facto de l'article soumis. Nonobstant toutes ces controverses, des 31 chercheurs qui ont répondu à cette question (4 chercheurs n'y ont pas répondu), 20 soit $65 \%$ ont estimé que cet anonymat était normal alors que 10 soit $33 \%$ chercheurs ont au contraire estimé cet anonymat anormal et qu'un chercheur soit $2 \%$ a répondu en estimant cet anonymat normal et aussi anormal selon les cas. On peut conclure donc que les $2 / 3$ chercheurs investigués (rejoignant en cela les résultats de la question précédente concernant le jugement objectif de leur soumission) ne voient pas dans l'anonymat de l'expert matière à controverses et que cette pratique avait ses raisons d'être.

\section{Question n²8-}

Au cours de vos soumissions, avez-vous pris en ligne de compte les correctifs que l'on vous a demandé d'apporter?

-Oui

-Non

Il est connu qu'il est très rare qu'un manuscrit soumis soit accepté sans aucune demande de corrections la part des experts, correctifs pouvant varier du plus superficiel au plus radical selon les demandes des experts. Ce genre d'opération peut être sous la forme de plusieurs aller et retours entre les experts et l'auteur afin que l'article soumis réponde aux critères scientifiques en vigueur et à ceux de la revue ciblée. Il semble que les chercheurs du CERIST aient compris cette donne incontournable dans la quête d'être publié étant donné que sur les 35 répondants, 30 des chercheurs soit $86 \%$ ont pris en ligne en compte les correctifs demandés alors qu'un seul chercheur soit $3 \%$ ne les a pas pris en ligne de compte (4 chercheurs n'ont pas répondu à cette question).Le taux serait même plus haut (97\%) si nous prenions le nombre de chercheurs ayant répondu oui par rapport aux chercheurs ayant effectivement répondu à la question. 


\section{Question n²9-}

\section{L'avez-vous fait convaincu des arguments de l'expert ou tout simplement pour être publié ?}

Face à l'obligation de publier et être reconnu des chercheurs, nous avons posé cette question pour savoir est ce que le but est de publier à tout prix ou au contraire le chercheur, convaincu des arguments présentés par les experts, les a pris en ligne de compte et les a appliqué. Sur les 31 répondants (les 4 chercheurs n'ayant pas répondu à la question précédente n'ont pas aussi répondu à cette question), 22 soit $71 \%$ l'ont fait convaincu des arguments des experts, 7 chercheurs soit $23 \%$ l'ont fait dans le but d'être publié seulement alors que 2 chercheurs soit $6 \%$ l'ont fait convaincus des arguments mais aussi pour être publiés. Ce qui nous donne presque les $3 / 4$ des chercheurs qui pensent que le contrôle par les pairs améliore leur manuscrit. Cette approche (le contrôle par les pairs améliore le manuscrit) est une des raisons que les pro contrôle par les pairs utilisent pour justifier son maintien.

\section{Question n³0-}

\section{Pouvez-vous indiquer le pourcentage de vos soumissions acceptées par rapport à celles soumises?}

Des 35 répondants du questionnaire, 14 soit $40 \%$ n'ont pas répondu à la question (nous pensons que c'est les chercheurs n'ayant pas publié cf. question 19).Des 21 chercheurs ayant répondu, nous avons des pourcentages très hétéroclites allant de $00 \%$ ( 1 chercheur soit $5 \%$ ), $33 \%$ ( 1 chercheur soit 5\% ), 50\% (5 chercheurs soit 24\%), 60\% ( 2 chercheurs soit 9\%),70\% ( 5 chercheurs soit 24\%),80\% ( 3 chercheurs soit 15\%), $90 \%$ ( 2 chercheurs soit $9 \%$ ) à 100\% ( 2 chercheurs soit 9\%).Ces chiffres peuvent être interprétés de plusieurs manières : si le chercheur qui a avoué ne pas avoir de recherches acceptés peut être considéré comme une exception ( quoique le nombre de soumissions ne soit pas connu dans ce cas ), les autres réponses qui s'échelonnent de $50 \%$ à $70 \%$ semblent constituer les pourcentages les plus fréquents et en même temps les plus logiques ( étant donné le haut taux de rejet en sciences humaines et sociales comparativement aux sciences exactes selon différentes recherches).Ils ( les pourcentages ) constituent de ce fait, un peu plus de la moitié (exactement 57,14\%) des réponses données et traduisent la réalité de la soumission en sciences humaines et sociales. Quant aux taux d'acceptation de $0 \%$ et de $100 \%$, ils constituent, peut être, des cas extrêmes qui ne peuvent être pris en ligne de compte dans les moyennes générales. 


\subsection{7-NOUVELLES FORMES DE CONTROLE DE L'INFORMATION}

Question n³1-

Êtes-vous au courant des nouvelles formes de contrôle par les pairs des publications scientifiques sur Internet?

-Oui

-Non

La communication scientifique et technique est depuis quelques années et surtout depuis l'avènement d'Internet complètement changée tant du point de vue de son approche que de son maniement. Le scientifique ne se contente plus de faire de la recherche et la soumettre à une revue scientifique et attendre les décisions du staff éditorial, mais la communication scientifique et technique est devenue beaucoup plus interactive, ouverte et internationale grâce à Internet. On ne peut plus communiquer ou soumettre un article par exemple de la manière traditionnelle, tout se passe par Internet et implique une rapidité plus grande et un échange constant et continu de l'information. De ce fait cette question avait pour but d'essayer de savoir le degré de connaissance des chercheurs dans des schémas de communication qu'on peut appeler novateurs. Sur les 35 répondants, 1 seul n'a pas répondu à la question. Des 34 autres chercheurs ayant répondu, 29 chercheurs soit $85 \%$ ont reconnu ignorer ces nouvelles formes alors que seuls 5 soit $14 \%$ chercheurs ont affirmé être au courant de ces nouvelles formes. Cette sorte " d'ignorance " peut être interprétée de deux manières : soit on y voit une ignorance coupable de la part de nos chercheurs ou alors, ces schémas sont tellement nouveaux et même révolutionnaires qu'ils sont largement méconnus par les chercheurs. Nous pencherions plutôt pour la deuxième explication car même dans les pays développés ces formes ne sont pas très connues ${ }^{*}$ ni même acceptés.

\section{Question n³2-}

Accepteriez vous que votre soumission soit mise sur Internet et jugée par toute la communauté ?

-Oui

-Non

Parmi les nouvelles formes de contrôle de l'information que la question précédente a essayé d'investiguer, se trouve ce qui est communément appelé le contrôle par les pairs ouvert (open peer review), où la soumission est mise sur Internet et c'est la communauté des chercheurs dans son entièreté qui la juge. De toute évidence, cette pratique n'a pas que des supporteurs ${ }^{* *}$.

\footnotetext{
* Nous avons eu l'occasion de constater cette ignorance au cours d'un colloque international au Canada où nous avions présenté les trois cas de contrôle par les pairs ouvert abordés en supra dans cette partie. Bien que l'assistance se composait de chercheurs de pays développés, ces cas étaient méconnus et nombre de participants ont exprimé leur étonnement devant l'extrême ouverture et l'aspect révolutionnaire de ces schémas non encore intériorisés.

${ }^{* *}$ Nombre de recherches ont fait ressortir des griefs comme par exemple le fait que le chercheur ne veut pas que sa recherche soit connue de tout le monde et aussi et surtout le fait qu'un nombre de chercheurs confirmés aient refusé que leur recherche soit jugé par des novices ou, pire, par n'importe quelle personne ayant un accès Internet.
} 
De ce fait, ces nouveaux schémas, en plus d'être méconnus, sont loin d'être acceptés. Les chercheurs du CERIST, par contre, semblent ne pas y voir un obstacle à leur effort de publication. Ainsi sur les 34 répondants ( 1 chercheur n'a pas donné son avis) ,23 chercheurs soit $68 \%$ seraient prêt à se faire expertiser par la communauté dans un cadre ouvert alors que 10 chercheurs soit $29 \%$ sont contre et qu'un chercheur soit $3 \%$ a répondu en même temps par oui et non selon les cas.

\section{Question n 33-}

Accepteriez vous que ces commentaires soient publiés sur Internet?

-Oui

-Non

En complément à la question précédente, cette question investigue plus l'ouverture des chercheurs quant à l'éventualité que les commentaires faits dans le cadre du contrôle par les pairs ouvert (open peer review) soient publiés sur Internet d'une manière totalement ouverte. Ainsi sur les 33 répondants ( 2 chercheurs n'ont pas répondu à la question), 19 chercheurs soit $58 \%$ ont affirmé qu'ils ne voyaient aucun problème à ce que ces commentaires soient publiés sur Internet, 13 chercheurs soit 39\% quant à eux n'accepteraient pas ce genre d'ouverture alors qu' 1 chercheur soit 3\% a répondu que cela dépendait des cas. A l'instar de la question précédente, les chercheurs du CERIST ont répondu d'une manière assez prononcée pour ce genre d'ouverture.

\section{Question n³4-}

\section{Accepteriez-vous en cas d'acceptation que ces commentaires soient publiés sur Internet avec la version finale et définitive?}

-Oui

-Non

Une des plus révolutionnaires et nouvelles formes de publication fait paraitre les commentaires que la soumission a reçu durant sa période d'expertise dans le contrôle par les pairs ouvert avec la version définitive et acceptée de la recherche. Cette forme de publication permet de voir les différentes réactions que la soumission a engendré et aussi les changements et correctifs apportés en réaction à ces commentaires. A l'instar des deux questions précédentes ayant investigué des problématiques analogues, les chercheurs du CERIST ont répondu à cette avec des taux très proches. Ainsi sur les 34 chercheurs ayant répondu à la question, 22 soit $65 \%$ ont répondu positivement et ne verraient aucun inconvénient à ce que les commentaires soient publiés avec la version finale. De leur coté, 11 chercheurs soit $32 \%$ n'accepteraient pas que ces commentaires paraissent avec la version finale. Enfin, 1 chercheur soit 3\% a répondu que ceci était sujet à débat selon les cas. 


\title{
4.3.8-POSITION DES CHERCHEURS VIS-A-VIS DES NOUVELLES FORMES DE CONTROLE DE L'INFORMATION
}

\section{Question $n^{\circ} 35-$}

Pensez vous que ces nouvelles formes de certification plus ouvertes peuvent contribuer à atténuer les accusations de subjectivité et de préjudice qui semblent, des fois, entacher le processus?

\section{-Oui \\ -Non}

Comme il a été amplement discuté dans ce travail, le contrôle par les pairs a focalisé (et focalise toujours) l'ire des chercheurs à cause d'accusations multiples de subjectivité et de préjudice. Avec l'ouverture qu'Internet a permis, ces griefs peuvent être combattus étant donné que le contrôle par les pairs se fait (ou du moins devra se faire) au vu et au su de toute la communauté. Bien que de multiples recherches aient donné des résultats contradictoires, il n'en demeure pas moins que ces nouvelles formes promettent de " démocratiser " et inclure plus transparence dans la publication scientifique. C'est dans ce cadre que cette question a été posée aux chercheurs du CERIST et leurs réponses ont en général abondé dans le même sens que les questions précédentes ayant abordé des problématiques similaires. Ainsi sur les 32 répondants (3 chercheurs n'ayant pas répondu à la question), 21 chercheurs soit $66 \%$ ont répondu par oui à la question ,10 chercheurs soit $31 \%$ ont répondu par non alors qu'un chercheur soit $3 \%$ a répondu par oui et non selon les cas. On peut donc conclure, que les $2 / 3$ des chercheurs voient en cette ouverture une manière de rendre le contrôle par les pairs plus ouvert et moins sujet à des manipulations d'ordre subjectif qu'il ne l'a été ou accusé de l'avoir été auparavant.

\section{Question n’36-}

Pensez vous que cette subjectivité peut être d'ordre :

\author{
-Sexuelle (homme ou femme) \\ -Linguistique (selon la langue utilisée) \\ -Religieuse (précisez) \\ -Personnelle \\ -Autres (précisez)
}

Ces accusations de subjectivité dont est accusé le contrôle par les pairs ont été largement documenté dans la littérature du sujet .Elles peuvent prendre des formes multiples et variées et des fois insoupçonnables mais les plus connues et les plus documentées sont soit sexuelle (selon que l'on soit un homme ou une femme) linguistique (quand la langue de communication n'est soit pas connue ou alors lorsque le soumissionnaire ne la maîtrise pas ), religieuse ( si l'expert a, par exemple, des idées préconçues à l'encontre ou en faveur d'une religion donnée ) personnelle ( quand l'expert a un contentieux personnel avec une personne 
donnée ou a intérêt à retarder une publication donnée émanant d'une personne de la même spécialité), etc....... Cette question posée aux chercheurs du CERIST a permis de cerner l'approche qu'ils en ont. Sur les 28 répondants ( 7 chercheur n'ont pas répondu à la question), 10 chercheurs soit $36 \%$ ont répondu par une subjectivité linguistique autrement dit qu'il étaient victimes de jugements subjectifs et négatifs à cause de la langue dans laquelle ils soumettaient, 6 chercheurs soit $21 \%$ ont répondu par une subjectivité personnelle ( connu sous l'appellation de " subjectivité ad hominem " ou contre une personne donnée) ce qui implique qu'ils pensent que leur soumission aurait été refusée ou critiquée parce qu'elle est la leur. Enfin, 12 chercheurs soit $43 \%$ ont évoqués des raisons multiples dont nous citerons :

-le manque d'expérience et de compétence dans le domaine (c'est le cas très fréquent où le chercheur soumet une recherche soit novatrice ou, plus souvent, très spécialisée).

-la renommée de l'encadreur ou directeur de laboratoire (on a tendance à accepter une soumission si elle faite sous la direction d'un chercheur renommé ou connu).

-l'appropriation des œuvres des autres (c'est le cas de la fraude et plagiat qui est plus prépondérant qu'on ne l'imagine pour preuve les différents scandales qui de temps à autre secouent le monde académique et scientifique).

Enfin les chercheurs ont cité différentes combinaisons de raisons telles que linguistique et personnelle, personnelle et religieuse, religieuse et linguistique ( 2 fois) et linguistique et sexuelle.

\section{Question n 37-}

\section{Pensez que cette ouverture peut aider à combattre le plagiat et la fraude ?}

\section{-Oui \\ -Non}

Le plagiat et la fraude sont malheureusement deux aspects qui font partie de la vie d'un scientifique et d'un chercheur. Il existe un nombre de cas assez documentés (les plus connus sont ceux du Coréen Hwang Woo-suk et de l'Allemand Jan Hendrik Schön) qui fait que la Science est elle aussi traversée par des courants de fraudes et de plagiats que le contrôle par les pairs est supposé détecter (alors que certains ne voient pas le contrôle par les pairs comme étant un outil de détection de la fraude mais plus un outil de certification et de labellisation). Encore une fois, Internet et son ouverture permettrait non pas d'éliminer mais peut être d'atténuer cet aspect par son ouverture et sa facilité d'accès à l'information. A cette question, les chercheurs du CERIST ont répondu majoritairement par l'affirmative. Ainsi sur les 29 répondants, 20 soit $69 \%$ ont répondu par l'affirmative et pensent que cette ouverture permettra de combattre les accusations de fraude et de plagiat. De leur coté, 7 chercheurs soit $24 \%$ ne pensent pas que cette ouverture puisse atténuer le fraudes et les plagiats. Enfin, 2 chercheurs soit $7 \%$ ont des réponses ambiguës où ils n'ont pas d'idée et répondent que ceci est selon les cas. 


\section{Question n³8-}

Dans le cadre de votre éventuel travail de certification, accepteriez-vous d'être connu de celui dont vous expertisez le travail ?

-Oui

-Non

Le chercheur qui soumet un travail à expertiser est aussi un expert potentiel étant donné que s'il atteint une certaine notoriété, il lui sera fait appel pour juger et certifier le travail de ses pairs. Nous avons vu que l'un des problèmes du contrôle par les pairs se situe dans l'anonymat dont jouissent les experts dans le cadre de leur travail. Dans une question précédente les chercheurs du CERIST étaient au 2/3 (65\%) d'accord sur l'anonymat des experts certifiant leur travail. Si la question est inversée (anonymat du chercheur quand il expertise versus anonymat de l'expert quand il expertise le chercheur), les chercheurs du CERIST répondent à cette question d'une manière plus prononcée étant donné que sur les 33 répondants ( 2 chercheurs n'ont pas répondu ), 23 soit 70\% sont d'accord à ce que leur nom soit dévoilé alors que seuls 9 chercheurs soit $27 \%$ ne voudraient pas leur nom connu par le chercheur qu'ils expertisent ( la plupart ont exprimé une peur de représailles s'ils refusaient et rejetaient une recherche ) alors qu'un chercheur a donné une réponse ambiguë en disant que ceci dépendait de la situation. On peut dire que les taux concernant l'anonymat en tant qu'expert ou soumissionnaire sont pratiquement les mêmes et traduisent une certaine gène (et même rejet) de cet aspect controversé de la communication scientifique.

\section{Question n³9-}

Pensez-vous que les recherches publiées dans les archives ouvertes et les journaux électroniques peuvent être prise en ligne de compte au même titre que les publications sur papier?

\section{-Oui \\ -Non}

Les journaux électroniques en libre accès et les archives ouvertes sont des media nouveaux sur la scène de la recherche scientifique. Comme nous l'avons vu, certains chercheurs en ignorent même l'existence (surtout en ce qui concerne les archives ouvertes).De plus, Internet étant (encore) considéré comme un terrain d'expérimentation, nombre de chercheurs l'assimilent à un no man's land scientifique et ne lui confieraient pas leur communication ou recherche de peur de se faire déposséder ou voler. Il existe de ce fait une sorte de méfiance et surtout de dévalorisation de ces deux media. Cette méfiance se vérifie d'ailleurs dans les réponses des chercheurs étant donné que sur les 31 répondants (4 chercheurs n'ont pas répondu à cette question), seuls 18 soit $58 \%$ ont estimés que les journaux électroniques et les archives pouvaient être pris en ligne de compte au même titre que les publications traditionnelles sur papier alors que 13 soit $42 \%$ ne le pensent pas. Une claire méfiance se dessine concernant le medium électronique qui, s'il est plébiscité pour sa facilite de maniement et d'accès, devient suspect quand il doit être pris en ligne de compte dans les 
promotions et financements. Il apparaît d'ailleurs clairement d'après les réponses que les chercheurs semblent assumer que sur Internet, il n'existe pas de contrôle et que de ce fait tout ce qui y est sujet à caution. Ceci était peut être vrai au début mais la tendance s'est inversée depuis.

\section{4-ANALYSE DES REPONSES DU QUESTIONNAIRE ADRESSE AU CHERCHEURS DU CDTA}

\subsection{1- RENSEIGNEMENTS PERSONNELS}

\section{Question n¹-}

\section{Age :}

La tranche d'âge de ceux et celles ayant répondu au questionnaire s'échelonne entre 1952 et 1981 alors que 8 chercheurs n'ont pas jugé opportun de donner d'indication sur leur âge .Ce qui nous donne sur l'ensemble des répondants un taux de $74 \%$.Il ne se dégage pas un consensus quant à l'âge des chercheurs ayant répondu étant donné qu'à part 5 chercheurs nés en 1965 soit $22 \%$ et 3 chercheurs nés en 1969 soit $14 \%$ ( soit un peu plus du 1/3 des répondants), le reste des répondants ne représentent qu'1 ou 2 personnes de chaque tranche d'âge. On peut conclure que les répondants à la question sont dans leur majorité (du moins à $36 \%$ ) dans la quarantaine, âge où peut être les chercheurs de cette institution atteignent la plénitude de leurs moyens.

\section{Question n²-}

\section{Sexe :}

Ceux et celles qui ont répondu se repartissent de la manière suivante

-20 chercheurs de sexe masculin soit $65 \%$

-11 chercheurs de sexe féminin soit $35 \%$

Cet échantillonnage peut eut être considéré équilibré étant donné que ces 31 répondants représentent $34 \%$ des chercheurs effectivement au Centre. L'annuaire des chercheurs recense quant à lui sur les 117 chercheurs sur le site (nous insistons sur le site car en les contactant personnellement sur leur boite électronique, nous nous sommes aperçu qu'en fait le nombre de chercheurs toujours au Centre était de 92 comme il nous a été donné l'occasion de le signaler plus haut) dont 74 soit $63 \%$ de sexe masculin et 43 soit $37 \%$ de sexe féminin. Comme on peut le voir, ces taux sont très prés des taux des chercheurs ayant répondu ( 20 chercheurs de sexe masculin soit $65 \%$ et 11 chercheurs de sexe féminin soit 35\%).Il est clair qu'un échantillon plus grand aurait été meilleur pour mieux appréhender la situation sur le terrain mais les difficultés et obstacles rencontrés (et que nous avons signalé) n'ont pu le permettre. 


\section{Question n³-}

\section{Spécialité}

Les quatre divisions de recherche investiguées du CDTA sont : Architecture des Systèmes et Multimédia, Productique et Robotique, Microélectronique et Nanotechnologie et Milieux Ionisés et Laser, qui comptent respectivement 23, 33 ,23 et 38 chercheurs. Les répondants ne semblent pas, malheureusement, représenter un bon échantillonnage étant donné que Architecture des Systèmes et Multimédia n'a eu un taux de réponses que de 2 chercheurs sur les 23 inscrits soit $9 \%$, Productique et Robotique n'a eu un taux de réponses que de 9 chercheurs sur les 33 inscrits soit 27\%, Microélectronique et Nanotechnologie n'a eu qu'un taux de réponses de 10 chercheurs sur les 23 inscrits soit $43 \%$ et enfin Milieux Ionisés et Laser avec un taux de 10 chercheurs sur les 38 inscrits soit $26 \%$.On peut néanmoins mitiger ces chiffres en voyant le nombre effectif des chercheurs encore au Centre (92) par rapport aux répondants qui nous donne un taux ( assez acceptable ) de $34 \%$.

\section{Question n4-}

\section{Fonction}

Dans cette question, nous avons voulu savoir quelle était la fonction occupée par les différents chercheurs ayant répondu au questionnaire. Nous avons obtenu un taux de réponse de 20 chercheurs ayant renseigné leur fonction sur 31 répondants soit $65 \%$.Sur ces 20 répondants, 7 chercheurs soit $35 \%$ sont des attachés de recherche, 4 soit $20 \%$ des chargés d'études ce qui nous donne $65 \%$ des fonctions généralement occupée par des titulaires de Magister. Quant aux autres répondants, ils ont indiqué Maître de recherche (2 occurrences), Chargé de recherche (1 occurrence), Ingénieur ( 2 occurrences), " chercheur " (2 occurrences), Ingénieur principal (1 occurrence) et Directeur adjoint (1 occurrence).

\section{Question n5-}

\section{Diplôme}

Le but de cette question est de déterminer le niveau d'instruction des différents chercheurs du CDTA et cela de par les diplômes obtenus. Sur les 31 répondants, 1 seul chercheur n'a pas indiqué son diplôme et 15 chercheurs soit $50 \%$ sont titulaires du diplôme de Magister, 6 chercheurs soit $20 \%$ du diplôme de Doctorat et 8 chercheurs soit $27 \%$ sont des Ingénieurs, alors qu' 1 chercheur soit 3\% a indiqué le diplôme de Magister Ingénieur. Comme on peut le voir $70 \%$ des chercheurs ont une première ou deuxième post graduation et $27 \%$ sont des Ingénieurs ce qui est normal pour un Centre dont le but est la recherche technologique 


\subsection{2-DEGRE DE CONNAISSANCE DU LIBRE ACCES}

\section{Question nº-}

\section{Êtes-vous au courant des efforts militant pour l'accès libre et gratuit à l'IST ?}

Sur les 31 chercheurs investigués, seuls 20 soit $65 \%$ ont affirmé être au courant des efforts en faveur du libre accès alors que 11 soit $35 \%$ n'ont en pas entendu parler. Le fait que $2 / 3$ seulement des chercheurs d'un centre dont la vocation la premier est de faire de la recherche technologique de pointe ont entendu parler d'un mouvement qui, nonobstant sa nouveauté, est entrain de révolutionner le monde académique est symptomatique d'un manque de sensibilisation. Nous avons à plusieurs reprises eu l'occasion de constater que le libre accès ne bénéficie pas en Algérie d'une grande sensibilisation bien que ses bienfaits soient largement évident. Ceci d'autant plus que sa philosophie est de mettre rendre l'information disponible à tout un chacun (à condition d'une connexion Internet) et surtout aux pays dont le niveau de vie ne permet pas de l'acquérir.

\section{Question n7-}

\section{Connaissez-vous l'O.A.I. ? (Open Access Initiative)}

Cette question a pour but de savoir est ce que les chercheurs qui bénéficient des bienfaits du libre accès en savent les fondements qu'est l'O.A.I. Les chiffres obtenus montrent que 22 chercheurs soit $71 \%$ ne la connaissent pas et que seuls 9 chercheurs soit $29 \%$ en connaissent l'existence. Cela indique clairement une faille dans la sensibilisation mais aussi les efforts des chercheurs eux-mêmes qui doivent être au courant des efforts internationaux dans ce sens.

\section{Question n'8-}

\section{Etes vous au courant de}

-l'Appel de Budapest en 2002

- l'Appel de Berlin en 2003

\section{- l’Appel de Berlin III en 2005}

Cette question n'a reçu de la part des chercheurs du CDTA aucune réponse positive ce qui est étonnant étant donné la prépondérance de ces appels dans le lancement et ensuite la réussite du libre accès. Il va sans dire aussi que les signataires aussi sont inexistants ce qui nous donne un résultat très étonnant et que les chercheurs et responsables du Centre doivent essayer de résoudre.

\section{Question n'9-}

\section{Savez vous qu'est ce qu'une archive ouverte?}

Le archives ouvertes ont été lancés la première fois en sciences exactes en 1991 par le biais de ArXiv et qui a ouvert le champ à de nombreuses expériences les une plus novatrices que les autre au point que les entrepôts d'archives sont devenus de véritables trésors de l'information 
en temps réel surtout pour des spécialités aussi précises que l'astronomie, la physique, l'informatique, etc..... D'ailleurs c'est dans cette branche du savoir où la vitesse est la plus prépondérante que les archives ouvertes se sont le plus développées .Malgré cela, sur les 31 répondants du CDTA, une quasi parité entre ceux qui connaissent et ceux qui ne connaissent pas ce qu'est une archive ouverte. Ils sont 15 chercheurs soit $48 \%$ à connaitre ce qu'est une archive ouverte et 16 soit 52\% à l'ignorer. Encore une fois tel que nous l'avons suggéré, un travail de sensibilisation et de recherche est indispensable afin de pouvoir profiter (dans le sens le plus noble du terme) de cette manne d'information.

\section{Question n¹0-}

\section{En avez-vous déjà utilisé une en dépôt ou consultation ?}

Etant donné que le taux de connaissance du concept d'archive ouverte dans la question précédente s'était avéré assez bas, il est naturel (du moins logiquement) à ce que l'utilisation (en dépôt ou en consultation) suive la même tendance. En fait, ceci s'est confirmé étant donné que sur les 31 répondants, 23 chercheurs soit $74 \%$ ont affirmé ne pas l'utiliser ni en dépôt ni en consultation alors que seuls 8 chercheurs soit $26 \%$ l'utilisent. Plus encore, parmi ces 8 chercheurs qui ont affirmé utiliser une archive ouverte, certains ont cité " Archive d'état civil de l'Algérie sous l'occupation française (1830-1904) ", opencores, opensource, open access journals, ce qui dénote une complète ignorance du concept même d'archives ouvertes. Un seul chercheur a cité ArXiv, 1 autre http://tel.archives-ouvertes.fr, 1 autre a cité TEL (These En Ligne) et enfin 1 chercheur a cité IN TECH. Comme on peut le voir, la méconnaissance des archive ouvertes et leur utilisation est même plus grave étant donné les réponses reçues comme " Archive d'état civil de l'Algérie sous l'occupation française (1830-1904) .....

\section{Question n¹1-}

\section{Connaissez vous ArXiv?}

Comme il est connu, ArXiv est le premier modèle d'archive ouverte et qui a servit (et sert toujours) de référence pour tout ce qui a trait aux archives ouvertes. De plus, elle est spécialisée dans les sciences exactes et même dures ce qui en fait un site incontournable pour tout chercheur en sciences physiques, Mathématiques, Informatique, Biologie quantitative, Finance quantitative et Statistiques. Malgré cela, 24 des répondants à cette question soit $87 \%$ ont répondu qu'ils ne connaissaient pas ArXiv et seuls 4 soit $13 \%$ chercheurs la connaissent. Ces chiffres ne font que corroborer les résultats des deux réponses précédentes et dans les quelles il est apparu clairement que les chercheurs du centre n'ont pas une grande connaissance ni sont sensibilisés à l'importance ni les bienfaits des archives ouvertes. Cet état de fait que nous avons constaté durant ce questionnaire (et même au cours de discussion informelle avec des chercheurs ou au cours de rencontre scientifiques nationales) traduit un manque de sensibilisation qui pourrait, peut être et très rapidement (vu l'extrême rapidité avec laquelle les modèles de transmission des résultats de la recherche évoluent) mettre les chercheurs hors des circuits de la recherche devenue, est il utile de le rappeler, internationale et interdisciplinaire. 


\section{Question n¹2-}

\section{Connaissez-vous:}

\section{-The Registry of Open Access Repositories (ROAR) \\ -The Directory of Open Access Repositories (DOAR)}

Les deux sites recensant les entrepôts en libre accès leur utilisation et leur importance n'est plus à démontrer. Encore une fois, il semblerait que les chercheurs investigués du CDTA soient dans une ignorance quasi-totale de ces deux sites. En effet, sur les 31 répondants, seuls 2 chercheurs soit $6 \%$ connaissent ces deux sites alors que 28 soit $90 \%$ ne les connaissent pas (1 chercheur soit 3\% a répondu qu'il connaissait ROAR et pas DOAR). Ces chiffres traduisent une méconnaissance confirmée mais ne sont nullement étonnant si on examine les résultats des questions ayant précédé et ne sont qu'une suite logique des positions et réponses ayant été fournies.

\section{Question n¹3-}

\section{Avez-vous déjà utilisé des journaux électroniques en libre accès ?}

Si les archives ouvertes (parent pauvre du libre accès selon l'expression consacrée) sont moins connues que les journaux électroniques, on s'attendrait à ce que ces derniers soient mieux connus et utilisés que les archives ouvertes. Cette hypothèse se vérifie dans notre cas mais jusqu'à un certain niveau. En effet, les chercheurs ayant affirmé qu'ils utilisaient les journaux électroniques en libre accès sont au nombre de 17 soit $55 \%$ alors que ceux qui ne les ont pas utilisé sont au nombre de 14 chercheurs soit $45 \%$.Il est, à notre avis, très surprenant qu'en 2011, des chercheurs en spécialités aussi technologiquement avancés que la nanotechnologie, le laser etc...... n'aient pas utilisé des journaux électroniques en libre accès. Le chercheur de nos jours, très souvent tend à utiliser Internet pour ces recherches et de ce fait quand une information lui manque ou qu'il n'est pas sûr d'une information, d'une référence, d'une citation, Internet a l'avantage d'être rapide et sûr. De plus, les centres de recherches ont généralement des abonnements institutionnels permettant au chercheur de consulter sur son poste au travail (ou même à partir de son lieu de résidence) les journaux dont il a besoin.

\section{Question nº14-}

\section{Connaissez-vous}

\section{-African Journal On Line (AJOL)}

\section{- Directory of Open Access Journal (DOAJ)}

Les réponses à cette question confirment les tendances que nous avons observées dans les questions précédentes ayant trait aux habitudes documentaires des chercheurs. Seul 1 chercheur soit $3 \%$ sur les 31 investigués connait AJOL et DOAJ, 1 autre chercheur soit 3\% connait AJOL et ne connait pas DOAJ alors que 3 chercheurs soit $10 \%$ connaissent DOAJ et ne connaissent pas AJOL. Le reste des chercheurs investigués soit 26 chercheurs représentant 
$83 \%$ des répondants ne connaissent ni AJOL ni DOAJ. La question qui se pose est la suivante : est ce qu'il est normal que $83 \%$ de chercheurs d'un Centre dont le périodique scientifique " Technologies Avancées " est le seul périodique algérien avec RIST du CERIST à être référencé par $\mathrm{AJOL}^{*}$ ne connaissent même pas l'existence ces sites (surtout AJOL) et de ce fait ne les utilisent même pas.

\subsection{3-HABITUDES DOCUMENTAIRES ET DE RECHERCHE}

\section{Question n' 15-}

\section{Dans vos activités de recherche et de documentation utilisez-vous plus le support :}

\section{-Papier}

\section{-Electronique}

\section{-Indifféremment}

Comme il est devenu de plus en plus fréquent, le chercheur a tendance à utiliser de plus en plus des supports autres que le papier qui a été avec l'homme depuis des siècles. Parmi ces nouveaux supports, le support électronique occupe de plus en plus une place sans cesse croissante. A cette question, 19 répondants soit $61 \%$ ont répondu que le support électronique était leur support de prédilection alors que le papier n'était plus utilisé que par un seul chercheur soit $3 \%$.A un autre niveau, 8 chercheurs soit $26 \%$ ont répondu que leur l'utilisation n'obéissait pas à une préférence mais qu'ils utilisaient le papier et le support électronique indifféremment et 3 chercheurs soit 10\% utilisaient le papier et le support électronique de manière équilibrée. Une nette préférence de l'utilisation du support électronique se dessine chez les chercheurs du CDTA, ce qui est normal pour des chercheurs en technologie de pointe telle que la nanotechnologie et autres sujets de recherche que les chercheurs du CDTA abordent.

\section{Question nº 16-}

\section{Quel est le support que vous préférez?}

\section{-Papier}

\section{-Electronique}

\section{-Indifféremment}

Si les chercheurs du CDTA utilisent plus le support électronique de part sa facilité et aussi leur formation de base, il n'en demeure pas moins qu'ils ont tendance à préférez dans le faits le papier plutôt que le support électronique. En effet, la tendance s'est pratiquement inversée, étant donné que 17 chercheurs soit $55 \%$ préfèrent le papier et que 11 soit $35 \%$ préfèrent le support électronique. Enfin 3 chercheurs soit 10\% utilisent les deux supports indifféremment. Cette quasi inversion des résultats a été à plusieurs fois documenté dans différentes recherches

\footnotetext{
* Le seul périodique " algérien "en libre accès sur DOAJ est " Synergie Algérie " éditée par le GERFLINT (Groupe d'Études et de Recherches pour le Français Langue Internationale).Cette publication est édité par le base de revues du GERFLINT en France ....
} 
par le fait que la facilité d'accès fait que le chercheur utilise le support électronique mais que ces préférences vont vers le papier pour de multiples raisons dont - entre autres - la facilité du maniement du support (on a pas besoin de machine pour lire un article) et aussi l'habitude de l'utilisation du support papier.

\section{Question n¹7-}

\section{Dans quelle proportion estimez-vous votre utilisation des deux supports?}

-Papier

-Electronique

Si les chercheurs utilisant plus le support électronique mais préfèrent le support papier, le taux d'utilisation quant à lui nous donne des taux où le support papier est très rarement plus utilisé que le support électronique. C'est ainsi qu' 1 chercheur soit 3\% utilise le support électronique plus que le support papier à raison de 5\%-95\%,4 chercheurs soit $13 \%$ à raison $10-90 \%, 9$ chercheurs soit $30 \%$ à raison de $20 \%$ - $80 \%, 1$ chercheur soit 3\% à raison de $25 \%-75 \%, 2$ chercheurs soit $6 \%$ à raison de $30-70 \%, 1$ chercheur soit $3 \%$ à raison de $35 \%-65 \%, 6$ chercheurs soit $20 \%$ à raison de $40 \%-60 \%, 1$ chercheur soit $3 \%$ à raison de $50 \%-50 \% 2$ chercheurs soit $6 \%$ à raison de $60 \%-40 \%, 1$ chercheur soit $3 \%$ à raison de $70 \%-30 \%, 1$ chercheur soit $3 \%$ à raison de $80 \%-20 \%$. Enfin 1 chercheur soit $3 \%$ a répondu que son utilisation dépendait de la disponibilité. Comme on peut le voir, le support électronique est largement plébiscité par rapport au support papier.

\section{Question $n^{\circ} 18-$}

\section{Quelle (s) langue (s) utilisez-vous dans vos soumissions?}

\section{-Français}

- Arabe

-Anglais

\section{-Autres (précisez)}

Il est un fait qui s'est imposé sans conteste à la communauté des chercheurs : la prépondérance et l'importance la langue anglaise dans la communication scientifique. Il est estimé selon certaines études que le taux de ce qui est globalement publié mondialement avoisine les $80 \%$.C'est dire son importance et la presque obligation de sa maitrise afin de s'insérer dans le monde la recherche. Cet état de fait est, un tant soit peu, vérifié par les résultats du questionnaire étant donné que 14 chercheurs soit $45 \%$ des répondants déclarent publier en Français et en Anglais, alors que 13 chercheurs soit 42\% communiquent en Anglais enfin 4 chercheurs soit 13\% communiquent en Français seulement. Ces résultats nous donnent un taux de $87 \%$ de chercheurs qui utilisent et communiquent en langue anglaise. On remarque enfin la non inclusion de l'Arabe comme langue de soumission des recherches contrairement aux chercheurs du CERIST et dont certains avaient évoqué et cité la langue arabe en tant que vecteur de communication. 


\subsection{4-PRODUCTION SCIENTIFIQUE}

Question n¹9-

Avez-vous déjà publié dans des journaux scientifiques?

- Oui

-Non

La publication dans des revues nationales et internationales est une des conditions de promotion et d'avancement pour le chercheur surtout en sciences exactes où les brevets et leurs droits d'usufruit obligent le chercheur à publier. A cet effet, les réponses obtenues du questionnaire soumis aux chercheurs du CDTA indiquent que sur les 31 répondants, 22 chercheurs soit $71 \%$ des chercheurs ont publié dans des revues internationales, 6 chercheurs soit $19 \%$ dans des revues nationales et enfin 3 chercheurs soit 10\% n'ont pas publié du tout. On peut en conclure que $90 \%$ des chercheurs participent aux efforts de publication de leur Centre et que les $10 \%$ restants sont, peut être, des chercheurs novices n'ayant pas encore entamé leur carrière de recherche en publiant.

\section{Question n²0-}

\section{Quel est le nombre de ces publications?}

$$
\begin{array}{r}
-1-3 \\
-3-5 \\
-5+
\end{array}
$$

Si publier fait partie des obligations du chercheur, le nombre de publications est quant à lui révélateur de l'activité du chercheur. Sur les 28 répondants à cette question (les 3 chercheurs ayant affirmé ne pas avoir publié dans la question précédente n'ayant pas répondu à cette question), 15 chercheurs soit $54 \%$ ont répondu avoir publié plus de 5 fois, 9 autres chercheurs soit $32 \%$ ont publié entre 3 et 5 fois alors que 4 chercheurs soit $14 \%$ ont publié entre 1 et 3 fois. Il semblerait d'après ces chiffres que les chercheurs du CDTA ont un rythme de publication assez soutenu et aussi assez volumineux ${ }^{*}$ étant donné que $86 \%$ ont publié entre 5 fois et plus de 5 fois, ce qui est un taux assez encourageant pour la recherche.

\section{Question n²1-}

\section{Avez-vous déjà participé à des conférences dans votre spécialité ?}

\section{-Oui}

-Non

Si publier dans des revues est indispensable pour le chercheur, la participation aux conférences l'est aussi. Ce genre de participation en plus de permettre au chercheur d'être au courant des dernières innovations dans sa spécialité, lui permet aussi de bénéficier de contacts

\footnotetext{
${ }^{*}$ Voir à cet effet $:$ http://www.cdta.dz/communicationscdta_periode.asp
} 
personnels, directs et informels (collège invisible) avec ses collègues et de pouvoir échanger les informations. A cet effet, 26 des répondants soit $84 \%$ du questionnaire ont répondu avoir participé à des conférences nationales et internationales alors que 5 chercheurs soit $16 \%$ n'ont participé qu'à des conférences au niveau national. Ces chiffres confirment les chiffres des questions précédentes qui avaient fait ressortir une production scientifique importante de la part des chercheurs du Centre.

\section{Question n²2-}

\section{Quel est le nombre de ces publications?}

$$
\begin{array}{r}
-1-3 \\
-3-5 \\
-5+
\end{array}
$$

Encore une fois, il apparaît que les activités de participation aux conférences des chercheurs du Centre soient assez soutenues du point de vue quantitatif. Ainsi, 25 chercheurs soit $81 \%$ ont déclaré avoir participé plus de 5 fois alors que 3 chercheurs soit $9 \%$ ont affirmé avoir participé entre 3 et 5 fois et enfin 3 autres chercheurs soit $9 \%$ ont participé entre 1 et 3 fois. Force est de reconnaitre que les efforts de participation aux conférences des chercheurs du Centre tendent à être assez soutenus et ceci surtout de la part de ceux qui publient assez souvent ( + de 5 participations) .

\subsection{5--ATTITUDE VIS-A-VIS DU CONTROLE PAR LES PAIRS}

\section{Question n²3-}

\section{Au cours de vos soumissions saviez-vous si elles étaient contrôlées par les pairs ?}

-Oui

-Non

Le chercheur essaye au cours de ses soumissions de viser les revues les plus prestigieuses de son domaine et sa spécialité. Que ce soit par prestige ou pour publier dans des revues au fort facteur d'impact (et que les décideurs d'octroi de subventions ou les experts décidant des promotions considèrent comme indispensables), le chercheur sait que sa publication est beaucoup plus " considérée " si elle passait l'examen et la vérification scrutatrice du contrôle par les pairs. Cette assertion se vérifie dans les réponses des chercheurs du Centre puisque sur les 31 répondants, seuls 3 chercheurs soit $10 \%$ ont soumis des recherches sans être au courant si elles étaient contrôlées ou pas. Par contre, 28 chercheurs soit $90 \%$ ont soumis tout en sachant que leur travail allait être contrôlé par des experts ce qui impliquerait une attente à ce que le travail soit de qualité supérieure que s'il était soumis à des revues dont le système de contrôle est plus laxiste et de ce fait au facteur d'impact moindre. 


\section{Question n²4-}

Avez-vous soumis vos recherches en tenant compte de ce paramètre ?

-Oui

-Non

Si le chercheur soumet aux revues les plus prestigieuses et les plus cotées pour obtenir de l'avancement et des subventions, il n'en demeure pas moins que ces raisons peuvent être autres. Ceci est, plus ou moins, vérifié dans ces réponses étant donné que si les chercheurs à $90 \%$ soumettent en sachant que leur soumission sera certifiée, ils ne sont que 21 chercheurs soit $68 \%$ à l'avoir fait pour cette raison (soumission certifiée) alors que 10 chercheurs soit 32 $\%$ l'ont fait pour des raisons autres. On peut conclure que si les chercheurs soumettent leurs travaux sachant qu'il sera contrôlé et de ce fait aura plus de valeur, ils ne le font pas automatiquement pour ce genre de raison purement scientifique.

\subsection{6-EXPERIENCE AVEC LE CONTROLE PAR LES PAIRS}

\section{Question n²5-}

Estimez vous que le contrôle par les pairs est indispensable à l'avancement de la Science?

\section{-Oui \\ -Non}

Si les chercheurs dans leur activités soumettent à des revues tout en sachant si elles sont contrôlées ou pas et en même temps le feraient pour d'innombrables et différentes raisons (comme par exemple l'avancement, être publié, etc......), il n'en demeure pas moins que ces mêmes chercheurs reconnaissent l'importance et l'inéluctabilité du contrôle par les pairs dans la recherche scientifique et technique. Ainsi, sur les 29 répondants ( 2 chercheurs n'ayant pas répondu à cette question) 27 chercheurs soit $93 \%$ ont répondu par l'affirmative quant à l'indispensabilité du contrôle par les pairs alors que seuls 2 chercheurs soit $7 \%$ ne pensent pas que le contrôle par les pairs soit indispensable.

\section{Question n²6-}

Avez-vous eu l'impression au cours de vos soumissions que votre travail a été jugé d'une manière objective?

$$
\begin{aligned}
& \text {-Oui } \\
& \text {-Non }
\end{aligned}
$$

Nous l'avons dans la partie réservée aux différents griefs adressés au contrôle par les pairs que le manque d'objectivité (pour nombre de raisons) constituait une des critiques les plus citées. Ce manque d'objectivité qui pouvait prendre des formes diverses et variées minaient les relations entre les experts et les auteurs. Cette question posée aux chercheurs du CDTA a élicité un taux de réponses assez élevé quant au jugement objectif qu'auraient reçus les 
soumissions des chercheurs du Centre. Ainsi sur les 30 répondants ( 1 chercheur n'ayant pas répondu à la question), 25 chercheurs soit $83 \%$ pensent avoir été jugés d'une manière objective lors de leur soumission alors que 3 chercheurs soit $10 \%$ ne le pensent pas. Deux chercheurs soit $7 \%$ quant à eux donnent des réponses où ils expriment en même temps une satisfaction et aussi une certaine déception avec la manière dont ils ont étés jugés.

\section{Question n²7-}

Estimez vous normal que dans les formes traditionnelles de certification, le nom de l'expert soit tenu secret?

- Oui

-Non

Parmi les autres griefs faits au contrôle par les pairs figure en bonne place l'anonymat dont bénéficie l'expert lors de son travail d'expertise. Cette manière de procéder à donné lieu à de nombreuses études dont la plupart avaient conclut qu'il (l'anonymat des experts) était propice aux dépassements. Malgré cela, les chercheurs du CDTA n'y voient pas un problème puisque sur les 30 répondants ( 1 chercheur n'ayant pas répondu à la question), 23 soit 77\% pensent que ceci est normal alors que seul 6 chercheurs soit $20 \%$ ne le pensent .Enfin, 1 chercheur soit $3 \%$ a répondu en même temps par oui et non expliquant que ceci dépendait d'un nombre de paramètres.

\section{Question n²8-}

Au cours de vos soumissions, avez-vous pris en ligne de compte les correctifs que l'on vous a demandé d'apporter?

-Oui

-Non

Comme il est connu, le manuscrit soumis fait très souvent l'objet d'aller - retour entre l'auteur et les experts afin d'y apporter les correctifs que ces derniers jugent opportuns. D'ailleurs de la (ou les) réponse qu'apporte l'auteur à ces demandes de corrections dépend la publication ou non de la recherche, ce qui démontre l'importance de cette étape. A cette question, sur les 30 répondants ( 1 chercheur n'ayant pas répondu à la question), 29 chercheurs soit $97 \%$ ont répondu qu'ils avaient pris en ligne de compte les correctifs demandés alors qu'1 seul chercheur soit 3\% ne l'a pas fait. Ces résultats nous indiquent clairement que les chercheurs du CDTA sont plutôt convaincus des arguments présentés par les experts et les ont appliqué et ont apporté les correctifs demandés afin de voir leur soumission publiée. 


\section{Question n²9-}

\section{L'avez-vous fait convaincu des arguments de l'expert ou tout simplement pour être publié ?}

De la décision des experts dépend la publication ou non de la soumission. L'auteur est donc " à la merci de l'expert" (si on peut s'exprimer de cette manière).L'auteur doit donc abonder dans le même sens que l'expert au risque de voir son travail rejeté ou alors le convaincre par ses propres arguments de la justesse de ces idées. Nous nous trouvons donc devant deux éventualités qu'a l'auteur : soit être convaincu des arguments de l'expert et faire les correctifs demandés ou alors les accepter juste pour se faire publier ( peut être pour des raisons de promotion dont la publication en question dépend ).Pour ce qui est de notre questionnaire, les chercheur du CDTA ont apporté les réponses suivantes : sur les 30 répondants ( 1 chercheur n'ayant pas répondu à la question), 20 chercheurs soit $67 \%$ l'ont fait convaincus des arguments des experts, 8 chercheurs soit $27 \%$ en même temps par les arguments des experts et pour être publié, 1 chercheur soit $3 \%$ pour tout simplement être publié enfin 1 autre chercheur 1'a fait " plutôt pour expliquer ce qui est ambigu ".On peut conclure que les résultats de cette question abondent dans le même sens que les résultats de la question précédente où la quasi totalité des chercheurs avaient apportés les correctifs demandés .

\section{Question n³0-}

Pouvez-vous indiquer le pourcentage de vos soumissions acceptées par rapport à celles soumises?

Le pourcentage des soumissions acceptées par rapport à celles soumises indique le taux de réussite des chercheurs dans leur effort de recherche. Les différentes recherches ayant investigué les taux d'acceptation dans les différentes sciences ont fait ressortir que les sciences exactes et dures ont toujours eu des taux plus élevés que les sciences humaines et sociales. Cette tendance semble se confirmer concernant les chercheurs du CDTA étant donné que sur le 27 chercheurs ayant répondu (4 chercheurs n'ayant pas répondu à cette question), 25 soit $93 \%$ ont des taux dépassant les $50 \%$, alors que 19 chercheurs soit $70 \%$ ont des taux entre 80 et $100 \%$ avec ce dernier taux (100\%) totalisant 5 chercheurs soit $19 \%$ des chercheurs ayant eu toutes leur recherches acceptées. Les réponses fournies sont comme suit: 4 chercheurs soit $16 \%$ (4\% pour chaque chercheur) ont eus leurs soumissions acceptées à hauteur de $10 \%, 50 \%, 60 \%$ et enfin 70\%.Deux chercheurs soit $7 \%$ ont eu leurs soumissions acceptées à hauteur de $75 \%$ et 2 soit $7 \%$ autres à raison de $77.77 \%$.Cinq chercheurs soit $19 \%$ ont eus un taux d'acceptation de $80 \%, 7$ autres chercheurs soit $26 \%$ ont enregistré un taux de $90 \%$ alors que les taux de 95 et $99 \%$ ont enregistré 1 réponse chacune soit $4 \%$ pour chacune. Enfin, tel que déjà signalé plus haut, un taux de $100 \%$ a été enregistré par 5 chercheurs soit 19\% de l'ensemble des répondants n'ont pas eu de soumissions rejetées. Ces chiffres, en plus de confirmer la tendance des taux d'acceptation habituellement haut dans les sciences exactes et dures, prouvent que les chercheurs du CDTA participent de manière positive aux progrès de la recherche dans leurs spécialités respectives. 


\subsection{7-NOUVELLES FORMES DE CONTROLE DE L'INFORMATION}

Question n`31-

Êtes-vous au courant des nouvelles formes de contrôle par les pairs des publications scientifiques sur Internet?

\section{-Oui \\ -Non}

Le chercheur ne peut de nos jours se suffire de publier, faire de la recherche et participer à des conférences dans sa spécialité sans avoir recours aux nouvelles technologies ayant révolutionné le monde de la publication scientifique. Alors qu'avant l'avènement des réseaux (et Internet en particulier), la vitesse de transmission de l'information était lente et dépendait de facteurs multiples, le chercheur de nos jours est connecté pratiquement en temps réel à ses collègues et à ses sites de prédilection. Malgré ces avantages, il semblerait que les chercheurs du CDTA ne soient pas très au courant de ces nouvelles formes et de ce fait ne peuvent pas en profiter. Les réponses sont à cet effet tout ce qui a de plus clair : 29 des chercheurs investigués soit $94 \%$ ne sont pas au courant de ces nouvelles formes de contrôle par les pairs et seuls 2 chercheurs soit $6 \%$ en sont au courant. Ces chiffres paraissent d'autant plus étonnants que dans la question précédente les chercheurs avaient affiché des taux d'acceptation très respectable qui auguraient d'une bonne connaissance de ce genre de certification de l'information.

\section{Question n³2-}

Accepteriez vous que votre soumission soit mise sur Internet et jugée par toute la communauté?

-Oui

-Non

Les nouvelles formes de certification de l'information ont introduits de nombreux aspects novateurs se prévalant tous de la rapidité, l'interactivité et surtout l'ouverture que les réseaux permettent et encouragent même. Parmi les plus révolutionnaires de ces aspects est ce qui est communément connu sous l'appellation de contrôle par les pairs ouvert (open peer review ) et dont un dossier a été publié par Nature en 2006 ( Voir supra dans cette partie ).Dans cette forme de certification, c'est toute la communauté qui juge le travail mis sur Internet et qui est ensuite accepté ou non selon ces expertises ou avis mais dont le dernier mot reste dans une expertise classique dans sa démarche telle qu'elle est actuellement opérée. Ces schémas bousculent de toute évidence nombre d'habitudes et comme toute révolution (car c'est des schémas révolutionnaires pour un processus aussi traditionnel et fermé que l'a été le contrôle par les pairs avant l'avènement d'Internet), ils rencontrent des résistances quant à son acceptation .Malgré cela, 20 des chercheurs du CDTA soit $65 \%$ ont répondu à cette question par l'affirmative alors que seuls 11 chercheurs soit $35 \%$ sont contre cette procédure. Si ceci est symptomatique de quelque chose, c'est l'ouverture des chercheurs du CDTA sur le monde 
et aussi le fait qu'ils soient prêts à affronter le jugement des autres chercheurs pour certifier leurs recherches.

\section{Question n 33-}

\section{Accepteriez vous que ces commentaires soient publiés sur Internet ?}

-Oui

-Non

Si avoir sa soumission mise sur Internet et jugée par toute la communauté des chercheurs de la spécialité (et même des fois des personnes ne faisant pas nécessairement partie de la communauté) est en lui-même révolutionnaire, un autre aspect plus radical fait appel à la publication de ces même commentaires sur Internet. Cet aspect fait partie de nouvelles manières de certification qui tendent toutes à le rendre (le contrôle par les pairs) de plus en plus ouvert et en même temps responsable et justifiable. Il va sans dire que cette manière n'a pas rencontré que des avis favorables de la part et des chercheurs soumissionnaires et aussi des experts (dans le sens traditionnel du terme).Ces critiques sont nombreuses mais on peut les résumer en deux catégories ; la première concerne les auteurs qui ne veulent pas avoir leur soumission (avec tous ses défauts et faiblesses) exposée au vu et su de tout le monde et la deuxième concerne les experts qui ne veulent pas que leur travail soit "dévalorisé " par l'intervention de personnes qui n'auraient pas les compétences requises pour pouvoir juger de la solidité ( méthodologique et scientifique ) de la soumission. Nonobstant toutes ces critiques et discussions somme toutes normales pour des nouvelles approches, les réponses des chercheurs du CDTA semblent être de concert avec les avis des chercheurs en général. Ainsi sur les 31 chercheurs investigués, 19 soit $61 \%$ n'accepteraient pas que les commentaires de leur soumission soient mis et publiés sur Internet lors que 12 chercheurs soit $39 \%$ l'accepteraient.

\section{Question n 34-}

Accepteriez-vous en cas d'acceptation que ces commentaires soient publiés sur Internet avec la version finale et définitive ?

-Oui

-Non

Si la certification des travaux et des recherches sur Internet ainsi que la publication des commentaires sont des étapes en elles-mêmes révolutionnaires, il existe une autre étape encore plus révolutionnaire et qui consiste à publier avec le manuscrit accepté les remarques et autres jugements que la communauté a pu émettre. Certains chercheurs ont comparé cette manière d'agir comme étant un article en perpétuel expertise vu que ces remarques peuvent donner lieu à d'autres remarques et ainsi de suite .De plus, certains chercheurs ont posé la question de savoir quelle est la version acceptée de l'article étant donné que les remarques émises peuvent durer un temps indéterminé. Face à ces différents problèmes et approches, les chercheurs du CDTA adoptent une position qu'on pourrait décrire de novatrice vu que sur les 
31 chercheurs, 23 soit $74 \%$ accepteraient que les commentaires soient publiés sur Internet alors que 8 chercheurs soit $26 \%$, ne le souhaiteraient pas. En conclusion, on peut dire que les chercheurs du CDTA adoptent des positions novatrices étant donné qu'ils acceptent ces nouveaux schémas à raison de $3 / 4$ pour et $1 / 4$ contre.

\title{
4.4.8-POSITION DES CHERCHEURS VIS-A-VIS DES NOUVELLES FORMES DE CONTROLE DE L'INFORMATION
}

\section{Question n 35-}

Pensez vous que ces nouvelles formes de certification plus ouvertes peuvent contribuer à atténuer les accusations de subjectivité et de préjudice qui semblent, des fois, entacher le processus?

$$
\begin{aligned}
& \text {-Oui } \\
& \text {-Non }
\end{aligned}
$$

Il est connu que le contrôle par les pairs est sujet à des comportements peu amènes et aussi et surtout il a été prouvé, à maintes reprises, qu'il était incapable (certains chercheurs ont même déclaré que cela n'était pas de son ressort ni même qu'il en était capable) de détecter les fraudes qui, malheureusement, ne sont pas des exceptions, preuve en est les différentes affaires ayant étés largement relayées et commentées par les medias ces dernières années et dont nous avons parlé plus tôt. Nous obtenons en ce qui concerne les chercheurs du CDTA, un résultat assez équilibré étant donné que sur les 30 répondants ( 1 chercheur n'ayant pas répondu à la question), 16 chercheur soit $53 \%$ pensent que ces nouvelles manières d'opérer peuvent atténuer les différentes accusations de subjectivité et de fraudes qui surfacent de temps à autre lors que 14 chercheurs soit $47 \%$ ne le pensent pas et tendent à ne pas considérer ces nouveaux schémas comme dissuasifs. On peut en conclure que les chercheurs du CDTA sont pratiquement égaux en taux quant à l'efficacité de ces nouveaux schémas dans la lutte contre la fraude et la subjectivité dans le jugement.

\section{Question n³6-}

Pensez vous que cette subjectivité peut être d'ordre :

\author{
-Sexuelle (homme ou femme) \\ -Linguistique (selon la langue utilisée) \\ -Religieuse (précisez) \\ -Personnelle \\ -Autres (précisez)
}

Les réponses obtenues pour cette question sont très diverses et peuvent être classées en quelques groupes de réponses assez disparates. Ainsi sur les 23 réponses obtenues (8 chercheurs n'ayant pas répondu à cette question), les préjudices d'ordre personnel sont les plus cités et ce à raison de 6 fois soit $26 \%$ suivi par les préjudices d'ordre linguistique et 
religieux avec 3 occurrences soit $13 \%$.Les préjudices d'ordre seulement linguistique sont cités à 2 reprises soit $9 \%$ ainsi que les préjudices contre les chercheurs du tiers monde ( les réponses ont indiqué que les " chercheurs du tiers monde sont marginalisés ") à 2 reprises soit $9 \%$ aussi. Le reste des réponses est, comme nous l'avons dit, assez disparate et comprend des réponses comme l'expert n'est pas assez expert dans la spécialité (argument assez cité dans la littérature du sujet d'ailleurs), religieux et culturel, géographique (préjuge contre monde arabe musulman alors que pour l'Algérie ceci arrive en de rares cas et un nombre d'autres raisons très divers). Nous remarquerons seulement que le préjudice linguistique et personnel est le plus cité sous différentes formes et combinaison avec d'autres raisons ce qui nous pousse à conclure que les chercheurs investigués ont étés victimes de préjudice soit personnellement ou plus plausiblement à cause de la langue utilisée ceci est plausible quant on voit que les chercheurs du CDTA publient à $87 \%$ en langue anglaise (dans la question posée au sujet de la langue utilisée, 14 chercheurs avaient déclaré qu'ils publiaient en français - anglais et 13 en anglais ) qui n'est pas à proprement parlé une langue très usitée en Algérie ( à part peut être dans les milieux de la recherche en sciences exactes comme les réponses à cette question pour le CDTA tendraient à le prouver ).

\section{Question n 37-}

\section{Pensez vous que cette ouverture peut aider à combattre le plagiat et la fraude ?}

\section{-Oui \\ -Non}

Une des grandes hantises du chercheur est de se voir dépossédé du fruit de son travail par une autre personne ou de voir un travail non scientifique frauduleux publié surtout si ceci se passe dans la spécialité du chercheur concerné. La littérature du sujet abonde, et ce depuis les premières manifestation de la recherche scientifique, d'histoires de fraude et de plagiat ayant causé du tort à la science .L'avènement des réseaux (surtout Internet) constitue une occasion pour le chercheur qui peut être au courant de ce qui se passe dans son domaine et aussi en même temps un moyen de contrôler l'information et son utilisation. Le chercheur peut en un clic de souris avoir pratiquement la totalité de la littérature du sujet à laquelle il est intéressé. Cette ouverture et disponibilité peut être une arme à double tranchant car soit le chercheur l'utilise pour acquérir du savoir ou dans le cas contraire, il peut l'utiliser afin de s'accaparer le savoir des autres. Quant aux chercheurs du CDTA, ils ont répondu majoritairement que cette ouverture pouvait conduire à atténuer le plagiat et la fraude et ce à $65 \%$ étant donné que 20 ont répondu par l'affirmative alors que 9 soit $29 \%$ ont répondu par la négative. Enfin 2 chercheurs soit $6 \%$ ont répondu qu'ils ne savaient pas. 


\section{Question n³8-}

Dans le cadre de votre éventuel travail de certification, accepteriez-vous d'être connu de celui dont vous expertisez le travail ?

-Oui

-Non

Il est de notoriété que l'anonymat dont bénéficie les experts dans le cadre du contrôle par les pairs traditionnel a été sujet à de nombreuses études et recherches afin de déterminer est ce qu'il est (l'anonymat) propice à des dépassements. Si les chercheurs sont généralement contre cet aspect (à part quelque cas rares) car ils y voient une sorte d'impunité pour l'expert qui peut juger un travail sans rendre compte (à part à l'éditeur) ni être inquiété alors que leur identités est elle connue, il est intéressant d'essayer de savoir leur position dans le cas contraire c'est-à-dire lorsqu' ils sont appelés à certifier eux mêmes un travail. Encore une fois nous obtenons une quasi égalité car sur les 31 chercheurs ayant répondu, 17 soit $55 \%$ accepteraient d'être connu alors que 14 chercheurs soit $45 \%$ ne l'accepteraient pas. Au delà de ces résultats quantitatif, il est intéressant de remarquer que les chercheurs ayant ajouté une remarque (10 chercheurs au total sur les 31 investigués) ont à 5 reprises soit $50 \%$ évoqué (sous différentes formes) le critère d'objectivité qui doit être la base du travail d'expertise. A un degré moindre, les cas de conflit potentiels entre chercheurs et de possible revanche ont étés aussi cites. Ces chiffres traduisent à notre avis des positions non tranchées car elles font rentrer en ligne de compte des acquis, des avantages assez importants pour que les chercheurs puissent y trancher d'une manière franche.

\section{Question n³9-}

Pensez-vous que les recherches publiées dans les archives ouvertes et les journaux électroniques peuvent être pris en ligne de compte au même titre que les publications sur papier?

\section{- Oui \\ -Non}

Il est une idée largement répandue que les journaux électroniques gratuits en libre accès sur Internet n'ont pas la même manière de certifier l'information scientifique et technique. Ceci est dû, entre autres, à la prolifération de sites et de la tendance un tant soit peu désordonnée et des fois libertaire des premières années d'Internet. L'idée que ce qui est sur Internet n'est pas contrôlé est très prévalent même chez les chercheurs au fait des progrès du libre accès. D'un autre côté, la pression mise sur le chercheur afin de publier dans des revues de référence ou noyaux (core periodicals) au fort facteur d'impact (connues aussi sous l'appellation de " revues de rang A") afin de progresser, lui fait considérer la publication dans ces revues avec circonspection. La même méfiance s'applique beaucoup plus même aux archives ouvertes où les critères de certification et de contrôle ne sont ni les mêmes ni, des fois, mêmes existantes. Cette question a pour but donc de voir est ce que le chercheur publierait dans ces revues et ces entrepôts afin de se faire considérer pour une éventuelle promotion ou financement. Force est de constater que les chercheurs du CDTA ne semblent pas accorder à cette nouvelle 
manière de publier toute la confiance qu'elle mérite .Ainsi sur les 30 répondants, seuls 11 chercheurs soit $37 \%$ pensent que les revues électroniques et les archives ouvertes peuvent être prises en ligne de compte dans les promotions, financements et octroi de poste. De leur coté, 19 chercheurs soit $67 \%$ ne pensent pas que ces nouveaux medias peuvent être prises en ligne de compte .Encore une fois au delà de cette quantification ( $1 / 3$ pour et $2 / 3$ contre), il est intéressant de remarquer qu'une l'idée centrale semble se détacher des réponses : libre accès $=$ absence de contrôle. Cette idée est donnée par un grand nombre de répondants mais est résumée de meilleure manière par la réponse d'un chercheur - que nous reproduisons in extenso - car elle nous semble synthétiser et cristalliser les points de vue d'un nombre de chercheur. Il dit : "Le problème qui se pose concernant les publications dans des journaux libres, est le fait que d'un côté, ça donne une impression de manque de rigueur et de sérieux, car j'ai l'impression que le correcteur n'ai pas assez connu, et il fait cela pour remplir son $C V$. Je pense que dans la communauté scientifique, on est très souvent attaché à la notion de "prestige" et de "reconnaissance" qui ne vient que par le fait de se retrouver dans un cocon de " noms" célébrés [célèbres] "ayant publié, et n'ayant jugé qu'une classe particulière de gens, en d'autre termes, les journaux libres donnent accès à tous, et font perdre de ce fait la notion d'élitisme ". Cette réponse résume, on ne peut mieux, cette méfiance de se retrouver avec des personnes qui ne font pas partie de notre classe. La notion d'élitisme est citée car le chercheur ne veut pas être cité, ni jugé que par ses pairs, des personnes avec les quels il partage un fonds commun. Internet, vecteur de base de ces nouveaux schémas, est considéré donc comme trop " populaire " car permettant l'accès à tout un chacun.

\section{5- Tableau comparatif des réponses des chercheurs du CERIST et du CDTA}

A l'issue de cette analyse des réponses des chercheurs des deux centres pris en exemple, nous mettrons les résultats sous la forme d'un tableau afin de voir quelles sont les différences et les analogies dans leur attitude et approche.

\begin{tabular}{|c|c|c|}
\hline Q. & CERIST & CDTA \\
\hline $1-$ & Entre 1955 et 1961 ( 17 sont dans la trentaine) & $\begin{array}{l}\text { Entre } 1952 \text { et } 1981 \text { ( } 8 \text { sont dans la quarantaine) }, 8 \\
\text { n'ont pas répondu }\end{array}$ \\
\hline $2-$ & 19 - masculin, 16- féminin & 20 -masculin, 11 - féminin \\
\hline $3-$ & 16 -D.R.S.I., 11- D.R, 6- D.S.I.S.M, 2 -D.T.I.S.M. & 2-A. S. M., 9- P. R., 10- M. N., 10- M.I. L. \\
\hline 4- & $\begin{array}{l}16 \text {-A.R., } 8 \text {-C.E., } 1 \text {-D.D.,1-M.R., 2- " chercheurs } \\
\text { " }\end{array}$ & $\begin{array}{l}\text { 7-A.R., 4-C.E., 2-M.R, 1-C.R., } \\
\text { " CHER."ING., } 1 \text { 2-ING.PR, 1-DIR. ADJ. }\end{array}$ \\
\hline 5- & 22 mag., 6 ing., 5 doct., 1 lic., 1 mast. 2 & $\begin{array}{l}15 \text {-mag, } 6 \text {-doct } 8 \text {-ing, } 1 \text {-mag ing., 1- n'a pas } \\
\text { répondu }\end{array}$ \\
\hline $6-$ & oui $-74 \%$, non- $26 \%$ & oui $-65 \%$, non- $35 \%$ \\
\hline $7-$ & oui- $49 \%$, non- $51 \%$ & oui- $29 \%$, non- $71 \%$ \\
\hline $8-$ & $\begin{array}{l}\text { oui- } 20 \% \text {, non- } 80 \% \text { ( } 2 \text { Budapest, } 1 \text { Budapest et } \\
\text { Berlin) }\end{array}$ & oui $-00 \%$, non $-100 \%$ \\
\hline 9- & oui- $71 \%$, non- $29 \%$ & oui- $48 \%$, non- $52 \%$ \\
\hline $10-$ & oui $29 \%$ ( généralement consultation), non $71 \%$ & oui- $26 \%$, non- $74 \%$ \\
\hline $11-$ & oui $34 \%$, non $66 \%$ & oui $-13 \%$, non $-87 \%$ \\
\hline
\end{tabular}

\footnotetext{
* C'est nous qui corrigeons car il nous semble que le mot célèbre est plus adéquat que célébrés.
} 


\begin{tabular}{|c|c|c|}
\hline $12-$ & oui $04 \%$, non $96 \%$ & $\begin{array}{l}\text { oui }-06 \% \text {, non - 90\%, 3\%-oui-non } \\
\text { DOAR) }\end{array}$ \\
\hline 13 & $\begin{array}{l}\text { OUI- } 49 \% \text { (B.B.F., } \\
\text { archimag), NON }-51 \%\end{array}$ & OUI- $55 \%$, NON $\%-45 \%$ \\
\hline $14-$ & oui $-11 \%$, non $74 \%$, oui-non $14 \%$ (AJOL), & $\begin{array}{l}\text { oui- } 03 \% \text {, non- } 83 \% \text {, oui-non-3\% (AJOL-DOAJ) } \\
\text { oui-non- } 10 \%(\text { DOAJ -AJOL) }\end{array}$ \\
\hline $15-$ & elec.- $68 \%$, pap.- $3 \%$, indif.- $23 \%$, elec.-pap.- $06 \%$ & elec. $-61 \%$, pap. $-3 \%$, indif.- $26 \%$, elec.-pap. $-10 \%$ \\
\hline $16-$ & elec.- $40 \%$, pap.- $43 \%$, indif.- $17 \%$, & elec.- $35 \%$, pap.- $55 \%$, indif.- $10 \%$, \\
\hline $17-$ & $\begin{array}{l}05-95 \%(3), 10-90 \%(4), 20-80 \%(10), 30-70 \%(9), \\
40-60 \%(2), 49 \%-51 \%(1), 50-50 \%(1), 60-40 \% \\
(1), 70-30 \%(1), 9-10 \%(1)\end{array}$ & $\begin{array}{l}05-95 \%(1), 10-90 \%(4), 20-80 \%(9), 25 \%-75 \%(1) \text {, } \\
30-70 \%(2), 35-65 \%(1), 40 \%-60 \%(6), 50-50 \% \\
(1), 60-40 \%(2), 70-30 \%(1), 80-20 \% \text { (1), selon } \\
\text { disponibilité (1) }\end{array}$ \\
\hline $18-$ & $\begin{array}{l}\text { anglais-français (16), anglais (10), français (3) } \\
\text { arabe-français (3), arabe (1), français-anglais- } \\
\text { arabe-allemand (1), français-arabe-anglais (1) }\end{array}$ & anglais-français (14), anglais (13), français (4) \\
\hline $19-$ & $\begin{array}{c}\text { non-46\%,oui-54\%, (14\%-internationales, } 17 \% \text { - } \\
\text { nationales, } 23 \% \text {-nationales- internationales) }\end{array}$ & $\begin{array}{c}\text { non-10\%, oui-90\%, (71\%-internationales, } 19 \% \text { - } \\
\text { nationales, }\end{array}$ \\
\hline $20-$ & $11(1-3), 4(3-5), 4(+5)$ & $4(1-3), 9(3-5), 15(+5)$ \\
\hline $21-$ & $\begin{array}{l}\text { non- } 17 \%, \quad \text { oui- } 83 \%(31 \% \text {-internationales, } 17 \% \text { - } \\
\text { nationales, } 35 \% \text { nationales- internationales }\end{array}$ & $\begin{array}{l}\text { non- } 00 \% \text {, oui- } 100 \% \text { ( } 84 \% \text { internationales, } 16 \% \\
\text { nationales) }\end{array}$ \\
\hline $22-$ & $12(1-3), 6(3-5), 11(+5)$ & $3(1-3), 3(3-5), 25(+5)$ \\
\hline $23-$ & oui-24, non-7, n'ont pas répondu -4 & oui- 28, non-3, \\
\hline $24-$ & $\begin{array}{l}\text { oui-26 (14 avancement, } 2 \text { prestige, } 2 \text { avancement- } \\
\text { prestige, } 8 \text { n'ont pas donné de raisons), non- } 9\end{array}$ & $\begin{array}{l}\text { oui- } 21 \text { (oui- pub avanc-10, } \\
\text { oui-pub avanc. et prestige- } 2 \\
\text { oui- } 2 \text {, divers }-7, \text { non- } 10\end{array}$ \\
\hline $25-$ & oui-31, non-1, n'ont pas répondu -3 & oui- 27, non- 2, n'ont pas répondu -2 \\
\hline $26-$ & oui-22, non-5, oui-non-1, n'ont pas répondu-7 & oui- 25, non-3, oui-non-2, n'a pas répondu-1 \\
\hline $27-$ & $\begin{array}{l}\text { 20-normal, 10-anormal, 4- n'ont pas répondu, 1- } \\
\text { normal-anormal }\end{array}$ & $\begin{array}{l}\text { 23-normal, 6-anormal, 1-normal-anormal, 1- n'a } \\
\text { pas répondu, }\end{array}$ \\
\hline $28-$ & oui-30, non-1, n'ont pas répondu-4 & oui-29, non-1, n'a pas répondu-1 \\
\hline 29 & $\begin{array}{l}\text { 22-arguments des experts, 7-être publié, 2- } \\
\text { arguments experts-être publié, 4-n'ont pas répondu }\end{array}$ & $\begin{array}{l}\text { 20-arguments des experts, 8-être publié, 1- } \\
\text { arguments experts- être publié, 1-n'a pas répondu }\end{array}$ \\
\hline $30-$ & $\begin{array}{l}1-00 \%, 1-33 \%, 5-50 \%, 2-60 \%, 5-70 \%, 3-80 \% \text {, } \\
2-90 \%, 2-100 \%\end{array}$ & $\begin{array}{l}1-10 \%, \quad 1-50 \%, 1-60 \%, 1-70 \%, \quad 2-75 \%, \quad 2- \\
77.77 \%, 5-80 \%, 7-90 \%, 1-95 \%, 1-99 \%, 5-100 \%, \\
\text { n'ont pas répondu }\end{array}$ \\
\hline $31-$ & oui-29, non-5, 1-n'a pas répondu & oui-29, non-2 \\
\hline $32-$ & oui-23, non-10, non-oui-1, n’a pas répondu-1 & oui-20, non-11 \\
\hline $33-$ & oui-19, non-13, 1-oui-non, 2-n'ont pas répondu & oui-19, non-12 \\
\hline $34-$ & oui-22, non-11, oui-non- 1, 1-n’a pas répondu & oui- 23, non- 8 \\
\hline $35-$ & $\begin{array}{l}\text { oui - } 21 \text {, non - } 10 \text {, oui - non - 1, 3-n'ont pas } \\
\text { répondu }\end{array}$ & oui - 16 , non - 14,1 - n’a pas répondu \\
\hline $36-$ & $\begin{array}{l}\text { linguistique-10, personnelle-6-diverses-12 } \\
\text { (manque d'expérience et de compétence dans le } \\
\text { domaine, renommée de l'encadreur ou directeur de } \\
\text { laboratoire, appropriation des œuvres des autres, } \\
\text { linguistique-personnelle, personnelle-religieuse, } \\
\text { religieuse-linguistique-2fois-et linguistique- } \\
\text { sexuelle) -7- n'ont pas répondu }\end{array}$ & $\begin{array}{l}\text { linguistique et religieux-3, personnelle }-6 \text {, } \\
\text { linguistique-2, chercheurs tiers monde }-2 \text {, diverses- } \\
10 \text { (manque d'expertise de l'expert, religieux et } \\
\text { culturel, géographique),8-n'ont pas répondu }\end{array}$ \\
\hline $37-$ & oui - 20, non - 7 , oui - non-2, 6-n'ont pas repondu & oui - 20, non - 9 , oui - non- 2 \\
\hline $38-$ & oui-23, non-9, oui-non-1, 2-n'ont pas répondu & oui -17, non -14 \\
\hline $39-$ & oui-18, non-13, n'ont pas répondu-4 & oui-11, non- 19, n'a pas répondu-1 \\
\hline
\end{tabular}

Tableau16-Tableau comparatif des réponses des chercheurs du CERIST et du CDTA 


\section{6-Libellé des questions et des abréviations :}

Q.1-Age des chercheurs

Les chercheurs investigués sont dans la plupart soit trentenaires (pour le CERIST) ou alors quadragénaires (pour le CDTA).On remarquera le haut taux de non réponses des chercheurs du CDTA ( 8 au total) qui au cours des différentes correspondances que nous avons eu avec eux (elles) ont semblés réticent quant à donner des informations concernant leur âge. Difficulté que nous n'avons pas rencontrée avec les chercheurs du CERIST.

\section{Q.2-Sexe des chercheurs}

Sur les 35 chercheurs du CERIST investigués, une quasi parité apparaît étant donné que 19 chercheurs sont de sexe masculin et 16 de sexe féminin. Quant au CDTA, sur les 31 répondants, un peu plus d'un 1/3 est de sexe féminin alors que les $2 / 3$ sont de sexe masculin. Encore une fois, cette proportion n'est peut être pas représentative mais les difficultés posées par le retour des questionnaires n'ont pas permis d'avoir ce qui aurait été considéré comme un échantillon représentatif.

\section{Q.3-Différentes Divisions}

\section{-CERIST:}

D.R.S.I.: Division Recherche et Développement en Sciences de l'information.

D.R : Division Réseau.

D.S.I.S.M : Division Systèmes d'information et Systèmes Multimédia.

D.T.I.S.M : Division Théorie et Ingénierie Systèmes Informatiques.

Les quatre divisions constituant le CERIST ont toutes répondues au questionnaire. Les pourcentages des divisions par rapport aux répondants totaux nous donne les chiffres suivants :

$46 \%$ des répondants sont de la Division Recherche et Développement en Sciences de l'information.

$31 \%$ des répondants sont de la Division Réseau

$17 \%$ des répondants sont de la Division Systèmes d'Information et Systèmes Multimédia.

$06 \%$ des répondants sont de la Division Théorie et Ingénierie des Systèmes Informatiques.

Etant donné que le chiffre total des chercheurs au CERIST - et celui de chaque division - ne nous est pas connu (bien qu'un responsable nous ait affirmé que ceux susceptibles de publier ne dépassait pas la soixantaine), ces pourcentages ne nous sont pas d'un grand secours à part indiquer le nombre de répondants de chaque division. 


\section{-CDTA :}

A. S. M. : Division Architecture des Systèmes et Multimédia

P. R. : Division Productique et Robotique

M. N. : Division Microélectronique et nanotechnologie

M.I. L. : Division Milieux Ionises et Laser.

Les quatre divisions constituant le CDTA ont toutes répondues au questionnaire. Les pourcentages des divisions par rapport aux répondants totaux nous donnent les chiffres suivants :

07\% des répondants sont de la Division Architecture des Systèmes et Multimédia.

$29 \%$ des répondants sont de la Division Productique et Robotique.

$32 \%$ des répondants sont de la Division Microélectronique et nanotechnologie.

$32 \%$ des répondants sont de la Division Milieux Ionises et Laser.

D'un autre coté, les différentes divisions représentent en termes de pourcentage les chiffres suivants (le pourcentage est calculé sur la base du nombre de chercheurs dans chaque division par rapport au nombre total de chercheurs du Centre* )

20\% des chercheurs sont de la Division Architecture des Systèmes et Multimédia.

28\% des chercheurs sont de la Division Productique et Robotique.

20\% des chercheurs sont de la Division Microélectronique et nanotechnologie.

$32 \%$ des chercheurs sont de la Division Microélectronique et nanotechnologie.

Ces chiffres indiquent que la proportion des répondants est un tant soit peu égale à leur nombre au Centre à part pour les chercheurs de la Division Architecture des Systèmes et Multimédia qui représentent $20 \%$ des chercheurs mais dont le taux de réponse est seulement de $7 \%$ des réponses totales. La même remarque s'applique, à un degré moindre, aux chercheurs de la Division Microélectronique et nanotechnologie qui représentent $32 \%$ des répondants alors qu'ils ne représentent que $20 \%$ des chercheurs du Centre. Encore une fois, cet échantillonnage est du aux aléas de la recherche et aussi aux difficultés rencontrées durant la distribution et la récupération du questionnaire.

\footnotetext{
* Il est à signaler que le nombre total des chercheurs répertoriés sur le site est de 118 mais qu'en fait nos différents contacts nous a fait descendre ce chiffre à quelque 90 chercheurs effectivement au Centre ( ou du moins dont les adresses électroniques étaient toujours valides). Certains chercheurs avaient soit leurs adresses non valides ou avaient quitté le Centre .Donc notre hypothèse de travail s'appuiera sur un échantillon de quelques 90 chercheurs effectivement ayant pu répondre.
} 
Q.4-Fonctions des chercheurs

-CERIST: A.R. : Attaché de Recherche, C.E.: Chargé d'études, D.D : Directeur de Division, M.R. : Maître de Recherche.

Sur les 35 répondants, $16(46 \%)$ sont des attachés de recherche et $8(23 \%)$ sont des chargés d'études. Ce qui nous donne un peu plus des $2 / 3$ des répondants qui seraient des titulaires de diplômes de première post graduation. Le reste des répondants sont 1 Directeur de Division et 1 Maître de Recherche qui seraient des titulaires de diplômes de deuxième post graduation. Notons que 2 chercheurs ont indiqués "chercheur" comme réponse et que 7 chercheurs n'ont pas répondu à la question.

-CDTA: A.R: Attache de Recherche, C.E.: Chargé d'Etudes, M.R: Maître de Recherche, C.R.; Chargé de recherche, Ing. : Ingénieur, Ing. Pr. : Ingénieur Principal, Dir. Adj. : Directeur Adjoint.

Sur les 31 chercheurs investigués, un peu plus du tiers $(35 \%)$ n'ont pas répondus à la question. Les 20 chercheurs l'ayant fait se repartissent de la manière suivante : 7 Attachés de Recherche,4 Chargés d'études, 2 Maîtres de Recherche,2 Ingénieurs, 2 Ingénieurs Principaux , 1 Ingénieur Principal et 2 "chercheurs". Ce qui nous donne encore une fois une majorité de détenteur de diplômes de première post graduation. Notons seulement que le faible taux de réponse pour une question somme toute banale, a faussé l'enquête car la fonction est importante dans ce genre d'entreprise.

Q.5-Diplômes des chercheurs Master 2

-CERIST : Mag : Magister, Ing. : Ingénieur, Doct. : Doctorat, Lic. : Licence, Mast.2 :

Cette question semble corroborer les résultats de la question précédente au sujet de la fonction des chercheurs. En effet, une majorité (63\%) de chercheurs sont titulaires de diplômes de première post graduation (Magister ou Master 2).D'autre part, 6 chercheurs sont titulaires de Doctorat et un nombre quasi analogue (5) de diplôme d'ingénieur. Enfin, un chercheur est titulaire d'une licence.

-CDTA : Mag : Magister, Ing. : Ingénieur, Doct. : Doctorat, Mag. Ing. : Magister Ingénieur

Les résultats que nous obtenons pour le CDTA semblent abonder dans le mêmes sens que ceux obtenus pour le CERIST. En effet, la moitie de ceux qui ont répondu sont titulaires de Magister, alors qu'un 1/5 est titulaire d'un doctorat. Le reste est composé d'Ingénieurs et de Magister Ingénieurs qui au nombre de 9 composent le $1 / 3$ du reste des chercheurs investigués.

Q.6-Degré de connaissance du libre accès.

Malgré son importance, le libre accès ne semble pas être très connu par les différents chercheurs investigués ces deux Centres. En effet, il est pour le moins étonnant alors que la 
recherche en général et le monde de la publication scientifique en particulier vivent au rythme des changements radicaux imposé par le libre accès et que $1 / 4$ des chercheurs du CERIST et un peu plus du 1/3 des chercheurs du CDTA ne soient pas au courant des efforts dont les bénéficiaires les premiers sont les chercheurs et, d'après notre expérience, les chercheurs des pays en voie de développement en premier lieu. Il existe une faille dans le monde de la recherche en ces deux Centres auquel il faudrait remédier.

\section{Q.7-Degré de connaissance de l'O.A.I. (Open Access Initiative)}

Si la question précédente avait pour but d'investiguer le degré de connaissance des efforts promouvant le libre accès en général, le but de cette question est de savoir le degré de conscience mêmes des chercheurs quant au coté technique de l'Initiative dont le but, est il besoin de le signaler, est de " développer et promouvoir les critères d'interopérabilité afin de faciliter la dissémination efficace de contenu " ${ }^{(123)}$.Force est de reconnaître que cette question est aussi largement ignorée (à l'instar des efforts promouvant le libre accès) étant donné que pour le CERIST un peu plus la moitié des chercheurs ignorent l'O.A.I. et que pour le CDTA c'est un peu plus des $2 / 3$ qui en est ignorant. Ces chiffres dénotent encore une fois la situation pas très reluisante des chercheurs des deux Centres (pourtant supposés être au courant de ces actions) quant à un pan important du libre accès.

\section{Q.8-Degré de connaissance des appels fondateurs du libre accès (Budapest, Berlin et Berlin}

La réponse à cette question résume à elle seule la gravité du degré de conscience des chercheurs des deux Centres quant au libre accès. En effet, pratiquement personne des chercheurs ne semblent connaitre des appels qui ont fait le libre accès. En effet, tel que nous l'avons montré dans la deuxième partie de ce travail, les Appels sus-cités ont lancés le libre accès et ont étés les premières actions entreprises par les organisations, les individus et ensuite les gouvernements pour faire du libre ce qu'il est aujourd'hui : un mouvement qui a profondément révolutionné le monde de la recherche scientifique et de l'édition. Nous ne voudrions pas extrapoler outre mesure, mais il est loisible de penser que si ces deux Centres (choisis spécialement pour leur supposé et hypothétique conscience de ce genre d'actions) sont dans cette quasi ignorance, les autres centres, organisations, bibliothèques et autres pôles de recherche ne doivent pas être logés à une meilleure enseigne.

\section{Q.9-Degré de connaissance du concept d'archive ouverte}

Au delà des chiffres qui ne font que confirmer la mauvaise appréhension des chercheurs des deux Centres des différents concepts du libre accès (ils sont d'un peu plus des $2 / 3$ pour le CERIST ce qui est très acceptable mais elle descend à la moitié pour les chercheurs du CDTA), il est un autre point qu'il est important de signaler. Il concerne la mauvaise, et même fausse, compréhension de ces mêmes concepts. En effet, même les spécialistes ne semblent pas accepter le terme d'archive ouverte comme étant (d'une manière générale et même superficielle) l'information sur Internet. Le terme en lui-même évoque l'univers papier et ne semble pas être accepté comme représentant les nouvelles technologies. Enfin, l'utilisation

\footnotetext{
${ }^{(123)} \mathrm{http}$ ://www.openarchives.org/
} 
" Archive d'état civil de l'Algérie sous l'occupation française (1830-1904) " par un chercheur est symptomatique de ce genre d'ignorance.

\section{Q.10-Degré d'utilisation des archives ouvertes}

Les chiffres obtenus pour les deux Centres indiquent encore une fois une méconnaissance et une utilisation très superficielle des archive ouvertes. D'ailleurs, les chercheurs du CERIST ont répondus qu'ils utilisaient plus les archives en consultation plus qu'en dépôt. Quant aux réponses, on obtient pratiquement les mêmes chiffres d'utilisation qui se situent aux environs d'un peu plus du $1 / 4$ du taux d'utilisation alors qu'aux environ des $3 / 4$ ne l'utilisent pas .

Q.11-Degré de connaissance de :

-ArchiveSIC

$-\operatorname{ArXiv}$

Toute spécialité a un nombre de références qui étaient (et est toujours mais à degré moindre) les journaux scientifiques. Les archives ouvertes sont beaucoup plus récentes et ont coïncidés avec l'avènement d'Internet. Elles sont devenues des outils indispensables pour le chercheur à coté des journaux électroniques sur Internet. Mais force est de reconnaître encore une fois que les chercheurs des deux centres investigués ne semblent pas connaitre les deux archives qui sont des références dans leur spécialités : Archive SIC et ArXiv ( arXiv étant LA référence et la première des archives ouvertes qui a ouvert la route aux autres expériences ).Si les chercheurs du CERIST présentent des chiffres un tant soit peu respectables avec un taux de $1 / 3$ des chercheurs qui connaissent Archive SIC, les chercheurs du CDTA quant à eux font preuve d'un quasi ignorance étant donné que seuls le 1/6 des chercheurs en connaissent l'existence. Ceci est d'autant plus grave qu' Ar Xiv fait figure de référence dans les sciences exactes et dures.

Q.12-Degré de connaissance de :

- The Registry of Open Access Repositories (ROAR)

-The Directory of Open Access Repositories (DOAR)

La réponse à cette question ne fait que confirmer les résultats des questions précédentes. En effet, on peut dire que les chercheurs investigués sont dans une quasi-totale ignorance de ces deux outils qui sont des véritables mines d'information et dont l'utilisation et la consultation faciliteraient l'accès à des bases de données de grande importance. Nous disons quasi-totale ignorance car quand seulement 2 chercheurs pour chaque Centre investigué sur un échantillon pratiquement égal (31 et 35 chercheurs) connaissent l'existence e ces deux sites, on peut parler de faille. Connaissant ces deux sites, on peut attester de leur grande utilité et surtout facilité et gratuité d'accès qui va dans la sens que la philosophie du libre accès promeut. Il est malheureux qu'un outil de recherche dont les bienfaits sont plus à même de rendre service à ceux qui peinent à obtenir de l'information soit ignoré par ceux là même qui ont en besoin. 
Q.13-Degré d'utilisation de journaux électroniques en libre accès

Si l'utilisation d'un medium aussi nouveau et méconnu que les archives ouvertes peut être comprise, l'autre aspect du libre accès (les journaux électroniques accessibles sur Internet) est, comparativement aux archives ouvertes du moins, plus rentré dans les mœurs des chercheurs. Malgré cela, les chiffres ne semblent pas être à la hauteur de attentes que ce genre de medium devrait susciter. Sinon comment interpréter des chiffres qui dans l'ensemble donnent la moitié des chercheurs n'ayant pas utilisé de journaux électroniques en libre accès? Il est, le moins que l'on puisse dire, étonnant qu'à l'heure où Internet est rentrée dans les mœurs et est devenue un outil indispensable, même pour les tâches les plus triviales, que des chercheurs ne l'utilisent pas dans leur recherche surtout que cela a l'avantage d'être dans beaucoup de cas - gratuit. On remarquera enfin que les chercheurs du CERIST ont indiqués qu'ils utilisaient B.B.F., Documentaliste, RIST et Archimag alors que les chercheurs du CDTA n'ont indiqués aucun titre.

Q.14- Degré de connaissance de :

-African Journal On Line (AJOL)

- Directory of Open Access Journal (DOAJ)

Les sites d'AJOL et du DOAJ sont eux des sites incontournables pour les journaux électroniques accessibles sur Internet. Si DOAJ est un site recensant les journaux au niveau international et est supposé être inclusif (il recensait en date du 26 mars 20127587 journaux), AJOL (qui à la même date recensait 417 journaux seulement) est quant à lui est expressément destiné à aider la recherche africaine à sortir de son ghetto. De plus, Revue de l'Information Scientifique et Technique (RIST) et Technologies Avancées (les deux journaux respectivement du CERIST et du CDTA) sont indexés dans AJOL et ne le sont pas sur DOAJ tel que signalé précédemment. Malgré cela, les taux et les résultats sont aussi décevants que les réponses des questions précédentes. Sinon comment expliquer alors que seuls 10\% des chercheurs d'un coté et $3 \%$ de l'autre connaissent ces deux sites ? (tout en soulignant qu'un pourcentage assez bas connait une combinaison ou une autre - connait AJOL et pas DOAJ ou connait DOAJ et pas AJOL-) Il est clair que ces résultats sont ne font que refléter le manque d'information et de conscience des chercheurs des deux Centres par rapport à des outils de recherche dont ils devraient être les premiers utilisateurs et aussi pourvoyeurs ce qui ne semble pas être le cas étant donné qu'un nombre assez élevé les ignorent.

Q.15- Degré d'utilisation pour la documentation et la recherche des supports :

-Electronique

-Papier

L'utilisation du support par lequel la recherche est faite a, de toute évidence, radicalement changé. Alors que le support électronique était quasiment ignoré il y a de cela une vingtaine d'années, il est devenu avec la démocratisation du matériel informatique un support de plus en 
plus utilisé. L'avènement d'Internet a accentué cette tendance étant donné qu'il est possible de télécharger un article (un livre même) pour une lecture à venir. Cette tendance s'est vérifiée pour notre cas puisque seuls 3\% des chercheurs investigués dans les deux centres utilisent le papier plus que le support électronique. De plus, un quart des chercheurs des deux Centres a déclaré qu'il utilisait indifféremment l'un des deux supports et qu'une autre portion utilisait aussi bien le papier que le support électronique en même temps. Il apparaît très clairement que les chercheurs investigués ont clairement intégré le support électronique dans leurs habitudes de recherche.

Q.16-Préférence du support

-Electronique

-Papier

Si les habitudes concernant l'utilisation du support ont montré une nette prépondérance du support électronique, il semblerait que la tendance ne soit pas la même quant à la préférence du support. Ainsi, les taux varient et sont en dessous de la moitié dans les deux cas pour le support électronique. Le support papier atteint dans les deux cas des taux avoisinant ou dépassant la moitié. Cet aspect est, si on peut dire, normal dans le sens où la maniabilité et surtout l'omniprésence du support électronique le fait plébisciter quant à l'utilisation mais le chercheur préfère le support papier pour de multiples raisons dont entre autres les habitudes qui ne peuvent être changées en un si court laps de temps.

\section{Q.17- Proportion d'utilisation des deux supports}

Cette question ne fait que confirmer par les chiffres ce que les chercheurs ont déclaré au sujet de leur taux d'utilisation des deux supports. Seule une infime partie des chercheurs des deux Centres investigués ( 3 et 4 respectivement pour le CERIST et le CDTA) utilisent effectivement le papier plus que le support électronique et encore à des taux quasi égaux (à part pour un chercheur qui a déclaré qu'il utilisait les deux supports à raison de $10-90 \%$ en faveur du papier). On peut donc conclure que les chercheurs des deux Centres utilisent effectivement majoritairement le support électronique par rapport au support papier.

Q.18-Langues utilisées durant soumission

$$
\begin{aligned}
& \text {-Français } \\
& \text { - Arabe } \\
& \text {-Anglais } \\
& \text {-Autres }
\end{aligned}
$$

Il a été prouvé que la langue de soumission pour le chercheur est importante car le style d'écriture et de rédaction de l'article scientifique sont normalisés. De plus, il existe, en Algérie, un bilinguisme de fait étant donné que la langue arabe et la langue française sont toutes deux utilisées par les chercheurs. Les résultats obtenus sont, contrairement aux questions précédentes, différentes en ce qui concerne la (ou les) langues utilisées. A cet effet, 
les langues de publication utilisées sont majoritairement anglais - français à raison de 46\% pour le CERIST et de $45 \%$ pour le CDTA. La langue anglaise quant à elle est utilisée à raison de $29 \%$ pour le CERIST et de $45 \%$ pour le CDTA. La langue française est utilisée à raison de 9\% pour le CERIST et de 13\% pour le CDTA. Les chercheurs du CERIST ont aussi indiqués qu'ils avaient publié en arabe et en français à raison de 9\%.D'autres combinaison de langues de publication (français-anglais-arabe-allemand, français-arabe-anglais) ont étés signalées ainsi qu'en langue arabe. Il apparaît clair d'après les chiffres que les langues française et anglaise sont les langues utilisées par les chercheurs des deux Centres pour communiquer. La langue arabe n'est que rarement utilisée (une seule fois par un chercheur du CERIST et pas une seule fois par les chercheurs du CDTA) ce qui tendrait à prouver que la langue arabe ne bénéficie pas des faveurs des chercheurs surtout en sciences exactes et dures. Les autres langues utilisées telles que la langue allemande par exemple ne sont que des exceptions et des cas marginaux.

\section{Q.19- Publication dans journaux scientifiques}

La publication dans des journaux scientifiques est pour le chercheur une condition sine qua none dans la progression de sa carrière scientifique. L'aune à laquelle est calculée sa contribution est la production scientifique et en premier lieu les articles publiés dans les journaux de sa spécialité. Les chercheurs des deux Centres investigués quant à eux présentent des chiffres qu'on pourrait décrire de normaux étant donné la plus grande importance qu'a l'article pour le chercheur en sciences exactes et dures par rapport aux sciences sociales en général. Ainsi, les chercheurs du CERIST ont répondu qu'ils avaient à hauteur de 54\% publié et que le reste $(46 \%)$ n'avait pas publié ce qui nous donne un taux qui dépasse de peu la moitié qui a publié. Quant aux chercheurs du CDTA, on peut dire que leur taux de publication est de loin plus haut que celui des chercheurs du CERIST et ce à raison de $90 \%$.D'un autre coté, les chercheurs du CERIST ont beaucoup plus publié au niveau national qu'international alors que ceux du CDTA ont publié beaucoup plus dans des revues internationales. Il semble que ces réponses soient conformes aux hypothèses quant à la publication scientifique : elle est plus prépondérante et plus internationale chez les sciences dures et exactes que les sciences humaines et sociales. .

\section{Q.20- Nombre des publications}

Si publier est, comme on l'a vu, primordial pour un chercheur, il n'en demeure pas moins que la carrière et l'avancement du chercheur dépend de la longueur de la liste de ces mêmes publications. Plus le chercheur publie et contribue de ce fait aux progrès de sa spécialité et plus il a prouvé que son travail est digne de faire partie du corpus certifié et accepté de la Science. De ce fait, il semblerait que les chercheurs du CDTA soient plus prolifiques et productifs que ceux du CERIST. En effet, la cadence de productivité des chercheurs du CERIST semble diminuer plus le nombre de publication augmente : ainsi 11 chercheurs ont publié entre 1 et 3 fois, 4 entre 3 et 5 fois enfin 4 plus de 5 fois. De leur côté, la cadence de productivité des chercheurs du CDTA emprunte exactement le chemin inverse : si 4 chercheurs ont publié entre 1 et 3 fois et que 9 autres ont publié entre 3 et 5 fois , 15 chercheurs soit presque de la moitié de ceux investigués ( et $54 \%$ de ceux ayant répondu ) ont 
publié plus de 5 fois .Ces réponses ajoutées à ceux concernant les habitudes de publication, montrent clairement la plus grande importance de la publication chez deux Centres dont les spécialités sont diamétralement opposées.

\section{Q.21- Participation à des conférences dans la spécialité}

La participation aux conférences est une autre des facettes de l'activité du chercheur qui en plus permet le contact et les échanges avec les collègues. A l'instar des questions précédentes concernant les publications et leur fréquence, le CDTA affiche des taux de participation plus élevés que ceux du CERIST.A cet effet, tous ceux qui répondu ont affirmé avoir participé à des conférences lors que les chercheurs du CERIST n'ont répondu qu'à hauteur de $83 \%$.D'un autre coté, les mêmes tendances en ce qui concerne ces participations se vérifient: les chercheurs du CDTA participent plus aux conférences internationales que celles nationales contrairement aux chercheurs du CERIST qui participent aux conférences internationales avec une moindre fréquence.

\section{Q.22- Nombre des participations}

Les réponses données à cette question ne font que confirmer les chiffres précédemment vus en ce qui concerne la fréquence de participation aux conférences et la différence entre les deux Centres investigués. Encore une fois, c'est la même tendance avec le phénomène inverse qui caractérise la fréquence de participation. A cet effet, sur les 29 chercheurs ayant répondu à la question pour le CERIST, seuls 11 soit $38 \%$ ont participé plus de 5 fois à des conférences. Ce taux (participation à plus de 5 conférences) se retrouve, par contre, chez $81 \%$ des chercheurs du CDTA. On a remarqué cette tendance dans les publications dans les journaux scientifiques et ceci se conforme pour la participation aux conférences.

Q.23-Degré de conscience du contrôle par les pairs de la soumission

On peut estimer que le chercheur qui soumet aux journaux veut publier dans le meilleur de sa spécialité. Ceci implique une envie de contribuer, se faire connaitre et aussi de bénéficier de la meilleure certification et contrôle. A cet effet, une majorité des chercheurs investigués des deux Centres ont répondu qu'ils avaient soumis en prenant en ligne de compte ce critère quoique ce critère n'ait pas été cité par les chercheurs du CERIST aussi souvent que ceux du CDTA (77\% de ceux ayant répondu et $69 \%$ pour l'ensemble des chercheurs pour le CERIST alors que $90 \%$ des chercheurs CDTA ont répondu positivement à la question). Il serait intéressant de savoir quelles sont les raisons de ceux qui ont soumis sans tenir compte du critère de la présence ou non du facteur de certification.

Q.24- Soumission en fonction du contrôle par les pairs

En plus de soumettre à de journaux contrôlés par les pairs et d'en être conscient, le chercheur a de toute évidence des raisons (quand c'est le cas).Premièrement, une majorité des chercheurs des deux Centres (26 chercheurs sur 35 soit à hauteur de $74 \%$ pour le CERIST et de 21 chercheurs sur 31 soit $68 \%$ pour le CDTA) a soumis en tenant compte de ce critère. Quant aux raisons de ceux qui ont répondu oui à cette question, il apparaît que l'avancement est le critère principal et ceci dans la majorité des cas .Ainsi, ils sont 14 chercheurs sur 26 soit 
$54 \%$ à publier pour obtenir de l'avancement pour le CERIST alors qu'ils sont 21 chercheurs sur 31 soit $68 \%$ à le faire pour le CDTA. Le reste des raisons invoquées sont une combinaison de publication pour l'avancement et le prestige (à 2 reprises pour les eux centres), de prestige (à 2 reprises pour le CERIST).Enfin, d'autres raisons diverses ont étés invoquées par les chercheurs dont nous citerons " bien valider le travail par des spécialistes " et une " évaluation crédible " pour le CERIST. Les chercheurs du CDTA ont évoqués : " plus de valeur à la publication ", " critiques et recommandations scientifiques " , " meilleure évaluation ", " spécialisation dans le domaine ", " journal doit vérifier l'authenticité et l'originalité des résultats qu'il publie ", " avoir un avis objectif et réel sur le travail ", " juger le travail à sa juste valeur ", " viser les revues de renommées pour donner plus de valeur aux travaux publiés " et enfin " connaitre la consistance du travail soumis ". Notons seulement que parmi les chercheurs des deux Centres ayant répondu à cette question positivement, 3 chercheurs pour le CERIST et 1 chercheur pour le CDTA n'ont pas donné de raisons. Enfin, 5 chercheurs pour le CERIST n'ont pas répondu à la question. Comme on peut le voir, les chercheurs (surtout ceux du CDTA) ont évoqués nombre de critères qu'ils estiment importants dans leur raisons de publier .Il n'en reste pas moins que la tendance qui est prépondérante par les chiffres est celle de la publication pour avancement et ce pour les deux Centres.

Q.25-Position quant à l'importance du contrôle par les pairs

Face aux différentes controverses, scandales et autres affaires ayant jalonné la courte histoire du contrôle par les pairs des temps modernes, nombre de courants de pensée ont mis en cause son essence et sa viabilité à cause des différents problèmes qu'il a engendré. Nous citerons les préjugés de toutes sortes (raciaux, religieux, personnels -ad hominem-, linguistique), les retards dans la publication, la suppression des idées novatrices, la résistance aux changements, le copinage etc.......Malgré ces critiques, la communauté scientifique est unanime à voir dans le contrôle par les pairs le seul barrage connu afin de séparer la graine de l'ivraie pour reprendre une expression utilisée par un chercheur du domaine. Les chercheurs de deux Centres investigués quant à eux se situent dans la même optique étant donné qu'une majorité (à raison de 31 chercheurs ayant répondu positivement à la question pour 1 seul ayant répondu négativement pour le CERIST et 27 chercheurs ayant répondu positivement pour 2 ayant répondu négativement pour le CDTA ce qui nous donne un taux de respectivement 97 et $93 \%$ pour le CERIST et le CDTA) pense que le contrôle par les pairs est indispensable à l'avancement de la Science.

Q.26- Position quant au jugement objectif de la soumission.

Parmi les griefs les plus récurrents que le contrôle par les pairs a engendré est l'accusation de subjectivité avec laquelle la soumission est jugée. D'autres part, cette accusation est moins récurrente en sciences exactes qu'en sciences humaines et sociales pour de multiples causes dont la plus plausible est le fait qu'en sciences exactes le résultat d'une recherche est plus mathématique et technique et ne peux souffrir de contestation comme pour par exemple une théorie ou une idée ou encore un point de vue. Cette donnée s'est un tant soit peu vérifié dans notre échantillon étant donné que les chercheurs du CDTA ont jugé que leur soumission avait 
été jugée d'une manière plus objectivement que les chercheurs du CERIST. En effet, sur les 28 répondants du CERIST , 22 soit $79 \%$ ont jugé que leur soumission avait bénéficié de toute l'objectivité voulue alors que 5 chercheurs soit $18 \%$ étaient d'avis que leur soumission n'avait pas été jugée avec objectivité (1 chercheur avait répondu qu'il estimait que sa soumission avait été jugée en même temps objectivement et subjectivement et 7 chercheurs n'avaient pas répondus à la question) .De leur coté, des 30 chercheurs du CDTA ayant répondu à la question, 25 soit $83 \%$ ont estimé avoir été jugés objectivement, alors que 3 soit $10 \%$ étaient d'avis que leur soumission n'avait pas été jugée avec objectivité, (2 chercheurs avaient répondu qu'ils estimaient que leur soumission avait été jugée en même temps objectivement et subjectivement et 1 chercheur n'avait pas répondu à la question).On peut estimer ces résultats confirmant les hypothèses déjà énoncées concernant l'avis des chercheurs quant à l'objectivité de l'expertise selon les spécialités.

\section{Q.27- Position quant à l'anonymat de l'expert durant la soumission}

Une des raisons les plus fréquemment évoquées quant à la subjectivité (effective ou supposée) des expertises a trait à l'anonymat dont bénéficie l'expert au cours de son travail. Nombre de recherches dans ce sens n'ont pu donner de réponses franches et conclusives quant à la question. Les derniers développements concernant l'ouverture du processus (surtout dans le cadre de l'expertise ouverte sur Internet) abonderaient dans ce sens quoique les avis divergent vu la nouveauté du medium et aussi de l'expérience. Quant aux chercheurs du CERIST, sur les 31 répondants, 20 chercheurs soit $65 \%$ trouvent cette situation normale et 10 chercheurs soit $32 \%$ pensent le contraire alors qu'1 chercheur trouve cette situation en même temps normal et anormale selon les cas et les situations. Les chercheurs du CDTA de leur côté, sur les 30 chercheurs ayant répondu, 23 soit $77 \%$ trouvent cette situation normale et 6 chercheurs soit $20 \%$ pensent le contraire alors qu' 1 chercheur trouve cette situation en même temps normal et anormale selon les cas et les situations. Ces chiffres indiquent que les chercheurs du CERIST seraient moins enclins à accepter l'anonymat tant décrié des experts et par delà confirmant l'hypothèse de la plus grande subjectivité (supposée ou réelle) des expertises en sciences sociales et humaines comparativement aux sciences dures et exactes.

\section{Q.28-Position quant aux correctifs demandés durant expertise}

Il est rare pour une publication d'être acceptée sans que les experts expriment une demande d'introduction de rectificatifs qui peuvent aller du plus superficiel au plus radical. Ces échanges peuvent durer et prendre la forme d'un dialogue entre l'auteur (ou les auteurs) et les experts. Bien que cette étape ne soit, pour des raisons évidentes, pas la bienvenue chez l'auteur, il n'en demeure pas moins qu'elle constitue une occasion d'améliorer le manuscrit et d'y apporter les correctifs que les experts voient indispensables. Il semble que les chercheurs de deux Centres investigués aient compris cette donne et l'aient intégré dans leurs habitudes de soumission. Ainsi, ils répondent majoritairement qu'ils ont apportés les correctifs demandés et ce à raison de 30 chercheurs sur les 31 soit $97 \%$ ayant répondu à cette question (4 chercheurs n'ont pas répondu à cette question) en ce qui concerne le CERIST et à raison de 29 chercheurs sur les 30 soit $97 \%$ ayant répondu à cette question (1 chercheur n'a pas répondu à cette question) pour les chercheurs du CDTA. Ces chiffres indiquent clairement 
que les chercheurs des deux Centres investigués croient en la place importante du contrôle par les pairs dans l'amélioration et l'échange de données que les différentes étapes du contrôle par les pairs impliquent.

\section{Q.29-Raisons de l'introduction des correctifs}

Si accepter d'introduire des correctifs, malgré le travail supplémentaire et des fois redondant que cela implique, est accepté par les chercheurs afin de voir les fruits de leur travail publié et reconnu, les raisons de l'acceptation de cette étape du contrôle par les pairs peuvent quant à elles être différentes. Ainsi les différentes études ont démontré que les chercheurs ont des approches des réactions et surtout des buts qui peuvent beaucoup varier. Ces raisons sont innombrables mais deux peuvent être considérées comme les plus importantes: être convaincu des arguments des experts quant aux correctifs à apporter et le désir (et même des fois l'obligation) d'être publié. Quant aux chercheurs investigués des deux Centres, il semblerait que les arguments des experts lors de la soumission les aient convaincus étant donné que pour le CERIST sur les 31 chercheurs ayant répondu, 20 soit $65 \%$ ont évoqués ce critère alors que 7 chercheurs soit $23 \%$ aient évoqués le désir ou peut être besoin d'être publié. Les 2 autres chercheurs soit $6 \%$ ont évoqués une combinaison des deux (arguments des experts et aussi besoin d'être publié).Les chercheurs du CDTA quant à eux, ont exprimé des avis pratiquement analogues que ce soit en chiffres ou en pourcentage. Ainsi sur les 30 chercheurs ayant répondu, 28 soit $67 \%$ ont évoqués avoir été convaincus par les arguments des experts, 8 chercheurs soit $27 \%$ avaient introduit le changements seulement pour être publié alors qu'1 chercheur avait répondu par une combinaison de deux critères (arguments et être publié).Notons que cette quasi similitude dans les résultats entre les deux Centres indiquerait un consensus quant à la justesse et objectivité des experts, consensus que les résultats des autres questions dont celle relative à l'objectivité lors de l'expertise (où les chercheurs des deux Centres avaient exprimés dans des taux dépassant les $80 \%$ en moyenne) confirmerait.

\section{Q.30- Pourcentage des soumissions acceptées}

Le taux d'acceptation d'un chercheur indique sa réussite à faire publier le résultat de ces recherches dans des journaux scientifiques contrôlés par les pairs. Il est de notoriété que les taux d'acceptation des soumissions dans les sciences exactes et dures sont de loin plus hauts que ceux des sciences humaines et sociales pour de multiples raisons déjà évoquées. Cette donnée s'est vérifiée pour les chercheurs du CERIST et du CDTA de par les chiffres qu'ils ont déclarés. Ainsi sur les 21 chercheurs du CERIST ayant répondu à cette question on remarque que les hauts taux d'acceptation ne sont pas aussi fréquent que ceux du CDTA. Par exemple, seul un chercheur du CDTA à eu un taux d'acceptation de $10 \%$ alors qu'un chercheur du CERIST a eu un taux d'acceptation de 00\%.Les autres chiffres quant à eux montrent un meilleur taux d'acceptation chez les chercheurs du CDTA comparativement à ceux du CERIST. Par exemple, les taux d'acceptation de $80 \%$ et + étaient présents chez les chercheurs du CDTA 19 fois alors que le même taux $(80 \%$ et +$)$ ne se retrouve chez les chercheurs du CERIST que 7 fois. De même pour le taux de 100\% (acceptation de toutes les soumissions) qui n'est présent que 2 fois chez les chercheurs du CERIST alors qu'il est 
présent 5 fois chez les chercheurs du CDTA. Ces chiffres ne font que confirmer la thèse du plus haut d'acceptation des soumissions dans les sciences dures et exactes comparativement aux sciences sociales et humaines.

Q.31- Degré de connaissance des nouvelles formes de contrôle par les pairs des publications scientifiques sur Internet

La publication scientifique a connu ces dernières années avec l'avènement de l'outil informatique et des réseaux, particulièrement Internet, des mutations qu'il aurait été difficile à d'imaginer seulement deux décennies avant. Alors que le périodique scientifique papier était l'apanage de la bibliothèque ou le centre de documentation qui l'acquerrait au nom des lecteurs, ce même périodique est devenu consultable à partir de l'ordinateur de l'utilisateur à la bibliothèque ou au domicile de ce dernier. De même que la soumission des manuscrits est devenue elle aussi possible par le biais d'Internet. Il en a résulté que le contrôle de ces mêmes soumissions est devenu lui aussi entrepris sur Internet. Les chercheurs des deux Centres investigués ont majoritairement répondu à cette question de manière positive. Ainsi 29 chercheurs du CERIST soit $83 \%$ ont répondu qu'ils étaient au courant de ces nouvelles formes de contrôle par les pairs sur Internet alors que 5 chercheurs soit $14 \%$ ont déclaré ne pas être au courant. Les chercheurs du CDTA quant à eux ont répondu dans les mêmes proportions étant donné que 29 chercheurs soit $94 \%$ ont répondu positivement à la question alors que seuls 2 chercheurs soit $6 \%$ ont déclaré ne pas être au courant de ces nouvelles formes de certification sur Internet. Ces chiffres prouvent que les chercheurs des deux Centres sont au courant ces nouvelles formes malgré le fait qu'ils ne soient pas très au courant des différents appels, entrepôts d'archives et autre sites indispensables dans le libre accès comme on l'a vu dans les réponses aux questions précédentes.

Q.32-Position vis-à-vis des nouvelles formes de contrôle par les pairs des publications scientifiques sur Internet

L'avènement d'Internet a permis avec sa propension à l'ouverture, entre autres, d'introduire de nouvelles formes dans la certification de l'information que l'univers papier ne pouvait même pas entrevoir. Parmi ces innovations, le fait d'avoir sa soumission non pas jugée par des experts d'une manière secrète mais que cette opération soit faite par tous ceux qui peuvent y accéder par l'intermédiaire d'Internet. Il existe nombre de variantes de ces contrôles par les pairs ouverts (open peer review) qui vont de l'ouverture la plus totale à l'accès par mot de passe ou encore par cooptation. Ces nouvelles formes révolutionnaires de certification n'ont pas reçus une acceptation franche de la part de la communauté des chercheurs pour des raisons multiples allant de la peur du plagiat à la peur du ridicule en passant par la méfiance et même condescendance qu'ont certains chercheurs ( surtout ceux d'un certain standing ) de se faire juger par des " non pairs ".Quant aux chercheurs des deux Centres, ont peut estimer leur position ambivalente étant donné que les taux se situent aux environs de $2 / 3$ pour et de $1 / 3$ contre. Ainsi 23 soit $67 \%$ chercheurs du CERIST ont répondu positivement à cette question alors que 10 soit $32 \%$ ont répondu négativement ( 1 chercheur a répondu que cela dépendait de la situation).Quant aux chercheurs du CDTA, ils ont étés 20 soit $65 \%$ a avoir répondu positivement et 11 soit $35 \%$ à avoir répondu négativement. Comme on peut le voir, ces taux 
de $2 / 3$ pour et $1 / 3$ contre ne permettent pas de dire que les chercheurs ont définitivement adopté ce nouveaux schémas mais sont plutôt en position attentiste ce qui est normal pour des procédures très nouvelles et novatrices.

Q.33- Position vis-à-vis publication des commentaires sur Internet.

Parmi les plus novatrices de ces nouvelles formes de certification de l'information sur Internet mais aussi parmi les plus controversées et débattues est celle qui fait publier les commentaires suscités au cours du contrôle par les pairs ouvert. Cette manière de procéder peut être considérée comme un pas de plus dans l'ouverture préconisée et qu'Internet a permis. Encore une fois les résultats des deux Centres investigués montrent une quasi similitude quant à la position des chercheurs. Ainsi sur les 33 chercheurs du CERIST ayant répondu, 19 soit 58\% accepteraient que les commentaires soient publiés avec l'article alors que 13 chercheurs soit $39 \%$ ne l'accepteraient pas ( 1 chercheur a répondu que cela dépendait de la situation). Quant aux chercheurs du CDTA, 19 soit $61 \%$ ont répondu par oui alors que 12 soit 39\% ont répondu par non. On peut conclure que face à la nouveauté et même l'aspect révolutionnaire du procédé les chercheurs ont certaines réticences à avoir leurs noms, les commentaires des autres et autres informations révélés sur Internet et que tout un chacun peut lire.

Q.34- Acceptation commentaires publiés sur Internet avec la version finale et définitive?

Une autre manière de rendre le contrôle par les pairs encore plus ouvert (et beaucoup plus novateur) et moins sujet aux controverses et autres critiques qu'il suscite consiste à publier les commentaires que le contrôle par les pairs ouvert a suscité durant l'expertise avec la version finale acceptée. Cette manière de procéder, encore plus révolutionnaire que celle de poster les commentaires que la soumission a engendré, a suscité de multiples réactions quant au devenir de l'article scientifique qui serait en perpétuel changement de même que s'est posé la question de sa version finale et définitive. Cette question a elle aussi reçu des réponses mitigées quoique assez similaires de la part des chercheur des deux Centres. Ainsi, 22 des chercheurs $\mathrm{du}$ CERIST soit $65 \%$ ont répondu positivement alors que 11 soit 32\% ont répondu négativement. Quant aux chercheurs du CDTA, 23 soit $74 \%$ ont répondu positivement alors que 8 soit $26 \%$ ont répondu négativement. Ces chiffres indiqueraient que les chercheurs du CDTA seraient plus ouverts à ce genre d'expérimentation beaucoup plus en vigueur dans leurs spécialités que dans les spécialités plus orientées vers les sciences humaines et sociales.

Q.35-Position quant à l'efficacité des nouvelles formes de contrôle par les pairs en ce qui concerne l'atténuation de la subjectivité et du préjudice.

Parmi les nombreuses critiques que le contrôle par les pairs a toujours engendré la subjectivité de l'expertise est très souvent citée. En effet, le travail de l'expertise étant par essence humain, il est presque normal que des interférences rentrent en ligne de compte. Ces interférences peuvent être d'ordre personnel, linguistique, méthodologiques, etc.....Cette propension qu'a le contrôle par les pairs à engendrer (et même encourager) la subjectivité sous toutes formes est aggravée par le secret et l'anonymat dans lequel cette opération se déroule. L'ouverture qu'Internet a permis a été vue comme une solution à ce genre de 
problèmes mais les expériences sont trop récentes et encore non conclusives pour en tirer des conclusions. Quant aux chercheurs du CDTA et du CERIST, leurs réponses ont étés, contrairement aux précédentes questions examinées un tant soit peu différentes .Ainsi 21 chercheurs du CERIST soit $66 \%$ pensent que cette ouverture pourrait atténuer la subjectivité et le préjudice alors que 10 autres soit $31 \%$ ne le pensent pense et qu'un chercheur a donné une réponse allant dans les deux sens (notons que 3 chercheurs n'ont pas répondu à la question).De leur coté ,les chercheurs du CDTA ont une position un peu moins prononcée et pratiquement égale quant aux réponses données. Ainsi, 16 chercheurs soit 53\% pensent que cette ouverture pourrait atténuer la subjectivité et le préjudice alors que 14 autres soit $47 \%$ ne le pensent pas (notons aussi qu'un chercheur n'a pas répondu à la question).Il semblerait que ces chiffres traduisent surtout une ignorance de ces pratiques, ignorance nullement imputable aux chercheurs mais beaucoup plus à une nouveauté de ces pratiques et expériences.

Q.36-Types de préjudices

Sexuelle (homme ou femme)

-Linguistique (selon la langue utilisée)

-Religieuse (précisez)

-Personnelle

-Autres

Les préjudices vis-à-vis des auteurs soumettant un manuscrit peuvent prendre des formes et des aspects très différents. Ils seraient très fastidieux de tous les énumérer mais quelques uns semblent revenir le plus souvent dont nous en citerons: sexuels, linguistique, religieux, national, personnel, etc...Les réponses que nous avons obtenu de la part des chercheurs des deux Centres indiqueraient que ces chercheurs sont majoritairement victimes (ou du moins se sentent victimes) soit d'un préjudice linguistique pour le CERIST et ce à raison de 10 réponses soit 36\% (ils ont aussi indiqué le préjudice personnel à 6 reprises soit $21 \%$ ) ou personnel pour le CDTA à raison de 6 réponses soit $26 \%$ ( les chercheurs du CDTA ont aussi indiqué une combinaison de préjudice :linguistique et religieux en même temps à 3 reprises soit $11 \%$ et aussi un préjudice vis-à-vis des chercheurs du Tiers Monde à 2 reprises soit 7\%). Les autres réponses sont diverses et sont pour le CERIST: manque d'expérience et de compétence dans le domaine, renommée de l'encadreur ou directeur de laboratoire, appropriation des œuvres des autres ainsi qu'une combinaison de raisons dont la plupart tourne autour de linguistique-personnelle, personnelle-religieuse, religieuse-linguistique et enfin linguistique-sexuelle. Les autres diverses réponses du CDTA se cristallisent en : manque d'expertise de l'expert, une combinaison religieux - culturel et un préjudice géographique. Notons enfin le haut taux de non réponse à cette question pour les deux Centres et qui est de 20 et $26 \%$ pour respectivement le CERIST et le CDTA. Notons enfin que les préjudices personnels et linguistiques semblent être une grande préoccupation des chercheurs des deux Centres étant donné qu'ils représentent respectivement $57 \%$ et $35 \%$ pour le CERIST et le CDTA. 
Q.37-Position vis-à-vis de l'efficacité des nouvelles formes de contrôle par les pairs pour combattre le plagiat et la fraude

Les problèmes de plagiat et de fraude sont parmi les aspects les plus inquiétants que rencontre la communauté scientifique dans sa quête de respectabilité et d'honnêteté. Bien que considérés comme marginaux, ces aspects sont, de temps de en temps, reportés par les journaux scientifiques et certains comme ceux de Hwang Woo-suk et de Jan Hendrik Schön ont eu un écho international de par la couverture médiatique qu'ils ont reçus. L'avènement d'Internet a suscité des espoirs quant à l'éradication de ces pratiques qui discréditent toute la communauté à cause de ce qui a été appelé des " brebis galeuses " ( black sheep) en même temps que cet avènement a suscité aussi des inquiétudes quant à des utilisations plutôt illégales. Les réponses obtenues de la part des chercheurs des deux Centres semblent encore une fois très similaires quant aux chiffres. Ainsi les chercheurs qui ont répondus positivement à cette question sont au nombre de 20 soit $69 \%$ pour le CERIST et de 20 aussi soit $65 \%$ pour le CDTA. Quant aux chercheurs ayant répondu négativement à la question, ils ont 7 soit $24 \%$ pour le CERIST et de 9 chercheurs soit 29\% pour le CDTA. Enfin, 2 chercheurs de chaque Centre ont répondu par oui et non selon les cas à la question ce qui représente respectivement $7 \%$ et $6 \%$ pour le CERIST et le CDTA. Notons que 6 chercheurs du CERIST n'ont pas répondu à la question. Ces taux montrent une étonnante similitude dans la position des chercheurs des deux Centres vis-à-vis des nouvelles formes de contrôle et le combat contre la fraude et le plagiat.

Q.38- Position vis-à-vis de l'anonymat dans le cas de l'expertise.

Le chercheur qui publie et atteint un certain niveau de notoriété dans sa spécialité est appelé après une certaine période à expertiser lui-même et juger de la scientificité, méthodologie, résultats d'un travail soumis. Les réponses des chercheurs des deux Centres quant à leur position de l'anonymat des experts vis-à-vis de leur travail avaient démontré qu'ils n'y étaient pas complètement contre cet aspect à raison de 65 et $77 \%$ pour respectivement le CERIST et le CDTA.

Est ce que le chercheur qui lorsque expertisé voudraient connaitre qui le " juge " aurait la même position lorsque il devient lui-même " juge " ? Les réponses des chercheurs des deux Centres tendraient à le prouver étant donné que pour le CERIST 23 chercheurs soit $70 \%$ ont répondu par l'affirmative alors que pour les chercheurs du CDTA 17 chercheurs soit 55\% ont aussi répondu par l'affirmative. Quant aux chercheurs ayant répondu par la négative à la question, ils sont 9 pour le CERIST soit $27 \%$ et 14 pour le CDTA soit $45 \%$. Notons que pour le CERIST 2 chercheurs n'ont pas répondu à la question. Contrairement aux questions précédentes similaires, il semblerait que les chercheurs du CDTA soient moins enclins à lever l'anonymat que leurs collègues du CERIST. Ceci est peut être dû à l'expérience des chercheurs de ce Centre quant à leur soumission et aussi à la prépondérance de la publication chez les chercheurs des sciences exactes par rapport aux chercheurs en sciences humaines et sociales. 
Q.39- Position vis-à-vis de la prise en ligne de compte des archives ouvertes et journaux électroniques quant à la promotion.

La publication scientifique a, selon ce que nous avons vu dans les parties précédentes, changé de manière drastique quant à sa forme et sa présentation. Les publications d'un auteur donné sont devenues très souvent électroniques qu'elles soient dans des revues accessibles par le Web ou dans des archives ouvertes. Bien que réticents quand à sa viabilité, certificabilité et d'autres bien d'autres raisons, les chercheurs ont dû accepter cette forme de publication comme étant l'égale de la publication traditionnelle sur papier. Malgré les avantages que cette forme de publication recèle (maniabilité, disponibilité, facilité d'accès, etc....), la communauté scientifique en général ne semble pas lui accorder toute sa confiance généralement pour des problèmes de certification (le contrôle sur Internet ayant été au début très lâche). Quant à l'échantillon de chercheurs du CDTA et du CERIST, il semble avoir une approche diamétralement opposée quant à l'acceptation de ces nouveaux media. Ainsi sur les 31 chercheurs du CERIST ayant répondu à cette question ,18 soit $58 \%$ pensent que les articles électroniques et les publications des archives ouvertes doivent pris en ligne de compte dans les promotions et avancement alors que 13 soit $42 \%$ ne le pensent pas .Quant aux chercheurs du CDTA, sur les 30 répondants, seuls 11 soit 37\% pensent que les articles électroniques et les publications des archives ouvertes doivent pris en ligne de compte dans les promotions et avancement alors que 19 chercheurs soit $63 \%$ ne le pensent pas. Nous trouvons tout de même étonnant ces chiffres car il est généralement admis que les chercheurs en sciences exactes sont plus ouverts et plus à même (de part leur formation de base) à utiliser les technologies que leurs collègues des sciences humaines et sociales. Ces réponses en fait, en plus d'infirmer (du moins pour notre cas) une donnée généralement acceptée, tendent à prouver que, nonobstant sa spécialité, le chercheur de nos jours a irrémédiablement inclut la publication électronique dans ses habitudes de recherche et de communication

\section{7-Conclusion générale sur les réponses du CERIST et du CDTA.}

Les questions posées aux chercheurs des deux Centres précités l'ont étés afin de savoir quelle est leur position quant aux nouvelles formes contrôle de l'information. L'analyse des réponses obtenues nous permettra de tirer des conclusions primaires qui, nous devons encore une fois le souligner, auraient pu être plus adéquates et surtout exploitables n'étais ce les mauvaises conditions de réception des questionnaires et le quasi refus de les remplir après l'accord en ce qui concerne le CDTA. Toujours est il que les difficultés rencontrées au cours de cette expérience (que nous pensons la première à l'échelle nationale) nous servira de leçon pour de futures recherches analogues afin de pouvoir mieux investiguer cette importante étape du processus de la publication et par delà essayer de le rendre plus compris et surtout en éviter les effets négatifs. Nous pouvons donc résumer les résultats des questionnaires dans les orientations suivantes :

1-les répondants au questionnaire sont la plupart soit dans la trentaine (CERIST) ou la quarantaine (CDTA) 
2-ils sont majoritairement de sexe masculin. Pour le CDTA, ils sont 20 de sexe masculin et 11 de sexe féminin alors que pour le CERIST, une quasi parité est remarquée à raison de 19 sexe masculin et 16 de sexe féminin.

3-la majorité de ceux ayant répondu sont titulaire soit d'un magister (22 pour le CERIST et 15 pour le CDTA) ou alors d'un doctorat (5 pour le CERIST et 5 pour le CDTA).Les titulaires d'un ingéniorat sont aussi représentés et ce à raison de 6 pour le CERIST et de 8 pour le CDTA.

4-les chercheurs ayant répondu au questionnaire (et plus particulièrement ceux du CDTA) ne semblent pas avoir une grande connaissance des différents efforts qui en général sont entrepris afin de permettre l'accès gratuit et libre à l'information scientifique et technique. Ainsi si les $3 / 4$ des chercheurs du CERIST connaissent ces efforts, seule une proportion de $2 / 3$ des chercheurs du CDTA les connaissent. Nous pensons personnellement que ces taux sont bas étant donné que ces efforts touchent tous les chercheurs et qu'en être au courant est une condition sine qua none pour avancer et utiliser toutes les fonctionnalités qu'offre le vecteur le premier de ces efforts : Internet.

5-les chercheurs des deux Centres ne semblent pas encore plus être au courant de l'Open Access Initiative qui est parmi les plus importantes étapes du libre accès. Il ne faut pas oublier qu'il est reconnu comme le premier rassemblement historique fondateur du mouvement libre accès. Malgré cela, seule la moitie des chercheurs du CERIST et moins du tiers du CDTA le connaissent. Cette quasi ignorance corrobore la réponse aux efforts concernant le libre accès et indique clairement que les chercheurs des deux Centres (et plus ceux du CDTA) sont peu au courant d'actions qui sont importantes et cruciales pour leur avenir.

6-le manque de connaissance des chercheurs des deux Centres est encore plus flagrant quant aux appels fondateurs du libre accès (Budapest, Bethesda et Berlin) qui sont pratiquement non connus soit par les chercheurs du CERIST et ce à hauteur de $20 \%$ et de $00 \%$ pour les chercheurs du CDTA. Quant à en être signataire, seuls 3 chercheurs du CERIST l'ont fait. Il est pour le moins difficile de croire que des chercheurs supposés être à la pointe de l'innovation dans leur spécialité ne connaissent pas du tout des appels qui ont révolutionné le monde de la publication scientifique et technique.

7-le concept d'archive ouverte ne semble pas quant à lui très compris par les chercheurs du CDTA (l'exemple du chercheur ayant répondu " archive coloniale de l'Algérie " est tout ce qu'il y a de plus édifiant) qui ne le connaissent qu'a hauteur de $48 \%$ alors que les chercheurs du CERIST semblent être plus au courant puisque $71 \%$ en sont conscient.

8-si le concept d'archive ouverte n'est pas très bien compris, il en résulte une utilisation (beaucoup plus en dépôt) très faible de l'ordre de moins d'un tiers des chercheurs des deux Centres.

9-la quasi ignorance des concepts de l'archive ouverte a induit une utilisation très faible des deux archives ouvertes connues des deux spécialités: ArchiveSIc et ArXiv et dont le taux d'utilisation est encore une fois bas et même très bas pour ArXiv (13\% qui la connaissent seulement) qui ne l'oublions pas est le première et même la plus réussie des archives jusqu'à ce jour. 
10-quant aux entrepôts d'archives ouvertes, il et clair que leur existence même n'est pas répertoriée par les chercheurs des deux Centres. Il est logique, à la suite de l'orientation des autres réponses, que les chercheurs avouent leur ignorance de ces outils qui, il faut le dire, ne sont pas très connus (à part des personnes spécialisées) mais qui rendent de grands services.

11-les journaux électroniques en libre accès sur Internet sont devenus des outils que le chercheur ne peut ignorer de par leur maniabilité, facilité d'accès, disponibilité, etc....Même plus, ils ont pratiquement supplanté les journaux papier dans nombre de spécialités. Malgré tous ces avantages, nos deux Centres ne les utilisent qu'a raison d'une moyenne d'à peu prés $50 \%$ pour les deux ce qui d'après nous est faible étant donné la prépondérance de ce medium dans la vie du chercheur moderne.

12-AJOL et DOAJ sont deux sites d'une importance primordiale pour l'utilisation des journaux électroniques que ce soit au niveau international ou régional. Les revues des deux Centres sont indexées par AJOL malgré cela une infime partie des chercheurs des deux Centres connait ces deux sites qui ne fait que confirmer les réponses précédentes et qui montrent une quasi totale ignorance de media dont l'utilisation est devenue non seulement banale mais plutôt indispensable et incontournable.

On peut sans risque de se tromper dire que les chercheurs investigués des deux Centres sont loin d'être au courant des multiples efforts tels que les Appels ainsi que des plus importants outils du libre accès que ce soit les archives ouvertes en particulier celles de la spécialité ou alors les journaux électroniques, les sites les indexant et encore moins les entrepôts d'archives ouvertes. Cette méconnaissance est dûe selon notre propre expérience à de multiples raisons mais que nous résumerons en une passivité de la part des chercheurs quant à savoir ce qui se passe dans le domaine de la recherche et des nouvelles manières de faire de la recherche mais aussi de la part des institutions dont les effort de sensibilisation ne sont pas, le moins que l'on puisse dire, très à propos. Nos contacts avec ces institutions nous ont fait découvrir le peu d'empressement et d'intérêt que les responsables semblent accorder au libre accès et surtout leur propre ignorance quant aux bienfaits que l'on peut en tirer.

13-1'avènement de l'outil informatique et des réseaux a profondément changé les supports de recherche. Alors que le papier était le seul support de prédilection des chercheurs, il a commencé à être sérieusement concurrencé dés le fin du $20^{\mathrm{e}}$ siècle par le support électronique. On remarquera pour notre cas que les chercheurs des deux Centres ont inclus cet aspect dans leurs habitudes documentaires et ce à raison d'à peu prés les $2 / 3$ d'utilisation du support électronique alors qu'à peu $1 / 3$ quant à eux utilisent soit les eux supports indifféremment ou alors les eux supports en même temps. On remarque qu'une infime partie (3\%) de chaque Centre utilise le papier en premier lieu. Ces chiffres ne font que traduire une vérité que tout chercheur expérimente : la prépondérance du support électronique de nos jours dans la vie des chercheurs.

14-si l'utilisation du support électronique est un fait qu'on peut difficilement occulter, il n'en demeure pas moins que le support préféré est différent. En effet, il existe une différence entre le support utilisé et celui avec lequel le chercheur est plus à l'aise .Cette dichotomie est vérifiée dans notre cas car les chercheurs des deux Centres expriment des avis différents par rapport au support utilisé. Ainsi ils préfèrent dans une proportion entre $2 / 5$ et $1 / 3$ le support 
électronique et à peu prés la moitié préfère le support papier pour respectivement le CERIST et le CDTA (alors qu'une proportion variant entre le 1/10 et le 1/6 est indifférente au support). Il existe de ce fait une différence entre la disponibilité du support et la préférence du support qui dans notre cas se vérifie. Enfin, il est tout de même étonnant que les chercheurs du CDTA, en principe plus enclins à être utilisateurs de technologie, soient moins versés vers cette option que les chercheurs du CERIST.

15- les pourcentages d'utilisation obtenus ne permettent pas de se faire une claire idée de la préférence qui enregistre de grandes différences. Ainsi les taux d'utilisation varient de 05$95 \%$ à $90-10 \%$ et de $05-95 \%$ à $80-20 \%$ en faveur du support électronique et ce respectivement pour le CERIST et le CDTA. Malgré cette dispersion de taux, on remarque que le taux 20$80 \%$ en faveur du support électronique est le plus prépondérant et ce à raison de 10 et 9 occurrences soit $30 \%$ pour respectivement le CERIST et le CDTA.

16-les deux Centres sont dans l'ensemble très ressemblants quant aux langues utilisées. La combinaison Anglais - Français est la plus prépondérante avec respectivement 16 et 14 occurrences pour respectivement le CERIST et le CDTA, alors que l'Anglais est aussi présent avec respectivement 10 et 13 occurrences. Enfin, le Français est aussi présent (bien qu'à un degré moindre) avec respectivement 4 et 3 occurrences. Ces deux langues représentent $83 \%$ pour le CERIST et $100 \%$ pour le CDTA des langues utilisées. Quant au reste des langues pour le CERIST et qui sont une combinaison de différentes langues utilisées par les chercheurs, elles ne représentent que $17 \%$ on peut affirmer ans risque de se tromper que les chercheurs des deux Centres communiquent et soumettent dans la langue internationale de la science par excellence et ceci est très encourageant pour la recherche nationale qui se voit sortir de la mainmise et la dichotomie des langues arabes et françaises. Publier en Anglais veut dire avoir une plus grande exposition dans le concert universel de la recherche.

On peut dire concernant les habitudes documentaires des chercheurs des deux Centres qu'ils sont assez ressemblant quant aux points examinés .Ainsi les chercheurs deux Centres utilisent plus le support électronique que le support papier avec des taux assez haut et, fait assez étonnant, les chercheurs du CDTA préfèrent le support papier plus que ceux du CERIST. D'un autre coté, un grand nombre des chercheurs deux Centres semblent affectionner le support électronique à $80 \%$ (ce qui veut dire qu'ils préfèrent le support papier qu'à 20\%).Enfin, la langue anglaise qui ne bénéficie pas en Algérie des avantages des langues arabes et française est utilisée massivement par les chercheurs des deux Centres, ce qui est dans le milieu linguistique algérien est un fait assez intéressant et encourageant à souligner.

17-il n'est plus besoin de faire ressortir l'importance de la publication dans la vie du chercheur qui doit publier pour de différentes raisons dont la plus importante est l'avancement et la reconnaissance des siens. D'un autre coté, il est de notoriété aussi que les chercheurs en sciences exactes et dures publient beaucoup plus des articles dans des journaux que des monographies à cause de leur rapidité comparativement aux livres. Cet aspect se trouve conforté dans notre échantillon, étant donné que les chiffres des deux Centres ne sont pas les mêmes. Si pratiquement la moitié des chercheurs du CERIST ayant répondus n'ont pas publié, les chercheurs du CDTA ayant répondus affichent quant à eux un taux de $90 \%$ de 
chercheurs ayant publié contre $10 \%$ ne l'ayant pas fait. Il se confirme, du moins pour notre enquête, que les chercheurs des sciences exactes tendent à publier plus que leurs collègues des sciences humaines et sociales. De plus, ces chercheurs préfèrent (ou sont dans l'obligation) de publier plus dans des journaux internationaux que les chercheurs du CERIST qui tendent à publier dans des journaux nationaux.

18- si moins de chercheurs du CERIST ont tendance à publier que leur collègue du CDTA, ils ont aussi tendance à moins publier. Ainsi on peut voir que sur les 19 chercheurs ayant répondu à cette question pour le CERIST, plus de la moitié ne l'ont fait qu'entre 1 et 3 fois alors que ceux qui ont publié plus de 5 fois ne représentent qu'aux environs du $1 / 5$ (21\%).Quant aux chercheurs du CDTA, la tendance est complètement inversée, plus de la moitié $(54 \%)$ a publié entre 1 et 5 fois alors que seuls $14 \%$ ont publié entre 1 et 3 fois. Une claire différence apparaît dans ces chiffres et ne fait que confirmer les chiffres de la question précédente.

19- les participations aux conférences des chercheurs des deux Centres sont une confirmation quasi parfaite des réponses aux précédentes questions ayant trait aux habitudes documentaires des chercheurs des deux Centres. Si un 1/6 seulement des chercheurs du CERIST a participé à des conférences la plupart internationales, la tendance pour les chercheurs du CDTA est complètement différente : tous les chercheurs ayant répondu ont déclaré avoir participé à des conférences, la plupart internationale .De surcroît, la même tendance quant au nombre de participations se confirme : ainsi si 12 chercheurs n'ont publié qu'entre 1 et 3 fois et que 11 autres ont publié plus de 5 fois pour le CERIST, $81 \%$ des chercheurs du CDTA ont publié plus de 5 fois alors que les autres chercheurs ( ayant publié entre 1 et 3 fois ou entre 3 et 5 fois) ne représentent que $20 \%$.

On peut conclure que les habitudes documentaires des chercheurs des deux Centre sont à l'opposé les uns des autres et se résument en les points suivants : les chercheurs du CERIST publient moins que ceux du CDTA alors que les deux tendent à publier beaucoup plus dans des journaux internationaux. On remarque aussi qu'un plus grand nombre de chercheurs du CDTA publient plus de fois que les chercheurs du CERIST. Les chercheurs du CDTA ont tous participé à des conférences surtout internationales alors que ceux du CERIST y ont beaucoup moins participé et encore une fois le nombre de ces participations dépasse et de loin celui des chercheurs du CERIST. Les chercheurs du CDTA sont beaucoup plus actifs en matière de publication et participation à des conférences que leurs collègues du CERIST et ceci serait dû à la plus grande importance qu'occupe la publication dans le cursus du chercheur en sciences exactes par rapport à celui du chercheur en sciences humaines et sociales.

19-les chercheurs des deux Centres investigués semblent accorder une grande importance au contrôle par les pairs que ce soit en amont (au moment de la soumission) où entre les $3 / 4$ pour le CERIST et les 9/10 pour le CDTA ont soumis en sachant que leur travail leur travail sera soumis au contrôle. En aval aussi car cette décision de soumettre était tributaire de ce critère de contrôle. Les chercheurs ont aussi exprimé à de très fortes proportions que ceci (soumettre en sachant que la publication est contrôlée) était majoritairement pour l'avancement. Critère 
qui nous fait dire que les chercheurs sont en même temps conscients de l'importance de publier et aussi de " bien publier " (dans des revues à fort facteur d'impact).

20- les chercheurs des deux Centres investigués semblent accorder au contrôle par les pairs une grande importance et ce à différents niveaux : que ce soit quant à son importance où une majorité pense qu'il est indispensable à l'avancement de la Science. D'un autre coté, ces mêmes chercheurs ont majoritairement estimé aussi qu'ils avaient été jugés d'une manière objective lors de leurs soumissions. Les chercheurs des deux Centres ont aussi exprimé une concordance quant au problème de l'anonymat des experts qu'ils ont estimé normal à des proportions assez ressemblantes (65\% pour le CERIST et $77 \%$ pour le CDTA).L'autre point de concordance concerne l'acceptation des correctifs demandés et leur intégration dans le travail révisé. Enfin, le dernier aspect sur lequel les chercheurs des deux Centres ne divergent pas est celui concernant les raisons de l'intégration des correctifs demandés: il s'agit majoritairement des arguments des experts qui ont convaincus les chercheurs et à un degré moindre le besoin ou envie d'être publié. Le seul point sur lequel les deux Centres présentent des chiffres différents sont ceux des taux d'acceptation où les chercheurs du CERIST ont des taux bien moindres que ceux des chercheurs du CDTA ce qui ne fait que confirmer les chiffres précédents concernant le taux de chercheurs ayant entrepris de publier pour les deux Centres. Ainsi à titre d'exemple, les taux dépassant les $80 \%$ représentent 19 cas soit $70 \%$ alors que le même taux ne se retrouve que chez 7 cas soit $33 \%$.Ces chiffres tendraient à confirmer une constante dont nous avons déjà parlé : le taux d'acceptation des soumissions en sciences exactes qui est plus levé qu'en sciences humaines.

21-les chercheurs des deux Centres semblent avoir une approche assez ouverte vis-à-vis des nouvelles formes de certification de l'information sur Internet. Ils sont très au courant de ces nouvelles formes de publication à hauteur de 85 et $95 \%$ pour respectivement le CERIST et le CDTA. Ils ne sont pas, par contre, très ouvert à l'idée d'avoir leur soumission jugée ouvertement par la communauté des chercheurs comme cela se fait et ce à hauteur des $2 / 3$ environs ( $68 \%$ et $65 \%$ pour respectivement le CERIST et le CDTA).Ils affichent pratiquement les mêmes réticences quant à l'éventualité d'avoir ces commentaires affichés sur Internet et ce respectivement à 58 et $61 \%$.Quant à la dernière question concernant un aspect encore plus révolutionnaire et qui consiste à afficher ces commentaires avec la publication finale, ils présentent encore des similitudes étant donné que les taux d'acceptation de ces changements se situent entre 67 et $74 \%$ pour respectivement le CERIST et le CDTA. Les réponses obtenues indiquent une claire ouverture des chercheurs des deux Centres quant à ces nouvelles et parfois même révolutionnaires formes de certification de l'information.

22- les chercheurs investigués ont répondus concernant leurs positions vis à vis des nouvelles formes de contrôle de l'information d'une manière très peu ressemblantes. Ainsi, les chercheurs du CERIST semblent faire plus confiance à ces nouvelles formes pour diminuer les accusations de préjudice et de subjectivité et ce à hauteur de $68 \%$ alors que les chercheurs du CDTA n'y croient qu'a hauteur de 53\%.D'un autre coté, les chercheurs du CERIST ont cités en premier lieu le critère linguistique ensuite personnel comme raison du préjudice alors que les chercheurs du CDTA citent le critère personnel en premier lieu comme raison du préjudice ( le critère linguistique n'étant que faiblement cité ).En fait, une multitude de 
critères est citée par les chercheurs des deux Centres que l'on peut résumer en : manque d'expérience et de compétence dans le domaine, renommée de l'encadreur ou directeur de laboratoire, appropriation des œuvres des autres, linguistique-personnelle, personnellereligieuse, religieuse-linguistique et linguistique-sexuelle pour le CERIST et chercheurs tiers monde, manque d'expertise de l'expert, religieux et culturel, géographique pour le CDTA. Notons qu'un nombre assez important de chercheurs n'a pas répondu à la question ( 7 et 8 respectivement pour le CERIST et le CDTA).Par contre, ils ont exprimé une convergence de point de vue concernant l'efficacité des nouvelles formes de contrôle de l'information quant à l'atténuation des accusations de fraude et de plagiat et ce à hauteur de $69 \%$ pour les deux Centres. Ils divergent enfin quant à la question de l'anonymat lorsqu'ils sont experts où les chercheurs du CERIST accepteraient d'être connu à hauteur de $72 \%$ alors que les chercheurs du CDTA ne l'accepteraient qu'à hauteur de 55\%.Enfin, les chercheurs du CERIST semblent accepter les nouvelles formes de publication (archives ouvertes et journaux électroniques) comme aune par laquelle on accéderait aux promotions plus que ceux du CDTA et ce à hauteur de 58\% alors que les chercheurs du CDTA ne l'accepterait qu'à hauteur de $37 \%$.Ces chiffres ( concernant l'acceptation des archives ouvertes et des journaux électroniques pour les promotions) sont étonnant à plus d'un titre et dont nous avons relevé deux faits saillants : la plus grande ouverture et acceptation des archives ouvertes et des journaux électroniques par les chercheurs du CERIST par rapport à ceux du CDTA et aussi et surtout les ( relatifs ) faibles taux d'acceptation par les chercheurs des deux Centres de ces nouveaux media qui ne se situent qu'à des taux bas pour des media qui sont rentrés dans les mœurs des chercheurs avec l'avènement et la propagation d'Internet. Ces chiffres indiquent, à notre avis, une sorte d'ignorance qui n'est pas très réconfortante pour des Centres supposés être à la pointe de la recherche en Algérie dans leurs domaines respectifs.

Ces chiffres que nous avons obtenus sont, il faut le dire, ambivalent. En effet, certaines des réponses sont étonnantes de par leurs résultats alors que d'autres réponses vont complètement à l'encontre des résultats précédents. On peut en conclure ( ou du moins supputer ) que ceci est du soit à une ignorance de schémas nouveaux et même révolutionnaires ou alors à un manque de sensibilisation de la part des autorités responsables. Il est encore tôt d'y répondre mais il est clair que des investigations plus poussées et futures doivent être entreprises afin d'avoir une plus claire idée. Efforts dont nous espérons cette enquête est le prélude car il est clair qu'on ne peut rester en retrait de ces nouvelles technologies si on veut progresser. 
Bien que récent dans sa forme actuelle et n'ayant été appliqué plus ou moins uniformément qu'après la deuxième guerre mondiale, le contrôle par les pairs a engendré une foisonnante documentation (surtout anglo-saxonne) de par l'extrême importance qu'il revêt aux yeux des chercheurs. En effet, dans un système résolument tourné vers la publication et obnubilé par la production chiffrée, le chercheur est engagé dans une course poursuite afin de publier et surtout publier en quantité. Il en a pas toujours de même avant l'avènement de ce qui est communément appelé " l'explosion documentaire " qui a coïncidé avec l'essor qu'a connu l'Occident dans tous les domaines (et la recherche scientifique en particulier) au lendemain de la deuxième guerre mondiale. En effet, le contrôle par les pairs a agit et a eu deux fonctions primordiales : filtrer les publications de par la qualité et aussi les filtrer de par le nombre. Si la première fonction a plus ou moins toujours existé sous des formes différentes et remonte à bien avant l'avènement des périodiques après la seconde moitié du $17^{\mathrm{e}}$ siècle (comme on l'a vu pour le livre d'Ishak Ibn Ali Arrahwi au $9^{\mathrm{e}}$ siècle) et est en fait inhérente à la nature même de la science qui ne peut progresser que par incrémentations successives, chacune s'appuyant sur celle (s) l' (es) ayant précédé (s). La deuxième quant à elle découle d'un postulat numérique de l'augmentation quasi incontrôlée de la production scientifique et qui a conduit à une saturation difficile à gérer. C'est dans ce contexte que les pays vainqueurs de la Guerre (à leur tête les Etats Unis et sur fonds de guerre froide) investirent lourdement dans les programmes de recherche afin de reconstruire ce que la guerre a causé comme dégât. S'ensuit alors une injection formidable dans la recherche surtout fondamentale afin de reconstruire le monde d'après guerre. C'est alors que ces même pays entreprirent de développer leur capacités de publication des résultats de cette recherche par le lancement (et aussi la continuation) de nouveaux périodiques scientifiques et techniques dont la fonction était de faire connaitre ces résultats. Comme nous l'avons vu dans la deuxième partie de ce travail, il impossible d'avoir le nombre total de ces périodiques pour de multiples raisons dont la définition même de ce qu'est un périodique scientifique est la plus prégnante (les autres raisons communément évoquées sont le taux de mortalité, les changements de nom, les fusions, les cessations de parution, etc....) mais une recherche récente a estimé le nombre d'articles publié en 2006 à quelques $1350000{ }^{{ }^{(124)}}$.On remarquera que c'est le nombre d'articles qui est estimé et non pas le nombre périodiques. Ceci a été fait par les auteurs en se basant sur l'Ulrich's Periodical Directory et l'Institute for Scientific Information (ISI) ainsi que des échantillonnages et Google Search. Une autre estimation met le nombre d'articles publiés depuis les premiers périodiques scientifiques à 50000000 (cinquante millions) ${ }^{(125)}$.Ces chiffres mirobolants donne une idée de la tâche titanesque qu'avait les périodiques à absorber cette masse d'information.

C'est alors que la deuxième fonction (filtre quantitatif) est entrée en jeu sous la forme d'un choix de plus en plus drastique des soumissions. En fait, les deux fonctions (filtre quantitatif et qualitatif) vont de pair et ne sont pas antinomiques. Pour être plus explicite, face à

${ }^{(124)}$ Björk, B-C., Roos, A. \& Lauri, M., Scientific journal publishing: yearly volume and open access availability, Information Research, Vol. 14, ${ }^{\circ} 1$, paper 391[Accessible à partir du 12 Janvier 2009 à http://nformationR.net/ir/14-1/paper391.html] (Accédé le 25 Juillet 2012)

${ }^{(125)}$ http://duncan.hull.name/2010/07/15/fifty-million/ (Accédé le 25 Juillet 2012) 
l'augmentation exponentielle des données et des résultats de la recherche, et afin de juguler cette masse, il fallait choisir les meilleurs travaux.

Au début cette opération n'était nullement normalisée et chaque éditeur appliquait ses propres critères. Ce n'est que lorsque le travail devint de plus en plus sophistiqué qu'il le fut normalisé. " Ce ne fut qu'après la période post deuxième guerre mondiale, soit quelques deux cent ans après ses débuts que le processus devint universellement accepté. Comme il n'y a pas de temps spécifique quand le concept de contrôle par les pairs a été adopté, de même le processus n'a jamais pris une forme normalisée et continue de varier de journal à journal " (126). A l'instar de Henry Oldenburg (première personne à avoir assumé et balisé le rôle d'éditeur au $17^{\mathrm{e}}$ siècle), les premiers éditeurs des temps modernes ont utilisé ce qui s'apparentait à un contrôle interne qui consistait à expertiser la soumission sans l'envoyer à des experts hors du comité éditorial, l'éditeur servant même de premier filtre. Dans ce cas, l'éditeur doit voir est ce que la soumission rentre dans les sujets que le journal couvre, est scientifiquement et méthodologiquement acceptable, est acceptable par les critères de qualité du journal en question (critères de toutes évidence différents de journal à journal - voir à cet effet le facteur d'impact qui différencie les revues de classe A et celles de moindre prestige).Bien que l'on ne puisse mettre de date précise à la naissance du contrôle par les pairs actuel ( du moins dans sa forme traditionnelle papier ) tel que l'a explicité Manske, il semblerait que le début de l'envoi systématique des soumissions à des experts extérieurs marque le début du contrôle par les pairs tel qu'on le connait de nos jours. Bien que de datation difficile comme c'est le cas pour le contrôle par les pairs lui-même, on peut le situer au début des années 60, plus précisément à 1962, quand Harvey Blank dans un éditorial dans Archives of Dermatology ${ }^{(127)}$ avoua le besoin d'avoir recours à des experts extérieurs afin d'assurer une bonne marche du journal dont il était l'éditeur en ces termes: " Malheureusement, l'information scientifique significative augmentant, une plus grande expansion du banal, du trivial et même du faux menace apparemment d'occulter le savoir important dont on a besoin pour porter des jugements judicieux et rationnels". Il continue plus loin parlant des experts dont son journal a eu besoin afin d'assurer : " Dans un domaine aussi vaste que la dermatologie, on doit souvent chercher à une large échelle des autorités en la matière avec un savoir expert détaillé suffisant pour faire office d'experts éditoriaux critique et constructifs. Au cours de la dernière année, le comité éditorial de Archives of Dermatology s'est lancé dans un programme visant à rechercher ce genre d'expertise pour chaque manuscrit soumis ". Il termine son éditorial en ces termes : "Durant l'année 1962, plus de 450 manuscrits furent soumis et le Comité [éditorial] voudrait remercier les personnes suivantes qui, en consacrant généreusement leur temps et leur savoir, ont permis d'expertiser un si grand nombre de contributions : .... [Noms des experts ayant contribué] ". D’un autre coté, si le contrôle par les pairs est largement accepté et appliqué, certaines des parties de tout journal n'y sont pas soumises comme les informations en général et les colonnes périodiques. De même les éditoriaux, les symposia, les actes de conférences les manuscrits sollicités, les commentaires et, en général, tout matériel n'ayant pas besoin d'un contrôle impératif en sont

\footnotetext{
(126) Manske, P.R., A Review of peer review, Journal of hand surgery, Vol. 22 A ,n 5, p 767-771, 1997

${ }^{(127)}$ Blank,Harvey., The Summary move forward, Archives of Dermatology, Vol.87, n 4 , p.413-414, Avril 1963
} 
exempts. De leur coté, certains journaux publiés par les sociétés savantes donnent la priorité aux recherches présentées aux conférences annuelles de la société et, de toute évidence, ne sont pas sujettes une deuxième fois à une certification ayant déjà passé cette étape pour leur acceptation à la conférence.

Une fois accepté et (plus ou moins) normalisé, l'application et les modalités d'exécution du contrôle par les pairs allaient commencer à poser des problèmes .Pour être plus clair, et pour situer le processus dans un contexte historico-politique, la manne financière que la période post deuxième guerre mondiale engendra allait aiguiser les convoitises des scientifiques. En effet, à côté des traditionnelles satisfactions ressenties par le chercheur (telles que la reconnaissance des pairs, la satisfaction du travail et efforts récompensés, la contribution au progrès humain - surtout dans des domaines comme la médecine -, etc....), la réussite académique ouvrait la porte à de nombreux avantages plus palpables sous formes de financements, avancements dans la carrière scientifique et même postes de responsabilités aux avantages très alléchants. Ces attraits aux mains d'être humains somme toute vulnérables ont engendré des polémiques à ne plus cesser ayant éclaboussé l'establishment scientifique. Harnad a peut être résumé cette situation dans un de ces plus incisives contributions ${ }^{(128)}$ et dans laquelle il appelle à la disparition du support papier et des ses coûts mais défend le maintien du contrôle par les pairs en ces termes :" La nature humaine étant ce qu'elle est, on ne peut s'y fier afin de s'auto contrôler et s'auto surveiller. Des exceptions individuelles existent mais les traiter comme la règle serait sous estimer le degré auquel notre indiscipline potentielle est contrôlée par les contraintes collectives, mises en œuvre formellement. Plus loin, il introduit le concept de "Quis custodiet ipsos custodes ? "* ; il continue son argumentaire en ces termes :" c'est le cas pour les affaires civiques et il n'y a pas de différence dans le monde de la recherche savante. Le problème du Quis custodiet entre les chercheurs a été traditionnellement résolu par un système de certification et de contrôle de qualité $[\mathrm{C} / \mathrm{CQ}]$ appelé le contrôle par les pairs ". On peut sentir dans la définition de Harnad une sorte de résignation dans les problèmes posés par le contrôle par les pairs. L'utilisation de l'expression Quis custodiet ne veut elle pas dire que le contrôle par les pairs n'a jamais été et ne sera, peut être, jamais complètement loin des controverses car il est tout simplement le fait d'être humain et ceci est presque une assurance qu'il ne sera jamais objectif. D'ailleurs, la littérature sur le sujet abonde de références à la comparaison faite de la célèbre expression de Winston Churchill au sujet de la démocratie et dans laquelle il dit " qu'elle est [la démocratie] la pire forme de gouvernement exception faite de toutes les autres formes essayées de temps à autre ". Le contrôle par les pairs est imparfait, critiquable, ouvert aux dépassements de tous genres mais il n'y a pas eu d'alternative viable pour le remplacer. Nombre d'accusations furent dirigés à l'encontre du contrôle par les pairs .Dans certains cas

\footnotetext{
${ }^{(128)}$ Harnad, Stevan, The Invisible hand of peer review. Disponible à http://users.ecs.soton.ac.uk/harnad/nature2.html (Accédé le 27 Juillet 2012)

"Expression voulant dire grosso modo :" Mais qui gardera ces gardiens ?" expression utilisé par le poète romain Juvenal dans ses satires et dans laquelle il visait, dans le texte original, les gardiens que les citoyens romains chargeaient de veiller sur leur maison en leur absence et que l'auteur accusait d'infidélité ( http://fr.wikipedia.org/wiki/Quis custodiet ipsos_custodes\%3F) .L'allusion est claire ici dans son utilisation par Harnad quant à l'auto contrôle qu'exerceraient les scientifiques sur eux-mêmes dans le cas de l'expertise scientifique d'une soumission.
} 
(heureusement rares), les règles les plus élémentaires de la bienséance furent dépassées et donnèrent une piètre et dévalorisante idée de la recherche scientifique. La discussion scientifique supposée être polie et policée devint un échange d'insultes, d'invectives, de menaces et d'accusations très préjudiciable pour la Science. Un des exemples les plus extrêmes fut celui du Docteur Carlo Castro avec Paul Ginsparg intitulé " My Struggle with Ginsparg (arXiv.org) and the Road to Cyberia: A Scientibic-Gulag in Cyberspace "(Mon combat avec Ginsparg (arXiv.org) et le chemin vers Cyberia : un goulag scientifique dans le cyber espace). et dont Castro a publié une sorte de dénonciation des pratiques dans un site et avec un titre très évocateur ${ }^{(129)}$ Dans cette dénonciation, Carlos Castro dénonce les pratiques de censure que Paul Ginsparg lui aurait appliqué à cause, d'après lui, de ses méthodes scientifiques non conventionnelles. La dénonciation est une suite de faits, de mails, etc.... qui prouveraient l'acte de censure de Ginsparg à l'encontre de Castro (et même de ces coauteurs). Toujours d'après Castro, Ginsparg (et derrière lui ArXiv.org) a même utilisé le système d'endossement qu'applique ArXiv.org (système institué depuis Janvier 2004 par ArXiv.org et stipulant qu'un nouveau soumissionnaire devait avoir l'endossement d'une personne ayant déjà publié avec ArXiv.org pour être permis de publier sur l'archive) pour le bloquer (le terme exact utilisé est " liste noire "). Ils auraient même mis en doute l'affiliation académique de Castro (et ses co-auteurs) afin de prouver une quelconque fraude. Enfin, un des défendeurs de Castro, Carlos R.Handy, professeur associé de physique et Co-Directeur du Center for Theoretical Studies of Physical Systems at Clark Atlanta University dans sa lettre aux modérateurs du site d'ArXiv utilisa les termes suivants: "Nous n'aimons pas cette impression d'une mafia intellectuelle qui semble être engagée dans une censure scientifique ". Un autre exemple de cette lutte que se livrent les savants afin de pouvoir publier dans les conditions les meilleures et surtout d'être jugés sur leur valeurs intrinsèques et non pas leur opinions ou tout autre critère autre que scientifique est un livre collectif détaillent la censure des idées scientifiques non conventionnelles intitulé " Against the tide : a critical review by scientists of how physics and astronomy get done " (130) (Contre le courant: une revue critique de la manière dont les sciences physiques et l'astronomie sont gérées ).La synthèse donnée par l'éditeur sur son site nous a paru très à propos. Nous la reproduisons in extenso car elle nous a paru résumer cette guerre larvée que se livre les scientifiques sur des bases non pas scientifiques mais beaucoup plus subjectives et matérielles. " Personne ne devrait avoir le monopole de la vérité dans cet univers. La censure et la suppression des idées provocantes allant à l'encontre des courants dominants de la recherche, la mise sur liste noire de scientifiques, par exemple, ne sont ni la meilleure manière de pratiquer et filtrer la science, ni de promouvoir le progrès dans le savoir humain. La suppression des idées intéressantes et inédites de la scène scientifique est très préjudiciable à la quête de vérité. Il existe des cas où une simple croyance sans réserve peut parfois être transformée en une théorie scientifique globalement acceptée à travers les actions de filtrage de la littérature expertisée et les rencontres organisés par la communauté scientifique et aussi par le biais de la distribution des financements contrôlés par des " clubs d'opinions ". Ceci conduit à une pensée et des paradigmes unitaires pas nécessairement associés à la vérité unique. C'est le but de ce livre :

\footnotetext{
(129) $\mathrm{http}: / /$ www.archivefreedom.org/freedom/Cyberia.html (Accédé le 29 Juillet 2012)

(130) Corredoira, Martín López \& Perelman, Carlos Castro Ed., Against the tide: a critical review by scientists of how physics and astronomy get done, Boca Raton [FL, U.S.A.], Universal Publishers, 2008, 256 p.
} 
analyser d'une manière critique les mécanismes officiels (et parfois illicites ) sous lesquels la science ( particulièrement les sciences physiques et l'astronomie ) est administrée et filtrée de nos jours avec les lourdes conséquences que ces mécanismes ont sur nous tous.[Les auteurs de ce livre ] tous des chercheurs professionnels expriment une vue pessimiste des problèmes du système actuel alors qu'une lueur d'espoir reste dans le leitmotiv de la revendication pour la liberté dans l'exercice de la recherche et la réalisation d'un niveau acceptable d'éthique de la science " ${ }^{(131)}$.Bien que ce livre soit l'œuvre d'un nombre de chercheurs reconnus ( à l'instar de Juan Miguel Campanario) il est dans le contenu le même que la diatribe de Carlos Castro (qui en est un des deux éditeurs scientifiques ) contre Ginsparg. Il traduit un ras le bol des chercheurs face à une sorte de mandarinat de la part de ceux qui occupent les postes de responsabilité. Ce mandarinat - qui serait acceptable la science étant par définition méritocratique - est doublé d'une position de cartellisation et d'indu pouvoir que cette caste détient. De cette position de force, un certain nombre de privilèges ont découlé parmi les quels l'accès à la publication.

De là est née cette constante bataille du contrôle par les pairs, enjeu primordial des aspirants chercheurs dans leur quête à percer et publier. Les différentes recherches ayant abordés cette problématique ont détecté d'innombrables failles et préjugés biaisant le travail, supposé objectif, des experts (qui sont aussi des auteurs soumettant et publiant mais auxquels il est dévolu la tache de juger, filtrer, choisir les travaux les meilleurs grâce à leur niveau). Parmi les plus critiqués et les plus cités de ces préjugés, on citera les préjugés idéologiques, raciaux, sexuels, régionaux, et même personnels. Ces préjugés (supposés ou véridiques) détaillés auparavant ne peuvent être comparés à un préjugé que toute personne sortant du moule a expérimenté. En effet, la science est faite de consensus, de découvertes, de théories, de règles et de théorèmes acceptés par la communauté. Cette suite de lois est respectée par ceux qui font la recherche afin de faire progresser et avancer la science. Dans certains cas, un chercheur ou groupe de chercheurs, faisant fi de ces règles, innove et sort de " l'orthodoxie " du domaine et va à contre courant de ce qui est communément accepté. Galilée, Copernic et d'autres ont essuyé les foudres des tenants de l'orthodoxie et plus près de nous d'autres chercheurs comme Immanuel Velikowsky dont nous avons parlé ou alors Carlos Castro ont aussi été soit ridiculisés ou vilipendés. Une des meilleures contributions à ce genre de préjudice sont deux publications de Juan Miguel Campanario sur le sujet. Dans la première, ${ }^{(132)}$, Campanario étudie les résistances que les chercheurs rencontrent quand ils font des recherches innovantes ou essayent de publier des articles qui devinrent plus tard parmi les plus cités. Sur 205 commentaires par les auteurs des articles les plus cités de tous les temps ayant été examiné afin d'en identifier les articles dont les auteurs ont eu des difficultés à publier leur travaux, 22 exprimèrent certaines difficultés ou résistance en faisant ou en essayant de publier les recherches publiées dans l'article. Trois de ces articles ayant expérimenté des difficultés à être publié sont les plus cités de leurs journaux respectifs. Selon

(131) http://www.universal-publishers.com/book.php?method=ISBN\&book=1599429934 (Accédé le 29 Juillet 2012)

${ }^{(132)}$ Campanario, Juan Miguel, Have referees rejected some of the most-cited articles of all times?, Journal of the American Society of information Science, Vol. 47 , n4 , p.302-310, Avril 1996

(Resumé disponible à http://onlinelibrary.wiley.com/doi/10.1002/(SICI)1097-4571(199604)47:4\%3C302::AIDASI6\%3E3.0.CO;2-0/abstract (Accédé le 31 Juillet 2012) 
les auteurs concernés, bien que les remarques négatives des experts peuvent, dans certains cas, aider à améliorer les articles, dans d'autres cas experts et éditeurs rejetèrent injustement les articles qui s'avérèrent être fortement cités. Dans le deuxième ${ }^{(133)}$, Campanario étudia le point de vue de scientifiques qui expérimentèrent de la résistance à leurs idées innovatrices en sondant un échantillon de 815 scientifiques auteurs d'articles fortement cités. Des 132 réponses reçues, seuls 47 n'ont eu aucun problème avec les éditeurs, les experts et aussi les autres scientifiques. Les causes les plus communes de difficultés rencontrées sont rejet du manuscrit, scepticisme, ignorance et incompréhension. Les arguments les plus communément cités par les experts contre la publication étaient que les résultats étaient considérés comme une avance insuffisante pour justifier une publication, les articles manquaient d'impact pratique, étaient basés sur une hypothèse fausse ou alors étaient basés sur un concept faux. Les stratégies adoptées par les auteurs pour contourner variaient de l'obtention de l'aide pour publier ces recherches problématiques, faire des changements dans le texte à la simple persistance. Malgré ces difficultés, certains des chercheurs ayant répondus ont reconnu l'effet positif du contrôle par les pairs. Campanario fait ressortir dans ces contributions (et d'autres relatives au même sujet de la non fiabilité et surtout frilosité du contrôle par les pairs face à l'innovation) que le chercheur qui sort des sentiers battus et challenge ce qui est communément accepté, et même s'il est dans le vrai, a de fortes chances d'être rejeté et même des fois ostracisé par la communauté scientifique.

De son coté, et dans un éditorial ${ }^{(134)}$ publié dans le très respecté Nature, l'éditeur du périodique, pourtant considéré avec Science comme le périodique scientifiques le plus prestigieux, reconnaît en ces termes que le contrôle par les pairs est, parfois, coupable de rejeter des recherches qui devinrent plus tard très citées et très importantes dans la recherche du domaine. Il dit au début de son éditorial : " Des cas de rejet de découvertes couronnées par le Prix Nobel mettent en évidence le conservatisme en science. Malgré des erreurs de jugement historiques, les éditeurs de journaux peuvent aider mais, par dessus tout, les auteurs auront besoin de persistance continue ". Défendant son journal, il reconnaît cependant "....[qu'] il y a eu d'incontestables faux pas dans notre histoire. Il s'agit notamment du rejet des radiations de Cerenkov, les mésons de Hideki Yukawa, les travaux sur la photosynthèse de Johann Deisenhofer, Robert Huber et Hartmut Michel et le rejet initial (mais éventuelle acceptation) de la théorie des radiations des trous noirs de Stephen Hawkings". Le fait que l'éditeur d'un périodique scientifique aussi prisé pour la publication reconnaisse ce genre de faux pas nous renseigne sur l'état du contrôle par les pairs face à ces nombreux détracteurs et ses nombreuses contradictions.

\footnotetext{
(133) Campanario ,Juan Miguel, Acedo, Erika, Rejecting highly cited papers: the views of scientists who encounter resistance to their discoveries from other scientists, Journal of the American Society of information Science, Vol. 58 , n5, p.734.-743, Mars 2007

(Résumé disponible à http://onlinelibrary.wiley.com/doi/10.1002/asi.20556/abstract (Accédé le 31 Juillet 2012)

(134) Editorial, Coping with peer rejection, Nature, Vol.425, $\mathrm{n}^{\circ} 6959, \mathrm{p} .645,16$ Octobre 2003 (Disponible à http://www2.uah.es/jmc/peer\%20rejection.pdf) (Accédé le 2 Août 2012)
} 
De son coté l'étude de Peters et Ceci, citée en supra et devenue célèbre depuis sa publication en 1982 et malgré des failles méthodologiques qu'elle comportait quant à la conception et l'administration et que certains chercheurs ont décelé, reste une référence quant aux préjugés Ad Hominem (personnels) et surtout institutionnel. Comme nous avons vu, les auteurs de cette étude ont ressoumis des articles en psychologie qui avaient été acceptés dans des journaux au haut facteur d'impact et aux critères d'acceptation parmi les plus sélectifs Quand les resoumissions furent effectuées, de légers changements furent apportés. Le résultat fut que des articles précédemment acceptés, expertisés, publiés et faisant partie du corpus de la science furent rejetés par les mêmes périodiques les ayant accepté la première fois. De plus, certaines raisons évoquées prouvaient que certains des experts n'étaient au fait des derniers progrès du domaine en rejetant des recherches précédemment acceptées et publiées dans les meilleurs périodiques scientifiques américains de la spécialité. Mais la conclusion la plus importante est le préjugé institutionnel qui est de mise. Sinon comment expliquer qu'une même recherche quand elle est soumise au nom d'un auteur d'une université prestigieuse est acceptée mais soumise sous un nom - fictif - non prestigieux, elle est sujette au rejet ? Il est vrai que certains chercheurs ont justifié cette manière de procéder par le fait qu'il est logique qu'une recherche émanant d'un chercheur de Harvard, par exemple, est supposée être de haute facture étant donné la réputation de Harvard. Cela $n$ 'empêche qu'une soumission est supposée être jugée sur ces valeurs intrinsèques et non sur sa provenance. De même, un chercheur au $\mathrm{CV}$ étoffé et à la réputation bien établie, est en droit de s'attendre à plus de respect et de considération (Voir l'argument de Stanley Fish en supra) quoique le chercheur novice peut lui aussi ne pas accepter cet état de fait (Voir l'argument de Jeffrey Skoblow en supra) et demander à être jugé sur sa valeur intrinsèque.

Toutes ces embûches que le contrôle par les pairs rencontre, ont fait réfléchir les scientifiques quant à des solutions afin de le rendre plus ouvert, moins sectaire et surtout plus juste. Les différentes tentatives dont la plus probante est le contrôle ouvert où le soumissionnaire est connu de l'expert et où l'expert est connu du soumissionnaire n'a eu que des résultats mitigés ou du moins n'a pas allégé les accusations précédemment évoqués. Au contraire, ces nouvelles formes ont engendré des problèmes qui n'existaient pas auparavant comme par exemple la peur des représailles de la part des nouveaux experts dans le cas ils critiquaient, rejetaient les soumissions des chercheurs plus expérimentés et plus connus. Ces derniers pouvaient en retour faire acte de représailles pour les postes à pourvoir et /ou les demandes de financements pour lesquels ils ont la main haute. De même que ces experts novices se sentiraient, peut être, inhibés à l'idée de critiquer le travail d'une personne considérée comme une sommité dans la spécialité, situation qui serait impossible dans le système traditionnel où l'anonymat le plus complet (dans les deux sens) est de mise.

L'avènement d'Internet dans les années 90 (du moins sa démocratisation surtout dans les pays développés) a fait passer le contrôle par les pairs à une forme, une approche, une philosophie même d'ouverture complète. La Toile mondiale, comme elle est aussi connue, permet en effet de permettre cette extrême ouverture - et par delà un espoir de diminution des débordements et des travers inhérents à l'univers papier et son contrôle fermé - et de rendre la certification 
et le contrôle plus responsable devant ceux la même qui en sont les sujets. Le magazine Nature entrepris alors en 2006 une expérience pionnière et unique jusqu'à ce jour dans laquelle le magazine demande à des chercheurs et à des éditeurs leur point de vue sur l'expertise ouverte sur Internet. Les différentes contributions (au nombre de 22) abordèrent les différents aspects du contrôle par les pairs sur Internet allant des avis les plus enthousiastes aux plus pessimistes sur la question. Certains comme Erik Sandewall, Ulrich Pöschl et Thomas Koop ou encore Eugene Koonin, Laura Landweber, David Lipman et Ros Dignon qui présentèrent des expériences connues et ayant fait leur preuve dans le domaine. En effet, ETAI (Electronic Transactions On Artificial Intelligence), ACP (Atmospheric Chemistry and Physics) et Biology Direct sont les premières expériences dans le domaine et ont eu des résultats mitigés (ETAI) ou ont enregistrés des succès et ont fait de leur système des modèles que les autres périodiques prennent en exemple à l'instar d'ACP. Biology Direct quant à lui promeut une approche assez originale où - pour reprendre l'article - ".......une recherche publiée représenterait une hypothèse résolument non conventionnelle, accompagnée d'expertises intéressées mais dûment sceptiques, un autre [recherche] inclurait des spéculations extrêmement larges d'une interprétation de résultats de génomique comparative. Cependant, la plupart des recherches ressembleraient aux affaires courantes (business as usual) ce sont des travaux sérieux qui pourraient facilement être publiés dans des journaux plus traditionnels. C'est peut être un signe que Biology Direct attirera un heureux mélange de bonne " normale "science et d'idées audacieuses". ${ }^{(135)}$ L'approche de Biology Direct semble la plus à même de réussir car elle concerne un domaine (la biologie) où les enjeux sont très grands (brevets, découvertes, royalties etc....) et où, de ce fait, il est très fructueux de publier non seulement pour des motifs seulement académiques mais aussi et surtout pour des avantages purement pécuniaires, l'attribution de brevets, la paternité de découvertes étant synonymes de récompenses financières très alléchantes. Malgré cela, cette expérience qui date maintenant de six ans ne semble pas avoir eu de répercussions ni de suites positives. D'ailleurs, Nature donna le résultat des différentes enquêtes ayant accompagnés les différentes contributions et il en ressort que les éditeurs et surtout les auteurs ne sont pas près à accepter cette ouverture. Il y a une sorte de réticence à s'engager et " se découvrir " surtout afin de ne pas antagoniser la communauté. Les éditeurs se sont plaints que les commentaires donnés par les concernés soient du genre " déclarer que [le contrôle par les pairs ouvert] c'est [du]" bon travail " au lieu d'analyser et donner des avis qui auraient pu être utilisés pour aider à la prise de décision. La conclusion générale fut que malgré l'intérêt porté à l'expérimentation, les résultats indiquent que la communauté n'est pas prête à faire le saut et à adopter le contrôle pour les pairs ouvert. En conséquence, Nature et ses éditeurs continueront à explorer les utilisations participatives du web mais n'appliqueront pas le contrôle par les pairs ouvert, pour le moment du moins.

Les autres expériences que nous avons clairement et longuement analysées dans ce travail (ACP, F1000, ETAI et Philica) représentent les formes de certification les plus innovantes dans le monde de la publication scientifique. Formes, résultant des nombreuses et

${ }^{(135)}$ Koonin, Eugene [et al], Can open peer review work for biologists? Biology Direct is hopeful.Disponible à : http://www.nature.com/nature/peerreview/debate/nature05005.html 
ankylosantes critiques que le contrôle par les pairs a suscité (et suscite toujours) et aussi permis par l'extraordinaire développement et fonctionnalités qu'Internet permet. Cet outil devenu pour la communauté scientifique une forme inévitable de recherche, de communication, de partage, d'évaluation, etc.... a été introduite dans la sphère de la certification comme gage d'ouverture et de probité et aussi d'égalité et de combat des dérives dont il est affublé. Les quatre exemples choisis (qui ne représentent qu'une partie infime des expériences entreprises par les différents périodiques scientifiques) l'ont étés pour de multiples raisons dont la possibilité de voir le processus en direct sur le Net était la plus importante. En effet, si ces formes de certifications sont expérimentées depuis l'avènement d'Internet, elles ne sont pas totalement accessibles et ouvertes pour toutes.

Ainsi The Journal of Interactive Media in Education, The British Medical Journal (BMJ)Rapid response, The Biology Direct, The Medical Journal of Australia, Naboj, The Annals of Reumathic Diseases, The Journal of Medical Ethics, The Emergency Medical Journal ,The Journal of Medical Internet Research ${ }^{*}$ et d'autres tous pratiquent cette forme de certification mais elle n'est pas aussi ouverte que dans les quatre exemples présentés. D'ailleurs trois des quatre exemples cités font l'objet d'articles dans le fameux " Open peer review trial " de Nature, le seul qui n'y figure pas est F 1000, lancé après 2006, date l'étude de Nature. Ces quatre exemples peuvent être, schématiquement, départagés en deux catégories quant aux résultats que ces expériences ont donnés.

Les deux premiers sont ACP et $\mathrm{F} 1000$ qui ont atteints des critères de professionnalisation et de sérieux méthodologiques certains. Ainsi ACP est reconnu par la communauté des géo scientifiques comme un site où le contrôle par les pairs profite de l'ouverture offerte par Internet et donne des recherches et des études de haute qualité scientifiques que le haut facteur

\footnotetext{
* Ce journal pratique une autre forme de contrôle par les pairs ouverte appelée " open peer review articles " (articles en expertise ouverte). Cette forme permet aux utilisateurs de Journal of Medical Internet Research (JMIR) de s'auto inscrire comme experts pour des articles spécifiques actuellement en expertise par JMIR (en plus des auteurs et éditeurs sélectionnés pour l'expertise traditionnelle).Pour ce faire, il existe sur le site une liste d'articles dont les auteurs n'ont pas refusé l'expérimentation de l'expertise ouverte et où l'éditeur n'a pas encore pris de décision ( Il est à noter que cette particularité concerne seulement des articles spécifiques. Pour s'inscrire, et attendre que l'éditeur assigne des articles correspondant avec les centres d'intérêt de l'expert, il est demandé d'utiliser le profil sur le site. Pour s'auto attribuer (c'est les mots utilisés sur les site) un article en tant qu'expert, l'expert doit avoir un compte sur les site, être connecté. L'expert peut alors ajouter son nom en cliquant sur le lien "+ Expertise moi ! + " se trouvant sous chaque article. Des instructions complètes sur la manière de procéder seront envoyées à l'adresse électronique peu de temps après. Enfin, il est à signaler que les articles publiés auront leur experts reconnus par nom alors que les articles non publiés ne le seront pas. La manière de procéder de " The Journal of Medical Internet Research " ressemble dans plusieurs points à celle de Philica notamment dans l'auto attribution de la fonction d'expert et sa propension à attirer les experts (il est dit expressément " les résumés sur cette page sont des études non publiées- prière de ne pas les citer - pour le moment - .Si vous souhaitez les citer /les voir publiés, rédigez votre opinion sous la forme d'une expertise ! ") Mais diffère dans plusieurs autres points tels que le conflit d'intérêt et l'inscription sous une fausse identité qui, d'après le site, serait sévèrement puni et exposerait son auteur à des sanctions au niveau de son établissement de rattachement. Encore une fois, ces nouvelles formes sont toujours au stade d'expérimentations et ne sont pas définitivement établies ouvrant la porte à des pratiques qui, des fois, ne sont ni acceptables ni usités que ce soit méthodologiquement ou alors dans le domaine de la recherche scientifique et technique. Informations disponibles à http://www.jmir.org/reviewer/openReview/abstracts (Accédé le 12 Août 2012)
} 
d'impact de la revue prouve. De plus, ACP couvre les sujets aussi sensibles et spécialisés que l'atmosphère de la terre et leur processus chimiques et physiques sous jacents ainsi que la turbo pause y compris la troposphère, stratosphère et la mésosphère. Sujets pointus et spécialisés ,s'il en est, qui font que les expertises ne peuvent être entreprises que par des spécialistes dont le niveau de connaissance, d'érudition et de scientificité est reconnus. De plus, ACP est indexé dans Science Citation Index, Science Citation Index Expanded (Web of Science), Scopus, ADS, Chemical Abstracts, Current Contents, GeoRef, Google Scholar et J-Gate. Il est aussi inclut dans the Directory of Open Access Journals (DOAJ) ainsi que dans The Bodleian Library (UK), Deutsche Digitale Bibliothek (D) et The Library of Congress (USA) .Il est enfin archivé électroniquement à long terme dans Portico. Cette longue liste de références bibliographiques atteste de la solidité du travail entrepris par les éditeurs, chercheurs d'ailleurs reconnus dans le domaine à l'instar d'Ulrich Pöschl.

De son coté, F 1000 pratique le contrôle par les pairs ouvert mais n'est pas à proprement parler un périodique électronique dans le sens où il est connu. Le site va en fait au-delà du fait d'être un périodique scientifique pratiquant le contrôle par les pairs électronique et innove en repérant les meilleurs recherches des domaines biologiques et médical et en les ré expertisant une deuxième fois par le choix qu'il en fait .Nous avons, peut être, utilisé un néologisme en disant reexpertisée, mais c'est ce que ce site a innové. Les recherches choisies ont déjà passé le filtre de leur périodiques respectifs et sont de nouveau choisis car les chercheurs et la Faculté des 1000 (F 1000) composant le site a jugé qu'ils étaient digne d'intérêt et les élisent de nouveau. On peut supputer et dire que F 1000 a élevé le contrôle par les pairs à un autre niveau en faisant expertiser des recherches déjà acceptées, faisant partie du corpus du domaine et les faisant passer une deuxième fois le filtre qualitatif du contrôle par les pairs. De plus, dans " perles cachées ", les membres de F 1000 repèrent des recherches qui auraient dû être mieux classées et connues et les mettent à la disposition de leur collègue.

ETAI quant lui ressemble dans certaines de ces fonctionnalités à ACP mais semble en fait victime de sa non continuité et de son interruption de publication. Avant ceci, les informations disponibles sur le site semble indiquer un processus de certification ouvert auquel les différents protagonistes participent et échangent opinions, critiques, additions aux recherches soumises. Le site donne l'impression d'une sorte d'arrêt et ne présente plus de nouvelles soumissions depuis 2006 malgré que le site comporte les anciennes soumissions et leurs différentes phases de discussion. Erik Sandewall, l'éditeur et principal responsable du site nous a indiqué dans une correspondance électronique personnelle en réponse à une question concernant le statut d'ETAI que ".....le site n'était actif pour le moment ".... et ce en date du $1^{\text {er }}$ Juin 2012. Il nous parait, en fait, qu' ETAI à l'instar de Psycholoquy pourtant lancé comme l'équivalent électronique de BBS ( Brain and N Behavioral Sciences ) par Harnad, n'a pas trouvé sa niche dans ces différentes expérimentations soit pour des raisons financières ( l'exemple de Psycholoquy ) ou alors pour des raisons simplement de management ou alors plus plausiblement de réactions des chercheurs qui ont, d'une certaine manière, peur de l'inconnu que ces nouvelles formes présentent. D'ailleurs, le dossier de Nature fait foi quant à cette assertion et même des éditeurs aussi ouverts à l'innovation ont décidés de ne pas 
appliquer le contrôle ouvert sur Internet et d'investiguer le sujet plus en profondeur dans le temps avant d'appliquer cette nouvelle et innovante forme de certification.

Le dernier site examiné est Philica qui présente, du moins pour nous, un cas assez flagrant de sites crées par des scientifiques et qui ne présentent nullement les conditions les plus élémentaires de scientificité, de contrôle, de hiérarchisation et de sérieux dans la publication. De prime abord, le site se présente d'une manière tout ce qu'il y a de plus commerciale en affichant " où les idées sont libres ", qui voudrait dire (free pouvant dire soit " libre " ou alors " gratuit " en anglais selon le contexte) que le site applique la liberté de publier pour toute personne le voulant. En d'autres termes, Philica permet aux nombreux chercheurs privés de publication par la faute d'un contrôle par les pairs inique, d'avoir l'occasion de présenter leur travail au monde. Cette manière de procéder, louable à plus d'un titre et qui serait plus que bienvenue dans un monde - comme il a été déjà démontré - où l'équité, l'égalité des chances, l'objectivité et même l'honnêteté ont étés mis à mal, se heurte cependant à des manquements d'ordre surtout méthodologiques flagrants. Parmi ces failles, le contrôle par les pairs, pierre angulaire de la publication scientifique et de la Science en général, est tout simplement évacué. En son lieu et place, une sorte d'absence totale de contrôle est instaurée et même porté au pinacle comme la panacée. Le site publie instantanément tout ce qui lui est présenté et laisse le soin à la communauté de juger si la soumission est digne ou non de publication. Cette manière serait acceptable (elle se pratique ailleurs comme pour ArXiv par exemple) et obéirait à des critères scientifiques si la communauté était définie et surtout connue. A la place, toute personne avec une connexion Internet peut devenir expert et juger les soumissions. Comme précédemment expliqué, ce genre d'expertises existe (et est même le sujet de ce travail) mais sous certaines conditions. La plus importante - et en même temps logique - est que l'expert soit expert en d'autres termes qu'il (ou elle) ait fait ses preuves, prouvé ses capacités, et enfin être accepté par ses pairs sur la base de ses publications et ses découvertes. La Science est ainsi faite : elle obéit à des considérations méritocratiques et on avance et on gravit les échelons grâce au travail présenté (que ce soit article, monographie, enquête, questionnaire, encadrement, projet de recherche, etc.....). Ces gages de sérieux sont jugés et, dépendant de leur viabilité, sérieux et faisabilité, ils font la carte de visite du chercheur. Tous ces critères (à quelques nuances près) ne sont nullement présents sur Philica car la " démocratisation " est telle que toute personne qui le désire est expert. Plus, si une personne veut devenir un membre avec un statut de chercheur confirmé, elle doit envoyer des renseignements très basiques (nom, prénom, nom d'utilisateur, adresse électronique, affiliation professionnelle, poste occupé, etc....) et elle obtient le statut. Statut qui dans le système Philica permet au membre d'être plus considéré et dont les expertises ont plus de poids. De plus les personnes qui font des donations, seront affublées d'une sorte d'icône de donateur à coté de leur nom afin de signaler qu'ils ont contribués à aider la dissémination du Savoir. Rien dans ce système n'est méritocratique et il existe même des chercheurs dont le site doute de l'affiliation. Tous ces défauts font que Philica ne peut prétendre au titre de site promouvant la science au même titre que les autres sites qui observent un minimum de probité intellectuelle et font appel à des véritables experts pour donner leur avis et ne se basent pas sur des expertises dont la teneur et le sérieux sont sujet à controverses. Le résultat sont des 
articles dont les titres prêteraient à rire n'était ce le supposé sérieux de l'entreprise. Nous avons signalé les différents titres et soumissions les unes plus saugrenues que les autres mais nous ne pouvons omettre de signaler la dernière en date dont le sujet est True Blood-The Vampire Mysteries par une certaine Ms ahana baby [sic] et dont l'établissement d'affiliation est " kykjkopklko, CEMA Instituto Universitario, Argentina ". De plus, cette personne est d'affiliation douteuse et ceci est reconnu par Philica qui lui accole un ? voulant dire qu'ils ne sont pas sur de l'affiliation (kykjkopklko ne nous semble pas de prime abord un nom d'institut ou de département de recherche). Toutes ces spécificités, ne militent nullement en faveur de la prise en compte sérieusement de ce site qui semble beaucoup plus agir pour des raisons soit de vanité personnelle ou peut être financières.

Enfin, nous avons dans cette partie du travail décidé de faire une enquête par questionnaire sur les habitudes et les positions des chercheurs algériens face à ces nouvelles manières de certifier l'information. Le chercheur algérien est de toute évidence, partie prenante dans le concert mondial de la recherche et ce questionnaire avait pour but de donner un tant soit peu un ancrage local à ce travail. Pour ce faire, nous avons choisi deux centres de recherches (le CERIST et le CDTA ) pour différentes raisons dont la plus importante est la disponibilité de ces deux centres à nous aider (quoique ce critère s'est avéré inopérant face aux blocages et retournements de situations que nous dûmes endurer de la part du CDTA), la réputation des deux Centres comme étant des Centres de recherche activant dans le domaine de l'information scientifique et technique (surtout le CERIST). Nous avons tenu en ligne de compte aussi le fait que ces deux Centres étaient ( et sont toujours ) les seuls dont les périodiques scientifiques font partie d'A.J.O.L. (African Journal On Line ).Nous avons structuré ce questionnaire de telle sorte à connaître en premier lieu le degré de connaissance de ces chercheurs des nouvelles formes de contrôle par les pairs et ensuite leur expérience en tant que chercheur publiant des articles scientifiques pour leur promotion ou dans le cadre de leur travail au sein de ces centres de recherche. Bien que cela a signalé dans la conclusion au sujet du questionnaire, il est important et utile de le répéter et de signaler certains dysfonctionnements qui entravent les efforts des chercheurs algériens (du moins ceux investigués) comme la faiblesse relative de publication, le manque de connaissance de certains sites, actions, appels et concepts qui gouvernent la publication scientifique moderne en réseau. Sinon comment expliquer le chercheur qui à la question de connaissance d'archive ouverte répondit " les archives de la période coloniale en 1830 ".D'un autre côté, il est signalé une quasi ignorance (et par delà une quasi absence de signature) des différents appels qui ont fait et font le libre accès. L'occasion nous a été donnée de constater que la signature et la participation dans ces appels pouvaient ouvrir des horizons pour le chercheur en plus du fait qu'en tant chercheur, on doit défendre les idées que la communauté porte et promeut. Nous avons eu l'occasion de constater que nos institutions à titre de collectivité et nos chercheurs à titre individuel ne participent et ne profitent pas (dans le sens noble du terme) de certains programmes qui ont pour but d'aider à l'accès à l'information. Cette ignorance a pour résultat une perte d'opportunité de participer et de contribuer au progrès et aux avancées de la spécialité et d'avoir un sens d'appartenance. Toutes ces caractéristiques sont malheureusement peu usitées par nos chercheurs tels que les réponses l'ont démontré. D'un autre coté, il 
semblerait que la publication et la recherche prennent de plus en plus d'importance pour nos chercheurs qui y voient une possibilité de participer au progrès de la Science et aussi une opportunité de promotion scientifique et matérielle d'autant plus que les réponses ont agréablement indiqué une propension des chercheurs à utiliser la langue anglaise en tant que vecteur de recherche et de publication. Cette donne indique une prise de conscience des chercheurs quant à l'importance de s'insérer dans le concert mondiale de la recherche et, il faut le reconnaître, la langue anglaise est dans ce cas primordiale. Enfin, malgré une ignorance quasi totale des appels et de certains concepts, les chercheurs des deux centres semblent être au diapason des nouvelles formes de certification qui font partie de la vie du chercheur d'aujourd'hui et ont intériorisés ces nouvelles donnes. Cette partie du travail est importante à plus d'un titre car le monde du libre accès (et de la science en général) est occidental à ne pas en douter ce qui nous impose en tant que chercheur de pays en développement à investiguer et essayer d'être au diapason. Ceci est primordial car cette énième révolution a cette particularité : elle se passe sous nos yeux et à une vitesse telle que les retards et autres omissions seront très difficiles à rattraper. Nous nous devons d'être des leaders et non pas des attentistes car cette dernière position est synonyme de dépendance et d'auto satisfaction, situation qui ne pardonne pas en ce $21^{\mathrm{e}}$ siècle à la vitesse de développement décuplée. 
Bibliographie

Bailar, John C. III, and Patterson, Kay, Journal peer review: the need for a research agenda, New England Journal of Medecine, Vol. 312, p.654-657, 7 Mars 1985

Bauer, Henry H., Beyond Velikovsky: the history of a public controversy: University of Illinois Press, Urbana [Ill., USA] 1999, xiii - 354 p.

Björk, B-C., Roos, A. \& Lauri, M., Scientific journal publishing: yearly volume and open access availability, Information Research,, Vol. 14, n²1, paper 391

Blank,Harvey., The Summary move forward, Archives of Dermatology , Vol.87, n 4, p.413414.

Bourne , Charles P., The World 's technical journal litterature : an estimate of volume , origin , language, field, indexing and abstracting, American Documentation, Vol.13,n2, 159168, Avril 1962

British Academy (The), Peer review: the challenges for the humanities and social science: a British Academy report, The British Academy, London, 2007

Disponible à : $\underline{\text { http://www.britac.ac.uk/policy/peer-review.cfm }}$

Broad, William, Wade, Nicholas, La Souris truquée : enquête sur la fraude scientifique Paris, Ed. du Seuil,1987,288p.

Burnham, J.C., The Evolution of editorial peer review, Journal of the American Medical Association, Vol. 263, n 10 , p. 1323-1329, 9 Mars 1990

Campanario, Juan Miguel, Have referees rejected some of the most-cited articles of all times? Journal of the American Society of information Science, Vol. 47 , nº4 , p.302-310, Avril 1996 (Résumé disponible à http://onlinelibrary.wiley.com/doi/10.1002/(SICI)10974571(199604)47:4\%3C302::AID-ASI6\%3E3.0.CO;2-0/abstract (Accédé le 31 Juillet 2012)

Campanario, Juan Miguel, Acedo, Erika, Rejecting highly cited papers: the views of scientists who encounter resistance to their discoveries from other scientists, Journal of the American Society of information Science, Vol. 58 , n5 , p.734.-743, Mars 2007

(Résumé disponible à http://onlinelibrary.wiley.com/doi/10.1002/asi.20556/abstract (Accédé le 31 Juillet 2012)

Coping with peer rejection (Editorial), Nature, Vol.425, n6959, p.645, 16 Octobre 2003 (Disponible à http://www2.uah.es/jmc/peer\%20rejection.pdf ) (Accédé le 2 Août 2012)

Corredoira, Martín López \& Perelman, Carlos Castro Ed., Against the tide: a critical review by scientists of how physics and astronomy get done, Boca Raton [FL, U.S.A.], Universal Publishers, 2008, 256 p.

Corredoira, Martín López \& Perelman, Carlos Castro Ed., Against the tide: a critical review by scientists of how physics and astronomy get done, Boca Raton [Florida,], Universal 
Publishers, 2008, 256 p.(Résumé disponible à http://onlinelibrary.wiley.com/doi/10.1002/asi.20556/abstract (Accédé le 31 Juillet 2012)

Dahdouh-Guebas, Farid [et al], Neo-colonial science by the most industrialized upon the least developed countries in peer-reviewed publishing, Scientometrics, Vol.56, $\mathrm{n}^{\circ} 3$, p.340-341, 2003

De Grazia, Alfred,,Ed, The Velikovsky Affair: The warfare of science and scientism, University books, New York, 260p.

De Solla-Price, Derek, Little science, big science, Columbia University Press, New York, 1963, XVI-119 p.

Dolby, R. G. A., What Can We Usefully Learn from the Velikovsky Affair ? $\underline{\text { Social Studies of }}$ Science, Vol. 5, No. 2, May 1975, p. 165-175

Drummond, Rennie, Editorial peer review:its development and rationale, $\underline{I n}$ :Peer review in health sciences, Goodlee, Fiona and Tom Jefferson Ed., BMJ Books,London, 2003, VIII$392 p$.

Ellenberger, Leroy, A lesson from Velikovsky, Skeptical Inquirer, , Vol. X, n²4, Summer 1986, p. 380-381.

Fabiato, Alexandre, Anonymity of reviewers, Cardiovascular Research, Vol.28, n8, 1994, p.1134-1139

Fish, Stanley, No bias, no merit :the case: the case against blind submission, Publications of the Modern Language Association, Vol.103, n5, p. 739-748, October 1988

Fish, Stanley, Doing what comes naturally: change, rhetoric and the practice of theory in literature and legal studies,[Durham, N.C.]: Duke University Press, 1989, 624 p.

Godlee, F, [et al], Effect on the quality of peer review of blinding reviewers and asking them to sign their reports: a randomized controlled trial, Journal of the American Medical Association, 1998, Vol.280,p.37-40

Godlee F, The Ethics of peer review In Hudson Jones A., McLellan F. Ed., Ethical Issues in Biomedical Publication, Johns Hopkins University Press, Baltimore, 2000,400 p.

Goldsmith, Donald W. Ed, Scientists Confront Velikovsky, W. W. Norton, New York, 1977, $188 \mathrm{p}$

Guédon, Jean-Claude, Open Access Archives : from scientific ploutocraty to the republic of science, IFLA Journal, Vol 29, n²,p.129-140

Hall, J., Author review of reviewers, American Psychologist, Vol .34, 1979, p.798

Harnad, Stevan, Implementing Peer Review on the Net: scientific Quality Control in scholarly electronic journals, In: Peek, R., Newby, G, Ed, Scholarly publication: the electronic frontier, Cambridge [Ma, U.S.A.]: MIT Press. p. 103-108. 
Harnad, Stevan, The Invisible hand of peer review. Disponible à http://users.ecs.soton.ac.uk/harnad/nature2.html (Accédé le 27 Juillet 2012)

Harnad, Stevan Post-Gutenberg Galaxy: The Fourth Revolution in the Means of Production of Knowledge, Public-Access Computer Systems Review, Vol.2, n¹: p.39 - 53, 1991

Harnad, Stevan Scholarly Skywriting and the Prepublication Continuum of Scientific Inquiry, Psychological Science, Vol. 1, p.342 - 343, 1990

International Committee of Medical Journal Editors, Statements on Electronic Publication and on Peer-Reviewed Journals, Annals of internal medecine, Vol. 116, no. 12, p.1030

Katzen, M.F., The Changing appearance of research journal in science and technology: an analysis and case study, In: Meadows, A.J. Ed., Development of science publishing in Europe, Amsterdam, Elsevier, IX -269 p.

Kronick, David A., Litterature of the life sciences : historical background, Bulletin of the New York Academy of Medicine, Vol 60, n9, p.869-870,9 Novembre 1984

Kronick D.A., Peer review in 18th-century scientific journalism, Journal of the American Medical Association, Vol. 263, $\mathrm{n}^{\circ}$,p. 1321-1322, 1990

Lewis, E.P. ,A Peerless publication, Nursing outlook,Vol.28, n²,p.225-226, Avril 1980

Lock, S., Quality assurance in medical publication, Proceedings of The Royal Society of Edinburgh, Section b-biological sciences, 101, p.359-375, 1993.

Mac Nutt R.A., [et al], The Effects of blinding on the quality of peer review, Journal of the American Medical Association, 1990, Vol.263,p.1371-1376.

Manske, P.R., A Review of peer review, Journal of hand surgery, Vol. 22 A ,n5, p 768

Meadows, A.J., Communicating research, Academic Press, London, 1998, 280 p.

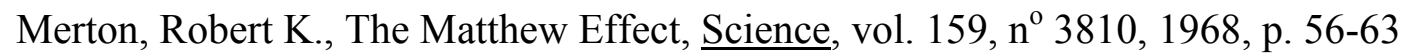

Morrow Brown, Harry, Peer review should not be anonymous, British Médical Journal, Vol.32,. 12 April 2003, p.824.

Pearlman, Dale Ann, Ed., Stephen J. Gould and Immanuel Velikovsky: essays in the continuing Velikovsky Affair, Ivy Press, Surrey [England], 1996, 832 p.

Peters, Douglas P. and Ceci, Stephen C., Peer review practice of psychological journals: the fate of published articles, submitted again, Behavioural and Brain Science, Vol. 5, n²,1982, p.187-195

Repository Interface for Overlaid Journal Archives (RIOJA).Project definition. Disponible à http://www.ucl.ac.uk/ls/rioja/. accédé le 12 Juin 2012 
Richard T.De George, Fred Woodward, Ethics and manuscript reviewing, Journal of scholarly publishing, Vol.25, n³, 1994, p.133-145

Schaffner, A.C., The Future of scientific journals: lessons from the past, Information Technology Libraries, Vol.13, n²4, p.239-248, 4 Dec.1994

Schmelkin, L. P., Peer review: Standard or delusion? Presidential Address delivered at The annual meeting of the American Psychological Association, Division 5, Toronto, Canada (August 2003).

Shatz, David, Peer review: a critical inquiry, Rowman \&Littlefield, Lanham [Md., USA], 2004, 264 p.

Shilling, C.W., Requirement for a scientific mission - oriented information center, American Documentation, Vol.14, n²1, p.49-53, Janvier 1963

Skoblow, Jeffey Fish on blind submission, Publications of the Modern Language Association, Vol.104, n² p.215-216, 1989

Sojka, R.E. , Mayland, H.F., Driving science with one eye on the peer review mirror, Forum proceedings, "Ethics, Values, and the Promise of Science", San Francisco, 25-26-02-1993, p.202 (Disponible à http://eprints.nwisrl.ars.usda.gov/1062/1/816.pdf )

Spier, Ray, The History of the Peer-Review Process in Science, Trends in biotechnology, Vol.20, n॰8, p.357-358, 8 August 2002

Suber, Peter, Guide to the open access movement, 2003 Guide to the Open Access Movement, 2003. Disponible à http://www.earlham.edu/ peters/fos/guide.htm, accédée 12 Juin 2012

Swanson, E.A., McCloskey, J., The Manuscript review process of nursing journals, Image, Vol.14, n³, p.72-76, Octobre 1982

Tenopir, Carol and King, Donald W., Towards electronic journals: realities for scientists, librarians and publishers, Special Libraries Association, Washington, DC, 2000

Tracey, Brown Ed.,Science Peer review and the acceptance of new scientific ideas :Discussion paper from a Working Party on equipping the public with an understanding of peer review, London, Sense about science, 2004, 61p.

Disponible à : $\underline{\text { http://www.senseaboutscience.org/data/files/resources/17/peerReview.pdf }}$

Van Rooyen S, [et al], The effect of blinding and masking on the quality of peer review: a randomized trial, Journal of the American Medical Association, 1998, Vol.280, p.234-237 
Van Rooyen, Susan [et al], Effect of open peer review on quality of reviews and on reviewers' recommendations: a randomised trial, British Médical Journal, Vol. 318, 2 January 1999, p.23-27.

Weinberg, Alvin M., Science, Government and information: the responsabilities of the technical community and the government in the transfer of information, President's Science Advisory Committee, Washington , D.C., 55p., 1963 (Accessible à : http://eric.ed.gov/PDFS/ED048894.pdf)

Weller, Ann C., Editorial peer review : its strengths and weaknesses, Information Today, Medford [N.J., USA], 2002, 344 p.

Ziman , John M. , Public knowledge : the social dimension of science, Cambridge [Ma., U.S.A.]: Cambridge University Press,1968, 168 p.

Zuckerman, Harriet, Merton, Robert K., Patterns of evaluation in science: institutionnalisation, structure and functions of the referee system, Minerva, Vol.9, $\mathrm{n}^{\circ} 1, \mathrm{p} .76$, Janvier 1971

\section{Webographie}

http://openaccess.inist.fr/spip.php?mot28

$\underline{\text { http://www.minerva-ebm.be/articles/FR/woordenlijst_fr/regression_vers moyenne.htm }}$ http://homepages.vub.ac.be/ clvidal/writings/Vidal_2005a_Le_commentaire_ouvert.pdf) http://journals.cambridge.org/action/displayJournal?jid=BBS

http://richardfmasters.wordpress.com/2011/06/02/the-humble-beginnings-of-peer-reviewalternatives-stevan-hanard-and-open-peer-commentary/

http://richardfmasters.wordpress.com/2011/06/02/the-humble-beginnings-of-peer-reviewalternatives-stevan-hanard-and-open-peer-commentary/

http://www.cogsci.ecs.soton.ac.uk/cgi/psyc/newpsy

http://journals.cambridge.org/action/displayJournal?jid=BBS

www.atmospheric-chemistry-and-physics.net/

http://www.atmospheric-chemistry-andphysics.net/review/review_process_and_interactive_public_discussion.html\#interactivepublic discussion

$\underline{\mathrm{http}: / / \mathrm{f} 1000 . \mathrm{com} /}$

http://f1000.com/tour

http://f1000.com/rankings/journals/year/current

http://f1000.com/rankings/journals/year/2010

http://f1000.com/rankings/journals/year/2009

http://f1000.com/about/whatis/posters

http://f1000.com/posters/journalresponses 
$\underline{\text { http://f1000.com/posters }}$

http://f1000.com/my/searches/manage

$\underline{\text { http://bbf.enssib.fr/consulter/bbf-2010-02-0088-009 }}$

$\underline{\text { http://blog.f1000.com/ }}$

http://www.ida.liu.se/ext/etai/

$\underline{\text { http://www.philica.com/ }}$

http://www.philica.com/tutorial.php

http://www.philica.com/noreviews.php (Consulté le 16 Juillet 2012)

http://www.philica.com/display_article.php?article id=20 (Consulté le 16 Juillet 2012)

http://www.philica.com/display_article.php?article_id=62 (Consulté le 16 Juillet 2012)

http://www.philica.com/display article.php?article id=29 (Consulté le 16 Juillet 2012)

http://www.philica.com/display_observation.php?observation_id=34 (Consulté le 16 Juillet 2012)

http://www.philica.com/display observation.php?observation id=36 (Consulté le 16 Juillet 2012)

http://www.philica.com/display article.php?article id=28 (Consulté le 16 Juillet 2012)

http://www.philica.com/display_article.php?article id=78 (Consulté le 16 Juillet 2012)

http://www.philica.com/display observation.php?observation $\mathrm{id}=53$ (Consulté le 17 Juillet 2012)

http://www.philica.com/display observation.php?observation $\mathrm{id}=57$ (Consulté le 17 Juillet 2012)

http://www.philica.com/display_observation.php?observation_id=64 (Consulté le 17 Juillet 2012)

http://www.philica.com/display_observation.php?observation_id=65 (Consulté le 17 Juillet 2012

http://www.philica.com/display observation.php?observation id=74 (Consulté le 17 Juillet 2012)

http://www.philica.com/display_observation.php?observation_id=66 (Consulté le 17 Juillet 2012)

http://www.philica.com/top10pop.php (Consulté le 18 Juillet 2012)

http://www.philica.com/display_article.php?article id=65 (Consulté le 18 Juillet 2012)

http://www.philica.com/top10rate.php (Consulté le 21 Juillet 2012)

http://www.philica.com/display_article.php?article_id=24 (Consulté le 22 Juillet 2012)

http://www.philica.com/display_article.php?article_id=14 (Consulté le 22 Juillet 2012)

http://www.philica.com/display_article.php?article_id=161 (Consulté le 22 Juillet 2012)

http://www.philica.com/display_article.php?article_id=110 (Consulté le 22 Juillet 2012)

http://www.philica.com/display_article.php?article_id=24 (Consulté le 22 Juillet 2012) 
http://www.philica.com/display observation.php?observation_id=10 (Consulté le 22 Juillet 2012)

http://www.philica.com/recentreviews.php (Consulté le 22 Juillet 2012)

http://www.biology-direct.com/authors/peerreview

http://www.bmj.com/comment/rapid-responses

http://homepages.vub.ac.be/ clvidal/writings/Vidal_2005a_Le commentaire_ouvert.pdf,

http://www-jime.open.ac.uk/

http://homepages.vub.ac.be/ clvidal/writings/Vidal_2005a_Le commentaire ouvert.pdf,

http://www.cdta.dz/communicationscdta_periode.asp

http://www.cdta.dz/publications cdta_periode .asp

http://www.openarchives.org/

http://InformationR.net/ir/14-1/paper391.html] (Consulté le 25 Juillet 2012)

http://duncan.hull.name/2010/07/15/fifty-million/ (Consulté le 25 Juillet 2012)

http://fr.wikipedia.org/wiki/Quis_custodiet_ipsos_custodes $\% 3 \mathrm{~F}$

http://www.archivefreedom.org/freedom/Cyberia.html (Consulté le 29 Juillet 2012)

http://www.universal-publishers.com/book.php?method=ISBN\&book=1599429934 (Consulté le 29 Juillet 2012)

http://www.jmir.org/reviewer/openReview/abstracts (Consulté le 12 Août 2012) 


\section{Conclusion générale}

Au terme de ce travail dans lequel nous avons essayé de répondre à la question du devenir d'une étape incontournable de la science publiée, le contrôle par les pairs, il convient de s'interroger sur les conclusions que nous pouvons tirer en réponse à la problématique de notre étude. Nous avons voulu, de par cette recherche, contribuer à éclairer le futur du contrôle par les pairs dans la science aujourd'hui : le libre accès. Il s'agit donc d'une étude historique et évolutive à l'aune d'un autre phénomène (révolution même) qui à l'orée de ce $21^{\mathrm{e}}$ siècle a bouleversé notre vie en général mais surtout celle des chercheurs et des savants : Internet. En effet, parler de libre accès implique automatiquement parler d'Internet .Plus même, nous oserons cette expression : le libre accès est Internet.

Ce travail est sujet d'actualité car le libre accès date, au plus, que des deux dernières décennies et l'avènement d'Internet grand public. Si au cours de cette courte période (et avant), nombre de recherches, d'articles et d'études furent entrepris sur le sujet du contrôle par les pairs et ses différents problèmes, il est indéniable que le sujet en question à l'ère d'Internet n'a pas été abordé d'une manière exhaustive et inclusive. De plus, ce sujet au niveau national est carrément occulté et même ignoré comme les résultats du questionnaire l'ont démontré. Notre étude descriptive et analytique de l'état de l'art de la question a été scindée en trois axes principaux et qui sont :

1-Une relation aussi précise que possible de l'évolution de la communication scientifique en faisant ressortir la sophistication sans cesse grandissante auquel est parvenu l'être humain en passant de la parole à l'écriture et l'alphabet qui a culminé avec l'invention de l'imprimerie. L'impact de cette révolution a eu une portée telle que même de nos jours (alors qu'Harnad parle de la société post gutenberguienne) le sujet est encore étudié. De même, le papier a conditionné le progrès de l'imprimerie en lui donnant un support pratique comparativement à certains matériaux très hétéroclites utilisés alors. Ces accélérations (imprimerie et papier) ayant induit une forte demande d'information et une certaine "démocratisation" du savoir, le livre qui jusqu'alors était le seul medium fut supplanté par un medium plus rapide et plus en accord avec la relative rapidité de la transmission de l'information. Le périodique scientifique a permis, surtout dans une l'Europe sortant d'un long Moyen Age, d'accélérer la cadence et la rapidité des informations scientifiques. Ce medium aussi révolutionnaire que l'imprimerie, est toujours avec nous sur support papier mais aussi et surtout de plus en plus sur support électronique. Cette ambivalence, des fois dichotomique, n'en a pas moins fait du périodique scientifique le medium préféré des scientifiques de par sa rapidité et, à la lumière des développements électroniques et l'omniprésence des réseaux, sa disponibilité aussi bien matérielle que temporelle.

2-Une historiographie du libre accès qui reste le mouvement sous tendant le soubassement de notre travail étant donné que nous investiguons sa relation au contrôle par les pairs. S'il est récent quant à son inception, le libre accès a accompli en quelques deux décennies qu'a duré sa vie des progrès, des réalisations tels que le monde de la publication scientifique et technique en est irrémédiablement et profondément changé. Les premiers pas balbutiants de l'informatique datant du lendemain de la deuxième guerre mondiale, les progrès accomplis sont allés depuis crescendo et surtout exponentiellement induisant une sorte de fuite en avant quant à la quantité d'information produite et disponible. L'avènement d'Internet a de son coté accéléré le tempo au point qu'il est devenu impossible de savoir la quantité de données produites et disponible mais a aussi créé les outils pour gérer cette masse formidable de données. 
3-Un état des lieux d'une étape primordiale et incontournable de la science publiée : le contrôle par les pairs; étape importante mais inégalement et tardivement appliquée. L'application tardive du contrôle par les pairs et qui se situe au lendemain de la deuxième guerre mondiale résulte selon toute vraisemblance (et à coté d'autres raisons moins primordiales) à l'explosion documentaire extraordinaire qui a suivi les efforts de recherche post 1945. Cette application a, de par les enjeux qu'elle renferme et les avantages qu'elle procure, toujours posé des problèmes dans le monde de la publication scientifique .Elle a été accusée de maux diverses dont les plus prégnants sont le conservatisme, le secret du processus, le cout, la lenteur, les passe droits, l'arrogance des experts, son inefficacité à détecter les fraudes, etc..... Ces maux maintes fois signalés et étudiés n'ont pas trouvés de solutions à cause de raisons multiples (nouveauté du processus, son secret, sa quintessence purement humaine sujet à des manipulations, l'absence d'alternatives, etc....) et le monde de la publication scientifique a du prendre son mal en patience. L'avènement d'Internet a présenté une solution alternative de par son ouverture, sa rapidité et son coté démocratique. Cette option, bien que très prometteuse, est trop récente pour pouvoir porter un jugement .La seule vérité est que le contrôle par les pairs se fera sur Internet et dont la question nodale et primordiale est : comment? C'est sur la base de cette question quintessentielle que s'est basée la problématique de notre travail

Parler du contrôle par les pairs c'est parler de la Science. L'expression " Il n'est de science que publiée " est à ce titre symptomatique de l'étroite relation entre la publication certifiée, expertisée, labélisée et la science en construction qui progresse et avance par incrémentations. Le contrôle par les pairs, accepté et devenu une étape incontournable dans le monde scientifique, n'en véhicule pas moins une réputation d'iniquité et d'arbitraire à la base de tous les griefs qui lui sont (à tors ou à raison) imputés. Il est loin de répondre, dans un monde très compétitif et surtout lucratif, aux besoins d'une communauté scientifique très attentive aux enjeux de la recherche scientifique et surtout bien loin des clichés des philosophes et amateurs des $17-18^{\mathrm{e}}$ siècles. La science est devenue (de par son étroite relation avec le monde de l'industrie) une affaire économique aux enjeux financiers et même politiques dont les ramifications dépassent le monde feutré et policé de la Science. Ce changement dans l'optique a induit un changement de la philosophie de la science et de ses paradigmes au centre desquels le contrôle par les pairs en occupe une partie prépondérante.

D'un processus appliqué tardivement en raison surtout d'une explosion documentaire ayant rendu l'exploitation des données scientifiques pratiquement impossible mais aussi et surtout en tant que filtre qualitatif de ce qui est publiable et pas (avec des gradations progressives), le contrôle par les pairs a connu des orientations et des utilisations peu recommandables l'ayant discrédité aux yeux de la communauté scientifique. D'un terrain où seuls des critères tels que le mérite, l'excellence, l'ingéniosité et l'effort devaient récompensés, il est devenu un terrain de luttes et d'antagonismes. Cette état de tension entre auteurs, experts et éditeurs (qui il faut le dire sont les mêmes personnes) résulte de la nature même du contrôle par les pairs et de sa propension au secret. En effet, si le circuit et le cheminement d'une soumission est relativement connu et balisé, il demeure empreint d'une culture de secret et de zones d'ombres une fois dans le bureau de l'éditeur. Sur quelles bases sont sélectionnés les experts? Pourquoi sont ils sélectionnés? Quelle est l'incidence de leur choix quant au devenir du manuscrit soumis ? Ont-ils des instructions comment expertiser ou alors, comme certaines études l'ont confirmé, ils apprennent sur le tas ? N'y a t il pas des conflits d'intérêt non déclarés? L'expert n'utiliserait-il pas sa position privilégiée pour retarder (ou même rejeter) une soumission concurrente ? Dans un monde hyperspécialisé où le réservoir d'experts est 
réduit, l'option de l'anonymat a-t-elle sa raison d'être ? La fameuse expertise en double aveugle est elle aussi efficace qu'elle a été décrite ? Autant de questions et d'autres qui font que le contrôle par les pairs a atteint un niveau conflictuel tel qu'il touchait aux fondements même de la science.

De part son importance, le contrôle par les pairs a, sous une forme ou une autre, toujours été présent avec l'être humain. Parler à une personne, communiquer avec l'autre, entreprendre une action sociale quelconque, enseigner, se présenter à un vote etc.... sont des actes où on soumet et on attend la réaction, l'approbation (ou désapprobation) de l'autre. Ce contrôle permanent et naturel est une forme de communication inhérente à l'activité quotidienne humaine. Quand cette activité devient scientifique, les critères la régissant sont tout autres et engagent l'avenir, le devenir même de l'Homme. La Science étant par définition méritocratique et même élitiste, les experts, les Gardiens du Temple (ou de la Science) comme ils ont étés si adéquatement décrits, ont une responsabilité et sont investis d'une mission quasi mystique. Leur fonction est non seulement technique et obéit à des critères de scientificité, de rigueur méthodologique et d'objectivité mais elle les dépasse et devient une fonction de construction de la Science. En d'autres termes, l'expert non seulement juge, choisit et accorde le quitus (on serait tenté de dire l'Imprimatur) mais aussi impulse et indique la direction que la Science prend. L'expert, par cette fonction, dépasse le simple acte de juger le mérite d'un travail et de choisir s'il est publié ou pas et forge, façonne et oriente le Savoir.

On peut voir de par cette responsabilité l'immense pouvoir qu'un expert peut avoir. Il est facile aussi de voir, l'être humain n'ayant jamais été parfait - bien loin s'en faut - les tentations que cette puissance pourrait avoir sont multiples. L'histoire de la science est une longue litanie de ce genre de comportement plus que trouble. A titre d'exemple, la découverte de l'évolution des espèces de Darwin au $19^{\mathrm{e}}$ siècle ou la théorie de la dérive des continents (ou des plaques tectoniques) d'Alfred Wegener au début du $20^{\mathrm{e}}$ siècle ou alors en 1903 le premier vol motorisé contrôlé d'un avion par les frères Wright ne furent pas reconnus à leur juste valeur que bien plus tard. Ces exemples démontrent une constante du contrôle par les pairs à travers les âges : le conservatisme. Cette constante, maintes reprises dénoncée a son origine dans la nature humaine. En effet, l'être humain tend d'une manière innée à ne pas accepter, intégrer ce qui est nouveau, révolutionnaire ou sortant de l'orthodoxie. Cette situation résulte de multiples raisons dont la plus plausible est que les experts faisant en principe partie de l'élite de la science et de leur spécialités, ils ont des intérêts et tendent à les défendre. Toutes théories, idées ou orientations nouvelles seraient contraires aux idées qu'ils défendent et les discréditeraient. Si cette idée de conservatisme est à ne pas en douter présente, la question qui vient à l'esprit est la suivante : ce conservatisme est il conscient ou alors inconscient? De la réponse à cette question découle une autre hypothèse qui taraude les études sur le contrôle par les pairs et qui touche même à l'ethos de la science. Le conservatisme est il une réponse à une idée de la construction de la science ou alors est une tentative délibérée d'étouffer dans l'œuf une nouvelle idée ? Nous ne pensons pas tenir la réponse mais il semblerait que cela soit un amalgame des deux. Campanario ${ }^{(1)}$ a analysé le phénomène et a conclut que ...." certaines découvertes importantes étaient précoces dans la mesure où elles ne correspondaient pas aux paradigmes existants ou alors leurs implications n'ont pas pu être reliés par une série de mesures logiques et simples à la connaissance scientifique existante de l'époque ". Il continue plus loin : " Dans d'autres cas, les nouvelles théories ou découvertes entrèrent en collision avec les paradigmes dominants de la science et

(1) Campanario, Juan Miguel, Rejecting and resisting Nobel class discoveries: accounts by Nobel laureates, Scientometrics, Vol. 81, n ${ }^{\circ}$ 2, p.549-565, 2009 
rencontrant du mépris même et une généreuse dose de scepticisme". Campanario reconnait donc dans son analyse le coté conservateur (frileux même) de la science qui ne s'attaque pas aux dogmes ou du moins ne les conteste pas. L'autre accusation, plus grave et ébréchant l'image idyllique d'une science dépourvue de toute pensées malsaines est que le blocage est la résultante d'une action délibérée et malsaine. Ainsi dans un cas devenu célèbre dans la communauté des physiciens (présenté en supra dans la troisième partie), un certain Carlos Castro Perelman et son co auteur Martín López Corredoira éditèrent un livre dans lequel ils critiquent - entre autres - le contrôle par les pairs qu'ils accusent de nombreux maux. Le livre est en fait un ensemble de contributions de scientifiques de renom (dont Campanario) qui dénoncent tous l'orthodoxie des institutions et des chercheurs confirmés qui de par leur position suppriment les idées non conformes aux canons de la spécialité. Dans sa contribution, (2) Castro dépeint une situation quasi kafkaïenne où il est (d'après lui) victime d'une véritable cabale et mis sur une liste noire par Paul Ginsparg (fondateur d'ArXiv).Il décrit avec force détails les difficultés qu'il commença à rencontrer à publier après qu'il fut mis sur une liste noire par Ginsparg en 1999. Les détails présentés par Castro trahissent des agissements très préjudiciables et, en même temps, étonnant dans un monde supposé être peuplé de chercheurs dont la première mission est de faire avancer la Science. Malheureusement, les chercheurs sont des êtres humains aussi avec tous leurs défauts et parmi lesquels la cupidité, l'envie et la mauvaise foi. On ne peut leur faire confiance quand il s'agit d'être en position de profiter des situations où ils sont en positions de force.

Cette situation de mainmise, de comportements iniques et contraires à l'éthique, de positions dominantes et d'agissements préjudiciables est le résultat d'un nombre de raisons largement documentées et étudiées par les chercheurs. Parmi celles là, on peut citer le secret entourant le processus et ce depuis son début .Secret qui est beaucoup plus appliqué du coté de l'expert et beaucoup moins que coté du soumissionnaire. On se retrouve donc dans une situation de quasi impunité de la part de l'expert qui peut agir à sa guise tout en étant couvert par le secret .Cette situation a fait décrire le contrôle part les pairs par Richard Smith, l'éditeur en chef du respecté B.M.J. (British Journal of Médecine) de la manière suivante ......." il parait injuste pour quelqu'un qui émet un jugement important sur le travail des autres de le faire en secret. Une cour avec un juge non dentifié nous fait immédiatement penser à un régime totalitaire et au monde de Franz Kafka.". Il poursuit en ces termes :.... " identifier l'expert lie privilège et responsabilité, rappelant à l'expert que le pouvoir implique la responsabilité : l'expert qui revêt l'habit du juge ne peut être arbitraire dans son jugement et doit être un critique constructif " ${ }^{(3)}$.Le nom du soumissionnaire de son coté est connu et est, de ce fait, ouvert à toutes les manipulations les plus subjectives (revanche sur un concurrent dans la spécialité, retardement de parution des résultats surtout quand ils sont en concurrence avec ceux de l'expert, utilisation illicite des données pour des buts personnels, etc...). Cette situation est le terreau qui a fait du contrôle par les pairs le terrain de conflits, luttes et de controverses qu'il est et dont les conséquences se font sentir dans les milieux de la recherche dans son ensemble. La situation est telle qu'elle ressemble à un accusé qui est jugé mais ne connait ni son juge, ni les accusations auxquelles il doit répondre. Cette situation a accéléré le passage à un système en principe beaucoup plus ouvert, plus juste et surtout moins enclin aux dépassements que le secret du processus encourageait. Cette " révolution " (et le vocable n'est pas trop fort) a été rendue possible par Internet. De par son ouverture, sa disponibilité et surtout sa propension à être continuellement amélioré et adapté aux différentes situations, Internet a constitué la

\footnotetext{
(2) Castro,Carlos,My struggle with Ginsparg (arxiv.org) and the road to cyberia : a scientibc-gulag in cyberspace (Disponible à http://www.archivefreedom.org/freedom/Cyberia.html )

(3) Smith, Richard, Opening up BMJ peer review : a beginning that should lead to complete transparency, British Medical Journal ,Vol 318,p.4 , 02 Janvier 1990
} 
réponse immédiate à ces attentes (quoique les expériences étant très récentes et partielles, elles ne peuvent décemment être considérées comme des exemples ou du moins des canevas sur lesquels les conclusions peuvent être tirées). De par leur courte histoire, les expériences tendant à ouvrir un processus qui a toujours été connu pour son excessive secret ou du moins sa tendance à évoluer dans un univers élitiste et sélectif, ne sont pas assez nombreuses, n'ont pas eus assez de recherches les contredisant mais, elles peuvent être considérées comme relativement réussies. Par exemple, l'étude Nature sur le contrôle par les pairs ouvert en 2006 a conclut entre autres que le magazine, malgré le retour d'écoute positif de la part des auteurs et des éditeurs, n'allait pas mettre en œuvre le processus. Cette décision de la part de Nature fait transparaître le malaise, la gêne de la part des auteurs et des éditeurs contactés quant à une mise en œuvre du processus. Les différents points de vue exposés dénotent une peur de ce qui est nouveau (peur somme toute normale et qui nous fait revenir aux théories de Thomas Kuhn sur les révolutions scientifiques et les changements de paradigme) malgré l'enthousiasme que cette expérience suscite chez les chercheurs. En effet, il a été remarqué une constante qui, paraîtrait, de prime abord bizarre et contradictoire mais qui bien examinée révélerait la juste nature des choses : les chercheurs, les éditeurs, les scientifiques - presque - unanimement réclament une refonte, un changement, un bouleversement du contrôle par les pairs car il les concerne tous et surtout de ses résultats dépendent le devenir de leurs carrières. Cette demande de changement est motivée par les résultats sujets à controverses que le contrôle par les pairs a toujours suscité. Malgré cela, et l'expérience de Nature en est la meilleure preuve, ces mêmes chercheurs ne semblent pas être à accepter cette ouverture. Ceci est du au simple fait que d'un processus empreint du secret le plus complet, le processus de contrôle par les pairs est passé à une ouverture totale et complète. Ceci a engendré des réactions de rejet normales qui ont trait à de multiples raisons. Il est loisible de penser que l'auteur qui soumet une recherche médiocre s'expose ainsi à la vindicte (et même le sarcasme) de ses collègues, situation qui ne se passerait pas dans le secret du contrôle par les pairs traditionnel. D'un autre coté, Smith dans son éditorial précédemment cité indique un problème que le contrôle par les pairs ouvert a déjà enfanté et il a trait à la peur des experts débutants quant à leur éventuelle critique des chercheurs confirmés et la peur de représailles de la part de ces derniers. Cette éventualité était absente dans le contrôle par les pairs fermé traditionnel mais se pose avec acuité dans le contrôle par les pairs ouvert. Ceci est encore aggravé par le fait que les recherches ont prouvés que les experts débutants (ne dépassant pas la quarantaine) donnent les meilleures expertises ${ }^{(4)}$. Leur peur des représailles (surtout pour ceux qui dépendent des subventions pour leur travail) peut avoir des conséquences inhibitrices très négatives sur le processus, déjà affecté par une pénurie d'experts de haut niveau. Cette donne est cependant, selon Smith, un petit prix à payer et l'emporte sur les éventuels problèmes cités qu'elle engendrerait.

L'avènement de l'outil informatique a de son coté représenté une grande avancée pour le monde de la publication et surtout le segment scientifique avec toutes les fonctionnalités qu'il représente. Les différentes taches précédemment manuelles devinrent graduellement automatisées et surtout plus rapide et précises dans leur traitement, dissémination et analyse. C'est l'époque des grandes bases de données bibliographiques qui rendent le travail du bibliothécaire plus facile et plus inclusif. A cet effet, les années 60 voient les ancêtres de nos bases de données et autres programmes actuels tels que CDS /ISIS, Medlars et autres MARC envahir les services des bibliothèques et autres centre de documentation. Ces systèmes et programmes ne sont que le prélude à ce qui est devenu une constante et une étape incontournable dans le monde de la publication scientifique : Internet. Il serait long, rébarbatif

\footnotetext{
(4) Goldbeck - Wood, S., What makes a good reviewer of manuscripts ?, British Medical Journal ,Vol. 316,p.86, 10 Janvier 1990
} 
et en même temps redondant d'essayer de faire ressortir la place qu'occupe Internet dans notre vie car la question que nous nous poserions serait: comment a-t-on pu faire de la recherche sans Internet? En d'autres termes, cet outil qui au début des années 90 n'était pas très connu (même aux Etats-Unis) et était réservé à une élite universitaire dans les années 70, est devenu une partie de la vie de tous les jours et en particulier de la vie de ceux qui font la recherche. L'histoire retiendra que le premier log in (connexion) eut lieu en 1969 quand la première tentative de connexion se fit entre l'Université of California at Los Angeles (UCLA) et le Stanford Research Institute (SRI).Depuis cette date, les progrès réalisés par Internet se passent de tout commentaire (on estime qu'en date du 30 Juin 2012, $34.3 \%$ de la population mondiale est connecté avec des régions comme l'Amérique du Nord, l'Océanie/Australie et l'Europe affichant des pourcentages de $78.6 \%, 67.6 \%$ et $63.2 \%$ respectivement. De plus la même source indique que la progression de la connectivité a augmenté de 566.4\% entre 2000 et $\left.2012^{(6)}\right)$.

Ces chiffres, ces données et les avantages qui vont avec ont induit une prise de conscience de la part des scientifiques et des chercheurs et aussi et surtout de la part de la communauté des bibliothécaires qui souffraient (et souffrent toujours) d'une augmentation inconsidérée et en même temps insoutenable des prix des périodiques et dont la résultante a été une vague de désabonnement aux titres les plus chers (et par delà les plus importants). Cette situation à la limite du ridicule (un chercheur d'une université donnée ne pouvait accéder à sa propre recherche si sa bibliothèque n'était pas abonnée à la revue dans laquelle il avait publié ou ne pouvait se permettre d'acquérir les droits sur la recherche) a poussé les chercheurs, les scientifiques et les responsables des bibliothèques à se réapproprier (ou du moins avoir plus de contrôle) sur le fruit de leur production intellectuelle et scientifique. Internet, qui a adéquatement épousé en terme de timing cette augmentation, a donné aux promoteurs du libre accès matière à pousser leur agenda et à demander des comptes aux grands éditeurs (tels que Elsevier, Wiley \& Sons, Blackwell, Springer Verlag, etc...).En effet, ces derniers à la faveur d'une politique de désengagement des gouvernements suivi d'une politique de privatisation tout azimuts dans les années 80 (surtout aux Etats-Unis et en Grande Bretagne) ont augmenté les prix des abonnements des périodiques dans des proportions démesurées. A titre d'exemple, Reed Elsevier, la plus grande compagnie éditrice de périodiques scientifiques au monde a quadruplé, entre 1984 et 1990, son revenu net et ceci en changeant du segment moins profitable, moins international et moins spécialisé des magazines grand public vers le segment international, plus spécialisé et surtout plus profitable de la publication scientifique pour lequel le marché ne tendait pas à être très affecté par les fluctuations de l'économie en général. Par exemple en 1989 seulement, Elsevier Science Publishers ajouta 30 autres titres à sa liste de périodiques, la plupart achetés plutôt que nouvellement lancés. A la suite de ces opérations et d'autres, les profits nets d'Elsevier atteignirent 500 millions de Florin hollandais en $1990^{(7)}$.D'ailleurs, ces chiffres et ces profits insolents et la situation de plus en plus désespérée des bibliothèques engendrèrent une guerre entre les protagonistes (les promoteurs $\mathrm{du}$ libre accès et les grands groupes éditoriaux internationaux). Pour situer un peu le ton guerrier de cette contribution (Poynder utilise au début de son article les mots suivants "Alors que la bataille pour le libre accès à la littérature scientifique s'est intensifiée, des différents fronts de conflit se sont ouverts "), il faut savoir que 2007 est une année où nombre de propositions et de lois étaient encore à l'étude mais que les promoteurs du libre accès avaient déjà fait de grands progrès quant à l'acceptation de leurs idées. On se retrouve donc à une jonction de deux événements qui de prime abord sont contradictoires mais qui en fait vont permettre une plus grande accessibilité aux ressources de la recherche scientifique. Des

\footnotetext{
${ }^{(6)} \mathrm{http} / / / \mathrm{www}$. internetworldstats.com/stats.htm (Accédé le 13/12/2012)

(7) http://www.company-histories.com/Reed-Elsevier-plc-Company-History.html (Accédé le 13/12/2012)
} 
chercheurs (à l'image de Stevan Harnad, Peter Suber, Herbert Von Sompel, Harold Varmus etc....), forts de leur conviction et militant pour une plus juste répartition des résultats des recherches financées par les gouvernements ainsi qu'un accès libre à ces mêmes recherches vont entreprendre un ensemble d'actions toutes dirigées vers l'acceptation, la reconnaissance et l'institutionnalisation du libre accès. Ces actions sont multiples et très nombreuses mais on peut citer parmi celles qui en sont les références :

-La Lettre ouverte de Public Library of Science (PLoS) en 2003 et dans laquelle des chercheurs prennent position pour "la création d'une bibliothèque publique en ligne qui fournirait le contenu intégral des résultats publiés de la recherche et des textes scientifiques dans le domaine de la médecine et des sciences du vivant sous une forme en libre accès ".

-Les déclarations de Budapest, Béthesda et Berlin, (connues sous le vocable des B) représentatives des évolutions du libre accès.

*L'Initiative de Budapest pour l'Accès Ouvert (BOAI) recommande deux voies pour atteindre le libre accès, l'auto-archivage et les revues alternatives.

*La Déclaration de Béthesda donne une définition de la publication en libre accès mais aussi des rôles des différents acteurs œuvrant dans la communication scientifique.

*La Déclaration de Berlin, signée par des institutions, élargit la notion du libre accès à tous les biens culturels et à toutes les données issues d'un travail de recherche

Malgré toutes ces actions et les résultats obtenus par les chercheurs qui ont pu, par exemple, imposer le droit de mettre dans les archives ouvertes ce qu'ils publient dans les périodiques électroniques en libre accès (alors que ceci était difficile à obtenir lors du dépôt dans un périodique scientifique et demandait la permission dudit périodique), obtenir l'obligation pour les périodiques de mettre en ligne sans embargo les recherches financées par l'argent publique, écourter de plus en plus les délais de latence, imposer le système auteur payeur, faire appliquer les différents programmes intergouvernementaux ( HINARI, OARE , AGORA, etc.... ) qui permettent aux pays en voie de développement d'accéder gratuitement aux bases de données ( ou en payant une somme forfaitaire) selon leur adresse IP, etc....., le monde des éditeurs, voyant des revenus substantiels et assurés lui échapper, a entrepris de réagir aux avancées du libre accès. Si dans certains cas, et répondant à la déclaration de guerre de Poynder, les éditeurs eurent recours à des stratagèmes pas très en symbiose avec le monde feutré de la publication scientifique (En 2007, un certain Eric Dezenhall -surnommé le pit bull des relations publiques- est engagé par de grands groupes éditoriaux afin de discréditer le libre accès. Dans sa stratégie, il leur conseille de se concentrer sur des messages simples tels que " l'accès public [gratuit] équivaut à la censure gouvernementale " et il leur suggéra d'essayer mettre sur le même pied les modèles de publication traditionnels et contrôle par les pairs et " brosser un tableau de ce que serait le monde sans articles contrôlés par les pairs), les éditeurs ont essayé de lâcher du lest afin de ne pas perdre face à la déferlante du libre accès .Ces concessions vont de l'offre d'accès gratuite à certaines bases de données et à la publication en libre accès sans contrepartie financière (à l'instar de SAGE Open) ou permettre le libre accès sous certaines conditions ou après une certaine période, l'allocation de sommes d'argent pour aider les auteurs à publier dans le cadre de l'option auteur - payeur, etc.....) et elles ont pour seul but d'abonder dans le même sens que le libre accès .Il faut dire que les éditeurs ont mauvaise presse de la part des chercheurs qui les accablent (à juste titre en bien des cas) de tous les maux dans leur difficultés à accéder à l'information alors que la santé financière insolente de ces compagnies prête le flanc à ce genre de réactions .Les chercheurs (même dans les pays développés mais surtout ceux dans les pays en voie développement) ne comprennent pas qu'ils n'arrivent pas accéder à l'information, théoriquement si abondante et 
disponible, alors qu'Internet le leur permettrait avec une connexion minimale. D'ailleurs, il faut comprendre que contrairement à l'offre de l'information sur support mobile telle qu'elle était avant l'avènement d'Internet, cette dernière permet une désintermediarisation de la chaîne de la publication scientifique et ceci aussi bien que du point de vue du consommateur ( lecteur ) que celui du producteur ( auteur ) qui, en fait, sont un seul et unique personne, l'auteur étant appelé à consulter le travail des autres alors que le lecteur est lui-même partie de la chaîne de production de l'information. C'est cette fonction de désintermediarisation qui est au centre même du processus du libre accès et de ses défis. Elle permet, en principe, au chercheur de court-circuiter le maillon intermédiaire - l'éditeur - et de passer directement l'information au consommateur. Si cette forme de communication est de prime abord facile et solutionnerait tous les problèmes, elle n'est en fait pas applicable. Le métier d'éditeur qui a été avec la publication scientifique depuis ses débuts sous une forme ou une autre (et qui en fait en est une partie indissociable) a acquis tout au long de ces années un savoir faire, une technicité et une approche professionnelle que le commun des mortels, aussi doué soit il dans la manipulation informatique, ne peut décemment avoir ni même égaler. Contrairement à certaines positions jusqu'au-boutiste et qui voient en la présence des éditeurs un frein à la dissémination de l'information, la plupart des recherches ont plutôt axé leur méthodologie vers une refonte, un changement et une adaptation du rôle de l'éditeur aux nouvelles donnes et aux nouvelles manières de disséminer l'information.

Nous avons présenté le contrôle par les pairs de par ses différentes étapes, approches, problèmes ainsi que l'avènement du libre accès et d'Internet (évoquer le libre accès implique automatiquement Internet) et l'influence que ce dernier a eu sur le contrôle par les pairs et le construction de la Science en général. Nous avons aussi essayé de contribuer à ce sujet de par une approche locale et nationale afin de voir l'état d'avancement de l'Algerie dans le domaine de la publication scientifique, Internet, les programmes en libre accès et les projets en cours. Force est de reconnaitre que la situation n'est guère reluisante malgré les investissements aussi bien matériels qu'humains après plus de cinquante ans d'indépendance. L'université algérienne en plus de la crise endémique, structurelle et systémique dans laquelle elle se débat est, il est malheureux de le dire, parmi les universités les plus mal classées au monde. The Academic Ranking of World Universities (ARWU) plus connu sous le nom de Classement de Shanghai ne liste même pas Algeria dans la liste des pays recensés . Comment ceci n'est il pas possible quand un autre site que nous avons déjà cité (netindex.com) et qui recense les performances des pays en matière de bande passante Internet classe l'Algerie à la $182^{\mathrm{e}}$ place sur 186 pays . Ou quand un medium aussi important qu'AJOL ou le DOAJ ne recensent que respectivement que 2 et 5 titres algériens alors qu'à titre d'exemple un pays comme le Nigeria enregistre respectivement 182 et 39. Enfin, DOAR et ROAR, des entrepôts d'archives ouvertes ne recensent qu'un seul site pour DOAR et aucun pour ROAR. Le seul projet digne de ce nom est Webreview qui est un portail des périodiques scientifiques algériens. Cette initiative du CERIST, dont nous avons signalé l'importance et le dynamisme dans le contexte algérien, n'est pas à notre avis suffisante. Quant au seul dépôt numérique celui de l'Université d'Alger I, nous avons déjà signalé dans l'examen de la situation du libre accès en Algerie son incongruité. En effet, ce dépôt numérique est premièrement signalé sur le site comme " Bibliothèque Virtuelle " sachant la volatilité et la différence de ces concepts. Ce dépôt est en fait un réservoir de documents hétéroclites datant de 1883 à 2007 et qui n'ont de relation que le fait que la bibliothèque virtuelle (ou dépôt c'est selon) possède. Ce projet louable à plus d'un titre aurait gagné à être lancé et supervisé par des connaisseurs du domaine car une bibliothèque virtuelle n'est pas un dépôt de documents n'ayant aucune suite logique à part leur présence à la bibliothèque. 
Le questionnaire distribué quant à lui n'a fait que corroborer la situation du libre accès en Algerie. En effet, les chercheurs du CERIST et du CDTA investigués ne semblent pas être assez au fait de certains concepts. D'un autre coté, cette ignorance est reflétée dans la quasi absence de participation dans les pétitions et différents appels que la communauté des chercheurs a lancé. Il faut savoir que le libre accès se passe sur Internet et la participation même virtuelle du chercheur est primordiale. Le collège invisible qui unissait dans l'univers papier les chercheurs d'une même communauté s'est considérablement élargi et est sur Internet avec tout ce que cela implique comme exposition extrême. Idée que semble peu partager et appréhender notre communauté (du moins celle investiguée). Il semblerait que publier, participer et contribuer à un mouvement universel de propagation de l'information ne soit pas parmi les priorités de nos chercheurs à qui n'incombe pas toute la faute. Si l'Algerie de part ses institutions universitaires, premières bénéficiaires potentiels du libre accès à l'information scientifique technique, sont si en retrait la faute incomberait aux décideurs et planificateur de la politique universitaire algérienne. Avec tous les chiffres précédemment cités (Internet, périodiques scientifiques - papier ou électronique -, archives ouvertes institutionnelles, etc....), il est loisible de comprendre le marasme que vit le libre accès en Algerie.

La solution est une politique tout azimut de promotion d'Internet (amélioration du taux de pénétration, généralisation du Wi Fi sachant surtout la superficie du pays et surtout la topographie de certaines régions, politique de prix à la baisse, ouverture effective du marché au privé, etc....) au niveau national mais surtout universitaire. Ceci passerait par une bande

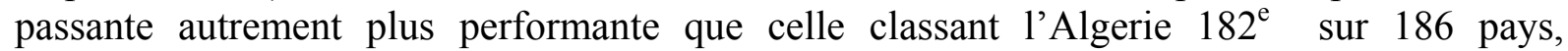
l'institution d'une politique de numérisation et de mise à disposition sur Internet des fonds de périodiques scientifiques, la création de dépôts d'archives institutionnelles au niveau de chaque université et qui seraient le réceptacle de la production scientifique des dites institutions, des campagnes de sensibilisation au libre accès au niveau des universités afin d'en faire profiter et surtout sensibiliser la communauté universitaire, etc.... Tout cet ambitieux programme ne pourrait être entrepris sans un ancrage centralisateur qui servirait de catalyseur à tous ces projets. S'il est de notoriété que les ministères de l'enseignement supérieur et celui des postes et télécommunications sont tout indiqués de par leur domaine de couverture, au niveau institutionnel et universitaire, le CERIST de par sa composante humaine, matérielle et aussi son expérience serait (plus qu'il ne l'est déjà) l'institution qui piloterait cette nouvelle politique qui impulserait les leviers de la recherche scientifique en Algerie. L'Algérie pouvant se targuer actuellement d'une santé financière sans précédant que nous envient nombre de pays, devrait investir cette manne dans la recherche et développement afin de se prévenir d'un quelconque retournement de situation qui mettrait en péril tout effort d'investissement. L'exemple de " petits pays " comme Le Botswana, Le Cap Vert et l'Ile Maurice à qui l'appellation de " Tigres d'Afrique " a été donnée (en une claire allusion aux " Tigres d'Asie " tels que La Corée du Sud et Singapour) devrait être pris en exemple. Ces derniers ont entrepris une ambitieuse politique de développement et de privatisation du secteur des télécommunications Malgré leurs ressources limités (et des fois une situation géographique désavantagée comme l'enclavement du Botswana), ils ont su investir dans le facteur humain au lieu d'adopter une politique dont le souci est la sauvegarde de la paix sociale. De fait, il s'agit de promouvoir la politique du développement humain de concert avec le développement technologique. Il est, peut être, suranné de dire que nous vivons dans un monde où la richesse n'est plus matérielle mais informationnelle et que celui qui la possède est le plus riche mais dans le sujet qui nous concerne cette expression prend tout son poids et c'est à l'aune de sa maitrise des intrants informationnels et non pas aux richesses naturelles épuisables qu'une nation est jugée. 


\section{Bibliographie Générale}


Acosta, [...et al], Evolution of scientific communication, Food Science and Technology International, Vol. 7 n 2, p.183-184, April 2001

Acosta - Cazares, Benjamin, [....et al], Scientific colonialism and safari research, Clinical medecine and health research, 11 January 2000

(Disponible à http://clinmed.netprints.org/cgi/content/full/2000010008v1 )

Albanese,Andrew Richard, Life after the NIH, Library Journal Archive Content April 15, 2005

Disponible à : http://lj.libraryjournal.com/2005/04/academic-libraries/life-after-the-nih/

Alexander, Cynthia J. . Pal, Leslie A., Ed ,Digital democracy : policy and politics in the wired world, Toronto, Oxford University Press,1998, 256 p.

Alexandrov, Georgii A., The Purpose of peer review in the case of an open-access publication, Carbon Balance and Management, Vol.1, $\mathrm{n}^{\circ} 10,2006$

Disponible à : http://www.cbmjournal.com/content/pdf/1750-0680-1-10.pdf

Altbach, Philip G., Teferra, Damtew Ed., Knowledge dissemination in Africa: the role of scholarly journals, Oxford, African Books Collective, 1997, 95 p.

Altman , Patrick, Un Nouveau modèle économique pour l'édition électronique

Disponible à : http://www.bmlisieux.com/colloque/altman.htm

Ambrosi , Alain,[...et al], Enjeux de mots : Regards multiculturels sur les sociétés de l'information, Caen, C\&F Editions 2008, 649 p

American Council of Learned Societies, Our Cultural commonwealth, Report of the American Council of Learned Societies Commission on Cyberinfrastructure for the Humanities and Social Sciences, New York, ACLS, 2006, iii-43p.

Disponible à : http://www.acls.org/cyberinfrastructure/OurCulturalCommonwealth.pdf

Andersen, Deborah Lines, Digital scholarship in the tenure, promotion, and review process, Armonk [NY, USA], M.E. Sharpe, 2004, 264 p.

Anderson, Kent,[....et al],Publishing online-only peer-reviewed biomedical literature: three years of citation, author perception, and usage experience, Journal of electronic publishing, Vol. $6, \mathrm{n}^{\circ}$ 3, March, 2001.

(Disponible à : http://quod.lib.umich.edu/j/jep/3336451.0006.303?rgn=main;view=fulltext )

Andrews, Judith, Law, Derek, Ed. , Digital libraries: policy, planning, and practice, Farnham [Grande Bretagne ], Ashgate Publishing Limited,2004, 180 p.

Antelman, Kristin, Do Open access articles have a greater research impact? College \& Research Libraries, Vol. $65 \mathrm{n}^{\circ}$ 5, p. 372-382, September 2004

(Disponible à http://crl.acrl.org/content/65/5/372.full.pdf) 
Arms, William Y, What are the alternatives to peer review ? quality control in scholarly publishing on the web, Journal of Electronic Publishing, Vol. 8, $\mathrm{n}^{\circ}$ 1, August, 2002

(Disponible à : http://quod.lib.umich.edu/cgi/t/text/text-

idx?c=jep; view=text;rgn=main;idno=3336451.0008.103)

Aronson, Barbara,Improving online access to medical information for low-income countries

New England Journal of Medecine, Vol. 350, n³10, p.966-968, March 4, 2004

(Disponible à : http://www.nejm.org/doi/full/10.1056/NEJMp048009\#t=article )

Arunachalam, Subbiah Information and knowledge in the age of electronic communication: a developing country perspective,Journal of Information Science, Vol. 25, n.6,p.465-476, 1999 (Disponible à: http://jis.sagepub.com/content/25/6/465.abstract)

Association of Research Libraries, Current models of digital scholarly communication: results of an investigation conducted by Ithaka for the Association of Research Libraries, Washington DC, Association of Research Libraries, 2008,49 p.

(Disponible à : http://www.arl.org/storage/documents/publications/digital-sc-models-report2008.pdf)

Atkinson, Malcom, Peer review’ culture, Science and engineering ethics, Vol. 7, $\mathrm{n}^{\circ} 2$, p 193-204, 2001

Bachrach, Steven, [....et al], Who should own scientific papers? $\underline{\text { Science, }}$ Vol. $281 \mathrm{n}^{\circ} .5382$, p. 1459-1460, 4 September 1998

(Disponible à : http://www.sciencemag.org/content/281/5382/1459.full )

Baldwin, Christine, Pullinger, David, What readers value in academic journals, Learned Publishing, Vol. 13, n 4, p. 229-239, 1 October 2000

(Disponible à:

http://docserver.ingentaconnect.com/deliver/connect/alpsp/09531513/v13n4/s5.pdf?expires=1386 $\underline{\text { 834649\&id }=76599461 \& \text { titleid }=885 \& \text { accname }=\text { Guest }+ \text { User\&checksum }=E 3 D 383 \text { D52FF4A6D78 }}$ 95FD0943EA53340)

Bazerman, Charles, Shaping written knowledge: the genre and activity of the experimental article in science, Madison, [Wisconsin, U.S.A.], University of Wisconsin Press, 1988, 400 p.

(Disponible à : http://wac.colostate.edu/books/bazerman_shaping/)

Baum, Rudy M., The open-access myth, Chemical \& Engineering News, Vol. 82, $\mathrm{n}^{\circ}$ 08, p.3, February 23, 2004

(Disponible à : http://pubs.acs.org/email/cen/html/032804112410.html )

Des Bibliothèques aux archives ouvertes, Documentaliste - Sciences de l'information, Vol.43, n०5-6, 87p.

Benkler Jochai, The Wealth of networks: how social production transforms markets and freedom,New Haven [Ct., USA], Yale University Press, 2006, 528 p

(Disponible à http://www.benkler.org/wonchapters.html) 
Berry, R. Stephen, Is Electronic publishing being used in the best interests of science ? the scientist's view, Presented at the Second Conference on Electronic Publishing in Science held in Unesco House,Paris, France 19 - 23 February 2001

Borgman, Christine L., From Gutenberg to the global information infrastructure: access to information in the networked world (digital libraries and electronic publishing), Cambridge [MA, USA], The MIT Press, 2003, 344 p.

Boscq, Hélène, La Communication scientifique revue et corrigée par Internet,Paris, INRIA, 14p. (Disponible à : $\underline{\text { http://open-access.infodocs.eu/OAI_helene_bosc.pdf) }}$

Bot, Marjolein, Burgemeester, Johan, Roes, Hans, The Cost of publishing an electronic journal :a general model and a case study, D-Lib Magazine, November 1998

(Disponible à : http://www.dlib.org/dlib/november98/11 roes.html )

Bourne, P.E., Ten simple rules for getting published, PLoS Computational Biology, Vol. 1, $\mathrm{n}^{\circ}$ 5, 2005

(Disponible à : http://www.ploscompbiol.org/article/info:doi/10.1371/journal.pcbi.0010057)

Bouvier-Ajam, Laurent, Synthèse de l'étude d'évaluation de l'expérimentation de la mise à disposition d'ouvrages sous droits via la bibliothèque numérique de la BnF Gallica 2, Paris, Ourouk ,2009, 11 p.

(Disponible à : http://www.bnf.fr/documents/evaluation_gallica2.pdf)

Briggs, Asa Burke, Peter, Social history of the media: From Gutenberg to the Internet, Cambridge [Grande Bretagne ],Polity Press, 2010, 320 p.

Brody, Tim, Harnad, Stevan, Carr, Leslie, Earlier Web usage statistics as predictors of later citation impact, Journal of the American Society for Information Science and Technology, Vol. 57, $\mathrm{n}^{\circ}$ 8, p. 1060-1072, June 2006

(Disponible à: http://arxiv.org/ftp/cs/papers/0503/0503020.pdf)

Brown, Douglas, Scientific communication and the dematerialization of scholarship, Proquest CSA, Discovery Guides, March 2007

Brown, Tracey, Ed. Peer review and the acceptance of new scientific ideas: discussion paper from a Working party on equipping the public with an understanding of peer review, November 2000-May 2004, London, Sense about Science, XI-51 p.

(Disponible à : http://www.senseaboutscience.org/data/files/resources/17/peerReview.pdf)

Buckland,Michael and Liu, Ziming, History of information science, p.272-295 $\underline{\text { In}}$, Historical studies in information science, Hahn, Trudi Bellardo and Buckland, Michael, Ed., Silver Spring,[Md],Association for Information Science and Technology, 1998, 326 p.

Burke, Peter, A Social history of knowledge : from Gutenberg to Diderot, Cambridge [Grande Bretagne], Polity Press, 2000, VIII-246 p

Butterworth, Ian Ed., The Impact of electronic publishing on the academic community, [workshop organised by] Wenner - Gren Foundation [and] Academia Europaea, Portland, Portland Press, 1998,214 p. 
Butler,Declan The Writing is on the web for science journals in print, Nature, Vol.397, $\mathrm{n}^{\circ} 6716$, p.195-200,21 January 1999

Cain, Joe, Why be my colleague's keeper? Moral justifications for peer review, Science and engineering ethics, Vol. 5, n 4, p 531-540, 1999

Canessa , Enrique, Zennaro, Marco, Ed., Science dissemination using Open Access : a compendium of selected literature on Open Access, Trieste, ICTP - The Abdus Salam International Centre for Theoretical Physics,2008, 207 p.

(Disponible à : , http://sdu.ictp.it/openaccess/SciDissOpenAccess.pdf)

Cetto, Ana Maria, Sharing scientific knowledge through publications : what do developing countries have to offer? World conference on science : Science for the twenty first century : a new commitment, Forum I - Programme thematic meetings-I.6 Sharing scientific knowledge, Organised by UNESCO and International Council on Science,Budapest, June 26-July1st ,1999. (Disponible à : http://www.unesco.org/science/wcs/abstracts/I_6_sharing.htm )

Chartron, Ghislaine, Acteurs du "marché" de l'article scientifique, impacts du numérique, L'Edition électronique : vers de nouveaux modes d'évaluation de la recherche? Séminaire du 21 octobre 2005 - URFIST de Toulouse/SCD de l'université Toulouse 1 Sciences sociales Disponible à : http://archivesic.ccsd.cnrs.fr/docs/00/06/27/10/PDF/sic_00001687.pdf

Chartron, Ghislaine, L'Edition scientifique face à Internet : Bibliothèques numériques, cours INRIA, 9-13 octobre 2000

(Disponible à : http://urfist.enc.sorbonne.fr/anciensite/archives/inria2000/inriasupport.htm

Chartron, Ghislaine, Salaün, Jean-Michel, Expertise de ressources pour l'édition de revues numériques

(Disponible à : http://revues.enssib.fr/pdf/Accueil.pdf)

Chartron, Ghislaine Nouveaux modèles pour la communication scientifique ? Colloque Une nouvelle donne pour les revues scientifiques? 19-20 Novembre 1997, ENSSIB

(Disponible à : http://urfist.enc.sorbonne.fr/anciensite/archives/enssib97.htm)

Chartron, Ghislaine, Salaün, Jean-Michel, La Reconstruction de l'économie politique des publications scientifiques, Bulletin des Bibliothèques de France, $n^{\circ}$ 2, p. 32-42, 2000

(Disponible à : http://bbf.enssib.fr/consulter/bbf-2000-02-0032-003)

Chubin , Daryl E. , Hackett, Edward J., Peerless science: peer review and U. S. science policy Albany [NY, USA], SUNY Press, 1990, 267 p.

Constantine, Norman A., Peer review process, Encyclopedia of Epidemiology, 9 March 2009,p. 795-797

The Coming revolution in scholarly communications\& cyberinfrastructure, CT Watch Quarterly, Vol.3, n³ August 2007, 76 p.

Disponible à : $\underline{\text { http://www.ctwatch.org/quarterly/pdf/ctwatchquarterly-12.pdf }}$ 
Daniel, Hans-Dieter, Guardians of science: fairness and reliability of peer review, Weinheim [Allemagne], Wiley-VCH Verlag, 1993,140p.

De La Vega, Josette F, La Communication scientifique à l'épreuve de l'Internet : l'émergence d'un nouveau modèle, Villeurbanne, presses de l'Enssib, 2000, 253p.

De Vries , Jaap, Peer review: the holy cow of science ,p.231-244, In Fredriksson, E.H. Ed, A Century of science publishing, Amsterdam, IOS Press, 2001, 312p.

Dominy, Margaret, Bradley, Jean-Claude, Bhatt, Jay, Peer Review in the Google Age: Is technology changing the way science is done and evaluated ?, 2006. (Unpublished) [Presentation PPT]

(Disponible à : http://eprints.rclis.org/7411/1/Peer_Review in the Google_Age\%5B1\%5D.ppt)

Doueihi, Milad, La Grande conversion numérique, Ed. du Seuil,2008, 288p.

Dowler, Lawrence, Gateways to knowledge - the role of academic libraries in teaching, learning \& research, Cambridge [MA, USA], MIT Press, 1997, 256 p.

Doyle, Helen, Gass, Andy, Kennison Rebecca, Open Access and scientific societies, PLoS Biology, Vol 2, n 5, p.549-550, May 2004

Duff, Alistair S., Some post-war models of the information chain, Journal of Librarianship and Information Science, Vol. 29 n², p. 179-187, December 1997

Duncan, Lindsay, Scientific publication system in social science: a study of the operation of leading professional journals in psychology, sociology, and social work, San Francisco,JosseyBass, 1979,169 p.

Eason, Ken, Richardson, Sue, Yu, Liangzhi, Patterns of use of electronic journals, Journal of Documentation, Vol. $56 \mathrm{n}^{\circ}$ 5, p.477 - 504, 2000

Eisenstein, Elizabeth L. , The Printing press as an agent of change,Cambridge [Grande Bretagne],Cambridge University Press, 1982,2 vol. 820 p.

Ekman, Richard, Quandt, Richard E. Ed., Technology and scholarly communication, Berkeley,University of California Press, 1999, 431p.

(Disponible à : http://publishing.cdlib.org/ucpressebooks/view?docId=ft5w10074r\&brand=ucpress)

Electronic publishing: new schemes in electronic publishing in physics, $2^{\text {nd }}$ CERN Workshop on Electronic Publishing in Physics, Geneva, March 1st 2000

(Disponible à : http://indico.cern.ch/conferenceDisplay.py?confId=a99231)

E-Publishing in science, $2^{\text {nd }}$ ICSU-UNESCO conference, Paris, 19 to 23 February 1996

Esposito, Joseph J., The Devil you don't know: the unexpected future of Open Access publishing, First Monday, Vol. 9, no 8 - 2 August 2004

(Disponible à : http://firstmonday.org/ojs/index.php/fm/article/view/1163/1083) 
Fanelli, Daniele, Do pressures to publish increase scientists' bias? an empirical support from us states data, PLOS ONE April 21, 2010

Forsyth, Ann, On Writing and tenure, Journal of Planning Education and Research, Vol. 19, n ${ }^{\circ}$, p.98-103, September 1999

Fjällbrant, Nancy, Scholarly communication - historical development and new possibilities, Proceedings of the IATUL Conferences, Paper 5.

(Disponible à : http://docs.lib.purdue.edu/cgi/viewcontent.cgi?article=1389\&context=iatul)

Fletcher, Robert H., Fletcher, Suzanne W., Evidence for the effectiveness of peer review, Science and engineering ethics, Vol. 3, n ${ }^{\circ}$ 1, p. 35-50, 1997

Fredriksson, E.H. Ed, A Century of science publishing, Amsterdam, IOS Press, 2001, 312p.

(Disponible à : http://ebooks.iospress.nl/book/a-century-of-science-publishing)

Gargouri,Yassine....[et al], Self-selected or mandated, open access increases citation impact for higher quality research, PLOS One, October 18, 2010

Garvey, W.D. Communication : the essence of science: facilitating information exchange among librarians, scientists, engineers, and students,Oxford [Grande Bretagne] ,Pergamon Press 1979, $340 \mathrm{p}$.

Gdoura, Wahid, Le Libre accès dans les universités arabes: opinions et pratiques des chercheurs et des éditeurs, World Library and Information Congress, 75th IFLA General Conference and Council, 23-27 August 2009, Milan, Italy

(Disponible à : http://conference.ifla.org/past/2009/142-gdoura-fr.pdf)

Gehringer, Edward F., Strategies and mechanisms for electronic peer review, 30th ASEE/IEEE Frontiers in Education Conference, October 18 - 21, 2000, Kansas City, Session F1B-2

(Disponible à :

$\underline{\text { http://citeseerx.ist.psu.edu/viewdoc/download?doi=10.1.1.18.8807\&rep=rep1\&type=pdf) }}$

Ginsparg, Paul,First steps towards electronic research communication in physics, $\underline{\text { Solaris, }}, \mathrm{n}^{\mathrm{o}} 3$, Juin 1996.

(Disponible à : http://gabriel.gallezot.free.fr/Solaris/d03/3ginspar.html)

Goldman, Alvin, Knowledge in a social world, Oxford, Clarendon Press ,1999, 422 p.

Gomes, Suely, Meadows, Jack, Perceptions of electronic journals in British universities, $\underline{\text { Journal }}$ of Scholarly Publishing, Vol.29, n³, p.174-181.

Gorman, G. E., Scholarly publishing in an electronic era, London, Facet Pub., 2005219 pages

Grosch, E., Reply to "ten simple rules for getting published". PLoS Computational Biology, Vol. 3, n 9,2007

(Disponible à :

http://www.ploscompbiol.org/article/info\%3Adoi\%2F10.1371\%2Fjournal.pcbi.0030190). 
Gross, Alan G., Harmon Joseph E., Reidy, Michael S.,Communicating science: the scientific article from the 17th century to the present, Anderson [SC, USA],Parlor Press, 2009, 280 p.

Guédon, Jean-Claude, Beyond core journals and licenses: the paths to reform scientific publishing, Association of Research Libraries , $\mathrm{n}^{\circ}$. 218, p. 1-8, October 2001 (Disponible :http://www.arl.org/resources/pubs/br/br218/br218guedon.shtml).

Haank, Derek, Is Electronic publishing being used in the best interests of science ? the publisher's view, Presented at the Second Conference on Electronic Publishing in Science held in Unesco House,Paris, France 19 - 23 February 2001.

Hague, Barry N. , Loader, Brian D. , Ed, Digital democracy: discourse and decision making in the information age, London, Routledge, 1999, 296 p.

Hajjem, C. , Harnad, S., Gingras, Y., Ten-year cross-disciplinary comparison of the growth of open access and how it increases research citation impact

(Disponible à : $\underline{\text { http://arxiv.org/abs/cs/0606079) }}$

Harnad, Stevan, ....[et al] ,The Access/impact problem and the green and gold roads to open access, Serials Review, Vol. 30, n 4, p. 310-314,2004

Harnad, Stevan, Advancing science by self-archiving refereed research

(Disponible à : http://eprints.soton.ac.uk/252619/1/science-bloom.pdf)

Harnad,Stevan, E-KNOWLEDGE: freeing the refereed journal corpus online, Computer Law \& Security Report, Vol. 16, n², p. 78-87, 2000

(Disponible à : http://www.cogsci.soton.ac.uk/ harnad/Papers/Harnad/harnad00.scinejm.htm)

Harnad, Stevan, For whom the gate tolls ? how and why to free the refereed research literature online through author/institution self-archiving, now

(Disponible à : http://users.ecs.soton.ac.uk/harnad/Tp/resolution.htm)

Harnad, Stevan, Free at last: the future of peer-reviewed journals, D-Lib Magazine,

Vol. 5, n 12 , December 1999

(Disponible à : http://www.dlib.org/dlib/december99/12harnad.html)

Harnad, Stevan, Open access to peer-reviewed research through author/institution self-archiving: maximizing research impact by maximizing online access

(Disponible à : http://users.ecs.soton.ac.uk/harnad/Temp/digital-libraries.htm)

Harnad, Stevan, Ed., Peer commentary on peer review: a case study in scientific quality control, Cambridge [UK], Cambridge University Press, 1982,71 p.

Harnad, Stevan, The Self-archiving initiative : freeing the refereed research literature online Nature , 410, p.1024 - 1025, (26 April 2001)

(Disponible à : http://eprints.soton.ac.uk/255947/1/nature4.html) 
Harnad, Stevan, Universal FTP archives for esoteric science and scholarship: a subversive

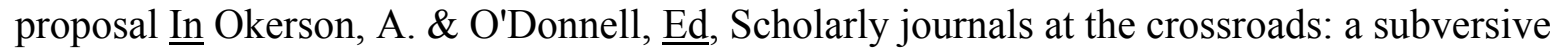
proposal for electronic publishing J., Washington DC,Association of Research Libraries, 1995 (Disponible à : http://www.arl.org/storage/documents/publications/subversive-proposalelectronic-publishing-jun05.pdf)

Harnad, Stevan, Brody, Tim, Comparing the impact of open access (OA) vs. non-OA articles in the same journals, D-Lib Magazine, Vol.10, ${ }^{\circ} 6,2004$

(Disponible à : http://eprints.soton.ac.uk/260207/)

Hatch, Robert A., The Scientific revolution-paradigm lost ? OAH Magazine of History, Vol.4, $\mathrm{n}^{\circ} 2$, p. 34-39,1989

(Disponible à : http://web.clas.ufl.edu/users/ufhatch/pages/03-Sci-Rev/SCI-REV-Home/08sr$\underline{\text { htch.htm) }}$

Heck, Andre, Ed., Electronic publishing for physics and astronomy, Berlin, Springer, $250 \mathrm{p}$. Hoffman, Eric, Guidebook for publishing philosophy, Bowling Green,[OH, USA] Bowling Green State Univ Philosophy, 1997, 256 p.

Horowitz, Irving Louis, Communicating ideas: the crisis of publishing in a post-industrial society, New York, Oxford University Press 1986, 251 p.

House of Commons.Science and Technology Committee (Tenth report of session 2003-04), Scientific publications: free for all ?, London, The Stationery Office, 2004, 188p.

(Disponible à :

http://www.publications.parliament.uk/pa/cm200304/cmselect/cmsctech/399/399.pdf)

Hudson Jones, Anne, McLellan, Faith Ed, Ethical issues in biomedical publication, Baltimore [Md., USA], Johns Hopkins University Press, 2000, 400 p.

Huet, Sylvestre (2013). " Open access : du rêve au cauchemar ", Libération, 4 octobre 2013 (Disponible à : http://sciences.blogs.liberation.fr/home/2013/10/open-access-du-r\%C3\%AAveau-cauchemar-.html)

Hurd, Julie M., The Transformation of scientific communication: a model for 2020, Journal of the American Society for Information Science, Vol.51, n¹4, p.1279-1283, 2000

(Disponible à : http://www.ou.edu/ap/lis5703/sessions/hurd.pdf)

The Ins and outs of the peer-review process,Partnership : The Canadian Journal of Library and Information Practice and Research, Vol. 2, $\mathrm{n}^{\circ} 22007,5 \mathrm{p}$.

(Disponible à : https://journal.lib.uoguelph.ca/index.php/perj/article/view/399/591\#.UqoaJxt3vIV)

International Association of Scientific, Technical \& Medical Publishers, Brussels Declaration on STM publishing, Oxford, International Association of STM Publishers

(Disponible à : http://www.stm-assoc.org/2007_11_01_Brussels_Declaration.pdf)

International Network for the Availability of Scientific Publications, African Journals On Line (AJOL): a second internal evaluation: 2003-2005, Oxford [Grande Bretagne], INASP, 2006,44 p. (Disponible : http://www.inasp.info/uploads/filer_public/2013/04/09/ajol_evaluation_20032005.pdf) 
Jackson, Douglas N., Rushton, J. Philippe, Ed., Scientific excellence: origins and assessment, Newbury Park, [CA, USA], SAGE, 1987, 360 p.

Johns,Adrian, The Nature of the book: print and knowledge in the making,Chicago, University of Chicago Press, 2000, 776 p.

Johnson, Richard K. , A Question of access :SPARC, BioOne, and society-driven electronic publishing, D-Lib Magazine, Vol. 6, $\mathrm{n}^{\circ}$ 5, May 2000

(Disponible à : http://www.dlib.org/dlib/may00/johnson/05johnson.html)

Kahin, Brian , Varian, Hal R._Ed ,Internet publishing and beyond : the economics of digital information and intellectual property, Cambridge [MA, USA] ,The MIT Press, 2000, 236 p.

Kaser,Richard T., If Information wants to be free . . . then who's going to pay for it ? D-Lib Magazine, Vol. 6, $\mathrm{n}^{\circ}$ 5, May 2000

(Disponible à : http://www.dlib.org/dlib/may00/kaser/05kaser.html)

King, Donald W., Tenopir , Carol, Economic cost model of scientific scholarly journal publishing, Proceedings of ICSU Press Workshop on Economics, real costs and benefits of electronic publishing in science- a technical study, Session 2 : Economics factors and costs analyses of online access to primary scientific research and databases, Keble College, University of Oxford ,31 March to 2 April 1998

(Disponible à : http://www.bodley.ox.ac.uk/icsu/)

Kitcher,Philip ,Science, truth and democracy,Oxford, Oxford University Press, 2003, 234 p.

Kling, Rob , Callahan, Ewa, Electronic journals, the internet, and scholarly communication (Disponible à : https://scholarworks.iu.edu/dspace/bitstream/handle/2022/1087/wp01-04B.html)

Kling, Rob, McKim, Geoffrey, Not just a matter of time: field differences and the shaping of electronic media in supporting scientific communication (1999), Journal of the American Society for Information Science. Vol. 51, n¹4, p.1306-1320, 2000

(Disponible à : https://scholarworks.iu.edu/dspace/bitstream/handle/2022/1098/wp99 02B.html)

Kuchma ,Iryna ,Open Access policies in developing and transition countries, Presented at Interactive Facilitation Meeting on Open Access, WSIS 2011, May 19, Geneva

(Disponible à : http://www.ifla.org/files/assets/wsis/forum/2011_iryna kuchma.pdf)

LaFollette, Marcel C.,Stealing into print: fraud, plagiarism, and misconduct in scientific publishing paperback, Berkeley, [CA, USA], University of California Press, 1996, 293 p.

Lawrence, Steven, Online or invisible ? Nature, Vol. 411, n 6837, p. 521, 2001.

Lankhorst, G.J., Franchignoni F., The Impact factor : an explanation and its application to rehabilitation journals, Clinical Rehabilitation, Vol.15, n², p.115-118, April 2001

Le Saux, Annie, La Communication scientifique électronique, Bulletin des bibliothèques de France, 2001, t.46, nº 6, p. 118-119, 2001

(Disponible à : http://bbf.enssib.fr/consulter/bbf-2001-06-0118-007) 
Le Crosnier, Hervé, Bibliothèques numériques In Enjeux de mots : regards multiculturels sur les sociétés de l'information, Caen, C\&F Editions 2008, 649 p.

Le Crosnier, Hervé, Documents numériques et cyberinfrastructure, Introduction à la table ronde Atelier ARTIST sur les pratiques du document numérique scientifique, $10^{\mathrm{e}}$ Colloque International sur le Document Electronique (CIDE), Nancy , 2 - 4 juillet 2007

Le Crosnier, Hervé, Economie de l'immatériel : abondance, exclusion et biens communs, Hermès, La Revue, Vol. 2, n 45, p. 51-59, 2006

(Disponible à : http://www.cairn.info/revue-hermes-la-revue-2006-2-page-51.htm)

Le Crosnier, Hervé, Les Journaux scientifiques électroniques ou la communication de la science à l'heure du réseau mondial, Colloque La communication de l'IST dans l'enseignement supérieur et la recherche : l'effet Renater/Internet, 16, 17 et 18 mars 1995, Bordeaux

(Disponible à : http://gabriel.gallezot.free.fr/Solaris/d03/3lecrosnier.html)

Le Crosnier, Hervé, Moteurs de recherche et bibliothèques numériques, Introduction à une tableronde, Troyes le 3 juin 2005

(Disponible à : http://vecam.org/article461.html)

Le Crosnier, Hervé, Numériser, Colloque Numériser et valoriser en région le patrimoine écrit et Graphique : bibliotheques, musées, archives, Rennes, 22-23 Septembre 2005

Le Crosnier, Hervé, Tentative de definition du vectorialisme, p. 133-152 In : Traitements et pratiques documentaires : vers un changement de paradigme? Actes de la deuxième conférence Document numérique et Société, 2008 Sous la direction d'Evelyne Broudoux et Ghislaine Chartron, Paris, ADBS, 2008, 456 p.

Li Lin, Liu Xiwen, Zhang Xiaolin, Open Access practice in national science library, Chinese Academy of Science,World Library and Information Congress: 75th IFLA General Conference and Council, 23-27 August 2009, Milan, Italy, 5p.

(Disponible à : http://conference.ifla.org/past/2009/142-lin-en.pdf)

Lin,Angela E., Writing for scientific publication: tips for getting started, Clinical Pediatrics, Vol.45, nº 4 , p.295-300, May 2006

Lock, Stephen, A Difficult balance: edotorial peer review in medecine, Londres, Nuffield Provincial Hospitals Trust, 1985,172 p.

Lustig, Harry, Electronic publishing: the role of a large scientific society, Joint ICSU Press/UNESCO Expert Conference on Electronic publishing in science, UNESCO, Paris, 19-23 February 1996

(Disponible à : http://www.library.illinois.edu/icsu/lustig.htm)

Lynch, Clifford A., Institutional repositories essential infrastructure for scholarship in the digital age, Association of Research Libraries Bimonthly Report, $n^{\circ} 226$, p.327-336, February 2003

(Disponible à : http://www.arl.org/storage/documents/publications/arl-br-226.pdf)

MacClure, Charles R. , Hernon, Peter, U.S. scientific and technical information policies (STI) : views and perspectives, Westport, [CT, USA], Praeger 1989, 432 p. 
MacKenzie Owen, John, The New dissemination of knowledge: digital libraries and institutional roles in scholarly publishing, Journal of economic methodology, Vol.9, n³, p. 275-288, 2002

(Disponible à :

http://dspace.library.uu.nl/bitstream/handle/1874/1139/2004-jed9-3.pdf?sequence=2)

MacKnight,Cliff, Electronic journals : what do users think of them ? In Proceedings of the International symposium on research development and preactice in digital libraries, 18-21

November 1997, Tsukuba (Japan)

(Disponible à : http://www.dl.slis.tsukuba.ac.jp/ISDL97/proceedings/mcknight.html)

Mahé, Annaïg, Andrys, Christine, Chartron, Ghislaine, How French researchers are making use of electronic journals : a case study conducted at the Pierre et Marie Curie and Denis Diderot universities, Journal of Information Science, Vol. 26, n 5, p.291-302, 2000

(Disponible à : http://archivesic.ccsd.cnrs.fr/docs/00/06/21/63/PDF/sic 00000399.pdf)

Mahoney, Michael J., Publication prejudices: an experimental study of confirmatory bias in the peer review system, Cognitive therapy and research, Vol. 1, n 2, p 161-175, June 1977

Mainguy, Gaell , Motamedi, Mohammad R., Mietchen ,Daniel , Peer review - the newcomers'perspective, PLos Biology, Vol.3, n9, p.1534-1535, September 2005

(Disponible à

http://www.plosbiology.org/article/info\%3Adoi\%2F10.1371\%2Fjournal.pbio.0030326)

Martin, Brian, Suppression stories, Wollongong, [Australia], Fund for intellectual dissent, 1997, $175 \mathrm{p}$.

(Disponible à http://www.bmartin.cc/dissent/documents/ss/ss.pdf )

Melero, R., López-Santoveña, F., Referees'attitudes toward open peer review and electronic transmission of papers, Food Science and Technology International, Vol. 7, $\mathrm{n}^{\circ}$. 6, p.521-527, December 2001

Merceur, Frederic, Fonctionnements et usages d'une archive institutionnelle, Paris, IFREMER, 2008, 16 p.

(Disponible à : http://archimer.ifremer.fr/doc/2008/rapport-4632.pdf)

Miller, Cass T. , Harris , Julianna C., Scholarly journal publication: conflicting agendas for scholars, publishers, and institutions, Journal of Scholarly Publishing, Vol. 35, $\mathrm{n}^{\circ}$ 2, p. 73-91, January 2004

Molz ,Redmond Kathleen, Dain, Phyllis, Civic space/Cyberspace: The American public library in the information age, Cambridge [MA, USA], The MIT Press, 2001, 275 p.

Moran, Gordon, Silencing scientists and scholars in other fields: power, paradigm controls, peer review, and scholarly communication (contemporary studies in information management, policy, and services), Westport [CT, USA], Greenwood Publishing Group, 1998, 187 p.

Moses, Joel, Dertouzous , Michael , The Computer age: a twenty-year view , Cambridge [MA,USA] The MIT Press, 1980, 510 p. 
Nawrocki, François, Le Protocole OAI et ses usages en bibliothèque, Rapport de recherche, étude, Infotheque francophone, ressources en ligne et actualités scientifiques francophones Disponible à : http://www.infotheque.info/cache/7877/www.culture.gouv.fr/culture/dll/OAIPMH.htm

Nikitenko,Charlotte, Ed., La Publication en ligne, Cahiers du numérique, Vol.1, n ${ }^{\circ}$ 5, 222 p.

Nielsen Norman Group, How users read on the web, October 1, 1997

(Disponible à : http://www.nngroup.com/articles/how-users-read-on-the-web/)

Nielsen Norman Group,PDF: unfit for human consumption, July 14, 2003

(Disponible à : http://www.nngroup.com/articles/pdf-unfit-for-human-consumption/)

Neustadt,Richard M., The Birth of electronic publishing: legal and economic issues in telephone, cable and over-the-air teletext and videotext, Torndike, [ME,USA] G. K. Hall 1982, 160 pages

Odlyzko, Andrew, Competition and cooperation: libraries and publishers in the transition to electronic scholarly journals, Journal of Electronic Publishing, Vol. 4, n 4, May, 1999

(Disponible à : http://quod.lib.umich.edu/j/jep/3336451.0004.411/--reprint-competition-andcooperation-libraries-and-publishers?trgt $=\mathrm{p}$ Evans W;view=fulltext)

Odlyzko, Andrew, The Economics of electronic journals, Journal of Electronic Publishing, Vol 4, $\mathrm{n}^{\circ} 1$, September 1998

(Disponible à : http://www.dtc.umn.edu/ odlyzko/doc/economics.journals.pdf)

Odlyzko , Andrew M., Tragic loss or good riddance ? the impending demise of traditional scholarly journals, International Journal of Human-Computer Studies, Vol., $\mathrm{n}^{\circ} 1$, p. 71-122, January 1995

(Disponible à : http://www.jucs.org/jucs 0 0/tragic loss or good/Odlyzko A.pdf)

Okerson, Ann Ed. Filling the pipeline and paying the piper, Washington, DC., Association of Research Libraries, 1995, 251 p., Proceedings of the 4th Symposium on Scholarly publishing on the electronic networks, Washington, DC, Nov. 5-7 1994.

Okerson, Ann, Six flavors of open access: successes and possibilities for STM journals, Liber Quarterly, Vol 14, n 3/4,p.339-347,2004

(Disponible à : http://liber.library.uu.nl/index.php/lq/article/view/7785)

Parks, Robert P., The Faustian grip of academic publishing, Journal of Economic, Methodology, Vol. 9, $\mathrm{n}^{\circ} 3$, p.317-335, 2002

Parliamentary Office of Science and Technology, Peer review : postnote , London , POST, $\mathrm{n}^{\circ} 182$, September 2002, 4 p.

(Disponible à : http://www.parliament.uk/documents/post/pn182.pdf)

Peters,John, The Hundred years war started today: an exploration of electronic peer review,Journal of Electronic Publising, Volume 1, n 1\&2, May 1996

(Disponible à : $\underline{\text { http://quod.lib.umich.edu/j/jep/3336451.0001.117?rgn=main;view=fulltext) }}$ 
Peugeot, Valérie, Ed, Pouvoir Savoir : santé-agriculture-industrie-culture : le développement face aux biens communs de l'information et de la propriété intellectuelle, Caen, C\&F Editions, 2005,254 p.

Pignard, Nathalie, Les Nouvelles formes de publication scientifique sur Internet : la remise en cause du modèle éditorial traditionnel, [s.1.], [s.n.], 2000, 101 p.

Diplôme d'Etudes Approfondies : Sciences de l'Information et de la Communication : Grenoble $3: 2000$

Piolat, A., Vauclair, J., Le Processus d'expertise éditoriale avant et avec Internet $=$ The Editorial peer revious process before and with Internet, Pratiques psychologiques, Vol. 10, p. 255-272, 2004

(Disponible à : http://sites.univ-

provence.fr/ wpsycle/documentpdf/DocPiolat/Publications/PiolatVauclairPP04.pdf)

Peer review in PLoS medecine (Editorial), PLos Médecine, Vol.4, n¹, p.1-2, 2007

Pöschl, Ulrich, Interactive open access publishing and peer review: the effectiveness and perspectives of transparency and self-regulation in scientific communication and evaluation, Liber Quarterly, Vol.19, n³/4, p.293-314,2010

(Disponible à :

http://liber.library.uu.nl/index.php/lq/article/viewFile/URN\%3ANBN\%3ANL\%3AUI\%3A10-1$\underline{113571 / 8265})$

Pöschl,Ulrich, Open access interactive peer review enhances journal quality

(Disponible à : http://www.researchinformation.info/risepoct04openaccess.html)

Pöschl, Ulrich, Open Access: scientific quality assurance by interactive peer review \& public discussion, Learned Publishing, 2004, 17p.

(Disponible à : http://www.atmospheric-chemistry-and-

physics.net/pr_acp_open_access_scientific quality_assurance.pdf)

Pöschl, Ulrich, Koop, Thomas, Interactive open access publishing and collaborative peer review for improved scientific communication and quality assurance, Information Services \& Use, Vol.28, p.105-107, 2008

(Disponible à : http://www.atmospheric-chemistry-andphysics.net/pr_acp_poeschl_koop_infoservuse_2008_intoapub.pdf)

Polastron ,Lucien X., La Grande numérisation: y a-t-il une pensée après le papier ? , Paris, Denoël, 2006, 208 p.

Poynder, Richard, The Answer to the machine is in the machine, In Caught in a web: intellectual property in cyberspace, London, Derwent Thomson Scientific, 2001, ix, 191 p.

Poynder, Richard,Ed. Caught in a web: intellectual property in cyberspace, London, Derwent Thomson Scientific, 2001, ix, 191 p.

Poynder, Richard, Ten years after, Information Today, Vol. $21 n^{\circ} 9$ - October 2004

(Disponible à : http://www.infotoday.com/it/oct04/poynder.shtml) 
Poynder, Richard, The War in Europe

(Disponible à : http://poynder.blogspot.com/2007/03/open-access-war-in-europe.html)

Prévot, Michel, La Publication scientifique à accès libre: de l'idéal aux modalités concrètes : application aux sciences de la terre, $14 \mathrm{p}$.

(Disponible à : http://archivesic.ccsd.cnrs.fr/docs/00/06/26/32/PDF/sic 00001492.pdf)

PubMed Central : an NIH-operated site for electronic distribution of life sciences research reports

(Disponible à : http://www.nih.gov/about/director/pubmedcentral/pubmedcentral.htm)

Pupique, M., Peer review my foot ! Journal of Biological Rhythms, Vol. 17 , n 2, p.194, April 2002

(Disponible à : http://www.srbr.org/Documents/12 Apr 2002 Peer Review MP.pdf)

Rennie, Drummond Guarding the guardians: research on editorial peer review, Selected proceedings from the First International Congress on Peer Review in Biomedical Publication. May 10-12, 1989, Chicago, Ill., Journal of the American Medical Association, 1990, Vol.263, $\mathrm{n}^{\circ} 10, \mathrm{p} .1317-1441$

Research Information Network, E-journals: their use, value and impact, [a report of] Research Information Network, April 2009, 52 p.

(Disponible à : http://www.rin.ac.uk/our-work/communicating-and-disseminating-research/ejournals-their-use-value-and-impact)

Rice, Michael, Toward enhancing the social benefits of electronic publishing: report of an Aspen Institute planning meeting, Feb. 25-26, 1987, Queenstown, [Md]

Roberts, Peter, Scholarly publishing peer review and the Internet, First Monday, Vol. 4, $\mathrm{n}^{\circ}$ 4, 5 April 1999

(Disponible à : $\underline{\text { http://ojs-prod-lib.cc.uic.edu/ojs/index.php/fm/article/view/661/576) }}$

Robinson ,Lyn , Karamuftuoglu, Murat, The Nature of information science : changing models, Information Research, Vol 15, $\mathrm{n}^{\circ} 4$, December 2010 - Proceedings of the 7th International Conference on Conceptions of Library and Information Science -" Unity in diversity " - Part 2 (Disponible à: http://www.informationr.net/ir/15-4/colis717.html)

Roger T. Pedauque, Le Document à la lumière du numérique :forme, texte, medium comprendre le rôle du document numérique dans l'émergence d'une nouvelle modernité, Caen , C\&F Editions ,2006, 220 p.

Rosenzweig, Roy, The Road to Xanadu: public and private pathways on the history web, Journal of American History, Vol.88, n², p.548-579,2001

(Disponible à :

http://chnm.gmu.edu/digitalhistory/links/cached/introduction/link0.27a.pathwaysonhistweb.html)

Rusch-Feja, Diann, Siebeky,Uta, Evaluation of usage and acceptance of electronic journals : results of an electronic survey of max planck society researchers including usage statistics from Elsevier, Springer and Academic Press, D-Lib Magazine, Vol. 5, n¹0, October 1999

(Disponible à : http://www.dlib.org/dlib/october99/rusch-feja/10rusch-feja-summary.html) 
Salaün, Jean-Michel, Que cache l'augmentation des tarifs des revues scientifiques ? Les transformations de la circulation des articles scientifiques, CERSI/ENSSIB, Document de travail - version du 02-10-97

(Disponible à : http://www.abhatoo.net.ma/maalama-textuelle/sciences-de-1information/industries-de-l-information/edition/que-cache-l-augmentation-des-tarifs-des-revuesscientifiques-les-transformations-de-la-circulation-des-articles-scientifiques)

Sandewall, Erik, Maintaining live discussion in two-stage open peer review, Frontier in Computational Neuroscience, Vol.6, $\mathrm{n}^{\circ} 9,2012$

(Disponible à : http://www.ncbi.nlm.nih.gov/pmc/articles/PMC3282940/)

Sathe,Nila A. , Grady,Jenifer L., Giuse, Nunzia B. , Print versus electronic journals: a preliminary investigation into the effect of journal format on research processes, Journal of the Medical Library Association, Vol. 90, n², p. 235-243, April 2002

(Disponible à : http://www.ncbi.nlm.nih.gov/pmc/articles/PMC100770/)

Scaria, Vinod, Peer review of scholarly communication in health: Perspectives in the Internet age, Online Journal of Health and Allied Sciences, Vol.1, n², 2003

(Disponible à : http://cogprints.org/3228/1/2003-1-2.pdf)

Schaffner, Ann C., The Future of scientific journals: lessons from the past, Information Technology and Libraries, Vol. 13, n 4, December 1994, p.239-247

(Disponible à : http://faculty.washington.edu/jwj/lis520/schaffner.html)

Schauder, Don, Electronic publishing of professional articles: attitudes of academics and implications for the scholarly communication industry, Journal of the American Society for Information Science, Vol. 45, $\mathrm{n}^{\circ}$ 2, p. 73-100, March 1994

(Disponible à : http://docs.lib.purdue.edu/cgi/viewcontent.cgi?article=1002\&context=iatul)

Schmidt, Jessica, Reflexions sur quelques nouveaux modeles de communication scientifique [s.1.],[s.n.], 2003, 133 p.

Diplome d'Etudes specialisées : Sciences et technologies de l'Information : Université Libre de Bruxelles: 2003

Scholarly Publishing \& Academic Resources Coalition, Declaring independance : a guide to creating community controlled journals, Washington, DC, SPARC, 2004, 4p.

(Disponible à :

http://www.sparc.arl.org/sites/default/files/Declaring_Independence_Selection.pdf)

Smith, Richard, Opening up BMJ peer review (Editorial), British Medical Journal, Vol. 318, p.45,1999

Soete, George, Salaba, Athena, Measuring the cost effectiveness of journals : ten years after Barschall

(Disponible à : http:/ www.wisc.edu/projects/glsdo/cost.html)

Solomon, David, Talking past each other: making sense of the debate over electronic publication, First Monday, Vol. 7, $\mathrm{n}^{\circ}$ 8, 5 August 2002

(Disponible à : http://firstmonday.org/ojs/index.php/fm/article/view/978/899\#author) 
Sosteric, Mike, Shi, Yuwei, Wenker, Olivier, Electronic first: the upcoming revolution in the scholarly communication system, Journal of Electronic Publishing,Vol.7, n 2, December 2001 (Disponible à : http://quod.lib.umich.edu/j/jep/3336451.0007.203?rgn=main;view=fulltext)

SQW Ltd., Economic analysis of scientific research publishing (a report commissioned by the Wellcome Trust), Histon [Grande Bretagne], SQW, 2004,41p.

Suber, Peter, Who should control access to research literature ? SPARC Open Access Newsletter, $\mathrm{n}^{\circ} 79,2$ November 2004

(Disponible à :

http://dash.harvard.edu/bitstream/handle/1/4725018/suber_researchaccess.htm?sequence=1)

Suls, Jerry, Martin, René The Air we breathe: a critical look at practices and alternatives in the peer-review process, Perspectives on Psychological Science, January 2009 , Vol. 4 n .1 , p. 4050

(Disponible à: http://mres.gmu.edu/pmwiki/uploads/Main/Suls2009.pdf)

Sumner, Tamara and Buckingham Shum, Simon, Open peer review \& argumentation: loosening the paper chains on journals, Ariadne , $\mathrm{n}^{\circ}$ 5, September 1996

(Disponible à : http://www.ariadne.ac.uk/issue5/jime)

Svenonius,Elaine ,The Intellectual foundation of information organization (digital libraries and electronic publishing), Cambridge [MA, USA], The MIT Press, 2000, 264 p.

Swan, Alma, What authors want': the ALPSP research study on the motivations and concerns of contributors to learned journals, Learned publishing, Vol.12, ${ }^{\circ}{ }^{\circ} 3, \mathrm{p} .170-172$, July 1999

(Disponible à :

http://image.sciencenet.cn/olddata/kexue.com.cn/upload/blog/file/2009/12/200912271418712849 $\underline{4 . p d f})$

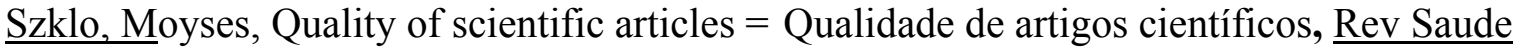
Publica, Vol.40, p.30-35, 2006 ( Numéro Spécial )

(Disponible à : http://www.scielo.br/pdf/rsp/v40nspe/30619.pdf)

Tan-Torres Edejer, Tessa, Disseminating health information in developing countries: the role of the internet, British Medical Journal, Vol.321, n797, 30 September 2000)

Thelwall, Mike, Digital libraries and multi-disciplinary research skills (Disponible à : http://libres.curtin.edu.au/libres14n2/Thelwall final.htm)

Tenopir, Carol, Hitchcock, Brenda, Pillow, Ashley, Use and users of electronic library resources: an overview and analysis of recent research studies, Washington, D.C ,Council on Library and Information Resources, 2003,72 p.

(Disponible à : http://www.clir.org/pubs/reports/pub120/pub120.pdf)

Tenopir, Carol, King, Donald W., Lessons for the future of journals, Nature, Vol. 413, p. 672-674 (Disponible à : http://www.nature.com/nature/debates/e-access/Articles/tenopir.html) 
Thiel, Shayla, The Online newspaper: a postmodern medium, The Journal of electronic publishing, Vol. 4, n 1, September 1998

(Disponible à : http://quod.lib.umich.edu/cgi/t/text/textidx?c=jep; view=text;rgn=main;idno=3336451.0004.110)

Till, J.E., Peer review in a post-eprints world: a proposal, Journal of Medical Internet

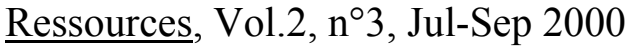

(Disponible à : http://intl-clinmed.netprints.org/cgi/content/full/2000010010v1)

Toffler Alvin, Future shock, New York, Bantam 1984, 576 p.

United Kingdom National Commission for UNESCO, Improving access to scientific information for developing countries: UK learned societies and journal access programmes : report by improving access to scientific information working group (Natural Sciences Committee), London, United Kingdom National Commission for UNESCO secrétariat, 2008, 31 p.

United Kingdom Serial Group, The Future of electronic information intermediaries, Whitney, [England], United Kingdom Serials Group, 1996, 348 p.

Van de Sompel , Herbert ,... [et al ] Rethinking scholarly communication : building the system that scholars deserve, D-LibMagazine, Vol.10, n9 9 , September 2004

(Disponible à : http://webdoc.sub.gwdg.de/edoc/aw/dlib/dlib/september04/vandesompel/09vandesompel.html)

VECAM Ed. Libres savoirs: les biens communs de la connaissance : produire collectivement, partager et diffuser les connaissances au XXIe siecle, Caen , C\&F Editions , 2011,352 p.

Voorbij, Henk J., Searching scientific information on the Internet: a Dutch academic user survey, Journal of the American Society for Information Science, Vol. 50, n 7, p. 598-615, 1999

Wager,Elizabeth, Godlee,Fiona, Jefferson, Tom, How to survive peer review, London, BMJ Books , 2002, [68 ] p.

(Disponible à : http://www.bmj.com/sites/default/files/attachments/resources/2011/07/wager.pdf)

Walker, T.J., Free internet access to traditional journals, American Scientist, Vol 86, n 5, 1998

(Disponible à : http://users.ecs.soton.ac.uk/harnad/amlet.html)

Warren, Kenneth S., Coping with the biomedical literature : a primer for the scientist and clinician, Westport [CT, USA], Greenwood Publishing Group, 1981, 233 p.

Washington DC Principles for Free Access to Science

(Disponible à : www.dcprinciples.org)

Wellerstein, Aurélie,Panorama de l'offre éditoriale en matière de revues électroniques en sciences humaines et sociales disponibles via Internet, Conservatoire National des Arts et Métiers, Institut National des Techniques de la Documentation,103p.

Diplôme Supérieur en Sciences de l'Information et de la Documentation Spécialisées : Science de l'Information et Documentation :CNAM : 2003 
Westfall, Richard S, The Scientific Revolution, History of science society newsletter, Vol. 15, $\mathrm{n}^{\circ}$ 3, July 1986

(Disponible à : http://web.clas.ufl.edu/users/ufhatch/pages/03-Sci-Rev/SCI-REV-Home/05-

RSW-Sci-Rev.htm)

Willinsky, John, The Access principle: the case for open access to research and scholarship, Cambridge [MA, USA], The MIT Press, 2005

(Disponible à

http://kuramoto.files.wordpress.com/2008/09/theaccessprinciple themitpress 0262232421.pdf )

Willinsky, John, Copyright contradictions in scholarly publishing, First Monday, Vol. 7, $\mathrm{n}^{\circ} 11,4$ November 2002

(Disponible à : http://firstmonday.org/ojs/index.php/fm/article/view/1006/927)

Wojciechowska, Anna, Usage des archives ouvertes dans les domaines des mathématiques et de l'informatique, Documentaliste-Sciences de l'Information, Vol. 43, n ${ }^{\circ}$ 5-6 p. 294-302, 2006

(Disponible à : http://www.cairn.info/revue-documentaliste-sciences-de-l-information-2006-5page-294.htm)

Yaşar, Tonta, and Güleda, Düzyol, Mapping the structure and evolution of electronic publishing as a research field using co-citation analysis., 14th International Conference on Electronic Publishing, Helsinki (Finland), 16-18 June 2010

(Disponible à : http://eprints.rclis.org/14695/1/tonta_duzyol_ELPUB2010-helsinki-epublishing.pdf)

Yiotis, Kristin, The Open Access initiative: a new paradigm for scholarly communications, Information Technology \& Libraries, vol. 24, n 4, December 2005, p. 157.

Young, Jeffrey R., High Wire press transforms publication of scientific journals, The Chronicle of higher education,May 161997 


\section{Annexe}

\section{Questionnaire adressé aux chercheurs du CERIST et du CDTA}

\section{Observation}

Le même questionnaire a été adressé aux chercheurs des deux centres. Seule la question 11 au sujet de la connaissance de l'archive de référence de la spécialité (Archive Sic pour le CERIST ou ArXiv pour le CDTA) est différente. 


\section{UNIVERSITE ALGER 2-FACULTE DES SCIENCES HUMAINES ET SOCIALES-DEPARTEMENT DE BIBLIOTHECONOMIE ET DES SCIENCES DOCUMENTAIRES}

\section{QUESTIONNAIRE}

Dans le cadre d'une recherche faisant partie d'un doctorat en bibliothéconomie intitulé "L'avenir de la publication scientifique contrôlée par les pairs face aux défis du libre accès », nous vous soumettons ce questionnaire afin que vous y répondiez. Le but de cette enquête est de savoir quelles sont les attitudes des chercheurs de votre institution face au contrôle par leurs pairs des résultats de leurs travaux de recherche. Il n'échappe à personne que le chercheur et tenu(e)dans toutes les disciplines de publier (d'où la célèbre expression «publias or perish »-publier ou périr) dans des journaux contrôlés par les pairs afin d'accéder à un certain nombre d'avantages découlant de cette activité tels que le prestige et la reconnaissance au sein de la spécialité, l'allocation de fonds de recherche, , un statut meilleur, etc...D'un autre coté, les recherches ayant abordé le sujet du contrôle par les pairs (appelé aussi évaluation, certification, expertise, révision ,ou aussi validation du contenu par un comité d'experts ),étape indispensable de la Science publiée, font ressortir un certain nombre de dysfonctionnements dans son application (idées préconçues, résistance au changements, préjudice personnel ou autre, etc.... ).Avec l'avènement d'Internet, le contrôle par les pairs a radicalement changé, passant d'un travail fermé, en vase clos à un système de certification ouvert où nombre de données sont complètement changés.

Nous voudrions donc dans ce questionnaire savoir quelle a été votre expérience personnelle lors de la publication de vos travaux de recherche et aussi quelle est votre position par rapport à ces nouveaux schémas de certification sachant que de nos jours l'utilisation des réseaux avec leur esprit d'ouverture est une réalité que l'on ne peut occulter. Nous attirons votre attention sur l'importance des résultats de ce questionnaire, non seulement pour la recherche en cours, mais aussi et surtout pour la communauté nationale des chercheurs qui doit s'insérer et être au courant de ces nouveaux schémas de certification. Nous vous demandons, en conséquence, de bien vouloir accorder la plus grande importance aux réponses que vous donnerez car de leur précision dépendra la justesse des résultats de l'enquête.

Enfin, nous tenons à vous remercier de votre collaboration et de votre diligence et nous ne doutons pas de l'application avec laquelle vous répondrez à ce questionnaire qui, nous l'espérons, servira la communauté des chercheurs algériens face à de nouveaux schémas de communication sans cesse en évolution constante.

\section{Samir Hachani}

Doctorant en bibliothéconomie 


\section{I-Renseignements personnels :}

Q.1- Date de naissance : JJ- MM-AAAA

Q.2-Sexe : M

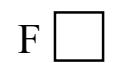

Q.3-Spécialité :

1-Réseaux $\square$

2-Systèmes d'information et systèmes multimédia

3-Recherche et développement en sciences de l'information

4- Théorie et ingénierie des systèmes documentaires

Q.4-Fonction :

Q.5-Diplôme

Licence $\square \quad$ Magister $\square \quad$ Doctorat $\square \quad$ Ingénieur $\square \quad$ Autre :

\section{$\underline{\text { II-Degré de connaissance du libre accès }}$}

Q.6-Etes vous au courant des efforts militant pour l'accès libre et gratuit à l'Information Scientifique et Technique?

Oui $\square \quad$ Non $\square$

Q.7- Connaissez vous 1'O.A.I. (Open Access Initiative)

Oui $\square \quad$ Non $\square$

Q.8- Etes vous au courant de :

L'Appel de Budapest en 2002

Oui

Non $\square$

L'Appel de Berlin en 2003

Oui

Non $\square$

L'Appel de Berlin III en 2005

Oui

Non 
Si vous avez répondu à oui à une de ces questions, prière indiquer le(s)quel(s) en êtes vous signataire?

L'Appel de Budapest en 2002

L'Appel de Berlin en 2003

L'Appel de Berlin III en 2005

Q.9-Savez vous qu'est ce qu'une archive ouverte?

Oui

Non

Q.10-En avez-vous déjà utilisé une en dépôt ou en consultation ?

Oui $\square$

Non $\square$

Si vous avez répondu par oui, pourriez vous indiquer la(es)quelle(s)?

L'avez-vous consulté ou avez-vous fait un dépôt?

Dépôt $\square \quad$ Consultation $\square$

Q.11- Connaissez vous Archive Sic?

Si vous avez répondu par oui, l'avez-vous utilisé ?

Oui

Non $\square$

Q.12-Connaissez vous :

The Registry of Open Access Repositories (ROAR)

Oui

Non

The Directory of Open Access Repositories (DOAR)

Oui

Non

Q.13-Avez vous déjà utilisé des journaux électroniques en libre accès ?

Si vous avez répondu par oui, pourriez vous citer lesquels

Q.14-Connaissez vous :

African Journal On Line (AJOL)

Oui

Non

Directory of Open Access Journals (DOAJ) Oui

Non 


\section{III-Habitudes documentaires et de recherche}

Q.15-Dans vos activités de recherche et de documentation, utilisez vous plus le support :

Papier $\square \quad$ Electronique $\square \quad$ Indifféremment $\square$

Q.16-Quel est le support que vous préférez?

Papier $\square \quad$ Electronique $\square \quad$ Indifféremment $\square$

Q.17-Dans quelle proportion estimez vous votre utilisation des deux supports?

Papier $\% \quad$ Electronique $\%$

Q.18-Quelle (s) langue (s) utilisez-vous lors de vos soumissions ?

Français $\bigotimes \quad$ Arabe $\square \quad$ Anglais $\bigotimes \quad$ Autres $\square$

Si vous avez répondu par autres, pouvez vous préciser?

\section{IV-Production scientifique}

Q.19-Avez-vous déjà publié des articles dans des journaux scientifiques ?

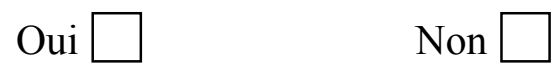

Si vous avez répondu par oui, sont ils

Nationaux

Internationaux

Q.20-Quel est le nombre de ces publications?
$1-3 \square$
$3-5 \square$
$5+\square$

Q.21-Avez vous aussi participé à des conférences dans votre spécialité ?

Si vous avez répondu par oui, sont elles

Nationales

Internationales

Q.22- Quel est le nombre de ces participations ?
$1-3$
$3-5$
$5+\square$ 


\section{$\underline{\text { V-Attitude vis-à-vis du contrôle par les pairs }}$}

Q.23-Au cours de vos soumissions de recherches à des manifestations scientifiques ou journaux, saviez vous ou non est ce que ces soumissions étaient contrôlées par les pairs?

Oui

Non

Q.24-Aviez vous soumis vos recherches en tenant compte de ce paramètre (recherche contrôlée ou non par les pairs)

Oui

Non

Si vous avez répondu par oui, est ce pour :

Facilité de publication

Prestige de publier

Publier pour avancement

Autres

Préciser brièvement

\section{VI-Expérience avec le contrôle par les pairs}

Q.25-Estimez vous que le contrôle par les pairs est indispensable à l'avancement de la Science?

Oui $\square \quad$ Non $\square$

Pourquoi ?

Q.26-Avez-vous eu l'impression, au cours de vos soumissions, que votre travail a été jugé d'une manière objective?

Oui $\square \quad$ Non $\square$

Commentez brièvement

Q.27-Estimez vous normal que dans les formes traditionnelles de certification, le nom de l'expert soit tenu secret?

Oui $\square \quad$ Non $\square$

Q.28-Au cours de vos soumissions, avez-vous pris en ligne de compte les correctifs que l'on vous a demandé d'apporter?

Oui $\square \quad$ Non $\square$ 
Q.29-L'avez-vous fait convaincu des arguments de l'expert ou pour être tout simplement publié ?

Convaincu par les arguments de l'expert

Pour être publié

Q.30- Pourriez vous indiquer le pourcentage de vos soumissions acceptées par rapport à celle soumises?

$\%$

\section{VII-Nouvelles formes de contrôle de l'information}

Q.31-Etes vous au courant des nouvelles formes de contrôle par les pairs des publications scientifiques sur Internet?

Oui

Non $\square$

Q.32-Accepteriez vous, par exemple et dans ce cadre, que votre soumission soit mise sur Internet et jugée, contrôlée par la communauté des chercheurs?

Oui $\square \quad$ Non $\square$

Q.33-Accepteriez vous que ces commentaires soient publiés sur Internet, comme cela se fait dans certaines spécialités?

Oui $\square \quad$ Non $\square$

Q.34-Accepteriez vous, en cas d'acceptation de votre recherche pour publication, que ces commentaires soient publiés sur Internet avec la version finale et définitive ?

Oui $\square \quad$ Non $\square$

\section{VIII-Position des chercheurs vis-à-vis des nouvelles formes de contrôle}

Q.35-Pensez vous que ces nouvelles formes de certification plus ouvertes peuvent contribuer à atténuer les accusations de subjectivité et de préjudice qui, semblent des fois, entacher le processus?

Oui

Non $\square$

Q.36-Pensez vous que cette subjectivité peut être d'ordre :

Sexuelle (homme ou femme) $\square \quad$ Linguistique (selon la langue utilisée)

Religieuse (Précisez) $\square \quad$ Personnelle $\square \quad$ Autres (Précisez brièvement) 
Q.37-Pense vous que cette ouverture peut aider à combattre les formes de plagiat et de fraude tel que le cas du scientifique sud coréen Hwang Woo-Suk en clonage humain et celui de l'Allemand J.Hendrik Shon en physique?

Oui

Non

Q.38-Dans le cadre de votre éventuel travail de certification de recherches, accepteriez vous d'être connu(e) de celui (ou celle) dont vous expertisez le travail ?

Oui Non

Dans le cas où vous auriez répondu par non, pourriez-vous donner vos raisons très brièvement?

Q.39-Pensez vous que les recherches publiées dans les archives ouvertes ou dans les journaux électroniques en libre accès sur Internet peuvent être pris en ligne de compte dans les promotions au même titre que les publications traditionnelles sur papier?

Oui

Non

Donnez brièvement votre avis 